\title{
Abstracts
}

$19^{\text {th }}$ European Congress of Trauma and Emergency Surgery

May, 6-8, 2018

Valencia, Spain

\section{Congress President}

Isidro Martínez Casas

Jaen, Spain 
European Journal of Trauma and Emergency Surgery

Official Publication of the European Society for Trauma and Emergency Surgery

Abstracts for the $19^{\text {th }}$ European Congress of Trauma and Emergency Surgery

May, 6-8, 2018

Valencia, Spain

Organized by

European Society for Trauma \& Emergency Surgery

Spanish Surgeons Association

Socieded Española de Medicina de Urgencias y Emergencias

Sociedad Española de Cirurgía Ortopédica y Traumatología

Semicyuc - Los Profesionales Del Enfermo Crítico

Sociedad Española de Anestesiología, Reanimación y Terapéutica del Dolor

\section{Contents}

S276 Clinical Research | Oral Presentation

S321 Clinical Research | Poster

S512 Case Study | Poster

S599 Author Index 


\section{Welcome Message from ECTES President}

Dear colleagues and friends,

It is my honor and a privilege to welcome you to the 19th European Congress of Trauma and Emergency Surgery from May $6-8,2018$ in Valencia, Spain.

The Congress is organized by the European Society for Trauma and Emergency Surgery, in close cooperation with the Spanish Surgeons Association (AEC) and other Spanish Societies like the Spanish Society of Emergency Physicians (SEMES), Spanish Society for Anesthesia and Reanimation (SEDAR), Spanish Society for Intensive Care Medicine (SEMICYUC) and the Spanish Society for Traumatology and Orthopedic Surgery (SECOT) all linked by their common interest in the polytraumatized and emergency surgical patient.

The leading theme of the congress is

\section{"Bringing the light"}

as the aim of the meeting is to illuminate and focus on the multidisciplinary approach to our patients. Colleagues of different European and non-European countries and representatives of many scientific societies like IATSIC, ESS, WAIOT, DGU, ATLS, ATCN, Küntscher Society, AOTrauma and this year guest society: the Indian Trauma Society, will share their experience to light the broad field of Trauma and Emergency Surgery.

During this 3 days, there will be discussion on a wide variety of topics, from high energy trauma management to intraabdominal infections, trauma registries, complex hip and pelvic fractures, damage control radiology, point of care ultrasound, the politraumatized child, minimally invasive techniques in trauma and emergency surgery, new strategies in wound care and acute elderly patients. The sessions, interactive case presentations free paper presentations, poster walks and guest symposia have been structured to confront different points of view and assure the audience participation.

Several pre-congress courses are organised: ATLS, DSTC, ITLS, MUSEC, ATCN, EASC, MRMI, ETC, Medical Writing course and Polytrauma course, all them to introduce young specialists in the philosophy of our field of interest. Furthermore, the European Board of Surgery Qualification Exams in Emergency Surgery will be held in Valencia on may the 9th.

Valencia, a city of arts and science, its a place where traditional and modern architecture, hospitality, gastronomy and climate will offer also networking and leisure moments in the Mediterranean.

Proud to welcome you,

Isidro Martínez Casas 


\section{Clinical Research | Oral Presentation}

\section{COMPLEX HIP AND PELVIC FRACTURES}

\section{$\mathbf{O 0 0 1}$ \\ QUALITY OF LIFE IN MULTIPLY INJURED PATIENTS WITH LOWER EXTREMITY TRAUMA 20 AND 30 YEARS AFTER TRAUMA: A COMPARISON STUDY}

\author{
R. Pfeifer, G. Osterhoff, K. Sprengel, E. Zilkens, H. Pape
}

Trauma Surgery, University Hospital Zürich, Zürich/ SWITZERLAND

Introduction: Injuries of the lower extremities are known to be associated with negative long term outcome. In this analysis, we aimed to analyze the quality of life and the dynamics in multiply injures patients sustained injuries of lower extremities.

Material and methods: A standardized SF (Short Form) 12 questionnaire was administered to multiply injured patients at least 10 years (time point 1) and 20 years (time point 2) after trauma. Using this score, we could calculate the "Physical Health Score" (PHS) and "Mental Health Score" (MHS). Following anatomical regions were distinguished: Pelvis (Acetabulum + proximal femur), femoral shaft, knee joint (distal femur + proximal tibia), tibia shaft and foot injuries. Results: In total 299 patients completed the SF 12 questionnaire at both time points. Demographic parameters of patients with lower extremity trauma $(n=87)$ were as follows: male $(n=70) 80 \%$, ISS mean $21 \pm 9.6$ points; age $26 \pm 12.1$ years at time of injury, mean follow up (time point I) 18 years and (time point II) 28 years. Only patiens with pelvic injuries have shown a significant decrease of the PHS over the observation period ( 20 years: 44,6 points) and ( 30 years $37,7$ points $)(p=0.025)$. There were no changes in quality of life in patients with femoral shaft, knee joint, tibia shaft and foot injuries during the observation period.

Conclusion: We did not find any worsening of quality of life in patient with lower extremity trauma in our cohorts. Only patients sustained pelvic injuries were associated with significant reduction of the PHS at long term follow up.

References:

Disclosure: No significant relationships.

\section{2}

\section{FRACTURE PROGRESSION MAY CAUSE PROLONGED PAIN IN PATIENTS WITH FRAGILITY FRACTURES OF THE PELVIS}

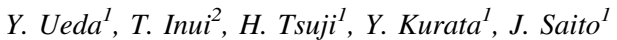

${ }^{1}$ Division Of Orthopedic Trauma, Sapporo Tokushukai Hospital, Sapporo/JAPAN, ${ }^{2}$ Department Of Orthopaedic Surgery, Teikyo University Postgraduate School of Medicine, Tokyo/JAPAN

Introduction: Most fragility fractures of the pelvis (FFPs) are conservatively treated in the early phase. However, the criterion for surgical treatment after the failure of conservative treatment is controversial. At our institution, we provide surgical treatment if prolonged pain exists in FFPs conservatively treated in the early phase. On the other hand, fracture progression (FP) occurs during treatment course in some FFPs. FP usually means increasing instability that leads to prolonged pain. There has been no report on the relationship between FP and prolonged pain. The purpose of this study was to evaluate the relationship between FP and prolonged pain observed in FFPs conservatively treated in the early phase.

Material and methods: From August 2013 to July 2017, 192 consecutive FFP patients were enrolled in this study. In these patients, 79 cases that were diagnosed with CT and MRI were finally included. FP was diagnosed with $\mathrm{CT}$, which confirmed bony union in the follow-up period. The relationship between FP and prolonged pain was analyzed using Fisher's exact test.

Results: Of 79 patients, 18 showed FP. Pain was prolonged in 4 of the 18 patients with FP. Two of 61 patients without FP developed prolonged pain $(\mathrm{p}<0.05$; odds ratio 9.23).

Conclusion: FP in FFPs was considered a risk factor for prolonged pain. If FP is observed, surgical treatment may be required with careful follow-up observation.

References: 1) Rommens, P. M. \& Hofmann, A. Comprehensive classification of fragility fractures of the pelvic ring: Recommendations for surgical treatment. Injury 44, 1-12 (2013). 2) Rommens, P., Ossendorf, C. \& Pairon, P. Clinical pathways for fragility fractures of the pelvic ring: personal experience and review of the literature. $J$. Orthop. Sci. Jan, 1-11 (2015). 3) Wagner, D., Ossendorf, C., Gruszka, D., Hofmann, A. \& Rommens, P. M. Fragility fractures of the sacrum: how to identify and when to treat surgically? Eur. J. Trauma Emerg. Surg. 41, 349-362 (2015). 4) Hopf, J. C., Krieglstein, C. F., Müller, L. P. \& Koslowsky, T. C. Percutaneous iliosacral screw fixation after osteoporotic posterior ring fractures of the pelvis reduces pain significantly in elderly patients. Injury 46, 1631-1636 (2015). 5) Arduini, M., Saturnino, L., Piperno, A., Iundusi, R. \& Tarantino, U. Fragility fractures of the pelvis: treatment and preliminary results. Aging Clin Exp Res27 (Supl 1), S61-S67 (2015). 6) Maier, G. S. et al. Risk factors for pelvic insufficiency fractures and outcome after conservative therapy. Arch. Gerontol. Geriatr. 67, 80-85 (2016).

Disclosure: No significant relationships.

\section{3}

\section{PREPERITONEAL PELVIC PACKING FOR INITIAL HEMORRHAGIC CONTROL IN HEMODYNAMIC UNSTABLE PATIENTS DUE TO COMPLEX PELVIC FRACTURES IN AN IRANIAN BUSY TRAUMA CENTER}

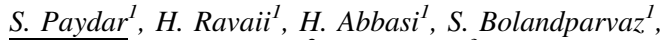 \\ R. Askarynezhadbehzady ${ }^{2}$, S. Mohseni ${ }^{3}$
}

${ }^{1}$ Trauma Research Center, Shiraz University of Medical Sciences, Shiraz/IRAN, ${ }^{2}$ Student Research Center, Shiraz University of Medical Sciences, Shiraz/IRAN, ${ }^{3}$ Division Of Trauma And Emergency Surgery, Department Of Surgery, Örebro University Hospital, School of Medical Sciences, Örebro University, Örebro, Sweden, Orebro/SWEDEN

Introduction: Pelvic fractures are still a challenge for trauma surgeons with a mortality rate up to $32 \%$. Preperitoneal pelvic packing (PPP) has been adopted widely in Europe and at some centers in North America for initial hemorrhage control. We have adopted this approach at our center in recent years. We hypothesized that this approach would decrease blood transfusion and mortality in centers without angioembolization facilities. 
Material and methods: A retrospective review hemodynamic instable patients due to pelvic fracture between 2011-2016 was conducted in a hospital without angioembolization facilities. Hemodynamic instability was defined as a blood pressure less than 90 $\mathrm{mmHg}$ or $\mathrm{BE}<-8$.

Patient demographics, clinical characteristics at arrival, type and severity of the pelvic fracture Tile Classification, blood transfusions (RBS units), other injuries, all surgical managements, ICU and hospital length of stay, and overall outcome were collected for analysis. Results: A total of 86 hemodynamic unstable patients due to complex pelvic fractures were admitted during the study period. Of these, 41 (48\%) patients were subjected to PPP.

There was no difference in the other surgical interventions and severity of the pelvic fractures and also arrival GCS between groups . Comparing PPP (+) group with the PPP (-) there was a significant difference in blood transfusion requirements (RBC: 7.4 Units vs. 10.2 Units, $\mathrm{p}<0.001)$, renal failure $(2.4 \%$ vs. $11.1 \%, \mathrm{p}=0.035)$, and mortality $(39 \%$ vs. $64 \%, \mathrm{p}=0.019)$.

Conclusion: Preperitoneal packing for initial control of hemorrhagic shock was associated with significant decrease in blood transfusion and reduced mortality in our center without angioembolization facilities.

References: 1. Biffl WL, Smith WR, Moore EE, Gonzalez RJ, Morgan SJ, Hennessey T, et al. Evolution of a multidisciplinary clinical pathway for the management of unstable patients with pelvic fractures. Annals of surgery. 2001;233 (6):843-50. 2. Scaglione M, Parchi P, Digrandi G, Latessa M, Guido G. External fixation in pelvic fractures. Musculoskeletal surgery. 2010;94 (2):63-70. 3. Katsoulis E, Giannoudis PV. Impact of timing of pelvic fixation on functional outcome. Injury. 2006;37 (12):1133-42. 4. Demetriades D, Karaiskakis M, Toutouzas K, Alo K, Velmahos G, Chan L. Pelvic fractures: epidemiology and predictors of associated abdominal injuries and outcomes. Journal of the American College of Surgeons. 2002;195 (1):1-10. 5. Sathy AK, Starr AJ, Smith WR, Elliott A, Agudelo J, Reinert CM, et al. The effect of pelvic fracture on mortality after trauma: an analysis of 63,000 trauma patients. J Bone Joint Surg Am. 2009;91 (12):2803-10. 6. Cullinane DC, Schiller HJ, Zielinski MD, Bilaniuk JW, Collier BR, Como J, et al. Eastern Association for the Surgery of Trauma practice management guidelines for hemorrhage in pelvic fracture-update and systematic review. Journal of Trauma and Acute Care Surgery. 2011;71 (6):1850-68. 7. Jang JY, Shim H, Jung PY, Kim S, Bae KS. Preperitoneal pelvic packing in patients with hemodynamic instability due to severe pelvic fracture: early experience in a Korean trauma center. Scandinavian journal of trauma, resuscitation and emergency medicine. 2016;24 (1):3. 8. Huittinen V-M, Slätis P. Postmortem angiography and dissection of the hypogastric artery in pelvic fractures. Surgery. 1973;73 (3):454-62. 9. Tötterman A, Madsen JE, Skaga NO, Røise O. Extraperitoneal pelvic packing: a salvage procedure to control massive traumatic pelvic hemorrhage. Journal of Trauma and Acute Care Surgery. 2007;62 (4):843-52. 10. Burlew CC, Moore EE, Smith WR, Johnson JL, Biffl WL, Barnett CC, et al. Preperitoneal pelvic packing/external fixation with secondary angioembolization: optimal care for life-threatening hemorrhage from unstable pelvic fractures. Journal of the American College of Surgeons. 2011;212 (4):628-35. 11. Cothren CC, Osborn PM, Moore EE, Morgan SJ, Johnson JL, Smith WR. Preperitonal pelvic packing for hemodynamically unstable pelvic fractures: a paradigm shift. Journal of Trauma and Acute Care Surgery. 2007;62 (4):834-42. 12. Osborn PM, Smith WR, Moore EE, Cothren CC, Morgan SJ, Williams AE, et al. Direct retroperitoneal pelvic packing versus pelvic angiography: a comparison of two management protocols for haemodynamically unstable pelvic fractures. Injury. 2009;40 (1):54-60. 13. Surgeons A. ATLS, Advanced Trauma Life Support for Doctors. Chicago, Illinois: Amer College of Surgeons. 2008. 14. Chiara O, di Fratta E, Mariani A, Michaela B, Prestini L, Sammartano F, et al. Efficacy of extra- peritoneal pelvic packing in hemodynamically unstable pelvic fractures, a Propensity Score Analysis. World Journal of Emergency Surgery. 2016;11 (1):22. 15. Burlew CC, Moore EE, Stahel PF, Geddes AE, Wagenaar AE, Pieracci FM, et al. Preperitoneal pelvic packing reduces mortality in patients with life-threatening hemorrhage due to unstable pelvic fractures. Journal of Trauma and Acute Care Surgery. 2017;82 (2):233-42. 16. Heetveld MJ, Harris I, Schlaphoff G, Balogh Z, D'Amours SK, Sugrue M. Hemodynamically unstable pelvic fractures: recent care and new guidelines. World journal of surgery. 2004;28 (9):904-9. 17. Glass NE, Burlew CC. Preperitoneal Pelvic Packing: How and When. Current Trauma Reports. 2015;1 (1):1-7. 18. Mohan P, Sandhu J, Mehta A, Cohen D, Abrahams R, Narayanan G. Factors predicting positive angiogram and the role of pre-peritoneal packing in pelvic trauma. Journal of Vascular and Interventional Radiology. 2017;2 (28):S150. 19. Li Q, Dong J, Yang Y, Wang G, Wang Y, Liu P, et al. Retroperitoneal packing or angioembolization for haemorrhage control of pelvic fractures-Quasi-randomized clinical trial of 56 haemodynamically unstable patients with Injury Severity Score $\geq 33$. Injury. 2016;47 (2):395-401. 20. Salerno F, Gerbes A, Ginès P, Wong F, Arroyo V. Diagnosis, prevention and treatment of hepatorenal syndrome in cirrhosis. Gut. 2007;56 (9):1310-8. 21. Wong F, O’Leary JG, Reddy KR, Kamath PS, Garcia-Tsao G, Maliakkal B, et al. A cut-off serum creatinine value of $1.5 \mathrm{mg} / \mathrm{dl}$ for AKI-To be or not to be. Journal of hepatology. 2015;62 (3):741-3. 22. Piano S, Rosi S, Maresio G, Fasolato S, Cavallin M, Romano A, et al. Evaluation of the Acute Kidney Injury Network criteria in hospitalized patients with cirrhosis and ascites. Journal of hepatology. 2013;59 (3):482-9. 23. Zuckerman GR, Cornette GL, Clouse RE, Harter HR. Upper gastrointestinal bleeding in patients with chronic renal failure. Annals of internal medicine. 1985;102 (5):588-92. 24. Chertow GM, Levy EM, Hammermeister KE, Grover F, Daley J. Independent association between acute renal failure and mortality following cardiac surgery. The American journal of medicine. 1998;104 (4):343-8.

Disclosure: No significant relationships.

\section{4}

\section{COMPARATIVE BIOMECHANICAL STUDY OF PRECONTOURED ANGULAR PLATE AND RECONSTRUCTION PLATE IN TREATING POSTERIOR WALL FRACTURES OF THE ACETABULUM}

\author{
G. Saka ${ }^{l}$, G. Altun ${ }^{2}$
}

${ }^{1}$ Orthopedics And Traumatology, Fulya Orthopedics and Spine Center, Istanbul/TURKEY, ${ }^{2}$ Orthopedics And Traumatology, Umraniye Research and Education Hospital, Istanbul/TURKEY

Introduction: Most posterior wall fractures are comminuted and associated with an impaction injury of the articular surface. Few surgical approaches and techniques to repair of these fractures have advanced and most patients had good outcomes after anatomical reduction and rigid internal fixation ${ }^{1}$.Little information is available about mechanical properties of fixation materials used for surgical treatment of fractures of this region ${ }^{2}$.Conventional Curved Reconstruction Plates (CCRP) cause prolongation in duration of surgery and ideal anatomical adaptation can not be provided. This condition makes precontoured anatomic buttress plates (PABP) attractive for surgeons. The purpose of this study was to evaluate the biomechanical properties of two fixation systems.

Material and methods: Twelve pelvis models in which fracture was created in same type and dimension in posterior wall of the 
acetabulum as biomechanical test material were used. Two different types of fixation materials were used for fixation: 5-hole reconstruction plate and PABP. Fixated pelvis models were embedded in a polyurethane block.Rigidity was calculated for two different plates benefiting from load-displacement distribution graph obtained in consequence of tests.

Results: Rigidity of PABP was found to be statistically significantly higher than CCRP $(p=0.022)$. Static tests performed, the amount of total displacement was found to be significantly less in PABP than conventional curved reconstruction plate.

Conclusion: Application of PABP in posterior wall fractures of the acetabulum provides a more stable, effective and safe fixation. Anatomic contour of the plate, low profile feature, not allowing joint penetration of locking screws due to their design, to allow placement of much more screws in proximal and distal part of fracture fragment upon request are among important advantages.

References: 1. Matta JM. Fractures of the acetabulum: accuracy of reduction and clinical results in patients managed operatively within three weeks after the injury. J Bone Joint Surg Am 1996;78: 1632-1645. 2. Zha G, Sun J, Chen L, et al. Late reconstruction of posterior acetabular wall fractures using iliac crest. J Trauma Acute Care Surg. 2012;72:1386-1392.

Disclosure: No significant relationships.

\section{5}

\section{COMPARISON OF OPERATION TIME AND FLUOROSCOPY TIME IN SUBTROCHANTERIC FRACTURES TREATMENT BETWEEN SIF (SELFDYNAMISABLE INTERNAL FIXATOR) GROUP AND LITERATURE DATAS FOR OTHER INTERNAL FIXATION TYPES}

\author{
M.M.Mitkovic $^{1}$, S. Milenkovic ${ }^{2}$, I. Micic ${ }^{2}$, I. Kostic ${ }^{2}$, P. Stojiljkovic ${ }^{2}$, \\ M.B. Mitkovic
}

${ }^{1}$ Clinic For Orthopaedic And Traumatology, Clinical Centre Nis, Nis/ SERBIA, ${ }^{2}$ Clinic For Orthopaedics And Traumatology, Clinical Center Nis, Nis/SERBIA, ${ }^{3}$ Medical Faculty, University of Nis, Nis/ SERBIA

Introduction: Subtrochanteric fractures are unstable fractures of proximal femur, tending to varus and antecurvatum displacement with overlapping of the fracture fragments. The purpose of this study was to compare operation time and fluoroscopy time between the use of different internal fixation implants in the treatment of subtrochanteric fractures.

Material and methods: There was a group of 27 consecutive patients with subtrochanteric fracture treated by the internal fixation using SIF (Self-dynamisable Internal Fixator) implant. Followed parameters from this group were compared to same parameters data from the literature for Gamma Nail (GN), PF-LCP, DCS and Blade Plate (BP). Results: Operation time in SIF group was 60 (35-140) min, and fluoroscopy time was 43 (20-95) s. Average operation time from the literature for other implants: 104 (43-166) $\mathrm{min}$ for GN, $78 \mathrm{~min}$ for PFLCP, $105 \mathrm{~min}$ for DCS and $221 \mathrm{~min}$ for BP. Average fluoroscopy time: $91 \mathrm{~s}$ for GN, $180 \mathrm{~s}$ for PF-LCP, $238 \mathrm{~s}$ for DCS.

Conclusion: Average values of followed parameters were higher in GN, PF-LCP, DCS and BP comparing to SIF method. This difference could be explained by the influence of required precision degree in the first operative technique step, using screws number and required type of the fracture reduction manevar in the operative time and fluoroscopy time duration. Operation time for GN can be shorter than average operation time for SIF method and it could be explained by the need for good closed reduction skills of the surgeon in an intramedullary nailing of subtrochanteric fractures.

References: 1. Halwai MA, Dhar SA, Wani MI, Butt MF, Mir BA, Ali MF, Dar IH. The dynamic condylar screw in the management of subtrochanteric fractures: does judicious use of biological fixation enhance overall results?. Strategies Trauma Limb Reconstr 2007; 2 (2):77-81. 2. Rahme DM, IA Harris. Intramedullary nailing versus fixed angle blade plating for subtrochanteric femoral fractures: a prospective randomised controlled trial. J Orthop Surg (Hong Kong) 2007; 15 (3):278-81. 3. Brein WW, Wiss DA, Becker V Jr, Lehman T. Subtrochanteric femur fractures: a comparison of the Zickel nail, 95 degrees blade plate, and interlocking nail. J Orthop Trauma 1991; 5 (4):458-64. 4. El-Desouky II, Mohamed MM, Kandil AE. Clinical outcome of conventional versus biological fixation of subtrochanteric fractures by proximal femoral locked plate. Injury 2016; 47 (6):130917. 5. Rahme DM, IA Harris. Intramedullary nailing versus fixed angle blade plating for subtrochanteric femoral fractures: a prospective randomized controlled trial. J Orthop Surg (Hong Kong) 2007; 15 (3):278-81. 6. Brein WW, Wiss DA, Becker V Jr, Lehman T. Subtrochanteric femur fractures: a comparison of the Zickel nail, 95 degrees blade plate, and interlocking nail. J Orthop Trauma 1991; 5 (4):458-64.

Disclosure: No significant relationships.

\section{6}

\section{LONG-TERM QUALITY OF LIFE IN PATIENTS WITH PELVIC FRACTURES; A MULTICENTER STUDY}

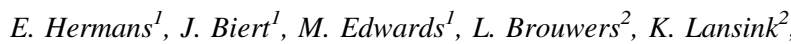 \\ T. Van Gent ${ }^{1}$
}

${ }^{1}$ Surgery, Radboudumc, Nijmegen/NETHERLANDS, ${ }^{2}$ Surgery, Elisabeth Tweesteden Ziekenhuis, Tilburg/NETHERLANDS

Introduction: Pelvic fractures are often caused by high energy trauma (HET) and are therefore associated with a lot of concomitant injuries which can have an effect on patient outcomes. Many studies focus on radiological or functional outcome. Little is known however on long term Health-related quality of life (HRQoL). The purpose of this study was to conduct a cross-sectional analysis of patients using HrQoL-instruments and to identify factors that are predictive for worse outcome

Material and methods: We identified in our hospital databases all patients who were involved in a HET with a pelvic fracture and were admitted to 2 level 1 trauma centers in The Netherlands in the period 2007-2012. Data concerning patient characteristics, fracture type according to Tile, concomitant injuries, treatment and complications were analyzed. All patients were asked to complete the Majeed Pelvic Score (MPS) questionnaire, the EuroQol 5D (EQ5D) and the Short Musculoskeletal Function Assessments (SMFA). A multivariate analysis was performed to determine predictors for worse outcome. Results: In total, 108 completed the questionnaires. 19 patients had a Tile A fracture, 49 a type B fracture and 40 a type C fracture. Mean age was 40 years and mean ISS was 26 . Concomitant injuries were seen in $87 \%$ of the patients. The EQ5D index score and SMFA score were not lower compared to the general Dutch population. On all items of the MPS, no significant differences were seen between the different fracture types. However, patients with a Tile $\mathrm{C}$ fracture had a significant lower mean total MPS than patients with a type A or B fracture $(P=0.04)$. Using multivariate analysis, factors that contributed to a significant worse outcome were neurological injuries and injuries to the lower extremity $(\mathrm{P}<0.03)$. 
Conclusion: Health-related Quality of Life in patients after a pelvic fracture is good and comparable to the general population. However, concomitant injuries to the lower extremity or neurologic injuries are predictors for worse outcome.

References:

Disclosure: No significant relationships.

\section{7}

\section{THE BONE QUALITY IN PATIENTS WITH SACRUM FRACTURES}

\section{J. Berger-Groch, D.M. Thiesen, M.J. Hartel}

Department Of Trauma-, Hand- And Reconstructive Surgery, University Hospital Hamburg Eppendorf, Hamburg/GERMANY

Introduction: Aim of the study was to evaluate the prevalence and extent of osteoporosis in patients admitted with pelvic ring fractures to the investigating institution. $\mathrm{CT}$ is a standard diagnostic tool to detect injuries to the pelvic ring. Pickhardt et al. described a CT-scan based method to analyze bone quality. In this study this methodology is used on a large cohort of patients to evaluate the prevalence of osteopenia and osteoporosis.

Material and methods: Retrospective analysis of all patients treated from 2004-2014 with sacral fractures. A total of 531 patients (398 women, 133 men) were included. To evaluate the bone density the established methods published by Pickhardt et al. was used. We measured the Hounsfield Units (HU) in the vertebral body L5. The assessment was done by placing a single oval over the trabecular bone in the axial view. In a sagittal plane the correct position in the middle of the vertebral body was double-checked.

Results: We defined: HU < 99 osteoporosis, osteopenic bone 100-150 HU, normal bone $150>$ HU. In $71.6 \%$ of the cases reduced bone density was encountered. $75 \%$ of the cases age 65 and above showed a manifest osteoporosis. With each additional year of age bone density is decreasing by 2.7 Hounsfiled units $(p<0.001)$. Female gender was significant predominant in osteoporotic bone $(\mathrm{p}<0.001)$.

Conclusion: There is a significant prevalence of osteoporosis in the patient group investigated. The methodology used in this study is an uncomplicated way to further assess the bone quality in patients with fractures of the pelvic ring.

References: 1. Gauthier A, Kanis JA, Jiang Y, et al. Epidemiological burden of postmenopausal osteoporosis in the UK from 2010 to 2021: estimations from a disease model. Arch. Osteoporos. 2011;6:179-188. 2. Kannus P, Parkkari J, Niemi S, et al. Low-Trauma Pelvic Fractures in Elderly Finns in 1970-2013. Calcif. Tissue Int. 2015 Dec;97 (6):577-580. 3. Pickhardt PJ, Lee LJ, del Rio AM, et al. Simultaneous screening for osteoporosis at CT colonography: bone mineral density assessment using MDCT attenuation techniques compared with the DXA reference standard. J. Bone Miner. Res. Off. J. Am. Soc. Bone Miner. Res. 2011 Sep;26 (9):2194-2203. 4. Nüchtern JV, Hartel MJ, Henes FO, et al. Significance of clinical examination, CT and MRI scan in the diagnosis of posterior pelvic ring fractures. Injury. 2015 Feb;46 (2):315-319.

Disclosure: No significant relationships.
0008

\section{A BIOMECHANICAL STUDY OF SACROILIAC ROD FIXATION FOR UNSTABLE PELVIC RING INJURIES: VERIFICATION OF THE "WITHIN RING" CONCEPT}

\section{$\underline{\text { K. Futamura }}$}

Trauma Center, Shonan Kamakura General Hospital, kamakura city/ JAPAN

Introduction: The aim of this study was to compare the fixation power of sacroiliac rod fixation (SIRF), which was developed based on "within ring" concept to exclude the lumbar vertebra from the fixation range, and Spinopelvic fixation (SPF) in a biomechanical experiment.

Material and methods: SPF and SIRF were applied to the posterior element in 4 bones each with the pelvic ring fracture model (AO/OTA classification 61-C1.3). A 300-N axial force was loaded on the 5th lumbar vertebra of the simulated pelvis. Then the stiffness $(\mathrm{N} / \mathrm{m})$ and deformation $(\mathrm{mm})$ of SPF and SIRF were determined, and the final displacement $(\mathrm{mm})$ of the fracture region and angular deformity (degrees) were measured. Displacements were measured using the markers at two sites of the sacral fracture (upper margin of the sacral ala (Ala) and second sacral vertebra level (S2)) and one site of the pubic symphysis (PS), and angular deformity was measured at Ala and PS .Statistical evaluation was performed at a level of significance $\mathrm{p}<0.05$ for all statistical tests.

Results: In SPF and SIRF, the mean stiffness's and deformations showed no statistical significant difference. Only the vertical displacement at Ala differed significantly between SPF and SIRF ( $\mathrm{p}=0$. 045), and the fixing force of SPF was higher. There was no other significant difference in vertical and horizontal displacement. The mean angular deformities also showed no significant difference between the two methods .

Conclusion: In biomechanics experiments, vertical resistance was stronger in SPF-treated than SIRF-treated bone, but stiffness and deformation, horizontal resistance, and angular deformity did not differ significantly.

References:

Disclosure: No significant relationships.

\section{9}

\section{PREVENTION OF MEDIAL RE-DISPLACEMENT IN GERIATRIC ACETABULAR FRACTURES WITH DISRUPTION OF THE QUADRILATERAL PLATE - A BIOMECHANICAL STUDY}

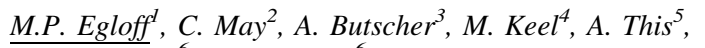
K. Siebenrock ${ }^{6}$, J.D. Bastian ${ }^{6}$

${ }^{1}$ Department Of Orthopaedic And Trauma Surgery, , University of Bern, Inselspital, Bern, , Bern/SWITZERLAND, ${ }^{2} \mathrm{Fe}$ Simulation, RMS Foundation, Reutlingen/GERMANY, ${ }^{3} \mathrm{Head}$ Of Bio- \& Structural Mechanics, RMS Foundation, Bettlach/SWITZERLAND, ${ }^{4}$ Trauma Zentrum, Hirslanden Zürich, Zürich/SWITZERLAND,

${ }^{5}$ Trauma/cmf R\&d, Synthes GmbH, Zuchwil/SWITZERLAND,

${ }^{6}$ Department Of Orthopaedic And Trauma Surgery, Inselspital Bern, Bern/SWITZERLAND 
Introduction: In the elderly acetabular fractures frequently involve the anterior column and the quadrilateral plate $(\mathrm{QLP})^{1}$. Open reduction of displaced acetabular fractures has to achieve a congruent joint. Fracture fixation has to prevent from medial re-displacement of the QLP to avoid secondary displacement with subsequent incongruency of the hip joint during fracture healing. Thus, the aim of this biomechanical study was to compare the capability in preventing medial re-displacement of the QLP in two different osteosynthetic constructs.

Material and methods: An anterior column posterior hemitransverse (ACPHT) fracture pattern with disruption of the QLP in synthetic hemipelvises $(n=4)$ was assessed. In group 1 , for fixation a suprapectineal reconstruction plate without periarticular screws along the QLP but an infrapectineal buttress plate was used. In group 2, a suprapectineal reconstruction plate with additional periarticular screws along the QLP was used. These constructs were loaded with linearly raising forces using a Zwick testing machine. The amount of QLP medial re-displacement was locally assessed by a digital image correlation system. The level of significance was set at $\mathrm{p}<0.05$.

Results: Applied loads resulting in failure of the osteosynthetic constructs were lower in group 1 compared to group 2 with statistical significance.

Conclusion: The fixation strength in patients might be enhanced by the use of additional periarticular screws instead of infrapectineal buttress plates.

References: 1. Ferguson TA, Patel R, Bhandari M, Matta JM. Fractures of the acetabulum in patients aged 60 years and older. J Bone Jt Surg 2009;250-7.

Disclosure: No significant relationships.

\section{O010}

\section{MID-TERM FUNCTIONAL RESULTS AFTER INTERNAL FIXATION OF ACETABULAR FRACTURES INVOLVING THE ANTERIOR COLUMN USING THE PARARECTUS APPROACH}

C. Von Rüden, L. Wenzel, J. Becker, A. Thannheimer, A. Woltmann, V. Bühren, M. Perl

Trauma Surgery, BG Trauma Center Murnau, Murnau/GERMANY

Introduction: The intrapelvic Pararectus approach has become more and more popular for surgical treatment of acetabular fractures of the anterior column including medialized quadrilateral plate. Aim of this prospective cohort study was to evaluate mid-term results at least one year after open reduction and internal fixation of displaced acetabular fractures through the Pararectus approach.

Material and methods: Fifty-two consecutive patients (median age: 55 (range 17-90) years; 13 female, 39 male) with displaced acetabular fractures involving the anterior column were surgically treated between January 2012 and July 2016 in a single European level I trauma center using the Pararectus approach. Fractures were classified according to the AO/OTA classification. Failure was defined as the need for total hip arthroplasty. Twelve months postoperatively the functional outcome was rated according to LEFS, WOMAC, and SF36 scores.

Results: Thirty patients suffered polytraumatization and 22 a monotrauma. Six out of 52 patients obtained total hip arthroplasty. Nineteen out of 52 patients were available for a complete follow-up at regular intervals, at least 12 months postoperatively. The median midterm functional results demonstrated 98 points according to the LEFS score, $6 \%$ according to the WOMAC score, and $70 \%$ according to the physical functioning part of the SF-36 score.

Conclusion: In this prospective clinical trial, functional outcome demonstrated good to excellent mid-term results following open reduction and internal fixation of comminuted acetabular fractures involving the anterior column addressed through the Pararectus approach. Our encouraging results reinforce to raise the use of the Pararectus approach as a rational alternative in these challenging fracture configurations.

References: 1. Letournel E: The treatment of acetabular fractures through the ilioinguinal approach. Clin Orthop Relat Res 1993; 292: 62-76 2. Keel MJ, Ecker TM, Siebenrock KA, Bastian JD: Rationales for the Bernese approaches in acetabular surgery. Eur $\mathrm{J}$ Trauma Emerg Surg 2012; 38: 489-498 3. Keel MJ, Bastian JD, Büchler L, Siebenrock KA: Anterior approaches to the acetabulum. Unfallchirurg 2013; 116: 213-220 4. Bastian JD, Tannast M, Siebenrock KA, Keel MJ. Mid-term results in relation to age and analysis of predictive factors after fixation of acetabular fractures using the modified Stoppa approach. Injury 2013; 44: 1793-1798 5. Keel MJ, Ecker TM, Cullmann JL et al.:The Pararectus approach for anterior intrapelvic management of acetabular fractures: an anatomical study and clinical evaluation. J Bone Joint Surg Br 2012; 94: 405-411 6. Keel MJ, Tomagra S, Bonel HM, Siebenrock KA, Bastian JD. Clinical results of acetabular fracture management with the Pararectus approach. Injury 2014; 45: 1900-1907 7. Märdian S, Schaser KD, Hinz P, Wittenberg S, Haas NP, Schwabe P: Fixation of acetabular fractures via the ilioinguinal versus pararectus approach: a direct comparison. Bone Joint J 2015; 97-B: 1271-1278 8. Bastian JD, Savic M, Cullmann JL, Zech WD, Djonov V, Keel MJ: Surgical exposures and options for instrumentation in acetabular fracture fixation: Pararectus approach versus the modified Stoppa. Injury 2016; 47: 695-701 Disclosure: No significant relationships.

\section{MINIMALLY INVASIVE TECHNIQUES IN TRAUMA AND EMERGENCY SURGERY}

\section{1}

\section{ECONOMIC IMPACT OF USE OF ENDOLOOP IN SURGICAL TREATMENT OF ACUTE APPENDICITIS}

V.M. Durán Muñoz-Cruzado $^{1}$, L. Tallón-Aguilar ${ }^{2}$, J. Tinoco-González ${ }^{2}$, M.J. Tamayo-Lopez ${ }^{2}$, A. Sánchez-Arteaga ${ }^{2}$, I. Alarcón-Del Agua ${ }^{2}$, J. Padillo-Ruíz, F. Pareja-Ciuró ${ }^{2}$

${ }^{1}$ Emergency Surgery, Hospital Universitario Virgen del Rocío, Seville/SPAIN, ${ }^{2}$ Emergency Surgery Unit, Virgen del Rocío Universitary Hospital, Seville/SPAIN, ${ }^{3}$ Hepatobiliary And Transplantant Surgery Unit, Virgen del Rocío Universitary Hospital, Seville/SPAIN

Introduction: Acute appendicitis is the most frequent surgical pathology in Emergency Department. It is because of the important prevalence of this disease, so it could be a field of efficiency improvement. The systematization and optimization of its surgical techniques improves the results and could reduce costs without this affecting the safety of patients.

Material and methods: Prospective analytical study from January 2015 to September 2017. All patients with diagnosis of acute appendicitis operated by a laparoscopic approach have been prospectively collected. We analyzed demographic data, intraoperative findings, use of endoloop Vs GIA, stay and postoperative 
complications. The endpoint of our study was to analyze costs with each one of laparoscopic techniques (endoloop Vs endoGIA).

Results: During this period, 444 patients $(58 \%$ women, 36'27 \pm $16^{\prime} 51$ years) were included. 92 patients $\left(20^{\prime} 72 \%\right)$ had complicated appendicitis. Percentage of conversion was 3'37\%. 176 patients were operated on with endoloops (39'63\%). The mean postoperative stay was $2.68 \pm 3.14$ days for those treated with endoloop and $2.65 \pm 3.15$ days for endoGIAs group. Two patients operated with GIAs had postoperative collection and 5 patient operated with endoloops. There were only two patients with surgical wound infection in GIAs group. We have identified that in each procedure performed with endoloop we saved more than $€ 200$.

Conclusion: The use of endoloop for treatment of acute appendicitis could be a significant saving for the Health System. His use seems to be as safe and effective than endoGIA.

References: 1. Antoniou SA et al. Optimal strump management in laparoscopic appendectomy: A network meta-analysis by the Minimally Invasive Surgery Synthesis of Interventions and Outcomes Network. 2017. doi: 10.1016/j.surg.2017.07.013. [Epub ahead of print] 2. Mehdorn $\mathrm{M}$ et al. Intended cost reduction in laparoscopic appendectomy by introducing the endolopp: A single centre experience.2017;17 (1):80. 3. Lucchi A et al. Laparoscopic appendectomy: Hem-o-lok versus Endoloop in strump closure. 2017;69 (1):61-65.

Disclosure: No significant relationships.

\section{2}

\section{EXPERIENCE IN DIAGNOSTIC AND THERAPEUTIC LAPAROSCOPY FOR PATIENT WITH ABDOMINAL STAB WOUNDS}

\section{H. Lin, Y. Chen}

Surgery, Far-Eastern Memorial Hospital, Banqiao District, New Taipei/TAIWAN

Introduction: Laparoscopy has been gradually accepted as a diagnostic tool for hemodynamically stable patients with abdominal stab wounds, but less has been reported as a therapeutic modality. This study collected our experience in diagnostic and therapeutic laparoscopy for patients with abdominal stab wounds.

Material and methods: All patients sustaining abdominal stab wounds over an 11-year period (2006-2017) at a tertiary medical center were included. Patients either underwent wound repairs only, diagnostic laparoscopy, diagnostic and therapeutic laparoscopy, converted laparotomy, or exploratory laparotomy. The clinical outcomes of the groups were described

Results: There were 173 patients sustaining abdominal stab wounds in the study period. Fifty-two patients underwent wound repairs only and 29 patients underwent a mandatory laparotomy successfully. Ninetytwo hemodynamically stable patients with anterior abdominal stab wounds and without bowel eviscerations underwent a diagnostic laparoscopy initially. Among them, 40 patients underwent a diagnostic laparoscopy only because no significant intra-abdominal injuries. Forty-six patients with significant injuries underwent both a diagnostic and successful therapeutic laparoscopy whereas the other 6 patient underwent a converted laparotomy for complex lesions. The accuracy rate of diagnostic laparoscopy was $98.9 \%$ (91/92) with 1 complication of missed injuries requiring a re-operation. The rate of conversion to laparotomy was $6.5 \%(6 / 92)$. There was no mortality in the diagnostic laparoscopy group. The complication rate was $5.4 \%$ (5/92) with 1 reoperation, 2 intra-abdominla abscesses, and 2 wound infections.
Conclusion: Laparoscopy is feasible and safe for the diagnosis and treatment of hemodynamically stable patients with abdominal stab wounds.

References: 1.Ahmed N, Whelan J, Brownlee J, Chari V, Chung R. The contribution of laparoscopy in evaluation of penetrating abdominal wounds. J Am Coll Surg. 2005;201:213-6. 2. Koto MZ, Matsevych OY, Motilall SR. The Role of Laparoscopy in Penetrating Abdominal Trauma: Our Initial Experience. J Laparoendosc Adv Surg Tech A. 2015;25:730-6 3. Uranues S, Popa DE, Diaconescu B, Schrittwieser R. Laparoscopy in penetrating abdominal trauma. World J Surg. 2015;39:1381-8. 4.Kawahara NT, Alster C, Fujimura I, Poggetti RS, Birolini D. Standard examination system for laparoscopy in penetrating abdominal trauma. J Trauma. 2009;67:589-95. 5. Lin HF, Wu JM, Tu CC, Chen HA, Shih HC. Value of diagnostic and therapeutic laparoscopy for abdominal stab wounds. World J Surg. 2010;34:1653-62. 6.Chestovich PJ, Browder TD, Morrissey SL, Fraser DR, Ingalls NK, Fildes JJ. Minimally invasive is maximally effective: Diagnostic and therapeutic laparoscopy for penetrating abdominal injuries. J Trauma Acute Care Surg. 2015;78:1076-83; discussion 83-5.

Disclosure: No significant relationships.

\section{3}

\section{HYBRID TREATMENT IN SEVERE TRAUMA: EXPERIENCE AT A LEVEL 1 TRAUMA CENTER IN A UPPER-MIDDLE INCOME COUNTRY}

\author{
J.P. Carbonell ${ }^{1}$, A.M. Del Valle ${ }^{1}$, H.E. Munevar ${ }^{1}$, A.F. Garcia ${ }^{l}$, \\ R. Ferrada ${ }^{2}$, J.C. Puyana ${ }^{3}$, C. Navarro ${ }^{4}$, J.C. Bayona ${ }^{5}$, J.P. Trochez ${ }^{5}$
}

${ }^{1}$ Valle Del Cauca, Fundación Valle del Lili, Cali/COLOMBIA, ${ }^{2}$ Valle Del Cauca, Clinica Imbanaco, Cali/COLOMBIA, ${ }^{3}$ Department Of Surgery, University of Pittsburgh Medical Center, Pittsburgh/PA/ UNITED STATES OF AMERICA, ${ }^{4}$ Talca, Servicio de salud del Maule, Talca/CHILE, ${ }^{5}$ Valle Del Cauca, Hospital Universitario del Valle, Cali/COLOMBIA

Introduction: Combination of open and endovascular procedures in the treatment of trauma patients has emerged as a new option that could improve the outcome. The published experience is limited to small series and case reports. We describe a case series of trauma patients with hybrid therapy (HT) in a Level I Trauma Center in an upper-middle income country.

Material and methods: Retrospective study of the patients who received HT from 2008 to 2017. Demographic data, trauma mechanism, severity and details of the lesions and the treatment were registered.

Results: A total of 41 patients, $36(87,8 \%)$, were included. Median age, inter-quartile rank (IQR) was 25 years $(20-41)$. HT was performed emergently in 25 cases $(61.0 \%)$. In 7 patients, endovascular treatment was performed before an open procedure, while in 34 $(82.9 \%)$, it was undertaken as complement. The mechanism of trauma was penetrating in 23 subjects $(56.1 \%)$. Trauma severity was higher in blunt trauma, median ISS 40,5 IQR $(29-50)$ vs 25 IQR (16 - 36), median RTS 6,4 vs 7,1 (IQR 5,1 - 7,1 vs 6,4-7,5). Injuries were located more frequently in the pelvis $(33.3 \%)$, liver $(27,8 \%)$, and aorta $(22.2 \%)$ in blunt trauma, and in head \& neck, in penetrating trauma $(52.2 \%)$. Laparotomy was performed in 20 patients, orthopedic procedures in 12, thoracotomy in 7 , and other surgeries in 10 . Six patients died, all of them, in the blunt trauma group. Mortality in penetrating trauma was less than expected. 
Conclusion: HT is a viable option that could reduce mortality in penetrating trauma.

References: 1. Kirkpatrick, A. W., Vis, C., Dubé, M., Biesbroek, S., Ball, C. G., Laberge, J., ... \& Kortbeek, J. (2014). The evolution of a purpose designed hybrid trauma operating room from the trauma service perspective: The RAPTOR (resuscitation with angiography percutaneous treatments and operative resuscitations). Injury, 45 (9), 1413-1421. 2. Kataoka, Y., Minehara, H., Kashimi, F., Hanajima, T., Yamaya, T., Nishimaki, H., \& Asari, Y. (2016). Hybrid treatment combining emergency surgery and intraoperative interventional radiology for severe trauma. Injury, 47 (1), 59-63. 3. Fehr A, Beveridge J, D'Amours, Kirkpatrick A, Ball Ch. The potential benefit of a hybrid operating environment among severely injured patients with persistent hemorrhage : How often could we get it right? J . Trauma Acute Care Surg 2016; 80 (3) :457 - 460. 4. Branco BC, DuBose JJ. Endovascular solutions for the management of penetrating trauma : an update on REBOA and axillo- subclavian injuries .Eur J Trauma Emerg Surg . 2016. 5. DuBose JJ, Rajani R, Gilani R, Arthurs ZA, Morrison JJ, Clouse WD, Rasmussen TE. Endovascular management of axillo-subclavian arterial injury: a review of published experience.Injury. 2012; 43:1785-92.

Disclosure: No significant relationships.

\section{4}

\section{ACHIEVING HIGHER RATES OF TRANSCYSTIC CHOLEDOCHOSCOPY DURING LAPAROSCOPIC COMMON BILE DUCT EXPLORATION (LCBDE)}

\author{
J. Almusawi, L. Navaratne, K. Qurashi, A. Isla
}

General Surgery, Northwick Park Hospital, London/UNITED KINGDOM

Introduction: Transcystic choledochoscopy results in lower complication rates when compared to laparoscopic common bile duct exploration (LCBDE) via choledochotomy. ${ }^{1}$ The objective was to review our practice of LCBDE over time and describe contributing factors which enabled higher rates of transcystic choledochoscopy.

Material and methods: We performed a retrospective review of our prospectively kept database of 350 LCBDEs. The primary outcome measure was the route used to access the CBD when performing choledochoscopy (transcystic vs transductal). Cases were divided chronologically in blocks of 50 cases to demonstrate technical progression over time.

Results: After the first 200 cases of LCBDE our group published its results and found that we utilised the transcystic approach in just $8.3 \%$ of cases. This is in contrast to the last 50 cases in our series $(n=350)$ with transcystic choledochoscopy accounting for over $90 \%$ of cases. The last 250 cases of LCBDE in blocks of 50 cases utilised transcystic choledochoscopy in $4 \%, 20 \%, 36 \%, 74 \%$ and $91.2 \%$ respectively $(\mathrm{p}<$ $0.05)$. We describe four factors that contribute to high rates of transcystic choledochoscopy: (1) retraction of the cystic duct (2) availability of a $3 \mathrm{~mm}$ choledochoscope (3) transinfundibular approach (TIA) to the CBD when the hilum is frozen and (4) the laser-assisted bile duct exploration by laparoendoscopy (LABEL) technique.

Conclusion: High success rates in transcystic choledochoscopy can be achieved given the right technical expertise and equipment available to the operating team. We have found that adhering to four basic principles significantly improves the likelihood that the CBD can be explored via the transcystic route.
References: 1. Reinders JSK, Gouma DJ, Ubbink DT, van Ramshorst B, Boerma D. Trancystic or transductal stone extraction during single-stage treatment of choledochocystolithiasis: A systematic review. World J Surg. 2014;38:2403-2411.

Disclosure: No significant relationships.

\section{5}

\section{IS COMPUTED TOMOGRAPHY TRACTOGRAPHY RELIABLE IN PATIENTS WITH ANTERIOR ABDOMINAL STAB WOUNDS?}

\section{I.S. Sarici, M.U. Kalayci}

General Surgery, Kanuni Sultan Suleyman Training and Reseach Hospital, İstanbul/TURKEY

Introduction: Current literature and guidelines recommended that determination of peritoneal violation is to be done first in cases of anterior abdominal stab wounds. The primary endpoint of this study was to determine reliability of CT tractography to assessing peritoneal violation in anterior abdominal stab wounds. Secondary end point is to compare of local wound exploration, conventional CT and CT tractography for evaluate the peritoneal violation.

Material and methods: Totally of 252 patients who referred with anterior abdominal stab wounds included in this prospective observational study. Three techniques (local wound exploration, conventional abdominal tomography and computed tomography tractography)applied to all patients respectively. Sensitivity, specificity, positive predictive value (PPV), negative predictive value (NPV)and accuracy calculated for each technique for determine peritoneal violation.

Results: The results for the local wound exploration is $100 \%$ sensitivity, $100 \%$ specificity, $100 \%$ PPV, $100 \%$ NPV and $100 \%$ accuracy. CT tractography was $95 \%$ sensitivity, $100 \%$ specificity, $100 \%$ PPV, $80 \%$ NPV and $96 \%$ accuracy. Conventional abdominal tomography results were $87 \%$ sensitivity, $50 \%$ specificity, $91 \%$ PPV, $40 \%$ NPV and $82 \%$ accuracy.

Conclusion: Local wound exploration is $\% 100$ safe and effective in determine peritoneal violation with anterior abdominal stab wounds. CT tractography is better than conventional CT to detect peritoneal violation. However we do not recommend CT tractography in anterior abdominal stab wounds due to the false negative results and adversely affect in clinical follow-up.

References:

Disclosure: No significant relationships.

\section{6}

\section{IS LAPAROSCOPIC TREATMENT IN PERFORATED PEPTIC ULCER SAFE FOR ALL PATIENTS?}

\author{
K. Thorsen ${ }^{1}$, J.A. Søreide ${ }^{2}$, K. Søreide ${ }^{I}$
}

${ }^{1}$ Gastrointestinal Surgery, Stavanger University Hospital, stavanger/ NORWAY, ${ }^{2}$ Clinical Medicine, University of Bergen, bergen/ NORWAY

Introduction: Perforated peptic ulcer (PPU) is a surgical emergency associated with high mortality. Laparoscopy in PPU has been studied in 6 randomized controlled trials (RCT) and further 6 metaanalyses 
yielding favourable outcomes for the laparoscopy group. However patients with high degree of comorbidity have not been included in the RCTs.

Material and methods: This is a consecutive cohort including 255 patients operated for a perforated peptic ulcer (PPU) at Stavanger University Hospital (SUH) in Southwest Norway. Surgical approach was chosen by the responsible surgeon. In this observational study the aim was to evaluate and compare outcomes after routine treatment for PPU by laparoscopic or open surgery.

Results: A total of 255 patients were operated for PPU during the study period. Females constituted $52.5 \%(134 / 255)$ and males $47.5 \%$ (121). Median age was 67 years (range 18-100). The 30-day mortality was $15.7 \%$ (40/255) with no significant difference observed between those treated with laparotomy $(24 / 145(16.6 \%))$ and patients in the laparoscopy group $(16 / 110(14.5 \%))(\mathrm{p}=0.66)$. There were no statistical significant differences in age $(\mathrm{p}=0.80)$, ASA score $(\mathrm{p}=$ $0.138)$, Boey score $(\mathrm{p}=0.08)$ or Charlton comorbidity index (CCI) score $(\mathrm{p}=0.46)$ between the laparotomy and the laparoscopy group. The Laparotomy group had significantly increased length of stay (LOS) compared to the laparoscopy group $(\mathrm{p}<0.001)$.

Conclusion: This observational study comprising two well balanced treatment groups with regard to clinical characteristics including age, ASA score and CCI, shows that even high risk PPU patients can be treated safely with a laparoscopic surgical technique. In addition, LOS is almost half in the laparoscopy group.

References:

Disclosure: No significant relationships.

\section{7}

\section{ADHESIVE SMALL BOWEL OBSTRUCTION: SINGLE ADHESIVE BANDS OR MATTED ADHESIONS? THE ANSWERS ARE ON CT SCAN}

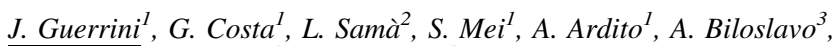

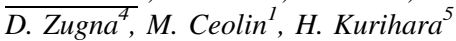

${ }^{1}$ Emergency Surgery And Trauma Unit, Humanitas Research Hospital, Rozzano, Milano/ITALY, ${ }^{2}$ General Surgery, Humanitas Research Hospital, Rozzano, Milano/ITALY, ${ }^{3}$ Chirurgia Generale, Ospedale di Cattinara, Trieste/ITALY, ${ }^{4}$ Scienze Mediche, Sezione Di Statistica Medica, Università di Torino, Torino/ITALY, ${ }^{5}$ Emergency Surgery And Trauma Unit, Humanitas Research Hospital, Rozzano/ ITALY

Introduction: To differentiate adhesive small-bowel obstruction (ASBO) due to single adhesive bands (SAB) from ASBO by matted adhesions (MA) by CT scan.

Material and methods: CT scans of patients with ASBO caused by either surgically confirmed SAB or MA were analyzed. Following CT findings were compared between the two groups: kind of obstruction (simple or closed-loop SBO, complete or partial), location of the transition zone, presence of whirl sign, "small-bowel feces" sign, beak sign, "fat-notch" sign, C-shaped bowel's configuration, decreased bowel wall enhancement sign and mesenteric fluid sign. Predictive models based on logistic regression were developed to estimate the risk of SBO due to $\mathrm{SAB}$, intestinal ischemia and necrosis according to CT findings. Models' predictive ability was quantified by ROC curve.

Results: 116 patients were included in the study: $65.5 \%$ developed SBO due to SBA, 40.5\% developed intestinal ischemia, 9.5\% developed intestinal necrosis. By multivariable analysis, the risk of SBO due to SBA was positively associated with complete obstructions $(\mathrm{OR}=4.14,95 \% \mathrm{CI}: 1.43-11.93)$ and "fat notch" sign (OR $=7.40,95 \%$ CI: $1.64-33.28)$. Using 0.5 as cut-point probability of SBA for the predictive model, sensibility was $85.5 \%$, specificity was $70.0 \%$, positive and negative predictive values were $84.4 \%$ and $71.8 \%$ respectively, area under ROC curve was 0.86 . The risk of intestinal ischemia was positively associated with mesenteric fluid sign $(\mathrm{OR}=6.17,95 \% \mathrm{CI}: 2.28-16.69)$, the risk of necrosis with decreased bowel wall enhancement sign $(\mathrm{OR}=27.90,95 \% \mathrm{CI}: 2.21$ 353.04).

Conclusion: Specific CT findings may predict whether ASBO is caused by SAB or MA and potentially influence clinical pathway (conservative versus operative, laparoscopic versus open).

References: 1. Maung AA, Johnson DC, Piper GL, Barbosa RR, Rowell SE, Bokhari F, Collins JN, Gordon JR, Ra JH, Kerwin AJ. Evaluation and management of small-bowel obstruction: an Eastern Association for the Surgery of Trauma practice management guideline. J Trauma Acute Care Surg. 2012 Nov;73 (5 Suppl 4):S362-9. 2. Millet I, Ruyer A, Alili C, Curros Doyon F, Molinari N, Pages E, Zins M, Taourel P. Adhesive small-bowel obstruction: value of CT in identifying findings associated with the effectiveness of nonsurgical treatment. Radiology. 2014 Nov;273 (2):425-32. 3. Delabrousse E, Lubrano J, Jehl J, Morati P, Rouget C, Mantion GA, Kastler BA. Small-bowel obstruction from adhesive bands and matted adhesions: CT differentiation. AJR Am J Roentgenol. 2009 Mar;192 (3):693-7. 4. Di Saverio S, Coccolini F, Galati M, Smerieri N, Biffl WL, Ansaloni L, Tugnoli G, Velmahos GC, Sartelli M, Bendinelli C, Fraga GP, Kelly MD, Moore FA, Mandal V, Mandal S, Masetti M, Jovine E, Pinna AD, Peitzman AB, Leppäniemi A, Sugarbaker PH, Goor HV, Moore EE, Jeekel J, Catena F.Bologna guidelines for diagnosis and management of adhesive small bowel obstruction (ASBO): 2013 update of the evidence-based guidelines from the world society of emergency surgery ASBO working group. World J Emerg Surg. 2013 Oct 10;8 (1):42.

Disclosure: No significant relationships.

\section{8}

\section{CHARACTERISTICS AND PREVALENCE OF ACUTE CHOLECYTITIS IN PATIENTS WITH HEMATOLOGIC MALIGNANCIES}

\section{A.Y. El Rifai, K. Naja, M. El Hechi, A. Hallal}

Surgery, The American University of Beirut Medical Center, Beirut/ LEBANON

Introduction: The purpose of this study is to describe the natural history and outcome of acute cholecytitis and symptomatic cholelithiasis in patients who have lymphoproliferative diseases.

Material and methods: This study is a retrospective review of patients admitted to the American University of Beirut Medical Center (AUBMC) for treatment of hematologic malignancies from January2008 to December2016, who developed gallbladder symptoms requiring an intervention during their treatment course. The charts of the patients were reviewed and data related to patient demographics, chemotherapeutic regimen, symptomatology, management strategies and complications were collected. Data was entered and analyzed using SPSS.

Results: Out of 2426 patients with hematologic malignancies sixteen patients were included in the study with a prevalence of gallbladder disease of $0.7 \%$. Sixty-two percent were males and the mean age was 53 years. Forty-four percent had leukemia, thirty-seven percent had lymphoma and nineteen percent had multiple myeloma. Thirteen 
patients had acute cholecystitis and three had symptomatic cholelitiasis. Thirty-eight percent were on Doxorubicin, twenty-five percent on Vincristine and nineteen percent were on Cytarabine as part of their chemotherapeutic regimen. Twelve out of the sixteen $(75 \%)$ patients underwent cholecystectomy upon diagnosis and four $(25 \%)$ were treated with antibiotics. Out of the four, three required operative intervention. Mean length of hospital stay was 9 days. One patient developed postoperative sepsis and there were no postoperative mortalities.

Conclusion: Acute cholecystitis and symptomatic cholelithiasis do not occur more often in patients with hematologic malignancies than the general population and can be safely treated surgically with good outcomes if diagnosis and intervention were done promptly.

References: 1- Gibney et al. Asymptomatic Gallstones, British Journal of Surgery, 1990, Vol 77 April, 368-372. 0007-1323/90/ 040368-05 2- Strasberg et al. Acute Calculous Cholecytitis, N Engl J Med June 2008;358:2804-11. 3- Sandoval et al. Cholelithiasis and Choledocholithiasis After Sequential Cytarabine and Asparaginase Journal of Pediatric Hematology/Oncology, Vol. 25, No. 8, August 2003 4- Ates et al. Prevalence of Gallstones in Patients with Chronic Myelocytic Leukemia, Med Princ Pract 2009;18:175-179 DOI: 10.1159/000204346 5- Berrevoets et al. Atypical presentation of 'acalculous cholecystitis' with marked isolated hyperbilirubinaemia in patients treated for haematological malignancies, European Journal of Haematology 94 (182-185), 14 April 2014, doi:10.1111/ ejh.12357 6- Büyükasik et al. Acalculous acute cholecystitis in leukemia, Journal of Clinical Gastroenterology, Issue: Volume 27 (2), September 1998, pp 146-148

Disclosure: No significant relationships.

\section{9}

\section{TRANSCATHETER ARTERIAL EMBOLIZATION VERSUS SPLENECTOMY IN PATIENTS WITH ISOLATED BLUNT SPLENIC INJURY}

\author{
T. Suzuki $i^{1}$ A. Shiraishi ${ }^{2}$, Y. Otomo ${ }^{3}$
}

${ }^{1}$ Department Of Emergency And Critical Care Medicine, Kimitsu Chuo Hospital, Chiba/JAPAN, ${ }^{2}$ Emergency And Trauma Center, Kameda Medical Center, Kamogawa/JAPAN, ${ }^{3}$ Emergency And Disaster Medicine, Tokyo Medical and Dental University Hospital, Tokyo/JAPAN

Introduction: In patients with blunt splenic injury, transcatheter arterial embolization (TAE) can achieve hemostasis with organ preservation and is increasingly being performed instead of splenectomy $[1,2]$. However, the comparative survival benefit of TAE and splenectomy remains unclear in the population with blunt splenic injury. This study compared hospital mortality between patients undergoing TAE or splenectomy for blunt splenic injury.

Material and methods: Out of patients registered in the Japan Trauma Data Bank (JTDB), this study included trauma patients who underwent TAE or splenectomy for isolated blunt splenic injury. Patients with out-of-hospital cardiac arrest were excluded. A logistic regression analysis was performed to estimate the propensity score for TAE from known predictors of in-hospital mortality. Then propensity score matching analysis was used to compare in-hospital mortality between patients who received TAE or splenectomy.

Results: Of 236,698 trauma patients registered in the JTDB, 1,191 patients were selected and propensity score matching was performed for 315 patients each receiving TAE or splenectomy. Hospital mortality was similar between patients undergoing TAE or splenectomy (6.7\% versus $9.1 \%$, odds ratio $0.72,95 \%$ CI $[0.40,1.31], \mathrm{P}=0.283)$. Forty-two patients receiving primary TAE $(5.5 \%)$ required subsequent splenectomy.

Conclusion: This propensity score-matched observational study found that TAE did not significantly reduce hospital mortality compared with splenectomy. Further studies are needed to compare the effectiveness of TAE and splenectomy in patients with isolated blunt splenic injury.

References: 1) Aiolfi A et al. J Trauma Acute Care Surg. 2017 Sep;83 (3):356-360. 2) Wallis et al. World Journal of Emergency Surgery 2010, 5:18 3) Stassen NA et al. J Trauma Acute Care Surg. 2012 Nov;73 (5 Suppl 4):S294-300.

Disclosure: No significant relationships.

\section{O019A}

\section{FACTORS ASSOCIATED WITH MORTALITY IN PATIENTS UNDERGOING EMERGENCY LAPAROTOMY FOR NON- TRAUMATIC INTRABDOMINAL PATHOLOGY; A STATISTICAL ANALYSIS}

\section{N. Misra, M. Campbell, J.V. Taylor}

Emergency And Trauma Surgery, Aintree University Hospital, Liverpool/UNITED KINGDOM

Introduction: Mortality associated with emergency laparotomy remains high in the UK, but is improving. This single centre, prospectively gathered database of emergency laparotomy patients evaluated the factors associated with the post-operative mortality. Material and methods: A prospective database of emergency laparotomy patients in a teaching hospital between December 2015 and November 2016 was retrospectively analysed. Primary endpoint was 30 day post-operative mortality. Variables examined were time to laparotomy (defined as time from admission to surgery to theatre), ASA grade, age, PPOSSUM score, presence of generalised peritonitis, Presence of Consultant Surgeon or Anaesthetist in the OR, and post-operative admission to ICU. A retrograde, stepwise multi-variable logistic regression analysis was used to construct adjusted odds ratio of mortality.

Results: 177 consecutive patients were included. The crude mortality was $14.7 \%$. Mortality in those who underwent surgery within and after 48hours from admission was identical (14.7\%). In both the unadjusted (OR 0.99, $\mathrm{p}=0.96$ ) and adjusted (OR 0.68, $\mathrm{p}=0.47$ ) analysis there was no statistically significant relationship between time to laparotomy and mortality. This remained true when time was treated as a continuous variable $(\mathrm{P}=0.24)$. There was a statistically significant relationship with mortality and age (OR 1.065, $\mathrm{p}=0.003)$, ASA grade $(\mathrm{OR}-1.88, \mathrm{p}=0.021)$ and admission to ICU (OR 2.97, $\mathrm{p}$ $=0.038$ ).

Conclusion: In our cohort increasing age, ASA grade and admission to ICU were associated with higher mortality in patients undergoing emergency laparotomy. There did not appear to be an association with Consultant presence nor time from admission to theatre.

\section{References:}

Disclosure: No significant relationships. 


\section{THORACIC AND SPINE INJURIES}

$\mathbf{O 0 2 0}$

\section{EXTENSIVE THORACO-ABDMINAL SURGERY CAUSES INTRA-OPERATIVE NEUTROPENIA DESPITE THE EMERGE OF BANDED (CD16DIM/CD62LBRIGHT) NEUTROPHILS IN PERIPHERAL BLOOD}

\section{M.P.J. Teuben}

Traumatologie, university hospital zuerich, zuerich/SWITZERLAND

Introduction: Polymorphonuclear neutrophils (PMNs) are essential effector cells in both the local and systemic inflammatory response to surgery. However, excessive activation of PMNs forms the basis for severe complications such as ARDS and MODS. The aim of this study was to describe the early neutrophil response to extensive thoraco-abdominal surgery.

Material and methods: A total of 12 pigs were utilized and these animals were exposed to a standardized model of combined extensive thoraco-abdominal surgery. Samples were analyzed at baseline and after 60, 120 and 180 minutes of ongoing surgery. We compared neutrophil activation (FcyRIII (CD16), L-selectin (CD62L) as well as Mac-1 (CD11b)) over time. Furthermore absolute PMN numbers and neutrophil subset mobilization were determined.

Results: Eleven out of 12 animals survived the observation period. A significant drop of systolic blood pressure and $\mathrm{pH}$ (7.48 vs 7.37), as well as an increase of pulse rate was observed. Absolute neutrophil numbers dropped gradually during surgery (from $8.6 \times 10^{\wedge} 6$ cells/l to $2.4 \times 10^{\wedge} 6$ cells $\left./ 1, \mathrm{p}<0.05\right)$. However a prominent increase of banded (CD16dim/CD62Lbright) in peripheral blood was observed. Furthermore the mean cell surface expression of CD16 on blood neutrophils decreased, whereas the expression of CD62L and CD11b increased over time

Conclusion: We showed that extensive thoraco-abdominal surgery in pigs results in early neutropenia, despite the emerge of young (CD16dim/CD62Lbright) PMNs in peripheral blood. The shifts in subsets appearance in peripheral blood impact the cell surface expression of activation markers in the blood neutrophil population. References:

Disclosure: No significant relationships.

\section{1}

\section{SELECTING CERVICAL SPINAL CORD INJURY PATIENTS FOR EARLY TRACHEOSTOMY}

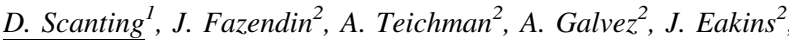
B. Mcracken ${ }^{2}$

${ }^{1}$ Surgery, Drexel University College of Medicine/Hahnemann University Hospital, Philadelphia/PA/UNITED STATES OF AMERICA, ${ }^{2}$ Surgery, Drexel University College of Medicine/ Hahnemann University Hospital, Philadelphia/UNITED STATES OF AMERICA

Introduction: Cervical spinal cord injuries (CSCI) often necessitate ventilator support (VS). Endotrachial tubes convey substantial morbidity long term. Tracheostomy is recommended if VS is anticipated to be 7 days. Identifying these patients and performing early tracheostomy could prevent substantial morbidity and mortality while lowering hospital costs. Our study is unique.

Material and methods: We queried the Pennsylvania Trauma Outcome Study database for patients with cervical spine fractures and CSCI from 2005-2014. Patients surviving $\geq 7$ days and requiring any VS were included. Patients were excluded for incomplete data or death within 6 days. C1-C4 injuries and C5-7 injuries made separate cohorts. Within groups, we compared patients needing ventilator support $\geq 7$ days ( $\geq 7 \mathrm{D}$-cohort) to those needing less VS. ISS, age, GCS, LOS, highest cervical fracture level (H-CFL) and tracheostomy placement were evaluated. Analysis utilized logistic regression, Mann-Whitney U and Fisher's exact tests.

Results: Within the $\mathrm{C} 1-\mathrm{C} 4$ cohort $(\mathrm{N}=258)$, age difference was not significant $(\geq 7 \mathrm{D} 52 \mathrm{y}-\mathrm{vs}-59 \mathrm{y}, \mathrm{p}=0.064)$. H-CFL $(\geq 7 \mathrm{D}$ median $\mathrm{C} 3-$ vs-C4, p = 0.0394), ISS ( $\geq$ 7D median 35-vs-21, p $<0.0001)$, GCS ( $\geq$ 7D median 8.5 -vs-15, $\mathrm{p}<0.0001$ ), GCS motor ( $\geq$ 7D median 1-vs$6, \mathrm{p}<0.0001)$, mean VS $(\geq 7 \mathrm{D}$ mean 25.9 -vs-3.2days, $\mathrm{p}<0.0001)$ and tracheostomy placement $(\geq 783 \%$-vs-6\%, p $<0.0001)$ were significantly different. For the C1-C5 cohort $(\mathrm{N}=625)$, H-CFL was not significant $(\geq 7 \mathrm{D}$ median C5-vs-C5, $\mathrm{p}=0.091)$. Age $(\geq 7 \mathrm{D}$ mean 51y-vs-55y, $\mathrm{p}=0.0257$ ). ISS $(\geq 7 \mathrm{D}$ median 29-vs-21, $\mathrm{p}<0.0001$ ), GCS $(\geq 7 \mathrm{D}$ median 14-vs-15, $\mathrm{p}<0.0001)$, GCS motor $(\geq 7 \mathrm{D}$ median 4-vs-5, p $<0.0001)$, mean VS ( $\geq 7 \mathrm{D}$ mean 23.4-vs-2.7days, $\mathrm{p}<$ $0.0001)$ and tracheostomy placement $(\geq 780 \%$-vs- $8 \%, p<0.0001)$ were significantly different.

Conclusion: Surgeons can confidently talk with families and schedule severely injured CSCI patients presenting with low GCS and GCS motor scores for early tracheostomy. We anticipate reduced hospital costs and complication rates.

References: Branco et al. Incidence and clinical predictors for tracheostomy after cervical spinal cord injury: a national trauma databank review. J Trauma, 2011;70:111-115.

Disclosure: No significant relationships.

\section{2}

\section{BONE MASS AND LENGTH DEPENDING ON SCREW PATHWAY IN PEDICLE SCREW FIXATION IN S1}

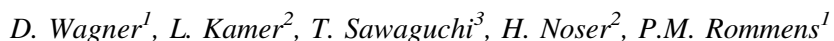

${ }^{1}$ Department Of Orthopedics And Traumatology, University Medical Center Mainz, Mainz/GERMANY, ${ }^{2}$ Ao Research Institute, AO Foundation, Davos/SWITZERLAND, ${ }^{3}$ Department Of Orthopedics \& Joint Reconstructive Surgery, Toyama Municipal Hospital, Toyama/ JAPAN

Introduction: Fractures of the lower lumbar spine sometimes need dorsal fixation to S1. However, implant loosening at this level is observed especially in osteoporotic individuals.

Material and methods: The mean bone mass distribution in Hounsfield units (HU) was calculated with Computed Tomography (CT) scans using statistical computational methods: Included were 92 sacra without fracture (44 males and 48 females, mean age 61.5years, SD +/-11.2). Mean bone mass along following pedicle screw paths for S1 were studied: trans-pedicular, mid-sacral, mid-sacral superior, and parallel.

Results: The highest bone mass along screw path was demonstrated for the trans-pedicular screw through the pedicle and along the subchondral bone of S1 endplate. The superior mid-sacral screw ending in the promontorium had a longer trajectory with moderate bone mass than the mid-sacral screw, which ended better cortical bone at level of 
sacral vertebra S1. The parallel S1 screw ended in very dense cortical bone.

Conclusion: Especially in osteoporotic patients or in revision cases, a trans-pedicular screw path in S1 starting at the superior S1 facet and traversing a long distance subchondrally along the endplate of $\mathrm{S} 1$ is favorable. The parallel screw path anchored in dense cortical bone above foramen S1, however, there is a risk of neurovascular damage. For the mid-sacral screw, the tip should anchor in the promontory

\section{References:}

Disclosure: No significant relationships.

\section{3}

\section{STABILITY STUDIES OF COMBINED FRACTURES OF THE THORACIC SPINE AND THE STERNUM ON THE HUMAN CORPSE PREPARATION (PMHB)}

\section{S. Schulz-Drost $^{1}$, A. Langenbach ${ }^{2}$, F.F. Hennig ${ }^{2}$, S. Krinner ${ }^{2}$}

${ }^{1}$ Trauma- And Orthopedic Surgery, Unfallkrankenhaus Berlin, Berlin/ GERMANY, ${ }^{2}$ Trauma And Orthopedic Surgery, University Hospital Erlangen, Erlangen/GERMANY

Introduction: The combined sternum and thoracic spine-injury (SVI) means a high degree of stability loss of the trunk with the necessity of an osteosynthetic care whose operative strategies are discussed to prevent kyphosis and implant failure. The aim of this study is to determine the stabilizing component of sternal fractures in SVI.

Material and methods: 5 cadavers were examinated. The 6th thoracic vertebral body (T6) had been displaced and dorsally stabilized by internal fixator T5-T7. An electronic pressure gauge had been applied for measurement in the sitting position of the preparation and repetition after a transverse sternotomy angulus. After obelisc application, the pressure in the sternal osteotomy gap is measured (sitting) as well as after of its removal. The vertebral pressure measurement had been repeated after longitudinally sternal locked plateosteosynthesis. Subsequently, the analogous sequence is performed for the $1^{\text {st }}$ lumbar vertebra with ventro-dorsal stabilization.

Results: Dorsal stabilization reduces the pressure in the thoracic vertebral body to $64 \%$ (32-100\%). The sagittal pressure in T6 is then 1.7 times (1.1-2.9) higher in unstable sternum than after its plateosteosynthesis. Conversely, the sternal pressure is increased 1.9 times (1.4-2.6) if T6 is completely unstable ventrally. In LWK 1 fracture, the effects without sternum-stabilization with $1.4(1.0-2.2)$ and the sternum without ventral vertebral replacement with 1.2 (1.01.6) fold pressure are significantly less marked.

Conclusion: All three regions of the trunk influence its stability in SVI. Thus, a sternal stabilization in addition to the BWS can reduce the vertebral pressure conditions which means to avoid a kyphosis

References:

Disclosure: Advisory Member of AO TK Thoracic Expert Group and Consultant Agreement with DePuySynthes
0024

\section{LUNG RESECTION FOR TRAUMA: AN UPDATED ANALYSIS FROM THE NATIONAL TRAUMA DATA BANK}

\author{
A. Aiolfi, K. Inaba, D. Khor, K. Matsushima, S. Siboni, E. Benjamin, \\ D. Demetriades
}

Acute Care Surgery And Surgical Critical Care, LAC+USC Medical Center, Los Angeles/CA/UNITED STATES OF AMERICA

Introduction: Lung operation for trauma is relatively uncommon and only a limited number of patients might require resection ${ }^{1-3}$. Purpose of this study was to analyze the presentation and injury patterns among a cohort of patients who underwent lung resection for trauma and to compare outcomes stratified by the extent of resection. Material and methods: National Trauma Data Bank (NTDB) retrospective study (2007-2014). Adult patients ( $\geq 16$ years-old) who underwent lung resection (wedge, lobectomy, pneumonectomy) for thoracic trauma.

Results: During the study period, 3,433 patients met the inclusion criteria. Overall, 1,006 patients $(29.3 \%)$ sustained a blunt trauma while $2,427(70.7 \%)$ sustained a penetrating injury. Wedge resection was the most commonly performed procedure $(54.3 \%)$ followed by lobectomy $(38.2 \%)$, and pneumonectomy $(7.5 \%)$. Patients who underwent major resections showed longer in-hospital length of stay $(\mathrm{p}=0.011)$, ICU length of stay $(\mathrm{p}=0.002)$, and mechanical ventilation days $(p=0.038)$. By extent of resection, a stepwise increase for overall $(\mathrm{p}<0.001)$, infectious $(\mathrm{p}=0.001)$, pulmonary complications $(\mathrm{p}=0.001)$ and mortality $(\mathrm{p}<0.001)$ was observed. Logistic regression analysis identified blunt trauma, hypotension, GCS $<9$, lobectomy and pneumonectomy as independent predictors of mortality and overall complications. After excluding patients with severe associated injuries (head, abdomen, heart, great vessels), there were 1,979 patients with an isolated lung injury. Again, a stepwise increase in the overall, infectious, pulmonary complications and mortality was observed. Extent of lung resection remained an independent predictor of mortality.

Conclusion: Lung resection is infrequent for traumatic injury, but carries substantial associated morbidity and mortality. The extent of lung resection is an independent predictor of postoperative overall complications and mortality.

References: 1. Huh J, Wall MJ Jr, Estrera AL, et al. Surgical management of traumatic pulmonary injury. Am J Surg. 2003 Dec;186 (6):620-624. 2. Martin MJ, McDonald JM, Mullenix PS, et al. Operative management and outcomes of traumatic lung resection. J Am Coll Surg. 2006 Sep;203 (3):336-344. 3. Matsushima K, Aiolfi A, Park C, et al. Surgical outcomes after trauma pneumonectomy: Revisited. J Trauma Acute Care Surg. 2017 May;82 (5):927932.

Disclosure: No significant relationships. 
0025

\section{RIB FIXATION VERSUS CONSERVATIVE TREATMENT FOR FLAIL CHEST AND MULTIPLE RIB FRACTURES AFTER BLUNT THORACIC TRAUMA. A MULTICENTER COHORT STUDY}

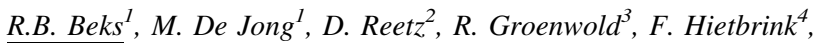
M. Edwards ${ }^{2}$, L.P.h. Leenen ${ }^{4}$, R.M. Houwert ${ }^{5}$, J.P. Frolke ${ }^{2}$

${ }^{1}$ Utrecht Traumacenter, University Medical Center Utrecht, Utrecht/ NETHERLANDS, ${ }^{2}$ Surgery, Radboudumc, Nijmegen/

NETHERLANDS, ${ }^{3}$ Julius Center For Health Sciences And Primary Care, UMC Utrecht, Utrecht/NETHERLANDS, ${ }^{4}$ Surgery, University Medical Center Utrecht, Utrecht/NETHERLANDS, ${ }^{5}$ Surgery, UMC Utrecht, Utrecht/NETHERLANDS

Introduction: A trend towards operative treatment of flail chest has evolved although evidence is limited. For patients with multiple rib fractures without a flail chest, there is even less data available. Therefore, the aim of this study was to compare rib fixation with nonoperative treatment for both patients with a flail chest and patients with multiple rib fractures.

Material and methods: All patients admitted with $\geq 3$ rib fractures between 2014 and 2017 were retrospectively included in this multicenter cohort study. Primary outcome measures were intensive care and hospital length of stay (ILOS and HLOS) for patients with a flail chest and patients with multiple rib fractures, respectively. To control for potential confounding, propensity score matching was applied.

Results: A total of 332 patients were included. The mean age was 56 (SD 17) years and $257(77 \%)$ were male. The overall mean ISS was 23 (SD 11) and the average number of rib fractures was 8 (SD 4). There were 92 patients with a flail chest, $37(40 \%)$ had rib fixation and $55(60 \%)$ conservative treatment. There were 240 patients with multiple rib fractures, $28(12 \%)$ had rib fixation and $212(88 \%)$ conservative treatment. For both patient groups, after propensity score matching, rib fixation was not associated with ILOS (for flail chest patients) nor with HLOS (for multiple rib fracture patients).

Conclusion: No advantage could be demonstrated for operative fixation of rib fractures, despite a clinical based treatment algorithm. Future studies are needed to optimize the indication and describe long-term outcome after rib fixation.

References:

Disclosure: No significant relationships.
0026

\section{CLINICAL AND RADIOLOGICAL MIDTERM RESULTS AFTER HYBRID STABILIZATION OF UNSTABLE OSTEOPOROTIC VERTEBRAL FRACTURES OF THE THORACOLUMBAR SPINE}

\author{
U. Spiegl, J. Jarvers, C. Josten, C. Heyde
}

Department Of Orthopeadics, Trauma Surgery, And Plastic Surgery, University of Leipzig, Leipzig/GERMANY

Introduction: Unstable osteoporotic fractures at the thoracolumbar mid spine can be stabilized using bisegmental fixateur interne in combination with cement augmentation of the fractured vertebral body (hybrid). However, midterm results of hybrid stabilization remain unclear.

Material and methods: This retrospective study was performed at a level I trauma center. 113 patients aged 61 and older were stabilized using hybrid stabilization after suffering an unstable osteoporotic vertebral body fractures at the thoracolumbar spine. All patients were treated by hybrid stabilization. The minimum follow-up has been two years. The operating time and complications were documented. The primary outcome parameter was the ODI score. Additionally, the VAS pain score was measured. Radiologic outcome parameters have been the following: Bisegmental reduction loss and sagittal alignement.

Results: 72 women and 41 men $(75 \pm 6.8$ years $)$ were included. Fractures consisted:24* A1 fractures, $5 *$ A2 fractures, 71 incomplete fractures, $9 \mathrm{~A} 3$ and $4 \mathrm{~A} 4$ fractures. 65 patients $(58 \%)$ were re-evaluated after a mean of 48 months. 17 patients died already (15\%). The mean operating time was 108 minutes $( \pm 35 \mathrm{~min}$ ). A total of 5 complications were documented. There were statistically significant correlations between the amount of reduction loss and ODI (correlation cofficent: $0.337, \mathrm{p}=0.033$ ), pelvic incidence and the latest bisegmental cobb angle $(p=0.043)$, and between the extent of the disability defined by ODI and the lumbar lordosis $(\mathrm{p}=0.027)$

Conclusion: Large reduction losses correlated directly with inferior outcomes. Consequently, further studies are necessary to identify potential risk factors leading to high reduction losses.

References:

Disclosure: No significant relationships. 


\section{WITHDRAWN}

0028

\section{1-YEAR OUTCOME OF PLATE VERSUS INTRAMEDULLARY FIXATION OF ROBINSON 2B.1 AND 2B.2 CLAVICLE FRACTURES}

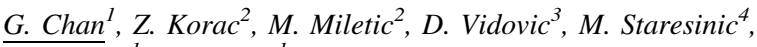
J. Phadnis ${ }^{l}$, B. Bakota ${ }^{I}$

${ }^{1}$ Department Of Trauma \& Orthopaedics, Brighton \& Sussex University Hospitals NHS Trust, Brighton/UNITED KINGDOM, ${ }^{2}$ Department Of Trauma \& Orthopaedics, General Hospital Karlovac, Karlovac/CROATIA, ${ }^{3}$ Clinic For Traumatology, , University Clinical Centre "Sisters of Mercy", Zagreb/CROATIA, ${ }^{4}$ Department Of Trauma \& Orthopaedics, University Hospital Merkur, Zagreb/ CROATIA

Introduction: Surgical fixation of displaced midshaft clavicle fractures (DMCF) has been shown to reduce rates of non-union; plate and intramedullary (IM) fixation remain the two operative modalities of choice. This is the first published study comparing the outcomes of Robinson 2B.1 (displaced two-part) and 2B.2 (displaced multifragmentary) fractures treated with plate or IM fixation.

Material and methods: Between 2000 and 2012133 patients were treated for a DMCF, 80 patients with $2 \mathrm{~B} .1$ injuries; 64 IM fixation ( $2.5 \mathrm{~mm} \mathrm{~K}$-wire), 16 plate fixation (8-hole DCP plate) and 53 patients with 2 B. 2 injuries; 27 IM fixation, 26 plate fixation. All patients were followed-up for 12-months.

Results: Non-union for 2B.1 fractures was $2 / 61$ (3.13\%) for IM fixation and $0 / 16(0.00 \%)$ for plate fixation $(\mathrm{p}=0.477)$. For $2 \mathrm{~B} .2$ injuries non-union rates were IM; $2 / 27(7.41 \%)$ and, plate; $0 / 26(0.00 \%)(\mathrm{p}=$ $0.161)$. There was no significant difference in reoperation rates between IM and plate fixation for $2 \mathrm{~B} .1(\mathrm{p}=0.559)$ and for $2 \mathrm{~B} .2$ fractures $(p=0.969)$. No instances of deep infection were recorded for either IM or plate fixation for both injuries. No significant difference in reoperation rates between IM and plate fixation for 2 B. 1 (p $=0.559)$ and $2 \mathrm{~B} .2$ fractures $(\mathrm{p}=0.969)$ was recorded.

Conclusion: IM fixation of 2B.1 and 2B.2 clavicle fractures results in no significantly increased rates of non-union, deep infection and reoperation rates compared to plate fixation. The higher rates of nonunion observed with IM fixation of 2B.2 fractures lead us to recommend consideration of IM fixation for 2-part DMCF while plate fixation would be favoured for multifragmentary DMCF.

References:

Disclosure: No significant relationships.

\section{GUNSHOT PENETRATING CHEST WOUNDS ACCOMPANIED BY SHOCK - SURGICAL TACTICS}

\author{
D. Ivchenko ${ }^{1}$, A. Koltovich $^{2}, K$. Zhestkov $^{3}$, K. Nikolaev $^{3}$
}

${ }^{1}$ Thoracic Surgery, The Main military clinical hospital National Guard troops of Russian Federation, Balashicha, Russian Federation, Moscow/RUSSIAN FEDERATION, ${ }^{2}$ Surgery, The main clinical hospital Ministry of interior, Moscow, Moscow/RUSSIAN FEDERATION, ${ }^{3}$ Surgery, The Main military clinical hospital National Guard troops of Russian Federation, Balashicha, Russian Federation, Moscow/RUSSIAN FEDERATION

Introduction: In modern armed conflicts chest damage occurs in 4-11\%.

Material and methods: Total of 138 records were reviewed. Gunshot chest penetrating wounds were obtained during the counter-terrorist operation in the North Caucasus in the period from 2000 to 2016. All the wounded were healthy men (mean age $25 \pm 3.2$ years), fit for military service. After getting injured, all the injured were in a state of traumatic shock. The main group included 71 (51.5\%) wounded, who underwent a hardware atypical resection of the lung to stop bleeding, treatment of combined injuries was carried out using the "damage control" tactics. The control group included 67 (48.5\%) of the injured, who underwent lobectomy, pneumonectomy or suturing lung wounds to stop the bleeding with lung lesions. With combined wounds, a full-fledged surgical intervention was performed on the principle of "total care". The groups were homogeneous by sex, age and severity of the lesions.

Results: All wounded with the lung root vessels damage died on the battlefield or on evacuation routes. In the main group 7 (9.9\%) were died: 2 - from heart lesions, 4 - from severe massive blood loss and shock, 1 - from a combined wound of the chest and head. In the control group $16(23.9 \%)$ died, $p<0.05$, one - from heart lesions, the rest - from blood loss and shock.

Conclusion: The use of surgical tactics aimed at reducing operational aggression in injured with gunshot penetrating chest wounds, accompanied by shock, has reduced postoperative mortality from $23.9 \%$ to $9.9 \%$.

Disclosure: No significant relationships.

\section{ADVANCES IN ANESTHESIA AND ERAS IN TRAUMA AND EMERGENCY SURGERY}

\section{$\mathbf{O 0 3 0}$}

\section{STATINS MAY BE A BETTER AGENT FOR THROMBOEMBOLIC PROPHYLAXIS}

J.M. Samuels ${ }^{1}$, J.R. Coleman ${ }^{2}$, G.R. Stettler ${ }^{2}$, G.R. Nunns ${ }^{2}$,

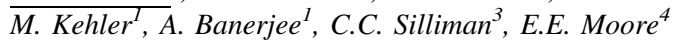

${ }^{1}$ General Surgery, University of Colorado Denver, Denver/CO/ UNITED STATES OF AMERICA, ${ }^{2}$ General Surgery, University of Colorado Denver, Denver/UNITED STATES OF AMERICA, ${ }^{3}$ Pediatrics-hem Onc And Bone Marrow Transplantation, Children's Hospital Colorado, Aurora/CO/UNITED STATES OF AMERICA, ${ }^{4}$ Surgery, Denver Health Medical Center, Denver/CO/UNITED STATES OF AMERICA 
Introduction: Statins have been associated with a decreased risk of venous thromboembolic events, but the mechanism behind this benefit remains unclear. Previous studies reported that statins likely modulate hepatic synthesis and endothelial activation. Therefore, we hypothesize that statins will not affect coagulation on whole blood from healthy volunteers in an in vitro study.

Material and methods: Citrated whole blood (WB) from healthy volunteers $(n=11)$ were treated with pravastatin to final concentrations of $50 \mathrm{nM}, 100 \mathrm{nM}$, and $200 \mathrm{nM}$. Samples were incubated for five minutes and native thrombelastography (cnTEG) was performed. Time to clot initiation (R-time), rate of clot formation (angle), maximum clot strength (MA), and clot breakdown at thirty minutes (LY30) were measured.

Results: Pravastatin treatment resulted in a statistically significant increase in LY30. WB median Ly30 was $2.9 \%$, while median Ly30 for $50 \mathrm{~nm}, 100 \mathrm{nM}$, and $200 \mathrm{nM}$ pravastatin were $7.5 \%, 9.4 \%$, and $7.7 \%$, respectively ( $\mathrm{P}<0.05$ for all). The $\mathrm{R}$ time was prolonged at the two higher doses (R time WB $9.25 \mathrm{sec}, 100 \mathrm{nM}$ pravastatin, $10.8 \mathrm{sec}$; $\mathrm{P}=0.11$, and $200 \mathrm{nM}$ pravastatin $11.5 \mathrm{sec} ; \mathrm{P}=0.02$ ). MA and Angle were not significantly different after treatment with pravastatin at all doses.

Conclusion: These data suggest that pravastatin's influence on coagulation is rapid and independent on endothelial or hepatic effects. The increase in fibrinolysis, as demonstrated by the increased Ly30, would help explain statins' ability to decrease VTE events.

References:

Disclosure: No significant relationships.

\section{1}

PERIPHERAL NERVE BLOCKADE IN THE ELBOW VERSUS FRACTURE HEMATOMA INFILTRATION FOR PAIN RELIEF DURING REDUCTION OF DISPLACED DISTAL RADIUS FRACTURES; RESULTS OF A RANDOMIZED TRIAL

\author{
T. Haak ${ }^{1}$, M. Bronkhorst ${ }^{1}$, J. Keizer ${ }^{2}$
}

${ }^{1}$ Traumasurgery, Haaglanden Medical Center, The Hague/ NETHERLANDS, ${ }^{2}$ Traumasurgery, St Antonius Ziekenhuis, Nieuwegein/NETHERLANDS

Introduction: Displaced fractures of the distal radius are commonly seen in the emergency department. In most cases, patients are treated with closed reduction and plaster immobilisation. Different options for pain relief during closed reduction have been investigated in history. The objective of this study was to determine whether a peripheral nerve block in the elbow gives better pain relief during fracture reduction then standardized infiltration of the fracture hematoma, both using lidocaine.

Material and methods: Patients with an isolated displaced fracture of the distal radius were randomized using computer-randomization in two different groups. One group recieved peripheral nerve blockade in the elbow and the other group the standardized infiltration of the fracture hematoma (Böhler anesthesia), both using lidocaine. VAS-pain scores were collected at multiple times and compared between the two groups. Primary outcome was VAS pain score during fracture reduction. Length of follow up was one week, to check for possible complications and observe secundairy fracture redislocation Results: A total of 34 patients were randomized in this study. The mean VAS pain scores at time of fracture reduction was 4,75 for the peripheral nerve blocking group and 7,67 for the fracture hematoma infiltration group. These findings were statistically different and relevant ( $\mathrm{P}<0,001)$. No complications of peripheral nerve blocking were observed.

Conclusion: Peripheral nerve blocking at the level of the elbow used for pain relief during reduction of displaced distal radial fractures seems to be a safe method en gives treated patients significantly more pain relief compared to infiltration of fracture hematoma with local anestheticum.

References: Anaesthesia for treating distal radius fractures in adults. Cochrane Library, 2009. Use of a nerve stimulator does not improve the efficacy of ultrasound guided supraclavicular blocks. Beach et al. Journal of Clinical Anesthesia, 2006 Hematoma block or Biers block for colles fracture reduction in the accident and emergency department - which is best? Kendall et al. J Accid Emerg Med 1997 Disclosure: No significant relationships.

\section{2}

\section{HIGHER TRANSTHYRETIN LEVELS OVER TIME AS AN INDEPENDENT PREDICTOR FOR BETTER OUTCOMES IN CRITICALLY ILL TRAUMA PATIENTS}

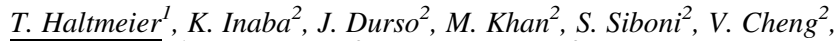

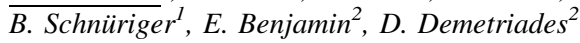

${ }^{1}$ Department Of Visceral Surgery And Medicine, Division Of Acute Care Surgery, Inselspital, Bern University Hospital, Bern/ SWITZERLAND, ${ }^{2}$ Acute Care Surgery And Surgical Critical Care, LAC+USC Medical Center, Los Angeles/CA/UNITED STATES OF AMERICA

Introduction: Transthyretin (TTR) is a known biomarker for the nutritional status and outcomes in critically ill patients. Low TTR levels have been shown to be associated with poorer clinical outcomes in trauma patients specifically. However, no data exists about the impact of TTR levels over time on outcomes in this patient population.

Material and methods: Prospective observational study including trauma patients admitted to the Intensive Care Unit (ICU) of a large US Level I trauma center 05/2014-05/2015. TTR levels at admission and all subsequent values obtained during the hospital course were recorded. Patients were observed for 28 days or until hospital discharge. The impact of TTR levels over time on clinical outcomes was analyzed using Generalized Estimating Equation (GEE) analysis.

Results: TTR was measured in 237 patients at admission, 69 patients had repeated TTR measurements and were included in the analysis. Median age was 45.0 years (IQR 29.5), 79.7\% were male sex, median ISS was 18 (IQR 15), and median TTR at admission $19.5 \mathrm{mg} / \mathrm{dl}$ (IQR 8.2) with $47.8 \%$ of the measured values below the normal range (19.0-38.0 mg/dl). Multivariable GEE analysis revealed higher TTR levels over time as an independent predictor for better outcomes, including fewer surgical site infections (OR 0.919, CI 0.871-0.970, p $=.002)$ ) and lower in-hospital mortality (OR 0.868, CI 0.764-0.986, $\mathrm{p}$ $=0.030$ ).

Conclusion: In critically ill trauma patients, higher TTR levels over time were independently associated with fewer surgical site infections and lower mortality. Based on these results, TTR levels should be considered as a prognostic and nutritional follow-up marker in this patient population.

References: 1. Cheng V, Inaba K, Haltmeier T, Gutierrez A, Siboni $\mathrm{S}$, Benjamin E, Lam L, Demetriades D: Serum transthyretin is a predictor of clinical outcomes in critically ill trauma patients. Surgery 2015, 158 (2):438-444. 2. Devakonda A, George L, Raoof S, Esan A, Saleh A, Bernstein LH: Transthyretin as a marker to 
predict outcome in critically ill patients. Clinical biochemistry 2008, 41 (14-15):1126-1130.

Disclosure: No significant relationships.

\section{$\mathbf{0 0 3 3}$}

\section{KETAMINE DURING TRACHEAL INTUBATION OF TRAUMA PATIENTS- A SYSTEMATIC REVIEW AND META-ANALYSIS}

\section{J.S. Bakgaard, T.G. Eskesen, M. Sillesen, L.S. Rasmussen, J. Steinmetz}

Department Of Anaesthesiology, Rigshospitalet, COPENHAGEN/ DENMARK

Introduction: The choice of drug used to facilitate endotracheal intubation in trauma patients during rapid sequence induction (RSI) may have an impact on survival. In this systematic review we sought to investigate whether ketamine should be preferred over other induction agents.

Material and methods: Databases were systematically searched for studies reporting RSI of adult trauma patients with ketamine compared to another induction agent (etomidate, propofol, thiopental or midazolam). The primary outcome was 30-day mortality and secondary outcomes included information on blood transfusions, length of hospital stay, and hospital mortality.

Results: Four studies were included.

A cohort study from 1976 compared thiopental $(n=26)$ with ketamine $(\mathrm{n}=14) .{ }^{1}$ The primary outcome was number of blood transfusions, and no significant difference was found. A randomized controlled trial from 2009 compared etomidate $(n=57)$ with ketamine $(\mathrm{n}=47)$ and found no significant difference in 28-day mortality (ketamine, OR 0.8 [0.4 to 2.0]). ${ }^{2}$ Two cohort studies from 2015 and 2017 also compared etomidate $(\mathrm{n}=116$ and $\mathrm{n}=526)$ with ketamine $(\mathrm{n}=145$ and $\mathrm{n}=442){ }^{3,}{ }^{4}$ No significant difference in hospital mortality between the groups was observed (etomidate, OR 1.11 [0.38 to 3.27] and OR 1.41 [0.91 to 2.16], respectively), and a fixed effect meta-analysis on those two studies did not alter this finding (etomidate, OR 1.36 [0.92 2.03]).

Conclusion: Few studies have compared induction agents for RSI in trauma patients. No significant differences have been found in mortality, length of hospital stay, or number of blood transfusions after induction with ketamine compared to other induction agents.

References: 1. Cromartie RS. Rapid anesthesia induction in combat casualties with full stomachs. Anesth Analg. 1976 Feb;55 (1):74-6. 2. Jabre P, Combes X, Lapostolle F, Dhaouadi M, Ricard-Hibon A, Vivien $B$, et al. Etomidate versus ketamine for rapid sequence intubation in acutely ill patients: a multicentre randomised controlled trial. The Lancet. 2009 Jul;374 (9686):293-300 3. Significant modification of traditional rapid sequence induction improves safety and effectiveness of pre-hospital trauma anaesthesia. Crit Care [Internet]. 2015 Dec [cited 2017 Aug 30];19 (1). Available from: http://ccforum.com/content/19/1/134 4. Upchurch CP, Grijalva CG, Russ S, Collins SP, Semler MW, Rice TW, et al. Comparison of Etomidate and Ketamine for Induction During Rapid Sequence Intubation of Adult Trauma Patients. Ann Emerg Med. 2017 Jan;69 (1):24-33.e2. Disclosure: No significant relationships.

\section{4}

\section{THROMBOELASTOGRAPHY (TEG) AND CONVENTIONAL COAGULATION TESTS (CCTS) IN TRAUMA PATIENTS: DO WE ABANDON CCTS IN TRAUMA PATIENTS?}

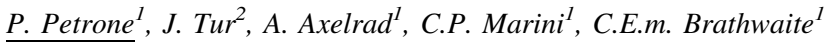 \\ ${ }^{1}$ Trauma Surgery And Critical Care, NYU Winthrop Hospital, \\ Mineola, Long Island/NY/UNITED STATES OF AMERICA, \\ ${ }^{2}$ General Surgery, Hospital Universitari Mútua de Terrassa, Terrassa/ \\ SPAIN
}

Introduction: TEG provides in-vivo assessment of viscoelastic clot strength in whole blood. CCTs (PTT, PT, INR) performed in serum do not reflect the influence of platelets. Primary aim of this study: compare TEG to CCTs in trauma patients stratified by mechanism of injury (MOI) and pre-existent coagulation status.

Material and methods: Cross-sectional study of 226 trauma patients admitted to a Level 1-trauma center (1/1/2016-12/31/2016) that had TEG and CCTs on admission. Data included demographics, comorbidities, MOI, ISS, shock, anticoagulants (AC) \& anti-platelets agents (AP). Patients stratified as: multiple trauma-135, TBI-33, and MT+TBI-62. Statistical analysis included correlation of TEG and CCTs in all groups, and a subgroup of patients on AC. Hypercoagulability was defined as moderate if $\mathrm{R} \leq 5$; severe if $\mathrm{R} \leq 5, \alpha$ angle $\geq 72$ and hypercoagulable/hyperthrombotic if $\mathrm{R} \leq 5, \alpha$ angle $\geq 72$ and $M A \geq 72$. Analysis: ANOVA, Spearman correlation, linear regression. Statistical significance $\mathrm{p}<0.05$.

Results: Patient's mean age, 75. Total of $58 \%$ were on AC, AP or both: $15 \%$-AC, $38.1 \%$-AP and $4.4 \%$ both. Mean ISS, 13.6. Patients' coagulation status by TEG: normal-27.8\%, hypercoagulable-70.3\%, hypocoagulable-1.8\%. Coagulation status not affected by: age, ISS, shock. CCTs were abnormal in $63.6 \%$ with normal-TEG. Analysis of the 23 Coumadin and 11 new oral-AC (NOAC) stratified by INR showed a normal/hypercoagulable-TEG in $21 / 23$ and 10/11, respectively. Mean INR, 2.9 (1.9-9.3). Two patients had hypocoagulableTEG, mean INR 8.8 (5.4-12.1).

Conclusion: TEG is more useful than CCTs including Coumadin/ NOAC patients. CCTs are not useful for patients on AC/NOAC, should be abandoned as standard tests. How to approach patients with TBI/visceral injuries with abnormal CCTs but normal or hypercoagulable-TEG, requires further investigation to potential medico-legal consequences.

References: 1. Hartert H.. Klin Wochenschr. 1948;26 (37-38):57783

2. Kang YG, Martin DJ, Marquez J, et al. Intraoperative changes in blood coagulation and thrombelastographic monitoring in liver transplantation. Anesth Analg. 1985;64 (9):888-96.

3. Tuman KJ, Spiess BD, McCarthy RJ, Ivankovich AD. Comparison of viscoelastic measures of coagulation after cardiopulmonary bypass. Anesth Analg. 1989;69 (1):69-75.

4. Gibbs NM, Crawford GP, Michalopoulos N. Thrombelastographic patterns following abdominal aortic surgery. Anaesth Intensive Care. 1994;22 (5):534-8.

5. Chen A, Teruya J. Global Hemostasis Testing Thromboelastography: Old Technology, New Applications. Clin Lab Med. 2009;29 (2):391-407.

6. Frith D, Goslings JC, Gaarder C, et al. Definition and drivers of acute traumatic coagulopathy: clinical and experimental investigations. J Thromb Haemost. 2010;8 (9):1919-25.

7. Kaufmann CR, Dwyer KM, Crews JD, Dols SJ, Trask AL. Usefulness of thrombelastography in assessment of trauma patient coagulation. J Trauma. 1997;42 (4):716-22. 
8. Cullinane DC, Morris JA Jr. The impact of age and medical comorbidities on the outcome following severe trauma. J Intensive Care Med 1999;14:86-94.

9. Ali JT, Daley MJ, Vadiei N, et al. Thromboelastogram does not detect pre-injury anticoagulation in acute trauma patients. Am J Emerg Med. 2017;35 (4):632-6.

10. Wikkels $\varnothing$ A, Wetterslev J, Møller AM, Afshari A. Thromboelastography (TEG) or rotational thromboelastometry (ROTEM) to monitor haemostatic treatment in bleeding patients: a systematic review with meta-analysis and trial sequential analysis. Anaesthesia. 2017;72 (4):519-31.

11. Chitlur M, Lusher J. Standardization of thromboelastography: Values and challenges. Semin Thromb Hemost. 2010;36 (7):707-11.

12. Inaba K, Rizoli S, Veigas PV, et al. 2014 Consensus conference on viscoelastic test-based transfusion guidelines for early trauma resuscitation. J Trauma Acute Care Surg. 2015;78 (6):1220-9.

13. Whiting $\mathrm{P}, \mathrm{Al} \mathrm{M}$, Westwood M, et al. Viscoelastic point-ofcare testing to assist with the diagnosis, management and monitoring of haemostasis: A systematic review and cost-effectiveness analysis. Health Technol Assess (Rockv). 2015;19 (58):1-228.

Disclosure: No significant relationships.

\section{$\mathbf{O 0 3 5}$ \\ METABOLIC CHANGES IN THE ACUTE PERIOD AFTER SEVERE PEDIATRIC TBI}

\author{
P. Menshchikov, A. Manzhurtsev, M. Ublinskiy, I. Melnikov, \\ N. Semenova, T. Akhadov
}

Radiology, Clinical and Research Institute of Emergency Pediatric Surgery and Trauma, Moscow/RUSSIAN FEDERATION

Introduction: Proton magnetic resonanse spectroscopy is unique mrthod that allows in vivo measurments of metabolic concentrations in the brain. For the first time new method [1] for simulteneous Aspartate (Asp) and glutamate (Glu) concentrations were used for evaluate excitatory neurotransmitter excititoxicity effects after severe pediatric traumatic brain injury.

Material and methods: Two groups of participants were studied: eight patients with acute severe TBI (mean age $=13.2 \pm 1.8$, mean time between MRI examination and injury $=23 \pm 4$ hours) and eleven healthy volunteers (mean age $=14.4 \pm 1.7$ ). Besides standart MR images research protocol contains proton PRESS and MEGA-PRESS MR spectra for in vivo NAA, Asp, Glu, Cr, Cho concentrations quantification. Spectra were ascuired from cubic voxel (size $25 * 25 * 30 \mathrm{~mm}$ ) in the frontal lobe (uninjured brain tissue). All investigations were performed on a Phillips 3.0T Achieva TX MRI scanner

Results: Intergroup analysis of the data showed a significant simultaneous decrease of N-acetyl aspartate (NAA) $(p<0.001)$, Glu $(p<$ $0.05)$, Creatine $(\mathrm{Cr})(p<0.001)$ and $\operatorname{Asp}(p<0.001)$ concentrations in patients with acute sTBI as compared to control group. Choline concentration was unchanged ( $\mathrm{p}=\mathrm{n} . \mathrm{s}$.).

Conclusion: Revealed Glutamate and Aspartate decrease is associated with excititoxicity (rapidly release of Glu and Asp from vesicles). Also Asp reduction might result from reduced availability of Glu. NAA, marker of neuronal activity, reduction may be associated with synthesis disruption due to reduction of major NAA precussor (Aspsrtate). Decrease in concentration may indicate increased energy costs to combat the consequences of injury. Further investigations of metabolite concentrations (espesialy Asp and Glu) and comparison with outcome indexes will help find metabolic markers for trauma outcome.

References: 1. P.E. Menshchikov, N.A. Semenova, T.A. Akhadov. Quantification of cerebral aspartate concentration in vivo using proton magnetic resonance spectroscopy. Bulletin of the Lebedev Physics

Institute, 2017, Vol. 44 (3), pp. 56-60

Disclosure: No significant relationships.

\section{6}

\section{LOW PHYSICAL PERFORMANCE IS ASSOCIATED WITH DEVELOPING PULMONARY COMPLICATIONS FOLLOWING ACUTE HIGH-RISK ABDOMINAL SURGERY}

\author{
L.T. Tengberg ${ }^{1}$, L.R. Jønsson ${ }^{2}$, N.B. Foss ${ }^{3}$, L.H. Ingelsrud ${ }^{4}$, \\ T.Q. Bandholm ${ }^{2}$, M.T. Kristensen ${ }^{2}$
}

${ }^{1}$ Surgical Department, Zealand University Hospital Køge, Køge/ DENMARK, ${ }^{2}$ Department Of Physiotherapy, Copenhagen University Hospital Hvidovre, Hvidovre/DENMARK, ${ }^{3}$ Department Of Anesthesiology And Intensive Care, Copenhagen University Hospital Hvidovre, Hvidovre/DENMARK, ${ }^{4}$ Department Of Orthopedic Surgery, Copenhagen University Hospital Hvidovre, Hvidovre/ DENMARK

Introduction: Acute high-risk abdominal (AHA) surgery is associated with high mortality rates and multiple postoperative complications, of which pulmonary complications are common. The knowledge of the association between pulmonary complications and functional performance following AHA surgery is limited. We evaluated the association between physical performance and pulmonary complications in patients following AHA surgery in a prospective cohort study.

Material and methods: Fifty consecutive patients undergoing AHA surgery was enrolled. In the first postoperative week, all patients were evaluated daily with regards to physical performance, using the Cumulated Ambulation Score (CAS, 0-6 points) for basic mobility and the activePAL ${ }^{\mathrm{TM}}$ monitor for 24-hour physical activity level. A postoperative pulmonary complication was only considered relevant if it had a Clavien Dindo Classification grade above 1 .

Results: During hospitalization, 17 patients (34\%) developed a pulmonary complication, within a mean of 3.3 days after surgery. Seven patients died within the first postoperative week, of which 5 developed a pulmonary complication. 15 out of $43(35 \%)$ of the patients who survived were still not independently mobilized (CAS $<6$ ) on postoperative day 7 . Not being independently mobilized was associated with pulmonary complications $(67 \%$ vs $33 \% \mathrm{P}=0.01$, Odds ratio $=0.15,95 \% \mathrm{CI}: 0.03-0.63)$. Patients developing pulmonary complications also lay or sat more minutes on postoperative day 7 (P $=0.04$ ).

Conclusion: Patients developing pulmonary complications were less independent and more sedentary within the first week following AHA. Causality cannot be determined, but further studies investigating strategies for an enhanced early mobilization program in the immediate postoperative period following AHA surgery, seems urgently needed.

References: 1. Pearse RM, Harrison DA, James P, et al. Identification and characterisation of the high-risk surgical population in the United Kingdom. Crit Care. 2006;10 (3):1-6. 2. Arenal JJ, BengoecheaBeeby M. Mortality with emergency abdominal surgery in the elderly. Can J Surg. 2003;46 (2):111-116. 3. Svenningsen P, Manoharan T, Foss NB, et al. Increased mortality in the elderly after emergency abdominal surgery. Dan Med J. 2014;61 (7):5-8. 4. Saunders DI, 
Murray D, Pichel AC, et al. Variations in mortality after emergency laparotomy: the first report of the UK Emergency Laparotomy Network. BJA. 2012;109 (3):368-375. 5. Vester-andersen M, Lundstrøm LH, Møller MH, et al. Mortality and postoperative care pathways after emergency gastrointestinal surgery in 2904 patients: a population-based cohort study. BJA. 2014;112 (5):860-870. 6. Barrow E, Anderson ID, Varley S, et al. Current UK practice in emergency laparotomy. Ann R Coll Surg Engl. 2013;95:599-603. 7. Tengberg LT, Bay-Nielsen M, Bisgaard T, et al. Multidisciplinary perioperative protocol in patients undergoing acute high-risk abdominal surgery. BJS. 2017;104:463-471. 8. Møller MH, Adamsen S, Thomsen RW, et al. Multicentre trial of a perioperative protocol to reduce mortality in patients with peptic ulcer perforation. Br J Surg. 2011;98:802-810. 9. Kehlet H, Wilmore DW. Evidence-Based Surgical Care and the Evolution of Fast-Track Surgery. Ann Surg. 2008;248:189-198. 10. Kehlet H, Mythen M. Why is the surgical high-risk patient still at risk? Br J Anaesth. 2011;106 (3):289-291. 11. Kehlet H, Wilmore DW. Multimodal strategies to improve surgical outcome. Am J Surg. 2002;183:630-641. 12. Adamina M, Kehlet H, Tomlinson GA, et al. Enhanced recovery pathways optimize health outcomes and resource utilization: A meta-analysis of randomized controlled trials in colorectal surgery. Surgery. 2011;149:830-840. 13. Dorcaratto D, Grande L, Pera M. Enhanced Recovery in Gastrointestinal Surgery: Upper Gastrointestinal Surgery. Dig Surg. 2013;30:70-78. 14. Peden C, Scott MJ. Anesthesia for Emergency Abdominal Surgery. Anesthesiol Clin. 2015;33:209-221. 15. Jakobsen DH, Sonne E, Andreasen $\mathrm{J}$, et al. Convalescence after colonic surgery with fast-track vs conventional care. Color Dis. 2006;8:683-687. 16. Castelino T, Fiore JF, Niculiseanu $P$, et al. Outcomes The effect of early mobilization protocols on postoperative outcomes following abdominal and thoracic surgery: A systematic review. Surgery. 2016;159 (4):991-1003. 17. Lawrence VA, Hilsenbeck SG, Mulrow CD, et al. Incidence and Hospital Stay for Cardiac and Pulmonary Complications after Abdominal Surgery. J Gen Intern Med. 1995;10:671-678. 18. Suetta C, Magnusson SP, Beyer N, et al. Effect of strength training on muscle function in elderly hospitalized patients. Scand J Med Sci Sport. 2007;17:464-472. 19. Haines KJ, Skinner EH, Berney S. Association of postoperative pulmonary complications with delayed mobilisation following major abdominal surgery: an observational cohort study. Physiotherapy. 2013;99:119-125. 20. Bautmans I, Njemini R, De Backer J, et al. Surgery-Induced Inflammation in Relation to Age, Muscle Endurance, and Self-Perceived Fatigue. $J$ Gerontol A Biol Sci Med Sci. 2010;65 (3):266-273. 21. Clavien PA, Barkun J, de Oliveira ML, et al. The Clavien-Dindo Classification of Surgical Complications. Ann Surg. 2009;250 (2):187-196. 22. Levy MM, Fink MP, Marshall JC, et al. 2001 SCCM/ESICM/ACCP/ATS/ SIS International Sepsis Definitions Conference. Crit Care Med. 2003;29:530-538. 23. Oken MM, Creech RH, Tormey DC, et al. Toxicity and response criteria of the Eastern Cooperative Oncology Group. Am J Clin Oncol. 1982;5 (6):649-655. 24. Owens WD, Felts JA, Spitznagel EL. ASA Physical Status Clssifications: A Study of Consistency of Ratings. Aneshesiology. 1978;49:239-243. 25. Parker MJ, Palmer CR. A New Mobility Score for Predicting Mortality after Hip Fracture. J Bone Jt Surg [Br]. 1993;755 (75):797-798. 26. Kristensen MT, Kehlet H. Most patients regain prefracture basic mobility after hip fracture surgery in a fast-track programme. Dan Med J. 2012;59 (6):A4447. 27. Kristensen MT, Bandholm T, Foss $\mathrm{NB}$, et al. High Inter-Tester Reliability of the New Mobility Score in Patients with Hip Fracture. J Rehabil Med. 2008;40:589-591. 28. von Elm E, Altman DG, Egger M, et al. The strengthening the reporting of observational studies in epidemiology (STROBE) statement: Guidelines for reporting observational studies. Int J Surg. 2014;12 (12):1495-1499. 29. Foss NB, Kristensen MT, Kehlet H. Prediction of postoperative morbidity, mortality and rehabilitation in hip fracture patients: the cumulated ambulation score. Clin Rehab. 2006;20:701708. 30. Kristensen MT, Jakobsen TL, Nielsen JW, et al. Cumulated
Ambulation Score to evaluate mobility is feasible in geriatric patients and in patients with hip fracture. Dan Med J. 2012;59 (7):A4464. 31. Kristensen MT, Andersen L, Bech-Jensen R, et al. High intertester reliability of the Cumulated Ambulation Score for the evaluation of basic mobility in patients with hip fracture. Clin Rehabil. 2009;23:1116-1123. 32. Kristensen MT, Foss NB, Ekdahl C, et al. Prefracture functional level evaluated by the New Mobility Score predicts in-hospital outcome after hip fracture surgery. Acta Orthop. 2010;81 (3):296-302. 33. Ryan CG, Grant PM, Tigbe WW, et al. The validation of a novel activity monitor in the measurement of posture and motion during everyday activities. Br J Sport Med. 2006;40:992997. 34. Sellers C, Dall P, Grant M, et al. Gait \& Posture Validity and reliability of the activPAL3 for measuring posture and stepping in adults and young people. Gait Posture. 2016;43:42-47. 35. Taraldsen K, Askim T, Sletvold O, et al. Evaluation of a Body-Worn Sensor System to Measure Physical Activity in Older People With Impaired Function. Phys Ther. 2011;91:277-285. 36. Grant PM, Granat MH, Thow M, et al. Analyzing Free-Living Physical Activity of Older Adults in Different Environments Using Body-Worn Activity Monitors. J Aging Phys Act. 2010:171-184. 37. Huskisson EC. Measurement of pain. Lancet. 1974;7 (21):1127-1131. 38. Williamson A, Hoggart B. Pain: a review of three commonly used pain rating scales. J Clin Nurs. 2005;14 (7):798-804. 39. Covinsky KE, Palmer RM, Fortinsky RH, et al. Loss of Independence in Activities of Daily Living in Older Adults Hospitalized with Medical Illnesses: Increased Vulnerability with Age. JAGS. 2003;51:451-458. 40. Gill TM, Allore HG, Zhenchao G. Restricted Activity and Functional Decline Among Community-Living Older Persons. Arch Intern Med. 2003;163:1317-1322. 41. Roubenoff R. Sarcopenia: Effects on Body Composition and Function. J Gerontol. 2003;58 (11):1012-1017. 42. Hirvensalo M, Rantanen T, Heikkinen E. Mobility Difficulties and Physical Activity as Predictors of Mortality and Loss of Independence in the Community-Living Older Population. J Am Geratr Soc. 2000;48:493-498. 43. Roubenoff R. Physical Activity, Inflammation, and Muscle Loss. Nutr Rev. 2007;65 (12):208-213. 44. Browning L, Denehy L, Scholes RL. The quantity of early upright mobilisation performed following upper abdominal surgery is low: an observational study. Aust J Physiother. 2007;53 (1):47-52. 45. Bailey JG, Davis PJB, Levy AR, et al. The impact of adverse events on the health care costs for older adults undergoing nonelective abdominal surgery. Can J Surg. 2016;59:172-179. 46. Gill TM, Allore HG, Holford TR, et al. Hospitalization, Restricted Activity, and the Development of Disability Among Older Persons. JAMA. 2004;292 (17):2115-2124. 47. Gill TM, Allore HG, Gahbauer EA, et al. Change in Disability After Hospitalization or Restricted Activity in Older Persons. JAMA. 2010;304 (17):1919-1928. 48. Lees MC, Merani S, Tauh K, et al. Perioperative factors predicting poor outcome in elderly patients following emergency general surgery: a multivariate regression analysis. Can J Surg. 2015;58:312-317. 49. Khuri SF, Henderson WG, Depalma RG, et al. Determinants of Long-Term Survival After Major Surgery and the Adverse Effect of Postoperative Complications. Ann Surg. 2005;242 (3):326-343. 50. Shea RA, Brooks JA, Dayhoff NE, et al. Pain intensity and postoperative pulmonary complications among the alderly after abdominal surgery. Hear Lung. 2002;31 (6):440-449. 51. Lawrence VA, Cornell JE, Smetana GW. Strategies To Reduce Postoperative Pulmonary Complications after Noncardiothoracic Surgery: Systematic Review for the American College of Physicians. Ann Intern Medicne. 2006;144 (8):596-608. 52. Smetana GW, Lawrence VA, Cornell JE. Preoperative Pulmonary Risk Stratification for Noncardiothoracic Surgery: Systematic Review for the American College of Physicians. Ann Intern Med. 2006;144:581-595. 53. Mackay MR, Ellis E, Johnston C. Randomised clinical trial of physiotherapy after open abdominal surgery in high risk patients. Aust J Physiother. 2005;51:151-159. 54. Nielsen KG, Holte $\mathrm{K}$, Kehlet $\mathrm{H}$. Effects of posture on postoperative pulmonary function. Acta Anaesthesiol Scand. 2003;47:1270-1276. 55. Havey R, 
Herriman E, O'Brien DO. Guarding the Gut - Early Mobility After Abdominal Surgery. 2013;36 (1):63-72. 56. Galvin E, Boesjes H, Hol $\mathrm{J}$, et al. Modafinil reduces patient-reported tiredness after sedation/ analgesia but does not improve patient psychomotor skills. Acta Anaesthesiol Scand. 2010;54 (2):154-161. 57. de la Motte L, Kehlet $\mathrm{H}$, Vogt K, et al. Preoperative Methylprednisolone Enhances Recovery After Endovascular Aortic Repair. Ann Surg. 2014;260 (3):540-549.

Disclosure: No significant relationships.

\section{7}

\section{P MRS STUDY OF BRAIN CELLULAR METABOLISM ACTIVATION BY A LOW-PRESSURE HYPERBARIC OXYGENATION SESSION}

A. Manzhurtsev ${ }^{1}$, O. Vasiukova ${ }^{2}$, P. Menshchikov ${ }^{3}$, N. Zaitseva $^{4}$, V. Sergeeva ${ }^{4}, M$. Ublinskiy ${ }^{3}$, T. Akhadov ${ }^{3}$, N. Semenova ${ }^{I}$

${ }^{1}$ 0501, N.M. Emanuel Institute of Biochemical Physics of RAS, Moscow/RUSSIAN FEDERATION, ${ }^{2}$ Medical Physics, NRNU MEPHi, Moscow/RUSSIAN FEDERATION, ${ }^{3}$ Radiology, Clinical and Research Institute of Emergency Pediatric Surgery and Trauma, Moscow/RUSSIAN FEDERATION, ${ }^{4}$ Hyperbaric Oxygenation,

Clinical and Research Institute of Emergency Pediatric Surgery and Trauma, Moscow/RUSSIAN FEDERATION

Introduction: Hypoxia causes disorders in brain energy metabolism. One of perspective ways of recovey is hyperbaric oxygenation (HBO). The in vivo studies of HBO influence on energy metabolism might be of interest, ${ }^{31} \mathrm{P}$ Magnetic Resonance Spectroscopy (MRS) is a unique noninvasive method for it. Aim: reveal the effect of one lowpressure (1.2 atmosphere absolute, ATA) HBO session on ${ }^{31} \mathrm{P}$ MRS metabolites of the normal human brain.

Material and methods: Participants: 11 healthy subjects aged 18-30. The plan of the study is as follows. MRI scanner Philips Achieva 3.0T and $31 \mathrm{P} / 1 \mathrm{H}$ Rapid Biomed head coil were used to perform a short anatomical study and collect two-dimensional ${ }^{31} \mathrm{P}$ MRS data (15 minutes). Then the subject proceeded to hyperbaric chamber Sechrist 3200 for 50 minutes. After that the MRS study was repeated. Spectroscopy data before and after $\mathrm{HBO}$ was processed in jMRUI program. The intensities of resonance lines (that are proportional to metabolite concentrations) in spectra after $\mathrm{HBO}$ were normalized on the according intensities in spectra before HBO.

Results: After HBO session there was a statistically significant ( $p<$ 0.01 ) decrease of creatine phosphate (PCr) by $\sim 3 \%$ and increase (by $\sim 6 \%$ ) of $\alpha$-ATP peak integral intensities.

Conclusion: Since $\beta$-ATP remains unchanged, [ATP] is constant. We suppose that we revealed $[\mathrm{NAD}+]$ and/or $[\mathrm{NADH}]$ increase, because these peaks underlay a massive $\alpha$-ATP peak. Decrease of PCr also happens due to NAD $(\mathrm{H})$ synthesis. [NAD $(\mathrm{H})$ ] plays an important role in cell energy metabolism[1], so the result obtained might be a confirmation of $\mathrm{HBO}$ effectiveness even at low pressures. Further investigations for trauma group are required.

References: 1. Berger F, Ramirez-Hernandez MH, Ziegler M. The new life of a centenarian: signalling functions of NAD (P). Trends Biochem Sci 2004;29:111-118.

Disclosure: No significant relationships.
0038

\section{A RISE IN NEUTROPHIL CELL SIZE PRECEDES ORGAN DYSFUNCTION AFTER TRAUMA}

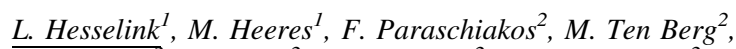

A. Huisman $^{2}$, I. Hoefer ${ }^{2}$, M. De Groot ${ }^{2}$, W. Van Solinge ${ }^{2}$,

M. Dijkgraaf ${ }^{3}$, P. Hellebrekers ${ }^{1}, K$. Van Wessem ${ }^{1}$, L. Koenderman ${ }^{4}$, L.P.h. Leenen ${ }^{1}$, F. Hietbrink ${ }^{1}$

${ }^{1}$ Trauma Surgery, University Medical Center Utrecht, Utrecht/ NETHERLANDS, ${ }^{2}$ Clinical Chemistry And Hematology, University Medical Center Utrecht, Utrecht/NETHERLANDS, ${ }^{3}$ Clinical

Research Unit, Academic Medical Center, Amsterdam/ NETHERLANDS, ${ }^{4}$ Laboratory For Translational Immunology, University Medical Center Utrecht, Utrecht/NETHERLANDS

Introduction: Organ dysfunction (ARDS and/or MODS) remains a major cause of morbidity after trauma. The development of organ dysfunction is determined by the inflammatory response, in which neutrophils are important effector cells. A femoral fracture particularly predisposes for the development of organ dysfunction. This study investigated the chronologic relation between neutrophil characteristics and organ dysfunction in trauma patients with a femoral fracture.

Material and methods: Patients with a femoral fracture presenting at the University Medical Centre Utrecht between 2007 and 2013 were included. Data of neutrophil characteristics from standard hematological analyzers were recorded on a daily basis until the 28th day of hospital stay or until discharge. Generalized Estimating Equations were used to compare outcome groups.

Results: In total 157 patients were analyzed, of whom 81 had polytrauma and 76 monotrauma. Eleven patients developed organ dysfunction. Patients with organ dysfunction showed a significant increase in neutrophil count $(p=0.02)$, neutrophil cell size $(p=0.03)$ and neutrophil complexity $(\mathrm{p}=0.00)$, and a significant decrease in neutrophil lobularity $(\mathrm{p}=0.00)$. The rise in neutrophil cell size preceded the clinical manifestation of organ dysfunction.

Conclusion: Patients who develop organ dysfunction post injury show changes in neutrophil characteristics before organ dysfunction becomes clinically evident. These findings may contribute to the development of prognostic tools for immune mediated complications in trauma patients.

References:

Disclosure: No significant relationships.

\section{9}

\section{SHOCK INDEX IN PATIENT WITH SOLID ORGAN INJURY TO PREDICT THE NEED FOR BLOOD TRANSFUSION AND LAPAROTOMY}

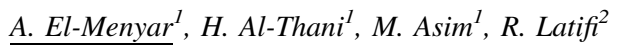

${ }^{1}$ Trauma Surgery, Hamad General Hospital, Doha/QATAR, ${ }^{2}$ Surgery, Westchester medical center, valhalla/NY/UNITED STATES OF AMERICA

Introduction: We aimed to assess the utility of shock index (SI) in patients with solid organ injury (SOI) post abdominal trauma to predict the need for blood transfusion and exploratory laparotomy (ExLap) in a Level 1 Trauma center. 
Material and methods: We conducted a retrospective analysis for patients with SOI between June 2011 and June 2014. Patients were categorized according to SI on admission (low SI $<0.8$ and high SI $\geq 0.8$ ).

Results: Of the total 4500 patients admitted with blunt trauma, 546 sustained solid organ injuries over 3 years. Half of the patients ( $\mathrm{n}=$ 273) had SI $\geq 0.8$. In comparison to low SI, patients with high SI significantly were younger, had lower pulse pressure (PP), higher ISS and lower GCS and fewer TRISS. Abdomen AIS, liver and splenic injury, positive FAST, and hemothorax were comparable in the 2 groups. High SI was associated with lower hematocrit, and higher serum lactate $(\mathrm{p}=0.001)$. Retroperitoneal hematoma, ExLap, blood transfusion, massive transfusion protocol (MTP), and sepsis were more likely in patients with high SI $(\mathrm{p}=0.01)$. Hospital length of stay (HLOS) and mortality (12.8\% vs 3\%) were greater in patients with high SI $(\mathrm{p}=0.001)$. SI was significantly correlated with serum lactate $(r=0.39)$, hematocrit $(r=-0.32)$, PP $(r=-0.44)$, HLOS $(r=0.22)$, ISS $(r=0.30)$, TRISS $(r=-0.20)$, and amount of transfused blood $(r=$ 0.25). After adjustment for age, ISS, abdomen AIS, lactate, hematocrit, and FAST, multivariate analysis showed that high SI is independent predictor of blood transfusion (OR 3.0; 95\%CI 1.615.19).

Conclusion: SI is a simple reliable predictor in patient with SOI post abdominal trauma.

References: American Journal of Emergency Medicine 31 (2013) 1260-1263

Disclosure: No significant relationships.

\section{O040}

\section{DETERMINATION OF THE INNATE IMMUNE STATUS BY A POINT-OF-CARE AUTOMATED LOAD-AND-GO FLOW CYTOMETER IN AN ACUTE TRAUMA SETTING: A FEASIBILITY STUDY}

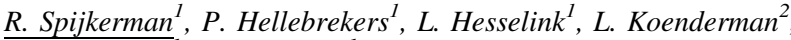 $\overline{\text { L.P.h. Leenen }}^{1}$, F. Hietbrink ${ }^{l}$}

${ }^{1}$ Trauma Surgery, University Medical Center Utrecht, Utrecht/ NETHERLANDS, ${ }^{2}$ Laboratory For Translational Immunology, University Medical Center Utrecht, Utrecht/NETHERLANDS

Introduction: Major trauma is followed by an overwhelming immune response. Changes in the functional phenotype of circulating neutrophils determined by flow cytometry is an adequate read-out of the amplitude and changes of the immune status after trauma. Until now, the technical and logistical difficulties of flow cytometry precluded clinical applicability of such an approach. Therefore, the aim of this study was to investigate the implementation of a point-of-care immunological test applying an automated load-and-go flow cytometer in an acute trauma care setting in the emergency bay.

Material and methods: We will perform a mono-center pilot study in a level one trauma center in The Netherlands. All trauma patients (between 18 and 80 years) will be included that visited the shock room. Blood will be drawn together with the diagnostic workup and the tube is placed directly in the load-and-go flow cytometer. This cytometer is able to mix, pierce the cap, prepare and analyse the sample. Our primary endpoint is the practical feasibility.

Results: When this pilot study shows that implementing a point-ofcare immunological test in the acute trauma setting is feasible, an international collaboration will be initiated.

Conclusion: Several level one trauma centers around the world will be equipped with the automated load-and-go flow cytometer. The aim of this international multicenter study is to provide enough power to build a model to identify the (trauma) patient at risk for infectious or inflammatory complications during hospital admission.

References:

Disclosure: No significant relationships.

\section{STRATEGIES IN BLEEDING}

\section{1}

\section{RESUSCITATIVE ENDOVASCULAR BALLOON OCCLUSION OF THE AORTA IN EXPERIMENTAL CARDIOPULMONARY RESUSCITATION - AORTIC OCCLUSION LEVEL MATTERS}

\author{
E.M. Dogan ${ }^{1}$, T.M. Hörer ${ }^{2}$, K.F. Nilsson ${ }^{2}$
}

${ }^{1}$ Department Of Cardiothoracic And Vascular Surgery, Faculty of Medicine and Health, Örebro University, Örebro, Sweden, Örebro/ SWEDEN, ${ }^{2}$ Department Of Cardiothoracic And Vascular Surgery, Faculty of Medicine and Health, Örebro University, Örebro, Sweden, ÖREBRO/SWEDEN

Introduction: Aortic occlusion during cardiopulmonary resuscitation (CPR) increases systemic arterial pressures (1). Correct thoracic placement during the resuscitative endovascular balloon occlusion of the aorta (REBOA) may be important for achieving effective CPR.

Material and methods: Cardiac arrest was induced in 27 anesthetized pigs. After $7 \mathrm{~min}$ of CPR with a mechanical compression device, REBOA in the thoracic descending aorta at heart level (zone $\mathrm{Ib}$, REBOA-Ib, $\mathrm{n}=9$ ), at diaphragmatic level (zone Ic, REBOA-Ic, $\mathrm{n}$ $=9$ ) or no occlusion (control, $\mathrm{n}=9$ ) was initiated. The primary outcome was systemic arterial pressures during CPR.

Results: During CPR, REBOA-Ic increased systolic blood pressure from $86 \mathrm{mmHg}$ (CI 71-101) to $128 \mathrm{mmHg}$ (CI 107-150, p < 0.001). Simultaneously, mean and diastolic blood pressures increased significantly in REBOA-Ic ( $<0.001$ and $p=0.006$, respectively), and were higher than in REBOA-Ib ( $p=0.04$ and $p=0.02$ respectively) and control ( $p=0.005$ and $p=0.003$, respectively). REBOA-Ib did not significantly affect systemic blood pressures. Arterial $\mathrm{pH}$ decreased more in control than in REBOA-Ib and REBOA-Ic after occlusion $(\mathrm{p}=0.004$ and $\mathrm{p}=0.005$, respectively).

Conclusion: Thoracic aortic occlusion in zone Ic during CPR may be more effective in increasing systemic arterial pressures than occlusion in zone Ib. The inflated balloon could interfere with the compression or filling of the heart during mechanical chest compressions. REBOA during CPR was found to be associated with a more favorable acid-base status of circulating blood. If REBOA is used as an adjunct in CPR, it may be of importance to carefully determine the aortic occlusion level, in both traumatic and non-traumatic causes of cardiac arrest.

References: 1. Gedeborg R, Rubertsson S, Wiklund L: Improved haemodynamics and restoration of spontaneous circulation with constant aortic occlusion during experimental cardiopulmonary resuscitation. Resuscitation 40: 171-180, 1999.

Disclosure: No significant relationships. 
0042

\section{„STOP THE BLEED - SAVE A LIFE!“ INTRODUCING CIVILIAN BYSTANDERS AS IMMEDIATE RESPONDERS IN THE NETHERLANDS}

\author{
L. Geeraedts $^{\text {}}$, G. Giannakopoulos ${ }^{1}$, I. Ambagtsheer ${ }^{2}$, D. Scholler ${ }^{3}$,
}

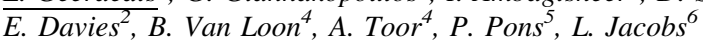

${ }^{1}$ Trauma Surgery, VU University Medical Center, Amsterdam/ NETHERLANDS, ${ }^{2}$ Education, VUmc Academy, Amsterdam/ NETHERLANDS, ${ }^{3}$ Business Development, Innovation Exchange Amsterdam, Amsterdam/NETHERLANDS, ${ }^{4}$ Public Relations, Acute Care Area Network Northwest Netherlands, Amsterdam/ NETHERLANDS, ${ }^{5}$ Emergency Medicine, Denver Health, Denver/ CO/UNITED STATES OF AMERICA, ${ }^{6}$ Surgery, Hartford Hospital, Hartford/CT/UNITED STATES OF AMERICA

Introduction: Due to terrorist attacks, we are increasingly confronted with civilian victims sustaining military-type of injuries with lifethreatening hemorrhage. Direct control of external bleeding is the most important life -saving measurement. The military principles of tourniquet application and wound-packing have been successfully translated to civilian prehospital care. However, to save more lives, by-standers at the scene should act as immediate responders to stop life-threatening external hemorrhage in victims.

Material and methods: After training in Denver, CO, USA, surgeons from VU University Medical Center introduced the non-profit Stop the bleed - save a life initiative and course for civilians in the Netherlands. The course includes the identification of life-threatening hemorrhage and bleeding control through direct pressure, tourniquet application and wound-packing with hemostatic gauzes. Proctored practical skills on tourniquet application and wound-packing form the core of the course.

Results: In 6 months, 892 immediate responders (citizens, first-aid providers) were trained as well as police/firefighter instructors. 493 persons (201 instructors) took the course. 399 persons have already been trained by these instructors. Instructors have free access to the educational material. The initiative provides for an informative website (www.stopdebloedingredeenleven.nl). Purchase of bleeding control-kits as well as wound-packing models and tourniquets for practice is facilitated. The initiative has the formal support of the Dutch Society for Trauma Surgery.

Conclusion: The enthusiasm is overwhelming. The course should be followed by all medical and nursing students, first aid providers, police-officers and firefighters. Further dissemination is planned through Acute Care Networks in The Netherlands. For further introduction throughout Europe, trauma surgeons may take the lead.

References: www.stopdebloedingredeenleven.nl

Disclosure: No significant relationships.

\section{3}

PREDICTIVE VALUE OF IL-1 AND IL-6 IN POLYTRAUMA PATIENTS WITH PELVIC FRACTURES - CAN THEY GUIDE THE TREATMENT?

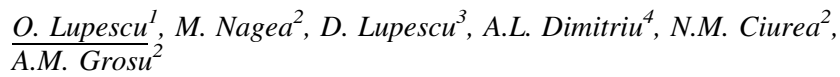

${ }^{1}$ Orthopaedics And Trauma, Clinical Emergency Hospital, University of Medicine and Pharmacy "Carol Davila" Bucharest, Bucharest/ ROMANIA, ${ }^{2}$ Orthopaedics And Trauma, CLINICAL EMERGENCY
HOSPITAL, BUCHAREST/ROMANIA, ${ }^{3}$ Anaesthesia And Intensive Care, BUFTEA HOSPITAL, BUFTEA/ROMANIA, ${ }^{4}$ Clinical Emergency Hospital, Orthopaedics And Trauma, University of Medicine and Pharmacy Carol Davila, BUCHAREST/ROMANIA

Introduction: The purpose is to evaluate potential correlations between IL- 1 and IL- 6 and the patients's outcome and the possibility to use them for guiding the treatment in polytrauma. This concept is part of the "Damage Control Surgery" (DCS), adapting the treatment to the patient status so as to avoid the "second hit phenomenon"

Material and methods: The authors prospectively evaluate 20 polytrauma patients with pelvic fractures treated between 01.06.2015 and 01.06.2016 in a Level 1 Trauma Centre. "Early total care" was used for 6 stable patients, while external fixation (DCS) for the other 14 borderline and unstable, followed by internal fixation only after the systemic inflammatory response subsided. General complications (mortality, ARDS, MSOF) and length of stay were evaluated parallel with IL-1 and IL-6

Results: The values of IL-1 and IL 6 were correlated with the incidence of complications and hospital stay, and persistant high values were associated with general complications. More than that, their values, especially IL-6, had guiding value: when the definitive treatment was performed on the decendent curve of IL, the outcome was favorable, while in patients where major surgery was performed on the ascending line of ILs, systemic complications appeared. This is a different aspect from that of the classical recommendation for definitive surgery in a fix interval, suggesting that individual assessment based on IL values can guide the treatment

Conclusion: This study demonstrated that the dynamics of IL-1 and IL-6 can have predictive value and should be used for individually guiding the treatment, instead of fix intervals, accepted today, as each patient has its own reactivity

References: Dekker A-BE, Krijnen P, Schipper IB. Predictive value of cytokines for developing complications after polytrauma. World Journal of Critical Care Medicine. 2016;5 (3):187-200. doi:10.5492/ wjccm.v5.i3.187.

Disclosure: No significant relationships.

\section{4}

\section{COMPLICATIONS IN PATIENTS WHO DID AND DID NOT UNDERGO RESUSCITATIVE ENDOVASCULAR OCCLUSION OF THE AORTA: A PROPENSITY SCORE MATCHING ANALYSIS}

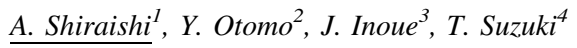

${ }^{1}$ Emergency And Trauma Center, Kameda Medical Center, Kamogawa/JAPAN, ${ }^{2}$ Emergency And Disaster Medicine, Tokyo Medical and Dental University Hospital, Tokyo/JAPAN, ${ }^{3}$ Emergency Department, Yamanashi Prefectural Central Hospital, Kofu/JAPAN, ${ }^{4}$ Department Of Emergency And Critical Care Medicine, Kimitsu Chuo Hospital, Chiba/JAPAN

Introduction: Mortality rates have been reported to be higher in Japanese trauma patients who did undergo emergency torso surgery with resuscitative endovascular occlusion of the aorta (REBOA) than that in those without REBOA. [1] We assessed the prevalence of subsequent complications in propensity-score matched, severely-injured and surgically-treated trauma patients who did and did not undergo REBOA.

Material and methods: Trauma subjects who underwent any kind of emergency torso surgery and were registered in the Japan Trauma 
Databank were retrospectively analyzed. A logistic regression analysis with 55 pretreatment variables was used to estimate a propensity score to predict use of REBOA. A 1:1 propensity-score matching analysis compared in-hospital mortality and morbidity rates.

Results: Propensity-score matching included 691 patients each who did and did not undergo REBOA. Use of REBOA was associated with significantly higher rates of in-hospital mortality $(59.2 \%$ versus $39.2 \%$, difference $+20.0 \%$ [95\% confidence interval $(\mathrm{CI})+14.7 \%$, $+25.3 \%], \mathrm{P}<0.001)$ and all-type complications $(47.3 \%$ versus $34.9 \%$, difference $+12.4 \%$ [95\% CI $+7.3 \%,+17.6 \%], \mathrm{P}<0.001$ ). REBOA was also significantly associated with complications in the circulatory system $(24.7 \%$ versus $13.5 \%$, difference $+11.3 \%$ [95\% CI $+7.2 \%,+15.4 \%], \mathrm{P}<0.001)$, digestive system $(9.1 \%$ versus $5.5 \%$, difference $+3.6 \%[95 \% \mathrm{CI}+0.9 \%,+6.4 \%], \mathrm{P}=0.010)$, and coagulation system $(25.0 \%$ versus $13.7 \%$, difference $+11.3 \%$ [95\% CI $+7.2 \%,+15.4 \%$ ], $\mathrm{P}=0.001$ )

Conclusion: Use of REBOA was associated with higher morbidity and mortality rates. The anatomical distribution of these morbidities suggests their association with ischemia after REBOA.

References: Inoue J, Shiraishi A, Yoshiyuki A, Haruta K, Matsui H, Otomo Y. Resuscitative endovascular balloon occlusion of the aorta might be dangerous in patients with severe torso trauma: A propensity score analysis. J Trauma Acute Care Surg. 2016;80:559-66 Disclosure: No significant relationships.

\section{5}

\section{A NEW OCCLUSION DEVICE FOR TEMPORARY HEMOSTASIS OF THE VENA CAVA IS AS EFFECTIVE AS TOTAL HEPATIC VASCULAR ISOLATION IN A PORCINE MODEL OF RETRO-HEPATIC INJURY}

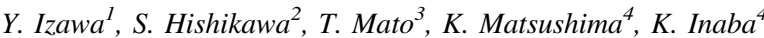

${ }^{1}$ Surgery And Emergency Medicine, Saiseikai Utsunomiya Hospital, Tochigi-ken/JAPAN, ${ }^{2}$ Center For Development Of Advanced Medical Technology, Jichi Medical University, Tochigi-ken/JAPAN, ${ }^{3}$ Emergency And Critical Care Medicine, Jichi Medical University, Tochigi-ken/JAPAN, ${ }^{4}$ Surgery And Emergency Medicine, University of Southern California, Los Angeles/CA/UNITED STATES OF AMERICA

Introduction: Injury to the retro-hepatic Inferior Vena Cava (IVC) is a highly lethal injury. A novel double balloon occlusion device with a hollow core for venous return was developed to provide temporary hemostasis. This aim of this study was to compare the hemostatic efficacy of Total Hepatic Vascular Isolation (THVI) against the novel Occlusion Device (OD).

Material and methods: Twelve swine were randomized to one of two groups, THVI versus OD. After a $5 \mathrm{~mm}$ incision was made on the supra-hepatic IVC, either THVI or insertion of the OD was performed. Temporary hemostasis was achieved and the injury was repaired primarily. Ability to achieve temporary hemostasis, time to hemostasis and repair of injury, blood loss, fluid requirements, mean arterial pressure, heart rate and stroke volume variation were compared.

Results: Temporary hemostasis was achieved in all cases, allowing direct suture repair of the injury. The time required to obtain temporary hemostasis (THVI: $4.3 \pm 0.8 \mathrm{~min}, \mathrm{OD}: 5.0 \pm 1.7 \mathrm{~min}$, mean \pm $\mathrm{SD})$, time to hemostasis $(6.5 \pm 2.9,5.3 \pm 2.3 \mathrm{~min})$, the volume of hemorrhage $(402 \pm 192,416 \pm 491 \mathrm{~g}$ at $10 \mathrm{~min}, 623 \pm 17,684 \pm 459$ at 1 hour), and fluid requirements $(1025 \pm 70,950 \pm 50 \mathrm{ml}$ at $10 \mathrm{~min}$, $1580 \pm 120,1675 \pm 650$ at 1 hour) were similar between groups.
Mean arterial pressures at the end of the repair, heart rate, and stroke volume variation did not differ between groups. All animals survived to the end point at one hour after injury.

Conclusion: The new device is as effective as total hepatic vascular isolation for obtaining temporary hemorrhage control and repair of major retro-hepatic venous injury in this porcine model.

References:

Disclosure: No significant relationships.

\section{6}

\section{REBOA: EVIDENCE FOR DIRECT BLEEDING CONTROL IN HEMODYNAMIC UNSTABLE PATIENTS}

B.L.s. Borger Van Der Burg ${ }^{1}$, T.T.c.f. Van Dongen ${ }^{2}$, J.J. Morrison ${ }^{3}$,

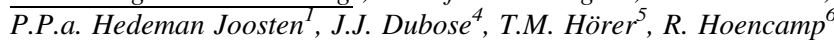

${ }^{1}$ Department Of Surgery, Alrijne Hospital, Leiderdorp/ NETHERLANDS, ${ }^{2}$ Defense Healthcare Organisation, Ministry of Defense, Utrecht/NETHERLANDS, ${ }^{3} \mathrm{R}$ Adams Cowley Shock Trauma Center, University of Maryland Medical System, Baltimore/ MD/UNITED STATES OF AMERICA, ${ }^{4}$ Division Of Vascular Surgery, David Grant Medical Center, Sacramento/CA/UNITED STATES OF AMERICA, ${ }^{5}$ Department Of Cardiothoracic And Vascular Surgery, Örebro University Hospital, Örebro/SWEDEN, ${ }^{6}$ Division Of Surgery, Leiden University Medical Center, Leiden/ NETHERLANDS

Introduction: Circulatory collapse is a leading cause of mortality among traumatic major exsanguination and in ruptured aortic aneurysm patients. Approximately $40 \%$ of patients die before hemorrhage control is achieved. Resuscitative endovascular balloon occlusion of the aorta (REBOA) is an adjunct designed to sustain the circulation until definitive surgical or endovascular repair. A systematic review was conducted for the current clinical use of REBOA in patients with hemodynamic instability and its potential role in improving prehospital and in-hospital outcome.

Material and methods: Systematic review and meta-analysis (19002017) using MEDLINE, Cochrane, EMBASE, Web of Science and Central \& Emcare using the keywords "aortic balloon occlusion", "aortic balloon tamponade", "REBOA", and "Resuscitative Endovascular Balloon Occlusion" in combination with hemorrhage control, hemorrhage, resuscitation, shock, ruptured abdominal, thoracic aorta, endovascular repair, and open repair. Original published studies on human subjects were considered.

Results: A total of 490 studies were identified; 89 met criteria for inclusion. Of the 1,436 patients, overall reported mortality was $49.2 \%$ $(613 / 1,246)$ with significant differences $(\mathrm{p}<0.001)$ between clinical indications. Hemodynamic shock was evident in $79.3 \%$, values between clinical indications showed significant difference $(\mathrm{p}<$ 0.001). Pooled analysis demonstrated an increase in mean systolic pressure by $50.1 \mathrm{mmHg}$ following REBOA use.

Conclusion: REBOA has been used in trauma patients and ruptured aortic aneurysm patients with improvement of hemodynamic parameters and outcomes for several decades. Formal, prospective study is warranted to clarify the role of this adjunct in all hemodynamic unstable patients.

References: Van Dongen TTCF, Idenburg FJ, Tan EC, Rasmussen TE, Hamming JF, Leenen LP, Hoencamp R. Combat related vascular injuries: Dutch experiences from a role 2 MTF in Afghanistan. Injury. 2016;47 (1):94-8. Stannard A, Eliason JL, Rasmussen TE. Resuscitative endovascular balloon occlusion of the aorta (REBOA) as an adjunct for hemorrhagic shock. J Trauma. 2011;71 (6):1869-72. Hörer 
TM, Skoog P, Pirouzram A, Nilsson KF, Larzon T. A small case series of aortic balloon occlusion in trauma: lessons learned from its use in ruptured abdominal aortic aneurysms and a brief review. Eur $\mathbf{J}$ Trauma Emerg Surg. 2016;42 (5):585-92. Karkos CD, Papadimitriou CT, Chatzivasileiadis TN, Kapsali NS, Kalogirou TE, Giagtzidis IT, Papazoglou KO. The Impact of Aortic Occlusion Balloon on Mortality After Endovascular Repair of Ruptured Abdominal Aortic Aneurysms: A Meta-analysis and Meta-regression Analysis. Cardiovasc Intervent Radiol. 2015;38 (6):1425-37. Morrison JJ, Galgon RE, Jansen JO, Cannon JW, Rasmussen TE, Eliason JL. A systematic review of the use of resuscitative endovascular balloon occlusion of the aorta in the management of hemorrhagic shock. Journal of Trauma and Acute Care Surgery. 2016;80 (2):324-34.

Disclosure: No significant relationships.

\section{O047}

\section{THE ASSOCIATION BETWEEN ABO BLOOD TYPE AND THE MORTALITY OF SEVERE TRAUMA PATIENTS}

\section{W. Takayama, A. Endo, Y. Otomo}

Emergency And Disaster Medicine, Tokyo Medical and Dental University Hospital, Tokyo/JAPAN

Introduction: Recent studies have implicated the difference of $\mathrm{ABO}$ histro-blood group system as a potential risk of various diseases. Especially, blood type $\mathrm{O}$ is indicated as a potentially important genetic risk factor for bleeding possibly due to lack of von Willebrand factor. To our knowledge, no reports to date have evaluated the association between the difference of $\mathrm{ABO}$ blood type and the mortality of severe trauma patients.

Material and methods: A retrospective observational study was conducted in two tertiary emergency critical care medical centres in Japan. Trauma patients who were transferred to the hospitals between April 2013 and March 2016 and had injuries of the injury severity score $>15$ were included. The association between the difference of $\mathrm{ABO}$ blood type and the outcomes of in-hospital mortality and total transfusion volume were evaluated with univariable and multivariable competing-risks regression models.

Results: The population by ABO blood type was as follows; O, 284 (32\%); A, 285 (32\%); B, 209 (23\%); and AB, 123 (13\%). Blood type $\mathrm{O}$ was associated with high mortality (O type vs non-O-type; $28 \%$ vs $11 \%, p<0.001)$. This association was also observed in a multivariable model. The trend of larger volume of red blood cell transfusion within 24 hours was observed in patients with blood type $\mathrm{O}$, though the difference was not statistically significant.

Conclusion: Blood type $\mathrm{O}$ was significantly associated with high mortality; however, the result was difficult to explain on account of only bleeding factor.

References: [1] K. Landsteiner. Zur kenntnis der antifermentativen, lytischen und agglutinierenden wirkungendes des blutserums und der lymphe. Zentralbl Bakteriol, 27 (1900), pp. 357-363 [2] M. Franchini, C. Rossi, C. Mengoli, et al. ABO blood group and the risk of coronary artery disease. J Thromb Thrombolysis, 36 (2013), pp. 286-287 [3] F. Dentali, M.N. Di Minno, S. Turato, et al. Role of ABO blood group and of other risk factors on the presence of residual vein obstruction after deep-vein thrombosis Thromb Res, 134 (2014), pp. 264-267 [4] A. Etemadi, F. Kamangar, F. Islami, et al. Mortality and cancer in relation to $\mathrm{ABO}$ blood group phenotypes in the Golestan Cohort Study BMC Med, 13 (2015), p. 8 [5] J.B. Harris, A.I. Khan, R.C. LaRocque, et al. Blood group, immunity, and risk of infection with Vibrio cholerae in an area ofendemicity. Infect Immun, 73 (2005), pp. 7422-7427 [6] Dentali F, Sironi AP, Ageno W, et al. Relationship between $\mathrm{ABO}$ blood group and hemorrhage: a systemic literature review and meta-analysis. Semin Thromb Hemost, 2013 Feb:39 (1):72-82. [7] Reilly JP, Anderson BJ, Mangalmurti NS, et al. The ABO Histlo-Blood Group and AKI in Critically Ill Patients with Trauma or Sepsis. Clin J Am Soc Nephrol. 2015 Nov 6;10 (11):191120 [8] JP Reilly, NJ Meyer, MGS Shashaty, et al. ABO blood type A is associated with increased risk of ARDS in whites following both major trauma and severe sepsis. Chest, 145 (4) (2014), pp. 753-761 Disclosure: No significant relationships.

\section{8}

\section{PROTECTIVE EFFECTS OF ALCOHOL ON COAGULOPATHY IS ASSOCIATED WITH ALTERATIONS IN THE INFLAMMATORY RESPONSE FOLLOWING TRAUMA}

\author{
A. Bonde, P. Svenningsen, M. Sillesen
}

Surgical Gastroenterology, Copenhagen University Hospital, Rigshospitalet, Copenhagen Ø/DENMARK

Introduction: Alcohol consumption may modulate trauma pathophysiology, and has previously been associated with a reduced risk of coagulopathy. We hypothesized that this could in part be mediated through a modulation of the immune response following trauma.

Material and methods: Secondary analysis of the Inflammation and Host Response to Injury Dataset. Data from 2002 blunt trauma patients were analyzed. Multiple regression associated ethanol (EOTH) levels on admission with coagulopathy (International Normalized Ratio, INR $>=1.3$ ) and volume of Packed Red blood Cells (PRBC) transfused over the initial 24 hours of treatment. For a subset $(\mathrm{n}=492)$ patients, a within-patient concentration change (WPCC) was used to calculate longitudinal trajectories during admission of 30 cytokines measured on days $0,1,4,7,21$ and 28 where applicable. Multiple regression associated WPCC with on-arrival ETOH levels while controlling for confounders.

Results: ETOH levels were negatively associated with both PRBC requirements over the first 24 hours $(\mathrm{p}=0.01)$ as well as Coagulopathy $\left(\mathrm{p}=3.6^{*} 10^{-8}\right)$. Furthermore, longitudinal trajectories of inflammatory cytokines Interleukin 12 (IL12, p = 0.03) and Interleukin 1 receptor antagonist (IL1RA, $\mathrm{p}=0.04$ ) were negatively associated with ETOH levels.

Conclusion: Alcohol, as measured ETOH, is associated with modulation of the trauma pathophysiology, including coagulopathy and inflammatory dynamics. These effects are furthermore associated with a reduced $\mathrm{PRBC}$ requirement.

References:

Disclosure: No significant relationships. 


\section{O049}

\section{THE USE OF THE SHOCK INDEX AS A PREDICTOR OF ACTIVE BLEEDING IN TRAUMA PATIENTS}

\author{
A. Campos Serra, S. Montmany Vioque, P. Rebasa Cladera, \\ H. Llaquet Bayo, R. Gracia Roman, S. Navarro Soto
}

\section{General And Digestive Surgery, Hospital Universitari Parc Taulí,} Sabadell/SPAIN

Introduction: Vital signs indicate the presence of bleeding only after large amounts of blood have been lost, with a high morbidity and mortality. The Shock Index (SI) is an indicator of bleeding with a cutoff point for the risk of bleeding at 0.9 . The aim of this study is to show that a cutoff of ${ }^{3} 0.8$ is more sensitive for detecting occult shock and allows early initiation of therapeutic maneuvers.

Material and methods: Descriptive, retrospective study of severe trauma patients $>16$ years of age. Vital signs and SI were recorded. The relation of the SI with five predictors of active bleeding was analyzed: need of massive transfusion, angiographic embolization, surgical bleeding control, death due hypovolemic shock and an overall predictor "active bleeding" (defined as the presence of one of the above variables).

Results: Data from 1402 trauma patients were collected prospectively over a period of 10 years. The mean Injury Severity Score was 20.9 (SD $=15.8)$, and the mortality rate was $10 \%$. The mean SI was $0.73(\mathrm{SD}=$ 0.29 ); SI was above 0.9 in 266 patients (19\%), and above 0.8 in 395 patients $(28.2 \%)$. The SI's area under the ROC curve for "active bleeding" was 0.749 ; its sensitivity with a cutoff point of 0.8 was 59.16 , its specificity was 78.95 , and its negative predictive value was 89.37 . Conclusion: A cut-off point of ${ }^{3} 0.8$ for the SI is more sensitive than one of ${ }^{3} 0.9$, and allows earlier initiation of resuscitation maneuvers in patients with occult active bleeding.

References: 1. Krug EG, Sharma GK, Lozano R. The global burden of injuries. Am J Public Health. 2000;90 (4):523-526. 2. Gruen RL, Brohi K, Schreiber M, et al. Haemorrhage control in severely injured patients. Lancet. 2012;380 (9847):1099-1108. 3. Cannon CM, Braxton CC, Kling-Smith M, Mahnken JD, Carlton E, Moncure M. Utility of the shock index in predicting mortality in traumatically injured patients. J Trauma. 2009;67 (6):1426-1430. 4. Bruijns SR, Guly HR, Bouamra O, Lecky F, Lee $\mathrm{W}$ a. The value of traditional vital signs, shock index, and age-based markers in predicting trauma mortality. $J$ Trauma Acute Care Surg. 2013;74 (6):1432-1437. 5. Kuster M, Exadaktylos A, Schnüriger B. Non-invasive hemodynamic monitoring in trauma patients. World J Emerg Surg. 2015:1-6. 6. Kortbeek JB, Al Turki S a, Ali J, et al. Advanced trauma life support, 8th edition, the evidence for change. J Trauma. 2008;64 (6):1638-1650. 7. Teixeira PGR, Inaba K, Salim A, et al. Preventable morbidity at a mature trauma center. Arch Surg. 2009;144 (6):536-41-2. 8. Yücel N, Lefering R, Maegele M, et al. Trauma Associated Severe Hemorrhage (TASH)-Score: probability of mass transfusion as surrogate for life threatening hemorrhage after multiple trauma. J Trauma Acute Care Surg. 2006;60 (6):1228-1236-1237. 9. Nunez TC, Voskresensky I V, Dossett L a, Shinall R, Dutton WD, Cotton B a. Early prediction of massive transfusion in trauma: simple as ABC (assessment of blood consumption)? J Trauma. 2009;66 (2):346-352. 10. McLaughlin DF, Niles SE, Salinas J, et al. A predictive model for massive transfusion in combat casualty patients. J Trauma. 2008;64 (2 Suppl):S57-S63; discussion S63. 11. Ardagh MW, Hodgson T, Shaw L, Turner D. Pulse rate over pressure evaluation (ROPE) is useful in the assessment of compensated haemorrhagic shock. Emerg Med. 2001:43-46. 12. Campbell R, Ardagh MW, Than M. Validation of the pulse rate over pressure evaluation index as a detector of early occult hemorrhage: a prospective observational study. J Trauma Acute Care Surg. 2012;73
(1):286-288. 13. Olaussen A, Blackburn T, Mitra B, Fitzgerald M. Review article: Shock Index for prediction of critical bleeding posttrauma: A systematic review. EMA - Emerg Med Australas. 2014;26 (3):223-228. 14. Vandromme MJ, Griffin RL, Kerby JD, McGwin G, Rue LW, Weinberg $\mathbf{J}$ a. Identifying risk for massive transfusion in the relatively normotensive patient: utility of the prehospital shock index. J Trauma. 2011;70 (2):384-388-390. 15. Demuro JP, Simmons S, Jax J, Gianelli SM. Application of the shock index to the prediction of need for hemostasis intervention. Am J Emerg Med. 2013;31 (8):1260-1263. 16. McNab A, Burns B, Bhullar I, Chesire D, Kerwin A. A prehospital shock index for trauma correlates with measures of hospital resource use and mortality. Surg (United States). 2012;152 (3):473-476. 17. Guyette F, Suffoletto B, Castillo J-L, Quintero J, Callaway C, Puyana J-C. Prehospital serum lactate as a predictor of outcomes in trauma patients: A retrospective observational study. $J$ Trauma Inj Infect Crit Care. 2011;70 (4):782-786. 18. Colombo J, Shoemaker WC, Belzberg H, Hatzakis G, Fathizadeh P, Demetriades D. Noninvasive monitoring of the autonomic nervous system and hemodynamics of patients with blunt and penetrating trauma. $J$ Trauma. 2008;65 (6):1364-1373. 19. Lu KJQ, Chien LC, Wo CCJ, Demetriades D, Shoemaker WC. Hemodynamic Patterns of Blunt and Penetrating Injuries. J Am Coll Surg. 2006;203 (6):899-907. 20. Zarzaur BL, Croce M a, Magnotti LJ, Fabian TC. Identifying lifethreatening shock in the older injured patient: an analysis of the National Trauma Data Bank. J Trauma. 2010;68 (5):1134-1138. 21. Heffernan DS, Thakkar RK, Monaghan SF, et al. Normal Presenting Vital Signs Are Unreliable in Geriatric Blunt Trauma Victims. J Trauma Inj Infect Crit Care. 2010;69 (4):813-820. 22. Pandit V, Rhee $\mathrm{P}$, Hashmi A, et al. Shock index predicts mortality in geriatric trauma patients: an analysis of the National Trauma Data Bank. J Trauma Acute Care Surg. 2014;76 (4):1111-1115. 23. McNab A, Burns B, Bhullar I, Chesire D, Kerwin A. An analysis of shock index as a correlate for outcomes in trauma by age group. Surg (United States). 2013;154 (2):384-387. 24. Toccaceli A, Giampaoletti A, Dignani L, Lucertini C, Petrucci C, Lancia L. The role of shock index as a predictor of multiple-trauma patients' pathways. Nurs Crit Care. 2016;21 (2):e12-e19. 25. Cotton B a, Dossett LA, Haut ER, et al. Multicenter validation of a simplified score to predict massive transfusion in trauma. J Trauma. 2010;69 Suppl 1 (1):S33-S39.

Disclosure: No significant relationships.

\section{0}

\section{TPA-CHALLENGED TEG: A NEW, MORE RELIABLE PREDICTIVE ASSAY FOR SCENE-OF-INJURY BLOOD SAMPLES}

M.P. Chapman
A. Sauaia
A. $^{3}$ E.E. Moore $^{4}$

${ }^{1}$ Vascular And Interventional Radiology, University of Colorado Denver, Aurora/CO/UNITED STATES OF AMERICA, ${ }^{2}$ Department Of Surgery, University of Colorado Denver, Aurora/CO/UNITED STATES OF AMERICA, ${ }^{3}$ Department Of Surgery, University of Colorado Denver, Aurora/UNITED STATES OF AMERICA, ${ }^{4}$ Surgery, Denver Health Medical Center, Denver/CO/UNITED STATES OF AMERICA

Introduction: The drive to achieve early triage by diagnosis of trauma-induced coagulopathy (TIC) has resulted in increased use of point-of-care viscoelastic assays such as TEG and ROTEM. Many centers sample the earliest possible time point, collecting blood at the scene-of-injury, but these samples may have limited utility, as TIC 
has not had time to develop. We hypothesized that the use of tPAChallenged $^{\mathrm{TM}}$ TEG would unmask evolving physiologic decompensation even in these early samples, and facilitate rapid triage by predicting TIC, using scene-of-injury samples.

Material and methods: We compared scene-of-injury blood to blood collected upon emergency department (ED) arrival, for 587 trauma activations, using citrated Rapid TEG (CRT) as well as tPA-Challenged TEG, using $75 \mathrm{ng} / \mathrm{mL}$ of exogenous tPA to promote fibrinolysis. To compare assay performance, receiver operating characteristic curves (ROC) were plotted for the prediction of massive hemorrhage $(\mathrm{MH})$, defined as $\geq 10$ units of PRBCs or death from bleeding in the first six hours.

Results: The area under the ROC curve of CRT maximum amplitude (MA) for prediction of $\mathrm{MH}$ was very good, at $0.87(\mathrm{P}<0.0001)$ for samples collected in the ED, but was unusably poor at 0.63 (not significantly different from identity) for scene-of-injury samples. Conversely, the area under the ROC curve of tPA-Challenged TEG 30-minute lysis (LY30) was as good as CRT MA for MH prediction, at $0.87(\mathrm{P}<0.0001)$ for ED samples, and only declined modestly to $0.78(\mathrm{P}=0.0001)$ for scene-of-injury samples.

Conclusion: tPA-Challenged TEG better predicts MH than traditional Rapid TEG, when samples are drawn at the scene-of-injury, and is therefore a better early triage tool.

References: 1. Moore HB, Moore EE, Chapman MP, Huebner BR, Einersen PM, Oushy S, et al. Viscoelastic Tissue Plasminogen Activator Challenge Predicts Massive Transfusion in 15 Minutes. Journal of the American College of Surgeons. 2017 Jul;225 (1):138-47. PubMed PMID: 28522144. Pubmed Central PMCID: 5527680. 2. Armand R, Hess JR. Treating coagulopathy in trauma patients. Transfusion medicine reviews. 2003 Jul;17 (3):223-31. PubMed PMID: 12881783. Epub 2003/07/26. eng. 3. Gonzalez E, Moore EE, Moore HB, Chapman MP, Silliman CC, Banerjee A. Trauma-Induced Coagulopathy: An Institution's 35 Year Perspective on Practice and Research. Scandinavian journal of surgery : SJS : official organ for the Finnish Surgical Society and the Scandinavian Surgical Society. 2014 Jun;103 (2):89-103. PubMed PMID: 24786172. Pubmed Central PMCID: 4214916. 4. Cohen MJ. Towards hemostatic resuscitation: the changing understanding of acute traumatic biology, massive bleeding, and damage-control resuscitation. The Surgical clinics of North America. 2012 Aug;92 (4):877-91, viii. PubMed PMID: 22850152. Epub 2012/08/02. eng. 5. Chapman MP, Moore EE, Moore HB, Gonzalez E, Gamboni F, Chandler JG, et al. Overwhelming tPA release, not PAI-1 degradation, is responsible for hyperfibrinolysis in severely injured trauma patients. The journal of trauma and acute care surgery. 2016 Jan;80 (1):16-23; discussion -5. PubMed PMID: 26491796. Pubmed Central PMCID: 4688194.

Disclosure: We receive research support from IL and Haemonetics.

\section{O050A}

\section{GOAL-DIRECTED FLUID MANAGEMENT DURING HIGH- RISK LAPAROTOMY, A META-ANALYSIS}

\section{G.A. Bass ${ }^{1}$, C.C. Duffy ${ }^{2}$, D.M. Honan ${ }^{3}$}

${ }^{1}$ Dept. Of Surgery, St. Vincent's University Hospital, Dublin/IRELAND, ${ }^{2}$ Dept Of Anaesthesia, Cork University Hospital, Cork/ IRELAND, ${ }^{3}$ Dept Of Anaesthesia \& Intensive Care, Wexford General Hospital, Wexford/IRELAND

Introduction: Advances in the determination of intravascular volume and cardiac function have unmasked the dangers of over-resuscitation. Fluid restriction is recommended as part of care-bundles for enhanced recovery after elective surgery, whilethe trauma literature now advocates active de-resuscitation in the primary and secondary prevention of intra-abdominal hypertension. Several RCTs examining goal-directed fluid management (GDFM) resuscitation strategies in the undifferentiated sepsis patient failed to demonstrate a survival advantage, although these studies were not designed to examine the high-risk surgical patient.

Material and methods: A systematic review of the literature was undertaken to identify trials of GDFM vs. unrestricted resuscitation. Meta-analysis was performed to determine if GDFM can prevent the morbidity associated with over-resuscitation during high-risk emergency or scheduled laparotomy.

Results: GDFM was associated with a significant reduction in 30-day overall mortality (79/491 (14.6\%)vs. $217 / 758$ (28.6\%)events, p < $0 \cdot 001)$ as well as resuscitation-associated morbidities. Respiratory failure requiring prolonged mechanical ventilation was seen less frequently following GDFM (15/288 (5.8\%) vs.32/427 (7.5\%) events, $\mathrm{p}=0.03)$, as was post-operative coagulopathy $(11 / 147(7.5 \%) v s$. $21 / 145(14.5 \%)$ events, $\mathrm{p}=0.04)$ and anastomotic leak requiring radiologic or operative intervention $(9 / 94(9.5 \%)$ vs. 18/91 (19.8\%) events, $\mathrm{p}=0.006)$. Renal failure requiring dialysis was more common following GDFM (41/255 (16.1\%) vs.28/427 (6.8\%) events, $\mathrm{p}<$ 0.001).

Conclusion: GDFM, irrespective of the monitoring modality employed, was shown to accrue a survival advantage over unrestricted resuscitation following high-risk laparotomy. GDFM was associated with a decrease in earlypost-operative morbidity such as respiratory failure, coagulopathy and anastomotic leak, but at the cost of renal failure requiring dialysis. These findings may have practicechanging implications, particularly for the management of the septic emergency surgical patient.

References:

Disclosure: No significant relationships.

\section{ADVANCES IN CIVILIAN AND MILITARY TRAUMA CARE}

\section{$\mathbf{0 0 5 1}$}

\section{THE BURDEN OF DISEASES AND NON-BATTLE INJURIES DURING DEPLOYMENT}

$\underline{\text { T.T.c.f. Van Dongen }}{ }^{1}$, E.p. Huizinga ${ }^{2}$, F.j. Idenburg ${ }^{3}$, R. Hoencamp ${ }^{2}$

${ }^{1}$ Defense Healthcare Organisation, Ministry of Defense, Utrecht/ NETHERLANDS, ${ }^{2}$ Surgery, Central Military Hospital, Ministry of Defense, Utrecht/NETHERLANDS, ${ }^{3}$ Surgery, Haaglanden Medical Center, Den Haag/NETHERLANDS

Introduction: Todays armed conflicts pose a substantial risk for exposure of combat events to units deployed. Many battlefield casualties have been reported in the recent conflict in Afghanistan. However, during deployments there is also a substantial burden of diseases and non-battle injuries (DNBI). During the Dutch involvement in Afghanistan, the amount of DNBIs that needed medical evacuation out of theater $(60 \%)$ exceeded the number of battle injuries (BI, 40\%). The aim of this study is to describe the burden of DNBI in the period 2003-2014 during combat operations in Afghanistan and identify possible trends/patterns.

Material and methods: Observational cross-sectional cohort study in a selected group of Dutch servicemembers that deployed to Afghanistan (2003-2014) and were repatriated due to illness or sustaining a non-battle injury. Using the SF-36, EQ-6D, SCL-90 and PDRS questionnaires, their outcome was compared with a control group of deployed servicemembers who did not sustain any injury or illness. 
Results: Groups were comparable in age, rank, number of deployments and social status. There were significant differences found in terms of physical functioning, pain and health perspective (SF-36 and EQ-6D). No differences were seen in emotional or psychological outcomes.

Conclusion: Sustaining a DNBI can pose a significant physical burden on the deployed military population. Medical and operational planners must be aware of, and anticipate for potential significant implications for the military medical support organization. Continuous registration of DNBI during deployment will provide more insight and more accurate predictions of this potential burden. Further research and follow up is needed to determine the longterm outcome . References: Huizinga E, Hoencamp R, van Dongen T, Leenen L. Cross-sectional analysis of Dutch repatriated service members from southern Afghanistan (2003-2014). Mil Med. 2015 Mar;180 (3):3104. Patel AA, Hauret KG, Taylor BJ, Jones BH. Non-battle injuries among U.S. Army soldiers deployed to Afghanistan and Iraq, 2001-2013. J Safety Res. 2017;60:29-34. Toman E, Beaven A, Naumann DN, Myatt RW, Parker PJ, Kay AR. Non-battle injury among repatriated UK armed forces since cessation of combat operations: a prospective observational study. J R Army Med Corps. 2017 Aug 23. van Dongen TTCF, de Graaf J, Plat MJ, Huizinga EP, Janse J, van der Krans AC, Leenen LPH, Hoencamp R. Evaluating the Military Medical Evacuation Chain: Need for Expeditious Evacuation Out of Theater? Mil Med. 2017 Sep;182 (9)

Disclosure: No significant relationships.

\section{2}

\section{8-DAY THAWED PLASMA MAINTAINS CAPACITY TO INHIBIT TPA-INDUCED FIBRINOLYSIS SIMILAR TO FRESHLY THAWED PLASMA}

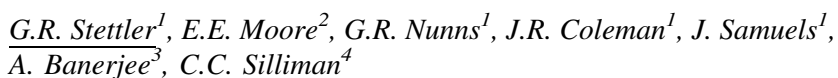

${ }^{1}$ Surgery, University of Colorado, Aurora/CO/UNITED STATES OF AMERICA, ${ }^{2}$ Surgery, Denver Health Medical Center, Denver/CO/ UNITED STATES OF AMERICA, ${ }^{3}$ Surgery, University of Colorado, Aurora/UNITED STATES OF AMERICA, ${ }^{4}$ Pediatrics, University of Colorado, Aurora/CO/UNITED STATES OF AMERICA

Introduction: Plasma-first resuscitation ameliorates trauma-inducedcoagulopathy (TIC). The current standard for storage of thawed plasma (TP) is based on Factor XIII's half-life. However, TP has the capacity to reduce tPA-mediated fibrinolysis 14-days after thawing. We hypothesize that TP maintains significant hemostatic potential to inhibit tPA-induced hyperfibrinolysis at 28-days of storage similar to FFP.

Material and methods: Plasma was thawed and stored at $4{ }^{\circ} \mathrm{C}$ for the following intervals: day of experiment, 5, 7, 14, 21 and 28 days. Healthy volunteers underwent blood draws followed by $50 \%$ dilution with TP, FFP, normal saline (NS), and whole blood (WB). Citrated native $(\mathrm{CN})$ and tPA-challenge $(75 \mathrm{ng} / \mathrm{ml})$ thrombelastography (TEG) were performed on all samples. Differences across dilution groups were detected using a non-parametric paired Friedman test and Dunn's multiple comparisons.

Results: 28-day TP inhibited tPA-induced hyperfibrinolysis (median LY30 13.5\%) similar to FFP $(10.8 \%, \mathrm{p}>0.9990)$ and WB $(7.85 \%, \mathrm{p}$ $=0.364)$ but superior to NS $(36 \%, \mathrm{p}=0.0355) .28$-day TP enhanced $\mathrm{CN}$-angle compared to $\mathrm{WB}\left(57.35^{\circ}\right.$ vs. $\left.43.1^{\circ}, \mathrm{p}=0.110\right)$ and NS $\left(57.35^{\circ}\right.$ vs. $\left.44.35^{\circ}, \mathrm{p}=0.003\right)$ but was not different from FFP $\left(57.35^{\circ}\right.$ vs. $\left.59.6^{\circ}, \mathrm{p}>0.9999\right)$. There were no differences in 28-day TP compared to WB, NS, or FFP for CN MA or LY30. All TP intervals demonstrated similar clot characteristics to FFP on CN and tPAchallenge TEG.

Conclusion: TP stored for 28 days contains enough hemostatic potential to inhibit tPA-induced hyperfibrinolysis and resist clot degradation similar to FFP and WB. This provides evidence that the use of TP at periods beyond the current 5-day standard may have beneficial effects to reducing TIC, specifically systemic hyperfibrinolysis.

References: Cotton BA, Harvin JA, Kostousouv V, et al. Hyperfibrinolysis at admission is an uncommon but highly lethal event associated with shock and prehospital fluid administration. J Trauma Acute Care 2012;73 (2):365-70. Moore HB, Moore EE, Gonzalez E, et al. Plasma is the physiologic buffer of tissue plasminogen activatormediated fibrinolysis: rationale for plasma-first resuscitation after lifethreatening hemorrhage. Journal of the American College of Surgeons 2015;220 (5):872-9. Huebner BR, Moore EE, Moore HB, et al. 14-Day thawed plasma retains clot enhancing properties and inhibits tPA-induced fibrinolysis. Journal of Surgical Research 2017;219:14550. Tholpady A, Monson J, Radovancevic R, et al. Analysis of prolonged storage on coagulation Factor (F)V, FVII, and FVIII in thawed plasma: is it time to extend the expiration date beyond 5 days? Transfusion 2013;53 (3):645-50.

Disclosure: No significant relationships.

\section{3} 'THINKING LEAN'- THE USE OF QUALITY
IMPROVEMENT MODELS TO IMPROVE TRAUMA CLINIC
SERVICE PROVISION IN A SINGLE CENTRE IN ENGLAND

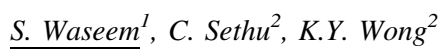

${ }^{1}$ Vascular Surgery, St Thomas' Hospital, London/UNITED KINGDOM, ${ }^{2}$ Plastic Surgery, Salisbury District Hospital, Salisbury/ UNITED KINGDOM

Introduction: Quality improvement models such as 'lean thinking' and rapid cycle change ${ }^{2}$ have successfully streamlined service provision in the industrial sector, with increasing interest in the application of these models to health services ${ }^{3}$. We tested these models in improving a hand trauma clinic in one UK hospital.

Material and methods: We undertook a 6-month period of prospective data collection including patient demographics, injury modality, diagnosis, treatment delays, theatre efficiency and nil by mouth time. After our $1^{\text {st }} 2$-month period of data collection, we identified sources of 'lean waste' in service provision. Changes intended to remove 'lean waste' were enacted using the 'Study-PlanAct-Do' model. We completed a further audit cycle to assess the impact of these changes.

Results: Within 6 months, 478 patients attended the trauma clinic. 329 $(68.8 \%)$ underwent a procedure; $189(57.4 \%)$ of which were performed in the outpatient clinic, and $140(42.6 \%)$ in theatre. When patients went to theatre, $55(39.2 \%)$ were performed as emergency procedures and 85 $(60.8 \%)$ were performed on elective lists. The most common primary injury modality was lacerations, accounting for $302(63.2 \%)$ injuries. The largest sources of lean waste identified were the training of junior doctors in trauma clinic and the delay in time to theatre. Our changes reduced the time to surgery by $73 \%$ (30 days to 8 ).

Conclusion: Our study showed that a lack of target driven practice has contributed to areas of inefficiency in health service provision. We give preliminary evidence that the adoption of quality improvement models has some beneficial effects on health service provision. 
References: 1. Lean Enterprise Institute. Principles of Lean. Lean Enterprise Institute, 2015. 2. Langley GJ, Moen R, Nolan KM, et al. Changes that result in improvement. In: The improvement guide: a practical approach to enhancing organizational performance. 2nd edn. San Francisco: Jossey-Bass, 2009:15-25. 3. D’ Andreamatteo A, Ianni L, Lega F, Sargiacomo M. Lean in healthcare: A comprehensive review. Health Policy. 2015 Sep;119 (9):1197-209. doi: 10.1016/ j.healthpol.2015.02.002. Epub 2015 Feb 11.

Disclosure: No significant relationships.

\section{4}

\section{EVALUATION OF THE CLINICAL COMPETENCIES OF YOUNG FRENCH SURGEONS IN PENETRATING TRAUMA MANAGEMENT AND DAMAGE CONTROL SURGERY}

$\frac{\text { J. Gaudric }^{1} \text {, B. Malgras }}{2}$, A. De Carbonnieres ${ }^{3}$, S. Bonnet ${ }^{3}$,

${ }^{1}$ Vascular Surgery, Hopital pitié Salpétriere, Paris/FRANCE ${ }^{2}$ Digestive Surgery, HIA Begin, Saint Mandé/FRANCE, ${ }^{3}$ Department Of Digestive Surgery, Percy Military hospital, Clamart/FRANCE

Introduction: Recent terrorist attacks in France have demonstrated a large diversity of operating modes. The threat is not limited to the big cities. French national Health authorities have highlighted the necessity of a large diffusion of the surgical "damage control" concept. This study aims to evaluate the level of the clinical skills of young French surgeons in penetrating trauma management.

Material and methods: A descriptive epidemiological study based on a questionnaire sent to the civilian and military residents and assistants of the hospitals in digestive, vascular and urologic surgery Results: 506 surgeons have responded. The response rate was $84 \%$. $58 \%$ were digestive surgeons, $25 \%$ vascular and 11 urologists. $57 \%$ were working in a teaching hospital and $76 \%$ in a trauma center. $8,5 \%$ were military surgeons. $85 \%$ of the surgeons have completed the oneday basic university course in trauma surgery $(85 \%), 53 \%$ an additional course. $58,4 \%$ of the surgeons estimated that their clinical competencies in damage control surgery were "low" or "very low". $14 \%$ have followed the course organized by the French health minitry after the terrorist attack in Paris. 75\% estimated that a complementary learning was needed. The military surgeons were more familiar with damage control surgery and $100 \%$ had a deep initial training

Conclusion: Despite the terrorist threat and the risk of mass casualties incident, the level of the clinical skills in trauma surgery of the young French surgeons remains very low. The current reform of the internship in surgery will lead to an early hyper-specialization of the residents and will probably increase the problem

References:

Disclosure: No significant relationships.

\section{5}

\section{E-TRIAGE IN MASS CASUALTIES ON THE SEA - AN INNOVATIVE BACKPACK-SYSTEM FOR MARITIME OFFICERS} S. Schulz-Drost
M. J. Unigeldt ${ }^{2}$, H. Rankerkofler

${ }^{1}$ Trauma- And Orthopedic Surgery, BG Hospital Unfallkrankenhaus Berlin gGmbH, Berlin/GERMANY, ${ }^{2}$ Zentrum Für Klinische Forschung, Unfallkrankenhaus Berlin, Berlin/GERMANY, ${ }^{3}$ Department Of Trauma, Reconstructive Surgery And Rehabilitation Medicine, University Medicine Greifswald, Greifswald/GERMANY

Introduction: Effective paramedic-TRIAGE in the event of mass casualties can significantly improve the effectiveness of the management. Through structured algorithms, such as mSTART, it can also be performed by non-medical staff at the earliest time. In support of this, computer-based systems are increasingly establishing themselves in order to speed up the processes and at the same time to generate an overview documentation.

What is the possibility to have an e-triage system practically packaged for ship crews?

Material and methods: In the KOMPASS Project, a concept was developed to provide a mobile, well-portable PC system with radio connection to the coordination station on the country side. The Triage is to be carried out at the same time by 4 helpers with tablets, which are connected by W-LAN with the laptop. The power supply should be autonomous for the first hours.

Results: Together with the industry, a water- and shock-resistant backpack concept with high wearing comfort could be developed. Laptop and 4 tablets are kept as secure as a printer, a beamer and a set of Triage cards.

The operation is intuitive by layperson, the devices are wind and water protected.

First practical evaluations show simple operation and a drastic reduction of the Triage time through simultaneous triage by 4 helpers in parallel.

Conclusion: This highly innovative E-Triage system improves paramedic Triage significantly. The data center will be informed about the situation in real-time via data transmission and can plan further management at an early stage. On land, this could also very effectively support the maneuvering process.

References: 1 www.kompassprojekt.de 2 N. Ellebrecht, L. Latasch. Vorsichtung durch Rettungsassistenten auf der Großübung SOGRO MANV 500. Eine vergleichende Analyse der Fehleinstufungen. Notfall Rettungsmed 2012 • 15:58-64 DOI 10.1007/ s10049-011-1477-1

Disclosure: The KOMPASS Project had been funded by the German federal ministry of Education and Science. 


\section{6}

\section{COPING MASS CASUALTY INCIDENCES AT SEA - RESULTS OF A TABLE-TOP EXERCISE}

\author{
E.Henning ${ }^{\text {I }}$, T. Görig ${ }^{2}$, S. Schulz-Drost ${ }^{2}$, A. Ekkernkamp ${ }^{2}$, \\ D. Gümbel
}

${ }^{1}$ Department Of Trauma, Reconstructive Surgery And Rehabilitation Medicine, University Medicine Greifswald, Greifswald/GERMANY, ${ }^{2}$ Department Of Trauma, reconstructive Surgery And Rehabilitation Medicine, University Medicine Greifswald, Greifswald/GERMANY

Introduction: Mass casualty incidents (MCI) at sea occur with low probability but high consequences that overwhelm local emergency resources stressing the need for a structured emergency plan. Along with a continuously growing shipping industry including leisure cruises and passenger vessels a higher risk for MCI is expected. Therefore, specific training for the shipping crew is crucial for increasing preparedness and resilience. The aim of the current study was to evaluate a designed table-top exercise for MCI's at sea and to identify training potential for crew members.

Material and methods: A five-phases-timed-schedule table top exercise involving 60 dynamic patients was developed simulating a MCI caused by a fire on a RoPax ferry. Following a introduction to the scenario non-medical personnel was asked to triage patients, initiate treatment and prioritize transport. Patient characteristics including age, sex, vital signs and injury pattern were given on patient charts. Initiated treatment influenced health status of the patients during the scenario.

Results: In one day 8 trainees completed the table-top exercise. $35 \%$ patients were triaged regardless of patient vital signs. $74,6 \%$ of the triages were correct. Initiated treatment involved $3 \%$ of the patients. Deterioration of patients' status was observed throughout the exercise.

Conclusion: As medical resources at sea are scarce there is still an unmet need for MCI training of crew members. Despite a high number of correctly triaged patients in this scenario coordinative efficiency in identifying life threatened patients and transport prioritization can be improved with the implementation of MCI training and triage support systems.

Disclosure: No significant relationships.

\section{7}

\section{FASCIOTOMY FOR COMPARTMENT SYNDROME AFTER ARTERIAL REPAIR IN LOWER LIMB TRAUMA}

\section{J. Klocker}

Vascular Surgery, Medical University Innsbruck, Innsbruck/ AUSTRIA

Introduction: Proper management of ischemia-reperfusion injury leading to compartment syndrome after arterial reconstruction in limb trauma includes fasciotomy. Previously published data on frequency, associated morbidity, need for re-do surgery and outcome of patients after fasciotomy in lower limb arterial trauma are limited.

Material and methods: All patients that underwent arterial repair in lower limb trauma during a 25 -years period were included and further evaluated. Endpoints were: mortality; limb salvage; re-do operations (re-do arterial repair; re-do fasciotomy; wound management and/or debridement; secondary wound closure); renal function; length of hospital stay.

Results: A total of 141 patients underwent arterial repair in lower limb trauma, and $71(50.4 \%)$ had fasciotomy. Thirty-day mortality after fasciotomy was $1.4 \%$, and 30 -day limb loss rate was $17 \%$. Re-do operations were frequently performed for: revision of vascular repair ( $\mathrm{n}=10 ; 14 \%)$; extended fasciotomy $(\mathrm{n}=16 ; 23 \%)$; wound management $(n=45 ; 63 \%)$ or debridement $(n=21 ; 30 \%)$. Wound closure was performed after median 11 days and required skin grafting $(31 \%)$ or myocutaneous flaps $(7 \%)$. Transient elevations of serum creatinine above normal $(1.2 \mathrm{md} / \mathrm{dl})$ were seen in $38 \%$ of patients, however, only 1 patient required long-term renal replacement therapy. Median length of hospital stay in patients undergoing fasciotomy was 26 days. Conclusion: Fasciotomy was performed in approximately 50\% of patients after arterial repair in leg trauma. Despite fasciotomy, limb loss was frequently seen (17\%) and transient renal function impairment affected $38 \%$ of the patients. After fasciotomy, re-do operations including re-do fasciotomy, wound management and debridement are common. As a consequence, patients after fasciotomy require a prolonged hospital stay.

References:

Disclosure: No significant relationships.

\section{8}

\section{KOMPASS: AN E-LEARNING TOOL FOR MARITIME OFFICERS FOR TRIAGE IN MASS CASUALTY INCIDENTS AT SEA}

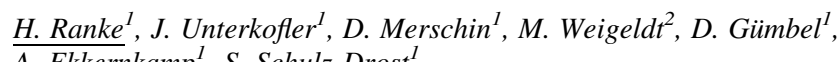
A. Ekkernkamp ${ }^{1}$, S. Schulz-Drost ${ }^{1}$

${ }^{1}$ Trauma- And Orthopedic Surgery, Unfallkrankenhaus Berlin, Berlin/ GERMANY, ${ }^{2}$ Zentrum Für Klinische Forschung, Unfallkrankenhaus Berlin, Berlin/GERMANY

Introduction: Mass casualty incidents (MCI) are a rare and highly challenging situation. A special case is represented by MCI at sea, because of the delay of time until alerted medical staff is arriving and the limitation of space and equipment. Therefore medically untrained personnell or maritime officers need to be trained as "first-aiders" to initiate the very first steps to coordinate and successfully handle a MCI at sea.

Material and methods: The well established triage system mSTaRT, which can be run by non-doctors, is the core of the triage system of $\mathrm{MCI}$ at sea. A particular software was programmed to maintain tablet based triage steps and summarize all single cases for later analyses. A special web based learning platform was created for maritime officers to learn and practice triaging people and initiate basic life support.

Results: The learning platform teaches nautical staff, location-independent, to perfect triage and medical support, to minimize the amount of fatalities and optimize the given resources. With the help of tutorials and specific cases users are taught in procedures and mSTaRT triage system. Training and teaching of nautical staff is extraordinarily important because of the increasing worldwide ship traffic, reduction of safety precaution by increasing cost pressure and increasing risk of terrorist attacks.

Conclusion: No golden triage pathway for MCI at sea has been established yet. The learning platform of the KOMPASS project represents a perfect system to prepare people for MCI on sea and initialize the first right steps. 
References: Projekt KOMPASS Germany (Capacities and Organization in Mass Casualties at Sea); Notfall + Rettungsmedizin 05/2006 (mSTaRT), Der Notarzt 02/2016

Disclosure: No significant relationships.

\section{9}

\section{IS THERE DIFFERENCE BETWEEN MILITARY, CIVILIAN TERRORIST ATTACKS AND CIVILIAN BLAST ACCIDENT - OUR EXPERIENCE WITH THREE MASS CASUALTY EVENTS}

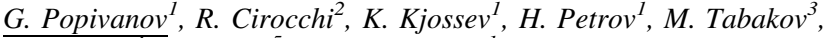 \\ R. Ribarov ${ }^{4}$, B. Zlatev, V. Mutafchiyski ${ }^{1}$
}

${ }^{1}$ Surgery, Military Medical Academy, Sofia/BULGARIA, ${ }^{2}$ General And Surgical Oncology, University of Perugia, Perugia/ITALY, ${ }^{3}$ Surgery, University Hospital, Sofia/BULGARIA, ${ }^{4}$ Public Health, Medical University, Sofia/BULGARIA, ${ }^{5}$ Orthopedic And Reconstrutive Surgery, Military Medical Academy, Sofia/ BULGARIA

Introduction: The unceasing terrorist attacks became a constant threat for our society directed toward innocent people. Blast trauma is frequently associated with mass casualty events and continues to challenge our preparedness.

Material and methods: Three mass casualty events were analyzed and compared. The terrorist events were combat attack against Bulgarian base in Kerbala, Iraq with 3 tones TNT, and civilian attack against a civilian bus at Bourgas Airport, Bulgaria. The third event was an accidental blast of six train tanks in populated area. The following variables were analyzed - total number of casualties, number of dead-on-scene, dead/wounded ratio, first ambulance arrival, Injury Severity Score, hospitalized, need for major surgery, overall mortality, rate of overtriage.

Results: The distribution of results in terrorist attacks (combat and civilian) and civilian accident was following: number of casualties 65 vs 39 vs 45 , dead-on-scene - $6 \%$ vs $15 \%$ vs $13 \%$, dead:wounded ratio $-1: 13$ vs $1: 6$ vs $1: 6$, fisrt ambulance arrival -25 vs 15 vs 17 min, hospitalized $-54 \%$ vs. $97 \%$ vs $41 \%$, Injury Severity Score $>16-$ $14 \%$ vs $12 \%$ vs $23 \%$, major surgery - $14 \%$ vs $8 \%$ vs $23 \%$, overall mortality $-8 \%$ vs $18 \%$ vs $16 \%$, overtriage $-72 \%$ vs $90 \%$ vs $43 \%$. Conclusion: Civilian mass casualty events are associated with higher rate of dead-on-scene, dead/wounded ratio probably due to structural collapse in our cases. Lessions learned lowers significantly the rate of overtriage. The unpredictability of these events warrants the proper staff's training of each hospital.

References: Almogy G, Belzberg H, Mintz Y, et al. Suicide bombing attacks. Update and modifications to the protocol. Ann Surg 2004;239:295-303. Einav S, Aharonson-Daniel L, Weissman C, et al. In-hospital resource utilization during multiple casualty incidents. Ann Surg 2006;243:533-540. Frykberg ER. Medical management of disasters and mass casualties from terrorist bombings: How can we cope? J Trauma 2002;53:201-212.

Disclosure: No significant relationships.

\section{TRAUMA REGISTRIES}

\section{$\mathbf{0 0 6 0}$}

\section{PATIENTS' PERSONALITY AND LONG TERM OUTCOME IN SEVERELY INJURED TRAUMA PATIENTS}

\author{
K. Van Delft-Schreurs ${ }^{1}$, M.A.c. De Jongh ${ }^{1}$, K. Lansink ${ }^{2}$ \\ M. Verhofstad ${ }^{3}$, J. De Vries ${ }^{4}$
}

${ }^{1}$ Nazb, ETZ Elisabeth, Tilburg/NETHERLANDS, ${ }^{2}$ Surgery, Elisabeth Tweesteden Ziekenhuis, Tilburg/NETHERLANDS, ${ }^{3}$ Surgery, Erasmus MC, Rotterdam/NETHERLANDS, ${ }^{4}$ Medical Psychology, ETZ Elisabeth, Tilburg/NETHERLANDS

Introduction: In recent years more studies focus on the outcome parameter (Health Related) Quality of Life ( (HR)QOL) after a severe injury. Psychological complaints are known to be associated with (HR)QOL.[1, 2] However, real long-term QOL was more or less unknown. Besides, the patients' personality might also be associated with long-term QOL. The aim of this study was to evaluate QOL, psychological complaints and physical limitations after a severe injury about ten years after trauma and to compare this with the patients' situation 6 years ago. Besides we investigated the association of long-term QOL with the patients' personality.

Material and methods: 156 patients who were included in the trauma registry of the ETZ-Elisabeth Hospital in the years 2006 to 2008 after a severe injury (ISS > 15), and participated six years ago in a study in which quality of life, psychological problems and physical limitations were measured, were reassessed to determine their current situation by questionnaires. Besides, the patients' personality was determined.

Results: The response rate was $58 \%$. Compared to their situation six years ago, no significant difference in the patients' QOL, psychological complaints and physical limitations has been found, except for the social component, which decreased. Personality was an important confounder in the interaction between psychological complaints and long-term QOL.

Conclusion: The QOL, psychological and physical situation of severely injured patients ten years after their injury is comparable to their situation four years after their injury. Personality is an important factor for long-term QOL. Therapy for useful coping strategies may be helpful.

References: 1 Van Delft-Schreurs, C.C.H.M., J.J.M. van Bergen, M.A.C. de Jongh, P. van de Sande, M.H.J. Verhofstad \& J. de Vries, 2014. Quality of life in severely injured patients depends on psychosocial factors rather than on severity or type of injury. Injury 45 (1): 320-6. 2 Van Delft-Schreurs, C.C.H.M., M.A. van Son, M.A. de Jongh, K.W.W. Lansink, J. de Vries \& M.H.J. Verhofstad, 2017. The relationship between physical and psychological complaints and quality of life in severely injured patients. Injury 48 (9): 1978-1984. Disclosure: No significant relationships. 
0061

\section{WHAT YOU DON'T KNOW CAN HURT YOU: CHRONIC DISEASE PORTENDS INCREASED MORTALITY AFTER INJURY IN LOW RESOURCE SETTINGS}

\author{
S.A. Christie $^{1}$, F. Dissak-Delon ${ }^{2}$, A. Chichom-Mefire ${ }^{3}$, \\ G.A. Etoundi Mballa ${ }^{4}$, R.A. Dicker ${ }^{5}$, C. Juillard ${ }^{1}$
}

${ }^{1}$ Surgery, University of California San Francisco, San francisco/CA/ UNITED STATES OF AMERICA, ${ }^{2}$ Littoral Region Delegation, Ministry of Public Health, Cameroon, Yaounde/CAMEROON, ${ }^{3}$ Surgery And Gynecology-obstetrics, University of Buea, Faculty of Health Sciences, Buea/CAMEROON, ${ }^{4}$ Director Of Disease Control, Ministry of Public Health, Cameroon, Yaounde/CAMEROON, ${ }^{5}$ Surgical Critical Care, University of California Los Angeles, Los Angeles/CA/UNITED STATES OF AMERICA

Introduction: Chronic disease states are known to confer increased risk for poor trauma outcomes in high-income settings (1). Despite increasing burden of both injury and chronic disease in low-and middle-income countries (LMIC) $(2,3)$, lack of prospective data has limited understanding of the impact of these conditions leading to ongoing under-prioritization by policymakers (4). We hypothesize that chronic disease is significantly under-recognized but portends poor trauma outcomes in LMIC.

Material and methods: We performed a multi-center prospective observational cohort study of all injured patients presenting to three regional referral hospitals in Cameroon between July 2015 and January 2017. History of chronic illness was identified at admission along with demographic, socio-economic, and injury characteristic data. Injured patients were followed throughout hospitalization for critical outcomes including death.

Results: Of 7,897 injured patients, only 195 persons reported having a diagnosis of one or more chronic illness. Patients endorsing chronic diseases were older (51 vs. 31 years), more frequently female $(46 \%$ vs. $37 \%)$ and from rural areas ( $21 \%$ vs. $3 \%$, all p < 0.01$)$. Chronically ill patients were more likely to sustain fall mechanisms than patients without chronic illness $(22 \%$ vs. $15 \%, \mathrm{p}=0.011)$, but physiologic and anatomic severity scores were clinically similar between groups. Multiple logistic regression adjusted for age, sex, mechanism and injury severity demonstrated chronic disease to be an independent predictor of in-hospital mortality after injury (OR $2.67 \mathrm{p}=0.03)$.

Conclusion: Chronic disease is likely under-recognized in LMIC, but strongly predicts increased mortality after injury. Policymakers should prioritize strengthening of health systems capable of diagnosis and management of chronic disease to improve outcomes after injury. References: 1. Patel MS, Malinoski DJ, Nguyen XM, et al. The impact of select chronic diseases on outcomes after trauma: a study from the National Trauma Data Bank. J Am Coll Surg. 2011;212 (1):96-104. 2. Abegunde DO, Mathers CD, Adam T, et al. The burden and costs of chronic diseases in low-income and middle-income countries. Lancet. 2007;370 (9603):1929-1938. 3. Mathers CD, Loncar D. Projections of global mortality and burden of disease from 2002 to 2030. PLoS Med. 2006;3 (11):e442. 4. Chichom Mefire A, Etoundi Mballa GA, Azabji Kenfack M, et al. Hospital-based injury data from level III institution in Cameroon: retrospective analysis of the present registration system. Injury. 2013;44 (1):139-143.

Disclosure: No significant relationships.
0062

\section{EVALUATING TRAUMA MANAGEMENT, OUTCOMES AND ECONOMIC DATA IN A SYSTEM IN CRISIS: CHANGES REQUIRED FOR IMPROVEMENT}

\author{
A. Prionas, A. Tooulias, A. Tsolakidis, C. Christou, G. Tsoulfas, \\ $\overline{V . \text { Papadopoulos }}$
}

First General Surgery Department, Aristotle University of Thessaloniki, Papageorgiou General Hospital, Thessaloniki/GREECE

Introduction: The goal is to record and evaluate trauma management in our University Hospital, while laying the foundations for a national database.

Material and methods: Retrospective study of trauma patients $(\mathrm{N}=$ 2320) between 2014 and 2015, through our single-center registry. Demographic information, injury patterns, hospital transfer, diagnostic and therapeutic procedures, duration of hospitalization, ISS, outcomes, complications and cost were recorded.

Results: For the total 2,320 trauma patients, RTAs (23.2\%) were the most common injury pattern after fall traumas $(36.3 \%)$ and presented the highest associated injury severity (mean ISS $=5.49$ ). RTAs were decreased during the study period (from $25.6 \%$ in 2014 to $21 \%$ in 2015).Regarding prehospital management, after applying the EMS triage algorithm criteria[1], we found that over-triage of trauma patients to our facility was high $(52.1 \%)$ and was not reduced during the study period. Hospital transfer by the National Emergency Medical Services was provided in $6.4 \%(\mathrm{n}=76 / 1192)$ of patients in 2015 and in $9,7 \%(n=109 / 1128)$ in 2014.Overall, 3.9\% $(n=91)$ of our trauma patients received operative management $(4.2 \%$ in 2015 and $3,5 \%$ in 2014 , with 30 days surgical mortality been $7,5 \%$ and $7,3 \%$ respectively).2.8\% $(n=64)$ of our patients were considered polytrauma (ISS > 17) and their mortality was $37.5 \%$. Overall non-salary cost for trauma management was 623,322 Euros. $50 \%$ of overall costs was attributed to managing RTAs' victims. A significant reduction of costs (mean 24\%, CI95\%:12\%-60\%) could have been achieved if prehospital triage was more effective.

Conclusion: There is a need for improved prehospital triage in order to increase the cost-effectiveness of trauma care. Road safety programs and further training of medical personnel in treating critical traumas are necessary.

References: 1.Sasser SM, Hunt RC, Faul M, Sugerman D, Pearson WS, Dulski T, Wald MM, Jurkovich GJ, Newgard CD, Lerner EB; Centers for Disease Control and Prevention (CDC)/"Guidelines for field triage of injured patients: recommendations of the National Expert Panel on Field Triage, 2011"/MMWR Recomm Rep. 2012 Jan 13

Disclosure: No significant relationships. 
0063

\section{VALUE OF INFERIOR VENA CAVA DIAMETERS AS PREDICTOR OF HEMODYNAMIC DETERIORATION IN SEVERE TRAUMA PATIENTS: HOW MUCH "FLATNESS"?}

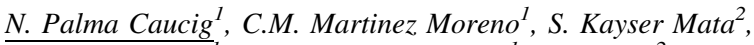 \\ C. Rey Varcalcel' $^{1}$, L. Garciafilia Cabrera ${ }^{1}$, J. Zulueta ${ }^{2}, F$. Turégano \\ Fuentes $^{2}$
}

${ }^{1}$ Cirugia General, HOSPITAL GENERAL UNIVERSITARIO GREGORIO MARAÑON, Madrid/SPAIN, ${ }^{2}$ Cirugia General, HOSPITAL UNIVERSITARO GREGORIO MARAÑON, Madrid/ SPAIN

Introduction: A collapsed IVC can be seen in CT scans of trauma patients with hypovolemic shock, but whether or not a "flat" IVC can predict hemodynamic deterioration is still controversial. Our aim was to evaluate the predictive value of the IVC ratio for hemodynamic deterioration in the first 6 hours after admission.

Material and methods: Retrospective cohort study of patients included in our Trauma Registry between 2013 and 2016. Inclusion criteria were stability at admission or after fluid resuscitation, and CT scan performed within three hours of arrival. We excluded patients with clinical pneumothorax or cardiac tamponade. A blinded radiologist measured the transverse-to-anteroposterior IVC ratio immediately above the right renal vein. Hemodynamic deterioration was defined as a SBP $<90 \mathrm{mmHg}$ in the first 6 hours after admission. Results: 246 patients ( $84 \%$ blunt) met the inclusion criteria. The median ISS and NISS were of 17 (IQ 10-29) and 22 (IQ 14-34), respectively. 59 were excluded because of pneumothorax. An IVC ratio $\geq 2.2$ was chosen according to the ROC results of a previously reported cohort study performed in hour center with 426 patients between 2007 and 2012. 48 patients (24\%) suffered hemodynamic deterioration in the first 6 hours after CT scan, $41 \%$ of the IVC ratio $\geq$ 2.2 group and $16 \%$ of the IVC ratio $<2.2$ group $(p<0,001)$. Sensitivity and negative predictive value were $60 \%$ and $85 \%$ respectively. Conclusion: Trauma patients with an IVC ratio $<2.2$ had a NPV of $85 \%$ for hemodynamic deterioration. This could be a helpful tool in selecting patients for less invasive treatments.

References: Shokei Matsumoto, MD, Kazuhiko Sekine, MD, Motoyasu Yamazaki, MD, Kenihiro Sasao, MD, Tomohiro Funabiki, MD, Masayuki Shimizu. Predictive Value of a Flat Inferior Vena Cava on Initial Computed Tomography for Hemodynamic Deterioration in Patients With Blunt Torso Trauma. The Journal of TRAUMA®. 2010; 69 (6) 1398-1402 Michal Radomski, MD, Ritesh Agnihothri, Stephanie Knapp, Daniel Scher, MD, Nadia Khati, MD, Kathleen Brindle. Inferior vena cava size is not associated with shock following injury. J Trauma Acute Care Surg. 2014; 77 (1) 34-37

Disclosure: No significant relationships.

\section{4}

\section{MAIN ERRORS IN THE MANAGEMENT OF TRAUMA PATIENTS: ANALYSIS OF A HOSPITAL TRAUMA REGISTRY}

\author{
M. Burneo Esteves ${ }^{1}$, A. Sanchez Arteaga ${ }^{2}$, A. Lusilla López, \\ A. Garcia Sevilla ${ }^{4}$, R. Orti Rodriguez, ${ }^{5}$ M.D. Pérez Díaz, \\ C. Rey Varcalcel ${ }^{3}$, F. Turégano Fuentes ${ }^{3}$
}

${ }^{1}$ Cirugía General Y Del Ap. Digestivo, Hospital Quirónsalud Sur, Madrid/SPAIN, ${ }^{2}$ Cirugía General Y Del Ap. Digestivo, Hospital Universitario Virgen del Rocio, Sevilla/SPAIN, ${ }^{3}$ Cirugia General Y Del Ap. Digestivo, Hospital General Universitario Gregorio Marañón, Madrid/SPAIN, ${ }^{4}$ Cirugía Maxilo Facial, Hospital General Universitario de Ciudad Real, Ciudad Real/SPAIN, ${ }^{5}$ Cirugía General Y Del Ap. Digestivo, Hospital Universitario Nuestra Señora de Candelaria, Santa Cruz de Tenerife/SPAIN

Introduction: One of the main components of Performance Improvement programs in trauma care is the periodic analysis of errors incurred in this process of care. A standard classification of these errors could facilitate comparison between registries and promote initiatives to reduce them. We aimed to assess and classify these errors by the Joint Commission (JC) taxonomy.

Material and methods: Retrospective, descriptive observational study of patients included in our Severe Trauma Registry for a period of 20 years (June 1993-December 2012). The characteristics and process of care of all patients dead with a Ps $>0.5$ were analyzed with the aim of defining possible errors and opportunities for improvement. These errors were classified in accordance to the five domains of the JC taxonomy.

Results: 2120 patients with severe trauma were reviewed. The mechanism was blunt (BT) in $75.7 \%$ of them, and penetrating (PT) in $24.3 \%$. Most errors were clinical and were incurred mainly in therapeutic decisions at the emergency department; mistakes were made mainly by medical personnel, and were mainly of two types: in the follow-up of instructions, and errors of knowledge.

Conclusion: Using the JC taxonomy we were able to identify the main type of error as clinical. The limitation of this classification is its complexity.

References: Montmany S, Pallisera A, Rebasa P, Campos A, Colilles $\mathrm{C}$, Luna A, et al. Preventable deaths and potentially preventable deaths. What are our errors? Injury. 2016;47 (3):669-73. Vioque SM, Kim PK, McMaster J, Gallagher J, Allen SR, Holena DN, et al. Classifying errors in preventable and potentially preventable trauma deaths: a 9-year review using the Joint Commission's standardized methodology. Am J Surg. 2014;208 (2):187-94. O'Reilly D, Mahendran K, West A, Shirley $\mathrm{P}$, Walsh M, Tai N. Opportunities for improvement in the management of patients who die from haemorrhage after trauma. Br J Surg. 2013;100 (6):749-55. Runciman W, Hibbert P, Thomson R, Van Der Schaaf T, Sherman H, Lewalle P. Towards an International Classification for Patient Safety: key concepts and terms. Int J Qual Health Care. 2009;21 (1):18-26. Ivatury RR, Guilford K, Malhotra AK, Duane T, Aboutanos M, Martin N. Patient safety in trauma: maximal impact management errors at a level I trauma center. J Trauma. 2008;64 (2):265-72. Divi C, Koss RG, Schmaltz SP, Loeb JM. Language proficiency and adverse events in US hospitals: a pilot study. Int J Qual Health Care. 2007;19 (2):60-7. Teixeira PG, Inaba K, Hadjizacharia P, Brown C, Salim A, Rhee $\mathrm{P}$, et al. Preventable or potentially preventable mortality at a mature trauma center. J Trauma. 2007;63 (6):1338-47 Gruen RL, Jurkovich GJ, McIntyre LK, Foy HM, Maier RV. Patterns of errors contributing to trauma mortality: lessons learned from 2,594 deaths. Ann Surg. 2006;244 (3):371-80.

Disclosure: No significant relationships. 


\section{5}

DOES ASA ADD VALUE TO THE PREDICTIVE ABILITY OF TRISS IN THE AGEING TRAUMA POPULATION?

\author{
L. De Munter ${ }^{1}$, S. Polinder ${ }^{2}$, N.C.w. Ter Bogt ${ }^{3}$, M.A.c. De Jongh ${ }^{1}$
}

${ }^{1}$ Trauma Topzorg, Elisabeth-Twee Steden Hospital, Tilburg/ NETHERLANDS, ${ }^{2}$ Department Of Public Health, Erasmus Medical Centre, Rotterdam/NETHERLANDS, ${ }^{3}$ Network Emergency Care Euregio, Trauma Registry, Enschede/NETHERLANDS

Introduction: It has been shown previously that the Trauma and Injury Severity Score (TRISS) is not an adequate tool to benchmark hospitals for the ageing trauma population, although it showed accurate results in the general trauma population. With the ageing population, benchmark numbers will be negatively biased in hospitals that mainly treat elderly people. Therefore, the aim of this study was to assess performance of TRISS with simple and minimal adjustments for age and the addition of comorbidity. Because predictors should be easily available, age is incorporated as continuous variable or restructured in categories. In addition, the predictive value for the American Society of Anesthesiologists Physical Status (ASA) will be assessed.

Material and methods: This research was conducted in Network Emergency Care Brabant. Missing values were imputed and subsets were created based on age and injury type (i.e. hip fractures). Model performance was assessed by discrimination and calibration. After applying a shrinkage factor, the models were externally validated in Network Emergency care Euregio.

Results: A total of 23,591 observations were used for model development and a total of 7,787 observations were used for external validation. The discriminative ability improved significant after the adjustment of age, and increased even further after incorporating ASA into the model. Calibration of the newly developed models were all accurate for the general population and significantly improved when assessed in the elderly.

Conclusion: Performance of the newly developed models improved significantly and showed accurate and reliable predictions in the total population and in the elderly, without major adjustments.

References:

Disclosure: No significant relationships.

\section{6}

INTRACRANIAL PRESSURE MONITORING IN ISOLATED SEVERE TRAUMATIC BRAIN INJURY: AN EVALUATION OF OUTCOMES AND COMPLIANCE WITH GUIDELINES USING A MATCHED COHORT FROM THE TRAUMA IMPROVEMENT QUALITY PROGRAM (TQIP) DATABASE

\author{
R. Ahl ${ }^{1}$, B. Sarani ${ }^{2}$, G. Sjolin ${ }^{3}$, S. Mohseni ${ }^{3}$
}

${ }^{1}$ Department Of Surgery, Karolinska University Hospital, Stockholm/ SWEDEN, ${ }^{2}$ Center For Trauma And Critical Care, George Washington University, Washington/WA/UNITED STATES OF AMERICA, ${ }^{3}$ Department Of Surgery, Orebro University Hospital, Orebro/SWEDEN

Introduction: Although recommended by the Brain Trauma Foundation, the use of intracranial pressure (ICP) monitoring in patients with severe traumatic brain injury (TBI) is still debated. We sought to determine compliance with guidelines and impact of ICP-monitoring on outcomes in patients with isolated severe TBI.

Material and methods: This is a retrospective observational study using data from the Trauma Quality Improvement Program (TQIP) database from 2014. Patients $\geq 18$ years with an isolated severe TBI, defined as GCS score $\leq 8$ with an intracranial AIS $\geq 3$ and all other body regions AIS $=0$, due to blunt injury were recruited. Patients who had an AIS $=6$ or died $<48$ hours were excluded to control for nonsurvivable injuries. Patients with and without ICP-monitoring were propensity score matched 1:1 based on variables indicating need for monitoring and factors known to influence outcome.

Results: 3,289 patients met inclusion criteria. Of these, $601(18.3 \%)$ patients were subjected to ICP-monitoring. 557 matched pairs $(\mathrm{n}=$ $1,154)$ were available for analysis. No discrepancies were noted with regards to patient and injury characteristics or required neurosurgical intervention. Both ICU (median [Q1, Q4]: 12 [6, 18] vs. 6 [3, 12] days, $\mathrm{p}<0.001$ ) and hospital length of stay (median [Q1, Q4]: 16 [9, $26]$ vs. $10[5,19]$ days, $\mathrm{p}<0.001)$ were longer in the ICP-monitored group. Patients subjected to ICP-monitoring had an increased mortality risk with OR 1.6, CI 95\% 1.1-2.5, $\mathrm{p}=0.038$.

Conclusion: No survival benefit of monitoring was noted. This finding needs further investigation since ICP-monitoring is associated with increased resource utilization leading to higher costs, longer ICU and hospital length of stay.

References:

Disclosure: No significant relationships.

\section{7}

\section{A CLINICAL AND FORENSIC STUDY INTO MORTALITY FROM ROAD TRAFFIC ACCIDENTS IN THE U.K}

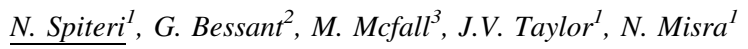

${ }^{1}$ Surgery, Aintree University Hospital, Liverpool/UNITED KINGDOM, ${ }^{2}$ Emergency Medicine, Yorkshire and Humber Deanery, Leeds/UNITED KINGDOM, ${ }^{3}$ Road Traffic Collision Unit, Merseyside Police, Liverpool/UNITED KINGDOM

Introduction: Understanding the pathology of fatal road traffic injuries can better inform clinicians managing blunt traumatic cardiac arrest.

Material and methods: Data of fatal road traffic accidents (RTA) in Merseyside from January 2013 to June 2016 was collected retrospectively. Dynamics of the RTA and the effect on the patient were evaluated by means of police roadside reports and post mortem reports.

Results: There were 80 fatal RTAs over the 42 months. Main contributing factors were dangerous vehicle movement $(36.25 \%)$, driver judgement error $(33.75 \%)$ and over-speeding $(30 \%)$, with alcohol/drugs $(12.5 \%)$ and road/environmental factors directly affecting accident $(6.25 \%)$ only featuring in a minority. 38 of the 80 fatalities had a post mortem carried out and this subset of patients were evaluated. Only $8 \%$ (3) deaths identified an isolated single significant injury. Skull fractures and/or intracranial haemorrhage were the leading injuries identified in $44 \%$ (17). Haemothoraces and liver lacerations were both identified in $32 \%$ (12) of cases. 6 (75\%) out of the 8 patients found to have significant splenic injury also suffered liver injury and $88 \%$ (7 out of 8 ) of significant cardiac injuries who also had a haemothorax. Cervical cord injury was significant in $24 \%$ (9) of deaths. While aortic arch injury (18\%) and pelvic fractures $(16 \%)$ were common, abdominal aortic $(1 \%)$ and caval injuries $(0)$ were much less so. $5(13 \%)$ post mortems commented on the 
contributing factor of a concurrent medical condition leading directly to death; such as splenomegaly secondary to haematological malignancy that lead to increased chance and consequences of visceral injury.

Conclusion: Most fatalities secondary to road traffic accidents may occur due to human error. Clinically, the insult is multi-systemic and the clinician managing a traumatic cardiac arrest should be aware of this.

References:

Disclosure: No significant relationships.

\section{8}

\section{REGAIN OF THE PRE-FRACTURE BASIC MOBILITY AT THE TIME OF ACUTE HOSPITAL DISCHARGE IS ASSOCIATED WITH THE RISK OF 30-DAY POST- DISCHARGE MORTALITY - A 1-YEAR NATIONWIDE REGISTER STUDY OF 5,154 DANISH PATIENTS WITH HIP FRACTURE}

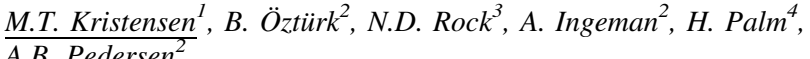

${ }^{1}$ Physical Medicine And Rehabilitation Research - Copenhagen (pmrc), Departments Of Physical Therapy And Orthopedic Surgery, Copenhagen, Denmark, Amager-Hvidovre University Hospital, Hvidovre/DENMARK, ${ }^{2}$ Department Of Clinical Epidemiology, Aarhus University Hospital, Denmark., Aarhus/DENMARK, ${ }^{3}$ Department Of Orthopedic Surgery O, Odense University Hospital, Denmark, Odense/DENMARK, ${ }^{4}$ Hip Fracture Unit, Department Of Orthopedics, Hvidovre University Hospital, Copenhagen, Denmark, Hvidovre/DENMARK

Introduction: The regain of pre-fracture basic mobility status at acute hospital discharge is considered an important first step for patients recovering from a hip fracture (HF). The Cumulated Ambulation Score (CAS) is a validated measure of basis mobility used in all patients with HF in Denmark and registered in the nationwide Danish Multidisciplinary Hip Fracture Database (DMHFD). We examined the association between the regain of prefracture basic mobility at time of acute hospital discharge and 30-day post-discharge mortality after HF.

Material and methods: Using the DMHFD we identified 5,154 patients, aged $\geq 65$ years admitted with an acute first time HF during the year 2015, at all the 25 Danish HF operating hospitals, and with both a pre-fracture and discharge CAS score. Regain of basis mobility was defined as achieving $\geq$ the total pre-fracture CAS at discharge. Results: Overall 30-day post-discharge mortality was $6.6 \%(n=340)$, while this was $2.6 \%(n=54)$ for the $40 \%(n=2,051)$ of patients who regained their pre-fracture CAS score compared with $9.2 \%(n=286)$ for those who did not $(\mathrm{p}<0.001)$. Correspondingly in adjusted analyses, the risk of 30-day mortality was 2.7 times higher if the prefracture CAS was not regained.

Conclusion: In this large national HF registry study of 5,154 Danish patients we found that the regain of pre-fracture basic mobility level before acute hospital discharge was strongly related to the 30-day post-discharge mortality. This indicates the importance of basic mobility independence as a primary early rehabilitation goal, and that the CAS could be considered as an outcome parameter in other HF registries.

References:

Disclosure: No significant relationships.

\section{O068A}

\section{LEAVING THE HOSPITAL AGAINST MEDICAL ADVICE AFTER TRAUMATIC INJURY}

N. Balakrishnan, M. Mohammed, J. Blansfield, H. Wing, S. Sanchez, C. Narsule, R. Schulze, P. Burke, G. Kasotakis

Trauma Surgery, Boston University Medical Center, Boston/MA/ UNITED STATES OF AMERICA

Introduction: Medical patients with who leave Against Medical Advice (AMA) suffer greater morbidity and readmission rates. However, the phenomenon is not well studied in the trauma population. With this project, we aim to identify risk factors for leaving AMA and assess related outcomes.

Material and methods: All trauma activations from an urban academic level I trauma center over a period of 6.5 years were reviewed, and the incidence, reason and location of leaving AMA recorded. Patient and injury characteristics, as well as hospital course information were studied and a forward stepwise regression model was fitted for leaving AMA. Statistical significance was declared at $\mathrm{p}<$ 0.05 .

Results: Out of 13,416 consecutive trauma activations, $3.74 \%$ left AMA and the most common location the regular ward (46\%). Patients who left AMA quoted other commitments (14.5\%), were unhappy with care $(8.4 \%)$, had other reasons $(63 \%)$, or eloped $(14.1 \%)$ Younger African-American males, victims of assault or penetrating injuries, and those with comorbidities, substance abuse, and government/no insurance were more likely to leave AMA. Conversely, those with brain injury, fractures, higher ISS, requiring transfusions, immediate intubation, operative management or other major interventions, and those who suffered complications were less likely to leave AMA. Of the AMA discharges, $22.5 \%$ returned to the emergency department within 7 days, $36.3 \%$ of whom required readmission. There were no mortalities.

Conclusion: Efforts to target those with the aforementioned factors should be undertaken to minimize discharges AMA, although they may be of lower clinical significance.

References: Glasgow JM, Vaughn-Sarrazin M, Kaboli PJ. "Leaving against medical advice (AMA): Risk of 30-day mortality and hospital readmission.” J Gen Intern Med. 2010; 25 (9): 926-9. Hwang SW, Li J, Gupta R, Chien V, Martin RE. "What happens to patients who leave hospital against medical advice?" Canadian Medical Association Journal. 2003; 168 (40): 417-20. Parreco J, Buicko J, Cortolillo N, Namias N, Rattan R. "Risk factors and costs associated with nationwide nonelective readmission after trauma." J Trauma Acute Care Surg. 2017; 83: 126-134. Saia M, Buja A, Mantoan D, Bertoncello C, Baldovin T, Callegaro G, Baldo B. "Frequency and trends of hospital discharges against medical advice (DAMA) in a large administrative database." Ann $1^{\text {st }}$ Super Sanità. 2014; 50 (4): 356-362. Spooner KK, Salemi JL, Salihu HM, Zoorob RJ. "Discharge against medical advice in the United Stated, 2002-2011." Mayo Clinic Proceedings. 2017; 92 (4): 525-535.

Disclosure: No significant relationships. 


\section{ACUTE ELDERLY PATIENTS}

\section{9}

\section{ENHANCEMENT OF DETERIORATED WOUND HEALING IN DIABETIC MICE BY LIPID MEDIATOR 11, 12 EPOXYEICOSATRIENOIC ACID (EET)}

\section{K. Sommer, A.L. Sander, D. Henrich, I. Marzi, J. Frank}

Klinik Für Unfall-, Hand- Und Wiederherstellungschirurgie, Uniklinik Frankfurt am Main, Frankfurt am Main/GERMANY

Introduction: Wound healing is often complicated in chronic diabetic patients and still a challenge in modern medicine. EETs have so far been a promising tool to ameliorate wound healing. They regulate inflammation, angiogenesis, and vascular tone. As their effect on diabetic wounds has not been evaluated, we examined the effects of local 11, 12 EET application on wounds in diabetic mice in this study. Material and methods: Diabetes was induced one week prior to wounding by streptozotocin injection. Standardized full thickness dermal wounds were created on the dorsum of the ears $(n=10$ per group). Epithelialization was measured every second day. CD31 and VEGF were evaluated as markers for neovascularization on day 3,6 , and 9 after wounding ( $\mathrm{n}=8$ per group). SDF1 $\alpha$ was measured as important homing factor on the same days.

Results: Local 11, 12 EET application significantly reduced time to wound epithelialization to 8.40 days $\pm 0.31 \mathrm{SD}$ (standard deviation) compared to non-treated diabetic wounds that closed on day $13.00 \pm$ 0.49 SD. 11, 12 EET treatment also significantly enhanced CD31 and VEGF expression on day 3 and day 3 and 6 respectively. Furthermore SDF- $1 \alpha$ expression was significantly elevated after 11, 12 EET treatment on day 3 .

Conclusion: 11, 12 EET application enhances deteriorated wound healing in diabetic mice by promoting neovascularization as wounds exhibit an augmented expression of CD31 and VEGF. Moreover, 11, 12 EET application seem to improve homing of progenitor and immune cells by enhancing SDF- $1 \alpha$ expression. Thus, lipid mediators as 11, 12 EET should be considered a promising therapeutic tool in chronic diabetic ulcers as well as in posttraumatic situations in diabetic patients.

References: Liu, Zhao-Jun; Velazquez, Omaida C. (2008): Hyperoxia, endothelial progenitor cell mobilization, and diabetic wound healing. In: Antioxidants \& redox signaling 10 (11), S. 1869-1882. DOI: 10.1089/ars.2008.2121. Nissen, N. N.; Polverini, P. J.; Koch, A. E.; Volin, M. V.; Gamelli, R. L.; DiPietro, L. A. (1998): Vascular endothelial growth factor mediates angiogenic activity during the proliferative phase of wound healing. In: The American journal of pathology 152 (6), S. 1445-1452. Panigrahy, Dipak; Kalish, Brian T.; Huang, Sui; Bielenberg, Diane R.; Le, Hau D.; Yang, Jun et al. (2013): Epoxyeicosanoids promote organ and tissue regeneration. In: Proceedings of the National Academy of Sciences of the United States of America 110 (33), S. 13528-13533. DOI: 10.1073/ pnas.1311565110. Sander, Anna Lena; Jakob, Heike; Sommer, Katharina; Sadler, Christian; Fleming, Ingrid; Marzi, Ingo; Frank, Johannes (2011): Cytochrome P450-derived epoxyeicosatrienoic acids accelerate wound epithelialization and neovascularization in the hairless mouse ear wound model. In: Langenbeck's archives of surgery 396 (8), S. 1245-1253. DOI: 10.1007/s00423-011-0838-z.

Disclosure: No significant relationships.
0070

\section{FIBRINOLYSIS PHENOTYPES IN THE ELDERLY: IMPLICATION FOR EMPIRIC ANTI-FIBRINOLYTICS}

\author{
G.R. Nunns ${ }^{1}$, H.B. Moore ${ }^{1}$, E.E. Moore ${ }^{1}$, G.R. Stettler ${ }^{2}$, \\ J.J. Sumislawski, B.A. Cotton ${ }^{3}$, C.C. Silliman ${ }^{l}$, A. Banerjee ${ }^{I}$, \\ M.J. Cohen ${ }^{1}$, A. Sauaia ${ }^{1}$
}

${ }^{1}$ Department Of Surgery, University of Colorado Denver, Aurora/CO/ UNITED STATES OF AMERICA, ${ }^{2}$ Surgery, University of Colorado, Aurora/CO/UNITED STATES OF AMERICA, ${ }^{3}$ The Center For Translational Injury Research, The University of Texas Health Science Center at Houston, Houston/TX/UNITED STATES OF AMERICA

Introduction: Post-injury fibrinolysis can present as shutdown (SD), physiologic $(\mathrm{PF})$, and hyperfibrinolysis $(\mathrm{HF})^{1}$. Both $\mathrm{SD}$ and $\mathrm{HF}$ are associated with increased mortality compared to PF, with SD being the most frequent while HF is the most lethal ${ }^{1,2}$. Age has been shown to be a risk factor for SD, but it is not known whether age modifies the association between fibrinolysis and death ${ }^{2}$. We hypothesized that SD incidence would be higher in the elderly, while PF would maintain its survival benefit.

Material and methods: Patients presenting to three trauma centers were enrolled from 2010-2016. Patients with ISS $>15$ and admission rapid thrombelastography (rTEG) were stratified by age: 18-44, 45-64, and $>65$ years. Fibrinolysis phenotypes were defined TEGLY30: $\mathrm{SD} \leq 0.8 \%$, PF $0.9-2.9 \%$, HF $>3 \%$. We adjusted for confounders using Poisson and conditional logistic regression models.

Results: 2682 patients were included: $58 \%$ aged $18-44,28 \%$ aged $45-64,14 \% \geq 65$ years. All age groups showed the previously described U-shaped trend in mortality, with $\mathrm{HF}>\mathrm{SD}>\mathrm{PF}(\mathrm{p}<0.05)$. Compared to the 18-44 age group, the $45-64$ and $\geq 65$ age groups were significantly more likely to develop SD (45-64, Relative risk, RR: $1.22,95 \%$ CI: $1.21-1.24 ; \geq 65$ RR 1.40, 95\% CI: 1.34-1.47). Age did not significantly modify the association between $\mathrm{PF}$ and death in the multivariate model (interaction $\mathrm{PF}^{*}$ age $\mathrm{p}=0.79$ ).

Conclusion: While increasing age was independently associated with increased incidence of SD, age did not affect the survival benefit associated with PF. These data suggest empiric anti-fibrinolytics in the elderly may be hazardous and reinforces the need for point-of-care testing to individualize therapy.

References: 1. Moore HB, Moore EE, Gonzalez E, Chapman MP, Chin TL, et al. Hyperfibrinolysis, physiologic fibrinolysis, and fibrinolysis shutdown: the spectrum of postinjury fibrinolysis and relevance to antifibrinolytic therapy. J Trauma Acute Care Surg 2014:77:811-817; discussion 817. 2. Moore HB, Moore EE, Liras IN, Gonzalez E, Harvin JA, et al. Acute Fibrinolysis Shutdown after Injury Occurs Frequently and Increases Mortality: A Multicenter Evaluation of 2,540 Severely Injured Patients. J Am Coll Surg 2016:222:347-355.

Disclosure: We receive research support from Haemonetics LLC and TEM GmbH, but have no financial interests in these companies. Drs. E.E. Moore and H.B. Moore have shared intellectual property with Haemonetics LLC 
0071

\section{DEVELOPMENT AND VALIDATION OF A NEW PRE- OPERATIVE IDEX TO ASSESS FRAILTY IN ELDERLY PATIENT UNDERWENT EMERGENCY SURGICAL PROCEDURES: EMERGENCY SURGERY FRAILTY INDEX (EMSFI)}

\author{
G. Massa, G. Costa, P. Fransvea, B. Frezza, L. Bragaglia, \\ G. Balducci
}

Chirurgia Generale E D’urgenza, Facoltà di Medicina e Psicologia Università "Sapienza" Roma, Rome/ITALY

Introduction: Several studies suggest that frailty predisposes elderly to worsening outcome after surgery, so it is paramount to have an accurate stratification of surgical risk. The aim of this study is to identify a new pre-operative Frailty Index to assess risk of elderly population underwent emergency surgical procedure.

Material and methods: Preliminary results of the nationwide multicenter Italian observational study FRAILESEL were considered, involving patients over 65 years of age who require emergency surgical procedures. With the aim of developing an index of frailty in emergency surgery called "EmSFI" (Emergency Surgery Frailty Index), an accurate study of comorbidities and clinical factors was carried out, evaluating its statistical significance in terms of morbidity and mortality. ROC curve analysis was then performed to test the sensitivity and specificity of our Frailty Index and other pre -operative scores.

Results: EmSFI was calculated on a heterogeneous population of 784 patients. The mean value of this was $3.81 \pm 2.29$ (range 1 to 12 ). Analyzing the frequencies of morbidity and mortality compared to the various EMSFI classes, these have increased as the value of the index increases. Both the score of P-POSSUM and EmSFI proved to be moderately accurate predictors of mortality (AUC 0.750 and AUC 0.731 respectively). While as regards morbidity, only P-POSSUM proved to be a moderately accurate test (AUC 0.727).

Conclusion: EmSFI it is asimple, easy-to-use tool in the emergency setting and at the same time reliable enough to calculate the clinical fragility of the elderly patient. Further studies are needed to validate the Index in a wider population

References: 1 - ROCKWOOD K, STADNYK K, MACKNIGHT C, MCDOWELL I, HÉBERT R, HOGAN DB. A brief clinical instrument to classify frailty in elderly people. Lancet. 1999 Jan 16;353 (9148):205-6. 2 - FRIED LP, TANGEN CM, WALSTON J, NEWMAN AB, HIRSCH C, GOTTDIENER J, SEEMAN T, TRACY R, KOP WJ, BURKE G, MCBURNIE MA, CARDIOVASCULAR HEALTH STUDY COLLABORATIVE RESEARCH GROUP. Frailty in older adults: evidence for a phenotype. J Gerontol. A Biol Sci Med Sci. 2001 Mar; 56 (3):M146-56. 3 - SEARLE SD, MITNITSKI A, GAHBAUER EA, GILL TM, ROCKWOOD K. A standard procedure for creating a frailty index. BMC Geriatr. 2008 Sep 30;8:24. 4 - CHIMUKANGARA M, HELM MC, FRELICH MJ, BOSLER ME, REIN LE, SZABO A, GOULD JC. A 5-item frailty index based on NSQIP data correlates with outcomes following paraesophageal hernia repair. Surg Endosc. 2017 Jun;31 (6):25092519. 5 - VELANOVICH V, ANTOINE H, SWARTZ A, PETERS D, RUBINFELD I. Accumulating deficits model of frailty and postoperative mortality and morbidity: its application to a national database. J Surg Res. 2013 Jul;183 (1):104-10. 6 - OROUJI JOKAR T, IBRAHEEM K, RHEE P, KULAVATUNYOU N, HAIDER A, PHELAN HA, FAIN M, MOHLER MJ, JOSEPH B. Emergency general surgery specific frailty index: A validation study. J Trauma Acute Care Surg. 2016 Aug; 81 (2):254-60.

Disclosure: No significant relationships.
0072

\section{MANAGEMENT OF ELDERLY BLUNT HEAD TRAUMA PATIENTS TAKING ANTITHROMBOTICS: WHO REALLY NEEDS REPEAT IMAGING?}

\author{
D. Scanting, J. Williamson, R. Kucejko, B. Mccracken
}

Surgery, Drexel University College of Medicine/Hahnemann University Hospital, Philadelphia/PA/UNITED STATES OF AMERICA

Introduction: Many elderly patients suffer from intracranial hemorrhage (ICH) after blunt head trauma and many are utilizing antithrombotic (AT) medications. There is no relevant literature evaluating delayed CT head (D-CTH) to guide management in this population. We hypothesized that D-CTH in this group would not change clinical management.

Material and methods: We reviewed patients $\geq 65$ years sustaining blunt head injuries from January 2010-July 2017 using our institutional trauma database. AT-patients who had ICH on initial CT and received D-CTH were included. AT-patients with worsened $\mathrm{ICH}$ on D-CTH were compared to those with stable to improved ICH on D-CTH and those taking AT were compared to a matched cohort of elderly non AT-patients. Chi Square, logistic regression and Mann Whitney U tests were utilized.

Results: 143 AT-patients were compared with 39 non AT-patients. 54 had worsened bleeds on D-CTH versus 18 non AT-patients $(37.8 \%$ vs-47.4\%, $\mathrm{p}=0.351) .22$ AT and 10 non AT-patients had new bleeds on D-CTH $(15.4 \%$-vs-26.3\%, $\mathrm{p}=0.149) .1$ comatose AT-patient had change in management from D-CTH $(0.7 \%$-vs- $0 \%, \mathrm{p}=1)$. There was no difference for surgical intervention or GCS, though AT-patients were older (80years-vs-76years, $p=0.001$ ). 54 AT-patients had worsened bleeds while 89 did not. There was no difference between these cohorts in age or GCS. Progression of hemorrhage increased with warfarin use $(27.8 \%$-vs-13.5\%, p $=0.0468)$, prevalence of subdural hematoma $(72.2 \%$-vs-55.1\%, $\mathrm{p}=0.0347)$ and multiple types of ICH $(55.5 \%$-vs- $19.1 \%, \mathrm{p}<0.0001)$.

Conclusion: D-CTH resulted in charges of $\$ 4,350 \mathrm{USD} /$ patient and did not alter management; clinical examination did. Patients with unreliable neurologic examination may benefit from D-CTH, especially those using warfarin and those with subdural hematoma or multiple ICH types.

References: 1. Centers for Disease Control and Prevention, National Center for Injury Prevention and Control. Web-based Injury Statistics Query and Reporting System (WISQARS) [online]. Accessed January 17th, 2017. Available from URL: https://www.cdc.gov/injury/wisqars/index.html.

2. United States Census Bureau. QuickFacts [online]. Accessed January 17th, 2017. Available from URL: https://www.census.gov/ en.html.

3. Lonner JH, Koval KJ Polytrauma in the elderly. Clinical orthopaedics and related research 1995:136-143.

4. Spaniolas K, Cheng JD, Gestring ML, Sangosanya A, Stassen NA, et al. Ground level falls are associated with significant mortality in elderly patients. The Journal of trauma 2010:69:821-825.

5. Heffernan DS, Thakkar RK, Monaghan SF, Ravindran R, Adams CA, Jr., et al. Normal presenting vital signs are unreliable in geriatric blunt trauma victims. The Journal of trauma 2010:69:813820.

6. Patel VI, Thadepalli H, Patel PV, Mandal AK. Thoracoabdominal injuries in the elderly: 25 years of experience. Journal of the National Medical Association 2004:96:1553-1557. 
7. Siracuse JJ, Robich MP, Gautam S, et al. Antiplatelet agents, warfarin, and epidemic intracranial hemorrhage. Surgery 2010:148:724-729; discussion 729-730.

8. Peck KA, Calvo RY, Schechter MS, et al. The impact of preinjury anticoagulants and prescription antiplatelet agents on outcomes in older patients with traumatic brain injury. The journal of trauma and acute care surgery 2014:76:431-436.

9. Scantling D, Fischer C, Gruner R et al. The role of delayed head $\mathrm{CT}$ in evaluation elderly blunt head trauma victims taking antithrombotic therapy. Eur J Trauma Emerg Surg. 2017; e-published ahead of print.

10. Joseph B, Sadoun M, Aziz H, et al. Repeat head computed tomography in anticoagulated traumatic brain injury patients: still warranted. The American surgeon 2014:80:43-47. 11. Hylek EM, Singer DE. Risk factors for intracranial hemorrhage in outpatients taking warfarin. Annals of internal medicine. 1994 Jun 1;120 (11):897-902. 12. Kreitzer N, Lyons MS, Hart K, et al. Repeat Neuroimaging of Mild Traumatic Brain- injured Patients With Acute Traumatic Intracranial Hemorrhage: Clinical Outcomes and Radiographic Features. Academic emergency medicine : official journal of the Society for Academic Emergency Medicine. 2014;21 (10):10831091. 13. Joseph B, Aziz H, Pandit V et al. A Three-Year Prospective Study of Repeat Head Computed Tomography in Patients with Traumatic Brain Injury. J Am Coll Surg. 2014; 219 (1)45-51. 14. Joseph B, Aziz H, Pandit V et al. Low-dose aspirin therapy is not a reason for repeating head computed tomographic scans in traumatic brain injury: a prospective study. J Surg Res. 2014;186 (1): 287-291. 15. Uccella L, Zoia A, Perlasca F et al. Mild Traumatic Brain Injury in Patients on Long-Term Anticoagulation Therapy: Do They Really Need Repeated Head CT Scan? World Neurosurg. 2016;93:100-103. Disclosure: No significant relationships.

\section{3}

\section{INTESTINAL ISCHEMIA IN THE ELDERLY SURGICAL PATIENT}

\section{P. Colsa ${ }^{1}$, D. Morales ${ }^{2}$}

${ }^{1}$ Servicio De Cirugía General Y Del Aparato Digestivo, Hospital Grande Covián, Arriondas/SPAIN, ${ }^{2}$ Servicio De Cirugía General Y Del Aparato Digestivo, Hospital Universitario Marqués de Valdecilla, Santander/SPAIN

Introduction: Intestinal ischemia is a common cause of acute abdomen in the elderly. Under this entity, ischemic colitis and mesenteric ischemia are grouped together. The objective of the present study was to describe the characteristics of patients older than 70 years who were treated urgently for intestinal ischemia.

Material and methods: A retrospective population-based study of patients older than 70 years who were operated on for urgent abdominal pathology. A total of 675 patients undergoing surgery from January 2012 to December 2013 in the community of Cantabria were included. Interventions for complications immediate to scheduled surgery and those that did not require hospitalization were excluded from the study. In the $4.8 \%$ of patients the diagnosis was intestinal ischemia. Data were analyzed using the X2 test with SPSS 17.0 statistical software. They were considered significant at $\mathrm{p}<0.05$.

Results: Intestinal ischemia predominates in the group of patient under 80 , with a mean age of 79.4 years and a standard deviation of 4.7 years. Of these, $61.3 \%$ were male and $38.7 \%$ female. $45.2 \%$ were diagnosed with mesenteric ischemia and $54.8 \%$ with ischemic colitis.
$67.7 \%$ took part in the Hospital Marqués de Valdecilla; 29\% in Hospital Sierrallana and 3.2\% in Laredo.

They had a history of hypertension $77.4 \%$ and $19.4 \%$ of peripheral arteriopathy. The proportion of patients with polymedication was $62.5 \%$.

At the time of surgery $96.6 \%$ met criteria of sepsis and $80 \%$ of shock

In almost half of the patients, exploratory laparotomy was performed, with total colectomy in $16.1 \%$, right hemicolectomy in $16.1 \%$, left in $9.7 \%$, enteric segmental resection 9.7\%, Hartmann intervention $3.2 \%$, and AMS embolectomy $3.2 \%$.

The surgical morbidity was $22.6 \%$ and the medical morbidity was $64.5 \%$. $54.8 \%$ in the form of cardiac complications; $48.4 \%$ were respiratory and $48.4 \%$ were urinary.

$72.7 \%$ developed postoperative shock, and mortality reached $54.8 \%(78.6 \%$ of mesenteric ischemias and $55.6 \%$ of ischemic colitis).

Conclusion: - Although intestinal ischemia is more frequent at advanced ages, the surgical indication was preferred at younger ages. - These patients are especially vulnerable because of their comorbidity. . At the time of the intervention, many patients have an immune and hemodynamic compromise. - Cardiac, respiratory, nephrouretic complications, shock and early mortality were frequent in the ischemic processes.

References: Feuerstadt P, Brandt LJ. Colon Ischemia. Recent Insights and Advances. Curr Gastroenterol Rep.2010 Diagnosis and Management.9th edition. Saunders-Elsevier. Philadelphia, 2010: 2027-2048. Kärkkäinen J, Lehtimäki T, Manninen H, Paajanen H. Acute Mesenteric Ischemia Is a More Common Cause than Expected of Acute Abdomen in the Elderly. J GastrointestSurg.2015; 19:1407-1414. 2027-2048.

Disclosure: No significant relationships.

\section{4}

\section{ABDOMINAL INJURIES AFTER FALL FROM STANDING - INTERVENTION IS PREDICTABLE WITHOUT ROUTINE IMAGING}

N. Hagan, H.O. Berdel, A. Tefft, A.C. Bernard

Department Of Surgery, University of Kentucky, Lexington/KY/ UNITED STATES OF AMERICA

Introduction: As the population has aged, falls from standing (FFS) have become the most common mechanism of injury at many centers. Liberal imaging of high-energy trauma is common but of questionable value in FFS. We hypothesize that abdominal trauma requiring intervention is rare in FFS and that history and physical examination are sufficient screening for abdominal injuries that require intervention.

Material and methods: We queried the trauma registry at our ACSverified Level 1 trauma center for FFS from 1/1/14-12/31/16. Demographics, historical details, hemodynamics, injuries, injury severity, procedures, and outcome were recorded.

Results: 1,914 falls were analyzed. Fracture hematomas were excluded. 62 patients $(3.2 \%)$ had 77 injuries. Average age was 65 years. Mean abdominal abbreviated injury score (AIS) was 2.6 (range 1-5). Mean injury severity score (ISS) was 13.1. Spleen injury was most common (Table). Table. Abdominal Injuries in FFS Area of Injury Number of Injuries Mean AIS ABD Mean ISS Spleen 32 3.0 15.3 Retroperitoneum 12 2.0 9.3 Renal/Adrenal 7 2.0 8.4 Liver 5 2.0 16.4 Abdominal Wall 4 1.8 13.2 Bowel 13.0 3.0 Gyn 13.0 9.0 All 
cases requiring intervention (4 splenic, 1 bowel) had abnormal abdominal physical examination. Fall against objects (FAO) was common in those requiring intervention ( 3 of 5). Neither age nor use of anti-platelet/anti-coagulants was associated with intervention. Conclusion: Abdominal injuries in FFS are rare. We found no injuries requiring intervention that were not evident on exam. FAO may carry a higher risk of intervention. Screening patients by history and physical examination is sufficient and allows for selective abdominal CT.

References: Lavingia KS, Collins JN, Scoult MC, Terzian WH, Weireter LJ, Britt LD. Torso Computed Tomography Can Be Bypassed after Thorough Trauma Bay Examination of Patients Who Fall from Standing. Am Surg 2015; 81 (8): 798-801. Ong AW, Castor L, Figueredo R, Butler S, Fernandez F. Pan-body computed tomographic scanning for patients with intracranial hemorrhage after lowenergy falls. Am J Surg 2015; 209: 521-525.

Disclosure: No significant relationships.

\section{5}

\section{FIBRINOLYSIS SHUTDOWN IN HEMODIALYSIS PATIENTS: EMPIRIC TXA MAY HOLD UNFORESEEN RISKS IN THIS POPULATION}

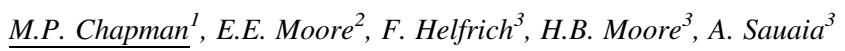

${ }^{1}$ Vascular And Interventional Radiology, University of Colorado Denver, Aurora/CO/UNITED STATES OF AMERICA, ${ }^{2}$ Surgery, Denver Health Medical Center, Denver/CO/UNITED STATES OF AMERICA, ${ }^{3}$ Department Of Surgery, University of Colorado Denver, Aurora/CO/UNITED STATES OF AMERICA

Introduction: With an increasingly elderly trauma population, the impact of preexisting medical disease on the practice of hemostatic resuscitation must be considered. In particular, the rise in type II diabetes has driven an epidemic of renal failure in this population. The hemostatic disorders in end stage renal disease (ESRD) are complex and poorly understood. ESRD patients are conventionally thought to have a bleeding diathesis, but paradoxically suffer from frequent thrombotic events. We sought to characterize, in detail, the pathophysiology of coagulation and fibrinolytic disorders in ESRD, to determine if baseline derangements exist, which should alter the empiric hemostatic resuscitation algorithm for these patients.

Material and methods: We compared 75 consecutive ESRD patients to 134 healthy volunteers. We utilized multi-channel thrombelastography (TEG) to characterize the patients' coagulation and fibrinolytic systems. The TEG Functional Fibrinogen assay was used to assess fibrinogen levels. The fibrinolytic system was tested with tPA-Challenged TEG, wherein exogenous tPA is added to the TEG cup, and fibrinolysis quantified as the 30 minute lysis (LY30).

Results: ESRD patients were resistant to exogenous tPA fibrinolysis, with a clot lysis in 30 minutes (TEG LY30) of $34.2 \%$ compared to $52.6 \%$ for healthy controls ( $\mathrm{p}<0.0001$, two-tailed Mann-Whitney test). ESRD patients also had significantly higher levels of functional fibrinogen than healthy controls at 575 versus $471 \mathrm{mg} / \mathrm{dL}(\mathrm{p}<$ 0.0001).

Conclusion: ESRD patients have profoundly impaired fibrinolysis and elevated fibrinogen levels compared to healthy controls. This new insight suggests that trauma patients with ESRD may be at increased risk of thromobosis if treated with antifibronolytics. Thus, removal of TXA from empiric hemostatic resuscitation algorithms in ESRD patients is worthy of investigation.
References: 1) Galbusera M, Remuzzi G, Boccardo P. Treatment of bleeding in dialysis patients. Seminars in dialysis. 2009;22 (3):27986. Epub 2009/07/04. 2) Gaspari F, Vigano G, Orisio S, Bonati M, Livio M, Remuzzi G. Aspirin prolongs bleeding time in uremia by a mechanism distinct from platelet cyclooxygenase inhibition. The Journal of clinical investigation. 1987;79 (6):1788-97. Epub 1987/06/ 01. 3) Astrup AS, Tarnow L, Pietraszek L, Schalkwijk CG, Stehouwer $\mathrm{CD}$, Parving $\mathrm{HH}$, et al. Markers of endothelial dysfunction and inflammation in type 1 diabetic patients with or without diabetic nephropathy followed for 10 years: association with mortality and decline of glomerular filtration rate. Diabetes care. 2008;31 (6):11706. Epub 2008/03/12. 4) Huang Y, Noble NA. PAI-1 as a target in kidney disease. Current drug targets. 2007;8 (9):1007-15. Epub 2007/09/28.

Disclosure: No significant relationships.

\section{6}

\section{RISK FACTORS OF POSTOPERATIVE DILIRIUM IN $>65$ YEARS PATIENTS UNDERGOING EMERGENCY SURGERY}

A. Hernando Sanz ${ }^{l}$, B. Fernandez-Velilla ${ }^{1}$, I. Eguaras ${ }^{2}$, J. Mateo $^{l}$,

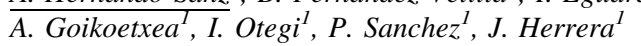

${ }^{1}$ Cirugía General, Complejo Hospitalario de Navarra, Pamplona/ SPAIN, ${ }^{2}$ General Surgery, CH-navarra, Pamplona/SPAIN

Introduction: Postoperative delirium (DPO) corresponds to that which occurs in patients who are undergoing a surgical procedure. The aim of this study is to determine the main risk factors for the presence of postoperative delirium in $>65$ years old patients after an emergency surgery.

Material and methods: A cohort prospective study was performed between January and August 2017 with 161 patients excluding patients who died during the hospital stay. Two groups of patients were formed: those with delirium and those without it. Intergroup comparison of data, including age, gender, ASA grade, comorbidities, Barthel index, nutritional status, cognitive impairment, frailty, MEWS scale (modified early warning score), type of surgery and hospital stay and was done.

Results: The incidence of postoperative delirium was 15\% (28 delirium and 133 no delirium). Risk factors found were: older than 65 years, ASA $\geq I V$, comorbidities, Barthel index, nutritional status, cognitive impairment, frailty.

Conclusion: The reported incidence of postoperative delirium was $15 \%$. The main risk factors found in this study were the presence of emergency surgery, the presence of renal failure and the presence of sepsis. In the multivariate analysis the only risk factor was postoperative stay.

References: 1. Robinson TN, Eiseman B. Postoperative delirium in the elderly: diagnosis and management. Clinical Interventions in Aging. 2008;3 (2):351-355. 2. Flinn DR, Diehl KM, Seyfried LS, Malani PN. Prevention, diagnosis, and management of postoperative delirium in older adults. J Am Coll Surg. 2009 Aug;209 (2):261-8. 3. Vijayakumar B, Elango P, Ganessan R. Post-operative delirium in elderly patients. Indian Journal of Anaesthesia. 2014;58 (3):251-256. 4. Marcantonio ER. Postoperative Delirium: A 76-Year-Old Woman With Delirium Following Surgery. JAMA: the journal of the American Medical Association. 2012;308 (1):73-81. 5. Núñez Ureña JM, Lopez Carrillo L, Hrnández Luna A. Factores de riesgo de delirium postoperatorio en la unidad de cuidados intensivos. Universidad Autónoma del Estado de México. Uri: 6. Martínez-Velilla N, Alonso 
Bouzón C, Ripa Zazpe C, Sánchez-Ostiz R. Acute postoperative confusional syndrome in the elderly patient. Cir Esp 2012;90:75-84. Disclosure: No significant relationships.

\section{7}

\section{EMERGENCY SURGERY ACUITY SCORE: IS IT A USEFUL TOOL IN OUR POPULATION?}

\section{Garciafilia Cabrera, J. Gil Rodriguez, L. Martin Roman, \\ C. Rey Varcalcel, M. Sanz Sánchez, F. Turégano Fuentes}

Cirugia General Y Aparato Digestivo, Hospital General Universitario Gregorio Marañon, Madrid/SPAIN

Introduction: Emergency Surgery (ES) carries a high morbidity and mortality, and is steadily increasing with the growing elderly population. Recently a new score was developed by the Massachusetts G.H. group in order to predict ES outcomes. The Emergency Surgery Acuity Score (ESAS) and Physiological Emergency Surgery Acuity Score (PESAS) are based on the ACS-NSQIP database, and are advocated as a user-friendly tool to predict the risk of 30-day mortality in ES patients. Our aim was to evaluate ESAS and PESAS accuracy in our population.

Material and methods: Patients who underwent ES at our centre during 6 consecutive weeks were enrolled in a prospective open cohort study. The 22 independent preoperative variables highlighted in ESAS/PESAS were recorded, and a 30-day postoperative follow-up was performed in order to assess complications according to ClavienDindo classification, and mortality. Analysis was performed with the IBM SPSS statistics 23.0.0.2 software.

Results: 101 patients needed ES, with a 30-day mortality and morbidity of $4,9 \%$ and $25,4 \%$, respectively. Only 9 out of the 22 ESAS/ PESAS variables had a significant association with mortality and morbidity. AUROC values predicting 30-day mortality for ESAS and PESAS were of 0.894 (CI 0,72-1) and 0.757 (CI 0,28-1), respectively. Conclusion: ESAS and PESAS in our experience are not as accurate as in the original description, although ESAS has a good mortality predictive value. More studies are needed to justify the additional cost in lab tests required in our population.

References: Sangji NF et al. Derivation and validation of a novel Emergency Surgery Acuity Score (ESAS). J Trauma Acute Care Surg, 2016; 81 (2):213-220.

Disclosure: No significant relationships.

\section{8}

\section{PREDICTING MORTALITY IN GERIATRIC TRAUMA ICU PATIENTS: A COMPARISON OF THE GERIATRIC TRAUMA OUTCOME SCORE AND THE TRISS METHODOLOGY}

J. Barea Mendoza ${ }^{1}$, M. Chico Fernández ${ }^{1}$, M. Sánchez Casado ${ }^{2}$, I. García Sáez , M.A. Ballesteros Sanz ${ }^{4}$, F.i. Pino Sánchez,
I. Molina Díaz ${ }^{6}$, K. Nanwani ${ }^{7}$, J.m. Jiménez Moragas ${ }^{8}$, J.A. Llompart-Pou ${ }^{9}$

${ }^{1}$ Servicio De Medicina Intensiva, Hospital Universitario 12 de Octubre, Madrid/SPAIN, ${ }^{2}$ Servicio De Medicina Intensiva, Hospital Virgen de la Salud, Toledo/SPAIN, ${ }^{3}$ Servicio De Medicina Intensiva, Hospital Universiario de Donostia, Donostia/SPAIN, ${ }^{4}$ Servicio De Medicina Intensiva, Hospital Marqués de Valdecilla, Santander/ SPAIN, ${ }^{5}$ Servicio De Medicina Intensiva, Complejo Hospitalario de Granada, Granada/SPAIN, ${ }^{6}$ Servicio De Medicina Intensiva, Hospital Universitario Nuestra Señora de Candelaria, Santa Cruz de Tenerife/ SPAIN, ${ }^{7}$ Servicio De Medicina Intensiva, Hospital Universitario La Paz, Madrid/SPAIN, ${ }^{8}$ Servicio De Medicina Intensiva, Hospital Puerta del Mar, Cádiz/SPAIN, ${ }^{9}$ Servei De Medicina Intensiva, Hospital Universitari Son Espases, Palma de Mallorca/SPAIN

Introduction: Geriatric patients constitute an increasing population in trauma Intensive Care Units (ICUs). Most of these patients will ultimately die, in some cases following limitation of life support therapies (LLS). Scores to predict mortality are necessary. We compared the newly developed Geriatric Trauma Outcome (GTO) score with the Probability of Survival using the TRISS methodology (PSTRISS) in a sample of severely injured geriatric patients from the RETRAUCI.

Material and methods: We included all patients admitted for traumatic disease in the participating ICUs aged $\geq 65$ years-old. Quantitative data were reported as median (Interquartile Range (IQR)), categorical data as number (percentage). We analyzed the GTO score and the PS-TRISS to predict mortality performing Receiving Operating Curves (ROC) to evaluate the area under the curve (AUC) with its 95\% confidence interval (CI), taking into consideration the LLS therapies. A $p$ value $<0.05$ was considered statistically significant.

Results: The cohort included 1417 patients aged $\geq 65$ years-old. Median age was 75.5 (70.5-80.5), male 1003 (68.2\%), median ISS 18 (13-25). Mechanical ventilation was used in $61 \%$ of the patients. Falls were the mechanism of injury in 659 patients $(44.8 \%)$. In-hospital mortality was $18.2 \%$. The AUC were PS-TRISS 0.69 (95\% CI $0.66-$ 0.73 ) and GTO 0.66 (95\% CI 0.62-0.70); $\mathrm{p}<0.05$. After excluding 228 patients with LLS, the AUC were PS-TRISS 0.72 (95\% CI $0.67-$ 0.77 ) and GTO 0.69 (95\% CI 0.64-0.74); p NS.

Conclusion: In our sample of severely injured geriatric patients, the GTO was not superior to the PS-TRISS to predict mortality.

References: 1. Zhao FZ, Wolf SE, Nakonezny PA, et al. Estimating Geriatric Mortality after Injury Using Age, Injury Severity, and Performance of a Transfusion: The Geriatric Trauma Outcome Score. J Palliat Med. 2015; 18:677-81. 2. Cook AC, Joseph B, Inaba K, et al. Multicenter external validation of the Geriatric Trauma Outcome Score: A study by the Prognostic Assessment of Life and Limitations After Trauma in the Elderly (PALLIATE) consortium. J Trauma Acute Care Surg. 2016; 80:204-9. 3. Madni TD, Ekeh AP, Brakenridge $\mathrm{SC}$, et al. A comparison of prognosis calculators for geriatric trauma: A Prognostic Assessment of Life and Limitations After Trauma in the Elderly consortium study. J Trauma Acute Care Surg. 2017;83:90-6.

Disclosure: No significant relationships. 


\section{NEW DEVELOPMENTS IN FRACTURE CARE}

\section{O079}

\section{BIOMECHANICAL TESTING ON EX-VIVO MODEL OF FRACTURED PIG FEMUR FIXED WITH NEW BIODEGRADABLE POLYMER COMPOSITE GLUE}

M. Krticka ${ }^{\text {I }}$ L. Michlovska ${ }^{2}$, V. Nekuda ${ }^{3}$, M. Zboncak ${ }^{2}$, K. Sojka ${ }^{2}$, J. Kaiser ${ }^{2}$, L. Vojtova ${ }^{2}$

${ }^{1}$ Department Of Trauma Surgery, University Hospital Brno, Brno/ CZECH REPUBLIC, ${ }^{2}$ Department Of Biomaterials, CEITEC VUT Brno, Brno/CZECH REPUBLIC, ${ }^{3}$ Trauma Surgery Department, University Hospital Brno, Brno/CZECH REPUBLIC

Introduction: In recent years, there has been a growing interest, in many fields of medicine, in the use of bone adhesives that are biodegraded to non-toxic products and resorbed after fulfilling their function in contact with living tissue. Biomechanical properties of newly developed bone glue, such as adhesion to bone and elastic modulus were tested in our study.

Material and methods: Newly developed injectable biodegradable "self-setting" bone adhesive prepared from inorganic tricalcium phosphate powder and aqueous solution of organic thermogelling polymers was used for ex-vivo fixing fractured pig femur. Ex-vivo biomechanical tests were performed on 45 fresh pig femurs. Tested group were divided to following 4 subgroups (sg); $\mathrm{sg} 1$ - $\mathrm{O}$ fracture (n = 15) glued together with 3 different type of bone adhesives, $\operatorname{sg} 2 \mathrm{BW}$ fracture $(n=5)$ glued together with bone adhesive $(n=5)$; sg3-BW fracture fixed with locking compression plate (LCP), $n=5$; sg4 - BW fracture fixed with LCP in combination with bone adhesive. Threepoint bending force and shear compression tests were performed on linear electrodynamic test instrument (ElectroPuls E10000, Instron). Femurs from $\mathrm{sg} 1, \mathrm{sg} 2$ and $\mathrm{sg} 4$ was tested on Micro-CT before and after biomechanical testing.

Results: Shear compression tests in $\mathrm{sg} 1$ without amino acids modification showed that is needed force of $0,5 \mathrm{mPa}$ to recreate fracture, modification with amino acids increase force to $3 \mathrm{mPa}$. Three-point bending force test in $\mathrm{sg} 2$ showed decreased force $250 \mathrm{~N}$ to recreate fracture, but in $\mathrm{sg} 4$ force to recreate fracture was increased to $5000 \mathrm{~N}$. Conclusion: Newly developed injectable biodegradable bone adhesive represents new possibility how to fix comminuted fracture.

References:

Disclosure: No significant relationships.

\section{0}

\section{THE EFFECTIVENESS OF INTRAOPERATIVE 3D FLUOROSCOPY IN THE TREATMENT OF INTRA- ARTICULAR DISTAL RADIUS FRACTURES: A RANDOMIZED CONTROLLED TRIAL}

\author{
C.A. Selles ${ }^{l}$, R.j.o. De Muinck Keizer ${ }^{l}$, S. Beerekamp ${ }^{l}$, \\ P.a. Leenhouts ${ }^{1}$, M. Maas ${ }^{1}$, J.c. Goslings ${ }^{1}$, N.W.l. Schep ${ }^{2}$
}

${ }^{1}$ Surgery, Academic Medical Center, Amsterdam/NETHERLANDS, ${ }^{2}$ Trauma Unit, Department Of Surgery, Maasstad Ziekenhuis, Rotterdam/NETHERLANDS

Introduction: Three-dimensional (3D) fluoroscopy is thought to be beneficial in the operative reduction and fixation of intra-articular distal radius fractures. The goal of this multicenter randomized controlled trial was to investigate the effectiveness of the additional use of intraoperative 3D fluoroscopy compared to conventional 2D fluoroscopy in patients requiring operative treatment for intra-articular distal radius fractures.

Material and methods: We performed a multicenter prospective randomized controlled trial where patients were randomized between $3 \mathrm{D}$ or conventional fluoroscopy during operative treatment of their distal radius fracture. The primary outcome measure was quality of fracture reduction and fixation on a postoperative CT scan. Secondary outcome measures were the number of revision operations and the number of complications after one year of follow up, and functional outcome measured with the Patient-Rated Wrist Evaluation (PRWE) questionnaire.

Results: A total of 207 distal radius fracture were included in the study. The postoperative CT-scan showed an indication for additional revision of reduction or implant position in $25 \%$ of the 3D group versus $31 \%$ in the $2 \mathrm{D}$ group $(\mathrm{p}=0.34)$. There was no significant difference in the number of complications and revision surgeries performed within one year. The median PRWE score was 7 for the 3D group and 8 for the $2 \mathrm{D}$ group $(\mathrm{p}=0.8)$.

Conclusion: The use of intraoperative 3D fluoroscopy does not improve the quality of reduction and fixation in the management of intra-articular distal radius fractures. There was no benefit of intraoperative 3D fluoroscopy with regard to postoperative complications, quality of life, or functional outcome.

References: Beerekamp, M. S. H. et al. Fracture surgery of the extremities with the intra-operative use of 3D-RX: a randomized multicenter trial (EF3X-trial). BMC Musculoskelet. Disord. 12, 151 (2011). Atesok, K. et al. The use of intraoperative three-dimensional imaging (ISO-C-3D) in fixation of intraarticular fractures. Injury. 38, 1163-1169 (2007).

Disclosure: No significant relationships.

\section{O081}

\section{IN-VIVO TESTING OF A LOW-ALLOYED BIODEGRADABLE MAGNESIUM ALLOY}

M. Humenberger ${ }^{1}$, M. Eilenberg ${ }^{2}$, S. Pajenda ${ }^{3}$, L. Schwitter ${ }^{3}$,

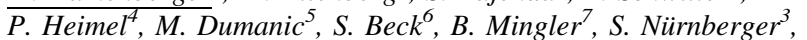
S. Tangl ${ }^{4}$, P. Uggowitzer ${ }^{8}$, W. Mayr ${ }^{9}$, H. Bergmeister ${ }^{10}$

${ }^{1}$ Department Of Trauma Surgery, Medical University of Vienna, Vienna/AUSTRIA, ${ }^{2}$ Surgery, Medical University of Vienna, Vienna/ AUSTRIA, ${ }^{3}$ Trauma Surgery, Medical University of Vienna, Vienna/ AUSTRIA, ${ }^{4}$ School Of Dentistry, Medical University of Vienna, Vienna/AUSTRIA, ${ }^{5}$ Department Of Radiology, Medical University of Vienna, Vienna/AUSTRIA, ${ }^{6}$ Biomaterials Europe, Synthes GmbH, Oberdorf/SWITZERLAND, ${ }^{7}$ Health \& Bioresources, Austrian Institute of Technology, Wr. Neustadt/AUSTRIA, ${ }^{8}$ Department Of Materials, ETH Zürich, Zürich/SWITZERLAND, ${ }^{9}$ Center For Medical Physics And Biomedical Engineering, Medical University of Vienna, Vienna/AUSTRIA, ${ }^{10}$ Biomedical Research, Medical University of Vienna, Vienna/AUSTRIA

Introduction: Magnesium alloys are promising materials for biodegradable medical implants for medium load-bearing applications in orthopedic and trauma surgery. A suitable alloy has to combine highest biocompatibility, an appropriate and homogeneous degradation rate and good mechanical properties, especially a high strength. After promising results in in-vitro testing, in-vivo testing for 
degradation and biocompatibility of a low-alloyed magnesium alloy was performed in a rat model.

Material and methods: Pins made of a new $\mathrm{Mg}-\mathrm{Zn}-\mathrm{Ca}$ alloy were implanted in a bicortical manner in the femora of Sprague Dawley rats and were compared to pins made of the established magnesium alloy WE43, which contains small amounts of potentially problematic rare earth elements with a not yet fully understood toxicology. The degradation of the pins was followed via X-ray, micro-CT and microPET-CT for up to 15 months. The femora were then tested biomechanically and in histologic sections for degradation of the pin, bony healing, gas formation and signs of inflammation.

Results: The degradation rate of the new $\mathrm{Mg}-\mathrm{Zn}-\mathrm{Ca}$ alloy was similar to the WE43 alloy and showed no signs of inflammation in histological examinations of the bone and surrounding tissue. Micro-CT showed good osteoconductive properties and low gas formation.

Conclusion: The new Mg-Zn-Ca alloy showed good results in-vivo and is therefore a promising candidate for future applications in orthopedic and trauma surgery.

References:

Disclosure: No significant relationships.

\section{2}

\section{D NAVIGATION TECHNIQUE IN TREATMENT PELVIC FRACTURES}

\author{
M. Křivohlávek, V. Herrmann, R. Harcuba, J. Sram
}

Traumacentre, Regional Hospital Liberec, Liberec/CZECH REPUBLIC

Introduction: Percutaneous screw fixation in the treatment of pelvic fractures represents a highly effective stabilization, especially in posterior segment of pelvic ring - injuries of sacroiliacal joint, sacral fractures, selected acetabular fractures or pelvic ring injuries. Due to anatomical localization, the possibility of iatrogenic injury or malposition of the screws using only fluoroscopic control up to $12 \%$.The limit of iso-3D navigation is only a small area of the pelvis $(12 \times 12$ $\mathrm{cm})$. Intraoperative CT represents a high radiation load and high economic cost of operating room equipment. Combining preoperative CT scans and perioperative iso 3D scans allows us to improve the quality and size of the navigated images while maintaining accuracy and low radiation dose.

Material and methods: From October 2015 to October 2017 was applied 45 navigated screws using iso-3D (Ziehm 3D FD) CT matching in 33 pelvis injuries (SI screws - 24, IS on both sides or transsacral screw - 5, pubic screws - 3, acetabulum 1). In total, 16 men and 17 women with an average age of 50.3 years were treated. The duration of the surgical procedure was $62(45-120)$ minutes, the mean value of emitted radiation was 1925 (744-3410) cGycm2.

Results: There was no malposition of the screws or iatrogenic neurological injury. One times it was not possible to match $3 \mathrm{D}$ and $\mathrm{CT}$ images. Once we recorded a hematoma in a surgical wound requiring revision surgery.

Conclusion: The use of perioperative iso-3D in combination with preoperative CT significantly improves the clarity of iso-3D scans and accuracy insertion of percutaneous screws.

References: Behrendt, Daniel, Maria Mütze, Hanno Steinke, Martin Koestler, Christoph Josten, a Jörg Böhme. 2012. „Evaluation of 2D and 3D Navigation for Iliosacral Screw Fixation”. International Journal of Computer Assisted Radiology and Surgery 7 (2):249-55. https://doi.org/10.1007/s11548-011-0652-7. Li, Bin, Jiliang He, Zexing Zhu, Dongsheng Zhou, Zhenhai Hao, Yonghui Wang, a Qinghu
Li. 2015. „Comparison of 3D C-Arm Fluoroscopy and 3D ImageGuided Navigation for Minimally Invasive Pelvic Surgery". International Journal of Computer Assisted Radiology and Surgery 10 (10):1527-34. https://doi.org/10.1007/s11548-015-1157-6. Matityahu, Amir, David Kahler, Christian Krettek, Ulrich Stöckle, Paul Alfred Grutzner, Peter Messmer, Jan Ljungqvist, a Florian Gebhard. 2014. „Three-Dimensional Navigation Is More Accurate than TwoDimensional Navigation or Conventional Fluoroscopy for Percutaneous Sacroiliac Screw Fixation in the Dysmorphic Sacrum: A Randomized Multicenter Study". Journal of Orthopaedic Trauma 28 (12):707-10. https://doi.org/10.1097/BOT.0000000000000092. Takao, Masaki, Takashi Nishii, Takashi Sakai, a Nobuhiko Sugano. 2013. „CT-3D-Fluoroscopy Matching Navigation Can Reduce the Malposition Rate of Iliosacral Screw Insertion for Less-Experienced Surgeons". Journal of Orthopaedic Trauma 27 (12):716-21. https://doi.org/10.1097/BOT.0b013e31828fc4a5. Thakkar, Savyasachi C., Rashmi S. Thakkar, Norachart Sirisreetreerux, John A. Carrino, Babar Shafiq, a Erik A. Hasenboehler. 2017. „2D versus 3D Fluoroscopy-Based Navigation in Posterior Pelvic Fixation: Review of the Literature on Current Technology". International Journal of Computer Assisted Radiology and Surgery 12 (1):69-76. https://doi.org/10.1007/s11548-016-1465-5.

Disclosure: No significant relationships.

\section{3}

\section{CAN WE EASILY PREDICT THE OPTIMAL IMPLANT POSITION ON THE CLAVICLE?}

\author{
M. Herteleer ${ }^{\text {I }}$, S. Vancleef ${ }^{2}$, T. Tollens ${ }^{3}$, P. Herijgers ${ }^{4}$, I. Jonkers $^{2}$, \\ S. Nijs ${ }^{1}$, J. Vander Sloten ${ }^{2}$
}

${ }^{1}$ Traumatology, UZ Leuven, Leuven/BELGIUM, ${ }^{2}$ Biomechanical Engineering, KU Leuven, Leuven/BELGIUM, ${ }^{3}$ General Surgery, Imelda Ziekenhuis, Bonheiden/BELGIUM, ${ }^{4}$ Faculty Of Medicine, KU Leuven, Leuven/BELGIUM

Introduction: Current clavicle fracture fixation plates are suboptimal, leading to reoperation rates up to $53 \%$. Plate irritation, potentially caused by a bad geometric fit and plate prominence, has been found to be the most important factor for reoperation. Therefore, thin plate implants that do not interfere with muscle attachment sites (MAS) would be beneficial in reducing plate irritation.

Material and methods: 14 Cadaveric clavicles were dissected and reverse engineered so they could be used to make a statistical shape model. This re-engineering was done with the help of optical laser scanning, photogrammetry and segmentation of CT scan images. Next, a statistical shape model (SSM) of the clavicles was created. The average clavicle was then registered to all original clavicle meshes. The MAS of individual muscles of all 14 bones were indicated on the average clavicle.

Results: Visualization of all MAS on the average clavicle resulted in $72 \%$ coverage of the surface. Heat mapping of the clavicle's MAS showed that there are regions which are less at risk of interfering with a plate. Mean area was $602 \mathrm{~mm} 2 \pm 137 \mathrm{~mm} 2$ for the deltoid muscle, $1022 \mathrm{~mm} 2 \pm 207 \mathrm{~mm} 2$ for the trapezius muscle, and $683 \mathrm{~mm} 2 \pm 132$ $\mathrm{mm} 2$ for the pectoralis major muscle. The sternocleidomastoid muscle has a mean area of $513 \mathrm{~mm} 2 \pm 190 \mathrm{~mm} 2$ and the subclavius muscle had the smallest mean area of $451 \mathrm{~mm} 2 \pm 162 \mathrm{~mm} 2$.

Conclusion: Design of generic plates that do not interfere with individual MAS is challenging. Hence, patient-specific clavicle fracture fixation plates should be considered to minimally interfere with MAS. 
References: [1] T. Huttunen et al. Injury, 44, 12: 1899-1903, 2013 [2]

J. G. Wijdicks et al, Arch. Orthop. Trauma Surg, 5;617-625, 2012 [3]

B. D. Ashman et al, Injury, 10:1549-1553, 2014

Disclosure: No significant relationships.

\section{4}

\section{THE EFFECT OF 3D MODELING ON SURGICAL DECISION IN TRIMALLEOLAR FRACTURES}

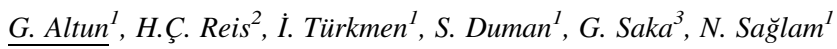

${ }^{1}$ Orthopedics And Traumatology, Umraniye Research and Education Hospital, Istanbul/TURKEY, ${ }^{2}$ Nature Science And Technical Mapping Engineer, Gumushane University, Gumushane/TURKEY, ${ }^{3}$ Orthopedics And Traumatology, Fulya Orthopedics and Spine Center, Istanbul/TURKEY

Introduction: Posterior malleolar fractures range from small extraarticular lip fractures to large triangular fragments extending to the medial or lateral malleolus. The aim of this study is, to compare the $3 \mathrm{D}$ distal tibia articuler surface with the lateral ankle rontgenographies to measure the posterior malleol fracture fragments.

Material and methods: 28 patients were included in the study. All fractures were classified according to Haraguchi and Bartonicek classification. The rate of PF to the articular surface at the distal end of the tibia was calculated with traditional methods by two different senior surgeons on lateral ankle radiographs.Additionally, 3D modeling was performed by resizing computed tomography scans of the ankles in the digital environment and the rate of fracture fragment to the articular surface was measured via 3D Doctor Computer software. Results: When the rate of PF to the articular surface at the distal end of the tibia on lateral ankle radiograph was compared with values at 3D measurements especially in large fragments, it was seen that there was a statistical inconsistency. Additionally, it was seen that there was a less suitability between the values calculated on lateral ankle radiographs by two surgeons.

Conclusion: Measurements performed on lateral ankle radiographs are taken into consideration in the decision-making process for treatment. Our study showed that conventional measurements performed on radiographs were not reliable for both relevant percentage of articular surface. Since the rate of area of fracture fragment to especially the entire articular surface can be calculated near real with measurements performed by using 3D modeling method, we think that it is more realistic, effective and applicable for indication.

References:

Disclosure: No significant relationships.

\section{5}

\section{PERCUTANEOUS RETROGRADE TRANSPUBIC SCREW FIXATION - A STATISTICAL ANALYSIS OF AVAILABILITY, LENGTH OF THE SCREW CORRIDOR AND LOCAL BONE STOCK BASED ON A 3D STATISTICAL MODEL OF THE PELVIC RING}

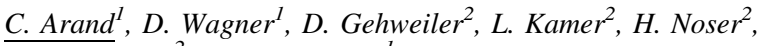 \\ G.R. Richards ${ }^{2}$, P.M. Rommens ${ }^{l}$
}

${ }^{1}$ Centre For Orthopaedics And Traumatology, University Hospital Mainz, Mainz/GERMANY, ${ }^{2}$ Ao Research Institute, AO Foundation,

\section{Davos/SWITZERLAND}

Introduction: In fractures of the anterior pelvic ring (ramus superior ossis pubis) without major dislocation percutaneous, retrograde trans pubic screw fixation is a common method of treatment. It is a minimally invasive technique with the aim of pain reduction and early mobilization of the patient. In general, a $7.3 \mathrm{~mm}$ partially threaded, cannulated screw is used. The corridor for the retrograde trans pubic screw is usually quite narrow, often relevantly curved and therefore not always available. The aim of this study was to perform a statistical analysis regarding the availability and length of the screw corridor as well as the local bone stock.

Material and methods: Based on a 3D statistical model of the pelvic ring 50 individual 3D CT models (25 males, 25 females) were examined.

Results: A transpubic corridor reaching into the supraacetabular area was present in 34 (20 male, 14 female). The average corridor length was $131.7 \mathrm{~mm}( \pm 10.2)$. The bone stock distribution given in Hounsfield units showed a consistent pattern with the highest grey values in the supraacetabular area.

Conclusion: Due to individual anatomy, a minimal invasive retrograde transpubic screw fixation is not always possible. In females, the corridor was shown to be too narrow or curved for screw positioning more often than in males (availability in $80 \%$ of the male and $56 \%$ of the female samples). As the cancellous bone stock shows the highest values in the supraacetabular area, the target should be to reach this region with the screw for a more stable fixation.

References: "Clinical pathways for fragility fractures of the pelvic ring: personal experience and review of the literature", Rommens et al., J Orthop Sci (2015) "Surgical management of osteoporotic pelvic fractures: a new challenge", Rommens et al., Eur J Trauma Emerg Surg (2012) "Biomechanical principles for treatment of osteoporotic fractures of the pelvis", Gänsslen, Der Unfallchirurg (2010) "Are two retrograde $3.5 \mathrm{~mm}$ screws superior to one $7.3 \mathrm{~mm}$ screw for anterior pelvic ring fixation in bones with low bone mineral density?", Acklin et al., Bone Joint Res (2017) "Gun barrel view of the anterior pelvic ring for percutaneous anterior column or superior pubic ramus screw placement", Quercetti et al., Eur J Orthop Surg Traumatol (2017)

Disclosure: Charlotte Arand received a Research Fellowship from the AO Foundation, partially funded by the AO TK System.

\section{6}

\section{NEW MINIMALLY INVASIVE REDUCTION AND FIXATION TECHNIQUE FOR ILIUM FRACTURES (ROMMENS' CLASSIFICATION FFP TYPE IIIA)}

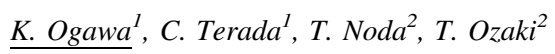

${ }^{1}$ Division Of Orthopaedic Trauma, Fukuyama City Hospital Emergency Medical Center, Hiroshima/JAPAN, ${ }^{2}$ Orthopaedic Surgery, Okayama University, OKAYAMA/JAPAN

Introduction: The ilium fractures mean that fracture line runs from iliac crest to greater sciatic notch leaving sacroiliac joint intact ${ }^{1) 2}$. The aim of this study is to introduce our brand-new, unique, and simple technique and to report the results.

Material and methods: 12 patients were included. There were four males and eight females. The mean age of injury was 80.3 years. Surgical technique Patient was placed in supine position. Fracture site of iliac crest was exposed with $3-5 \mathrm{~cm}$ skin incision. Exposure was developed to arcuate line. Next, anterior inferior iliac spine (AIIS) 
was exposed with $3 \mathrm{~cm}$ skin incision, and false pelvis was exposed at the level of AIIS. Following all three procedures maintain reduction; 1) The leg of injured side was pulled with slightly abducted and internally rotated. 2) Ball spike pusher was inserted through iliac window, and acetabular fragment was compressed on arcuate line. 3) Retractor was inserted through AIIS window and retracted. The tip of retractor was placed on arcuate line. Iliac crest was fixed with screw or plate. Three LC2 screws were inserted ${ }^{3)}$ leaving the fracture reduced as shown above. Anterior rami fracture was not fixed.

Results: Acceptable reduction was achieved in 11 cases. All fractures were united within 3 months. Reduction was maintained in all cases. Skin ulcer of buttock was observed because of the irritation of extruded screw tip.

Conclusion: Our new reduction technique is unique and reliable. Three LC2 screws can keep reduction until bone union without fixing rami fractures. Definition of correct screw length is mandatory to avoid skin complication.

References: 1) Rommens, P. M., \& Hofmann, A. (2013). Comprehensive classification of fragility fractures of the pelvic ring: recommendations for surgical treatment. Injury, 44 (12), 1733-1744. 2) Rommens, P. M., Dietz, S. O., Ossendorf, C., Pairon, P., Wagner, D., \& Hofmann, A. (2015). Fragility fractures of the pelvis: should they be fixed?. Acta Chir Orthop Traumatol Cech, 82 (2), 101-12. 3) Starr, A. J., Walter, J. C., Harris, R. W., Reinert, C. M., \& Jones, A. L. (2002). Percutaneous screw fixation of fractures of the iliac wing and fracture-dislocations of the sacro-iliac joint (OTA Types 61-B2. 2 and 61-B2. 3, or Young-Burgess "lateral compression type II" pelvic fractures). Journal of orthopaedic trauma, 16 (2), 116-123.

Disclosure: No significant relationships.

\section{7}

\section{THE DETERMINATION OF SACROILIAC SCREW LOOSENING USING 2D AND 3D TECHNIQUES IN CADAVERS: INTRA- AND INTERRATER RELIABILITY AND METHOD COMPARISON}

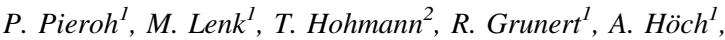 \\ D. Wagner ${ }^{3}$, C. Josten ${ }^{1}$, J. Böhme
}

${ }^{1}$ Department Of Orthopedics, Trauma And Plastic Surgery, University of Leipzig, Leipzig/GERMANY, ${ }^{2}$ Department Of Anatomy And Cell Biology, Martin Luther University Halle-Wittenberg, Halle (Saale)/ GERMANY, ${ }^{3}$ Department Of Orthopedics And Traumatology, University Medical Center Mainz, Mainz/GERMANY

Introduction: Persistent instability or an impaired bone stock may lead to sacroiliac screw (SI) loosening. Although this loosening might indicate non-union, the clinical relevance and causes for SI loosening are still unknown. Moreover, this condition might be overlooked due to the less reliable measurement techniques for the posterior pelvic ring and missing measurement techniques for implant failure.

Material and methods: A both-sided transalar sacral fracture with additional symphysiotomy was set in 9 cadaveric pelves and fixed with SI screws. Following a CT scan and fluoroscopy (anterior-posterior, inlet, outlet), one SI screw was approximately $2 \mathrm{~cm}$ manually turned back. Optical measurements were performed using referenced photographs $(n=9)$. Fluoroscopy (anterior-posterior, inlet, outlet) and a 3D scan were obtained. Mimics was used to determine SI screw loosening in fluoroscopy $(n=5)$ and $3 \mathrm{D}$ scans $(\mathrm{n}=9)$. Segmented CT scans were used for a contour based registration to fluoroscopies in all available views (X-ray module) and SI screw loosening was measured $(n=9)$. All measurements were performed three times by three independent observers.

Results: The X-ray module and two fluoroscopic measurement techniques yielded a difference less than $5 \mathrm{~mm}$ compared to 3D scan. Intrarater reliability was for two observers for almost all techniques very good $\geq 0.81$. However, only three fluoroscopic measurement techniques and optical measurement displayed a very good interrater reliability $\geq 0.81$. Method comparison determined X-ray module and two fluoroscopic measurement techniques comparable to the 3D scan assessing SI screw loosening.

Conclusion: 3D techniques most precisely determine SI screw loosening but also fluoroscopic measurement techniques can yield reliable results.

References:

Disclosure: No significant relationships.

\section{8}

\section{RAPID PROTOTYPING IN MIDSHAFT CLAVICLE FRACTURE REPAIR} R.A.Van Der Linde
S.H. Van Helden

${ }^{1}$ Surgery, Isala Hospital, Zwolle/NETHERLANDS, ${ }^{2}$ Biomechanical Engineering, University of Twente, Enschede/NETHERLANDS

Introduction: Clavicle fractures are common injuries, often treated using plate fixation. Clavicle plates rarely fit properly, requiring perioperative alterations. The aim of this study was to test a method in which plates were bent preoperatively, enabling custom fitted clavicle plates for every individual clavicle. We hypothesized this would shorten the process of perioperative plate handling, and reduce the number of complications and reoperation rate.

Material and methods: A non-blinded, prospective cohort study was performed. The control group received conventional treatment using standard precontoured clavicle plates. The intervention group was treated using customized plates, which were preoperatively adjusted with the use of reconstructed physical three dimensional models. Clinical records were examined in order to obtain patient characteristics and information about the course of treatment. Primary outcome was reduction in plate handling time. Secondary outcome were complications and reoperation rate, both after $\geq 1$ year.

Results: Seven patients were included in both the control- and intervention group. The process of peroperative plate alteration was reduced by $3: 22$ minutes $(\mathrm{p}=0.430)$, though the overall average fracture repair time increased by $3: 35$ minutes $(p=0.450)$. One patient in the intervention group developed an infection, requiring reoperation. Furthermore, significantly more patients in the control group required a reoperation for plate removal because of implant related complaints $(\mathrm{p}=0.032)$.

Conclusion: Preoperative adjustments of clavicle plates can successfully be implemented in daily practice. However, it does not reduce overall operation time. A significant decrease in reoperations due to implant related complications was observed in the invention group.

References: None

Disclosure: No significant relationships. 


\section{COMMON PROBLEMS IN ACUTE CARE SURGERY}

\section{9}

THE EMERGENCY SURGERY SCORE (ESS) AS A TRIAGE TOOL PREDICTING THE POSTOPERATIVE ICU NEED OF EMERGENCY GENERAL SURGERY PATIENTS

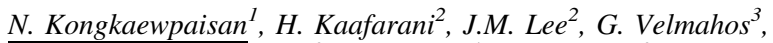
D. King , P.J. Fagenholz ${ }^{3}$, N. Saillant ${ }^{4}$, A. Mendoza ${ }^{3}$, M.G. Rosenthal ${ }^{3}$, A.T. Nordestgaard ${ }^{3}$, A.I. Eid ${ }^{5}$, S. Van Wijck, M. Kongwibulwut ${ }^{3}, \mathrm{~K} . \mathrm{Han}^{3}$, N.F. Sangji $i^{2}$

${ }^{1}$ Division Of Acute Care And Ambulatory Surgery, Siriraj Hospital, Mahidol University, Khet Bangkok Noi, Bangkok/THAILAND, ${ }^{2}$ Division Of Trauma, Emergency Surgery And Surgical Critical Care, Department Of Surgery, Massachusetts General Hospital, Harvard Medical School, Boston/MA/UNITED STATES OF AMERICA, ${ }^{3}$ Division Of Trauma, Emergency Surgery And Surgical Critical Care, Department Of Surgery, Massachusetts General Hospital, Harvard Medical School, Boston/UNITED STATES OF AMERICA, ${ }^{4}$ Department Of Surgery - Division Of Trauma, Emergency Surgery, And Surgical Critical Care, Massachusetts General Hospital, BOSTON/UNITED STATES OF AMERICA, ${ }^{5}$ Surgery, Massachusetts General Hospital, Boston//UNITED STATES OF AMERICA

Introduction: The emergency surgery score (ESS) is a preoperative risk calculator recently validated as a mortality predictor in emergency general surgery (EGS) patients. We sought to evaluate the utility of ESS as an ICU admission triage tool.

Material and methods: A four-step methodology was designed. First, the 2007-2015 ACS-NSQIP database was examined to identify all EGS patients using the "emergent" variable and CPT codes for "digestive system". Second, we created a composite variable called ICU need, defined as death or the development of $\geq 1$ postoperative complication warranting critical care (e.g. intubation, ventilator $>48$ hours, cardiac arrest, septic shock). Third, for each patient, ESS was calculated. Fourth, the correlation between ESS and ICUneed was assessed by calculating the model c-statistics (AUROC).

Results: Out of a total of 4,456,809 patients, 65,989 patients were included. The mean population age was 56 years; $51 \%$ were female, and $71 \%$ were white. The overall 30 -day postoperative mortality and morbidity were $8.2 \%$ and $31.7 \%$, respectively. ESS gradually and accurately predicted ICUneed, with $1 \%, 40 \%$ and $98 \%$ of patients with ESS of 2, 9 and 16 requiring critical care, respectively. Only $6.2 \%$ of patients with ESS $\leq 7$ had an ICUneed, while $97.2 \%$ of patients with ESS $\geq 15$ had an ICUneed. The c-statistic of the predictive model was 0.90 .

Conclusion: ESS accurately predicts the need for postoperative critical care and ICU admission. In resource-limited settings, ESS may prove useful as an ICU triage tool ensuring a prompt rescue of the clinically deteriorating patient without unnecessary and burdensome ICU admissions.

References: 1. Sangji N, Bohnen J, Ramly E, et al. Derivation and validation of a novel Emergency Surgery Acuity Score (ESAS). $J$ Trauma Acute Care Surg. 2016;81:213-220 2. Nandan A, Bohnen J, Sangji N, et al. The Emergency Surgery Score (ESS) accurately predicts the occurrence of postoperative complications in emergency surgery patients. J Trauma Acute Care Surg. 2017;83:84-89

Disclosure: No significant relationships.
0090

\section{THE NATURE COURSE OF PATIENTS WITH PERCUTANEOUS TRANSHEPATIC GALLBLADDER DRAINAGE WITHOUT LATER ELECTIVE LAPAROSCOPIC CHOLECYSTECTOMY}

\author{
S.Y. Wang, Y.L. Hung
}

Trauma And Emergency Surgery, Chang Gung Memorial Hospital, Taoyuan/TAIWAN

Introduction: Percutaneous Transhepatic Gallbladder Drainage (PTGBD) is an alternative treatment for acute cholecystitis. Elective surgery, usually laparoscopic cholecystectomy (LC), is arranged 6 to 8 weeks later. However, there are still some patients not undergoing LC later. We investigated the clinical outcome of those patients.

Material and methods: From 2009 to 2016, an 8-year-period, there were 724 patient undergoing PTGBD and then admitted to service of Trauma and Emergency Surgery. There were 624 patients enrolled in this study with the indication of acute cholecystitis (86.2\%). For the clinical outcome of patients without LC, we tried review medical record of our institute and interview by telephone for adverse events for which hospitalization is indicated, including recurrent acute cholecystitis, cholangitis, or biliary pancreatitis.

Results: Based on Tokyo Guideline 2013 (TG13), 405 patients were classified as severity of grade II and III (64.9\%). There were 367 patients $(58.8 \%)$ undergoing LC later in the course while 257 patients $(41.2 \%)$ without further surgical treatment. Over half of both grade I and grade II patients underwent later elective LC while only $30 \%$ of patient with grade III received surgery later. Over half of patients without elective LC were noted with adverse event later.

Conclusion: PTGBD is an alternative treatment for acute cholecystitis. However, definite treatment is still necessary for symptom free of symptomatic cholecystolithiasis.

References:

Disclosure: No significant relationships.

\section{1}

\section{SURGEON-PERFORMED ULTRASOUND (SPUS) IN SUSPECTED APPENDICITIS}

A. Natroshvili, A. Shulutko, F. Nasirov

Department Of Surgery \#2, I.M. Sechenov First Moscow State Medical University, Moscow/RUSSIAN FEDERATION

Introduction: Acute appendicitis is one of the most common diseases in daily surgical practice. Traditionally, diagnosis and decisionmaking are based on history and clinical examination, leading to high negative appendectomy rate. CT has improved diagnostic quality, but its routine use is questioned because of high costs and long-term effects of radiation exposure. Limited use of CT makes ultrasound first line imaging modality in patients with RLQ pain. The role of surgeon-performed ultrasound (SPUS) in endocrine and vascular surgery has been well documented and showed good results. Increasing role of bedside ultrasonography led us to study the role of SPUS in the diagnosis of appendicitis.

Material and methods: 1866 patients with RLQ pain underwent surgeon-performed ultrasonography. Decision to perform an open or laparoscopic appendectomy was based on the complete clinical 
evaluation, which included clinical presentation and US results. Final diagnosis of appendicitis was confirmed by operative findings and histologic examination of removed appendix.

Results: SPUS was true positive in $734(39.3 \%)$ cases, true-negative in 951 (50.9\%), false-positive in 23 (1.2\%) and false-negative in 156 $(8.3 \%)$ cases. The sensitivity of SPUS for diagnosing of acute appendicitis was $82.5 \%$, specificity $97.6 \%$, overall accuracy 94.4 , positive and negative predictive value were $97 \%$ and $85.9 \%$ respectively.

Conclusion: Surgeon-performed ultrasonography is a valuable, safe and readily available adjunct to clinical impression in patients with RLQ pain. Negative result of ultrasonography does not rule out appendicitis, surgeon decision-making about appendectomy/laparoscopy should be based on complete clinical evaluation.

References:

Disclosure: No significant relationships.

\section{2}

\section{ACUTE CHOLECYSTITIS IN HIGH RISK PATIENTS: CHOLECYSTOSTOMY VS CHOLECYSTECTOMY VS EUS- GUIDED DRAINAGE. RESULTS OF A RETROSPECTIVE STUDY}

A. La Greca ${ }^{1}$, V. Cozza ${ }^{2}$, G. Pepe ${ }^{2}$, M. Di Grezia ${ }^{2}$, C. Lodoli ${ }^{2}$, A. Di Giorgio ${ }^{2}$, M. Foco $^{2}$, G. Di Flumeri ${ }^{2}$, F. Attili ${ }^{3}$, M. Bossola ${ }^{2}$, S. Magalini ${ }^{2}, G$. Costamagna ${ }^{3}, D . G^{2} i^{2}$

${ }^{1}$ Emergency Surgery, Fondazione Policlinico Universitario “A. Gemelli", rome/ITALY, ${ }^{2}$ Emergency Surgery, Fondazione Policlinico Universitario "A. Gemelli”, ROME/ITALY, ${ }^{3}$ Surgical Endoscopy, Fondazione Policlinico Universitario "A. Gemelli”, ROME/ITALY

Introduction: The aim of this retrospective study is to compare clinical outcomes of percutaneous cholecystostomy, cholecystectomy and EUS-guided drainage for acute cholecystitis in high risk patients. Material and methods: We have analysed 655 patients with acute cholecystitis admitted in the last 8 years to our Institution and compared the outcomes in terms of mortality and morbidity (according to the Clavien classification). We have also compared the mean lenght of stay (LOS).

Results: In-hospital mortality and postoperative complications are higher in patients treated with percutaneous cholecystostomy (PC), especially in low and mild grade cholecystitis and ASA I-II. The outcomes are similar in ASA III patients and severe cholecystitis. EUS-guided drainage shows encouraging results and should be considerated in high risk patients and severe cholecystitis.

Conclusion: PC does not offer advantages compared to cholecystectomy in acute cholecystitis. A larger, multicenter, randomised trial is needed to assess the best treatment for severe cholecystitis in high risk patients and EUS-guided draiange should be considered among those treatments.

References: 1. Zarour S, Imam A, Kouniavsky G, Lin G, Zbar A, Mavor E. Percutaneous cholecystostomy in the management of highrisk patients presenting with acute cholecystitis: Timing and outcome at a single institution. Am J Surg. 2017 Sep;214 (3):456-461. 2. Dimou FM, Adhikari D, Mehta HB, Riall TS. Outcomes in Older Patients with Grade III Cholecystitis and Cholecystostomy Tube Placement: A Propensity Score Analysis. J Am Coll Surg. 2017
Apr;224 (4):502-511. 3. Kortram K, van Ramshorst B, Bollen TL, Besselink MG, Gouma DJ, Karsten T, Kruyt PM, Nieuwenhuijzen GA, Kelder JC, Tromp E, Boerma D. Acute cholecystitis in high risk surgical patients: percutaneous cholecystostomy versus laparoscopic cholecystectomy (CHOCOLATE trial): study protocol for a randomized controlled trial. Trials. 2012 Jan 12;13:7.

Disclosure: No significant relationships.

\section{3}

\section{DAILY USE OF SURGEON-PERFORMED ULTRASOUND IN A GENERAL AND EMERGENCY SURGERY UNIT. OVERALL ANALYSIS OF A TWO TEARS PROSPECTIVE} REGISTRY

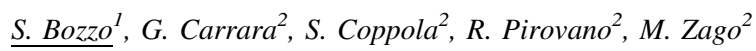

${ }^{1}$ Chirurgia Generale, POLICLINICO SAN PIETRO, Ponte San Pietro/ITALY, ${ }^{2}$ Minimally Invasive Surgery Unit - General Surgery Dept, Policlinico San Pietro, Bergamo/ITALY

Introduction: The value of clinical ultrasound (US) has been explored for many surgical diseases and in trauma setting. The global impact of surgeon-performed US in daily surgical practice is underestimated. In order to address this issue, a prospective registry of all US performed in a General and Emergency surgical unit was established.

Material and methods: Since October 2015, all bedside US exams performed for patients hospitalized or admitted to the ED for surgical advice were prospectively registered. Data set included descriptive decision-making effect of the US results too. Four out of 10 surgeons had adequate US skills and performed US. Data were analyzed to study the effect of US on time-to-clinical-decision (TCD) and on saving-of-further-imaging (SFI). A cost-effectiveness analysis was also provided.

Results: A total of 536 bedside point-of-care US were performed. In 27 cases US was performed for trauma patients; 509 US were performed in non-traumatic patients, 68 of which (13.3\%) to assess and manage post-operative course and complications; 37 between PTX and pleural effusions were managed which US; also, in 117 cases $(21.8 \%)$ US was useful for diagnostic/therapeutic interventional procedures. In 132 cases it was performed in ED. A detailed list of indications and results will be shown. Bedside US allowed to shorten TCD in 112 cases $(20,8 \%)$ and impacted in SFI in 86 cases $(16 \%)$.

Conclusion: Daily use of bedside surgeon-performed US has a tremendous potential impact on decision-making, TCD and SFI. The estimation of the economical implications is demanding and ongoing. Awareness about this reality should entail the need for systematic training programs

References: - Rozycki GS et al. A prospective study of surgeonperformed ultrasound as the primary adjuvant modality for injured patient assessment. J Trauma. 1995;39 (3):492-8. - Jeanmonod R, Stawicki SP, Bahner DP, Zago M. Advancing clinician-performed sonography in the twenty-first century: building on the rich legacy of the twentieth century pioneers. Eur J Trauma Emerg Surg (2016) 42:115-118

Disclosure: No significant relationships. 


\section{4}

\section{AN OPTIMUM ANTIBIOTIC REGIMEN FOR PEDIATRIC COMPLICATED APPENDICITIS CAN MINIMIZE INFECTION RATE}

\section{S.C. Ha, C. Koh, Y. Chen}

Pediatric Surgery, Far Eastern Memorial Hospital, New Taipei City/ TAIWAN

Introduction: Appendicitis is the most common abdominal emergency in children. Infection rate may increase when perforation has occurred. Wound infection and intraabdominal abscess were noted in some cases of complicated appendicitis (CA) in our hospital under three regimen antibiotics treatment (Cefmetazole, Gentamycin, and Metronidazole). Pseudomonas was reported in the appendix culture result $(29 \%)$ in our patients and which was not covered by 3 regimen antibiotics. Therefore new antibiotic regimen was set up since April 2013. We want to determine if this protocol is effective for infection prevention.

Material and methods: All charts of CA under 18 years old in our hospital from Jan 2010 to Aug 2016 were reviewed. Laparoscopic appendectomy with a 7-mm JP drainage was applied to all cases. Three regimen antibiotics followed by oral-form antibiotics were applied before April 2013 and followed by implementation of protocol with Tazocin + Metronidazole and subsequent oral-form ciprofloxacin after April 2013. Patients were divided into two groups according to their antibiotics regimen. Group 1 was the patients treated with three regimen antibiotics. Group 2 were the patients treated with new protocol.

Results: There were 42 patients in group 1 compared to 45 patients in group 2. 9 patients developed an infection in group 1 compared with none of group $2(\mathrm{P}=0.001)$. Hospital cost was USD1473 in group 1 compared with USD1601 in group $2(\mathrm{P}=0.21)$. Re-admitted patients spend USD2288 $(\mathrm{P}=0.022)$ in average.

Conclusion: A hospital-based protocol of antibiotic regimen for complicated appendicitis can minimize complication rate without increasing the expense.

References:

Disclosure: No significant relationships.

\section{5}

ENTEROATMOSPHERIC FISTULA INCIDENCE AND INFLUENCE OF VISCERAL PROTECTIVE LAYER IN OPEN ABDOMEN THERAPY DUE TO PERITONITIS A PROPENSITY SCORE MATCHED CASE CONTROL ANALYSIS

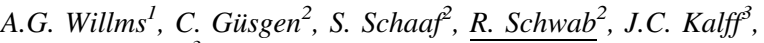 M. Von Websky}

${ }^{1}$ General-, Visceral- And Thoracic Surgery, German Armed Forces Central Hospital, Koblenz/GERMANY, ${ }^{2}$ General-/visceral- And Thoracic Surgery, German Armed Forces Central Hospital, Koblenz/ GERMANY, ${ }^{3}$ General-/visceral-, Thoracic And Vascular Surgery, University Hospital Bonn, Bonn/GERMANY

Introduction: Enteroatmospheric fistula (EAF) is a devastating complication of open abdomen therapy (OAT). Since there are no recommendations EAF prevention available, this study sought to elucidate whether a visceral protective layer (VPL) prevents EAF in patients with OAT due to peritonitis.

Material and methods: Overall, 120 consecutive patients with peritonitis and OAT from two clinics were included. Patients with application of VPL were considered the cases, patients without the use of VPL were the controls, respectively. Propensity score matching was performed for malign disease, emergency surgery, mortality, duration of OAT and fascial closure. Thus, the matched population was comprised of 34 cases and an equal number of controls. A logistic regression analysis was conducted to reveal the influence of VPL on EAF incidence.

Results: Cases and controls did not differ in distribution of age, sex and matching variables. However, statistically significant differences were found for use of negative pressure wound therapy (NPWT; VPL: $100.0 \%$ vs. noVPL: $14.7 \% ; \mathrm{p}=.00$ ) and for EAF occurrence (VPL:2.9\% vs. noVPL:23.5\%; $\mathrm{p}=.03$ ). Both the use of VPL and NPWT showed similar negative correlations with EAF (VPL:r $=-0.33$; NPWT:r $=-0.35 ; \mathrm{p}=.00)$. VPL and NPWT showed a strong autocorrelation $(r=0.97 ; p=.00)$. Thus, only VPL was included in the logistic regression model and its application was found to be highly protective in terms of EAF prevention (OR:0.10; CI: 0.01-0.84; $\mathrm{p}=.03$ ). Conclusion: The application of VPL was protective of EAF occurrence in this study. Regarding the high EAF-associated morbidity and reduced quality of life, the prevention of EAF should be a prioritized goal in OAT.

References:

Disclosure: No significant relationships.

0096

UNPLANNED READMISSION TO HOSPITAL POSTAPPENDECTOMY; A SYSTEMATIC REVIEW AND METAANALYSIS

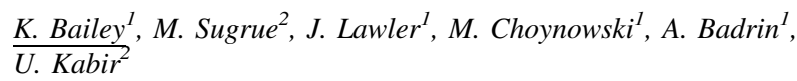

${ }^{1}$ Donegal Clinical Research Academy, Letterkenny University Hospital, Donegal/IRELAND, ${ }^{2}$ Department Of Surgery, Letterkenny University Hospital, Donegal/IRELAND

Introduction: Appendicitis is the most common cause of acute abdominal pain requiring surgical intervention ${ }^{1}$. Variable outcomes following appendectomy, including complications, contribute to readmission ${ }^{2}$. While many studies report readmission, a meta-analysis of readmission post-appendectomy has not been published. This study undertook a meta-analysis to determine a rate of readmission post appendectomy and to identify predictors of readmission.

Material and methods: An ethically approved PROSPERO-registered (ID CRD42017069040) meta-analysis following PRISMA guidelines using databases, PubMed and Scopus for studies published between January 2012 and June 2017 was undertaken. Articles relating to outcomes and readmissions after appendectomy were identified. Those scoring $>15$ for comparative studies and $>10$ for non-comparative studies using MINORS criteria were included in the final analysis. The odds ratio (OR) using random-effects, MantelHaenszel method with $95 \%$ confidence intervals (CI) were computed for each risk factor with RevMan5.

Results: 1,757 articles reviewed were reduced to 45 qualifying studies for a final analysis of 836,921 appendectomies. $4.3 \%$ (range: $0.0 \%-14.4 \%$ ) of patients were readmitted within 30 days. Significant pre-operative patient factors for increased readmission are diabetes mellitus $(\mathrm{OR}=1.93, \mathrm{CI}=1.63-2.28, \mathrm{P}<0.00001)$, complicated 
appendicitis $(\mathrm{OR}=3.6, \mathrm{CI}=2.43-5.34, \mathrm{p}<0.00001)$ and open surgical technique $(\mathrm{OR}=1.39, \mathrm{CI}=1.08-1.79, \mathrm{p}<0.00001)$. Increased readmission was not associated with gender, obesity, or paediatric versus general surgeons or centres.

Conclusion: This meta-analysis identified that readmission is not uncommon, occurring in 1 in 25 patients post-appendectomy. the mean readmission rate of $4.3 \%$ may act as a quality benchmark for improving emergency surgery care. Targeting high risk groups with diabetes, complicated appendicitis and increasing laparoscopic approach may reduce readmission.

References: 1. Graffeo CS, Counselman FL. Appendicitis. Emerg Med Clin North Am. 1996; 14 (4):653-671. 2. Lautz T, Reynolds M. Context and significance of emergency department visits and readmissions after pediatric appendectomy. J Ped Surg. 2011; 46: 1918-1922.

Disclosure: No significant relationships.

\section{7}

\section{SURGEON-PERFORMED ULTRASOUND ON MANAGEMENT OF PATIENTS WITH ACUTE ABDOMINAL PAIN}

\section{A. Natroshvili, A. Shulutko, F. Nasirov, E. Osmanov}

Department Of Surgery \#2, I.M. Sechenov First Moscow State Medical University, Moscow/RUSSIAN FEDERATION

Introduction: Patients with acute abdominal pain account for up to $10 \%$ of emergency department visits. The routine management of this patients includes taking medical history and clinical examination, followed by laboratory test and radiological investigations. Abdominal ultrasound is one of the most frequently performed investigations in surgical practice. The use of bedside ultrasound performed by the surgeon is increasing and has been described as the stethoscope of the new millennium. A study was performed to evaluate the benefits of surgeon-performed ultrasound for the routine management of the acute abdomen.

Material and methods: 3919 patients with acute abdominal pain underwent US in emergency department performed by trained in ultrasonography surgeon after taking history and physical examination. Surgical decision-making was based on the complete clinical evaluation. Final diagnosis was confirmed by operative findings, histologic examination or safe discharge without readmission in a month after initial visit.

Results: Surgeon-performed ultrasonography was useful in diagnosis and decision making in 734 patients with acute appendicitis, 632 with acute cholecystitis, 39 with peptic ulcer perforation, 213 with acute pancreatitis and 237 patients with bowel obstruction. In 138 cases other acute abdominal pathology was diagnosed. In 1687 patients SPUS helped to correctly exclude acute abdominal emergency. Ultrasonography was false-positive in 37 cases and false-negative in 202 patients with acute abdominal pain.

Conclusion: Surgeon-performed ultrasonography is a valuable, safe and readily available adjunct to clinical impression in patients with acute abdominal pain that improves surgeon decision making about treatment strategy.

References:

Disclosure: No significant relationships.

\section{8}

\section{HOSPITAL COMPLICATIONS OF INTESTINAL ANASTOMOSIS AFTER BLUNT ABDOMINAL TRAUMA}

\author{
I. Mahmood, F. Mustafa, B. Younis, K. Ahmed, A. El-Menyar, \\ M. Asim, A. Alhassani, R. Peralta, H. Al-Thani
}

Trauma Surgery, Hamad General Hospital, Doha/QATAR

Introduction: We aimed to analyze the postoperative outcomes following intestinal injury due to blunt abdominal trauma.

Material and methods: We retrospectively reviewed all intestinal injuries in adult patients who underwent laparotomy for blunt abdominal trauma between 2008 and 2015 at Level I trauma center. Data included demographics, mechanism of injury, site, type of repair, type of anastomosis, associated injuries, and management.

Results: One hundred sixty patients with bowel injuries were identified with a mean age of 33.1 years, $95.6 \%$ were males, and $55.6 \%$ sustained motor vehicle collision. Hypotension on admission was found in $16.3 \%$. The average base deficit was $5.1 \mathrm{mmol} / \mathrm{L}$ with a mean lactate level of $3.4 \mathrm{mg} / \mathrm{dL}$. Small bowel injuries were found in $56 \%$ whereas colon injuries comprised $32.5 \%$ and combined small and large bowel injuries were seen in $9.4 \%$. Only two duodenal and one rectal injuries were present. Most of the repairs $(88.5 \%)$ were performed at the initial surgery and $21.3 \%$ required damage control laparotomy. Anastomoses were hand-sewn in 51.3\%, stapled in $44.4 \%$ and combined in $3.8 \%$ of patients. The overall complication was $17.5 \%$ (wound infection (10\%), intra-abdominal abscess $(8.1 \%)$, and anastomotic leak (3.8\%)). Number of re-laparotomy, blood transfusion requirement, and high lactate level were associated with worse outcomes.

Conclusion: Morbidity after repair of traumatic bowl injury remains a challenge with high incidence of abdominal complications. Type of repair and site of injury do not seem to affect the outcome.

References: doi:

Disclosure: No significant relationships. 


\section{Clinical Research | Poster}

\section{ANESTHESIA}

\section{PR001}

\section{IS $\alpha$-MACROGLOBULIN A NEW BIOMARKER FOR ARDS OCCURRENCE IN POLYTRAUMA PATIENTS?}

\author{
O. Arnaut $^{1}$, R. Baltaga ${ }^{2}$, G. Rojnoveanu ${ }^{3}$, S. Shandru ${ }^{2}$
}

${ }^{1}$ Human Physiology And Biophysics, SUMPh „Nicolae Testemitanu”, Chisinau/MOLDOVA, ${ }^{2}$ Anesthesiology And Critical Care Medicine Nr. 1 “valeriu Ghereg”, SUMPh „Nicolae Testemitanu", Chisinau/MOLDOVA, ${ }^{3}$ Surgery No.1 "nicolae Anestiadi”, Nicolae Testemitanu State University of Medicine and Pharmacy, Chisinau/MOLDOVA

Introduction: Polytrauma and ARDS still remain important problems in medicine $[1,2]$. The aim of this study was to elaborate a model/models that are able to predict ARDS appearance in polytrauma using plasmatic concentration of $\alpha 2$-macroglobulin (neutrophylic proteases inactivator) as covariates [3].

Material and methods: A prospective research, 67 polytrauma patients (injury to at least 2 body regions with AIS $\geq 3$ and SIRS on at least one day during the first 72 hours [4]). Plasma concentration of $\alpha 2$-macroglobulin was determined at 3, 6, 12, 24, 48, 72 after traumatic event. ARDS occurrence was considered according to Berlin definition [5].

Results: Using logistic regression we have created a model $(\chi 2$ ( $\mathrm{df}=$ $1)=47.715 \mathrm{p}<0.001)$ that uses the plasmatic level of $\alpha 2$ macroglobulin collected at 3 hours after trauma ( $\alpha 2 \mathrm{M} 3$ hours): $\mathrm{p}=1 /$ $\left(1+\mathrm{e}^{\wedge}(-5,651+5,783 \times \alpha 2 \mathrm{M} 3\right.$ hours $\left.)\right)$ where $p$ - probability to develop ARDS in polytrauma, $e$ - constant (2.71828) The model has following characteristics: Nagelkerke R Square $=0.695(69.5 \%)$; Hosmer and Lemeshow Test $\chi^{2}(\mathrm{df}=8)=12.024, \mathrm{p}=0.15$; Sensibility $(97.6 \%, 41$ from 42) and Specificity (80\%, 20 from 25); Bootstrap (B = -5.783, CI95\% -9.810; -4.146).

Conclusion: $\alpha 2$-macroglobulin collected at 3 hours after trauma can be considered a potential biomarker for ARDS occurrence in polytrauma. Besides prognostic value the model has a perspective to be a base for ARDS prophylaxis strategy for polytrauma patients. The validation of model follows.

References: 1. World Health Organization. Global status report on road safety 2013: supporting a decade of action. Geneva: WHO Press; 2013. 2. John G. Laffey. Epidemiology, Patterns of Care, and Mortality for Patients With Acute Respiratory Distress Syndrome in Intensive Care Units in 50 Countries JAMA. 2016;315 (8):788-800. 3. Moore A.R., Appelboam A., Kawabata K., et al. Destruction of articular cartilage by alpha 2 macroglobulin elastase complexes: role in rheumatoid arthritis. În: Ann Rheum Dis. 1999 Feb;58 (2):109-13 4. Butcher N., Enninghorst N., Sisak K., et al. The definition of polytrauma: variable interrater versus intrarater agreement-a prospective international study among trauma surgeons. In: J Trauma Acute Care Surg, 2013 Mar; 74 (3): 884-9. 5. Gordon D. Rubenfeld. Acute Respiratory Distress Syndrome. The Berlin Definition In: JAMA. 2012;307 (23):2526-2533

Disclosure: No significant relationships.
PR003

\section{OPERATIVE PROCEDURES IN VENO-VENOUS EXTRACORPOREAL MEMBRANE OXYGENATION (VV- ECMO) IS OFTEN NECESSARY AND SAFE}

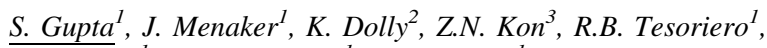
D.M. Stein ${ }^{1}$, J.V. O'Connor ${ }^{I}$, T.M. Scalea ${ }^{I}$

${ }^{1}$ Shock Trauma, University of Maryland, Baltimore/MD/UNITED STATES OF AMERICA, ${ }^{2}$ Peri-operative Services, University of Maryland, Baltimore/MD/UNITED STATES OF AMERICA, ${ }^{3}$ Cardiac Surgery, University of Maryland School of Medicine, Baltimore/MD/UNITED STATES OF AMERICA

Introduction: Surgical procedures are often indicated for patients on VV-ECMO. Concern for high risk of mortality may lead to reluctance to offer surgical intervention. The purpose of this study was to describe surgeries performed and outcomes in adult patients on VVECMO.

Material and methods: All patients on VV-ECMO between August 2014 and September 2017 were identified. Records were reviewed for demographics, ECMO related data, surgical procedures, complications and outcomes. Chi-square analysis was used to evaluate differences in survival.

Results: 181 patients were included. Mean age was 42 years (STD 14.6); $63.5 \%$ were males. Common indications for ECMO included acute respiratory distress syndrome $(33.1 \%)$ and bacterial pneumonia (18.8\%). Survival was $73.5 \%$. 118 patients $(65.2 \%)$ underwent 309 surgeries; median of 2 (IQR 1-10) per patient. 23 (7.4\%) operations were performed at the bedside, including a pericardial window and cesarean section. 200 operations $(64.7 \%)$, including tracheostomy (n $=78$ ) and non-index abdominal surgeries $(n=67)$, were classified as non-emergent. 109 (35.2\%), including 17 decompressive laparotomies and 46 for bleeding (including 8 post tracheostomy and 24 post-operative abdominal hemorrhage), were classified as emergent surgeries. There was no difference in survival between patients with and without an operation $(76.1 \%$ vs $68.3 \%, p=0.44)$. Patients requiring an emergent surgery had a lower survival than those who only underwent non-emergent surgery $(57.9 \%$ vs $85.3 \% \mathrm{p}=0.001)$. Conclusion: Most patients on VV-ECMO require surgery. We demonstrated no increased mortality for patients who required surgeries while on ECMO. Although patients who require an emergent operation had increased mortality, the majority survived. VV-ECMO should not be considered a contraindication for surgery.

References:

Disclosure: No significant relationships.

\section{PR004}

\section{INHERITED THROMBOPHILIA - AS A MARKER OF VENOUS THROMBOEMBOLISM IN THE WOUNDED WITH COMBAT GUNSHOT INJURIES}

$\underline{\text { K. Nikolaev }}^{1}$, V. Zubritskij ${ }^{2}$, S. Kapustin ${ }^{3}$, D. Ivchenko ${ }^{4}$, A. Koltovich ${ }^{2}$

${ }^{1}$ Surgery, The Main military clinical hospital National Guard troops of Russian Federation, Balashicha, Russian Federation, Moscow/ RUSSIAN FEDERATION, ${ }^{2}$ Surgery, The main clinical hospital Ministry of interior, Moscow, Moscow/RUSSIAN FEDERATION,

${ }^{3}$ Laboratory, Russian Research Institute of Hematology and Blood

Transfusion, Federal Agency of Medicine and Biology, St.

Petersburg, St. Petersburg/RUSSIAN FEDERATION, ${ }^{4}$ Thoracic 
Surgey, The Main military clinical hospital National Guard troops of Russian Federation, Balashicha, Russian Federation, Moscow/ RUSSIAN FEDERATION

Introduction: Combat casualties are usually at high-risk conditions for developing venous thromboembolism (VTE).The objective of this study was to evaluate the possibility of using congenital thrombophilia tests as a marker of VTE.

Material and methods: A 5-year cohort analysis performed to determine the possibility of using the study of congenital thrombophilia as a marker of VTE in the wounded with combat gunshot trauma, admitted to the hospital from the North Caucasus. Continuous and categorical variables were studied with the Student's t test, Fisher's exact test or $\chi^{2}$ test, multivariate analysis was performed using a stepwise regression logistic model.

Results: A total of 46 records were reviewed. All patients - men, mean age - 29,5 $\pm 5,1$ years. Mine-blast injured 33 (71,7\%), bullet $13(28,3 \%)$ patients. All the wounded were classified as high risk for venous thromboembolic complications and had 3 - 4 risk factors for their development. The study group included $13(28,3 \%)$ patients who during treatment at the hospital were diagnosed with venous thrombosis \& the control group - 33 (71,7\%) - with no signs of thrombosis. Studied allelic polymorphism of genes: the factors I, II, V, XII, PAI-1, HPA-1, HPA-2, P2Y12, GpIa C677T (MTHFR). It has been found that hereditary thrombophilia Prt G20210-A, HPA-2 and Fibrinogen $\mathrm{G} / \mathrm{A}-455$ are risk factors for thrombosis $(\mathrm{p}<0.05)$.

Conclusion: We have found that, despite the high prevalence of thrombophilia not all lead to the development of thrombosis. The greatest probability of developing clinical manifestations of thrombosis must be expected for the wounded with combinations of several genetic mutations.

References:

Disclosure: No significant relationships.

\section{PR005}

PRE AND POST IMPLEMENTATION OF RAPID SEQUENCE INDUCTION PROTOCOL IN THE EMERGENCY MEDICAL SERVICES

\section{B.M. Wahlen ${ }^{1}$, N. Castle ${ }^{2}$, A. El-Menyar ${ }^{3}$, H. Al-Thani ${ }^{3}$}

${ }^{1}$ Trauma Surgery, Hamad General Hospital, Doha/QATAR, ${ }^{2}$ Ems Services, Hamad General Hospital, Doha/QATAR, ${ }^{3}$ Trauma Surgery Section, Department Of Surgery, Hamad Trauma Center, Doha/ QATAR

Introduction: We aimed to evaluate the safety of a newly implemented Rapid sequence induction (RSI) protocol in the emergency medical services.

Material and methods: A retrospective analysis was performed for all prehospital intubated trauma patients from September 2015 to August 2017. Patients were divided into three groups according to their body weight. 50-60 $\mathrm{kg}(\mathrm{S}), 80-90 \mathrm{~kg}(\mathrm{M}),>100 \mathrm{~kg}(\mathrm{~L})$ as well as their clinical appearance (stable versus unstable). Fentanyl and Ketamine dosages were given based on the body weight and stability. The muscle relaxant choice was left between Suxamethonium or Rocuronium according to body weight. The pre- and post - implementation data were analyzed with respect to the used induction, paralytic and analgesic agents

Results: There were 192 patients included in the analysis; 99 in the pre-implementation (Group I) and 93 in the post-implementation phase (Group II). A drop in MAP of more than $20 \%$ from the baseline value could be observed in $12 \%$ of the patients of group I whereas only $4 \%$ of the patients in group II displayed such a drop in MAP. A rise in MAP above $20 \%$ from the baseline value was calculated in almost $29 \%$ of the patients of group I whereas only in $20 \%$ of the patients of group II.

Conclusion: After implementation of the new RSI protocol more patients remain stable with respect to cardio-circulatory parameters. However, further evaluation of the hospital settings and protocols would be beneficial.

References: Lyon et al. Critical Care (2015) 19: 134. DOI 10.1186/ s13054-015-0872-2

Disclosure: No significant relationships.

\section{PR006}

\section{ADMINISTRATION OF HYPERTONIC SALINE (23.4\%) DOES NOT PROVOKE COAGULOPATHY}

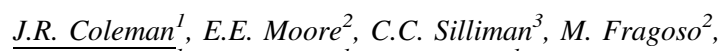
$\overline{J . M . ~ S a m u e l s}^{1}$, G.R. Nunns ${ }^{1}$, A. Banerjee ${ }^{1}$

${ }^{1}$ Surgery, University of Colorado Medical Center, Denver/CO/ UNITED STATES OF AMERICA, ${ }^{2}$ Surgery, Denver Health Medical Center, Denver/CO/UNITED STATES OF AMERICA, ${ }^{3} \mathrm{Hematology,}$ University of Colorado Medical Center, Denver/CO/UNITED STATES OF AMERICA

Introduction: Administering a bolus of hypertonic saline $(23.4 \%)$ (HTS) is common practice for refractory intracranial hypertension following trauma but effects on coagulation are unknown. Coagulopathic changes from HTS only occur at $5 \%$ whole blood dilution, significantly higher than volumes administered in the clinical setting. Therefore, we hypothesize that there will be no significant changes in coagulation in a rat tail bleeding time (TBT) model with clinically relevant doses of HTS.

Material and methods: Ten Sprague-Dawley rats were randomized to receive a bolus of normal saline $(0.9 \%$, NS) $(n=5)$ or HTS $(n=5)$ at a dose equivalent to $2.5 \%$ blood dilution. Baseline sodium, osmolarity and citrated native thrombelastography (TEG) were measured. Then, a $0.6 \mathrm{~mL}$ bolus was administered and the tail was transected $0.5 \mathrm{~cm}$ from tip. At five, 20 and 40 minutes, sodium, osmolarity and TEG were measured.

Results: Administration of HTS did not result in statistically significant changes in TEG measurements as compared to NS. There was a higher median TBT in the HTS group (37.5 versus 29.5 minutes in NS group), though also not statistically significant $(\mathrm{p}=0.15)$. The HTS group demonstrated a higher sodium after bolus administration, with a median rise from $141 \mathrm{meq}$ to a maximum $153 \mathrm{meq}$ at five minutes (no change in NS group) $(\mathrm{p}=0.008)$ and a higher serum osmolarity, with a median rise from 300 to $326 \mathrm{mOsm}$ at five minutes (no change in NS group) $(\mathrm{p}=0.11)$.

Conclusion: Even at bolus doses equivalent to $2.5 \%$ blood dilution (three times the normal bolus volume), there is no statistically significant effect on bleeding time or clotting activity.

References:

Disclosure: No significant relationships. 
PR007

\section{OUT-OF-HOSPITAL AIRWAY MANAGEMENT IN TRAUMA PATIENTS - IS THE MISPLACED INTUBATION AN UNDERESTIMATED DANGER?}

\section{O. Özkurtul $^{l}$, J.K.M. Fakler ${ }^{2}$, C. Josten ${ }^{3}$}

${ }^{1}$ Klinik Für Orthopädie, Unfallchirurgie Und Plastische Chirurgie, Universitätsklinikum Leipzig, Leipzig/GERMANY, ${ }^{2}$ Orthopaedic, Trauma And Plastic Surgery, University Clinic Leipzig, Leipzig/ GERMANY, ${ }^{3}$ Department Of Orthopeadics, Trauma Surgery, And Plastic Surgery, University of Leipzig, Leipzig/GERMANY

Introduction: Establishing and maintaining an adequate airway by endotracheal intubation (ETI) is an essential component of advanced trauma life support (ATLS $\AA$ ) to ensure best possible oxygenation and ventilation. Orotracheal intubation remains the gold standard for securing a definitive airway in trauma care. When performed in the out-of-hospital environment special attention has to be paid to cervical spine protection, the risk of aspiration and possible side-effects of analgesia and sedation. The aim of our study was to determine the influence of airway management associated problems on neurological outcome after out-of-hospital intervention by EMS physicians in patients suffering from severe and multiple injuries.

Material and methods: In this retrospective study (01/2011-12/ 2013), all patients admitted to the trauma room of a level I trauma center were analyzed consecutively. The incidence of unrecognized esophageal misplaced tube, endobronchial intubation and massive aspiration of stomach content were recorded as airway associated problems. The neurological outcome at hospital discharge was evaluated using the Glasgow Outcome Scale (GOS).

Results: During the study period 1,176 patients were admitted. Out of these, 151 patients received advanced airway management in the outof-hospital setting. Airway associated problems were identified in 12 cases $(8 \%)$. Esophageal intubation was recorded in 5 patients $(3 \%)$ and other problems were identified in 5\%. Airway associated problems was significant associated with vegetative state or severe disability (GOS 2/3) in 36,3\%.

Conclusion: Out-of-hospital airway management problems contribute to the neurological outcome of severe and multiple injured patients. Intensive and routine trauma and airway management trainings seem to be a prerequisite for successful trauma management. References:

Disclosure: No significant relationships.

\section{PR008}

\section{DOES PREOPERATIVE HEMODYNAMIC PRECONDITIONING (PHP) IMPROVE MORBITITY AND MORTALITY AFTER TRAUMATIC HIP FRACTURE IN GERIATRIC PATIENTS}

F.J..P. Beeres ${ }^{1}$, B. Schafroth ${ }^{2}$, F. Eckhart ${ }^{2}$, C. Konrad ${ }^{2}$, M. Rohner ${ }^{1}$, R. Babst ${ }^{1}$, B. Poblete $^{2}$

${ }^{1}$ Department Of Orthopaedic And Trauma Surgery, Luzerner Kantonsspital, Luzern/SWITZERLAND, ${ }^{2}$ Department Of Anaesthesia, Luzerner Kantonsspital, Luzern/SWITZERLAND

Introduction: Traumatic hip fracture (THF) in geriatric patients has a high morbidity and mortality. Goal directed therapy helped to reduce morbidity/mortality. However, patients need invasive cardiac output monitoring. Therefore we investigated a treatment protocol for PHP using clinical parameters. Aim: Improving quality of care and reduction of the $\mathrm{CR}$ as well as mortality in geriatric patients by means of PHP.

Material and methods: P-Possumscore (pP) (2) of all patients $>70 y$ admitted to our hospital for THF was calculated. Patients with a $\mathrm{pP}>$ 5 were supposed to receive a PHP in our intermediate care unit (IMCU). For capacity reasons not all patients could be included in the IG. Patients not admitted to the IG served as a quality control group (CG). Blood pressure, PPV or peripheral circulation, diuresis, time to operation, CR, 30 and 90d mortality and length of stay (LOS) were recorded. CR mainly included presence or absence of pneumonia, urinary tract infections, decubitus, wound infections, delirium, myocardial infarction, stroke and anemia that required blood transfusions.

Results: In 2015 a total of 158 patients were admitted for THF to our hospital. 57 patients had a $\mathrm{pP}>5$ of which 39 patients entered the IG and 18 the CG. There was a trend toward a lower CR in the IG.

Conclusion: Geriatric patients with a $\mathrm{pP}>5$ could benefit from PHP in terms of a lower $90 \mathrm{~d}$ mortality. There was a trend toward a lower $\mathrm{CR}$ in the IG at the cost of a longer LOS.

References: 1. Cecconi et al. Critical Care 2013, 17:209 Clinical review: Goal-directed therapy - what is the evidence in surgical patients? The eff ect on different risk groups 2 . www.riskprediction.org.uk

Disclosure: No significant relationships.

PR009

EARLY VENOUS THROMBOEMBOLIC PROPHYLAXIS IS SAFE IN PATIENTS WITH SEVERE TRAUMATIC BRAIN INJURY

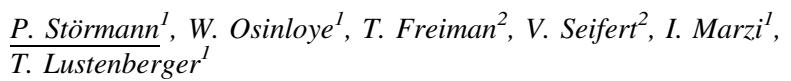

${ }^{1}$ Department Of Trauma, Hand And Reconstructive Surgery, University Hospital Frankfurt, Goethe-University, Frankfurt am Main/GERMANY, ${ }^{2}$ Department Of Neurosurgery, University Hospital Frankfurt, Goethe-University, Frankfurt am Main/ GERMANY

Introduction: Traumatic brain injury (TBI) patients are known to be at high risk for venous thromboembolic events (VTE). Prophylactic anticoagulation should be used to prevent VTE complications, however, there is an increased risk of a progression of the intracranial hemorrhage with VTE prophylaxis. The aim of this study was to determine whether early VTE prophylaxis is safe for patients with severe TBI.

Material and methods: Retrospective review of all patients admitted over a 4-year period with evidence of an acute intracranial hemorrhage. Type of intracranial bleeding, occurrence of VTE events and progression of bleeding after start of prophylactic anticoagulation was investigated. Patients were categorized according to the time point of VTE prophylaxis start (early: $\leq 24$ hours, intermediate: $>24$ hours until $\leq 48$ hours, late: $>48$ hours after admission).

Results: A total of 296 patients were included. Overall, 116 patients received VTE prophylaxis early, 105 patients intermediate, and 75 patients late. Only 1 patient suffered a proven deep venous thrombosis. Evaluating the cranial CT-scans, 134 patients $(45.0 \%)$ presented with a progression of the intracranial hemorrhage before start of prophylaxis and 46 patients $(15.5 \%)$ showed a progression of 
the intracranial hemorrhage after start of VTE prophylaxis. Regarding bleeding progression, no statistically significant difference was found comparing the different groups (early VTE prophylaxis $15.5 \%$ vs. intermediate $14.3 \%$ vs. late $17.3 \%, \mathrm{p}=0.857$ ).

Conclusion: In patients with severe traumatic brain injury, early VTE prophylaxis does not increase the rate of intracranial hemorrhage progression.

References:

Disclosure: No significant relationships.

\section{PR010}

USEFULNESS OF INCISIONAL CATHETERS OF CONTINUOUS PERFUSION OF LOCAL ANESTHETIC IN THE MANAGEMENT OF POSTOPERATIVE PAIN IN PATIENTS SUBMITTED TO INSTRUMENTED LUMBAR ARTHRODESIS

\section{Auñon Martin, F.J. Ortega Garcia}

Ortopédica Surgery Service, Hospital 12 de Octubre, Madrid/SPAIN

Introduction: Pain is a common event in patients after a spinal surgery. Poor pain control can lead to morbidities and hamper optimal outcome. An analgesic modality has not been developed that fulfills all the ideal characteristics that are supposed to it, for this reason multimodal analgesia techniques have been introduced to improve the results of the treatment. Our objective is to evaluate if postoperative continuous wound infiltration of levo-bupivacaine through two submuscular catheters connected to two elastomeric pumps after lumbar instrumented arthrodesis is more effective or associated with more adverse effects than intravenous patient controlled analgesia alone.

Material and methods: An observational, prospective cohorts study was carried out. The Visual Analogue scale (VAS), the need for additional rescue analgesia and the onset of adverse effects were recorded. Inclusion criteria were patients aged 18 to 85 years who underwent posterior-instrumentated lumbar arthrodesis using PosteroLateral or Transforaminal Intersomatic Fusion.

Results: Pain records measured with VAS scale were significantly lower in the 48 hours postoperative record at rest $(\mathrm{p}=0.032)$. The other records of VAS showed a clear tendency to lower levels of pain in the group treated with incisional catheters. No significative differences were found in the rescue analgesia demands of the patients. The adverse effects were clearly lower in the catheter group (6 cases versus 11 cases) but without statistical differences.

Conclusion: A clear trend to a lower pain records was found in the group treated with incisional catheters, although differences were not statistically significant.

References: Kehlet $\mathrm{H}$ : Postoperative opioid sparing to hasten recovery: What are the issues? Anestehesiology 2005; 102:1083-5 EDITORIAL VIEWS. Continuous Local Anesthetic Wound Infusion to Improve Postoperative Outcome. Back to the Periphery? Anesthesiology 2007; 107: 369-71. Méndez Gómez RM, Ortiz Buiza L, Vázquez Chena P, Álvaro Hernando J, Hernández Cabeza B. Analgesia Postoperatoria Mediante Sistema Elastomérico. XXIV Congreso Nacional de la Asociación Española de Enfermería de Anestesia, Reanimación y Terapia del Dolor (ASEEDAR-TD). Madrid: Hospital
12 de Octubre; 2010. James B. Elder, MD, Daniel J. Hoh, MD, and Michael Y. Wang. Postoperative Continuous Paravertebral Anesthetic Infusion for Pain Control in Lumbar Spinal Fusion Surgery. Spine 2008; 33: 210-218. Hollmann MW, Strumper D, Durieux ME. The poor man's epidural: systemic local anesthetics for improving postoperative outcomes. Med Hypotheses 2004; 63: 386-9. Postoperative Analgesia: Comparing Continuous Epidural Catheter Infusion of Local Anesthetic and Opioid and Continuous Wound Catheter Infusion of Local Anesthetic. Maria Cristina Simões de Almeida, Giovani de Figueiredo Locks, Horácio Pereira Gomes, Guilherme Muriano Brunharo, Ana Laura Colle Kauling. Rev Bras Anestesiol 2011;61:293-303.

Disclosure: No significant relationships.

\section{PR011}

\section{ANESTHETIC MANAGEMENT OF EARTHQUAKE VICTIMS WITH MULTI SYSTEM MECHANICAL INJURY IN INFANTS}

\author{
M. Kovalenko
}

Anesthesiology, Clinical and Research Institute of Emergency Pediatric Surgery and Trauma, Moscow/RUSSIAN FEDERATION

Introduction: Clinical care and diagnostics including anesthetic management of catastrophe victims are relevant worldwide. Specialized medical aid aimed at the elimination of earthquake health effects is impossible without anesthesia.

Material and methods: Data from 4 earthquakes (Indonesia, 2006, 2009; Haiti, 2010; Nepal, 2015) were analyzed retrospectively. Special care was performed by the team from our institute. The team consisted of 2 anesthesiologists, 2 or 3 traumatologists, 2 surgeons and a neurosurgeon. In total, 1340 anesthesias of various complexity have been performed for 275 patients aging from 1 month to 18 years. General health status was defined according to ASA score (II-IV).The retrospective analysis of provided anesthesias was performed.

Results: The orthopedic trauma prevailed with limb fractures dominating $(53 \%)$. Head injuries of various complexity amounted to $19 \%$. $10 \%$ of traumas was the combined injuries, $4 \%$ - back injury, $5 \%$ pelvic injury. The wide range of anesthesia models were applied including combined and mixed anesthesias. 1340 anesthesias were performed for 275 patients: $556(41,5 \%)$ during urgent and delayed surgery and $784(58,5 \%)$ during dressing. The percentage distribution of performed anesthetic models was: inhalation anesthesia 45,7\% (612), intravenous anesthesia 13,9\% (186), endotracheal anesthesia combined with inhalation anesthetics $15,4 \%$ (206), block anesthesia $3,9 \%$ (52), mixed anesthesia 10,2\% (137).

Conclusion: The choice of anesthetic model to provide special care for earthquake victims depended on the type and the time of surgical treatment. Inhalation and intravenous anesthesias were the method of choice for minimally invasive surgery and extended dressings. Long term, large urgent surgery required endotracheal anesthesia combined with block anesthesia.

References:

Disclosure: No significant relationships. 


\section{EMERGENCY SURGERY}

\section{PR012}

\section{SCORING TOLL PREDICTING MORTALITY IN ELDER PATIENTS}

\author{
I. Eguaras ${ }^{1}$, P. Sanchez ${ }^{1}$, B. Fernandez-Velilla ${ }^{1}$, A. Galbete ${ }^{2}$, \\ A. Goikoetxea ${ }^{3}$, J. Herrera ${ }^{3}$, J. Mateo $^{1}$, I. Otegi ${ }^{3}$
}

${ }^{1}$ General Surgery, CH-navarra, Pamplona/SPAIN, ${ }^{2}$ Stadistic, Navarrabiomed, pamplona/SPAIN, ${ }^{3}$ General Surgery, chnavarra, pamplona/SPAIN

Introduction: Improvements in living conditions and progress in medical and surgical management have resulted in an increase in life expectancy. The number of elderly patient requiring emergency surgical intervention has raised dramatically. The main objective was to elaborate a score to define morbimortality in patients $65>$ years old undergoing emergency abdominal surgery.

Material and methods: Retrospective study using a prospective database. We include subjects $>65$ years old undergoing emergency abdominal surgery between 1994-2016. Risk factor for 30 days morbimortality (using Calvien Dindo classification) were identified using multivariante regression and weights assigned using odds ratios. A morbimortality score was derived from the aggregate of weigthed scores

Results: Data were collected from 4255 patients. We analyse separately mortality and morbility (Clavien I and II). Mortality (8.2\%); risk factor associated significally were: ASA, Age, Preoperatory diagnostic (OR:23 for intestinal isquemia, OR:3.17; colorectal perforation). From this data we elaborate a risk score. We can estimate the probability of survival in each patient $(\mathrm{p}=-6.26+(0.17 \mathrm{Age})+$ $(0.59$ ASA $)+(-1.02))$. The AUROC for this score was 0.82. Morbility $(37.6 \%)$ : risk factor associated significally were: ASA, Sex, preoperatory diagnostic, type of surgery, open surgery (versus laparoscopic surgery). From this data we elaborate a risk score; $\mathrm{p}=$ $4.864+(0.30 \mathrm{men})+(0.70$ ASA $)+($ B Diagnostic $)+(B$ Type of surgery $)+(0.72$ open surgery $)$. The AUROC for this score was 0.71 . Conclusion: The most significant predictors of mortality were: ASA, Age, Preoperatory diagnostic. Risk factor associated significally with morbility were: ASA, sexe, Preoperatory diagnostic, type of surgery, open surgery. This models developed have a high discriminative ability to stratify the operative risk in a broad range of acute abdominal emergencies.

References: Introduction Improvements in living conditions and progress in medical and surgical management have resulted in an increase in life expectancy. The number of elderly patient requiring emergency surgical intervention has raised dramatically. The main objective was to elaborate a score to define morbimortality in patients $65>$ years old undergoing emergency abdominal surgery.

Material and methods Retrospective study using a prospective database. We include subjects $>65$ years old undergoing emergency abdominal surgery between 1994-2016. Risk factor for 30 days morbimortality (using Calvien Dindo classification) were identified using multivariante regression and weights assigned using odds ratios. A morbimortality score was derived from the aggregate of weigthed scores.

Results Data were collected from 4255 patients. We analyse separetly mortality and morbility (Clavien I and II). Mortality (8.2\%); risk factor associated significally were: ASA, Age, Preoperatory diagnostic (OR:23 for intestinal isquemia, OR:3.17; colorectal perforation). From this data we elaborate a risk score. We can estimate the probability of survival in each patient $(\mathrm{p}=-6.26+(0.17$ Age $)+$ $(0.59$ ASA $)+(-1.02))$. The AUROC for this score was 0.82 .
Morbility (37.6\%): risk factor associated significally were: ASA, Sex, preoperatory diagnostic, type of surgery, open surgery (versus laparoscopic surgery). From this data we elaborate a risk score; $\mathrm{p}=$ $4.864+(0.30$ men $)+(0.70$ ASA $)+($ B Diagnostic $)+(B$ Type of surgery $)+(0.72$ open surgery). The AUROC for this score was 0.71 .Conclusion The most significant predictors of mortality were: ASA, Age, Preoperatory diagnostic.Risk factor associated significally with morbility were: ASA, sexe, Preoperatory diagnostic, type of surgery, open surgery.This models developed have a high discriminative ability to stratify the operative risk in a broad range of acute abdominal emergencies.

Disclosure: No significant relationships.

\section{PR013}

\section{THE MISSING DENOMINATOR: AN ANALYSIS OF PATIENTS WHO DIED WITH INTRA-ABDOMINAL PATHOLOGY WITHOUT UNDERGOING AN EMERGENCY LAPAROTOMY}

\section{$\underline{\text { K. Broughton }}{ }^{1}$, R.J. Aitken ${ }^{2}$}

${ }^{1}$ Colorectal, Western General Hospital, Edinburgh/UNITED KINGDOM, ${ }^{2}$ General Surgery, Sir Charles Gairdner, Perth/WA/ AUSTRALIA

Introduction: The unknown denominator in Emergency Laparotomy (EL) audits are the patients who present with an intra-abdominal surgical pathology, but do not undergo surgery. No prospective data has been collected on such patients in EL audits. The aim was to examine mortality audit records to ascertain this denominator in Western Australia (WA).

Material and methods: Data from the Western Australian Audit of Surgical Mortality (WAASM) was examined for deaths under a general surgeon during the years 2010, 2015 and 2016. Records were reviewed for patient demographics, reasons surgery was not performed, surgical diagnosis and length of stay.

Results: In the three years of WAASM audit data examined, there were a total of 392 patients that met the inclusion criteria with $68 / 140$ in 2010, 62/126 in 2015 and 60/116 in 2016 dying without having undergo an EL. Patients who did not undergo operative intervention were older, with the most common diagnoses being mesenteric ischaemia and small bowel obstruction. In 2016, the average length of stay for non-operative patients was 8.5 days, and for operative patients 16.6 days. Extrapolation from administrative data demonstrates the percentage of patients who died without surgery was 4.1 per cent.

Conclusion: Prospective data collection of non-operative deaths needs to be included in EL audits. The low 30-day mortality following ELs reported in WA may partly be due to avoidance of surgery that has limited quality or quantity of life benefit. Whether differences in practice elsewhere explains the higher mortality internationally is unclear without local data.

References: 1. Pearse RM, Harrison DA, James P, Watson D, Hinds $\mathrm{C}$, Rhodes $\mathrm{C}$ et al. Identification and characterization of the high-risk surgical population in the United Kingdom. Crit Care 2006; 10: R81. 2. Symons NR, Moorthy K, Almoudaris AM, Bottle A, Aylin P, Vincent CA et al. Mortality in high-risk emergency general surgical admissions. Br J Surg 2013; 100 (10): 1318-1325. 3. Ingraham AM, Cohen ME, Bilimoria KY, Feinglass J, Richards E, Hall BL et al. Comparison of hospital performance in non-emergency versus emergency colorectal operations at 142 hospitals. J Am Coll Surg 2010; 210 (2):155-165. 4. Singh R, Kumar N, Bhattacharya A, 
Vajifdar H. Preoperative predictors of mortality in adult patients with perforation peritonitis. Indian Journal of Critical Care Medicine: Peer-reviewed, Official Publication of Indian Society of Critical Care Medicine. 2011;15 (3):157-163. doi:10.4103/0972-5229.84897. 5. Altemimi, Mohammed \& Griffee, Matthew \& M Enniss, Toby \& Preston, Robert \& Vargo, Daniel \& Overton, Sean \& Kimball, Edward \& Barton, Richard \& Nirula, Raminder. (2012). When Is Death Inevitable after Emergency Laparotomy? Analysis of the American College of Surgeons National Surgical Quality Improvement Program Database. Journal of the American College of Surgeons. 215. 503-11. 10.1016/j.jamcollsurg.2012.06.004. 6. Saunders DI, Murray D, Pichel A C, Varley S, Peden C J. Variations in mortality after emergency laparotomy: the first report of the UK emergency laparotomy network. Br J Anaesth 2012; 109 (3): 368-375. 7. Racz J, Dubois L, Katchky A, Wall W. Elective and emergency abdominal surgery in patients 90 years of age or older. Canadian Journal of Surgery. 2012;55 (5):322-328. doi:10.1503/cjs.007611. 8. National Confidential Enquiry into Patient Outcome and Death Peri-operative Care: Knowing the Risk, 2011. 2017 Jun;64 (6). pii: A5371. 16. Protopapa KL, Simpson J, Smith N, Moonesinghe S. Development and validation of the Surgical Outcome Risk Tool (SORT). BJS 2014; 101: 1774-1783. 17. Oliver CM, Walker E, Giannaris S, Grocott M, Moonesinghe S. Risk assessment tools validated for patients undergoing emergency laparotomy: a systematic review. $\mathrm{Br} J$ Anaesth 2015; 115 (6): 849-860. 18. Semmens JB, Sanfilippo F, Mukhtar S A, Haynes N, Mountain J, Aitken R J.. The Western Australian Audit of Surgical Mortality: Advancing Surgical Accountability. Med J Aust 2005; 183: 545-546. 19. Azzam DG, Neo C A, Itotoh F, Aitken R J. The Western Australian Audit of Surgical Mortality: outcomes from the first 10 years. Med J Aust 2013; 199 (8): 539-542. 20. Ghaferi AA, Birkmeyer JD, Dimick JB. Complications, Failure to Rescue, and mortality with major inpatient surgery in Medicare patients. Ann Surg 2009; 250: 1029-1034.

Disclosure: No significant relationships.

\section{PR014}

\section{PERITONITIS IN VERY ELDERLY: THE IMPORTANCE OF TIME}

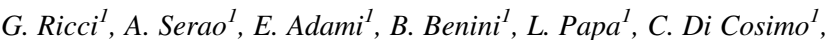
P. Fransvea ${ }^{2}$, R. Mancuso $^{I}$, J.R.M. Casella ${ }^{I}$, P. Marini $^{I}$

${ }^{1}$ Emergency Department - Division Of General And Emergency Surgery, San Camillo - Forlanini Hospital, Rome/ITALY, ${ }^{2}$ Chirurgia Generale E D'urgenza, Facoltà di Medicina e Psicologia Università "Sapienza" Roma, Rome/ITALY

Introduction: This study is intended to ascertain the risk factors for mortality in patients with age $\geq 80$ years operated for diffuse peritonitis.

Material and methods: 543 patients aged $\geq 80$ years underwent emergency abdominal surgery between November 2012 and March 2017 in our division. Emergency reoperations of prior elective surgery were excluded, a total of 98 patients with diffuse peritonitis were then included in our retrospective analysis. Mannhein peritonitis index has been used to assess peritonitis. Multivariable logistic regression was used to identify predictors of in-hospital mortality.

Results: Mean patient age was 84,8 years (Range 80-95), 53 were females and 35 males. 30-day morbility and mortality rate was respectively $71,4 \%$ and $30,6 \%$, median hospital stay was 16 days (range 5-66). At multivariate regression analysis ASA score (OR 3,57 95\% CI 0,992-8,366 p <0,01), Mannhein peritonitis index (OR 4,91
95\% CI 0,861-21,826 p <0,01), Malignancy (OR 2,97 95\% CI 1,042$3,943 \mathrm{p}<0,01)$ and Green or Red code at triage (OR 2,75 95\% CI $1,012-2,986 \mathrm{p}<0,01)$ were independent predictors of mortality. 39 patients received yellow code and 55 green code at traige, postoperative mortality rate was $17,9 \%$ for yellow and $36,3 \%$ for green code (p 0,05).

Conclusion: Respect to patients that received yellow code, patients with green code at triage spent a mean of 5,6 hours more in emergency department before operation, this could explain why patients with green code had a mortality rate almost double. Time is a killer in very elderly people with peritonitis, routine assignment of yellow code to patients aged $\geq 80$ years with acute abdominal pain should be considered.

References: 1. Okubo R, Yajima K, Sakai Y, Kido T, Hirano K, Musha N, Tsubono T, Hatakeyama K. Short- and Long-term Outcomes of surgery for diffuse peritonitis in patients 80 years of age and older. Surg Today 2008;38:413-419. 2. Ukkonen M, Kivivuori A, Rantanen T, Paajanen H. Emergency abdominal operations in the elderly: a multivariate regression analysis of 430 consecutive patients with acute abdomen

Disclosure: No significant relationships.

\section{PR015}

\section{CHARACTERISTICS OF POSTOPERATIVE ILEUS IN SURGICAL ELDERLY PATIENT}

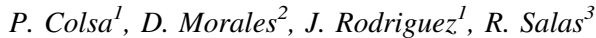

${ }^{1}$ Servicio De Cirugía General Y Del Aparato Digestivo, Hospital Grande Covián, Arriondas/SPAIN, ${ }^{2}$ Servicio De Cirugía General Y Del Aparato Digestivo, Hospital Universitario Marqués de Valdecilla, Santander/SPAIN, ${ }^{3}$ Residencia Sanitas, Residencia Sanitas, Santander/SPAIN

Introduction: One of the physiological changes typical of aging is the slowing of intestinal peristalsis. The aim of the present study was to describe the incidence and risk factors of postoperative ileus in elderly surgical patients.

Material and methods: Retrospective study of 675 patients over 70 undergoing surgery for abdominal pathology in Cantabria. The ileus was considered as the absence of oral intake after the seventh postoperative day. Cases were not included for deliberate absence of oral intake, intended to protect a digestive anastomosis in the immediate postoperative period. Data were analyzed using the X2 test with SPSS 17.0 statistical software. Subsequently, a multivariate analysis was performed by means of logistic regression of statistically significant covariates in univariate analysis. Significance was set at $\mathrm{p}<0.05$.

Results: A total of $15.8 \%$ of patients had postoperative ileus. This incidence is close to the described in studies with a broad population base $(17.4 \%$ and $15 \%)$.

These patients had a significantly higher number of antecedents of COPD 23.6\% ( $\mathrm{p}=0.011)$, rheumatologic pathology $15,1 \%(\mathrm{p}=$ $0.047)$ and endocrine pathology $1.9 \%$ vs $7.4 \%(p=0.035)$. From the functional point of view they led an independent life $61.7 \%$ vs $53.2 \%$ $(\mathrm{p}=0.004)$. Similarly, patients admitted with sepsis $60.6 \% \mathrm{p}=0.002$ or shock $37.7 \% \mathrm{p}=0.001$ presented a higher proportion of postoperative ileus. The development of ileus was accompanied in many cases by surgical complications such as postoperative hemorrhage ( $13.1 \%$ vs $5.7 \%), \mathrm{p}=0.013$, wound infection $(18.9 \%$ vs $9.2 \%), \mathrm{p}=$ 0.003 organ-espace infection $(34 \%$ vs $6.7 \%) \mathrm{p}=0.001$; fistula $(21 \%$ vs $3.9 \%) \mathrm{p}=0.001$ and evisceration $(7.5 \%$ vs $2.3 \%) \mathrm{p}=0.001$. The most frequent medical complications were nephrourea $(42.5 \%$ vs 
$17.4 \%) \mathrm{p}=0.001$; respiratory $(40.6 \%$ vs $14 \%) \mathrm{p}=0.001$; cardiac $(31.4 \%$ vs. $16.1 \%) \mathrm{p}=0.001$; and delirium $(23.2 \%$ vs $11 \%) \mathrm{p}=0.001$. $29.2 \%$ of the cases required reintervention $\mathrm{p}=0.001$; and $34.9 \%$ admission to intensive care unit $\mathrm{p}=0.001$. Of all patients with ileus, $29.2 \%$ of patients died $\mathrm{p}=0.001$. The hospital stay was greater than 11 days in $69.8 \%$ of cases $p=0.001$.In the multivariate analysis, ileus was related to reinterventions $(\mathrm{p}=0.016)$; respiratory complications $(\mathrm{p}=0.001)$; history of COPD $(\mathrm{p}=0.042)$ and fistula $(\mathrm{p}=0.001)$.

Conclusion: Knowledge of the physiological characteristics and basal functional status is essential in the elderly surgical patient. These variables can guide us in preventing, diagnosis and management of postoperative ileus and influence surgical decision making in emergency situations.

References: Artinyan A, Nunoo-Mensah J, Balasubramaniam S, Gauderman J, Essani R, Gonzalez-Ruiz C et al. Prolonged Postoperative Ileus-Definition, Risk Factors, and Predictors after Surgery. World J Surg. 2008; 32 (7):1495-1500. Chapuis P, Bokey L, Keshava A, Rickard M, Stewart P, Young C et al. Risk Factors for Prolonged Ileus After Resection of Colorectal Cancer. Annals of Surgery.2013; 257 (5):909-915. uage: EN-US; mso-fareast-language: EN-US;msobidi-language:AR-SA' > Prolonged Postoperative Ileus-Definition, Risk Factors, and Predictors after Surgery. World J Surg. 2008; 32 (7):1495-1500. Kronberg U, Kiran R, Soliman M, Hammel J, Galway $\mathrm{U}$, Coffey $\mathrm{J}$ et al. A Characterization of Factors Determining Postoperative Ileus After Laparoscopic Colectomy Enables the Generation of a Novel Predictive Score. Annals of Surgery. 2011; 253 (1):78-81. ed Postoperative Ileus-Definition, Risk Factors, and Predictors after Surgery. World J Surg. 2008; 32 (7):1495-1500. Lyer S, Saunders W, Stemkowski S. Economic Burden of Postoperative Ileus Associated With Colectomy in the United States. JMCP. 2009; 15 (6):485-494. ive Score. Annals of Surgery. 2011; 253 (1):78-81. ed Postoperative Ileus-Definition, Risk Factors, and Predictors after Surgery. World J Surg. 2008; 32 (7):1495-1500.

Disclosure: No significant relationships.

\section{PR016}

\section{LENGTH OF HOSPITAL STAY AFTER URGENT ABDOMINAL SURGERY IN ELDERLY PATIENTS}

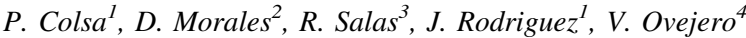

${ }^{1}$ Servicio De Cirugía General Y Del Aparato Digestivo, Hospital Grande Covián, Arriondas/SPAIN, ${ }^{2}$ Servicio De Cirugía General Y Del Aparato Digestivo, Hospital Universitario Marqués de Valdecilla, Santander/SPAIN, ${ }^{3}$ Residencia Sanitas, Residencia Sanitas, Santander/SPAIN, ${ }^{4}$ Servicio De Cirugía General Y Del Aparato Digestivo, Hospital sierrallana, Torrelavega/SPAIN

Introduction: Population aging is a social reality with repercussions on surgical practice. The aim of present study was to describe the postoperative length of stay and associated factors in patients over 70 who underwent urgent abdominal surgery.

Material and methods: A retrospective population-based study of patients older than 70 who underwent urgent abdominal surgery. We included 675 patients operated from January 2012 to December 2013 in the community of Cantabria.

Interventions for complications immediate to scheduled surgery and those that did not require hospitalization were excluded from the study.

Data were analyzed using the SPSS17.0 statistical software using the t-student test and later binary logistic regression: forward stepwise method. The $\mathrm{p}$-value for inclusion was $<0.05$; for exclusion $>0,1$.
Results: The mean length of stay during the postoperative period was 11.43 days, standard deviation (s.d.)11.52 days. Patients older than 80 had longer stays (12.36 days, $\mathrm{p}=0.042$ ), also those with oncological antecedents (11.29 days, $\mathrm{p}=0.050)$, preoperative sepsis (14.35 days, $\mathrm{p}=0.001)$ and shock (18.42 days, $\mathrm{p}=0.001)$. Pancreatitis surgery (25.84 days, $\mathrm{p}=0.001)$, open operative access (12.26 days, $\mathrm{p}=$ $0.001)$, operative time greater than 90 minutes $(14.5$ days, $\mathrm{p}=0.001)$ and reinterventions (26.38 days, $\mathrm{p}=0.001)$.

Some complications during the postoperative period had a significant influence on length of stay, such as ileus (20.35 days, $\mathrm{p}=0.001)$, hemorrhage (23.5 days, $\mathrm{p}=0.001)$, surgical wound infection $(21.12$ days; $=0.001)$, organ infection (26.06 days, $\mathrm{p}=0.001)$, fistula $(24.97$ days, $\mathrm{p}=0.001$ ), evisceration (21.68 days, $\mathrm{p}=0.008)$, cardiac morbidity, $\mathrm{p}=0.002)$, respiratory morbidity $(17.68$ days, $\mathrm{p}=0.001)$, and nephrourine morbidity (16.26 days, $\mathrm{p}=0.001)$.

Similarly, hospital stay was significantly longer in cases of intensive care (24.27 days, $\mathrm{p}=0.001)$, delirium (16.15 days, $\mathrm{p}=$ 0.002 ) and shock (16.65 days, $\mathrm{p}=0.003)$. In the multivariate analysis, the following were confirmed as independent factors of longer stay: open surgical access $(p=0.021)$, operative time greater than 90 minutes $(p=0.001)$, reinterventions $(p=0.001)$, ileus $(p=0.001)$, surgical wound infection $(\mathrm{p}=0.009)$, nephrouretic complications $(\mathrm{p}=$ $0.013)$, postoperative shock $(\mathrm{p}=0.001)$ and admission to the intensive care unit $(\mathrm{p}=0.001)$.

Conclusion: Postoperative length of hospitalization has important implications in elderly patients, so we should try to reduce it: . Minimizing surgical invasiveness and operative time. Optimizing treatment of the ileus, wound infections and nephrological complications. - Preventing the development of postoperative shock

References: Caglar Bilgin B, Kahramanca S, Akin T, Emre Gokce I, Akin M, Kucukpinar T. Factors influencing cost, length of hospital stay and mortality in colorectal cancer. Journal of the Balkan Union of Oncology. 2015; 20 (4):1023-1029. Ivatury S, Louden C, Schwesinger W. Contributing Factors to Postoperative Length of Stay in Laparoscopic Cholecystectomy. JSLS. 2011; 15 (2):174-178. tay and mortality in colorectal cancer. Journal of the Balkan Union of Oncology. 2015; 20 (4):1023-1029. Arenal JJ, Bengoechea-Beeby M. Mortality associated with emergency abdominal surgery in the elderly. Can J Surg. 2003; 46:111-116. span > tay and mortality in colorectal cancer. Journal of the Balkan Union of Oncology. 2015; 20 (4):1023-1029. Svenningsen P, Manoharan T, Foss NB, Lauritsen ML, Bay-Nielsen M. Increased mortality in the elderly after emergency abdominal surgery. Dan Med J. 2014; 61 (7):4876.

Disclosure: No significant relationships.

\section{PR017}

\section{FEASIBILITY AND SAFETY OF LAPAROSCOPIC EMERGENCY PROCEDURE IN THE ELDERLY: PRELIMINARY DATA OF FRAILESEL STUDY}

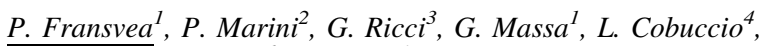

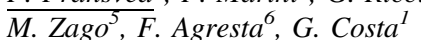

${ }^{1}$ Chirurgia Generale E D’urgenza, Facoltà di Medicina e Psicologia Università "Sapienza" Roma, Rome/ITALY, ${ }^{2}$ Chirurgia Generale E D'urgenza, Ospedale San Camilllo Forlanini, rome/ITALY, ${ }^{3}$ Emergency Department - Division Of General And Emergency Surgery, San Camillo - Forlanini Hospital, Rome/ITALY, ${ }^{4}$ Chirurgia D'urgenza, Az Ospedaliero Universitaria Pisana, Pisa/ITALY, ${ }^{5}$ Chirurgia Generale Ed Oncologica, Policlinico San Pietro, Bergamo/ ITALY, ${ }^{6}$ Chirurgia Generale, Ospedale civile di Adria, Adria/ITALY 
Introduction: Introduction: life expectancies are increasing and consequently there is an increasing elderly population with more complex co-morbidity. Emergency surgery in the elderly is challenging in terms of decision making, managing co-morbidity and post-operative rehabilitation with high morbidity and mortality rate. New physiopathology acknowledgement, improved surgical and anesthetic skills allowed the surgeon to achieve better results in treating these high risk patients. The aim of the present study is to evaluate the feasibility and safety of laparoscopic approach in the elderly population needing emergency surgical procedure

Material and methods: Materials and Methods: preliminary data of the Italian nationwide, multicenter prospective FRAILESEL study were analyzed. Variability considered for analysis were: age, sex, pre - operative comorbidities and patient fragility, type of procedure performed, timing, morbidity and mortality rate

Results: Results: a total of 450 emergency procedure were included in the database during the study period. Of these 150 were performed laparoscopically. For this study, data from the centers of Roma San Camillo, Roma S. Andrea, Roma San Filippo Neri, AbanoTerme, Adria, Arezzo and Pisa were used. The conversation rate was of $16.4 \%$. The most common diagnosis was cholecystitis. Overall morbidity rate was of $23.9 \%$ cases, laparoscopy group morbidity rate was of $22.5 \%$. Overall mortality rate was of $11.7 \%$, with no deaths occurred in the laparoscopy group.

Conclusion: Conclusion: from a preliminary assessment of the data, it emerged that laparoscopic approach is no longer closely linked to the patient's clinical status but to hospital resources and surgical skill and therefore it is feasible and safe even in the elderly patient

References: 1 - STUDY PROTOCOL Frailty and emergency surgery in the elderly: Protocol of a prospective, multicenter study in Italy for evaluating perioperative outcome (The FRAILESEL Study. Gianluca Costa, Giulia Massa, Genoveffa Balducci, Barbara Frezza, Pietro Fransvea, Giuseppe Nigri, Stagnitti Franco, Stefano Miniello, Mauro Zago, Rinaldo Marzaioli, Diego Piazza, Pierluigi Marini, Ferdinando Agresta, Gianluigi Luridiana, Antonio Crucitti, Piergaspare Palumbo, Francesco Gabrielli, Sergio Sandrucci, Luca Ansaloni, Fausto Catena, Osvaldo Chiara, Federico Coccolini, Alessandra Spagnoli, ERASO (Elderly Risk Assessment And Surgical Outcome) Collaborative Study Group. F1000Research 2017, 6:1162 Last updated: 04 AUG 20172 - Agresta F, Ansaloni L, Baiocchi GL, Bergamini C, Campanile FC, Carlucci M, Cocorullo G, Corradi A, Franzato B, Lupo M, Mandalà V, Mirabella A, Pernazza G, Piccoli M, Staudacher C, Vettoretto N, Zago M, Lettieri E, Levati A, Pietrini D, Scaglione M, De Masi S, De Placido G, Francucci M, Rasi M, Fingerhut A, Uranüs S, Garattini S. Laparoscopic approach to acute abdomen from the Consensus Development Conference of the Società Italiana di Chirurgia Endoscopica e nuove tecnologie (SICE), Associazione Chirurghi Ospedalieri Italiani (ACOI), Società Italiana di Chirurgia (SIC), Società Italiana di Chirurgia d'Urgenza e del Trauma (SICUT), Società Italiana di Chirurgia nell'Ospedalità Privata (SICOP), and the European Association for Endoscopic Surgery (EAES). Surg Endosc. 2012 Aug;26 (8):2134-64. doi: 10.1007/s00464-012-2331-3. Epub 2012 Jun 27. 3 - Kenig J, Wałęga P, Olszewska U, Konturek A, Nowak W. Geriatric Assessment as a qualification element for elective and emergency cholecystectomy in older patients. orld J Emerg Surg. 2016 Jul 29;11:36. doi: 10.1186/s13017-016-0094-1. 4 - Søreide K, Desserud KF. Emergency surgery in the elderly: the balance between function, frailty, fatality and futility. Scand J Trauma Resusc Emerg Med. 2015 Feb 3;23:10. doi: 10.1186/s13049-015-0099-x. Disclosure: No significant relationships.

\section{PR018}

\section{IS CHRONOLOGICAL AGE A LIMITING FACTOR IN THE ELDERLY'S URGENT SURGERY?}

\author{
P. Colsa ${ }^{1}$, D. Morales García ${ }^{2}$
}

${ }^{1}$ Servicio De Cirugía General Y Del Aparato Digestivo, Hospital Grande Covián, Arriondas/SPAIN, ${ }^{2}$ Servicio De Cirugía General Y Del Aparato Digestivo, Hospital Universitario Marqués de Valdecilla, Santander/SPAIN

Introduction: There is some evidence that the increase in mortality that occurs in the surgery of elderly patients does not emanate from the age itself, but from the greater comorbidity that these patients have. The aim of the present study was to describe the predictive value of chronological age in elderly surgical patients.

Material and methods: Retrospective study of 675 patients over 70 years of age who were urgently operated on for abdominal pathology. The sample was divided into two groups according to age (70-79 years) and ( $>80$ years). Data were analyzed using the X2 test with SPSS 17.0 statistical software. They were considered significant at $p$ $<0.05$. The results were compared with those published by other authors in this type of patients.

Results: At higher age, a higher proportion of females was identified $(\mathrm{p}=0.001)$; anemias $(\mathrm{p}=0.035)$; osteoarticular disease $(\mathrm{p}=0.029)$; atrial fibrillation $(\mathrm{p}=0.002)$; and heart disease $(\mathrm{p}=0.013)$.

We also found a higher polypharmacy index $(\mathrm{p}=0.036)$; and use of prosthetic devices $(\mathrm{p}=0.047)$.

The older the proportion of interventions for pancreatitis and ischemic pathology, while those for intestinal obstruction $(p=0.006)$ increase. The hemorrhagic pathology is maintained at levels unchanged with age.

The group of patients older than 80 years had a significantly lower mean stay $(2.36$ days vs. 10.53 days, $\mathrm{p}=0.042)$; and less surgical morbidity $(46.5 \%$, vs $57.3 \% \mathrm{p}=0.009)$. On the contrary, cardiac morbidity $(\mathrm{p}=0.028)$ and postoperative delirium $(\mathrm{p}=0.001)$ increased.

In our series there was no difference between the two groups in terms of mortality.

Conclusion: - Although cardiac complications increase, the stay is shorter and mortality does not vary.

- Age should not be understood as a criterion for denying urgent intervention.

- Comorbidity and functional status are more important in these patients.

References: C. Modini, F. Romagnoli, R. De Milito, et al. Octogenarians: an increasing challenge for acute care and colorectal surgeons. An outcomes analysis of emergency colorectal surgery in the elderly. Colorectal Disease. 2012:2027-48 Arenal JJ, BengoecheaBeeby M. Mortality associated with emergency abdominal surgery in the elderly. Can J Surg. 2003; 46:111-116. Bardram L, Funch-Jensen $\mathrm{P}$, Kehlet $\mathrm{H}$. Rapid rehabilitation in elderly patients after laparoscopic colonic resection. Br J Surg. 2000; 87:1540-1545. Kettunen J, Paajanen H, Kostiainen S. Emergency abdominal surgery in the elderly. Hepatogastroenterol. 1995; 42:106-108. Leardi S, De Santis C, Ciccarelli O, Valenti M, D’Alessandro A, Pietroletti R, Simi M: Risk of surgery in geriatric age: prospective evaluation of risk factors. Ann Ital Chir 1998, 69:575-579. Lidsky ME, Thacker JKM, LagooDeenadayalan SA, Scarborough JE. Advanced age is an independent predictor for increased morbidity and mortality after emergent surgery for diverticulitis. Surgery. 2012; 152 (3):465-472. Massarweh N. Impact of advancing age on abdominal surgical outcomes. Arch Surg 2009;144:1108-1114.

Disclosure: No significant relationships. 
PR019

\section{CHARACTERISTICS OF SURGICAL SITE INFECTION IN ELDERLY EMERGENCY PATIENTS}

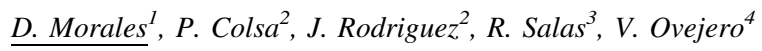

${ }^{1}$ Servicio De Cirugía General Y Del Aparato Digestivo, Hospital Universitario Marqués de Valdecilla, Santander/SPAIN, ${ }^{2}$ Servicio De Cirugía General Y Del Aparato Digestivo, Hospital Grande Covián, Arriondas/SPAIN, ${ }^{3}$ Sanitas Residencial, Sanitas residencial, Santander/SPAIN, ${ }^{4}$ Servicio De Cirugía General Y Del Aparato Digestivo, Hospital Sierrallana, Torrelavega/SPAIN

Introduction: The term "immunosenescence" refers to the physiological changes characteristic of aging on the immune system. The result is an increase in infections, neoplasms, autoimmune disorders, normocytic anemias, and weakness-sarcopenia syndromes. The aim of the present study was to describe the incidence and risk factors of surgical site infection in elderly surgical patients.

Material and methods: Retrospective study of 675 patients older than 70 years who underwent urgent surgery due to abdominal pathology in Cantabria. Clinical criteria (purulent secretion) or microbiological criteria (bacteria in culture) were taken for the diagnosis of surgical site infection. Data were analyzed using the X2 test with SPSS 17.0 statistical software. Subsequently, a multivariate analysis was performed by means of logistic regression of statistically significant covariates in univariate analysis. Significance was set at $p$ $<0.05$.

Results: In 72 of the patients $(10.7 \%)$ there was surgical wound infection during the postoperative period. Among the patients infected with the wound, there was a higher proportion of subjects younger than 80 years $(p=0.023)$, with a history of neuropathy $(p=0.035)$ and preoperative impairment of the ability to understand $(\mathrm{p}=0.047)$. On the contrary, the history of urological pathology $(\mathrm{p}=0.011)$ was less common. Surgical access was fundamentally open $(p=0.001)$ and significantly longer operative time $(\mathrm{p}=0.001)$. The surgical wound infection was also statistically associated with a higher proportion of reinterventions $(\mathrm{p}=0.001)$, readmissions $(\mathrm{p}=0.008)$, postoperative ileus $(\mathrm{p}=0.003)$, haemorrhages $(\mathrm{p}=0.044)$, organspace infections $=0.001)$, fistulas $(\mathrm{p}=0.001)$, eviscerations $(\mathrm{p}=$ $0.001)$ and admissions to intensive care units $(p=0.009)$. These patients often also presented Clavien I $(\mathrm{p}=0.022)$, II $(\mathrm{p}=0.001)$ and III $(\mathrm{p}=0.001)$ morbidity. As a result, prolonged hospital stay $(\mathrm{p}=$ 0.001 ) was also common, but not precocious mortality - ClavienV- ( $p$ $=0.032$ ). In the multivariate analysis, surgical wound infection was related to impaired preoperative comprehension level $(\mathrm{p}=0.038)$ and to prolonged interventions $(\mathrm{p}=0.027)$. The absence of wound infection was shown as a protective factor against evisceration $(\mathrm{p}=$ 0.003 ).

Conclusion: Knowledge of the physiological characteristics and basal functional status is essential in the elderly surgical patient. These variables can guide us in the prevention, diagnosis and management of surgical site infection and influence decision making in emergency situations.

References: Greif R, Akça O, Horn E, Kurz A, Sessler D. Supplemental Perioperative Oxygen to Reduce the Incidence of SurgicalWound Infection. New England Journal of Medicine.2000;342 (3):161-167. Haley RW, Culver DH, Morgan WM, White JW, Emori TG, Hooton TM. Identifying patients at high risk of surgical wound infection. A simple multivariate index of patient susceptibility and wound contamination. Am J Epidemiol.1985; 121:206-215. table > 2000;342 (3):161-167. Moro ML, Carrieri MP, Tozzi AE, Lana S, Greco D. Risk factors for surgical wound infections in clean surgery: a multicenter study. Italian PRINOS Study Group. Ann Ital
Chir.1996; 67:13-19. National Nosocomial Infections Surveillance (NNIS) System Report, Data Summary from January 1992-June 2001, Issued August 2001. American Journal of Infection Control. 2001; 29 (6):404-421.

Disclosure: No significant relationships.

\section{PR020}

\section{EMERGENCY VERSUS ELECTIVE SURGERY FOR COLORECTAL CANCER: COMPARABLE OUTCOMES?}

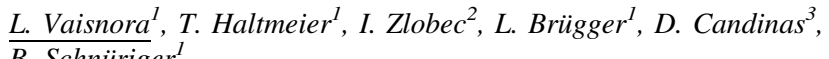 \\ B. Schnüriger
}

${ }^{1}$ Department Of Visceral Surgery And Medicine, Division Of Acute Care Surgery, Inselspital, Bern University Hospital, Bern/ SWITZERLAND, ${ }^{2}$ Institute Of Pathology, University of Bern, Bern/ SWITZERLAND, ${ }^{3}$ Visceral Surgery And Medicine, Inselspital, Bern University Hospital, Bern/SWITZERLAND

Introduction: Clinical outcomes in patients undergoing emergency versus elective resection for colorectal cancer (CRC) are discussed controversially.

Material and methods: Patients undergoing emergency or elective surgery for CRC from 07/2002-02/2013 were extracted from our institution's prospectively maintained CRC-database. Patient- and injury-characteristics were analyzed in univariable analysis, outcomes were compared using Cox-regression analysis.

Results: Overall, 492 patients were included. Mean age was $65 \pm 22$ years. A total of 154 patients were operated for rectal cancer and 338 patients for colon cancer. Median follow-up was 439 days (IQR 49-1409). Emergency resection was performed in 108 (22\%) patients due to obstruction, perforation or bleeding. T4 tumor stages and ASAscores $\geq 3$ were significantly more frequent in the emergency than the elective resection group $(41.7 \%$ vs. $15.9 \%, p<0.001 ; 74.6 \%$ vs. $57.8 \%, p=0.014)$. The total number of harvested lymph nodes and the rate of patients with positive lymph nodes were similar in the two groups $(23.8 \pm 12.7$ vs. $22.8 \pm 13.0, p=0.479 ; 46.2 \%$ vs. $45.5 \%, p=$ 0.895). Positive lymph nodes and metastases were significantly associated with lower survival, whereas T4 tumors were associated with significantly higher local recurrence. 5-year survival [adjusted hazard ratio (aHR) 0.925 (95\%CI 0.567-1.511)] and local tumor recurrence [aHR 1.104 (95\%CI $0.317-3.840)$ ] were not significantly different in patients undergoing emergency and elective surgery for CRC.

Conclusion: In the current study, 5-year survival and local recurrence after surgery for CRC were determined by the tumor stage, but not by the emergency vs. elective surgical setting. The close collaboration of the emergency surgery and colorectal team in our institution may have contributed to this finding.

References: 1. An acute care surgery service expedites the treatment of emergency colorectal cancer: a retrospective case-control study. Anantha RV, Brackstone M, Parry N, Leslie K. World J Emerg Surg. 2014 Mar 21;9 (1):19. doi: 10.1186/1749-7922-9-19. 2. Risk factors for obstruction, perforation, or emergency admission at presentation in patients with colorectal cancer: a population-based study. Rabeneck L, Paszat LF, Li C. Am J Gastroenterol. 2006 May;101 (5):1098103. 3. Risk factors in patients presenting as an emergency with colorectal cancer. Scott NA1, Jeacock J, Kingston RD. Br J Surg. 1995 Mar;82 (3):321-3. 4. Elective versus emergency surgery for patients with colorectal cancer. Anderson JH1, Hole D, McArdle CS. Br J Surg. 1992 Jul;79 (7):706-9. 5. Early and late outcome after surgery for colorectal cancer: elective versus emergency surgery. 
Ascanelli S1, Navarra G, Tonini G, Feo C, Zerbinati A, Pozza E, Carcoforo P. Tumori. 2003 Jan-Feb;89 (1):36-41.

Disclosure: No significant relationships.

\section{PR021}

\section{PERCUTANEOUS CHOLECYSTOSTOMY: A SAFE OPTION IN THE TREATMENT OF ACUTE CHOLECYSTITIS IN ELDERY FRAIL PATIENTS}

\section{Seisdedos ${ }^{l}$, J. Morales Bernaldo De Quirós ${ }^{1}$, F. Turégano Fuentes ${ }^{2}$, J. De Tomás Palacios ${ }^{3}$}

${ }^{1}$ Cirugía General Y Del Aparato Digestivo, Hospital General Universitario Gregorio Marañon, Madrid/SPAIN, ${ }^{2}$ Cirugia General Y Del Ap. Digestivo, Hospital General Universitario Gregorio Marañón, Madrid/SPAIN, ${ }^{3}$ Surgery, Hospital Gregorio Marañón, Madrid/ SPAIN

Introduction: Urgent cholecystectomy for acute cholecystitis (AC) in elderly frail patients is fraught with potential serious morbidity and mortality. The aim of this study was to assess the outcome of percutaneous cholecystostomy (PC) as a less invasive option.

Material and methods: Retrospective review of patients aged $\geq 70$ with $\mathrm{AC}$ who underwent a PC at a University Hospital during a 6-year period (2009-2015). The Tokyo 2013 criteria (TG13) and the ASA classification were used to grade severity.

Results: A total of 64 patients that met the inclusion criteria required PC. The mean age was 81 (70-102). Patients were admitted to the ED after an average period of 3.3 days with symptoms at home. According to the TG13 criteria, 6 patients were diagnosed as mild (Grade I), 34 as moderate (Grade II), and 24 as severe cholecystitis (Grade III). According to the ASA classification, 15 patients were classified as ASA II, 27 as ASA III, and 22 as ASA IV. 76.5\% of Grade II patients were ASA III or IV. The mean hospital delay to PC was of 2.6 days, and the average length of hospital stay was of 21.7 days. PC was successful in $85.9 \%$ of patients. Only $35.9 \%$ underwent a scheduled cholecystectomy later on. Three patients required an urgent cholecystectomy due to failure of PC, and $6(9.4 \%)$ died after PC. $10.9 \%$ of patients were readmitted due to a new episode of AC. Conclusion: $\mathrm{PC}$ is an effective procedure in the treatment of $\mathrm{AC}$ in elderly frail patients, although the mortality and readmission rates are not negligible.

References:

Disclosure: No significant relationships.

\section{PR022}

\section{THE IMPORTANCE OF COMPUTER TOMOGRAPHY IN THE RIGHT ILIAC FOSSA SYNDROME IN ELDERLY PATIENTS}

\section{Beuran, B. Stoica, I. Tanase, I. Negoi, S. Paun}

General Surgery, Emergency Hospital of Bucharest, Bucharest/ ROMANIA

Introduction: Introduction: Appendicitis is often thought of as a disease of the young but it has now become a disease of the elderly because of increasing life expectancy of the modern day man resulting from improved medical attention. It is the most common cause of acute surgical condition of the abdomen.

Material and methods: Method: All patients admitted to the general surgery department in Bucharest Emergency Hospital between January 2014 and September 2017 were identified, yielding a total of 146 patients with right iliac fossa pain aged over 50 years old.

Results: Results: One hundred forty six patients were studied aged over 50 years old admitted for right iliac fossa pain in emergency setting. Dividing the cases by year we noticed that the number of the patients admitted were roughly equal, with a low incidence in 2014 (only 31 cases). Out of 146 cases, 98 patients (67\%) abdominal CT was performed before surgery or discharging. Ninety-two of 146 $(63 \%)$ patients underwent surgery. Half of this patients $(52.05 \%)$ were diagnosed with acute appendicitis in different histopathological varaints. Second was non specific abdominal pain in wich nothing was found in investigations, and the pain was disappeared after medical treatment. Colonic neoplasia was found only in 8 patients $(5.5 \%)$.

Conclusion: Conclusion: Clinicians should be aware that appendicitis remains an important differential diagnosis in elderly adults with abdominal pain or unspecific symptoms localized in right iliac fossa, and performing preoperative abdminal CT should help surgeon to define the surgery approach.

References: Gammeri E, Catton A, van Duren BH, Appleton SG, van Boxel GI. Towards an evidence-based management of right iliac fossa pain in the over 50-year-old patient. Annals of the Royal College of Surgeons of England. 2016;98 (7):496-9.

Disclosure: No significant relationships.

PR023

\section{FRAILTY IN OLDER PATIENTS UNDERGOING} EMERGENT ABDOMINAL SURGERY

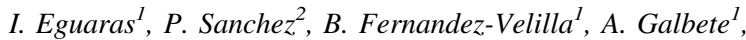 A. Goikoetxea $^{1}$, J. Herreral ${ }^{1}$, I. Otegi ${ }^{1}$}

${ }^{1}$ General Surgery, CH-navarra, Pamplona/SPAIN, ${ }^{2}$ General Surgery, chnavarra, pamplona/SPAIN

Introduction: As population ages, increasing numbers of older adults are undergoing surgery. Frailty has been described in many studies as a risk factor of postoperatories complications, major complications, and Hospital length of stay The objective of our study was to describe the prevalence of frailty in elder patient undergoing abdominal emergency surgery. The second objective was to correlate frailty with mortality.

Material and methods: We conducted a prospective observational study. All patients older than 65 admitted to a general surgery ward from the emergency department that underwent emergent surgery. We register frailty, using the FRAIL scale (fatigue, resistance, aerobic, illnesses, loss of weight). Functional evaluation was register using Barthel index. The outcome was mortality (30 days postoperatorie). Cross sectional observations were tested using the chisquare test. We performed a multivariante analyse to identify risk factor of mortality. Results: We studied 179 participants with an average of 78 years 7.18 (standar deviation), $81(45.3 \%)$ women. 75 patients were ASA IV (41.9\%). 80 patients were prefrail $(44 \%)$, and 33 were frail patients (18.4\%). 113 patients were totally independent (68.2), and 56 Partially dependent or totally dependent $(30.3 \%)$. The overall mortality rate of $13.5 \%$. The frail group didn't show higher mortality rates, compare with non-frail group (p: 0.157 OR: 0.42 ). Totally dependent patients did not have higher mortality in comparison with independent patients. 
Conclusion: Although frailty has been identified as a risk factor of mortality. In our database, frailty is not a risk factor of mortality.

References: Frailty in older patients undergoing emergent abdominal surgery Inés Eguaras Córdoba, Pablo Sánchez Acedo, Beatriz Fernandez-Velilla, Arkaitz Galbete Jimenez, Javier Herrera Cabezón, Ibai Otegi Altolaguirre, Andrea Goikoetxea Urdiáin, Janire Mateo Retuerta. Introduction As population ages, increasing numbers of older adults are undergoing surgery. Frailty has been described in many studies as a risk factor of postoperatories complications, major complications, and Hospital length of stay The objective of our study was to describe the prevalence of frailty in elder patient undergoing abdominal emergency surgery. The second objective was to correlate frailty with mortality. Methods: We conducted a prospective observational study. All patients older than 65 admitted to a general surgery ward from the emergency department that underwent emergent surgery. We register frailty, using the FRAIL scale (fatigue, resistance, aerobic, illnesses, loss of weight). Functional evaluation was register using Barthel index. The outcome was mortality (30 days postoperatorie). Cross sectional observations were tested using the chisquare test. We performed a multivariante analyse to identify risk factor of mortality. Results. We studied 179 participants with an average of 78 years 7.18 (standar deviation), 81 (45.3\%)women. 75 patients were ASA IV (41.9\%). 80 patients were prefrail $(44 \%)$, and 33 were frail patients $(18.4 \%) .113$ patients were totally independent (68.2), and 56 Partially dependent or totally dependent $(30.3 \%)$. The overall mortality rate of $13.5 \%$. The frail group didn't show higher mortality rates, compare with non-frail group (p: 0.157 OR: 0.42). Totally dependent patients did not have higher mortality in comparison with independent patients. Conclusion Although frailty has been identified as a risk factor of mortality. In our database, frailty is not a risk factor of mortality.

Disclosure: No significant relationships.

\section{PR024}

\section{EMERGENCY VASCULAR SURGERY PROCEDURES AFTER CATHETERIZATION FOR CADIAC INTERVENTIONS. 10 YEARS EXPERIENCE}

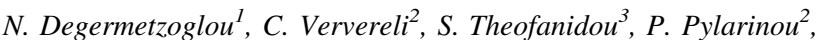 \\ C. Kotakos $^{2}$, I. Bountouris ${ }^{2}$
}

${ }^{1}$ Vascular Surgery Clinic, 251 HELLENIC AIR FORCE GENERAL HOSPITAL, ATHENS/GREECE, ${ }^{2}$ Cadiology Clinic, 251 HELLENIC AIR FORCE GENERAL HOSPITAL, ATHENS/ GREECE, ${ }^{3} 1 \mathrm{~b}$ Wing, AROGI REHABILITATION CENTRE, THESSALONIKI/GREECE

Introduction: Catheterization of radial and femoral artery, femoral and subclavian vein for cardiac interventions is nowadays a routine procedure in a role 3 hospital. Although most of the cases are uncomplicated as far as the vessels interruption is concerned, the consequences of a vessel complication maybe fatal for the patient, especially if it is not diagnosed on time. We describe our experience in emergency treatment of such cases the last 10 years.

Material and methods: During the last 10 years in our clinics of 97 emergency treated. The complications were established in the common or the bifurcation of the femoral artery (39), the common femoral vein (18), the radial artery (32) and the subclavian vein (8). Complications included haemorrhage (64), thrombosis (27) and arteriovenous fistula (6).

Results: Conservative treatment was indicated in 43 cases, while vascular operation needed in 39 . In 15 cases there was thrombin infusion under ultrasound guidance in haematoma with active haemorrhage of pseudo aneurysm of the femoral artery. Mean extra hospitalization time was $2,3( \pm 0,6)$ days for conservative treatment and 3,7 $( \pm 1,2)$ for vascular intervention. Analysis of the parameters measured and types of vascular intervention are described.

Conclusion: Cardiac catheterization is a routine practice in Role 3 Hospitals. Vascular complications in experienced centres are rare but one must be on the alert for the early, acute diagnosis of them and collaboration between intervention cardiologists and vascular surgeons is necessary in order to minimize the complication consequences, morbidity and mortality that are caused from them. In our study, both complication rates and types are in accordance to the international literature. However more effort is needed in order to minimize them in the optimum level.

References: 1. Lee JT, Bongard FS. Iliac vessel injuries. Surg Clin North Am 2002; 82 (1):21-48 2. Rich NM. Management of venous trauma. Surg Clin North Am 1988; 68 (4):809-821 3. Rowe VL, Salim A, Lipham J, et al. Shank vessel injuries. Surg Clin North Am 2002; 82 (1):91-104

Disclosure: No significant relationships.

\section{PR025}

\section{MULTICENTER STUDY OF INTESTINAL INTUSSUSCEPTION IN ADULTS}

\author{
J. Tinoco-González ${ }^{1}$, B. De-Soto-Cardenal ${ }^{2}$, I. Ramallo Solís ${ }^{3}$, \\ J.A. Lopez Ruiz ${ }^{2}$, L. Tallón-Aguilar ${ }^{1}$, B. Marenco De La Cuadra ${ }^{4}$, \\ V. Durán-Muñoz-Cruzado ${ }^{1}$, M. Sanchez Ramirez ${ }^{5}$, \\ A. Sánchez-Arteaga ${ }^{l}$, I. Alarcón-Del Agua ${ }^{1}$, M.J. Tamayo-Lopez ${ }^{1}$, \\ F. Pareja-Ciuró ${ }^{1}, F$. Oliva Mompean ${ }^{2}$, J. Padillo-Ruíz ${ }^{6}$
}

${ }^{1}$ Emergency Surgery Unit, Virgen del Rocío Universitary Hospital, Seville/SPAIN, ${ }^{2}$ General Surgery, University Hospital Virgen Macarena, Seville/SPAIN, ${ }^{3}$ Coloproctology Surgery Unit, Virgen del Rocío Universitary Hospital, Seville/SPAIN, ${ }^{4}$ Cirugía General, Hospital Virgen Macarena, Seville/SPAIN, ${ }^{5}$ Emergency Surgery Unit, University Hospital Virgen Macarena, Sevilla/SPAIN, ${ }^{6}$ Hepatobiliary And Transplantant Surgery Unit, Virgen del Rocío Universitary Hospital, Seville/SPAIN

Introduction: Intestinal intussusception (II) is a rare cause of abdominal pain in adults. It happens in less than $1 \%$ of cases of intestinal obstruction of small bowel. In adults the majority of cases are the result of an intestinal lesion that acts as an invaginating head. Material and methods: Observational, descriptive, cross-sectional and retrospective multicenter study ( 2 third-level hospitals) from Jan.09 to Sep.17. We collect demographic variables, location of intussusception, diagnostic test, surgical intervention, comorbidity and type of histology.

Results: Twenty-five patients with II (17 of Universitary Hospital Virgen Macarena and 8 of Universitary Hospital Virgen del Rocío) were included. The mean age was $45.9 \pm 20.2$ years, and $14(56 \%)$ were male. In $24(96 \%)$ the onset of the clinic was abdominal pain, however, only $9(36 \%)$ developed an obstructive. Diagnosis was obtained in $15(60 \%)$ cases by CT and $8(32 \%)$ by ultrasonography. The location was 16 ileo-ileal, 7 ileo-colic and 2 colo - colic, with 17 $(68 \%)$ urgents interventions, $4(16 \%)$ scheduled, and 4 with conservative treatment. There were 14 small bowel resections and 6 right hemicolectomies. Three lipomas and 3 benign polyps were the most frequent histological diagnoses, with 7 malignant cases of different types. 
Conclusion: Despite its infrequency, intestinal intussusception in adults is a pathology that must be included in the differential diagnosis of intestinal obstruction. It should be considered as a potentially serious pathology due to an important number of cases secondary to a malignant disease, so resection should be the procedure of choice if exist invaginating head.

References:

Disclosure: No significant relationships.

\section{PR026}

\section{SURGICAL MANAGEMENT IN ELDERLY PATIENTS; OUR EXPERIENCE}

B.L. López Durán ${ }^{1}$, J.A. López Ruíz ${ }^{2}$, B. De Soto Cardenal ${ }^{3}$, L.T. Aguilar, B.M. De La Cuadra , M.S. Ramírez, E.P. Margallo ${ }^{5}$, J. López Pérez ${ }^{6}, F$. Oliva Mompean ${ }^{6}$

${ }^{1}$ General Surgery, HOSPITAL UNIVERSITARIO VIRGEN MACARENA, SEVILLA/SPAIN, ${ }^{2}$ Cirugía General Y Del Aparato Digestivo, Hospital Universitario Virgen Macarena, Seville/SPAIN, ${ }^{3}$ General Surgery, HOSPITAL UNIVERSITARIO VIRGEN MACARENA, sevilla/SPAIN, ${ }^{4}$ General Surgery, H.U.V.Rocio, Sevilla/SPAIN, ${ }^{5}$ General Surgery, H.U.V.Macarena, Sevilla/SPAIN, ${ }^{6}$ Emergency Surgery Unit, Hospital Universitario Virgen Macarea, Seville/SPAIN

Introduction: Due to the increase of expectations regarding quantity and quality of life, it's now common for the surgeon to face difficult surgical decision regarding elderly patients. We aim to expose our experience in emergency surgery in patients above 80 years old.

Material and methods: Descriptive observational study. We collected cases in our center from June 2014 to June 2016.

Results: 144 cases were collected, with a mean age of 84.46 years, $45 \%$ of men and 55\% women. The most frequent pathology was acute cholecystitis $(20 \%)$, followed by complicated incisional hernias and primary hernias $(17 \%)$ and colorectal neoplasms complications. In Twenty-seven cases $(18.75 \%)$ a laparoscopic approach was performed, with a conversion rate of $11.11 \%$ ( 3 cases). Postoperative morbidity was mainly related to Surgical Site Infections (SSI) Incisional SSI in $19.4 \%$, with few cases of deep SSI (5.5\%), fistulas (4.16\%), bleeding $(2.7 \%)$ or evisceration $(2 \%)$. Twenty-two intestinal anastomoses were performed, with anastomotic leakage in 2 cases (9\%). Five patients of the total cases needed to be reoperated. The most relevant complications not related to surgery were respiratory in $7.6 \%$ patients, cardiological in $6.9 \%$ and catheter infection in $2 \%$. Mortality rate was $9.3 \%$ (14 patients), all of them underwent an open surgery.

Conclusion: It's not all about chronological age but the physiological reserve and physical state of the patient what must help us decide regarding the surgical treatment of the elderly patient. We can conclude that the risk of surgery is a minor problem regarding the need to offer a proper treatment according to the pathology.

References: Svensson O, Gustafson L, Gustafson Y, Olofsson N, Söderqvist A, Wallin G., Geriatric surgery discriminated. Health care badly prepared to meet old patients, Lakartidningen. 2009 Jun 24-Jul 7;106 (26-27):1722-5 Martín LG., Peculiarities of the surgery in the elderly. I. General considerations, An R Acad Nac Med (Madr). 2008;125 (2):277-309. Popa F, Mihai P., Geriatric surgery-present and perspective, J Med Life. 2008 Jul-Sep;1 (3):239-46. Cheng EY, Wang-Cheng RM., Impact of aging on preoperative evaluation, J Clin Anesth. 1991 Jul-Aug;3 (4):324-43. Martin WL., Surgery in aged patients, Hahnemann Hosp Tidings. 1946 Nov;51 (8):199-203.

Disclosure: No significant relationships.
PR027

\section{MANAGEMENT OF ADHESIVE SMALL-BOWEL OBSTRUCTION IN A HOSPITAL LEVEL III}

\author{
J.C. Gamero Huamán ${ }^{1}$, J.A. López Ruíz ${ }^{2}$, B. De Soto Cardenal ${ }^{3}$, \\ B. Marenco De La Cuadra ${ }^{4}$, M. Sanchez Ramirez, \\ L. Tallón-Aguilar ${ }^{6}$, E.P. Margallo ${ }^{2}$, J. López Pérez ${ }^{7}$, \\ F. Oliva Mompean ${ }^{7}$
}

${ }^{1}$ Sevilla, Hospital Univeristario Virgen Macarena, Sevilla/SPAIN, ${ }^{2}$ Cirugía General Y Del Aparato Digestivo, Hospital Universitario Virgen Macarena, Seville/SPAIN, ${ }^{3}$ General Surgery, HOSPITAL UNIVERSITARIO VIRGEN MACARENA, sevilla/SPAIN, ${ }^{4}$ Cirugía General, Hospital Virgen Macarena, Sevilla/SPAIN, ${ }^{5}$ Unidad De Cirugía De Urgencias, Hospital Universitario Virgen Macarena, Sevilla/SPAIN, ${ }^{6}$ Emergency Surgery Unit, Virgen del Rocío Universitary Hospital, Seville/SPAIN, ${ }^{7}$ Emergency Surgery Unit, Hospital Universitario Virgen Macarea, Seville/SPAIN

Introduction: Postoperative adhesions are a common cause of smallbowel obstruction and represent a common surgical emergency. Material and methods: Retrospective study that includes 75 patients described the hospital stay, demographic data, treatment, complications and mortality from June 2014 to December 2016.

Results: In total, 75 patients were included in this study, 41 were men and 34 were female. The mean age is 68.3 years and the median hospital stay was 2 days. Immediate surgical treatment was performed in 2 patients for signs of peritonitis. The remaining 73 patients were treated with conservative measures (absolute diet, NG Tube and Gastrografin). From this group, 18 patients failed with this treatment (2 due to lack of progression of the gastrografin, 10 due to clinical worsening and 6 for both reasons) and had emergency surgery. Finally, the conservative treatment was performed in 55 patients and surgical in 20 patients. 19 patients had a laparotomy approach and 1 patient had laparoscopy. Adhesiolysis was performed in 15 patients and intestinal resection in 5 , with 4 small bowel anastomosis and 1 ileostomy. The wound infection rate was $12 \%$, hemorrhage $1.3 \%$, evisceration $2.1 \%$ and reintervention $2.7 \%$. The total mortality was $9.3 \%$, in the conservative treatment of $6.6 \%$ and the surgical treatment $2.7 \%$.

Conclusion: In our hospital, we initially performed a conservative management for adhesive small-bowel obstructions with good results. However, a strict control of the evolution of the symptoms should be followed. In case of treatment failure or clinical worsening, the approach will change to surgical.

References: Lorentzen L, Oines $M$ et al. Recurrence after operative treatment of adhesive small-bowel obstruction. J. Gastrointest. Surg. 2017 Oct 13. Smolarek S, Shalaby M et al. Small-bowel obstruction secondary to adhesive after open or laparoscopic colorectal surgery. JSLS. 2016 Oct-Dec 20. Colonna AL, Byrge $\mathrm{N}$ et al. Noonoperative manamegement of adhesive small bowel obstruction: what is the break point?. Am. J. Surg. 2016 Dec:212 (6):1214-1221.

Disclosure: No significant relationships. 


\section{PR028}

\section{MANAGEMENT OF BOWEL OBSTRUCTION FOR COLORECTAL CANCER IN A LEVEL III HOSPITAL}

J.C. Gamero Huamán ${ }^{1}$, J.A. Lopez Ruiz ${ }^{2}$, B. Marenco De La Cuadra ${ }^{3}$, B. De Soto Cardenal ${ }^{4}$, M. Sanchez Ramirez ${ }^{5}$, E.P. Margallo, L. Tallón-Aguilar ${ }^{7}, J$. López Pérez ${ }^{8}, F$. Oliva Mompean ${ }^{8}$

${ }^{1}$ Sevilla, Hospital Univeristario Virgen Macarena, Sevilla/SPAIN, ${ }^{2}$ Cirugía De Urgencia, Hospital Universitario Virgen Macarena, Sevilla/SPAIN, ${ }^{3}$ Cirugía General, Hospital Virgen Macarena, Sevilla/ SPAIN, ${ }^{4}$ General Surgery, HOSPITAL UNIVERSITARIO VIRGEN MACARENA, sevilla/SPAIN, ${ }^{5}$ Unidad De Cirugía De Urgencias, Hospital Universitario Virgen Macarena, Sevilla/SPAIN, ${ }^{6}$ Cirugía General Y Del Aparato Digestivo, Hospital Universitario Virgen Macarena, Seville/SPAIN, ${ }^{7}$ Emergency Surgery Unit, Virgen del Rocío Universitary Hospital, Seville/SPAIN, ${ }^{8}$ Emergency Surgery Unit, Hospital Universitario Virgen Macarea, Seville/SPAIN

Introduction: Describe the results of management and complications of bowel obstruction for colorectal cancer.

Material and methods: Retrospective study that includes 37 patients described the hospital stay, demographic data, treatment, complications and mortality from June 2014 to December 2016.

Results: The analysis of 37 patients with a homogeneous gender distribution was 19 males and 18 females. The median duration of hospital stay was 7 days and the mean age was 66.6 years. 37 patients were operated on urgently and delayed urgently, 34 by laparotomy and 3 by laparoscopic of which 2 cases required conversion to an open surgery due to technical difficulty. Twenty-three bowel resections were performed with primary anastomosis (all cases of colon cancer), 2 hartmann ( 1 case of colon and 1 case of rectum), 2 ileostomies ( 1 case of colon and 1 case of rectum) and 10 colostomies (6 cases of colon and 4 cases of rectum). There was a $17 \%$ infection rate, $2.1 \%$ of evisceration ( 1 case of evisceration contained), $0 \%$ of reintervention and $2.1 \%$ of bleeding. Mortality was $8.1 \%$ secondary to to respiratory failure.

Conclusion: In our experience colorectal cancer represent one of the most frequent cause of bowel obstruction, with different surgical options to treat it. The surgical approach should be individualized and protocolized.

References: Bear C, Menon R et al. Emergency presentations of colorectal cancer. Surg. Clin. North. Am. 2017; 97: 529-545 Tsung-Ming $\mathrm{C}$, Yen-Ta $\mathrm{H}$ et al. Outcome of colon cancer initially presenting as colon perforation and obstruction. World J. Surg. Oncol. 2017; 15: 164.

Disclosure: No significant relationships.

\section{PR029}

\section{FOURNIER'S GANGRENE IN ELDERLY PATIENTS. MANAGEMENT BY GENERAL SURGEONS IN A RURAL HOSPITAL}

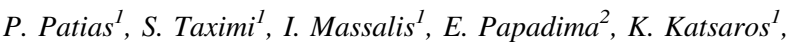 \\ G. Konstantoudakis ${ }^{1}$, G. Euthymiou ${ }^{1}$, P. Gkanas ${ }^{I}$
}

${ }^{1}$ Surgical Department, NAFPLION GENERAL HOSPITAL, NAFPLION/GREECE, ${ }^{2}$ Radiology Department, NAFPLION GENERAL HOSPITAL, NAFPLION/GREECE

Introduction: Fournier's gangrene (FG) is a gas-forming, rapidly evolving necrotizing fascitis of the perineum and the genital area.
Case series reported $20-40 \%$ mortality rates .The most frequent predisposing factors include diabetes mellitus, alcoholism and immunosuppression. With a growing elderly population with comorbidities, the frequency of severe cases of FG is rapidly increasing.

Material and methods: We retrospectively reviewed 10 patients diagnosed with FG in our General Surgery Department focused in elderly patients during the last 6 years. The clinical presentation, demographics, laboratory data and etiology were recorded. Collected data for elderly patients were further analyzed.

Results: In our series we treated a total of 10 patients with severe FG at our institution from 2011 to 2016 and 5 of them (50\%) were over 65 years old. All of the patients had diabetes mellitus. Comorbidities also were present in all of them. Overall mortality was $20 \%$ but the two deaths were among the elderly population (40\%). All patients were submitted to multiple extensive surgical debridements and received broad-sprectum antibiotics. Diverting colostomy was performed on two of them and the wide wounds of two patients were healed using improvised vacuum-assisted closure (VAC).

Conclusion: Early diagnosis and an aggressive surgical treatment is the cornerstone of therapy for FG, especially in elderly population.

References: 1. Hong KS et al, Int Wound J. 2017 "Prognostic factors and treatment outcomes for patients with Fournier's gangrene: a retrospective study." 2. Osbun N, et al, J Am Coll Surg. 2017 "LowVolume vs High-Volume Centers and Management of Fournier's Gangrene in Washington State."

Disclosure: No significant relationships.

PR030

\section{EMERGENCY ABDOMINAL SURGERY IN OCTOGENARIANS: THE NEED FOR A MULTIDIMENSIONAL DECISION MAKING}

$\frac{\text { J.L. Lavanchy }}{\text { B. Schnüriger }}{ }^{1}$, M. Holzgang ${ }^{2}$, T. Haltmeier ${ }^{1}$, D. Candinas ${ }^{2}$,

${ }^{1}$ Department Of Visceral Surgery And Medicine, Division Of Acute Care Surgery, Inselspital, Bern University Hospital, Bern/ SWITZERLAND, ${ }^{2}$ Department Of Visceral Surgery And Medicine, Inselspital, Bern University Hospital, Bern/SWITZERLAND

Introduction: Due to the aging population emergency abdominal surgery (EAS) has become more frequent in elderly patients. However, the benefit of EAS in these patients may be questionable. The aim of this study was, therefore, to assess outcomes of octogenarian patients undergoing EAS.

Material and methods: Single center, retrospective observational study. Patients $\geq 80$ years undergoing EAS 12/2011-12/2016 were included. Survivors and patients with early mortality (death within 7 days after surgery) were compared using univariable and multivariable regression analysis.

Results: Overall, 131 patients with a mean age of $84.9 \pm 3.6$ years and a mean Charlson-Comorbidity-Index of $2.65 \pm 1.8$ were included. EAS was performed for cholecystitis (26.7\%), ileus $(23.6 \%)$, hollow viscus perforation (16.8\%), diverticulitis $(11.5 \%)$, mesenteric ischemia $(10.7 \%)$, incarcerated hernia $(9.2 \%)$ and appendicitis (1.5\%). Mean hospital and ICU length of stay was $11.1 \pm 7.8$ and 1.5 \pm 2.8 days, respectively. Overall and early mortality was $17.6 \%(\mathrm{n}=$ $23)$ and $10.7 \%(n=14)$, respectively. The reasons for early mortality were multi-organ-failure $(n=7)$, postoperative withdrawal of care (n $=5)$, cardiac arrest $(n=1)$, and respiratory insufficiency $(n=1)$. In univariable analysis early mortality was associated with mesenteric 
ischemia $(3.7 \%$ vs. $42.9 \%, p<0.001)$ and ASA-Scores $>3$ (33.3\%vs.100\%, p < 0.001), whereas patients with cholecystitis (32.4\%vs.0\%, $\mathrm{p}=0.010)$ and admission from home $(20.8 \% \mathrm{vs} .5 .4 \%$, $p=0.017$ ) were more likely to survive. Multivariable analysis revealed mesenteric ischemia as an independent predictor for early mortality [OR 27.75 (CI95\% 2.92-263.47), p = 0.004].

Conclusion: In octogenarians undergoing EAS, withdrawal of care was the reason for early mortality in over a third of the patients. To prevent ethically questionable surgical interventions, future studies assessing a comprehensive and interdisciplinary approach in this patient population are warranted.

References:

Disclosure: No significant relationships.

\section{PR031}

\section{ACUTE DIVERTICULITIS: CAUSE OF HOSPITALIZATION IN MEDITERRANEAN POPULATIONS}

\author{
I. Lintzeris ${ }^{1}$, X. Agrogianni ${ }^{2}$
}

${ }^{1}$ Surgery, General Hospital Of Athens "Evaggelismos", Athens/ GREECE, ${ }^{2}$ Medical School, University of Athens, Athens/GREECE

Introduction: Acute diverticulitis represents a common disease among patients with advanced age. A low fiber diet, obesity, lack of exercise, smoking and immunosuppression are predisposing factors to this clinical situation. The current study attempts to feature the incidence of diverticulitis treated surgically compared to the one conservatively treated in a Mediterranean subgroup.

Material and methods: A retrospective, single-centered study was conducted. Medical records of patients hospitalized because of diverticulitis were thoroughly evaluated. Clinical course, complications, imaging and epidemiology data such as age, gender, origin and treatment applied, were registered.

Results: There was a registration of 93 patients being hospitalized because of diverticulitis in a six year period in the under study center. Surgery was performed to 15 patients, diagnosed with complicated diverticulitis $-15 \%$ - and 5 patients presenting with recurrent diverticulitis-5.37\%. The remaining 73 patients were treated conservatively under the prism of uncomplicated disease.

Conclusion: There is little known about the true incidence and epidemiology of mild and complicated diverticulitis in Mediterranean population groups where fiber based diet should easily be more feasible. Further epidemiological research would be beneficial and enlightening and therefore should be proceded.

References:

Disclosure: No significant relationships.

\section{PR032}

\section{CASE SERIES OF ESOPHAGEAL RUPTURE IN ELDERLY PATIENTS IN JAPAN}

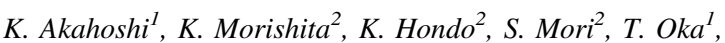 \\ J. Aiboshi ${ }^{I}$, M. Kaji ${ }^{1}$, Y. Otomo ${ }^{2}$
}

${ }^{1}$ Shock Trauma And Emergency Medical Center, Tokyo Medical and Dental University Hospital, Tokyo/JAPAN, ${ }^{2}$ Trauma And Acute

Critical Care Medical Center, Tokyo Medical and Dental University,
Medical Hospital, Tokyo/JAPAN

Introduction: Causes of esophageal rupture are mainly iatrogenic, idiopathic, and traumatic. Alcohol intake and successive vomiting is also a frequent cause. The mortality rate is still high accounting for $20 \%$. Japan is one of the world's most aging societies, and therefore, the number of esophageal rupture is expected to increase. It is important for physicians to know the rate of etiology, characteristics, and the appropriate treatment of choice to face this imminent problem.

Material and methods: For 11 years from November 2006 to November 2017, elderly people aged 65 years and older who were brought to our hospital by ambulance and diagnosed as esophageal rupture were included. We made a case series study based on their causes, symptoms, onset to surgery time, and the number of hospitalization days.

Results: There were 17 cases for 11 years. 4 cases (24\%) with "unknown cause of vomiting" were the most frequent case, followed by 3 cases $(18 \%)$ of esophageal ulcer and 3 cases (18\%) of accidental foreign body ingestion such as a denture. There were also 2 cases $(12 \%)$ caused by alcohol intake. With regard to the time of onset to surgery, cases caused by alcohol intake marked very short with mean 7.5 hours, whereas it took mean 20 hours for other causes. Duration of hospital stay of accidental ingestion of foreign body cases was shorter ( $\mathrm{n}=3$, mean 20 days) than that of other cases (31days) with significant differences. $(\mathrm{p}<0.01)$

Conclusion: Esophageal perforation in the elderly is more frequently caused by non-alcoholic etiologies, such as ulcers and accidental ingestion.

References:

Disclosure: No significant relationships.

\section{PR033}

\section{THE EFFECT OF THE OPERATION TYPE MADE ON SIGMOID COLON VOLVULUS (SCV)ON MORTALITY AND LENGTH OF HOSPITAL STAY (LOS)}

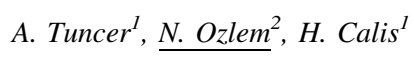

${ }^{1}$ Faculty Of Medicine, General Surgery, Ahi Evran University, Kırsehir/TURKEY, ${ }^{2}$ Faculty Of Medicine; General Surgery, Ahi Evran University, Kirsehir/TURKEY

Introduction: $5 \%, 50 \%$ respectively of all bowel obstruction in developed, in developing countries is SCV

Material and methods: 21 patients $(15 \mathrm{M} / 6 \mathrm{~F})$ with age;64.9 (2083)are emergently operated for SCV. intraoperatively blood supply of twisted bowel (BSTSC), operation type (hartman's (H), resection+ anastomosis $(\mathrm{R}+\mathrm{A})$ ), the effect of operation type on mortality and LOS in patients with SCV was recorded.BSTSC is insufficient in 9of21patients, 1of them is only died. Being insufficient BSTSC is no statically significant effect on mortality.15patients have $\mathrm{R}+\mathrm{H}, 6$ $\mathrm{R}+\mathrm{A}$. 6 patientswith $\mathrm{R}+\mathrm{H}$ was colostomy retrivied $(\mathrm{CR}) .2 \mathrm{R}+\mathrm{H}$ patients have no follow up, 3of are waiting to CR.LOS of with $\mathrm{R}+\mathrm{H}$ is 8.1 days, are 17.2 days for $\mathrm{R}+\mathrm{H}+\mathrm{CR} . \mathrm{LOS}$ forwithR+A is 9.5 days. Results: There is statistically significant difference between LOS with $\mathrm{R}+\mathrm{A}$, with $\mathrm{R}+\mathrm{H}(\mathrm{H}+$ retrivial $)(0.004=\mathrm{p})$ Operative time $(\mathrm{OT})$ for $(\mathrm{R}+\mathrm{H})$ is mean 114 mins. $\mathrm{R}+\mathrm{A}$ constructed in 132 mins. OT (colostomy opening + retrivial) of the patients with $\mathrm{H}+\mathrm{A}$ is 266 mins. All of 4 patientsundertaken $\mathrm{H}$ are died in p.o. All patientswith $\mathrm{R}$ $+\mathrm{A}$ are alive. The mortality rate in the patients with Hartmanns is $26.6 \%$.the operation type have no statistically significant effect on 
mortality $(\mathrm{P}=0.2)$. The bowel has necrosis is not associated related with dead risk according to some authors, us.

Conclusion: The effect of selected operation for operative therapy of SCV on mortality is not clear, there is only few data about it. $33 \%$ of the patients of Akcan et'l have $\mathrm{R}+\mathrm{H}, 67 \%$ of are with $\mathrm{R}+\mathrm{A}$.Ahmad et'l operate their patients in rate of $85 / 15 \%=\mathrm{R}+\mathrm{H} / \mathrm{R}+\mathrm{A}$.Oren et' 1 ; if stabil patient no tensile anastomosis; then first choice should be $\mathrm{R}+\mathrm{A}$.our first choice is $\mathrm{R}+\mathrm{A}$.the surgeonsinourclinic on due maychoose $\mathrm{R}+\mathrm{H}$; to take no anastomotic leakrisk.confront a patient with hemodynamic instability, could not achieve a tension-free anastomosis. $\mathrm{R}+\mathrm{A}$ rate of us increase. a $\mathrm{R}+\mathrm{H}$ reconstruction is longer than time for $\mathrm{R}+\mathrm{A}$ statistically significant.LOS is longer in patients with $\mathrm{R}+\mathrm{H}$.Our total mortality is $18 \%$, ocur in all patients with Hartmann. Literature rate is $5-14 \%$.Kassi et'l, we have the same results; dead patient are in age $>80$ of $3 / 4$.The surgery of SCV are debate to continue. ourconclusion; $\mathrm{R}+\mathrm{A}$ should be chosen. This work was supported by the Ahi Evran University Scientific Research Projects. Coordination Unit. Project Number: TIP E2.17.021.

References: 1. Clinical outcomes of sigmoid colon volvulus: identification of the factors associated with successful endoscopic detorsion Tomoya Iida, Suguru Nakagaki, Shuji Satoh, Haruo Shimizu, Hiroyuki Kaneto, Hiroshi Nakase2pISSN 1598-9100 • eISSN 2288-1956 https://doi.org/10.5217/ir.2017.15.2.215 Intest Res 2017;15 (2):215220 2. Evaluation of the Primary Anastomosis Side Effects in Patients with Sigmoid Volvulus in Imam Hossein and Firoozgar Hospitals in 2014-2015 Mahdi Alemrajabi, Mostafa Hosseini, Behzad Nemati Honar, Mahdi Kayyal, Adnan Tizmaghz, Amirmohsen Jalaeefar J Acute Dis 2017; 6 (3): 103-106 3. Minilaparotomy without general anesthesia for the treatment of sigmoid volvulus in high-risk patients: A case series of 4 patients Ernesto L. Enrique, Kareem A. Hamdy

Disclosure: No significant relationships.

\section{PR034}

\section{ELDERLY PATIENTS AND OTHER MORTALITY PREDICTIVE FACTORS IN OBSTRUCTIVE COLON CANCER}

\author{
J. Tinoco-González ${ }^{1}$, M. Rubio-Manzanares-Dorado ${ }^{1}$, \\ S. Martínez-Nuñez, V. Durán-Muñoz-Cruzado ${ }^{1}$, \\ A. Sánchez-Arteaga ${ }^{I}$, I. Alarcón-Del Agua ${ }^{1}$, M.J. Tamayo-Lopez ${ }^{1}$, \\ F. Pareja-Ciuróo ${ }^{1}$, L. Tallón-Aguilar ${ }^{1}$, J. Padillo-Ruíz ${ }^{2}$
}

${ }^{1}$ Emergency Surgery Unit, Virgen del Rocío Universitary Hospital, Seville/SPAIN, ${ }^{2}$ Hepatobiliary And Transplantant Surgery Unit, Virgen del Rocío Universitary Hospital, Seville/SPAIN

Introduction: Intestinal obstruction represents $20-30 \%$ of urgent admissions in surgery, being the most usual cause of acute abdomen in elderly people. This becomes especially relevant as ageing of western countries population constitutes a prime social attention focus.

Material and methods: An analytic, observational, transversal, retrospective study is performed in patients who underwent emergency surgery due to obstructive colon cancer (OCC) from Jan.09 to Dec.15. A multivariate analysis is accomplished with the statistically significant variables in the bivariant analysis $(\mathrm{p}<0.25)$, adding the clinical relevant ones and creating a mortality-predictive model for patients undergoing OCC emergency surgery.

Results: A total of 160 cases of OCC were collected, $94(58,8 \%)$ males, $41(25,6 \%)$ above 80 , and $86(53,8 \%)$ with Charlson index $\geq 5$. As mortality predictors age over 80 : OR $2,42(1,07-5,50) \mathrm{p}=0,032$, anastomosis leakage: OR 9,55 (2,93-31,09) $\mathrm{p}<0,001$, cardiologic complication: OR 20,52 (4,48-93,92) $\mathrm{p}<0,001$; and ICU admission: OR 4,83 (1,55-15,06) were identified, Harrell's c-index $0,84(0,76-$ $0,93)$. In addition, male gender: OR 4,46 $(1,54-12,92) \mathrm{p}=0,006$, Charlson index $\geq 5$ : OR 2,59 $(1,12-6,05) \mathrm{p}=0,024$; and stage IV 4,98 $(1,55-16,04) \mathrm{p}=0,007$ were attached to global death predictors, Harrell's c-index 0,87 (0,81-0,93).

Conclusion: Age over 80, comorbidity (Charlson index $\geq 5$ ), anastomosis leakage, cardiologic complications, ICU admission, stage IV and male gender are OCC mortality predictors.

\section{References:}

Disclosure: No significant relationships.

\section{PR035}

\section{URGENT ATTENDANCE OF ELDERY PATIENTS BY GENERAL SURGERY}

\author{
C. Alegre Torrado, M.C. Nevado, M. Gutierrez Andreu, \\ G. Supelano Eslait, O. García Villar, L. Brandariz Gil, \\ J. Bernal Tirapo, F. Cruz Vigo, P. Yuste García
}

General And Digestive Surgery, 12 DE OCTUBRE UNIVERSITY HOSPITAL, MADRID/SPAIN

Introduction: The progressive aging of the population and the improve in life expectancy is causing an increase in the percentage of elderly patients attended in the emergency department. It is difficult to establish the age limit from which patients are considered elderly, and frailty is a concept we have to keep in mind to treat this patients. Material and methods: A retrospective, descriptive study of urgently elderly patients treated by general surgery service of " 12 de Octubre" University Hospital during the last 2 years was performed, being considered eldery those patiens older than 75 years old.

Results: During 2 years, 4,276 elderly patients were treated by general surgery, $24 \%$ of all patients treated urgently. The mean age was $82.45 \pm 4.94$ years $(75-103), 51.1 \%$ males and $48.9 \%$ females. The most frequent source was the emergency department (59.5\%). Among the patients evaluated by other specialties, the most frequent were those from internal medicine $(37.82 \%)$. The most frequently diseases attended were: colorectal $(21.65 \%)$ and hepatobiliary (19.13\%) diseases and hernias (10\%). Thirty-two percent of patients attended in the emergency department were admitted and $11.7 \%$ were operated on urgently. Acute cholecystitis $(22.19 \%)$ and colorectal surgery $(17.95 \%)$ were the most common surgeries performed.

Conclusion: The progressive aging of the population is producing an important increase of the patients urgently attended and operated by general surgery. Due to the improvement in the quality of life of elderly patients, there is more controversy to establish the age limit to consider them as such, being frailty a very important concept to keep in mind.

\section{References:}

Disclosure: No significant relationships. 


\section{PR036}

\section{LAPAROSCOPIC APPROACH IN ADHESIVE SMALL BOWEL OBSTRUCTION: A MATTER OF TIME}

\author{
R. Menè, J. Guerrini, L. Viggiani, G. Costa, S. Mei, M. Ceolin, \\ H. Kurihara
}

Acute Care And Trauma Surgery Unit, Humanitas Research Hospital, Rozzano (MI)/ITALY

Introduction: Advantages of laparoscopic over open adhesiolysis are being extensively investigated. Aim of this study is to evaluate differences in outcome between laparoscopic and open approaches in patients presenting for adhesive Small Bowel Obstruction (aSBO).

Material and methods: We retrospectively identified patients who underwent adhesiolysis for aSBO from 2005 to 2017 from the internal registry of our center. Data on type of intervention, operative duration, length of hospital stay, bowel perforation and mortality were obtained. Univariate analysis (Chi-square and Wilcoxon test) and logrank test were performed to compare outcomes.

Results: 116 patients (54 females, mean age 68) were identified. Initial approach was laparoscopy in $54.3 \%$ and open in $45.7 \%$ of cases; conversion rate was $44.4 \%$. Interestingly, when analyzing the 27 surgeries performed by two highly specialized emergency surgeons, laparoscopic approach was more frequently preferred $(85.2 \%$ of cases; $\mathrm{P}<0.005$ ) and conversion rate decreased to $30.4 \%$. Comparing the laparoscopic, conversion and open groups, median operative times were 65,123 and 87 minutes while median length of hospital stay were 4, 6 and 8 days respectively, both resulting significantly shorter $(\mathrm{P}<0.001)$ in the laparoscopic group; log-rank test confirmed the shorter duration of hospital stay in this group. No difference in perforation or mortality rate was noted.

Conclusion: In our study, laparoscopic approach for aSBO is associated to shorter operative duration and hospital stay, although conversion poses the risk of longest operative times. Notably, surgeon expertise covers a crucial role in choosing the appropriate approach. References: Di Saverio S, Coccolini F, Galati M, Smerieri N, Biffl WL, Ansaloni L, Tugnoli G, Velmahos GC, Sartelli M, Bendinelli C, Fraga GP, Kelly MD, Moore FA, Mandal V, Mandal S, Masetti M, Jovine E, Pinna AD, Peitzman AB, Leppäniemi A, Sugarbaker PH, Goor HV, Moore EE, Jeekel J, Catena F.Bologna guidelines for diagnosis and management of adhesive small bowel obstruction (ASBO): 2013 update of the evidence-based guidelines from the world society of emergency surgery ASBO working group.World J Emerg Surg. 2013 Oct 10;8 (1):42. Sajid MS, Khawaja AH, Sains P, Singh KK, Baig MK. A systematic review comparing laparoscopic vs open adhesiolysis in patients with adhesional small bowel obstruction. Am J Surg. 2016 Jul;212 (1):138-50.

Disclosure: No significant relationships.

\section{PR037}

\section{ENDOLOOP VERSUS STAPLER: RESULTS OF A PROPENSITY SCORE ANALYSIS}

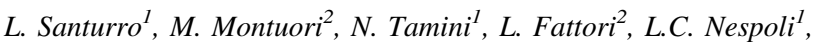 \\ L.V. Gianot $t i^{I}$
}

${ }^{1}$ Department Of Surgery, University of Milano Bicocca - San Gerardo Hospital, Monza/ITALY, ${ }^{2}$ Department Of Surgery, University of Milano-Bicocca - San Gerardo Hospital, Monza/ITALY
Introduction: In laparoscopic appendectomy, closure of the appendix stump is important to avoid severe complications. The aim of this study was to compare two main technique (endoloop versus stapler) in terms of operating time, complication rates and hospital stay.

Material and methods: We analyzed all patients (450) undergoing appendectomy for suspected appendicitis in University of MilanoBicocca - San Gerardo Hospital, between September 2013 and October 2016. We excluded open and converted appendectomy. The technique used for appendiceal stump closure was chosen based on surgeon's personal preference.

Results: Three hundred and five patients (males 58,9\%; females $47.7 \%)$ were included in this study. The data were divided in stapler $(36.7 \%)$ and endoloop (63.3\%) groups. Stapler was most used in older patients $(\mathrm{p}=0.001)$, in high BMI $(\mathrm{p}=0.001)$ and in gangrenous appendicitis $(\mathrm{p}=0.001)$. Instead, complication rates were comparable in loop-treated and stapler-treated $(6.7 \%$ vs $11.6 \%)$. Propensity score analysis was performed on the bases of age, BMI, CRP and severity of appendicitis to eliminate differences in the groups. The results was two groups of 108 patients omogeneus. The Chi-square test showed no differences in complications rates $(\mathrm{p}=0.368)$ or in Clavien-Dindo classification $(\mathrm{p}=0.191)$. However, differences was found in operative time $(\mathrm{p}=0.002)$ and in hospital stay $(\mathrm{p}=0.043)$.

Conclusion: Our study showed no differences in terms of complication rates. Differences in operative time after propensity score analysis is difficult to explain, in consideration that the propensity score analisys eliminate the bias of severity of appendicitis. Differences in hospital stay, instead, is probably due to a type I error.

References: 1. Beldi G, Vorburger SA, Bruegger LE, Kocher T, Inderbitzin D, Candinas D. Analysis of stapling versus endoloops in appendiceal stump closure. Br J Surg. 2006;93 (11):1390-1393. 2. Sahm M, Kube R, Schmidt S, Ritter C, Pross M, Lippert H. Current analysis of endoloops in appendiceal stump closure. Surg Endosc. 2011;25 (1):124-129. 3. Safavi A, Langer M, Skarsgard ED. Endoloop versus endostapler closure of the appendiceal stump in pediatric laparoscopic appendectomy. Can J Surg. 2012;55 (1):37-40. 4. Kazemier G, in't Hof KH, Saad S, Bonjer HJ, Sauerland S. Securing the appendiceal stump in laparoscopic appendectomy: evidence for routine stapling? Surg Endosc. 2006;20 (9):1473-1476.

Disclosure: No significant relationships.

\section{PR038}

\section{PERFORATED PEPTIC ULCER, ADVANCED AGE AND DIFFUSE PERITONITIS - IS LAPAROSCOPY SAFE?}

\author{
A. Sazhin, G. Ivakhov, V. Petukhov, E. Stradymov
}

Faculty Surgery \#1, Russian State Medical Research University, Moscow/RUSSIAN FEDERATION

Introduction: Laparoscopy surgery for perforated peptic ulcer (PPU) becomes the procedure of choice for cases of local peritonitis and non-comorbidities patients. The aim of our study is to assess the safety and feasibility of laparoscopic surgery for elderly patients with PPU and diffuse peritonitis and to analyze surgical prognosis in patients older than 60 years who were operated for perforated peptic ulcer.

Material and methods: A total of 127 patients after laparoscopic suturing of PPU were included in this study. In $114(89,8 \%)$ cases patients suffered from diffuse peritonitis. $23(20,2 \%)$ patients over 60 years with PPU and diffuse peritonitis were operated laparoscopically. In all cases we performed simple closure of PPU. Boey 0,1 and 
2 was confirmed for $7(30,5 \%), 13(56,5 \%)$ and $3(13 \%)$ patients respectively.

Results: All patients with Boey 0 have mortality rate $0 \%$. Two patients with Boey 1 died (mortality $-15,4 \%$ ). Mortality rate in group with Boey 2 was $66,7 \%$ (2/3 patients). Postoperative mortality rate was $3.1 \%$ in the whole series but $17.4 \%$ (4 patients) among the elderly. Early complications occurred in $8(34,8 \%)$ patients of laparoscopic group. Complications were divided according to Clavien-Dindo classification. 4 patients $(50 \%)$ had major complication: Clavien-Dindo grade $3 \mathrm{~b}$ (75 \%) and grade $4(25 \%)$. A suture leak rate in this group was $8,7 \%$ and $3,5 \%$ in the whole series.

Conclusion: Laparoscopic repair of PPU may be operation of choice for elderly (greater than 60 years) patients with Boey 0 and diffuse peritonitis.

References:

Disclosure: No significant relationships.

\section{PR039}

\section{RECURRENT INTUSSUSCEPTION IN CHILDREN}

D.E. Golikov ${ }^{1}$, O. Karaseva ${ }^{2}$, A.L. Gorelik ${ }^{3}$, A.V. Timofeeva ${ }^{4}$,

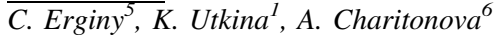

${ }^{1}$ Intensive Care And Polytrauma Unit, Clinical and Research Institute of Emergency Pediatric Surgery and Trauma, Moscow/RUSSIAN FEDERATION, ${ }^{2}$ Polytrauma, Clinical and Research Institute of Emergency Pediatric Surgery and Trauma, Moscow/RUSSIAN FEDERATION, ${ }^{3}$ Department Of Polytrauma, Clinical and Research Institute of Emergency Pediatric Surgery and Trauma (CRIEPST), Moscow/RUSSIAN FEDERATION, ${ }^{4}$ Department Of Polytrauma, Clinical and Research Institute of Emergency Pediatric Surgery and Trauma (CRIEPST) Department of Health of Moscow, Moscow/ RUSSIAN FEDERATION, ${ }^{5}$ Radiology, Clinical and Research Institute of Emergency Pediatric Surgery and Trauma, Moscow/ RUSSIAN FEDERATION, ${ }^{6}$ Endoscopia, Clinical and Research Institute of Emergency Pediatric Surgery and Trauma, Moscow/ RUSSIAN FEDERATION

Introduction: There is no single point of view on the management of children with recurrent intussusception. The aim of this study is optimize the protocol for the diagnosis and treatment of intussusception in children.

Material and methods: The study included 13 children (boys $61.5 \%$, girls $-38.5 \%$ ) with recurrent ileocecal intussusception. All children were older than 1 year, the average age of children was $2.9 \pm$ 2.02 years., Ultrasound-guided hydrostatic enema was used as a method of conservative treatment.

Results: Recurrent intussusception were diagnosed in $11.4 \%$ of the total number of observations. In $92.3 \%$ of cases recurrent intussusception occurred in the early period (up to 72 hours), in $7.7 \%$ - 1 month after conservative treatment. Laparoscopy was performed, with the exception of 2 children, who were repeatedly performed with ultrasound-guided hydrostatic enema. At 3 children there was a second recurrent and at 2 of them - after a laparoscopic reduction of intussusception. The anatomical cause was established in $30,7 \%$ (4) observations: at laparoscopy - in 3 cases (Meckel's diverticulum -1, Burkitt's lymphoma-1, juvenile polyps of the caecum-1), at colonoscopy - in 1 (Burkitt's lymphoma) after laparoscopic reduction of intussusception.

Conclusion: Conservative treatment is contraindicated in recurrent intussusception. Laparoscopy and colonoscopy used step-by-step allows timely diagnosis of the cause of the disease and determine the volume of surgical treatment.

References:

Disclosure: No significant relationships.

\section{PR040}

\section{THREE-PORT VERSUS STANDARD FOUR-PORT LAPAROSCOPIC CHOLECYSTECTOMY: A PROSPECTIVE COHORT STUDY}

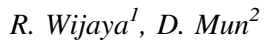

${ }^{1}$ General Surgery, Changi General Hospital, Singapore/SINGAPORE,

${ }^{2}$ Yong Loo Lin School Of Medicine, National University of Singapore, Singapore/SINGAPORE

Introduction: Laparoscopic cholecystectomy is conventionally done using four ports. In recent years, some surgeons have explored the safety and reducibility of reduced port techniques and its advantages are still being debated. This study aims to prospectively compare the clinical outcomes of three-port versus four-port laparoscopic cholecystectomy. Material and methods: Between 2014 and 2016, 75 consecutive patients prospectively underwent laparoscopic cholecystectomy with either the three-port or four-port technique. The primary outcome measure was length of post-operative hospital stay and secondary outcome measures compared included operative time and amount of analgesia. Other outcome data collected included conversion to open surgery as well as morbidity. Statistical analysis was performed with SPSS statistical software, version 19.0 using appropriate methods.

Results: Demographics were similar for both groups (age, sex and comparison of elective versus emergency surgery). Patients in the three-port group had shorter median hospital stay as compared to the four-port group (1.0 vs $2.0, \mathrm{p}=0.001)$. There was also shorter mean operative time $(113.1 \pm 45.9$ vs $143.4 \pm 52.6, \mathrm{p}=0.011)$ as well as amount of paracetamol used on Day 1 was lower in the three-port group ( 1.0 vs $2.0, \mathrm{p}=0.022)$. There were no intra-operative complications in both groups and no statistical difference in conversion to open surgery.

Conclusion: In our study, the three-port laparoscopic cholecystectomy had shorter hospital stay and operative time as compared to the traditional 4-port technique. Thus, it can be recommended as a safe alternative to the standard four-port technique with a potential additional advantage of requiring less analgesia.

\section{References:}

Disclosure: No significant relationships.

\section{PR041}

\section{REDUCED-PORT LAPAROSCOPIC EMERGENCY SURGERY: ONGOING EXPERIENCE}

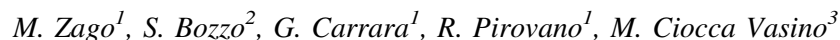

${ }^{1}$ Minimally Invasive Surgery Unit - General Surgery Dept, Policlinico San Pietro, Bergamo/ITALY, ${ }^{2}$ Chirurgia Generale, POLICLINICO SAN PIETRO, Ponte San Pietro/ITALY, ${ }^{3}$ Chirurgia Generale, POLICLINICO SAN PIETRO, Ponte san pietro/ITALY

Introduction: Reduced-port laparoscopy and single-port laparoscopic surgery (SILS) have been successfully employed in many acute 
abdominal disorders. Compared to SILS, mini-laparoscopy requires a minimal learning curve, is cheaper and reproducible by all staff surgeons.

Material and methods: Out of a series of 59 mini-laparoscopic operations, 28 patients (M 14, F 14 - mean age 52 yr [range 11-88], $\mathrm{BMI}<28)$ prospectively underwent mini-laparoscopic emergency operation in a tertiary community hospital with the following indications: 8 cholecystitis (6 uncomplicated, 2 gangrenous), 17 appendicitis ( 9 uncomplicated, 3 complicated, 4 diffuse peritonitis, 1 localized peritonitis), 3 small bowel obstructions. Standard trocars position was employed, with $3.5 \mathrm{~mm}$ operating ports, mini-laparoscopic instruments $\left(\mathrm{ab}-\mathrm{medica}^{\circledR}\right)$ and a conventional first trocar (Hasson or optic trocar), used for specimen extraction.

Results: No conversions to open or conventional laparoscopy were needed. In 10 cases (3 appendectomies, 6 cholecystectomies, 1 adhesiolysis) one mini-trocar was replaced by a $5 \mathrm{~mm}$ trocar (hybrid mini-laparoscopy) for technical reasons (clipping of hilar structures, unavailability of $3 \mathrm{~mm}$ bipolar forceps, inadequate lighting with the $3 \mathrm{~mm}$ camera). Five out of 9 staff surgeons (55\%) performed at least one mini-laparoscopic operation. Intraoperative cholangiography was performed in 2 cases. No statistical difference on operative time and length of stay (LOS) was found between mini-laparoscopic operations and conventional emergency laparoscopies in the same surgical team. No major complication occurred.

Conclusion: Mini-laparoscopic emergency surgery is a safe approach in acute abdomen even in complicated cases. It could be applied without specific training or increased costs in surgical staffs with standard laparoscopic skills.

References:

Disclosure: No significant relationships.

\section{PR042}

\section{ESOPHAGEAL PERFORATIONS AT A REFERRAL MEDICAL FACILITY: A 17-YEARS PERSPECTIVE}

\section{S. Saar, A. Reinsoo, P. Talving, T. Vanakesa, P. Saluvere, I. Almre}

Department Of Surgery, North Estonia Medical Centre, Tallinn/ ESTONIA

Introduction: Esophageal perforation is a relatively rare entity resulting in delayed management and poor outcomes. Nevertheless, contemporary studies on esophageal perforations are scarce. Thus, we set out to review esophageal perforations in a 17 -year study period at a referral medical facility in Estonia.

Material and methods: After the ethics review board approval, all consecutive patients with esophageal perforation admitted to the North Estonia Medical Centre between 1/2000 and 12/2016 were retrospectively reviewed. Data collection included demographics, etiology, management, complications per Clavien-Dindo (CD) classification, hospital length of stay (HLOS), intensive care unit (ICU)LOS, and mortality. Primary outcome was in-hospital mortality. Also, outcomes comparison between early (2000-2008) and late study period (2009-2016) was performed.

Results: A total of 52 cases were included during the study period. Mean age was $58.7 \pm 16.3$ years and $67.3 \%$ were male. Etiology of perforations was Boerhaave syndrome, iatrogenic, foreign body, and other/unknown perforations in $38.5 \%, 30.8 \%, 13.5 \%$, and $17.3 \%$ of patients, respectively. Esophagectomy was performed in $35 \%$ and $6.3 \%$ of patients during early and late study period, respectively. Overall, $36 \%$ of patients had complications per CD. Mean HLOS and ICU-LOS was $30.5 \pm 31.2$ and $15.4 \pm 21.2$ days, respectively.
Overall in-hospital mortality was $21.2 \%$. Significantly improved primary outcome was observed during the late study period $(40.0 \%$ vs. $9.4 \%, \mathrm{p}<0.05)$.

Conclusion: Esophageal perforation is a highly lethal condition with a total of $21.2 \%$ mortality in our investigation. Advancements in surgical and endoscopic management resulting in esophageal preservation and improved survival were noted during the study period.

References:

Disclosure: No significant relationships.

\section{PR043}

\section{EARLY DISCHARGE AFTER URGENT APPENDECTOMY: LESS THAN 24 HOURS OF POSTOPERATIVE STAY, IS THIS A SAFE PROCEDURE?}

\section{B. Bascuas Rodrigo ${ }^{I}$, J.A. Lopez Ruiz ${ }^{2}$, B.M. De La Cuadra ${ }^{I}$, L.T. Aguilar ${ }^{I}$, M. Sanchez Ramirez ${ }^{1}$, Y. Lara Fernández, ${ }^{1}$, J. Lopez Perez ${ }^{I}$ E. Perez Margallo ${ }^{I}, F$. Oliva Mompean ${ }^{I}$}

${ }^{1}$ Unidad De Cirugía De Urgencias, Hospital Universitario Virgen Macarena, Sevilla/SPAIN, ${ }^{2}$ Cirugía De Urgencia, Hospital Universitario Virgen Macarena, Sevilla/SPAIN

Introduction: Acute appendicitis is the most common cause of acute abdomen requiring surgical intervention in the world. Nowadays, standard treatment of acute appendicitis involves a surgical approach, either laparoscopic or open. The purpose of the present study is to evaluate the safety of a discharge within less than 24 hours after performing appendectomy as a result of an uncomplicated acute appendicitis.

Material and methods: We retrospectively reviewed our database of patients who underwent surgery for acute uncomplicated appendicitis at our institution between January 2015 and January $2017(\mathrm{n}=261)$. Patients have been divided into 2 groups according to the length of postoperative stay (Group $1 \leq 24$ hours, Group $2>24$ hours). The following data concerning patient and surgery were recorded: age, gender, operative time, surgical approach (open or laparoscopic), rate of post-operative complications, reoperation and readmissions within 30 days after surgery.

Results: No significant differences were observed in age, gender, operative time, surgical approach, post-operative complications (intraabdominal abscess, surgical wound infection, bleeding, paralytic ileus and evisceration), reoperation or readmission rate between Group 1 and 2 ( $\mathrm{p}>0.05$ ). Nevertheless, the difference in post-operative stay between the two groups was statistically significant $(\mathrm{p}<$ $0.05)$.

Conclusion: Patients who undergo appendectomy for acute uncomplicated appendicitis, without surgical incidents and an adequate social-family network, can be discharged in less than 24 hours without a higher risk of post-operative complications or readmissions than patients with longer postoperative stays. Therefore, it will be necessary to conduct more prospective studies with higher level of evidence in order to corroborate our results.

References:

Disclosure: No significant relationships. 
PR044

\section{FOREIGN BODIES INGESTION}

B.L. López Durán ${ }^{1}$, J.A. Lopez Ruiz ${ }^{2}$, A. Suárez Cabrera', B.M. De La Cuadra ${ }^{3}$, L. Tallón-Aguilar ${ }^{4}$, M.S. Ramírez, E.P. Margallo ${ }^{5}$, J. López Pérez ${ }^{6}, F$. Oliva Mompean ${ }^{6}$

${ }^{1}$ General Surgery, HOSPITAL UNIVERSITARIO VIRGEN MACARENA, SEVILLA/SPAIN, ${ }^{2}$ Cirugía De Urgencia, Hospital Universitario Virgen Macarena, Sevilla/SPAIN, ${ }^{3}$ General Surgery, H.U.V.Macarena, Sevilla/SPAIN, ${ }^{4}$ Emergency Surgery Unit, Virgen del Rocío Universitary Hospital, Seville/SPAIN, ${ }^{5}$ Cirugía General Y Del Aparato Digestivo, Hospital Universitario Virgen Macarena, Seville/SPAIN, ${ }^{6}$ Emergency Surgery Unit, Hospital Universitario Virgen Macarea, Seville/SPAIN

Introduction: While accidental ingestion of strange bodies is mostly related to feeding, voluntary intake is usually due to psychiatric disorders or attempted autolysis. We aim to expose our experience in the management of the ingestion of foreign bodies.

Material and methods: Descriptive study of 132 cases collected between January 2012 and April 2016 in our center.

Results: The average number of cases was 31 per year. $17.7 \%$ had an intestinal obstruction at admission, requiring surgery for resolution in $78.3 \%$ of them. $12.9 \%$ (17 cases) had a perforation. 16 of them $(94.1 \%)$ required surgery. The remaining cases were solved by colonoscopy. Of the 16 surgical cases, in $8(50.0 \%)$ of them the object was extracted and a simple suture was made, in 4 cases $(25.0 \%)$ an enterotomy was performed, in 3 cases $(18.7 \%)$ an intestinal resection with anastomosis, and in one case $(6.3 \%)$ a Hartmann procedure was done Overall, $54.2 \%$ were solved by endoscopic approach, $31.3 \%$ by surgery and $14.5 \%$ by spontaneous resolution. The morbidity was $26.5 \%$, more frequent in the surgical treatment (46.3 vs $21.1 \%$ endoscopic). There was no mortality directly related to the cases.

Conclusion: A conservative management may be used as a first option of treatment in some cases, but sometimes, a surgical approach is mandatory specially in cases when a perforation is settled, for in our experience perforation is related to a higher rate of morbidity and mortality. A tailored approach for each case is advised, and a minimally invasive technique may be a feasible and safe procedure

References: Iwamuro M, Okada H, Matsueda K, Inaba T, Kusumoto $\mathrm{C}$, Imagawa A, Yamamoto $\mathrm{K}$, Review of the diagnosis and management of gastrointestinal bezoars, World J Gastrointest Endosc. 2015 Apr 16;7 (4):336-45. Orting M, Lundbech PE, Bjerregaard NC, Jensen LS, Presentation, diagnosis and management of swallowed foreign bodies, Ugeskr Laeger. 2014 Jan 20;176 (2):157-62. Mairose U, Intentional ingestion, Dtsch Arztebl Int. 2013 Apr;110 (17):297. Ambe P, Weber SA, Schauer M, Knoefel WT, Swallowed foreign bodies in adults, Dtsch Arztebl Int. 2012 Dec;109 (50):869-75. Jayachandra S, Eslick GD, A systematic review of paediatric foreign body ingestion: presentation, complications, and management, Int $\mathbf{J}$ Pediatr Otorhinolaryngol. 2013 Mar;77 (3):311-7. Antoniou D, Christopoulos-Geroulanos G, Management of foreign body ingestion and food bolus impaction in children: a retrospective analysis of 675 cases, Turk J Pediatr. 2011 Jul-Aug;53 (4):381-7. Jiménez-Rodríguez RM, Flores-Cortés M, Méndez C, Valera-Sánchez Z, López-Bernal F, Pareja-Ciuro F, Perforation of the rectosigmoideal junction by ingestión of foreign bodies, Rev Esp Enferm Dig. 2009 Dec;101 (12):886-8. Syrakos T, Zacharakis E, Antonitsis P, Zacharakis E, Spanos C, Georgantis G, Kiskinis D, Surgical intervention for gastrointestinal foreign bodies in adults: a case series, Med Princ Pract. 2008;17 (4):276-9. Lee J, Bezoars and foreign bodies of the stomach, Gastrointest Endosc Clin N Am. 1996 Jul;6 (3):605-19.

Disclosure: No significant relationships.
PR045

\section{EMERGENCY TREATMENT OF AORTOENTERIC FISTULAE AFTER ABDOMINAL ANEURYSM REPAIR. 15 YEARS EXPERIENCE}

N. Degermetzoglou ${ }^{1}$, K. Stavrides ${ }^{2}$, S. Theofanidou ${ }^{3}$, K. Tigkiropoulos ${ }^{2}$, N. Zacharopoulos ${ }^{2}$, M. Mantelas ${ }^{2}$, D. Karamanos ${ }^{2}$, I. Lazaridis $^{2}$, G. Tsoulfas ${ }^{2}$, I. Bountouris ${ }^{1}$, N. Saratzis $^{2}$

${ }^{1}$ Vascular Surgery Clinic, 251 HELLENIC AIR FORCE GENERAL HOSPITAL, ATHENS/GREECE, ${ }^{2}$ Vascular Surgery Department, AUTH 1st SURGERY CLINIC, PAPAGEORGIOU GENERAL HOSPITAL, THESSALONIKI/GREECE, ${ }^{3} 1 \mathrm{~b}$ Wing, AROGI REHABILITATION CENTRE, THESSALONIKI/GREECE

Introduction: Aortoenteric Fistulae (AEF) is a direct fistula between abdominal aorta and intestine, usually duodenum. Mass hematemesis and hematochesia with hemodynamic instability is the most common and dramatic clinical condition. Thus, it is of bad prognosis and high mortality and morbidity rates. AEF after Abdominal Aortic Aneurysm Repair (AAA) is a rare, but known delayed complication, especially after open surgery. We describe our experience in emergency treatment of such cases the last 15 years.

Material and methods: During the last 15 years in our clinics 12 cases of AEF after AAA repair were emergency treated, 6 with open repair and the rest 6 with an endovascular urgent procedure before final repair. In open surgery, ligation of the aorta, debridement of surrounding tissues, use of the omentum for covering the duodenum fistula and an auxillo-femoral bypass was used, while in the endovascular procedure a cuff was used for temporal sealing of the fistula.

Results: From the 12 patients, $11(91,6 \%)$ survived the operational procedure. $5(41,6 \%)$ were discharged from the hospital after a mean hospitalization of $18,3( \pm 6,8)$ days, with a mean life expectance of 2,3 years $( \pm 1,2)$. Only $1(8,4 \%)$ patient is still alive, while the other 4 that were discharged from hospital died from causes not relevant with the disease. From the patients that died during hospitalization, 4 $(66,6 \%)$ died of Multi Organ Failure (MOF), 1 (16,7\%) from Acute Respiratory Disorder Syndrome (ARDS) and the last one from sepsis $(16,7 \%)$, with a mean time of life 2,6 days $( \pm 1,7)$.

Conclusion: The management and handling of mass casualties bleeding during AEFs after AAA repair is vital for the prognosis of these cases in accordance to the repair technique. Prognosis is very heavy, with a high morbidity and mortality rate, due to the dramatic clinical condition and the hemodynamic instability that usually these patients suffer. It seems that endovascular emergency procedures have a better impact in prognosis that primary open repair, but a numerous number of cases is needed in order to justify that.

References: S Gnus J, Ferenc S, Kościelna M et al. Secondary Aortoenteric Fistula After Abdominal Aortic Graft Implementation in Our Own Material. Adv Clin Exp Med. 2016 Nov-Dec;25 (6):12651271. Heinola I, Kantonen I, Jaroma M et al. Treatment of Aortic Prosthesis Infections by Graft Removal and In Situ Replacement with Autologous Femoral Veins and Fascial Strengthening. Eur J Vasc Endovasc Surg. 2016 Feb;51 (2):232-9. Silingardi R, Coppi G, Saitta $\mathrm{G}$ et al.Use of Aortic Occluders and Endovascular Plugs in a 2-step Hybrid Treatment of Secondary Aortoenteric Fistulas. Ann Vasc Surg. 2016 Jan;30:310.e9-15

Disclosure: No significant relationships. 


\section{PR046}

\section{GENERAL SURGERY SERVICE EMERGENCY ACTIVITY REGISTRY IN A LEVEL III HOSPITAL}

\author{
C. Alegre Torrado, M.C. Nevado, M. Gutierrez Andreu, \\ G. Supelano Eslait, O. García Villar, L. Brandariz Gil, \\ A. Moreno Bargueiras, E. Ferrero, P. Yuste García
}

\section{General And Digestive Surgery, 12 DE OCTUBRE UNIVERSITY HOSPITAL, MADRID/SPAIN}

Introduction: Emergency services in level III hospitals present a high volume of patients, many of them of high complexity. The general surgery department of these centers attend a large number of these patients with different degrees of complexity, presenting a high rate of admittances and emergency surgeries.

Material and methods: A retrospective, descriptive study of urgently treated patients by general surgery service of " 12 de Octubre" University Hospital was performed during the last 2 years.

Results: During 2 years 17,819 patients were treated, $57.1 \%$ male, $42.9 \%$ female, with a mean age of $58.47 \pm 19.57$ years (11-103), $40.8 \%$ over 65 years old. The most frequent source of attended patients was the emergency department (58.2\%). Among the patients evaluated by other specialties, the most frequent were those from internal medicine $(18.1 \%)$. The most frequent diseases attended were: anorectal $(18.5 \%)$, colorectal $(15.5 \%)$ and hepatobiliary $(13.8 \%)$ diseases. Twenty-six percent of the patients attended in the emergency department were admitted, being hepatobiliary pathology $(30.4 \%)$, appendicitis $(23 \%)$ and diverticulitis $(10.6 \%)$ the most common causes. Of all the patients evaluated in the emergency room, $17.5 \%$ were operated, with a mean age of $51.36 \pm 21.09$ years (14-97) .The most frequent surgeries were anorectal surgeries $(29.12 \%)$, appendectomy $(23.6 \%)$, cholecystectomy $(10.91 \%)$, and colorectal surgery $(10.19 \%)$.

Conclusion: General surgery attend a high volume of patients in emergency service. Twenty-six percent of patients seen in the emergency room were admitted, with $17.5 \%$ of emergency surgeries being performed. The attended diseases in the emergency room are very heterogeneous, being the most frequent surgeries performanced anorectal surgery, appendectomy and cholecystectomy.

References:

Disclosure: No significant relationships.

\section{PR047}

\section{OBTURADOR HERNIA - ONLY IN BOOKS?}

S.M. Silva, A. Melo, C. Ferreira, N. Tenreiro, J.C. Lage, P. Avelar

Surgery, Centro Hospitalar Trás-os- Montes e Alto Douro, Vila Real/ PORTUGAL

Introduction: The obturator hernia is a rare pathology, consisting of the entrance of pelvic or abdominal contents through the obturator foramen.

Material and methods: We present the case of an 85-year-old man who came to the emergency department with a history of nausea, vomiting, abdominal pain and constipation with 3 days of evolution. Results: On admission the patient was stable and without fever, with distended abdomen, painful to palpation, with increased bowel sounds. Analytically with increased inflammatory parameters and abdominal radiography with small bowel air-fluid levels. Computed tomography revealed distension of small bowel, which indicates occlusion secondary to incarcerated obturator hernia. We propose urgent surgery. The patient was submitted to an exploratory laparotomy, with confirmation of a right obturator hernia, containing small bowel loop with necrosis and perforation. We did an enterectomy, mechanical anastomosis and herniorraphy.

Conclusion: The obturator hernia is an uncommon type of hernia (0.1-1\% of all hernias), with only 0.2 to $1.6 \%$ complicating with mechanical obstruction of the small bowel.

It is a challenging diagnosis, usually confirmed with an exploratory laparotomy. It is important to early recognise de need to operate, since the mortality rate is the highest among all hernias, approximately $13-40 \%$.

References: N. Hodgins et al. Obturator hernia: a case report and review of the literature. International Journal of Surgery Case Reports 4 (2013) 889-892.

Mottin CC et al. Hérnia obturadora recidivada encarcerada: um relato de caso. Scientia Medica (Porto Alegre) 2011; volume 21, número 1, p. 20-22.

Sanjeev R. Kulkarni et al.: Obturator hernia: A diagnostic challenge. International Journal of Surgery Case Reports. 2013; 4 (7): 606- 608. A.

Mendonça et al. Diagnóstico e tratamento da hérnia obturadora encarcerada: relato de caso. Rev. Ciênc. Saúde Nova Esperança Dez. 2014; 12 (2).

Disclosure: No significant relationships.

PR048

\section{PERFORATED GALLBLADDER WITH BILIARY PERITONITIS? - LAPAROTOMY TIME IS GONE, DARE TO DO IT LAPAROSCOPICALLY!}

\author{
A. Mihailescu, A.L. Bubuianu
}

General/emergency Surgery, Tameside General Hospital, Ashton-uLyne/UNITED KINGDOM

Introduction: Laparoscopic cholecystectomy has become the standard in treating elective biliary pathology and is being introduced for treatment of acute cholecystitis in the emergency setting with very good outcomes. The perforated gallbladder is still an indication for laparotomy or percutaneous drainage in unfit individuals. There is reluctance in a laparoscopic approach for perforated gallbladder, with free bile contamination in the abdominal cavity.

Material and methods: We will present a case of acute cholecystitis, with free perforation of the gallbladder in the abdominal cavity, managed laparoscopically.

Results: The laparoscopic approach has proven a viable way of treating a perforation of the gallbladder, with morbidity similar for a laparoscopic cholecystectomy for acute cholecystitis, and much better than an open approach.

Conclusion: Management of the perforated gallbladder with diffuse biliary peritonitis can be performed through a laparoscopic approach in experienced hands, with results comparable with any other treatment for acute cholecystitis.

References:

Disclosure: No significant relationships. 
PR049

\section{INTRAABDOMINAL ABSCESS RATE OF LAPAROSCOPY IN COMPLICATED ACUTE APPENDICITIS PERFORMED BY JUNIOR RESIDENTS}

\author{
L. Martin Roman, C. Ruiz, S. Kayser, M.D. Pérez Díaz, \\ C.M. Martinez Moreno, F. Turégano Fuentes
}

General Surgery, Hospital General Universitario Gregorio Marañón, Madrid/SPAIN

Introduction: Laparoscopic appendectomy has yet to be generalized as the technique of choice in acute appendicitis (AA). Its claimed association with a much higher intraabdominal abscess (IAA) rate, despite recent evidence to the contrary, has restrained its implementation, among other likely factors. We aimed to assess the morbidity of laparoscopy in complicated appendicitis in our hospital.

Material and methods: Retrospective descriptive study of a prospective database of laparoscopic appendectomies between July 2015 and September 2017. The laparoscopic approach has been the standard in our hospital for many years. Phlegmonous AA were considered as uncomplicated, whereas all other macroscopic findings were considered as complicated AA. Variables studied were age, gender, need of conversion, operating surgeon (resident vs attending), morbidity, length of hospital stay, and readmission rate. Results were compared to those of a recent meta-analysis.

Results: Sixteen out of 500 patients were excluded due to misdiagnosis. Among the 484 patients, $326(67 \%)$ had uncomplicated AA. The IAA rate in the remaining 158 with complicated AA was of $6.4 \%$. The conversion rate was of $1.9 \%$, and $3.2 \%$ needed readmission. Mortality in this group was of $1.3 \%$ ( 2 patients). No reinterventions were needed in either group, and junior residents performed $97 \%$ of all procedures. Our $6.4 \%$ IAA rate compares favorably with the results of a very recent meta-analysis comparing open and laparoscopic appendectomies in complicated AA, which reports a $6 \%$ and $8 \%$ rate of IAA, respectively.

Conclusion: The laparoscopic approach in complicated AA in our hospital seems warranted in terms of postoperative IAA

References: Athanasiou Ch et al. WJS DOI 10.1007/s00268-0174123-3.

Disclosure: No significant relationships.

\section{PR050}

\section{PREDICTING CONVERSION FROM LAPAROSCOPIC TO OPEN CHOLECYSTECTOMY: A SINGLE INSTITUTION RETROSPECTIVE STUDY}

\author{
S. Almasri, A. Hallal
}

Surgery, The American University of Beirut Medical Center, Beirut/ LEBANON

Introduction: Laparoscopic cholecystectomy (LC) is the standard surgical treatment for benign gallbladder disease. Nevertheless, conversion to open cholecystectomy (OC) is needed in some cases. The aim of this study is to calculate our institutional conversion rate, and to identify the variables that are implicated in increasing the risk of conversion (LC-OC).

Material and methods: We carried out a retrospective study of all the cases of LC performed at the American University of Beirut
Medical Center between 2000 and 2015. Each (LC-OC) case was randomly matched to a laparoscopically completed case by the same consultant within the same year of practice, as the LC-OC case, in a 1 to 5 ratio. Forty-eight parameters were compared between the two study groups.

Results: 48 out of 4668 LC were converted to OC over the 15 years study period; the conversion rate in our study was $1.03 \%$. The variables that were found to be most predictive of conversion were male gender, advanced age, prior history of laparotomy, especially in the setting of prior gunshot wound, a history of restrictive or constrictive lung disease, and anemia $(\mathrm{Hb}<9 \mathrm{~g} / \mathrm{dl})$. The most common intraoperative reasons for conversion were perceived difficult anatomy or obscured view secondary to severe adhesions or significant inflammation. Patients who were in the LC-OC arm had a longer length of hospital stay.

Conclusion: Advance age, male gender, significant comorbidities and history of prior laparotomies have a high risk of conversion. Patients with these risk factors should be counseled for the possibility of conversion to open surgery preoperatively. Further research is needed to determine whether these high risks patients should be operated on by surgeons with more extensive experience in minimal invasive surgery.

References: 1. Ashfaq, A., Ahmadieh, K., Shah, A., et al (2016). The difficult gall bladder: Outcomes following laparoscopic cholecystectomy and the need for open conversion. The American Journal of Surgery, 212 (6), pp.1261-1264. 2. Beksac K., Turhan, N., Karaagaoglu, E. and Abbasoglu, O. (2016). Risk Factors for Conversion of Laparoscopic Cholecystectomy to Open Surgery: A New Predictive Statistical Model. Journal of Laparoendoscopic \& Advanced Surgical Techniques, 26 (9), pp.693-696. 3. Csikesz N, Ricciardi R, Tseng J, Shah S (2008). Current Status of Surgical Management of Acute Cholecystitis in the United States. World J Surg;32 (10):2230-2236. doi:10.1007/s00268-008- 9679-5. 4. Bittner R (2006). Laparoscopic surgery: 15 years after clinical introduction. World J Surg.;30:1190203, doi: 10.1007/s00268-005- 0644-2. 5. Ballal M, David G, Willmott S, et al (2009). Conversion after laparoscopic cholecystectomy in England. Surgical Endoscopy;23 (10):2338- 2344. doi:10.1007/ s00464-009- 0338-1.

Disclosure: No significant relationships.

\section{PR051}

\section{LAPAROSCOPIC ASSISTED SIGMOIDECTOMY IN THE TREATMENT OF VOLVULUS AS DELAYED URGENCY}

C. Domínguez Sánchez ${ }^{1}$, J.A. Lopez Ruiz ${ }^{1}$, L. Tallón-Aguilar ${ }^{2}$, J.C. Gamero Huamán ${ }^{\text {, Y. Lara Fernández }}{ }^{l}$, B.M. De La Cuadra ${ }^{l}$, M. Sanchez Ramirez ${ }^{I}$, E. Perez Margallo ${ }^{I}$, J. López Pérez ${ }^{I}$, F. Oliva Mompean ${ }^{1}$

${ }^{1}$ General Surgery, University Hospital Virgen Macarena, Seville/ SPAIN, ${ }^{2}$ Emergency Surgery Unit, Virgen del Rocío Universitary Hospital, Seville/SPAIN

Introduction: There are two essential methods for treating sigmoid volvulus, which are not in any way counteracted, complement each other: endoscopic devolution and surgical treatment. Our objective is to design a valid algorithm for volvulus management, as well as to evaluate the utility of elective sigmoidectomy assisted by endoscopic laparoscopy in the algorithm we propose.

Material and methods: After designing a management algorithm, thirteen consecutive sigmoid volvulus patients treated by endoscopic devolution were included, followed by laparoscopy assisted 
sigmoidectomy at $48-72 \mathrm{~h}$. Patients with ASA IV, previous fecal incontinence and those in whom endoscopic devolution did not show or showed necrosis of the colon were excluded, following the scheme implanted in our service.

Results: Our population sample included 13 patients ( 7 women and 6 men) aged between 67 and 93, all of whom were ASA III, and were independent for activities of daily living. The operative time was 60 to 90 minutes. Endoscopic devolution was successful in all patients. Complications included an infection of the operative wound, a seroma, an anastomotic leak, and postoperative bleeding. In the reviews made so far, there has been a relapse at 3 years.

Conclusion: A sigmoidectomy with laparoscopic assisted primary anastomosis in a delayed emergency is a safe and effective therapeutic option for patients with sigmoid volvulus in whom endoscopic devolution has been effective. Proper patient selection is a crucial point for the success of this procedure. Although the results of this study are encouraging, broader studies are needed to confirm this initial success.

References: - Liang JT1, Lai HS, Lee PH. Elective laparoscopically assisted sigmoidectomy for the sigmoid volvulus. Surg Endosc. 2006 Nov;20 (11):1772-3. - Masami Ueda, Tadashi Onishi, et al. Usability of elective laparoscopic sigmoidectomy and feasibility of single incision laparoscopic surgery for sigmoid volvulus: Report of Three Cases. Int Surg 2015;100:408-413.

Disclosure: No significant relationships.

\section{PR052}

\section{APPENDICEAL LENGTH PREDICTS RISK OF PERFORATION IN APPENDICITIS - DOES SIZE MATTER?}

\section{A.L. Moynihan, B. Dumbrava, G.A. Bass}

Dept Of Surgery, Royal College of Surgeons in Ireland, Dublin/ IRELAND

Introduction: While recent reports suggest a paradigm change in the management of early appendicitis, the prediction, prevention and management of complicated perforated appendicitis remains a significant diagnostic and therapeutic challenge. Considerable variation exists in the length of the human vermiform appendix, and this has physical implications in relation to the forces acting both across the wall and along the length of the cylinder. It is hypothesised that this heterogeneity in organ length may contribute to the variability in the natural history of the simple-to-perforated appendicitis progression.

Material and methods: A retrospective analysis was undertaken of 414 consecutive appendicectomy specimens where the appendix was removed for a clinical diagnosis of appendicitis. Logistic regression analysis correlated appendiceal length with the histopathologic findings (inflammation, suppuration, gangrene or perforation).

Results: Four-hundred and fourteen appendicetomies were performed between 2010 and 2012; of these, 61/414 (14.7\%) were histologically normal, 216/414 (52.2\%) demonstrated simple appendicitis, 37/414 (8.9\%) were gangrenous and 100/414 (24.2\%) were perforated. The mean length of the resected appendix in patients with perforated appendicitis $(59.1 \mathrm{~mm}+/-18 \mathrm{~mm})$ was significantly shorter than that seen in either simple appendicitis $(65.1 \mathrm{~mm}+/-15.75 \mathrm{~mm} ; \mathrm{p}=0.006)$ or gangrenous appendicitis $(84.19 \mathrm{~mm}+/-19.97 \mathrm{~mm} ; \mathrm{p}=0.001)$.

Conclusion: Appendiceal length was demonstrated to inversely-correlate with likelihood of perforation in acute appendicitis, placing patients with an anatomically-short appendix at a greater risk of progression to complicated disease $(\mathrm{OR}=4.81)$. We thus suggest consideration of removal of the macroscopically-normal short appendix on diagnostic laparoscopy for appendicitis to minimise future risk.

References:

Disclosure: No significant relationships.

\section{PR053}

\section{DETERMINATION OF THE ENDOTOXEMIA DEGREE IN CHOLELITHIASIS WITH OBSTRUCTIVE JAUNDICE}

\section{Streltov, G. Rojnoveanu}

Surgery No.1 "nicolae Anestiadi", Nicolae Testemitanu State University of Medicine and Pharmacy, Chisinau/MOLDOVA

Introduction: Disturbances of hepatic metabolism activate proteolysis, causing the accumulation of average molecular weight peptides in the blood, considered to be the main endotoxin group which causes the development of toxemia in obstructive jaundice syndrome.

Material and methods: The study group comprised 94 patients with cholelithiasis and obstructive jaundice with absolute surgical indications. Determination of the endotoxemia degree was performed by assessing the presence of inflammatory syndrome and average molecular weight peptides (AMWP) with comparative assessment at admission and post-decompression. Parameters were compared in the following groups: chronic cholelithiasis and jaundice $(n=30)$, acute cholelithiasis and jaundice $(n=33)$, acute biliary pancreatitis and jaundice $(\mathrm{n}=31)$.

Results: Control batch $16.8 \pm 1.37$ (uc/ml). At hospitalization, the highest values of AMWP were recorded in acute cholelithiasis and jaundice $-26.95 \pm 1.63(\mathrm{uc} / \mathrm{ml})$, where values increased with $+60.41 \%(\mathrm{p}<0.01)$ and in acute biliary pancreatitis and obstructive jaundice $-24.23 \pm 1.5(\mathrm{uc} / \mathrm{ml})$, where values increased with $+44.22 \%(\mathrm{p}<0.01)$. Postoperatively in all groups the values were elevated in the first two days $-2.4 \pm 0.8(\mathrm{uc} / \mathrm{ml})$ from baseline with a decrease in 4-5 days. Increased levels of average molecular weight peptides were consistent with variable inflammatory syndrome in the study groups, confirmed by leukocytosis and marked erythrocyte sedimentation rate.

Conclusion: Endotoxemia is most pronounced in the inflammatory complications of cholelithiasis associated with jaundice. Surgery for early decompression prevents the progression of both endogenous intoxication and liver and kidney failure.

References:

Disclosure: No significant relationships.

\section{PR054}

\section{USING OF INTERVENTIONAL ULTRASOUND PROGRAM} IN AN EMERGENCY SURGERY DEPARTMENT

\author{
J. Tinoco-González $^{1}$, G. Suárez-Artacho ${ }^{2}$, L. Tallón-Aguilar ${ }^{1}$, \\ $\overline{\text { A. Sánchez-Arteaga }}^{1}$, V. Durán-Muñoz-Cruzado ${ }^{1}$, \\ I. Alarcón-Del Agua ${ }^{1}$, M.J. Tamayo-Lopez ${ }^{1}$, F. Pareja-Ciuró ${ }^{1}$
}

${ }^{1}$ Emergency Surgery Unit, Virgen del Rocío Universitary Hospital, Seville/SPAIN, ${ }^{2}$ Hepatobiliary And Transplantant Surgery Unit, Virgen del Rocío Universitary Hospital, Seville/SPAIN

Introduction: Interventional ultrasound emerge as a natural improvement of conventional ultrasound, enabling secure invasive 
procedures by visualization of neighboring structures. These sorts of procedures are usually performed by a radiologist in most of thirdlevel hospitals in Spain.

Material and methods: Observational, descriptive study from a prospective cohort (April 2016-September 2017) at a third-level hospital. We analyzed all interventional ultrasound-guided procedures regarding type of procedure, success of the technique and outcomes. Results: We analyzed 73 ultrasound-guided procedures (68 patients) performed in 21 different medical conditions. Most common procedures were cholecystostomies $(31,42.5 \%)$, primary infectious abscesses $(16$, $21.9 \%$ ), and postoperative abscesses $(15,20.5 \%)$. In 56 cases $(76.7 \%)$ a pig-tail catheter was placed, and puncture-aspiration technique was performed in 3 patients $(4.1 \%)$. A successful procedure was registered in 71 cases $(97.2 \%) .2$ failed cholecystostomies were reported, requiring urgent cholecystectomy. Mean age was 69,2 ( $\pm 13,2)$. Regarding the cases of cholecystostomy, mean age was reported as older (77,4 $\pm 9,5 \mathrm{vs.}$ $65,8 \pm 12,7, \mathrm{p}<0,001)$. Procedure-related complications were $5.1 \%$ : bleeding in 2 cases (without need of any further intervention), 1 pneumothorax (needing a chest tube) and 1 colonic perforation (requiring surgery). Procedure-related mortality was $0 \%$, and mortality of the primary disease was report in 9 patients $(12.3 \%)$.

Conclusion: Ultrasound-guided interventional procedures in abdominal emergencies can be safetly performed by specifically trained surgeons, as a feasible, secure technique with low rates of morbidity and mortality. A correct selection of patients, and a proper indication of the procedure, might be determinant for success.

References:

Disclosure: No significant relationships.

\section{PR055}

\section{LAPAROSCOPIC REPAIR OF THE DUODENAL PERFORATED ULCER}

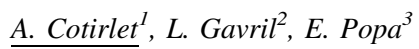

${ }^{1}$ Surgery, University "Vasile Alecsandri” Bacau/Moinesti Hospital, Moinesti/ROMANIA, ${ }^{2}$ Intensive Care, UMF Gr T POPA/IRO IASI, MOINESTI/ROMANIA, ${ }^{3}$ Surgery, MOINESTI HOSPITAL, MOINESTI/ROMANIA

Introduction: Laparoscopic management of perforated ulcer disease has clearly potential advantages over classical laparotomy. The aims of this study were to analyze the reasons for, and outcome of, conversion from laparoscopic to open surgery and to identify factors that may predict the need for conversion.

Material and methods: In total, 63 patients who underwent laparoscopic simple closure for perforated ulcer disease from January 2012 to December 2016 were retrospectively analyzed. Patients were divided into totally laparoscopic and conversion groups. The characteristics of patients, intraoperative findings, postoperative complications, conversion rates and suture leakage rates of each group were investigated.

Results: 18 women and 45 men with a mean age 32.8 (range: $22-46$ ) were included in our study. A laparoscopic treatment was performed successfully in 58 patients $(92.06 \%)$. Five patients $(8 \%)$ needed a conversion to laparotomy, all of them being discovered with an ulcer perforation size greater than $15 \mathrm{~mm}$. The mean operative time was 57 min (range $37-84 \mathrm{~min}$ ) There were 8 postoperative complications $(9.65 \%)$ and the mean hospital stay was 4.8 days (3-16). No malignant pathology was identified at the biopsy during the control upper endoscopy that was made 6 weeks after disharge. No postoperative mortality was recorded.
Conclusion: Perforated ulcer can be treated safely by laparoscopy. Ulcer size greater than $15 \mathrm{~mm}$ is a significant risk factor for predicting conversion to open surgery. The risk of postoperative morbidity was associated with late onset of symptoms (more than 24 hours) and the association of comorbidities.

References: laparoscopic, perforated, ulcer

Disclosure: No significant relationships.

\section{PR056}

\section{A COMPARiSON OF PRE INTRA POSTOPERATIVE DIAGNOSIS OF ACUTE APPENDiCiTIS}

\author{
N. Ozlem ${ }^{1}$, S.S. Gultekin ${ }^{2}$
}

${ }^{1}$ Faculty Of Medicine, General Surgery, Ahi Evran University, Kırsehir/TURKEY, ${ }^{2}$ General Surgery, gazi state hospital, ankara/ TURKEY

Introduction: The most common diagnoses in reproductive-aged women with acute pelvic pain are idiopathic pelvic pain, pelvic inflammatory disease, acute appendicitis, ovarian cysts, ectopic pregnancy, and endometriosis. Among postmenopausal women, cancer must be considered. Findings from the history and physical examination can point to likely diagnoses, and laboratory testing and imaging can help confirm.

Material and methods: At Ahi Evran University Medical Faculty Education and Research Hospital General Surgery Clinic the records of 208 patients with an age range of 3-74 years, which were operated between 2015 and 2017 with a diagnosis of suspicious acute appendicitis, were reviewed and the pre, per, post operative diagnosis of these patients were evaluated.

Results: Acute appendicitis, acute disease needing surgical intervention is still seen in the same frequency as the past. Since its diagnosis and treatment needs prompt action, action must be taken as quick as possible. Leucocyte counts more than 10000/mm3 are in indicators of acute and perforated appendicitis.

Conclusion: Its been shown that usg and diagnostic laparoscopy can be used in the diagnosis and treatment of appendicitis but its use is mostly advised in women of child bearing age to prevent the risk of infertility. The fact that the patient with perforated appendicitis comes to medical attention much more later than others must make us a ware that to prevent the negatice effects of perforation, the potantial patients meaning all the citizens should be educated and informed, first step physicians who first examine the patient mostly should be educated well and the general surgeons must be motivated to improve themselves both technically and in knowledge. Lastly the family physicians, the members of speciality which is new in our country, who will examine the patient in the first step should have through knowledge and capability in dealing with such diseases.

References: 1 Cho, Jinbeom; Lee, Dosang; Sung, Kiyoung; et al. ANNALS OF SURGICAL TREATMENT AND RESEARCH Volume: 93 Issue: 1 Pages: 43-49 Published: JUL 2017 2.Emergency appendicectomy in Australia: findings from a multicentre, prospective study Volume: 41 Issue: 1 Pages: 70-74 Published: JAN 201 3. 3. Acute Appendicitis: A Weak Concordance Between Perioperative Diagnosis, Pathology and Peritoneal Fluid Cultivation Tind, Sofie; Qvist, Niels WORLD JOURNAL OF SURGERY Volume: 41 Issue: 1 Pages: 70-74 Published: JAN 201 4.Common Questions About the Evaluation of Acute Pelvic Pain ] AMERICAN FAMILY PHYSICIAN Volume: 93 Issue: 1 Pages: 41-48 Published: JAN 12016

Disclosure: No significant relationships. 
PR057

\section{OPEN EXPLORATION CONVERTED TO LAPAROSCOPIC: A USEFUL TECHNIQUE IN EMERGENCY HERNIA SURGERY}

\section{Gerogiannis}

General Surgery, Kingston Hospital NHS Foundation Trust, Kingston/UNITED KINGDOM

Introduction: In Emergency Surgery, strangulated abdominal and groin hernias can be proven challenging. Sometimes, reduction of the hernia can occur intraoperatively while manipulating the hernia sac leading to uncertainty about viability of its contents. We describe a useful technique that helps identifying any possible ischaemic structure after an accidental reduction of previously strangulated hernia.

Material and methods: An open exploration of a strangulated hernia is performed followed by identification of the strangulated hernia sac, dissection of the sac and identification of the defect. Sac is opened to explore its contents; however, this leads to a reduction of the contents of the hernia intraperitoneally. Insertion of a $10 \mathrm{~mm}$ port through the defect. It can be either through the abdominal wall or through the inguinal canal in case of a strangulated inguinal hernia. Establishment of pneumoperitoneum. Insertion of a $10 \mathrm{~mm}$ camera and laparoscopy of the abdominal cavity. The reduced contents should be just below the port site however sometimes a full laparoscopy and insertion of a $5 \mathrm{~mm}$ port through the abdominal wall is needed.

Results: Full visualisation of the reduced hernia sac contents is achieved. In case of a finding of ischaemic bowel, there are two options. Either insert a $5 \mathrm{~mm}$ abdominal port and try with a laparoscopic atraumatic grasper to guide the loop of bowel through the hernia defect or make a mini-laparotomy extending the original incision, externalise the bowel and perform a resection and anastomosis.

Conclusion: The above technique is safe and avoids unnecessary laparotomies in case of intraoperative accidental reduction of a strangulated hernia content.

References: Yang GP. Laparoscopy in emergency hernia repair. Ann Laparosc Endosc Surg 2017;2:107.

Disclosure: No significant relationships.

\section{PR058}

\section{IMPACT OF PLATELET RICH FIBRIN ON BONE MARROW MONONUCLEAR CELLS (IN VITRO)}

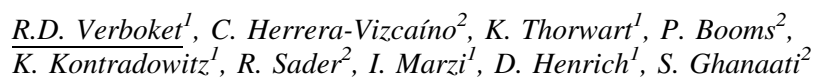

${ }^{1}$ Klinik Für Unfall-, Hand- Und Wiederherstellungschirurgie, Uniklinik Frankfurt am Main, Frankfurt am Main/GERMANY, ${ }^{2}$ Department Of Oral And Maxillofacial Surgery, Uniklinik Frankfurt am Main, Frankfurt am Main/GERMANY

Introduction: Huge bone defects after trauma are a big challenge. In actual work the use of bone marrow mononuclear cells (BMCs) combined with an osteoconductive scaffold has been demonstrated as a good treatment of large bone defects $[1,2,3]$. Another biomaterial for tissue engineering is platelet rich fibrin (PRF). $P R F$ is produced in a one-step procedure through a physiologic clot formation [4]. The matrix of the clot serves as a reservoir of growth factors [5]. To boost the healing potential of BMCs the combination with PRF could be a comfortable solution.

Material and methods: After a dose-effect study on with different speeds centrifuged PRF (medium speed and low speed) and BMC we found no further cell activity atop $10 \%$ concentration. We performed MTS tests and rtPCR analysis after 2, 7, and 14 days as control group the same concentration of blood serum was used.

Results: The metabolic activity of BMC increased in all groups significantly on day 14 (median serum: $110 \times 10^{\wedge} 3$ metabolic equivalents; median PRF-low: $80 \times 10^{\wedge} 3$ metabolic equivalents; median PRF-medium: $76,7 \times 10^{\wedge} 3$ metabolic equivalents). Also, we were able to find an upregulation of the gene expression of matrix metalloproteinases (MMP2/7/9) in PRF-low and PRF-medium.

Conclusion: In conclusion, we were able to show a positive effect in our in vitro model. Different effects in metabolic activity and MMPsynthesis were discoverable. These variations could be reasoned through supporting effects of VEGF, TGF-b and EGF in the PRF clot. Further in vivo testing of BMC combined with PRF and scaffolds could lead to a better bone healing.

References: 1. Henrich D, Verboket R, Schaible A, Kontradowitz K, Oppermann E, Brune JC, et al. Characterization of Bone Marrow Mononuclear Cells on Biomaterials for Bone Tissue Engineering In Vitro. Biomed Res Int. Hindawi; 2015;2015:1-12. 2. Seebach C, Henrich D, Schaible A, Relja B, Jugold M, Bonig H, et al. Cell-based therapy by implanted human bone marrow-derived mononuclear cells improved bone healing of large bone defects in rats. Tissue engineering. Part A. Mary Ann Liebert, Inc. 140 Huguenot Street, 3rd Floor New Rochelle, NY 10801 USA; 2015;21:1565-78. 3. Seebach C, Henrich D, Meier S, Nau C, Bonig H, Marzi I. Safety and feasibility of cell-based therapy of autologous bone marrow-derived mononuclear cells in plate-stabilized proximal humeral fractures in humans. J Transl Med. BioMed Central; 2016;14:314. 4. Dohan DM, Choukroun J, Diss A, Dohan SL, Dohan AJJ, Mouhyi J, et al. Plateletrich fibrin (PRF): A second-generation platelet concentrate. Part I: Technological concepts and evolution. Oral Surgery, Oral Medicine, Oral Pathology, Oral Radiology, and Endodontology. 2006;101:e37e44. 5. Fujioka-Kobayashi M, Miron RJ, Hernandez M, Kandalam U, Zhang Y, Choukroun J. Optimized Platelet-Rich Fibrin With the LowSpeed Concept: Growth Factor Release, Biocompatibility, and Cellular Response. J. Periodontol. 2017;88:112-21.

Disclosure: No significant relationships.

\section{PR059}

\section{IS DAMAGE-CONTROL SURGERY WITH OPEN ABDOMEN (OA) USEFUL IN EMERGENCY GENERAL SURGERY? (A RETROSPECTIVE STUDY OVER THE PAST 5 YEARS)}

\section{K. Ueda, Y. Okishio, T. Nasu, S. Kawashima, K. Kunitatsu, S. Kato}

Emergency And Critical Care Medicine, Wakayama Medical University, Wakayama/JAPAN

Introduction: Damage-control surgery with open abdomen (OA) is described for trauma, but little exists regarding in the emergency general surgery. The purpose of this research is to clarify the usefulness of OA in emergency general surgery by looking at cases over the past 5 years.

Material and methods: A total of 201 cases of emergency abdomen surgeries required ICU post-operative management during the period between September 2012 and August 2017 at Department of Emergency and Critical Care Medicine, Wakayama Medical University Hospital (excluding 28 cases involving exploratory laparotomy and 5 
cases involving death within 24 hours of surgery). Of these 201 cases, 58 cases $(29 \%)$ of OA were the subjects of this study, with 143 cases (71\%) of single-stage surgeries making up the control group. The type of temporary abdominal closure was a Modified Opsite sandwich method (Ann R Coll Surg Engl 2007; 89:57-61) in all of 58cases.

Results: The median age of the OA group was 74 (IQR, 67-85), indicating no significant difference with the median age of 76 (IQR, 69-85) in the control group. The length of stay in the intensive care unit (ICU) was significantly longer in the OA group at 7 days (IQR, 4-13.5) compared to 4 days (IQR, 3-6) in the control group. The APACHE-II and SOFA scores on the first day of admission to the ICU were 25.5 (IQR, 19-31) and 9 (IQR, 7-13), respectively, in the OA group, representing significantly higher values than the scores of 18 (IQR, 15-23) and 6 (IQR, 4-9) in the control group, meaning OA was clearly performed on critically ill patients. On the other hand, fewer cases required second surgery after abdominal closure in the OA group at $9 \%$ compared to $17 \%$ in the control group $(\mathrm{p}=0.130)$. The 90 -day mortality rates were $21 \%$ and $15 \%$ in the OA group and control group, respectively, indicating no significant difference $(\mathrm{p}=$ 0.301). As for the indication for OA management, 45 cases were damage control, 29 cases were second look, 17 cases were excessive contamination, and 9 cases were decompression (including overlapping). Of the 58 cases of OA, 3 cases succumbed to death as abdominal closure could not be performed. Of the remaining 55 cases, the median number of surgeries performed before abdominal closure (including the primary operation) was 3 (IQR, 2-4), with a median period until abdominal closure of 2 days (IQR, 1-5). Moreover, of the 55 cases, 54 cases underwent successful fasciorrhaphy, and 1 case underwent a skin suture only. The 12 cases that did not survive past 90 days in the OA group were significantly older in age (median 85.5) compared to the 46 cases $(\mathrm{p}=0.036)$ that survived, in addition to having higher SOFA scores (median 13.5) $(\mathrm{p}=0.001)$.

Conclusion: Although the cases of OA were clearly more serious than the cases of single-stage surgeries, there was no difference in mortality rate, with a tendency for fewer second operations. It was indicated that the lifesaving rate may potentially be improved by performing OA in a proactive manner. However, extra care must be taken with very elderly patients and those with high SOFA scores.

References:

Disclosure: No significant relationships.

\section{PR060}

\section{TOWARDS A PRE-DIAGNOSE OF ABDOMINAL SURGICAL WOUNDS THROUGH THE ANALYSIS OF VISUAL 3D}

\author{
J.J. Segura-Sampedro $^{1}$, N. Muntaner ${ }^{2}$, F. Bonin-Font ${ }^{2}$, \\ $\overline{\text { A. Jiménez-Ramírez }}^{2}$, R. Morales-Soriano ${ }^{I}$, F.J. González-Argenté ${ }^{1}$
}

${ }^{1}$ General \& Digestive Surgery, University Hospital Son Espases, Palma de Mallorca/SPAIN, ${ }^{2}$ Systems, Robotics \& Vision Group. Dptment De Matemàtiques I Informàtica, Universitat Ills Balears, Palma de Mallorca/SPAIN

Introduction: The objective is to develop an App able to filter, automatically, those wounds that potentially present inflammation as sign of infection and need a face-to-face evaluation in the hospital from those that course a normal evolution and can be managed at home, saving time and medical resources.

Material and methods: Pipeline for Wound Evaluation

Video Recording

Feature Matching
Structure From Motion for 3D reconstruction Dense Point Cloud and Texture Mesh

Polyline and Plane Fitting

Map of Distances

Results: Real Inflamed Wound with Template

Although there is a clear gradient of distances between some parts of the center and the sides of the evaluated area, susceptible of being pre-diagnosed as inflamed, the shape of the wound is not clearly identified, being necessary a final and definitive diagnose given by the surgeon

Real Non Inflamed Wound

This type of plot would suggest to the patient and to the doctor, in principle, an unnecessary face-to-face revision.

Real Infected Wound with Template

This distance gradient marks an anomaly in the area.

Conclusion: This paper presents an innovative methodology to estimate a previous diagnose of post-surgical abdominal wounds using visual data. Experiments with simulated scenes reveal a clear discrimination between a simulated infected wound and the background. The method is also able to discriminate clearly the sane wounds. However, results of experiments with real inflamed wounds are still on a preliminary stage, but pointing in a clear good direction.

References: Barnes, C., Shechtman, E., Finkelstein, A., and Goldman, D. (2009). PatchMatch: A Randomized Correspon- dence Algorithm for Structural Image Editing. ACM Transactions on Graphics, 28 (3):24:1-24:11. cDcSeacave (2017). OpenMVS. Open Multi-View Stereo Reconstruction Library. https://github.com/cdcseacave/openMVS. Girardeau-Montaut, D. (2017). Cloud Compare, 3D Point Cloud and Mesh Processing Software Open Source Project. http://www.danielgm.net/cc/. Hartley, R. and Zisserman, A. (2003). Multiple View Geom- etry in Computer Vision. Cambridge University Press. Jancosek, M. and Pajdla, T. (2014). Exploiting Visibil- ity Information in Surface Reconstruction to Preserve Weakly Supported Surfaces. International Scholarly Research Notices, 2014. Lowe, D. G. (2004). Distinctive Image Features from Scale-Invariant Keypoints. Int. Journal on Computer Vision, 60 (2):91-110Moulon, P., Monasse, P., and Marlet, R. (2013). Global Fusion of Relative Motions for Robust, Accurate and Scalable Structure from Motion. In Proceedings of the International Conference on Computer Vision. Moulon, P., Monasse, P., Marlet, R., and Others (2017). OpenMVG. An Open Multiple View Geometry Li- brary. https://github.com/ openMVG/openMVG. Muja, M. and Lowe, D. (2009). Fast Approximate Nearest Neighbors with Automatic Algo- rithm Configuration. Source code available at https://github.com/mariusmuja/flann/. Nephroflow (2017). Nephroflow. https://nephroflow.com/. Nordheim, L., Haavind, M., and Iversen, M. (2014). Ef- fect of Telemedicine Follow-up Care of Leg and Foot Ulcers: a Systematic Review. BMC Health Services Research, 14 (565):58-62. Rublee, E., Rabaud, V., Konolige, K., and Bradski, G. (2011). ORB: An Efficient Alternative to SIFT or SURF. In Proceedings of the 2011 International Conference on Computer Vision, pages .Segura-Sampedro, J., I.RiveroBelenchon, Pino-Diaz, V., Sánchez, M. R., Pareja-Ciuro, F., PadilloRuiz, J., and Rodriguez, R. J. (2017). Feasibility and Safety of Surgical Wound Remote Follow-up by Smart Phone in Appendectomy: A Pilot Study. Annals of Medicine and Surgery, 21:58-62. Topdoctors (2017). Topdoctors. www.topdoctors.es/. Vu, H., Labatut, P., and Pons, J. (2012). High Accuracy and Visibility-Consistent Dense Multiview Stereo. IEEE Transactions on Pattern Analysis and Machine Intel-ligence, 34 (5):889-901. Waechter, M., Moehrle, N., and Goesele, M. (2014). Let There Be Color! Large-Scale Texturing of 3D Recon- structions. In Proceedings of the European Confer- ence on Computer Vision, pages 836-850.

Disclosure: No significant relationships. 


\section{PR061}

\section{NEW FRONTIERS IN COMBAT WOUND MANAGEMENT TECHNOLOGIES - EXPANDING THE LIMITS BY INTENSIVE WOUND CARE}

\author{
M. Topaz ${ }^{1}$, O. Barzel ${ }^{2}$, S. Ezra ${ }^{2}$, M. Motiei ${ }^{3}$, G. Topaz ${ }^{4}$, N. Zilony ${ }^{3}$, \\ R. Katzengold ${ }^{5}, A$. Gefen $^{5}$, I. Ziev_Ner ${ }^{2}$, R. Popovtzer ${ }^{3}$, I. Ashkenazi $^{6}$
}

${ }^{1}$ Plastic Surgery Unit, Hillel Yaffe Medical Center, Hadera/ISRAEL, ${ }^{2}$ Rehabilitation Center, Haim Sheba Medical Center, Ramat Gan/ ISRAEL, ${ }^{3}$ Faculty Of Engineering \& The Institute Of Nanotechnology And Advanced Materials, Bar Ilan University, Ramat Gan/ISRAEL, ${ }^{4}$ Department Of Internal Medicine G, Meir Medical Center, Kfar Saba/ISRAEL, ${ }^{5}$ Department Of Biomedical Engineering, Faculty Of Engineering, Tel Aviv University, Tel Aviv/ ISRAEL, ${ }^{6}$ Not Applicable, Hillel Yaffe Medical Center, Hadera/ ISRAEL

Introduction: Prolonged duration from injury to full wound closure is associated with infection, increased morbidity and mortality, increased costs and poor outcome functionality. The purpose of this study was to evaluate the yield of a combination treatment composed of the TopClosure ${ }^{\circledR}$ Tension Relief System (TRS) and simultaneous administration of supplemental oxygen and antibiotic irrigation within a dedicated negative pressure-assisted wound therapy (ROINPT) in the treatment of soldiers injured in combat suffering of significant soft tissue injuries.

Material and methods: Retrospective study of 7 soldiers injured in combat in July-August 2014.

Results: Complete wound closure was achieved in all seven soldiers within 2 to 4 days without skin grafts or flaps. Cases will be presented.

Conclusion: 1. TRS is a novel device for stretching, and securing wound closure, applying stress relaxation and mechanical creep for primary closure of large skin defects that otherwise would have required closure by skin grafts, flaps, free flaps or tissue expanders. 2 . Irrigation may accelerate evacuation of infectious material from the wound and may provide a novel method for antibiotic administration. 3. Supplemental oxygen to the wound reverses reduced $\mathrm{pO}_{2}$ levels in the wound's atmosphere inherent to treatment with conventional vacuum therapy limiting anaerobic contamination and possible infection. 4. Combined ROI-NPT and TRS may transform the traditional 3-phase text-book wound healing sequence into a short, more efficient 2-phase method. The TRS - ROI-NPT system allowed for reduced surgical complexity, short hospital stay, early postoperative mobilization and physiotherapy with good to excellent functional results in victims of combat soft tissue injury.

References:

Disclosure: No significant relationships.

\section{PR062}

\section{LAPAROSTOMY}

\section{Gumaa}

Surgical Emergency Unit, Oxford university hospitals, oxford/ UNITED KINGDOM

Introduction: Background : Laparostomy is currently used in many severely ill or injured patients to facilitate healing or prevent complications. With improved understanding of the pathophysiology of common abdominal emergencies, such as abdominal sepsis, and major abdominal trauma, the number of patients with laparostomy can be expected to increase in general and surgical intensive care units. Material and methods: Methodology: Retrospective study covering all patients who underwent laparostomy in SEU in JR hospital in Oxford over 30 months .

Results: Results: 51 patients underwent laparostomy over 30 months. The average length of stay in hospital was 17 days. The most common indications for laparostomy was bowel ischemia and abdominal contamination. All patients were admitted to ITU post operatively. Temporary abdominal coverage was achieved by Abthera vac dressing in $100 \%$ of patients. Average number of operations before definitive abdominal closure was 2.6, with average wait of 5 days. $68 \%$ of patients had primary abdominal closure, while $8 \%$ had closure assisted with prolene mesh and $7 \%$ had closure with vicryl mesh. $14 \%$ of patients developed incisional hernia, and $2 \%$ required abdominal wall reconstruction. The incidence of incisional hernia was lowest with primary closure, $20 \%$ of patients developed incisional hernia. And it was the highest after closure with vicryl mesh, 33\% of patients had incisional hernia. Mortality rate was $22 \%$.

Conclusion: Conclusion: Laparostomy has a relatively good outcome, taking in consideration that it is the solution for severely ill or injured patients.

References:

Disclosure: No significant relationships.

\section{PR063}

\section{SOMETIMES WE HAVE FORCE BUT NOT CONTROL. A NEW DEVICE EXPLOITS THE PIPED WALL VACUUM SYSTEM FOR THE NEGATIVE PRESSURE WOUND THERAPY (NPWT)}

\author{
S. Magalini ${ }^{1}$, A. La Greca ${ }^{2}$, G. Pepe ${ }^{1}$, M. Foco ${ }^{I}$, A. Di Giorgio ${ }^{1}$, \\ C. Lodoli ${ }^{I}$, M. Di Grezia ${ }^{I}$, V. Cozza ${ }^{I}$, D. Gui ${ }^{l}$
}

${ }^{1}$ Emergency Surgery, Fondazione Policlinico Universitario “A. Gemelli”, ROME/ITALY, ${ }^{2}$ Emergency Surgery, Fondazione Policlinico Universitario "A. Gemelli”, rome/ITALY

Introduction: NPWT devices are pump driven. MINIVACUUM ${ }^{\circledR}$ is a low-cost programmable and allarmed electronic system utilizing piped wall vacuum system (PWVS) as driving force. Many "handy" practitioners use non-commercial systems to build "homemade VACs" with hospital stocks of low-cost material such as gauzes, adhesive drapes, tubes, canisters and electrical aspirators or directly PWVS. Most crucial element recognized by all, also for security reasons, is regulation and stability of the applied vacuum force often uncontrollable in the longterm.

Material and methods: MINIVACUUM ${ }^{\circledR}$ was utilized in a preliminary clinical study on medium and large surgical wounds and entero-atmospheric fistulas of ten patients.

Results: Ten patients (4 with major infections of the surgical abdominal wound, 2 with necrotizing fasciitis of the limbs and 1 of the face, 3 with entero-atmospheric intestinal fistulas) were treated, with good results, not different from those expected with known commercial devices.

Conclusion: MINIVACUUM was especially useful in case of high output wounds and enteric fistulas, because it is easily adaptable to large bore tubing necessary to remove the thick secretions. High flow sustainable by the device is useful in case of "extreme" medications with air leaks such as these. 
References: Huang C, Leavitt T, Bayer LR, Orgill DP. Effect of negative pressure wound therapy on wound healing. Curr Probl Surg. 2014 Jul;51 (7):301-31. Orgill DP, Bayer LR. Negative pressure wound therapy: past, present and future. Int Wound J. 2013 Dec;10 Suppl 1:15-9.

Disclosure: No significant relationships.

\section{PR064}

\section{USE OF THE SMALL STITCH TECHNIQUE IN LAPAROTOMY CLOSURE IN EMERGENCY SURGERY}

C.G. De Pedro $^{\text {I }}$, J.T. Gonzalez ${ }^{1}$, L. Tallón-Aguilar ${ }^{2}$, M. Flores Cortes ${ }^{I}$, M. Bustos Jimenez ${ }^{1}$, M.J. Tamayo López ${ }^{I}$, V. Duran Muñoz-Cruzado ${ }^{1}$, F. Pareja Ciuró ${ }^{1}$, S. Martínez Núñez ${ }^{1}$, L. Navarro Morales ${ }^{1}$

${ }^{1}$ CirugÍa General Y Digestiva, HOSPITAL UNIVERSITARIO VIRGEN DEL ROCIO, SEVILLA/SPAIN, ${ }^{2}$ Emergency Surgery Unit, Virgen del Rocío Universitary Hospital, Seville/SPAIN

Introduction: The classic technique of laparotomy closure is the Large Stitch technique that uses double-strand monofilament sutures with a SL/WL ratio of $4: 1$ and a separation of $1 \mathrm{~cm}$ between each point. Nowadays, the Small Stitch technique is accepted as the technique of choice in the closure of laparotomies in programmed surgery because the long-term incisional hernia rate is reduced, but there is very little experience in the field of surgical emergencies.

Material and methods: In our surgery service of Virgen del Rocío University Hospital, we are beginning to use the Small Stitch technique for the closure of laparotomies in Emergency Surgery. We use a very long-term monofilament absorbable synthetic suture of Poly-4hydroxybutyrate (Monomax) 2/0 with HR 26 needle. In the technique should be given at least 2 points for each wound $\mathrm{cm}$, with a distance to the alba line of $0^{\prime} 5 \mathrm{~cm}$ and $0^{\prime} 5 \mathrm{~cm}$ of separation between stitches. The closure starts from both ends, ending in the middle of the laparotomy with an overlap of at least $2 \mathrm{~cm}$. The ratio between the length of the suture and the length of the wound should be at least 4:1. Results: The small stitch technique can reduce the incisional hernia in Emergency Surgery.

Conclusion: The closure of laparotomy is one of the most important challenges facing the surgeon especially in the field of Emergency Surgery where the increased morbidity of the patient and the higher rate of surgical wound infection can play a fundamental role, and can be improved with the use of new abdominal wall closure techniques. References: Israelsson LA, Millbourn D. . Cost analysis of the use of small stitches when closing midline abdominal incisions. Hernia. 2014;18 (6):775-80. O’Dwyer PJ, Courtney CA. Factors involved in abdominal wall closure and subsequent incisional hernia. Surgeon. 2003 Feb;1 (1):17-22. Jenkins TP. The burst abdominal wound: a mechanical approach. Br J Surg. 1976 Nov;63 (11):873-6.

Disclosure: No significant relationships.

\section{PR065}

\section{ABDOMINAL EVISCERATION TREATED IN AN EMERGENCY SURGERY UNIT}

M.J. Tamayo-López ${ }^{I}$, J. Tinoco-González ${ }^{1}$, N. García-Fernández ${ }^{1}$, V. Durán-Muñoz-Cruzado ${ }^{l}$, A. Sánchez-Arteaga ${ }^{l}$, I. Alarcón-Del Agua ${ }^{2}$, F. Pareja-Ciuró ${ }^{2}$, L. Tallón-Aguilar ${ }^{1}$, J. Padillo-Ruíz ${ }^{3}$
${ }^{1}$ Emergency Surgery Unit, Virgen del Rocío Universitary Hospital, Seville/SPAIN, ${ }^{2}$ Digestive And General Surgery Unit, Virgen del Rocío Universitary Hospital, Seville/SPAIN, ${ }^{3}$ Hepatobiliary And Transplantant Surgery Unit, Virgen del Rocío Universitary Hospital, Seville/SPAIN

Introduction: Eviscerations need to be treated as quickly as posible to restore abdominal physiology, and accordingly, to reduce associated complications and mortality.

Material and methods: We analyzed 126 patients with evisceration treated in the Emergency Surgery Unit of Virgen del Rocío University Hospital, from January 2010 to July 2017. Evaluated variables were wound infection, precocity of treatment, closure techniques, use of mesh, need of ICU and mortality.

Results: All patients were diagnosed and treated in less than 12 hours from suspected diagnosis. Wound infection was present in 63 cases $(50 \%)$, in which mesh was not used. According to the wound infection classification: $1(0.8 \%)$ case was clean surgery, 44 were potentially contaminated surgery (34.9\%), 78 contaminated surgery and 3 infected surgery $(2.3 \%) .27$ patients $(21.4 \%)$ need intensive care after surgery. Mortality of the serie was $2.4 \%$. Using of mesh prosthesis were need in 52 cases $(41 \%)$, while $74(59 \%)$ were treated with simple closure and $7(5,5 \%)$ with Abthera system. We observed an increase in the use of mesh prosthesis from 2013 to 2017.

Conclusion: Our Emergency Surgery Unit manages to treat $100 \%$ of patients diagnosed in less than 12 hours. Primary closure was the most used, however, last years the use of mesh is increasing. Mortality in our serie is within the quiality standards.

References:

Disclosure: No significant relationships.

\section{PR066}

\section{RECURRENT INTUSSUSCEPTION IN CHILDREN}

\section{O.V. Karaseva}

Polytrauma, Clinical and Research Institute of Emergency Pediatric Surgery and Trauma, Moscow/RUSSIAN FEDERATION

Introduction: There is no single point of view on the management of children with recurrent intussusception.

Material and methods: The study included 13 children (boys $61.5 \%$, girls $-38.5 \%$ ) with recurrent ileocecal intussusception. All children were older than 1 year, the average age of children was $2.9 \pm$ 2.02 years. As a method of conservative treatment ultrasound-guided hydrostatic reduction of intussusception was used.

Results: Relapses of the disease were diagnosed in $11.4 \%$ of the total number of observations. In $92.3 \%$ of cases relapse occurred in the early period (up to 72 hours), in $7.7 \%$ - 1 month after conservative treatment. In the diagnosis of recurrence laparoscopy was performed, with the exception of 2 children, who were repeatedly performed with ultrasound-guided hydrostatic reduction of intussusception. At 3 children there was second relapse, and at 2 of them - after a laparoscopic reduction of intussusception. The anatomical cause was established in $30,7 \%$ (4) observations: at laparoscopy - in 3 cases (Meckel diverticulum-1, Burkitt's lymphoma-1, juvenile polyps of the cecum-1), at colonoscopy - in 1 (Burkitt's lymphoma) after relapse after laparoscopic reduction of intussusception. In the remaining observations lymphadenopathy of the ileocecal angle was identified and confirmed in the biopsy material. 
Conclusion: With a relapse of intussusception conservative treatment is contraindicated. Step-by-step use of laparoscopy and colonoscopy allows timely diagnosis of the cause of the disease and determine the scope of surgical treatment

References:

Disclosure: No significant relationships.

\section{PR067}

\section{MINIMALLY INVASIVE LAPAROSCOPIC TECHNOLOGIES IN THE TREATMENT OF PATIENTS WITH PERFORATED DUODENAL ULCER}

\section{O. Shepetko-Dombrovskyi, G. Shepetko-Dombrovskyi}

\section{Department Of Surgery №1, Kyiv City Clinical Emergency Care} Hospital, Kyiv/UKRAINE

Introduction: Aim of this study was to compare the result of open and laparoscopic repair of perforated peptic ulcers in terms of operation time, postoperative pain, hospital stay.

Material and methods: Clinical notes of 266 patients who underwent the operative closure of perforated peptic ulcers from 2007 to 2015 were available for study. The study of 2 group included 160 and 106 patients, who underwent of laparoscopic and open approach. Results were analyzed in time of operation, terms of requirement of analgesia, hospital stay, complications.

Results: Repair was done using simple suturing closure of the ulcer perforation and excision of perforated duodenal ulcer with duodenoplasty. There was no conversion to open in laparoscopic group. The mean operation time was less in laparoscopic versus open $(\mathrm{p}<$ $0,01)$. Post operative pain were less in laparoscopic group and number of analgesic injection given were 2 the corresponding figure in laparotomy were $5(\mathrm{p}<0,01)$. Total numbers of complication in laparoscopic repair were 3 compared to 11 in open, this was due to the size of the perforativ hole and ulcerous destruction of the walls of duodenum.

Conclusion: The use of minimally invasive laparoscopic technologies gave the possibility to improve the postoperative period for patients with perforated duodenal ulcer, namely the study group showed a decrease in the number of analgesics doses, postoperative dynamics of recovery, patient's stay in the hospital.

References: 1.A prospective randomized controlled trial of laparoscopic repair versus open repair for perforated peptic ulcers/[B. Ge, M. Wu, Q. Chen at al..]. //Surgery (United States). - 2016. - №159. P. 451-458. 2. Enhanced postoperative recovery pathways in emergency surgery: a randomised controlled clinical trial/[M. Gonenc, A. Dural, F. Celik et al.]. //The American Journal of Surgery. - 2014. №6. - P. 807-814. 3. Laparoscopic Finney pyloroplasty in the emergency setting: first case report in the literature and technical challenges [Electronic resource]/E.Moggia, P. G. Athanasopoulos, C. Hadjittof, S. Berti //Annals of Translational Medicine. - 2016. Access mode to resource: http://atm.amegroups.com/article/view/ 10244 .

Disclosure: No significant relationships.

\section{PR068}

\section{THE INCIDENCE AND REASONS FOR CONVERSION OF LAPAROSCOPIC CHOLECYSTECTOMY}

\author{
G. Rojnoveanu, I. Gagauz, R. Gurghis, M. Vozian
}

Surgery No.1 "nicolae Anestiadi", Nicolae Testemitanu State University of Medicine and Pharmacy, Chisinau/MOLDOVA

Introduction: Though it has many advantages, laparoscopic cholecystectomy is not risk-free and in difficult situations there is the need to convert to open cholecystectomy, the aim is to minimize the postoperative complications.

Material and methods: 51 cases of conversion to open cholecystectomy out of 2685 laparoscopic cholecystectomies performed within the Institute of Emergency Medicine between 2010-2016. The $\mathrm{F} / \mathrm{M}$ ratio was $24 / 27$, the mean age was $60.15 \pm 1.66$ years. The analyzed criteria were: time period between the onset of acute cholecystitis and operation, the duration of cholelithiasis history, intraoperative morphologic status and the results of histopathologic examination.

Results: Emergency interventions were performed in 39 (7.47\%) cases, elective $-12(23.52 \%)$. The rate of conversion was $1.89 \%$. In 41 $(80.39 \%)$ cases the conversions were deliberate, while conversions of necessity in $10(19.6 \%)$ cases, $\mathrm{p}<0.01$. The factors that led to deliberate conversion were: biliary fistulas $-11(21.56 \%)$, inflammatory mass -9 $(17.64 \%)$, gangrenous cholecystitis - $9(17.64 \%)$, scleroatrophic gallbladder - $4(7.84 \%)$, purulent cholangitis - $2(3.92 \%)$, choledocholithiasis - 1 (1.96\%), pancreonecrosis - 1 (1.96\%), chronic duodenal ulcer penetrated in the gallbladder $-1(1.96 \%)$. Conversion of necessity were imposed by: iatrogenies $-4(7.84 \%)$, hemorrhage -3 $(5.88 \%)$, bile leakage $-3(5.88 \%)$. The results of histopathologic examinations revealed: acute inflammation $-27(52.94 \%)$, chronic -22 $(43.13 \%)$, adenocarcinomas $-2(3.92 \%)$ cases.

Conclusion: The deliberate causes were the main reasons for conversion to open cholecystectomy. We consider that surgical attention should be focused on the remaining $20 \%$ of cases of imposed conversions. The assessment of morphological particularities and surgical possibilities in these cases would minimize the intraoperative incidents and postoperative complications.

References:

Disclosure: No significant relationships.

\section{PR069}

\section{GOOD, BUT NO PERFECT: ULTRASOUND IN THE EMERGENCY DEPARTMENT AVERTS THE NEED FOR CT IN MOST PATIENTS BUT DOES NOT IMPROVE TIME TO SURGERY AND THE INCIDENCE OF COMPLICATED APPENDICITIS}

\section{Ashkenazi ${ }^{1}$, A. Zeina ${ }^{2}$, R. Alfici ${ }^{3}$, O. Olsha ${ }^{4}$}

${ }^{1}$ Not Applicable, Hillel Yaffe Medical Center, Hadera/ISRAEL, ${ }^{2}$ Radilogy Department, Hillel Yaffe Medical Center, Hadera/ ISRAEL, ${ }^{3}$ Surgery Department, Hillel Yaffe Medical Center, Hadera/ ISRAEL, ${ }^{4}$ Surgery Department, Shaare Zedek Medical Center, Jerusalem/ISRAEL

Introduction: We hypothesized that the increased use of early ultrasound done during the first 6 hours from admission to the 
emergency department would hasten surgery and decrease the the incidence of complicated appendicitis (gangrene with or without perforation).

Material and methods: Retrospective study of patients having surgery for acute appendicitis from December 2006 to December 2016. Patients undergoing early ultrasound were compared to patients undergoing CT within six hours and to patients without any preoperative imaging.

Results: 2427 patients were included of which 1573 underwent ultrasound. The proportion of patients undergoing preoperative imaging increased from $14.9 \%$ in 2007 to $100 \%$ in 2016 , mostly due to increased use of preoperative ultrasound. The proportion of negative appendectomies decreased from $10.5 \%$ to $5.0 \%(\mathrm{p}=0.0031)$. Increased use of ultrasound was not associated with a real decrease in proportion of patients operated beyond 12 and 24 hours from admission ( $\mathrm{p}=0.5270$ and $\mathrm{p}=0.6903$ respectively). Preoperative imaging in the emergency department did not impact on the proportion of complicated appendicitis compared to no preoperative imaging $(\mathrm{p}=0.166)$. In 1475 appendicitis patients evaluated with ultrasound, this was diagnostic of acute appendicitis in $80.5 \%$. Patients with negative or inconclusive ultrasound had delays in surgery and an increased incidence of complicated appendicitis $(\mathrm{p}<0.001)$.

Conclusion: Ultrasound averts the need for CT in most of the patients with acute appendicitis. While reducing the number of negative appendectomies, ultrasound does not appear to reduce the proportion of patients with complicated appendicitis. Complicated appendicitis is in part due to delays in surgery following negative or inconclusive ultrasound.

References:

Disclosure: No significant relationships.

\section{PR070}

\section{BLUNT TRAUMATIC ABDOMINAL WALL HERNIA - IS IT MANDATORY TO OPERATE?}

\section{R. Bar Haim, Z. Bramnik, I. Waksman}

General Surgery, galillee medical center, naharia/ISRAEL

Introduction: Traumatic abdominal wall hernias resulting from blunt trauma are uncommon, accounting for only $1 \%$ of all blunt trauma admissions. $(1,2,3,4)$ Reviewing the literature, it is seen that there are only small series and few case reports, and there is no consensus on the treatment, the timing of repair and whether repair is actually needed. (5)

Traumatic abdominal wall hernias have a high rate of associated injurie. The probability of intra-abdominal injuries is key to the decision of operative intervention.

We present our experience of three cases of blunt traumatic abdominal wall hernia which were diagnosed only on CT scan, and were managed differently in accordance with CT scan findings.

Material and methods: Our experience with 3 trauma patients that where diagnosed with traumatic abdominal wall hernias, that we had to operate on two of them, have presented a challenge in the abdominal wall reconstruction point of view.

Results: Two of the patients undergone surgery - and were operated on the additional injuries (devitalized bowel, vascular tear), and on the same operation the hernia was repaired. The repair was layered and used mesh.

One patient had a conservative management, with serial clinical examination, hemoglobin level follow-up, and after 24 hours observation, was allowed to eat, and after total of 72 hours of observation, was discharged to ambulatory follow-up in the outpatient clinic. Without any additional abdominal complains.

Conclusion: We believe that whenever there is a risk to hollow viscous these patients should be operated immediately and we believe that repair of abdominal wall defect with mesh should be performed if feasible and the patient is stable.

References: Reference Netto FA, Hamilton P, Rizoli SB, Nascimento BN, Brenneman FD, Tien H, Tremblay LN. Traumatic abdominal wall hernia: epidemiology and clinical implications. J Trauma. 2006; 61:1058-1061. Brenneman FD, Boulanger BR, Antonyshyn O. Surgical management of abdominal wall disruption after blunt trauma. J Trauma. 1995; 39:539-544. Burt BM, Hazem YA, Wantz GE, Barie PS. Traumatic lumbar hernia: report of cases and comprehensive reviewof the literature. J Trauma. 2004; 57:1361-1370. Belgers HJ, Hulsewe KW, Heeren PA, Hoofwijk AG. Traumatic Abdominal Wall Hernia: Delayed Presentation in Two Cases and a Review of the Literature. Hernia 2005; 9:388-391. Drew Honaker, John Green. Blunt traumatic abdominal wall hernias: Associated injuries and optimal timing and method of repair. J Trauma Acute Care Surg. 2014; 77 (5): 701-704 Esposito TJ, Fedorak I. Traumatic lumbar hernia: case report and literature review. J Trauma. 1994; 37:123-126. Traumatic anterior abdominal wall hernia: A report of three rare cases. J Emerg. Trauma Shock. 2011; 4 (1): 142-145. Shiomi H, Hase T, Matsuno S, Izumi M, Tatsuta T, Ito F, . Handle bar hernia with intra-abdominal extra luminal air presenting as novel form of traumatic abdominal wall hernia: report of a case. Surgery Today. 1999; 29: 1280-1284. Wood RJ, Ney AL, Bubrick MP. Traumatic abdominal hernia: a case report and review of the literature. Am Surg. 1988; 54:648-651. Liasis L, Tierris I, lazarioti F, Clark CC, Papaconstantinou HT. Traumatic abdominal wall hernia: is the treatment strategy a real problem? J Trauma Acute Care Surg. 2013; 74: 1156-1162. Lane CT, Cohen AJ, Cinat ME. Management of traumatic abdominal wall hernia. Am Surg. 2003; 69:73-76. Mahajna A, Ofer A, Krausz MM. Traumatic abdominal hernia associated with large bowel strangulation: case report and review of the literature. Hernia. 2004; 8:80-82. Gill IS, Toursarkissian B, Johnson SB, Kearney PA. Traumatic ventral abdominal hernia associated with small bowel gangrene: case report. J Trauma. 1993; 35:145-147

Disclosure: No significant relationships.

\section{PR071}

\section{THE LONG TERM OUTCOME OF OPEN ABDOMEN MANAGEMENT IN JAPAN}

\author{
K. Sekiya ${ }^{1}$, M. Suzuki ${ }^{1}$, Y. Otomo ${ }^{2}$
}

${ }^{1}$ Trauma And Acute Critical Care Medical Center, Tokyo Medical and Dental University Hospital of Medicine, Tokyo/JAPAN,

${ }^{2}$ Emergency And Disaster Medicine, Tokyo Medical and Dental University Hospital, Tokyo/JAPAN

Introduction: Recently, development of temporary abdominal closure (TAC) improved the mortality of patients who were performed open abdomen management (OAM). Although, it is not clear whether OAM also improves long term outcome?

Material and methods: We retrospectively reviewed records of OAM patients from April 2007 to March 2015, and researched the incidence of abdominal wall hernia, adhesive intestinal obstruction, mortality by telephone and letter survey.

Results: From April 2007 to March 2015, total of 79 patients were underwent OAM. 59.5\% $(\mathrm{n}=47)$ patients survived $23.4 \%(\mathrm{n}=11)$ were trauma, $76.6 \%(n=36)$ were non-trauma patients. All patients were 
underwent TAC, 3 patients were skin closure, 14 patients were silo closure and 30 patients were vacuum closure. $78.7 \%(n=37)$ succeeded typical primary fascial closure (PFC) without using mesh. On the other hand, $21.3 \%(n=10)$ failed PFC. Among the 10 patients, 3 patients achieved ventral hernia, 7 patients were performed our unique technique named the anterior rectus abdominis sheath turnover flap method. Furthermore, we researched complications after the surgery of 2-5 years and 6-9 years. In the period of 2-5 years after surgery, compared with typical PFC and anterior rectus abdominis sheath turnover flap method, morbidity and mortality were not change, significantly. In our study, by comparing periods and method of fascial closure, we want to reveal which incidence affect patients' life after the OAM..

Conclusion: In short term, we achieved about $80 \%$ PFC, without using mesh and special devices. This time, we will show both the long term outcome in Japan, and advantage of the anterior rectus abdominis sheath turnover flap method.

References:

Disclosure: No significant relationships.

\section{PR072}

\section{CAUSTIC INGESTION INJURIES, ¿IS A PROBLEM FOR THE SURGEON?}

\section{Pérez-Santiago, M. Garcés-Albir, M.D.C. Fernandez-Moreno, R. Martí-Obiol, F. Lopez-Mozos, J. Ortega}

Cirugia General Y Del Aparato Digestivo, Hospital Clinico Universitario, Valencia/SPAIN

Introduction: Injury from caustic ingestion often occurs involuntarily or as an attempted suicide. Anamnesis, clinical manifestations and endoscopy are essential for assessing the severity of the ingestion that will sometimes require emergency surgery.

Material and methods: A retrospective study of caustic ingestion injuries was carried out in a tertiary hospital between January 2011 and December 2016.

Results: A total of 67 patients were diagnosed in the study period. The mean age was 49years (range17-84), 46 (68.7\%) women and 21 (31.3\%) men.An endoscopy was performed on 63 (94\%) patients. The classification by endoscopy of the lesions was: ZargarI $(50,74.6 \%)$, ZargarIIA (4, 5,9\%), ZargarIIB (2, 2,9\%), ZargarIIIA (2, 2,9\%) and ZargarIIIB $(5,7.5 \%)$. Patients with a ZargarI classification were discharged from the hospital with home surveillance. Of the 13 patients with a Zargar classification major of II: 7 (53,8 \%) were admitted to Intensive Care, $4(30,7 \%)$ in the Digestive Unit and 2 $(15,5 \%)$ in the Psychiatry Unit. The5 patients with the diagnosis of ZargarIIIB required emergency surgery, and $3(60 \%)$ died as a result of surgical complications. The two patients who survived emergency surgery underwent reconstructive surgery in the subsequent months. A coloplasty was performed in both cases without major complications. Of the 62 patients who did not require surgical intervention, 5 $(7,4 \%)$ required several sessions of endoscopic esophageal dilations due to stenosis.

Conclusion: Endoscopy is essential in the diagnosis and subsequent treatment of the complications of these patients. There is a good correlation between the grades of the Zargar classification and the prognosis of the injuries. Most cases do not require hospital admission; instead, when they require an emergent surgery, mortality increases exponentially.

References:

Disclosure: No significant relationships.

\section{PR073}

\section{TREATMENT OPTIONS IN CATASTROPHIC ACUTE ABDOMEN}

\author{
B. Ugarte-Sierra ${ }^{l}$, A. Landaluce-Olavarria ${ }^{l}$, S. Postigo-Morales ${ }^{2}$, \\ M. Arzuaga-Esquino ${ }^{2}, F . j$. Ibáñez-Aguirre ${ }^{l}$
}

${ }^{1}$ Surgery, GALDAKAO HOSPITAL, GALDAKAO-USANSOLO/
SPAIN, ${ }^{2}$ Anaesthesia, GALDAKAO HOSPITAL, GALDAKAO-
USANSOLO/SPAIN

Introduction: INTRODUCTION. The catastrophic acute abdomen implies a challenge for a surgeon. Ideally it should be prevented detecting early the complications. There is no scientific evidence gathered about how to approach those cases where an acute abdomen appears.

Material and methods: MATERIAL AND METHODS. Retrospective study on the catastrophic acute abdomen cases treated in our department from November 2011 to November 2016. A total of 27 patients were included. The variables analysed in the study were: preoperative predicted mortality (P-Possum and Manheim's peritonitis index), catastrophic abdomen cause, use of negative pressure therapy, number of replacements, primary wall closure, use of retraction mesh, postoperative eventration occurrence, and mortality after 30 days. The acute abdomen causes were as follows. 3 patients (11.1\%) evisceration, 2 patients $(7.4 \%)$ abdominal compartment syndrome, 1 patient $(3.7 \%)$ intestinal ischemia, 1 patient $(3.7 \%)$ hypovolemic shock, 1 patient $(3,7 \%)$ gunshot wound to the abdomen, 6 patients $(22.2 \%)$ anastomotic dehiscence, 2 patients $(7.4 \%)$ acute infection of soft parts and 11 patients $(40.7 \%)$ abdominal sepsis caused by acute peritonitis. The study was divided into: use of damage control surgery on patients with septic shock of abdominal origin, abdominal wall closure, suprafascial space treatment, and treatment of the intestinal fistulae in hostile abdomen.

Results: RESULTS. The preoperative mortality was $42.3 \%$. The postoperative mortality was $11.1 \%$. This means a decrease over $50 \%$. The replacements media was 3.88 (1-11), being higher in those patients for whom primary closure was not possible. A primary closure was performed in 22 patients $(81.5 \%)$. In 5 patients $(18.51 \%)$ primary closure failed. In 2 patients a kugel mesh was placed and in other 2 a retraction mesh. In every case the borders separation was higher than 10 centimetres. 3 patients $(11.1 \%)$ suffered from postoperative eventration. In the beginning of the series, the suprafascial space handle was carried out by large section suction drainages but they got obstructed from the 15th postoperative day on. In a second phase of the series, negative pressure therapy was used, obtaining a reduction higher than $90 \%$ of the suprafascial space. The intestinal fistulae in the hostile abdomens were handled step by step attending to the intestinal debt: negative pressure therapy, negative pressure therapy + guided fistula, negative pressure therapy + Ring technique and negative pressure therapy+ ileostomy bag fixed to the abdomen with Corega ${ }^{\circledR}$. In those patients with a high intestinal debt, this last option managed the local control of the fistula and to avoid the sepsis. Conclusion: CONCLUSIONS. 1. The damage control surgery in patients with septic shock decreases mortality notoriously. 2. The retraction mesh associated with a negative pressure system helps to close the wall. 3. Suprafascial space decreases dramatically with the negative pressure therapy.4. The intestinal fistulae in the hostile abdomen can be handled successfully with the use of Corega ${ }^{\circledR}$ together with the negative pressure therapy.

References: Reider, Kersten E. Fistula Isolation and the Use of Negative Pressure to Promote Wound Healing: A Case Study. Journal of Wound, Ostomy, \& Continence Nursing. 44 (3):293-298, 2017 May/Jun Bobkiewicz, Adam; Walczak, Dominik; Smolinski, 
Szymon; Kasprzyk, Tomasz; Studniarek, Adam; Borejsza-Wysocki, Maciej; Ratajczak, Andrzej; Marciniak, Ryszard; Drews, Michal; Banasiewicz, Tomasz. Management of enteroatmospheric fistula with negative pressure wound therapy in open abdomen treatment: a multicentre observational study. International Wound Journal. 14 (1):255-264, 2017 Feb Nicodemi, Sara; Corelli, Sergio; Sacchi, Marco; Ricciardi, Edoardo; Costantino, Annarita; Di Legge, Pietro; Ceci, Francesco; Cipriani, Benedetta; Martellucci, Annunziata; Santilli, Mario; Orsini, Silvia; Tudisco, Antonella; Stagnitti, Franco. Recurrent incisional hernia, enterocutaneous fistula and loss of the substance of the abdominal wall: plastic with organic prosthesis, skin graft and VAC therapy. Clinical case. Annali Italiani di Chirurgia. 86 (2):172-6, 2015 Mar-Apr. Layton, Brian; Dubose, Joe; Nichols, Shawnn; Connaughton, James; Jones, Tracy; Pratt, Jerry. Pacifying the open abdomen with concomitant intestinal fistula: a novel approach. American Journal of Surgery. 199 (4):e48-50, 2010 Ap Disclosure: No significant relationships.

\section{PR074}

\section{EXPERIENCE IN ABDOMINAL WALL CLOSURE IN CATASTROPHIC ABDOMEN IN AN EMERGENCY SURGERY UNIT}

M.J. Tamayo-Lopez ${ }^{l}$, J. Tinoco-González ${ }^{1}$, N. García-Fernández ${ }^{l}$, V. Durán-Muñoz-Cruzado ${ }^{I}$, A. Sánchez-Arteaga ${ }^{l}$,

I. Alarcón-Del Agua ${ }^{2}$, F. Pareja-Ciuró ${ }^{2}$, L. Tallón-Aguilar ${ }^{1}$,

J. Padillo-Ruíz ${ }^{3}$

${ }^{1}$ Emergency Surgery Unit, Virgen del Rocío Universitary Hospital, Seville/SPAIN, ${ }^{2}$ Digestive And General Surgery Unit, Virgen del Rocío Universitary Hospital, Seville/SPAIN, ${ }^{3}$ Hepatobiliary And Transplantant Surgery Unit, Virgen del Rocío Universitary Hospital, Seville/SPAIN

Introduction: Early closure of catastrophic abdomen is difficult because the possibility of producing compartimental syndrome, abdominal wall infection or loss of abdominal wall substance. However our goal should be getting it in the first 7-10 days.

Material and methods: Sixty patients were treated in the Emergency Surgery Unit of Virgen del Rocío University Hospital, from January 2010 to July 2017. Evaluated variables were survival, causal pathology, closure methods, hospitalization time and death cause.

Results: This serie included 13 cases $(21.7 \%)$ in $2010,4(6,7 \%)$ in 2011, $5(8,3 \%)$ in 2012 and 2013, $3(5 \%)$ in 2015, $10(16,7 \%)$ in 2016 and $12(20 \%)$ in 2017 . Thirty five of them $(58.3 \%)$ were males. Causal pathologies were polytrauma in 13 cases $(21.7 \%)$, anastomotic leakage in $9(15 \%)$, severe acute pancreatitis in $6(10 \%)$, abdominal evisceration in $3(5 \%)$ and miscellaneous causes in the other 29 cases. Closure techniques employed were Abthera and VAC systems in 36 patients $(68 \%)$, skin graft in $1(2 \% \%)$, Leppäniemi in $3(5,8 \%)$ and hernia reconstruction using mesh in $48(92 \%)$. Nineteen pacients $(31.6 \%)$ death because of progress of the causal patohlogy, such as pancreatits in 3 patients, abdominal evisceration in 3, trauma in 1 and abdominal wall tumor in the other one. The complete abdominal fascial closure was getting in all of survivors (41 patients, 68,4\%), after controlling primary pathology or derived complications.

Conclusion: Primary closure was not posible in any patient, requering progresive closure in all cases, with a global survival of $68 \%$.

References:

Disclosure: No significant relationships.

\section{PR075}

\section{MULTICENTER STUDY OF THE OPEN ABDOMEN IN SPAIN}

M. Jiménez Fuertes ${ }^{1}$, F. Turégano Fuentes ${ }^{2}$, J.M. Jover Navalón ${ }^{3}$,

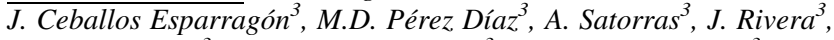
R. Gómez Pérez ${ }^{3}, F$. Fernández García ${ }^{3}$, I.M. Goena Iglesias ${ }^{3}$, S. Linacero Martín ${ }^{3}$, A. Gómez De La Portilla , F. García-Moreno Nisa ${ }^{3}$, R.M. Jiménez Rodríguez, C. Rey Varcalcel ${ }^{3}$, M. Prieto Calvo ${ }^{3}$, C. Olona Casas ${ }^{3}$, M. Alvite Canosa ${ }^{3}$, L. Delgado Plasencia ${ }^{3}$,

J. Ruiz-Tovar ${ }^{3}$, Y. Sosa Quesada ${ }^{3}$, Z. Madrazo ${ }^{3}$, F.M. González Valverde $^{3}$, M. Ruiz Marín ${ }^{3}$, A. Landaluce Olavarría ${ }^{3}$, B. Ugarte ${ }^{3}$, M. Beltrán ${ }^{3}$, C. González Gómez $z^{3}$, J.T. Castell Gómezz, I. Rey Simó ${ }^{3}$, I. Isabel Grifo Albalat ${ }^{3}$, M. Bruna ${ }^{3}$, A. Rodríguez Padilla ${ }^{3}$, L. Ponchietti ${ }^{3}$, P. Fernández Balaguer ${ }^{3}, M$. Caballero ${ }^{3}$, P. Trillo Parejo ${ }^{3}$, M.D.C. Alvarez Sánchez ${ }^{3}$, F.J. Sánchez Manuel ${ }^{3}$, M.L. De La Hoz ${ }^{3}$ M.A. García Ureña ${ }^{3}$, P. Jiménez Morillas ${ }^{3}$, J.C. Magdalena López ${ }^{3}$, J.j. Espert Ibáñez ${ }^{3}$, I. Cendoya ${ }^{3}$, F. Mata Sancho ${ }^{3}$, P. Patricia Pérez De Villarreal ${ }^{3}$, J. Aguiló Lucía ${ }^{3}$, A. Roig Bataller ${ }^{3}$, A. Etxart Lopetegi ${ }^{3}$, E. Lopez De Heredia

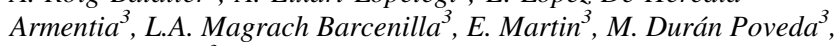
D. García Olmo ${ }^{3}$

${ }^{1} \mathrm{Hpb}$ Surgery. Emergency Surgery, Rey Juan Carlos University Hospital, Móstoles/SPAIN, ${ }^{2}$ Cirugia General Y Del Ap. Digestivo, Hospital General Universitario Gregorio Marañón, Madrid/SPAIN, ${ }^{3}$ Aec, Proyecto ABAB, Mostoles/SPAIN

Introduction: Leaving the abdominal cavity open for longer than necessary outside of the operating room is not something desirable for either patient or surgeon. This study aim is to evaluate the rate of primary fascial closure (PFC) and the rate of complications among patients who undergo open abdomen, by the type of TAC used in our country. Studied as secondary objectives were the clinical/epidemiological characteristics, the techniques used for the TAC and final closure, complications and the factors which affect PFC and the occurrence of complications.

Material and methods: A prospective multicenter observational cohort study of patients treated using temporary abdominal closure from January 2014 through July 2015. The inclusion criteria were as follows: patients 18 years old or older, men and non-pregnant women, with no prior eventrations or loss of any portion of the abdominal wall, who underwent temporary abdominal closure within the context of damage control surgery in trauma or non-trauma event (severe abdominal infection, need for re-evaluation or the patient's hemodynamic instability) or as a treatment for abdominal compartmental syndrome or sustained IAH.

Results: In all, the study included 292 patients treated with TAC at 37 hospitals Factors which affect primary fascial closure: PFC was achieved in $51.4 \%$ of patients, though the rate was 1.4 times greater when active techniques were used.

Conclusion: Active temporary abdominal closure methods are associated with higher rates of fascial closure and fewer complications when compared with passive techniques used in our country.

References:

Disclosure: No significant relationships. 


\section{PR076}

\section{ENCAPSULATED PERITONEAL SCLEROSIS - AN ABDOMINAL DRAMA ASSOCIATED WITH ACUTE ABDOMEN IN PATIENTS WITH PERITONEAL DYALISIS}

\author{
C.R. Iorga, V. Strambu, A. Aldea, C. Iorga, P.A. Radu, M. Zurzu \\ I. Bengulescu
}

General Surgery, carol davila hospital bucharest, bucharest/ ROMANIA

Introduction: Encapsulated peritoneal sclerosis is a clinico-morphological entity that is associated with long term peritoneal dyalisis. it significantly influences survival in patients with chronical kidney disease, even if is not characteristic for these patients.

Material and methods: The study, retrospective, was conducted in Surgery Hospital "Dr. Carol Davila “, between 2011 and 2017. 60 patients with extraction of peritoneal dialysis catheters and 30 patients with placement of this catheter were enrolled, 7 of them with various stages of EPS.

Results: EPS represents the wrapping of the entire intestine in a fibrous capsule, characterized by peritoneal hypertrophy, degenerative sclerosis of the capillars and capillary neo-angiogenesis. It usually occurs in more than two years after the initiation of peritoneal dialysis. Surgical treatment of EPS is preponderant in emergency due to haemoperitoneum, perforations or occlusions. We tried ablation of the fibrotic capsule, which was accompanied with bleeding due to rupture of large vessels and intestinal perforations. Other interventions mentioned are limited intestinal resections with anastomosis, multiple incisions of fiber membrane for stress relief and suture of the perforations.

Conclusion: EPS is a surgical challenge by itself. It is oftened diagnosed as an acute abdomen, putting the surgeon in face with a real problem : what should I do? Hemoperitoneum in EPS represents an aggravating factor in evolution of the patient with peritoneal dialysis, representing both a marker of occurance of sclerosys and a complication of surgical treatment. Mortality is high, perioperatively and after a long time, by continuing the process of fibrosis despite stopping the peritoneal dialysis and transition to hemodialysis

References: 1. Oran Ebru et all - Encapsulating peritoneal sclerosis as a late complication of peritoneal dialysis. Ann Med Surg, sep 20152. Kitamura M. et all - New insights into therapeutic strategies for the treatment of peritoneal fibrosis: learning from histochemical analyses of animal models.Acta Histochem Cytochem aug 2014 3. Koc Y et all - Is there impact of mortality prior hemodialysis therapy in peritoneal dialysis patients? Nefrologia, may 2012

Disclosure: No significant relationships.

\section{PR077}

\section{NEGATIVE PRESSURE THERAPY WITH INTRAPERITONEAL SALINE INSTILLATION IN THE OPEN SEPTIC ABDOMEN}

\author{
M. Jiménez Fuertes ${ }^{1}$, M. Durán Poveda ${ }^{1}$, D. García Olmo ${ }^{2}$
}

${ }^{1} \mathrm{Hpb}$ Surgery. Emergency Surgery, Rey Juan Carlos University Hospital, Móstoles/SPAIN, ${ }^{2}$ Hpb Surgery. Emergency Surgery, Fundación Jimenez Díaz, Móstoles/SPAIN

Introduction: Open abdomen has become a common technique in the management of abdominal sepsis. We present a series of cases of 12 open septic abdomen treated by using intermittent therapy with intraabdominal irrigation with a modified VAC- Ulta ${ }^{\circledR}$ associated with ABThera ${ }^{\circledR}$ dressing, comparing with ABThera ${ }^{\circledR}$ dressing suction therapy alone.

Material and methods: Pilot prospective study of 12 consecutive patients with temporary abdominal closure for abdominal sepsis. During the initial laparotomy washing of the peritoneal cavity was performed with warm saline. Six patients (Goup 1) were treated by KCI ABThera ${ }^{\circledR}$ dressing, and keeping constant suction pressure -125 $\mathrm{mmHg}$. For the second group (Group 2) the mentioned dressing was used, and we performed also intermittent intra-abdominal instillation with modified VAC-Ulta ${ }^{\circledR}$.

Results: Primary fascial closure was achieved in $100 \%$ of patients with instillation therapy, 4 of them in the first change after 3 days, and the other 2 patients in the second change (6th day). In the group 1, only 3 of them were closed (at 3th, 6th and 11th day). Two of them remained with the open abdomen and the other one died. In patients with instillation therapy, there were no intra-abdominal complications after closure.

Conclusion: The results obtained by combining the intra-abdominal instillation therapy with suction seem to be promising, regarding the rate of abdominal closure, reducing the time to primary fascial closure and consequently in fewer complications associated with open abdomen.

References: Moore-Olufemi SD, Xue H, Allen SJ, Moore FA, Stewart RH, Laine GA, Cox CS: Effects of primary and secondary intraabdominal hypertension on mesenteric lymph flow: implications for the abdominal compartment syndrome. Shock 2005; 23:571-575. Amin AI, Shaikh IA: Topical negative pressure in managing severe peritonitis: a positive contribution? World J Gastroenterol 2009, 15 (Suppl 27): 3394-3397 Rev Gastroenterol Peru. 2010; 30: 211-5. Gabriel A, Shores J, Heinrich C et al. Negative pressure wound therapy with instillation: a pilot study describing a new method for treating infected wounds. Int Wound J. 2008 5:399-413

Disclosure: No significant relationships.

\section{PR078}

\section{CERVICAL ABSCESS: DIFFERENTIAL ASPECTS BETWEEN COMMUNITY AND NOSOCOMIAL INFECTION}

\author{
S.T. Arieta-Araunabeña ${ }^{l}$, J.J. Fraile ${ }^{1}$, L. Grande-Posa ${ }^{2}$, \\ E. Membrilla-Fernández ${ }^{3}$, J.A.P. Rodriguez ${ }^{3}$, J. Sancho-Insenser ${ }^{3}$
}

${ }^{1}$ Cirugía General Y Aparato Digestivo, Hospital del Mar, Barcelona/ SPAIN, ${ }^{2}$ General Surgery, Hospital del Mar, Barcelona/SPAIN, ${ }^{3}$ Emergency Unit. General Surgery Service., Hospital del Mar, Barcelona/SPAIN

Introduction: Cervical abscesses (CA) are uncommon and they usually require surgical debridement and broad spectrum empiric antibiotics administration. AIM: It describes the source and microbiological aspects of CA and their postoperative course depending on the type of infection.

Material and methods: Retrospective study including cervical abscess patients from 2006 to 2015. The origin of the abscess, microbiology and evolution was assessed.

Results: 24 CA patients were operated: $13(54.2 \%)$ of their had a community infection (CI) and $11(54.8 \%)$ nosocomial origin (NI). In the CI group, the infection source was indeterminate in 5, ENT infection in 4, traumatic wound in 3 and neoplasia related in 1 . In NI patients, the predominate origin was a surgical operation. All patients required surgical debridement. Eight of then required reoperations for 
source control. In CI patients, infections were predominantly produced by coco Gram-positive $(69.3 \%)$ and in 3 patients the cultures were negative. In NO patients cultures, bacilli gram-negative isolation predominated $(54.5 \%)$ and in 4 of them cultures were negative. Three patients died due to neoplasm progression and none due to cervical infection. The complications rate, readmissions and reoperations was similar in both groups.

Conclusion: In the CI, the inability to find the origin of the infection focus is frequent when the ENT focus has been discard. Regarding empirical antibiotic therapy, it is important to think about the CA source to choose the treatment, given the divergence in the abscess microbiology.

References:

Disclosure: No significant relationships.

\section{PR079}

\section{CLASSICAL AND MODERN PRINCIPLES IN THE MANAGEMENT OF ABDOMINAL TRAUMA}

D. Cartu, A.M. Goganau, M. Radulescu, S.I. Bordu, M.C. Lazar, M.L. Manea, S.C. Mucenic, E.F. Georgescu, V. Surlin

First Surgical Clinic, Emergency Clinical Hospital Craiova, CRAIOVA/ROMANIA

Introduction: . Abdominal trauma is a major cause of morbidity and mortality and their management has experienced significant changes in the last years.

Material and methods: Material and method. This retrospective study was performed on a sample of 142 patients with blunt abdominal trauma $(70.4 \%)$ and penetrating abdominal trauma (29.6\%) on a period of 10 years (2007-2016).

Results: . The leading cause of abdominal trauma was represented by road accidents $(27.5 \%)$, followed by individual aggression $(23.2 \%)$. In $54.2 \%$ of cases were associated injuries of other anatomical areas. In the last years has gained ground the CT performed in the emergency area $(61.8 \%$ in the last 5 years vs $20.3 \%$ in the first part of the study). The "FAST" ultrasound has gained ground, being used to $1 / 3$ of the cases $(33.1 \%)$. The non surgical management of parenchymatous organs injuries has been introduced in our service in the last 6 years, $5.3 \%$ of spleen injuries and $23.1 \%$ of liver injuries grade I and grade II have been treated conservatively. Laparoscopic approach has been used on 4 cases: 2 diagnostic laparoscopies, a hepatorrhaphy and a splenectomy for a ruptured hematoma. The morbidity and mortality in our clinic were low (7.0\% and $2.1 \%)$.

Conclusion: s. The modern algorithm of associated diagnosis with the current therapeutic principles can lead to avoidance of unnecessary laparotomies, improving at the same time the results of the management of this group of diseases.

References:

Disclosure: No significant relationships.

\section{INFECTION}

\section{PR080}

\section{EFFECT OF MELATONIN INTRAVENOUS ADMINISTRATION ON IMMUNOLOGICAL RECOVERING IN SEPTIC PATIENTS AFTER ABDOMINAL SURGERY}

P. Bueno-Larano ${ }^{1}$, C. Plata-Illescas ${ }^{2}$, P. Notario-Fernandez ${ }^{3}$, A. Vico-Arias ${ }^{2}$, D. Acuña-Castroviejo ${ }^{4}$, G. Escames-Rosa ${ }^{4}$, A. Comino-Pardo ${ }^{1}$, A. Mansilla-Rosello ${ }^{2}$

${ }^{1}$ Experimental Surgery Unit, SAS, Granada/SPAIN, ${ }^{2}$ General And Digestive Surgery, Hospital Virgen de las Nieves (SAS), Granada/ SPAIN, ${ }^{3}$ General And Digestive Surgery, Hospital Virgen de las Nieves (SAS), GRANADA/SPAIN, ${ }^{4}$ Biochemical Research Center, Granada University, Granada/SPAIN

Introduction: Melatonin is an hormone involved in multiple physiological processes. The purpose of this study has been to evaluate the effect of melatonin injectable under patent determining effects on blood and biochemical parameters in septic patients after abdominal surgery.

Material and methods: Prospective, randomized, double-blind clinical trial (EUDRACT 2008-006782-83) was performed in 30 septic patients after abdominal surgery. Patients were randomly assigned to 2 study groups, Melatonin and placebo. Melatonin-treated group patients $(\mathrm{n}=15)$ received conventional treatment and melatonin as an injectable under patent (ES2392903B1) at a dose of $40 \mathrm{mg} /$ day/5 days. Placebo group $(\mathrm{n}=15)$ received the conventional treatment. Treatment started $12 \mathrm{~h}$ after surgery and ended 5 days later. Peripheral blood samples were obtained before surgical intervention (T0) and $24 \mathrm{~h}$ after treatment (T1) until day 5 (T5). Blood samples were obtained to determinate leukocytes (neutrophils and lymphocytes percentage), erythrocytes, platelets and biochemical determinations (AST, ALT, GGT, creatinin, urea, alkaline phosphatase, and LDH).

Results: show differences in lymphocytes percentage, being enhanced in melatonin-treated patients compared to placebo group at $48 \mathrm{~h}$ and $72 \mathrm{~h}$ of treatment initiation (times T2 and T3). Concerning neutrophils percentage, there are differences between groups being reduced in melatonin-treated compared to placebo group. A decrease in leukocyte levels in both groups of study was also observed. Melatonintreated patients showed a difference in red blood cells number as well as in hemoglobin and hematocrit values at time T3 after starting treatment. Conclusion: These data are an indication of a positive immunological recovering in septic patients treated with a melatonin injectable.

References:

Disclosure: No significant relationships.

\section{PR081}

\section{INTRAVENOUS MELATONIN. NEW ROUTE OF ADMINISTRATION}

\author{
A. Mansilla-Rosello ${ }^{l}$, C. Plata-Illescas ${ }^{l}$, A. Vico-Arias ${ }^{l}$, \\ P. Notario-Fernandez ${ }^{I}$, D. Acuña-Castroviejo ${ }^{2}$, G. Escames-Rosa ${ }^{2}$, \\ A. Comino-Pardo ${ }^{3}$, P. Bueno-Larano ${ }^{3}$
}

${ }^{1}$ General And Digestive Surgery, HOSPITAL VIRGEN DE LAS NIEVES, SERVICIO ANDALUZ DE SALUD (SAS), GRANADA/ SPAIN, ${ }^{2}$ Biochemical Research Center, Granada University, Granada/SPAIN, ${ }^{3}$ Experimental Surgery Unit, SAS, Granada/SPAIN 
Introduction: Melatonin plays a key role in regulating the inflammatory response. In fact it's a potent "scavenger", regulates the cytokines production and inhibits apoptosis. However, its intravenous use has not been used until now. The objective of this study is to evaluate the safety of the administration of a new intravenous melatonin formula.

Material and methods: A prospective, randomized, double-blind clinical trial (EUDRACT 2008-006782-83) was performed in 30 septic patients after abdominal surgery. The study protocol was approved by the local Clinical Research (Ethics) Committee. The use of intravenous melatonin was authorized by the Spanish Medicines Agency. Patients were randomly assigned to 2 study groups: Melatonin-treated group $(n=15)$ who received conventional treatment and melatonin as an injectable under patent (ES2392903B1) at a dose of $40 \mathrm{mg} /$ day $/ 5$ days. Placebo group $(\mathrm{n}=15)$ who received the conventional treatment. Treatment started $12 \mathrm{~h}$ after surgery and ended 5 days later. During treatment, possible adverse reactions to melatonin, clinical course (SOFA) and functional status of the various organs by biochemical determinations in peripheral blood were monitored.

Results: The groups were similar in their clinical conditions. No adverse effects were observed resulting from the administration of intravenous melatonin. On the SOFA scale, there is a tendency for improvement in the treated group. There was no deterioration in the biochemical parameters, and in some cases there was a tendency for improvement in patients treated with intravenous melatonin.

Conclusion: At doses administered in this study, intravenous melatonin is well tolerated, without adverse effects and without dysfunction in the organs studied.

References:

Disclosure: No significant relationships.

\section{PR082}

\section{WHAT IS THE RESISTANCE PROFILE OF GERMS INVOLVED IN COMMUNITY COMPLICATED INTRA - ABDOMINAL INFECTIONS?}

V.M. Durán Muñoz-Cruzado ${ }^{1}$, L. Tallón-Aguilar ${ }^{2}$ J. Tinoco-González ${ }^{2}$, M.J. Tamayo-Lopez ${ }^{2}$, I. Alarcón-Del Agua ${ }^{2}$, A. Sánchez-Arteaga ${ }^{2}$, J. Molina ${ }^{2}$, J.M. Cisneros ${ }^{2}$, J. Padillo-Ruíz, F. Pareja-Ciuró ${ }^{2}$

${ }^{1}$ Emergency Surgery, Hospital Universitario Virgen del Rocío, Seville/SPAIN, ${ }^{2}$ Emergency Surgery Unit, Virgen del Rocío Universitary Hospital, Seville/SPAIN

Introduction: In recent years, alerts have been extended by the increase in bacterial resistance. That can put compromise the therapeutic treatment for community infections. So it is important to know the profile of germs from our community to properly treat our patients by reducing antibiotic pressure.

Material and methods: Prospective descriptive study from September 2016 to February 2017. We analyzed the cultures obtained from patients that underwent emergency surgery for community complicated intra - abdominal infections (secondary to appendicitis, cholecystitis or non-tumor intestinal perforation). The endpoint of our study was to identify the sensitivity of our community germs to different antibiotics.

Results: A total of 15 germs were found (17 E. Coli, $10 \mathrm{~K}$. Pneumoniae, 3 P. Aeruginosa, 5 large negative bacilli -BGN-). $94 \%$ of E. coli were sensitive to cephalosporin of 3 rd generation and $100 \%$ to piperacillin-tazobactam; $80 \%$ of K. pneumoniae to cephalosporin with same percentage for piperacillin -tazobactam. BGN were $100 \%$ sensitive to both drugs.

Conclusion: Given the resistance profile of germs in our community, the use of narrow-spectrum antibiotics versus the broad spectrum seems to be an appropriate option. With this we could safely support strategies to prevent the growth of multiresistant germs.

References: 1 . Cälina D et al. Antimicrobial resistance development following surgical site infections. MOl Med Rep. 2017;15 (2):681688. 2. MIssaoui $\mathrm{K}$ et al. [Bacteriological profile and antibiotic treatment of postoperative peritonitis. Arch Inst Pasteur Tunis. 2014;91 (1-4):57-66. 3. Herzog T, Chromik AM, UhlW. Treatment of complicated intra-abdominal infections in the era of multi-drg resistant bacteria. Eur J Med Res. 2010; 15 (12):525-32.

Disclosure: No significant relationships.

\section{PR083}

\section{TREATMENT PROTOCOL FOR ACUTE LEFT SIDE COLONIC DIVERTICULITIS BASED ON WORLD SOCIETY OF EMERGENCY SURGERY GUIDELINES}

\author{
A. Sanchez Arteaga ${ }^{l}$, J. Tinoco-González ${ }^{l}$, \\ V. Durán-Muñoz-Cruzado ${ }^{2}$, I. Alarcón-Del Agua ${ }^{2}$, \\ M.J. Tamayo-Lopez ${ }^{I}$, L. Tallón-Aguilar ${ }^{1}$, F. Pareja-Ciuró ${ }^{1}$, \\ J. Padillo-Ruíz ${ }^{2}$
}

${ }^{1}$ Emergency Surgery Unit, Virgen del Rocío Universitary Hospital, Seville/SPAIN, ${ }^{2}$ Digestive And General Surgery Unit, Virgen del Rocío Universitary Hospital, Seville/SPAIN

Introduction: Acute left sided colonic diverticulitis (ALCD) is a frequent clinical condition admitted at Emergency Surgery Department. Hinchey's classification has been widely accepted for outlining grades of severity. Since the World Society of Emergency Surgery (WSES) Consensus Conference on acute diverticulitis, a new classification has been proposed. We present our clinical expertise in the management of ALSD through a protocol based in the WSES classification.

Material and methods: Retrospective analysis of ALCD from Trauma and Emergency Surgery Department of Universitary Hospital Virgen del Rocío. We report grade of severity according to WSES classification, management strategy and outcomes.

Results: From January 2016 to present, 110 patients have been admitted with ALCD. For non-complicated ALCD in 11 healthy patients, home treatment with a 5 days antibiotics therapy has been preferred, with 2 readmissions for exacerbation. Localized complicated ALCD have been managed with inpatient antibiotic treatment for 2-3 days in 94 patients, with PCR monitoring and clinical followup. If clinical improvement is observed, the patient can be discharged to complete antibiotic treatment for 10 days. If $>4 \mathrm{~cm}$ abscess is visualized, percutaneous drainage has been intend ( 2 patients). For 16 patients with distant complicated ALCD, surgical approach has been considered suitable. Laparoscopic lavage has been reserved for selected patient with purulent peritonitis and non-viewable perforation, in 4 cases. Otherwise, Hartmann operation has been the most common strategy.

Conclusion: Despite medical/surgical management for ALCD should be individualized, WSES classification can be a valid grading system for ALCD, and it might be useful in the creation of treatment protocols for daily practice.

References: Sartelli M, Catena F, Ansaloni L, et al. WSES Guidelines for the management of acute left sided colonic diverticulitis in the emergency setting. World J Emerg Surg. 2016;11:37.

Disclosure: No significant relationships. 


\section{PR084}

\section{CLINICAL PRESENTATION OF ACUTE APPENDICITIS DURING PREGNANCY IN EMERGENCY CARE}

J. Tinoco-González ${ }^{I}$, M. Rubio-Manzanares-Dorado ${ }^{1}$, A. Senent-Boza ${ }^{I}, F$. Pareja-Ciuróo ${ }^{l}$, V. Durán-Muñoz-Cruzado ${ }^{l}$, A. Sánchez-Arteaga ${ }^{l}$, I. Alarcón-Del Agua ${ }^{1}$, M.J. Tamayo-Lopez ${ }^{1}$, A. Navas-Cuéllar ${ }^{1}$, L. Tallón-Aguilar ${ }^{1}$, J. Padillo-Ruíz ${ }^{2}$

${ }^{1}$ Emergency Surgery Unit, Virgen del Rocío Universitary Hospital, Seville/SPAIN, ${ }^{2}$ Digestive And General Surgery Unit, Virgen del Rocío Universitary Hospital, Seville/SPAIN

Introduction: To analyze clinical expression of acute appendicitis (AA) during pregnancy and associated materno-fetal risk.

Material and methods: An analytical, longitudinal and ambispective study in women of childbearing age with AA from 2000 to 2017. We divided the patients into two groups: pregnant $(\mathrm{P})$ and fertile nonpregnant woman (NP), paired by propensity score matching. Collected variables were age, Alvarado's score, imaging test, leukocytosis, anesthesia, surgical approach, surgical site infection, maternal-fetal alterations and trimester of pregnancy.

Results: A total of 153 women of childbearing age with diagnosis of AA were included, $102 \mathrm{NP}$ and $51 \mathrm{P}$, with a mean age of $28.79 \pm 6.52$ years (P: $29.67 \pm 5.81$ vs NP: $28.35 \pm 6.83$; $=0.242$ ). Alvarado's score was $7.09 \pm 1.62$ (P: $6.65 \pm 1.67$ vs NP: $7.31 \pm 1.55 ; \mathrm{p}=0.016$ ). Mean stay was $2.84 \pm 3.29$ days (P: $5.10 \pm 4.80$ vs NP: $1.72 \pm 1.03$; $<0.001)$, highest in the third trimester with $10.45 \pm 6.95$ days $(\mathrm{p}<$ 0.001 ). Risk of labor when using general anesthesia was 0.16 (IC $95 \%$ 0.04-0.60, $\mathrm{p}=0.016$ ). When using laparotomic approach, risk of preterm birth was $1.6(95 \%$ CI $1.22-2.09, \mathrm{p}=0.009)$ and risk of low birth weight $1.42(95 \%$ CI $1.13-1.81, \mathrm{p}=0.004)$. Pregnancy poses a risk of complicated AA of 8.06 (95\% CI 2.11-30.30, $\mathrm{p}=0.002)$. Conclusion: AA during pregnancy has less obvious clinical manifestations than in non-pregnant women. Presence of complicated AA is more frequent during pregnancy, especially on third trimester.

References:

Disclosure: No significant relationships.

\section{PR085}

\section{SIMULATION OF SUPRALEVATOR ABSCESSES AND FISTULA IN CADAVERS: LOCATION, PELVIC DISSEMINATION CHANNELS AND DRAINAGE ROUTES}

A. Garcia-Granero ${ }^{1}$, J. Sancho Muriel ${ }^{1}$, L. Sánchez-Guillén ${ }^{1}$, $\overline{E . ~ A l v a r e z ~ S a r r a d o ~}^{1}$, D. Fletcher-Sanfeliu ${ }^{2}$, M. Frasson ${ }^{1}$, A. Valverde Navarro ${ }^{3}$, F. Martinez Soriano ${ }^{4}$

${ }^{1}$ Colorectal Unit, HOSPITAL UNIVERSITARIO Y POLITECNICO LA FE. VALENCIA, VALENCIA/SPAIN, ${ }^{2}$ Cardiovascular Surgery, Hospital Universitario Son Espases, Mallorca/SPAIN, ${ }^{3}$ Anatomy And Embryology, Valencia University, valencia/SPAIN, ${ }^{4}$ Anatomy And Embryology, Valencia University, Valencia/SPAIN

Introduction: Most of the abscesses of the ano-rectal zone have a cryptoglandular origin, following the pathogenesis described by Parks, in 1961. Supraelevator abscesses are the most uncommon location among ano-rectal abscesses. Nevertheless, such pathology results in a high mortality due to its difficult diagnosis and treatment.
Our objective is to assess its pathogenesis and management, as well as the complications derived from its incorrect treatment.

Material and methods: Anatomic Studio of 4 cadavers (three corps in formalin, 1 fresh). Three pelvises ( 2 men, 1 woman) sagittally sectioned and perineal dissection in a man. Anatomo-surgical description of pelvic and perianal zone, simulation of the different types of supraelevator abscesses and its feasible drainage pathways. Secondary complex fistulas due to inadequate drainage pathways.

Results: We have accomplished to realistically reproduce the origin of the different types of supraelevator abscesses, as well as its location and possible drainage pathways, definite treatment of secondary fistulas and morbidity due to its incorrect drainage.

Conclusion: A deep anatomo-surgical knowledge of the pelvis and perineal zone is fundamental to comprehend the etio-pathogenesis of supraelevator abscesses. Cadaver simulation may help accomplishing the correct treatment of such pathology and thus, avoiding the difficult management of complex fistulas.

References: Parks A.G, Gordon P.H, Hardcastle J.D. A classification of fistula-in-ano. Br J Surg. 1976;63:1-12. Prasad ML, Read DR, Abcarian H. Supralevator abscess: diagnosis and treatment. Dis Colon Rectum. 1981;24:456-461. Vasilevsky CA, Gordon PH. Benign Anorectal: Abscess and Fistula: In Wolff BG, Fleshman JW, Beck DE, Pemberton JH, Wexner SD eds, The ASCRS Textbook of Colon and Rectal Surgery. New York: Springer; 2007:192-214. GarciaGranero A, Granero-Castro P, Frasson M, Flor-Lorente B, Carreño O, Espí A, Puchades I, Garcia-Granero E. . Supralevator anorectal abscess presenting as acute low back pain and sciatica. Ann Emerg Med. 1994;23:132-5. Sanyal S, Khan F, Ramachandra P. Successful management of a recurrent supralevator abscess: a case report. Case report in surgery. 2012; Article ID 871639, 3 pages. Buchan R, Grace RH. Anorectal suppuration: the resuhs of treatment and the factors intlueneing the recurrence rate. Br J Surg 1973;60:537-40. Beck DE, Fazio VW, Lavery IC, Jagelman DG, Weakley FL (1988) Catheter drainage of ischiorectal abscesses. South Med J 814:444-446. Garg P. Supralevator extension in fistula-in-ano is almost always in the intersphincteric plane: easy solution for a complex disease. Dis Colon Rectum. 2016;59:e41-e42. Zhang H, Zhou Z, Hu B, Liu D, Peng H, $\mathrm{Xie} S$ et al. Clinical Significance of 2 deep posterior perianal spaces to complex cryptoglandular fistulas. Dis Colon Rectum2016; 59:766774. Kurihara H, Kanai T, Ishikawa T, et al. A new concept for the surgical anatomy of posterior deep complex fistulas: the posterior deep space and the septum of the ischiorectal fossa. Dis Colon Rectum. 2006;49 (10 suppl):S37-S44. Hanley PH. Conservative surgical correction of horseshoe abscess and fistula. Dis Colon Rectum. 1965;8:364-368. Gage KL, Deshmukh S, Macura KJ, Kamel IR, Zaheer A. MRI of perianal fistulas: bridging the radiological-surgical divide. Abdom Imaging 2013;38:1033-1042.

Disclosure: No significant relationships.

\section{PR086}

\section{THE ROLE OF ABDOMINAL CT SCAN IN PREDICTION OF} SEPSIS IN SECONDARY PERITONITIS

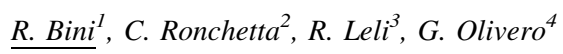

${ }^{1}$ General And Emergency Surgery, SG Boscon Hospital, Torino/ ITALY, ${ }^{2}$ Mecau, ASL TO4, Chivasso/ITALY, ${ }^{3}$ General And Emergency Surgery, SG Bosco Hospital, Torino/ITALY, ${ }^{4}$ Emergency Surgery, ASL Ciità della Salute e della Scienza, Torino/ITALY

Introduction: Peritonitis secondary to perforation of hollow viscous or anastomotic dehiscence are at risk of developing sepsis in turn 
burdened with high mortality and morbidity. Abdominal CT (Computed Tomography) is the diagnostic tool of choice in these cases. The end point of this study is to evaluate the correlation between abdominal free fluid on CT scan and sepsis and major surgical outcomes.

Material and methods: A retrospective study was conducted on 65 patients with intraperitoneal effusion at CT scan subjected to urgent laparotomy. A CT score (CTs) rating was calculated for each patient from 1 to 7 based on the number of abdominal quadrants involved. We used a Logistic Regression Models to investigate the associations of CTs with Variables. P-values under 0,05 have been considered significant in Univariate and Multivariate analysis.

Results: . 55.38\% of perforations were primitive. The average ASA score was 2.9 and the average age was 65 years. Mortality and morbidity rates were $26.15 \%$ and $47.69 \%$ respectively. There is a significant difference in value of CTs among for patients with preoperative septic shock ( $\mathrm{p} 0.003$ ) and postoperative ( $\mathrm{p} 0.043$ ). In the case of preoperative septic shock, the ROC curve is accurate in correlating it with a diffuse 4 or more quadrant effusion (AUC $<0.764$ ). A diffuse effusion correlates with a higher risk of preoperative shock (OR 12.32, $\mathrm{p}<0.017)$. CTs $\geq 4$ relates with P-Possum morbidity ( $\mathrm{p}<$ $0.005)$, mortality $(\mathrm{p}<0.016)$, and prolonged patient length $(\mathrm{p}<$ $0.031)$

Conclusion: CTs correlates significantly with the clinical severity of the patient. It is capable of predicting mortality and morbidity.

References: Sartelli et al. World Journal of Emergency Surgery 2013, 8:3 WSES 2013 guidelines for management of intr abdominal infections.

Disclosure: No significant relationships.

\section{PR087}

\section{IT'S TIME TO FORGET THE GOLDEN 72 HOURS RULE FOR ACUTE CHOLECYSTITIS}

\author{
M. Ceresoli, L. Degrate, L. Fattori, L.V. Gianotti
}

General Surgery, Milano-Bicocca University, Monza/ITALY

Introduction: The treatment of acute cholecystitis (AC) is laparoscopic cholecystectomy; guidelines suggest surgical intervention as soon as possible, but the golden 72 hours rule is often followed in clinical practice. The aim of this study is to investigate the outcome difference in patients operated within and beyond 72 hours from hospital admission.

Material and methods: Patients undergoing choelcystectomy for AC, graded as mild-moderate according to Tokyo guidelines from 2006 to 2015 were divided in two group basing on the timing of intervention: immediate cholecystectomy (IC) within 72 hours from admission and index admission cholecystectomy (IAC), beyond 72 hours in the index admission. The two groups were compared for short term outcomes.

Results: 448 consecutive patients were studied. 183 (40.8\%) were in the IC group and $265(59.2 \%)$ in the IAC group. The two groups were similar for ASA score and gender, but differed for an higher average age in IAC (57 years vs 61 years, $\mathrm{p}=0.006$ ). IC patients were operated after a mean of 1.5 days compared to 8.1 of IAC patients ( $p$ $<0.001)$. Laparoscopic surgery, conversion rate, complication rate and post-operative length of stay did not differ significantly between the two groups.

Conclusion: As suggested by guidelines, cholecystectomy for AC should be performed as soon as possible. It should be taken into account that an operation performed beyond the golden 72 hours limit is safe and feasible with similar results.

References:

Disclosure: No significant relationships.

\section{PR088}

\section{ANTIMICROBIAL RESISTANT ISOLATES FROM PATIENTS WHO REQUIRE EMERGENCY LAPAROTOMY - AN URGENT NEED TO STANDARDISE PRACTICE}

\author{
M. Campbell, N. Misra, E. Fadden, N. Hettiarachchi, J. Mason, \\ B. Morton
}

Surgery, Aintree University Hospital, London/UNITED KINGDOM

Introduction: Patients undergoing emergency laparotomy typically receive empirical antibiotics Failure to send appropriate samples for culture may lead to suboptimal treatment and may be a contributing factor in development of antimicrobial resistance.

Material and methods: A retrospective analysis of the prospectively multiclinicain inputted emergency laparotomy database for our institution from 2014-15 was carried out. Microbiological samples received by the laboratory within 48 hours of laparotomy were defined as the primary endpoint for this analysis.

Results: 202 patients underwent emergency laparotomy. Blood cultures were sent in 35 cases (17.3\%), an abdominal sample in 19 cases $(9.4 \%)$ and both in four cases $(1.98 \%)$. Blood cultures were positive in $22.9 \%$ ( $8 / 27$ cases). Abdominal samples were positive in $63.3 \%$ of cases (12/19). Table 1 shows culture results: Resistance to amoxicillin was present in nine isolated organisms, with the majority of these growing $E$. coli. Tazocin (Aintree $1^{\text {st }}$ line for hospital acquired abdominal infection) was apparent in four cases. Table 2 shows betalactam resistance patterns according to organisms cultured (all organisms were sensitive to a beta-lactam in combination with gentamicin).

Conclusion: Samples were sent infrequently for microbiology from patients requiring emergency laparotomy and resistant organisms were detected in patients who would ordinarily receive empirical antibiotics for community acquired abdominal infections. This lack of sampling reduces our ability to guide antimicrobial therapy, particularly if a patient becomes unwell in the days after surgery.

References:

Disclosure: No significant relationships.

\section{PR089}

\section{CRITICAL ASSERSMENT OF TOKYO GUIDELINES FOR ACUTE CHOLECYSTITIS}

$\underline{\text { E. Membrilla-Fernández }}^{l}$, V. Ruíz-Artola ${ }^{l}$, M.J. Pons-Fragero ${ }^{l}$, J. Sancho-Insenser ${ }^{I}$, L. Grande-Posa ${ }^{2}$

${ }^{1}$ Emergency Unit. General Surgery Service., Hospital del Mar, Barcelona/SPAIN, ${ }^{2}$ General Surgery, Hospital del Mar, Barcelona/ SPAIN

Introduction: Background: The Tokyo International Guidelines (TG13) advice different initial treatments of acute cholecystitis (AC) based on severity, with no clear evidence that all patients benefit from the recommended treatment. Aim: To analyse factors for morbidity 
and mortality in patients with CA and their relationship with the severity scale of TG13.

Material and methods: Patients and methods: A retrospective study of 320 patients with CA in 18 months of whom 250 were selected for analysis of 166 variables, including the Charlson, Büchler and TG13 scales, and complications (Clavien).

Results: $31.6 \%$ of ACs were Grade I, $48 \%$ Grade II and $28.4 \%$ Grade III. The initial treatment was cholecystectomy in $97.6 \%$ of patients, cholecystostomy in $1.2 \%$ and antibiotic therapy in $5.6 \%$, in clear discordance with TG13. Non-operated patients had higher ASA scores $(\mathrm{P}=0.009)$ and Charlson $(\mathrm{P}=0.055) .12 \%$ of cholecystectomies were by laparotomy, with differences between grades (I: $5.1 \%$, II: $9.4 \%$, III: $21.2 \%, \mathrm{P}<0.001)$. $43 \%$ of the patients suffered complications, a significantly lower rate for CA Group I vs. Groups II and III $(\mathrm{P}<0.001)$. Complications were more frequent in patients with renal insufficiency (OR:24.5), GOT $>40 \mathrm{mg} / \mathrm{dL}$ (OR:4.1) or CRP $>3$ $\mathrm{mg} / \mathrm{dL}$ (OR:3.9), initial subcostal laparotomy (OR:8.4), or converted (OR:2.6) and a surgical time $>2 \mathrm{~h}(\mathrm{OR}: 4.1)$. Mortality was $3.2 \%$, significantly higher for Group III $(7.0 \%$; $\mathrm{P}=0.011)$ and was associated with diabetes with tissue damage (OR: 7.73) and renal failure (OR: 9.8).

Conclusion: s: Initial treatment of CA is mostly surgical, diverging from TG13, with competitive rates of complications and mortality.

References:

Disclosure: No significant relationships.

\section{PR090}

\section{PROCALCITONIN VALUES AND PREDICTION OF COMPLICATIONS AFTER COLORECTAL SURGERY}

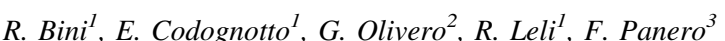

${ }^{1}$ General And Emergency Surgery, SG Bosco Hospital, Torino/ ITALY, ${ }^{2}$ Emergency Surgery, ASL Ciità della Salute e della Scienza, Torino/ITALY, ${ }^{3}$ Emergency Medicine, SG Bosco Hospital, Torino/ ITALY

Introduction: Despite recent advances in surgical techniques and perioperative care, infectious complications remain a major clinical problem in colorectal surgery. Surgical trauma induces systemic inflammatory response, which can confuse the diagnosis of postoperative infections. We analyzed procalcitonin (PCT) as early marker of infection after colo-rectal surgery

Material and methods: We prospectively analyzed consecutive patients who underwent colorectal surgery at our department in a period of 24 months, operated in elective or urgency surgery with open and laparoscopic approach. PCT was evaluate in the $\mathrm{III}^{\circ}$ post op day as an ordinal variable according to three strata of usual clinical interest $(<0.5 / 0.5-1.0 />1.0)$. We performed a multivariate analysis between PCT and other variables. The $p$ values lower than 0.05 were considered statistically significant

Results: Our cohort included 144 patients, $54.9 \%$ of whom were males with a median age of 72 years. One in four underwent an emergent/urgent surgical intervention. $40.3 \%$ was treated with a laparoscopic technique. $22.2 \%$ of patients experienced either a medical complication or needed a second surgical intervention. The association between PCT and type of surgical approach resulted statistically significant $(\mathrm{p}<0.05)$. Also correlations with complications, re-intervention and death were significant. Sex, comorbidity, ASA score, neoplastic pathology didn't affect PCT

Conclusion: Laparoscopic or open approach affected PCT differently and it is useful in this context to distinguish between infection and systemic inflammatory response in elective surgery. Furthermore PCT is able to predict the onset of complications, need to re intervention and death.

References: Philipp Schuetz et al. Procalcitonin for diagnosis of infection and guide to antibiotic decisions: past, present and future. BMC Medicine 2011, 9:107

Disclosure: No significant relationships.

\section{PR091}

\section{ITS BETTER THE USE OF ENDO GIA VS ENDOLOOP IN LAPAROSCOPIC APPENDECTOMY? A RETROSPECTIVE STUDY}

\section{A. Craus Miguel ${ }^{1}$, J.J. Segura-Sampedro ${ }^{2}$, P. Jimenez Morillas ${ }^{3}$, L. Fernández Vega ${ }^{I}$, C. Pineño Flores ${ }^{I}$, A. Ochogavia Segui ${ }^{2}$, M.B. Gamundi Cuesta ${ }^{2}$, F.X. Gonzalez Argente ${ }^{2}$}

${ }^{1}$ Resident Of General Surgery, Hospital Universitario Son Espases, Palma de Mallorca/SPAIN, ${ }^{2}$ General \& Digestive Surgery, University Hospital Son Espases, Palma de Mallorca/SPAIN, ${ }^{3}$ General And Digestive Surgery, Hospital de Manacor, Manacor/SPAIN

Introduction: Is not so clear in the literature which is the best technique (EndoloopvsEndoGIA) in laparoscopy appendecectomy (LAE). The objective of this study is to evaluate their economic and morbidity differences.

Material and methods: Retrospective cohort study which included all the LAE between 2011-2015. The main outcome was the costs. Baseline sociodemographic, hospital stay, histology characteristics of the appendix, presence of free fluid, intraoperative rupture, surgical material and postoperative complications according to Clavien Dindo were collected. Statistical analysis was performed using IBMSPSS21.

Results: A total of 345 patients underwent LAE, 241 patients with Endo GIA (81,7\%) and 54 with Endoloop (18.3\%). Patients' characteristics and outcomes did not differ significantly. No significant differences were found analyzing the type of appendix (18.7\% perforated in the EndoGIA group vs 7.4\% in the Endoloop group) and the presence of purulent fluid $(30.7 \%$ vs $22.2 \%$, respectively). The median duration of operation did not differ significantly. 30 patients underwent LAE had postoperative complications, of which 12 were Clavien Dindo I and 11 were Clavien Dindo II. The hospital stay was higher in cases treated by Endo Gia (3.41days) compared to patients treated with endoloop (2.59 days), without finding significant differences. The price of the EndoGIA is $€ 550.50$ while Endoloop is $€$ 52.71 .

Conclusion: Although only $14.2 \%$ of all appendicitis was perforated, the use of Endo GIA was $81.7 \%$, when both techniques present similar morbidity and complications. Since the cost of the endoloop is significantly lower, its use seems to be justified, however, prospective randomized studies are necessary to confirm this hypothesis.

References: Sauerland S, Jaschinski T. Laproscopic versus open surgery for suspected appendicitis. Cochrane Colorectal Group 2010; 10.1002/14651858.CD001546.pub3 Jaschinski T., Mosch C et al. Laparoscopic vs open appendectomy in patients with suspected appendicitis: a systematic review of meta-analyses of randomised controlled trials. BMC Gastroenterol 2015; 10.1186/s12876-0150277-3 2016 Oct 18;21:87. doi: 10.4103/1735-1995.192503. eCollection 2016.

Disclosure: No significant relationships. 


\section{PR092}

\section{CHOLECYSTECTOMY IN TREATMENT OF ACUTE CHOLECYSTITIS: THE IMPORTANCE OF TIMING}

\section{Y. Lara Fernández ${ }^{1}$, J.A. Lopez Ruiz ${ }^{2}$, B.M. De La Cuadra ${ }^{1}$, M.S. Ramírez ${ }^{I}$, E.P. Margallo ${ }^{I}$, L. Tallón-Aguilar ${ }^{1}$, B.B. Rodrigo ${ }^{I}$, J. López Pérez ${ }^{l}, F$. Oliva Mompean ${ }^{l}$}

${ }^{1}$ Emergency Surgery Unit, Hospital Universitario Virgen Macarea, Seville/SPAIN, ${ }^{2}$ Emergency Surgery Unit, Hospital Universitario Virgen Macarena, Seville/SPAIN

Introduction: Optimal timing of emergency cholecystectomy for acute cholecystitis is usually considered within the first 72 hours of symptoms. The aim of this study is to compare clinical outcomes between early cholecystectomy (within 72 hours from admission) and delayed cholecystectomy (after 72 hours to 7 days).

Material and methods: We performed a single center retrospective study of patients with diagnosis of acute cholecystitis with less than 7 days from symptoms onset, who underwent surgical treatment the period between June 2014-June $2016(\mathrm{~N}=241)$. Group $1(\mathrm{~N}=74)$ was composed of patients treated with early cholecystectomy; group 2 $(\mathrm{N}=167)$ included the patients treated with delayed cholecystectomy. Results: In group 1, surgical time was 95 minutes, and length of hospital stay was 3 days; 64 (85\%) patients underwent laparoscopy cholecystectomy, $4(6.2 \%)$ of them turned into open surgery. $2(2.7 \%)$ patients suffered bile duct injury, $6(8.1 \%)$ suffered bile leakage; there were $4(6.2 \%)$ wound infection, $7(9.4 \%)$ intraabdominal collections, $1(1.4 \%)$ postoperative ileus; 1 (1.4\%) patient was re-operated, and 2 $(2.7 \%)$ were re-admitted. In group 2, surgical time was 90 minutes, and length of hospital stay was 3 days.149 (89\%) patients underwent laparoscopy cholecystectomy, $8(5.36 \%)$ of them turned into open surgery. $1(0.5 \%)$ patient suffered bile duct injury, $5(3 \%)$ suffered bile leakage; there were $5(3 \%)$ wound infection, $8(4.8 \%)$ intraabdominal collections, $2(1.2 \%)$ postoperative ileus; $2(1.2 \%)$ patients were re-operated, and $5(3 \%)$ were re-admitted. There were no significant differences between both groups.

Conclusion: Cholecystectomy in the treatment of acute cholecystitis within 7 days of the onset of symptoms appears to be as safe and effective as early cholecystectomy, regarding postoperative complications, length of hospital stay and surgical time.

References: 1.Cao AM, Eslick GD, Cox MR. Early laparoscopic cholecystectomy is superior to delayed acute cholecystitis: a metaanalysis of case - control studies. Surg Endosc. 2016;30 (3):1172-82. 2.Degrate L, Ciravegna AL, Luperto M. Acute cholecystitis: the golden 72-h period is not a strict limit to perform early cholecystectomy . Results from 316 consecutive patients. 2013;1129-36. 3.Gönenç MABM, Yırgın KDPH. Admission after the gold interval in acute calculous cholecystitis: Should we really cool it off? 2017;73-7. 4.Gurusamy K, Campus RF, Hospital RF, Street RH. Early laparoscopic cholecystectomy appears better than delayed laparoscopic cholecystectomy for patients with acute cholecystitis Early laparoscopic cholecystectomy appears better than delayed laparoscopic cholecystectomy for patients with acute cholecyst. 2016;21 (1):2016-7. 5.Kolla SB, Aggarwal S, Kumar A, Kumar R, Chumber S, Parshad R, et al. Original articles Early vs delayed laparoscopic cholecystectomy for acute cholecystitis A prospective randomized trial. 2004; (November 2002):1323-7. 6.Menahem B, Mulliri A, Fohlen A, Guittet L, Alves A, Lubrano J. Delayed laparoscopic cholecystectomy increases the total hospital stay compared to an early laparoscopic cholecystectomy after acute cholecystitis: an updated meta-analysis of randomized controlled trials. Am J Med Sci [Internet]. 2015;17 (10):857-62. Available from: http://dx.doi.org/10.1111/ hpb.12449 7.Minutolo V, Licciardello A, Arena M, Nicosia A,
Stefano BDI. Laparoscopic cholecystectomy in the treatment of acute cholecystitis: comparison of outcomes and costs between early and delayed cholecystectomy. 2014;18 (Suppl 2):40-6. 8.Pisano M, Ceresoli M, Allegri A, Belotti E, Coccolini F, Colombi R, et al. Single center retrospective analysis of early vs . delayed treatment in acute calculous cholecystitis: application of a clinical pathway and an economic analysis. 2015;21 (5):373-9. 9.Prospective A, Study R, Ersin G. Early Versus Delayed Laparoscopic Cholecystectomy for Acute Cholecystitis: 2014;56-61. 10.Rajcok M, Bak V, Danihel L, Kukucka M, Schnorrer M. Early versus delayed laparoscopic cholecystectomy in treatment of acute cholecystitis. 2016;117 (6):328-31. 11.Review S. Laparoscopic cholecystectomy for acute cholecystitis: early or delayed? 2016;23 (May):1-8. 12.Sutton AJ, Vohra RS, Hollyman M, Marriott PJ, Buja A, Alderson D. Cost-effectiveness of emergency versus delayed laparoscopic cholecystectomy for acute gallbladder pathology. 2017;98-107. 13.Trial AR. Early Versus Delayed Cholecystectomy for Acute Cholecystitis, Are the 72 hours Still the Rule? 2016;264 (5):717-22. 14.Versus E, Cholecystectomy D, Trial AMR, Kipfm K. Acute Cholecystitis. 2013;258 (3):385-93. 15.Wu X, Tian X, Liu M, Wu L, Zhao S, Zhao L. Meta-analysis comparing early versus delayed laparoscopic cholecystectomy for acute cholecystitis. 2015;1302-13. 16.Zhou M, Gu X, Xiang J, Chen Z. Comparison of Clinical Safety and Outcomes of Early versus Delayed Laparoscopic Cholecystectomy for Acute Cholecystitis: A Meta-Analysis. 2014;2014. 17.Hospital W, Territories N, Kong H. Randomized trial of early. 1998; (October 1997):764-7.

Disclosure: No significant relationships.

\section{PR093}

\section{PERFORATIONS FOLLOWING ENDOSCOPIC RETROGRADE CHOLANGIOPANCREATOGRAPHY (ERCP)}

\author{
J. Mateo Retuerta, C. Chaveli Diaz, A. Goikoetxea, B. Sainz, \\ M.J. Sara, I. Eguaras, J.J. Ínigo
}

Cirugía General, Complejo Hospitalario de Navarra, Pamplona/ SPAIN

Introduction: Endoscopic retrograde cholangiopancreatography (ERCP) associated with sphincterotomy is a useful procedure that is widely used in cases of choledocholithiasis and bile duct obstruction. In spite of being a safe test, there is a risk of complications like pancreatitis, hemorrhaging or perforation. The management must be individualized and constantly reevaluated. In those cases where a conservative attitude is adopted, close clinical vigilance will be maintained.

Material and methods: In a period of 22 months (from January 2015 to October 2016), our Surgery Service was consulted for the surgical evaluation of 12 post-ERCP perforations. Given the increasing number of these procedures and the consequent increase in complications, we believe it is reasonable to present several type cases as an example of possible therapeutic management.

Results: Each of the patients have received a different management depending of the lesion mechanism, the place and extension of the perforation, as well as the clinical status and radiographic findings, with a good response to the treatments established.

Conclusion: In conclusion, post-ERCP duodenal perforation is a rare event that has serious consequences if it is not treated early. The time between perforation and surgical assessment is crucial because the delay in treatment significantly increases mortality. In general, patients with type I perforations require surgery invariably and those with type II and type III perforations can be managed with 
conservative treatment. Anyway, all will require observation and close reassessment to confirm the satisfactory progress of the clinical status.

References: 1. Yilmaz B, Roach EC, Koklu S, Aydin O, Unlu O, Alper Kilic Y. Air leak syndrome after endoscopic retrograde cholangiopancreatography: A rare and fatal complication. World $\mathbf{J}$ Gastroenterol. 2015 Apr 21; 21 (15): 4770-4772. 2. García-Cano J, González JA, Morillas J, Pérez A. Complications of endoscopic retrograde cholangiopancreatography. A study in a small ERCP unit. REV ESP ENFERM DIG (Madrid) Vol. 96. N. ${ }^{\circ} 3$, pp. 163-173, 2004 3. Ozgonul A, Cece H, Sogut O, Demir D, Kurkcuoglus IC. Pneumoperitoneum, pneumoretroperitoneum and bilateral pneumothorax caused by ERCP. J Pak Med Assoc. 2010 Jan;60 (1):60-1. 4. Palomeque A, González C, Pérez D, Jiménez JA. Conservative treatment of duodenal perforation after therapeutic endoscopic retrograde cholangiopancreatography . Gastroenterol Hepatol. 2015 Apr;38 (4):285-6. 5. Lee SM, Cho KB. Value of temporary stents for the management of perivaterian perforation during endoscopic retrograde cholangiopancreatography. World J Clin Cases. 2014 Nov 16; 2 (11): 689-697. 6. Stapfer M, Rick R, Stain CS, Katkhouda N, Parekh D, Jabbour N, et al. Management of Duodenal Perforation After Endoscopic Retrograde Cholangiopancreatography and Sphincterotomy. Annals of Surgery 2000 Vol. 232, No. 2, 191-198. 7. Brueck M, Bandorski D, Rauber K, Lotterer E. Pneumoretroperitoneum and bilateral pneumothorax after endoscopic biliary sphincterotomy. Dtsch Med Wochenschr. 2010 Apr;135 (17):853-6. doi: 10.1055/s-0030-1253667. Epub 2010 Apr 20. 8. Howard TJ, Tan T, Lehman GA, Sherman S, Madura JA, Fogel E, et al. Classification and management of perforations complicating endoscopic sphincterotomy. Surgery. 1999 Oct;126 (4):658-63. 9. Machado NO. Management of Duodenal Perforation Post-Endoscopic Retrograde Cholangiopancreatography. When and Whom to Operate and What Factors Determine The Outcome? A Review Article. JOP. J Pancreas (Online) 2012 Jan 10; 13 (1):18-25. 10. Makni A, Chebbi F, Ben Safta Z. Pneumoretroperitoneum, bilateral pneumothorax and emphysema following endoscopic biliary sphincterotomy. Acta Chir Belg. 2012 Jul-Aug;112 (4):307-9. 11. Cho KB. The management of endoscopic retrograde cholangiopancreatography-related duodenal perforation. Clin Endosc. 2014 Jul;47 (4):341-5. 12. Armas MD, Ojeda V, Roque C, Cabrera JC, Mathías MP, Ceballos D, et al. Duodenal perforations after endoscopic retrograde cholangiopancreatography . Cir Esp. 2015 Jun-Jul;93 (6):403-10. 13. Turner RC, Steffen CM, Boyd P. Endoscopic duodenal perforation: surgical strategies in a regional centre. World J Emerg Surg. 2014 Jan 24;9 (1):11. doi: 10.1186/1749-79229-11. 14. Völgyi Z, Szenes M, Gasztonyi B. Types and management of perforations occurring during endoscopic retrograde cholangiopancreatography . Orv Hetil. 2014 Feb 16;155 (7):248-54. 15. Vowinkel T, Senninger N. Retroperitoneal emphysema after endoscopic retrograde cholangiopancreatography. Chirurg. 2015 May;86 (5):462-7. 16. Fatima J, Baron TH, Topazian MD, Houdhton SG, Igbal CW, Ott BJ, et al. Pancreaticobiliary and duodenal perforations after periampullary endoscopic procedures: Diagnosis and management. Arch Surg., 142 (2007), pp. 448-454 17. Knudson K, Raeburn CD, McIntyre RC Jr, Shah RJ, Chen YK, Brown WR, et al. Management of duodenal and pancreaticobiliary perforations associated with periampullary endoscopic procedures. Am J Surg.2008;196 (6):975-81. 18. John AK, Thandassery RB, Alam SH, Al Kaabi SR. Extensive cervical, thoracic, and abdominal wall emphysema and pneumomediastinum following ERCP: a rare scenario. Ann Gastroenterol. 2014; 27 (4): 438-439.

Disclosure: No significant relationships.

\section{PR094}

\section{MORBIDITY AFTER NEGATIVE APPENDECTOMY: SINGLE CENTER EXPERIENCE ON 432 CASES}

\author{
L. Santurro, N. Tamini, M. Montuori, M.I. Gattuso, C. Barbieri, \\ L. Fattori, L.V. Gianotti
}

Department Of Surgery, University of Milano Bicocca - San Gerardo Hospital, Monza/ITALY

Introduction: Acute appendicitis (AA) is one of the most common causes of abdominal pain in emergency departments but remains one of the most difficult diagnostic challenge due to multiple, different and atypical clinical presentations. The optimal strategy to adopt when a non pathological appendix (NA) is recognized during surgery for suspected appendicitis is debated. The aim of this study is to analyze intraoperative and postoperative outcomes of histologically NA in order to underline the burden associated with avoidable procedures.

Material and methods: We analyzed all patients undergoing appendectomy for suspected acute appendicitis in University of Milano-Bicocca, San Gerardo Hospital, between September 2013 and October 2016. On the base of histologic report, patients were divided in $\mathrm{AA}$ and NA groups.

Results: 432 patients undergoing an emergency procedure for suspected appendicitis (362 patients in AA group, 70 in NA). NA group was constituted of more females $(p=0.028)$ and median age was lower $(\mathrm{p}=0.003)$. Preoperative White Blood Cells count and C-reactive protein were more elevated in AA group (respectively, $\mathrm{p}=$ 0.001 and $p=0.005$ ). There weren't differences in laparoscopically approach, in conversion rate and in operative time. Furthermore, no significant difference were noticed in complications rate $(9.4 \%$ vs $12.9 \%$ ), in Clavien-Dindo classification and in hospital stay.

Conclusion: The results of this study suggest that the removal of a macroscopically normal appendix carries a intraoperative and postoperative outcomes comparable with that associated with the removal of an inflamed appendix. Therefore, every surgeon must carefully consider risk/benefit ratio before performing an appendectomy during a diagnostic laparoscopy showing a normal appendix.

References: 1. van den Broek WT, Bijnen AB, de Ruiter P, Gouma DJ (2001) A normal appendix found during diagnostic laparoscopy should not be removed. Br J Surg 88:251-254. 2. Di Saverio S, Birindelli A, Kelly MD, et Al. (2016) WSES Jerusalem guidelines for diagnosis and treatment of acute appendicitis. World J Emerg Surg 11:34. 3. Hamminga JTH, Hofker HS, Broens PMA, Kluin PM, Heineman E, Haveman JW (2013) Evaluation of the appendix during diagnostic laparoscopy, the laparoscopic appendicitis score: a pilot study. Surg Endosc 27:1594-1600. 4. Güller U, Rosella L, McCall J, Brügger LE, Candinas D (2011) Negative appendicectomy and perforation rates in patients undergoing laparoscopic surgery for suspected appendicitis. Br J Surg 98:589-595.

Disclosure: No significant relationships. 


\section{PR095}

\section{COMPARISON OF IMMEDIATE RESULTS BETWEEN LAPAROSCOPY AND OPEN APPROACH IN 1500 APPENDECTOMIES}

\author{
A.M. Solà ${ }^{1}$, L. Torrent-Jansà̃ ${ }^{2}$ E. Membrilla-Fernández ${ }^{3}$, \\ A. Gallego-Vila ${ }^{3}$, M.P. Fraguero ${ }^{1}$, J. Guzmán-Ahumada ${ }^{2}$, \\ L. Grande-Posa ${ }^{2}$, J. Sancho-Insenser ${ }^{3}$
}

${ }^{1}$ General Surgery And Digestive Department, Hospital del Mar, BARCELONA/SPAIN, ${ }^{2}$ General Surgery, Hospital del Mar, Barcelona/SPAIN, ${ }^{3}$ Emergency Unit. General Surgery Service., Hospital del Mar, Barcelona/SPAIN

Introduction: To compare the results between laparoscopic and open approach for acute appendicitis.

Material and methods: Retrospective study on a prospectively maintained database of 1537 consecutive appendectomies. Sex, age, experience of the surgeon, complications (Clavien-Dindo scale) and hospital stay were evaluated.

Results: The mean age was $34.5 \pm 0.5$ years, predominantly male (59.3\%). 24 (1.5\%) patients with initial approach by laparotomy were excluded, leaving 1513 for analysis. The approach was open (ABI) in $848(56 \%)$ and laparoscopic (LAP) in $665(44 \%)$ patients. Fifty (7.5\%) laparoscopic open approaches were converted. LAP was more common in women $(52 \%$ vs $38.4 \%, \mathrm{P}<0.0001) .285$ patients presented complications $(19.2 \%)$, with small differences between ABI and LAP (17.9\% vs. $20.0 \% ; \mathrm{P}=0.00001)$. There were no statistically significant differences between ABI and LAP for Clavien complication types $(P=0.28)$, although the rate of reintervention was lower in the $\mathrm{ABI}$ group $(0.9 \%$ vs. $1.6 \% ; \mathrm{P}=0.25)$ and the rate of surgical site infections was similar $(5.4 \%$ vs.5.2\%, $\mathrm{P}=0.9)$. The mean postoperative stay was $4.0 \pm 0.1$ days, with a surprisingly longer stay after LAP ( $3.6 \pm 0.1$ vs. $4.2 \pm 0.2$ days; $\mathrm{P}=0.011)$. Experience since first year of training $(\mathrm{R} 1=1)$ showed significant differences between $\mathrm{ABI}$ and LAP (5.3 \pm 0.3 vs.7. \pm 0.3 years; $\mathrm{P}<0.001)$. Residents had fewer complications than surgeons $(16.3 \%$ vs. $24.9 \% ; \mathrm{P}<0.001)$, for both the LAP $(17.8 \%$ vs. $23.6 \% ; \mathrm{P}<0.04)$ and ABI $(15.4 \%$ vs.26.7\%;P $<0.001)$.

Conclusion: In sharp contrast to clinical trials comparing $\mathrm{ABI}$ and LAP approaches, this observational study of consecutive patients questions the superiority of the LAP.

References:

Disclosure: No significant relationships.

\section{PR096}

\section{NONSPECIFIC MESENTERIC LYMPHADENITIS IN THE STRUCTURE OF AN ACUTE ABDOMEN IN CHILDREN}

\author{
E. Sologub ${ }^{1}$, O.V. Karaseva ${ }^{1}$, E. Horchinova ${ }^{2}$
}

\author{
${ }^{1}$ Emergency Pediatric Surgery, Clinical and Research Institute of \\ Emergency Pediatric Surgery and Trauma, Moscow/RUSSIAN \\ FEDERATION, ${ }^{2}$ Radiology, Clinical and Research Institute of \\ Emergency Pediatric Surgery and Trauma, Moscow/RUSSIAN \\ FEDERATION
}

Introduction: Nonspecific mesenteric lymphadenitis is manifested with a complex of symptoms typical for acute abdomen what defines its importance in differential diagnostics of acute appendicitis.
Material and methods: 1860 children were included into the trail. Boys $46.6 \%$, girls $53.4 \%$, average age $-7,8 \pm 2,3$. All children were observed in dynamics. They also had blood analysis, urine analysis, ultrasound examination.

Results: Mesenteric lymphadenitis was diagnosed in $11 \%$ of children who were hospitalized with suspected acute appendicitis. In $0.9 \%$ of cases, diagnostic laparoscopy was made to exclude acute surgical pathology and confirm diagnosis of mesenteric lymphadenitis. Basic clinical manifestations of mesenteric lymphadenitis were: complains of periodic abdominal pains, pain during palpation in the right mesogastric area; positive McFadden and Sternberg symptoms. In most children, colds and dyspepsia were absent; temperature was subfebrile. Ultrasound examination revealed no surgical pathology; multiple lymph nodes were localized in the right mesogastric region, size 0.7 to $2.5 \mathrm{~cm}$, oval shape, with reduced echogenicity and increased blood flow under Doppler examination; in $80 \%$ of cases they formed a conglomerate .

Conclusion: Nonspecific mesenteric lymphadenitis has nonspecific clinical picture. Non-invasive diagnostics of this disease should include identification of local symptoms (McFadden and Sternberg) and ultrasound picture, if acute surgical pathology is excluded. In case of doubts, the only objective technique for mesenteric lymphadenitis diagnostics is laparoscopy.

References:

Disclosure: No significant relationships.

\section{PR097}

\section{EFFECT OF ADEGUATE EMPIRIC THERAPY IN INFECTIVE COMPLICATION RATE IN ACUTE APPENDICITIS}

\author{
M. Montuori, L. Santurro, L.V. Gianotti, L. Fattori
}

Department Of Surgery, University of Milano-Bicocca - San Gerardo Hospital, Monza/ITALY

Introduction: The aim of this study was to evaluate bacterial etiology and differences in terms of complication rates and lenght of stay respect adequacy of empiric antibiotic therapy.

Material and methods: We analyze a prospectively constructed database was performed of all patients who underwent appendectomy in our hospital from september 2013 to November 2016 at University of Milano-Bicocca - San Gerardo Hospital, Monza, Italy.

Results: 450 patients underwent emergency appendectomy (median age 23 years, IQR 14:43, . Male 56,1\%). 101 patients $(22,5 \%)$ had intraoperative swab taken, and was positive in 58 patients $(57,4 \%)$. The most commonly recovered species were E. Coli $(67,9 \%)$ with 2 $(5,3 \%)$ ESBL positive, Streptococcus $(38,6 \%)$, Bacteroides $(30,4 \%)$, Proteus $(23,2 \%)$, Klebsiella Pneumoniae $(19,4 \%)$ and Pseudomonas Aeruginosa $(16,1 \%)$. The flora was in $51,9 \%$ aerobic, in $9,6 \%$ anaerobic and in the remain $38,5 \%$ mixed. There was no differences in terms of surgical site infection $(5,2 \%$ vs $7 \%)$, deep infection $(10,3 \%$ vs $4,7 \%)$, and in clavien-dindo. An appropriate empiric therapy was set in $88,5 \%$ and inappropriate in $11,5 \%$. We found no differences in terms of surgical site infection, but a differences in deep site infection $(\mathrm{p}=0,036)$ with an increased rate in case of unsuitable antibiotic empiric therapy, but this didn't affect the lenght of stay $(\mathrm{p}=0,114)$.

Conclusion: The postoperative patient outcome maybe dependent on the severity of the appendicitis than on the results of the swab at time of surgery, but inappropriate empiric therapy may affect the incidence 
of deep site infection. Swabbing during appendectomy should be limited to only selected high-risk groups.

References: 1. Kenig J, Richter P. The need for culture swabs in laparoscopically treated appendicitis. Surg Infect (Larchmt). 2012 Dec;13 (6):383-90. doi: 10.1089/sur.2011.135. Epub 2012 Dec 11. 2. Jeon HG, Ju HU, Kim GY, Jeong J, Kim MH, Jun JB. Bacteriology and changes in antibiotic susceptibility in adults with communityacquired perforated appendicitis. PLoS One. 2014 Oct 24;9 (10):e111144. doi: 10.1371/journal.pone.0111144. eCollection 2014. 3. Chen CY, Chen YC, Pu HN, Tsai CH, Chen WT, Lin CH. Bacteriology of acute appendicitis and its implication for the use of prophylactic antibiotics. Surg Infect (Larchmt). 2012 Dec;13 (6):38390. doi: 10.1089/sur.2011.135. Epub 2012 Dec 11.

Disclosure: No significant relationships.

\section{PR098}

\section{RESULTS ON NEGATIVE APPENDECTECTOMIES: A DECADE STUDY}

L. Torrent-Jansà ${ }^{2}$, E. Membrilla-Fernández ${ }^{2}$, M.J. Pons-Fragero ${ }^{2}$, Á. Gallego-Vila ${ }^{2}$, A. Martínez-Solà ${ }^{2}$, L. Grande-Posa ${ }^{2}$,

J. Sancho-Insenser ${ }^{2}$

${ }^{1}$ General Surgery, Hospital del Mar, Barcelona/SPAIN, ${ }^{2}$ Emergency Unit. General Surgery Service., Hospital del Mar, Barcelona/SPAIN

Introduction: The aim of this study was to evaluate the clinical outcomes associated with negative appendectomies (NA) in a General Surgery Department.

Material and methods: A retrospective study on a prospectively maintained database of 2059 consecutive appendectomies, among them 68 negatives (3.3\%). Gender, age, surgical approach and postoperative complications were analysed and classified according to the Clavien-Dindo scale.

Results: The mean age was $34 \pm 0.5$ y/o $59 \%$ of them men. Surgical approach was 904 laparoscopic (44\%) and 1113 open approach $(54 \%)$. In the NA group, laparoscopy was used more often (74\% vs. $26 \% ; \mathrm{P}=0,0001)$. Women were more represented in the NA performed by laparoscopy (70\%), accounting for $68 \%$ of all the NA, thus, being more prone to get a negative surgical result $(\mathrm{P}=0.0001)$. $31 \%$ of NA suffered postoperative complications, with no differences in the approach (30\% vs. $31 \%$; P = 1). The Clavien-Dindo I, II and IIIa were $38 \%, 24 \%$ and $33 \%$, respectively. Bowel adhesions and ileitis were the most common conditions mistaken for appendicular disease. Around one fifth of all NA appendectomies did not show any pathological finding, being this outcome more frequent in women ( $26 \%$ vs. $17 \% ; \mathrm{P}=0.52$ ). Since 2011 , there was a consistent decrease in NA rates, from $5 \%$ to $2 \%$. These results correlate to the decrease in the use of radiologic diagnostic methods.

Conclusion: NA rates showed a progressive decrease in the last 6 years. It was more common approached by laparoscopy. Further investigations are needed to understand the inner differences in order to improve acute appendicitis diagnosis.

References:

Disclosure: No significant relationships.

\section{PR099}

\section{CRP AS A PREDICTIVE MARKER FOR GALLBLADDER NECROSIS AND EMPYEMA}

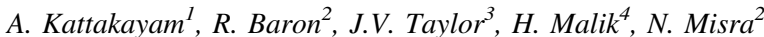

${ }^{1}$ Emergency General Surgical Unit, Aintree University Hospital, Liverpool/UNITED KINGDOM, ${ }^{2}$ Emergency General Surgery, University Hospital Aintree, Liverpool/UNITED KINGDOM, ${ }^{3}$ Emergency General Surgery And Trauma Unit, Aintree University Hospital, Liverpool/UNITED KINGDOM, ${ }^{4}$ Hepatobiliary Surgery, University Hospital Aintree, Liverpool/UNITED KINGDOM

Introduction: Laparoscopic cholecystectomy (LC) in the presence of an empyema or a necrotic inflammatory mass carries significant perioperative risk, which may decrease with earlier intervention. The aim of this study was to see if pre-operative serum CRP can predict the presence of gallbladder necrosis or empyema.

Material and methods: A retrospective study of a database of all acute cholecystectomys was undertaken between June 2016 and June 2017. Data including patient demographics, pre-operative physiology, pre-operative CRP, operative findings, post-operative course and histology were collected.

Results: From a database of 634 patients, a cohort of 128 acute cholecystectomy were examined. $78 \%$ patients with an empyema or necrosis of the gallbladder had a CRP $>90$ as compared to $15 \%$ of patients with a $\mathrm{CRP}<90 ; \mathrm{Chi}^{2}=45.7, \mathrm{p}<0.0001$. Receiver Operator Curve analysis of CRP compared to pathology showed a CRP of 90 resulted in a sensitivity of $80 \%$ and specificity of $86 \%$ (area under the curve of 0.88).

Conclusion: CRP is a useful marker in the pre-operative setting for patients requiring laparoscopic cholecystectomy. A CRP $>90$ on admission or later during the period of medical management may be a sign for urgent operative management due to empyema or gall bladder necrosis

\section{References:}

Disclosure: No significant relationships.

\section{PR100}

\section{CORRELATION BETWEEN INTRAOPERATIVE SURGICAL DIAGNOSIS OF ACUTE APPENDICITIS AND THE PATHOLOGY REPORT}

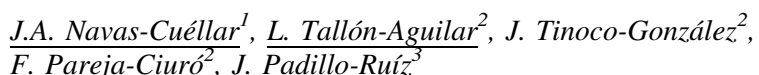

${ }^{1}$ Digestive Surgery Department, Quirónsalud Campo de Gibraltar Hospital, Palmones, Los Barrios, Cádiz/SPAIN, ${ }^{2}$ Emergency Surgery Unit, Virgen del Rocío Universitary Hospital, Seville/SPAIN,

${ }^{3}$ Digestive And General Surgery Unit, Virgen del Rocío Universitary Hospital, Seville/SPAIN

Introduction: The postoperative management of appendectomy for acute appendicitis is based primarily on intraoperative findings. Surgeons describe the severity of disease and antibiotic therapy accordingly it. The histologic findings are not considered in the immediate postsurgical period. The aim of this study is to assess the agreement between Surgeon's intraoperative description and Pathologist's report in a consecutive series of patients with acute appendicitis. 
Material and methods: An analytic observational study was performed in 1947 patients who underwent surgery for acute appendicitis at our hospital during a five-year period (2012-2016). Surgeon's classification of severity of appendicitis was compared with Pathologist's report using the Cohen's Kappa coefficient.

Results: 1947 patients underwent emergency surgery for acute apendicitis (55'8\% male, mean age 33'8 years old). 57'6\% of histological normal appendix was described by the surgeon (the rest was described as not complicated appendicitis). $88^{\prime} 6 \%$ of histological non complicated appendix was previously described by the surgeon. In complicated appendicitis (gangrenous and perforated) the degree of agrement was higher in histological perforated appendicitis (82'6 vs $\left.65^{\prime} 7 \%\right)$. Only in one case $\left(4^{\prime} 5 \%\right)$ the surgeon suspected a tumoral appendix, which was confirmed by the pathologist's exam. The overall degree of agreement was $75^{\prime} 1 \%$. The Cohen's Kappa concordante coefficient was 0 ' 482 .

Conclusion: We found a moderate overall correlation between Surgeons' and Pathologists' descriptions. The higher level of agreement was found in non complicated appendicitis. The strength of agreement was very poor in tumoral appendix. In conclusion, we recommend a systematic histological study of all the surgical specimens of appendectomy for acute appendicitis

References:

Disclosure: No significant relationships.

\section{PR101}

\section{NECROTISING FASCIITIS: TECHNIQUES AND OUTCOMES}

B. Diaconescu, M.R. Bratu, R. Marian, R. Ciocan, D. Ene, M. Beuran

Surgery Department, Emergency Clinical Hospital, Bucharest/ ROMANIA

Introduction: Despite advances in antibiotic and surgical management and supportive care, necrotizing fasciitis are aggressive infections, multimicrobial in origin, due to the synergistic action of anaerobes and aerobes and associated with significant morbidity and organ failure, and high mortality. The rapid progression makes quick diagnosis and treatment critical. The two commonest pitfalls in management are failure of early diagnosis and inadequate surgical debridement.

Material and methods: We present a series of 5 patients with necrotizing fasciitis. Predisposing factors present in the group was diabetes mellitus, alcoholism, immunosuppression, renal and hepatic disease, vascular disease and obesity. For two patients we use VAC therapy in comparative with classical therapy.

Results: The essentials of successful treatment include early diagnosis, aggressive surgical debridement, antibiotics, and supportive intensive treatment unit care. Incision and drainage is an inappropriate surgical strategy for necrotizing soft tissue infections; excisional debridement is needed. Hyperbaric oxygen therapy may be useful, but it is not as important as aggressive surgical therapy.

Conclusion: Survival is higher among patients who receive surgical treatment. Although there are clinical practice guidelines in place, there still remains much variability in choice and duration of antibiotic therapy, time to initial surgical debridement, and use of adjuvant medical therapies.

References:

Disclosure: No significant relationships.

\section{PEDIATRIC TRAUMA}

\section{PR102}

\section{ALTERED CEREBRAL NEUROTRANSMITTERS CONCENTRATIONS AFTER PEDIATRIC MILD TBI}

\author{
P. Menshchikov, A. Manzhurtsev, M. Ublinskiy, N. Semenova, \\ T. Akhadov
}

Radiology, Clinical and Research Institute of Emergency Pediatric Surgery and Trauma, Moscow/RUSSIAN FEDERATION

Introduction: The vast majority of mild traumatic brain injury (mTBI) cases do not lead to abnormalities of brain structures. The correlation between early structural neuroimaging findings and longterm clinical outcomes is weak. The main aim of this work was to estimate changes in in vivo cerebral GABA and Glutamate concentrations after acute mTBI using ${ }^{1} \mathrm{H}$ MRS.

Material and methods: Two groups of participants were included in the study: patients $(\mathrm{n}=11$, mean age $-16 \pm 2$ years, Glasgow Coma Score (GCS) - 15) with acute mTBI (mean time between trauma and MRI examination $40 \pm 20$ hours); 8 healthy children (mean age - 16 \pm 1 years) without history of any TBIs. MR acquisition: $1 \mathrm{H}$ MR spectra were acquired on scanner Phillips 3.0T Achieva TX using PRESS (NAA, Creatine, Choline signals) and MEGA PRESS (GABA and Glutamate signals) pulse sequences. All voxels in size of $25 \times 25 \times 30 \mathrm{~mm}$ were located in the frontal lobe

Results: The main effect on the [GABA] was found $(\mathrm{Z}=2.03, p<$ $0.05)$, with the patients having higher [GABA] as compared to the control group $(36 \%)$. Absolute concentrations of NAA+NAAG, tCho, $\mathrm{tCr}$ and glutamate were unchanged

Conclusion: This study for the first time revealed increased cerebral $[\mathrm{GABA}]$ as well as disorders in the $[\mathrm{GABA}] /[\mathrm{GLX}]$ balance in the pediatric acute mTBI. The most likely cause of [GABA] increase is growth of free pool of GABA (non-related to GABA receptors). Postconcussion changes of neurotransmitter revealed in the present study could be promising for understanding of functional consequences of MRI negative TBI.

References:

Disclosure: No significant relationships.

\section{PR103}

\section{MINIMALLY INVASIVE MANAGEMENT OF PEDIATRIC PANCREATIC INJURIES}

\section{A.L. Gorelik, O.V. Karaseva, A.V. Timofeeva, T. Ahadov}

Department Of Polytrauma, Clinical and Research Institute of Emergency Pediatric Surgery and Trauma (CRIEPST), Moscow/ RUSSIAN FEDERATION

Introduction: At the present time there is not consistent act of treatment of pediatric pancreatic injuries.

Material and methods: 53 children were treated at CRIEPST with pancreatic injuries in the period 2008-2017. Median age was 7,9 \pm 6,8 years old. The boys composed $69,8 \%$, girls $-30,2 \%$. Median ISS was $24,7 \pm 2,08$. The polytrauma predominated $(90,5 \%)$, isolation pancreatic injury was diagnosed in $9,5 \%$ finding.

Results: $83 \%$ of the children had minor injury - AAST grade I - II. Injuries with the damage of Wirsung's duct composed 17\% (AAST 
grade III $11,3 \%$, grade IV $-5,7 \%$ ). The basic guidelines of treatment injuries grade III-IV were: antisecretory therapy, installation of the intestinal tube for enteral nutrition feeding, percutaenous drain under ultrasound control in case omentobursitis (77,8\% finding); laparoscopic drain in case of enzymatic peritonitis (22,2\% finding). All children resumed one's health. There aren't any complications, as of pseudocysts in catamnesis.

Conclusion: Using of the minimally invasive technic coupled with medication allows to elude of the aggressive operative therapy.

References:

Disclosure: No significant relationships.

\section{PR104}

\section{ENDOSCOPIC ASSISTANCE IN ORBITAL TRAUMA IN CHILDREN}

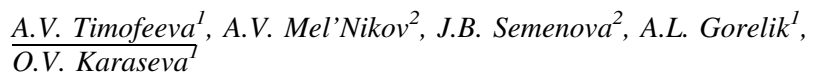

${ }^{1}$ Department Of Polytrauma, Clinical and Research Institute of Emergency Pediatric Surgery and Trauma (CRIEPST) Department of Health of Moscow, Moscow/RUSSIAN FEDERATION, ${ }^{2}$ Department Of Neurosurgery And Neurotrauma, Clinical and Research Institute of Emergency Pediatric Surgery and Trauma (CRIEPST) Department of Health of Moscow, Moscow/RUSSIAN FEDERATION

Introduction: Aim: To assess opportunities and effectiveness of endoscopic assistance in treatment of orbital trauma in children.

Material and methods: Methods: A total of $98.2 \pm 24$ patients with trauma of the face admit to our hospital in a year. Orbital trauma dominates $(62.9 \%)$ : fractures of the orbital floor account for $48.1 \%$, orbital roof $-7.4 \%$, medial and later wall $-3.7 \%$ each.

Results: Results: Due to limited space for work in orbital trauma endoscopy was used often - in $72 \%$. In orbital roof fractures endoscopy was used in all cases to visualize the fracture line, presence of bone fragments, basal cerebrospinal fluid leakage, encephalocele, continuing bleeding from fracture line. In case of fracture of the optic nerve canal endoscopy allows to perform save decompression of the nerve using lateral eyebrow approach. $69.2 \%$ of orbital floor fractures we operate through the maxillary sinus using intraoral approach. Endoscopy allows to make a thorough sanation of the sinus, visualize the interposition of the tissues, perform their correction and make reduction and fixation of the fracture. In the age group up to 12 years, due to the small size of the sinus, endoscopy is used in $100 \%$ of cases and up to $70 \%$ in the older age group. There were no intraoperative complications.

Conclusion: Conclusion: Videoendoscopic assistance in surgical treatment of orbital wall fractures provides an optimal visualization, what enables to specify injury pattern and to perform anatomic reduction and fixation of the fracture using optimal surgical approach, what by-turn reduces surgical trauma and provides better aesthetic and functional outcome.

References:

Disclosure: No significant relationships.

\section{PR105}

\section{RESTING STATE NETWORK ALTERATIONS IN NORMAL- APPEARING CHILDREN BRAIN CORTEX IN ACUTE PERIOD OF MILD TBI. RSFMRI STUDY}

\author{
M. Ublinskiy, N. Semenova, T. Akhadov, I. Melnikov, P. Menshchikov, \\ A. Manzhurtsev
}

Radiology, Clinical and Research Institute of Emergency Pediatric Surgery and Trauma, Moscow/RUSSIAN FEDERATION

Introduction: The aim of this study is to examine functional connectivity of resting state network in normal-appearing cortex in acute period of mild traumatic brain injury (mTBI) using fmRI.

Material and methods: 34 patients were studied in age from 12 to 17 years (mean age - 14.5 years). Group of patients consisted of 17 children with mild traumatic brain injury in acute stage. 17 agematched healthy volunteers comprised control group. Phillips Achieva 3.0T scanner was used. fMRI studies were conducted using EPI BOLD $(\mathrm{TR}=3000, \mathrm{TE}=30)$. fMRI data were processed using functional connectivity toolbox CONN. Statistical processing was performed using software package Statistica 6.0.

Results: Seed-based analysis using default mode network (DMN) parts demonstrated alterations in connectivity between areas of posterior cingulate cortex (PCC) and medial prefrontal cortex (MPFC) and anterior parts of cerebellum in patients. While in control group our analysis showed statistically significant correlations in respective areas connectivity.

Conclusion: Our data demonstrate alterations of DMN network at the resting state, particularly disrupted functional connectivity between MPFC and PCC areas and anterior parts of cerebellum. This fact may indicate a functioning violation in normal-appearing cerebellum as a result of concussion. Resting-state functional connectivity of the DMN could serve as a potential marker for mTBI improved analysis. References:

Disclosure: No significant relationships.

\section{PR106}

\section{IS PLATING OF A SINGLE BONE ADEQUATE IN BOTH BONE FOREARM FRACTURE OF CHILDREN}

\section{K.X. Lim}

Orthopedic Department, Far Eastern Memorial Hospital, New Taipei City/TAIWAN

Introduction: Both bone forearm fractures are common orthopedic injuries. In children, acceptable alignment can tolerate greater fracture displacement and angulation due to the immature bone's remodeling potential. The optimal management and method of fixation in both bone forearm fracture (BBFF) of children have not been clearly established. The goal of this manuscript is to compare the outcome to determine whether fixation of single bone is adequate in BBFF of children.

Material and methods: We studies, retrospectively, 31 both bone forearm fracture in skeleton immature children treated by plating. Group A (11 children) had receive plating of both broken forearm bones and Group B (20 children) had plating of single bone (radius in distal third fracture and ulnar in middle and proximal third fracture). The mean age was 12 years in both groups. In group B an 
acceptable alignment of the unfixed bone was regarded as less than 15 degrees of angulation in both anteroposterior (AP) and lateral planes. Results: Union was achieved in all patients, the mean time being 5.8 weeks in group A and 8 weeks in B. 7 children in group A and 13 in group B had their fixation devices removed, the mean interval was 6.9 months in both group. Complications were noted in three patients (27\%) after fixation in Group A and one patient $(6 \%)$ in Group B. No complications occurred after removal of implants in both group.

Conclusion: We consider that in children with both bone forearm fracture, plating of single bone produces excellent results with reduction of complication rate

References: 1. Treatment of unstable fractures of the forearm in children. Is plating of a single bone adequate? Bhaskar AR, Roberts JA Bone Joint Surg Br 2001 Mar;83 (2):253-8. 2.Non-operative treatment of both-bone forearm shaft fractures in children: Predictors of early radiographic failure Eric N. Bowman, MPH . Author manuscript; available in PMC 2012 Jan 1.

Disclosure: No significant relationships.

\section{PR107}

\section{HIGH INCIDENCE OF RE-DISLOCATION OF PAEDIATRIC DISTAL BOTH-BONE FOREARM FRACTURES WARRANTS PRIMARY STABILIZATION WITH KIRSCHNER WIRES}

\author{
E.A.k.Van Delft ${ }^{1}, J$. Vermeulen $^{2}$, G.J. Van Der Bij ${ }^{2}$ \\ ${ }^{1}$ Trauma Surgery, VUmc, Amsterdam/NETHERLANDS, ${ }^{2}$ Trauma \\ Surgery, Spaarne Gasthuis, Haarlem/NETHERLANDS
}

Introduction: Metaphyseal fractures of the distal forearm are among the most frequent encountered fractures in children. The typical both bone forearm fracture is displaced and as such reduction is commonly necessary. Reduction of the fracture followed by cast immobilization alone has been the standard of care, while stabilization with Kirschner wires is used more and more nowadays. Recent literature shows that up to $91 \%$ of reduced fractures re-dislocate over time when stabilized by a cast alone, supporting the use of Kirschner wires. However ongoing debate exists regarding the optimal treatment for these fractures. As such we determined the incidence of secondary dislocation in AO type 23-M/3.1 paediatric both-bone forearm fractures, and, more importantly, we investigated weather primary use of Kirschner wires prevented re-dislocation and secondary operations in paediatric patients.

Material and methods: To assess the clinical controversy of the treatment of both-bone metaphyseal paediatric forearm fractures we conducted a retrospective cohort study of all consecutive paediatric patients who presented at the emergency department of a large teaching hospital in the Netherlands, throughout a 2-year period (2015-2016). We retrospectively analysed the patient files. Radiographic characteristics, OTC/AO-classification were assessed. Type of treatment, reduction technique, surgical interventions, removal of hardware and complications were recorded.

Results: 51 patients (boy/girl 32/19; mean age 9 years) with dislocated both bone forearm fractures requiring reduction were included in the study. The vast majority ( $>95 \%$ ) where initially treated in the operation room under general anaesthesia. $40 \%$ of all patients were primarily treated with reduction and Kirschner wire fixation. No secondary dislocations occurred in this group. $58 \%$ of the children who received upper arm cast immobilization alone after reduction had re-dislocation and 38\% off all patients treated without $\mathrm{K}$-wires required a secondary intervention in the operating room.
Conclusion: Paediatric distal both-bone forearm fractures have an unacceptable high rate of secondary dislocation when solely stabilized using an upper-arm cast after reduction. As such we strongly recommend direct stabilization using Kirschner wires in all paediatric patients suffering from these fractures.

References: -

Disclosure: No significant relationships.

\section{PR108}

\section{DIFFUSION TENSOR IMAGING FINDINGS IN CHILDREN WITH ACUTE MILD TRAUMATIC BRAIN INJURY}

\author{
I. Melnikov, M. Ublinskiy, M. Akhlebinina, T. Akhadov
}

Department Of Radiation Diagnostics, Clinical and Research Institute of Emergency Pediatric Surgery and Trauma (CRIEPST), Moscow/ RUSSIAN FEDERATION

Introduction: TBI is a leading cause of disability and death in the world. About $75-85 \%$ of all TBIs are classified as "minor", "mild", or "concussions", and can be accompanied with post-concussive symptoms (PCS $)^{1}$. The lack of radiological evidence in mild TBI leads to underestimation of brain injury, which is critical for developing pediatric brain. Diffusion tensor imaging (DTI) is a relatively new neuroimaging technique that is sensitive to subtle changes in white matter fiber tracts and is capable of revealing microstructural axonal injuries ${ }^{2}$.

Material and methods: We retrospectively evaluated clinical and MRI data of 19 pediatric patients with mTBI. The severity of clinical condition of each patient was evaluated with the use of the Glasgow Coma Scale. Mild injury was considered in the cases when the score was not less than 13 points. The control group consisted of 19 agematched non-traumatized children. In all cases MRI was performed with the use of Philips Achieva 3T scanner not later than in 2 days from the moment of trauma. Our basic brain MRI protocol was supplemented with DTI sequence with subsequent FA and ADC analysis.

Results: We identified statistically significant differences in quantitative diffusion MRI parameters for patients with mTBI compared to those without trauma.

Conclusion: Diffusion-tensor imaging is one of the most useful imaging tools in the acute phase of mTBI, which helps to investigate the subtle traumatic brain alterations. Further investigation is needed to determine radiological markers of mTBI, which potentially may help to understand the basis of PCS and define effective treatment variants.

References: 1) Bigler ED. Neuropsychology and clinical neuroscience of persistent post-concussive syndrome. J Int Neuropsychol Soc. 2008; 14 (1):1-22. 2) Pierpaoli C, Basser PJ. Toward a quantitative assessment of diffusion anisotropy. Magn Res Med. 1996; 36:893-906

Disclosure: No significant relationships. 
PR109

\section{MONITORING OF CEREBRAL HYPERTHERMIA IN SEVERE COMBINED TRAUMA INCLUDING TRAUMATIC BRAIN INJURY IN CHILDREN}

\section{Berezhnaya, V. Amcheslavskii, S. Averianov, Y. Berezhnoy, T. Sabinina}

Anaesthesiology And Intensive Care, Clinical and Research Institute of Emergency Pediatric Surgery and Trauma, Moscow/RUSSIAN FEDERATION

Introduction: It is well-known that cerebral hyperthermia is a secondary factor of cerebral damage but it's correction features is poorly covered in the literature in pediatric practice.

Material and methods: Forty patients with severe concomitant injury, including TBI, were observed. The mean value of the ISS scale was $27.3 \pm 2.8$ and the Glasgow Coma Pediatric Scale was $5 \pm 2$. The mean age was $9.5 \pm 8.5$ years. The microwave thermometry (MT) method was used to control thermoregulatory effects on the patient's body. The temperature analysis recorded by the implanted thermal sensor (Tinv) and the MT (Tmt) values was made. Sixteen patients underwent a craniocerebral hypothermia $(\mathrm{CCH})$ session in hypothermia mode (decreased brain temperature to $34{ }^{\circ} \mathrm{C}$ ) and the normothermia mode (decreased brain temperature to $36.5-37.5^{\circ} \mathrm{C}$ ). All patients underwent multiple measurements of the glomerular filtration rate (GFR), using a simple growth-independent Pottel formula. Results: There was no significant differences (Spearman correlation coefficient $=0.845)$ between $\operatorname{Tinv}\left(37.2 \pm 0.2{ }^{\circ} \mathrm{C}\right)$ and Tmt $(36.7 \pm$ $0.2{ }^{\circ} \mathrm{C}$ ). When $\mathrm{CCH}$ was used an increase of the temperature differences between preceding and concomitant hypothermia $\mathrm{T}$ was detected $\left(\Delta \mathrm{Tmt}=2.7{ }^{\circ} \mathrm{C}, \Delta \mathrm{Tinv}=1.9^{\circ} \mathrm{C}\right)$. Assessing the $\mathrm{CCH}$ normothermia mode adverse impact, a statistically significant risk factor of reducing GFR was elicited according to the exact (twosided) Fisher test $(\mathrm{p}=0.02 ; \mathrm{p}<0.05)$.

Conclusion: Non-invasive microwave thermometry is a critical care method of cerebral temperature monitoring at the acute period of severe brain injury. The method has definitely advantages for using in pediatricy. In turn, $\mathrm{CCH}$ is an effective method of cerebral hyperthermia correcting, However, being a risk factor for reducing GFR. References:

Disclosure: No significant relationships.

\section{PR110}

\section{PEDIATRIC SURGEON VS GENERAL SURGEON: DOES SUBSPECIALTY TRAINING AFFECT THE OUTCOME OF USING ESIN METHOD OF PEDIATRIC FOREARM FRACTURES?}

\author{
K. Ivković ${ }^{1}$, D. Divković ${ }^{2}$, T. Galo ${ }^{1}$
}

\section{${ }^{1}$ Department Of Pediatric Surgery, UNIVERSITY HOSPITAL CENTRE OSIJEK, Osijek/CROATIA, ${ }^{2}$ Department Of Pediatric Surgery, UNIVERSITY HOSPITAL CENTRE OSIJEK, OSIJEK/ CROATIA}

Introduction: Elastic stable intramedullary nailing (ESIN) has been established as the standard treatment for forearm shaft fractures in children, if operative stabilization is required. The aim of this study was to compare the outcomes of children treated for forearm fractures using ESIN method by pediatric surgeons (PS) and by general surgeons (GS)

Material and methods: This was a retrospective review of the charts of all patients 4-16 years old who underwent operative treatment forearm fractures with ESIN method during 5 years (2012.-2016.) Outcome measures included length of hospital stay (LOS) and overall rate of complications.

Results: A total of 55 patients (PS group, $\mathrm{n}=37$; GS group, $\mathrm{n}=18$ ) were included. Median LOS was significantly different between the two groups. Patients had longer LOS when treated by GS (7,3 vs 5,8 days). Overall complications were significantly more prevalent in the GS group (8 vs 4). Complications were 2 extensor pollicis longus tendon ruptures, 1 lesion of the superficial radial nerve, 1 wound infection (Enterococcus), 2 malplacement of ESIN, 1 refracture with ESIN in situ and 1 limited range of motions in GS group. In PS group complications were 1 wound infection (Staphylococcus aureus), 2 refractures (1 with ESIN in situ and 1 after ESIN removal) and 1 delayed union.

Conclusion: Complications were significantly more prevalent in patients who were treated by GS $(14,5 \%$ vs. $7,2 \%)$. It is important to pay attention to biomechanical principles and correct technical procedure.

References: 1. F.F. Fernandez, M.Langendorfer. Failures and complications in intramedullary nailing of children's forearm fractures. J Pediatr Orthop. 2010.4:159-167 2. Matthew L.Vopat, P.M.Kane, Melissa A.Christino, J.Truntzer, P.McClure, Julia Katarincic, Bryan G.Vopat. Treatment of diaphyseal forearm fractures in children. Orthopedic Reviews. 2014; vol.6:5325 3. John M.Flynn, Kristofer J.Jones, Matthew R.Garner, Jennifer Goebel. Eleven years experience in the operative menagement of pediatric forearm fractures. J Pediatr Orthop. 2010; 30;313-319 4. C.Kruppa, P.Bunge, T.A.Schildhauer, M.Dudda. Low complication rate of elastic stable intramedullary nailing (ESIN) of pediatric forearm fractures. Medicine.2017;96:16 5. S.-N. Kang, J. Mangwani, M. Ramachandran, J. M. H. Paterson, M. Barry. Elastic intramedullary nailing of paediatric fractures of the forearm - A decade of experience in a teaching hospital in the United Kingdom. The Journal of bone and joint surgery. 2011. vol. 93-B Disclosure: No significant relationships.

\section{PR111}

\section{THE SEVERITY OF COMPRESSION VERTEBRAL FRACTURE MAY BE RELATED TO THE INITIAL FAT FRACTION AND BONE MINERAL DENSITY. 1H MRS STUDY}

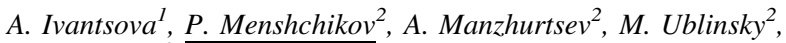 N. Semenova ${ }^{2}$, T. Akhadov ${ }^{2}$}

${ }^{1}$ Institute Of Engineering Physics For Biomedicine, NRNU MEPhI, Moscow/RUSSIAN FEDERATION, ${ }^{2}$ Radiology, Clinical and Research Institute of Emergency Pediatric Surgery and Trauma, Moscow/RUSSIAN FEDERATION

Introduction: The purpose of the study was to explore the relationship between fat fraction (FF), bone mineral density (BMD) and severity of compression vertebral fracture (CVF) caused by injury in children

Material and methods: Twenty patients $(10.9 \pm 2.4$ years $)$ with CVF were studied. Quantitative CT (QCT) was used to determine BMD $\left[\mathrm{mg} / \mathrm{cm}^{3}\right]$ in vertebrae L3, L4 using Philips Brilliance $16 .{ }^{1} \mathrm{H} \mathrm{MR}$ spectra $($ STEAM, TE $=12.8 \mathrm{~ms}, \mathrm{TR}=3000 \mathrm{~ms}$, voxel size $=$ $20 \times 15 \times 10 \mathrm{~mm}$ ) were acquired from the cancellous bone of lumbar 
vertebrae L3, L4 using MRI Philips AchievaTX 3.0 T. FF index was calculated using following equation: $\mathrm{FF}=\mathrm{I}_{\mathrm{fat}} /\left(\mathrm{I}_{\mathrm{fat}}+\mathrm{I}_{\mathrm{water}}\right)$

Results: There were no patients with osteoporosis according to the CT-densitometry results. Correlation analysis revealed significant inverse correlation link ( $\mathrm{p}<0,05, \mathrm{R}=-0,51)$ between FF and BMD for all vertebrae of all patients. Patients were classified into two groups: 8 mild CVF patients (1-2 damaged vertebrae) and 12 severe CVF patients (more than 2 damaged vertebrae). Intergroup analysis revealed significant increase $(\mathrm{p}<0,005) \mathrm{FF}$ and a reduction $(\mathrm{p}<$ $0,005)$ of BMD in patients with severe CVF as compared to mild CVF.

Conclusion: Dependence FF and BMD on degree of the disturbance was revealed in patients without osteoporosis. So even small changes in FF and BMD can increase the risk of more serious injury. Therefore, these parameters must be controlled especially in childhood. Revealed correlation between FF and BMD in children without osteoporosis suggests that the processes of increasing FF and lowering BMD are parallel. Therefore ${ }^{1} \mathrm{H}$ MRS could be good alternative to QCT without radiation dose in osteoporosis detection.

References: 1. EFFO and NOF (1997) Who are candidates for prevention and treatment for osteoporosis? Osteoporos Int 7:1 2. Griffith JF, Yeung DK, Antonio GE, Lee FK, Hong AW, Wong SY, et al. Vertebral bone mineral density, marrow perfusion, and fat content in healthy men and men with osteoporosis: dynamic contrast-enhanced MR imaging and MR spectroscopy. Radiology (2005) 236 (3):945-51. 3. Karampinos DC, Ruschke S, Gordijenko O, Grande Garcia E, Kooijman H, Burgkart R, et al. Association of MRS-based vertebral bone marrow fat fraction with bone strength in a human in vitro model. J Osteoporos (2015) 2015

Disclosure: No significant relationships.

\section{PR112}

\section{COMPARISON OF SPINAL BMD IN PATIENTS WITH VERTEBRAL FRACTURES, A PROLONGED PERIOD OF IMMOBILITY AND HEALTHY CHILDREN}

\section{U. Polyakova ${ }^{1}$, P. Menshchikov $^{1}$, M. Ublinskiy ${ }^{2}$, A. Manzhurtsev $^{3}$, T. Akhadov ${ }^{2}$}

${ }^{1}$ Radiology, Clinical and Research Institute of Emergency Pediatric Surgery and Trauma, Moscow/RUSSIAN FEDERATION,

${ }^{2}$ Department Of Radiation Diagnostics, Clinical and Research Institute of Emergency Pediatric Surgery and Trauma (CRIEPST), Moscow/RUSSIAN FEDERATION, ${ }^{3} 0501$, N.M. Emanuel Institute of Biochemical Physics of RAS, Moscow/RUSSIAN FEDERATION

Introduction: Osteoporosis is a systemic metabolic skeletal disease characterised by reduced bone mass, microarchitectural bone disruption and skeletal fragility, resulting in increased risk of fractures. It is much more common in older people, but osteoporosis can occur in children too. Now there are some studies that proving that fracture risk in childhood is associated with lower trabecular BMD. Also known, that without osteoporosis, bone loss may caused by prolonged immobility leading to decreased bone formation and uncoupling of bone resorption. We compared spinal BMD in patients with vertebral fractures, a prolonged period of immobility and healthy children.

Material and methods: Data from 135 children (80 patients with vertebral fractures, mean aged $10 \pm 3,15$ patients with long period of immobility, mean aged $11 \pm 4$, and 40 healthy children in control group, mean aged $11 \pm 4$ ) were analyzed. All the CT examinations were performed with a 16 and 64-slice CT system. A phantom-less quantitative computed tomography and The Extended Brilliance Workspace (Philips) were used.

Results: A statistically significant decrease in BMD was revealed in patients with compression fractures $(108 \pm 20 \mathrm{~g} / \mathrm{cm} 2)$ and with a long period of immobility $(101 \pm 29 \mathrm{~g} / \mathrm{cm} 2)$ in comparison with the control group $(125 \pm 19 \mathrm{~g} / \mathrm{cm} 2)$ : $\mathrm{t}$-value $=4,2 \mathrm{p}$-value $<0,0001$ and $\mathrm{t}$-value $=$ 3,5 p-value $<0,0001$ respectively.

Conclusion: Children with vertebral compression fractures have lower BMD than conventionally healthy patients. This means that the risk of injury in patients with reduced bone mineral density is actually higher than that of healthy children. Long-term immobile patients also significantly lose bone mass.

References:

Disclosure: No significant relationships.

\section{PR113}

\section{VOMITING AS AN INDICATION FOR IMAGING IN PAEDIATRIC TRAUMATIC BRAIN INJURY - QUESTIONABLE SIGNIFICANCE!}

\section{D.P.C.K.A. Lal}

Surgery, The National Hospital of Sri Lanka, Colombo/SRI LANKA

Introduction: Vomiting is a nonspecific clinical feature of traumatic brain injury and considered as an indication for imaging the brain in suspected traumatic brain injury. Number of vomiting episodes is discussed as a tool to predict the severity of injury and currently three episodes of vomiting is accepted as an indication for computerized tomography in paediatric head injury assessment. The objective of this study was to assess the validity of three times vomiting as an indication for imaging in suspected paediatric traumatic brain injury. Material and methods: This is a retrospective descriptive study. Paediatric head injury patients with vomiting more than three times after the injury was referred for non-contrast computerized tomography of the brain as part of the protocol based management. All the CTs were assessed by the neurosurgical team.

Results: There were 32 patients having 3 to 8 times post traumatic vomiting following accidental falls, sports injury and road traffic accidents. Only 3 patients had CT evidence of traumatic brain injury and managed conservatively without any neurosurgical interventions. 29/32 (90\%) patients had no CT evidence of traumatic brain injury though they had more than three times post traumatic vomiting.

Conclusion: Significant number of paediatric patients undergoes unnecessary transportation and radiation when more than three episodes of vomiting is considered an indication for CT brain in the absence of other indications. Therefore further audit and research are needed to find the real significance of vomiting in the assessment of paediatric traumatic brain injury.

References: Jim B, Simon C. Vomiting and serious head injury in children. J Accid Emerg Med 2000;17:400-402. Hugenholtz H, Izukawa D, Shear P, et al. Vomiting in children following head injury. Childs Nerv Syst1987;3:266-70.

Disclosure: No significant relationships. 
PR114

\section{THE TREATMENT POLICY FOR "PULSELESS PINK HAND” FOLLOWING HUMERAL SUPRACONDYLAR FRACTURES IN CHILDREN}

$\underline{\text { K. Sato }^{l}}, Y . \operatorname{Ueda}^{2}$

${ }^{1}$ Orthopaedic Trauma Center, Sapporo Tokushukai Hospital, Sapporo/ JAPAN, ${ }^{2}$ Division Of Orthopedic Trauma, Sapporo Tokushukai Hospital, Sapporo/JAPAN

Introduction: No treatment policy has been established yet for "Pulseless pink hand" following humeral supracondylar fractures in children. Some reports have stated that if the peripheral perfusion circulation is well perfused, even though the radial artery is not palpable, open surgery, including vessel repair, is unnecessary.

Material and methods: Between April 2011 and March 2017, 116 cases of humeral supracondylar fracture were treated in our trauma centre in children under 14 years of age. Among these cases, there were nine cases of a pulseless pink hand. We investigated the operative procedures, intraoperative findings and, postoperative complications, and considered the treatment policy for the pulseless pink hand.

Results: Eventually, open surgery was performed in all nine cases. In one case, a closed reduction and percutaneous pinning were performed, but following the procedure the radial artery was not palpable, so an arterial repair was done with open surgery. In addition, four cases were complicated by median nerve paralysis, and the nerve was found to be entrapped by peripheral soft tissue in two of these four cases. No deep infection, ischemic muscle contracture of the forearm, or compartment syndrome was observed, nor did any restriction of the range of motion persist.

Conclusion: Since the treatment outcome of open surgery for the pulseless pink hand is good and it allows for the release of an entrapped nerve, it is recommended to pursue open surgery for the pulseless pink hand widely.

References: (1)R. Omid, P. Choi, D Skaggs. Supracondylar humeral fractures in children. J Bone Joint Surg Am. 2008;90:1121-1132 (2)H. Badkoobehi, P. Choi, D. Bae et al. Management of the pulseless pediatric supracondylar humeral fracture. J Bone Joint Surg Am. 2015;97:937-43

Disclosure: No significant relationships.

\section{PR115}

BLUNT CEREBROVASCULAR ARTERY INJURY (BCVI) IN CHILDREN - AN INTERNATIONAL MULTICENTER ANALYSIS

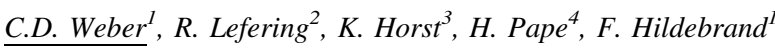

${ }^{1}$ Dept. Of Trauma Surgery, RWTH Aachen University Medical Center, Aachen/GERMANY, ${ }^{2}$ Institut Für Forschung In Der Operativen Medizin, Akademie der Unfallchirurgie GmBH, Cologne/ GERMANY, ${ }^{3}$ Department For Trauma And Reconstructive Surgery, University Hospital Aachen, Aachen/GERMANY, ${ }^{4}$ Division Of Traumatology, University Hospital Zürich, Zürich/SWITZERLAND

Introduction: The purpose of this study is to characterize blunt cerebrovascular artery injury (BCVI) in the pediatric trauma population. We evaluated prevalences, CT imaging, associated injuries and outcome parameters in this multicenter analysis.

Material and methods: The TraumaRegister DGU ${ }^{\circledR}$, a prospectively maintained database, was used for analysis (01/2002-12/2015). Inclusion criteria: Children (0-17years) with severe injuries (ISS $\geq 16$ points) with and without BCVI. Subgroups: carotid artery and vertebral artery injury. Data of demographic, injury, advanced imaging, therapy and outcome characteristics were collected and analyzed using SPSS statistics (Vs.23, IBM Inc., Armonk, NY).

Results: The final study cohort included 8128 children from Germany, Austria and Switzerland. BCVI prevalence in children was $0.5 \%(n=42)$, carotid injuries $(n=30,0,4 \%)$ were found more often than vertebral injuries $(n=12,0.1 \%)$. No significant differences in age and sex were observed. BCVI patients sustained more head $(\mathrm{p}=$ $0.028)$, facial $(\mathrm{p} \leq 0.001)$; chest $(\mathrm{p} \leq 0.001)$ and spinal injuries $(\mathrm{p} \leq 0.001)$. The majority underwent head/neck CT $(85.3 \%$ vs. $94.4 \%$, $\mathrm{p}=0.16)$ or whole-body CT $(64.6 \%$ vs. $86.1 \%, \mathrm{p}=0.008)$. Two BCVI cases did not undergo CT, both deceased early after ER admission. There was an increased risk for thrombo-embolic complications in children with BCVI (1\% vs. $8.3 \%, \mathrm{p}=0.026)$ and in-hospital mortality $(7.7 \%$ vs. $38.1 \%, \mathrm{p} \leq 0.001)$.

Conclusion: Pediatric BCVI is less frequently diagnosed, when compared to adults, despite a high utilization of advanced imaging techniques. Carotid artery injuries are observed more often when compared to injuries of the vertebral artery. The risk factors seem to mimic those reported for adult trauma patients. BCVI in children is associated with a significant burden of complications and excessive mortality.

References: 1) Cook MR et al. A Cohort Study of Blunt Cerebrovascular Injury Screening in Children: Are They Just Little Adults? J Trauma Acute Care Surg. 2017 2) Dewan MC et al. Treatment Practices and Outcomes After Blunt Cerebrovascular Injury in Children. Neurosurgery. 2016 3) Ravindra VM et al. Predicting Blunt Cerebrovascular Injury in Pediatric Trauma: Validation of the "Utah Score". J Neurotrauma. 2017 4) Ravindra VM et al. Risk factors for traumatic blunt cerebrovascular injury diagnosed by computed tomography angiography in the pediatric population: a retrospective cohort study. J Neurosurg Pediatr. 2015

Disclosure: No significant relationships.

\section{PR116}

\section{COMPENSATORY PROCESSES IN NORMAL-APPEARING CHILDREN BRAIN CORTEX IN ACUTE PERIOD OF SEVERE TBI}

\section{Ublinskiy, N. Semenova, T. Akhadov, I. Melnikov, A. Manzhurtsev, P. Menshchikov}

Radiology, Clinical and Research Institute of Emergency Pediatric Surgery and Trauma, Moscow/RUSSIAN FEDERATION

Introduction: The aim of this study is to reveal and characterize compensatory processes in normal-appearing cortex in acute and subacute period of traumatic brain injury (TBI)

Material and methods: 34 patients were studied in age from 5 to 16 years (mean age -12.7 y.). Group of patients consisted of 18 children with severe brain injury (volume of injured tissue was 30-50 ml). 16 age-matched healthy volunteers comprised control group. Phillips Achieva 3.0T scanner was used. MRS-studies were conducted in acute and subacute period of trauma. The area of interest in intact frontal - parietal cortex (volume $=3 \mathrm{~cm}^{3}$ ) was studied using PRESS $(\mathrm{TE}=35 \mathrm{~ms}, \mathrm{TR}=2000 \mathrm{~ms}, \mathrm{NSA}=32)$. 
Results: In comparison with control group significant decrease of NAA, increase of Cho, $\mathrm{mI}$ and $\mathrm{Cr}+\mathrm{PCr}$ was found in patients. A direct statistically significant correlation $(p<0,05)$ between NAA, $\mathrm{Cr}+\mathrm{PCr}$ and $\mathrm{Cho}$ was revealed in both groups: in control group $\mathrm{R}_{\mathrm{NAA}-\mathrm{Cr}}=0.65, \mathrm{R}_{\mathrm{NAA}-\mathrm{Cho}}=0.64, \mathrm{R}_{\mathrm{Cr}-\mathrm{Cho}}=0.61$; in patients group $\mathrm{R}_{\mathrm{NAA}-\mathrm{Cr}}=0.82, \mathrm{R}_{\mathrm{NAA}-\mathrm{Cho}}=0.53, \mathrm{R}_{\mathrm{Cr}-\mathrm{Cho}}=0.66$

Conclusion: Increase of $\mathrm{Cr}+\mathrm{Pcr}$, Cho signal intensities indicates activation of compensatory processes of choline and creatine synthesis in brain cells. At the same time NAA level is reduced. Existing of NAA-Cr and NAA-Cho correlations could mean that activation of $\mathrm{Cr}$ and Cho synthesis causes the NAA decrease. The scheme of metabolism which iexplains NAA, Cr, Cho level changes in TBI is proposed. It follows from the scheme that enhancement of compensatory processes activity requires activation of Krebs cycle.

\section{References:}

Disclosure: No significant relationships.

\section{PR117}

\section{NEW DECADE - DIFFERENT INJURY PATTERN? A STUDY ABOUT THE INCIDENCE AND AETIOLOGY OF FOREARM FRACTURES REQUIRING SURGICAL TREATMENT IN CHILDREN AND ADOLESCENTS FROM 2010 TO 2016}

\author{
D. Sauter, M. Schüler
}

Orthopedics, Kantonsspital Münsterlingen, Münsterlingen/ SWITZERLAND

Introduction: The incidence of fractures in children/adolescents in Europe is $21-25 / 1000$ per year, whereby the data cited is primarily from the end of the 20th century. Forearm fractures are the most common injuries in the paediatric population with 59\%. There is limited information on the aetiology. It is also unclear how often the forearm fractures had to be treated surgically nowadays.

Material and methods: As a retrospective study, we analyzed all forearm fractures in children/adolescents $(<16 y)$ who were treated at our clinic from 2010-2016. Our service area encompassing approx. 26,400 children/adolescents.

Results: 738 forearm fractures were diagnosed. Of these, 296 fractures $(190 \mathrm{~m} / 106 \mathrm{f})$ had to be either set using closed reduction or osteosynthesis (K-wires/TENS/plate). This corresponds to an incidence of forearm fractures requiring treatment of 20/10,000 per year for boys and 12/10,000 for girls. The injury patterns show an age- and gender-specific distribution. Bicycle accidents are found predominantly in the 10-16 years age group, with $20 \%$ for boys and girls. Sports injuries are more common among boys and start at the age of 5-9 years with $27.5 \%$ and rise to $48 \%$ between the ages of 10 and 16 years. Among infants up to the age of 4 years, $56 \%$ of the boys were injured in the playground, as compared with only $27 \%$ of the girls. Conclusion: A prevailing incidence of forearm fractures requiring surgical treatment could be calculated. In the last two years of the observation period, a trend has become apparent which shows that more young people between 10 and 16 years need to have surgery. References: An epidemiological evaluation of pediatric long bone fractures - a retrospective cohort study of 2716 patients from two Swiss tertiary pediatric hospitals. Joeris A, Lutz N, Wicki B, Slongo T, Audigé L. BMC Pediatr. 2014 Dec 20;14:314.

Disclosure: No significant relationships.

\section{PR118}

\section{LEG LENGTH DISCREPANCY AFTER PEDIATRIC LOWER LIMB FRACTURES}

\author{
C. Lee, T. Lan
}

Orthopedic, Far Eastern Memorial Hospital, New Taipei City/ TAIWAN

Introduction: Post traumatic overgrowth is a common cause of pediatric leg length discrepancy. It's common to allow fractures healing in a somewhat shortened position. Our aim was to find how much initial excessive fracture overlap could be done that the discrepancy would be less than $1 \mathrm{~cm}$.

Material and methods: Total of 27 patients aging between 0-15 years old experiencing from either femoral shaft or tibia shaft fracture between 2010- 2015 were reviewed. They were managed by close or open reduction, according to age and fracture pattern. Initial excessive fracture overlap was recorded. Leg length discrepancy was measured utilizing the scanogram after bone healing and at least one year follow-up were achieved.

Results: Femoral shaft group (12 cases) conducted an average overgrowth of $1.06 \mathrm{~cm}$ after one year. Eight of them (8/12) received excessive fracture overlap initially with $0.5 \mathrm{~cm}$ in five cases, $1 \mathrm{~cm}$ in two cases and $1.5 \mathrm{~cm}$ in one case. Two of those not receiving excessive fracture overlap (2/4) required further epiphysiodesis due to ongoing discrepancy of more than $1.5 \mathrm{~cm}$. Tibia shaft group (16 cases) conducted an average overgrowth of $0.67 \mathrm{~cm}$ after one year. Five of them (5/16) received excessive fracture overlap with $0.5 \mathrm{~cm}$ in four cases and $1 \mathrm{~cm}$ in one case. Two of them (2/16) required further epiphysiodesis due to ongoing discrepancy of more than $1 \mathrm{~cm}$.

Conclusion: Our findings indicate that an initial excessive fracture overlap of $0.5 \mathrm{~cm}$ in tibia and $1 \mathrm{~cm}$ in femur may be reasonable to diminish the leg length discrepancy.

References: 1. Stephens MM, Hsu LC, Leong JC., "Leg length discrepancy after femoral shaft fractures in children. Review after skeletal maturity", J Bone Joint Surg Br, 1989; 71:615-618. 2. SangHee Lee, Jae-Young Hong, "Factors related to leg length discrepancy after flexible intramedullary nail fixation in pediatric lower-extremity fractures", Journal of Pediatric Orthopaedics, 2015, Vol 24 No 33. Shapiro F, "Fractures of the femoral shaft in children: the overgrowth phenomenon", Acta Orthop 1981; 52:649-655. 4. Corry IS, Nicol RO. Limb length after fracture of the femoral shaft in children. $J$ Pediatr Orthop 1995; 15:217-219. 5. Martinez AG, Carroll NC, Sarwark JF, Dias LS, Kelikian AS, Sisson GA Jr, "Femoral shaft fractures in children treated with early spica cast", J Pediatr Orthop 1991; 11:712-716. 6. Flynn JM, Skaggs DL. Femoral shaft fractures. In: Rockwood CA, Beaty JH, Wilkins KE, Kasser JR, editors. Rockwood and Wilkins' fractures in children. Philadelphia, PA: Lippincott Williams \& Wilkins, 8th ed 7. James F. Mooney, III and William L. Hennrikus. Tibia shaft fractures. In: Rockwood CA, Beaty JH, Wilkins KE, Kasser JR, editors. Rockwood and Wilkins' fractures in children. Philadelphia, PA: Lippincott Williams \& Wilkins, 8th ed Disclosure: No significant relationships. 
PR119

\section{NEGATIVE PRESSURE THERAPY OF ABDOMINAL CAVITY IN EMERGENCY PEDIATRIC SURGERY AND TRAUMA}

\section{K. Utkina, O.V. Karaseva, A.L. Gorelik, A.V. Timofeeva, D.E. Golikov}

Intensive Care And Polytrauma Unit, Clinical and Research Institute of Emergency Pediatric Surgery and Trauma, Moscow/RUSSIAN FEDERATION

Introduction: Severe traumatic injuries of the abdominal cavity and complicated course of a number of surgical diseases often require to proceed open abdominal management (laparostomy) with delayed programmed revisions, especially in cases of concussion lesions with delayed tissue necrosis. One of the most advanced techniques for temporary closure of abdominal cavity is negative pressure therapy of the abdominal cavity, which is relatively rarely used in pediatric surgery. The aim of our study is to assess advantages of this method in pediatric emergency surgery.

Material and methods: Eight patients were treated using negative pressure abdominal systems at our unit since January 2013 till September 2017. Four patients had traumatic abdominal injuries and four patients had complicated course of surgical diseases (appendicular peritonitis, intestinal malrotation, duodenal ulcer, strangulated inguinal hernia). $62.5 \%$ of patients were male, $37.5 \%$ - female; age varied from 1 month to 17 years old. In all cases we applied special negative pressure abdominal kit, designed for adult patients and adapted for pediatric patients by ourselves.

Results: The number of abdominal cavity revisions varied from 1 to 7 during the treatment period. We observed lethal outcomes in 3 cases: 2 patients died due to severity of primary abdominal lesions, 1 patient - due to non-abdominal severe sepsis. In 5 patients we achieved a successful abdominal sanitation with good recovery.

Conclusion: Using negative pressure abdominal system as a method of temporary closure of abdominal cavity has many significant advantages and allow creating conditions for abdominal cavity sanitation, timely revisions and surgical correction of delayed lesions.

References:

Disclosure: No significant relationships.

\section{PR120}

\section{A NOVEL SURGICAL TECHNIQUE FOR LATERAL HUMERAL CONDYLAR FRACTURES IN CHILDREN: TENSION BAND FIXATION WITH AN ABSORBABLE SUTURE}

\author{
T. Waki, K. Sawauchi, K. Ito, Y. Nakanishi, R. Kato, T. Yano, \\ S. Matsushima
}

\section{Orthopaedic Surgery, Akashi Medical Center, Akashi/JAPAN}

Introduction: Lateral humeral condylar fractures are the second most frequent elbow fractures in children. These displaced fractures require surgical interventions to prevent nonunion. ${ }^{1}$ We report on a novel surgical technique for these fractures using tension band fixation with two removable Kirschner wires and an absorbable suture.

Material and methods: From 2008 to 2016, 17 patients (12 boys and 5 girls), with lateral humeral condylar fractures displaced more than 2 $\mathrm{mm}$, treated with open reduction and tension band fixation. Once anatomical reduction was achieved, tension band fixation was performed using two Kirschner wires (1.0-1.6 mm) and an absorbable suture (1 or 2 Vicryl, Ethicon). Kirschner wires left protruding the skin were removed 4 to 8 weeks after surgery under no anesthesia. Carrying angle and range of motion limitations were assessed, and complications were investigated. The Flynn criteria were used to assess the cosmetic and functional outcomes.

Results: The average limitation angle of elbow joint was (3.5+/-6.3) degrees, and the Carrying angle was (8.2+/-3.2) degrees. According to Flynn criteria, the cosmetic outcomes were excellent in 14 and good in 3 , and the functional outcomes were excellent in 14, good in 1 and fair in 2. Nonunion and avascular necrosis were not observed.

Conclusion: In this study, we used tension band fixation with an absorbable suture. Cadaveric study have shown that tension band fixation with an absorbable suture is superior in maintenance of reduction to fixation with two Kirschner wires only. ${ }^{2}$ Furthermore, subsequent reoperation is avoided by the use of an absorbable suture instead of a metal cable. Good clinical results were achieved and nonunion was not observed using our technique. Open reduction and tension band fixation with Kirchner wires and absorbable suture could be an effective treatment for displaced lateral humeral condylar fractures in children.

References: 1. Tejwani N et. al. J Am Acad Orthop Surg 2011; 19: 350-358. 2. Elliott MJ et. al. J Pediatr Orthop B 2005; 14: 444-447. Disclosure: No significant relationships.

\section{PR121}

\section{SPECIALIZED SURGICAL CARE TO CHILDREN SUFFERED IN EARTHQUAKES}

\section{Mitish, P. Medinskiy, R. Nalbandyan, L. Roshal}

Pediatric Surgery, Clinical and Research Institute of Emergency Pediatric Surgery and Trauma, Moscow/RUSSIAN FEDERATION

Introduction: The study demonstrate the effectiveness of the method of active surgical treatment in case of huge limb injury that are complicated by infection in children suffered in earthquakes.

Material and methods: This study based on the treatment of 226 children suffering in earthquakes in Pakistan (2005), Indonesia (2006, 2009), Haiti (2010) and Nepal 2015. The children were admitted to the organized surgical centers (based on undestroyed local hospitals). All patients had open wounds of the extremities. Open fractures were $23,0 \%$, deep infection of the amputation stump were $15,5 \%$ and crush syndrome were $13,8 \%$.

Results: Typical mistakes for the first stage of surgical care (performed by other surgeons) have been detected: refusal to make a debridement, performing semiclosed fasciotomy without lesion focus revision, putting primary sutures on the crashed tissues, finishing the surgery of extremity amputation with the primary stump formation, performing internal osteosynthesis under surgical infection. Our method of active surgical treatment included: radical wound debridement, intensive therapy, wound treatment with multicomponent ointments; external osteosynthesis (include Ilizarov method) and early reconstructive and plastic surgeries (skin grafting - 22,1\%, plastics with local tissues $-77,9 \%$ ). Healing with primary intension was seen in $96.5 \%$ cases. All damaged limbs planned to amputations early were saved. All the children with huge open wounds gave satisfactory results.

Conclusion: Specialized surgical treatment to child victims of earthquakes with huge injuries of limbs should be provided by experienced surgeons and anesthesiologists. Primary and deferred 
reconstructive plastic surgeries restore anatomical and functional integrity of the damaged limbs and minimize the rate of disability. References: 1. Mitish V.A., Roshal L.M., Medinskiy P.V. (2016) Amputations in Children with Severe Trauma. In: Wolfson N., Lerner A., Roshal L. (eds) Orthopedics in Disasters. Springer, Berlin, Heidelberg. pp 489-500. 2. Mitish V., Roshal L., Medinskiy P., Nalbandyan R. Role of mobile surgical team in treating children with serious injuries after the earthquakes. Difficulties in organizing the mission. Prehospital and Disaster Medicine 2013; 28: Suppl 1: 41. 3. Roshal L.M. Problems of rendering medical aid to children in disasters, wars and terrorist events. Prehospital and Disaster Medicine 2007; 22: 2: 92-93.

Disclosure: No significant relationships.

\section{PR122}

\section{GASTROINTESTINAL BLEEDING IN CHILDREN WITH SEVERE COMBINED TRAUMA}

\author{
A. Kharitonova, O.V. Karaseva, A.L. Gorelik, M. Kovalenko
}

Endoscopy, Clinical and Research Institute of Emergency Pediatric Surgery and Trauma, Moskow/RUSSIAN FEDERATION

Introduction: Severe trauma refers to the types of childhood traumatism with high mortality. One of the causes of fatal outcomes is bleeding from acute gastrointestinal ulcers. To research the frequency, structure, and macroscopic manifestations of gastrointestinal lesions in patients with ST.

Material and methods: 124 children aged 0 to 18 years were examined. The severity of the lesions was assessed on the ISS scale. The average value is 27.5 points. All patients were divided into 6 groups on the basis of leading damage. The largest group consisted of 69 children with a predominance of craniocerebral trauma. Esophagogastroduodenoscopy (EGDS) with examination of the initial parts of the jejunum was performed at 7-10 days of posttraumatic period. To children with severe spinal trauma, EGDS were conducted at 1-2 days. The presence of pathological impurities, the state of the mucosa, peristaltic activity was assessed. In the diagnosis of bleeding, combined endoscopic hemostasis was performed.

Results: Erosions and ulcers were identified as the main endoscopic pathology in groups of children with leading skeletal, combined and abdominal trauma. The group with abdominal trauma was the leader in the number of gastrointestinal bleeding.

Conclusion: For the critical period ST is characterized by destructive changes in the upper gastrointestinal tract, which determines the obligatory planned conduct of EGDS in the monitoring regime.

References:

Disclosure: No significant relationships.

\section{PR123}

\section{IDENTIFYING BARRIERS TO CHILD PASSENGER RESTRAINT USE IN QATAR: INITIAL RESULTS FROM THE YKISS KAP SURVEY}

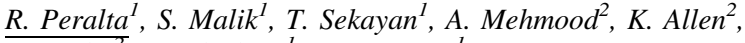

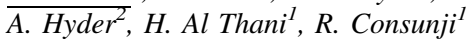

${ }^{1}$ Surgery, Hamad General Hospital \& Hamad Medical Corporation,
Doha/QATAR, ${ }^{2}$ Johns Hopkins Bloomberg School Of Public Health,
Johns Hopkins University, Baltimore/MD/UNITED STATES OF AMERICA

Introduction: Motor vehicle crashes are one of the leading causes of death and injury among young children, under 5 years, in Qatar. The objective of this survey is to examine knowledge, attitudes and usage of Child Passenger Restraint Systems [CRS] in a sample of parents in Doha, Qatar. It was conducted as part of a larger grant [NPRP 7-16813-429] funded by the Qatar Foundation designed to measure current CRS knowledge, attitudes and practices and test two potential interventions to improve their use in Qatar

Material and methods: A survey, containing 25 questions that tested CRS knowledge, attitudes and practices of participants, was applied by trained health communicators in 3 Clinics of the Primary Health Care Corporation.

Results: There were 602 parents, of children under 5 years consulting at well-baby clinics, that responded to the survey. Seventy five percent needed instructions on when to change from rear to forward facing CRS, only $47 \%$ used a CRS for their children; $53 \%$ either did not have or did not use a CRS. The most common reason CRS nonuse was child crying when in CRS (56\%). Moreover, nearly all [94\%] reported never witnessing enforcement of child passenger laws prohibiting children from riding in the front.

Conclusion: Half of young children, in this population, are not benefiting from safety provided by CRS. There is a need to develop awareness programs that educate caregivers on the proper use of CRS. Another priority area for intervention includes the consistent enforcement of laws banning child passengers in the front seat.

References: 1. Keay L, Brown J, Hunter K, Ivers R. Adopting child restraint laws to address child passenger injuries: Experience from high income countries and new initiatives in low and middle income countries. Injury. 2015;46 (6):933-4. 2. Bener A, Hussain SJ, Ghaffar A, Abou-Taleb H, El-Sayed HF. Trends in childhood trauma mortality in the fast economically developing State of Qatar. World J Pediatr WJP. 2011 Feb;7 (1):41-4.

Disclosure: No significant relationships.

\section{PR124}

\section{ESIN METHOD IN PEDIATRIC FOREARM FRACTURES: DOES THE OPERATION TIME AFTER INJURY AFFECTS METHODS (OPEN VS. CLOSED REDUCTION WITH ESIN) OF OPERATIVE TREATMENT?}

\author{
K. Ivković ${ }^{1}$, D. Divković ${ }^{2}$, T. Galo ${ }^{1}$
}

${ }^{1}$ Department Of Pediatric Surgery, UNIVERSITY HOSPITAL CENTRE OSIJEK, Osijek/CROATIA, ${ }^{2}$ Department Of Pediatric Surgery, UNIVERSITY HOSPITAL CENTRE OSIJEK, OSIJEK/ CROATIA

Introduction: The aim of this study was to assess does the operation time after injury affects methods of operative treatment (open vs. closed reduction with ESIN method).

Material and methods: This was a retrospective review of the charts of all patients 4-16 years old (age averaged 9,1 years) who underwent operative treatment forearm fractures with ESIN method during 5 years (2012.-2016.). This study included 55 patient who underwent operative treatment at the day of injury at our Department, several days ( $2-9$ days) after injury or failed closed reduction at our Department and failed operative treatment at another Department of surgery (9 - 23 days after injury). 
Results: Four of 14 patients who underwent operative treatment at the day of injury at our Department were treated with open reduction with ESIN (28\%). Six of 16 patients were treated several days after injury or failed closed reduction at our Department with open reduction with ESIN (37\%). Twenty of 25 patients who was sent at our Department from another Department of surgery were treated with open reduction with ESIN (80\%)

Conclusion: The analysis shows that later time of operative treatment after injury significantly increase need for open reduction with ESIN. References: 1. F.F. Fernandez, M.Langendorfer. Failures and complications in intramedullary nailing of children's forearm fractures. J Pediatr Orthop. 2010.4:159-167 2. Matthew L.Vopat, P.M.Kane, Melissa A.Christino, J.Truntzer, P.McClure, Julia Katarincic, Bryan G.Vopat. Treatment of diaphyseal forearm fractures in children. Orthopedic Reviews. 2014; vol.6:5325 3. John M.Flynn, Kristofer J.Jones, Matthew R.Garner, Jennifer Goebel. Eleven years experience in the operative menagement of pediatric forearm fractures. J Pediatr Orthop. 2010; 30;313-319 4. C.Kruppa, P.Bunge, T.A.Schildhauer, M.Dudda. Low complication rate of elastic stable intramedullary nailing (ESIN) of pediatric forearm fractures. Medicine.2017;96:16 5. S.-N. Kang, J. Mangwani, M. Ramachandran, J. M. H. Paterson, M. Barry. Elastic intramedullary nailing of paediatric fractures of the forearm - A decade of experience in a teaching hospital in the United Kingdom. The Journal of bone and joint surgery. 2011. vol. 93-B Disclosure: No significant relationships.

\section{PR125}

\section{PATHOLOGICAL DIAPHYSIAL FRACTURES IN CHILDREN}

\section{N. Serova $^{l}$, S. Nikishov ${ }^{1}, \underline{\text { M. Ushakov }}{ }^{2}$}

\begin{abstract}
${ }^{1}$ Traumatology Department, Clinical and Research Institute of Emergency Pediatric Surgery and Trauma (CRIEPST), Moscow/ RUSSIAN FEDERATION, ${ }^{2}$ Pediatric Department, Pirogov Russian National Research Medical University, Moscow, Russia, Moscow/ RUSSIAN FEDERATION
\end{abstract}

Introduction: Diagnostics and treatment of pathological fractures in children is an actual problem for nowadays because pathological fractures is a frequent complication in patients with bone and skeleton diseases. There is no uniform protocol for treating pathological fractures either in national or foreign literature. Therefore currently, pediatric traumatologists-experts are looking for an optimal technique for treating pathological fractures in the acute period.

Material and methods: 60 children with pathological diaphysial fractures were examined and operated on in the Clinical and Research Institute of Emergency Pediatric Surgery and Trauma from 2010 till 2016.

Results: After retrospectively analyzing a group of 60 children with pathological diaphysial fractures in 2010- 2016 the researchers found out that the percentage of initially revealed osseous cysts was $61.6 \%$. Trauma is the primary reason when patients are seeking for medical help. The most frequent trauma location is the femur $(60 \%)$.Skeletal traction is the most often surgical technique for treating fractures in their acute period in the top third of femur diaphysis $(10.02 \%, 6$ cases); in the middle third, it is little-invasive intramedullary osteosynthesis with TENs (titan elastic nails) (11.6\%, 7 cases); in the lower third, it is closed fracture reposition with osteosynthesis needles (8.3\%, 5 cases).

Conclusion: As far as there is no any uniform protocol on selecting a curative modality in children, each case needs an individual approach which depends on fracture's type and patient's basic disease. The authors consider that little-invasive intramedullary osteosynthesis with TENs is a preferable approach. While using intramedullary osteosynthesis, social and economic efficiency of treatment is increased.

References:

Disclosure: No significant relationships.

\section{PR126}

\section{DIAGNOSTIC EFFICIENCY OF COMPUTED TOMOGRAPHY IN PEDIATRIC ANKLE JOINT INJURY}

\author{
I. Melnikov, T. Kostikova, M. Ublinskiy, T. Akhadov, M. Akhlebinina
}

Department Of Radiation Diagnostics, Clinical and Research Institute of Emergency Pediatric Surgery and Trauma (CRIEPST), Moscow/ RUSSIAN FEDERATION

Introduction: Fractures of the distal departments of the peroneal and tibial bones in children account for about $5 \%$ of all fractures. The frequency of the distal tibial growth plate damage, among others, is about $15-20 \%$, second only to the distal radius. A significant portion of the fractures also includes damage to the distal epiphysis of the tibia, that is, these fractures are regarded as intra-articular fractures. Incorrect assessment of the growth zones deformation and the presence of diastases of fragments of the articular surface and the incorrect choice of treatment tactics may lead to growth disorders, changes in joint biomechanics, development of posttraumatic osteoarthritis.

Material and methods: We performed the retrospective data analysis of 63 patients with ankle joint fractures who admitted in the CRIEPST in 2016. The age of the patients varied from 9 to 17 years. All patients underwent classical radiography and CT.

Results: Bone lesions evaluation mismatch was revealed in $36 \%$ patients. In 13 cases, X-ray did not visualize the line of tibial distal epiphysis fracture. Diastasis of the articular surface of more than 2 $\mathrm{mm}$ was observed in six patients. Damage to the distal intercostal syndesmosis was diagnosed in two patients; in one child osteochondral fragments were revealed; in 2 children a subluxation of the foot was detected. For seven patients, on which X-ray showed distal epiphysis injury, CT found diastases between the fragments more than 2 $\mathrm{mm}$. In 16 cases, the above-mentioned findings on CT forced trauma surgeons to change the tactics of treatment, i.e. to choose other than on the results of X-ray. Thus, regarding to our data the specificity of $\mathrm{X}$-ray for detecting ankle joint damage was $75 \%$, and for fractures of the distal epiphysis - only $35 \%$.

Conclusion: For detailed diagnosis, which allows to choose the optimal surgical tactic, X-ray is not effective enough. Our results allow us to recommend including $\mathrm{CT}$ in the diagnostic algorithm in patients with ankle injury.

References:

Disclosure: No significant relationships. 


\section{PR127}

\section{PROXIMAL RADIAL FRACTURE IN CHILDHOOD}

\section{S. Nagy, Z. Kincses, P. Marosán}

Traumatology, Pándy Kálmán Hospital, Gyula/HUNGARY

Introduction: Radial head and neck fractures are very rare in childhood. The cases which need operation are rarer. An American multicenter study found 167 operative cases in a 5 years long period with retrospctive analysis. The patient were operated by 23 different surgeons, and only 5 doctors make operation more than 10 times.

Material and methods: We will show in some slide the pathophysiology of the fracture, and the indications of the conservative and operative treatment. We will present the expected complications. We would like to present 4 cases in our departement. I would like to speak about our patients 's first X-rays, the methods and the late functional results. In one case we made CT scan, in another we made MRI, so we can compare these 2 different diagnostical methods.

Results: Making statistical analysis is impossible if you have only 4 cases. Even so our resuslts are the same, as it is published in international literature.

Conclusion: In the international literature's authors pay attention: if we decided making operation, we have to do in the day of the accident or maximum at the end of the 3rd day. Otherwise the complication rate will increase.

References: Brian Weatherford: Radial Head and neck fractures Pediatric Ujash Sheth: Elbow dislocation - Pediatric

Disclosure: No significant relationships.

\section{PR128}

\section{PROLONGED HOSPITALIZATION AFTER ORTHOPEDIC INJURY IN UGANDAN CHILDREN: RESULTS FROM A PROSPECTIVE TRAUMA REGISTRY}

\section{D.J. Zheng $^{1}$, S..A. Christie ${ }^{1}$, M.M. Ajiko ${ }^{2}$, C. Juillard ${ }^{1}$, R.A. Dicker ${ }^{3}$}

${ }^{1}$ Surgery, University of California, San Francisco, San Francisco/CA/ UNITED STATES OF AMERICA, ${ }^{2}$ Surgery, Soroti Regional Referral Hospital, Soroti/UGANDA, ${ }^{3}$ Surgical Critical Care, University of California, Los Angeles, Los Angeles/CA/UNITED STATES OF AMERICA

Introduction: Orthopedic injuries in children pose a particular challenge in low-and middle-income countries due to unavailability of definitive surgical management. ${ }^{1}$ We sought to describe patterns of injury and predictors of prolonged hospitalization among pediatric orthopedic patients using trauma registry data from Uganda to identify opportunities for resource optimization.

Material and methods: All injured patients presenting to a regional referral hospital in rural Uganda between October 2016 and April 2017 were included in a prospective trauma registry. Demographic, injury, treatment, and outcome information including length of hospitalization were recorded using a standardized form.

Results: Of 1824 injured patients, $43 \%$ were age 18 or younger. Of these, $460(59 \%)$ presented with orthopedic injuries. The median age of pediatric orthopedic patients was 7 and $65 \%$ were male. Falls comprised the most frequent mechanism of injury (78\%). Among patients admitted for fractures, $48 \%$ underwent closed reduction and casting, $28 \%$ received no treatment, and $23 \%$ underwent traction. No children underwent definitive open reduction and internal fixation. Multiple logistic regression analysis adjusted for injury severity, mechanism, and presence of other injuries demonstrated that use of traction significantly predicted prolonged hospitalization ( $>30$ days) (OR 42, p = 0.004).

Conclusion: Use of traction for pediatric fractures in Uganda is associated with prolonged length of hospital admission, indicating a potential target for improved resource utilization. ${ }^{2}$ Cost-effectiveness analysis of fixation in pediatric orthopedic trauma should be considered to identify optimal care strategies to reduce length of stay and improve outcomes for children with fractures in rural Uganda.

References: [1] Kramer EJ, Shearer D, Morshed S. The use of traction for treating femoral shaft fractures in low- and middle-income countries: a systematic review. Int Orthop. 2016;40 (5):875-83. [2] Parkes RJ, Parkes G, James K. A systematic review of costeffectiveness, comparing traction to intramedullary nailing of femoral shaft fractures, in the less economically developed context. BMJ Glob Health. 2017;2 (3):e000313.

Disclosure: No significant relationships.

\section{PR129}

\section{A QUESTIONNAIRE FOLLOW-UP STUDY ON THE OVERALL INFECTIOUS PROBLEMS IN PEDIATRIC TRAUMA PATIENT AFTER BLUNT SPLENIC INJURY}

R. Spijkerman, M.P.J. Teuben, T. Blokhuis, W.L.m. Kramer, L.P.h. Leenen

Trauma Surgery, University Medical Center Utrecht, Utrecht/ NETHERLANDS

Introduction: Splenectomy used to be the main treatment modality for blunt splenic injuries (BSI). It is known that asplenic patients are at increased risk of developing bacterial infections, that sometimes develop in an overwhelming post-splenectomy infection (OPSI) syndrome. However little evidence is available about the overall long term infectious problems that asplenic patients experience. Therefore the aim of this study was to analyze long term infectious problems after a blunt splenic injury.

Material and methods: A study of all pediatric patients sustaining BSI managed at our level I trauma center from January 1979 to March 2012 was performed. A questionnaire was sent to all included patients to determine the amount of fever episodes, amount of antibiotic treatments, amount of general practitioner (GP) visits for fever and overall increase of GP visits for fever. Furthermore hospital visits and admissions due to fever or infections were noted.

Results: We included 116 children with BSI. A total of 93 completed interviews were eligible for analysis, resulting in a total response rate of $80 \%$ and 1116 patient years. Twenty-seven patients were splenectomized, and 66 patients were treated by a spleen preserving therapy. No differences were found between both study groups concerning fever, antibiotic treatments and GP visits. There were more hospital admissions seen in the splenectomy group (4/27) compare to the spleen preserving group (1/66).

Conclusion: Traumatic splenectomy in pediatric patients with BSI is not associated with more GP visits, antibiotic treatments or fever episodes. However hospital admissions for infectious complications are more often seen in splenectomized patients.

References:

Disclosure: No significant relationships. 
PR130

\section{A DESCRIPTIVE ANALYSIS OF AMBULANCE RECORDS ON EMERGENCY PEDIATRIC TRANSPORT IN OSAKA CITY, JAPAN: A POPULATION-BASED STUDY}

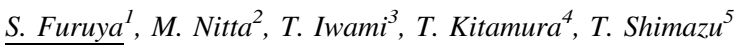

${ }^{1}$ Emergency Medical Center, Osaka City General Hospital, Osaka/ JAPAN, ${ }^{2}$ Emergency Medicine, Osaka Medical University, Takatsuki/JAPAN, ${ }^{3}$ Health Service, Kyoto University, Kyoto/ JAPAN, ${ }^{4}$ Social A and Environmental Service, Osaka Unversity, Suita/JAPAN, ${ }^{5}$ Traumatology And Acute Critical Medicine, Osaka Unversity Graduate School of Medicine, Suita/JAPAN

Introduction: Recently, the transport to a hospital by ambulances has been increasing in Japan, and the number of transported emergency children was 240,918 cases in 2014 . However, the current situation of pediatric emergency transport has not been sufficiently understood. The aim of this study was to conduct descriptive analyses of ambulance records on pediatric emergency patients in Osaka City from January 2013 to December 2014.

Material and methods: Excluding the patient request transport or intra-hospital transport, we analyzed a total of 18,891 emergency pediatric patients aged $<=15$ years old from ambulance records of Osaka Municipal Fire Department by age group (newborn: 0-28 days, infant: 29 days - 1 year old, and children: 1-15 years old).

Results: Of 18,891 emergency pediatric patients, the proportion of children was $90.2 \%$ (17031 cases), infants 9.4\% (1781 cases), and newborns $0.4 \%$ (79 cases), respectively. In whole children, the transport number of boys was larger than girls (11193 cases versus 7698 cases), and the number of infants aged 1 year old was largest (3484 cases) in these age groups. The proportion of severity degree among pediatric patients were mild $89.8 \%$ (16956 cases), moderate $9.9 \%$ ( 1872 cases), severe $0.2 \%$ ( 39 cases), and death $0.1 \%$ ( 24 cases). Conclusion: From a population-based ambulance records, we described characteristics of pediatric emergency patients in Osaka City. This fundamental data would be of help to improve medical care system for the transport of children.

References:

Disclosure: No significant relationships.

\section{PR131}

\section{PAEDIATRIC ARTHROSCOPIC SURGERY: OUR EXPERIENCE IN THE PAST 5 YEARS}

\section{Bustamante, E. Vacas, R. Marti, R. Viña}

Orthopaedic Surgery And Traumatology, Hospital Universitario 12 de Octubre, Madrid/SPAIN

Introduction: Indications of arthroscopic surgery in the paediatric population are increasing progressively, mostly in knee pathology. The most frequent diagnoses are meniscal and ligamentous lesions. Our objective is to present an epidemiological study based on our experience in paediatric arthroscopy in the last 5 years.

Material and methods: We performed a retrospective study between 2011 and 2016. Patients who underwent arthroscopic surgery in the paediatric area, including epidemiological, clinical and surgical data, were reviewed.

Results: A total of 33 knees were reviewed in 32 patients (18 males/ 17 females). The mean age at which patients were intervened was
14.6 years (1-17). Forced rotation movements were the main cause of injury (13 cases), followed by trauma (10 cases). The mean follow-up time was 36 months. Regarding the arthroscopic findings, the most frequent were meniscal lesions ( 14 cases//42\%), followed by ruptures of the anterior cruciate ligament ( 8 cases $/ / 24 \%)$. The degree of correlation between preoperative diagnosis and the arthroscopic findings was $87.8 \%$. Concerning the misdiagnosis cases, no lesions were found in 1 patient. In the other 3 cases, the meniscal lesions found differed from the ones described in the Magnetic Resonance. In 5 cases, recurrence of their pathologies was assessed, from which 2 patients required reintervention.

Conclusion: Paediatric arthroscopy is increasing its indications progressively, reaching the level of adult arthroscopic surgery. The peculiarities of these patients, such as smaller joints and the presence of the growing plates, sometimes involve surgery of high complexity and a steep learning curve. Nevertheless, it presents a low rate of morbidity and associated complications.

References: Utukuri MM1, Somayaji HS, Khanduja V, Dowd GS, Hunt DM. Update on paediatric ACL injuries. Knee 2006 Oct;13 (5):345-52. Good CR1, Green DW, Griffith MH, Valen AW, Widmann RF, Rodeo SA. Arthroscopic treatment of symptomatic discoid meniscus in children: classification, technique, and results. Arthroscopy 2007 Feb;23 (2):157-63. Hagino T, Ochiai S, Watanabe Y, Senga S, Saito M, Wako M, Ando T, Sato E, Haro H. Usefulness of knee arthroscopy for diagnosis of knee pain in pediatric patients: comparison with preoperative clinical diagnosis. Arch Orthop Trauma Surg (2013) 133:669-673 Accadbled F. Arthroscopic surgery in children. Orthop Traumatol Surg Res 2010 Jun;96 (4):447-55

Disclosure: No significant relationships.

\section{PR132}

\section{PEDIATRIC INJURY PROFILE FOR CHILDREN AGES 0-14 YEARS OLD IN HONDURAS}

$\underline{\text { K. Long }}{ }^{1}$, F.J. Bonilla-Escobar ${ }^{2}$, C. Rodriguez ${ }^{3}$, J.C. Puyana ${ }^{4}$

${ }^{1}$ School Of Medicine, University of Pittsburgh, Pittsburgh/PA/ UNITED STATES OF AMERICA, ${ }^{2}$ Department Of Ophthalmology, UPMC Mercy Hospital, Pittsburgh/PA/UNITED STATES OF AMERICA, ${ }^{3}$ Direccion General, Hospital Escuela Universitario (HEU), Tegucigalpa/HONDURAS, ${ }^{4}$ Department Of Surgery, University of Pittsburgh Medical Center, Pittsburgh/PA/UNITED STATES OF AMERICA

Introduction: Worldwide, approximately 2000 children die daily due to injuries ${ }^{1}$. Honduras had the highest global homicide rate from 2013-2016 ${ }^{2}$, yet no comprehensive pediatric injury profile exists for Honduras. A paper-based injury surveillance system (InSS) registered all cases of injuries in the emergency department at Honduras' largest hospital in Tegucigalpa; however, data from this registry has never been analyzed. This study aims to determine the injury profiles for children 0-14 years old.

Material and methods: A cross-sectional study of injuries in patients 0-14 years old was completed from the 2013 InSS database using descriptive data analysis.

Results: Out of the 17,971 total patients, 5,873 (32.68\%) patients were 0 -14 years old, with an average age of $7 \pm 4$ years, and 3,790 $(64.5 \%)$ were male. Unintentional injuries constituted for 5,539 (94.2\%) of the total injuries, with $9.9 \%$ as traffic collisions, $59.0 \%$ as falls, and $11.7 \%$ as blunt force trauma. There were $109(1.9 \%)$ selfinflicted and 230 (3.9\%) interpersonal injuries. The mortality rate was $0.24 \%$, and $84 \%$ of the children spent $\leq 3$ days in the hospital. 
Conclusion: Childhood injuries are highly prevalent and are $1.8 \mathrm{x}$ more likely in males, similarly seen in the $\mathrm{US}^{3}$ and Nicaragua ${ }^{4}$. The most typical unintentional injuries in Honduras were also seen in Nicaragua ${ }^{4}$ and in the $\mathrm{US}^{5}$. A similar percentage of intentional injuries compared to total injuries in Honduras (5.8\%) were seen in Mozambique $^{6}(6 \%)$, while the percentages in the US were $0.27 \%$ for nonfatal and $0.002 \%$ for fatal injuries ${ }^{10}$. With this knowledge, injury prevention strategies can be focused on Honduras' specific needs.

References: 1. World Health Organization. World Report on Child Injury Prevention. Geneva2008. 2. United Nations Office on Drugs and Crime. Global Study on Homicide. Vienna2013. 3. Odetola, F. O., \& Gebremariam, A. (2015). Paediatric trauma in the USA: patterns of emergency department visits and associated hospital resource use. International journal of injury control and safety promotion, 22 (3), 260-266. 4. Martínez T, M. D. L., Rocha C, J., Clavel-Arcas, C., \& Mack, K. A. (2010). Nonfatal unintentional injuries in children aged $<15$ years in Nicaragua. International journal of injury control and safety promotion, 17 (1), 3-11. 5. Centers for Disease Control and Prevention. Web-based Injury Statistics Query and Reporting System (WISQARS) [Online]. (2003). National Center for Injury Prevention and Control, Centers for Disease Control and Prevention (producer). Available from: URL: www.cdc.gov/ncipc/wisqars. [2017 Oct 17]. 6. de Sousa Petersburgo, D., Keyes, C. E., Wright, D. W., Click, L. A., Macleod, J. B., \& Sasser, S. M. (2010). The epidemiology of childhood injury in Maputo, Mozambique. International journal of emergency medicine, 3 (3), 157-163. 7. Birken CS, Macarthur C. Socioeconomic status and injury risk in children. Paediatr Child Health. 2004;9 (5):323-325. 8. Bonilla-Escobar FJ RC, Puyana JC. Trauma Care and Surveillance: International "eCapacity" Efforts and Honduras Experience. World Journal of Surgery. 2017. 9. Center for Disease Control and Prevention. Child Development. 2016. Accessed 11/25/2016, 2016. 10. Haagsma JA, Graetz N, Bolliger I, et al. The global burden of injury: incidence, mortality, disability-adjusted life years and time trends from the Global Burden of Disease study 2013. Inj Prev. 2016;22 (1):3-18. 11. Harvey A, Towner E, Peden M, Soori $\mathrm{H}$, Bartolomeos K. Injury prevention and the attainment of child and adolescent health. Bull World Health Organ. 2009;87 (5):390-394. 12. Pan American Health Organization. Honduras - Health in the Americas. Washington, D.C.2007. 13. World Health Organization. injuries and Violence- The Facts. Geneva2014.

Disclosure: No significant relationships.

\section{PR133}

\section{POLYTRAUMA MENAGEMENT IN CHILDREN}

\section{O. Karaseva $^{1}$, A.L. Gorelik ${ }^{2}$, A.V. Timofeeva ${ }^{2}$, D.E. Golikov ${ }^{1}$, K. Utkina ${ }^{I}$}

${ }^{1}$ Polytrauma, Clinical and Research Institute of Emergency Pediatric Surgery and Trauma, Moscow/RUSSIAN FEDERATION,

${ }^{2}$ Department Of Polytrauma, Clinical and Research Institute of Emergency Pediatric Surgery and Trauma (CRIEPST), Moscow/ RUSSIAN FEDERATION

Introduction: Severe injury in children is characterized by high rates of mortality and disability.

Material and methods: The study included 331children with polytrauma (ISS-25.4 \pm 7.0 ). Boys accounted for $59 \%$, girls $-41 \%$. The average age of children was $10.69 \pm 5.4 .56 .6 \%$ of children were injured as a result of an accident; $28.9 \%$ - of catatrauma.The study included 331children with polytrauma (ISS-25.4 \pm 7.0). Boys accounted for $59 \%$, girls $-41 \%$. The average age of children was
$10.69 \pm 5.4 .56 .6 \%$ of children were injured as a result of an accident; $28.9 \%$ - of catatrauma.

Results: The basic principles of medical care for polytrauma are hospitalization in the trauma center, diagnosis based on the whole body $\mathrm{CT}$ with contrast enhancement, surgical treatment in accordance with the stability of the patient's condition and the period of traumatic illness, within the principles of damage control and fast track, multiparametric monitoring of the critical period, acute rehabilitation and multidisciplinary interaction at all stages of traumatic disease.

Conclusion: Compliance with the protocol of treatment led to a decrease in mortality from $12.4 \%$ to $5.6 \%$ /

References:

Disclosure: No significant relationships.

\section{PR134}

\section{MUSCULOSKELETAL DISORDERS AMONG ADOLESCENT GREEK COMPETITIVE-LASER 4.7M SAILING ATHLETES}

\author{
P. Gkrilias, M. Tsekoura, C. Matzaroglou, E. Tsepis
}

Department Of Physical Therapy, TEI of Western Greece, Aigio Achaias/GREECE

Introduction: The highly repetitive nature of competitive sailing associated with overuse injury ${ }^{1}$. The purpose of this study was to record the musculoskeletal symptoms per anatomical body region in adolescent Greek elite competitive-laser $4.7 \mathrm{~m}$ sailing athletes.

Material and methods: The Greek version of the Standardized Nordic Questionnaire (SNQ), was given to adolescent participants in the 2017 Pan-Hellenic Laser Championship 4.7m. Participants in SNQ were asked whether they had pain/discomfort in 9 different anatomical regions (neck, shoulder, elbow, wrist/hand, upper back, lower back, hip/thigh, knee and ankle/foot) during the preceding 12 months and if those symptoms prevented their normal activity during the last year as well as the 7 previous days. Analysis consisted of descriptive statistics.

Results: 118 (75 Male, 43 Female) sailing athletes (Age:15,3 $\pm 1,2$ years, Training age:6,9 $\pm 2,4$ years, Training hours/week:13,0 $\pm 4,3$ hours, Height:171,5 $\pm 8,3 \mathrm{~cm}$, Weight: $59,9 \pm 7,3 \mathrm{~kg}$, BMI: $20,3 \pm$ $1,7 \mathrm{Kg} / \mathrm{m}^{2}$ ) completed the SNQ. The 12-month prevalence rate of pain/discomfort was $52.5 \%$ in knees, followed by the wrists/hands (37,3\%), shoulders $(36,4 \%)$, lower back $(33,9 \%)$, neck $(32,2 \%)$, upper back $(22,9 \%)$, foots/ankles $(23,7 \%)$ and hips/thighs $(21,2 \%)$ and elbows $(11,0 \%)$. Those symptoms prevented athlete's normal activity (functionality) during the last 12 months with different prevalence rate per anatomical body region (knees:33,9\% neck: $23,7 \%$, lower back: $22,9 \%$, shoulders: $22,0 \%$, wrists/hands: $22,0 \%$, hips/thighs: 16,9\%, upper back:16,1\%, ankles/foots: $16,1 \%$ and elbows: $5,1 \%$ ). Conclusion: The high prevalence of musculoskeletal pain/discomfort in adolescent sailing athletes, highlights the need for specific injury prevention programs.

References: 1. Neville V and Folland JP. The epidemiology and aetiology of injuries in sailing. Sports Med.39 (2):129-145, 2009. Disclosure: No significant relationships. 
PR135

\section{5-YEAR EXPERIENCE OF TREATING CHILDREN WITH CLAVICULAR FRACTURES IN CLINICAL AND RESEARCH INSTITUTE OF EMERGENCY PEDIATRIC SURGERY AND TRAUMA (CRIEPST)}

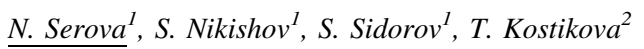

${ }^{1}$ Traumatology Department, Clinical and Research Institute of Emergency Pediatric Surgery and Trauma (CRIEPST), Moscow/ RUSSIAN FEDERATION, ${ }^{2}$ Department Of Radiation Diagnostics, Clinical and Research Institute of Emergency Pediatric Surgery and Trauma (CRIEPST), Moscow/RUSSIAN FEDERATION

Introduction: Statistical indexes demonstrate the increase in the number of children, especially adolescents, with injuries of musculoskeletal system in Russia. Literature data show that claviclular fractures are one of the most common injuries in children. However, up to now there is no consensus on surgical tactics.

Material and methods: Annually, more than 60 children with clavicle fractures are admitted to the traumatological department in CRIEPST. However, less than $50 \%$ of opened surgical interventions were performed by our traumatologists. Among all children, older patients (aged 13-17) predominate.

Results: Currently, we use a differentiated approach in choosing a technique for treatment with the severity of injury kept in mind. In case of fractures with slight fragment dislocation and with satisfactory fragment position on control X-ray images, conservative treatment with immobilization using eight-gypsum dressing or an orthosis are usually indicated. In fragmented fractures or in dislocation more than $2 \mathrm{~cm}$, opened reposition, metal-osteosynthesis with a blocked plate and screws is performed. If the fracture with significant dislocation of fragments is localized in the distal part of the clavicle, we also prefer surgical intervention with fixation using special plates and not frequent a crochet suture. Consolidation of fractures in all children took place in their age period.

Conclusion: Surgical intervention must be performed only under strict indications: dislocation of claviclular fragments more than $2 \mathrm{~cm}$, fragmented fracture, high risk of damage to the skin or to a neurovascular bundle. The chosen tactics of treatment improves outcomes and accelerate the recovery period of children with clavicular fractures.

References:

Disclosure: No significant relationships.

\section{PR136}

\section{TRAUMATISM IN CHILDHOOD}

\section{A. Filipov, Y. Parvanov, B. Ninov}

Surgery, UMHAT “D-r Georgi Stranski”, Pleven/BULGARIA

Introduction: Traumatism in childhood is widely spread in all age groups and is common in daily practice of Pediatric surgeons. With this report authors want to share their experience and observation in the field of childhood traumatism as well as the principles of overall behavior - diagnostics and treatment in different cases.

Material and methods: A retrospective analysis of the treated children for a 15-year period (2002-2016) showed total number of 287 patients. The study included patients from 2 months to 18 years and
67 of them were operated. The main diagnostic approaches used: Full blood count, Ultrasound, CT, X-Ray. Procedures used: blood and plasma transfusions, preventing an acute respiratory failure, easing the pain and normalizing the electrolyte balance.

Results: 287 children, 180 boys $(62.72 \%)$ and 107 girls (37.28\%), 67 (23.34\%) operated. Age groups: $4-8$ years $(32.52 \%)$, 9-13 years (28.72\%), 14-18 years (27.68\%), 0-3 years (11.08\%). Affected organs: spleen $(43.15 \%)$, liver $(31.1 \%)$, lungs $(10.54 \%)$, small intestine $(6.8 \%)$, retroperitoneum $(5.2 \%)$, other organs and systems $(3.21 \%)$. Traumas by type: generalized (57.09\%), abdominal $(32.52 \%)$, thoracic $(4.5 \%)$, combined $(4.15 \%)$, gunshot $(1.74 \%)$. The number of conservatively treated children is 220 . We preferred organ - preserving operations in case of minor traumas and an urgent operation for generalized ones with severe trauma. Two patients were not transported in time to receive surgical treatment and they died on arrival in ER.

Conclusion: Traumatism in childhood requires urgent transport, diagnosis and treatment. Extensive hospitalization of traumatic pediatric patients and urgent surgical treatment with organ - preserving and easing the patient - operations is established.

References: 1. Fundamentals of Pediatric Surgery 2011, Part 3, 145-150, DOI:10.1007/978-1-4419-6643-8_19 Thoracic trauma Martin S.Keller 2. European Journal of Trauma and Emergency Surgery Vol.33, Number 5 (2007), 476-481, DOI: 10.1007/s00068007-7124-3 Focus on Spinal Injuries in Multiple Trauma Patients. Distribution of Spinal and Associated Injuries in Multiple Trauma Patients Helmut Laurer, Bernd Maier, Andre El Saman, Mark Lehnert, Hendrick Wyen and Ingo Marzi 3. Ballistic Trauma 2005, Section 2, 396-417, DOI:10.1007/1-84628-060-5_19 Ballistic Trauma in Children Graeme J.Pitcher 4. Dickinson E, Limmer D, O'Keefe MF, Grant HD, Murray R (2008). Emergency Care (11th Edition). Englewood Cliffs, New Jersey: Prentice Hall. pp. 848-52. . Disclosure: No significant relationships.

\section{POLYTRAUMA}

\section{PR137}

\section{THE EFFECT ON ADMISSIONS AND MORTALITY BY BECOMING A MAJOR TRAUMA CENTRE IN NORTH WEST ENGLAND, 2012 - 2016}

\section{B. Allen, N. Misra, R. Lunevicius, K. Shahzad, J.V. Taylor, R.G. Ward}

General Surgery Department, Emergency General Surgery And Major Trauma Centre, Merseyside, Aintree University Hospital NHS Foundation Trust, Liverpool/UNITED KINGDOM

Introduction: In 2012, Aintree was a busy urban hospital. By 2016, it became the single Major trauma centre for Merseyside and Cheshire. We studied the effect of this re-organisation through retrospective analysis of assessment, admissions, and mortality of Major Trauma patients over this period.

Material and methods: Data was gathered from Aintree Trauma Register and Trauma Audit and Research Network (TARN). Major Trauma was defined as Injury Severity Score $>15$, more than a 72 hours admission to the hospital, critical care bed use or death. Ten categories of injury mechanism were studied: blast, assault, burns, crush, fall $<2 \mathrm{~m}$, fall $>2 \mathrm{~m}$, shooting, stabbing, road traffic accidents (RTA), and other (not classified). Case-fatality rates were calculated for each mechanism of injury.

Results: Major Trauma Team activations rose by a factor of 3.8 from 305 in 2012 to 1,169 in 2016. Major trauma admissions increased by a 
factor of 2.9 from 180 in 2012 to 522 in 2016 . The biggest increases were seen in RTAs by a factor of 2.4, falls $>2 \mathrm{~m}$ (factor of 2.8), and stabbings (factor of 1.8). Case-fatality rate showed a marked improvement from $2.7 \%$ to $1.14 \%$ in RTAs, but for falls greater than $2 \mathrm{~m}$ there was case-fatality rate increase from $6.9 \%$ to $8.7 \%$. There was no mortality from stabbings.

Conclusion: Becoming a Major Trauma Centre caused a massive increase in Trauma Team activations and hospital admissions with implications for staffing levels. We demonstrate a significant improvement in outcome from RTAs justifying centralisation of care for trauma patients.

References:

Disclosure: No significant relationships.

\section{PR138}

\section{INFLUENCE OF REBOA ON PROFOUND HYPOTENSION WITH IMPENDING CARDIAC ARREST: A 3-YEAR REPORT FROM THE ABO TRAUMA REGISTRY}

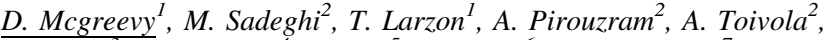
P. Skoog ${ }^{3}, K$. Idoguchi ${ }^{4}, Y . K^{2} n^{5}$, T. Ishida ${ }^{6}, Y$. Matsumura $^{7}$, J. Matsumoto ${ }^{8}$, M. Maszkowski $^{9}$, A. Bersztel ${ }^{9}$, E. $_{\text {Caragounis }}{ }^{10}$, M. Falkenberg ${ }^{11}$, L. Handolin ${ }^{12}$, G. Oosthuizen ${ }^{13}$, T. Wannatoop ${ }^{14}$, S.W. Chang ${ }^{15}$, B. Kessel ${ }^{16}$, D. Hebron ${ }^{17}$, F. Coccolini ${ }^{18}$

L. Ansaloni ${ }^{18}$, M.J. Madurska ${ }^{19}$, V. Manchev ${ }^{13}$, J. Marttala ${ }^{2}$,

A. Kastbergl, E.M. Dogan ${ }^{2}$, V.A. Reva ${ }^{20}$, J. Manning ${ }^{21}$,

J.J. Morrison ${ }^{22}$, K.F. Nilsson ${ }^{2}$, T.M. Hörer ${ }^{2}$

${ }^{1}$ Cardiothoracic And Vascular Surgery, Örebro University Hospital, Örebro/SWEDEN, ${ }^{2}$ Department Of Cardiothoracic And Vascular Surgery, Örebro University Hospital, Örebro/SWEDEN, ${ }^{3}$ Department Of Hybrid And Interventional Surgery, Sahlgrenska University Hospital, Göteborg/SWEDEN, ${ }^{4}$ )senshu Trauma And Critical Care Center, Rinku General Medical Center, Izumisano/JAPAN, ${ }^{5}$ Emergency And Critical Care Center, Hachinohe City Hospital, Hachinohe/JAPAN, ${ }^{6}$ Emergency And Critical Care Center, Ohta Nishinouchi Hospital, Koriyama/JAPAN, ${ }^{7}$ Department Of Emergency And Critical Care Medicine, Chiba University Graduate School of Medicine, Chiba/JAPAN, ${ }^{8}$. Department Of Emergency And Critical Care Medicine, St. Marianna University School of Medicine, Kawasaki/JAPAN, ${ }^{9}$ Department Of Vascular Surgery, Västmanlands Hospital, Västerås/SWEDEN, ${ }^{10}$ Department Of Trauma Surgery, Sahlgrenska University Hospital, Göteborg/SWEDEN, ${ }^{11}$ Department Of Radiology, Salgrenska Academy, Göteborg/SWEDEN,

${ }^{12}$ Department Of Orthopedics And Traumatology, Helsinki University Hospital, Helsinki/FINLAND, ${ }^{13}$ Department Of Surgery, Pietermaritzburg Metropolitan Trauma Service, Pietermaritzburg/ SOUTH AFRICA, ${ }^{14}$ Department Of Surgery, Siriraj Hospital, Mahidol University, Bangkok/THAILAND, ${ }^{15}$ Department Of Thoracic And Cardiovascular Surgery, Dankook University Hospital, Dongnam-gu/KOREA, DEMOCRATIC PEOPLE'S REPUBLIC OF,

${ }^{16}$ Trauma Unit, Hillel Yaffe Medical Center, hadera/ISRAEL,

${ }^{17}$ Department Of Surgery, Hillel Yaffe Medical Centre, Hadera/ ISRAEL, ${ }^{18}$ Department Of Surgery, Papa Giovanni XXIII Hospital, Bergamo/ITALY, ${ }^{19}$ Department Of Vascular Surgery, Queen Elizabeth University Hospital, Glasgow/UNITED KINGDOM, ${ }^{20}$ War Surgery Department, Kirov Military Medical Academy, SaintPetersburg/RUSSIAN FEDERATION, ${ }^{21}$ Department Of Emergency Medicine, University of North Carolina, North carolina/UNITED STATES OF AMERICA, ${ }^{22} \mathrm{R}$ Adams Cowley Shock Trauma Center, University of Maryland Medical System, Baltimore/MD/UNITED STATES OF AMERICA
Introduction: Resuscitative endovascular balloon occlusion of the aorta (REBOA) is an evolving technique for gaining hemodynamic control in hemodynamically unstable patients. The use of REBOA in traumatic cardiac arrest may potentially increase survival rates by controlling distal bleeding and sustaining carotid and coronary perfusion, paramount in achieving return of spontaneous circulation (ROSC). The aim of this study is to report on the use of REBOA in patients with profound hypotension and impending cardiac arrest (ICA) to aid ROSC during initial trauma management.

Material and methods: The ABO Trauma Registry has collected retrospective and prospective data on the use of REBOA from centers around the world. Hemodynamic, access, complications and survival data were reported. Profound hypotension has been defined as systolic blood pressure $<40 \mathrm{~mm} \mathrm{Hg}$.

Results: A total of 115 patients were reported between $2011-2017.36$ patients had profound hypotension and ICA, of which $64 \%$ received CPR. Vascular access was successful in $83 \%, 94 \%$ while ongoing CPR in the emergency department. ROSC was achieved in $44 \%$, with a mean SBP increase of $55 \mathrm{~mm} \mathrm{Hg}$. 17\% survived past 30-days.

Conclusion: This study indicates that arterial vascular access is achievable despite profound hypotension and ICA or even ongoing $\mathrm{CPR}$, and that REBOA might play a part in achieving ROSC with survivors past 30 -days.

References:

Disclosure: No significant relationships.

\section{PR139}

\section{REPAIRING GREAT DUODENAL DEFECTS IN RABBITS BY EPTFE PATCH. REPAIRING GREAT DUODENAL DEFECTS IN RABBITS BY EPTFE PATCH}

\author{
N. Ozlem ${ }^{1}$, H. Calis $^{2}$
}

${ }^{1}$ Faculty Of Medicine; General Surgery, Ahi Evran University, Kursehir/TURKEY, ${ }^{2}$ Faculty Of Medicine, General Surgery, Ahi Evran University, Kırsehir/TURKEY

Introduction: The aim of this experimental study was to evaluate the results of repairing large duodenal defects in rabbits by an ePTFE (Gore-Tex) soft tissue patch graft.

Material and methods: A defect as large as $50 \%$ of the total circumference of the duodenum (i.e. Grade 3 injury) on anterior surface of the second part in 14 white New Zealand rabbits was done and repaired by $1 \mathrm{~mm}$ ePTFE graft.

Results: The stomach and duodenum were inspected for evidence of either leak or obstruction, and completeness of the mucosa covering the defect. Neither leak nor obstruction were observed and the whole grafted area was covered by the mucosa at the end of the sixth month. Conclusion: The main conclusion is that the method we used, is easy and reliable. Further experience will appropriate animal models is needed before this technique can be considered for application (in human duodenal injuries).

References: 1. Minerva Chir. 1986 Jun 30;41 (11-12):1055-60. [Repair of duodenal ruptures with synthetic patches. An experimental study in the rabbit]. [Article in Italian] Franchini R, Romagnoli S, Francesca F, Angeli G, Gullotti M, Di Lorenzo B. 2. Int Surg. 1974 May;59 (5):274-8. Experimental closure of large duodenal defects. Tobik S. 3. Br J Surg. 1982 Feb;69 (2):82-3. Dacron patch for closure of experimental stomach defects. Smyrnis SA. 4. . 5.Br J Surg. 1993;80:1013-1014.Çağa, T., Gürer, F. Polytetrafluoroethylene patch grafting for the closure of stomach defects in the rat. Disclosure: No significant relationships. 


\section{PR140}

\section{CHRONIC PAIN AFTER A MAJOR TRAUMA INJURY}

H. Llaquet Bayo, S. Montmany Vioque, A. Campos Serra, N. Alegret, S. Navarro Soto

General Surgery, Hospital Universitari de Sabadell. Corporació Sanitària Parc Taulí., Sabadell/SPAIN

Introduction: After a major trauma, quality of life improves but do not return to previous levels. Pain has been identified as the worst rated dimension, with $55-65 \%$ of patients suffering pain one year after injury.

Material and methods: A prospective cohort study was conducted in which all major trauma patients admitted to the intensive care unit were included. Follow-up assessments of quality of life were performed using EQ-5D-5L questionnaire. Patients with pain score $\geq 3$ were identified and initiated follow-up by pain clinics. Sociodemographic characteristics and risk factors for chronic pain after major trauma are described.

Results: 200 patients were included and 168 completed quality of life assessment at 12 months after the injury. 80 patients (48\%) described a moderate, severe or incapacitating pain/discomfort. Most of them were men $(58.7 \%$ ), mean age $49.0 \pm 19.5$, mean ISS $16,1 \pm 9,3$, blunt injury $88.7 \%$. EQ-VAS (health status index) of patients suffering chronic pain was 59.7 at 12 months while EQVAS cohort at 12months was 70,7 and EQVAS Spanish general population norm was 78,0. EQ-US (utility score) of patients suffering chronic pain was 0.490 at 12 months while EQ-US cohort at 12 months was 0.671 and EQ-US Spanish general population norm was 0.949 . Female gender, anxiety or depression problems, severe chest injury, ISS $\geq 25$ and ICU length of stay $>7$ days were all factors associated to chronic pain at 12 months in the univariate analysis. In the multivariate analysis, only anxiety or depression problems (OR 7.69, CI 3.18-18.59), severe chest injury (OR 2.51, CI 1.13-5.57) and female gender (OR 2.40, IC 1.05-5.52) were associated to chronic pain. Half of the patients with chronic pain had a concomitant anxiety or depression problem.

Conclusion: We are still waiting for quality of life outcomes in trauma patients with chronic pain after being followed-up by pain clinics. However, and given the important component of anxiety/ depression associated, a psychological support should be also offered to improve outcomes.

References:

Disclosure: No significant relationships.

\section{PR141}

\section{OVERUSE OF COMPUTED TOMOGRAPHY FOR PENETRATING NECK INJURIES: WHAT HAPPENED TO THE GOOD OLD CLINICAL EXAM?}

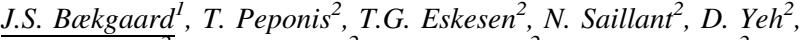 \\ H. Kaafarani ${ }^{2}$, P. Fagenholz ${ }^{2}$, M. De Moya ${ }^{2}$, G.C. Velmahos ${ }^{2}$
}

${ }^{1}$ Department Of Anaesthesiology, 4231, Rigshospitalet, COPENHAGEN/MA/DENMARK, ${ }^{2}$ Department Of Surgery Division Of Trauma, Emergency Surgery, And Surgical Critical Care, Massachusetts General Hospital, BOSTON/UNITED STATES OF AMERICA
Introduction: Computed Tomography (CT) has become a panacea for penetrating neck injuries (PNI). We sought to evaluate if CT improves the diagnostic accuracy of clinical exam.

Material and methods: The records of PNI patients admitted to a Level 1 trauma center between 1996-2016 were reviewed. Hard and soft signs of significant organ/vessel injury were recorded. The sensitivity and specificity of CT in predicting the need for neck exploration were calculated.

Results: Of 155 PNI (24\% gunshot and 76\% stab wounds) 80 (52\%) were explored urgently and $75(48 \%)$ were selected for non-operative management (SNOM). All cases of SNOM were successful.

Among 61 patients with hard signs, CT was performed in 20. Of them 12 patients had a positive CT resulting in $10(83 \%)$ delayed, but therapeutic, explorations. Eight patients had a negative CT, yet 2 (25\%) had therapeutic explorations.

Of the 32 patients with soft signs, CT was performed in 28. Of them 11 were positive, but only $3(27 \%)$ had clinically significant injuries. All 17 negative CTs allowed SNOM.

Of the 62 patients with no signs of PNI, CT raised suspicion of injury in 6; 2 were explored and one was found to have an injury.

Overall, the sensitivity of CT was 0.88 and the specificity 0.79 .

Conclusion: While CT may confirm the presence or absence of clinically significant injuries, it also results in delays and unnecessary explorations. Its greatest role seems to exist among patients with soft signs. Most patients with hard or no signs of PNI can be managed by clinical exam alone.

References:

Disclosure: No significant relationships.

\section{PR142}

\section{OPEN BUMPER FRACTURE: A MARKER FOR SERIOUS ASSOCIATED INJURIES}

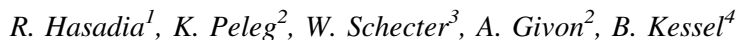

${ }^{1}$ Division Of Surgery, Hillel Yaffe Medical Center, hadera/ISRAEL, ${ }^{2}$ National Center For Trauma And Emergency Medicine Research, Gertner Institute For Epidemiology And Health Policy Research, Tel Hashomer, Israel, National Center for Trauma and Emergency Medicine Research, Gertner Institute for Epidemiology and Health Policy Research, Tel Hashomer, Israel, Ramat Gan/ISRAEL, ${ }^{3}$ Division Of Surgery, University of California, san francisco/SC/ UNITED STATES OF AMERICA, ${ }^{4}$ Trauma Unit, Hillel Yaffe Medical Center, hadera/ISRAEL

Introduction: A significant proportion of pedestrians hit by a car (PHBC) suffer from bumper fractures (BF). BF patients requiring urgent orthopedic surgery may be rushed to theatre without additional imaging.

Material and methods: A retrospective cohort of PHBCs 18 years of age or older with BFs, between the years 1997 and 2014, registered in the Israeli National Trauma Registry.

Results: 3351 PHBC suffered from BFs between the years 1997-2014. 1212 (36.17\%) patients had isolated BFs compared with $2139(63.83 \%)$ with concomitant injuries (AIS $\geq 3) .2637(78.7 \%)$ of these patients had closed BFs, and $714(21.3 \%)$ had open BFs. The mortality rate for open BF patients was $10.2 \%(n=73)$ compared with $4.6 \%(\mathrm{n}=122)$ for closed BF patients $(\mathrm{p}<0.001)$. The incidence of chest and abdomen injuries was almost two times higher with open compared to closed BFs

Conclusion: Almost two thirds of BF patients have serious concomitant injuries which require evaluation and treatment. Open BFs 
are associated with twice the risk of death compared with closed BFs. The incidence of severe head, chest and abdominal injuries is significantly higher in the open BF group. The presence of BF's is an indication for full trauma team activation and an aggressive search for associated injuries, including a whole body CT scan, prior to orthopedic surgery.

References: World-Health-Organization. Road traffic injuries, Factsheet no 358. 2013. The contribution of the Israeli trauma system to the survival of road traffic casualties. 2008; 16 (7):369-75. Prodromidis $\mathrm{AD}$, Charalambous $\mathrm{CP}$. The 6-hours rule for surgical debridement of open tibia fractures: A systematic review and metaanalysis of infection and nonunion rates. J Orthop Trauma 2016;30 (7):397-402

Disclosure: No significant relationships.

\section{PR143}

\section{NEW PERIL ON OUR ROADS}

\author{
J.H. Pek, Y.K. Liew
}

Acute Care Clinic, Sengkang Health, SingHealth, Singapore/ SINGAPORE

Introduction: Electronic scooter has been gaining popularity on Singapore roads, with associated injuries presented to the Emergency Department in varying severity. We aimed to understand the current landscape of these injuries.

Material and methods: A retrospective review of medical records from 2015 to 2016. Patient demographics, circumstances of accident, injuries sustained and clinical progress were collected for analysis.

Results: Thirty six cases were identified, all of whom were users of electronic scooter. The median age was 34 (range 17-70) and 67\% (n $=24$ ) were male. There was a 2.3 -fold increase in number of cases from 11 in 2015 to 25 in 2016. Another road user was implicated in $31 \%(\mathrm{n}=11)$ of cases. Reported use of protective equipment was observed in only 2 cases. Fourteen cases were conveyed by Emergency Medicine Services. In the Emergency Department, $11 \%(\mathrm{n}=4)$, $47 \%(\mathrm{n}=17)$ and $42 \%(\mathrm{n}=15)$ were triaged as P1 (emergent), P2 (urgent) and P3 (ambulatory) cases respectively. Investigations were performed in the ED for $86 \%(\mathrm{n}=31)$ cases. Superficial injuries were the most commonly sustained injury in $42 \%(\mathrm{n}=15)$, followed by fractures in $31 \%(\mathrm{n}=11)$, and head injury in $14 \%(\mathrm{n}=5)$. Nine patients were admitted to the hospital with 3 requiring surgery. The median length of stay was 2 days (range 1-6).

Conclusion: The incidence of electronic scooter related injuries appear to be on the rise, with a potential for incurring significant morbidity and healthcare costs. Further efforts at safety education and enforcement should be taken to prevent these accidents and minimize the impact of these injuries.

References:

Disclosure: No significant relationships.

\section{PR144}

\section{A MIXED PSYCHIATRIC AND SOMATIC CARE UNIT FOR TRAUMA PATIENTS: A TEN YEAR EXPERIENCE IN AN URBAN LEVEL I TRAUMA CENTER IN THE NETHERLANDS}

L. Dekker $^{l}$, H. Heller ${ }^{2}$, E.j. Toor $^{2}$

${ }^{1}$ Trauma Surgery, VU University Medical Center, HV/ NETHERLANDS, ${ }^{2}$ Trauma Surgery, VU University Medical Center, HV/NETHERLANDS

Introduction: A Medical-Psychiatric Unit (MPU) is a special unit where staff is trained in taking care for patients with psychiatric or behavioural problems needing hospitalisation for their physical problems. It is well-known that these patients are at higher risk of complications, have a longer length of stay and higher costs than patients without psychiatric comorbidity. The objective of this study was to analyse the trauma patient population of the first ten years of existence of the MPU and comparing them with the overall trauma patient population in VUmc.

Material and methods: A retrospective analysis was performed in cohorts of 2 years from 2006 through 2016. Data (psychiatric diagnosis, substance abuse, trauma scores, surgical interventions, complications, mortality) were extracted from individual patient notes and the Regional Trauma Registration.

Results: 258 patients were identified. The proportion of men was $64 \%$ and the median age was 46 (range 18-94). $36 \%$ of all patients had a history of previous admission at a psychiatric ward and $30 \%$ had attempted suicide at least once in their lifetime. Substance abuse was the most common psychiatric disorder (39\%), together with a psychotic disorder (28\%) in the second place. 35\% of all patients used alcohol, drugs or a medication overdose at time of admission. The median hospital stay was 21 days. Median length of stay at the MPU was 10 days (range 1-160). The traumatic injury was in $57 \%$ selfinflicted. The most common mechanism of injury was falling from height with intentional jumping in second place. In $24 \%$ the trauma was penetrating and $33 \%$ had an ISS $\geq 16$, while this was $5 \%$ and $15 \%$ respectively in the overall trauma patient population. The most common injuries were those of the head and neck. Complications were encountered in $49 \%$.

Conclusion: This study shows that trauma patients that were admitted to the MPU of an urban level I trauma center had serious psychiatric comorbidity as well as high injury severity. Penetrating injury was more common than in the overall trauma patient population. A high complication rate was noted (when compared the literature). These findings warrants combined psychiatric and somatic (nursing) care for this subpopulation of trauma patients. This should be taken into account in the prehospital triage to a hospital of this type of trauma patients. And therefore, the institution of a MPU in level 1 trauma centers is recommended.

References:

Disclosure: No significant relationships. 


\section{PR146}

\section{MEASURING THE IMPACT OF TRAUMA IN GREECE: A SYSTEMATIC REVIEW OF GREEK TRAUMA REGISTRIES. IS QUALITY IMPROVEMENT ACHIEVABLE?}

\section{A. Prionas, A. Tooulias, G. Tsoulfas, V. Papadopoulos}

First General Surgery Department, Aristotle University of Thessaloniki, Papageorgiou General Hospital, Thessaloniki/GREECE

Introduction: The goal was to identify all trauma registries that were established after 1990 in Greece and to assess the methodology used in these registries. We, also, sought to determine the demographics, the mechanisms of injury, the severity and the mortality of Greek trauma patients.

Material and methods: We performed a systematic review of published trauma registry literature from Greece according to PRISMAguidelines. We assessed the registries' methodology[1] based on the physical and human resources, the administration strategies, the processes of data collection and analysis reported. SPSS was used for the synthesis of the quantitative data (demographics, mechanisms of injury, ISS, mortality).

Results: The initial search retrieved 1732 abstracts. After screening the abstracts, 81 full-text articles were scrutinized for their suitability for inclusion. Fourteen papers from six registries, including 159,763 patients, were deemed eligible. Half of the publications (7/14) derived from registries that received funding. Regarding analyses, epidemiological analysis was the commonest (12/14), followed by comparisons (internal /external) (6/14). Quality improvement analyses were not demonstrated. The mean age of patients was $41 \pm 7.3$ years old and $63.17 \% \pm 3.96 \%$ among them were males. RTAs were the commonest mechanism of injury $(38.19 \% \pm 18.79 \%)$, followed by falls $(36.1 \% \pm 16.83 \%)$. The mean ISS of patients was $8.4 \pm 4.4$ and their average mortality was $3.85 \% \pm 2.94 \%$.A different ISS threshold for considering a trauma patient severely injured was used in each registry; the mean ISS threshold was ISS $>12 \pm 5$.

Conclusion: There is a need for road safety programs and fall prevention strategies. Trauma auditing in Greece is in an embryonic state. Trauma care quality improvement can be achieved through the introduction of a National Greek Trauma Database.

References: 1.O'Reilly GM, Joshipura M, Cameron PA, Gruen R (2013)Trauma registries in developing countries: a review of the published experience. Injury 44 (6), 713-721.

Disclosure: No significant relationships.

\section{PR147}

TRANSLATION AND VALIDATION OF TRAUMA-SPECIFIC QUALITY-OF-LIFE MEASUREMENT INTO FINNISH LANGUAGE

\section{J. Kuorikoski, T. Brinck, R. Roine, H. Sintonen, L. Handolin,} T. Söderlund

Töölö Hospital, Helsinki University Hospital, Helsinki/FINLAND

Introduction: After trauma, patients have remaining pain and functional deficits. Quality of life is lowered and it is usually measured with generic quality of life instruments. Studies suggest that specific instruments are more accurate compared to generic instruments. In this study, we translate and make the cultural adaptions to trauma specific quality of life instrument (TQoL) published in 2014.

Material and methods: We included severely injured patients from Helsinki University Hospital's trauma registry between the years 2012-14. Patients answered Finnish version of TQoL (TQoL-FIN), $15 \mathrm{D}$ quality of life questionnaire and questions concerning everyday life after injury and working status.

Results: After exclusion of seven patients, a total of 417 patients were included. Questionnaires were returned by 222 patients (53.2\%). The patients returning the surveys matched the whole study cohort, only age was statistically significant difference between these two groups, $47.5 \pm 19.5$ years for whole cohort and $50.6 \pm 18.7$ in the cohort that answered the survey $(\mathrm{p}<0.05)$. Missing answers were adjusted to compose Relative TQoL-FIN. TQoL-FIN and Relative TQoL-FIN have a very strong correlation with $15 \mathrm{D}$ quality of life questionnaire and separate questions in Finnish concerning injury's affection to everyday life. In TQoL-FIN every question, except one, was answered with a good percentage and we didn't need to exclude any of the questionnaires.

Conclusion: The Finnish translation of TQoL seems valid for evaluation of quality of life in trauma patients. As the only quality-of-life questionnaire translated into Finnish, it has a potential to be a valuable tool in Finnish trauma research.

References:

Disclosure: No significant relationships.

\section{PR148}

\section{STRENGTHENING PRIMARY RESUSCITATION MAY REDUCE TRAUMA DEATHS IN LOW-RESOURCE SETTINGS: KEY FINDINGS FROM A MULTI-CENTER TRAUMA REGISTRY IN FRANCOPHONE AFRICA}

\author{
S.A. Christie ${ }^{1}$, F. Dissak-Delon ${ }^{2}$, G.A. Etoundi Mballa ${ }^{3}$, \\ $\overline{\text { M. Carvalho }}^{1}$, G. Motwani ${ }^{l}$, O. Nwanna-Nzewunwa ${ }^{1}$, \\ A. Chichom-Mefire ${ }^{4}$, R.A. Dicker ${ }^{5}$, C. Juillard ${ }^{1}$
}

${ }^{1}$ Surgery, University of California San Francisco, San francisco/CA/ UNITED STATES OF AMERICA, ${ }^{2}$ Littoral Region Delegation, Ministry of Public Health, Cameroon, Yaounde/CAMEROON,

${ }^{3}$ Director Of Disease Control, Ministry of Public Health, Cameroon, Yaounde/CAMEROON, ${ }^{4}$ Surgery And Gynecology-obstetrics, University of Buea, Faculty of Health Sciences, Buea/CAMEROON, ${ }^{5}$ Surgical Critical Care, University of California Los Angeles, Los Angeles/CA/UNITED STATES OF AMERICA

Introduction: Over $90 \%$ of trauma deaths occur in low- and middleincome countries, but prospective injury data in low-resource settings are scarce (1). We analyzed an ongoing multisite trauma registry in Cameroon to identify initial targets for health policy recommendations to improve injury care.

Material and methods: Between July 2015 and January 2017 prospective observational data including demographics, injury characteristics, clinical data, emergency department (ED) treatment, and in-hospital outcomes were collected on all injured patients presenting to three regional referral hospitals in Cameroon.

Results: Data were collected on 7,897 injured patients. Patients were young (mean 31.4 years) and predominantly male (72.6\%). Over half of injuries $(58 \%)$ were caused by road traffic collisions. On arrival to the ED, $60 \%$ of patients had active external bleeding and $30 \%$ had hemodynamic abnormalities. Frequently performed initial resuscitation maneuvers included placing intravenous lines (41\%), airway repositioning (12\%), and patient exposure (13\%). Interventions 
requiring more training and equipment, such as blood transfusion $(0.1 \%)$, intubation $(<0.1 \%)$ and tube thoracostomy $(<0.1 \%)$ were rarely performed when medically indicated. Importantly, 55\% of patients did not obtain recommended medical treatment due to cost constraints, despite a low median cost of emergency care (\$30 USD, IQR18-51). Overall, $85 \%$ of hospital deaths due to injury occurred in the ED.

Conclusion: The vast majority of in-hospital injury deaths in Cameroon occur in the emergency department, but primary survey resuscitation is extremely limited. Adequate training and staffing during initial trauma resuscitations could substantially increase survival after injury. Financial restructuring of initial trauma and emergency care also has potential to improve clinical outcomes.

References: (1) Gosselin RA, Charles A, Joshipura M, Mkandawire N, Mock CN, Price RR, et al. Surgery and Trauma Care. In: Debas HT, Donkor P, Gawande A, Jamison DT, Kruk ME, Mock CN, editors. Essential Surgery: Disease Control Priorities, Third Edition (Volume 1). Washington DC, 2015.

Disclosure: No significant relationships.

\section{PR149}

\section{TRENDS OF TRAUMA IN ELDERLY: INSIGHTS FROM A LEVEL 1 TRAUMA CENTER OVER 5 YEARS}

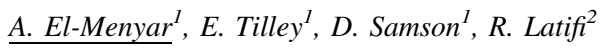

${ }^{1}$ Surgery, Westchester medical center, valhalla/UNITED STATES OF AMERICA, ${ }^{2}$ Surgery, Westchester medical center, valhalla/NY/ UNITED STATES OF AMERICA

Introduction: We aimed to study the pattern, trends and outcomes of trauma over the years among the elderly in a level 1 Trauma center in Westchester Medical Center in New York.

Material and methods: We conducted a retrospective analysis of all trauma patients $\geq 60$ years old between January 2012 and December 2016. Data were analyzed and compared across the last 5 years.

Results: Out of 8528 trauma in adults, there were 3052 (36\%) patients $\geq 60$ years old ( 600 in 2012, 642 in 2013, 590 in 2014, 589 in 2015 and 631 in 2016), of them 1512 (49.5\%) were females. The trends for trauma in males or females did not change over the 5 years $(p=0.12)$. Also, the trends among Whites or Blacks did not change significantly; however, trauma was much higher among whites in comparison to Blacks. Among Hispanics, the trend for trauma increased from $4.3 \%$ in 2012 to $8.4 \%$ in 2016 . There were no significant changes in the mechanisms of injuries over the years. However, the majority of trauma was related to falls from height $(\mathrm{n}=$ 2098; 68\%). There were significant decreases in the Injury Severity scoring (ISS), New Injury Severity scoring (NISS), the need for blood transfusion, and hospital length of stay across the study duration. The overall mortality showed statistically non-significant decrease in 2016 in comparison to the previous years $(9 \%, 9.7 \%, 8 \%$ and $6.7 \%$, respectively, $\mathrm{p}=0.36$ ).

Conclusion: Trauma in elderly constitutes more than one third of the injured adult population. However, the risk of falls in this population remains a public health challenge.

References: http://www.jems.com/articles/print/volume-40/issue-11/ features/complexities-of-geriatric-trauma-patients.html

Disclosure: No significant relationships.

\section{PR150}

\section{PROTOCOLIZATION OF ASSISTANCE TO THE OBSTETRIC PATIENT WITH POLITRAUMATISM IN A THIRD LEVEL HOSPITAL}

A. Valle Beltran, $N$. Alegret, C. Tremps

Anestesiologia, Hospital Parc Tauli, sabadell/SPAIN

Introduction: Maternal mortality is an important healthcare system quality indicator. Polytrauma complications account for $7-8 \%$ of pregnancies, being the first cause of non-obstetric fetal mortality and presenting a maternal mortality rate between 20-46\%. Maternal mortality secondary to trauma can be prevented with coordinated medical care, in which simultaneous care should be given to two patients with a complex physiological relationship between them.

Material and methods: After an exhaustive bibliographical review, a clinical guide was carried out by a multidisciplinary commission formed by: anesthesiologists, radiologists, intervencionist radiologists, obstetricians, neonatologists, surgeons, traumatologists and intensivists. Consensus was established in the management of these patients and intrahospital circuits were created to expedite their management.

Results: A management algorithm has been created in which the classical initial assessment is modified to include an $\mathrm{F}$ point for fetal assessment, corrected for physiological variations typical of the pregnant patient, and clearly sits the basis of complementary radiological examinations, indications and risks. As well as the coordination of the different surgical specialties in case it requires simultaneous attention.

Conclusion: The development of the clinical guide has contributed to the best care of the obstetric patient with polytrauma, homogenizing their attention in our center.

References: Jain V, Chari R, Maslovitz S, et al. Guidelines for the Management of a Pregnant Trauma Patient. J Obstet Gynaecol Canada JOGC. 2015;37 (6):553-74. Raja AS, Zabbo CP. Trauma in pregnancy. Emerg Med Clin North Am. 2012;30 (4):937-48 Lucia A, Dantoni SE. Trauma Management of the Pregnant Patient. Crit Care Clin. 2016;32 (1):109-17.

Disclosure: No significant relationships.

\section{PR151}

\section{RELIABILITY, CONSTRUCT VALIDITY AND RESPONSIVENESS OF THE SHORT MUSCULOSKELETAL FUNCTION ASSESSMENT IN INJURED PATIENTS}

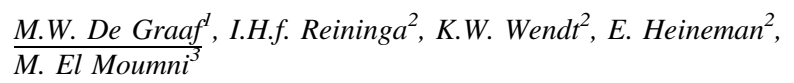

${ }^{1}$ Trauma Surgery, University Medical Centre Groningen, Groningen/ NETHERLANDS, ${ }^{2}$ Trauma Surgery, University Medical Center Groningen, Groningen/NETHERLANDS, ${ }^{3}$ Traumasurgery, University Medical Center Groningen, Groningen/NETHERLANDS

Introduction: The Dutch version of the Short Musculoskeletal Function Assessment (SMFA) questionnaire consists of four subscales. The aim was to assess test-retest reliability, construct validity and responsiveness for the four subscale configuration of the SMFA$\mathrm{NL}$ in a population of injured patients. 
Material and methods: Injured patients, treated at the emergency room, completed the SMFA-NL at 6 weeks, 8 weeks and 6 months post-injury. Internal consistency was evaluated using Cronbach's alpha. Test-retest reliability was assessed using intra-class correlation coefficient $\left(\mathrm{ICC}_{\text {agreement }}\right)$. Standard error of measurement (SEM) and smallest detectable change (SDC) were calculated. Construct validity and responsiveness were evaluated by testing pre-defined hypotheses and were considered sufficient when $\geq 75 \%$ were confirmed.

Results: A total of 232 patients (mean age: 46.9 (SD: 13.5)) participated. Lower extremity fractures were most prevalent $(n=124)$. Patients had a median injury severity score of 5 (range 1-42). Cronbach's alpha ranged from 0.81 to 0.94 . The ICCs ranged from 0.72 to 0.90 . The SEM ranged from 7.23 to 9.19 ; SDC ranged from 20.5 to 25.4 at individual level and 1.96 to 2.59 at group level. A total of $85 \%$ of all construct validity hypotheses, and $81 \%$ of all responsiveness hypotheses were confirmed.

Conclusion: This study showed that the four-subscale configuration of the SMFA-NL is a reliable, valid and responsive measurement instrument for reporting levels of physical functioning in injured patients.

References:

Disclosure: No significant relationships.

\section{PR152}

\section{MORBIDITY AND MORTALITY IN A DEDICATED TRAUMA CENTER DURING THE MOSUL CAMPAIGN: IRAQI KURDISTAN, 2016-2017}

M.P. Nerlander ${ }^{1}$, H. Rawand ${ }^{2}$,W. Moayad ${ }^{3}$, A. Älgåa ${ }^{I}$, J. Von Schreeb ${ }^{4}$

${ }^{1}$., Karolinska Institutet, Stockholm/SWEDEN, ${ }^{2}$., Emergency Management Center, Erbil/IRAQ, ${ }^{3}$., World Health Organization, Erbil/IRAQ, ${ }^{4}$ Public Health Sciences, Karolinska Institutet, Stockholm/SWEDEN

Introduction: Commencing October 2016, an Iraqi-led coalition began retaking Mosul from Islamic State of Iraq and Syria. Emergency Management Center (EMC) in Erbil, Iraqi Kurdistan, is a dedicated trauma hospital and one of two Ministry of Health hospitals receiving patients from Mosul. Data on admitted casualties was analyzed to define the epidemiology, morbidity and mortality of this patient group with a view to guide medical preparedness and response in future conflicts.

Material and methods: This retrospective study utilized routinely collected data from EMC and included all patients with conflict-related injuries presenting between October 16, 2016 and July 10, 2017. Data was analyzed using JMP v13.

Results: Analysis included 1,726 patients. The in-hospital death rate was $0.47 \%$. A majority (55\%) of patients were males aged 20-34. Ordnance accounted for most injuries (68\%), followed by gunshot wounds (GSWs) (18\%), and Improvised Explosive Devices (IEDs) $(14 \%)$. Injuries to the head and neck were more common in explosives-related injuries $(24 \%)$ than in GSWs $(9 \%)$. Surgery was least likely in IED-related injuries (19\%) compared to GSWs (34\%) and ordnance $(31 \%)$. ICU care $\geq 10$ days was exclusive to explosivesrelated injuries $(90 \%)$.

Conclusion: The very low in-hospital death rate is likely the result of long transportation times and limited pre hospital care. Military-age males were overrepresented, in keeping with the wartime context. The overwhelming majority of patients presented with explosives-related injuries. IED-related injuries were least likely to require surgery.
Explosives-related injuries were overrepresented among patients with long ICU stays, likely reflective of the severe wounding potential of these weapons.

References:

Disclosure: No significant relationships.

\section{PR153}

FACTORS ASSOCIATED WITH PREHOSPITAL DEATH BY TRAFFIC ACCIDENTS IN URBAN AND RURAL AREAS OF JAPAN: A NATIONWIDE OBSERVATIONAL STUDY

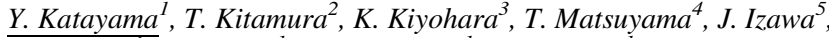

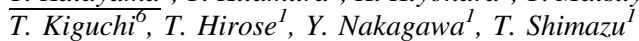

${ }^{1}$ Department Of Traumatology And Acute Critical Medicine, Osaka University Graduate School of Medicine, Suita/JAPAN, ${ }^{2}$ Social Aand Environmental Service, Osaka Unversity, Suita/JAPAN, ${ }^{3}$ Department Of Public Health, Tokyo Women's Medical University, Tokyo/ JAPAN, ${ }^{4}$ Department Of Emergency Medicine, Kyoto Prefectural Medical University, Kyoto/JAPAN, ${ }^{5}$ Depertment Of Anesthesiology, Intensive Care Unit, The Jikei University school of Medicine, Tokyo/ JAPAN, ${ }^{6}$ Health Service, Kyoto University, Kyoto/JAPAN

Introduction: Although it is important to assess the factors associated with traffic accident fatalities to decrease them as a matter of public health, these factors have not been fully revealed.

Material and methods: Using the Japanese Trauma Data Bank as the large hospital-based trauma registry in Japan, we retrospectively analyzed the traffic accident patients transported to participated facilities treated severe trauma patients from 2004 to 2015 . This study we defined prehospital death as the patients whose systolic blood pressure was $0 \mathrm{mmHg}$ or heart rate was $0 \mathrm{bpm}$ at hospital arrival. Prehospital factors associated with prehospital death due to traffic accidents were assessed by multivariable logistic regression analysis. Results: A total of 66,243 traffic accident patients were eligible for our analysis, of whom 3,390 patients were cardiac pulmonary arrest at hospital arrival. In a multivariable logistic regression model, 60-74 years old (adjusted odds ratio $[\mathrm{AOR}]=1.256 ; 95 \%$ confidence interval $[\mathrm{CI}], 1.142-1.382),>=75$ years old $(\mathrm{AOR}=1.487 ; 95 \% \mathrm{CI}$, 1.336-1.654), male sex (AOR $=1.234,95 \%$ CI, 1.139-1.338), nighttime $(\mathrm{AOR}=1.575 ; 95 \% \mathrm{CI}, 1.458-1.702)$, holiday including weekends $(\mathrm{AOR}=1.078 ; 95 \% \mathrm{CI}, 1.001-1.161)$, rural area $(\mathrm{AOR}=$ $1.181 ; 95 \% \mathrm{CI}, 1.097-1.271)$, backseat passenger $(\mathrm{AOR}=1.227,95 \%$ CI, 0.985-1.528) and pedestrian (AOR = 1.754; 95\%CI, 1.580-1.947) as type of patients were associated with prehospital death.

Conclusion: In this population, the factors associated with prehospital death due to traffic accidents were elderly people, male sex, nighttime, holiday and weekend, and backseat passenger and pedestrian as type of patients.

References: 1) Yusuke Katayama, Tetsuhisa Kitamura, Kosuke Kiyohara, et al. Factors associated with prehospital death among traffic accident patients in Osaka City, Japan: A population-based study. Traffic Inj Prev. 2017 Jun 28:1-5. 2) Bouaoun L, Haddak MM, Amoros E. Road crash fatality rates in France: a comparison of road user types, taking account of travel practices. Accid Anal Prev. 2015;75:217-225. 3) Loo BP, Tsui KL. Pedestrian injuries in an ageing society: insights from hospital trauma registry. J Trauma Acute Care Surg. 2009;66:1196-1201. 4) Dunbar G. The relative risk of nearside accidents is high for the youngest and oldest pedestrians. Accid Anal Prev. 2012;45:517-521. 5) Rodgers GB. Bicyclist deaths and fatality risk patterns. Accid Anal Prev. 1995;27:215-223. 6) Damsere-Derry J, Ebel BE, Mock CN, Afukaar F, Donkor P. 
Pedestrians'injury patterns in Ghana. Accid Anal Prev. 2010;42:1080-1088. 7) Stone M, Broughton J. Getting off your bike: cycling accidents in Great Britain in 1990-1999. Accid Anal Prev. 2003;35:549-556. 8) Sabreena Anowar, Shamsunnahar Yasmin, Richard Tay. Comparison of crashes during public holidays and regular weekends. Accid Anal Prev. 2015; 51: 93-97 9) Ming-Der Li, Ji-Liang Doong, Kai-Kuo Chang, et al. Differences in urban and rural accident characteristics and medical service utilization for traffic fatalities in less-motorized societies. Journal of Safety Research. 2008; 39: 623-630

Disclosure: No significant relationships.

\section{PR154}

\section{CYCLISTS AND MAJOR TRAUMA, A UK MAJOR TRAUMA CENTRE EXPERIENCE}

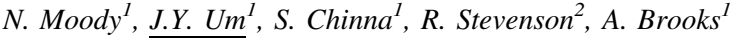

${ }^{1}$ Major Trauma, Queens medical centre, Nottingham/UNITED KINGDOM, ${ }^{2}$ Medical School, Nottingham University, Nottingham/ UNITED KINGDOM

Introduction: Road cycling has enjoyed an increasingly high profile over recent years and as a result there has been an increase in participation in the UK. The objective of this study was to evaluate the pattern of injuries sustained by cyclists admitted to a Major Trauma Centre in the UK over a 5-year period; to assess the likely resources required, and develop appropriate prevention and awareness strategies.

Material and methods: All patients admitted to East Midlands Major Trauma Centre (EMMTC) from January 2012 to September 2017 were included. The prospectively maintained database for the EMMTC was analysed for all patients involved in a cycle related incident. Data collected included mechanism of injury, time of day, severity, injuries and outcome.

Results: A total of 354 patients were included. All cyclists sustained injury as a result of interaction with a motor vehicle and $86 \%$ were male. There were 40 deaths $(11 \%)$. The median injury severity score was 16 (range 4 to 75 ). The most common areas of injury were the head (38\% of injuries) spinal injuries $(20.5 \%)$ and thoracic injuries $(18.5 \%)$. The median length of stay was 16 days. $27 \%$ of injuries occurred at dusk or dawn.

Conclusion: There is a substantial burden of cycle injury presenting to the MTC as a result of cyclist vs car incidents. Cyclists are extremely vulnerable to severe injury and utilise significant healthcare resources. A considerable proportion of incidents occur at dawn and dusk when visibility of the cyclist is likely to be reduced. Further work is required to develop prevention strategies.

References:

Disclosure: No significant relationships.

\section{PR155}

\section{COMPARISON OF RECALIBRATED TRISS VS NTRISS AS A PREDICTOR OF SURVIVAL IN A HOSPITAL TRAUMA REGISTRY}

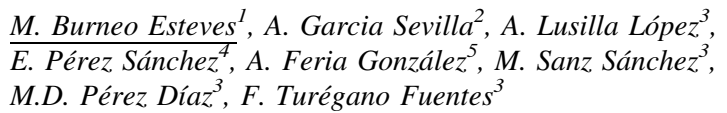

${ }^{1}$ Cirugía General Y Del Ap. Digestivo, Hospital Quirónsalud Sur, Madrid/SPAIN, ${ }^{2}$ Cirugía Maxilo Facial, Hospital General

Universitario de Ciudad Real, Ciudad Real/SPAIN, ${ }^{3}$ Cirugia General Y Del Ap. Digestivo, Hospital General Universitario Gregorio Marañón, Madrid/SPAIN, ${ }^{4}$ General Surgery, Hospital Universitario Nuestra Señora de Candelaria, Santa Cruz de Tenerife/SPAIN, ${ }^{5}$ Cirugia General Y Del Ap. Digestivo, Hospital Universitario Nuestra Señora de Candelaria, Santa Cruz de Tenerife/SPAIN

Introduction: NTRISS was born on the premise that the NISS predicts more reliably the mortality than ISS. Differences in demographics and epidemiology among populations have prompted the use of their own logistic regression models, adjusting " $b$ " coefficients accordingly. We aimed at improving our prediction capability by assessing those recalibrated models in both blunt (BT) and penetrating (PT) trauma

Material and methods: Retrospective, descriptive observational study of patients included in our Severe Trauma Registry for a period of 20 years (June 1993-December 2012). We have compared the predictive power of both original TRISS and NTRISS, and then using recalibrated models derived from our own population of patients. Recalibration was done using the same primary variables on half of the random sample, and the comparison of recalibrated models against the classic models was made in the other half of the sample for BT, using Wald's "steps backward" strategy. ROC type II curves were used to compare the models

Results: NTRISS had an AUC higher than TRISS. The random distribution of cases in two subsamples obtained a fairly homogenous balance of the deaths in the two groups for BT. We observed that our recalibrated models have a higher AUC ROC than the original models for BT. The number of PT deaths were insufficient for Recalibration Conclusion: NTRISS has a better prediction of survival than TRISS in our population. Both recalibrated models outperformed the original ones in relation to BT

References: Domingues Cde A, Nogueira Lde S, Settervall $\mathrm{CH}$, Sousa RM. Performance of Trauma and Injury Severity Score (TRISS) adjustments: an integrative review. Rev Esc Enferm USP. 2015;49:138-46. Schluter PJ, Cameron CM, Davey TM, Civil I, Orchard J, Dansey R, et al. Contemporary New Zealand coefficients for the Trauma Injury Severity Score: TRISS (NZ). N Z Med J. 2009;122 (1302):54 Bouillon B, Lefering R, Vorweg M, Tiling T, Neugebauer E, Troidl H. Trauma score systems: Cologne Validation Study. J Trauma. 1997;42 (4):652-8. Schluter PJ. Trauma and Injury Severity Score (TRISS): is it time for variable re-categorisations and re-characterisations? Injury. 2011;42 (1):83-9 Chico-Fernández M, Sánchez-Casado M, Alberdi-Odriozola F, Guerrero-López F, MayorGarcía MD, Egea-Guerrero J, et al. Validation of the probability of survival using the triss methodology in the Spanish Trauma ICU registry (RETRAUCI). Intensive Care Med Exp. 2015;3 (1):856-7. Schiowitz M, Stanovich H. The impact of volume on outcome in seriously injured trauma patients: two years' experience of the Chicago trauma system. J Trauma. 1991;31 (8):1176. Deshmukh VU, Ketkar MN, Bharucha EK. Analysis of Trauma Outcome Using the TRISS Method at a Tertiary Care Centre in Pune. Indian J Surg. 2012;74 (6):440-4. Jung K, Huh Y, Lee JC, Kim Y, Moon J, Youn 
SH, et al. The Applicability of Trauma and Injury Severity Score for a Blunt Trauma Population in Korea and a Proposal of New Models Using Score Predictors. Yonsei Med J. 2016;57 (3):728-34. Gunning AC, Leenen LP. Applicability of the predictors of the historical trauma score in the present Dutch trauma population: modelling the TRISS predictors. J Trauma Acute Care Surg. 2014;77 (4):614-9. Wong GK, Teoh J, Yeung J, Chan E, Siu E, Woo P, et al. Outcomes of traumatic brain injury in Hong Kong: validation with the TRISS, CRASH, and IMPACT models. J Clin Neurosci. 2013;20 (12):1693-6. Kimura A, Chadbunchachai W, Nakahara S. Modification of the Trauma and Injury Severity Score (TRISS) method provides better survival prediction in Asian blunt trauma victims. World J Surg. 2012;36 (4):813-8. Jurkovich GJ, Mock C. Systematic review of trauma system effectiveness based on registry comparisons. J Trauma. 1999;47 (3):46-55. Darbandsar Mazandarani P, Heydari K, Hatamabadi H, Kashani P, Jamali Danesh Y. Acute Physiology and Chronic Health Evaluation (APACHE) III Score compared to Trauma-Injury Severity Score (TRISS) in Predicting Mortality of Trauma Patients. Emerg (Tehran). 2016;4 (2):88-91. Chawda MN, Hildebrand F, Pape HC, Giannoudis PV. Predicting outcome after multiple trauma: which scoring system? Injury. 2004;35 (4):347-58

Disclosure: No significant relationships.

\section{PR156}

\section{CARDIAC COMPLICATIONS AND FAILURE TO RESCUE AFTER INJURY IN A MATURE STATEWIDE TRAUMA SYSTEM: IDENTIFYING OPPORTUNITIES FOR IMPROVEMENT}

\author{
D.N. Holena ${ }^{1}$, R.P. Dumas ${ }^{2}$, E.J. Kaufman ${ }^{3}$, Y. Huang ${ }^{4}$, D.J. Wiebe ${ }^{5}$, \\ P.M. Reilly' ${ }^{2}$ J.D. Christie ${ }^{6}$
}

${ }^{1}$ Department Of Surgery And Epidemiology, University of Pennsylvania, Philadelphia/PA/UNITED STATES OF AMERICA, ${ }^{2}$ Department Of Surgery, University of Pennsylvania, Philadelphia/ PA/UNITED STATES OF AMERICA, ${ }^{3}$ Department Of Surgery, Weil Cornell Medical College, New York/NY/UNITED STATES OF AMERICA, ${ }^{4}$ Department Of Biostatistics, Epidemiology, And Informatics, University of Pennsylvania, Philadelphia/PA/UNITED STATES OF AMERICA, ${ }^{5}$ Department Of Biostatistics, Epidemiology, And Informatics, University of Pennsylvania, Philadelphia/UNITED STATES OF AMERICA, ${ }^{6}$ Departments Of Medicine, University of Pennsylvania, Philadelphia/PA/UNITED STATES OF AMERICA

Introduction: Cardiac complications after injury are uncommon but contribute disproportionately to mortality. Variability in rates of cardiac complications (CC) and failure to rescue after cardiac complications (FTR-C) within trauma systems may suggest opportunities for improvement. We therefore examined center-level rates of $\mathrm{CC}$ and FTR-C in a statewide trauma system with the hypothesis that highperforming centers for each of these outcomes could be identified.

Material and methods: Using the Pennsylvania State Trauma Outcomes Study from 2007-2015 (inclusion criteria: age $>16$ years, Abbreviated Injury Score 2, and non-burn mechanism), we developed multivariable logistic regression models on CC and FTR-C including patient demographics, physiology, comorbidity, and injury data. Predicted probabilities of each outcome were summed to generate expected event rates, which were compared to observed event rates to generate center observed-to-expected (O:E) ratios. We measured internal consistency between CC and FTR-C for centers using Cronbach's alpha.
Results: Cardiac complications occurred in 5,079/278,042 (1.8\%; center-level range: $0.9-3.8 \%$ ) of included patients (median age 55 (IQR 34-76), 84\% Caucasian, 60\% male, 92\% blunt, median ISS 9 (IQR5-16)). Death after CC occurred in 982/5,097 patients for an FTR-C rate of $19.3 \%$ (center-level range: $7.8-30.4 \%$ ). For CC, 10/27 centers were high-performers $(95 \% \mathrm{CI}$ for $\mathrm{O}: \mathrm{E}$ ratio $<1)$ and $8 / 27$ were low performers. For FTR-C, 2/27 centers were high-performers and $3 / 27$ were low performers. Internal consistency between these metrics was poor (alpha $=0.31$ ).

Conclusion: Rates of CC and FTR-C vary significantly by center, but high-performing centers can be identified. Inconsistent performance between metrics suggests different institutional factors may underlie performance for CC and FTR.

References:

Disclosure: No significant relationships.

\section{PR157}

DEVELOPMENT OF A NATIONWIDE TRAUMA REGISTRY. A LESSON LEARNED THE HARD WAY

C.E.M. Pothmann, K. Sprengel, M.K. Schmelzer, H.P. Simmen, L. Mica, K.O. Jensen

Department Of Trauma Surgery, University Hospital Zurich, Zürich/ SWITZERLAND

Introduction: To optimally utilize the medical resources of the swiss health system all 26 regional health ministers decided in the year 2009 to evolve a concept of concentrating highly specialized medicine, HSM, in Switzerland. This comprises especially rare medical conditions, medical fields with high innovation potential and considerably higher expenditure on technology and human resources. These were concentrated to only a few specialized centers, covering Switzerland supra-regional.

Material and methods: The final government decision was presented by the IVHSM in 2011. It implied 12 trauma-centers within Switzerland being allowed to treat adult ( $>=16$ years) polytraumatized patients and patients with severe traumatic brain injuries. Including patients with an ISS $>=20$ or AIS-Head $>=3$. A descriptive, retrospective analysis of 24.576 patients of the Traumaregister DGU® (TR-DGU) 2009-2012 was performed.

Results: Despite a lot of equalities (mean age, distribution of gender, mean ISS 24.8 respective 24.0) we saw differences in the trauma mechanism with more falls from great height in Switzerland $32.3 \%$ vs. $16.9 \%$ ), whereas more traffic accidents happened in Germany (37.4\% vs. $54.2 \%)$. Preclinical interventions were less performed in Switzerland (1.97 vs. 2.25). This might be due to different rescue systems. Status at admission showed equal physiological parameters, while the rate of severe traumatic brain injuries was higher in the swiss cohort ( $62.7 \%$ vs. $49.5 \%)$. The standardized mortality ratio was comparable (CH 0.88 vs. D 0.82 ).

Conclusion: These results have to be seen as a trend, as at the time of evaluation only 3 swiss trauma-centers took part in the TR-DGU. A detailed analysis is to follow.

Disclosure: No significant relationships. 


\section{PR158}

\section{RESULTS OF THE IMPLEMENTATION OF DONATION PROGRAM IN MAASTRICHT IIA ASYSTOLE}

\author{
J. Tinoco-González ${ }^{1}$, C. García-Rivera ${ }^{1}$, L. Tallón-Aguilar ${ }^{1}$, \\ V. Durán-Muñoz-Cruzado ${ }^{1}$, A. Sánchez-Arteaga ${ }^{l}$, \\ I. Alarcón-Del Agua ${ }^{l}$, M.J. Tamayo-Lopez ${ }^{I}$, F. Pareja-Ciuróo ${ }^{1}$ \\ M.Á. Gómez-Bravo ${ }^{2}$, J. Padillo-Ruíz ${ }^{2}$
}

${ }^{1}$ Emergency Surgery Unit, Virgen del Rocío Universitary Hospital, Seville/SPAIN, ${ }^{2}$ Hepatobiliary And Transplantant Surgery Unit, Virgen del Rocío Universitary Hospital, Seville/SPAIN

Introduction: Although the transplant activity has practically reached 90 procedures per million population today, this is insufficient to cover the transplant needs of our population. Donation in asystole (DA) type IIa appears as an alternative to increase the number of grafts.

Material and methods: It is a cross-sectional, observational, prospective study of a single group. All possible donors from an outof-hospital cardiorespiratory arrest (CRA) who received unsuccessful care were included (2012-16). We collected the demographic variables of the possible donors, the causes of non-donation, out-patient resuscitation time, in-hospital, cannulation time, perfusion time, and overall time.

Results: We collected 72 candidates for donation, of which 40 $(54.8 \%)$ were finally donors. The median age of the donors was 42 (IQ: 10.7) years. The proportion of men was 9: 1 compared to women. Out-of-hospital cardiac arrest time was 71 (32-95) minutes, 25 (1351) in-hospital cardiac arrest, cannulation time was 30 (12-45), extracorporeal perfusion time of 126.5 (98-149). A median of 2 organs per donor was obtained, with a donor organ index of 1.1 in 2012 (11 organs), 1.8 in 2013 (9 organs), 1.7 in 2014 (12 organs), 1.6 in 2015 (8 organs) and 1.5 in 2016 (21 organs). From the 61 valid organs, 32 were from kidney donors, 4 liver donors and, as tissues, 47 corneas, 27 bone donors and 8 vascular grafts were extracted.

Conclusion: Warm ischemia times (out-of- and in-hospital CRA) as well as the overall time is similar to those of the national registry. Cannulation time of our group is 30 minutes, obtaining on average 1.68 organs/donor.

References:

Disclosure: No significant relationships.

\section{PR159}

CT SCANS IN TRAUMAS: DOES USING WHOLE BODY CT INSTEAD OF TARGETTED CT LEAD TO EARLIER IDENTIFICATION OF ALL INJURIES SUSTAINED?

\section{J.D. Chapman ${ }^{1}$, C.A. Findlay ${ }^{1}$, S. Scott ${ }^{2}$, N. Misra ${ }^{3}$, A. Sattout ${ }^{4}$}

${ }^{1}$ Postgraduate, University Hospital Aintree, Liverpool/UNITED KINGDOM, ${ }^{2}$ Trauma \& Orthopaedics, University Hospital Aintree, Liverpool/UNITED KINGDOM, ${ }^{3}$ Emergency General Surgery, University Hospital Aintree, Liverpool/UNITED KINGDOM, ${ }^{4}$ Emergency Medicine, University Hospital Aintree, Liverpool/ UNITED KINGDOM

Introduction: Computed Tomography $(\mathrm{CT})$ plays a crucial part in the initial management of major trauma patients, usually as a whole body CT (WBCT) or targeted CT (TCT).
Material and methods: We conducted a retrospective study at our level 1 regional Major Trauma Centre (MTC) from January till June 2016. The use of either WBCT or TCT was examined, and previously unidentified injuries (PUIs) or new investigations requested from the tertiary clinical survey (TCS) were used as primary end points.

Results: 289 major trauma patients were identified with $86.9 \%$ having CT imaging; $61.6 \%$ were WBCTs and $25.3 \%$ TCTs'. 166 TCS were identified and 32 PUIs or new investigations were found in 26 patients; 21 had WBCTs on admission, 2 TCTs and 3 no CTs. Hence PUIs or new investigations were either found or requested following $11.8 \%$ of WBCTs and $2.7 \%$ of TCTs. Mechanisms of PUIs found on TCS following WBCT were mostly RTA, fall down stairs or assault. $65.4 \%$ of PUIs were peripheral limbs (injuries to the arm, hand or distal to the femur were most common). Greater than $15 \%$ was investigations to monitor known injuries, with the remaining being possible axial skeleton or spinal injuries.

Conclusion: In conclusion WBCT should be the investigation of choice in patients where there is a high impact mechanism of injury. Peripheral limb examination during secondary survey should be comprehensive to identify possible injuries missed on initial CT imaging. TCTs should be ordered by the trauma physician as dictated by expertise and clinical presentation.

References:

Disclosure: No significant relationships.

\section{PR160}

\section{IN-HOSPITAL TRAUMA MORTALITY IN CATALONIA. A THREE YEAR ANALYSIS OF THE CATALONIAN TRAUMA REGISTRY}

\author{
S. Prat-Fabregat ${ }^{1}$, A. Placer $^{2}$, E. Balcells ${ }^{1}$, C. Medina ${ }^{3}$, \\ T. Traumcat Working Group ${ }^{4}$
}

${ }^{1}$ Traumatology, HOSPITAL CLÍNIC DE BARCELONA, BARCELONA/SPAIN, ${ }^{2}$ Emergencies, HOSPITAL CLÍNIC DE BARCELONA, BARCELONA/SPAIN, ${ }^{3}$ Epidemiology, CatSalut, BARCELONA/SPAIN, ${ }^{4}$ Catsalut, CatSalut, BARCELONA/SPAIN

Introduction: TraumCat (Catalonian Major Trauma Registry) started hospital trauma cases data collection in July 2012. Data collection follows Utstein style guidelines (1).

Material and methods: TraumCat collects trauma patients from 18 hospitals representing the main trauma care facilities in Catalonia.

Death cases at scene or before hospital arrival are excluded from the registry.

Results: The period analized is 2014-2016. Three hundred patients (over a 4239 patient population) died from trauma related conditions $(7,1 \%) .87 .7 \%$ of deaths occurred during the hospitalary emergency care, while $8.3 \%$ of deaths were during the hospital admission. $40.7 \%$ of the exitus were secondary to falls from a height, while $40.3 \%$ were related to road traffic accidents. Max-AIS was 4 in $21 \%$ of deaths, 5 in $54 \%$, and 6 in $16.7 \%$. NISS and ISS were $>44$ in 55\% of deaths, $35-44$ in $12 \%$ and $25-34$ in $22.7 \%$. GCS was $3-8$ in $54 \%$ of deaths, $9-13$ in $13 \%$, and $14-15$ in $23.3 \%$. Mortality rate is higher as the population gets older. Death rate is $24 \%$ in patients $>75$ y.o. whereas it is $5 \%$ in patients $40-59$ y.o. Mortality rate in men with NISS $>44$ is $54 \%$ whereas in women is $58 \%$

Conclusion: Death rate is $7.1 \%$ but considering different age groups, it can rise up to $24 \%$ in patients older than 75 y.o. Fall from a height and road traffic accidents are the main cause of death in our population. Death is related to injury severity but death rate seems to be higher in women than in men with the same severity level. 
References: (1) Dick WF, Baskett PJF. Recommendations for uniform reporting of data following major trauma - the Utstein style. A report of working party of the International Trauma Anaesthesia and Critical Care Society (ITACCS). Resuscitation. 1999;42: 81-100. (2) CDC Guidelines for field triage of injured patients. Recommendations of the National Expert Panel on Field Triage. MMWR. 2009;58 (No.RR-1): 1-35. (3) Prat S, Domínguez-Sampedro P, Koo M, Colilles C, Jiménez-Fábrega X, Espinosa L.TraumCat: preliminary analysis of a year of severe trauma registry in Catalonia. Emergencias 2014; 26: 267-274

Disclosure: No significant relationships.

\section{PR161}

\section{DIAGNOSIS AND TREATMENT OF CLAVICLE FRACTURES IN BELGIUM BETWEEN 2006 AND 2015}

\author{
M. Herteleer, H. Hoekstra, S. Nijs
}

Traumatology, UZ Leuven, Leuven/BELGIUM

Introduction: Clavicle fractures are common fractures of the shoulder girdle. The debate about whether these fractures should be treated conservatively or surgically is ongoing. The aim of this study was to describe the incidence of clavicle fractures in Belgium between 2006 and 2015 and how the surgical treatment rates have evolved over this time span

Material and methods: All patients who were diagnosed or surgically treated with a clavicle fracture in Belgium were included in the study. The Belgian National Institute for Health and Disability Insurance provided the data which included the patients' age, gender, location, time of injury and included the entire Belgian population. The fracture incidences and surgical treatment rates for different population groups were assessed.

Results: The incidence of clavicle fractures in Belgium increased from 56.5/100,000 persons/year in 2006 to 70.6/100,000 persons/year in 2015. The age-related incidence was U-shaped with high incidences in patients younger than 18 and older than 70 both seen in men and women. The rate of surgically treated clavicle fractures increased by $190 \%$ between 2006 and 2015 .

Conclusion: There has been an increasing incidence of clavicle fractures in Belgium between 2006 and 2015. In the male population the fracture incidence has increased among all age groups but in the female population the increase was most noted in elderly patients. Although the preferred treatment strategy of clavicle fractures remains under debate, there is a high and increasing rate of surgical treatment in Belgium with an increasing percentage of patients that are surgically treated in an outpatient setting.

References:

Disclosure: No significant relationships.

\section{PR162}

\section{SCORING MULTIPLE TRAUMA - IS THERE A SIMPLE WAY?}

R. Girshausen, K. Horst, F. Hildebrand, H. Andruszkow

Department For Trauma And Reconstructive Surgery, University Hospital Aachen, Aachen/GERMANY

Introduction: During the last decades, multiple scoring systems have been developed to predict outcome after multiple trauma. These scores are either based on anatomical patterns, physiological parameters or a combination. However, these scores differ considerably due to the complexity and availability of the parameters. In consequence, a conflict between predictive accuracy and usability in daily clinical practice might emerge.

Material and methods: To validate current scoring systems referring to their predictive accuracy considering daily clinical applicability a retrospective study including multiple trauma patients at a level one trauma center was performed. Inclusion criteria: ISS $\geq 9$, age $\geq 18$ years; $2010-2015$. In-hospital mortality was evaluated as the main outcome parameter.

Results: Overall, 465 patients were included. $72.3 \%$ were of male gender, mean age was $51.2 \pm 20.3$ years. The validated scoring systems (ISS, NISS, APACHE II, RTS, SOFA, MODS, RISC II, EAC) were associated with in-hospital mortality. Validation of predictive capabilities revealed the RISC II as strongest predictor regarding mortality (ROC AUC 0.91), followed by APACHE II (ROC AUC 0.81), MODS (ROC AUC 0.70), SOFA (ROC AUC 0.70), RTS (ROC AUC 0.66), NISS (ROC AUC 0.62), EAC (ROC AUC 0.60). Conclusion: In conclusion, the RISC II score provided the strongest predictive capability towards mortality. In comparison, more simple scores focusing on injury pattern (ISS, NISS) or physiological abnormalities (RTS, EAC) only provided a much less precise mortality prediction. Established ICU scores like APACHE II, SOFA and MODS were also proven to be helpful tools in multiple trauma patients.

\section{References:}

Disclosure: No significant relationships.

\section{PR163}

\section{ANALYSIS OF PREVENTABLE TRAUMA DEATHS IN A HOSPITAL REGISTRY OVER A PERIOD OF TWENTY YEARS}

M. Burneo Esteves ${ }^{1}$, A. Lusilla López ${ }^{2}$, A. Sánchez-Arteaga ${ }^{3}$, R. Orti Rodriguez ${ }^{4}$, L. Zarain Obrador ${ }^{5}$, E. Pérez Sánchez ${ }^{4}$, R. Gianchandani Moorjani ${ }^{6}$, F. Turégano Fuentes ${ }^{2}$

${ }^{1}$ Cirugía General Y Del Ap. Digestivo, Hospital Quirónsalud Sur, Madrid/SPAIN, ${ }^{2}$ Cirugia General Y Del Ap. Digestivo, Hospital General Universitario Gregorio Marañón, Madrid/SPAIN, ${ }^{3}$ Emergency Surgery Unit, Virgen del Rocío Universitary Hospital, Seville/SPAIN, ${ }^{4}$ General Surgery, Hospital Universitario Nuestra Señora de Candelaria, Santa Cruz de Tenerife/SPAIN, ${ }^{5}$ General Surgery, Hospital Virgen de la Salud, Toledo/SPAIN, ${ }^{6}$ Cirugía General Y Del Ap. Digestivo, Hospital Universitario Nuestra Señora de Candelaria, Santa Cruz de Tenerife/SPAIN

Introduction: The number of preventable trauma deaths (PD) remains high outside of specific trauma centers, but can be reduced 
with adequate medical management, quality controls, and a well organized trauma system. Three methods are used in the assessment of PD: statistical analysis of trauma scores, necropsy studies, and clinical review by an expert panel. We aimed to evaluate our PD rate using these methods, with the exception of necropsy studies.

Material and methods: Retrospective, descriptive observational study of patients included in a hospital severe Trauma Registry for a period of 20 years (June 1993-December 2012). The analysis of the PD rate has been based mainly on the NTRISS model (Ps $>0.5$ ), and the PD criteria of the ACSCOT, in addition to the evaluation by an expert panel.

Results: The registry included 2120 patients with severe trauma. The mechanism was blunt (BT) in $75.7 \%$ of them, and penetrating (PT) in $24.3 \%$. After the exclusion of "dead on arrival"patients, and deceased patients beyond 30 days, there remained 326 dead patients for analysis: The PD rate was of $26 \%$ ( 85 patients), and there were no differences in the number of PDs in two time periods analyzed. PDs were classified as clearly preventable deaths (CPD) $(3,4 \%)$, and possibly preventable deaths (PPD) $(22,6 \%)$.

Conclusion: Our $26 \%$ PD rate is high, although the clearly PD rate was only $3,4 \%$ and whithin the range reported by trauma centers. The PPD criteria remain a matter of interpretation.

References: Costa CD, Scarpelini S. Evaluation of the quality of trauma care service through the study of deaths in a tertiary hospital. Rev Col Bras Cir. 2012;39 (4):249-54. Kwon AM, Garbett NC, Kloecker GH. Pooled preventable death rates in trauma patients : Meta analysis and systematic review since 1990. Eur J Trauma Emerg Surg. 2014;40 (3):279-85. Esposito TJ, Sanddal TL, Reynolds SA, Sanddal ND. Effect of a voluntary trauma system on preventable death and inappropriate care in a rural state. J Trauma. 2003;54 (4):663-70 Turégano F, Ots J, Martín J, Bordons E, Perea J, Vega D, et al. Mortalidad hospitalaria en patients con traumatismos graves: análisis de la mortalidad evitable. Cir Esp. 2001;70 (1):21-6 Ghorbani P, Falken M, Riddez L, Sundelof M, Oldner A, Strommer L. Clinical review is essential to evaluate 30-day mortality after trauma. Scand J Trauma Resusc Emerg Med. 2014;22:18-24. Van Haren RM, Thorson CM, Curia E, Schulman CI, Namias N, Livingstone AS, et al. Impact of definitions on trauma center mortality rates and performance. J Trauma Acute Care Surg. 2012;73 (6):1512-6. Afuwape OO, Okolo CA, Akinyemi OA. Preventable trauma deaths in Ibadan: a comparison of revised trauma score and panel review. West Afr J Med. 2011;30 (1):19-23. Gorman DF, Teanby DN, Sinha MP, Wotherspoon J, Boot DA, Molokhia A. Preventable deaths among major trauma patients in Mersey Region, North Wales and the Isle of Man. Injury. 1996;27 (3):189-92. Boman H, Bjornstig U, Hedelin A, Eriksson A. "Avoidable" deaths in two areas of Sweden - analysis of deaths in hospital after injury. Eur J Surg. 1999;165 (9):828-33. Papadopoulos IN, Bukis D, Karalas E, Katsaragakis S, Stergiopoulos S, Peros G, et al. Preventable prehospital trauma deaths in a Hellenic urban health region: an audit of prehospital trauma care. J Trauma. 1996;41 (5):8649. Esposito TJ, Sanddal ND, Hansen JD, Reynolds S. Analysis of preventable trauma deaths and inappropriate trauma care in a rural state. J Trauma. 1995;39 (5):955-62. Stelfox HT, Straus SE, Nathens A, Bobranska-Artiuch B. Evidence for quality indicators to evaluate adult trauma care: a systematic review. Crit Care Med. $2011 ; 39$ (4):846-59. Zafarghandi MR, Modaghegh MH, Roudsari BS. Preventable trauma death in Tehran: an estimate of trauma care quality in teaching hospitals. J Trauma. 2003;55 (3):459-65. Llullaku SS, Hyseni N, Bytyci CI, Rexhepi SK. Evaluation of trauma care using TRISS method: the role of adjusted misclassification rate and adjusted w-statistic. World J Emerg Surg. 2009;4:2-7. Gruen RL, Jurkovich GJ, McIntyre LK, Foy HM, Maier RV. Patterns of errors contributing to trauma mortality: lessons learned from 2,594 deaths. Ann Surg. 2006;244 (3):371-80. Sanddal TL, Esposito TJ, Whitney JR, Hartford D, Taillac PP, Mann NC, et al. Analysis of preventable trauma deaths and opportunities for trauma care improvement in utah. J Trauma. 2011;70 (4):970-7.

Disclosure: No significant relationships.

\section{PR164}

\section{IS FEMALE GENDER PROTECTIVE IN SEVERE TRAUMA? A RETROSPECTIVE COHORT STUDY IN THREE LEVEL ONE TRAUMACENTERS IN THE NETHERLANDS}

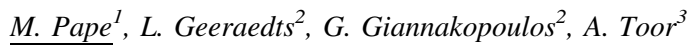

${ }^{1}$ Surgery, Section Traumasurgery, VU University Medical Center, Amsterdam/NETHERLANDS, ${ }^{2}$ Trauma Surgery, VU University Medical Center, Amsterdam/NETHERLANDS, ${ }^{3}$ Public Relations, Acute Care Area Network Northwest Netherlands, Amsterdam/ NETHERLANDS

Introduction: Some studies suggest that female gender is associated with a lower risk of mortality in severely injured patients, especially in premenopausal women. Gender is known to be an important risk factor in several diseases. The objective of this research was to evaluate the association between gender and mortality or ICU admission among severely injured patients in the Netherlands.

Material and methods: A retrospective multicenter study was performed including all polytrauma patients (Injury Severity Score (ISS) $\geq 16$ ) admitted to three level 1 trauma centers between 2006 and 2014 . Data regarding age, gender, injury mechanism, ISS, prehospital intubation, Revised Trauma Score (RTS), systolic blood pressure (SBP) and Glasgow Coma Scale upon ED admission were collected. To determine whether gender was an independent predictor of mortality and ICU admission, logistic regression analysis was performed. Results: Among 6,865 trauma patients, females aged 16- to 44-years had a significant lower in-hospital mortality rate compared to males (10.4\% vs $13.4 \%, \mathrm{P}=0.046)$. ICU admission rate was significantly lower for females $(49.3 \%$ vs $54.5 \%, \mathrm{P}=<0.0001)$. Logistic regression did not show gender as an independent predictor for in-hospital mortality or mortality within $24 \mathrm{hrs}$. However, male sex was associated with an increased likelihood for ICU admission (OR 1.205 (95\% CI $1.046-1.388), \mathrm{P}=0.010$ ).

Conclusion: This study shows that in this population, female gender is not a predictor for mortality. Furthermore, female gender is independently associated with a decreased likelihood for ICU admission. More research is needed to examine the possible protective effect of female gender in severe trauma.

References: 1. Choudhry M, Bland K, Chaudry I. Trauma and immune response-Effect of gender differences. Injury. 2007;38 (12):1382-1391. 2. Sperry J, Nathens A, Frankel H, Vanek S, Moore $\mathrm{E}$, Maier $\mathrm{R}$ et al. Characterization of the gender dimorphism after injury and hemorrhagic shock: Are hormonal differences responsible?*. Critical Care Medicine. 2008;36 (6):1838-1845. 3. Angele M, Frantz M, Chaudry I. Gender and sex hormones influence the response to trauma and sepsis: potential therapeutic approaches. Clinics. 2006;61 (5). 4.Knöferl M, Angele M, Diodato M, Schwacha M, Ayala A, Cioffi W et al. Female Sex Hormones Regulate Macrophage Function After Trauma-Hemorrhage and Prevent Increased Death Rate From Subsequent Sepsis. Annals of Surgery. 2002;235 (1):105-112. 5. Magnotti L, Fischer P, Zarzaur B, Fabian T, Croce M. Impact of Gender on Outcomes after Blunt Injury: A Definitive Analysis of More Than 36,000 Trauma Patients. Journal of the American College of Surgeons. 2008;206 (5):984-991. 6. Trentzsch H, Nienaber U, Behnke M, Lefering R, Piltz S. Female sex protects from organ failure and sepsis after major trauma haemorrhage. Injury. 2014;45:S20-S28. 7. Yang K, Zhou M, Sperry J, Rong L, Zhu X, Geng $L$ et al. Significant Sex-Based Outcome Differences in Severely Injured Chinese Trauma Patients. Shock. 2014;42 (1):11-15. 8. Croce M, Fabian T, Malhotra A, Bee T, Miller P. Does Gender Difference Influence Outcome? The Journal of Trauma: Injury, Infection, and Critical Care. 2002;53 (5):889-894. 9. Bird M, Karavitis J, Kovacs E. 
Sex differences and estrogen modulation of the cellular immune response after injury. Cellular Immunology. 2008;252 (1-2):57-67. 10. George R, McGwin G, Windham S, Melton S, Metzger J, Chaudry I et al. Age-Related Gender Differential in Outcome After Blunt or Penetrating Trauma. Shock. 2003;19 (1):28-32. 11. Wohltmann C, Franklin G, Boaz P, Luchette F, Kearney P, Richardson J et al. A multicenter evaluation of whether gender dimorphism affects survival after trauma. The American Journal of Surgery. 2001;181 (4):297300. 12. Rappold J, Coimbra R, Hoyt D, Potenza B, Fortlage D, Holbrook T. Female Gender Does Not Protect Blunt Trauma Patients from Complications and Mortality. The Journal of Trauma: Injury, Infection, and Critical Care. 2002;53 (3):436-441. 13. Napolitano L, Greco M, Rodriguez A, Kufera J, West R, Scalea a. Gender Differences in Adverse Outcomes after Blunt Trauma. The Journal of Trauma: Injury, Infection, and Critical Care. 2001;50 (2):274-280. 14. Trentzsch H, Lefering R, Nienaber U, Kraft R, Faist E, Piltz S. The Role of Biological Sex in Severely Traumatized Patients on Outcomes. Annals of Surgery. 2015;261 (4):774-780. 15. Coimbra R, Hoyt D, Potenza B, Fortlage D, Hollingsworth-Fridlund P. Does Sexual Dimorphism Influence Outcome of Traumatic Brain Injury Patients? The Answer Is No!. The Journal of Trauma: Injury, Infection, and Critical Care. 2003;54 (4):689-700. 16. George R, McGwin G, Metzger J, Chaudry I, Rue L. The Association between Gender and Mortality among Trauma Patients as Modified by Age. The Journal of Trauma: Injury, Infection, and Critical Care. 2003;54 (3):464-471. 25. Mutschler M, Nienaber U, Münzberg M, Wölfl C, Schoechl H, Paffrath $\mathrm{T}$ et al. The Shock Index revisited $-\mathrm{a}$ fast guide to transfusion requirement? A retrospective analysis on 21,853 patients derived from the TraumaRegister DGU®. Critical Care. 2013;17 (4):R172. 26. Mutschler M, Nienaber U, Brockamp T, Wafaisade A, Fabian T, Paffrath $\mathrm{T}$ et al. Renaissance of base deficit for the initial assessment of trauma patients: a base deficit-based classification for hypovolemic shock developed on data from 16,305 patients derived from the TraumaRegister DGU®. Critical Care. 2013;17 (2):R42. 27. Rainer $\mathrm{T}$, Li Y, Chan C, Agarwal N, Sin K, Chan S et al. Validating a pragmatic definition of shock in adult patients presenting to the emergency department. Resuscitation. 2014;85:S113. 28. Haider A, Crompton J, Chang D, Efron D, Haut E, Handly N et al. Evidence of Hormonal Basis for Improved Survival Among Females With Trauma-Associated Shock: An Analysis of the National Trauma Data Bank. The Journal of Trauma: Injury, Infection, and Critical Care. 2010;69 (3):537-540. 29. FarmacotherapeutischKompas [Internet]. Farmacotherapeutischkompas.nl. 2017 [cited 2 June 2017]. Available from: https://www.farmacotherapeutischkompas.nl/bladeren/ preparaatteksten/f/flutamide 30. Catania R, Angele M, Ayala A, Cioffi W, Bland K, Chaudry I. Dehydroepiandrosterone restores immune function following trauma-haemorrhage by a direct effect on T-lymphocytes. Cytokine. 1999;11 (6):443-450. 31. Jason Bowles B, Roth B, Demetriades D. Sexual dimorphism in trauma? A retrospective evaluation of outcome. Injury. 2003;34 (1):27-31. 32. Frink M, Pape H, van Griensven M, Krettek C, Chaudry I, Hildebrand F. Influence of sex and age on MODS and cytokines after multiple injuries. Shock. 2007;27 (2):151-156. 33. Oberholzer A, Keel M, Zellweger R, Steckholzer U, Trentz O, Ertel W. Incidence of Septic Complications and Multiple Organ Failure in Severely Injured Patients Is Sex Specific. The Journal of Trauma: Injury, Infection, and Critical Care. 2000;48 (5):932-937. 34. Liu T, Xie J, Yang F, Chen J, $\mathrm{Li} \mathrm{Z,} \mathrm{Yi} \mathrm{C} \mathrm{et} \mathrm{al.} \mathrm{The} \mathrm{influence} \mathrm{of} \mathrm{sex} \mathrm{on} \mathrm{outcomes} \mathrm{in} \mathrm{trauma} \mathrm{patients:}$ a meta-analysis. The American Journal of Surgery. 2015;210 (5):911921. 35. Dossett L, Swenson B, Heffernan D, Bonatti H, Metzger R, Sawyer R et al. High Levels of Endogenous Estrogens are Associated With Death in the Critically Injured Adult. The Journal of Trauma: Injury, Infection, and Critical Care. 2008;64 (3):580-585. 36. Wichmann M, Inthorn D, Andress H, Schildberg F. Incidence and mortality of severe sepsis in surgical intensive care patients: the influence of patient gender on disease process and outcome. Intensive Care
Medicine. 2000;26 (2):167-172. 37. Brattström O, Granath F, Rossi P, Oldner A. Early predictors of morbidity and mortality in trauma patients treated in the intensive care unit. Acta Anaesthesiologica Scandinavica. 2010;54 (8):1007-1017.

Disclosure: No significant relationships.

\section{PR165}

\section{EVALUATION OF THE QUALITY OF CARE IN TRAUMA PATIENTS BY USING PS14 AND TRISS SCORING SYSTEMS IN A SPANISH TRAUMA INTENSIVE CARE UNIT}

\author{
J. Sainz Cabrejas ${ }^{I}$, C. García Fuentes ${ }^{1}$, C. Garcià Juarraz ${ }^{2}$, \\ L. Maure Blesa ${ }^{2}$, A. Gonzàles Lòpez ${ }^{2}$, J.C. Montejo González ${ }^{1}$, \\ M. Chico Fernández ${ }^{l}$ \\ ${ }^{1}$ Uci De Trauma Y Emergencias, H. U. 12 de Octubre, Madrid/ \\ SPAIN, ${ }^{2}$ Facultad De Medicina, Universidad Coplutense de Madrid, \\ Madrid/SPAIN
}

Introduction: In order to evaluate the quality of medical trauma care, several predictive models of mortality have been developed. [1] The most widely used is TRISS model. Its coefficients were recalculated in 2010 (TRISS2010 model). [2] PS14 model is used in the UK as a standard of quality in trauma care. [3, 4] Our main objective is to evaluate the ability of these systems to predict mortality rates in our medical system and population.

Material and methods: We performed a retrospective observational study during a 66-month-period. Inclusion criteria were severe trauma (Injury-severity-score $\geq 16$ and/or Revised-Trauma-Score $<12$ ), and age $>14$ y.o. Information from medical care was recorded prospectively. "W" statistic (difference between expected and observed mortality for each 100 patients) and its significance were calculated for each model. Subgroup analysis was performed. Discrimination and calibration were evaluated by means of Receiver operating characteristic (ROC) curves, and Hosmer-Lemeshow test; and the GiViTI-calibration-belt respectively.

Results: We enrolled 1240 patients. Survival to hospital discharge was $81.9 \%$. W score for TRISS, TRISS2010 and PS14 models were respectively $+6.72(\mathrm{p}<0.01),+1.48(\mathrm{p}=0.08)$ and $+2.74(\mathrm{p}<$ 0.01 ). Subgroup analysis revealed significant favorable results for some populations. AUROC for TRISS, TRISS2010 and PS14 models were respectively $0.915,0.919$ and 0.914 . We found no significant differences between them $(p>0.05)$. Both, Hosmer-Lemeshow test and GiViTI-calibration-belt demonstrated poor calibration for the three models.

Conclusion: $\$$ These models are a suitable tool to assess quality of care in a Trauma ICU. They present an excellent discrimination but poor calibration. In our institution, survival rates higher than expected have been observed.

References: 1] Pino Sánchez FI, Ballesteros Sanz MA, Cordero Lorenzana L, Guerrero López F; Trauma and Neurointensive Care Work Group of the SEMICYUC. Quality of trauma care and trauma registries. Med Intensiva. 2015 Mar; 39 (2):114-23 [2] Muntera L., Polinderb S., Lansinka KKW, . Cnossenb MC., Steyerbergb EW, de Jongha MAC. Mortality prediction models in the general trauma population: A systematic review. Injury, Int. J. Care Injured 48 (2017) 221-229 [3]Bouamra O, Jacques R, Edwards A, Yates DW, Lawrence T, Jenks T, Woodford M, Lecky F. Prediction modelling for trauma using comorbidity and 'true' 30-day outcome. Emerg Med J. 2015 Dec;32 (12):933-8. [4] Trauma Audit and Research Network. TARN. Available online at: https://www.tarn.ac.uk/

Disclosure: No significant relationships. 
PR166

\section{THE INCIDENCE, MANAGEMENT AND OUTCOME OF TRAUMATIC VASCULAR INJURIES IN A MAJOR TRAUMA CENTRE}

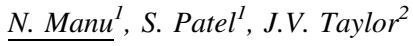

${ }^{1}$ Emergency General Surgery And Major Trauma Unit, Aintree University Hospital, Liverpool/UNITED KINGDOM, ${ }^{2}$ General Surgery Department, Emergency General Surgery And Major Trauma Centre, Merseyside, Aintree University Hospital NHS Foundation Trust, Liverpool/UNITED KINGDOM

Introduction: Reconfiguration of the major trauma service within the UK has resulted in 27 major trauma centres, which has proven to significantly improve the management of trauma in accordance with the ATLS guidelines. Aintree University Hospital has become the regional major trauma centre for Merseyside.

Material and methods: A single centre retrospective observational study of patients with traumatic vascular injury who presented to a major trauma centre between January 2014 and December 2016 were identified using TARN.

Results: Vascular injuries were identified in 37 trauma admissions; the majority sustained poly-trauma. Of these, 29 were male and 7 were female, ranging from the age of 16-93. Blunt trauma $(61 \%)$ accounted for a higher proportion of vascular trauma admissions compared with penetrative trauma $(39 \%)$. Patients who sustained blunt vascular injuries were more severely injured compared to penetrative vascular injuries. Blunt vascular injuries were also associated with higher critical care admission $(66.6 \%)$ and greater mortality $(77.7 \%) .11 \%$ patients underwent a thoracotomy, of which 3 went on to have a laparotomy. $42 \%$ patients had surgical repair of the injured vessel; on only one occasion an off-site vascular surgeon was required. $11 \%$ patients underwent IR and $17 \%$ patients were managed conservatively. $17 \%$ patients were transferred to a tertiary centre for management of the vascular injury.

Conclusion: Traumatic Vascular injuries only account for a small number of trauma admissions. Despite this, these patients have high mortality and morbidity. Prompt assessment and management of these patients in a major trauma centre is imperative to outcome due to associated poly-trauma.

References:

Disclosure: No significant relationships.

\section{PR167}

\section{ALCOHOL CONSUMPTION IS ACCOMPANIED BY ACIDOSIS IN INJURED TRAUMA PATIENTS - A MATCHED-PAIR ANALYSIS}

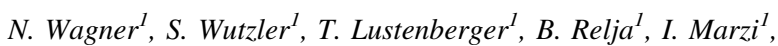
A. The Traumaregister $\mathrm{Dgu}{ }^{2}$

${ }^{1}$ Department Of Trauma, Hand And Reconstructive Surgery, University Hospital Frankfurt, Goethe-University, Frankfurt am Main/GERMANY, ${ }^{2}$ Intensive Care And Trauma Management (section Nis) Of The German Trauma Society (dgu), Committee on Emergency Medicine, Committee on Emergency Medicine Intensive Care and Trauma Management (Section NIS) of the German Trauma Society (DGU)/GERMANY
Introduction: Introduction: The current situation of studies regarding the influence of alcohol on injured trauma patients is inconsistent. Here, we investigate the influence of alcohol on injured patients in view of complications and mortality.

Material and methods: Material \& Methods: A retrospective matched-pair analysis of the multi-centre database of the TraumaRegister DGU ${ }^{\circledR}$ of the German Trauma Society (Deutsche Gesellschaft für Unfallchirurgie, DGU) was performed. Patients with a maximum Abbreviated Injury Scale (MAIS) of 3 or greater from the years 2015 and 2016 with measured alcohol values on admission were included. Matched-pair analysis between patients with positive blood alcohol concentration ( $\geq 0.5 \%$, BAC) and with negative BAC (no $\mathrm{BAC}$ ) according to age, gender, AIS body regions and Revised Injury Severity Classification II Score intervals was conducted.

Results: Results: 834 trauma patients were included, with 417 patients per group. BAC group showed an alcohol average level of $1.9 \%$ with a clear trend to penetrating traumata $(4.2 \%$ no BAC vs. $7.4 \%$ BAC, $\mathrm{p}=0.051)$ and low energy falls $(18.1 \%$ no BAC vs. $31.3 \%$ BAC). No difference between both groups were found regarding length of hospitalization, length of ICU treatment, Injury Severity Score (20.24 vs. 19.22$)$ and mortality rate $(8.2 \%$ no BAC vs. $5.8 \%$ BAC). Significantly more often hypotension (10.3\% no BAC vs. $15.2 \%$ BAC, $\mathrm{p}=0.045)$ and acidosis $(6.1 \%$ no BAC vs. $17.2 \%$ BAC, $p$ $<0.001)$ were observed in the BAC group.

Conclusion: Conclusions: This study indicates that positive BAC leads to acidosis in severely injured trauma patients, which might be relevant during initial therapy, but does not worsen the final outcome. References: TR-DGU project ID: 2017-001 Data provision was carried out by TraumaRegister DGU $\AA$.

Evaluation and interpretation are in the author's responsibility and haven't yet accomplished the review process of TraumaRegister DGU®.

Disclosure: No significant relationships.

\section{PR168}

\section{IMPLEMENTING AN ELECTRONIC TRAUMA REGISTRY IN GUATEMALA: LESSONS LEARNED FROM MISSING DATA}

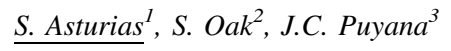

${ }^{1}$ Facultad De Medicina, Universidad Francisco Marroquin, Guatemala/GUATEMALA, ${ }^{2}$ Surgery, University of Pittsburgh, Pittsburgh/PA/UNITED STATES OF AMERICA, ${ }^{3}$ General \& Trauma Surgery, UPMC Presbyterian Hospital, Pittsburgh/PA/ UNITED STATES OF AMERICA

Introduction: Trauma is a worldwide health care problem, where outcomes are significantly conditioned on the infrastructure and resources at hand. In low middle income countries, like Guatemala, trauma devastates an already deplorable health care system by consuming a large portion of the public health budget. Here we describe the implementation process of an electronic trauma registry, through a pilot plan, in Guatemala city, and the impact it created on the health care system. The main objective of the implementation was to substitute a hand written admit note for an electronic tablet that automatically created a real-time data registry.

Material and methods: Thirteen electronic wireless tablets were installed throughout the hospital. Surgical residents were trained to use the electronic trauma health record software installed in each tablet. We divided the software in three main data point sets: surgical admission notes, operative notes and discharge notes. After assessing 
the data collected in a five month period, we conducted semi-structured interviews.

Results: $100 \%$ of the surgical residents were satisfied with the transition from paper to an electronic device and $100 \%$ referred the admission note to be a faster and easier way to admit patients. As for the operative note and discharge note there was a $0 \%$ compliance rate due to the fact that it represented double work.

Conclusion: Mobile electronic devices are a solution for the lack of data problem in LMIC's. The key components to a successful trauma registry implementation are the custom-made software, the pre evaluation of the work flow and the selection of data registrars.

References: Moore L, Clark DE. Injury. 2008 Jun;39 (6):686-95.

Disclosure: No significant relationships.

\section{PR169}

\section{CT MEASUREMENT OF ILIAC CREST MORPHOLOGY FOR APPROPRIATE PIN PLACEMENT IN ILIAC CREST EXTERNAL FIXATION}

\author{
S. Yokoo, T. Noda, Y. Shimamura, Y. Yamakawa, M. Kiyono, \\ Y. Mochizuki, M. Yoshimura, T. Uchino, K. Demiya, T. Ozaki
}

Orthopaedic Surgery, Okayama University, OKAYAMA/JAPAN

Introduction: External fixation (EF) plays an important role in the treatment of unstable pelvic ring injuries. Of the three basic configurations of pin placement, iliac crest (IC) has become the most popular method. This study's purpose was to investigate optimal insertion placement of the IC EF pin.

Material and methods: 18 Japanese unilateral iliac bones were scanned with multislice computed tomography (CT). After measuring the length of the IC on a three-dimensional CT, a tangential line was drawn on the IC 2 and $5 \mathrm{~cm}$ behind the anterior superior iliac spine (ASIS), and an image of a cross section perpendicular to the tangent was reconstructed. We measured the width of the IC, the narrowest medullary distance, and made a line through the midpoint of the intramedullary and measured its pathway.

Results: The length from the ASIS to the iliac tuberosity was $62.2 \pm$ $8.6 \mathrm{~mm}$, and the average maximum width of the IC was 16.0 and $19.1 \mathrm{~mm}$ at 2 and $5 \mathrm{~cm}$ behind the ASIS respectively. The average narrowest medullary distance was 5.6 and $4.5 \mathrm{~mm}$ respectively. The straight lines connecting the midpoints at depths of 2 and $4 \mathrm{~mm}$ passed almost at the center of the IC $(52.4 \%$ and $49.7 \%$ of maximum width respectively); however, the straight lines connecting the midpoints at 3 and $5 \mathrm{~mm}$ depth passed slightly to the outer side of the IC $(39.7 \%$ and $43.4 \%$ of maximum width respectively).

Conclusion: Making a pin insertion point slightly lateraly from the middle of the IC enables to pass the EF pin longer and surely through the medullary cavity.

References: Solomon LB, Pohl AP, Chehade MJ, et al. Surgical anatomy for pelvic external fixation. Clin Anat. 2008 Oct;21 (7):67482.

Disclosure: No significant relationships.

\section{PR170}

\section{THE DANGERS OF PETS AND HORSES ANIMAL RELATED INJURIES AT THE EMERGENCY DEPARTMENT}

\author{
E.A.k. Van Delft, M. Schreuder, I. Thomassen, N. Sosef
}

Surgery, Spaarne Gasthuis, Hoofddorp/NETHERLANDS

Introduction: Every year many patients present to the emergency department due to bites, scratches and falls caused by animals. Although bite and scratch injuries have often been described, the amount of patients who visit the emergency department due to all animal related injuries has never been described before.

Material and methods: A retrospective analysis of all emergency department visits throughout a 1-year period was performed from April 2015 until March 2016.

Results: 516 Patients were treated at the emergency department because of animal related injuries. Most were female and median age was 38 years. The animals causing most injuries were horses, followed by dogs and cats. Animal related injuries more often caused fractures $(n=165)$ or contusions $(n=171)$ compared to wounds $(n=$ 135). No lethal injuries were recorded. However, three animals did not survive the event.

Conclusion: The incidence of animal related injuries is high with $2.8 \%$, and this is probably an underestimation. Many of the injuries have a noteworthy origin and many injuries have a physical injury as result. Awareness in the general population on the potential dangers of pets and horses could reduce the amount and severity of animal related injuries.

References:

Disclosure: No significant relationships.

\section{PR171}

\section{BALCONING: NEW INJURY MECHANISM FOR ALCOHOL- RELATED FALLS FROM HEIGHTS}

C. Pineño Flores, J.J. Segura Sampedro, J.M. Garcia Perez, P. Jimenez Morillas, R. Morales Soriano, C. Jimenez Viñas, F.X. Gonzalez Argente

Resident Of General Surgery, Hospital Universitario Son Espases, palma de mallorca/SPAIN

Introduction: Balconing was defined the practice of jumping from a hotel balcony towards a swimming pool or from one balcony to another, during holidays after alcohol and/or drug abuse consumption with no intention of self-infliction.

Material and methods: A 5 years retrospective study in a tertiary referral center for severe trauma in the Balearic Islands, where balconing is endemic. Demographic data such as age, sex, nationality, alcohol or drug consumption, height of the fall and season of the year were collected. Scales of trauma severity and mortality rates were also included. Data were analysed using IBM SPSS Statistics, version 21.0.

Results: Were included 46 patients. Most of the patients were males, $45(97.83 \%)$, aged $24.20 \pm 5.98$ years and 28 of them of British nationality $(60.87 \%)$. In $44(95.65 \%)$ cases, alcohol consumption was present accompanied by other drugs in $17(36.96 \%)$. The mean height of the fall was approximately 3 floors. Only $6(13.04 \%)$ were intentional jumpers whereas $40(86.96 \%)$ fell while trying to reach another 
balcony. The most common injuries were head and neck injuries, 37 $(80.43 \%)$. There were 34 cases $(70 \%)$ of major trauma (NISS $\geq 16$ ) and almost $50 \%$ required major surgery. The length of stay (LOS) was $17.52 \pm 17.94$ days with only one death $(2.17 \%)$

Conclusion: Balconing is a new injury mechanism for alcohol-related falls from heights. The rate of head/neck injury among these patients is much higher $(80.43 \%)$ than those described in other falls from height $(54.8 \%)$. Other regions' injuries also have higher rates in balconing.

References: Pérez-Bovet J, Lorencio C, Taché A, Pujol Valverde P, Martín Ferrer S. Traumatic brain injury caused by 'balconing'. Br J Neurosurg 2014; 29: 1-5. 2 Sampedro L, Sáenz H. Shields BJ, Burkett E, Smith G a. Epidemiology of balcony fall-related injuries, United States, 1990-2006. Am J Emerg Med 2011; 29: 174-180. La siniestra lista del balconing suma y sigue. El Mundo. 2010.http:// www.elmundo.es/elmundo/2010/09/12/baleares/128429140 3.html (accessed 24 Jan2016). Jorro I. UK warns their tourists of 'balconing' risks in Spain. Zerotwobe. 2013.http://www.02b.com/en/notices/ 2013/07/uk_warns_their_tourists_o f_balconing_risks_in_spain_5243.php (accessed 24 Jan2016). Osler T, Baker SP, Long W. A modification of the injury severity score that both improves accuracy and simplifies scoring. J Trauma 1997; 43: 922-5-6 Carroll JT, Chapman AJ, Davis AT, Rodriguez CH. The impact of tree-stand falls on a Level 1 trauma center in West Michigan. Am J Surg 2015; : 6-9. Parreira JG, Kanamori LR, Valinoto GCJ, Perlingeiro J a. G, Soldá SC, Assef JC. Comparative analysis between identified injuries of victims of fall from height and other mechanisms of closed trauma. Rev Col Bras Cir 2014; 41: 285-291. Papadopoulos IN, Bonovas S, Kanakaris NK, Nikolopoulos G, Kotsilianou O, Konstantoudakis G et al. Alcohol and psychoactive drugs increased the prehospital mortality in 655 fall-related fatalities in Greece: A call for management protocols. Injury 2012; 43: 1522-1526.

Disclosure: The authors declare that they have no competing interests

\section{PR172}

\section{PROPOSAL FOR A NEW METHOD TO EVALUATE CRITICALLY INJURED PATIENTS}

\author{
M. El Moumni, A. Andela, K.W. Wendt
}

Trauma Surgery, University Medical Center Groningen, Groningen/ NETHERLANDS

Introduction: There is no consensus in defining 'polytrauma', while the terms multiple injured patients, major trauma, polytrauma, severely or critically injured patients are used interchangeably. We propose a new method to evaluate the (critically) injured patients.

Material and methods: We conceptualize that injury (either blunt or penetrating) results in tissue damage and tissue hypoxia causing organ injury. This in turn invokes a physiological response. Host factors, such as age and comorbidity, may influence, confound or mediate the outcome.

The physiological response puts a patients in a certain 'state' that can be a threat to life. Since this state cannot be measured directly, observed variables are used in a confirmatory factor analysis to analyse this conceptualized model.

Results: In this retrospective study, a total of 2461 trauma patients were included. A one-factor model with systolic blood pressure, temperature, hemoglobine, base excess, and fibrinogen was associated with good indices of fit: RMSEA $=0.029, \mathrm{CFI}=0.99, \mathrm{TLI}=0.98$, and SRMR $=0.035$. Factor loadings of $>0.5$ were observed for all observed variables. Internal consistency was good (Cronbach's alpha $=0.81$ ).

Conclusion: A one-factor model with 5 indicators (systolic blood pressure, temperature, hemoglobin, base excess, and fibrinogen) can be used to evaluate the physiological condition of (critically) injured patients.

References:

Disclosure: No significant relationships.

\section{PR173}

\section{ANALYSIS OF THE POLYTRAUMATIZED PATIENTS WITH SEVERE ABDOMIAL PELVIC TRAUMA AT "12 DE OCTUBRE” HOSPITAL, MADRID}

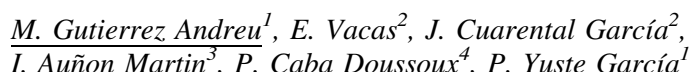

${ }^{1}$ General And Digestive Surgery, 12 DE OCTUBRE UNIVERSITY HOSPITAL, MADRID/SPAIN, ${ }^{2}$ Orthopaedic Surgery And Traumatology, Hospital Universitario 12 de Octubre, Madrid/SPAIN, ${ }^{3}$ Ortopédica Surgery Service, Hospital 12 de Octubre, Madrid/SPAIN, ${ }^{4}$ Cirugia Ortopedica Y Traumatologia, Hospital 12 de Octubre, Madrid/SPAIN

Introduction: The frequency of trauma in our environment has increased in recent decades, becoming a public health problem derived from its high rate of morbidity and mortality. We analyzed the patients with abdominal pelvic trauma in our hospital through an injury database.

Material and methods: Retrospective, descriptive, observational study performed over 10 years. (2004-2014). The following variables are evaluated: age, sex, injury mechanism, severity scale (ISS), type of injuries, average stay and mortality.

Results: 118 patients with abdominal pelvic trauma were attended with ISS $>13$, males $68,1 \%$, mean age of 37 years. The most common causes of trauma were traffic accidents $(38,46 \%)$, high-energy falls $(34,19 \%)$ and hit by car $(21,37 \%) .51,69 \%$ of the patients were hemodynamically unstable, $100 \%$ of the patients had abdominal and pelvic trauma, $85,5 \%$ had head trauma and $92 \%$ had thoracic trauma. Laparotomy was performed in 34 patients $(28.81 \%)$ : hepatic resection: $1,69 \%$ (2), abdomen packing 7,63\% (9), splenectomy 5,93\% (7). Thoracotomy was performed in 8 patients, pelvic invasive external fixation $33,05 \%$ (39) and 3 patients with extraperitoneal packing. Arteriography, $36,61 \%$ (41). The mean stay in the ICU was 13 days and in the ward 32 days. The mortality rate was $8.55 \%$, (10 patients). 6 patients died for exanguination and 3 for multi organ failure.

Conclusion: s: Polytrauma registries help to quantify the problem and evaluate the function of the current systems. Their main purpose is improving the quality of care, implementation of preventive measures and evaluation of new technologies.

References:

Disclosure: No significant relationships. 


\section{PR174}

IS THE CLINICAL AND PARACLINICAL FINDINGS HAVE THE POWER TO PREDICT THE CRITICAL CONDITIONS OF INJURED AFTER RESUSCITATION OF TRAUMATIC INJURIES? A CLINICAL STUDY BY USING DATA MINING ARTIFICIAL INTELLIGENCE

E. Parva, Z. Ghahramani, $\underline{\text { S. Paydar }}$

Shahid Rajaee (emtiaz) Trauma Hospital, Trauma Research Center, Shiraz/IRAN

Introduction: The triage and initial care of injured patient and subsequent right level of care is paramount for overall outcome after traumatic injury. Early recognition of patients with risk for worse prognosis is of importance in such decision-making. Utilizing data mining we sought to answer if clinical and paraclinical signs after initial resuscitation could predict outcome.

Material and methods: 1106 trauma patients 16 years and older were included in the study. The patients were in level I and II triage and admitted to Shahid Rajaee (Emtiaz) trauma hospital, Shiraz between the years 2014-2015. The CRISP metrology and modeling was used for assessing the early clinical and paraclinical variables to find the best predictive variables. The variables includes GCS, base deficit and diastolic blood pressure.

Results: Learning algorithms can predict the deterioration of the injured patients with monitoring the Bagging and SVM models with 99\% accuracy. The most fitted variables, especially after initial resuscitation, in the algorithms for overall outcome predictions were: Glasgow Coma Scale score, base deficit and diastolic blood pressure. Conclusion: Data mining for outcome measures in trauma patients could help in triage, initial treatment, and further decision-making. Clinical and paraclinical variables after resuscitation could predict overall outcome. Using artificial intelligence monitoring may have a role in trauma care and should be further investigated.

References:

Disclosure: No significant relationships.

\section{PR175}

\section{EVALUATION OF THE APPLICABILITY OF A TRIAGE SYSTEM BASED ON AMERICAN FIELD TRIAGE GUIDELINES IN ORDER TO ESTABLISH THE NEED FOR IN-HOSPITAL PARTIAL TRAUMA TEAM ACTIVATION}

\author{
J. Sainz Cabrejas ${ }^{1}$, C. García Fuentes ${ }^{1}$, J. Barea Mendoza ${ }^{2}$, \\ J.C. Montejo González ${ }^{1}$, M. Chico Fernández ${ }^{2}$
}

${ }^{1}$ Uci De Trauma Y Emergencias, H. U. 12 de Octubre, Madrid/ SPAIN, ${ }^{2}$ Uci De Trauma Y Emergencias, Hospital Universitario 12 de Octubre, Madrid/SPAIN

Introduction: In our hospital, we have been using for 5 years a triage system based on American Field Triage Guidelines [1] to indicate Hospital Partial Trauma Team activation (HPTTA), which consists of four sequential steps: 1) Physiological, 2) Anatomical, 3) Mechanism of Injury and 4) Special considerations. Our objective was to evaluate the accuracy of the system to predict ICU admission requirement.

Material and methods: We performed an observational retrospective study. All patients older than 16 y.o who required HPTTA were recruited.

For each step (that includes all the preceding criteria), we calculated under and overtriage rates (proportion of patients admitted to ICU who do not fulfill criteria and proportion of patients who fulfill criteria but do not require ICU admission respectively). We also calculated for each criterion, the number of patients needed to be evaluated so that one ICU admission is required (NND).

Results: We enrrolled 1548 patients, 163 of which (10.5\%) were admitted to ICU. HPTTA was indicated according to the first step in 97 patients, 164 to the second, 1101 to the third and 34 to the fourth. Over and undertriage rates were respectively: $3.42 \%$ and $71.26 \%$ for the first step, $12.81 \%$ and $51.15 \%$ for the second, $88.88 \%$ and $7.47 \%$ for the third and $90.97 \%$ and $6.32 \%$ for the fourth one. NND for each criterion, were $1.94 ; 3.7 ; 8.67$ and 8.66 respectively.

Conclusion: Our triage system is a suitable tool to indicate HPTTA.

४ Steps 1 and 2 present low overtriage rates, while third and fourth ones confer a high sensitivity to the system.

References: [1]Sasser SM, Hunt RC, Faul M, Sugerman D, Pearson WS, Dulski T, et al. Centers for Disease Control and Prevention $(C D C)$. Guidelines for field triage of injured patients: recommendations of the National Expert Panel on Field Triage, 2011. MMWR Recomm Rep. 2012 Jan 13;61 (RR-1):1-20. PubMed PMID: 22237112

Disclosure: No significant relationships.

\section{PR176}

\section{PREHOSPITAL DIAGNOSTIC VALUE OF SUSPECTED PELVIC AND ABDOMINAL INJURIES BY EMERGENCY MEDICAL SERVICES PROVIDERS IN TRAUMA PATIENTS}

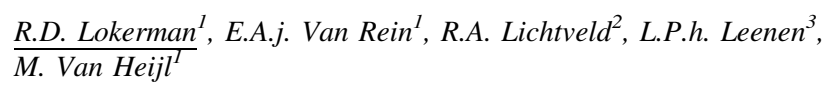

${ }^{1}$ Traumatology, University Medical Center Utrecht, Utrecht/ NETHERLANDS, ${ }^{2}$ Regional Ambulance Facilities Utrecht, Regional Ambulance Facilities Utrecht, Bilthoven/NETHERLANDS, ${ }^{3}$ Surgery, University Medical Center Utrecht, Utrecht/NETHERLANDS

Introduction: Abdominal and pelvic injuries have a relatively low incidence $^{1-4}$, but do show a high mortality rate ${ }^{3,5,6}$. Transport of patients with abdominal or pelvic injuries to high-level trauma centers is crucial to increase chances of survival. Although previous studies $^{7-9}$ suggest that both are difficult to recognize on-scene, this had never been investigated in emergency medical services (EMS) providers within an entire region. The purpose of this study is to evaluate the diagnostic value of EMS provider judgment in the identification of abdominal and pelvic injuries in the region Central Netherlands.

Material and methods: In this multicenter cohort study, all patients older than 15 transported by an ambulance with the highest priority between January 2015 and December 2016 to a hospital in the Central Netherlands region were included. The diagnostic value of the EMS provider judgment in the identification of abdominal or pelvic injury was used as primary outcome. In-hospital diagnosed abdominal and pelvic injuries were defined as an Abbreviated Injury Scale $\geq 2$ and were used as reference standard.

Results: A total of 2,766 patients were included of which $64(2 \%)$ were in-hospital diagnosed with an abdominal injury and $77(3 \%)$ with a pelvic injury. The EMS providers' prehospital judgment had a sensitivity and specificity of $39 \%$ and $95 \%$ for abdominal injury and $32 \%$ and $99 \%$ for pelvic injury.

Conclusion: In the prehospital phase EMS providers show a relatively low sensitivity for detection of abdominal and pelvic injuries. Prehospital detection of these injuries needs improvement, in order to transport the right patient to the right type of hospital. 
References: 1 . Twijnstra MJ, Moons KG, Simmermacher RK, Leenen LP. Regional Trauma System Reduces Mortality and Changes Admission Rates: A Before and After Study. 2010 Feb;251 (2):33943.

2. Landelijke Traumaregistratie 2011-2015: Rapportage Nederland. Landelijk Netwerk Acute Zorg. 2016 [cited 17 October 2017]. Available from: http://www.lnaz.nl/cms/LTR_landelijk_jaarrapport_2011-2015.pdf

3. Chang MC. National Trauma Data Bank 2016, Annual Report [Internet]. American College of Surgeons. [cited 17 October 2017] Available from: https://www.facs.org/ /media/files/quality\%20programs/trauma/ntdb/ntdb\%20annual\%20report\%202016.ashx

4. Giannakopoulos GF, Saltzherr TP, Lubbers WD, Christiaans HM, van Exter P, de Lange-de Klerk ES, Bloemers FW, Zuidema WP, Goslings JC, Bakker FC. Is a maximum Revised Trauma Score a safe triage tool for Helicopter Emergency Medical Services cancellations? Eur J Emerg Med. 2011 Aug;18 (4):197-201.

5. Balogh Z, King KL, Mackay P, McDougall D, Mackenzie S, Evans JA, et al. The epidemiology of pelvic ring fractures: a population-based study. J Trauma. 2007;63 (5):1066-73; discussion 72-3.

6. Smith J, Caldwell E, D'Amours S, Jalaludin B, Sugrue M. Abdominal trauma: a disease in evolution. ANZ J Surg. 2005;75 (9):790-4.

7. Helm M, Faul M, Unger T, Lampl L. [Reliability of emergency medical field triage : Exemplified by traffic accident victims]. Anaesthesist. 2013 Dec;62 (12):973-80.

8. Hasler RM, Kehl C, Exadaktylos AK, Albrecht R, Dubler S, Greif R, et al. Accuracy of prehospital diagnosis and triage of a Swiss helicopter emergency medical service. J Trauma Acute Care Surg. 2012;73 (3):709-15

9. Mulholland SA, Cameron PA, Gabbe BJ, Williamson OD, Young K, Smith KL, et al. Prehospital prediction of the severity of blunt anatomic injury. J Trauma. 2008;64 (3):754-60.

Disclosure: No significant relationships.

\section{PR177}

\section{LONG-TERM MORTALITY OF SEVERELY INJURED TRAUMA PATIENT}

\section{J. Kuorikoski, T. Brinck, L. Handolin, T. Söderlund}

Töölö Hospital, Helsinki University Hospital, Helsinki/FINLAND

Introduction: The long-term mortality after a major trauma is not well known. The emphasis is usually on the trauma patients as a large cohort, including trauma patients with minor injuries, and not only major trauma patients. In this study, we report long-term mortality after a major trauma.

Material and methods: Helsinki University Hospital's trauma registry (HTR) was used for patient identification from 1.1.2006 to 31.12.2015. Inclusion criteria were: NISS $\geq 16$ and blunt mechanism of injury. Exclusion criteria were: penetrating injury, isolated burn injury or patient living outside Finland. The patients were matched with 10 control persons by Population Register Center. Control persons were matched by age, sex and city of residency. Socioeconomic status and information of the highest degree of education were obtained from Statistics Finland for patients and control person. Injury date was the beginning of follow-up for patients and control persons.

Results: We identified 3557 patients from our trauma registry, $27.4 \%$ females $(\mathrm{n}=2581)$ and $72.6 \%$ males $(\mathrm{n}=976)$. The mean $(\mathrm{SD})$ age in years was $50.5(20.5)$, range 0-98. Mean (SD) NISS was $31.2(11.8)$ and ISS 24.5 (9.6). These patients are compared to 35502 controls. The outcome analysis was adjusted for socioeconomic status and education.

Conclusion: We report Kaplan-Meier analysis of major trauma patients up to 11.5-year follow-up time. These patients are compared to control persons to evaluate the effect of major trauma on long-term mortality. A Cox regression analysis is used to study the factors associated to long-term survival.

References:

Disclosure: No significant relationships.

\section{PR178}

\section{FREQUENCY, PRESENTATION AND MANAGEMENT OF TRAUMATIC ADRENAL INJURY}

H. Al-Thani ${ }^{1}$, A. El-Menyar ${ }^{2}$

${ }^{1}$ Trauma Surgery Section, Department Of Surgery, Hamad Trauma Center, Doha/QATAR, ${ }^{2}$ Trauma Surgery, Hamad General Hospital, Doha/QATAR

Introduction: We aimed to examine the clinical presentation, management and outcome of blunt adrenal injuries in a single institution Material and methods: A retrospective study of traumatic adrenal injury between 2011 and 2014 was conducted in a Level 1 trauma center.

Results: A total of 504 patients with solid organ injury were identified, of them 116 cases had adrenal injury (23\%) with a mean age of $32.5 \pm 13.7$ years. The majority of cases were males $(90 \%)$ and $70 \%$ were traffic-related injuries. The most frequent associated injuries included liver (47\%), ribs (42\%), lung (37\%), head (35\%), spleen $(23 \%)$, and kidney $(22 \%)$. Adrenal injury was mainly on the right side $(86 \%)$ and in $11.5 \%$ of cases it was bilateral. Based on the CT findings it was gross adrenal hemorrhage (82\%) and focal hematoma $(18 \%)$. Grading according to the adrenal gland injury scale (AAST): contusion (46\%), laceration extending into medulla $>2 \mathrm{~cm} \mathrm{(26 \% ),}>$ $50 \%$ parenchymal destruction $(23 \%)$ cortex laceration $<2 \mathrm{~cm}(4 \%)$ and total parenchymal destruction (1\%). Overall median Injury Severity Score (ISS) was 20.5 (IQR 16). Ninety percent of cases were treated conservatively and $10 \%$ has surgical interventions. Overall mortality was $14.7 \%$ (non-operative $12 \%$ vs $45.5 \%$ in surgical treated group, $\mathrm{p}=0.003$ ). Multivariate regression analysis after adjustment for age, ISS, trauma room systolic blood pressure (SBP) and Glasgow Coma Scale (GCS) and surgical intervention for adrenal injury showed that the predictors of mortality were SBP, GCS and ISS.

Conclusion: Traumatic adrenal injury is not rare in traffic related abdominal trauma. Polytrauma and patient stability play a prognostic role in these cases.

References: 2014 Jun 10;94 (22):1733-5

Disclosure: No significant relationships. 
PR179

\section{PROGNOSTIC FACTORS IN PATIENTS OPERATIVELY MANAGED FOR ADULT FEMORAL SHAFT FRACTURES - A RETROSPECTIVE STUDY}

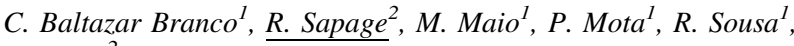 M. Coxo $^{2}$}

${ }^{1}$ Orthopedics And Traumatology, Centro Hospitalar Trás os Montes e Alto Douro, Vila Real/PORTUGAL, ${ }^{2}$ Serviço De Ortopedia E Traumatologia, Centro Hospitalar de Trás-os-Montes e Alto Douro, Vila Real/PORTUGAL

Introduction: Femoral shaft fractures (FSF) are severe injuries. Overall morbidity and mortality of FSF and its optimal treatment continues to be discussed. We aimed to identify predictors of outcome in patients operatively managed for FSF.

Material and methods: A retrospective study was conducted for patients admitted for FSF between July/2013 and December/2016. Patients over 18-years-old and in whom operative stabilization was performed were included. Mean follow-up time was 10months. Predictors for morbidity and mortality were evaluated.

Results: A total of 53patients were included, with a median age of $52.0 y e a r s$ and female predominance $(60.4 \%)$. The most frequent mechanism of injury was ground-level falls (45.3\%). Atypical fractures associated with bisphosphonates use were identified in 19patients. AO type 32-A was the most common $(75.5 \%)$. The most frequent associated bone injuries (BI) were pelvic, tibial shaft and rib fractures $(7.5 \%)$. Lung was the most affected internal organ injury (IOI) $(7.5 \%)$. Only 3patients were submitted to external fixation and 2 to plate fixation as initial treatment, all others were treated with intramedullary nailing. Postoperative complications (as in nonunion, infection and implant complications) were identified in $24.5 \%$ of the patients. They were related to the presence of associated BI and IOI, New Injury Severity Score, time to surgery and operative time. Three patients died within 30 days after surgery. The determinants of mortality were American Society of Anesthesiologists classification and presence of associated $\mathrm{BI}$ and IOI.

Conclusion: In our study, prognosis was mainly related to patient medical condition and additional injuries. Recognition of clinical variables associated with an adverse outcome can aid in optimal approach to FSF.

References: 1. Neumann MV, Sudkamp NP, Strohm PC. Management of femoral shaft fractures. Acta chirurgiae orthopaedicae et traumatologiae Cechoslovaca. 2015;82 (1):22-32. Epub 2015/03/10. 2. Byrne JP, Nathens AB, Gomez D, Pincus D, Jenkinson RJ. Timing of femoral shaft fracture fixation following major trauma: A retrospective cohort study of United States trauma centers. PLoS medicine. 2017;14 (7):e1002336. Epub 2017/07/06. 3. Anandasivam NS, Russo GS, Fischer JM, Samuel AM, Ondeck NT, Swallow MS, et al. Analysis of Bony and Internal Organ Injuries Associated With 26,357 Adult Femoral Shaft Fractures and Their Impact on Mortality. Orthopedics. 2017;40 (3):e506-e12. Epub 2017/03/31. 4. Pape HC, Hildebrand F, Pertschy S, Zelle B, Garapati R, Grimme K, et al. Changes in the management of femoral shaft fractures in polytrauma patients: from early total care to damage control orthopedic surgery. The Journal of trauma. 2002;53 (3):452-61; discussion 61-2. Epub 2002/09/28. 5. Eliezer EN, Haonga BT, Morshed S, Shearer DW. Predictors of Reoperation for Adult Femoral Shaft Fractures Managed Operatively in a Sub-Saharan Country. The Journal of bone and joint surgery American volume. 2017;99 (5):388-95. Epub 2017/03/01.

Disclosure: No significant relationships.

\section{PR180}

\section{THROMBOEMBOLIC EVENTS IN TRAUMA PATIENTS}

\author{
H. Al-Thani ${ }^{1}$, M. Asim ${ }^{2}$, A. El-Menyar ${ }^{3}$
}

${ }^{1}$ Trauma Surgery Section, Department Of Surgery, Hamad Trauma Center, Doha/QATAR, ${ }^{2}$ Trauma Surgery Section, Department Of Surgery, Hamad General Hospital, Doha/QATAR, ${ }^{3}$ Trauma Surgery, Hamad General Hospital, Doha/QATAR

Introduction: We aimed to assess the clinical presentation, risk factors and outcomes of thromboembolism among trauma patients over 5 years

Material and methods: We conducted a retrospective analysis of all trauma patients with documented thromboembolic events post-injury in a level 1 trauma center. Patients were categorized into 2 groups based on their main initial presentation post-trauma (deep vein thrombosis [DVT] or pulmonary embolism [PE]).

Results: Across the study period there were 662 patients diagnosed to have thromboembolism, of them 82 patients were identified from the trauma database. Out of these 82 trauma patients, 56 had DVT and 28 presented with PE. Two patients initially presented with DVT developed PE on the follow-up. There were 38 females and 46 males with a mean age of $46 \pm 18$ years. Abnormal coagulation profile was reported as 7 protein $\mathrm{C}$ deficiency, 5 protein $\mathrm{S}$ deficiency, 6 homocystinemia, 4 Antithrombin III deficiency, 4 lupus anticoagulant and 2 had Factor V leiden. Age, sex, obesity, and D-Dimer level were comparable between the 2 groups, whereas protein $\mathrm{S}$ deficiency, prior history of PE, history of recent surgery and history of recent travelling were more evident in the PE group. Overall mortality was $8.3 \%$ (DVT;8.9\% vs PE;7.1\%, respectively $=0.78$ ).

Conclusion: Coagulation profile plays an important role in the posttrauma thromboembolism. Thorough assessment of thromboembolic events is warranted to avoid missing cases in trauma patients.

References: Al-Thani H, El-Menyar A, Asim M, Kiliyanni AS.Angiology. 2016 Jul;67 (6):587-95. doi: 10.1177/0003319715604265. Epub 2015 Sep 6

Disclosure: No significant relationships.

\section{PR181}

\section{HONDURAS INJURY PROFILE: UNDERSTANDING INJURIES IN ONE OF THE MOST VIOLENT COUNTRIES IN THE WORLD}

A.V. Markovtsova $^{l}$, C. Rodriguez ${ }^{2}$, F. Bonilla-Escobar ${ }^{3}$, C. Restrepo-Lopera ${ }^{4}$, E. Medina ${ }^{5}$, J.C. Puyana ${ }^{4}$

${ }^{1}$ Trauma And Surgery, University of Pittsburgh School of Medicine, Pittsburgh/PA/UNITED STATES OF AMERICA, ${ }^{2}$ Direccion General, Hospital Escuela Universitario (HEU), Tegucigalpa/ HONDURAS, ${ }^{3}$ Department Of Ophthalmology, UPMC Mercy Hospital, Pittsburgh/PA/UNITED STATES OF AMERICA, ${ }^{4}$ Department Of Surgery, UPMC, Pittsburgh/PA/UNITED STATES OF AMERICA, ${ }^{5}$ School Of Medicine, Universidad Nacional Autonoma de Honduras, Tegucigalpa/HONDURAS

Introduction: Honduras is one of the most violent countries in the world, but epidemiological data describing injuries in Honduras is scarce. Our study is the largest injury profile on Honduras and 
provides valuable information not captured by crime statistics that must be used to generate effective injury prevention strategies.

Material and methods: A cross-sectional study was conducted using a paper-based injury surveillance system (InSS) from Honduras' largest referral medical center, the University Medical School Hospital $^{1}$. Descriptive statistics and bivariate analyses were carried out using data from 2013.

Results: Of the 17,971 injuries reported in the InSS, intentional injuries made up $18.15 \%$ (39.27 per 100,000 inhabitants; 95\% CI: 37.93-40.64) and included injuries from interpersonal violence $(14.68 \%)$ and self-infliction $(3.46 \%)$. Sexual attacks were reported in $0.27 \%$ of cases $(n=48)$. Unintentional injuries made up $81.79 \%$ of all injuries (188.63 per 100,000 inhabitants, $95 \%$ CI: $185.59-191.70)$ and were largely due to falls (37.9\%) and road traffic collisions (RTC) $(9.61 \%)$. Overall injury mortality was $0.65 \%(\mathrm{n}=117)$.

Conclusion: Low- and middle-income countries (LMIC) like Honduras disproportionately experience injuries compared to high-income countries $^{2}$. In Honduras' main trauma hospital, the injury mortality rate in 2013 was higher than that reported globally $(0.65 \%$ vs. $0.49 \%)^{3}$. Unintentional injuries were more prevalent than intentional ones, but violence-related injuries were still common, reflective of the country's high homicide rates. Sexual attacks were rare, most likely due to global underreporting by victims of sexual abuse ${ }^{4}$. Our study provides essential epidemiological data that can be used to create targeted policies in Honduras and other LMICs with high rates of violence.

References: 1. Bonilla-Escobar FJ, Rodriguez C, Puyana JC. Trauma Care and Surveillance: International "eCapacity" Efforts and Honduras Experience. World J Surg. 2017 Sep;41 (9):2415-2416. 2. Chandran, A., A.A. Hyder, and C. Peek-Asa, The global burden of unintentional injuries and an agenda for progress.Epidemiol Rev, 2010. 32: p. 110-20. 3. Haagsma, J.A., et al., The global burden of injury: incidence, mortality, disability-adjusted life years and time trends from the Global Burden of Disease study 2013. Injury Prevention, 2016. 22 (1): p. 3-18. 4. Garcia-Moreno, C., et al., Prevalence of intimate partner violence: findings from the WHO multi-country study on women's health and domestic violence. Lancet, 2006. 368 (9543): p. 1260-9.

Disclosure: No significant relationships.

\section{PR182}

\section{THE MORTAL FALL; INCIDENCE AND MORTALITY FROM MECHANISMS OF INJURY AT A UK MAJOR TRAUMA CENTRE 2012-16}

\section{B. Allen, R. Lunevicius, K. Shahzad, J.V. Taylor, R.G. Ward, N. Misra}

General Surgery Department, Emergency General Surgery And Major Trauma Centre, Merseyside, Aintree University Hospital NHS Foundation Trust, Liverpool/UNITED KINGDOM

Introduction: The reports on incidence and mortality from falls have rarely been the resources for initiation and development of the fall prevention programmes. This paper provides an overview of admissions and mortality from falls at designated adult Aintree Major Trauma Centre for Merseyside and Cheshire regions between 2012 and 2016.

Material and methods: Data from a National Trauma Audit and Research Register for patients admitted with a major trauma between 2012 and 2016 were reviewed.

Results: 4,136 patients were admitted to Aintree Major Trauma Centre in 2012-2016: 305 in 2012, 779 in 2013, 931 in 2014, 952 in
2015, and 1,169 in 2016. $3 / 4$ of major trauma patients sustained injuries due to falls and RTAs. Fall was the commonest cause of admissions: 218 patients (54.0\%) in 2012, and 413 patients $(59.3 \%)$ in 2016. The biggest increase was seen in falls $>2$ meters: from 58 to 161 (factor of 2.8). Trauma admissions from falls $<2$ meters increased by a factor of 1.6 , from 160 in 2012 to 252 in 2016. Casefatality rate improved from $10 \%$ to $9.13 \%$ in falls $<2$ metres. In contrast, case-fatality rate showed a marked increase from $6.9 \%$ to $8.7 \%$ in falls greater than 2 meters. Falls accounted for $71.4 \%$ of all deaths in 2012, and $78.7 \%$ in 2016

Conclusion: Falls continue to be a leading cause of major trauma and mortality. The findings of this study are a valuable resource to prioritise the fall prevention programmes in North West England.

References:

Disclosure: No significant relationships.

\section{PR183}

\section{THE VOLUME-OUTCOME RELATIONSHIP AMONG SEVERELY INJURED PATIENTS IN ENGLAND AND WALES: DOES IT EXPLAIN BETWEEN-CENTRE DIFFERENCES?}

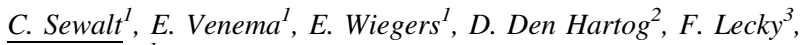 H. Lingsma ${ }^{l}$}

${ }^{1}$ Public Health, Erasmus MC, Rotterdam/NETHERLANDS, ${ }^{2}$ Surgery, Erasmus MC, Rotterdam/NETHERLANDS, ${ }^{3}$ School Of Health And Related Research, University of Sheffield, Sheffield/ UNITED KINGDOM

Introduction: The volume-outcome relationship in severely injured patients remains unclear and under debate. The aim of this study was to determine whether between-centre differences could be explained by the relationship between volume of severely injured patients in Major Trauma Centres (MTCs) and different patient outcomes.

Material and methods: A retrospective cohort study including severely injured adults (ISS > 15) admitted to a MTC between 2013 and 2016 was performed using the Trauma Audit and Research Network (TARN). The outcome measures were in-hospital mortality, LOS, critical care LOS, time to operation and time to CT scan. Hospital volume was defined as the mean number of severely injured patients treated in one MTC per year. Between centre differences were assessed with random effects logistic regression models and expressed with a $95 \%$ range of ORs compared to the average, based on the variance of random effects. Analysing hospital volume was done using mixed effects logistic and linear regression models with hospital volume as a continuous variable.

Results: A total of 47,157 severely injured patients from 28 MTCs were included in this study. Hospital volume varied from 69 to 781 severely injured patients per year. There were small between-centre differences in mortality (adjusted 95\% centre range: 0.91-1.06). Overall, after adjusting for important demographic and injury severity characteristics, higher hospital volume was not associated with lower mortality (OR $1.02,95 \%$ CI $0.68-1.54, \mathrm{p}=0.923$ ). No association was found between hospital volume and outher patient outcomes.

Conclusion: This study suggests that small between-centre differences could not be explained by the volume-outcome relationship in severely injured patients.

References:

Disclosure: No significant relationships. 


\section{PR184}

\section{ANALYSIS OF THE POLYTRAUMATIZED PATIENTS WITH SEVERE ABDOMINAL TRAUMA AT "12 DE OCTUBRE" HOSPITAL, MADRID}

\author{
M. Gutierrez Andreu, J. Bernal Tirapo, A. Moreno Bargueiras, \\ G. Supelano Eslait, C. Alegre Torrado, F. Cruz Vigo, E. Ferrero, \\ P. Yuste García
}

General And Digestive Surgery, 12 DE OCTUBRE UNIVERSITY HOSPITAL, MADRID/SPAIN

Introduction: Our objective is to describe the epidemiological characteristics, injury mechanism, techniques performed, surgical interventions, average stay and mortality of patients with severe trauma in our environment, through an injury database.

Material and methods: Retrospective, descriptive, observational study performed over 24 months. (Sep 2015-sep 2017). 180 patients with abdominal trauma were attended, $83(46,1 \%)$ with ISS $>9$.

Results: 83 polytraumatized patients with abdominal trauma and ISS $>9(46,1 \%)$ were attended, males $85.5 \%$, with a mean age of 42 years. The most common causes of trauma were traffic accidents (47\%), followed by stabs injuries (24.1\%) and high-energy falls $(21.7 \%)$. At prehospital care $55.4 \%$ of the patients were hemodynamically stable. The mass transfusion protocol was activated in $25.3 \%$ of the cases. ECO-FAST was performed in $27.7 \%$ of patients and CT in $78,5 \%$. About injuries: Thoracic trauma $62,5 \%$, cranioencephalic trauma $34,2 \%$, pelvic trauma $25,6 \%$, spleen injury $27.5 \%$, liver injury $39.5 \%$. $58 \%$ of the patients had other abdominal lesions. Surgical intervention was performed in 56 patients $(69.1 \%)$, with the initial most frequent approach being laparotomy $(52.7 \%)$, followed by invasive external pelvic fixation (25.5\%). Damage control surgery was performed in 19 cases (34.5\%), 6 hepatic packing, 4 extraperitoneal packing. 12 arteriography-embolization. The mean stay in the ICU was 11.4 days and in the ward 13.7 days. The mortality rate was $8.4 \%$, (7 patients).

Conclusion: s: Trauma registries are a useful tool for surgeons as they provide better understanding of injury epidemiology and contribute to improve the care of these patients.

References:

Disclosure: No significant relationships.

\section{PR185}

\section{RETROSPECTIVE EVALUATION OF PELVIC FRACTURES CONTROLLED WITH TAE AT EMERGENCY ROOM}

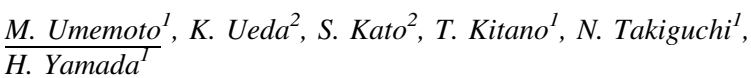

${ }^{1}$ Emergency And Critical Care Medicine, Wakayama medical university, Wakayama/JAPAN, ${ }^{2}$ Emergency And Critical Care Medicine, Wakayama Medical University, Wakayama/JAPAN

Introduction: The current management of pelvic fractures with hemodynamically unstable consists of aggressive resuscitation, angioembolization and mechanical stabilisation (external fixation). We use TAE (transcatheter arterial embolization) for exsanguination at the emergency room and external fixation is followed if needed. Material and methods: Between November 2006 and November 2016, a retrospective study was performed. 91 PRAF (pelvic ring and acetabular fractures) patients including 20 unstable pelvic bleeding were controlled with TAE at ER.

TAE had been performed in 40 patients (43.95\%). 20 out of 91 patients had hemodynamic instability and pelvic fractures who underwent TAE (10 patients) and external fixation (20 patients) at the Emergency Room.

Results: The TAE group (40 patients) and the nonTAE group (51 patients) were compared, there was no significant difference in the age, gender, ISS score $(\mathrm{p}=0.16)$ Of all the patients, the number of gender is the same $(50 \%)$ with a mean age of $61.29 \pm 22.56$ years And a mean ISS of $38.2 \pm 12$. The mean systolic blood pressure at the ER was $80.85 \pm 43.12 \mathrm{~mm} \mathrm{Hg}$. Heart rate was $95.7 \pm 25.25 \mathrm{bpm} .40$ patients who underwent TAE had a mean ISS of $40.4 \pm 13.7$, and the age $62.85 \pm 22.77$.

The mean sBP at ER was $93.43 \pm 37.34$.

Whereas 51 patients who did not need TAE had a mean ISS of $36.4 \pm 13.2$, the age $60.06 \pm 22.54$ and sBP at ER was $107.78 \pm$ 32.76 .

Conclusion: TAE is a rapid and effective method for controlling hemodynamically unstable patients with pelvic fractures. TAE is performed at the emergency room and then external fixation is done. References:

Disclosure: No significant relationships.

\section{PR186}

\section{A RETROSPECTIVE \& COMPARATIVE ANALYSIS OF PELVIC AND ACETABULAR FRACTURES IN A GERIATRIC POPULATION USING THE DGU TRAUMA REGISTER}

\section{Follet, G. Putzeys}

Orthopaedics, AZ Groeninge, Kortrijk/BELGIUM

Introduction: An increase of fragility fractures is pending. Though hip fractures are declining, pelvic fractures are growing in incidence. $7 \%$ of osteoporotic fractures is a pelvic fracture. Incidence pelvic injuries USA: increased from $10.6 \%$ (1993) to $15.2 \%$ (2010). Incidence pelvic fractures: $20-37$ per $100000 \mathrm{PPY}$, increased to 448 in $85+$. We performed a retrospective analysis on pelvic registry (DGU). Our emphasis is with the epidemiology, pathomechanisme, mean age, mortality, type of fracture, ... of pelvic fractures in $65+$. Material and methods: Cases from 2009-2014 registered in the Pelvic registry, including Belgian add on questions. All hospitalized cases with informed consent. Data processing: Memdoc and Excel. Comparison by propensity score based 1:1 matched case control.

Results: $65+$ pelvic injuries (623). Mean age: men (81.4) women (82.2). Sacral (384) and transpubic (363) fractures are most frequent. Increasing incidence: (2009: 93; 2010: 98; 2011: 109; 2012: 128; 2013: 114; 2014 uncomplete). 55\% originated from standing height. Sacroiliac and acetabular in men (high energy), sacral and transpubic in women (low energy). Mortality: men $(9.03 \%)$ women $(5.42 \%)$. Therapy: primary treatment (19) specialized treatment (65). Acetabular fractures most specialized treatment $(31.65 \%)$. Specialized treatment: 2009: 7; 2014: 15. Mortality: German pool (1.98\%) AZ Groeninge $(5.92 \%)(\mathrm{p}<0.0001)$.

Conclusion: In $65+$ fractures originate from low energetic trauma. Frequently female sacral and transpubic fractures. Higher mortality in men, but more high energetic trauma and fractures. Increasing incidence in pelvic fractures. Acetabulum most operated fracture. Increasing need for specialized treatment. Higher mortality AZ Groeninge vs. German hospitals, without explaining variable, possible due to social status, excellence... 
References: 1. Bouxsein ML, Kaufman J, Tosi L, Cummings S, Lane $\mathrm{J}$, Johnell O. Recommendations for optimal care of the fragility fracture patient to reduce the risk of future fracture. J Am Acad Orthop Surg. 2004;12 (6):385-95. 2. Friedman SM, Mendelson DA. Epidemiology of fragility fractures. Clin Geriatr Med. 2014;30 (2):175-81. 3. O'Connor TJ, Cole PA. Pelvic Insufficiency Fractures. Geriatr Orthop Surg Rehabil. 2014;5 (4):178-90. 4. Krappinger D, Kammerlander C, Hak DJ, Blauth M. Low-energy osteoporotic pelvic fractures. Arch Orthop Trauma Surg. 2010;130 (9):1167-75. 5. Government BF. Bevolking - Demografische indicatoren 2015-2060. In: FOD Economie KMO, Middenstand en Energie, editor. Statbel; 2015. 6. Breuil V, Roux CH, Carle GF. Pelvic fractures: epidemiology, consequences, and medical management. Curr Opin Rheumatol. 2016;28 (4):442-7. 7. Sullivan MP, Baldwin KD, Donegan DJ, Mehta S, Ahn J. Geriatric fractures about the hip: divergent patterns in the proximal femur, acetabulum, and pelvis. Orthopedics. 2014;37 (3):151-7. 8. Benzinger P, Becker C, Kerse N, Bleibler F, Buchele G, Icks A, et al. Pelvic fracture rates in community-living people with and without disability and in residents of nursing homes. J Am Med Dir Assoc. 2013;14 (9):673-8. 9. Humphrey CA, Maceroli MA. Fragility fractures requiring special consideration: pelvic insufficiency fractures. Clin Geriatr Med. 2014;30 (2):373-86. 10. Studer P, Suhm N, Zappe B, Bless N, Jakob M. Pubic rami fractures in the elderly-a neglected injury? Swiss Med Wkly. 2013;143:w13859. 11. Buller LT, Best MJ, Quinnan SM. A Nationwide Analysis of Pelvic Ring Fractures: Incidence and Trends in Treatment, Length of Stay, and Mortality. Geriatr Orthop Surg Rehabil. 2016;7 (1):9-17. 12. Munting E, Röder C, Sobottke R, Dietrich D, Aghayev E, Contributors ST. Patient outcomes after laminotomy, hemilaminectomy, laminectomy and laminectomy with instrumented fusion for spinal canal stenosis: a propensity score-based study from the Spine Tango registry. Eur Spine J. 2015;24 (2):358-68. 13. Rommens PM, Hofmann A. Comprehensive classification of fragility fractures of the pelvic ring: Recommendations for surgical treatment. Injury. 2013;44 (12):1733-44. 14. Dodd AC, Bulka C, Jahangir A, Mir HR, Obremskey WT, Sethi MK. Predictors of 30-day mortality following hip/pelvis fractures. Orthop Traumatol Surg Res. 2016;102 (6):70710. 15. Wang H, Phillips JL, Robinson RD, Duane TM, Buca S, Campbell-Furtick MB, et al. Predictors of mortality among initially stable adult pelvic trauma patients in the US: Data analysis from the National Trauma Data Bank. Injury. 2015;46 (11):2113-7.

Disclosure: No significant relationships.

\section{PR187}

\section{AN EVALUATION OF TRAUMATIC BRAIN INJURY IN HONDURAS: THE USE OF A PAPER-BASED SURVEILLANCE SYSTEM TO CHARACTERIZE INJURY PATTERNS}

$\underline{\text { E.D. Johnson }}^{\text {I }}$, F.J. Bonilla-Escobar ${ }^{2}$, C. Rodriguez ${ }^{3}$, J.C. Puyana ${ }^{4}$

${ }^{1}$ School Of Medicine, University of Pittsburgh, Pittsburgh/PA/ UNITED STATES OF AMERICA, ${ }^{2}$ Department Of Ophthalmology, UPMC Mercy Hospital, Pittsburgh/PA/UNITED STATES OF AMERICA, ${ }^{3}$ Direccion General, Hospital Escuela Universitario (HEU), Tegucigalpa/HONDURAS, ${ }^{4}$ Department Of Surgery, University of Pittsburgh Medical Center, Pittsburgh/PA/UNITED STATES OF AMERICA

Introduction: Traumatic brain injuries (TBI) are a leading cause of death and disability worldwide. ${ }^{1-6}$ Violent, intentional trauma is the leading cause of mortality in Honduras. However, the incidence and impact of TBI in this LMIC is unknown. ${ }^{5-7}$ The aim of this study is to describe the epidemiology of TBI in Honduras, as captured by an injury surveillance tool in the country's major referral center.

Material and methods: We conducted a cross-sectional review of all TBI-related emergency department visits at the main referral hospital in Honduras from January to December, 2013. We calculated descriptive statistics from Injury Surveillance System (InSS) data.

Results: Of 17,791 injuries seen in $2013,20 \%$ were traumatic brain injuries $(3,588)$. Most TBI were unintentional $(83.39 \%)$; the main mechanisms of injury were falls $(41.11 \%)$, road traffic $(23.91 \%)$, blunt trauma $(20.82 \%)$, knife $(5.85 \%)$, and firearm injuries $(2.26 \%)$. Most TBI were mild ( $99.69 \%$ of patients presented with a Glasgow Coma Scale of 15). Emergency room mortality was low (1.11\%). The modified Kampala Trauma Score mean was $7.65 \pm 0.52$, with a median of 8 and interquartile rank of 7-8.

Conclusion: Mild TBI accounts for a significant percentage of all injuries. Despite the high rates of violence in this country, most TBI were accidental, due to road traffic and falls. These data may be useful in directing the reallocation of healthcare resources: Public health strategies targeting motor vehicle accidents and falls could decrease the occurrence of TBI, while improving triage and diverting mild TBI to other facilities could allow for more efficient use of limited resources in managing trauma.

References: World Health Organization. Neurological Disorders: Public Health Challenges. WHO Press (2006). Injury Prevention and control: traumatic brain injury. Centers for Disease Control and Prevention web page. https://www.cdc.gov/traumaticbraininjury/index.html. Accessed September 30, 2017. Peden M, McGee K, Sharma G. The injury chart book: a graphical overview of the global burden of injuries. Geneva, World Health Organization, 2002. http://apps.who.int/iris/bitstream/10665/42566/1/924156220X.pdf Carroll, C. P., et al. (2012). "Are we underestimating the burden of traumatic brain injury? Surveillance of severe traumatic brain injury using centers for disease control International classification of disease, ninth revision, clinical modification, traumatic brain injury codes." Neurosurgery 71 (6): 1064-1070; discussion 1070. Hyder A.A., Wunderlich C.A., Puvanachandra P., Gururaj G., and Kobusingye O.C.: The impact of traumatic brain injuries: a global perspective. NeuroRehabilitation 2007; 22: pp. 341-353 Rubiano AM, Puyana JC, Mock CN, Bullock MR, Adelson PD. Strengthening neurotrauma care systems in low and middle income countries. Brain Inj 2013;27:262-72. Bryan-Hancock C, Harrison J. The global burden of traumatic brain injury: preliminary results from the Global Burden of Disease Project. Injury Prevention 2010;16:A17. Brain Trauma Foundation. Traumatic Brain Injury Statistics. Brain Trauma Foundation; https://www.braintrauma.org/uploads/05/02/Brain_Trauma_Foundation.pdf Centers for Disease Control. Traumatic Brain Injury Fact Sheet. Accessed October 18, 2017. https://www.cdc.gov/traumaticbraininjury/pdf/ bluebook_factsheet-a.pdf Peeters W, den Brande R, Polinder S, Brazinova A, Steyerberg EW, Lingsma HF, Maas AIR. Epidemiology of traumatic brain injury in Europe; Acta Neurochir (2015) 157:1683-1696.

Disclosure: No significant relationships. 
PR188

\section{INCIDENCE AND PREDICTORS OF LATE COMPLICATIONS IN TRAUMA PATIENTS IN THE SICUS (THAI-SICU)}

\section{O. Akaraborworn, B. Sangthong, K. Thongkhao, P. Chainiramol, K. Kaewsaengrueang}

Department Of Surgery, Faculty Of Medicine, Prince Of Songkla University, Prince of Songkla University, Hat Yai Songkhla/ THAILAND

Introduction: Late complications such as organ failure and sepsis are the most common causes of late death in trauma patients. This study aimed to investigate the incidence of late complications and predictors of complications in trauma patients admitted in the SICUs.

Material and methods: This study was an analysis of data from the THAI-SICU study which was a prospective cohort study that was conducted in nine university-based SICUs in Thailand. Only trauma patients were included in this study. Late complications were identified by major complications: acute kidney injury (AKI), acute lung injury (ALI) and acute respiratory disease syndrome (ARDS) and sepsis. The complications had to occur after 48 hours from SICU admission. Onset of complications and independent factors of late complications were identified. Patients who had late complications and patients who did not have late complications were compared.

Results: Among 4,652 patients in the cohort, 327 patients met the inclusion criteria. In 36 patients (11\%), major late complications were classified as AKI in 11 patients (3.4\%), ALI and ARDS in 7 patients $(2.2 \%)$ and sepsis in 24 patients $(7.3 \%)$. The admission SOFA score was significantly higher in patients who had late complications (5.9 vs $4.2, \mathrm{p}=0.01$ ). After adjusting for other potential factors in a logistic regression analysis, SOFA score at admission was a significant predictor of late complications in trauma patients (OR, 1.02; 95\% CI, 1.01-1.15).

Conclusion: The admission SOFA score was a significant predictor of late complications in trauma patients. Patients who have a high SOFA score on admission should be vigilantly monitored.

References: Probst C, Zelle BA, Sittaro NA. Late death after multiple severe trauma: what doese it occur and what are the causes? J Trauma. 2009;66 (4):1212-7.

Disclosure: No significant relationships.

\section{PR189}

\section{TRAUMA AND CRITICAL CARE: EPIDEMIOLOGY OF TRAUMA-RELATED CRITICAL ILLNESS IN A RURAL KENYAN HOSPITAL}

\section{S.M. Gichuki, E. Irungu, E. Langat, J. Macleod, S.W. Muthee}

\section{Surgery, Kijabe Hospital, Kijabe/KENYA}

Introduction: Kenya is among the developing countries where road traffic accidents (RTA) are common. But with insufficient care offered for victims of RTAs the outcome is often disproportionately poor. Further, trauma patients in low resource settings who need critical care are especially neglected with an inadequately determined burden of injury-related morbidity and mortality. Therefore, this study aims to assess the epidemiology of trauma patients presenting to
AIC Kijabe Hospital who required intensive care unit (ICU) admission and treatment.

Material and methods: AIC Kijabe Hospital is situated near one of the main Kenyan highways with several areas along the highway listed as especially dangerous where accidents are likely to occur. This contributes to a large trauma burden of seriously injured patients presenting to the Hospital. Therefore, we studied a retrospective cohort of trauma patients who presented to the AIC Kijabe Hospital and who required ICU Care between January 2014 to December 2016. We determined the pattern of injuries, complications and their 28-day outcomes.

Results: In the 3 year study period there was a total of 1,204 severely injured patients who presented to AIC Kijabe Hospital. Of these, 104 patients, $8.6 \%$ of the total cohort, required admission to the ICU. Of this ICU cohort of injured patients there were 23 deaths $(18 \%)$ recorded.

Conclusion: Trauma patients form a significant proportion of the patients admitted to the ICU. The nature of injury and delay in presentation contribute to the high mortality among this group of patients.

References:

Disclosure: No significant relationships

\section{PR190}

\section{EARLY HOSPITAL READMISSION AFTER ACUTE TRAUMATIC INJURI: EXPERIENCE AT A LEVEL - I TRAUMA CENTER}

\author{
A.M. Vargas Muñoz ${ }^{1}$, N. Alegret Monroig ${ }^{2}$, J. Martínez Cabañero $^{1}$
}

${ }^{1}$ Anesthesiology, Hospital Parc Tauli, Sabadell/SPAIN,

${ }^{2}$ Anesthesiology, Coorporació Sanitaria Parc Tauli, sabadell/SPAIN

Introduction: Imcidence of readmisiones in polytrauma patients (PTP) is between 6.6 and $7.6 \%$ and it's associated to an increase in morbidity. The PTP who present a higher readmission rate are those initially catalogued as non-life-threatening traumas with a low injury assessment and Injury Severity Score (ISS). They receive an early discharge from the hospital. These readmissions have relevant implications for health care costs. Awareness of the predisposing factors will allow setting criteria that would diminish the hospital readmission rate and therefore the morbimortality rate especially in patients with mild to moderate polytrauma.

Material and methods: This observational retrospective study included all PTP between July 2012 and July 2016 who did not require admission to critical care unit. Demographic variables, trauma characteristics, ISS, initial hospital stay (HS), date and reason of the readmission and total HS were analyzed.

Results: 923 patients were included $69.45 \%$ of which male with an average age of 41.02 years. $97.18 \%$ presented non-intentional origin trauma a $69.9 \%$ being secondary to traffic accidents. Medium ISS was 4.67points (DE 1-18 points) initial HS 2.76 days $26.3 \%$ were readmitted, 58\% during the first week after the trauma. Reasons of readmission were: pain $54.73 \%$, infection $3.7 \%$, others $37.45 \%$. The HS readmission was 1.4 days $10.3 \%$ needed surgical intervention, $4.53 \%$ antibiotic therapy and $56.4 \%$ analgesia.

Conclusion: The readmission rate in our center is higher than the one mentioned in the reviewed literature. Main reason is the lack of analgesic control at patients' home. We conclude, therefore, that we should optimize analgesic conditions when patients are discharged from hospital in order to reduce health care costs. 
References: - Porgo TV, Moore L, Tardif P-A. Evidence of data quality in trauma registries: A systematic review. J Trauma Acute Surg. 2016;80 (4):648-58. - Olubode A Olufajo, M.P.H., *, Zara Cooper, M.Sc The truth about trauma readmissions, The American Journal of Surgery (2016) 211, 649-655

Disclosure: No significant relationships.

\section{PR191}

\section{TRENDS AND PATTERNS OF WORK-RELATED ROAD TRAFFIC INJURIES IN HEAVY VEHICLES IN QATAR}

\author{
R. Consunji ${ }^{1}$, A. Abeid ${ }^{1}$, N. Hirani ${ }^{1}$, A. Mehmood $^{2}$, M. Mollazehi $^{l}$, \\ A. El-Menyar ${ }^{1}$, H. Al-Thani ${ }^{1}$, A. Hyder ${ }^{2}$, R. Peralta ${ }^{1}$
}

${ }^{1}$ Trauma Surgery Section, Department Of Surgery, Hamad Trauma Center, Doha/QATAR, ${ }^{2}$ Johns Hopkins Bloomberg School Of Public Health, Johns Hopkins University, Baltimore/MD/UNITED STATES OF AMERICA

Introduction: Injuries are the leading cause of death in Qatar, primarily those that occur at work or on the road, however, there is a paucity of data on work-related road traffic injuries [WRTIs] in Qatar. This study will describe the epidemiology of heavy-vehicle related WRTIs [HV-WRTIs] in Qatar and make recommendations for future prevention programs. It was conducted as part of a larger grant [NPRP 7-1120-3-288] funded by the Qatar Foundation designed to initiate and implement a targeted unified workplace injury registry to inform policies and programs to reduce the health burden, in terms of deaths and disabilities, and the healthcare costs from WRI's in Qatar.

Material and methods: Data, on patients with HV-WRTIs treated at the Hamad Trauma Center from 1 January 2015 to 31 December 2016, from the Trauma Registry was collected, analyzed according to road user type and other epidemiologic characteristics.

Results: There were 108 HV-WRTIs identified, $17 \%$ of all workrelated RTIs. The in-hospital mortality rate was $8.3 \%$. Majority [57\%] of the victims were involved in a transport occupation and $18 \%$ were laborers. Most [76\%] were drivers or passengers and 19\% were pedestrians. For drivers, $84 \%$ were in trucks, $32 \%$ in head-on-collisions, $22 \%$ hit fixed objects and $8 \%$ were restrained. Pedestrians had the highest mortality, $19 \%, 33 \%$ were hit by forklifts and $48 \%$ needed ICU admission.

Conclusion: HV-WRTIs are a major cause of severe WRTI and mortality. Priority must be focused on improving restraint use by drivers, worksite pedestrian environments and HV visibility, and maximum operating hours for HV drivers.

References: Heavy Vehicle Drivers Involved In Road Crashes In South Australia. Department of Planning, Transport and Infrastructure (DPTI). http://www.dpti.sa.gov.au/. Published August 2016. Smith S, Harris P. Truck Driver Job-Related Injuries in Overdrive. Truck Driver Job-Related Injuries in OverdrivelU.S. Department of Labor Blog. https://blog.dol.gov/2016/08/17/truck-driver-job-related-injuries-in-overdrive/. Published August 17, 16ADAD.

Disclosure: No significant relationships.
PR192

\section{TEMPORAL AND EPIDEMIOLOGIC TRENDS IN INJURY DEATHS: AN INITIAL REPORT FROM THE QATAR FATAL INJURY REPORTING STUDY GROUP}

\author{
$\underline{R . C O n s u n j i}^{l}$, S.M. Mouemel ${ }^{1}$, A. Ammar ${ }^{2}$, A. El-Menyar ${ }^{1}$, \\ H. Al-Thani ${ }^{I}$, R. Peralta ${ }^{l}$
}

${ }^{1}$ Trauma Surgery Section, Department Of Surgery, Hamad Trauma Center, Doha/QATAR, ${ }^{2}$ Clinical Pathology, Hamad Medical Corporation, Doha/QATAR

Introduction: Underreporting, misreporting and a lack of welltrained registers are some of the challenges faced by fatal injury reporting systems [FIRS] and analyses of the barriers and challenges for their quality are scarce. This study will describe the injury deaths reported in Qatar national vital statistics annual reports [NVSAR] and compare them with those from the FIRS of the Hamad Medical Corporation [HMC], Doha, Qatar.

Material and methods: This retrospective study extracted all injury deaths recorded in the HMC FIRS and compared them with those in NVSAR. Characteristics analyzed were: age, sex, mechanism of injury and the proportionate contribution to all injury deaths.

Results: The retrospective review extracted 20,870 deaths, from all causes, from the FIRS, from 1 January 2007 to 31 December 2015. During the study period there was a consistent pattern, with an annual average of 279 more deaths from all causes, in FIRS. The reverse was true for injury deaths. In FIRS, 3,391 (16.2\%) were due to injuries; this differed significantly from the NVSAR, with 4,054 reported injury deaths [22.1\%]. The difference between the reported FIRS injury deaths and the NSVAR followed a sigmoidal curve, from 2007-2015 with a nadir in 2012 and a peak in 2015. Comparisons by mechanism of injury did not reveal a consistent pattern of difference. Conclusion: The differential reporting pattern of deaths between the NSVAR and the FIRS was unique for injury deaths, with more deaths reported in the NSVAR. Further analysis must be done to better elucidate the cause/s of these differences.

References: 1. Global Health Observatory Data Repository 2012, World Health Organization: http://apps.who.int/gho/2. Injury Surveillance Guidelines, World Health Organization, 2001: http:// www.who.int/violence_injury_prevention/publications/surveillance/surveillance guidelines/en/

Disclosure: No significant relationships.

PR193

HELLO AGAIN: CHARACTERIZATION OF POPULATION AND FACTORS ASSOCIATED WITH EARLY READMISSION FOLLOWING TRAUMA

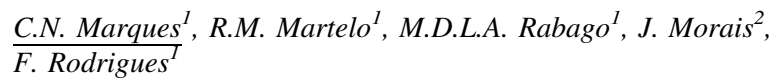

${ }^{1}$ Cirurgia Geral, Hospital Vila Franca de Xira, Vila Franca de Xira/ PORTUGAL, ${ }^{2}$ General Surgery, Hospital Vila Franca de Xira, Vila Franca de Xira/PORTUGAL

Introduction: Introduction The purpose of this study was to identify patient and hospital-based risk factors associated with 30 days readmission at a tertiary referral hospital. 
Material and methods: Patients and methods A retrospective analysis of 30 days post discharge trauma patients readmission was performed, from January 2012 to January 2016. Patient demographic data, Injury Severity Score (ISS), surgical procedure, ICU admission, length of stay (LOS) and readmission motive were collected. All patients discharged alive and readmitted within 30 days were included. Descriptive statistics were calculated and analyses using SPSS. Results: Results Of 623 patients admitted during the revised period, $23(3.7 \%)$ patients were readmitted within 30 days. 52 patients were excluded from analysis as the admissions were planned procedures. Of total 571 included patients, 54\% where male, caucasian (88\%), mean age 57, mean length of hospital stay of 24 days and mean length to readmission of 9 days. Of all patients, mean ISS was 9.1, multiple medical comorbidities were present in $61.9 \%$, mainly DM, COPD and Hypertension. Falls $(78 \%)$ were the most common mechanism of injury, followed by motor vehicle accident $(21 \%)$ and penetrating injury, including bullfight (6\%). Among in-hospital complications, the most common was wounds complications (mainly uncontrolled pain $(45 \%)$ and surgical site infection (37\%)). Preexisting COPD, DM, higher ISS, ICU admission were associated with readmission.

Conclusion: Conclusions Trauma patients have a low risk of readmission. Readmission was independently associated to ISS. Readmission population was mainly elderly men with multiple comorbidities. Modifiable factors to reduce readmissions were patient education of pain expectation and control and earlier wound surveillance.

References: INCLUDING COMORBIDITY IN THE ASSESSMENT OF TRAUMA PATIENTS: A REVISION OF THE TRAUMA INJURY SEVERITY SCORE; Melniker, L.a, W.m Briggs, and C.a Mancuso. Journal of Clinical Epidemiology 56.9 (2003): 921. WELCOME BACK: FACTORS ASSOCIATED WITH EARLY READMISSION FOLLOWING TRAUMA; Jennifer C. Roberts MD, MS, Jon Gipson MD, Joseph S. Farhat MD, Patty Reicks RN, Gregory Beilman; Session: XII: Quickshots; Paper 16: 9:46-9:52 am; American Association for the surgery ofTrauma, 2014 Annual Meeting Abstracts; TRAUMA CENTER CARE IS ASSOCIATED WITH REDUCED READMISSIONS AFTER INJURY Kristan Staudenmayer, Thomas G. Weiser, Paul M. Maggio, David A. Spain, Renee Y. Hsia; J Trauma Acute Care Surg. 2016 Mar; 80 (3): 412-418 TRAUMA PATIENT READMISSION: WHY DO THEY COME BACK FOR MORE?; Wright, Angela; The Journal of Emergency Medicine 50.4 (2016): 708-09. A REVISION OF THE TRAUMA SCORE; Champion, Howard $r$ et al; Journal of TraumaInjury Infection \& Critical Care; May 1989 TRAUMA CENTER PERFORMANCE INDICATORS FOR NONFATAL OUTCOMES. Moore, L., Stelfox, H. T., Boutin, A., \& Turgeon, A. F. (2013). Journal of Trauma and Acute Care Surgery, 74 (5), 1331-1343.

Disclosure: No significant relationships.

\section{PR193A}

\section{THE IMPORTANCE OF THE NATIONAL TRAUMA REGISTRY: IDENTIFICATION OF NOVEL COMBINATIONS OF PREDICTIVE FACTORS FOR MAJOR TRAUMA OUTCOMES AFTER A MASS-CASUALTY INCIDENT}

\author{
E.S. Tuboly ${ }^{1}$, E. Csonka ${ }^{2}$, E. Varga ${ }^{2}$
}

${ }^{1}$ Institute Of Surgical Research, University of Szeged, Szeged/ HUNGARY, ${ }^{2}$ Department Of Traumatology, University of Szeged, Szeged/HUNGARY

Introduction: The mortality rate of trauma complications has decreased during the past two decades, but is still around $30 \%$ from large registries (Frölich, 2014). To date, there was no existing trauma registry in our region thus we aimed to set up the Hungarian trauma registry and we further attempted to identify novel combinations of clinical or laboratory parameters with predictive value, following a large car accident.

Material and methods: 6 patients ( 4 male, 2 female, age: $43.83 \pm$ 2.97; serum lactate level: $3.22 \pm \mathrm{mmol} / \mathrm{l}$; base excess: $-2.13 \mathrm{mmol} / \mathrm{l}$ ) were simultaneously admitted to our institution with multiple fractures and blunt traumatic injuries. Important clinical parameters of shock and tissue oxygenation, markers of tissue necrosis and oxidative stress as well as blood test results were collected within the first 6 $\mathrm{h}$ after injury, $72 \mathrm{~h}$ after injury and then arranged into a database management software (Sigma Stat 12.0) and multiple linear regression analysis was performed.

Results: There was a strong and significant negative correlation found between leukocyte count at $6 \mathrm{~h}$ and the blood creatinine level at $72 \mathrm{~h}$ $\left(r_{s}=-0.949 ; p=0.0167\right)$ and a strong positive association was also observed between the blood creatinine level at $72 \mathrm{~h}$ and platelet count at $6 \mathrm{~h}\left(\mathrm{r}_{\mathrm{s}} 0.949 ; \mathrm{p}=0.0833\right)$ that might have a prognostic value in combination.

Conclusion: The first Hungarian trauma registry has been launched in our institution with a comprehensive dataset and seems valuable to characterize our major trauma burden and to provide novel combinations of predictive factors for complicated outcomes.

References: Fröhlich M, Lefering R, Probst C, Paffrath T, Schneider MM, Maegele M, Sakka SG, Bouillon B, Wafaisade A; Committee on Emergency Medicine, Intensive Care and Trauma Management of the German Trauma Society Sektion NIS. Epidemiology and risk factors of multiple-organ failure after multiple trauma: an analysis of 31,154 patients from the TraumaRegister DGU. J Trauma Acute Care Surg. 2014; 76 (4):921-7; discussion 927-8.

Disclosure: No significant relationships.

\section{PR194}

\section{APPROPRIATE USE OF D-DIMER CAN SAFELY REDUCE EXCESSIVE WHOLE-BODY COMPUTED TOMOGRAPHY (WBCT) FOR TRAUMA}

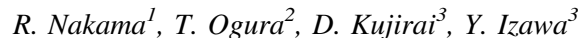

${ }^{1}$ Department Of Radiology, Saiseikai Utsunomiya Hospital, Utsunomiya-city/JAPAN, ${ }^{2}$ Advanced Medical Emergency Department And Critical Care Center, Japan Red Cross Maebashi Hospital, Maebashi-city/JAPAN, ${ }^{3}$ Department Of Emergency Medicine, Saiseikai Utsunomiya Hospital, Utsunomiya-city/JAPAN

Introduction: Whole-body computed tomography (WBCT) is widely used to evaluate trauma, but the indication for its use is unclear. The use of WBCT without reason may lead to excessive radiation exposure and high medical expenses. The level of the D-dimer after injury is reported to correlate with the injury severity score (ISS) [1]. We hypothesized that measurement of the D-dimer may help reduce excessive WBCT.

Material and methods: This was a retrospective review of electronic medical records from Saiseikai Utsunomiya Hospital. Patients seen from January 1, 2014 to April 30, 2017 with blunt trauma who underwent WBCT with a contrast agent and D-dimer measurement were included. Detection of multiple $(\geq 2)$ trauma sites on WBCT was defined as a positive finding. According to the result of WBCT evaluation, each traumatic injury with an abbreviated injury scale $\geq 3$ was defined as significant. We verified prediction accuracy of multiple trauma by D-dimer. 
Results: A total of 488 patients were included in this study. The areas under the receiver operating characteristic curve of D-dimer for multiple trauma (detected by WBCT) was 0.8226 (95\% CI: $0.7765-$ $0.8688)$. The cutoff value of the D-dimer was set to normal $(<1.0 \mu \mathrm{g} /$ $\mathrm{mL}$ ), the sensitivity and specificity values were $100 \%$ and $10.8 \%$.

Conclusion: If the D-dimer is low enough, WBCT may not be necessary for initial evaluation of trauma. Appropriate use of D-dimer may lead to decreased radiation exposure and lower medical expenses.

References: 1. Hagiwara S, et al: Usefulness of fibrin degradation products and d-dimer levels as biomarkers that reflect the severity of trauma. J Trauma Acute Care Surg. 2013, 74 (5):1275-8

Disclosure: No significant relationships.

\section{PR195}

\section{PREDICTION OF MASSIVE TRANSFUSION IN PATIENTS WITH PELVIC FRACTURE USING FIBRINOGEN AND BASE EXCESS LEVELS ON ADMISSION}

\section{N. Notani, S. Kanezaki, T. Sakamoto, T. Sone, H. Tsumura}

Emergency And Critical Care Center, Oita University Hospital, Yuhu/ JAPAN

Introduction: Prediction of massive transfusion (MT) requirement in patients with pelvic fracture is one of the greatest problems. There were some reports that fibrinogen (Fbg) and base excess (BE) might be indicators of the need for MT after blunt trauma. The aim of this study is to evaluate whether Fbg and BE levels on admission can be used to predict the need for MT in patients with pelvic fracture.

Material and methods: We conducted a retrospective observational study. Patients who were treated for pelvic fracture in our hospital were divided into 2 groups: MT and non-MT. MT was defined as the transfusion of packed red blood cells $\geqq 10$ units or death caused by bleeding within $24 \mathrm{~h}$ after admission. We compared variables, including vital signs, blood test and image inspection findings, scoring systems between two groups.

Results: There were 22 patients in MT, and 53 patients in non-MT. On univariate analysis, there were significant differences in injury severity score (ISS), Glasgow Coma scale (GCS), systolic blood pressure (sBP), unstable pelvic fracture, $\mathrm{Hb}, \mathrm{BE}, \mathrm{Fbg}$, lactate, TASH scores, FAST positive rate, extravasation positive rate. On multivariate analysis, the level of $\mathrm{Fbg}$ and $\mathrm{BE}$ were independent predictors of MT (odds ratio [OR] $0.801,95 \%$ confidence interval [CI] 0.6930.926 and OR $0.691,95 \%$ CI $0.525-0.909$, respectively).

Conclusion: In this study, we demonstrated that Fbg and BE levels on admission could be useful to predict the need for MT in patients with pelvic fracture.

References:

Disclosure: No significant relationships.

\section{PR196}

\section{IDENTIFYING MAJOR TRAUMA: REFINING THE TRAUMA TEAM ACTIVATION CRITERIA}

\author{
R. Crouch $^{1}$, S. Kellett ${ }^{2}$, T. Ringrose ${ }^{2}$
}

${ }^{1}$ Emergency Department, University Hospital Southampton, Southampton/UNITED KINGDOM, ${ }^{2}$ Trauma Anaesthetics, University Hospital Southampton, Southampton/UNITED KINGDOM

Introduction: Optimal Trauma Team Activation (TTA) criteria will identify patients who benefit from this while avoiding disruption by minimising unnecessary activation. Difficulties have been demonstrated achieving this ${ }^{(1-2)}$. We analysed triage outcome under our present system, and modelled the effect of refinement.

Material and methods: Retrospective analysis to quantify sensitivity and specificity of triage criteria predicting severe injury (ISS $>15$ ). All major trauma patients directly admitted to hospitals in the Wessex Trauma Network (WTN) UK from 1 January /2013 to 31 December 2014 ( $\mathrm{n}=4267)$ were included. TTA criteria were analysed with respect to sensitivity \& specificity, positive/negative predictive values. Data used to revise TTA criteria and model effect.

Results: Original criteria sensitivity was $87.42 \%$, over-triage $45.23 \%$. Physiological criteria were best predictors of severe injury, mechanism of injury (MOI) contributed most to over-triage. Following statistical analysis, revised criteria were retrospectively applied to the same data, resulting in sensitivity of $85.31 \%$ and over-triage of $30.75 \%$. The revised physiological criteria, with the evidence for individual components, will be presented.

Conclusion: Simplified TTA criteria, utilising predominantly physiological/anatomical criteria can safely reduce over-triage without significant decrease in sensitivity. Specific consideration to particular groups, such as the elderly, is important and we are currently modelling this.

References: 1. Bressan S, Franklin KL, Jowett HE, King SK, Oakley E, Palmer CS. Establishing a standard for assessing the appropriateness of trauma team activation: a retrospective evaluation of two outcome measures. Emergency medicine journal : EMJ. 2015 Sep;32 (9):716-21 2. Uleberg O, Vinjevoll OP, Eriksson U, Aadahl P, Skogvoll E. Overtriage in trauma - what are the causes? Acta anaesthesiologica Scandinavica. 2007 Oct;51 (9):1178-83.

Disclosure: No significant relationships.

\section{PR197}

\section{RISK FACTORS FOR SUBSEQUENT ANGIOGRAPHIC CONTRAST EXTRAVASATION DESPITE ITS ABSENCE ON INITIAL COMPUTED TOMOGRAPHY IN PATIENTS WITH PELVIC TRAUMA}

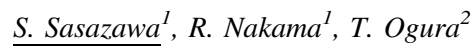

${ }^{1}$ Department Of Radiology, Saiseikai Utsunomiya Hospital, Utsunomiya-city/JAPAN, ${ }^{2}$ Advanced Medical Emergency Department And Critical Care Center, Japan Red Cross Maebashi Hospital, Maebashi-city/JAPAN

Introduction: Angiography and transcatheter arterial embolization (TAE) are widely used to control bleeding in pelvic fractures. Massive intrapelvic hematoma without contrast extravasation on 
computed tomography (CT-negative) is an indication for TAE. However, angiography may show contrast extravasation despite its absence on CT. We hypothesized that there may be factors that differentiate the CT-negative, angiography-positive group (AP) from the CT- negative, angiography-negative group (AN). Identification of these factors may enable appropriate selection of CT-negative cases requiring aggressive angiography.

Material and methods: This is a retrospective review of electronic medical records from Saiseikai Utsunomiya Hospital. The study included CT-negative patients with pelvic fractures who underwent subsequent angiography from January 1, 2012 to December 31, 2016. Basic clinical and injury data were obtained. Patients were grouped according to the presence of extravasation on angiography, and basic parameters were compared.

Results: Of 20 patients with pelvic fractures who met the inclusion criteria, $9(45 \%)$ showed contrast extravasation on angiography. Parameters compared between the AP and AN groups included age, systolic blood pressure on arrival, injury severity score, revised trauma score, Ps, and hemoglobin, platelet, fibrinogen, and D-dimer levels, and prothrombin time-international normalized ratio. There was no significant difference between the groups $(\mathrm{p}<0.05)$. Platelets tended to be lower in the AP group than in the AN group $(\mathrm{p}<0.11)$. Conclusion: There was no difference between the AP and AN groups. Additional cases are needed for review and factors such as the use of antiplatelet and anticoagulation drugs or concurrent head injury require further investigation.

\section{References:}

Disclosure: No significant relationships.

\section{PR198}

\section{THE IMPORTANCE OF AN ADDITIONAL STERNUM FRACTURE IN SPINAL COLUMN FRACTURES IN POLYTRAUMA - AN ANALYSIS FROM THE TRAUMA REGISTRY DGU®}

\author{
S. Krinner ${ }^{1}$, M. Hirschmann ${ }^{2}$, A. Langenbach ${ }^{2}$, F.F. Hennig ${ }^{2}$, \\ R. Lefering ${ }^{3}$, S. Schulz-Drost ${ }^{4}$
}

${ }^{1}$ Trauma And Orthopedic Surgery, University Hospital Erlangen, Erlangen/GERMANY, ${ }^{2}$ Trauma Surgery, University Hospital Erlangen, Erlangen/GERMANY, ${ }^{3}$ Institut Für Forschung In Der Operativen Medizin, Akademie der Unfallchirurgie GmBH, Cologne/ GERMANY, ${ }^{4}$ Trauma- And Orthopedic Surgery, Unfallkrankenhaus Berlin, Berlin/GERMANY

Introduction: Combined sternum and vertebral injuries may cause a severe loss of stability of the trunk what has been only described in small case series yet. The present study is intended to illustrate the importance of an additional sternum fracture in vertebral fractures by means of reliable, multicenter data in polytrauma patients.

Material and methods: A retrospective analysis from the data set of the TraumaRegistry DGU® (TR-DGU, Project ID 2015-004) included patients in $2009-2014$, ISS ${ }^{3} 16$, primary admission to a trauma center. Exclusion: primary transfer, isolated Brain injury. Evaluation and interpretation are the responsibility of the author and have not yet passed through the final review process of the TraumaRegister DGUß. Cervical, thoracic and lumbar spine fractures $(A I S \geq 2)$ without and with concomitant sternum fracture (SF) were compared Results: 3775 patients with cervical (407 SF), 5428 with thoracic (686 SF) and 5847 with lumbar spine fractures (576 SF) were included, An additional SF was highly associated with a more frequent car accident, a higher overall injury severity, prolonged respiratory, intensive and overall duration, as well as a poorer outcome. Patients with SF in all groups also had severe thoracic concomitant injuries and other fractures of the remaining spinal column sections. In the thoracic area, SF also significantly increased the rate of cross-sectional injuries.

Conclusion: In fractures of the thoracic spine as well as in those of the cervical and lumbar spine, an additional SF is multifactorially complicated, with the most severe manifestation in thoracic vertebral fractures.

\section{References:}

Disclosure: Advisory Member of AO TK Thoracic expert Group and Consultant Agreement with DePuySynthes

\section{PR199}

\section{HYPOTENSIVE RESUSCITATION IN TRAUMATIC HEMORRHAGIC SHOCK PATIENTS; A META-ANALYSIS}

\section{N. Owattanapanich}

Division Of Trauma, Surgery Department, Siriraj Hospital, Faculty of Medicine, Mahidol university, Bangkok/THAILAND

Introduction: Damage control strategies played an important role in trauma patient management. As a part of these strategies, hypotensive resuscitation had become increasingly utilized. While there were a number of randomized trials supporting hypotensive strategies, the mortality benefit has not been systematically reviewed.

Material and methods: PubMed, Medline-Ovid, Scopus, Science direct, EMBASE and CNKI data searching were conducted. Additional searching was also performed by reviewing of relevant primary literatures and review articles. Randomized controlled trials and cohort studies that reported the mortality associated with hypotensive resuscitation or limited resuscitation were selected. A fixed effect model was used to estimate mortality and other complications.

Results: Of the 2,114 initially reviewed studies, 24 were selected for this meta-analysis. There was statistically significant decrease mortality in hypotensive resuscitation group (95\% CI: $0.47,0.63$ ). Heterogeneity was observed with $\mathrm{I}^{2} 27 \%, \mathrm{df}=23(\mathrm{P}=0.11)$. Sensitivity analysis was performed and also showed the same result in mortality benefit of experimental group. Hypotensive resuscitation had less packed red cell transfusion and fluid resuscitation. These strategies had no statistically significant difference of the incidence of acute kidney injury. Interestingly, they showed protective effect in multi organ dysfunction and acute respiratory failure.

Conclusion: This meta-analysis showed that there is significant evidence to conclude that hypotensive resuscitation had a mortality benefit in traumatic hemorrhagic shock patients.

References: - Willium H Bickell, MD, Matthew J Wall, Paul E. Pepe, et al Immediate versus delayed fluid resuscitation for hypotensive patients with penetrating torso injuries. NEJM 1994;331:1105-1109 Matthew M. Carrick, Catherine A Morrison, Nicole M Tapia, et al Intraoperative hypotensive resuscitation for patients undergoing laparotomy or thoracotomy for trauma: Early termination of the randomized prospective clinical trial. J trauma Acute Care Surg 2016;80:886-896 - C. Anne Morrison, Matthew M. Carrick, Michael A Norman, et al Hypotensive Resuscitation Strategy Reduces transfusion requirements and severe postoperative coagulopathy in Trauma patients with hemorrhagic shock: Preliminary results of the randomized controlled trial. The Journal of TRAUMA Injury, Infection, and Critical Care 2011;70:652-663 - Martin A. Schreiber, Eric N. Meier, Samuel A. Tisherman, et al A controlled resuscitation strategy is feasible and safe in hypotensive trauma patients: results of a 
prospective randomized pilot trial. J trauma Acute Care Surg 2015;78 (4):687-697 - Wang Mei-tang, MEI bing, HE Jian, et al Effect of preoperative limited fluid resuscitation to the patients with traumatic shock. Journal of Medical Colleges of PLA 2007;22 (4):226-229 Richard P. Dutton, Colin F. Mackenzie, Thomas M. Scalea Hypotensive Resuscitation during Active Hemorrhage: Impact on InHospital Mortality. The Journal of TRAUMA Injury, Infection, and Critical Care 2002;52:1141-1146

Disclosure: No significant relationships.

\section{PR200}

\section{POLYTRAUMA IN SPECIAL SURROUNDING}

\section{Gavrankapetanovic}

Traumatology And Orthopaedic Clinic, University Clinical Center Sarajevo, Sarajevo/BOSNIA AND HERZEGOVINA

Introduction: During the wartime in Sarajevo, Bosnia and Herzegovina, a city that was under the siege for 1425 days, the longest in history after the World War II, we have seen and treated many polytraumas. It is estimated that in the period from 1992-1995 there were around 65000 wounded inhabitants. To treat a polytrauma in peace and in wartime is something completely different.

Material and methods: In this retrospective study we are presenting a 100 politraumatised patients that were treated at the Clinic for orthopedics and Traumatology of the University Clinical Center Sarajevo in the period of 1992-1995 with a 20 years follow up.

Results: From 65000 wounded inhabitants of Sarajevo, from which most of them were treated at the Clinic for Orthopedics and Traumatology of the University Clinical Center Sarajevo, we treated many polytraumatised patients from which we present a 100 patients with a 20 years follow up.

Conclusion: Despite one's best efforts, the overall difficult environment that war creates along with the experiences and problems which are faced on a daily basis, influence the outcome of every surgery. Surgeons in wartime ask themselves are they able to provide sufficient protection to their patients; are they safe and protected themselves; will they be able to perform the operation in the shortest period of time and in the best of their knowledge. It is essential to modify the surgical protocol because of the nature of the injuries as well as the circumstances under which every operation is performed. References:

Disclosure: No significant relationships.

\section{PR201}

THE PULMONARY NEUTROPHIL RESPONSE TO LONG BONE FRACTURES AND INTRAMEDULLARY NAILING IN RATS

\section{M.P.J. Teuben}

Traumatologie, university hospital zuerich, zuerich/SWITZERLAND

Introduction: Polymorphonuclear neutrophils (PMNs) are key effector cells in the development of ARDS after trauma. It has been shown that intramedullary nailing (IMN) for the treatment of femur fractures $(\mathrm{FF})$ leads to an increased risk at pulmonary complications in trauma patients. However it is unclear how IMN + FF alters neutrophil activation in blood and the lungs.

Material and methods: Female Whistar rats were subjected to IMN + FF. Groups $(\mathrm{N}=5)$ were sacrificed after 3, 7 and 14 days of observation. Neutrophils were isolated from peripheral blood and the lungs and thereafter analyzed by flowcytometry. The membrane receptor expression of Mac-1 (CD11b), LFA-1 (CD11a), L-selectin (CD62L) were measured and compared between groups

Results: FF/IMN is associated with statistically significantly reduced neutrophil numbers in blood ( $\mathrm{p}<0.01)$ after 72 hours of observation. However increased PMN counts in the lungs, as well as in the BALF are observed. Membrane receptor expression levels of CD11b and CD62L demonstrated a more activated status of pulmonary neutrophils. Moreover changes in neutrophil subpopulations (CD11a vs. CD11bsubtypes) in the lungs were also encountered. Seven days after surgery pulmonary neutrophil numbers dropped to baseline levels and these neutrophils do not express an increase of activation markers anymore. Conclusion: This study demonstrates that intramedullary nailing and a femur fracture in rats affects the pulmonary neutrophil pool. The post-interventional neutrophil alterations in the lungs may play a role in the development of ARDS. These new insights can form the basis for new therapeutic interventions to prevent systemic inflammatory complications after trauma and fracture stabilization.

References:

Disclosure: No significant relationships.

\section{PR202}

\section{IMPROVEMENTS OF POLYTRAUMA MANAGEMENT QUALITY USING A MODIFIED POLYTRAUMA ALGORITHM IN COMBINATION WITH A DOUBLE-ROOM SLIDING GANTRY CT}

\section{P. Kastner, T.S. Mueller}

\section{Chirurgie, Kantonsspital Graubünden, Chur/SWITZERLAND}

Introduction: Recent literature has shown the benefit of a CT scanner located in the shock room reducing the time for primary care of polytrauma patients. Designing our new trauma bay, we choose a format with a sliding gantry concept including one portable CT scanner. This enables us to have a CT scanner available $24 / 7$ for polytrauma patients as well as for our emergency department patients. Given by these new technical options, we introduce an adapted polytrauma algorithm as an integration of components of the ATLS ${ }^{\odot}$ concept and the ETC $^{\odot}$ concept. Our interdisciplinary and interprofessional crew resource management had this adapted shock room management algorithm training included. The aim of this study was to evaluate the impact of the new algorithm on the quality of the process and results for polytrauma management.

Material and methods: Single-center retrospective evaluation study (2015-2017) based on data, extracted from an evaluation form and records of shock room qualtity data.

Results: Subjective evaluation of trauma team members was overall positive. Objective performance indicators of shock room management improves over time. Also an increasing number of trained team members had a positive influence of reducing process time for initial polytrauma management.

Conclusion: A specialized human factor training and the use of a modified polytrauma algorithm in combination with latest technical equipment will improve the quality of trauma care.

References:

Disclosure: No significant relationships. 
PR203

\section{THE USE OF WHOLE BLOOD TRANSFUSION AS AN ALTERNATIVE TO BLOOD COMPONENT THERAPY IN SEVERE TRAUMA PATIENTS}

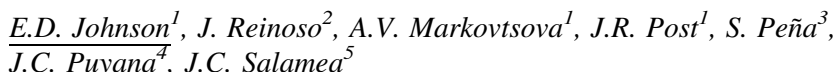

${ }^{1}$ School Of Medicine, University of Pittsburgh, Pittsburgh/PA/ UNITED STATES OF AMERICA, ${ }^{2}$ Direccione Generale, Universidad de Cuenca, Cuenca/ECUADOR, ${ }^{3}$ Direccione Generale, Hospital Vicente Corral Moscoso, Cuenca/ECUADOR, ${ }^{4}$ Department Of Surgery, University of Pittsburgh Medical Center, Pittsburgh/PA/ UNITED STATES OF AMERICA, ${ }^{5}$ Cirugía De Trauma Y Emergencias, Universidad de Azuay, Cuenca/ECUADOR

Introduction: Current guidelines for Damage Control Resuscitation (DCR) recommend using equal ratios of blood components to Packed Red Cells to emulate whole blood transfusion (WBT). Implementing WBT is difficult due to regional readiness of blood bank resources and donors as well as prevalent standardized practices for blood fractionation into its components (BCT). WBT is now being introduced in some regions of the world. Since 2012, our hospital implemented WBT for trauma patients. The aim of this study was to describe our preliminary experience using WBT strategy and to compare outcomes of severe trauma patients receiving BCT vs. WBT. Material and methods: We performed a retrospective matched casecontrol study comparing $31 \mathrm{WBT}$ versus $31 \mathrm{BCT}$ patients, from 2012-2015. The groups were matched by age, sex, and ISS. The inclusion criteria were patients with an ISS $\geq 25$ and age $>15$.

Results: WBT group characteristics included : MAP $=67.20( \pm$ 19.24); HR = 104.97 $( \pm 20.95) ; \mathrm{RR}=23.45( \pm 5.33) ; \mathrm{Temp}=36.03$ $\mathrm{C}( \pm 0.48)$; pre-op wait time $=106 \mathrm{~m}( \pm 190 \mathrm{~min})$; $\mathrm{LOS}=6.10 \mathrm{days}$ \pm 5.26 compared against $\mathrm{BCT}$ control group with a $\mathrm{MAP}=83.13( \pm$ $12.55) ; \mathrm{HR}=78.97( \pm 14.45) ; \mathrm{RR}=22.87( \pm 3.14)$; Temp $=36.26$ $\mathrm{C}$ ( \pm 0.63$)$; pre-op wait time $=60 \mathrm{~m}( \pm 70 \mathrm{~m})$ and a LOS of 8.63 days $\pm 4.69(\mathrm{p}<0,05)$. There were 2 deaths in the BCT group $(6.4 \%)$ and 3 deaths in the WBT $(9.6 \%)$.

Conclusion: WBT patients had a significantly higher ISS, and worse MAP and HR than BCT. WBT had shorter LOS with similar mortality rates. WBT is a feasible and promising DCR strategy in civilian trauma settings.

References: Holcomb JB, Tilley BC, Baraniuk S, Fox EE, Wade CE, Podbielski JM, del Junco DJ, Brasel KJ, Bulger EM, Callcut RA, Cohen MJ, Cotton BA, Fabian TC, Inaba K, Kerby JD, Muskat P, O'Keeffe T, Rizoli S, Robinson BRH, Scalea TM, Schreiber MA, Stein DM, Weinberg JA, Callum JL, Hess JR, Matijevic N, Miller CN, Pittet J, Hoyt DB, Pearson GD, Leroux B, van Belle G, for the PROPPR Study Group. Transfusion of Plasma, Platelets, and Red Blood Cells in a 1:1:1 vs a 1:1:2 Ratio and Mortality in Patients With Severe Trauma. The PROPPR Randomized Clinical Trial. JAMA. 2015;313 (5):471-482. doi:10.1001/jama.2015.12 Yazer, Mark H. MD; Jackson, Byron MD; Sperry, Jason L. MD; Alarcon, Louis MD; Triulzi, Darrell J. MD; Murdock, Alan D. MD: Initial safety and feasibility of cold-stored uncrossmatched whole blood transfusion in civilian trauma patients:. Journal of Trauma and Acute Care Surgery: July 2016 - Volume 81 - Issue 1 - p 21-26

Disclosure: No significant relationships.

\section{PR204}

\section{THE PREHOSPITAL MANAGEMENT OF HYPOTHERMIA - AN UP-TO-DATE OVERVIEW}

\author{
F. Haverkamp ${ }^{1}$, G. Giesbrecht ${ }^{2}$, E. Tan ${ }^{1}$
}

${ }^{1}$ Emergency Department, Radboud University Medical Center, Nijmegen/NETHERLANDS, ${ }^{2}$ Laboratory For Exercise And Environmental Medicine, University of Manitoba, Winnipeg/MB/ CANADA

Introduction: Accidental hypothermia is a serious threat to all injured patients. To prevent or manage deterioration, treatment of hypothermia should be initiated prehospital. The last review on this topic was performed 16 years ago. The aim of this study is to provide an up-to-date systematic overview of the currently available treatment modalities and their effectiveness for prehospital hypothermia management.

Material and methods: The databases PubMed, EMbase and MEDLINE were searched using the terms: "hypothermia", "accidental hypothermia", "Emergency Medical Services" and "prehospital". Articles with publications dates up to August 2017 were included and selected by the authors based on relevance.

Results: The literature search produced 921 articles, out of which 47 focused on passive insulation and/or active heating. The most effective passive insulation systems combined insulation with a vapor barrier; generally referred to as a hypothermia wrap. Active external rewarming interventions include chemical, electrical and charcoalburning heat packs; chemical or electrical heated blankets; and forced air warming. Mildly hypothermic patients, with significant endogenous heat production from shivering, will likely be able to rewarm themselves with only passive insulation, although active warming will still provide comfort and an energy-saving benefit. For colder, nonshivering patients, the addition of active warming is required for any significant core rewarming. The administration of warmed intravenous fluids reduces heat loss.

Conclusion: This thorough literature review reveals that there have been no major breakthroughs since the last review on this topic dating from 2001, but modern designs of hypothermia wraps have shown promise and battery-powered inline fluid warmers are practical devices to warm intravenous fluids.

References:

Disclosure: No significant relationships.

\section{PR205}

\section{CRUSHING INJURIES- SAME MECHANISM, DIFFERENT SYDROMES}

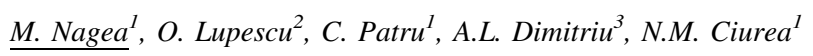

${ }^{1}$ Orthopaedics And Trauma, CLINICAL EMERGENCY HOSPITAL, BUCHAREST/ROMANIA, ${ }^{2}$ Orthopaedics And Trauma, Clinical Emergency Hospital, University of Medicine and Pharmacy " Carol Davila" Bucharest, Bucharest/ROMANIA, ${ }^{3}$ Clinical Emergency Hospital, Orthopaedics And Trauma, University of Medicine and Pharmacy Carol Davila, BUCHAREST/ROMANIA

Introduction: Due to high energy agents, complex crushing trauma became more frequent; they require early diagnosis and properly treatment, as local and general complications can be disastrous. 
Material and methods: This retrospective study analyses 80 patients admitted in a Level I Trauma Centre between 01.06.2012-01.01.2016 with a crushing injury described by diagnosis. The authors describe the algorithm for diagnosis (including laboratory findings and complementary examinations) and treatment (following MESS Score) for these cases, analyzing the most common confusions which can interfere.

Results: Different syndromes appeared: in $45 \%$ of cases, compartment syndrome of the shank appeared, in $85 \%$ of the cases with fracture. Overall, fractures appeared in $88 \%$ of the cases, but open injuries only in $78 \%$ of the cases. In $50 \%$ of the cases, complex trauma occurred, with vascular or nervous injury, while acute peripheral ischemia appeared in $15 \%$ of the trauma. The most important therapeutic problems are discussed concerning this patients: surgical treatment, general treatment, multiple steps therapy. The common identified problems were: neglecting the severity of the injury in closed trauma, incomplete assessment of the injury site, incomplete excision, improper closure and incomplete general monitoring.

Conclusion: Crushing is a severe trauma that has important local and general consequences that requires a complete and early treatment in several stages. Also, the flexible and complete evaluation of the patient and the treatment of associated injuries lead to a positive local and general evolution. Thorough debridement is essential and a multidisciplinary monitoring and treatment can generate a favorable outcome, with patient survival and optimal limb rehabilitation

References: Prasarn ML, Helfet DL, Kloen P. Management of the mangled extremity. Strategies in Trauma and Limb Reconstruction. 2012;7 (2):57-66. doi:10.1007/s11751-012-0137-4.

Disclosure: No significant relationships.

\section{PR206}

\section{DAMAGE CONTROL SURGERY IN JAPAN}

N. Urushibata, K. Murata, H. Hara, A. Yoshiyuki, M. Sugimoto, R. Nakamoto

Shock Trauma And Emergency Medical Center, Matsudo City General Hospital, Matsudo/JAPAN

Introduction: [Background] Surgical method in trauma is very diverse. The term "damage control surgery" is not necessarily universal, as the chosen surgical technique may be different according to the operating trauma surgeon. [Objective] Our objective was to analyze what surgical techniques were identified as "damage control surgery" in Japan.

Material and methods: [Material and Method] We used data from the Japanese Nationwide Administrative Database (DPC) from April 2010 to March 2015. Within the 5 years, we identified trauma patients who were admitted via ambulance, underwent laparotomy, and admitted to the ICU. We excluded patients under the age of 18, and also patients who were under cardiac pulmonary arrest before arrival. We divided the patients into damage control surgery group (DCS group), and laparotomy group (non-DCS group), and analyzed the two groups.

Results: [Results] The total number of trauma patients were 16,940 patients, and DCS group consisted of 648 patients, and non-DCS group consisted of 16,292 patients. The two groups showed no significant difference in the diagnosed disease, and the performed surgery. DCS group was significantly younger in age.

Conclusion: We seek to analyze the impact of DCS on outcome of trauma patients in Japan.

References:

Disclosure: No significant relationships.

\section{PR207}

\section{MANAGEMENT OF MULTIPLE VICTIM TRAFFIC ACCIDENT IN A REGIONAL HOSPITAL}

A. Nonnemacher San Julián, C. Cózar Lozano, L. Carrión, L. Oliver Garcia, O. Blasco, J.A. Perez.

General Surgery, Virgen del Puerto Hospital, Plasencia (Cáceres)/ SPAIN

Introduction: Management of traffic accident with multiple victims in a regional hospital, highlighting the importance of quick stabilization before definitive treatment or transfer to reference hospital if necessary.

Material and methods: Traffic accident occurred on EX-A1 highway, with multiple collisions due to a fog bank, involving 36 vehicles, causing one death. 25 victims were attended: 5 of them with severe thoracoabdominal trauma, one being refered to a higher level Centre. Results: Once the data were analyzed, we found an arrival time interval to the hospital from 45 minutes to 2 hours.

Triage, initial stabilization and management was undertaken in the emergency department. Concerning the most critical patient, he presented with multiple lower limbs fractures, left external iliac vein hematoma and splenic laceration, requiring right bellow-knee amputation, left femoral external fixation, stabilization with splint in right tibia and later admission to ICU.

Conclusion: After multiple victims traffic accident it is of vital importance the immediate attention, as well as the correct stabilization and initial management, not forgetting the possible need to refer the patient to a higher level center for specific treatments.

References: Lashoher A, Schneider EB, Juillard C, Stevens K, Kent: Implementation of the World Health Organization Trauma Care Checklist Program in 11 Centers Across Multiple Economic Strata: Effect on Care Process Measures. World J Surg (2017) 41:954-962. Up to date

Disclosure: No significant relationships.

\section{PR208}

\section{ASSOCIATED FACTORS WITH MORBIDITY AND MORTALITY IN POLYTRAUMA PATIENTS}

\author{
I. Perez-Nuñez ${ }^{1}$, L. Alonso Viana ${ }^{I}$, M. Fernandez Alvarez ${ }^{1}$, \\ P. Colsa Gutierrez ${ }^{2}$, V.J. Ovejero Gomez ${ }^{2}$, J.A. Blasco Velarde ${ }^{2}$, \\ D. Morales-Garcia ${ }^{2}$
}

\section{${ }^{1}$ Traumatology And Orthopedic Surgery, UNIVERSITY HOSPITAL "MARQUES DE VALDECILLA", SANTANDER/SPAIN, ${ }^{2}$ Department Of Surgery, UNIVERSITY HOSPITAL “MARQUES DE VALDECILLA”, SANTANDER/SPAIN}

Introduction: Polytrauma is the leading death cause in population from 1 to 45 years old. An early detection of patients with a high death or complications rate might help guide their initial care and lower such risks. The aim of this study was to analyze a group of variables, from injury mechanisms to analytic parameters, and indentify those which relate to an increased morbimortality rate. We will consider the value of a proper surgery times management and damage control surgery in these patients 
Material and methods: Retrospective study with polytrauma patients from HUMV between 2013 and 2016. Clinic histories review in order to get all suitable variables, and further statistic analysis

Results: There are certain variables which, if altered, imply a higher mortality or complications rate. It's the case of renal function parameters, blood pressure, haemoglobin, $\mathrm{pH}, \mathrm{O} 2 \mathrm{Sat}$ and rhabdomyolysis, among others. The most frequently found complications of our study are infectious, above all intravenous line/catheter or urinary catheter related infections.

Conclusion: Dyslipidemia, hypotension, altered renal function parameters, anaemia, acidosis, low O2Sat, low GCS or certain infections increase mortality rate in polytrauma patients. Similarly, the presence of rhabdomyolysis increases the likelihood to suffer from diverse infections and bacteriemia/sepsis during hospitalization.

References:

Disclosure: No significant relationships.

\section{REBOA}

PR209

BENEFICIAL CIRCULATORY AND METABOLIC EFFECTS OF PARTIAL REBOA COMPARED TO TOTAL REBOA IN HEMORRHAGIC SHOCK IN PIGS

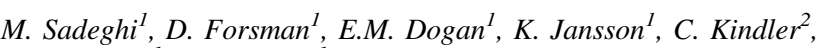 \\ K.F. Nilsson ${ }^{1}$, T.M. Hörer
}

${ }^{1}$ Department Of Cardiothoracic And Vascular Surgery, Örebro University Hospital, Örebro/SWEDEN, ${ }^{2}$ Pathology, Medicine, västerås/SWEDEN

Introduction: Resuscitative Endovascular Balloon Occlusion of the Aorta (REBOA) can be used as a bridge to intervention. REBOA reduces hemorrhage but causes ischemic injuries. This study investigates the hemodynamic and metabolic effects by partial REBOA (pREBOA) and total REBOA (tREBOA) during hemorrhagic shock in pigs.

Material and methods: The study was conducted in two parts. Protocol 1; 18 pigs were subjected to induced hemorrhage, SBP of 50 $\mathrm{mmHg}$ and randomized into three groups ( $\mathrm{n}=6$ group); $30 \mathrm{~min}$ pREBOA (SBP 80-100 mmHg), tREBOA or control. After intervention the animals were resuscitated by autologous transfusion and monitored for $3 \mathrm{~h}$. Hemodynamics, bloodgases, mesenteric bloodflow, visceral enzymes, inflammatory markers and intestinal histology were analyzed. Protocol 2; 12 pigs were used for induced hemorrhage, continuing until death. After $30 \mathrm{~min}$ hemorrhage, they were randomized to pREBOA, tREBOA or control. Hemorrhagic volume and time to death were measured.

Results: Hemorrhage induced shock in all groups. In pREBOA and tREBOA MAP increased during occlusion (p0.97). The bloodflow in superior mesenteric artery was higher in pREBOA than tREBOA during occlusion (p0.05). Arterial $\mathrm{pH}$ was lower, lactate and troponine levels were significantly higher in tREBOA compared to pREBOA during reperfusion. The cytokines and histological analyses from the small bowel were mostly affected in tREBOA without significance. Protocol 2: Total hemorrhagic volume until death was highest in pREBOA compared to tREBOA (p0.99) and control (0.53). Survival time was longest in tREBOA compared to control (0.03).

Conclusion: Partial REBOA decreases ongoing hemorrhage while maintaining tissue perfusion with less metabolic and inflammatory response than tREBOA, but reduces survival time without transfusions.
References: 1. Morrison JJ, Galgon RE, Jansen JO, Cannon JW, Rasmussen TE, Eliason JL. A systematic review of the use of resuscitative endovascular balloon occlusion of the aorta in the management of hemorrhagic shock. J Trauma Acute Care Surg. 2016;80 (2):324-34. 2. Horer TM, Skoog P, Pirouzram A, Nilsson KF, Larzon T. A small case series of aortic balloon occlusion in trauma: lessons learned from its use in ruptured abdominal aortic aneurysms and a brief review. Eur J Trauma Emerg Surg. 2015. 3. Russo RM, Williams TK, Grayson JK, Lamb CM, Cannon JW, Clement NF, et al. Extending the golden hour: Partial resuscitative endovascular balloon occlusion of the aorta in a highly lethal swine liver injury model. J Trauma Acute Care Surg. 2016;80 (3):372-80. 4. Russo RM, Neff LP, Lamb CM, Cannon JW, Galante JM, Clement NF, et al. Partial Resuscitative Endovascular Balloon Occlusion of the Aorta in a Swine Model of Hemorrhagic Shock. J Am Coll Surg. 2016. 5. Johnson MA, Neff LP, Williams TK, DuBose JJ, Group ES. Partial Resuscitative Balloon Occlusion of the AORTA (P-REBOA): Clinical Technique and Rationale. J Trauma Acute Care Surg. 2016. Disclosure: No significant relationships.

\section{PR210}

\section{RESUSCITATIVE ENDOVASCULAR BALLOON OCCLUSION OF THE AORTA IS ASSOCIATED WITH ELEVATION OF INTRACRANIAL PRESSURE IN AN OVINE MODEL OF HEMORRHAGIC SHOCK AND TRAUMATIC BRAIN INJURY}

\author{
E.A.Semenov ${ }^{l}$, V.A.Reva ${ }^{l}$, P.Y. Shevelev ${ }^{l}$, A.V. Denisov ${ }^{l}$, \\ I.P. Yablokov' ${ }^{1}$, S.Y. Telickiy ${ }^{I}$, N.A. Zhirnoval, I.S. Zheleznyak ${ }^{2}$, \\ S.S. zBagnenko ${ }^{2}$, J.J. Morrison ${ }^{3}$
}

${ }^{1}$ War Surgery Department, Kirov Military Medical Academy, SaintPetersburg/RUSSIAN FEDERATION, ${ }^{2}$ Department Of Radiology, Kirov Military Medical Academy, Saint-Petersburg/RUSSIAN FEDERATION, ${ }^{3} \mathrm{R}$ Adams Cowley Shock Trauma Center, University of Maryland Medical System, Baltimore/MD/UNITED STATES OF AMERICA

Introduction: The aim of this study is to evaluate changes in intracranial pressure (ICP) during Resuscitative Endovascular Balloon Occlusion of the Aorta (REBOA) in an ovine model of hemorrhagic shock and severe traumatic brain injury (TBI).

Material and methods: Twelve sheep weighing 22-36 kg underwent cranial ballistic injury ( 83 Joules; range $1 \mathrm{~m}$ ) followed by induction of hemorrhagic shock (35\% blood volume over 30 minutes) and the insertion of an ICP monitor. Animals were randomized to 60 minutes of either REBOA and no-REBOA, followed by whole blood resuscitation and 24 hours of neuro-monitoring.

Results: Following severe TBI, ICP $(\mathrm{mmHg})$ increased comparably in both REBOA (10.4 [7.9-14.3] to 28.7 [18.4-54.2]; $\mathrm{p}<0.001)$ and no-REBOA (10.3 [5.0-16.4] to 29.0 [17.2-40.0]; $\mathrm{p}=0.001)$ groups. During the controlled hemorrhage, ICP gradually decreased to 15.0 [12.9-19.8] $(\mathrm{p}<0.001)$ in the REBOA group and to 20.5 [13.4-28.7] $(\mathrm{p}=0.633)$ in the no-REBOA group. REBOA resulted in a significant increase of ICP in 10 minutes after inflation and remained unchanged until deflation when ICP dropped from 31.1 [23.9-52.6] to 16.5 [13.524.9] $(\mathrm{p}=0.002)$. Mortality was high, at $5 / 6(83.3 \%)$ animals in each group, with evidence of severe subarachnoid hemorrhage and intracranial hematomas identified at autopsy.

Conclusion: REBOA results in temporary elevation of ICP and may exacerbate severe TBI. Future research should focus on ameliorating 
this effect, while sustaining the favourable hemodynamic profile observed with REBOA.

References:

Disclosure: No significant relationships.

\section{PR211}

\section{BENEFIT OF HYBRID OPERATIVE ROOM IN MANAGEMENT OF SEVERE SURGICAL EMERGENCY}

C. Laroye, J.R. Nzamushe, I. Plénier, D.H. Garrigue, E.F. Pourbaix

Emergency Surgery, CHRU LILLE, Lille/FRANCE

Introduction: In order to optimize the management of surgical emergency, Hybrid operating rooms (HOR) had been installed in our trauma center since 2014. The aim of this study is to evaluate the benefit of combined surgical and radiological treatment performed in this HOR for the emergency patients.

Material and methods: We reviewed all the emergency procedures done in the HOR since its opening. We focused on patients who underwent both radiological and surgical management in the same moment. We evaluate the mortality within three months regarding the majors groups of severe emergency which required this combined management: Polytrauma patients (PP), Mesentric ischemia (MI), and bleeding caused by anticoagulant medication (BAC). This mortality was compared to the predictive prognosis score TRISS (Trauma and Injury Severity Score) for the PP and to the literature data for the two others groups.

Results: From april 2015 till june 2017, 632 emergency patients underwent a procedure in the HOR. Eighteen patients benefited of combined radiological and surgical treatment: PP $(n=7)$, MI $(n=7)$, BAC $(\mathrm{n}=4)$. The mean ISS among PP was 66 . The mortality was $42,8 \%$ vs $85,5 \%$ predicted by the TRISS for the PP; $28,5 \%$ for MI and $25 \%$ for BAC. Following the literature data, the average mortality due to MI and BAC represent respectively $60-80 \%$ and $42 \%$. Conclusion: Our experience shows that HOR improve the prognosis of severe polytrauma and emergency patient who need both interventional radiology and surgical treatment. Nevertheless, further study included cost-effectiveness could represent a real interest.

References:

Disclosure: No significant relationships.

\section{PR212}

\section{A 10 YEAR EVOLUTION OF A MASSIVE TRANSFUSION PROTOCOL IN A LEVEL 1 CIVILIAN TRAUMA CENTER WITH MODERATE EXPOSURE TO MASSIVE HEMORRHAGE. HAVE OUTCOMES IMPROVED IN TRAUMA PATIENTS UNDERGOING MASSIVE TRANSFUSION?}

\section{J.E. Van Der Meij}

Department Of Surgery, Section Trauma Surgery, VU University Medical Centre, Amsterdam/NETHERLANDS

Introduction: We aimed to evaluate the evolution and implementation of the massive transfusion protocol (MTP) in a level 1 trauma center with moderate exposure to life threatening hemorrhage in trauma patients by analyzing broad endpoints and the effect of a protocol update.

Material and methods: A retrospective before-and-after study of prospectively collected data was performed over a 14-year period. Three groups of trauma patients, who received more than 10 units of packed red blood cells (PRBC), were compared. A group before introduction of the MTP (January 2002 - January 2006), a group after implementation of the MTP-I (February 2006 - February 2010), and a group after implementation of the updated MTP-II (March 2010 July 2016).

Results: A total of 168 patients were included in this study. 54 preMTP patients were compared to 47 MTP-I and 67 MTP-II patients. After introduction of the MTP-II, fewer units of PRBC and platelets were administered within the first 24 hours: respectively, 17 vs. 14 (p $=0.01)$ and 12 vs. $8(\mathrm{p}<0.001)$. No difference was found regarding fresh frozen plasma usage. Less infections were noted after introduction of the MTP-I: $51.9 \%$ vs. $31.9 \%(\mathrm{p}=0.04)$.

Conclusion: Introduction of a MTP-II in our level 1 civilian trauma center significantly reduced the amount of PRBC and platelets used during damage control resuscitation. Introduction of the MTP did not seem to directly impact survival nor the incidence of complications. Nevertheless, this study reflects the complexity of real life medicine in a level 1 civilian trauma center, with moderate exposure to life threatening traumatic hemorrhage.

References: 1. Kauvar DS, Lefering R, Wade CE. Impact of hemorrhage on trauma outcome: an overview of epidemiology, clinical presentations, and therapeutic considerations. Journal of Trauma and Acute Care Surgery 2006 Jun;60 (6 Suppl):S3-11. 2. Hoyt DB, Dutton RP, Hauser CJ. Management of coagulopathy in the patients with multiple injuries: results from an international survey of clinical practice. Journal of Trauma and Acute Care Surgery 2008;65: 755-765. 3. Malone DL, Hess JR, Fingerhut A. Massive transfusion practices around the globe and a suggestion for a common massive transfusion protocol. Journal of Trauma and Acute Care Surgery 2006; 60: S91-S96. 4. O’Keeffe T, Refaai M, Tchorz K. A massive transfusion protocol to decrease blood component use and costs. Archives of Surgery 2008; 143 (7):686-691. 5. Davenport R, Manson J, De'Ath H. Functional definition and characterization of acute traumatic coagulopathy. Crit Care Med. 2011;39 (12):2652-8. 6. Geeraedts Jr LMG, Demiral H, Schaap NP. 'Blind' transfusion of blood products in exsanguinating trauma patients. Resuscitation. 2007; 73: 382-388. 7. Gonzales EA, Moore FA, Holcomb JB. Fresh frozen plasma should be given earlier to patients requiring massive transfusion. Journal of Trauma and Acute Care Surgery 2007; 62: 112-119. 8. Gunter OL, Au BK, Isbell JM. Optimizing outcomes in damage control resuscitation: identifying blood product ratios associated with improved survival. Journal of Trauma and Acute Care Surgery 2008; 65: 527-534. 9. Biswadev Mitra B, O’Reilly G, Cameron PA. Effectiveness of massive transfusion protocols on mortality in trauma: a systematic review and meta-analysis. ANZ Journal of Surgery 2013; 83 (12), 918-923. 10. Geeraedts Jr LMG, Kamphuis SJM. Introduction of a massive transfusion protocol: a before-and-after study in a level 1 trauma center. Management of trauma patients with life-threatening hemorrhage. Academisch Proefschrift VUmc 2013;6:117-133. 11. Ledgerwood AM, Lucas CE. A review of studies on the effects of hemorrhagic shock and resuscitation on the coagulation profile. Journal of Trauma 2003;54 (5Suppl):S68-74. 12. Ho AMH, Karmakar MK, Dion PW. Are we giving enough coagulation factors during major trauma resuscitation?. American Journal of Surgery 2005;190 (3):479-84. 13. Ketchum L, Hess JR, Hiippala S. Indications for early fresh frozen plasma, cryoprecipitate, and platelet transfusion in trauma. Journal of Trauma 2006;60 (6Suppl):S51-8. 14. Moore FA, Nelson T, McKinley BA, Moore EE, Nathens AB, Rhee $\mathrm{P}$, et al. Is there a role for aggressive use of fresh frozen plasma in massive transfusion of civilian trauma patients?. American Journal of Surgery 2008;196 (6):948-60. 15. 
Hess JR, Dutton RB, Holcomb JB, Scalea TM. Giving plasma at a 1:1 ratio with red cells in resuscitation: who might benefit?. Transfusion 2008;48 (8):1763-5. 16. Laudi S, Donaubauer B, Busch T, Kernen T, Bercker S, Bail $\mathrm{H}$ et al. Low incidence of multiple organ failure after major trauma. Injury 2007;38: 1052-8. 17. Geeraedts LM Jr, Kaasjager HA, van Vugt AB. Exsanguination in trauma: A review of diagnostics and treatment options. Injury. 2009 Jan;40 (1):11-20. 18. Mitra B, O'Reilly G, Cameron PA. Effectiveness of massive transfusion protocols on mortality in trauma: a systematic review and meta-analysis. ANZ J Surg 2013;83 (12):918-23. 19. Cotton BA, Gunter OL, Isbell J. Damage control hematology: the impact of a trauma exsanguination protocol on survival and blood product utilization. Journal of Trauma 2008;64 (5):1177-82. 20. Dente CJ, Shaz $\mathrm{BH}$, Nicholas JM. Improvements in early mortality and coagulopathy are sustained better in patients with blunt trauma after institution of a massive transfusion protocol in a civilian level I trauma center. Journal of Trauma 2009;66 (6):1616-1624. 21. Holcomb JB, del Junco DJ, Fox EE. The Prospective, Observational, Multicenter, Major Trauma Transfusion ( (PROMMTT) Study: comparative effectiveness of a time- varying treatment with competing risks. Arch Surg 2012;1-10. 22. Johansson PI, Stensballe J: Hemostatic resuscitation for massive bleeding: the paradigm of plasma and platelets-a review of the current literature. Transfusion 2010;50:701-710. 23. Bawazeer M, Ahmed N, Izadi H. Compliance with a massive transfusion protocol (MTP) impacts patient outcome. Injury 2015;46 (1):21-28. 24. Maegele M, Lefering R, Paffrath T. Red-blood-cell to plasma ratios transfused during massive transfusion are associated with mortality in severe multiple injury: a retrospective analysis from the Trauma Registry of the Deutsche Gesellschaft fur Unfallchirurgie. Vox Sanguinis 2008;95 (2):112-119. 25. Riskin DJ, Tsai TC, Riskin L. Massive transfusion protocols: the role of aggressive resuscitation versus product ratio in mortality reduction. Journal of the American College of Surgeons 2009;209 (2):198-205. 26. Geeraedts jr LMG, Kamphuisen PW, Kaasjager HAH. The role of recombinant factor VIIa in the treatment of life-threatening haemorrhage in blunt trauma. Injury 2005;36:495-500. 27. Kenet G, Walden R, Eldad A. Treatment of traumatic bleeding with recombinant factor VIIa. Lancet 1999;354 (9193):1879. 28. Martinowitz U, Kenet G, Segal E. Recombinant activated factor VII for adjunctive haemorrhage control in trauma. Journal of Trauma 2001;51:431-8. 29. Lin Y. Moltzan CJ, Anderson DR. The evidence for the use of recombinant factor VIIa in massive bleeding: revision of the transfusion policy framework. Transfusion Medicine 2012;22 (6):383-94. 30. Hauser CJ, Boffard K, Dutton R. Results of the CONTROL trial: efficacy and safety of recombinant activated factor VII in the management of refractory traumatic hemorrhage. The Journal of Trauma 2010;69:489-500. 31. Boyd JM, Moore L, Atenafu EG. A retrospective cohort study of the relationship between quality indicator measurement and patient outcomes in adult trauma centers in the United States. Injury 2016 pii: S0020-1383 (16)30717-3. 32. Johansson PI, Oliveri R, Ostrowski SR. Hemostatic resuscitation with plasma and platelets in trauma. A meta-analysis. $J$ Emerg Trauma Shock 2012;5:120-125. 33. Enticott JC, Jeffcott S, Ibrahim JE. A review on decision support for massive transfusion: understanding human factors to support the implementation of complex interventions in trauma. Transfusion 2012;52 (12):2692-705. 34. Stensballe J, Ostrowski SR, Johansson PI. Haemostatic resuscitation in trauma: the next generation. Current Opinion in Critical Care 2016;22 (6):591-597. 35. Gonzalez E, Moore EE, Moore HB. Goaldirected hemostatic resuscitation of trauma- induced coagulopathy: a pragmatic randomized clinical trial comparing a viscoelastic assay to conventional coagulation assays. Ann Surg 2016;263:1051-1059.

Disclosure: No significant relationships.

\section{PR213}

\section{VASCULAR ACCES TRAINING FOR REBOA PLACEMENT: A FEASIBILITY STUDY}

\author{
B.L.s. Borger Van Der Burg ${ }^{1}$, T.M. Hörer ${ }^{2}$, D. Eefting ${ }^{3}$, \\ T.T.c.f. Van Dongen ${ }^{4}$, J.F. Hamming ${ }^{5}$, J.J. Dubose ${ }^{6}$, M. Bowyer ${ }^{7}$, \\ R. Hoencamp ${ }^{8}$
}

${ }^{1}$ Department Of Surgery, Alrijne Hospital, Leiderdorp/ NETHERLANDS, ${ }^{2}$ Department Of Cardiothoracic And Vascular Surgery, Örebro University Hospital, Örebro/SWEDEN, ${ }^{3}$ Surgery, Haaglanden Medical Center, Den Haag/NETHERLANDS, ${ }^{4}$ Defense Healthcare Organisation, Ministry of Defense, Utrecht/ NETHERLANDS, ${ }^{5}$ Surgery, Leiden University Medical Center, Leiden/NETHERLANDS, ${ }^{6}$ F. r Adam Cowley Shock Trauma Center, University of Maryland Medical System, Baltimore/MD/UNITED STATES OF AMERICA, ${ }^{7}$ Surgery, Uniformed Services University of the Health Sciences, Bethesda/MD/UNITED STATES OF AMERICA, ${ }^{8}$ Surgery, Central Military Hospital, Ministry of Defense, Utrecht/NETHERLANDS

Introduction: The use of resuscitative endovascular balloon occlusion of the aorta (REBOA) in patients with severe hemorrhagic shock is increasing. Obtaining vascular access is a necessity to have the possibility for REBOA placement in these situations.

Material and methods: During the EVTM workshop (September 2017, Orebro, Sweden) twenty-one individuals participated in this study, 16 participants and 5 instructors. A formalized curriculum was constructed including basic anatomy of the femoral region and basic training in access materials for REBOA placement in zone 1. Key skills were: (1) Preparation of endovascular toolkit, (2) achieving vascular access in the model and (3) bleeding control with REBOA. Scoring ranged from $0-5$ for non-anatomical skills. Identification of anatomical structures was either sufficient (score $=1$ ), or insufficient $($ score $=0)$. Five specialists performed a second identical procedure as a post test.

Results: Medical specialists had significant better overall technical skills in comparison to the surgical residents $(p=0.008)$, understanding of surgical anatomy showed no significant difference. Procedure times differed significantly $(\mathrm{p}<0.01)$, residents having a median procedure time of 3 minutes and 24 seconds, specialists $2: 33$ and instructors 1:09.

Conclusion: This comprehensive training model using a porcine model can be used for femoral access and REBOA placement training in medical personnel with different prior training levels. Higher levels of training limit procedure times. Further research in open and percutaneous access training is necessary to simulate real life situations. This model can be used in a multi-step training program, in combination with a realistic moulage model and cadaver flow model.

References: 1 . Stannard A, Eliason JL, Rasmussen TE. Resuscitative endovascular balloon occlusion of the aorta (REBOA) as an adjunct for hemorrhagic shock. J Trauma. 2011;71 (6):1869-72. 2. Brenner M, Hoehn M, Pasley J, Dubose J, Stein D, Scalea T. Basic endovascular skills for trauma course: bridging the gap between endovascular techniques and the acute care surgeon. J Trauma Acute Care Surg. 2014;77 (2):286-91. 3. Mackenzie CF, Garofalo E, Puche A, Chen H, Pugh K, Shackelford S, Tisherman S, Henry S, Bowyer MW, Retention, et al. Performance of Vascular Exposure and Fasciotomy Among Surgical Residents Before and After Training Compared With Experts. JAMA Surg. 2017;152 (6):1-8.

Disclosure: Prytime Medical Devices, Inc - The REBOA Company ${ }^{\mathrm{TM}}$ (Bourne, Texas, USA), provided the catheters used for this animal study. 
PR214

\section{REBOA TECHNIQUE DURING EMERGENCY TREATMENT FOR RUPTURED ABDOMINAL AORTIC ANEURYSMS. 5 YEARS EXPERIENCE}

N. Degermetzoglou ${ }^{1}, K$. Stavrides ${ }^{2}$, S. Theofanidou ${ }^{3}$, K. Tigkiropoulos ${ }^{2}, N$. Zacharopoulos ${ }^{2}$, M. Mantelas ${ }^{2}$, D. Karamanos ${ }^{2}$, I. Lazaridis $^{2}$, I. Bountouris ${ }^{1}$, N. Saratzis ${ }^{2}$

${ }^{1}$ Vascular Surgery Clinic, 251 HELLENIC AIR FORCE GENERAL HOSPITAL, ATHENS/GREECE, ${ }^{2}$ Vascular Surgery Department, AUTH 1st SURGERY CLINIC, PAPAGEORGIOU GENERAL HOSPITAL, THESSALONIKI/GREECE, ${ }^{3} 1 \mathrm{~b}$ Wing, AROGI REHABILITATION CENTRE, THESSALONIKI/GREECE

Introduction: Introduction: The use of REBOA technique during management of mass bleeding situations is of crucial impact for life salvage. We describe our experience in our clinics during the last 5 years using this technique during operation procedures for emergency treatment of ruptured abdominal aortic aneurysms (RAAAs).

Material and methods: Material and Method: During the last 5 years in our clinics 117 cases of RAAAs were treated, 45 with open repair and the rest 72 with EVAR. REBOA technique was used in all cases with the insertion of an endovascular occlusion balloon in the suprarenal aorta through percutaneous femoral access. Insertion of the balloon was made as soon as the patients reached the operation table, under fluoroscopic supervision with a C-ARM.

Results: Results: From all 117 patients, $79(67,5 \%)$ survived the operation. 56 patients $(47,8 \%)$ were discharged from hospital. The balloon was deflated when sealing of the RAAA took place. 22 patients suffered from visceral ischemia or abdominal compartment syndrome for which secondary operation was needed. Need for blood restoration and transfusion was decreased by a mean of $27 \%$ in EVAR and $38,6 \%$ in open repair, compared to the non use of REBOA during previous years cases. Analysis of the parameters measured is described.

Conclusion: Conclusions: The management and handling of mass casualties bleeding during RAAAs is vital for the prognosis of these cases in accordance to the repair technique. REBOA technique is a crucial procedure for minimizing blood loss and hemodynamic stabilization these patients until perfect seal and repair of the rupture takes place. Comparison between the results from and without its use made us consider it as an obligatory procedure, no matter which the kind of repair will be, in the emergency treatment of RAAAs.

References: 1. Brenner M, Teeter W, Hoehn M et al. Use of Resuscitative Endovascular Balloon Occlusion of the Aorta for Proximal Aortic Control in Patients With Severe Hemorrhage and Arrest. JAMA Surg. 2017 Sep 27. doi: 10.1001/jamasurg.2017.3549. 2. Ologun G, Sharpton K, Granet P. Successful use of resuscitative endovascular balloon occlusion of the aorta in the treatment of ruptured 8.5-cm splenic artery aneurysm. J Vasc Surg. 2017 Sep 25. pii: S0741-5214 (17)32192-4. doi: 10.1016/j.jvs.2017.05.138. 3. Taylor JR 3rd, Harvin JA, Martin C et al. Vascular complications from resuscitative endovascular balloon occlusion of the aorta: Life over limb? J Trauma Acute Care Surg. 2017 Jul;83 (1 Suppl 1):S120-S123. Disclosure: No significant relationships.
PR215

\section{ANATOMICAL LANDMARKS FOR SAFELY IMPLEMENTING RESUSCITATIVE BALLOON OCCLUSION OF THE AORTA (REBOA) IN ZONE 1 WITHOUT FLUOROSCOPY}

\author{
Y. Okada, W. Ishii, R. Iiduka
}

Emergency And Critical Care Medicine, Japanese Red Cross Society Kyoto Daini Hospital, Kyoto/JAPAN

Introduction: Resuscitative balloon occlusion of the aorta (REBOA) can maintain hemodynamic stability during hemorrhagic shock after a following torso injury, although inappropriate balloon placement may induce brain or visceral organ ischemia. External anatomical landmarks [the suprasternal notch (SSN) and xiphoid process $(\mathrm{Xi})$ ] are empirically used to implement REBOA in zone 1. We aimed to confirm if these landmarks were useful for determining a balloon catheter length for safe implementation of REBOA in zone 1 without using fluoroscopy.

Material and methods: We selected 25 successive adult blunt trauma cases requiring contrast-enhanced chest/abdominal computed tomography (CT) treated at our emergency department between October 1, 2016 and January 31, 2017. We retrospectively evaluated anonymized CT images. We used three-dimensional multiplanar reconstructions to measure the length along the aorta's central axis, from the bilateral common femoral arteries (FA) to the celiac trunk (CeT) (FA-CeT) and to the origin of the left subclavian artery (LSCA) (FA-LSCA). Volume-rendering reconstruction images were used to measure the external distance from common FAs to SSN (FA$\mathrm{SSN})$ and to $\mathrm{Xi}(\mathrm{FA}-\mathrm{Xi})$.

Results: FA-LSCA was significantly longer than FA-SSN. FA-CeT was significantly shorter than FA-Xi.

Conclusion: Based on these results, the REBOA balloon catheter should be shorter than FA-SSN, and longer than FA-Xi to avoid placement outside zone 1 . The advantages of this method are that it can rapidly and easily predict a safe balloon catheter length, and it reflects each patient's individual torso height. We believe that these anatomical landmarks are good references for safe implementation of REBOA in zone 1 without radiographic guidance.

References: Scandinavian Journal of Trauma, Resuscitation and Emergency Medicine201725:63

Disclosure: No significant relationships

\section{PR216}

\section{TECHNICAL ADVANCES OF THE COMBAT STUDY: HOTTER EQUALS FASTER FOR PLASMA IN THE AMBULANCE}

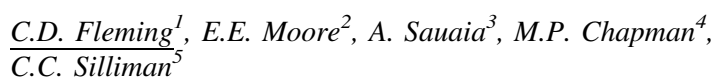

${ }^{1}$ Surgery/trauma Research, University of Colorado - Denver, Aurora/ CO/UNITED STATES OF AMERICA, ${ }^{2}$ Surgery, Denver Health Medical Center, Denver/CO/UNITED STATES OF AMERICA, 
${ }^{3}$ Department Of Surgery, University of Colorado Denver, Aurora/ UNITED STATES OF AMERICA, ${ }^{4}$ Vascular And Interventional Radiology, University of Colorado - Denver, Aurora/CO/UNITED STATES OF AMERICA, ${ }^{5}$ Pediatrics-hem Onc And Bone Marrow Transplantation, Children's Hospital Colorado, Aurora/CO/UNITED STATES OF AMERICA

Introduction: The recently completed COMBAT trial in the US validated the feasibility of delivering fresh frozen plasma (FFP) to trauma patients as the initial resuscitation fluid during ground ambulance transport. With transport times $<10$ minutes in urban areas, minimizing thawing times for FFP is critical, but this is limited by US regulations mandating that thawing be carried out at $37^{\circ} \mathrm{C}$. We hypothesized that thawing at higher temperatures would significantly decrease thawing times, without degrading clotting activity.

Material and methods: We thawed standardized units of FFP ( $\mathrm{n}=$ $10,223-253 \mathrm{~mL}$ ) in the Barkey Plasmatherm ${ }^{\circledR}$ device, of the type used in ambulances in the COMBAT trial. Thawing was conducted at 37 or $45^{\circ} \mathrm{C}$. Thawed FFP clotting activity was measured using the thrombelastography (TEG) reaction (R) times for the intrinsic (kaolin activated) and extrinsic (tissue factor activated) pathways.

Results: Thawing was approximately $40 \%$ faster (120 \pm 15 seconds) at $45{ }^{\circ} \mathrm{C}$ compared to $37{ }^{\circ} \mathrm{C}$ ( $200 \pm 10$ seconds). There was no significant difference in TEG R time between FFP thawed at high or low temperature. Kaolin (intrinsic pathway) yielded R times of $8.9 \pm$ 0.7 minutes for thawing at $45^{\circ} \mathrm{C}$, versus $9.3 \pm 1.8$ minutes for thawing at $37^{\circ} \mathrm{C}$. Tissue factor (extrinsic pathway) yielded $\mathrm{R}$ times of $1.7 \pm 0.1$ minutes for thawing at $45^{\circ} \mathrm{C}$, versus $1.8 \pm 0.4$ minutes for thawing at $37^{\circ} \mathrm{C}$.

Conclusion: Thawing FFP at higher temperatures of up to $45^{\circ} \mathrm{C}$ does not alter clotting activity, but is $40 \%$ faster compared to thawing at 37 ${ }^{\circ} \mathrm{C}$. This time savings is critical for FFP delivery in the ground ambulance or trauma bay.

References: 1. Chapman MP, Moore EE, Chin TL, Ghasabyan A, Chandler J, Stringham J, et al. Combat: Initial Experience with a Randomized Clinical Trial of Plasma-Based Resuscitation in the Field for Traumatic Hemorrhagic Shock. Shock. 2015 Aug;44 Suppl 1:6370. PubMed PMID: 25784527. Pubmed Central PMCID: 4504796. 2. D'Alessandro A, Moore HB, Moore EE, Wither MJ, Nemkov T, Morton AP, et al. Plasma First Resuscitation Reduces Lactate Acidosis, Enhances Redox Homeostasis, Amino Acid and Purine Catabolism in a Rat Model of Profound Hemorrhagic Shock. Shock. 2016 Aug;46 (2):173-82. PubMed PMID: 26863033. Pubmed Central PMCID: 4949141. 3. Davenport R, Curry N, Manson J, De'Ath H, Coates A, Rourke C, et al. Hemostatic effects of fresh frozen plasma may be maximal at red cell ratios of 1:2. The Journal of trauma. 2011 Jan;70 (1):90-5; discussion 5-6. PubMed PMID: 21217486. Epub 2011/01/11. eng. 4. Holcomb JB, Wade CE, Michalek JE, Chisholm GB, Zarzabal LA, Schreiber MA, et al. Increased plasma and platelet to red blood cell ratios improves outcome in 466 massively transfused civilian trauma patients. Annals of surgery. 2008 Sep;248 (3):447-58. PubMed PMID: 18791365. Epub 2008/09/16. eng.

Disclosure: No significant relationships.
PR217

\section{PROXIMAL HAEMODYNAMIC EFFECT OF TRANSITIONING FROM SHORT PERIOD RESUSCITIVE ENDOVASCULAR BALLOON OCCLUSION OF THE AORTA (REBOA) TO PARTIAL REBOA (P-REBOA) IN A PROGRESSIVE CONTROLLED HAEMORRHAGE SWINE MODEL}

E.R. Faulconer, M.A. Johnson, A. Davidson, M.A. Simon, E. Desoucy, E. Tibbits, G. Hoareau, L. Neff, T.K. Williams

Clinical Investigations Department, David Grant Medical Center, Travis USAFB/CA/UNITED STATES OF AMERICA

Introduction: Resuscitative Endovascular Balloon Occlusion of the Aorta (REBOA) increases proximal mean arterial pressure (pMAP) in haemorrhagic shock. Partial REBOA (P-REBOA) augments pMAP whilst maintaining distal perfusion to reduce ischaemia. The effects of transitioning from complete to partial REBOA are unknown. We report pMAP changes transitioning from short complete REBOA to P-REBOA in swine.

Material and methods: Methods: Five anaesthetised Yorkshire swine weighing $59-89 \mathrm{~kg}$ were prepared in a standard fashion with vascular access and an aortic flow sensor. Using an automated syringe driver to control balloon volume, animals were subjected to a series of phases of zone 1 REBOA and low flow P-REBOA in different states of haemodynamic shock $(0,10,20,30$ and $40 \%)$. Aortic flow, proximal and distal pressures and central venous pressures were recorded. After the $40 \%$ phase, animals were euthanized.

Results: During the long occlusive phase, all animals showed an initial rise in pMAP which plateaued before a secondary increase after 5 minutes. Two animals (59kg and $89 \mathrm{~kg}$ ) survived all phases of shock. In shock states of $0-30 \%$, these 2 animals showed a further rise in pMAP after transitioning from 3 minutes of REBOA to P-REBOA. A drop or no change in pMAP was observed in the 3 early deaths.

Conclusion: Transitioning from REBOA to P-REBOA after a short occlusion can lead to a temporary secondary rise in pMAP. This was associated with longer surviving animals and could be used as a good prognostic indicator during resuscitation. The mechanism behind this finding is unknown and warrants further investigation.

References:

Disclosure: No significant relationships.

\section{PR218}

\section{ISOLATED FEMORAL PRESSURE MONITORING CAN RELIABLY DEFINE A DEGREE OF OCCLUSION FOR PARTIAL REBOA: A COMPUTED TOMOGRAPHY STUDY ON LARGE ANIMALS}

V.A. Reva ${ }^{1}$, Y. Matsumura ${ }^{2}$, I.M. Samokhvalov ${ }^{1}$, A.A. Pochtarnik ${ }^{1}$, I.S. Zheleznyak ${ }^{3}$, E.M. Mikhailovskaya ${ }^{3}$, J.J. Morrison ${ }^{4}$

${ }^{1}$ War Surgery Department, Kirov Military Medical Academy, SaintPetersburg/RUSSIAN FEDERATION, ${ }^{2}$ Department Of Emergency And Critical Care Medicine, Chiba University Graduate School of Medicine, Chiba/JAPAN, ${ }^{3}$ Department Of Radiology, Kirov Military Medical Academy, Saint-Petersburg/RUSSIAN FEDERATION, ${ }^{4} \mathrm{R}$ Adams Cowley Shock Trauma Center, University of Maryland Medical System, Baltimore/MD/UNITED STATES OF AMERICA 
Introduction: The aim of this study is to define parameters that can reliably predict the degree of aortic occlusion during partial Resuscitative Endovascular Balloon Occlusion of the Aorta (pREBOA).

Material and methods: Six sheep weighing $35-46 \mathrm{~kg}$ were induced into hemorrhagic shock by exsanguination until SBP $<90 \mathrm{mmHg}$. Carotid (proximal) and femoral (distal) mean arterial pressure (MAP; $\mathrm{mmHg}$ ) was recorded while a REBOA balloon was inflated with saline in the thoracic aorta. CT angiography was performed following each $1 \mathrm{~mL}$ of balloon inflation to enable the percentage of occlusion to be calculated, which was correlated with femoral/carotid gradient, distal pressure and femoral pulse pressure.

Results: During incremental balloon inflation, proximal MAP increased and distal MAP decreased in agreement with the proportion of occlusion. At $>50 \%$ occlusion, the difference between the proximal and distal MAP was significantly different $(\mathrm{p}<0.001)$. The femoral/carotid pressure gradient linearly declined in accordance with percentage of occlusion and strongly correlated with distal MAP $\left(\mathrm{r}^{2}=\right.$ $0.988, \mathrm{p}<0.001)$. The femoral pulse pressure also decreased in agreement with the degree of occlusion $(p=0.048)$. Beyond $80 \%$ occlusion, there was a marked loss of pulsitility with a reduction in femoral pulse pressure (7.0 [5.5-16.5] to 2.0 [1.5-5.0]; $\mathrm{p}=0.028)$.

Conclusion: Femoral MAP and femoral pulse pressure may be reliable parameters that can help clinicians define the degree of aortic occlusion in pREBOA.

References:

Disclosure: No significant relationships.

\section{PR219}

\section{COMBAT RELATED TRUNCAL MAJOR HEMORRHAGE: WHAT ABOUT EVTM AND REBOA}

\author{
T.V. Dongen ${ }^{1}$, B.L.s. Borger Van Der Burg ${ }^{2}$, R. Hoencamp ${ }^{3}$
}

${ }^{1}$ Defence Healthcare Organisation, Ministry of Defence, Utrecht/ NETHERLANDS, ${ }^{2}$ Department Of Surgery, Alrijne Hospital, Leiderdorp/NETHERLANDS, ${ }^{3}$ Surgery, Central Military Hospital, Ministry of Defense, Utrecht/NETHERLANDS

Introduction: Major hemorrhage historically endures as the leading cause of preventable death, with truncal hemorrhage as major cause. During the Dutch deployment to Afghanistan (2006-2010) the Role 2E Medical Treatment Facility treated 307 major hemorrhage injuries. An adjunct in the initial treatment of major hemorrhage is the reinvented Resuscitative Endovascular Balloon Occlusion of the Aorta (REBOA). The aim of this study was to analyze potential indications for the use of REBOA as adjunct in resuscitation during the deployment of the Dutch Armed Forces in Afghanistan.

Material and methods: Cases were retrospectively collected, after the query of all major truncal hemorrhage during the deployment to Afghanistan 2006-2010. Thereafter all injuries were analyzed that would fit the criteria for REBOA use in prompt bleeding control, regardless of survivability.

Results: There were 208 possible indications for the use of REBOA. Of these 58 had a lethal outcome $(27.9 \%)$. Central thoracic/neck injuries accounted for $40 \%$ (83/208) of major vascular injuries and visceral injuries for $60 \%$.

Conclusion: Major hemorrhage sustained in combat are potentially salvageable when appropriate resuscitative techniques are used. REBOA could play a role as adjunct to manage major hemorrhage in a pre-hospital setting, especially in a remote environment. Solid registration is needed for identification and research, with the ultimate goal to save lives of wounded service members.
References: 1. Eastridge BJ, Mabry RL, Seguin P, Cantrell J, Tops T, Uribe P, Mallett O, Zubko T, Oetjen-Gerdes L, Rasmussen TE, et al. Death on the battlefield (2001-2011): implications for the future of combat casualty care. J Trauma Acute Care Surg. 2012;73 (6 Suppl 5):S431-7. 2. van Dongen TT, Idenburg FJ, Tan EC, Rasmussen TE, Hamming JF, Leenen LP, Hoencamp R. Combat related vascular injuries: Dutch experiences from a role 2 MTF in Afghanistan. Injury. 2016;47 (1):94-8. 3. Stannard A, Eliason JL, Rasmussen TE. Resuscitative endovascular balloon occlusion of the aorta (REBOA) as an adjunct for hemorrhagic shock. J Trauma. 2011;71 (6):1869-72. 4. Borger van der Burg BLS, van Dongen TTCF, Morrison JJ, Hedeman Joosten PPA, DuBose JJ, Hörer TM, Hoencamp R. A systematic review and meta-analysis of the use of resuscitative endovascular balloon occlusion of the aorta in the management of major exsanguination. Submitted. 2017.

Disclosure: No significant relationships.

\section{PR220}

\section{NON-TRAUMATIC HEMORRHAGE CAN BE CONTROLLED WITH REBOA IN ACUTE PHASE THEN MORTALITY INCREASES GRADUALLY BY NON-HEMORRHAGIC DEATH}

\author{
Y. Matsumura ${ }^{1}$, J. Matsumoto $^{2}$, K. Idoguchi $^{3}$, H. Kondo $^{4}$, T. Ishida ${ }^{5}$ \\ Y. Kon , K. Tomita ${ }^{1}$, K. Ishida ${ }^{7}$, T. Hirose ${ }^{8}$, K. Umakoshi ${ }^{9}$, \\ T. Funabiki $i^{10}$
}

${ }^{1}$ Department Of Emergency And Critical Care Medicine, Chiba University Graduate School of Medicine, Chiba/JAPAN, ${ }^{2}$. Department Of Emergency And Critical Care Medicine, St. Marianna University School of Medicine, Kawasaki/JAPAN, ${ }^{3}$ )senshu Trauma And Critical Care Center, Rinku General Medical Center, Izumisano/ JAPAN, ${ }^{4}$ Department Of Radiology, Teikyo University School of Medicine, Itabashi/JAPAN, ${ }^{5}$ Emergency And Critical Care Center, Ohta Nishinouchi Hospital, Koriyama/JAPAN, ${ }^{6}$ Emergency And Critical Care Center, Hachinohe City Hospital, Hachinohe/JAPAN, ${ }^{7}$ Department Of Acute Medicine And Critical Care Medical Center, Osaka National Hospital, Osaka/JAPAN, ${ }^{8}$ Department Of Traumatology And Acute Critical Medicine, Osaka University Graduate School of Medicine, Suita/JAPAN, ${ }^{9}$ Department Of Emergency And Critical Care Medicine, Ehime University Graduate School of Medicine, Ehime/JAPAN, ${ }^{10}$ Emergency And Critical Care Center, Saiseikai Yokohamashi Tobu Hospital, Yokohama/JAPAN

Introduction: Resuscitative endovascular balloon occlusion of the aorta (REBOA) is now a feasible and less invasive resuscitation procedure. This study aimed to compare the clinical course of trauma and non-trauma patients undergoing REBOA.

Material and methods: Demographics, etiology, bleeding sites, hemodynamic response, length of critical care, and cause of death were recorded. Characteristics and outcomes were compared between non-trauma and trauma patients. Survival analysis was also conducted.

Results: From August 2011 to December 2015, 142 cases were analyzed (36 non-trauma; 106 trauma). The abdomen was a common bleeding site $(69 \%)$, followed by the pelvis or extra-pelvic retroperitoneum. None of the non-trauma patients had multiple bleeding sites, whereas $45 \%$ of trauma patients did $(\mathrm{P}<0.001)$. Nontrauma patients presented a lower 24 -h mortality ( 19 vs. $51 \%, \mathrm{P}=$ $0.001)$. The non-trauma cases demonstrated a gradual but prolonged increased mortality, whereas survival in trauma cases rapidly declined $(\mathrm{P}=0.009)$. Non-trauma patients who survived for $24 \mathrm{~h}$ had 0 ventilator-free days and 0 ICU-free days vs. a median of 19 and 12, respectively, for trauma patients $(\mathrm{P}=0.33$ and 0.39 , respectively). 
Non-hemorrhagic death was more common in non-trauma patients (83 vs. $33 \%, \mathrm{P}<0.001)$.

Conclusion: Non-traumatic hemorrhagic shock often resulted from a single bleeding site, and resulted in better 24-h survival than traumatic hemorrhage among Japanese patients who underwent REBOA. However, hospital mortality increased steadily in non-trauma patients affected by non-hemorrhagic causes after a longer period of critical care.

\section{References:}

Disclosure: Yosuke Matsumura used to be a member of clinical advisory board member of Tokai Medical Products. None of the other authors have any conflicts of interest to declare.

\section{EDUCATION}

\section{PR221}

\section{SIMULATION TRAINING OF NON-TECHNICAL SKILLS FOR THE ACUTE MEDICINE}

\section{N. Shinyama, T. Noda, T. Hagiwara, Y. Mizobata}

Traumatology And Critical Care Medicine, Osaka City University, Osaka/JAPAN

Introduction: For teamwork to yield expected results, training of non-technical skills of the health-care providers is essential. We produced the simulation-based training of non-technical skills for the acute medicine (NoTAM) course. The aim of this study is to evaluate the effects of the NoTAM course for the clinical activities in the emergency department.

Material and methods: NoTAM course is the three-hour program, composed by didactic lecture, demonstration video, group discussion, and two case scenarios. Two attending physicians, one or two intern residents, and two nurses participated as a team. Between October and December in 2016, six NoTAM courses were carried for six teams. The surveys concerning the team performance were investigated. Primary treatments were recorded before $(n=27)$ and after $(n=27)$ the NoTAM course implementation. The performances of the patient care and non-technical skills were scored.

Results: The answer of the survey demonstrated that all the disciplines concern about the poor communication, leadership, and team performance in the daily practice. After the NoTAM course, they understood the team dynamics and predicted to be able to perform better non-technical skills. However, the performance scores did not show any changes in patient assessment, in non-technical skills, nor in total performance. Only the score of the briefing improved (2.1 [1.7 $2.7]$ vs. $2.4[2.1-2.9], \mathrm{p}=0.038$; pre vs. post, median $[25 \%-75 \%$ IQR]).

Conclusion: The teamwork training brought better understanding of the team management. The changes of the performances in the daily practical activities, however, may require repeated trainings and practical trials in the daily patient care.

References:

Disclosure: No significant relationships.
PR222

\section{PREHOSPITAL PREDICTION OF THORACIC INJURY BY EMERGENCY MEDICAL SERVICES PROVIDERS IN TRAUMA PATIENTS IN THE NETHERLANDS}

\author{
$\underline{\text { E.A.j.Van Rein }}^{1}$, R. Van Der Sluijs ${ }^{1}$, J. Hjortnaes ${ }^{2}$, R.A. Lichtveld ${ }^{3}$, \\ L.P.h. Leenen ${ }^{I}$, M. Van Heijl
}

${ }^{1}$ Traumatology, University Medical Center Utrecht, Utrecht/ NETHERLANDS, ${ }^{2}$ Cardiothoracic Surgery, University Medical Center Utrecht, Utrecht/NETHERLANDS, ${ }^{3}$ Regional Ambulance Facilities Utrecht, Regional Ambulance Facilities Utrecht, Bilthoven/ NETHERLANDS

Introduction: Severe thoracic injuries are time sensitive and triage to a facility with a high-level of trauma care is crucial. ${ }^{1}$ The emergency medical services (EMS) providers must identify patients with a severe thoracic injury and transport them to the right hospital. However, identifying these patients is challenging. ${ }^{2-4}$ The accuracy of the prehospital assessment of potential thoracic injury by EMS providers of the ground ambulance is unknown. Therefore, the aim of this study is to analyze the diagnostic value of the EMS provider judgment in the identification of a thoracic injury.

Material and methods: In this multicenter cohort study, all trauma patients aged 16 and over, transported with a ground ambulance to an emergency department in the Central Netherlands region, were evaluated. The primary outcome was the diagnostic value of EMS provider judgment in the identification of a thoracic injury. Thoracic injury according to the Abbreviated Injury Scale diagnosed at the trauma center was used as reference standard. The diagnostic value was determined using sensitivity and specificity.

Results: In total 2,766 patients were included, of which 465 (17\%) sustained a thoracic injury. Among the patients with a thoracic injury, the EMS providers suspected a thoracic injury in 52\%. The EMS providers' judgment had a sensitivity of $48 \%$ and a specificity of $93 \%$ for a thoracic injury.

Conclusion: The sensitivity for detection of the presence of thoracic injury by EMS providers needs improvement. Future research should focus on means -such as EMS provider education- to improve prehospital trauma triage of patients with a thoracic injury.

References: 1. Yu W, Chen H, Deng Q, Kang P, Zhang L. Comparison of influencing factors on outcomes of single and multiple road traffic injuries: A regional study in Shanghai, China (2011-2014). PLoS One. 2017;12:e0176907. 2. Candefjord S, Beundia R, Caragounis EC, Sjögvist BA, Fagerlind H. Prehospital transportation decisions for patients sustaining major trauma in road traffic crashes in Sweden. Traffic Inj Prev. 2016;17:16-20. 3. Scheetz LJ. Comparison of type and severity of major injuries among undertriaged and correctly triaged older patients. J Emerg Med. 2012;43:1020-1028. 4. Muhm M, Danko T, Madler C, Winkler H. [Preclinical prediction of prehospital injury severity by emergency physicians : approach to evaluate validity]. Anaesthesist. 2011;60:534-540.

Disclosure: No significant relationships. 
PR223

\section{NURSING WORKLOAD AND ASSESSMENT OF TRIAGE FUNCTION AT THE MIXED, (TRAUMA)-SURGICAL INTERMEDIATE CARE UNIT}

\section{J. Plate, L.P.h. Leenen, R.M. Houwert, F. Hietbrink}

Traumatology, University Medical Center Utrecht, Utrecht/ NETHERLANDS

Introduction: An important critique regarding the use of Intermediate Care Units (IMCU) is that they potentially admit patients who would otherwise be cared for on the regular ward. This would lead to an undesired waste of critical care resources. The objective of this study was to (1) describe the caseload and (2) assess the triage function at the IMCU.

Material and methods: This cohort study included all admissions from 2001 to 2015 at the mixed, surgical IMCU supervised by trauma-surgeons. The tertiary referral hospital serves as a level-1 trauma center. The Therapeutic Intervention Scoring System - 28 (TISS-28) was prospectively collected for all admissions, to describe the caseload at the IMCU and formulate medical criteria. These were combined with logistical criteria to assess the triage function at the IMCU.

Results: A total of 8816 admissions were included in the study. The average TISS-28 was 20.19 (CI 18.05-22.33), corresponding with 3.57 (CI 3.19-3.94) hours of direct patient-related work per patient per nursing shift. Over time, this increased with on average 0.27 points/ year $(\mathrm{p}<0.001)$. Of all admissions, $6539(74.17 \%)$ received medical care which could not have been provided at the hospital ward, whereas $7093(80.46 \%)$ was correctly admitted for logistical reasons. With these criteria combined, a total of 8324 (94.42\%) was correctly admitted.

Conclusion: The IMCU has the potential to admit patients with a high - and still increasing - nursing workload. Admissions are nearly always medically and logistically necessary. This finding contradicts the critique that the IMCU serves as a general hospital ward.

References: 1. Vincent JL, Rubenfeld GD. Does intermediate care improve patient outcomes or reduce costs? Crit Care. 2015;19:89. 2. Plate JDJ, Leenen LPH, Houwert M, Hietbrink F. Utilisation of Intermediate Care Units: A Systematic Review. Crit Care Res Pract. 2017:8038460. 3. Rosenthal GE, Sirio CA, Shepardson LB, Harper DL, Rotondi AJ, Cooper GS. Use of Intensive Care Units for Patients With Low Severity of Illness. Arch Intern Med. 1998;158:1133-51. 4. Arabi Y, Venkates S, Haddad S, Al Malik S, Al Shimemeri A. The characteristics of very short stay ICU admissions and implications for optimizing ICU resource utilization: the Saudi experience. International Journal for Quality in Health Care. 2004;16 (2). 5. Pirret AM. Utilizing TISS to differentiate between intensive care and high-dependency patients and to identify nursing skill requirements. Intensive and Critical Care Nursing. 2002;18 (1):19-26. 6. Zimmerman JE, Wagner DP, Knaus WA, Williams JF, Kolakowski D, Draper EA. The use of risk predictions to identify candidates for intermediate care units: implications for intensive care utilization and cost (Structured abstract). Chest. 1995;108:490-9. 7. Cullen DJ, Civetta JM, Briggs BA, Ferrara LC. Therapeutic intervention scoring system: a method for quantitative comparison of patient care. Crit Care Med. 1974;2 (2):57-60. 8. Miranda DR, De Rijk A, Schaufeli W. Simplified Therapeutic Intervention Scoring System: the TISS-28 items-results from a multicenter study. Crit Care Med. 1997;24 (1):64-73. 9. Garfield M, Jeffrey R, Ridley S. An assessment of the staffing level required for a high-dependency unit. Anaesthesia. 2000;55:137-43. 10. Yoo EJ, Damaghi N, Shakespeare WG, Sherman MS. The effect of physician staffing model on patient outcomes in a medical progressive care unit. J Crit Care. 2015;191. 11. Plate JD, Leenen LL, Houwert M, Hietbrink F. The Joint Management Format at the Intermediate Care Unit: An Interrupted Time Series Analysis. . 2017. 12. R Core Team. R: A Language and Environment for Statistical Computing. $R$ Foundation for Statistical Computing, Vienna, Austria. 2017. 13. van Buuren S, Groothuis-Oudshoorn K. Mice: Multivariate Imputation by Chained Equations in R. . Journal of Statistical Software. 2011;45 (3):1-67. 14. S original and from StatLib and by Rob Tibshirani R port by Friedrich Leisch. (2017). Bootstrap: Functions for the Book "An Introduction to the Bootstrap". R package version 2017. 15. Wickham H. ggplot2: Elegant Graphics for Data Analysis. Springer-Verlag New York. 2009;2009.

Disclosure: No significant relationships.

\section{PR224}

\section{MEASURING THE EFFECTIVENESS OF THE PREVENTION DAY PREVENT ALCOHOL AND RISK-RELATED TRAUMA IN YOUTH (P.A.R.T.Y.) AT AN UNIVERSITY HOSPITAL}

\section{E. Henning, T. Görig, R. Wendorf, S. Schulz-Drost, A. Ekkernkamp, D. Gümbel}

Department Of Trauma, Reconstructive Surgery And Rehabilitation Medicine, University Medicine Greifswald, Greifswald/GERMANY

Introduction: Reducing alcohol related harm in youth is a priority regarding the current accident statistics in Germany. However, there are few evidence-based prevention programs available for adolescents. The study aims to evaluate the P.A.R.T.Y. program at an university hospital. The main question is, increase the P.A.R.T.Y. program the awareness of alcohol and other risks in youth?

Material and methods: During an one-day course young people within the age of 13 to 17 got an overview over the treatment of an accident victim starting at the ambulance and ending at the rehabilitation centre. A systematic pre and post evaluation of the day was made by a structured written questionnaire.

Results: A total of 497 pupils from the grade 8-10 participated in the P.A.R.T.Y. program between 2013 and 2016. The average age of the youth was 15 years. After the program a higher number of the participants rated that they use the seatbelt always or frequent $(91,1 \%$ versus $95,2 \%$ ). Before the project $76 \%$ of the pupils mentioned that driving too fast is dangerous and $35,5 \%$ thought that riding a bike without a helmet is dangerous. After the project $83,5 \%$ consider fast driving as dangerous and $47 \%$ riding a bike without a helmet.

Conclusion: First results of the P.A.R.T.Y. program shows that the program endorses the accident prevention in youth. Specifically, it increases the risk awareness of the participated youth.

Disclosure: No significant relationships.

\section{PR226}

\section{REGULAR DAY-TIME SERVICE IN A UNIT FOR ACUTE CARE SURGERY MIGHT IMPROVE RESULTS FOR EMERGENCY PROCEDURES ON-CALL TIME}

\author{
I. Spanjol Pandelo ${ }^{1}$, K. Holmgren ${ }^{2}$, S. Redeen ${ }^{1}$, R. Sjodahl ${ }^{1}$, \\ P. Andersson ${ }^{3}$
}

${ }^{1}$ Depatment Of Surgery, University Hospial Linkoping, Linkoping/ SWEDEN, ${ }^{2}$ Department Of Primary Health Care, General Practice, Linkoping/SWEDEN, ${ }^{3}$ Department Of Surgery, Vrinnevi Hospital, 
Norrköping/SWEDEN

Introduction: In the era of highly specialized surgery, acute care surgery has evolved as a subspecialty aiming to improve emergency surgery. We investigated if there was any difference in outcomes after emergency surgery on-call time between surgeons working predominantly with either acute care surgery or elective surgery during daytime.

Material and methods: Prospectively collected routine register-data from 2011-2015 on emergency surgery performed on-call time by specialist surgeons were obtained. Surgeons were classified into acute care surgeons (A) or elective surgeons (E) based on service. Retrospectively postoperative 30-day morbidity according to ClavienDindo was analyzed. Appendectomy and repair of inguinal/femoral/ umbilical hernias were assigned minor, cholecystectomy and repair of incisional abdominal wall hernia intermediate and all regular laparotomies whether combined with bowel resection or not as well as all hernia repairs with bowel-resection were assigned major complexity. Differences in postoperative morbidity between groups were analyzed adjusted for confounders as sex, age, specialist-years, complexity and ASA by using logistic regression.

Results: 1588 operations were analyzed. Patients' median age was 50 (0-99), 790 were females. Postoperative morbidity between groups (A vs E) for all procedures did not differ for Clavien-Dindo 1-5 or Clavien-Dindo $\geq 3$ (OR 0.82; CI 0.61-1.10 and OR 0.84; CI 0.54-1.31 respectively) nor separately for minor-, intermediate-, and majorcomplexity procedures regarding Clavien-Dindo 1-5. However, for intermediate-complexity procedures results differed for ClavienDindo $\geq 3$ with less morbidity following surgery by acute care surgeons (OR 0.25; CI 0.07-0.90).

Conclusion: Working predominantly with acute care surgery daytime might improve results on-call time for emergent intermediatecomplexity procedures.

References:

Disclosure: No significant relationships.

\section{PR227}

\section{THE FRENCH APPLIED COURSE FOR DEPLOYMENT VASCULAR SURGERY (ACDVS) TRAINING OF MILITARY SURGEONS AT VASCULAR REPAIR ON ANATOMICAL PULSATILE SIMULATORS}

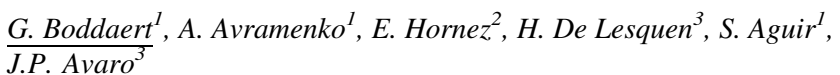

${ }^{1}$ Thoracic And Vascular Department, Percy Military Hospital, Clamart/FRANCE, ${ }^{2}$ Department Of Digestive Surgery, Percy Military Hospital, Clamart/FRANCE, ${ }^{3}$ Thoracic And Vascular Department, Sainte Anne Military Hospital, Toulon/FRANCE

Introduction: The increasing of the incidence of vascular trauma in warfare requires the knowledge and skill of vascular repair techniques by any deployed military surgeon. Applied Course for Deployment Vascular Surgery (ACDVS), based on the reproduction and repetition of gestures on anatomical pulsatile simulators, was created to meet this imperative. The aim of this work is to evaluate the relevance and results of this training through the participants' experience.

Material and methods: A retrospective analysis of 32 participants was conducted. Demographic data and prior surgical experience of participants were collected, self-Efficacy Questionnaires (SEQ) were completed before and after the course and a general course evaluation conducted.
Results: The mean age of the participants was $34 \pm 6.2$ years. The majority were men $(n=28,88 \%)$. The distribution of participants was mainly divided between visceral surgeons $17(53 \%)$ and orthopedists $13(40 \%)$. The majority $(\mathrm{n}=24,75 \%)$ had previously sone less than 5 vascular repairs. The average pre-course self-efficacy score was $2.7 \pm$ 0.4 and the average post-test score was $3.8 \pm 0.4$ on a scale of 5 , corresponding to a statistically significant increase $(\mathrm{p}<0.05)$. The majority of students were extremely satisfied with the training with an average score of $4.8 \pm 0.4$ on a scale of 5 .

Conclusion: Vascular trauma repair can be efficiently taught to nonvascular surgeons during a practical course on anatomical pulsatile simulators.

References: Simulation, surgery, vascular repair, trauma.

Disclosure: We declare conflict of interest with the society Vascular International School AG (Stansstad, Switzerland).

\section{PR228}

\section{THE VOLUME-OUTCOME RELATIONSHIP IN SEVERELY INJURED PATIENTS: A SYSTEMATIC REVIEW AND META- ANALYSIS}

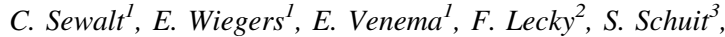 \\ D. Den Hartog ${ }^{4}, H$. Lingsma
}

${ }^{1}$ Public Health, Erasmus MC, Rotterdam/NETHERLANDS, ${ }^{2}$ School Of Health And Related Research, University of Sheffield, Sheffield/ UNITED KINGDOM, ${ }^{3}$ Emergency Medicine, Erasmus MC, Rotterdam/NETHERLANDS, ${ }^{4}$ Surgery, Erasmus MC, Rotterdam/ NETHERLANDS

Introduction: The volume-outcome relationship in severely injured patients remains under debate. In this systematic review and metaanalysis the aim was to evaluate the relationship between hospital or surgeon volume and health outcomes in severely injured patients.

Material and methods: Six electronic databases were searched up to February 2017 to identify studies that describe the relationship between hospital or surgeon volume and health outcomes in severely injured patients. Selection of relevant studies, data extraction and critical appraisal of the methodological quality were performed by two independent reviewers. Pooled adjusted and unadjusted estimates of the effect of volume on in-hospital mortality were calculated with random-effects meta-analysis. A mixed effects linear regression model was used to assess hospital volume in a continuous way.

Results: Seventeen observational cohort studies were included ${ }^{1-17}$. The majority of these studies reported an association between higher hospital or surgeon volume and lower mortality rate. Eight studies were included in the meta-analysis with a total of 222,418 patients ${ }^{1-5,9,10}$. High hospital volume (240 or more admitted severely injured patients per year) was associated with a lower risk of mortality (adjusted OR $0.85,95 \%$ CI $0.76-0.94$ ). Twelve studies were included in the regression model, providing a beta of -0.17 per 10 patients $(95 \% \mathrm{CI}-0.27--0.07, \mathrm{p}<0.01)$. There was no clear association between surgeon volume and mortality rates based on three available studies.

Conclusion: Our systematic overview reveals a modest association between high volume centers and lower mortality in severely injured patients, suggesting that designation of high volume centers might improve outcomes among severely injured patients.

References: 1. Bennett KM, Vaslef S, Pappas TN, Scarborough JE. The volume-outcomes relationship for United States Level I trauma centers. J Surg Res. 2011;167 (1):19-23. 2. Cooper A, Hannan EL, Bessey PQ, Farrell LS, Cayten CG, Mottley L. An examination of the 
volume-mortality relationship for New York State trauma centers. J Trauma. 2000;48 (1):16-23; discussion -4. 3. Cudnik MT, Newgard CD, Sayre MR, Steinberg SM. Level I versus Level II trauma centers: an outcomes-based assessment. J Trauma. 2009;66 (5):1321-6. 4. Demetriades D, Martin M, Salim A, Rhee P, Brown C, Chan L. The effect of trauma center designation and trauma volume on outcome in specific severe injuries. Ann Surg. 2005;242 (4):512-7; discussion 7-9. 5. Glance LG, Osler TM, Dick A, Mukamel D. The relation between trauma center outcome and volume in the National Trauma Databank. J Trauma. 2004;56 (3):682-90. 6. Konvolinka CW, Copes WS, Sacco WJ. Institution and per-surgeon volume versus survival outcome in Pennsylvania's trauma centers. Am J Surg. 1995;170 (4):333-40. 7. London JA, Battistella FD. Is there a relationship between trauma center volume and mortality? J Trauma. 2003;54 (1):16-24; discussion -5. 8. Lucas CE, Buechter KJ, Coscia RL, Hurst JM, Lane V, Meredith JW, et al. The effect of trauma program registry on reported mortality rates. J Trauma. 2001;51 (6):1122-6; discussion 6-7. 9. Margulies DR, Cryer HG, McArthur DL, Lee SS, Bongard FS, Fleming AW. Patient volume per surgeon does not predict survival in adult level I trauma centers. J Trauma. 2001;50 (4):597-601; discussion -3. 10. Marx WH, Simon R, O’Neill P, Shapiro MJ, Cooper AC, Farrell LS, et al. The relationship between annual hospital volume of trauma patients and in-hospital mortality in New York State. J Trauma. 2011;71 (2):339-45; discussion 45-6. 11. Nathens AB, Jurkovich GJ, Maier RV, Grossman DC, MacKenzie EJ, Moore $\mathrm{M}$, et al. Relationship between trauma center volume and outcomes. JAMA. 2001;285 (9):1164-71. 12. Olufajo OA, Metcalfe D, Rios-Diaz A, Lilley E, Havens JM, Kelly E, et al. Does Hospital Experience Rather than Volume Improve Outcomes in Geriatric Trauma Patients? J Am Coll Surg. 2016;223 (1):32-40 e1. 13. Sava J, Kennedy S, Jordan M, Wang D. Does volume matter? The effect of trauma surgeons' caseload on mortality. J Trauma. 2003;54 (5):82933; discussion 33-4. 14. Zacher MT, Kanz KG, Hanschen M, Haberle $\mathrm{S}$, van Griensven M, Lefering R, et al. Association between volume of severely injured patients and mortality in German trauma hospitals. Br J Surg. 2015;102 (10):1213-9. 15. Minei JP, Fabian TC, Guffey DM, Newgard CD, Bulger EM, Brasel KJ, et al. Increased trauma center volume is associated with improved survival after severe injury: results of a Resuscitation Outcomes Consortium study. Ann Surg. 2014;260 (3):456-64; discussion 64-5. 16. Freeman J, Nicholl J, Turner J. Does size matter? The relationship between volume and outcome in the care of major trauma. J Health Serv Res Policy. 2006;11 (2):101-5. 17. Pasquale MD, Peitzman AB, Bednarski J, Wasser TE. Outcome analysis of Pennsylvania trauma centers: factors predictive of nonsurvival in seriously injured patients. J Trauma. 2001;50 (3):465-72; discussion 73-4.

Disclosure: No significant relationships.

\section{PR229}

\section{IMPROVING ACUTE CARE SURGICAL ADMISSIONS; A TOTAL QUALITY MANAGEMENT APPROACH}

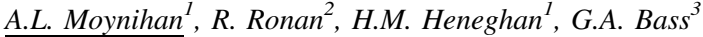

${ }^{1}$ Dept Of Surgery, St. Vincent's University Hospital, Dublin/IRELAND, ${ }^{2}$ Dept Of Nursing, St. Vincent's University Hospital, Dublin/ IRELAND, ${ }^{3}$ Dept. Of Surgery, St. Vincent's University Hospital, Dublin/IRELAND

Introduction: Standardising patient care, based on evidence-based guidelines, has been shown to improve patient outcomes. The exigencies of acute care surgery are such, however, that rigidlyprotocolled patient care is seldom possible. The quality of the process flow in admitting an unscheduled emergency surgery patient is variable and gaps in care can occur. We aimed to process-map the key points in initial surgical assessment and identify quality-improvement (QI) opportunities in our institution.

Material and methods: A standardised Acute Care Surgical Admission pro forma was introduced following physician and nursing stake-holder buy-in. A PDSA cycle was implemented and end-user experience was surveyed in order to close the QI audit loop.

Results: Documentation from 50 consecutive emergency surgical admissions to the Acute Surgical Unit (ASU) were assessed for completeness of documentation, adherence to NSQuIP guidelines and clarity of initial care plan. The pro forma was then introduced for a pilot period and 50 consecutive admissions were similarly graded. An anonymous survey of surgical residents and ASU and ED nursing staff was undertaken to assess satisfaction with the process. Iterative improvements were informed by qualitative and quantitative feedback.

Conclusion: Quality-improvement initiatives, often an aggregation of marginal gains, are important in acute surgical care delivery. We report our experience of implementation of a standardised admission pro forma designed to stream-line admission, error trap, and provide consistent, effective communication between care providers.

References:,

Disclosure: No significant relationships.

\section{PR230}

\section{A BOMB ATTACK IN THE NETHERLANDS; WHAT IS THE ROLE OF THE MAJOR INCIDENT HOSPITAL?}

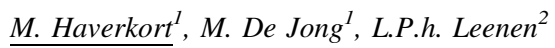

${ }^{1}$ Major Incident Hospital, University Medical Center Utrecht, Utrecht/NETHERLANDS, ${ }^{2}$ Traumatology, University Medical Center Utrecht, Utrecht/NETHERLANDS

Introduction: There is an ever-present threat of large-scale incidents and disasters, such as terrorism and natural disasters. The Netherlands has constructed a specific Major Incident Hospital (MIH) in order to cope with such incidents. This presentation explains the role of the MIH in the Dutch healthcare system.

Material and methods: Preparation and planning are the key to successful response to major incidents and disasters, which is why education, training sessions and exercises take place in the Netherlands.

Results: Analysis of recent large-scale incidents in Europe and the USA emphasises the importance of adequate distribution of the wounded to centres where the correct care can be provided.- A major step has been taken in the Netherlands to provide for such an eventuality with the introduction of distribution plans for the wounded, and other initiatives such as a regional hospital disaster plan.- If a large-scale incident should take place the Netherlands also has a Major Incident Hospital at its disposal; this is a unique facility that can guarantee availability of 200 spare beds to the Netherlands healthcare system within 30 minutes.

Conclusion: The MIH takes a central role in the Dutch major incident preparedness.The institute guarantees an additional capacity as well as expertise in preparedness.

References:

Disclosure: No significant relationships. 
PR231

\section{ADVANCED SURGICAL SKILLS FOR EXPOSURE IN TRAUMA COURSE: EXPERIENCES IN JAPAN}

\author{
K. Morishita ${ }^{1}, K$. Matsuhima $^{2}, R$. Abe $^{3}$, T. Suzuki $^{4}, K$. Hondo $^{1}$, \\ M. Bowyer ${ }^{5}$, K. Yanaga ${ }^{6}$, S. Oda ${ }^{3}$, Y. Otomo ${ }^{1}$
}

\begin{abstract}
${ }^{1}$ Trauma And Acute Critical Care Medical Center, Tokyo Medical and Dental University, Medical Hospital, Tokyo/JAPAN, ${ }^{2}$ Surgery, LAC+USC Medical Center, Los Angeles/CA/UNITED STATES OF AMERICA, ${ }^{3}$ Emergency And Critical Care Medicine, Chiba University Graduate School of Medicine, Chiba/JAPAN, ${ }^{4}$ Department Of Bioenvironmental Medicine (anatomy), Chiba University Graduate School of Medicine, Chiba/JAPAN, ${ }^{5}$ Surgery, Uniformed Services University of the Health Sciences, Bethesda/MD/UNITED STATES OF AMERICA, ${ }^{6}$ Surgery, Jikei University Graduate School of Medicine, Tokyo/JAPAN
\end{abstract}

Introduction: In Japan, there has been a significant reduction in open operative experience for trauma surgeons, necessitating additional training in these skills. The Advanced Surgical Skills for Exposure in Trauma (ASSET) course is a standardized, skills-based course developed by the American College of Surgeons Committee on Trauma. This scenario based cadaver course was developed to teach surgeons how to expose vital structures and control major hemorrhage in trauma. We conducted the ASSET courses in Japan in 2016 and 2017 and assessed the suitability and usefulness of this course for Japanese surgeon.

Material and methods: A retrospective analysis of the data collected from 3 ASSET courses conducted in 2016 and 2017 was performed. Results: A total of 36 students were enrolled and completed the course evaluation. Participants were asked evaluate the course on 7 specific areas using a 5 -point Likert scale with 1 being strongly disagree and 5 being strong agree. All 7 areas were rated highly with ranging from 4.5 to 4.8 . Overall, how would you rate this educational activity? (4.8 \pm 0.4$)$; Program topics and content met the standard objectives ( $4.8 \pm 0.5)$; Content was relevant to educational needs (4.6 \pm 0.5 ); Educational format was conductive to learning (4.6 \pm 0.5$)$; Acquired knowledge will be applied in my practice environment $(4.5$ $\pm 0.7)$. The most helpful area chosen by the largest number of participants was vascular exposure and fasciotomies of the extremites.

Conclusion: The ASSET course was highly rated by the course participants in Japan, suggesting that there is an important role for Japanese trauma surgeons.

References: Mackenzie CF, Garofalo E, Shackelford S, Shalin V, Pugh K, Chen H, Puche A, Pasley J, Sarani B, Henry S, Bowyer M. Using an Individual Procedure Score Before and After the Advanced Surgical Skills Exposure for Trauma Course Training to Benchmark a Hemorrhage-Control Performance Metric. J Surg Educ. 2015. NovDec;72 (6):1278-89.

Disclosure: No significant relationships.
PR232

\section{INTRODUCING TRAINING IN NON-TECHNICAL SKILLS FOR POLYTRAUMATIC PATIENTS ATTENTION AT A THIRD LEVEL HOSPITAL}

N. Alegret Monroig, A. Valle Beltran, L. Subirana, F. Martinez Lopez

Anesthesiology, Coorporació Sanitaria Parc Tauli, sabadell/SPAIN

Introduction: The training needed to treat polytrauma patients (PTP) is complex. It is obvious that trauma team members should have some theoretical and technical knowledge on PTP management clearly established in the specific training of courses such as ATLS or ETC. But the current management of PTP in a third level hospital requires the coordinated work of different professionals abandoning the vertical care model. Therefore, it is highly necessary to train all the team in non-technical skills for an optimal management of these patients. Material and methods: After conducting an audit of job comfort and training needs for personnel involved in PTP care, training needs in Crisis Resource Management were identified. We have designed specific training in non-technical skills in the management of PTP.

Results: We present the results of the audit as well as the algorithm to apply in the next 2 years with the aim of training our team. This training will have an on-line platform based on theoretical CRM that will be completed with exercises in our simulation center. Finally, the knowledge acquired by the participants will be evaluated on: rolls in the trauma team, distribution of tasks, communication, situational awareness and leadership.

Conclusion: In the current management of PTP, non-technical skills training is highly necessary. To carry out these programs, an individualized design for each hospital is required. The creation of a multidisciplinary team capable of auditing the current deficits and proposing specific solutions as well as the hospital's involvement in supporting the development of this type of training is necessary. References: Acta Anaesthesiol Scand. 2014 Aug;58 (7):775-87. doi: 10.1111/aas.12336. Epub 2014 May 14. Trauma Team Dynamics. Gillman, L.M., Widder, S., Blaivas, M., Karakitsos, D. 2016. Springer Disclosure: No significant relationships.

\section{PR233}

\section{LAPAROSCOPIC APPENDECTOMY FOR ACUTE APPENDICITIS: WHAT SPACE IS THERE FOR TRAINING RESIDENTS?}

\author{
A. Ussia, S. Vaccari, M. Cervellera, A. Romano, C. Cipressi, \\ S. Bianchini, V. Tonini
}

Dipartimento Di Scienze Mediche E Chirurgiche, Chirurgia d'Urgenza - Cervellera; Policlinico Sant'Orsola-Malpighi. Università degli Studi di Bologna., Bologna/ITALY

\section{Introduction:}

Material and methods: We performed a retrospective study examining the records of patients with acute appendicitis that underwent laparoscopy appendectomy at our institution from September 2011 to June 2017. Cases were divided in two groups: those performed by a trainee (G1) and those performed by the senior surgeon (G2). In each group we compared preoperative characteristics, operative data and postoperative morbidity and mortality. 
Results: During the study period, 922 patients with clinical diagnosis of appendicitis underwent laparoscopic appendectomy at our institution: of these, 386 cases were performed primarily by residents and 536 were performed by senior surgeons. There were no statistical differences in pre-operative characteristics of patients. The two groups did not differ significantly regarding operative times (G1:67 \pm 19 vs $\mathrm{G} 2: 70 \pm 26$ minutes), postoperative major complications (G1:9\% vs G2:19\%), length of stay ( $3 \pm 2$ days for both groups), and 30 -days mortality (none)., operative times $(67 \pm 19$ vs $70 \pm 26$ min; respectively), major complications ( $2 \%$ vs $3 \%$; respectively) and length of hospital stay ( $3 \pm 2$ vs $3 \pm 3$ ). Multivariate analysis confirmed that the type of main surgeon was not an independent risk factor for postoperative morbidity.

Conclusion: Postoperative results of laparoscopic appendectomies do not differ significantly if surgeries are performed by residents as opposed to specialists. Appendectomy can provide teaching opportunities without compromising results for patients.

References:

Disclosure: No significant relationships.

\section{PR234}

\section{IMPLANT AND INSTRUMENT SET MANAGEMENT IN TRAUMA AND ORTHOPEDIC SURGERY OF TODAY AND TOMORROW - COMPREHENSIVE RESTRUCTURING AT A GERMAN LEVEL 1 TRAUMA CENTER}

\section{S.L. Meier, B. Eisenhauer, I. Marzi}

Trauma-, hand- And Reconstructive Surgery, University hospital Frankfurt, Frankfut am Main/GERMANY

Introduction: Despite the advancements in design and handling of implants and instruments used in present trauma and orthopedic surgery, the way implants and instruments are managed has barely changed in the past decades. In the German speaking area instruments sets and implants are usually space-savingly stored together, needlessly limiting the maximum number of procedures possible. Furthermore restocking and implant logistics are complicated, timeconsuming and fault-prone and the frequent reprocessing of blood contaminated titanium surfaces within the operating sets is gradually seen discerningly. To meet the challenges of regulatory compliance and maximum flexibillity while maintaining cost efficiency a comprehensive restructuring of the existing implant and instrument set management was started in 2015 at the university hospital Frankfurt, Germany.

Material and methods: As a first step implant and instrument containers were split and separately reprocessed to avoid potential blood contamination. Moreover stepwise implants were replaced by individually packaged sterile implants and specific instruments sets were converted to standardized sets with a wider range of applications. Order management and serial number tracking were integrated into the hospital information system and the storage concept was optimized and colour coded for quick and easy retrival. To elucidate the effect of the transition on precedure times we performed a retrospective analysis of all open reduced distal radius fractures before (2014) and after (2016).

Results: Surprisingly we could not show any significant difference in procedure time before $(n=97$, median $=62 \mathrm{~min})$ and after the transition $(\mathrm{n}=135$, median $=58 \mathrm{~min})$.

Conclusion: A modern implant and instrument management is needed to maximise flexibility while maintaining cost-efficiency and regulatory compliance.
References: Kraft M, Wille F et al. Safe reprocessing of medical devices with a view of the entire process chain. Bundesgesundheitsbl 2014; 57 (12):1393-401.

Disclosure: No significant relationships.

PR235

\section{SWISS CHOCOLATE AND FREE BEVERAGES INCREASE THE MOTIVATION FOR SCIENTIFIC WORK AMONGST RESIDENTS - AN INSTRUCTION FOR THE PROSPEROUS REALISATION OF CLINICAL TRIALS IN A NON- ACADEMIC TEACHING HOSPITAL IN SWITZERLAND}

\author{
A. Ruehle, F. Oehme, B. Link, J. Metzger, H. Fischer, M. Stickel, \\ R. Babst, F.J..P. Beeres
}

Surgery, Luzerner Kantonsspital, Luzern/SWITZERLAND

Introduction: The success of a clinical trial depends on its recruitment of eligible patients, therefore this period requires special attention. We suppose that the recruitment rate can be improved using a new approach, consisting on continuous information and gratification increasing the residents motivation.

Material and methods: Two prospective randomised trials were initiated in our Swiss non-academic teaching hospital, initially randomisation was performed using sealed envelopes, later using a soft drink machine as a funny and motivational approach into scientific work and to compensate the resident with free soft drinks for each recruitment. Information about the trials and free Swiss chocolate was distributed to increase the knowledge about the trials and the motivation for scientific work amongst the residents regularly. With a special designed survey we investigated the success of our actions at two points, also the recruitment rate into the trials was evaluated.

Results: Our residents rated their awareness of the trials with median 8 and 9 on a scale to 10 and their motivation for scientific work with median 7 and 6 . The recruitment rates ranged from $72-90 \%$. These results confirm our approach of a good informed and recompensed resident as a first step towards a successful trial implementation.

Conclusion: This trial shows that positive enhancement, use of sugar as brain fuel and regularly information increases the motivation of surgical residents to participate in scientific work and therefore allows the prosperous realisation of a clinical trial in a non-academic-teaching hospital. References: Holmes E. G., A. C. (19. Jul 2016). Taking Care of Our Own: A Multispecialty Study of Resident. Acad Psychiatry. McDonald AM, K. R. (7:9 2006). What influences recruitment to randomised controlled trials? A review of trials funded by two UK funding agencies. Trials. Mergenthaler P, L. U. (Oct 2013). Sugar for the brain: the role of glucose in physiological and pathological brain function. Trends Neurosci (36 (10)), S. 587-97. Newington L, M. A. (23. Jan 2014). Factors influencing recruitment to research: qualitative study of the experiences and perceptions of research teams. $B M C$ Med Res Methodol. (14:10). Patterson S., H. M. (19. May 2011). Successful recruitment to trials: a phased approach to opening gates and building bridges. BMC Med Res Methodol. Ziebland S., K. F. (27. Jan 2007). Does it matter if clinicians recruiting for a trial don't understand what the trial is really about? Qualitative study of surgeons' experiences of participation in a pragmatic multi-centre RCT. Trials. Treweek S, L. P. (2013). Methods to improve recruitment to randomised controlled trials: Cochrane systematic review and metaanalysis. . BMJ Open, S. 3:e002360. Windholz, G. (Sept 1997). Ivan P. Pavlov: An Overview of His Life and Psychological Work. American Psychologist, S. 941-946.

Disclosure: No significant relationships. 
PR236

\section{COMPREHENSIVE COMPARISON OF THE EMERGENCY MEDICINE AMONG JAPAN, USA, AND EUROPEAN COUNTRIES}

\section{S. Mori, Y. Otomo}

Trauma And Acute Critical Care, Tokyo Medical and Dental University, Tokyo/JAPAN

Introduction: Japan, the United States of America (USA), and European Union (EU) have highly sophisticated medical systems of their own, but they are unique and different from each other due to national and geographic characteristics. Japan has allegedly had the highest life expectancy in the world for years. This study is to reveal how Japan keeps the high level of medicine, and in addition, to show why many of the physicians in the world are aiming at doing researches and a clinical training in USA.

Material and methods: Worldwide surveys and major articles describing medical systems in each country were referred to in this study. Based on the author's background which consists of working experiences both in Japan and USA, comparison of medicine between both countries is performed. In addition, thorough investigation of medical situation in EU is implemented as well.

Results: A worldwide survey showed Japan is superior in many points such as life expectancy, infant mortality rate, quality of health care system, medical cost, friendliness and courtesy of staff, and modern equipment, etc. Japan has totally free emergency medical systems (EMS) and a short ambulance response time. A doctor car system was invented to compensate the disability of EMS to perform procedures and treatments at scene and a research regarding the doctor car in our university hospital revealed its efficacy in increasing the 7-day and 30-day survival rate. $(\mathrm{P}<0.001)$

Conclusion: Japan, USA, EU have their unique medical systems contributing to amelioration of the quality. Japan is superior to the other countries in many aspects.

Disclosure: No significant relationships.

\section{PR237}

\section{WIDE VARIATION IN OPIOID PRESCRIBING PATTERNS IN ACUTE CARE SURGERY: TIME FOR STANDARDIZATION?}

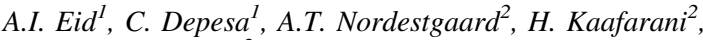 \\ N. Kongkaewpaisan ${ }^{2}$
}

${ }^{1}$ Surgery, Massachusetts General Hospital, Boston/MA/UNITED STATES OF AMERICA, ${ }^{2}$ Division Of Trauma, Emergency Surgery And Surgical Critical Care, Department Of Surgery, Massachusetts General Hospital, Harvard Medical School, Boston/MA/UNITED STATES OF AMERICA

Introduction: Diversion of unused prescription opioids is a major contributor to the current US opioid epidemic. We aimed to study the variation of opioid prescribing in emergency surgery.

Material and methods: Between October 2016 and March 2017, all patients undergoing laparoscopic appendectomy (LapAppy), laparoscopic cholecystectomy (LapChole), or inguinal hernia repair (InHR)] in the acute care surgery service at one academic center were included. For each patient, we systematically reviewed the electronic medical record and the prescribing pharmacy platform to identify: 1) history of opioid abuse, 2) opioid intake 3 months preoperatively, 3) number of opioid pills prescribed, 4) prescription of non-opioid pain medications (e.g. acetaminophen, ibuprofen) and 5) the need for opioid prescription refills. The mean and range of opioid pills prescribed, as well as their oral morphine equivalent (OME) was calculated.

Results: A total of 255 patients were included (43.5\% LapAppy, 44.3\% LapChole, and $12.1 \%$ InHR). The mean of age was 47.5 years, $52.1 \%$ were female, $11.4 \%$ had a history of opioid use, and $92.5 \%$ received opioid prescriptions upon hospital discharge. Only $70.9 \%$ of patients were instructed to use non-opioid pain medications. The mean and range of number of opioid pills prescribed were: LapAppy 17.4 (0-56), LapChole 17.1 (0-75), and InHR 20.9 (0-50) pills, while the range of prescribed OME was (0-600) $\mathrm{mg}$ for LapAppy/LapChole and (0-375) $\mathrm{mg}$ for InHR. No patients required any opioid medication refills.

Conclusion: Even within the same surgical service, wide variation of opioid prescription was observed. Guidelines that standardize pain management may help prevent opioid overprescribing.

\section{References:}

Disclosure: No significant relationships.

\section{PR238}

\section{URGENT COLORECTAL SURGERY: DOES SUBSPECIALIZATION CHANGE PATIENT MANAGEMENT?}

\section{$\underline{\text { S. Catarino }}^{\text {I }}$, J. Pereira $^{I}$, A. Matos $^{2}$, C. $_{\text {Henriques }}{ }^{2}$, C. $_{\text {Casimiro }}{ }^{l}$}

${ }^{1}$ Cirurgia Geral, Centro Hospitalar Tondela-Viseu, Viseu/ PORTUGAL, ${ }^{2}$ School Of Technology And Management, Polytechnic Institute of Viseu, Viseu/PORTUGAL

Introduction: Colorectal diseases requiring urgent surgery are commonly found on an acute care surgical unit. Colorectal subspecialist (CR) management of these patients could result, in theory, on decreased morbidity, stoma rates and mortality. The aim of this study was to compare management and outcomes between CR surgeons and general surgeons (GS) performing emergent colorectal surgery in the same acute care surgical unit.

Material and methods: This study shows a retrospective review of 143 consecutive patients submitted to emergent colorectal surgery between January 2012 and October 2016. Patients were divided into two groups: managed by a CR subspecialist or by a GS. Primary outcomes were morbidity and mortality and secondary outcomes were stoma and primary anastomosis rate and surgical timings (time interval from admission to surgery and time of surgical procedure). Statistical analysis was performed using IBM SPSS Statistics (version 24). Comparison between groups was analyzed using the MannWhitney test for numerical variables and the Chi-square test for categorical variables.

Results: Patients in the two groups were comparable for age, gender, ASA, Marshall and Charlson scores and diagnosis. There were no statistically significant differences found between groups in stoma rates, surgical timings, length of stay, Clavien-Dindo surgical complications score or mortality. The rate of residents performing urgent colorectal surgery was significantly higher in the CR group compared with the GS group (59.3\% versus $24.7 \%, P=0.000036$ ).

Conclusion: Management and outcomes of patients submitted to emergent colorectal surgery in our acute care surgical unit is similar whether it is performed by a CR specialist or a GS. 
References: 1 - Gibbons, G., Tan, C. J., Bartolo, D. C. C., Filgate, R., Makin, G., Barwood, N. and Wallace, M. (2015), Emergency left colonic resections on an acute surgical unit: does subspecialization improve outcomes?. ANZ J Surg, Vol 85; Issue 10; Pages 739-743. doi:10.1111/ans.13160 2 - Boyce, S. A., Bartolo, D. C. C., Paterson, H. M. and on behalf of the Edinburgh Coloproctology Unit (2013), Subspecialist emergency management of diverticulitis is associated with reduced mortality and fewer stomas. Colorectal Disease, Vol 15; Issue 4; Pages 442-447. doi:10.1111/codi.12022 3 - Teloken, P. E., Spilsbury, K., Levitt, M., Makin, G., Salama, P., Tan, P., Penter, C. and Platell, C. (2014), Outcomes in patients undergoing urgent colorectal surgery. ANZ J Surg, Vol 84; Issue 12; Pages 960-964. doi:10.1111/ans. 12580

Disclosure: No significant relationships.

\section{PR239}

\section{THE PERTH EMERGENCY LAPAROTOMY AUDIT (PELA)}

\author{
K. Broughton ${ }^{I}$, R.J. Aitken ${ }^{2}$
}

${ }^{1}$ Colorectal, Western General Hospital, Edinburgh/UNITED KINGDOM, ${ }^{2}$ General Surgery, Sir Charles Gairdner Hospital, Perth/ WA/AUSTRALIA

Introduction: Emergency laparotomies (ELs) are associated with high mortality and substantial outcome variation. There is no prospective Australian data on ELs. The aim of this study was to audit outcome after ELs in Western Australia.

Material and methods: A 12-week prospective audit was completed in 10 hospitals. Data collected included patient demographics, the clinical pathway, preoperative risk assessment and outcomes including 30-day mortality and length of stay.

Results: Data were recorded for $198(76.2 \%)$ of 260 patients. The 30-day mortality was $6.5 \%(17 / 260)$ in participating hospitals, and 5.4\% (19 of 354) across Western Australia. There was minimal variation between the three tertiary hospitals undertaking 220 of 354 $(62.1 \%)$ ELs. The median and mean post-operative lengths of stay, excluding patients who died, were 8 and 10 days, respectively. In the 48 patients with a prospectively documented risk of $\geq 10 \%$, both a consultant surgeon and anaesthetist were present for $68.8 \%, 62.8 \%$ were admitted to critical care and $45.8 \%$ commenced surgery within 2 h. The mortality in those retrospectively $(62 ; 31 \%)$ and prospectively risk-assessed was $9.5 \%$ and $5.2 \%$, respectively.

Conclusion: This prospective EL audit demonstrated low 30-day mortality with little interhospital variation. Individual hospitals have scope to improve their standards of care. The importance of prospective risk assessment is clear.

References: 1. Pearse RM, Harrison DA, James P et al. Identification and characterization of the high-risk surgical population in the United Kingdom. Crit. Care 2006; 10: R81. 2. Pearse RM, Moreno RP, Bauer $P$ et al. Mortality after surgery in Europe: a 7-day cohort study. Lancet 2012; 380: 1059-65. 3. Symons NR, Moorthy K, Almoudaris AM et al. Mortality in high-risk emergency general surgical admissions. Br. J. Surg. 2013; 100: 1318-25. 4. Ingraham AM, Cohen ME, Bilimoria $\mathrm{KY}$ et al. Comparison of hospital performance in nonemergency versus emergency colorectal operations at 142 hospitals. J. Am. Coll. Surg. 2010; 210: 155-65. 5. Vester-Andersen M,
Lundstrøm LH, Møller MH, Waldau T, Rosenberg J, Møller AM. Mortality and postoperative care pathways after emergency gastrointestinal surgery in 2904 patients: a population-based cohort study. Br. J. Anaesth. 2014; 112: 860-70. 6. Saunders DI, Murray D, Pichel AC, Varley S, Peden CJ. Variations in mortality after emergency laparotomy: the first report of the UK Emergency Laparotomy Network. Br. J. Anaesth. 2012; 109: 368-75. 7. National Confidential Enquiry into Patient Outcome and Death. Peri- Operative Care: Knowing the Risk. London, 2011. [Cited May 2017.] Available from URL: http://www.ncepod.org.uk/2011poc.html 8. National Confidential Enquiry into Perioperative Deaths. The 2003 report of the National Confidential Enquiry into Perioperative Deaths. London, 2003. [Cited May 2017.] Available from URL: http://www.ncepod.org.uk/2003report/Downloads/03full.pdf 9. The Royal College of Surgeons of England. The Higher Risk General Surgical Patient: Towards Improved Care for a Forgotten Group. London, 2011. [Cited May 2017.] Available from URL: https://www.rcseng.ac.uk/libraryand-publications/college-publications/docs/thehigher-risk-generalsurgical-patient/10. Huddart S, Peden CJ, Swart M et al. Use of a pathway quality improvement care bundle to reduce mortality after emergency laparotomy. Br. J. Surg. 2015; 102: 57-66. 11. Tengberg LT, Bay-Nielsen M, Bisgaard T, Cihoric M, Lauritsen M, Foss N. Multidisciplinary perioperative protocol in patients undergoing acute high-risk abdominal surgery. Br. J. Surg. 2017; 98: 802-10. 12. National Emergency Laparotomy Audit. The Second Patient Report of the National Emergency Laparotomy Audit (NELA). London, 2016. [Cited May 2017.] Available from URL: http://www.nela.org.uk/reports 13. National Emergency Laparotomy Audit. Organisational Report of the National Emergency Laparotomy Audit. London, 2014. [Cited May 2017.] Available from URL: http:// www.nela.org.uk/Organisational-Audit-Report\#pt 14. Shakerian R, Thomson BN, Gorelik A, Hayes IP, Skandarajah AR. Outcomes in emergency general surgery following the introduction of a consultantled unit. Br. J. Surg. 2015; 102: 1726-32. 15. von Elm E, Altman DG, Egger $\mathrm{M}$ et al. The Strengthening the Reporting of Observational Studies in Epidemiology (STROBE) statement: guidelines for reporting observational studies. J. Clin. Epidemiol 2008; 61: 344-9. 16. Singer M, Deutschman CS, Seymour CW et al. The Third International Consensus Definitions for Sepsis and Septic Shock (Sepsis3). JAMA 2016; 315: 801-10. 17. Protopapa KL, Simpson J, Smith N, Moonesinghe S. Development and validation of the Surgical Outcome Risk Tool (SORT). Br. J. Surg. 2014; 101: 1774-83. 18. Morris C. Oesophageal Doppler monitoring, doubt and equipoise: evidence based medicine means change. Anaesthesia 2013; 68: 677-83. 19. Semmens JB, Sanfilippo F, Mukhtar SA, Haynes N, Mountain J, Aitken RJ. The Western Australian Audit of Surgical Mortality: advancing surgical accountability. Med. J. Aust. 2005; 183: 545-6. 20. Azzam DG, Neo CA, Itotoh F, Aitken RJ. The Western Australian Audit of Surgical Mortality: outcomes from the first 10 years. Med. J. Aust. 2013; 199: 539-42. 21. National Emergency Laparotomy Audit. National Emergency Laparotomy Audit Top Tips. [Cited 19 Jul 2017.] Available from URL: http://www.nela.org.uk/download.php/?fn $=$ NELA\%20Hospital\%20Tips.pdf\&mime $=$ application $/$ pdf\&pureFn $=$ NELA\%20Hospital\%20Tips.pdf 22. Australian Commission on Safety and Quality in Health Care. Surgical Interventions. Australian Atlas of Healthcare Variation. 2017. [Cited 30. Jun 2017.] Available from URL: https://www.safetyandquality.gov.au/wp-content/uploads/2017/06/4.0-Introduction-and-key-recommendati ons1.pdf Disclosure: No significant relationships. 
PR240

\section{THE EFFECT OF PHYSICIAN-STAFFED HELICOPTER EMERGENCY MEDICAL SERVICES (P-HEMS) ON THE OUTCOME OF PATIENTS WITH SEVERE TRAUMATIC BRAIN INJURY. A SYSTEMATIC REVIEW OF THE LITERATURE}

\author{
Z. Popal ${ }^{1}$, S.M. Bossers ${ }^{2}$, M. Terral ${ }^{1}$, P. Schober ${ }^{2}$, M.A. De Leeuw ${ }^{2}$, \\ $\overline{F . W . ~ B l o e m e r s ~}^{1}$, G.F. Giannakopoulos ${ }^{I}$
}

${ }^{1}$ Trauma Surgery And Hems Lifeliner 1, VU University Medical Centre, Amsterdam/NETHERLANDS, ${ }^{2}$ Anesthesiology And Hems Lifeliner 1, VU University Medical Centre, Amsterdam/ NETHERLANDS

Introduction: The use of physician-staffed Helicopter Emergency Medical Services (P-HEMS) seems to show a substantial improvement on the survival of trauma patients. However, the effect of prehospital physician assistance for patients with specifically traumatic brain injury (TBI) is unclear. This provides a reason to determine the value of physician-staffed HEMS on the outcome of patients with severe TBI, compared to other forms of (non-physicianstaffed) EMS.

Material and methods: A systematic review of the literature, in accordance to PRISMA guidelines, was performed, including data from patients with severe TBI with a Glasgow Coma Score (GCS) below nine or a head Abbreviated Injury Score (AIS) equal to or higher than four, to investigate the difference in outcome between P-HEMS and other EMS. The effect of the different emergency services was examined by investigating the mortality and the neurological outcome, as well as hypotension, hypoxia, length of hospital stay and the number of neurosurgical interventions. The Newcastle-Ottowa Scale and Cochrane's Collaboration Tool was used for the quality assessment of the studies.

Results: After screening of 1484 articles, 13 articles were included in the systematic review. The majority of the studies ( 8 out of 13) concluded that physician-staffed assistance has a beneficial effect on the outcome of patients with severe TBI.

Conclusion: Most studies show beneficial results after the implementation of P-HEMS assistance for patients with severe traumatic brain injury. However, additional studies are required to gain a more accurate insight of the actual effects of P-HEMS assistance.

References: 1. Ramaiah, R., Grabinsky, A., Williamson, K., and Bhanankar, S.M. (2011). Trauma care today, what's new?, Int J Crit Illn Inj Sci 1, 22-26. 2. Cii, F., Series, I., WHO, Romualdez Jr., A. G., Dela Rosa, J. F. E., Flavier, J. D. A., Quimbo, S. L. A., Hartigan-Go, K. Y., Lagrada, L. P., David, L. C., Kongsap, A., Paphassarang, C., Phoxay, C., Vonglokham, M., Phommavong, C., Pholsena, S., World Health Organization (WHO), Hedges, J. P., Mock, C. N., Cherian, M. N., Universal, T., Machine, A., Hsia, R. Y., Mbembati, N. A., MacFarlane, S., Kruk, M. E., Care, L., Ventilator, E.C. (2015). Guidelines for essential trauma care, Health Syst. Transit. 34, 80. 3. Chi, J.H., Knudson, M. M., Vassar, M. J., McCarthy, M. C., Shapiro, M. B., Mallet, S., Holcroft, J. J., Moncrief, H., Noble, J., Wisner, D., Kaups, K. L., Bennick, L. D., Manley, G. T. (2006). Prehospital hypoxia affects outcome in patients with traumatic brain injury: a prospective multicenter study, J Trauma 61, 1134-1141. 4. Spaite, D. W., Hu, C., Bobrow, B. J., Chikani, V., Barnhart, B., Gaither, J. B., Denninghoff, K. R., Adelson, P. D., Keim, S. M., Viscusi, C., Mullins, T., and Sherrill, D. (2016). The effect of combined out-ofhospital hypotension and hypoxia on mortality in major traumatic brain injury, Ann. Emerg. Med. PubAh, 1-11. 5. Wells, G. A., Shea, B., O'Connell, D., Peterson, J., Welch, V., Losos, M., and Tugwell, P. (2013). The Newcastle-Ottawa Scale (NOS) for assessing the quality of nonrandomized studies in meta-analyses., Ottawa Hosp. Res. Inst., 1-4. 6. Higgins, J. P. T., Altman, D. G., Gotzsche, P. C., Juni, P., Moher, D., Oxman, A. D., Savovic, J., Schulz, K. F., Weeks, L., and Sterne, J. A. C. (2011), The Cochrane Collaboration's tool for assessing risk of bias in randomised trials, Bmj 343, d5928-d5928. 7. Hudak, A. M., Caesar, R. R., Frol, A. B., Krueger, K., Harper, C. R., Temkin, N. R., Dikmen, S. S., Carlile, M., Madden, C., and DiazArrastia, R. (2005). Functional outcome scales in traumatic brain injury: a comparison of the Glasgow Outcome Scale (Extended) and the Functional Status Examination., J. Neurotrauma 22, 1319-1326. 8. Kim, Y. (2011). A systematic review of factors contributing to outcomes in patients with traumatic brain injury., J Clin Nurs 20, 1518-32. 9. Jannot, A., Ehret, G., and Perneger, T. (2015). P $<5 \times 10$ $(-8)$ has emerged as a standard of statistical significance for genomewide association studies., J Clin Epidemiol 68, 460-5. 10. OCEBM Levels of Evidence Working Group, Durieux, N., Pasleau, F., and Howick, J. (2011). The Oxford 2011 Levels of Evidence, Group 1, 5653. 11. Garner, A., Crooks, J., Lee, A., and Bishop, R. (2001). Efficacy of prehospital critical care teams for severe blunt head injury in the Australian setting, Injury 32, 455-460. 12. Taylor, C., Jan, S., Curtis, K., Tzannes, A., Li, Q., Palmer, C., Dickson, C., and Myburgh, J. (2012). The cost-effectiveness of physician staffed Helicopter Emergency Medical Service (HEMS) transport to a major trauma centre in NSW, Australia, Injury 43, 1843-1849. 13. Franschman, G., Andriessen, T. M. J. C., Boer, C., Van Der Naalt, J., Horn, J., Haitsma, I., and Vos, P. E. (2013). Physician-based emergency medical service deployment characteristics in severe traumatic brain injury: A Dutch multicenter study, Injury 44, 1232-1236. 14. Franschman, G., Verburg, N., Brens-Heldens, V., Andriessen, T. M. J. C., Van der Naalt, J., Peerdeman, S. M., Vak, J. P., Hoogerwerf, N., Greuters, S., Schober, P., Vos, P. E., Christiaans, H. M. T., and Boer, C. (2012). Effects of physician-based emergency medical service dispatch in severe traumatic brain injury on prehospital run time, Injury 43, 1838-1842. 15. Pakkanen, T., Virkkunen, I., Kämäräinen, Huhtala, A., H., Silfvast, T., Virta, J., Randell, T., and Yli-Hankala, A. (2016). Pre-hospital severe traumatic brain injury - comparison of outcome in paramedic versus physician staffed emergency medical services., Scand. J. Trauma. Resusc. Emerg. Med. 24, 62. 16. Garner, A. A., Mann, K. P., Fearnside, M., Poynter, E., and Gebski, V. (2015). The Head Injury Retrieval Trial (HIRT): a single-centre randomised controlled trial of physician prehospital management of severe blunt head injury compared with management by paramedics only, Emerg Med J 32, 869-875. 17. Di Bartolomeo, S., Sanson, G., Nardi, G., Scian, F., Michelutto, V., and Lattuada, L. (2001). Effects of 2 patterns of prehospital care on the outcome of patients with severe head injury., Arch. Surg. 136, 1293-1300. 18. Aydin, S., Overwater, E., Saltzherr, T. P., Jin, P. H. P. F. K., Van Exter, P., Ponsen, K. J., Luitse, J. S. K., Goslings, J. C. (2010). The association of mobile medical team involvement on on-scene times and mortality in trauma patients., J. Trauma 69, 589-594; discussion 594. 19. Engel, D.C., Mikocka-Walus, A., Cameron, P. A., and Maegele, M. (2010). Prehospital and in-hospital parameters and outcomes in patients with traumatic brain injury: A comparison between German and Australian trauma registries, Injury 41, 901-906. 20. De Jongh, M.A., Van Stel, H. F., Schrijvers, A. J., Leenen, L. P., Verhofstad, M. H. (2012). The effect of helicopter emergency medical services on trauma patient mortality in the Netherlands, Injury 43, 1362-1367. 21. Bulger, E. M., Guffey, D., Guyette, F. X., MacDonald, R. D., Brasel, K., Kerby, J. D., Minei, J. P., Warden, C., Rizoli, S., Morrison, L. J., Nichol, G. (2012). Impact of prehospital mode of transport after severe injury: a multicenter evaluation from the Resuscitation Outcomes Consortium., J. Trauma Acute Care Surg. 72, 567-73; discussion 573-5; quiz 803. 22. Klemen, P., and Grmec, S. (2006). Effect of pre-hospital advanced life support with rapid sequence intubation on outcome of severe traumatic brain injury, Acta Anaesthesiol. Scand. 50, 1250-1254. 23. Baxt, W.G., and Moody, P. (1987). The impact of 
advanced prehospital emergency care on the mortality of severely brain-injured patients., J. Trauma 27, 365-369. 24. Garner, A., Rashford, S., Lee, A., and Bartolacci, R. (1999). Addition of physicians to paramedic helicopter services decreases blunt trauma mortality, Aust. N. Z. J. Surg. 69, 697-701. 25. Webb, H. (2013). The efficacy of pre-hospital endotracheal intubation in severe traumatic brain injury, Australas. J. Paramed. 10, 1-2. 26. Lansom, J. D., Curtis, K., Goldsmith, H., and Tzannes, A. (2016). The effect of prehospital intubation on treatment times in patients with suspected traumatic brain injury, Air Med. J. 35. 27. Bossers, S. M., Schwarte, L.A., Loer, S. A., Twisk, J. W. R., Boer, C., and Schober, P. (2015). Experience in prehospital endotracheal intubation significantly influences mortality of patients with severe traumatic brain injury: A systematic review and meta-analysis, PLoS One 10. 28. Mezue, W. C., Ndubuisi, C., Ohaegbulam, S. C., Chikani, M., and Erechukwu, U. (2013). Cranial bony decompressions in the management of head injuries: Decompressive craniotomy or craniectomy?, Niger. J. Clin. Pract. 16, 343-347. 29. Ringburg, A. N., Spanjersberg, W. R., Frankema, S. P. G., Steyerberg, E. W., Patka, P., and Schipper, I. B. (2007). Helicopter emergency medical services (HEMS): impact on on-scene times., J. Trauma 63, 258-262. 30. Geeraerts, T., Friggeri, A., Mazoit, J. X., Benhamou, D., Duranteau, J., and Vigué, B. (2008). Posttraumatic brain vulnerability to hypoxia-hypotension: The importance of the delay between brain trauma and secondary insult, Intensive Care Med. 34, 551-560.

Disclosure: No significant relationships.

\section{PR241}

\section{DIFFERENTIATION OF ON-SCENE FORCES IN MASS CASUALTY EVENTS AT SEA USING A COLOR BASED CONCEPT}

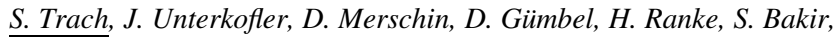
A. Ekkernkamp, S. Schulz-Drost

Trauma- And Orthopedic Surgery, Unfallkrankenhaus Berlin, Berlin/ GERMANY

Introduction: The KOMPASS project deals with the possibility of a mass casualty event at sea and it's handling. The presented work focuses on a color based concept to differentiate on-scene forces and through this providing a clear assignment of responsibilities.

Material and methods: We performed an internet based search of established mass casualty plans and interviews with experts in the field. We then developed a simple and easy to understand concept of designating on-scene forces. This was performed with the help of before mentioned experts and with the aim to keep as close as possible to already established concepts on land as possible.

Results: Our research showed a variety of different plans using different color schemes and different means of differentiation. Simply using a concept already in place was thus not possible. A different concept considering the needs and realities at sea was needed. We developed a concept based on colored approns establishing three groups. In accordance to established schemes with yellow for tactical/ technical leadership and blue for medical personnel. To recruit manpower we included a group of untrained personnel forming from capable and willing passengers for simple tasks e.g. carrying stretchers or relocating material. To establish a hierarchy within the designated groups the individual leader of the group should be marked with the word LEADER.

Conclusion: Our concept is designed to lay the foundation of successfully dealing with mass casualty events at sea. Time passing until professional help arrives can then be used to establish responsibilities, clear communication and hierarchy arriving personal can build upon. References: Konzeption des Ministeriums für Inneres (BaWü), Digitalisierung und Migration für die Einsatzplanung und Bewältigung eines Massenanfalls von Verletzten (ManV-Konzept) vom 1. August 2016, - Az.: 6-1441/73 Richtlinie zur Bewältigung von Ereignissen mit einem Massenanfall von Notfallpatienten und Betroffenen (MAN-RL) Bayer. Staatsministerium des Innern, für Bau und Verkehr vom 06.12.2016 Landeshauptstadt Magdeburg Der Oberbürgermeister Amt für Brand- und Katastrophenschutz Einsatzkonzept Massenanfall von Verletzten Erstellt von BR Dipl.-Ing. Armin Hilgers BF Magdeburg Ärztlicher Leiter Rettungsdienst Dipl. Med. Reinhardt Bondick Cimolino Ulrich; Zawadke Thomas.: Einsatzfahrzeuge für Feuerwehr und Rettungsdienst Technik.. 1. Auflage ecomed Verlag Landsberg 2005 Stab BTK 21; Mitarbeiter-Information der Berliner Feuerwehr Ausgabe 01/2016 vom 01. Oktober 2016;"Einsatzbezogene Kennzeichnung "Ministerium für Inneres und Sport, E4 - 4177-03, Erlass über die Kennzeichnung von Führungskräften an Einsatzstellen bei Großschadenslagen, Vom 10. April 2002 Einheitliche Kennzeichnung von Führungskräften und Führungsgremien im Land Brandenburg, vom 2.Dezember 2003; http://bravors.brandenburg.de/verwaltungsvorschriften/fkfg

Disclosure: No significant relationships.

\section{PR242}

\section{PRE-DEPLOYMENT PREPARATION REQUIREMENTS FOR MEDICAL PROFESSIONALS OF THE ICRC}

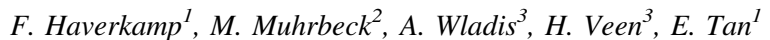

${ }^{1}$ Emergency Department, Radboud University Medical Center, Nijmegen/NETHERLANDS, ${ }^{2}$ Linköping University, Linköping University, Linköping/SWEDEN, ${ }^{3}$ Health, International Committee of the Red Cross, Genevea/SWITZERLAND

Introduction: The International Committee of the Red Cross (ICRC) is an independent humanitarian organisation, which provides medical assistance at conflict zones. Medical professionals who are deployed with the ICRC should be professionally prepared to work in these environments. The aim of this study is to gain insight into the selfperceived preparedness and learning needs of ICRC medical staff, and make recommendations on how to optimise future pre-deployment training.

Material and methods: All 284 ICRC medical staff members (as enlisted by the ICRC) were invited to participate in a digital questionnaire in March 2017. The survey contained questions about the personal background, pre-deployment training, deployment experiences, self-perceived preparedness and aftercare.

Results: The response rate was 54\% (153/284). The mean age of the respondents was 48 years (SD 10.5). The respondents mainly concerned nurses $(39 \%)$, surgeons $(30 \%)$ and anaesthesiologists $(24 \%)$, with a mean work experience of 23 years (SD 10.5). Respondents rated their self-perceived preparedness for treating trauma higher in case they had previously been deployed with other organisations, or attended at least one master class, e.g. ICRC surgical seminar on war wounds $(\mathrm{p}<0.05)$. Nurses had a lower feeling of self-perceived preparedness than anaesthesiologists and surgeons did $(\mathrm{p}<0.05)$. Additional training was requested most frequently about paediatrics $(65 / 150)$, fracture surgery $(46 / 150)$ and burn treatment $(45 / 150)$.

Conclusion: Overall, the medical staff of the ICRC felt sufficiently prepared for their deployments. However, additional learning needs 
were identified. These needs form the basis for recommendations on the content of future ICRC pre-deployment training.

References:

Disclosure: No significant relationships.

\section{PR243}

\section{OPTIMAL SURGICAL TIMING IN ACUTE BENIGN GALLBLADDER DISEASE: THE SOONER THE BETTER. A FULL COST ANALYSIS}

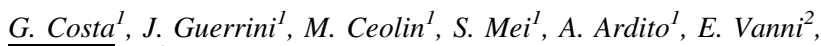
H. Kurihara

${ }^{1}$ Emergency Surgery And Trauma Unit, Humanitas Research Hospital, Rozzano, Milano/ITALY, ${ }^{2}$ Business Controlling, Humanitas Research Hospital, Rozzano, Milano/ITALY, ${ }^{3}$ Emergency Surgery And Trauma Unit, Humanitas Research Hospital, Rozzano/ ITALY

Introduction: Optimal timing of cholecystectomy in acute gallbladder pathology remains unclear. Some studies have shown that early cholecystectomy can be performed with good conversion rates, morbidity and mortality. By means of a full cost analysis, we aim to investigate the cost-effectiveness of surgical timing in acute benign gallbladder disease.

Material and methods: All patients that received a cholecystectomy for gallstone-related diseases between January 2014 and February 2017 were considered. For each patient, we recorded all admissions in the emergency department (ED) for gallstone-related complaints in the 4 years before surgery. Costs related to diagnostics, drugs, surgical procedures and hospital-stay for both surgical and any previous gallstone-related admission were collected.

Results: 845 patients were included in the analysis, among them 183 $(21,7 \%)$ received urgent surgery. Major morbidity and mortality were respectively $0,83 \%$ and $0,36 \%$. We found a positive relation between the number of previous admission in the ED and surgical costs (no admissions: $€ 1.374$; one admission: $€ 2.120$; two admissions: $€ 2.321$; three admissions: $€ 2.766$; four or more admissions: $€ 2.967$; median; $p$ $<0,001)$. Concerning timing of surgery, in patients operated within a month from the first ED admission, despite similar conversion rates, we found that sooner was surgery performed, fewer were the costs $(0$ 3 days: $€ 2.491$; $4-7$ days: $€ 2.684$; 8-15 days: $€ 2.726$; $16-30$ days: $€ 4.231$; median; $\mathrm{p}=\mathrm{ns}$ ).

Conclusion: By the means of a full cost analysis, we found that early surgery for acute benign gallbladder disease is less costly and as effective as delayed surgery. Choosing an early surgery approach can be beneficial to both patients and healthcare provides.

References: 1) Okamoto K, Suzuki K, Takada T, Strasberg SM, Asbun HJ, Endo I, Iwashita Y, Hibi T, Pitt HA, Umezawa A, Asai K, Han HS, Hwang TL, Mori Y, Yoon YS, Huang WS, Belli G, Dervenis C, Yokoe M, Kiriyama S, Itoi T, Jagannath P, Garden OJ, Miura F, Nakamura M, Horiguchi A, Wakabayashi G, Cherqui D, de Santibañes E, Shikata S, Noguchi Y, Ukai T, Higuchi R, Wada K, Honda G, Supe AN, Yoshida M, Mayumi T, Gouma DJ, Deziel DJ, Liau KH, Chen MF, Shibao K, Liu KH, Su CH, Chan ACW, Yoon DS, Choi IS, Jonas E, Chen XP, Fan ST, Ker CG, Giménez ME, Kitano S, Inomata M, Hirata K, Inui K, Sumiyama Y, Yamamoto M. Tokyo Guidelines 2018 flowchart for the management of acute cholecystitis. J Hepatobiliary Pancreat Sci. 2017 Oct 16. doi: 10.1002/jhbp.516. [Epub ahead of print] PubMed PMID: 29045062. 2) Sutton AJ, Vohra RS, Hollyman M, Marriott PJ, Buja A, Alderson D, Pasquali S, Griffiths EA; CholeS Study Group and the West Midlands Research Collaborative.
Cost-effectiveness of emergency versus delayed laparoscopic cholecystectomy for acute gallbladder pathology. Br J Surg. 2017 Jan;104 (1):98-107. doi: 10.1002/bjs.10317. Epub 2016 Oct 20. PubMed PMID: 27762448.

Disclosure: No significant relationships.

\section{PR244}

\section{THE STUDENT EMERGENCY ABDOMINAL SURGERY COURSE (SEASC) -AN IDEAL TEMPLATE IN UNDERGRADUATE SURGICAL EDUCATION}

\section{Sugrue}

Surgery, Letterkenny University Hospitlal, ramelton/IRELAND

Introduction: Undergraduate surgical education and enrolment into Surgery has suffered over the last decade. New strategies to enhance emergency surgery's profile and educational ability is urgently required (1).

Material and methods: Course curriculum consists of one day, 8 hours teaching, $40 \%$ didactic and $60 \%$ interactive. 12 short, 15 minute lectures and 11 twenty minute cases. A purpose designed slide set, with e manual. Cases include videos of presentation, examination, imaging and surgery. Participants complete detailed evaluation. The curriculum covers 8 common conditions including appendicitis, cholecystitis, diverticulitis, peptic ulceration and bowel obstruction. The multidisciplinary faculty are in surgery, gastroenterology, radiology, critical care and nursing

Results: 6 sEASCä courses have been held in 3 countries with 480 students taking part. Mean overall rating was $8.5 \pm 0.5$. Highlights were audio-visual clips of patients' findings and their surgery. Over $80 \%$ of medical students rated the course as the best educational experience in their undergraduate experience. The interdisciplinary discussion of cases at consultant level was also a highlight Conclusion: sEASC is an outstanding platform for final medical student surgical education, it could be used not only to address increasing knowledge gaps in acute surgical care but act a model undergraduate educational platform.

References: 12017 Sep 14. doi: 10.1007/s00268-017-4190-5. [Epub ahead of print]

Disclosure: No significant relationships.

\section{PR245}

\section{THE HUNGARIAN ATLS EXPERIENCE I.E. WHAT HAVE WE LEARNED?}

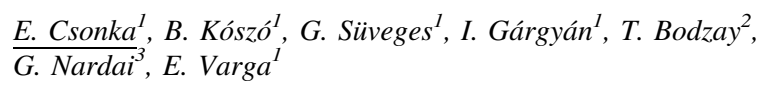

${ }^{1}$ Department Of Traumatology, University of Szeged, Szeged/ HUNGARY, ${ }^{2}$ Department Of Traumatology, Péterffy Hospital Trauma Center, Budapest/HUNGARY, ${ }^{3}$ Department Of Anaesthesiology, Péterffy Hospital Trauma Center, Budapest/ HUNGARY

Introduction: The history of ATLS started in 1976. The first course was held in 1978 and after 2 years an educational programme was accepted by American Colleges of Surgeons (ACS) and regular courses were introduced. In Hungary this procedure took a bit longer. 
Our colleagues got in touch with ACS in 1997, but the first Hungarian course was only held in 2005 . After 12 years we evaluated the success of the programme.

Material and methods: The success of a course depends on many factors. The students feedback evaluates the lectures, practices, it measures usefulness of the course. Is it worth the money, time? Do they learn something new? Since 2015 we survey the students' abilities before the course with a precourse assessment, and ask what level they would like to achieve after the course. After 4 weeks of the programme we evaluate the development with a postcourse assesment. Can these courses improve the management of polytrauma patients? Have the life expectancies of these patients got better? We are making a statistic analysis ultilizing European datas to evaluate the usefulness of these programmes.

Results: Students' satisfactions are above 4,5 points. There is a significant development in the magament of polytrauma patients. The life expectancy of a polytrauma patient in Hungary is close up to other ATLS trained countries.

Conclusion: The success of the programme can be measured by the feedbacks and statistical evaluations.

References: Warwick Chun-Wang Chan at al.: Patient-specific instrumentation versus conventional instrumentation in total knee arthroplasty Gomes N. Patient-specific instrumentation for total shoulder arthroplasty : Accuracy of patient-specific instrumentation in anatomic and reverse total shoulder arthroplasty

Disclosure: No significant relationships.

\section{PR246}

\section{THE EDINBURGH EMERGENCY SURGERY ORGANISATION: 'SPECIALIZATION' OF EMERGENCIES AS A WORKING MODEL}

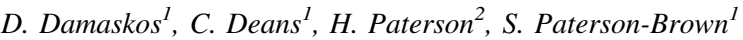

${ }^{1}$ General Surgery, Royal Infirmary of Edinburgh, Edinburgh/UNITED KINGDOM, ${ }^{2}$ Colorectal Surgery, Western General Hospital, Edinburgh/UNITED KINGDOM

Introduction: Emergency surgery has gained much attention following the publication of NELA. In Edinburgh we have adopted a unique approach to the organization and delivery of emergency surgical care.

Material and methods: Edinburgh has a split site emergency 'specialisation'. The Royal Infirmary (RIE) undertakes specialist upper GI surgery (oesophagogastric and HPB) and general surgery. The Western General Hospital (WGH) performs specialist colorectal surgery and general surgery. In addition, the RIE has the accident and emergency department. Both sites have a surgical assessment unit. Patients are triaged to the appropriate site upon pre-agreed protocols. Both sites are staffed 24 hours and consultant delivered ward rounds are conducted twice daily. An additional "hot" clinic is run daily to review ambulatory patients.

Results: An average 190 patients are assessed at RIE each week . On the target set by NELA for all patients to be reviewed within 14 hours by a consultant, RIE scores $90 \%$ (NELA 55\%). The introduction of the 'Hot' clinic has reduced admission rates from $85 \%$ to $54 \%$ releasing bed availability and reducing unnecessary admissions. During 2015, 259 emergency operations were performed after midnight at RIE with a Consultant present for $97 \%$ of cases (NELA $80 \%$ ). Conclusion: 'Specialization' of emergency services in Edinburgh has resulted in emergency patients receiving specialist input for the delivery of emergency care. The service is Consultant-delivered improving the standard of care and providing training in a supervised setting. The introduction of the 'hot clinic' has reduced unnecessary hospital admissions and improved patient satisfaction.

References:

Disclosure: No significant relationships.

\section{PR247}

\section{IMPROVING INFORMAL EDUCATION OF YOUNG RESIDENTS DURING SURGICAL ER ROTATION}

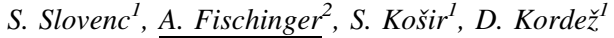 \\ ${ }^{1}$ Surgical Er, University Medical Centre Ljubljana, Ljubljana/ \\ SLOVENIA, ${ }^{2}$ Traumatology, University Medical Centre Ljubljana, \\ Ljubljana/SLOVENIA
}

Introduction: The amount of medical knowledge required from residents is increasing yet the time residents spend on surgical ER is decreasing. In practice this means longer waiting times and a possible increase in medical errors. With lack of formal resident education, informal education can play an important role in establishing an effective and patient safe ER. Experienced nurses can play an important role in junior ER residents training.

Material and methods: Residents rotating in surgical ER receive almost no formal education and rely on knowledge received during medical school and on knowledge gained with self directed learning. With absence of formal education, informal education usually evolves. Informal education is typically composed from traditional large group lectures and informal mentoring by older peers. Knowledge acquisition and retention after classical lectures remains low and since senior attending doctors usually do not work with minor trauma patients no practical mentorship is possible.

Results: One solution to this problem is to include experienced ER nurses in informal resident education. Nursing faculty is already included in teaching clinical skills to medical students but they can also improve informal resident education. ER nurses spend all their professional careers in the ER, have great practical knowledge and have witnessed same repeat junior resident mistakes countless of times. This puts them in an ideal position to help young residents from making common practical mistakes.

Conclusion: Informal resident education can improve resident performance in the surgical ER. Experienced nurses can play an important role in improving informal education of young residents and make ER safer for patients.

References: Abdallah B, Irani J, Dakessian Sailian S, George Gebran V, Rizk U. Nursing faculty teaching a module in clinical skills to medical students: a Lebanese experience. Adv Med Educ Pract. 2014, $5: 427-32$.

Disclosure: No significant relationships. 


\section{PR248}

\section{THE RESUSCITATION ROOM LENGTH OF STAY: IS THE TIME RUNNING OUT?}

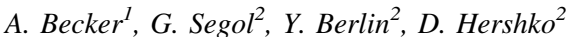

${ }^{1}$ B.rappaport School Of Medicine, Technion - Israel Institute of Technology, Haifa/ISRAEL, ${ }^{2}$ Surgery, Haemek Medical Center, Afula/ISRAEL

Introduction: The idea is that trauma patients have better outcomes if they are provided definitive care within 60 minutes of the occurrence of their injuries has been widely accepted . On the other hand, there is little evidence to directly support the "golden hour" concept. The purpose of this study is to examine the relationships between emergency department length of stay and hospital length of stay for hemodynamically stable trauma patients.

Material and methods: Data was prospectively collected from the trauma patients admitted to the trauma resuscitation area from February 1, 2013 to December 31, 2014. Only stable trauma patients with appropriated trauma triage criteria requiring trauma team activation were included in to the study. Exclusion criteria were as follow: patients died in the emergency department, patients taken emergently to the operating room on arrival.

Results: From 248 patients enrolled in to the study.. The total study population had a mean emergency department length of stay (ED LOS) of 125 minutes (SD 32). Mean EDLOS time for patients in short group was $78 \mathrm{~min}$ (range 45-115) versus $180 \mathrm{~min}$ (range 120- 215 for long disposition group).There was no difference in LOS in leveled trauma patient between short and long ED LOS groups. The mean hospital LOS for the two groups was: short 11.5 (SD 3.5 days) versus long group 12.8 (SD 4.6), (p-value 0.65).

Conclusion: There was no difference in LOS in leveled trauma patient between short and long ED LOS groups. Emergency department length of stay should not be used independently as a benchmark in stable trauma patients.

References: 1.Lerner EB, Moscati RM. The golden hour: scientific fact or medical "urban legend"? Acad Emerg Med. 2001; 8:758-760. 2. Harris B, Bai J, Kulstad EB. Crowding does not adversely affect time to percutaneous coronary intervention for acute myocardial infarction in a community emergency department. Ann Emerg Med. 2012; 59: 7-13. 3. National Voluntary Consensus Standards for Emergency Care: A Consensus Report.Washington, DC: National Quality Forum; 2009. 4. Chalfin DB, Trzeciak S, Likourezos A, et al DELAY-ED study group. Impact of delayed transfer of critically ill patients from the emergency department to the intensive care unit. Crit Care Med. 2007;35:1477-1483 5 Singer A, Thode H, Viccellio P, Pines JM. The association between length of emergency department boarding and mortality..Acad Emerg Med. 2011;18:1324-1329. 6. Parkhe M, Myles PS, Leach DS, Maclean AV. Outcome of emergency department patients with delayed admission to an intensive care unit. Emerg Med (Fremantle). 2002;14:50 -57. 7. Varon J, Fromm RE Jr, Levine RL. Emergency department procedures and length of stay for critically ill medical patients. Ann Emerg Med 1994; 23: 546-9 8. Sprung C, Geber D, Eidelman L, et al. Evaluation of triage decisions for intensive care admission. Crit Care Med 1999, 27:1073-1079. 9. Saukkonen K, Varpula M, Räsänen P, et al. The effect of emergency department delay on outcome in critically ill medical patients: evaluation using hospital mortality and quality of at 6 months. J Intern Med 2006, 260:586-591. 10.Tilluckdarry L, Tickoo $\mathrm{S}$, Amoateng-Adjepong $\mathrm{Y}$, et al. Outcomes of critically ill patients. Based on duration of emergency department. Am J Emerg Med 2005;23:336-339 11. Richardson DJ, Franklin G, Santos A, et al. Effective triage can ameliorate the deleterious effects of delayed transfer of trauma patients from the emergency department to the ICU. J Am Coll Surg. 2009;208: 671-81. 12. Mowery N, Dougherty S, Hildreth A, et al. Emergency department length of stay is an independent predictor of hospital mortality in trauma activation patients. J Trauma. 2011; 70: 1317-25. 13. Kinney E, Gursahani K, Armbrecht E, et al. Does emergency medicine length of stay predict trauma outcomes at a Level 1 Trauma Center?J Hospit Admin.2015;4:1-7 14. Bartolomeo S, Valent F, Rosolen V, et al. Are pre -hospital time and emergency department disposition time useful indicator for trauma care in Italy.Injury.2007;38:305-311. 15.Rivers E, Nguyen B, Havstad S, et al.; Early Goal-Directed Therapy Collaborative Group. Early goal-directed therapy in the treatment of severe sepsis and septic shock. N Engl J Med. 2001;345:1368 -1377. 16. Nguyen HB, Rivers EP, Havstad S, et al. Critical care in the emergency department: a physiologic assessment and outcome evaluation. Acad Emerg Med. 2000;7:1354 -1361.

Disclosure: No significant relationships.

\section{PR249}

\section{COCCYGECTOMY AND ITS RESULTS IN A DISTRICT GENERAL HOSPITAL}

\author{
A.K. Raghupathi ${ }^{1}, \underline{\text { A. Joshi }}^{2}$
}

${ }^{1}$ Trauma \& Orthopaedics, Southend University Hospital, SouthendOn-Sea/UNITED KINGDOM, ${ }^{2}$ Trauma \& Orthopaedics, Jersey General Hospital, jersey/UNITED KINGDOM

Introduction: Coccydynia is an uncommon, controversial condition, for which the results following non-operative treatment are mixed. When conservative treatment for chronic coccydynia has failed, coccygectomy may be considered, and favourable outcomes have been reported. The indications for coccygectomy are difficult to define as the cause of coccydynia in many patients is unknown. Neurosis and/or psychiatric disorders have often been associated with coccydynia and thought to be a contributing or magnifying factor.

Material and methods: A retrospective study in our hospital over a period of 6 years generated 7 patients who had coccygectomy where conservative measures have failed. Mean age of presentation is 18 to 52 years with an average age of 37 years. Conservative measures such as steroid injection, manipulation, physiotherapy, local heat, padding and cushioning were usually tried before deciding for surgical option. One patient had a history of fall, 3 cases had trauma during delivery and 2 cases had no relevant history.

Results: Coccygectomy was performed in knee elbow position. Midline approach with subperiosteal dissection was done. Sacrococcygeal junction was identified and any mobility was located on manual examination. Coccyx segments were removed taking adequate care to protect rectum. Surgery was performed from 8 to 24 months from the onset of symptoms. 3 out 7 patients developed complications such as superficial wound infection, wound dehiscence, hypersensitive scar, residual pain.

Conclusion: Coccygectomy is an effective operation in resistant cases with failed conservative treatment. Though it can give good results it is prone to certain complications.

References: 1. Surgery for Refractory Coccygodynia: Operative Versus Nonoperative Treatment. 2017 Apr 6. doi: 10.1007/s00590017-1947-3.

Disclosure: No significant relationships. 
PR250

\section{PSYCHIATRIC DISORDER ASSOCIATED WITH REPEATED BEHAVIOR OF FOREIGN BODY INGESTION. IS THERE AN EFFECTIVE PROTOCOL FOR ACTION TODAY?}

\section{T. Palau-Figueroa ${ }^{1}$, Y. Curbelo-Peña ${ }^{l}$, M. Saladich-Cubero ${ }^{1}$, N. Garcia-Leon ${ }^{2}$, M. Guixà-Gener ${ }^{1}, H$. Vallverdú-Cartie}

${ }^{1}$ General Surgery, Vic University Hospital, Vic/SPAIN, ${ }^{2}$ Psiquiatria, Vic University Hospital, Vic/SPAIN

Introduction: Introduction: Foreign body ingestion (FBI) usually occurs accidentally. The $70-80 \%$ of cases occur in children with ages between 6 months and 3 years.

Material and methods: Cases: The aim of this original article is present a case series of 4 patients with history of psychiatric disorders, all of whom are residents of the same institution, and who repeatedly perform FBI with self-injurious purposes. The treatment was different according to the episodes, from expectant management with radiological control in the most of cases, and upper digestive endoscopy until the finally surgical treatment.

Results: Discussion: Most of the objects use to be small inorganic objects such as piles, buttons, coins and pieces of toys. In adult patients, the objects are usually organics like bones, fish bones or food bowls. The most fragile are usually the elderly with dental prostheses ingestion. There is a special group, those patients who ingest voluntarily for different purposes. In one hand patients who have an intellectual disability, mental illness and/or substance abuse disorder, on the other hand, those who do it with economic purposes, also socalled "mules". The symptoms, complications and management depend on the object itself, evolution and anatomic localization.

Conclusion: Conclusion: The clinical performance is difficult because there is no protocoled management established currently to prevent, treat and most important, avoiding new episodes.

References: Errázuriz G. Ingestión de cuerpos extraños: Protocolo de estudio y tratamiento. Rev.Med, Clin.Condes. 2009; 60: 883-891. Banse C, Gorrón R, Cardemil D, Riquelme V, Cardemil P, Birrer AM. Ingestión de cuerpo extraño asociado a trastorno psiquiátrico. Cuad. Cir. 2003; 17 (1):47-51. Klein C. Intentional Ingestion and Insertion of foreign objects: A forensic perspective. J Am Acad Psychiatry Laq 2012; 40:119-126. Disponible en: http://citeseerx.ist.psu.edu/viewdoc/download?doi $=10.1 .1 .932 .7445 \&$ rep $=$ rep1\&type $=$ pdf Libuit, $\mathrm{J}$, Banez V. Repeated foreign body ingestion in a psychiatric patient. Journal of Interventional Gastroenterology 2014; 4:135-138. Disponible en: http://www.jigjournal.org/sites/default/files/135138\%20\%20JIG-2014.pdf Rodríguez J.I, Roig J, Ruiz B, Gironès J, Codina $\mathrm{A}$, Pont $\mathrm{J}$ y cols. Ingestión reiterada de cuerpos extraños y autoagresiones abdominales como intento de suicidio. Gastroenterol Hepatol 2004; 27 (9): 529-34. Disponible en: http:www.elsevier.es/ es-revista-gastroenterologia-hepatologia-14-pdf-S0210570503705214 Palta R, Sahota A, Bemarki A, et al: Foreign-body ingestion: characteristics and outcomes in a lower socioeconomic population with predominantly intentional ingestión. Gastrointest Endosc 2002; 69 (3):426-433. Disponible en: https://www.ncbi.nlm.nih.gov/pubmed/ 19019363 Artluri D, Veluru C, Chopra A, Mullen KD. Recurrent intentional foreign body ingestión: an endoscopist's dilema. Gastroenterol Hepatol 2012; 8:482-484. Disponible en: https:// www.ncbi.nlm.nih.gov/pmc/articles/PMC3533226/Poynter BA, Hunter JJ, Coverdale JH, Kempinsky CA. Hard to swallow: systematic review of deliberate foreign body ingestion. Gen Hosp Psychiatry. 2011; 33:518-524. Disponible en: http://dx.doi.org/10.1016/j.genhosppsych.2011.06.011 Palese C, Al-Kawas FH. Repeat Intentional Foreign Body Ingestion: The Importance of a Multidisciplinary Approach. Gastroenterology \& Hepatology. 2012;8 (7):485-486.
Disponible en: https://www.ncbi.nlm.nih.gov/pmc/articles/ PMC3533227/Liu L, Hunter J, Skorzewska A, Ovens H, Wright L. Care plan for patients who swallow foreign bodies. New York (NY): Mount Sinai Hospital, Psychiatry Patient Program. [Actualizado en 2017, Ene; consultado en 2017, Abr 9]. Disponible en: https:// www.mountsinai.on.ca/care/psych/on-call-resources/on-call-resources/foreign_bodies.pdf Gorospe E; Wong Kee Song L, Iyer P. Approach to Foreign Body ingestión, Food impaction and caustic injury. GI Endoscopic Emergencies. [Actualizado en 2016, Ene 1; consultado en 2017, Abr 9]. Disponible en: https://mayoclinic.pure.elsevier.com/en/publications/approach-to-foreign-bodyingestion-food-impaction-and-caustic-inj Ikenberry SO, Jue TL, Anderson MA, Appalaneni V, Banerjee S, Ben-Menachem T, et al. Management of ingested foreign bodies and food impactions. Gastrointest Endosc. 2011; 73 (6):1085-91. Disponible en: https:// www.ncbi.nlm.nih.gov/pubmed/21628009 Chinitz MA, Bertrand G. Endoscopic removal of toothbrushes. Gastrointest Endosc. 1990; 36 (5):527-30. Disponible en: McKinney PE. Acute elevation of blood lead levels within hours of ingestion of large quantities of lead shot. J Toxicol Clin Toxicol. 2000; 38 (4):435-40. Disponible en: https:// www.ncbi.nlm.nih.gov/pubmed/10930061 Mowad E, Haddad I, Gemmel DJ. Management of lead poisoning from ingested fishing sinkers. Arch Pediatr Adolesc Med. 1998; 152 (5):485-8. Disponible en: https://www.ncbi.nlm.nih.gov/pubmed/9605033 Beckley I, Ansari NS, Khwaja HA, Mohsen Y. Clinical management of cocaine body packers: the Hillingdon experience. Can J Surg. 2009;52 (5):417-21. Disponible en: https:/www.ncbi.nlm.nih.gov/pmc/articles/ PMC2769129/Mandava N, Chang RS, Wang JH, Bertocchi M, Yrad J, Allamaneni S, et al. Establishment of a definitive protocol for the diagnosis and management of body packers (drug mules). Emerg Med J. 2011; 28 (2):98-101. Disponible en: https://www.ncbi.nlm.nih.gov/ pubmed/21068166

Disclosure: No significant relationships.

\section{MILITARY \& DISASTER}

\section{PR251}

\section{'MAJOR INCIDENT DECLARED': ARE WE PREPARED?}

\section{Bernard, S.L. Tyler, G. Allen}

Accident And Emergency, Brighton and Sussex University NHS Trust, Brighton/UNITED KINGDOM

Introduction: To establish major incident (MI) staff preparedness within UK Major Trauma Centre's (MTC's) including level of training and their overall confidence in the event of an MI.

Material and methods: A questionnaire was sent via email to 26 MTC's in the UK (excluding Brighton and Sussex University Trust). It was disseminated via the ED secretaries to ED consultants, registrars and nurses.

Responses were captured between September and October 2017.

A Google form was used to capture responses.

Results: We confirmed that 18 MTC's had sent out the questionnaire. A total of 171 responses were gathered. 94 were from nurses, 24 from registrars and 53 from consultants.

138 had read their trusts major incident protocol. 133 were clear in their responsibilities in the event of an MI.

94 of respondents had not received any major incident training in the previous 12 months. Those who had received the most regular training was the nurses with 53 of them receiving training within the last 6 or 12 months. 
Regarding confidence in their own preparedness, 148 respondents felt neutral or better. The nurses are most confident with $68 \%$ of "very good' coming from them.

Regarding the department's preparedness, nurses again felt the most confident with just over half rating their department as "very good'.

Conclusion: Given the number of terror attacks in recent months it is concerning that $55 \%$ of respondents had not received any major incident training in the previous 12 months.

From this sample of clinicians and nursing staff it seems that more MI training is urgently required.

References:

Disclosure: No significant relationships.

\section{PR252}

\section{THE POSSIBILITY OF ULTRASONIC DIAGNOSTICS IN CASE OF MINE EXPLOSION INJURY}

\author{
S. Dvortsevoj ${ }^{1}$, A. Zubarev ${ }^{1}$, I. Obelchak ${ }^{2}$, K. Nikolaev $^{3}$
}

${ }^{1}$ Ultrasound, The Main military clinical hospital National Guard troops of Russian Federation, Balashicha, Russian Federation, Moscow/RUSSIAN FEDERATION, ${ }^{2}$ Radiology, The Main military clinical hospital National Guard troops of Russian Federation, Balashicha, Russian Federation, Moscow/RUSSIAN FEDERATION, ${ }^{3}$ Surgery, The Main military clinical hospital National Guard troops of Russian Federation, Balashicha, Russian Federation, Moscow/ RUSSIAN FEDERATION

Introduction: Ultrasound is often performed by the wounded after a mine blast injury.

Material and methods: A 15-year retrospective analysis performed to determine an opportunities and diagnostic value of ultrasound examination in casualties with mine blast injury, admitted to the medical detachment for special purposes and to the Main Military Clinical Hospital of Internal Troops from 2003 to 2017 year in the term from 1 to 6 hours after the injury. All the injured underwent ultrasound examination, X-ray, CT and (or) MRI. Continuous and categorical variables were studied with the Student's t test, Fisher's exact test or $\chi^{2}$ test, multivariate analysis was performed using a stepwise regression logistic model.

Results: A total of 271 records were reviewed. Head and neck injuries were at $42(15,5 \%)$, chest and abdomen - at $75(27,7 \%)$, extremity - at $154(56,8 \%)$ wounded. The sensitivity of ultrasound diagnostic method for wounds at superficial tissues of the head and trunk was $91 \%$, the detection of free fluid in the abdominal cavity $81 \%$, in the pleural cavity $-95 \%$, in wounds of the extremities $-91 \%$, in wounds of the abdominal cavity $-43 \%$. Specificity $-96 \%, 95 \%$, $98 \%, 96 \%, 94 \%$, accuracy $-95 \%, 84 \%, 97 \%, 95 \%, 91 \%$ respectively. Conclusion: Ultrasound is an informative method of visualizing lesions of extremities, soft tissues of the head and torso, intracavitary bleeding injuries in the acute period of mine blast injury. The use of portable ultrasound devices can diagnose damage in a field conditions when there is no possibility of evacuation of wounded.

Disclosure: No significant relationships.

\section{PR253}

\section{U.S. MILITARY FEMALE CASUALTIES IN THE IRAQ AND AFGHANISTAN WARS: A 12- YEAR RETROSPECTIVE ANALYSIS}

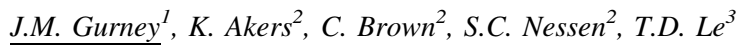

${ }^{1}$ Joint Trauma System, US Army Institute of Surgical Research, San Antonio/UNITED STATES OF AMERICA, ${ }^{2}$ Research Directorate, US Army Institute of Surgical Research, San Antonio/UNITED STATES OF AMERICA, ${ }^{3}$ Research Directorate, US Army Institute of Surgical Research, San Antonio/TX/UNITED STATES OF AMERICA

Introduction: Female service members (FSM) comprise 15\% of the active duty forces in the US military, and were authorized to deploy in direct combat roles in 2013. Traumatic injury in the Iraq and Afghanistan combat theaters is not comprehensively described in FSM. A precise characterization of the epidemiology of female military casualties and risk factors associated with mortality may elucidate differences from the predominantly male casualty population, and reveal opportunities to develop specific injury prevention strategies.

Material and methods: Data were extracted from the Department of Defense Trauma Registry for FSM injured while deployed from 2003 to 2014. Demographic and injury characteristics were described. Independent t-tests or Wilcoxon-Mann-Whitney test and chi-square or Fisher's exact test were used. Regression analysis and weighted moving average method (WMA) were performed to analyze injury outcome trends.

Results: 920 female casualties were analyzed, representing $3.1 \%$ of all combat casualties identified $(\mathrm{N}=29,289)$. FSM had a higher incidence of non-battle injury (NBI) than battle injury (BI), $61.9 \%$ vs. $38.1 \%, \mathrm{p}<0.0001$. Blunt injuries $(72.8 \%)$ were the leading injury type, followed by penetrating $(24.5 \%)$, and burn $(2.3 \%), \mathrm{p}<.0001$. Overall mortality amongst FSM was $3.6 \%(\mathrm{n}=33)$. The WMA shows that cumulative injury incidence declined from 2003 (5.2\%) to 2012 (2.9\%) then increased to $3.5 \%$ in 2014 .

Conclusion: Overall, the reported number of injuries for females decreased over time from 2003 to 2014, but it has increased since 2013. Identifying and understanding the causes, patterns and trends of injuries in FSMs may guide future measures to reduce preventable injury in this growing cohort of battlefield combatants.

References:

Disclosure: No significant relationships.

\section{PR254}

\section{AN INNOVATIVE SET OF UTILITIES FOR THE TREATMENT PLACE ON SHIPS IN MASS CASUALTIES}

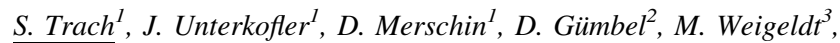
$\overline{\text { H. Ranke }}^{I}$, A. Ekkernkamp ${ }^{I}$, S. Schulz-Drost ${ }^{I}$

${ }^{1}$ Trauma- And Orthopedic Surgery, BG Hospital Unfallkrankenhaus Berlin gGmbH, Berlin/GERMANY, ${ }^{2}$ Department Of Trauma, Reconstructive Surgery And Rehabilitation Medicine, University Medicine Greifswald, Greifswald/GERMANY, ${ }^{3}$ Zentrum Für Klinische Forschung, Unfallkrankenhaus Berlin, Berlin/GERMANY

Introduction: Structured initial measures for mass casualties are crucial for a controlled procedure and a good outcome for as many 
patients as possible. In the first phase, the so-called chaosphase, a rapid order of space is particularly important. On the sea the often small experience of the maritime crew with mass casualties is additionally challenging. How could a treatment area for the initial medical treatment be established in order to provide sufficient assistance in the maritime environment?

Material and methods: Following the triage categories, the treatment area should contain the zones red, yellow, green and black as well as a minimum of medical equipment. This should be pre-sorted and marked for the respective treatment zone. The modules should be easy to transport by helpers. Through systematic literature research and expert interviews, an innovative standard of care has been defined.

Results: Four packages were established in colored bags with a trolley function. Each contains a stable tarp $(5 \times 6 \mathrm{~m})$ in the triage colour for laying on the ground for up to 10 patients on stretchers. For this purpose, 20 thermal blankets and medical equipment are provided. Red with emergency medical equipment and oxygen distribution module, yellow with medical equipment and green with first aid equipment. Prepared action charts contain clear algorithms as a help for the recommended treatment procedure.

Conclusion: Colored and easily portable packages allow easy and quick erection of a treatment area with colored marking of treatment zones by large tarpaulins. A graded equipment allows the necessary initial medical treatment respecting the triage category.

References:

Disclosure: The KOMPASS Project had been funded by the German Federal Ministry of Education and Science (BMBF)

\section{PR255}

\section{MSF EXPERIENCE TRAUMA FRONTLINE, TRIAGE MANAGEMENT IN A STABILIZATION CENTER IN MOSUL, IRAQ}

\section{L.B. Dominguez}

Medical Department, Medecins Sans Frontieres - Operational Center Brussels, Ixelles/BELGIUM

Introduction: In response to the Mosul crisis, MSF set up a stabilization center based in Hamam Al-Alil, 20 kilometers south of Mosul. The hospital was a mobile unit based in trailers on a truck and was called the MUST MUST stands for Mobile Unit Surgical Trailer. Material and methods: It performed life-saving emergency interventions (damage control surgery and resuscitation) from Feb. 19 to July 18 , 2017. It relied on a referral system to other health structures for post op care. Of the 3899 cases treated in the $\mathrm{ED}$, the average age was 24 years old, majority were more than 15 years old (64.2\%) and $35 \%$ were female. Based on the START triage, $15.6 \%$ of patients arriving were red, $61.9 \%$ yellow and $22.5 \%$ green.

Results: The first three months, $20 \%$ of cases were red and this decreased to $10 \%$ the last three months. $18.2 \%$ of cases were referred, $0.5 \%$ of cases died, while $32.8 \%$ of cases were referred. Of the dead, $(0.5 \%, 20$ cases $), 19$ were triaged as red and 1 was yellow. At the beginning, $90 \%$ of the cases treated were due to violence. Overall, $54.8 \%$ of the trauma cases was related to violence. Of the 1976 violent cases, $25.1 \%$ were admitted for surgery, $27 \%$ were discharged and $47 \%$ referred of which $37 \%$ were triaged as red, $55.6 \%$ yellow, and $7.4 \%$ green. 396 cases underwent surgery $-84 \%$ due to violent trauma. The highest number of visceral surgeries was performed during the first weeks.

Conclusion: Intra-op mortality was $1.8 \%$.
References: 2017 - HAMAM AL-ALIL[1] - SURGICAL CARE REPORT MSF OCB SAGE UNIT 2017 - HAMAM AL-ALIL EMERGENCY DEPARTMENT REPORT MSF OCB SAGE UNIT [1] Hamam Al-Alil is located around $30 \mathrm{~km}$ driving distance south of Mosul. It should be considered that the project is sometimes also called Mosul (Erbil) as it was intended to face the humanitarian crisis in Mosul city.

Disclosure: No significant relationships.

\section{PR256}

\section{EMERGENCY HEALTHCARE ACCESS FOR UNREGISTERED MIGRANTS IN HUNGARY}

\author{
I. Gárgyán, A. Mácsai, K. Csete, E. Varga
}

Department Of Traumatology, University of Szeged, Szeged/ HUNGARY

Introduction: Legal status is one of the most important determinants of the access of migrants to health services in a country. Each refugee and migrant must have full, uninterrupted access to a hospitable environment and, when needed, to high-quality health care, without discrimination on the basis of gender, age, religion, nationality or race. Migrants have the right to access emergency care free of charge in Hungary.

Material and methods: All the migrants in need of medical care were transported to our Emergency Department, because our institute is closest to the border. From January 2015 to September 2017, we collected the data from the migrants we were admitted.

Results: In 2015, 391,000 migrants entered Hungary. The ED provided a total of 320 migrants, $31 \%$ of whom were injured. 23 patients were admitted to our Traumatology Department. After closing the borders, the migratory phenomenon was spiraling down. We admitted only 8 severely injured patients from a single high-speed motor vehicle accident, all the patients had ISS $\leq 15$.

Conclusion: Emergency ambulances and our department were not burdened by the number of cases, but we had serious difficulties in providing professional care, because we had to deal with people of unknown origin, language. Migrants did not disclose their personal data, so in the absence of data, the dismissal of the injured was particularly difficult without a home address, without knowing the location of the relatives. The authorities have always helped to solve the problems, but the development of proper contacts with them took a longer time.

References:

Disclosure: No significant relationships.

\section{PR257}

\section{TEAM-BASED-LEARNING IN TEACHING DISASTER MEDICINE FOR UNDERGRADUATE MEDICAL STUDENTS}

\section{F.M.Abu-Zidan ${ }^{1}$, A.A. Cevik ${ }^{2}$}

${ }^{1}$ Surgery, College of Medicine, UAEU University, Al-Ain/UNITED ARAB EMIRATES, ${ }^{2}$ Internal Medicine, College of Medicine, UAEU University, Al-Ain/UNITED ARAB EMIRATES

Introduction: Team-Based Learning (TBL) is accepted as an active learning method with positive effects on learning outcomes (1-2). We 
aim to report our recent experience in using TBL in teaching disaster medicine for undergraduate medical students.

Material and methods: 5th year medical students triaged victims of a clinical scenario of a car bomb that resulted in 6 victims using TBL. The injuries ranged between psychological trauma to expectant death. TBL activity lasted for 2 hours. 98 medical students $(60$ females, 38 males) were taught in 24 teams having 3-6 students in 6 sessions (4 teams in each session) during the period of August 2016-September 2017. Triage was done according to a simple ABC approach. Initially each student made the triage alone, then with the team, and finally with the whole class. A team leader was chosen for each team in which she/he was asked to facilitate the discussion, present the findings, and defend the group decision in the class discussion.

Results: TBL sessions were dynamic, rich in debate, and interactive. The students were completely involved in the discussion and the decision making process. They showed maturity in understanding the scenario, following the rules, and making and defending their decisions. There were two clear observations on the triage of the students. Students over-triaged the victims and did not use the expectant category.

Conclusion: Principles of disaster medicine, like triage, can be included in the undergraduate curriculum. TBL was useful in promoting working within teams, critical decision making, and leadership; all are essential components of management of disasters. References: 1 . Sisk RJ. Team-based learning: systematic research review. J Nurs Educ. 2011; 50:665-9. 2. Thompson BM, Schneider VF, Haidet P, Levine RE, McMahon KK, Perkowski LC, Richards BF. Team-based learning at ten medical schools: two years later. Med Educ. 2007;41:250-7.

Disclosure: No significant relationships.

\section{PR258}

\section{ANALYSIS OF BATTLE AND NON-BATTLE TRAUMATIC RENAL INJURIES: A 6-YEAR EXPERIENCE IN A CONFLICT ZONE IN AFGHANISTAN}

\section{T. Zabkowski ${ }^{1}$, R. Brzozowski ${ }^{2}$}

${ }^{1}$ Urology Department, Military Institute of Medicine, Warsaw/ POLAND, ${ }^{2}$ Department Of Combat Medicine, Military Institute of Medicine, Warsaw/POLAND

Introduction: To analyze traumatic renal injuries and determine topography of the most common abdomen injuries. Gunshot wounds are the most common causes of penetrating injuries and have the tendency to become more severe and less predictable than blunt trauma. An average of $8-10 \%$ of blunt and penetrating abdominal injuries involve the kidneys.

Material and methods: A total of 1266 combat trauma patients were treated in two health centers in Afghanistan and the following features of renal injury were assessed: etiology or mechanism of trauma, PATI score in penetrating trauma, treatment method, and multiorgan injury. Results: Of the 44 renal injuries, 21 injuries were of the right whilst 23 injuries were of the left kidney. In 17 patients, nephrectomy was performed, nephrectomy of the left kidney in 13 patients and nephrectomy of the right kidney in 4 patients. The Kaplan-Meier survival analysis was applied to determine a salvage rate of kidney using PATI score as indicator of nephrectomy. The renal salvage rate was $61.36 \%$ and overall survival rate was $90.24 \%$. Battle injury died of wounds casualty occurred in 4 patients.
Conclusion: The left side of abdomen was most often subject to trauma therefore there are the numerous multiorgan injuries of the left side of abdomen.

References: Davis P, Bultitude MF, Koukounaras J, et al. Assessing the usefulness of delayed imaging in routine followup for renal trauma. J Urol 2010, 184:973-977. Bjurlin MA, Goble SM, Fantus RJ, et al. Outcomes in geriatric genitourinary trauma. J Am Coll Surg 2011, 213 (3):415-21. Najibi S, Tannast M, Latini JM. Civilian gunshot wounds to the genitourinary tract: incidence, anatomic distribution, associated injuries, and outcomes. Urology 2010, 76 (4):977-81.

Disclosure: No significant relationships.

\section{PR259}

\section{MASS CAUSALITY INCIDENCE AT SEA - A TRIAGE EXERCISE OF AN ANALOG AND DIGITAL SYSTEM}

\author{
R. Wendorf ${ }^{1}$, T. Görig ${ }^{2}$, E. Henning ${ }^{2}$, S. Schulz-Drost ${ }^{3}$, \\ A. Ekkernkamp ${ }^{3}$, D. Gümbel ${ }^{2}$
}

${ }^{1}$ Department Of Trauma, reconstructive Surgery And Rehabilitation Medicine, University Medicine Greifswald, Greifswald/GERMANY, ${ }^{2}$ Department Of Trauma, Reconstructive Surgery And Rehabilitation Medicine, University Medicine Greifswald, Greifswald/GERMANY,

${ }^{3}$ Trauma- And Orthopedic Surgery, Unfallkrankenhaus Berlin, Berlin/ GERMANY

Introduction: In the past the number of passengers travelling by ships has increased worldwide. On the one hand MCI at sea have a low-likelihood, on the other hand they are associated with high consequences for the crew and passengers. In this situation, a realistically patient triage is required to prioritize patient treatment before the transport organization of injured to medical facilities has to be made. Therefore a digital triage and transport priority system for nonmedical personnel were developed.

Material and methods: In a triage exercise the analog triage system LüDoG was compared with the developed digital triage doc-system "TOPAS". The aim of the exercise was to evaluate the performance and the usability of the TOPAS compared to an paper based triage system. Hence fifty non-medicals conducted the triage exercise with of 50 paper based patients for each system. The one half started with the analog \& the others with the digital system.

Results: In total 5000 paper based patients should have been triaged. $79,6 \%$ of the patients were triaged with the analog system and $71,5 \%$ with the digital version. With the analog system over $73,1 \%$ and digital $71,5 \%$ of the patients were correctly triaged. This difference might be caused by an over-categorizing at the digital system at $35,9 \%$. The transport priorization was more often proceeded by the digital process. The finale results will be presented.

Conclusion: The data lead us to the interim interpretation that the digital system is slower but increase the quality of the overall-organization of a MCI, especially when integrating non-professional is key.

\section{References:}

Disclosure: No significant relationships. 
PR260

\section{MANAGEMENT OF PENETRATING PELVIC TRAUMA. A FIVE YEARS EXPERIENCE OF FRENCH MILITARY HEALTH SERVICE}

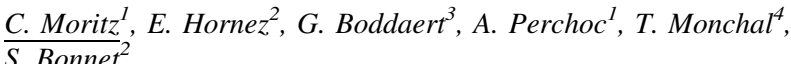

${ }^{1}$ Digestive Surgery, HIA Percy, Clamart/FRANCE, ${ }^{2}$ Department Of Digestive Surgery, Percy Military hospital, Clamart/FRANCE, ${ }^{3}$ Department Of Thoracic Surgery, Percy Military hospital, Clamart/ FRANCE, ${ }^{4}$ Digestive Surgery, HIA Sainte Anne, Toulon/FRANCE

Introduction: Penetrating pelvic trauma's are characterized by complex lesionnal associations in civilian and military setting. This study aims to report the experience and the lessons learned by the French military surgeons in the surgical management of pelvic trauma during the 5 last years.

Material and methods: This is a retrospective study of the civilian polytrauma and military medevac database fom 2011 to 2016. Up to 175 data points were queried for each patient and subjected to descriptive analysis. 4 subgroup were compared: war injuries, civilian injuries, rectum wounds and bladder wounds.

Results: 25 patients presented severe pelvic injuries, $64 \%$ were military and $32 \%$ civilian. The average age was 31.1. The median of ISS was 13 . There was $68 \%$ of "war injuries". Abdomen, thoracic, bladder and limbs injuries occurred in $56 \%, 24 \%, 20 \%$ and $60 \%$. The civilian subgroup included more hollow viscus injuries. The rectum injuries subgroup include more urologic injuries: $28.5 \%$ bladder. The rectum wound subgroup and the civilian subgroups had the most severity injuries with an ISS of $17.40 \%$ needed damage-control, $80 \%$ iterative surgeries. The median lenght of hospitalization was 33 days but 24.5 days for the civilian subgroup and 47 days for the rectum subgroup. The main complications were sepsis, chronic pain, neurological deficit and PTSD. The rectum injuries subgroup haved more sepsis (29\%) and neurologic complications (29\%).

Conclusion: Penetrating pelvic trauma need staged and iterative surgery. Rectal wound was a factor of severity (ISS, lenght of stay) and of complex lesionnal associations. Military and civilian cohorts was quite similar in the era of terrorism attack.

References:

Disclosure: No significant relationships.

\section{PR261}

\section{TELEMEDICINE SUPPORT FOR OFFSHORE WIND ENERGY PLANT - EXPERIENCE OF THE FIRST 24 MONTHS - THE VIEW OF THE MENTORING HOSPITAL}

M. Gondert ${ }^{1}$, M. Weigeldt ${ }^{2}$, J. Beneker ${ }^{3}$, A. Ekkernkamp ${ }^{4}$, S. SchulzDrost $^{4}$

${ }^{1}$ Klinik Für Unfallchirurgie Und Orthopädie, Unfallkrankenhaus Berlin, Berlin/GERMANY, ${ }^{2}$ Zentrum Für Klinische Forschung, Unfallkrankenhaus Berlin, Berlin/GERMANY, ${ }^{3}$ Rettungswesen, Unfallkrankenhaus Berlin, Berlin/GERMANY, ${ }^{4}$ Trauma- And Orthopedic Surgery, Unfallkrankenhaus Berlin, Berlin/GERMANY

Introduction: At offshore wind farms, the presence of numerous employees around the clock is necessary to ensure ongoing operations. Emergency medical care is supported by telemedical support.
Material and methods: We performed a retrospective evaluation of the telemedical consultations at the Unfallkrankenhaus Berlin from 01.07.2015 to 30.06 .2017

Results: During the observation period, 120 telemedicine consultations were held. The average age of patients was 39 years $(\min 4$, max 77 , SD 10.8), $7.2 \%$ were female, $92.8 \%$ male. Over the entire period, June and August were the most eventful months. In the "summer halfyear" (May-October), 74 (61.7\%) missions took place. The time of day statistics shows a maximum between 7 and 12 o'clock as well as between 18 and 22 o'clock.

Calls from offshore windfarm platforms took place in 108 cases (90\%), from merchant ships in $7(5.8 \%)$, from private boats in 4 $(3.3 \%)$, and from cruise ships in $1(0.8 \%)$ cases.

$42(35 \%)$ of the cases required disembarkation for on-shore supply, of which $34(81 \%)$ were evacuated by a helicopter.

Conclusion: The field of activity of the rescue personnel offshore could be adequately supported and secured by trained acute physicians of the interdisciplinary emergency department.

References:

Disclosure: No significant relationships.

\section{PR262}

\section{JUVENTUS FOOTBALL FANS DISASTER: LESSON LEARNED FROM HIGH VOLUME ADMISSION WITH LOW IMPACT INJURIES}

\section{E. Grosso}

Ortopedia E Traumatologia, città della salute, Torino/ITALY

Introduction: more and more frequent cases of massive inflow of patients to ER. June 3, 2017 during the championship leage final in Turin we had a catastrophic event with more than 1500 patients involved.

Material and methods: we describe the events that took place in our hospital and in the city that night

Results: the match attracted more people than expected, the organization was not prepared. the first catastrophic event in this city has highlighted shortages in the organization of relief.

Conclusion: the increase in cases of mass casualty requires a continuous updating of intervention protocols

References: [Mass casualty incidents : preparedness of German soccer arenas]. Luiz T, Preisegger T, Madler C. Anaesthesist. 2013 Apr;62 (4):278-84. doi: 10.1007/s00101-013-2162-1. Epub 2013 Apr 12. German. Lessons in planning from mass casualty events in UK. Moran CG, Webb C, Brohi K, Smith M, Willett K. BMJ. 2017 Oct 25;359:j4765. doi: 10.1136/bmj.j4765 Terrorism in France. Carli P, Telion C, Baker D. Prehosp Disaster Med. 2003 Apr-Jun;18 (2):92-9. Review.

Disclosure: No significant relationships. 
PR263

\section{EVALUATION OF AN EMERGENCY PRACTICE FOR A MASS CAUSALITY INCIDENCE AT SEE}

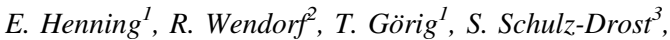 \\ A. Ekkernkamp $^{3}$, D. Gümbel ${ }^{1}$
}

${ }^{1}$ Department Of Trauma, Reconstructive Surgery And Rehabilitation Medicine, University Medicine Greifswald, Greifswald/GERMANY, ${ }^{2}$ Department Of Trauma, reconstructive Surgery And Rehabilitation Medicine, University Medicine Greifswald, Greifswald/GERMANY, ${ }^{3}$ Trauma- And Orthopedic Surgery, Unfallkrankenhaus Berlin, Berlin/ GERMANY

Introduction: The primary goal of the emergency practice was to investigate the logistic-organizational management of a MANV on a ship in the acute phase of a mass causality incidence (mci).

Material and methods: The emergency practice took place at the traditional ship. A number of 18 crew members and 39 injured actors were involved in the scenario. The crew were instructed to the scenario, the ship, developed guidelines regarding a mci, triage, a digital triage and transport organizing documentation system and existing medical equipment and medications for a mci. In this analyzes the focus will be on the result of the standardized semi structured and written questionnaire with the injured actors.

Results: About $59 \%$ of the injured actors were females. The average was 34 years old. $65,8 \%$ of the actors stated that they had already experience in acting as injured in other emergency practices. 92\% mentioned that "the exercise was useful". The support by the ship crew were ranked by $23 \%$ as very good and good, $35,9 \%$ as satisfying and $41 \%$ as sufficient and deficient. Significant results (95\%) were calculated by the difference of experience in acting as injured and the gender of the actors.

Conclusion: The results give a brief overview about the opinion and feeling of injured actors regarding their view of a mci at sea. To prepare a ship crew for a mci focusing on the treatment of injured, further emergency practices should be conducted by ship crews.

References:

Disclosure: No significant relationships.

\section{PR264}

\section{HEALTH RECORDS SYSTEM FOR TRIAGE AT MASS CASUALTY INCIDENCE AT SEA - OVERVIEW AND USABILITY OF EXISTING RECORD SYSTEMS}

\section{E. Henning $^{1}$, S. Schulz-Drost ${ }^{2}$, A. Ekkernkamp ${ }^{2}$, D. Gümbel ${ }^{2}$}

${ }^{1}$ Department Of Trauma, Reconstructive Surgery And Rehabilitation Medicine, University Medicine Greifswald, Greifswald/GERMANY, ${ }^{2}$ Department Of Trauma, reconstructive Surgery And Rehabilitation Medicine, University Medicine Greifswald, Greifswald/GERMANY

Introduction: To enable the leader of the first responder in the event of a mass casualty incidence (MCI) special documentation systems were developed to support organization. In an MCI at sea the crew is facing a tremendous challenge due to the lack of resources. Therefore, a review of analog documentation systems (ADS) and digital documentation systems (DDS) for triage and documentation was conducted to analyze the suitability of the different systems at sea.
Material and methods: Build on a web- and literature-based search an overview of ADS and DDS for MCI was given. The evaluation of the documentation systems was carried out by a developed tool regarding the usability at sea. This evaluation tool covered the categories technique, usability, contents and costs with an index of 13 points.

Results: Four ADS and five DDS were identified. Regarding the technical aspects ADS are waterproof compared to the DDS. No electricity or specific preparation is necessary. On the other hand DDS do not need daylight. An overview of the specific features of each report system is presented.

Conclusion: Both system groups have advantages and disadvantages. ADS have recognizable advantages in the area of usability and cost. In turn, DDS have explicit strengths in the content area. With them, it is possible to create an overview more quickly and to use other features such as transport organization. In summary, none of the identified documentation systems can be determined to be optimal for use at a mci at sea.

References:

Disclosure: No significant relationships.

\section{PR264A}

\section{TERRORIST ATTACKS AND THE MANAGEMENT OF THE PATIENT IN THE RESUSCITATION ROOM - ARE THERE ANY DIFFERENCES?}

\section{G. Achatz, B. Friemert}

Dept. For Orthopedics And Trauma Surgery, Reconstructive And Septic Surgery, Sportstraumatology, German Armed Forces Hospital Ulm, Ulm/GERMANY

Introduction: Terrorist attacks are now occurring in Western Europe in an abundance so that we have to estimate such scenarios at any time. Caused by the use of weapons and bombs these scenarios normally result in a large number of injured patients in the sense of a mass casualty incident (TerrorMASCAL). This is not only a major challenge for emergency responders and security forces at the event site, but also in the immediate aftermath for the hospitals. Especially because in many cases there are life-threatening injuries with not infrequently relevant bleeding.

Material and methods: From the efforts and the implementation of the Terror-and-Disaster-Surgical-Care - Course (TDSC®) in Germany we have dealt intensively with the topic of the initial care for the patients in the resuscitation room. There was the question to clarify, whether an ATLS ${ }^{\circledR}$ - based procedure is possible as normally or whether it must come to an adjustment due to the special situation. Results: On the basis of a comprehensive review of the literature and the implementation of the TDSC $\AA$ - Course in Germany, aspects and recommendations could be worked out which take the special circumstances into account.

Conclusion: The care for patients in the resuscitation room in TerrorMASCAL - situations requires a rethinking and adapted behaviour. This on the one hand in the way of the approach but also in the use of the available resources and frequently a distinction must be made between measures according to the Damage Control - Concept (DC) and the Tactial Abbreviated Surgical Care - Concept (TASC).

References:

Disclosure: No significant relationships. 


\section{VISCERAL TRAUMA}

\section{PR265}

\section{IMPACT OF CIRRHOSIS ON OUTCOMES IN TRAUMA}

\author{
M. Anastasiu, R. Dedu, D. Vicol, N. Micu
}

General Surgery, Emergency County Hospital, Buzau/ROMANIA

Introduction: The aim of this study is to evaluate the risk of mortality and complications in cirrhotic trauma patients undergoing laparotomy.

Material and methods: The trauma registry was queried for all patients with liver cirrhosis admitted and treated in our Level 2 Trauma Center between 2007 and 2016. Data analyzed included age, mechanism of injury, Injury Severity Score (ISS), Glasgow Coma Score (GCS), ICU length of stay, procedures performed, complications (acute renal failure, ARDS, intra-abdominal sepsis, trauma associated coagulopathy) and mortality. Multivariable analysis was utilized to compare the outcomes with the non-cirrhotic trauma patients; in addition for statistical comparison, Fischer's exact test and Student's $t$-Test were performed.

Results: During the 10-year study period were 2246 trauma laparotomies, of which $68(3 \%)$ had a diagnosis of cirrhosis. The overall mortality in the cirrhotic group is significantly higher than in noncirrhotic group ( $48 \%$ vs. $26 \%$; $\mathrm{OR}=6.80, \mathrm{p}=0.018$ ). Mortality in patients with ISS $\geq 16$ was $58 \%$ and $12 \%$, respectively in non-cirrhotic group $(\mathrm{p}=0.028)$. Concerning the complications, we have retained $41 \%$ in the cirrhotic group vs. $19 \%(\mathrm{p}=0.016)$ and the most common complication in the cirrhotic group was disseminated intravascular coagulopathy to 6 patients $(21 \%)$. The mean ICU stay was 14.2 days in cirrhotic group vs. 7.8 days $(\mathrm{p}=0.042)$ in noncirrhotic group. In non-survivors, the ICU stay was similar in the 2 groups (9.4 days vs. 10.6 days, $\mathrm{p}=0.994$ ).

Conclusion: Cirrhosis is an independent risk factor for increased mortality and a higher complication rate following trauma.

References: 1. Christmas AB, Wilson AK, Franklin GA et al (2005) Cirrhosis and trauma: a deadly duo. Am Surg 71:996-1000 2. Wahlstrom K, Ney AL, Jacobson S et al (2000) Trauma in cirrhotics: survival and hospital sequelae in patients requiring abdominal exploration. Am Surg 66:1071-1076

Disclosure: No significant relationships.

\section{PR266}

\section{ACUTE LITHIASIC PANCREATITIS IN ELDERLY SURGICAL PATIENT}

P. Colsa ${ }^{1},{\underline{\text { D. } \text { Morales }^{2}}}^{\text {, J. Rodriguez }}{ }^{1}$

${ }^{1}$ Servicio De Cirugía General Y Del Aparato Digestivo, Hospital Grande Covián, Arriondas/SPAIN, ${ }^{2}$ Servicio De Cirugía General Y Del Aparato Digestivo, Hospital Universitario Marqués de Valdecilla, Santander/SPAIN

Introduction: Acute lithiasic pancreatitis is a common cause of abdominal pain in elderly patinets. The aim of the present study was to describe the characteristics of patients over 70 years of age who were submitted to urgent surgery because of acute pancreatitis.

Material and methods: A retrospective population-based study of patientss older than 70 undergoing urgent surgery because of abdominal pain. We included 675 patients operated from January 2012 to December 2013 in the community of Cantabria.

In 8 of the patients, the diagnosis was acute pancreatitis. The data were analyzed using the $\mathrm{X} 2$ test with the statistical software SPSS 17.0. They were considered significant at $\mathrm{p}<0.05$.

Results: Lithiasic pancreatitis predominates in the group of patients under 80 , with a mean age of 76.5 years and standard deviation 4.6 years. Of these, $87.5 \%$ were male and $12.5 \%$ female. 7 patinets were involved in the Hospital Marqués de Valdecilla and 1 patient in the Hospital Sierrallana.

At the time of surgery $60 \%$ and $50 \%$ were ASA IV. All of them met sepsis criteria.

In one of the patients, laparoscopic cholecystectomy was performed. In the rest of patients, abdominal decompression surgery was performed.

Postoperative morbidity was $33.3 \%$ of postoperative ileus; $38.1 \%$ organ-space infection and $14.3 \%$ evisceration.

$47.6 \%$ of the patients required admission to an intensive care unit.

This pathology was associated with longer hospital stays compared to other causes of acute abdomen (63.2\% over 11 days). Different authors report a range between 5-25 days, higher than younger patients (3-10 days)

Mortality reached $76.9 \%$ in pancreatitis. According to some studies, diabetes and alcohol and tobacco use have been shown to be factors associated with severity.

Conclusion: Acute biliary pancreatitis is a common pathology in the elderly. These patients are especially vulnerable because of their baseline comorbidity, systemic compromise and delayed diagnosis.

References: Valdivieso V, Palma R, Wünkhaus R, Antezana C, Severín C, Contreras A. Effect of aging on biliary lipid composition and bile acid metabolism in normal Chilean women. Gastroenterology.1978; 74:871-874. Van Geloven AAW, Biesheuvel TH, Luitse JSK, Hoitsma HFW, Obertop H. Hospital admissions of patients aged over 80 with acute abdominal complaints. Eur J Surg 2000; 166:866-871. Cook TM, Day CJ. Hospital mortality after urgent and emergency laparotomy in patients aged $65 \mathrm{yr}$ and over. Risk and prediction of risk using multiple logistic regression analysis. Br J Anaesth.1998; 80:776-778. Arenal JJ, Bengoechea-Beeby M. Mortality associated with emergency abdominal surgery in the elderly. Can J Surg. 2003; 46:111-116 Br J Anaesth.1998; 80:776-781

Disclosure: No significant relationships.

\section{PR267}

\section{IMPLEMENTATION OF NEW STANDARD OPERATING PROCEDURES. ARE THEY ALSO WORKS FOR GERIATRIC TRAUMA PATIENTS WITH MULTIPLE INJURIES}

P. Lorenz, K.O. Jensen, C. Ossendorf, G. Osterhoff, H.P. Simmen, L. Mica, H. Pape, K. Sprengel

Department Of Trauma, University Hospital Zurich, Zurich/ SWITZERLAND

Introduction: The demographic changes towards ageing of the populations in developed countries imposes a challenge to trauma centres, as geriatric trauma patients require specific diagnostic and therapeutic procedures. This study investigated whether the integration of new standard operating procedures (SOPs) for the resuscitation room has an impact on the clinical course in geriatric patients. The new SOPs were designed for severely injured adult trauma patients, based on the Advanced Trauma Life Support (ATLS) and imply early 
whole-body computed tomography, damage control surgery, and the use of goal-directed coagulation management.

Material and methods: Single-center cohort study. We included all patients $>=65$ years of age with an Injury Severity Score (ISS) $>=9$ who were admitted to our hospital primarily via resuscitation room. A historic cohort was compared to a cohort after the implementation of the new SOPs.

Results: We enrolled 311 patients who met the inclusion criteria between 2000-2006 (Group PreSOP) and 2010-2012 (Group SOP). There was a significant reduction in the mortality rate after the implementation of the new SOPs $(P=.001)$. This benefit was seen only for severely injured patients (ISS $\geq 16$ ), but not for moderately injured patients (ISS 9-15). There were no differences with regard to infection rates or rate of palliative care.

Conclusion: We found an association between implementation of new ER SOPs, and a lower mortality rate in severely injured geriatric trauma patients, whereas moderately injured patients did not obtain the same benefit.

References: Champion HR, Copes WS, Buyer D, Flanagan ME, Bain L, Sacco WJ: Major trauma in geriatric patients. Am J Public Health 1989, 79:1278-1282. Perdue PW, Watts DD, Kaufmann CR, Trask AL: Differences in mortality between elderly and younger adult trauma patients: geriatric status increases risk of delayed death. $J$ Trauma 1998, 45:805-810. Taylor MD, Tracy JK, Meyer W, Pasquale M, Napolitano LM: Trauma in the elderly: intensive care unit resource use and outcome. $J$ Trauma 2002, 53:407-414. Vanzant EL, Hilton RE, Lopez CM, Zhang J, Ungaro RF, Gentile LF, Szpila BE, Maier RV, Cuschieri J, Bihorac A, et al: Advanced age is associated with worsened outcomes and a unique genomic response in severely injured patients with hemorrhagic shock. Crit Care 2015, 19:77. Butcher SK, Killampalli V, Chahal H, Kaya Alpar E, Lord JM: Effect of age on susceptibility to post-traumatic infection in the elderly. Biochem Soc Trans 2003, 31:449-451. Boltz MM, Podany AB, Hollenbeak CS, Armen SB: Injuries and outcomes associated with traumatic falls in the elderly population on oral anticoagulant therapy. Injury 2015, 46:1765-1771. Grimshaw JM, Russell IT: Effect of clinical guidelines on medical practice: a systematic review of rigorous evaluations. Lancet 1993, 342:1317-1322. Schoeneberg C, Schilling M, Burggraf M, Fochtmann U, Lendemans S: Reduction in mortality in severely injured patients following the introduction of the "Treatment of patients with severe and multiple injuries" guideline of the German society of trauma surgery-a retrospective analysis of a level 1 trauma center (2010-2012). Injury 2014, 45:635-638. HuberWagner S, Lefering R, Qvick LM, Körner M, Kay MV, Pfeifer KJ, Reiser M, Mutschler W, Kanz KG, Society WGoPotGT: Effect of whole-body CT during trauma resuscitation on survival: a retrospective, multicentre study. Lancet 2009, 373:1455-1461. Rossaint R, Bouillon B, Cerny V, Coats TJ, Duranteau J, Fernandez-Mondejar E, Filipescu D, Hunt BJ, Komadina R, Nardi G, et al: The European guideline on management of major bleeding and coagulopathy following trauma: fourth edition. Crit Care 2016, 20:100. Rotondo MF, Schwab CW, McGonigal MD, Phillips GR, Fruchterman TM, Kauder DR, Latenser BA, Angood PA: 'Damage control': an approach for improved survival in exsanguinating penetrating abdominal injury. $J$ Trauma 1993, 35:375-382; discussion 382-373. Richmond TS, Kauder D, Strumpf N, Meredith T: Characteristics and outcomes of serious traumatic injury in older adults. J Am Geriatr Soc 2002, 50:215-222.

Disclosure: No significant relationships.

\section{PR268}

\section{PROGNOSIS, AND ROLE OF ANGIOEMBOLIZATION AND SURGERY IN SPONTANEOUS RETROPERITONEAL AND RECTUS SHEATH HEMATOMAS: A SINGLE CENTER EXPERIENCE}

P. Ciriano Hernandez, M.L. Fernandez, Vazquez, M. Fernandez. Martinez, L. Martin Roman, E. Viejo Martinez, M. Sanz, Sanchez

\section{Surgery, HOSPITAL GREGORIO MARAÑON, MADRID/SPAIN}

Introduction: Retroperitoneal (RPH) and rectus sheath hematomas (RSH) pose a therapeutic challenge on occasions. Usually characterized by a benign course, they may present with hemodynamic instability and severe anemia, prompting invasive interventions. We wanted to review the prognosis and role of invasive procedures in the management of these entities, with the hypothesis that surgery is rarely, if ever, indicated nowadays.

Material and methods: Retrospective review of patients diagnosed of RSH or RPH between 2010 and 2017. We analized demographics, comorbidities, risk factors, antiplatelet or anticoagulation therapy, symptoms, lab values, treatment, length of hospital stay, time to reintroduction of anticoagulant therapy, and mortality.

Results: There were 91 patients, $70,3 \%$ of them female, with a median age of 82,5 years. Frequent comorbidities were high blood pressure $(80,2 \%)$, diabetes mellitus $(24,2 \%)$, and COPD $(27,5 \%)$. Most patients were on antiplatelets $(25,3 \%)$ or anticoagulation therapy $(82,4 \%)$ due to DVT, pulmonary embolism or arrhythmia. In most cases $(61,5 \%)$, no trigger factor was identified. Treatment is was most often conservative, requiring blood transfusion in $57,1 \%$ of patients. AE or surgical evacuation and hemostasis were required in $22 \%$ and $4,4 \%$, respectively. $61,5 \%$ resumed antiplatelet or anticoagulant therapy in a median time of 9,4 days. The median length of hospital stay was of 20 days, and mortality was of $13.2 \%$.

Conclusion: Spontaneous RPH and RSH rarely require surgery nowadays, and most are amenable to conservative management, with frequent use of angioembolization.

References: Clinical spectrum of patients with spontaneous retroperitonealhematomasAmada Sánchez-González, Leyre RianchoZarrabeitia, Zaida Salmón-González, José Antonio Riancho, Carmen Valero Evaluation of Risk Factors for Rectus Sheath Hematoma Heena S. Sheth, MD, MPH1, Rohit Kumar, MD2, Jeannine DiNella, DNP, RN3, Cheryl Janov, RN, BSN4, Hoda Kaldas, MD1, and Roy E. Smith, MD Massive retroperitoneal hematoma as a complication of anticoagulation therapy in a patient treated in a pulmonary intensive care unit Mihailo Stjepanović*, Ivana Buha*, Snežana Raljević*†, Uroš Babićł, Milan Savićł§, Jovana Mašković*, Marina Roksandić*, Dragana Marić SPONTANEOUS RETROPERITONEAL HEMATOMA: ETIOLOGY, CHARACTERISTICS, MANAGEMENT, AND OUTCOME Kharmene L. Sunga, MD, M. Fernanda Bellolio, MD, MS, Rachel M. Gilmore, MBBCHB, and Daniel Cabrera, MD

Disclosure: No significant relationships. 


\section{PR269}

\section{SUCCESSFUL SELECTIVE NON OPERATIVE MANAGEMENT OF PENETRATING STAB WOUNDS ACCORDING TO THE REGIONS OF THE ABDOMEN}

\author{
F. Turégano Fuentes ${ }^{I}$, M.D. Pérez Díaz ${ }^{1}$, M. Sanz Sánchez ${ }^{1}$, \\ P. Ciriano Hernandez ${ }^{2}$, A. Lusilla López ${ }^{2}$, M. Fernandez ${ }^{2}$
}

${ }^{1}$ Emergency Surgery, HOSPITAL GREGORIO MARAÑON, MADRID/SPAIN, ${ }^{2}$ Surgery, HOSPITAL GREGORIO MARAÑON, MADRID/SPAIN

Introduction: Our aims were to assess the success rate of SNOM in the different regions of the abdomen, as well as the rate of unnecessary laparotomy.

Material and methods: We reviewed all patients with penetrating abdominal stab wounds (SW) included in our trauma registry. Patients were excluded if penetration through the peritoneum was not confirmed. The abdomen was divided into 9 areas, and SNOM included the use of laparoscopy. Success was defined by avoidance of laparotomy.

Results: Overall, 83 (35\%) patients underwent SNOM which included $31(37 \%)$ laparoscopic explorations. In $7(8 \%)$ patients SNOM failed, including 4 of them due to a converted laparoscopy. In the other $153(65 \%)$ patients a laparotomy was done, with a $23 \%$ negative or non-therapeutic laparotomy rate. SNOM was most frequently attempted, by decreasing order, in the lumbar, flanks, RUQ, LUQ and RTHA areas. SNOM was the less performed in the hypogastric, mesogastric and epigastric areas. When taking into account unnecessary laparotomies and failures of SNOM, a laparotomy could have been most frequently prevented in $\mathrm{SW}$ in the flanks (67\%), epigastrium (64\%), lumbar (64\%), RUQ (59\%), and RTHA (44\%) areas, and less frequently prevented in the LTHA (27\%), LUQ (36\%), hypogastric $(36 \%)$, and mesogastric $(37 \%)$ areas

Conclusion: Our overall success rate was of $92 \%$, and SNOM was safest in SW of the flanks, epigastrium, lumbar, and RUQ areas, in $\geq$ $60 \%$ of patients in each region. Our $23 \%$ rate of unnecessary laparotomy is high, and SW in the epigastrium carried the highest unnecessary laparotomy rate

References: Como JJ, et al. Practice management guidelines for selective nonoperative management of penetrating abdominal trauma. J Trauma. 2010; 68:721-33.

Disclosure: No significant relationships.

\section{PR270}

\section{SPLENECTOMY IN ABDOMINAL TRAUMA}

\author{
M. Brito, C. Felicio, C. Lavado, A. Ramires, J. Malaquias, \\ J. Coutinho
}

Cirurgia, CHLN - Hospital de Santa Maria, Lisboa/PORTUGAL

Introduction: Spleen injuries are among the most frequent traumarelated injuries. Although nonoperative management (NOM) is the safest option in most patients with splenic injuries, some cases still need operative intervention.

Material and methods: We retrospectively analysed all patients with splenectomy performed for traumatic events between July 2012 and June 2017. Our primary outcome was the assessment of risk factors leading to early splenectomy.
Results: In our trauma centre, 30 splenectomies were performed in trauma patients. Of the spleen lesions, $23 \%$ were grade III of AAST Spleen Trauma Classification and the remaining being grade IV. 12 $(40 \%)$ patients were fall victims with a mean age of 66.6 years. 16 $(53,3 \%)$ were victims of road accidents with a mean age of 36.1 , one case of gunshot injury and one case of blast injury. $70 \%$ were males. At admission $43.3 \%$ of the patients were hemodynamically unstable. It is interesting to notice that in the group of road accident patients, there were more cases of instability than in the fall group $(\mathrm{p}=$ 0.0235). Most patients presented with a worsening of the Glasgow Coma Scale between the first out-of-hospital evaluation and the admission evaluation $(\mathrm{p}=0.0129)$. The overall mortality rate was $13.3 \%$ at 30 days. Regarding the diagnostic examinations of splenic lesion, abdominal CT was performed in $73.3 \%$, and the ecoFAST was performed in $23.3 \%$.

Conclusion: In our series, neither trauma mechanism nor administration-to-O.R. time lapse influenced the outcome.

References:

Disclosure: No significant relationships.

\section{PR271}

\section{ABDOMINAL STAB WOUNDS: SELF-INFLICTED "HARA-} KIRI” WOUNDS VERSUS NON-SELF-INFLICTED WOUNDS

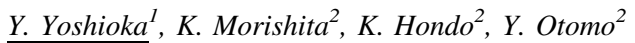

${ }^{1}$ Trauma And Acute Critical Care Medical Center, Tokyo Medical and Dental University, Medical Hospital, Tokyo/JAPAN, ${ }^{2}$ Shock Trauma And Emergency Medical Center, Tokyo Medical and Dental University, Medical Hospital, Tokyo/JAPAN

Introduction: The suicide rate in Japan is higher than other countries; suicide is the first leading cause of death among the youth. Japanese people have a unique way to commit suicide called "Hara-kiri," the traditional method for samurai to kill themselves using Japanese sword. Therefore, self-inflicted abdominal stab wounds are more common in Japan than other countries. The purpose of this study is to compare the clinical features between self-inflicted (Hara-kiri type) and non-self-inflicted abdominal stab wounds.

Material and methods: We proposed a retrospective single-center study that included patients receiving any managements for abdominal stab wounds in the emergency department at Tokyo Medical and Dental University Hospital of Medicine between April 2010 and August 2017. Patients' characteristics, patients' history, the number of wounds and injured organs, and injury severity score (ISS) were noted and compared.

Results: There were 30 patients who had abdominal stab wound. Of these cases, $83 \%$ were Hara-kiri type, and $27 \%$ patients was non-selfinflicted. There were significantly more psychiatric histories in Harakiri type patients. On the other hand, ISS and the number of injured organ were statistically significant higher in the non-self-inflicted group.

Conclusion: This study showed that patients with Hara-kiri type abdominal stab wound were less severe than those with non-selfinflicted stab wound.

References: Ministry of Health, Labour and Welfare. Comprehensive measures to prevent suicide 2017. Japan Trauma Care and Research, The Japanese Association for the Surgery of Trauma (Trauma Registry Committee), and The Japanese Association for Acute Medicine (Committee for Clinical Care Evaluation). Japan Trauma Data Bank Report 2016 (2011-2015) Venara A, et al. Abdominal stab wounds: Self-inflicted wounds versus assault wounds. J Forensic Leg Med 
(2013) 20:270-3 Inkinen J, et al. Penetrating trauma; experience from Southwest Finland between 1997 and 2011, a retrospective descriptive study. Eur J Trauma Emerg Surg (2015) 41:429-33 Leow JJ, et al. A review of stab wound injuries at a tertiary trauma centre in Singapore: are self-inflicted ones less severe? Singapore Med J (2015) 57 (1):13-17 Bugaev N, et al. Self-Inflicted Abdominal Stab Wounds Have a Higher Rate of Non-therapeutic Laparotomy/Laparoscopy and a Lower Risk of Injury. World J Surg (2017) 41:2681-8 Benerjee A, et al. Anterior abdominal stab injury: a comparison of self-inflicted and intentional third-party stabbings. Am J Surg (2013) 205:274-9 Ahn S, et al. A Comparison of Self-Inflicted Stab Wounds Versus Assault-Induced Stab Wounds. Trauma Mon (2016) 21 (5):e25304 Schatz DV. 2000. Manual of Trauma and Emergency Surgery. Saunders

Disclosure: No significant relationships.

\section{PR272}

\section{SURGICAL INTERVENTION FOR BLUNT BOWEL AND MESENTERIC INJURY: INDICATION AND TIME INTERVAL}

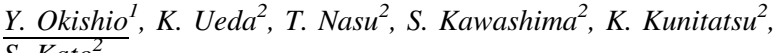 \\ S. Kato ${ }^{2}$
}

${ }^{1}$ Department Of Emergency And Critical Care Medicine, Wakayama Medical University, Wakayama/JAPAN, ${ }^{2}$ Emergency And Critical Care Medicine, Wakayama Medical University, Wakayama/JAPAN

Introduction: Decision making in management of blunt bowel and mesenteric injury (BBMI) is difficult. We aimed to study the indicators for laparotomy and time interval to surgery.

Material and methods: We retrospectively reviewed our hospital's trauma registry to identify patients with a diagnosis of BBMI from February 2011 to July 2017. Initially patients requiring therapeutic surgical treatment (OM group) were compared with those who didn't (NOM group). Factor with a $\mathrm{p}<0.05$ by univariate analysis were then further analyzed. Secondly in OM group we identify relevant factor for time interval to surgical intervention.

Results: Of the 2808 trauma patients who were admitted to our hospital, 83 (3.0\%) had Bowel and Mesenteric Injury. 6 with penetrating trauma, 2 lethal non-treated cases and 2 underwent exploratory laparotomy were excluded. There were 73 patients (47 males), with a mean Injury Severity Score (ISS) of 23. Results from the univariate analysis identified 3 relevant factors between OM group and NOM group: ISS score $(\mathrm{p}=0.036)$, hemodynamic instability $(\mathrm{p}=0.041)$ and free air $(\mathrm{p}=0.0018)$. Multivariate analysis revealed 1 relevant factor: free air $(\mathrm{p}=0.0002)$. The shorter interval between ED admission and intervention is associated with 7 days mortality $(\mathrm{p}=0.029)$, hemodynamic instability $(\mathrm{p}=0.0009)$, FAST positive $(\mathrm{p}<0.0001)$ and mesenteric extravasation $(\mathrm{p}=0.012)$; whereas the shorter interval between estimated onset and intervention is associated with 7 days mortality $(\mathrm{p}=0.037)$.

Conclusion: It is evident that early surgical intervention is essential to hemodynamically unstable BBMI and bowel perforation with free air. Nevertheless the earlier surgical intervention is associated with high mortality, more prompt transportation and intervention could avoid significant mortality.

References: T. Bege et al. Hollow viscus injury due to blunt trauma: A review. J Visc Surg. 2016; 153: 61-8.

Disclosure: No significant relationships.

\section{PR273}

\section{ABDOMINAL STAB WOUNDS: SELF-INFLICTED "HARA- KIRI" WOUNDS VERSUS NON-SELF-INFLICTED WOUNDS}

\author{
Y. Yoshioka, K. Morishita, K. Hondo, Y. Otomo
}

Shock Trauma And Emergency Medical Center, Tokyo Medical and Dental University, Medical Hospital, Tokyo/JAPAN

Introduction: The suicide rate in Japan is higher than other countries; suicide is the first leading cause of death among the youth. Japanese people have a unique way to commit suicide called "Hara-kiri," the traditional method for samurai to kill themselves using Japanese sword. Therefore, self-inflicted abdominal stab wounds are more common in Japan than other countries. The purpose of this study is to compare the clinical features between self-inflicted (Hara-kiri type) and non-self-inflicted abdominal stab wounds.

Material and methods: We proposed a retrospective single-center study including patients receiving any managements for abdominal stab wounds in the emergency department at Tokyo Medical and Dental University Hospital of Medicine between April 2010 and August 2017. Patients' characteristics, the location of the wounds, associated injuries, and severity of the injury as assessed by the injury severity score (ISS) were noted and compared.

Results: There were 30 patients who had abdominal stab wound. Of these cases, $83 \%$ were Hara-kiri type, and $27 \%$ patients was non-selfinflicted. There were significantly more psychiatric histories in Harakiri type injury group. On the other hand, the ISS of Hara-kiri type was less as compared with that of non-self-inflicted type injury. In all cases, no patients died.

Conclusion: This study showed that patients with Hara-kiri type abdominal stab wound were less severe than those with non-selfinflicted stab wound.

References: Ministry of Health, Labour and Welfare. Comprehensive measures to prevent suicide 2017. Japan Trauma Care and Research, The Japanese Association for the Surgery of Trauma (Trauma Registry Committee), and The Japanese Association for Acute Medicine (Committee for Clinical Care Evaluation). Japan Trauma Data Bank Report 2016 (2011-2015) Venara A, et al. Abdominal stab wounds: Self-inflicted wounds versus assault wounds. J Forensic Leg Med (2013) 20:270-3 Inkinen J, et al. Penetrating trauma; experience from Southwest Finland between 1997 and 2011, a retrospective descriptive study. Eur J Trauma Emerg Surg (2015) 41:429-33 Leow JJ, et al. A review of stab wound injuries at a tertiary trauma cetre in Singapore: are self-inflicted ones less severe? Singapore Med J (2015) 57 (1):13-17 Bugaev N, et al. Self-Inflicted Abdominal Stab Wounds Have a Higher Rate of Non-therapeutic Laparotomy/Laparoscopy and a Lower Risk of Injury. World J Surg (2017) 41:2681-8 Benerjee A, et al. Anterior abdominal stab injury: a comparison of self-inflicted and intentional third-party stabbings. Am J Surg (2013) 205:274-9 Ahn S, et al. A Comparison of Self-Inflicted Stab Wounds Versus Assault-Induced Stab Wounds. Trauma Mon (2016) 21 (5):e25304 Schatz DV. 2000. Manual of Trauma and Emergency Surgery. Saunders

Disclosure: No significant relationships. 
PR274

\section{THE EFFECT OF DELAYED DIAGNOSIS ON MORTALITY IN DOUDENAL INJURIES}

\author{
H. Calis, N. Ozlem
}

Faculty Of Medicine; General Surgery, Ahi Evran University, Kırsehir/TURKEY

Introduction: Duodenal injuries, most of which are caused by penetrant traumas, are generally seen around the age of 30 years old. These injuries may lead to serious complications and death if there is a delay in the diagnosis. In this study we have retrospectively evaluated the effect of diagnosis and treatment of duodenal injury on mortality in the examined cases.

Material and methods: In this study, 18 patients who were followed up because of duodenal injury during 1990-2015 were retrospectively examined. Four patients with missing information related to followup and treatment processes were excluded. Thus, 14 patients were included in the study. The records of the patients related to demographical characteristics, type of injury, duration until the surgical intervention, intra-operative data and the procedures were obtained. Results: Hospital records of 14 patients with duodenal injury who were followed-up were evaluated. Of these, 6 patients were injured after penetrant and 8 patients after blunt trauma (Table). One patient with mural hematoma on the first part of duodenum after a blunt trauma was medically followed-up. Sepsis-related late stage mortality was observed in 5 patients. Sepsis-related mortality was observed in 3 patients with delayed diagnosis and examination after getting stabbed. Mortality related to sepsis occurring after suture deficiency on the repair line was observed in one patient with duodenal injury after blunt trauma. Mortality related to sepsis after polytetraflouroethylene graft primary repairment was observed in one patient with iatrogenic duodenal injury. Table: Treatment Modalities Of Patients Variable No. Male/Female 5/9 Blunt trauma 8 Follow-up 1 Whipple procedure 1 Roux-en-Y diverticulization, cholecystectomy, bile duct drainage 1 Primary suture repairment, splenectomy, cholecystectomy, bile duct drainage 1 Primary repairment 3 Laparoscopic primary repairment 1 Penetrant trauma 6 Primary repairment 1 Primary repairment, cholecystectomy, bile duct drainage, 1 tube duodenostomy, feeding jejunostomy Lateral duodenostomy, feeding jejunostomy, tube gastrostomy 1 Primary suture repairment, pyloric exclusion 1 Gastrotomy, sponge extraction 1 Primary repairment using polytetrafluoroethylene (PTFE) graft 1

Conclusion: Following the stabilization and resuscitation of the patients after duodenal injuries, if the specialists are not sceptical in the first examination, there is usually a delay in the diagnosis. Early period vital sign and examination findings and radiological evaluations of the patients may not give exact results after trauma. In such a case, repeated examination and radiological evaluations may help. The relationship between an increase in radiological evaluation possibilities and lower levels of mortality is a remarkable detail. Repeated examinations and auxiliary imaging methods are important for diagnosis. Additionally, intra-operative skepticism is also important. In cases of duodenal injuries, it is important to mobilize the duodenum and evaluate each of the four parts. It should be kept in mind that a delay in diagnosis and treatment results in high levels of mortality and morbidity.

References: 1. Esther Garcia Santos, Ana Soto Sanchez, Juan M. Verde, Corrado P. Marini, Juan A. Asensio, Patrizio Petrone. Duodenal injuries due to trauma: Review of the literature. Cir Esp 93:6874, 2015. 2. Velmahos GC, Constantinou C, Kasotakis G. Safety of repair for severe duodenal injuries. World J Surg 32:7-12, 2008. 3. Fang JF, Chen RJ, Lin BC. Surgical treatment and outcome after delayed diagnosis of blunt duodenal injury. Eur J Surg 165:133-139, 1999. 4. Asensio JA, Demetriades D, Hanpeter DE, Gambaro E, Chahwan S. Management of pancreatic injuries. Curr Probl Surg 36:325-419, 1999. 5. Rotondo MF, Newell MA. Pancreatic and Duodenal Injuries. In: Current Surgical Therapy (Cameron JL, ed). 9 th Edition. CV Mosby, Missouri, pp 992-996, 2008. 6. Jayaraman MV, Mayo-Smith WW, Movson JS, Dupuy DE, Wallach MT. CT of the duodenum: An overlooked segment gets its due. Radiographics 21:147160, 2001. 7. Osuka A, Idoguchi K, Muguruma T, Ishikawa K, Mizushima Y, Matsuoka T. Duodenal disruption diagnosed 5 days after blunt trauma in a 2-year-old child: Report a case. Surg Today 37:984-898, 2007. 8. Hemanga K Bhattacharjee, Mahesh C Misra, Subodh Kumar, Virinder K Bansal. Duodenal perforation following blunt abdominal trauma. J Emerg Trauma Shock 4:514-517, 2011. 9. Degiannis E, Boffard K. Duodenal injuries. Br J Surg 87:1473-1479, 2000. 10. Rickard MJ, Brohi K, Bautz PC. Pancreatic and duodenal injuries: Keep it simple. ANZ J Surg 75:581-586, 2005.

Disclosure: No significant relationships.

\section{PR274A}

\section{INJURY PATTERNS AND MANAGEMENT OF BOWEL TRAUMA IN A UK TRAUMA CENTRE}

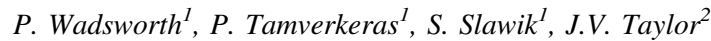

${ }^{1}$ Department Of Surgery, Aintree University Hospital, Liverpool/ UNITED KINGDOM, ${ }^{2}$ Emergency General Surgery And Trauma Unit, Aintree University Hospital, Liverpool/UNITED KINGDOM

Introduction: This review aims to evaluate one regional trauma centre's experience of colorectal and small bowel trauma. We aimed to identify associations between mechanism and injury patterns, and assess quality of management.

Material and methods: Trauma admissions to the unit are recorded on local and network databases. The database for April 2015-July 2017 was searched manually for all colorectal or small bowel injuries. Further details were acquired in patients' hospital records.

Results: 12 patients with relevant injuries were identified. All were male with median age of 30 (range 22-51). Mechanism of injury was predominantly penetrating trauma; three stabbed, five shot plus two fractured pelvis perforating the rectum. Of these, four patients underwent primary repair, two resection and anastomosis, one Hartmanns and three loop colostomy. Two blunt traumas (Road Traffic Collision) resulted in patients undergoing Hartmanns procedure (one also with resection and anastomosis of jejunal transection). One patient developed necrotising fasciitis due to late recognition of the rectal injury; no leaks were reported. Where follow up information was available all stomas were subsequently reversed.

Conclusion: Use of a comprehensive database facilitates review of practice within the unit. Colorectal and small bowel injuries are infrequent but there is no place for conservative management. Penetrating trauma is the predominant mechanism; the majority of penetrating injuries can be managed using simple repair $+/$ defunctioning depending on level. Blunt mechanism appears to cause injuries that are less amenable to primary repair or anastomosis. Failure to recognise complex wounds early resulted in significant morbidity, but patients generally received appropriate follow up.

References:

Disclosure: No significant relationships. 
PR275

\section{THE ROLE OF VIDEO-ASSISTED THORACOSCOPIC SURGERY IN THE TREATMENT OF FLAIL CHEST INJURIES}

N.C. Roozendaal $^{l}$, L.M. Van Roozendaal ${ }^{1}$, M.H. Van Gool ${ }^{1}$, A.M. Pijnenburg $^{2}$, P.A. Hustinx ${ }^{2}$, R. Van Vugt ${ }^{2}$, B. Meesters ${ }^{2}$, K.W.E. Hulsewé ${ }^{3}$ Y.L.J. Vissers ${ }^{3}$, E.R. De Loos $^{4}$

${ }^{1}$ General Surgery, Zuyderland medical center, Heerlen/ NETHERLANDS, ${ }^{2}$ Trauma Surgery, Zuyderland medical center, Heerlen/NETHERLANDS, ${ }^{3}$ Thoracic Surgery, Zuyderland medical center, Heerlen/NETHERLANDS, ${ }^{4}$ Thoracic/trauma Surgery, Zuyderland medical center, Heerlen/NETHERLANDS

Introduction: Rib fractures accompanied by hemothorax and pneumothorax are common and serious injuries following high-energy blunt chest trauma. There is limited evidence for the use of videoassisted thoracoscopic surgery (VATS) in the surgical stabilisation of rib fractures (SSRF), eg. for planning of incisions, direct visualisation of intrathoracic injuries and hemothorax evacuation. We performed a single center cohort study, with the aim to investigate the potential of VATS in the surgical treatment of flail chest.

Material and methods: From 2013 to 2017, all patients with VATSassisted SSRF were included. Three-dimensional CT reconstructions of the chest wall were obtained. Flail chest was defined as at least three fractured ribs with at least two fractures per rib. Patient related characteristics and complications related to VATS-assisted SSRF were reported.

Results: VATS-assisted SSRF was performed in 56 patients. The mean age was 63 years (range 21-92). Median ISS was 16 (range 9-45). Hemothorax was evacuated by VATS in 39 patients (median volume 200cc, range 100-1100). If needed, entrapped lung was freed from the fracture site. Stabilisation was performed using plates $(96 \%)$, splint fixation $(2 \%)$ or both $(2 \%)$. Median postoperative ICU admission was 2 days (range 0-41). Sixteen patients (28\%) had a postoperative complication (5\% wound infections, $14 \%$ pneumonia, $9 \%$ delirium). 30 -day mortality was $2 \%(\mathrm{n}=1$, cause respiratory failure, age $86 y$, ISS 16).

Conclusion: VATS-assisted SSRF is effective and safe in the treatment of flail chest. It enables fine tuning of planned incisions, direct visualisation and treatment of intrathoracic injuries and adequate evacuation of hemothorax.

References:

Disclosure: No significant relationships.

\section{PR276}

\section{VV-ECMO ALLOWS SAFE AND RELIABLE SURGICAL REPAIR OF TRAUMATIC MAIN BRONCHIAL INJURY}

\section{K. Okawara, M. Sawano, M. Matsuda}

Emergency And Critical Care Medicine, Saitama Medica Center, Kawagoe, Saitama-ken/JAPAN

Introduction: Single-lung ventilation (SLV) is a prerequisite for surgical repair of main bronchial injury (MBI). However, traumatic MBIs are frequently complicated with severe collateral lung injury, which impede sufficient oxygenation and ventilation by SLV. This study evaluates safety and reliability of veno-venous extra corporal membranous oxygenation (VV-ECMO) as a measure for respiratory management during diagnostic and surgical procedures for MBI. Material and methods: 3 cases underwent surgical repair of MBI under VV-ECMO in Saitama Medical Center from 2014 to 2017. Their ages, sexes and injury severity scores (ISS) were $40 \mathrm{M}, 20 \mathrm{M}, 8 \mathrm{~F}$, and $38,38,61$. MBI was in left side in the first case and right side in others. We evaluated their clinical courses and outcomes.

Results: In all cases, CT scan revealed massive subcutaneous and mediastinal emphysema, and pneumothorax. We suspected MBI based on CT findings and massive air leakage from a chest drainage tube. All cases were subject to sever hypoxia on arrival. SLV failed to provide sufficient oxygenation in the adults, and adequate endotracheal tube for SLV was unavailable for the child. Therefore, we introduced VV-ECMO prior to bronchial fibrescopy for accurate diagnosis of MBI, and surgical repair. Throughout these procedures, VV-ECMO achieved sufficient and stable oxygenation and ventilation, with no complications. They were successfully weaned from ECMO immediately after the surgery, and were discharged from hospital with no complication of the anastomosis.

Conclusion: VV-ECMO allows safe and reliable surgical repair of traumatic MBI in cases which SLV is insufficient or unable.

References:

Disclosure: No significant relationships.

\section{PR277}

\section{DOES BREAST SIZE INFLUENCE THE OVERALL OUTCOME OF SEVERELY INJURED WOMEN? A MORPHOMETRIC ANALYSIS UNDER SPECIAL CONSIDERATION OF SUBCUTANEOUS AND VISCERAL FAT TISSUE USING WHOLE BODY CT-SCANS}

\author{
O. Özkurtul, J.K.M. Fakler, T. Weber, S. Seinen, C. Josten
}

Department Of Orthopeadics, Trauma Surgery, And Plastic Surgery, University of Leipzig, Leipzig/GERMANY

Introduction: Previous studies analysed the influence of comorbidities using the BMI on the outcome of adipose trauma patients. However, special attention deserves to the soft tissue distribution regarding the protective effect due to inaccuracy of the BMI. The aim of our study was to determine soft tissue distinguishing visceral and subcutaneous fat in female breasts and its' influence on overall outcome.

Material and methods: Severely injured patients admitted to our level I trauma center and who received a whole body CT-scan were included into the study. Injuries severity and epidemiological data was acquired from our trauma registry data. Partially automated and manually volumetric segmentation was performed using image visualizing software Mimics ${ }^{\circledR}$.

Results: Between January 1st 2011 and December 31st 2012, a total of 110 polytrauma patients ( 26 female, 84 male, mean age $47 \pm 27$, age range 19 - 90) were referred to our hospital's trauma bay to receive a whole-body CT scan. Mean ISS was $29,76 \pm 14,23$. The mean BMI was $24,84 \pm 4,37 \mathrm{~kg} / \mathrm{m}^{2}$. The average volume of subcutaneous fat tissue in both breasts was $1256,81 \pm 541 \mathrm{~cm}^{3}$, the visceral fat tissue $629 \pm 334 \mathrm{~cm}^{3}$. The length of hospital stay was longer in the group $>1100 \mathrm{~cm}^{3}$ subcutaneous fat.

Conclusion: Traumatic impacts on the chest can be influenced by the volume of soft tissue which absorbs force directed to the lungs. BMI is not accurate enough to predict effects on the trauma outcome itself but segmented CT-measurements of the breast can be a reference to estimate impact on the respiratory system and overall outcome. 
References:

Disclosure: No significant relationships.

PR278

\section{TRAUMATIC AORTIC INJURY IN A LEVEL 1 TRAUMA CENTER}

\section{H. Al-Thani, A. El-Menyar}

Trauma Surgery, Hamad General Hospital, Doha/QATAR

Introduction: We aimed to evaluate the incidence, presentation and management of traumatic aortic injury in our institute over 16 years. Material and methods: We conducted a retrospective analysis of all patients presented with traumatic aortic injury from July 2000 to July 2017.

Results: A total of 69 cases of traumatic aortic Injury were identified with a mean age of $36.9 \pm 14.4$ years and $81 \%$ were males. Trauma was mainly traffic-related in a three-quarter of cases. Overall injury severity score was $29.6 \pm 9$ and head injury was found in $28 \%$ with a head AIS of $3.9 \pm 1$. Aortic injury score (Grade) was I $(n=8)$, II $(n=$ $11)$, III $(n=26)$ and IV $(n=24)$. Management was non-operative in 29 patients and operative in 40 patients (open surgical repair in 20 and endovascular stenting in 20). Paraplegia occurred in one patient of the open surgery group and asymptomatic partial subclavian artery cover happened in 3 patients of the stenting group). The overall mortality was $27.5 \%$ that varied according to the aortic injury grade $(12.5 \%$, $18.2 \% .19 .2 \%$, and $46 \%$ respectively) and management (38\% in nonoperative and $20 \%$ in the operative group).

Conclusion: The non-operative management of traumatic aortic injury is feasible in a subset of patients. The hospital outcomes vary according to the aortic injury grade and modality of management, however long-term follow-up is needed.

References: 2014;59 (2):334-41

Disclosure: No significant relationships.

\section{PR279}

\section{CLINICAL IMPLICATIONS OF THE CHEST TRAUMA SCORING SYSTEM IN POLYTRAUMA PATIENTS}

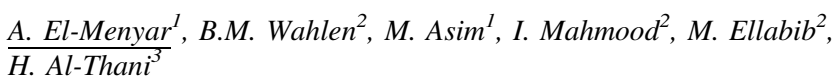

${ }^{1}$ Trauma Surgery Section, Department Of Surgery, Hamad General Hospital, Doha/QATAR, ${ }^{2}$ Trauma Surgery, Hamad General Hospital, Doha/QATAR, ${ }^{3}$ Trauma Surgery Section, Department Of Surgery, Hamad Trauma Center, Doha/QATAR

Introduction: Several scoring systems have been developed to predict complications and outcome in blunt chest trauma (BCT) patients, we aimed to evaluate the utility of chest trauma score (CTS) in BCT in a Level 1 Trauma center.

Material and methods: A retrospective analysis was performed for all patients admitted with blunt chest trauma between 2011 and 2015. Demographics, clinical presentation, complications and outcome were analyzed and compared in patients with CTS $<5$ and CTS $\geq 5$.

Results: Across the study period, 869 patients were included (mean age $37.2 \pm 15$ years and $791(91.0 \%)$ were males. There were 384 patients $(44.2 \%)$ with CTS $\geq 5$. In comparison to lower CTS, patients with CTS $\geq 5$ were older $(40.5 \pm 16$ vs $34.6 \pm 13.7)$, and were more likely to have sternal fracture (9.6\% vs $5.5 \%)$, pneumothorax $(35.7 \%$ vs $25.6 \%)$, hemothorax (26.8\% vs $15.7 \%)$, associated liver injury ( $17 \%$ vs $11 \%)$, higher ISS ( $19 \pm 9$ vs $15 \pm 9)$, greater chest AIS ( $3 \pm$ 0.5 vs $2.5 \pm 1)$, lower TRISS $(0.95 \pm 0.08$ vs $0.97 \pm 0.05)$. Intubation, blood transfusion, and hospital length of stay were significantly higher in patients with higher CTS. ARDS and pneumonia were comparable in the 2 groups. The mortality was almost double in patients with high CTS $(6.8 \%$ vs $2.9 \%, \mathrm{p}=0.001)$.On multivariable regression analysis, CTS was not independent predictor for mortality, however, massive blood transfusion, ISS and age were the predictors

Conclusion: In polytrauma patients including the chest, the CTS has clinical implications, however, bleeding and ISS are the main determinants of the survival.

References: Chen et al: A chest trauma scoring system to predict outcome. Surgery. 2014 Oct;156 (4):988-93

Disclosure: No significant relationships.

\section{PR280}

\section{ACUTE PAIN MANAGEMENT SCORE OF PATIENTS WITH MULTIPLE FRACTURED RIBS}

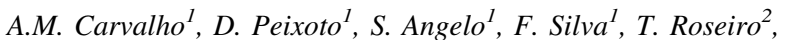 \\ C. Vale ${ }^{3}$, A. Mendes $^{1}$
}

${ }^{1}$ Ortopedia, Hospital Distrital da Figueira da Foz, Figueira da Foz/ PORTUGAL, ${ }^{2}$ Ortopedia, Hospital Distrital Figueira da Foz, Figueira/PORTUGAL, ${ }^{3}$ Serviço De Ortopedia, Hospital Distrital Figueira da Foz, Figueira da Foz/PORTUGAL

Introduction: Rib fratures extend across a broad spectrum of severity ranging from a single fractured rib to multiple fractured ribs that can result in a flail chest with respiratory failure. They cause severe pain that can seriously compromise respiratory mechanics and exacerbate underlying lung injury or pre-existing respiratory disease. Appropriate analgesia and early aggressive care appears to attenuate the development of pulmonary complications through enhancement of patients' functional capacity.

Material and methods: The most important determinants of morbility and mortality are the number of ribs frature and the age of the pacient. Together, they correlate with the severity of the injury. We present an useful algorithm that combines these determinants with the adequate level of analgesia recommended for each patient. The rib frature score $=($ breaks $\mathrm{x}$ sides $)+$ age factor, will determine which patients are at higher risk of complications and therefore in need of a higher level analgesia.

Results: Prompt multi-modal analgesia could be provided incorporating regional analgesia, i.v. opioids, and oral adjuncts. These are essential to reduce complications rates. Analgesia starts at step 1 from simple analgesics, weak opioids and anti-inflammatory drugs, and can be continue in step 2 with iv morphine. The $3^{\text {rd }}$ step consists in PCA and 4th step regional anaesthetic techniques and operative fixation . Conclusion: The management for the vast majority of rib fracture injuries remains supportive only, with analgesia and chest physiotherapy. A scoring system is important for adequate pain management in the early post-injury setting, to obtain a more favorable recovery and to prevent complications.

References: - Comprehensive approach to the management of the patient with multiple rib fractures: a review and introduction of a bundled rib fracture management protocol. Cordelie E Witt, Eileen M Bulger. Trauma Surg Acute Care Open Jan 2017, 2 (1) e000064; DOI: 10.1136/tsaco-2016-000064 - Acute Pain Management of Patients 
with Multiple Fractured Ribs. Karmakar, Manoj K. MD, FRCA; Ho, Anthony M.-H. MS, MD, FRCPC, FCCP. Journal of Trauma-Injury Infection \& Critical Care: .

Disclosure: No significant relationships.

\section{PR281}

\section{POSSIBILITIES OF MDCT WITH BLUNT THORACIC TRAUMA IN CHILDREN}

\author{
M. Ublinskiy ${ }^{I}$, T. Akhadov ${ }^{l}$, O.V. Karaseva ${ }^{2}$, N. Semenova ${ }^{l}$, \\ P. Menshchikov ${ }^{I}$, A. Manzhurtsev ${ }^{I}$, M. Akhlebinina ${ }^{3}$
}

${ }^{1}$ Radiology, Clinical and Research Institute of Emergency Pediatric Surgery and Trauma, Moscow/RUSSIAN FEDERATION, ${ }^{2}$ Surgery, Clinical and Research Institute of Emergency Pediatric Surgery and Trauma, Moscow/RUSSIAN FEDERATION, ${ }^{3}$ Department Of Radiation Diagnostics, Clinical and Research Institute of Emergency Pediatric Surgery and Trauma (CRIEPST), Moscow/RUSSIAN FEDERATION

Introduction: Thoracic injury ranks the third place in most common cause of trauma after head and extremities injuries. This type of trauma indicates a high level of morbidity and mortality: approximately $25 \%$ of deaths (2nd to traumatic brain injury). Lung damages occupy the first place in thorax injury structure. Multidetector computer tomography (MDCT) is method of choice for lung trauma evaluation.

Material and methods: We studied 195 children (age from 7 months to 18 years) with concomitant injury, 173 among them had serious lung injury. A follow-up study was performed in 40 patients. Studies were performed on 16 - and 64 - slice CT scanners (Philips). Intravenous injection of the contrast agent $1.5 \mathrm{ml} / \mathrm{kg}$ at a flow rate of 3-4 $\mathrm{ml}$ was required in the study of children with polytrauma. Axial slices were used for MPR, MIP and 3D rendering.

Results: Lung injuries were revealed in $70 \%$ of cases, in half of them - with massive hemorrhagic component. Hemothorax was determined in $50 \%$ of all cases, the source of hemothorax was lung gaps except for two cases of aortic rupture with pseudoaneurysm formation. All four types of lung gaps were diagnosed in $40 \%$ cases. Pneumothorax was revealed in $60 \%$ of all cases, mainly thanks to rip ruptures. Pneumomediastinum was detected in $10 \%$ in children with blunt thoracic trauma.

Conclusion: MDCT helps to identify lung injuries, to evaluate and calculate their sizes and to differentiate areas of atelectasis or aspiration. Thats why we can say that this method significantly improves detection of thoracic damage in children.

References:

Disclosure: No significant relationships.

\section{PR282}

\section{SURGICAL TREATMENT OF BRONCHIAL RUPTURE IN BLUNT CHEST TRAUMA: A SYSTEMATIC REVIEW}

N.C. Roozendaal ${ }^{1}$, L.M. Van Roozendaal ${ }^{1}$, M.H. Van Gool ${ }^{1}$, K.W.E.Hulsewé, Y.L.J. Vissers ${ }^{2}$, E.R. De Loos $^{3}$

${ }^{1}$ General Surgery, Zuyderland medical center, Heerlen/ NETHERLANDS, ${ }^{2}$ Thoracic Surgery, Zuyderland medical center, Heerlen/NETHERLANDS, ${ }^{3}$ Thoracic/trauma Surgery, Zuyderland medical center, Heerlen/NETHERLANDS

Introduction: A 19-year-old patient with a rupture of the left main bronchus after blunt chest trauma was treated with primary surgical repair in our hospital. Bronchial rupture by blunt chest trauma is rare and carries a high prehospital mortality. This systematic review provides insights into surgical treatment options and its outcome.

Material and methods: A systematic search was conducted to identify cohort studies of bronchial rupture after blunt chest trauma in adult patients between 1985 and $2016(n=215$ articles). Bronchial rupture by penetrating trauma, iatrogenic injuries and solitary tracheal injuries were excluded. Studies were included concerning four or more patients and in case patient data could be extracted. This resulted in 19 articles for final review, consisting of 155 patients.

Results: Mean age of 155 patients was 28 years (range 18-60). The mechanism of injury involved a car accident in $60 \%$, motorcycle accident in $16 \%$, other in $24 \%$. The main bronchus was mostly injured $(81 \%)$, in $5 \%$ including injury of trachea and in $14 \%$ lobar bronchi injury. Median time from injury to surgical treatment was 7 days. Surgical repair was performed in $95 \%$ of patients: primary anastomosis in $73 \%$, pneumonectomy in $15 \%$ and lobectomy in $9 \%$. Perioperative mortality rate was $10 \%$. Other complications occurred in $22 \%$ (e.g. empyema/bleeding/stenosis/fistula).

Conclusion: Bronchial rupture after blunt chest trauma varies in severity. It often involves a delayed diagnosis. Primary surgical repair seems mostly feasible with an acceptable perioperative mortality and morbidity risk.

References:

Disclosure: No significant relationships.

\section{PR283}

\section{TRAUMATIC STERNAL INJURY IN PATIENTS WITH AND WITHOUT RIB FRACTURE}

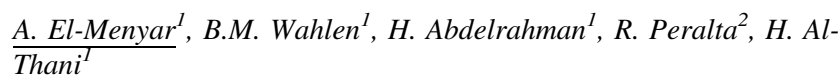

${ }^{1}$ Trauma Surgery, Hamad General Hospital, Doha/QATAR, ${ }^{2}$ Surgery, Hamad General Hospital \& Hamad Medical Corporation, Doha/ QATAR

Introduction: We aimed to study our experience with sternal injury with and without rib fracture in a level 1 trauma center

Material and methods: We conducted a retrospective review of trauma registry data to identify all patients who presented with sternal fracture between 2010 and 2017. Data were analyzed and compared in patients with and without rib fracture.

Results: We identified 212 patients with traumatic sternal injury, of them 110 had associated rib fractures (52\%).In comparison to those who had no rib fracture, patients with rib fractures were older $(40.5 \pm$ 13.8 vs $35.9 \pm 16)$, had higher chest AIS $(2.9 \pm 0.6$ vs $2.3 \pm 0.5)$, more likely to be intubated (33\% vs $21 \%$ ) and to receive blood transfusion (29\% vs $18 \%$ ). The mechanism of injury and rates of spine fracture, head injury, and solid organ injury were comparable in the 2 groups. Clavicular and scapular fractures, lung contusion, hemothorax and pneumothorax were significantly more evident with those who had rib fractures. ICU and hospital length of stay were prolonged in patients with rib fractures $(p=N S)$. The overall mortality was higher but not statistically significant in patients with rib fractures $(5.5 \%$ vs $2.9 \%)$. 
Conclusion: Combined sternal and rib fractures is more evident in a relatively older patients after chest trauma. This combination has certain clinical and prognostic implications.

References: 2014 Jan;186 (1):39-43. doi: 10.1016/j.jss.2013.08.025

Disclosure: No significant relationships.

\section{PR284}

CLINICAL ANALYSIS OF TRAUMATIC AORTIC INJURY IN SINGLE INSTITUTION

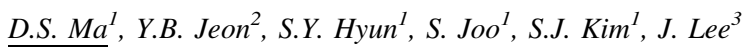

${ }^{1}$ Trauma Surgery, Gachon University Gil Medical Center, Incheon/ KOREA, REPUBLIC OF, ${ }^{2}$ Trauma Surgery, Gachon university Gil hospital, Incheon/KOREA, REPUBLIC OF, ${ }^{3}$ Department Of Trauma Surgery, Gachon University Gil Medical Center, Incheon/KOREA, REPUBLIC OF

Introduction: Traumatic thoracic aortic injuries were known for major cause of the trauma related mortality. The mortality rate of thoracic aortic injuries in the field is known as 80 to $90 \%$. Most patients with aortic injury were known to die before transfer to hospital, and up to $50 \%$ of those alive on arrival died within the first 24 hours. Recently, there has were progressive improved the management for trauma patients. The purpose of this study is to investigate the blunt aortic injury who visited the our trauma center.

Material and methods: This is a retrospective clinical analysis for patients with traumatic aortic injury in Trauma Center of Gachon University Gil Medical Center from January 2014 to December 2016. Demographics, physiologic data, mechanism of injuries, associated injury, timing of intervention and outcomes were reviewed.

Results: The patients with blunt aortic injury were 29. Male were 25 and female 4 . The mean age were 47.5 years old. The mean ISS were 31.72. According to injury mechanism, motor vehicle accident were major cause. The major injury site was directly below aortic isthmus. The TEVAR of management for aortic injury were peformed 22 cases. One patient with ascending aorta injury was surgical aortic reconstruction. One patient was undergone prophylactic arch debranching and left subclavian artery to left common carotid artery bypass. Maximal time lag of intervention from admission were 9 days.

Conclusion: Traumatic aortic injury is a life-threatening injury. However, if there is no impending sign of rupture, the intervention of aortic injury in multiple trauma thought be processed more carefully. References: 1. Jamieson WR, Janusz MT, Gudas VM, Burr LH, Fradet GJ, Henderson C. Traumatic rupture of the thoracic aorta: third decade of experience. Am J Surg 2002;183:571-575. 2. Burkhart HM, Gomez GA, Jacobson LE, Pless JE, Broadie TA. Fatal blunt aortic injuries: a review of 242 autopsy cases. J Trauma 2001;50:113-115. 3. Symbas PN, Tyras DH, Ware RE, Diorio DA. Traumatic rupture of the aorta. Ann Surg 1973;178:6-12. 4. Kato N, Dake MD, Miller DC, Semba CP, Mitchell RS, Razavi MK, et al. Traumatic thoracic aortic aneurysm: treatment with endovascular stent-grafts. Radiology 1997;205:657-662. 5. Demetriades D, Velmahos GC, Scalea TM, Jurkovich GJ, Karmy-Jones R, Teixeira PG, et al. Operative repair or endovascular stent graft in blunt traumatic thoracic aortic injuries: results of an American Association for the Surgery of Trauma Multicenter Study. J Trauma 2008;64:561-570; discussion 570-571.

Disclosure: No significant relationships.

\section{PR285}

\section{DESCRIPTIVE CASE SERIES OF 63 THORACIC PENETRATING INJURIES IN A SINGLE INSTITUTION FOR A YEAR}

F. Cózar Bernal, P. Carmona Soto, S. Sevilla López, A. Bravo Cerro

Thoracic Surgery, COMPLEJO HOSPITALARIO CIUDAD DE JAEN, JAEN/SPAIN

Introduction: Thoracic penetrating trauma (TPT) is a life threatening condition and fortunately rare in our country. The aim of this study is to make an epidemiological characterization of these condition in a country with high frequent.

Material and methods: A retrospective analysis of a series of patients experiencing TPT who presented in San José Hospital, Santiago, Chile was performed from January to December 2015.

Results: Of 63 patients with TPT, $57(90,4 \%)$ were male and $6(9,5 \%)$ were female. The median age was 25 (range 15-66) years. Stab wounds accounted for $47(74,6 \%)$ of the injuries, and $16(25,3 \%)$ suffered from gun shot wounds. $4(6,3 \%)$ emergent thoracotomy were performed and just 1 case was treated with observation. In the rest of patients 58 (92\%), pleurostomy was performed. Thoracic complications happened in 11 cases $(17,4 \%)$ after which a surgery was needed. 8 of them could be done by videothoracoscopy but the another 3 required a thoracotomy due to empiemas. Associated lesions in others organs were recorded in 10 cases $(15,8 \%)$. The median time of hospitalization was 4 days (range 2-93 days). 4 patients (6,3\%) died, 2 of them due to a penetrating heart lesions and another 2 for involved lesions of great vessels.

Conclusion: Young males are fundamentally affected. Stab wounds are more frequent than gun shot wound. Pleurostomy was the most frequent treatment and even the only one in the majority of these patients. Haemothoraxes was the most usual complications and it could be resolved by videothoracoscopy.

References:

Disclosure: No significant relationships.

\section{PR286}

\section{THE TREND IN TAIWAN'S SPLEEN INJURY TREATMENT}

\section{Liao}

Trauma And Emergency Surgery, Chang Gung memorial hospital, Linkou, Taoyuan City/TAIWAN

Introduction: Non-operative management of blunt spleen injury had been established as a reliable and effective method. Preserving the spleen as possible as we can had becoming the much emphasized in current treatment. And we review national insurance data base for past 12 years for discovering the trends of spleen injury treatment

Material and methods: This is a retrospective analysis using national medical insurance data bank in Taiwan. Almost all citizens in Taiwan had been enrolled in this medical insurance system in 1995. All the medical information is recorded in this data base especially those will cost.

Results: Total number of spleen injury during 2002 to 2013 is 11763 . 6565 patients had accepted non-operative treatment and the rest of them (5198) had accepted operative treatment. The mortality rate is higher in operative groups:5.4\% versus $11.7 \%, \mathrm{p}<0001$. And since 
there are only two categories of hospital: center, non-center, we calculate these different groups of patient's trends in spleen trauma. The operative rate in center had decreased from $44 \%$ to $27 \%$ (p < 0.001 ). And in non-center groups the operative rate decreased from $57 \%$ to $42 \%$. The outcome of spleen injury became better in both center or non-center groups. And ISS score is significantly higher in center group, but with better outcome.

Conclusion: Non-operative treatment had also become the main choice of treatment in spleen trauma with compatible outcome in Taiwan. And the rate of non-operative treatment is much higher in center, Taiwan with better outcome

References: Dent D, Alsabrook G, Erickson BA, et al. Blunt splenic injuries: high nonoperative management rate can be achieved with selective embolization. J Trauma. 2004;56 (5):1063-7. Dehli T, Bagenholm A, Trasti NC, et al. The treatment of spleen injuries: a retrospective study. Scand J Trauma Resusc Emerg Med. 2015;23 (1):85. Gaarder C, Dormagen JB, Eken T, et al. Nonoperative management of splenic injuries: improved results with angioembolization. J Trauma. 2006;61 (1):192-8. Olthof DC, van der Vlies CHCH, van der Vlies $\mathrm{CHCH}$, et al. Consensus strategies for the nonoperative management of patients with blunt splenic injury: a Delphi study. J Trauma Acute Care Surg. 2013;74 (6):1567-74. Sabe AA, Claridge JA, Rosenblum DI, Lie K, Malangoni MA. The effects of splenic artery embolization on nonoperative management of blunt splenic injury: a 16-year experience. J Trauma. 2009;67 (3):565.

Disclosure: No significant relationships.

\section{PR287}

\section{THE CONSERVATIVE MANAGEMENT VERSUS THE SURGICAL MANAGEMENT IN THE HEPATO-BILIARY TRAUMA -A 10 YEARS EXPERIENCE IN A LEVEL 1 TRAUMA CENTER}

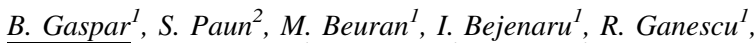
I. Gheju, A. Chiotoroiu', M. Avram ${ }^{I}$, S. Szabo ${ }^{I}$

${ }^{1}$ General Surgery, Clinical Emergency Hospital of Bucharest, Bucharest/ROMANIA, ${ }^{2}$ General Surgery, Emergency Hospital of Bucharest, Bucharest/ROMANIA

Introduction: The increasing of the car accidents in our country has arrived with an increasing of the hepatobiliary trauma.The experience of the medical teams has improved, the diagnosis techniques are in a big progress, but we still have a big rate of mortality with this cause. Material and methods: In the Clinical Emergency Hospital of Bucharest, the biggest level 1 trauma hospital in Romania, a number of 74 pacients with hepato-biliary trauma were admitted in the period January 2010-December 2015.. Grading for hepato-biliary injury was performed according to AAST. Diagnosis and severity were based on CT studies.

Results: $66 \%$ of the case were treated conservative, being associated with a smaller rarte of the complications. The surgical treatment was realised by many methods-from exploratory laparotomy with hepatoraphy to exploratory laparotomy with hepatic packing and selective hepatic embolisation. The postoperative complications started from haemobilia to sepsis and hemodinamic instability.

Conclusion: The diagnosis and also the therapy for hepatobiliary injury still remain challenging for the surgeons. An adequate diagnosis and therapy related to injury grading increase the changes of survival. The therapy varyed from complete non operative to major surgery according to the location and injury severity. The selective angioembolisation were very useful for stable patients.In the large majority of the patients we chose the conservative management, with a smaller rate of complications. Mortality is related to grading and complications.

References: 1.Oniscu, Gabriel C., Rowan W. Parks, -Classification of Liver and Pancreatic Trauma. HPB-THE OFFICIAL JOURNAL OF THE INTERNATIONAL HEPATO PANCREATO BILIARY ASSOCIATION 8.1 (2006) :4-9 PMC.Web.2 Feb.2016 2.Hongchi jiang, Jizhou Wang-Emergencies strategies and trends in the management of liver trauma in Front. Med, 2012, 225-233 3.K.D. Boffard-Manual of Definitive Surgical Trauma care, 3rd edition, Hodder Arnold, London, 2011 4.Luis Donizetti da Silva Stracieri, Sandro Scarpelini-Trauma hepatico in Acta Cirurgica Brasileira, vol 21, 2006, pag 85-88 5.Asensio JA, Demetriades D, Chahwan s et allOperative management and outcomes in 103 AAST-OIS grades IV and V complex hepatic injuries: Trauma surgeons still need to operate but angioembolisation helps. J Trauma, 2003 April;54 (4): 647-53 6.Kozar RA, Feliciano VD, Moore EE et all.-Western Trauma Association/Critical Decision in Trauma:Operative management of blunt Hepatic Trauma. J Trauma. 2011;71 (1):1-5

Disclosure: No significant relationships.

\section{PR288}

\section{THE RELATION BETWEEN CIS, MANAGEMENT, THE RESULTS OF COLONıC INJURY}

\section{N. Ozlem}

Faculty Of Medicine; General Surgery, Ahi Evran University, Kirsehir/TURKEY

Introduction: to determine whether a primary repair (PR)/colostomy reconstruction $(\mathrm{CR})$ should be performed.PR is now the preferred Surgical management (SM).to asses injury mechanism, severity, injured organ, management, mortality morbidity in our CRT patients. Material and methods: SM of 71pts (mean age:40.3 (10-84).M/F:57 $(80.2 \%) / 14(19.8 \%)$ with CRT is?.15 (21.1\%)with gun shot, 33 (46.4\%)with penetrating, 14 (19.8\%)with traffic accident, 9 (12.67\%) with iatrogenic injury, $49(69.01 \%)$ with colon, $11(15.49 \%)$ with rectum, $6(8.45 \%)$ with perine, $3(4.22 \%)$ with both, $2(2.81 \%)$ with colonic + rectal + perine injured patients. CIS:V in $6(8.45 \%), 6$ (8.45\%)IV, 9 (12.67\%)III, 46 (64.78\%)II, 4 (5.61\%)I.

Results: 2 (2.87\%)had perineal wound, sutured.35 (49.25\%);PR (4of them had GR I, 28 GR II, 3 GRIII, a CR were made in $29(40.84 \%)$ (14GRIIGRII, GRIII in5, 5 of 29 GRIV, 5of them GRV).5 (7.04\%)of 71patients had resection+anastomosis $(\mathrm{R}+\mathrm{A}) .2$ had GRII, 2 had GRIV, 1 GRV. medianLOS, 7.1/11.8days in the patients who had PR /CR respectively.The most common injured organs; colon, rectum, perinea respectively.The colonic injuries resulted from most commonly penetrating ones, followed by gun shot wound, traffic accident, iatrogenic injuries (II). The patients who had high grade injury had much more CR, R+A.7 (50\%)of 15 patients with gun shot woundwith more destruction on tissue had CR.23 (70\%)of33with penetrating injury had PR, 8 of them had CR, 2 of them had R+A.LOS were longer in $\mathrm{CR}$ than the patients with PR.Mortality rate were 8 (11.2\%);5of them had PR, 3of those had CR.There were no different effect on mortality between two treatment modality $(p=0.264)$.LOS are shorter in patients with PR who have less destructed trauma.

Conclusion: high grade injury pts had much more $\mathrm{CR}, \mathrm{R}+\mathrm{A}$, .effect of CR, PR on mortality is the same.thepatientswith.PR had shorter LOS, less destructed trauma.1.colon 2.rectum 3.perine injured in frequent. Colon injured from most commonly penetrating, secondly gun shot, 3rd traffic accident, 4th IIIs.Colostomy reconstructed in more 
than $50 \%$ of CRT patients with gun shot, the patients with penetrating injury had PR in a rate of more than $70 \%$ and LOS of them were shorter the others.

References: 1. Management of CRT: a review Ju Yong Cheong and Anil Keshava ANZ J Surg 87 (2017) 547-553 2.Factors affecting morbidity and mortality in traumatic colorectal injuries and reliability and validity of trauma Ay, Nurettin; Alp, Vahhac; Aliosmanoglu, Ibrahim; et al. World Journal of Emergency Surgery201510:21 Volume: 17 Issue: 9 Pages: 1712-1719 Published: SEP 2013

Disclosure: No significant relationships.

\section{PR289}

\section{STERNAL FRACTURE: ASSOCIATION WITH SEAT BELT WEARING AND SIGNIFICANCE - A RETROSPECTIVE ANALYSIS OF 903 CASES}

\author{
M. Apostolides ${ }^{1}$, P.A. Georgis ${ }^{2}$, F. Konstantara ${ }^{3}$, T.P. Michos ${ }^{4}$, \\ C. Chatziantoniou ${ }^{4}$, I. Gakidis ${ }^{4}$, P.T. Michos ${ }^{4}$
}

${ }^{1}$ Colorectal Surgery, Queen Alexandra Hospital, Portsmouth/ UNITED KINGDOM, ${ }^{2}$ Neurosurgery, "Agia Sofia" General Children's Hospital, Athens/GREECE, ${ }^{3}$ General Surgery, General Hospital of Attica "KAT", Athens/GREECE, ${ }^{4}$ Thoracic Surgery, General Hospital of Attica "KAT", Athens/GREECE

Introduction: Sternal fracture incidence has increased over the years, but their significance is largely debated. In this study, we reviewed the incidence of sternal fractures in the largest trauma-receiving hospital in Greece, over a period of 27 years. We also aimed to identify an association between sternal fractures and seatbelt wearing.

Material and methods: Patient data from the Thoracic Surgery Department at General Hospital of Attica "KAT" was reviewed retrospectively, from May 1989-2016. Patients admitted with a diagnosis of sternal fracture were included in this study, with no age, sex or mechanism of injury limitations. Restraint measures in RTC-related fractures, associated injuries, ISS score, complications and length of hospital stay (LOS) were recorded. Pearson's correlation test was used to find an association between sternal fractures and seatbelt wearing, as well as ISS and LOS.

Results: A total of 903 patients (male:female 1:0.81, mean age 53.6 years) were included in this analysis. The number of sternal fracture admissions fluctuated from 1989 to 2016 . By far the most common mechanism of injury was RTCs: 741 cases $(82 \%)$, the majority of whom had isolated sternal fractures $(61 \%)$. A statistically significant positive correlation was found, between sternal fractures and seatbelt wearing $(\mathrm{r}=0.8691, \mathrm{p}<0.00001) .61$ patients $(6.8 \%)$ had cardiacrelated complications and only 3 patients $(0.3 \%)$ were deceased. There was no significant correlation between ISS and LOS $(\mathrm{r}=$ $0.3627, \mathrm{p}=0.058$ ).

Conclusion: Sternal fractures occur more commonly in people involved in RTCs that wear seatbelts. However, seatbelt wearing seems to decrease the number of associated injuries and morbidity in this subgroup of patients.

References: D. Dante Yeh, J.O. Hwabejire, M.A. DeMoya, H.B. Alam, D.R. King, G.C. Velmahos. Sternal fracture - an analysis of the National Trauma Data Bank. Journal of Surgical Research 2014; 186: 39-43 R. Raghunathan, K. Porter. Sternal fractures. Trauma 2009; 11 : 77-92 K. Knobloch, S. Wagner, C. Haasper, C. Probst, C. Krettek, D. Otte, M. Richter. Sternal Fractures Occur Most Often in Old Cars to Seat-Belted Drivers Without Any Airbag Often With Concomitant Spinal Injuries: Clinical Findings and Technical Collision Variables Among 42, 055 Crash Victims. Ann Thorac Surg 2006; 82: 444-50
Al-achraf Khoriati, R. Rajakulasingam, R. Shah. Sternal fractures and their management. J Emerg Trauma Shock 2013; 6 (2): 113-116 Disclosure: No significant relationships.

\section{PR290}

\section{TRAUMATIC DIAPHRAGMATIC INJURIES: A RETROSPECTIVE STUDY OVER 12 YEARS}

\author{
H.B. Bahouth, S. Khuri, Y. Kluger
}

Syrgery, Rambam Health Care Campus, Haifa/ISRAEL

Introduction: Traumatic injury of the diaphragm is uncommon injury, yet underestimated. It can occur followingblunt or penetrating trauma, being more common after penetrating injuries. . Traumatic diaphragmatic injuries are usually occult, and diagnosis is often delayed. These injuries are a marker of a serious associated injuries, which are the main reason for mortality in this group of patients.

Material and methods: A retrospective study over 12 years, between January 1, 2005 and September 30, 2017 was performed at the general surgery department and trauma center at Rambam health care campus, Haifa, Israel. All patients with a diagnosis of traumatic diaphragmatic injuries included in the study.

Results: During the aforementioned period, data on 87 patients with a diagnosis of traumatic diaphragmatic injuries were reported. 57 patients $(65.51 \%)$ suffer diaphragm injury following penetrating trauma, and 30 patients (34.48\%) following blunt injury. Stab wounds were the most common, $43 \%$. The mean age of diagnosis was 34 year old. Left side injury $(74.71 \%)$, on the right $(21.83 \%)$, bilateral (3.44\%).Diagnosis mainly made during operative procedures $(59.77 \%)$. Pre-operative diagnosis $(40.22 \%)$.Diagnosis by CT scan in $34 \%$, by chest $\mathrm{x}$-ray in $6 \% .10 .34 \%$ had delayed diagnosis. 83 patients $(95.40 \%)$ underwent surgical repair. The spleen is the most common associated intra-abdominal injured organ

Conclusion: Traumatic diaphragmatic injuries are uncommon, yet underestimated and often diagnosed lately, Despite advances in imaging studies, diagnosis in the absence of herniation is difficult, and a high index of suspicion is warranted, especially following blunt thoraco-abdominal blunt trauma. Surgical repair is the most efficient management to prevent late complications secondary to herniated viscera.

References:

Disclosure: No significant relationships.

\section{PR291}

\section{CLASSIFICATION OF BONY CHEST WALL INJURIES -THE NEW AO/OTA CLASSIFICATION}

\section{S. Schulz-Drost}

Trauma- And Orthopedic Surgery, BG Hospital Unfallkrankenhaus Berlin gGmbH, Berlin/GERMANY

Introduction: The AO classification is widely known, especially with long tradition for fractures of the long bones of the extremities, but also for the body regions of the skull, spine, pelvis, hand and foot. To date, however, there has been no system for bony chest wall injuries. Material and methods: In the first step different regions of the bony thorax were defined and assigned a numbering system. Subsequently, 
various possible fracture forms were identified and these were classified according to the principles of the AO Foundation using $\mathrm{ABC}$ systematics according to the fracture morphology.

Results: The bony chest wall (16) is formed from the regions right ribs (16.1), left ribs (16.2) and sternum (16.3). Each rib receives its consecutive number (16.1.1-12) within this system. The sternum consists of the manubrium (16.3.1), the corpus (16.3.2) and the xiphoid (16.3.3). The ribs consist of a posterior endsegment (16._._.1) at the transition to the spine, the shaft segment (16._._.2) and the anterior, cartilaginous portion of the rib (16._._.3). The numbers were consented by the AO and the OTA

Conclusion: Fractures of each region of the chest wall can be described by a code for the first time.

For possible subtypes and unstable injury combinations of sternum and rib fractures or rib fractures series, the extension of this classification is still being developed. The challenge is to find a systematic description to possible injuries from 25 bones - the sternum and 12 pairs of ribs.

References:

Disclosure: The Author is an advisory member of the AO TK Thoracic Surgery Expert Group and he has a Consultant Agreement with DePuySynthes.

\section{PR292}

\section{LIFE SAVING SURGICAL TECHNIQUES FOR THORACIC TRAUMA: PREDICTORS FOR THE NEED OF DAMAGE CONTROL THORACOTOMY}

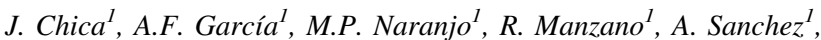 C.A. Ordoñez ${ }^{I}$, J.C. Puyana ${ }^{2}$, C.Y. Cevallos ${ }^{1}$}

\footnotetext{
${ }^{1}$ Trauma Surgery, Fundación Valle del Lili, Cali/COLOMBIA, ${ }^{2}$ Department Of Surgery, University of Pittsburgh Medical Center, Pittsburgh/PA/UNITED STATES OF AMERICA
}

Introduction: Damage control thoracotomy (DCT) has become a feasible and life saving procedure in patients suffering thoracic trauma. Our objective was to identify risk factors associated with DCT in patients suffering thoracic injuries.

Material and methods: Retrospective review in a level I trauma center. Patients were divided into two groups: 1 . Patients submitted to DCT and 2. Patients that not underwent DCT (non-DCT). Early predictors of DCT were identified by multiple logistic regression (MLR).

Results: A total of 116 patients were included of which 112 (96.6\%) were male. Penetrating injuries occurred in 108 (93.1\%). Median age was 27, (IQR 19.5 - 34) years. Median (IQR) of RTS and ISS were 7.0 (5.9-7.4) and 17.5 (16-25) respectively. The lungs were injured in $73(62.1 \%)$ patients, the heart in $35(30.2 \%)$ and major vessels in 4 (3.6\%). 23 patients (19.8\%) needed aortic occlusion, 75 patients $(83.3 \%)$ and 20 patients $(17.2 \%)$ had main lesion in high risk organ and Glasgow $<8$, respectively. Six $(9.5 \%)$ of the non-DCT and 13 (24.5\%) of the DCT died. MLR found GCS $<8$ (OR 5.5 (CI 95\% 1.2 - 24.8)), amount of blood loss (Lt.) (OR 2.1 (CI 95\% $1.4-3.2)$ ), need of aortic occlusion (OR $5.0(1.3-19.7)$ ) and lesion in high-risk organs (Lung AIS $\geq 3$, major vascular, trachea or main bronchus) OR 4.9 (CI 95\% $4.91 .5-16.6$ ) as risk factors for DCT.

Conclusion: We identified coma on admission, amount of blood loss, need of aortic occlusion and main lesion in high-risk structures as independent predictors of DCT.

References: 1. Rotondo MF, Schwab CW, Mc Gonigal MD. Damage control: an approach for improved survival in exanguinating penetrating abdominal injury. J Trauma 1993; 35: 375. 2. Moore EE, Burch JM, Francoise RJ et al. Staged physiologic restoration and Damage control surgery. World J Surg 1990; 22: 1184-91. 3. Matsumoto H, Mashiko K, Sakamoto Y et al. A new look at criteria for damage Control surgery. J Nippon Med Sch 2010; 77: 13-20. 4. Stawicki SP, Brooks A, Bilski T et al. The concept of damage control: extending the paradigma to emergency general surgery. J Care Injury 2008; 39: 93-101. 5. Rotondo MR, Zonies DH. The damage control sequence and underlying logic. Surg Clin North Am. 1997;77:761-77. 6. Sutton E, Bochicchio GV, Bochicchio K, et al. Long-term impact of damage control, surgery: a preliminary prospective study. J Trauma. 2006;61:831-6. 7. Bowley DMQ, Barker P, Boffard KD. Damage control surgery - Concepts and practice. J R Army Med Corps. 2000;146:176-82. 8. Hirshberg A, Mattox KL. Damage control surgery. Surg Clin North Am 1997; 77: 751-756. 9. Wall MJ, Soltero E. Damage control for thoracic injuries. Sur Clin North Am 1997; 77: 863-78. 10. Asensio JA, Petrone P, Costa D. Toracotomía de urgencia: Una revisión crítica. Cir Gen 2004; 26: 128-137. 11. Janchar T, Milzman D. Meta-analysis of the efficacy of resuscitativ thoracotomy following traumatic injury in the ED. Crit Care Med 1999; 27: 159. 12. Branney SW, Moore EE, Feldhaus KM. Critical analysis of two decades of experience with postinjury emergency department thoracotomy bin a regional trauma center. J Trauma 998; 45: 87-94. Shah AS, Forbess JM, Skaryak LA. Emergent thoracotomy for airway control after intrathoracic tracheal injury. J Trauma 2000; 48: 1163-1164. 13. Wall MJ, Villavicencio RT, Millar CC. Pulmonary tractotomy as an abbreviated thoracotomy technique. J Trauma 1998; 45: 1015-1023. 14. Huh J, Wall MJ, Estrera AL. Surgical management of traumatic pulmonary injury. Am J Surg 2003; 186: 620-624. 15. Garcia AF, Martinez J, Rodriguez J. Damage-control techniques in the management of severe lung trauma. AAST 2015 16. Beattie, Rory et al. Penetrating thoracic trauma .Surgery - Oxford International Edition, Volume 32, Issue 5, 249 - 253 17. Hunt, P.A. et al.Emergency thoracotomy in thoracic trauma-a review .Injury, Volume 37, Issue 1, 1 - 19

Disclosure: No significant relationships.

\section{PR293}

\section{AGE DISTRIBUTION AND CONCOMITANT INJURIES TO PULMONARY CONTUSION - AN ANALYSIS BASED ON ROUTINE DATA}

\section{R. Finkbeiner ${ }^{1}$, A. Langenbach ${ }^{1}$, F.F. Hennig ${ }^{1}$, S. Krinner ${ }^{1}$, S. Schulz- $\underline{\text { Drost }^{2}}$}

${ }^{1}$ Trauma And Orthopedic Surgery, University Hospital Erlangen, Erlangen/GERMANY, ${ }^{2}$ Trauma- And Orthopedic Surgery, BG Hospital Unfallkrankenhaus Berlin gGmbH, Berlin/GERMANY

Introduction: Pulmonary contusions (PC) and their common concomitant injuries are feared, especially in the elderly. This study describes prevalence and age distribution of chest injuries and PC, concomitant injuries and developments in management.

Material and methods: A retrospective analysis of german routine data (2009-2015) with 209.820 thoracic traumacases (main (MD) or secondary diagnose (SD); S27.X following ICD-10) was carried out. Analyses were done for the whole collective and the group of PC (S27.31).

Results: Out of 61.016 patients (MD S27.X) 7.558 (12,4\%) showed SD PC and among 148.804 patients (SD S27.X) 58.247 patients $(39,1$ $\%$ ) showed SD PC. Most common injury in the category S27.X was pneumothorax with cumulatively 66.762 cases $(31,8 \%)$. Among the 
most frequent SD of PC there were commotio cerebri $(25,7 \%)$, serial rib fractures $(16,9 \%)$, acute respiratory insufficiency $(16,6 \%)$ and pneumothoraces $(11,9 \%)$. Injuries to the heart, vessels and abdomen are comparatively rare. Within the observation period S27.X-diagnoses increased while the length of hospital stays in general decreased. The elderly show the longest stays. Haemothoraces and haemopneumothoraces, which are proportionaly most common among senior citizens augmented most, as well as pneumothoraces. Conclusion: The largest proportion of patients with a diagnosis S27.x are seniors, whereas the PC itself constitutes an injury of young adults. However older age proves to be an explicit risk factor for severe concomitant injuries and prolonged hospital stay. Clear influence by the demographic change will prospectively require structural changes in preparation for an older more vulnerable patient collective. References:

Disclosure: No significant relationships.

\section{PR294}

\section{THE EFFECT ON EARLY APPLICATION OF HEMOPERFUSION WITH POLYMXYIN-B IN SWINE THORACIC TRAUMA MODEL}

D. Kim, I. Jeong, G. Kim

Department Of Thoracic And Cardiovascular Surgery, Chonnam National Univeristy Hospital, GWANGJU/KOREA, REPUBLIC OF

Introduction: Hemoperfusion with adsorption filter is considered to potentially improve the clinical outcomes in septic patients. However little is known about the effect of immune-regulation by this device. The aim of our study is to evaluate the efficacy on early application of adsorption filter in swine trauma model.

Material and methods: Severe inflammation was experimentally induced by thoracic injury and hemorrhagic shock in 20 pigs. We managed the control group (10 pigs) with only fluid resuscitation and vasopressor, and the others were additionally treated with Polymyxin-B adsorption hemofilter (10pigs). In filter group, Hemoperfusion was started at 1 hour after induction of traumatic injury, and maintained for 6 hours. Blood samples were taken from the animals at baseline, 1 and 7 hours after injury. We applied the flow cytometric analyses to evaluate monocyte subtypes, and measured the mitochondrial function of monocyte with oxygen consumption rate (OCR).

Results: Mean body weight was $34.3 \pm 27.1 \mathrm{~kg}$. By comparison of 2 groups after cessation of treatment, the ratio of monocyte was statistically decreased in the adsorption group $(p=0.03)$, and the ratio of classical type in monocyte subsets (CD14+CD16+), which has been known as the pro-inflammatory mediator, also statistically decreased in the adsorption group $(p=0.003)$. In mitochondrial function test, the OCR was generally improved in adsorption group, whereas was decreased in control group $(\mathrm{p}=0.001)$.

Conclusion: We confirmed that the application of hemoperfusion with adsorption filter potentially improves the mitochondrial function in monocyte, and this type of modality should have an anti-inflammatory effect in early phase of traumatic injury. However further investigation will be needed.

References: Valparaiso AP, Vicente DA, Bograd BA, Elster EA, Davis TA. Modeling acute traumatic injury. J Surg Res. 2015;194 (1):220-32. Cruz DN, Antonelli M, Fumagalli R, Foltran F, Brienza $\mathrm{N}$, Donati A, et al. Early use of polymyxin B hemoperfusion in abdominal septic shock: the EUPHAS randomized controlled trial. JAMA. 2009;301 (23):2445-52. Dilektasli E, Inaba K, Haltmeier T,
Wong MD, Clark D, Benjamin ER, et al. The prognostic value of neutrophil-to-lymphocyte ratio on mortality in critically ill trauma patients. J Trauma Acute Care Surg. 2016;81 (5):882-888. Imtiaz F, Shafique K, Mirza SS, Ayoob Z, Vart P, Rao S. Neutrophil lymphocyte ratio as a measure of systemic inflammation in prevalent chronic diseases in Asian population. Int Arch Med. 2012;5 (1):2. Fan W, Zhang Y, Wang Y, Yao X, Yang J, Li J. Neutrophil-to-lymphocyte and platelet-to-lymphocyte ratios as predictors of survival and metastasis for recurrent hepatocellular carcinoma after transarterial chemoembolization. PLoS One. 2015;10 (3):e0119312. Mitaka C, Tomita M. Polymyxin B-immobilized fiber column hemoperfusion therapy for septic shock. Shock. 2011;36 (4):332-8. Lord JM, Midwinter MJ, Chen YF, Belli A, Brohi K, Kovacs EJ, et al. The systemic immune response to trauma: an overview of pathophysiology and treatment. Lancet. 2014;384 (9952):1455-65. Abe R, Hirasawa H, Oda S, Sadahiro T, Nakamura M, Watanabe E, et al. Up-regulation of interleukin-10 mRNA expression in peripheral leukocytes predicts poor outcome and diminished human leukocyte antigen-DR expression on monocytes in septic patients. J Surg Res. 2008;147 (1):1-8. Nishibori M, Takahashi HK, Katayama H, Mori S, Saito S, Iwagaki $\mathrm{H}$, et al. Specific Removal of Monocytes from Peripheral Blood of Septic Patients by Polymyxin B-immobilized Filter Column. Acta Med Okayama. 2009;63 (1):65-9.

Disclosure: No significant relationships.

\section{PR295}

\section{OUR EXPERIENCE WITH CHEST WALL STABILIZATION}

M. Reška ${ }^{l}$, J. Konecny ${ }^{2}$, I. Čapov ${ }^{l}, L$. Veverková $^{l}$, A. Peštál ${ }^{l}$

${ }^{1} 1$ st Department Of Surgery, St. Anne's University Hospital, Brno/ CZECH REPUBLIC, ${ }^{2} 1$ st Department Of Surgery, St. Anne‘s University Hospital Brno, Brno/CZECH REPUBLIC

Introduction: An unstable chest wall is defined as a fracture of at least three adjacent ribs broken at least in two planes. As a result of impaired mechanical strength of the chest wall, paradoxical movements occur, resulting in insufficient pulmonary ventilation with the development of respiratory insufficiency with typical consequences hypoxia, hypercapnia and acidosis.

Material and methods: We describe a group of 16 patients who underwent stabilization of the chest wall. The average age of the group was 56 years, the youngest patient was 23 and the oldest 76 years. There was an average of 6.1 broken ribs per patient, individually ranging from 4 to 8 . In 11 cases, the stabilization was performed for a block fracture, in 5 cases for a serial fracture with a thoracic wall deformity.

Results: The average length of hospital stay was 19 days, the time from admission to surgery was 4.46 days, the average duration of mechanical ventilation was 2.63 days. In our group, we had only one deep wound infection that was healed using vacuum therapy. Concerning other complications, there was one case of bronchopneumonia, one patient developed delirium. No redrainage for fluido or pneumothorax was necessary.

Conclusion: The main topic is the active surgical approach of treatment of serial and block fractures of ribs with deformation or manifest instability of the chest wall. Not all patients with the block fracture required stabilization, whereas 5 patients with serial fracture of the ribs were indicated for surgical revision for chest wall deformity and other complications.

References: 1. Molnar TF. Surgical Management of Chest Wall Trauma. Thoracic Surgery Clinics 2010;20 (4):475-485. 2. Vyhnánek 
F, Skála P, Škrabalová D. Př́ínos multidetektorové výpočetní tomografie hrudníku $\mathrm{k}$ indikaci stabilizace hrudní stěny u blokové zlomeniny žeber: první zkušenosti. Acta Chir Orthop Traum Čech 2011;78:258-261. 3. Sirmali M, Turut H, Topcu S, Gulhan E, Yazici $\mathrm{U}$, Kaya S, et al. A comprehensive analysis of traumatic rib fractures: morbidity, mortality and management. Eur J Cardiothorac Surg 2003;24 (1):133-8. 4. Voggenreiter G, Neudeck F, Aufmkolk M, Obertacke U, Schmit-Neuerburg KP. Operative chest wall stabilization in flail chest: outcomes of patients with or without pulmonary contusion. J Am Coll Surg 1998;187:130-138. 5. Richardson JD, Adams L Flint LM. Selective management of flail chest and pulmonary contusion. Ann Surg 1982;196:481-487. 6. Lardinois D, Krueger T, Dusmett M, Ghisletta M, Gugger N, Ris HP. Pulmonary function testing after operative stabilization of the chest wall for flail chest. Eur J Cardiothorac Surg 2001;20:496-501. 7. Ahmed Z, Mohyuddin Z. Management of flail chest injury: internal fixation versus endotracheal intubation. $J$ Thorac Cardiovasc Surg 1995;110:1676-1680. 8. Mouton W, Lardinois D, Ferrer M Regli B, Ris HB. Long-term follow-up of patients with operative stabilization of a flail chest. Thorac Cardiovasc Surg 1997;45:242-3. 9. Balci AE, Eren S, Cakir O, Eren MN. Open fixation in flail chest: review of 64 patients. Asian Cardiovasc Thorac Ann 2004;12:11-5. 10. Granetzny A, El-Aal MA, Emam E, et al. Surgical versus conservative treatment of flail chest. Evaluation of the pulmonary status. Interact Cardiovasc Thorac Surg 2005;4:583-87. 11. Khandelwal G, Mathur RK, Shukla $\mathrm{S}$, Maheshwari A. A prospective single center study to assess the impact of surgical stabilization in patients with rib fracture. Int J Surg 2011;9 (6):478-81. 12. Nirula R, Diaz Jr. JJ, Trunkey DD, Mayberry JC. Rib Fracture Repair: Indications, Technical Issues, and Future Directions. World Journal of Surgery 2009;33:14-22. 13. Jing-Quing X, Pei-Li Q, Rong-Guo Y, Shu-Rong G, Youg Y, Xiu-Ling S. Better short-term efficacy of treating severe flail chest with internal fixation surgery compared with conservative treatments. Eur J Med Res 2015;20 (1):55. 14. Chai X, Lin Q, Ruan Z, Zheng J, Zhou J, Zhang J. The clinical application of absorbable intramedullary nail and claw plate on treating multiple rib fractures. Minerva Chir 2013; 68:415-420. 15. 16. Vyhnánek F, Jirava D, Očadlík M, Škrabalová D. Chirurgická stabilizace u blokové zlomeniny žeber: indikace, technika a výsledky. Acta Chir Orthop Traum Čech 2015;82:303-307.

Disclosure: No significant relationships.

\section{PR296}

\section{THE CLINICAL INFLUENCE OF A CONCOMITANT LUNG CONTUSION IN POLYTRAUMA - AN ANALYSIS OF 18.892 PATIENTS OUT OF THE TRAUMA REGISTRY DGU®}

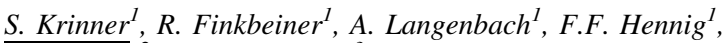 \\ ${ }_{\text {R. Lefering }}^{2}$, S. Schulz-Drost ${ }^{3}$
}

\begin{abstract}
${ }^{1}$ Trauma And Orthopedic Surgery, University Hospital Erlangen, Erlangen/GERMANY, ${ }^{2}$ Institut Für Forschung In Der Operativen Medizin, Akademie der Unfallchirurgie GmBH, Cologne/ GERMANY, ${ }^{3}$ Trauma- And Orthopedic Surgery, Unfallkrankenhaus Berlin, Berlin/GERMANY
\end{abstract}

Introduction: Combined sternum and vertebral injuries may cause a severe loss of stability of the trunk what has been only described in small case series yet. The present study is intended to illustrate the importance of an additional sternum fracture in vertebral fractures by means of reliable, multicenter data in polytrauma patients.

Material and methods: A retrospective analysis from the data set of the TraumaRegistry DGU® (TR-DGU, Project ID 2015-004) included patients in $2009-2014$, ISS ${ }^{3} 16$, primary admission to a trauma center. Exclusion: primary transfer, isolated Brain injury. Evaluation and interpretation are the responsibility of the author and have not yet passed through the final review process of the TraumaRegister DGU®. Cervical, thoracic and lumbar spine fractures (AIS $\geq 2$ ) without and with concomitant sternum fracture (SF) were compared

Results: 3775 patients with cervical (407 SF), 5428 with thoracic (686 SF) and 5847 with lumbar spine fractures (576 SF) were included, An additional SF was highly associated with a more frequent car accident, a higher overall injury severity, prolonged respiratory, intensive and overall duration, as well as a poorer outcome. Patients with SF in all groups also had severe thoracic concomitant injuries and other fractures of the remaining spinal column sections. In the thoracic area, SF also significantly increased the rate of cross-sectional injuries.

Conclusion: In fractures of the thoracic spine as well as in those of the cervical and lumbar spine, an additional SF is multifactorially complicated, with the most severe manifestation in thoracic vertebral fractures.

\section{References:}

Disclosure: No significant relationships

\section{PR297}

\section{A 5-YEAR OBSERVATIONAL STUDY OF CHEST TRAUMA MANAGEMENT WITH A SMALL-BORE CHEST TUBE; SINGLE EMERGENCY CENTRE IN A RURAL AREA IN JAPAN}

\section{Y. Yoshioka}

Emergency \& Critical Care Medicine, Tokushima Red Cross Hospital, Komatsushima City, Tokushima Prefecture/JAPAN

Introduction: Large-bore (28-32 Fr) chest tubes (CTs) are known to be optimal for patients with chest trauma. However, in Japan, because of the small body size of the people, small-bore (12-20 Fr) CTs tend to be used.

Material and methods: This is a retrospective observational study conducted in a tertiary emergency centre in a rural area in Japan. Cases of patients with chest trauma in whom a chest tube was inserted were selected via a chart review from April 2011 to March 2015. Age, gender, height and body weight, size of CT, duration of CT placement, type of chest trauma, need for additional tube insertion were described.

Results: In 5 years, 62 CTs were inserted in 50 patients with chest trauma. The mean age of the patients was 65.5 [interquartile range (IQR), 50.0-79.3] years. The mean height and body weight were 163 (IQR, 155-168) $\mathrm{cm}$ and 58.5 (IQR, 47.5-68) kg, respectively. The mean size of CT was 20 (IQR, 16-20) Fr. The type of chest trauma was pneumothorax in 22 patients, haemothorax in 11 and haemopneumothorax in 27. The mean duration of placement of CT was 6 (IQR, 4-9) days. Additional tube was inserted in 6 patients $(9.7 \%)$ due to failure in evacuating the pneumothorax in 5 patients and malposition in 1 . There was no case of failure with respect to the evacuation of the haemothorax.

Conclusion: This study described the treatment for chest trauma with a small-bore CT in Japan. Small-bore CTs for chest trauma may be optimal for patients with a small body size.

References:

Disclosure: No significant relationships. 
PR298

\section{THORACIC TRAUMA IN POLYTRAUMA IN CHILDREN}

\section{A.V. Timofeeva, O.V. Karaseva, A.L. Gorelik, D.E. Golikov}

Department Of Polytrauma, Clinical and Research Institute of Emergency Pediatric Surgery and Trauma (CRIEPST) Department of Health of Moscow, Moscow/RUSSIAN FEDERATION

Introduction: Aim: To study the features of thoracic trauma in polytrauma in children.

Material and methods: 262 patients with polytrauma (ISS $\geq 16$ ) went through the ICU within 3 years. Average age was $10 \pm 5.5$ years.

Results: Average ISS was $24.7 \pm 7.8$. Severe TBI dominated (87.4\%). Thoracic injury took second place (75.6\%). In 100\% of cases it was combined. Trauma caused by traffic accidents prevailed $(60.3 \%$ ). Mild trauma (AIS $=1-2$ ) was diagnosed in $71.2 \%$. More often we had bilateral trauma $(82.8 \%)$. Pneumothorax was twice as many as hemothorax $(30.3 \% / 13.1 \%)$. Fractures of the skeleton $28.3 \%$, fractures of the thoracic vertebras $-11,6 \%$, contusion of the heart $-5.1 \%$, traumatic bulls $-1.0 \%$, disruption of the main bronchus $-0.5 \%$. Primary chest injury required surgery in $31.3 \%$ of cases. $25.3 \%$ were operated on emergency. $2.5 \%$ were children who needed stabilizing surgeries on the vertebras. Tracheostomies were done in $25.3 \%$ of children with thoracic injury. In $1.0 \%$ of children posttraumatic abscess occurred. 54\% of children with thoracic trauma needed ALV. Pneumonia developed in $11.1 \%$ of children. The average length of hospitalization in the ICU was $13.2 \pm 11.4$ days. Mortality among children with polytrauma having a thoracic trauma was $10.6 \%$.

Conclusion: In $75.6 \%$ of cases polytrauma included thoracic trauma. Mainly it is bilateral minor lung injury with pneumothorax. Primary chest injury only in $31.3 \%$ of cases needs surgery. Hospitalization of children to a specialized emergency pediatric 1 level trauma hospital enables to diagnose in detail the severity and the pattern of thoracic and combined injuries and provide emergency treatment in maximum amount.

References:

Disclosure: No significant relationships.

\section{PR299}

\section{VALUE OF INJURY TOPOGRAPHY IN PENETRATING THORACIC INJURIES}

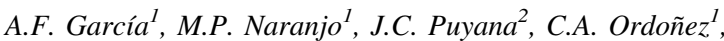
A. Sanchez $^{1}$, C.Y. Cevallos ${ }^{1}$, R. Manzano ${ }^{1}$, M. Vidal $^{1}$

\footnotetext{
${ }^{1}$ Trauma Surgery, Fundación Valle del Lili, Cali/COLOMBIA, ${ }^{2}$ Department Of Surgery, University of Pittsburgh Medical Center, Pittsburgh/PA/UNITED STATES OF AMERICA
}

Introduction: Topographic location of penetrating injuries can contribute to the decision-making process. However, this concept has not been well explored. We describe a series of patients with penetrating thoracic trauma with regards to the relevance of topography of the injury.

Material and methods: A retrospective review of patients submitted to thoracotomy at a level I trauma center. Patients were divided into three groups according to the injury location: 1) Precordial Injuries
(PI), 2) Multiple thoracic injuries (MT), 3) High-risk topography injuries (HR)

Results: 199 patients were included. Injury location was PI in 64 subjects, MT in 60 and HR in 68. The median (IQR) age was 27 (19.5 - 34) years, and 97\% were male. Anatomical (ISS median, [IQR]: PI: 19 [16-25] vs. HR: 25 [16-25] vs. MT: 25 [16-28]) and physiological severity scores (RTS median, [IQR]: PI: 6.72 [3.87-7.84] vs. HR: 5.88 [2.63-7.55] vs. MT: 6.98 [2.73-7.55]) did not differ significantly. A greater proportion of patients with HR topography had lung injuries (HR: $69 \%$ vs. MT: $66 \%$ vs. PI: $41 \%$; $\mathrm{p}=0.02$ ). These patients were less likely to present heart injuries (HR: $8.8 \%$ vs. MT: $26 \%$ vs. PI: $48.4 \% ; \mathrm{p}<0.01)$. Mortality was significantly higher in the MT and HR topography groups (Mortality: MT: $60 \%$ vs. HR: $44 \%$ vs. PI: $29 \% ; \mathrm{p}=0.003)$.

Conclusion: Patients with HR and MT topography were more like to present injuries that ended in worse physiologic compromise. Because mortality was significantly higher in these groups suggests an important role of injury topography in predicting poor outcomes among patients suffering penetrating thoracic injuries.

References: 1. Jhunjhunwala R, Mina MJ, Roger EI, Dente CJ, Heninger M, Carr JS, et al. Reassessing the cardiac box: A comprehensive evaluation of the relationship between thoracic gunshot wounds and cardiac injury. J Trauma Acute Care Surg. 2017;83 (3):349-55. 2. Bellister SA, Dennis BM, Guillamondegui OD. Blunt and Penetrating Cardiac Trauma. Surg Clin North Am. 2017;97 (5):1065-76. 3. Dennis BM, Bellister SA, Guillamondegui OD. Thoracic Trauma. Surg Clin North Am. 2017;97 (5):1047-64. 4. Beattie R, Mhandu PCE, McManus K. Penetrating thoracic trauma. Surgery - Oxford International Edition.32 (5):249-53. 5. Garcia A, Martinez J, Rodriguez J, Millan M, Valderrama G, Ordoñez C, et al. Damage-control techniques in the management of severe lung trauma. J Trauma Acute Care Surg. 2015;78 (1):45-50; discussion -1.

Disclosure: No significant relationships.

\section{PR300}

\section{THE INCIDENCE OF THORACIC TRAUMA IN A RURAL HOSPITAL. MANAGEMENT BY GENERAL SURGEONS EXPERIENCED IN TRAUMA}

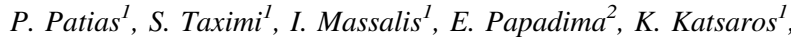 \\ G. Konstantoudakis ${ }^{I}$, G. Euthymiou ${ }^{1}$, P. Gkanas ${ }^{I}$
}

${ }^{1}$ Surgical Department, NAFPLION GENERAL HOSPITAL, NAFPLION/GREECE, ${ }^{2}$ Radiology Department, NAFPLION GENERAL HOSPITAL, NAFPLION/GREECE

Introduction: Thoracic trauma ranges from a simple rib fracture to the emergency department thoracotomy. Still, $25 \%$ of all trauma deaths are to be attributed to thoracic trauma. Trauma is the third leading cause of death in Western countries and yet relatively little is known about its epidemiology, especially in rural areas.

Material and methods: A retrospective study was done in our hospital to determine the incidence of chest trauma and its management by a team combined only by general surgeons. The study includes 165 trauma patients who were admitted to our hospital during a five year old period (2013-2017).

Each patient's injuries were rated according to the Injury Severity Score (ISS). Age, gender, mechanism of injury, clinical and radiological findings are analyzed for each patient. The management of each patient is also reviewed.

Results: Among 165 patients with chest trauma only $10(6 \%)$ were transferred to a bigger hospital due to lack of ICU facilities and four 
of them in order to undergo specific orthopaedic management. Even the most serious cases ( 2 cardiac tamponades- stab hearts) were initially successfully managed by our surgical team. Also, 2 cases of remained heamothorax were completely managed with VATS by the same surgeons.

Conclusion: Experienced in trauma, general surgeons, can properly manage the vast majority of thoracic injuries even in rural hospitals with limited facilities.

References: 1) The incidence of severe trauma in small rural hospitals.

Smith N.

J Fam Pract. 1987 Dec;25 (6):595-600. 2) Emergency thoracotomy in thoracic trauma-a review.

Hunt PA, Greaves I, Owens WA.

Injury. 2006 Jan;37 (1):1-19. Epub 2005 Apr 20. 3) The role of thoracoscopy in the management of retained thoracic collections after trauma.

Heniford BT, Carrillo EH, Spain DA, Sosa JL, Fulton RL, Richardson JD.

Ann Thorac Surg. 1997 Apr;63 (4):940-3. 4) Trauma to the chest wall and lung.

Livingston DH, Hauser CJ.

In: Moore E, Feliciano D, Mattox K (eds) Trauma, 6th ed 2004 Disclosure: No significant relationships.

\section{PR301}

\section{SUPERIOR PROLONGED MECHANICAL PROPERTIES OF COLLAGEN-BASED HEMOSTATIC PATCH COMPARED TO OXIDIZED CELLULOSE-BASED PATCH}

\author{
P. Slezak ${ }^{1}$, X. Monforte ${ }^{1}$, J. Ferguson ${ }^{1}$, S. Sutalo ${ }^{1}$, H. Redl ${ }^{1}$, \\ H. Gulle $^{2}, \underline{\text { D. Spazierer }}^{2}$
} ${ }^{1}$ Research, Ludwig Boltzmann Institute for Experimental and Clinical
Traumatology, Vienna/AUSTRIA, ${ }^{2}$ Research, Baxter Medical
Products GmbH, Vienna/AUSTRIA

Introduction: The use of local hemostats is the current standard of care. In order to choose the most appropriate hemostat for the clinical situation, it is essential to understand the mechanism of action, efficacy and possible adverse events of each material.

Material and methods: In this study two self-adhering hemostatic patches, either based on PEG coated collagen (PCC, Hemopatch) or PEG-coated oxidized cellulose (PCOC, Veriset), are compared in regard to maximum burst pressure, mechanical stability, and swelling. Additionally, the induction of tissue adhesions by the materials after implantation was assessed in a rabbit liver abrasion model.

Results: Both materials showed comparable sealing efficacy in a burst pressure test $(37 \pm 16$ vs $35 \pm 8 \mathrm{mmHg}, \mathrm{P}=0.73)$. After incubation in human plasma, PCC retained its mechanical properties while PCOC showed fast degradation leading to decreased force at break (minimum force at break $0.55 \mathrm{~N}$ during $8 \mathrm{~h}$ for PCC, $0.27 \mathrm{~N}$ for PCOC). PCC allowed higher deformation before break (52\% after $4 \mathrm{~h}$ and $50 \%$ after $8 \mathrm{~h}$ for PCC, $18 \%$ after $4 \mathrm{~h}$ and $23 \%$ after $8 \mathrm{~h}$ for PCOC) and showed less swelling in human plasma $(\sim 20 \%$ increase in thickness for PCC, $\sim 100 \%$ increase in thickness for PCOC). In vivo testing indicated a lower incidence of adhesion formation after PCC application.

Conclusion: In conclusion, the collagen-based Hemopatch has more reliable mechanical properties, reduced swell, and less adhesion formation than the oxidized cellulose-based Veriset. Collagen may offer greater clinical benefit for surgeons when using a hemostatic patch in procedures that require prolonged mechanical stability or that have potential leakage of body fluids.

References:

Disclosure: The study was funded by Baxter International Inc. Drs. Gulle and Spazierer are employees of Baxter Medical Products $\mathrm{GmbH}$. Studies were designed and performed using sound scientific methods.

\section{PR302}

\section{EFFECT OF FIBRINOGEN ON EXPERIMENTAL LIVER TRAUMA; A STEREOLOGICAL STUDY}

\section{G.S. Mahmoudi Nezhad, S. Paydar}

Trauma Research Center, Trauma Research Center, Shahid Rajaee (Emtiaz) Trauma Hospital, Shiraz University of Medical Sciences, Shiraz, Iran., Shiraz/IRAN

Introduction:

Material and methods:

Results:

Conclusion: Fibrinogen + gauze effectively controls liver bleeding and reduces the need to control bleeding for subsequent hemorrhage and biliary leakage in the post-operative days.

References:

Disclosure: No significant relationships.

\section{PR303}

\section{MAJOR VENOUS LIGATION RESULTS IN INCREASED RISK OF LATE COMPARTMENT SYNDROME: A MULTICENTER RETROSPECTIVE REVIEW}

E.D. Dauer $^{1}$, S. Yamaguchi ${ }^{1}$, K. Kelley ${ }^{1}$, H. Zhao ${ }^{1}$, J. Sharpe ${ }^{2}$,

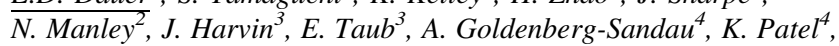

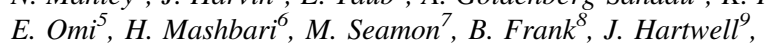
Z. Maher ${ }^{1}$

${ }^{1}$ Surgery, Temple University, Philadelphia/PA/UNITED STATES OF AMERICA, ${ }^{2}$ Surgery, University of Tennessee Health Science Center, Memphis/TN/UNITED STATES OF AMERICA, ${ }^{3}$ Surgery, University of Texas at Houston, Houston/TX/UNITED STATES OF AMERICA, ${ }^{4}$ Surgery, Cooper University Hospital, Camden/NJ/ UNITED STATES OF AMERICA, ${ }^{5}$ Surgery, Advocate Christ Medical Center, Oak Lawn/IL/UNITED STATES OF AMERICA, ${ }^{6}$ Surgery, University of Illinois at Chicago, Chicago/IL/UNITED STATES OF AMERICA, ${ }^{7}$ Traumatology, Surgical Critical Care And Emergency Surgery, University of Pennsylvania, Philadelphia/PA/ UNITED STATES OF AMERICA, ${ }^{8}$ Surgery, Geisinger Community Medical Center, Scranton/PA/UNITED STATES OF AMERICA, ${ }^{9}$ Surgery, Indiana University, Indianapolis/IN/UNITED STATES OF AMERICA

Introduction: The management of lower extremity venous injuries may contribute to the development of compartment syndrome not present at index operation and limb loss. We hypothesize that there is an increased risk of late compartment syndrome (LCS) and amputation following major venous ligation versus repair.

Material and methods: We conducted a multi-institutional retrospective review of patients with injury to the Inferior Vena Cava, 
External Iliac Vein, Common Iliac Vein and/or Femoral Vein from 2005-2015 at seven centers. Outcomes assessed were the development of LCS and the need for amputation.

Results: Patients that underwent venous ligation $(n=238)$ were compared to those having repair $(\mathrm{n}=285)$. The groups were similar with regards to age, gender, presenting HR/GCS, and time to OR (all p $>0.05) .231(44.2 \%)$ patients underwent fasciotomy at their index procedure. $31(10.6 \%)$ of the remaining patients developed LCS. Patients undergoing ligation were significantly more at risk $(\mathrm{p}<$ $0.0001) .27$ of the $523(5.2 \%)$ patients required amputation. Venous ligation $(\mathrm{p}<0.001)$, LCS $(\mathrm{p}<0.0001)$, associated fracture $(\mathrm{p}=$ $0.032)$, and combined arterial and venous injury $(<0.0001)$ were risk factors. In a multiple logistic regression model, patients managed with venous ligation were more likely to develop LCS (OR 3.8, CI 1.57$9.19, \mathrm{p}=0.003$ ), while patients with venous ligation (OR 3.76, CI 1.03-13.65, $\mathrm{p}=0.044)$, LCS (OR 11.67, CI 2.48-54.85, $\mathrm{p}=0.002)$, an associated fracture (OR 4.68, CI 1.46-14.99, $\mathrm{p}=0.009$ ) or combined arterial and venous injury (OR 16.93, CI 2.93-98.02, $\mathrm{p}=0.002$ ) were more likely to require amputation.

Conclusion: Ligation of major venous injuries leads to an increased risk of developing LCS. Patients undergoing venous ligation, with associated fractures or combined arterial and venous injury or who develop LCS are more likely to require amputation.

References:

Disclosure: No significant relationships.

\section{PR304}

\section{CRITERIA FOR DAMAGE CONTROL SURGERY IN BLUNT TRAUMA INJURIES}

\author{
M. Sugimoto, N. Urushibata, R. Nakamoto, A. Yoshiyuki, H. Hara, \\ K. Murata
}

Shock Trauma And Emergency Medical Center, Matsudo City General Hospital, Matsudo/JAPAN

Introduction: There are still many arguments regarding the criteria for damage control surgery (DCS). Previously, we analyzed the data from Japan Trauma Data Bank (JTBD) ' and created a score for damage control surgery (Damage Control Indication Detecting score; DECIDE score) based on body temperature" Glasgow coma scale (GCS) and type of injury. In the current study " we searched for a new score for DCS in blunt injuries.

Material and methods: Retrospective data from JTDB were used to analyze blunt trauma patients who underwent emergency laparotomy. Of those patients" we compared the patients that underwent DCS (DCS group) to the patients who underwent typical laparotomy (nonDCS group).

Results: Blood pressure" body temperature" GCS" FAST positive ratio" blood transfusion rate" Injury Severity Score and mortality rate were significantly different between the groups. Variables independently associated with DCS were blood pressure" body temperature" GCS and FAST positive ratio. From this data' we developed a DCS prediction score.

Conclusion: We propose a new criterion for DCS in blunt injury which can be scored with only vital signs on admission and FAST results.

References:

Disclosure: No significant relationships.

\section{PR305}

\section{OPEN VERSUS ENDOVASCULAR TEMPORARY SHUNTING IN AN OVINE MODEL OF ACUTE OCCLUSIVE ARTERIAL INJURY}

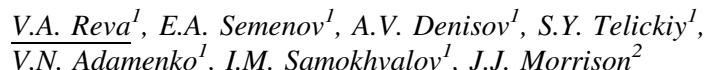

${ }^{1}$ War Surgery Department, Kirov Military Medical Academy, SaintPetersburg/RUSSIAN FEDERATION, ${ }^{2} \mathrm{R}$ Adams Cowley Shock Trauma Center, University of Maryland Medical System, Baltimore/ MD/UNITED STATES OF AMERICA

Introduction: The aim of this study is to evaluate endovascular temporary shunting (E-TS) for rapid revascularization in acute occlusive arterial injury.

Material and methods: Sixteen sheep weighing $30-55 \mathrm{~kg}$ underwent operative exposure of their left external iliac artery (EIA) and the induction of a thrombotic occlusion by means of repeated clamping. Animals were randomized into one of three groups: no-treatment (control group), endovascular temporary shunting (E-TS; 7-mm baremetal stent) or open temporary shunting (O-TS; 4.5-mm polyvinylchloride tube). Animals received no anticoagulants and were followed up for 3 days with serial doppler ultrasound and angiography to evaluate EIA patency. Function of the injured limb was evaluated using the ordinal Tarlov gait score.

Results: Following the EIA injury, the systolic velocity (SV) in all groups was reduced. This was sustained throughout the study in the control group, but SV was returned to pre-injury values in the E-TS and O-TS groups $(p>0.05)$. Three animals required euthanasia for bleeding complications ( $1 \mathrm{xE}-\mathrm{TS}$ and $2 \mathrm{xO}-\mathrm{TS}$ ). At the study conclusion, there was no difference in patency rates between the E-TS and O-TS groups ( $3 / 5$ versus $2 / 4$ respectively; $p>0.05$ ). Thrombosis development did not significantly affect limb function.

Conclusion: Stent implantation is at least as effective as a standard O-TS in emergent extremity revascularization in an ovine model of acute occlusive arterial injury.

References:

Disclosure: No significant relationships.

\section{PR306}

\section{SURGICAL TREATMENT OF THE TRAUMA OF THE SMALL BOWEL IN DAMAGE CONTROL OPERATIONS}

\section{F. Rodriguez, A.F. García}

Trauma Surgery, Fundación Valle del Lili, Cali/COLOMBIA

Introduction: Surgical treatment of trauma has evolved with nonoperative protocols and damage control procedures.

Information about surgical treatment of small bowel (SB) wounds is scarce.

We describe a contemporary series of SB lesions from a level 1 trauma center, emphasizing on damage control procedures.

Material and methods: Patients with trauma laparotomy, who survived 48 hours were included.

Damage control laparotomy (DCL), SB injury, trauma severity, treatment, and complications were registered.

Continuous variables were compared with nonparametric statistics. 
Results: One-hundred-sixty-two trauma laparotomies were performed. Hollow viscus lesions occurred in 131, (80.9\%). Eighty $(49.5 \%)$ had SB trauma. In this group, trauma mechanism was penetrating in 71 cases $(88.8 \%)$. DCL was performed in $40(50 \%)$. In the SB group, lesions were primary reconstructed (PR) in 15 cases and ligated (L) for posterior reconstruction in 25.

NISS was higher in L group [median (IQR) 43 (32 - 50) vs. 34 (27 - 35)], p = 0.04, as well as SB AIS, [median (IQR) 5 (5 - 5) vs.2 (1 5)]. Hemoperitoneum was bigger in the L group, median (RIQ) [2.5 (1.75 - 3.0), vs 1.5 (1.0 - 2.0) Lt.]

Ligation group required more procedures prior definitive abdominal closure [median $2.5(2-4.5)$ vs. $1(1-3)]$.

Seven patients $(28.0 \%)$ of the L group and one of the PR group $(6.7 \%)$ developed a fistula.

Two patients in the L group died.

Conclusion: In DCL, SB requiring ligation occurred in subjects with more severe trauma.

Patients who required ligation had a delay in the abdominal closure and higher risk of fistula.

References: 1. Ordonez C, Garcia A, Parra MW, Scavo D, Pino LF, Millan M, Badiel M, Sanjuan J, Rodriguez F, Ferrada R, et al. Complex penetrating duodenal injuries: less is better. The journal of trauma and acute care surgery. 2014 May;76 (5):1177-83. 2. Burlew $\mathrm{CC}$. The open abdomen: practical implications for the practicing surgeon. American journal of surgery. 2012 Dec;204 (6):826-35. 3. Burlew CC, Moore EE, Cuschieri J, Jurkovich GJ, Codner P, Crowell K, Nirula R, Haan J, Rowell SE, Kato CM, et al. Sew it up! A Western Trauma Association multi-institutional study of enteric injury management in the postinjury open abdomen. The Journal of trauma. 2011 Feb;70 (2):273-7 4. Olofsson P, Abu-Zidan FM, Wang J, Nagelkerke N, Lennquist S, Wikstrom T. The effects of early rapid control of multiple bowel perforations after high-energy trauma to the abdomen: implications for damage control surgery. The Journal of trauma. 2006 Jul;61 (1):185-91. 5. Kirkpatrick AW, Baxter KA, Simons RK, Germann E, Lucas CE, Ledgerwood AM. Intra-abdominal complications after surgical repair of small bowel injuries: an international review. The Journal of trauma. 2003 Sep;55 (3):399-406 Disclosure: No significant relationships.

\section{PR307}

\section{CONSERVATIVE MANAGEMENT OF LIVER LESIONS IN SERIOUS HEPATIC TRAUMA: BILIARY COMPLICATIONS FOLLOWING HEPATIC ARTERIAL EMBOLIZATION}

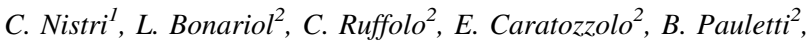 \\ ${\text { G. } \text { Marte }^{2}, \text { N. Bassi }}^{2}$, M. Massani ${ }^{2}$
}

${ }^{1}$ Iv Department Of Surgery - Regional Center For Hpb Surgery, Regional Hospital of Treviso, Ca Foncello - ULSS 2, Treviso/ITALY, ${ }^{2}$ Iv Department Of Surgery - Regional Center For Hpb Surgery, Regional Hospital of Treviso, Ca Foncello - ULSS 2, treviso/ITALY

Introduction: Conservative treatment of liver traumas has made important progress during last years and non-operative management (NOM) is the standard treatment in patients with hepatic blunt injuries and haemodynamical stability. The aim of this study is to analyse the conservative treatment of liver trauma, focusing on biliary complications.

Material and methods: This retrospective study analyses patients with serious hepatic trauma treated from January 2000 to September 2017 at the IV Department of Surgery, Regional Centre for HPB Surgery, in the regional Hospital of Treviso. Liver injuries were evaluated according to the American Association for the Surgery of Trauma.

Results: 104 patients with level III-IV-V liver injuries and treated in our Unit were included in the study ( 81 males, 23 females, median age 37 years). Patients that underwent NOM were 68 (65,38\%), 64 of them $(94,12 \%)$ were haemodynamically stable at admissions and 23 $(33,82 \%)$ underwent hepatic arterial embolization. NOM success rate was $77,94 \%$, and only 4 patients were treated with liver packing following NOM failure. Mortality rate among conservative-treated patients was $8,82 \%$, with no liver-related mortality. Among patients with failure of NOM, there were 7 biliary complications $(46,67 \%$ of failed NOM) and $57,14 \%$ of them occurred after hepatic arterial embolization. These included 3 biliary fistulas and 1 acute cholecystitis; all biliary fistulas needed a combination of endoscopic (nasobiliary drain) and surgical treatment (percutaneous drainage, biliostasis or cholecystectomy).

Conclusion: NOM is an effective treatment modality in most cases of serious hepatic trauma. We must be aware of biliary complications occurring after hepatic angioembolization and try to detect them in early stage to treat them correctly.

\section{References:}

Disclosure: No significant relationships

\section{PR308}

\section{INTOXICATION AND OXIDATIVE STRESS MANIFESTATION IN POSTTRAUMATIC HEMOPERITONEUM DURING NOM}

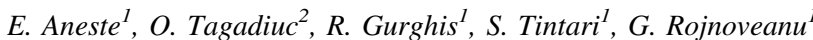

${ }^{1}$ Surgery No.1 "nicolae Anestiadi", Nicolae Testemitanu State University of Medicine and Pharmacy, Chisinau/MOLDOVA,

${ }^{2}$ Biochemistry, Nicolae Testemitanu State University of Medicine and Pharmacy, Chisinau/MOLDOVA

Introduction: Intoxication and oxidative stress biochemical indicators analysis in patients with traumatic hemoperitoneum during nonoperative management (NOM) presents interest in the context of the continuous increase in the incidence of trauma.

Material and methods: Prospective study (2011-2016) of 59 patients with posttraumatic hemoperitoneum. Intoxication indicators: necrotic substances (SN); substances with average molecular weight (SAMW) and oxidative stress indicators: advanced oxidation protein product (AOPP); advanced glycation end products (AGEs) were performed. $\mathrm{M}: \mathrm{F} / 2.7: 1$; average values: age $=37.6 \pm 15.2 ;$ ISS $=22.9 \pm 11.92$; RTS $=7.4 \pm 11.92 ;$ TRISS $=90.4 \% \pm 11.92$. Diagnosis established by: USG-59 (100\%), CT-48 (81.4\%). Division into 2 groups according to hemoperitoneum volume at admission. Gr.I $\leq 500 \mathrm{ml}\left(\mathrm{n}^{1}\right.$ $=38)$; gr.II $>500 \mathrm{ml}\left(\mathrm{n}^{2}=21\right)$.

Results: Mean hemoperitoneum volume at hospitalization: gr.I$299.74 \pm 182.26 \mathrm{ml}$; gr.II-788.1 $\pm 293.22 \mathrm{ml}$. Average values of SN, SAMW, AOPP and AGEs did not show statistically significant differences in dynamics at 1-st, 3-rd and 5-7-th days after admission, nor between gr.I and gr.II ( $\mathrm{p}>0.05)$.

Conclusion: The analyzed biochemical parameters showed that NOM in the treatment of posttraumatic hemoperitoneum in stable or metastable hemodynamic patients, in the absence of other absolute indications for surgical intervention, is a safe approach with favorable evolution.

References: Manolescu B. et all. Dynamic of oxidative and nitrosive stress markers during the convalescent period of stroke patients undergoing rehabilitation. Annals of Clinical Biochemistry. 2011;48 
(4):338-343; HawkinsCL, MorganPE, DaviesMJ. Quantification of protein modification by oxidants. Free Radical Biol Med 2009;46:965-88; Asif M., Egan J., Vasan S. An advanced glycation endproduct cross-link breaker can reverse age-related increases in myocardial stiffness. Proc Natl Acad Sci USA.2000;97 (6):28092813

Disclosure: No significant relationships.

\section{PR309}

\section{PRE-HOSPITAL RESUSCITATIVE THORACOTOMY: CURRENT VARIATION IN UK PRACTICE AND SYSTEMATIC REVIEW}

\author{
E.J. Nevins ${ }^{1}$, P.L. Moori ${ }^{2}$, J. Smith-Williams ${ }^{3}$, N. Bird ${ }^{\text {, S.J. Fadden }}$, \\ C. Jones ${ }^{4}$, J.V. Taylor ${ }^{1}$, N. Misra ${ }^{l}$
}

${ }^{1}$ Emergency General Surgery And Trauma Unit, Aintree University Hospital, Liverpool/UNITED KINGDOM, ${ }^{2}$ Liverpool Medical School, Liverpool Medical School, Liverpool/UNITED KINGDOM, ${ }^{3}$ Department Of Anaesthesia, Royal Liverpool University Hospital, Liverpool/UNITED KINGDOM, ${ }^{4}$ Department Of Anaesthesia, Aintree University Hospital, Liverpool/UNITED KINGDOM

Introduction: The indications for Pre-Hospital Resuscitative Thoracotomy (PHRT) remain undefined. The aim of this paper is to explore the variation in practice for PHRT in the UK, and review the published literature.

Material and methods: 22 UK Air-Ambulance Services were sent an electronic questionnaire to assess their PHRT practice. MEDLINE and, PUBMED search engines were then used to identify all relevant articles.

Results: 10 (45\%) Air-Ambulance Services responded, each service reported different indications for PHRT. All perform PHRT for penetrating chest trauma, however length of allowed pre-procedure down-time varied, ranging from 10-20 minutes. 7/10 services perform PHRT for blunt traumatic cardiac arrest, a procedure which is likely to require aggressive concurrent circulatory support, despite this only $5 / 10$ services carry pre-hospital blood products. 4 European publications report PHRT survival rates of $9.7 \%, 18.3 \%, 10.3 \%$ and $3.0 \%$ in $31,71,39$ and 33 patients respectively, all patients sustained penetrating chest injury. 6 case reports also detail survivors of PHRT, again all patients had penetrating thoracic injury. 1 Japanese paper presents 34 cases of PHRT following blunt trauma, of which $26.4 \%$ survived to ITU but none survived to discharge. A UK population report a single survivor of PHRT following blunt trauma but the case details remain unpublished.

Conclusion: Current indications for PHRT vary amongst different geographical locations, across the UK, and worldwide. Survivors are likely to have sustained penetrating chest injury with short downtime. There is only one published survivor of PHRT following blunt trauma, despite this, PHRT is still being performed in the UK for this indication

References: Athanasiou T, Krasopoulos G, Nambiar P, et al. Emergency thoracotomy in the pre-hospital setting: A procedure requiring clarification. In: European Journal of Cardio-Thoracic Surgery. Vol 26.; 2004:377-385. doi:10.1016/j.ejcts.2004.03.016. Davies GE, Lockey DJ. Thirteen survivors of prehospital thoracotomy for penetrating trauma: a prehospital physician-performed resuscitation procedure that can yield good results. J Trauma. 2011;70 (5):E75E78. doi:10.1097/TA.0b013e3181f6f72f. Coats TJ, Keogh S, Clark H, Neal M. Prehospital resuscitative thoracotomy for cardiac arrest after penetrating trauma: rationale and case series. J Trauma. 2001;50
(4):670-673. doi:10.1097/00005373-200104000-00012. Van Vledder MG, Van Waes OJF, Kooij FO, Peters JH, Van Lieshout EMM, Verhofstad MHJ. Out of hospital thoracotomy for cardiac arrest after penetrating thoracic trauma. Injury. 2017;48 (9):1865-1869.doi: 10.1016/j.injury.2017.04.002. Matsumoto H, Mashiko K, Hara Y, et al. Role of resuscitative emergency field thoracotomy in the Japanese helicopter emergency medical service system. Resuscitation. 2009;80 (11):1270-1274. doi:10.1016/j.resuscitation.2009.08.010. Lockey D, Crewson K, Davies. Traumatic Cardiac Arrest: Who are the Survivors?. Annals of Emergency Medicine. 2006;48 (3):240-244. Disclosure: No significant relationships.

\section{PR310}

\section{USE OF DAMAGE CONTROL SURGERY AND OPEN ABDOMEN IN PATIENTS WITH ACUTE MESENTERIC ISCHEMIA: 5-YEARS EXPERIENCE}

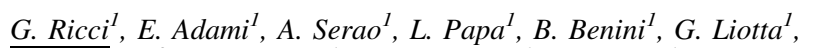
P. Fransvea ${ }^{2}$, R. Mancuso ${ }^{1}$, C. Di Cosimol ${ }^{1}$ P. Marini ${ }^{1}$

${ }^{1}$ Emergency Department - Division Of General And Emergency Surgery, San Camillo - Forlanini Hospital, Rome/ITALY, ${ }^{2}$ Chirurgia Generale E D'urgenza, Facoltà di Medicina e Psicologia Università "Sapienza" Roma, Rome/ITALY

Introduction: Acute mesenteric ischemia (AMI) is associated with a very high mortality due to multiple co-morbidities of these patients and to challenging diagnosis that can lead to delayed treatment. Damage control surgery and open abdomen (OA) have been extensively used in severe traumatic patients and nowadays has been proposed to improve the prognosis even in nontrauma setting. The purpose of our study is to verify the utility of the OA in patients operated for peritonitis secondary to AMI.

Material and methods: A prospectively mantained database of patients operated in emergency setting in our tertiary referral hospital was analysed: 4.588 emergency operations were performed between years 2012 and 2016. Patients with peritonitis due to AMI were identified, after exclusion of patients with non-occlusive mesenteric ischemia and patients with massive bowel necrosis in which only an explorative procedure was done, a total population of 98 patients was selected, 51 females and 47 males with mean age of 73 years (range 43-90).

Results: 48 OA procedures with second-look planned reoperation and 50 non-OA procedures with primary closure of the fascia were performed, OA was closed within a median of 2,7 days (range 1-7). Second look operations had a positive findings in $45,8 \%$ of cases. Total 30-days mortality rate was $41,8 \%$. OA versus non-OA decreased significantly re-operation rate, 30 -days morbility $(41,6 \%$ vs $66 \%, \mathrm{p}<0,01)$ and 30 -days mortality $(29,1 \%$ vs $54 \%, \mathrm{p}<0,01)$.

Conclusion: OA is effective in improving prognosis in patients with AMI, allowing a second-look operation, avoiding the development of intra-abdominal hypertension and helping in control of sepsis and shock.

References: 1. Tilsed JVT, Casamassima A, Kurihara H et al. ESTES guidelines: acute mesenteric ischaemia. Eur $\mathrm{J}$ trauma Emerg Surg 2016;42:253-270. 2. Meng X, Liu L, Jiang H. Indications and Procedures for Second-Look Surgery in Acute Mesenteric Ischemia. Surg Today 2010;40:700-705. 3. Ding W, Wang K, Liu B, Fan X, Wang S, Cao J, Wu X, Li J. Open abdomen improves survival in patients with peritonitis secondary to acute mesenteric aretry occlusion. J Clin Gastroenterol 2017;51 (9):e77-e82.

Disclosure: No significant relationships. 


\section{PR311}

\section{CLINICAL AND FUNCTIONAL OUTCOME OF ACUTE COMPARTMENT SYNDROME}

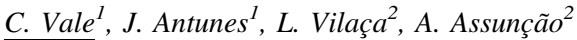

${ }^{1}$ Serviço De Ortopedia, Hospital Distrital Figueira da Foz, Figueira da Foz/PORTUGAL, ${ }^{2}$ Serviço De Cirurgia Vascular, Hospital de Braga, Braga/PORTUGAL

Introduction: Compartment Syndrome results from the increasing pressure in an osteofacial compartment, and is more often caused by a high-energy trauma, a vascular traumatic injury or an ischemiareperfusion injury. Symptoms include disproportional pain in a tense swollen compartment and paresthesia, wich later could evolve into paralysis and/or lack of pulse.

Material and methods: Retrospective cohort study including patients with Compartment Syndrome surgically treated in 2008-2016, without abdominal Compartment Syndrome cases. The aim was to perform a functional assessment through SF-36 Score.

Results: We included 16 patients ( $75 \%$ traumatic) with mean ages at the time of the study of $49 \pm 20$ years (13 male), with a 13 months average follow up. Located on leg $(62,5 \%)$, hand (25\%) and thigh $(12,5 \%)$, all were submited to emergent fasciotomy, with an associated bypass in $43,75 \%$. We report complications in $37,5 \%$, in wich $83 \%$ resulted from leg Compartment Syndrome, resulting in a longer stay-in-hospital. SF-36 Score (Mean 66,47 $\pm 15,8$ ) showed differences regarding location (p0,02): thigh $(32,3)$, leg $(68,2)$ and hand $(74,6)$. SF-36 Physical component varies depending on mechanism: 73,3 on post traumatic and 57,5 on ischemia-reperfusion injury.

Conclusion: Functional outcome presented an SF-36 of 66, $47 \pm$ 15,8 , being inferior when thigh-located, which corroborates the current literature. The longer stay in hospital for patients with leg compartiment syndrome may be related with the higher rate of complications in these cases. This study shall be widen in order to relate with Diabetes, Arterial Insufficiency or Associated Fractures. Compartiment Syndrome is a rare condition, but it represents a surgical emergency and it should be identified and timely treated with emergent Fasciotomy in order to achieve a better outcome.

References: - S. Terry Canale and James H. Beaty, , Campbell's Operative Orthopaedics, 2016 - Loreto Lollo, Andreas Grabinsky; Clinical and funtional outcome of acute lower extremity compartment syndrome at a Major Trauma Hospital, International Journal of Critical Illness \& Injury Science, 2016 Jul-Set - Joanne Pechar, M. Melanie Lyons; Acute Compartment Syndrome of the Lower Leg: A Review, Journal Nurse Pract. 2016 April

Disclosure: No significant relationships.

\section{PR312}

\section{CHARACTERISTICS AND TREATMENT STRATEGIES OF PENETRATING THORACOABDOMINAL INJURIES ON THE EXAMPLE OF GUNSHOT AND BLAST VICTIMS WITHOUT BALLISTIC BODY ARMOUR IN AFGHANISTAN (2009-2013)}

\author{
A.G. Willms ${ }^{1}$, C. Güsgen ${ }^{2}$, I. Richardsen ${ }^{2}$, R. Schwab ${ }^{2}$
}

${ }^{1}$ General-, Visceral- And Thoracic Surgery, German Armed Forces Central Hospital, Koblenz/GERMANY, ${ }^{2}$ General-/visceral- And Thoracic Surgery, German Armed Forces Central Hospital, Koblenz/ GERMANY
Introduction: Europe has recently seen many terrorist attacks in recent years. Penetrating torso trauma is associated with high mortality rates. This highlights the need for expertise in the treatment of penetrating torso trauma by bullets or explosions.

Material and methods: An analysis was performed based on data from patients who were treated in the German Military Hospital Mazar-e Sharif because of gunshot- or blast injuries of thorax and abdomen during 2009 to 2013. For better comparability with civilian attack scenarios, this study only included patients without ballistic body protection (body armour, protective vests).

Results: Out of 117 analysed patients, 58 were affected by firearms and 59 by explosive injuries of the thorax or abdomen. $60 \%$ of patients had a thoracic injury, $69 \%$ had an abdominal injury, and $25.6 \%$ had combined thoracic-abdominal injuries. Blast injury patients were significantly more affected by thoracic trauma. Furthermore patients with blast injuries had significantly more injured organs and a significantly higher ISS averaging $29.26 \%$ of the gunshot patients and $41 \%$ of the blast wounded patients received Damage Control Surgery (DCS). Overall mortality was $13.7 \%$. The ISS of deceased patients was significantly higher.. The highest mortality rate $(25.7 \%)$ was recorded for patients with combined thoracoabdominal injuries (vs. $8.3 \%$ for thoracic and $8.7 \%$ for abdominal injuries).

Conclusion: Patients without ballistic protection of the torso have high mortality rates, especially when suffering thoracoabdominal blast injuries. Blast injuries frequently lead to the DCS indication. The care of firearm and blast injury patients requires knowledge and competence in the damage control procedures for thorax and abdomen.

References:

Disclosure: No significant relationships.

\section{PR313}

\section{TRAUMA LAPAROTOMIES IN AN ERA OF DIFFERENTIATION}

D.P.J. Smeeing $^{1}$, J.J.M. Haverkort ${ }^{2}$, T.T.f. Saris ${ }^{1}$, K.J.p. Van Wessem $^{I}$, L.P.h. Leenen ${ }^{I}$, F. Hietbrink ${ }^{I}$

${ }^{1}$ Surgery, UMC Utrecht, Utrecht/NETHERLANDS, ${ }^{2}$ Major Incident Hospital, UMC Utrecht, Utrecht/NETHERLANDS

Introduction: Laparotomy can be a lifesaving procedure after abdominal trauma. In the past decade, incidence of penetrating trauma has declined in Western countries and the indication for a trauma laparotomy has decreased. The aim of this study was to describe the injuries and the performed surgical interventions after abdominal trauma followed by laparotomy in this present setting.

Material and methods: A single-center retrospective cohort study was performed in a Level 1 trauma center in the Netherlands. All patients who underwent a laparotomy directly related to trauma between January 2008 and January 2014 have been included. Patient demographics, trauma mechanism, traumatic injuries, surgical interventions and mortality were registered.

Results: A total of 121 trauma patients were included with a mean ISS of $29.2 \pm 14.4$. Males contributed for $77.7 \%$. The mean age was $42.5 \pm 21.3$ years. The most common trauma mechanisms were blunt (i.e. traffic accidents in cars $(30.6 \%)$, with cycling $(14.0 \%)$, with a scooter $(12.4 \%)$ and falls $(10.8 \%))$. The median number of laparotomies per patient was 2 . Most patients $(66.9 \%)$ underwent the first laparotomy on the first day. Thirteen patients $(10.7 \%)$ underwent an unplanned relaparotomy. A total of 27 patients (22.2\%) died, mainly 
of neurological injuries (29.6\%), multi organ failure (18.5\%) and retroperitoneal exsanguination $(22.2 \%)$.

Conclusion: This study shows that most patients who underwent a trauma laparotomy in the Netherlands are severely injured patients, often after high energy blunt trauma. A total of $8.3 \%$ deceased due to exsanguination. Despite a decreased exposure to this procedure, mortality and morbidity rates in a dedicated level 1 center are comparable to international literature.

References: -

Disclosure: No significant relationships.

\section{PR314}

\section{MULTIVARIATE ULTRASOUND CAN PREDICT VOLUME RESPONSIVENESS IN CRITICALLY ILL TRAUMA PATIENTS}

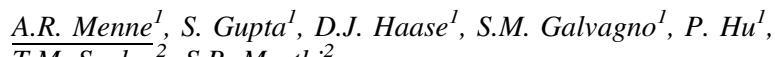
$\overline{\text { T.M. Scalea }}^{2}$, S.B. Murthi ${ }^{2}$

${ }^{1}$ Shock Trauma, University of Maryland, Baltimore/MD/UNITED STATES OF AMERICA, ${ }^{2}$ Shock Trauma, University of Maryland, Baltimore/UNITED STATES OF AMERICA

Introduction: Point of care ultrasound (POCUS) is increasingly utilized in real-time clinical decision making. The current standard of care POCUS assessment of volume responsiveness (VR) is respiratory variation of the inferior vena cava (rvIVC), however, there is conflicting data as to its accuracy.

Material and methods: This is a prospective observational study in critically ill surgical patients receiving a bolus of crystalloid or colloid. A transthoracic echocardiogram was performed before and after. Measurements obtained include rvIVC, respiratory stroke volume variation (SVV), left ventricular velocity time integral (VTI), positional internal jugular change $\left(0-90^{\circ} \mathrm{IJ}\right)$, and respiratory variation in the IJ lying flat $\left(\mathrm{rvIJ}^{\circ}\right)$ and sitting upright $\left(\mathrm{rvIJ} 90^{\circ}\right)$. In addition, age and body surface area (BSA) were recorded. VR was defined as $\geq$ $15 \%$ increase in stroke volume. Computer modeling decision trees were used to predict VR. Training and testing groups were used to establish and test the performance of the model. An area under the receiver operating characteristic curve (AUROC) was then assessed. Results: Over three years, 134 patients were enrolled; $68 \%$ were mechanically ventilated and $46 \%$ had undergone torso surgery. BSA and age were predictors of VR. US predictors of VR included all IJ assessments and VTI. The rvIVC and SVV were not predictive of VR. The training AUROC was $0.91(\mathrm{p}<0.0001)$ and this performed well on testing validation with AUROC $0.76(\mathrm{p}<0.001)$.

Conclusion: Multivariate assessment including both measures of inflow to the right ventricle (IJ assessments) and outflow from the left ventricle (VTI) is superior to rvIVC in critically ill trauma patients. References:

Disclosure: No significant relationships.

\section{PR315}

\section{A PROSPECTIVE ANALYSIS OF SHORT AND MID-TERM KNOWLEDGE RETENTION AFTER AN ULTRA-SHORT FAST COURSE FOR UNDERGRADUATE MEDICAL STUDENTS}

\author{
C.A.M. Menegozzo ${ }^{1}$, P.G. Cazolari ${ }^{2}$, F.C.F. Novo ${ }^{1}$, R. Colleoni ${ }^{2}$, \\ E.M. Uityama
}

${ }^{1}$ General Surgery And Trauma, Hospital das Clínicas, University of Sao Paulo, Sao Paulo/BRAZIL, ${ }^{2}$ Surgery, Federal University of Sao Paulo, Sao Paulo/BRAZIL

Introduction: Point-of-care ultrasound is becoming an invaluable tool in emergency surgery and is already considered as he new stethoscope by some authors. Ultrasound techniques may be taught early for undergraduates. FAST represents a simple exam that might be used to teach important aspects of ultrasound imaging. We sought to investigate knowledge retention on short and mid-term after an ultra-short FAST course for undergraduate students.

Material and methods: This prospective study involved medical students enrolled to the FAST course and included those who responded to a pre- and a post-course questionnaire. The same 8 -question form was presented previously to the course, 1 week and 3 months after, and were graded with one point to each question. The course duration was 50 minutes and involved theoretical and hands-on training. Mean values obtained in the questionnaires were compared to assess knowledge gain with the course and the retention after 1 and 3 months.

Results: A total of 52 students participated in the course. We obtained 67 questionnaire responses for prospective analysis, 37 pre-test (PRT) and 30 post-test (POT). Mean correct answers were 3 and 5,6 for PRT and POT, respectively, and 6 and 5 for 1-week (1POT) and 3-month post-tests (3POT), respectively. Comparison between PRT and POT, and between PRT and 3POT, were both significantly different $(\mathrm{p}<$ $0,0001)$. There was no difference between 1POT and 3POT results ( $\mathrm{p}$ $=0,13)$.

Conclusion: Despite limitations, this data show that even an ultrashort FAST course can effectively impact knowledge retention in undergraduate medical students. Efforts should be made to disseminate this knowledge early during medical education.

References: Walcher F. et al. Accuracy of prehospital focused abdominal sonography for trauma after a 1-day hands-on training course. Emerg J Med 2010 May;27 (5):345-9. McGahan JP et al. The focused abdominal sonography for trauma scan: pearls and pitfalls. J Ultrasound Med2002;21:789-800

Disclosure: No significant relationships. 
PR316

\section{LAPAROSCOPIC DIAPHRAGMATIC EVALUATION OF STABLE PATIENTS WITH THORACOABDOMINAL PENETRATING WOUNDS: A SINGLE CENTER EXPERIENCE}

C.A.M. Menegozzo, S.H.B. Damous, P.H.F. Alves, M.C. Rocha, $\overline{\text { C.O. Bernini, E.M. Utiyama }}$

General Surgery And Trauma, Hospital das Clínicas, University of Sao Paulo, Sao Paulo/BRAZIL

Introduction: Thoracoabdominal pentrating wounds (TAPW) are frequently associated with diaphragmatic injury. Patients without immediate indications for surgical exploration require investigation of such injuries since late diagnosis may result in high morbidity and mortality rates. The specificity of radiological studies tend to be insufficient to rule out such lesions. In such situations, laparoscopy may present an invaluable role in the evaluation of the diaphragm. The objectives of the present study are to analyze the role of laparoscopy in the management of stable patients with TAPW and to evaluate the accuracy of radiological studies regarding preoperative diagnosis of diaphragmatic lesions.

Material and methods: Retrospective analysis of stable patients with TAPW who underwent laparoscopic approach. Hemodynamically unstable patients or those who were operated by laparotomy were excluded. Demograpics, clinical and perioperative data were collected and analyzed. Two different radiologist reviewed the images without previous knowledge of surgical results. Direct and indirect signs of diaphragmatic injury were evaluated.

Results: Thirty patients were included, of which $95 \%$ were male and the mean age was 32 years. Median RTS and ISS were 7, 8404 and 13 , respectively. Sixteen procedures were therapeutic involving diaphragmatic repair while only 8 were negative laparoscopies. Direct or indirect signs of diaphragmatic injury were noted on plain radiographs in $50 \%$ of 24 images, and on CT in 33\% of 9 exames. Conversion rate was null and only one Clavien-Dindo 3 complication was observed.

Conclusion: This study further highlights the poor diagnostic accuracy of preoperative imaging regarding diaphragmatic injuries. Laparoscopy should be done to rule out those injuries in patients with thoracoabdominal penetrating wounds.

References: Parreira JG, Rasslan S, Utiyama EM. Controversies in the management of asymptomatic patients sustaining penetrating thoracoabdominal wounds. 2008;63 (5):695-700. Morales CH, Villegas MI, Angel W, Vásquez JJ. Value of digital exploration for diagnosing injuries to the left side of the diaphragm caused by stab wounds. Arch Surg 2001;136 (10):1131-5. Clarke DL, Greatorex B, Oosthuizen G V, Muckart DJ. The spectrum of diaphragmatic injury in a busy metropolitan surgical service. Inj Int $J$ Care Inj 2009;40:932-7.

Disclosure: No significant relationships.
PR317

\section{SPLENIC TRAUMA: SURGICAL VS. NON SURGICAL MANAGEMENT. A RETROSPECTIVE STUDY}

\author{
C. Pineño Flores ${ }^{1}$, J.J. Segura-Sampedro ${ }^{2}$, D. Ambrona Zafra ${ }^{1}$ \\ A. Craus Miguel ${ }^{1}$, C. Soldevila Verdeguer ${ }^{1}$, G.P. Martin Martin ${ }^{2}$, \\ F.X. Gonzalez Argente ${ }^{2}$
}

${ }^{1}$ Resident Of General Surgery, Hospital Universitario Son Espases, Palma de mallorca/SPAIN, ${ }^{2}$ General \& Digestive Surgery, University Hospital Son Espases, Palma de Mallorca/SPAIN

Introduction: The spleen is the most frequently injured solid organ following blunt abdominal trauma. We aimed to review the current practice for the evaluation, diagnosis and management of blunt splenic injury (BSI).

Material and methods: A 5 years retrospective review of spleen injuries in a tertiary referral center for severe trauma in the Balearic Islands. Demographic data, mechanism of injury, hemodynamic status, diagnostic approach, grading scale for BSI has been established by the American Association for the Surgery of Trauma (AAST), associated injuries, morbidity and mortality were collected. Data were analysed using IBM SPSS Statistics, 21.0.

Results: 69 patients were included. Most of the patients were males, $59(86 \%)$, aged $29 \pm 6$ ' 31 years. Injuries related to traffic accidents accounted for $29(42 \%)$ and falls $14(21 \%)$. In the emergency room, $16(23 \%)$ were hemodynamically unstable. $84 \%$ diagnosed by CT+FAST or FAST in 13\%. Degree of injury (ASST)was: I (35\%), II $(22 \%)$, III (17\%), IV (17\%). The median of ISS was $11,71 \%$ suffered concurrent injuries and the most frequent associated lesion were thoracic trauma (35 (51\%)). The 55\% (38) received non operative management (NOM). Twenty-seven $(45 \%)$ patients required surgical treatment for hemodynamic instability, free fluid or active bleeding in complementary tests. Splenectomy was the principal technique. But 9 $(13 \%)$ of these patients could be treated with splenic artery embolisation. The morbibity was lower in the NOM group (6\%). Mortality rate of $7 \%$.

Conclusion: In recent years, there has been an improvement in survival by standardization of the NOM in hemodynamically stable patients, reducing hospital stay and postoperative complications.

References: Frandon J. Rodiere M. et al. Blunt splenic injury: are early adverse events related to trauma, nonoperative management, or surgery? Diagn Interv Radiol 2015; 21: 327-333 Ruben Peralta d, Husham Abdelrahman d. Blunt splenic trauma: Assessment, management and outcomes. The surgeon 14 (2 $\left.0 \begin{array}{lll}2 & 1\end{array}\right) 5$ 2e5 8 Dominique C. Olthof Pieter Joosse Observation Versus Embolization in Patients with Blunt Splenic Injury After Trauma: A Propensity Score Analysis. World J Surg (2016) 40:1264-1271 DOI 10.1007/s00268-0153387-8 Mr JML Williamson. Splenic injury: diagnosis and management. British Journal of Hospital Medicine, April 2015, Vol 76, No 4 Oyo-Ita A, Chinnock P, Ikpeme IA. Surgical versus non-surgical management of abdominal injury. Cochrane Database of Systematic Reviews 2015, Issue 11. Art. No.: CD007383. DOI: 10.1002/ 14651858.CD007383.pub3. Dehli et al. Scandinavian The treatment of spleen injuries: a retrospective study. Journal of Trauma, Resuscitationand Emergency Medicine (2015) 23:85 DOI 10.1186/s13049015-0163-6

Disclosure: No significant relationships. 


\section{PR318}

\section{ASSESSMENT OF ABDOMINAL STAB WOUND INJURIES, A RETROSPECTIVE STUDY}

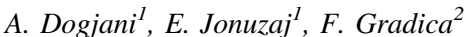

${ }^{1}$ General Surgery, University Hospital of Trauma, Tirana, , Tirana/ ALBANIA, ${ }^{2}$ Thoracic Surgeon, University Hospital of Lung Disease, Tirana/ALBANIA

Introduction: Penetrating trauma to the abdomen can seriously threaten patient' life because of high risk of internal bleeding or peritonitis. Patients with clinical pictures suggestive of these complications should immediately undergo surgical management. However, laparoscopy or laparotomy reveals no significant finding in many cases of abdominal stab wounds, showing that they could have been managed nonoperatively. Local wound exploration (LWE) was advocated by Markovchick in 1985 as the primary screening for the patients with anteriorabdominal penetrating trauma.

Material and methods: The case records of all patients with penetrating trauma (PT) diagnosed between January 2016 and August 2017. All patients who had performed or not emergency laparotomy (EL) after sustaining Stab wounds (SW), Gunshot wounds (GSW), Blast injuries (BI), were included in our study.

Results: We have recorded 102 patients $(\mathrm{Pt})[93(91 \%)$ male \& $9(9 \%)$ female] with PAT in this study. Of $102 \mathrm{Pt}, 47$ (46\%) were coming from Tirana and 55 (54\%) from another hospitals. The mean age was 34.6 (10-80) years. The distribution of data based on mechanism of injury was; 65 (64\%) of Pt was after SW, 34 (33\%) of Pt was after GSW, 2 (2\%) of Pt was after BI, were included in our study.

Conclusion: Although a high degree of diagnostic accuracy must be maintained to limit the frequency of missed injury. Abdominal stab wound exploration, serial clinical evaluation, or both are used to further assess patients in whom an exploration cannot definitively exclude peritoneal penetration and serial clinical evaluation is done as part of management.

References: 1- Como JJ, Bokhari F, Chiu WC, Duane TM, Holevar MR, Tandoh MA, et al. Practice management guidelines for selective nonoperative management of penetrating abdominal trauma. $J$ Trauma.2010; 68 (3):721-33. 2 -Biffl WL, Moore EE. Management guidelines for penetrating abdominal trauma. Curr Opin Crit Care. 2010;16 (6):609-17.

Disclosure: No significant relationships.

\section{PR319}

\section{THE IMPROVEMENT OF NON-OPERATIVE MANAGEMENT (NOM) OF BLUNT SPLENIC TRAUMA AFTER THE INTRODUCTION OF A HOSPITAL PROTOCOL: 9-YEARS EXPERIENCE OF A SINGLE UNIVERSITY HOSPITAL}

\author{
M. Di Grezia ${ }^{1}$, V. Cozza ${ }^{l}$, A. Di Giorgio ${ }^{I}$, G. Pepe ${ }^{l}$, C. Lodoli ${ }^{1}$, \\ G. Tropeano , A. Agnes ${ }^{I}$, F. Marzi ${ }^{I}$, R. Panzera ${ }^{I}$, A. Borrello $^{I}$, \\ S. Magalini ${ }^{1}$, A. La Greca ${ }^{2}$, D. Gui ${ }^{1}$
}

${ }^{1}$ Emergency Surgery, Fondazione Policlinico Universitario “A. Gemelli”, ROME/ITALY, ${ }^{2}$ Emergency Surgery, Fondazione Policlinico Universitario "A. Gemelli”, rome/ITALY

Introduction: The management of splenic blunt trauma has evolved in the last decades. The guidelined of the Eastern Association for the
Surgery of Trauma (EAST) suggest that non operative management (NOM) should be the treatment of choice. in hemodinamically stable patients. However, the criteria for selection of patients for NOM are not homogeneous. The aim of this retrospective study is to clarify the criteria for NOM and to examine the evolution of blunt splenic trauma treatment in our Institution.

Material and methods: Retrospective, cross-sectional study including all adult patients admitted with blunt splenic trauma between 2008 and 2016. Hemodinamic status, Grade of spleen injury and type of treatment were studied. The outcome was measured including percentage of NOM across the years, failure rate, complications.

Results: 138 cases of blunt splenic trauma were treated in the study period. We have observed a decrease of $30 \%$ in operative management, with stable failure rate $(15 \%)$. We have noticed that the adherence to the protocol has played a key role in improving the outcomes of these patients, reducing the percentage of avoidable splenectomies in hemodinamically stable patients.

Conclusion: The introduction of a hospital protocol improves salvage rates in blunt splenic trauma. NOM is safe in experienced centres and improves outcomes in hemodinamically stable patients.

References: 1. Tugnoli G, Bianchi E, Biscardi A, Coniglio C, Isceri S, Simonetti L, Gordini G, Di Saverio S. Nonoperative management of blunt splenic injury in adults: there is (still) a long way to go. The results of the Bologna-Maggiore Hospital trauma center experience and development of a clinical algorithm. Surg Today. 2015 Oct;45 (10):1210-7. 2. Olthof DC, van der Vlies CH, Goslings JC. EvidenceBased Management and Controversies in Blunt Splenic Trauma. Curr Trauma Rep. 2017;3 (1):32-37. 3. Watson GA, Hoffman MK, Peitzman AB. Nonoperative management of blunt splenic injury: what is new? Eur J Trauma Emerg Surg. 2015 Jun;41 (3):219-28.

Disclosure: No significant relationships.

\section{PR320}

\section{POINT OF CARE ULTRASOUND (POCUS):PERFORMING EXTENDED FAST (EFAST) ULTRASOUND ON PATIENTS WITH MINOR BLIND TRAUMA IN A DISTRICT GENERAL HOSPITAL OF GREECE}

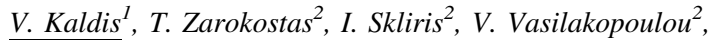 \\ C. Paspaliaris ${ }^{3}$, I. Mitrakos ${ }^{3}$, I. Poulos ${ }^{3}$, D. Giannakopoulos ${ }^{2}$
}

${ }^{1}$ Department Of General Surgery, PANARKADIKON GENERAL HOSPITAL OF TRIPOLI, TRIPOLI/GREECE, ${ }^{2}$ Department Of General Surgery Argos Medical Center, ARGOLIDAS GENERAL HOSPITAL, ARGOS/GREECE, ${ }^{3}$ Department Of Radiology Argos Medical Center, ARGOLIDAS GENERAL HOSPITAL, ARGOS/ GREECE

Introduction: CT scan is the cardinal diagnostic method on trauma. Performing Extended FAST (e-FAST) is the evolution in the ultrasound diagnosis on blind trauma triage ruling the surgical definitive care decision. The objective is to determine the necessity to perform eFAST on minor blind trauma.

Material and methods: Retrospective study 20 months period (2016/ 17) of consecutive trauma patients presenting in Trauma Bay undergone thru eFAST diagnosis recording demographic data, patern of injury, injury cause, ISS, outcome and diagnosis.

Results: Eligible were eFAST diagnosis trauma 159 patients ( $\precsim 120 /$ १39), managed thru ATLS/APLS/DSTC algorithm from certified surgeon, Median age 44 (4 -90) mostly reported and registered mostly with minor blind trauma (ISS < 16). Cause of injury caused from motor vehicle accidents (MCA)28\% $(n=44)$ following bicycle $34 \%$ 
$(n=53) \&$ ground falls in $25 \%(n=41)$.Predominant patern of injury was TBIs $48 \%$, combined with chest abdomen trauma in $24 \%$.Dismissed in 54\% ( $\mathrm{n}=86)$, admitted 30\% $(\mathrm{n}=49)$ and transferred $14 \%$ $(n=23)$, thru EMS, with severe trauma (ISS $>16$, ME 18) to the proper Trauma Service mostly with TBI under mechanical ventilation.Diagnosis confirmed, from board Radiologists, in all patients for the classical FAST ultrasound and in $35 \%(\mathrm{n}=55)$ more CT exam requested.One false negative eFAST case registrated.

Conclusion: In our district Hospital performing eFAST was beneficiary in the triage on minor blind trauma patients and life time keeping for the definitive surgical care.Abdomen exam requests were reduced.

References: 1.Montoya $\mathrm{J}$ et al. From FAST to E-FAST: an overview of the evolution of ultrasound-based traumatic injury assessment. Eur J Trauma Emerg Surg. 2015 Mar 14 2.Soult MC et al. Can routine trauma bay chest x-ray be bypassed with an extended focused assessment with sonography for trauma examination?. Am Surg. 2015 Apr. 81 (4):336-40.

Disclosure: No significant relationships.

\section{PR321}

\section{WHAT IS MORE IMPORTANT IN MANAGEMENT OF PENETRATE ABDOMINAL TRAUMA?}

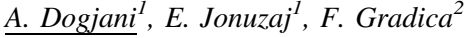

${ }^{1}$ General Surgery, University Hospital of Trauma, Tirana, , Tirana/ ALBANIA, ${ }^{2}$ Thoracic Surgeon, University Hospital of Lung Desease, Tirana/ALBANIA

Introduction: Over the past century, the management of penetrating abdominal trauma has engaged in repeated cycles involving both operative and nonoperative algorithms. In part, this sequence has been based on alterations in hospital resources, advances in diagnostic imaging and period-specific beliefs of well-known thought leaders. Nowadays penetrating trauma is increasing because of the growth of violence in our society. The penetrating injury (PI) constitutes about $6 \%$ of the hospitalized cases and $10 \%$ of visits to the emergency department (ED).

Material and methods: In this study was to evaluate the management results of patients with PAT in University Hospital of Trauma, Tirana, Albani, we have used a standardized data collection instrument, case records of all patients with PAT between August 2016 and August 2017.

Results: The time of presentation in emergency department (DE) was a- immediately - 89 (87\%) [58 (57\%) SW 29 (28\%) GSW; 2 (2\%) BI;]; b - after 6 hours - 10 (10\%) [5 (5\%) SW; 5 (5\%) GSW]; c - after $24 \mathrm{~h}-3(3 \%)$ [3 (3\%) SW] HdSt in admission of all Pt was; a Presence of shock on admission or Hemodynamic instability (HdInSt) in $49(48 \%)$ [22 (22\%) SW; 25 (25\%) GSW; 2 (2\%) BI] and b Hemodynamic stability (HdSt) 53 (52\%) [44 (44\%) SW; 9 (9\%) GSW;]....

Conclusion: The outcomes of treatment are depended from mechanism of injury, age, presence of shock in admission, number of injuries organ, and associations with extraabdominal injuries... The principle of guiding surgical management on the basis of a good clinical exam and appropriate imaging tests...

References: 1- FELICIANO D. V. Pattern of surgery. In:FELICIANO D. V., MOORE E. E., MATTOX K.L. (eds.). Connecticut: Trauma. Stamford, 1996. pp. 85-105. 2 - HOYT D. B. Trauma. In:GREENFIELD L. J., MULHOLLAND M. W., OLDHAM K. T., ZELENOCK G. B., LILLEMOE K. D. (eds.). Surgery: scientific principles and practice. 2nd ed. Philadelphia: Lippincott-Raven, 1997, pp. 267-79. 3 - Velmahos GC, Demetriades D, Toutouzas KG, Sarkisyan G, Chan LS, Ishak R, Alo K, Vassiliu P, Murray JA, Salim A, Asensio J, Belzberg H, Katkhouda N, Berne TV: Selective nonoperative managementin 1,856 patients with abdominal gunshot wounds: should routine laparotomy still be the standard of care? Ann Surg 2001, 234:395-402. 4 - Nelson R, Singer M: Primary repair for penetrating colon injuries. Cochrane Database Syst Rev 2002:CD002247. 5 - Demetriades D, Hadjizacharia P, Constantinou C, Brown C, Inaba K, Rhee P, Salim A: Selective nonoperative management of penetratingabdominal solid organ injuries. Ann Surg2006, 244:620-8.

Disclosure: No significant relationships.

\section{PR322}

\section{ROLE OF LAPAROSCOPIC APPROACH IN TRAUMA PATIENTS}

L. Tallón-Aguilar ${ }^{1}$, J.A. Lopez Ruiz ${ }^{2}$, C. Domínguez-Sanchez ${ }^{2}$, J. Tinoco-González ${ }^{l}$, B. Marenco De La Cuadra ${ }^{3}$, V. Durán-Muñoz-

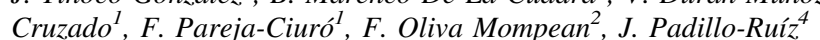

${ }^{1}$ Emergency Surgery Unit, Virgen del Rocío Universitary Hospital, Seville/SPAIN, ${ }^{2}$ General Surgery, University Hospital Virgen Macarena, Seville/SPAIN, ${ }^{3}$ Cirugía General, Hospital Virgen Macarena, Seville/SPAIN, ${ }^{4}$ Digestive And General Surgery Unit, Virgen del Rocío Universitary Hospital, Seville/SPAIN

Introduction: Laparoscopic approach in trauma patients is still very unusual. There are some indications as suspicion of diaphragmatic lesions, non massive haemorrhage in thoracic injuries or screening and treatment of selected abdominal injuries. It is more important to know its contraindications: unstable patients or severe cranial trauma. Our aim is to analyze our experience in laparoscopic approach in trauma patients.

Material and methods: Observational, descriptive, transverse and retrospective study in both hospitals. Demographic variables, type of trauma, injuries, diagnostic test, therapeutic actions and follow up have been collected.

Results: There were 13 patients ( 6 and 7 en each centre), with a mean age of 41 years and almost gender equality ( 7 women/6 men). Majority are open abdominal trauma $(9,70 \%)$ by white - bladed injuries, being the rest blunt abdominal trauma $(4,30 \%)$. In 3 cases (23\%), conversion to laparotomy was necessary due to diagnostic doubts or too complex lesions to be solved by laparoscopic approach, never by instability of patient. In 4 cases $(30 \%)$ exploratory laparoscopy without any surgical performance were performed. Laparoscopic procedures were diverse as diaphragmatic suture, gastric suture or intestinal suture.

Conclusion: Laparoscopy still plays a small role in trauma patients, but its diagnostic and therapeutic usefulness in selected cases should be considered. It has been shown to be useful in diagnosis of peritoneal violation in open trauma, in hemodynamically stable patients, evaluation and repair of diaphragm, examination in equivocal CT results and mainly in surgeons with extensive experience in laparoscopic approach and trauma care.

References:

Disclosure: No significant relationships. 
PR323

\section{BULL HORN INJURIES IN A SPANISH TERTIARY HOSPITAL}

\author{
L. Pérez-Santiago, M. Garcés-Albir, M.D.C. Fernandez-Moreno, \\ F. Lopez-Mozos, J. Ortega
}

Cirugia General Y Del Aparato Digestivo, Hospital Clinico Universitario, Valencia/SPAIN

Introduction: Bull horn injuries are quite common in some places in Spain, where bullfights are very normal in the patron saint festivities. These trauma injuries have some special characteristics that deserve to be analyzed.

Material and methods: A retrospective study was performed on a database of the emergency department between January 2011 and December 2016. The study variables were: age, sex, seasonality, location and number of lesions, diagnosis, treatment, mobility and mortality, and sequels.

Results: A total of 198 patients were evaluated in our hospital, 186 $(93.8 \%)$ men and $12(6.2 \%)$ women. Median age was 34 years (range, 19-63). Most patients $(144,73 \%)$ were admitted to the hospital between June and September. Usual locations of the lesions were: legs $(57,29.2 \%)$, arms $(37$,

$19 \%)$ and thorax $(28,14,2 \%)$. Total of $33(15 \%)$ patients, more than one location was affected. Orthopedic Unit attended more patients $(122,62.6 \%)$ followed by the General Surgery $(35,17.9 \%) .59$ $(29,2 \%)$ patients required surgery, 49 (25\%) emergently. The exploration, debridement and suturing of penetrating wounds were the most frequent interventions. A total of $15(7.7 \%)$ of the patients suffered complications, wound infection being the most frequent. A second intervention was necessary in $11,8 \%$ of the patients. Three deaths occurred in patients with penetrating chest wounds.

Conclusion: Bull horn injuries are one of the most trauma consultations in many places in Spain, especially in the summer months. Frequently, injuries are bruises that do not require surgical treatment. Approximately $29 \%$ of cases require surgery with a low morbidity and mortality rate. Penetrating thorax lesions, on the other hand, are associated with high mortality.

References:

Disclosure: No significant relationships.

\section{PR324}

\section{MANAGEMENT OF LIVER TRAUMA IN HPB UNIT OF TREVISO: A 24-YEAR EXPERIENCE}

\author{
C. Nistri ${ }^{1}$, L. Bonariol ${ }^{2}$, C. Ruffolo ${ }^{2}$, E. Caratozzolo $^{2}$, G. Marte $^{2}$, \\ N. Bassi ${ }^{2}$, M. Massani ${ }^{2}$
}

${ }^{1}$ Iv Department Of Surgery - Regional Center For Hpb Surgery, Regional Hospital of Treviso, Ca Foncello - ULSS 2, Treviso/ITALY, ${ }^{2}$ Iv Department Of Surgery - Regional Center For Hpb Surgery, Regional Hospital of Treviso, Ca Foncello - ULSS 2, treviso/ITALY

Introduction: Evolution of guidelines for the treatment of liver trauma has changed management practice and has reduced mortality. In this study we present the outcome of operative and non-operative management of patients with liver injury treated in a single institution.
Material and methods: The study was conducted retrospectively at the IV Department of Surgery, Regional Center for HPB Surgery, in the regional Hospital of Treviso and included patients with liver trauma admitted from January 1993 to September 2017 (246 patients). Liver injuries were evaluated according to the American Association for the Surgery of Trauma.

Results: Our group included 190 males $(77,24 \%)$ and 56 females $(22,76 \%)$, with a median age of 38 years at the time of trauma (range 15-91). The most common cause of liver injury was blunt trauma (93,09\%). At first observation, 180 patients $(73,17 \%)$ were hemodynamically stable, while 66 patients $(26,83 \%)$ were unstable. Level I-II liver traumas were $127(51,63 \%)$ and level III-IV-V liver lesions were $119(48,37 \%)$. Overall mortality was $14,63 \%$. Most patients were treated with non-operative management $(59,76 \%)$ with a high success rate $(74,15 \%)$. Use of hepatic arterial embolization technique started through the study period and 27 hepatic embolizations were performed (10,98\% of traumas), the $92,59 \%$ of which in serious hepatic traumas. Dividing patients into three periods (1993-2000, 2001-2006, 2007-2017), non-operative management increased from $36,76 \%$ to $78,85 \%$, with increasing success rates $(64 \%$ to $84,15 \%)$. Number of hepatic embolizations increased from $0 \%$ to $25 \%$.

Conclusion: Changes in liver trauma management have improved outcomes at our institution, favoring non-operative management, damage control surgery and hepatic embolization.

References:

Disclosure: No significant relationships.

\section{PR325}

\section{AN UNUSUAL CASE OF TRAUMATIC ABDOMINAL WALL HERNIA SECONDARY TO HANDLEBAR INJURY IN AN ADULT}

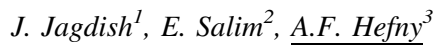

${ }^{1}$ Department Of Radiology, Al Rahba Hospital, AbuDhabi/UNITED ARAB EMIRATES, ${ }^{2}$ Department Of Surgery, Al Rahba Hospital, Abu Dhabi/UNITED ARAB EMIRATES, ${ }^{3}$ Department Of Surgery, College of Medicine and Health Sciences, Al Ain/UNITED ARAB EMIRATES

Introduction: Introduction: Traumatic Abdominal wall hernia is uncommon injury. Handlebar hernia is an even rarer type of traumatic hernia $(1,2)$. It is blunt enough to cause abdominal wall musculature disruption without penetrating the skin. Most of the literature describes abdominal wall herniation in children (3). Herein, we present a rare case of handlebar traumatic hernia in an adult lady.

Material and methods: Case Report 38-year-old woman presented to Emergency Department complaining of pain and bruising on the left abdominal wall following a fall from the bicycle. There was no past history of previous midline surgical incision or hernia. Clinical examination revealed a contusion mark that resembled the pattern of the end of the handlebar overlying a prominent abdominal swelling. The swelling increases in size on coughing and decreases on lying down.

Results: CT scan of the abdomen revealed a wide-necked left para midline abdominal wall hernia containing a loop of transverse colon. There was diffuse abdominal wall subcutaneous fat stranding centred on the hernia. There was no evidence of bowel injury. Patient was diagnosed to have a traumatic abdominal wall hernia secondary to bicycle handlebar injury. The patient was admitted to the surgical department and treated conservatively. She was discharged from the 
surgical department to be follow up in the surgical clinic for subsequent elective repair

Conclusion: Handlebar traumatic hernia is not often associated with other intra-abdominal injuries. CT scan confirmed the clinical suspicion and excluded intra-abdominal injuries. Urgent laparotomy may not be indicated in the early stage if no signs of intra-abdominal injuries were detected.

References: 1. Okamoto D, Aibe H, Hasuo K, Shida Y, Edamoto Y. Handlebar hernia: a case report. Emerg Radiol. 2007; 13:213-5. 2. Haimovici L, Papafragkou S, Kessler E, Angus G. Handlebar hernia: traumatic abdominal wall hernia with multiple enterotomies. A case report and review of the literature. J Pediatr Surg 2007; 42:567-9. 3. Decker S, Engelmann C, Krettek C, Müller CW. Traumatic abdominal wall hernia after blunt abdominal trauma caused by a handlebar in children: a well-visualized case report. Surgery 2012; 151:899-900. Disclosure: No significant relationships.

\section{SKELETAL TRAUMA}

\section{PR326}

\section{VALIDATION OF THE NOTTINGHAM HIP FRACTURE SCORE (NHFS) TO PREDICT 30-DAY MORTALITY IN PATIENTS WITH AN INTRACAPSULAR HIP FRACTURE}

\author{
L. De Jong ${ }^{1}$, T. Klem ${ }^{2}$, M. Kuijper ${ }^{1}$, G. Roukema ${ }^{I}$
}

${ }^{1}$ Chirurgie, Maasstad ziekenhuis, Rotterdam/NETHERLANDS,

${ }^{2}$ Surgery, Franciscus Gasthuis, Rotterdam/NETHERLANDS

Introduction: The Nottingham hip fracture score (NHFS) was developed to predict 30-day mortality following a fracture of the hip. While the NHFS has been validated in three hip fracture populations within Great Brittan, these studies make no distinction between the type of fracture and surgery yet. Literature however shows an increased risk for mortality after a hemi arthroplasty following an intra-capsular hip fracture. To verify whether the mortality after an intra-capsular hip fracture indeed antecedes the predicted mortality score according to the NHFS score, we performed a validation of the NHFS in patients with a hemi-arthroplasty after an intra-capsular hip fracture.

Material and methods: The NHFS was calculated for consecutive patients presenting with an intra-capsular fracture of the hip in two level II trauma teaching hospitals between 1 January 2011 and 1 May 2016. The observed 30-day mortality was compared with that predicted by the NHFS using several validation statistics.

Results: A total of 901 patients were included in the present study. Mean age in the patients was 83 years (SD 8) and $623(68 \%)$ of the patients were female. Almost $60 \%$ of the patients had an ASA score of $\geq 3$ and overall 30 -day mortality was $9.5 \%(n=86)$. The mortality rate in the studied population of hemi-arthroplasty patients was significantly higher than mortality rates predicted by the NHFS. $(\mathrm{P}=$ 0.0067)

Conclusion: Our findings suggest that for a patient with a hemiarthroplasty following an intra-capsular hip fracture, there could be an underestimation for the 30-day mortality rate following the NHFS.

References: Maxwell MJ, Moran CG, Moppett IK. Development and validation of a preoperative scoring system to predict 30 day mortality in patients undergoing hip fracture surgery. British journal of anaesthesia. 2008;101 (4):511-7. Moppett IK, Parker M, Griffiths R, Bowers T, White SM, Moran CG. Nottingham Hip Fracture Score: longitudinal and multi-assessment. British journal of anaesthesia. 2012;109 (4):546-50. Rushton PR, Reed MR, Pratt RK. Independent validation of the Nottingham Hip Fracture Score and identification of regional variation in patient risk within England. The bone \& joint journal. 2015;97-B (1):100-3. Nijmeijer WS, Folbert EC, Vermeer M, Slaets JP, Hegeman JH. Prediction of early mortality following hip fracture surgery in frail elderly: The Almelo Hip Fracture Score (AHFS). Injury. 2016;47 (10):2138-43.

Disclosure: No significant relationships.

\section{PR327}

\section{CLINICAL EXPERIENCE IN THE TREATMENT OF FEMORAL NECK FRACTURE WITH HANSSON PINLOC SYSTEM}

\author{
T. Iwakura, A. Sakurai, S. Sawamura
}

Department Of Orthopaedic Surgery, Awaji Medical Center, Sumoto/ JAPAN

Introduction: It has been suggested that posterior tilt of the femoral head may increase the risk of fixation failure in femoral neck fractures. Hansson Pinloc system (Pinloc, Swemac) can provide rotational stability and posterior support by locking 3 Hansson Pins and a plate into one dynamic unit, and allow the fracture to heal in its reduced position and reduce the occurrence of fracture healing complications. The purpose of this study is to evaluate the short term outcomes of femoral neck fractures treated with Pinloc.

Material and methods: Sixty-six patients were treated by internal fixation with Pinloc between 2014 and 2016. Forty-six patients followed up more than 6 months were retrospectively investigated. Radiographical and clinical outcomes were analyzed.

Results: Mean age was 80 years and $78 \%$ of the patients were women. There were 40 undisplaced (Garden's classification stage I or II) and 6 displaced fractures (stage III or IV). All fractures were united within 6 months after operation. Radiographic evaluation revealed that anterior-posterior and lateral Garden's alignment index (GAI) were 165.8 and 168.3 degrees pre-operatively, 163.5 and 179.2 degrees post-operatively and 163.0 and 179.3 degrees 6 months after operation. The mean amount of telescoping was $3.2 \mathrm{~mm}$. Lateral GAI was significantly improved post-operatively and was maintained until fracture union. Walking ability was maintained in 35 patients $(76 \%)$ at a final follow-up (average 14.5 months after operation). One patient underwent total hip arthroplasty due to late segmental collapse at 18 months after operation.

Conclusion: Hansson Pinloc system could allow the fracture to heal in its reduced position.

References: Garden RS, J Bone Joint Surg Br, 1961

Disclosure: No significant relationships.

\section{PR328}

\section{CLINICAL RESULTS OF FEMORAL TROCHANTERIC FRACTURES TREATED WITH PFNA-II -RELATION TO 3D- CT CLASSIFICATION}

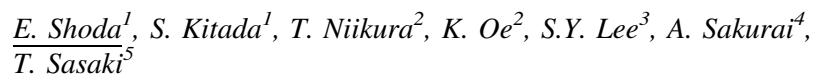

${ }^{1}$ Orthopaedic Surgery, Hyogo Prefectural Nishinomiya Hospital, Nishinomiya/JAPAN, ${ }^{2}$ Department Of Orthopaedic Surgery, Kobe University Graduate School of Medicine, Kobe/JAPAN, ${ }^{3}$ Department 
Of Orthopaedic Surgery, Showa University School of Medicine, Tokyo/JAPAN, ${ }^{4}$ Department Of Orthopaedic Surgery, Awaji Medical Center, Sumoto/JAPAN, ${ }^{5}$ Orthopaedic Surgery, Nishinomiya Watanabe Hospital, Nishinomiya/JAPAN

Introduction: In this study, femoral trochanteric fractures were classified with 3D-CT and the relationship between clinical results treated with PFNA-II and this 3D-CT classification was investigated. Material and methods: 140 cases over 65 years treated with PFNAII were investigated. Fracture was classified to 2part, 3part (5 subgroups), and 4part according to our 3D-CT classification. 5 subgroups of 3 part fracture were $\mathrm{G}(\mathrm{S})$ : small fragment of greater trochanter, $\mathrm{G}$ (B):big fragment of greater trochanter G-L: large fragment of greater trochanter including lesser trochanter, G (W) : whole fragment of greater trochanter, and L: lesser trochanter is the third fragment. Excessive sliding of blade, status of bone union and complications were evaluated in each group.

Results: 62 cases were united at 3 months follow up and other 48 cases have been united until 6 months. However, 30 cases were not united at 6 months. 13 cases were 3part G-L (48 cases), 4 cases were 3 part $\mathrm{G}(\mathrm{W})$ (10 cases) and 4 cases were 4 part ( 7 cases). Excessive sliding over $10 \mathrm{~mm}$ were seen in 11 cases; 2 cases were 2 part, 1 case was 3 part G (B), 4 cases were 3 part G-L, 3 case was 3 part L, and 1 case was 4 part. Cut out occurred in 2 cases and re-fracture at tip of the nail occurred in 1 case.

Conclusion: In this study, there were more non-united cases at 6 months follow up and excessive sliding over $10 \mathrm{~mm}$ cases in 3part $\mathrm{G}-\mathrm{L}, \mathrm{G}(\mathrm{W}), \mathrm{L}$ and 4 part. It is concluded that careful treatment is necessary in these types.

References: 1) Proposal of new classification of femoral trochanteric fracture by three-dimensional computed tomography and relationship to usual plain X-ray classification. Etsuo Shoda, Shimpei Kitada, Yu Sasaki et al. Journal of Orthopaedic Surgery, 25 (1) 1-5, 2017.

Disclosure: No significant relationships.

\section{PR329}

\section{OSTEOSYNTHETIC SURGERY OF PROXIMAL FEMORAL FRACTURES IN PATIENTS ON ANTIPLATELET THERAPY}

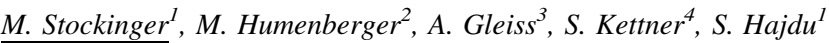

${ }^{1}$ Department Of Trauma Surgery, Medical University of Vienna, Vienna/AUSTRIA, ${ }^{2}$ Trauma Surgery, Medical University of Vienna, Vienna/AUSTRIA, ${ }^{3}$ Institute Of Clinical Biometrics, Medical University of Vienna, Vienna/AUSTRIA, ${ }^{4}$ Department Of Anaesthesia, Medical University of Vienna, Vienna/AUSTRIA

Introduction: Proximal femoral fractures represent one of the most common injuries among the elderly population. Affected patients are often multimorbid and require antiplatelet therapy (APT). Proximal femoral fractures are considered as semi-urgent-surgeries and surgery should be performed within 48 hours after the fracture occurs. For these reasons, early surgery of proximal femoral fractures along with continued APT should be considered. Due to a lack of prospective data, we performed a retrospective analysis of patients on APT who underwent early surgery by osteosynthesis for proximal femoral fractures.

Material and methods: This retrospective cohort study includes data from patients who suffered proximal femoral fractures and were treated by osteosynthesis between January 2013 and December 2015 at the Department of Trauma Surgery of the Medical University of Vienna.
Results: We included 401 patients with a mean age of 80.4 years. $77.8 \%$ of patients were operated within 24 hours after trauma, $22.2 \%$ had a longer time-to-surgery. Patients with ongoing APT exhibited a significantly shorter time to surgery compared to patients with interrupted APT. Patients with ongoing APT had no increased total blood loss compared to patients whose APT was interrupted. Surgical treatment within 24 hours resulted in significantly fewer complications, compared to later interventions. The total length of hospital stay was significantly shorter in patients with ongoing APT than in patients with interrupted APT.

Conclusion: Early osteosynthetic surgery of proximal femoral fractures in patients with ongoing APT is possible without increased blood loss and leads to a shorter time to surgery, a shorter hospitalization length and less common systemic complications.

References:

Disclosure: No significant relationships.

\section{PR330}

\section{COMPARISON OF OPERATION TIME OF SINGLE DOOR LAMINOPLASTY USING HYDROXYAPATITE BLOCK SPACER AND TITANIUM PLATE SPACER: A RETROSPECTIVE STUDY}

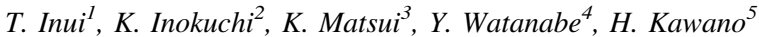

${ }^{1}$ Department Of Orthopaedic Surgery, Teikyo University Postgraduate School of Medicine, Tokyo/JAPAN, ${ }^{2}$ Emergency Medical Center, Saitama medical center of saitama medical university, Saitama/JAPAN, ${ }^{3}$ Department Of Orthopedic Surgery/ Trauma And Reconstruction Center, Teikyo University School of Medicine, Tokyo/JAPAN, ${ }^{4}$ Department Of Orthopaedic Surgery, Teikyo University School of Medicine, Tokyo/JAPAN, ${ }^{5}$ Orthopaedic Surgery, Teikyo University School of Medicine, Tokyo/JAPAN

Introduction: The efficacy of early surgical decompression of traumatic central cord syndrome has been reported. Hydroxyapatite block (HA) and titanium plate (TP) have been used as a spacer of open door laminoplasty, similar clinical outcome has been reported. The surgical procedure using TP is easier than HA, so that it may shorten the operation time. However, no report focused this point. The purpose of this study is to investigate whether the operation time differs depending on the type of spacer.

Material and methods: From January 2009 to April 2017, among patients, older than 60 years old, diagnosed with traumatic central cord injury classified as American Spinal cord Injury Association impairment scale $\mathrm{D}$, the patients received open door laminoplasty within 1 week from injury were included. The patients were divided into HA and TP group depending on type of spacer. The operation time was compared by t-test and multiple regression analysis adjusted by patients' baseline characteristics and covariates considered to have strong relationship with operation time. Incidence of complication requiring surgical procedure for each group was compared by chisquare test.

Results: HA group had 74 patients and TP group had 27 patients. The mean operation time was 159 (SD5.1) minutes and 123 minutes (SD6.1), respectively. The operation time using TP was significantly shorter than that using HA $(\mathrm{p}=0.001)$. Multiple regression analysis provided same result (regression coefficient: $-34.1, p=0.001$ ). Significant difference of the incidence of complication was not founded. Conclusion: Application of titanium plate spacer for open door laminoplasty would shorten the operation time without increasing complications. 
References: 1. Humadi A, Chao T, Dawood S, et al. A Meta-Analysis of Cervical Laminoplasty Techniques: Are Mini-Plates Superior? Global Spine Journal 2017;7:373-381

Disclosure: No significant relationships.

PR331

\section{ATYPICAL FEMORAL FRACTURES IN METASTATIC BONE TUMOR PATIENTS TREATED WITH ZOLEDRONIC ACID AND DENOSUMAB}

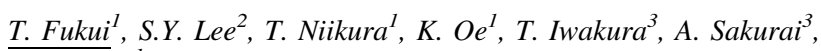
R. Kuroda ${ }^{I}$

${ }^{1}$ Department Of Orthopaedic Surgery, Kobe University Graduate School of Medicine, Kobe/JAPAN, ${ }^{2}$ Department Of Orthopaedic Surgery, Showa University, Tokyo/JAPAN, ${ }^{3}$ Department Of Orthopaedic Surgery, Awaji Medical Center, Sumoto/JAPAN

Introduction: Zoledronic acid and denosumab, those have an effect to suppress bone resorption, are widely used for treatment of skeletalrelated events in patients with bone metastases from solid tumors. However, atypical femoral fractures (AFF) which are supposed to be related to these drugs have been reported in recent years. In this study, we investigated the cases of AFF occurred in malignant tumor patients whom these drugs had been administered to.

Material and methods: Eight AFFs in 4 patients who were performed surgical treatment in our hospital were studied about age, gender, primary lesion, administration drug and administration period, presence of prodromal symptoms, fracture type, treatment, and bone union.

Results: One male and 3 females were studied and the mean age at the time of surgeries was 61.9 years old. Primary lesions were prostate cancer in the male patient and breast cancers in all female patients. Only denosumab was used in 2 patients and denosumab was used following zoledronic acid in 2 other patients. The mean drug administration period before surgeries was 53.5 months. Six fractures in 3 patients had prodromal symptoms before fractures. In all cases, osteosyntheses using intramedullary nails were performed. The operations were performed after the complete fractures in 5 limbs of 3 patients, and the period until fracture healing of the 2 limbs where bony fusion was obtained was 16 months and 29 months. In 3 limbs of 2 patients, surgeries were performed prophylactically before complete fractures.

Conclusion: In this study, all cases had bilateral AFF, and many cases showed prodromal symptoms. It is anticipated that the number of patients who are treated with these drugs will become greater in the future due to an increase in cancer patients. It is also conceivable that the occurrence of AFF in cancer patients may also increase. Moreover, in cancer patients, it is sometimes difficult to stop these drugs to maintain ADL, so care must be taken on the occurrence of the opposite side.

References:

Disclosure: No significant relationships.
PR332

\section{ANALYSIS OF HOSPITAL READMISSION AFTER OPERATIVE TREATMENT OF OSTEOPOROTIC FRACTURES OF THE PROXIMAL FEMUR}

\author{
C. Kopač, M. Tomazevic, G. Makovec, M. Cimerman
}

Traumatology, University Medical Centre Ljubljana, Ljubljana/ SLOVENIA

Introduction: Fracture of the proximal femur is one of the most serious complications of severe osteoporosis (1). The burden of proximal femur fractures is rising (2). At our department approximately one thousand fractures of proximal femur per year are treated operatively.

Material and methods: Retrograde analysis of the patients readmitted after operative treatment due to fracture of the proximal femur was done. During two year period 61 patients that were operatively treated for proximal femur fracture that resulted from a low energy fall were readmitted to our department. Analysis of the readmission cause was done.

Results: 17 (28\%) patients were readmitted due to surgical wound complications, $16(26 \%)$ patients were readmitted due to another low energy fracture, $13(21 \%)$ patients were readmitted due pneumonia or cystitis, $5(8 \%)$ were due to osteosynthesis break down, $4(7 \%)$ due to pain in previously conservatively treated proximal femur fracture, 3 (5\%) due to pain at the operated area and $3(5 \%)$ due to hip prosthesis dislocation. From the patients that were readmitted due to another fracture only one had written instructions to start the treatment of the osteoporosis, and from all the patients analyzed only 16 had written instruction for prevention and management of osteoporosis in their discharge paper.

Conclusion: In our analysis we found that another fracture is the second reason for rehospitalisation. Simple measures such as informing patients about osteoporosis and taking medication for prevention of osteoporosis could prevent multiple hospitalizations and expensive treatment of complications of osteoporotic fractures.

References: 1. Cummings SR, Melton LJ. Epidemiology and outcomes of osteoporotic fractures. Lancet (2002) 359:1761-1767. 2. Bleibler F., Konnopka A., Benzinger P. et al. Osteoporosis Int. (2013) 24: 835

Disclosure: No significant relationships.

\section{PR333}

\section{FEMORAL FRACTURES.: OUTCOMES OF OLDEST OLD}

\section{E. Grosso}

Ortopedia E Traumatologia, città della salute, torino/ITALY

Introduction: Hip fractures are serious injuries that often result in long term functional impairment and increase mortality. As our population ages, the number of hip fractures is likely to increase. Aim of our study was to analyze the treatment and the outcomes of the patient with hip fracture admitted to our hospital in oldest old patients.

Material and methods: We reviewed patients treated between february 2016 to february 2017 for proximal femur fractures and age over 85. Our case record included neck fractures, intertrochanterics, inter-subtrochanterics and subtrochanterics. We evaluated mortality, 
failure of the implant and functional recovery at a 6 months followup.

Results: Comorbidity is an important prognostic index for outcome. Age is a secondary factor

Conclusion: elderly patients with femoral fracture should be studied, and evaluated prior to surgical intervention.

References: One-year health and care costs after hip fracture for home-dwelling elderly patients in Norway: Results from the Trondheim Hip Fracture Trial. Hektoen LF, Saltvedt I, Sletvold O, Helbostad JL, Lurås H, Halsteinli V. Scand J Public Health. 2016 Dec;44 (8):791-798. doi: 10.1177/1403494816674162. Epub 2016 Oct 23. Predictors of noninstitutionalized survival 1 year after hip fracture: A prospective observational study to develop the Marburg Rehabilitation Tool for Hip fractures (MaRTHi). Buecking B, Eschbach D, Knobe M, Oberkircher L, Balzer-Geldsetzer M, Dodel R, Sielski R, Doering B, Ruchholtz S, Bliemel C. Medicine (Baltimore). $2017 \quad$ Sep;96 (37):e7820. doi: 10.1097/ MD.0000000000007820. Influence of proximal femur fractures in the autonomy and mortality of elderly patients submitted to osteosynthesis with cephalomedullary nail. Petros RSB, Ferreira PEV, Petros RSB. Rev Bras Ortop. 2017 Aug 26;52 (Suppl 1):57-62. doi: 10.1016/ j.rboe.2017.08.014. eCollection 2017. Predictors of noninstitutionalized survival 1 year after hip fracture: A prospective observational study to develop the Marburg Rehabilitation Tool for Hip fractures (MaRTHi). Buecking B, Eschbach D, Knobe M, Oberkircher L, Balzer-Geldsetzer M, Dodel R, Sielski R, Doering B, Ruchholtz S, Bliemel C. Medicine (Baltimore). 2017 Sep;96 (37):e7820. doi: 10.1097/MD.0000000000007820

Disclosure: No significant relationships.

\section{PR334}

\section{PATHOLOGIC FEMUR FRACTURES FOLLOWING LIMB- SALVAGE SURGERY AND RADIOTHERAPY FOR SOFT TISSUE SARCOMAS: THEY DON'T HEAL!}

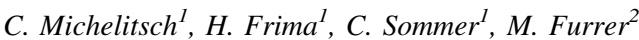

${ }^{1}$ Surgery, Trauma Unit, Kantonsspital Graubünden, Chur/ SWITZERLAND, ${ }^{2}$ Surgery, Thoracic/vascular Unit, Kantonsspital Graubünden, Chur/SWITZERLAND

Introduction: Combined limb-sparing surgery and radiation therapy are considered the standard of care for soft tissue sarcomas (STS) of the extremities. The correlation between radiation therapy and the risk of postradiation fracture is known but underestimated and can end up in serious long-term complications.

Material and methods: We reviewed the records of 3 patients with pathological femur fracture years after wide local excision of a STS of the proximal lower extremity with postoperative radiation therapy. All patients received more than 50 gray to the entire femur circumference; no one received perioperative chemotherapy, and during surgery, all patients had bone exposure, whereas only one patient had the periosteum stripped.

Results: The median time from radiation to fracture was 116 months (84 to 156 months). The median age at the time of diagnosis was 66 years (54 to 79 years). All fractures occurred within the radiation treatment field. Two fractures occurred after minimal trauma, one fracture occurred after a mountainbike fall. All three fractures developed a non-union. One patient died due to uncontrolled pulmonary metastasis and local recurrent disease. In the second case we had to perform an exarticulation at hip level due to uncontrolled infected non-union with soft tissue defect. The third patient is still under treatment of his non-union.

Conclusion: Local control rates after combined therapy for the treatment of soft-tissue sarcomas are high. However pathologic fractures after radiation therapy pose an extreme challenge in their treatment and may be associated with long-term complications that can cause physical disability and impairment of the quality of life.

References:

Disclosure: No significant relationships.

\section{PR335}

\section{THE IMPORTANCE OF A COMBINED CT OF HEAD AND CERVICAL SPINE IN ELDERLY PATIENTS AFTER LOW ENERGY TRAUMA TO THE HEAD}

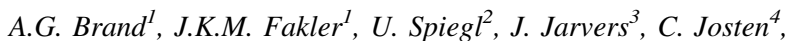 \\ C. Heyde ${ }^{2}$, T. Kahn ${ }^{5}$, P. Stumpp
}

${ }^{1}$ Orthopaedic, Trauma And Plastic Surgery, University Clinic Leipzig, Leipzig/GERMANY, ${ }^{2}$ Department Of Orthopeadics, Trauma Surgery, And Plastic Surgery, University of Leipzig, Leipzig/ GERMANY, ${ }^{3}$ Clinics Of Orthopeadics, Trauma Surgery, And Plastic Surgery, University of Leipzig, Leipzig/GERMANY, ${ }^{4}$ Department Of Orthopedics, Trauma And Plastic Surgery, University of Leipzig, Leipzig/GERMANY, ${ }^{5}$ Department Of Radiology, University of Leipzig, Leipzig/GERMANY

Introduction: In the recent past there was a rise in combined CT Scans oft he head and cervical spine in elderly patients after a low energy trauma to the head while under anticoagulating or platelet aggregation inhibiting medication. The aim of this study was to evaluate the incidence of cervical spine fractures in order see when a combined CT scan (head and cervical spine) is indicated.

Material and methods: Evaluation of prospectively collected data of 99 patients submitted to the emergency room of the University Clinic of Leipzig without previous consultation in other clinics between June and September of 2016 after low energy trauma to the head with a combined CT scan oft he head and cervical spine. We evaluated patients age, sex, antikoagulating/platelet aggregation inhibiting medication or dementia, a painful examination of the cervical spine and the detection of traumatic injuries the the head or spine.

Results: In 9 of 99 patients there was intracranial bleeding. 26,3\% (n $=26$ ) of patients either complained about pain of the cervical spine upon physical examination or the examination was not conclusive. In $4 \%(\mathrm{n}=4)$ patients we could find fractures of the cervical spine during CT scan.

Conclusion: The incidence of injuries to the cervical spine after low energy trauma to the head in elderly patients was a high as expected by the reference in literature. Because the clinical presentation of cognitiv impaired and/or patients under anticoagulating or platelet aggregation inhibiting medication is often inconclusive, combined CT scans of the head and cervical spine should be indicated more often. References: DGNC, S2 Leitlinie Schädelhirntrauma im Erwachsenenalter, 2015 Scherer et al., Intensivmedizin, 2014 DGNC, S2 Leitlinie Schädelhirntrauma im Erwachsenenalter, 2015 Wiedenhöfer et al., Kompendium der Orthopädischen Bildgebung, 2017 Hoffman et al., New England Journal of Medicine 343.2 (2000): 94-99 Stiell et al., JAMA. 2001;286 (15):1841-1848 Rybicki et al., American Journal of Roentgenology, 2002; Hikino et al., journal of trauma and acute care surgery, 2015

Disclosure: No significant relationships. 


\section{PR335A}

\section{NOT SOLELY CLASSIFICATION INFLUENCES DECISION- MAKING ON TREATMENT OF PELVIC RING FRACTURES IN THE ELDERLY. A REGISTRY STUDY}

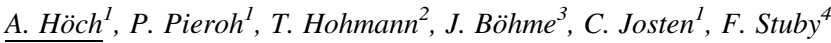

${ }^{1}$ Department Of Orthopedics, Trauma And Plastic Surgery, University of Leipzig, Leipzig/GERMANY, ${ }^{2}$ Department Of Anatomy And Cell Biology, Martin Luther University Halle-Wittenberg, Halle (Saale)/ GERMANY, ${ }^{3}$ Department Of Trauma And Septic Surgery, St. Georg Hospital, Leipzig/GERMANY, ${ }^{4}$ Department Of Traumatology And Reconstructive Surgery, BG Hospital Tübingen, Tübingen/ GERMANY

Introduction: Fragility fractures of the pelvis become more relevant in reasoned years. Beside the AO classification, new classifications with recommendations for treatment were published ${ }^{1}$. Nevertheless, there is no evidence for treatment strategies at the moment. Our hypothesis was that not solely fracture classifications leads to treatment decision in elderly patients.

Material and methods: Prospective collected registry data from 1813 patients aged over 65 years with pelvic ring fracture was evaluated. Excluded were patients with acetabular fractures and type $\mathrm{A} 1$ and $\mathrm{A} 3$ fractures not involving the pelvic ring. Patients were divided into 6 age groups. Besides baseline data, injury pattern, ISS, fracture classification according Tile/AO, LOS and mortality was evaluated. Correlations with treatment were calculated.

Results: Of 1814 patients in a mean age of $80.7 \pm 7.6$ years the majority was female $(79.0 \%)$. Mostly isolated pelvic ring fractures $(70.1 \%)$ occurred and only $8.2 \%$ were multiple injured (ISS $>16$ ). Most common were type A2 (35.4\%) and type B2 (38.0\%) followed by type $\mathrm{C} 1(7.3 \%)$. All other pelvic ring fracture types were represented less than 5\%. Especially pelvic ring fractures of type A2 (96.9\%) and type B2 $(83.0 \%)$ were treated non-operatively, overall $76.9 \%$. Mean LOS was 13 days and in hospital mortality rate was $3.8 \%$. Fracture classification did not differ in age groups. Nevertheless, the older patients were the lower surgery rate was.

Conclusion: Surgical treatment was carried out less than recommended by literature ${ }^{1}$. Data shows that other reasons, especially age influences decision-making on treatment. Existing fracture classifications alone might not be sufficient for treatment recommendation. References: ${ }^{1}$ Rommens, Wagner, Hofmann. Fragility Fractures of the Pelvis. (2017) JBJS Reviews

Disclosure: No significant relationships.

\section{PR336}

\section{SHOULD ASYMPTOMATIC ORTHOPAEDIC IMPLANT BE REMOVED AFTER FRACTURE HEALING?}

\section{S. Ko, S. Chae, W. Choi}

Orthopaedic Surgery, Daegu Catholic University Medical Center, Daegu/KOREA, REPUBLIC OF

Introduction: No universal agreement or clear guidelines exist with regards to routine removal of orthopaedic implant in asymptomatic patients after fracture healing. The aim of this study was to compare the functional outcomes of each patient prior to removal of implant with that of at least one year after the removal.
Material and methods: 87 adult patients underwent implant removal surgery between March 2004 to December 2011 at our hospital meeting the inclusion and exclusion criteria and were studied prospectively. The mean age of the patients at the time of implant removal surgery was $41.5 \pm 16.7$ years old (21-75 years old). There were 55 men and 32 women. The mean follow-up period was $27.0 \pm$ 17.7 months (12-64 months). All patients completed the Short Form36 (SF-36) questionnaire.

Results: The Physical Functioning (PF) and Role-Physical (RP) had statistically significant improvement from pre-implant removal to postoperatively $(\mathrm{p}<0.05)$. The overall Physical Component Score (PCS) had statistically significant improvement from pre-removal to postoperatively $(\mathrm{p}=0.001)$. The overall Mental Component Score (MCS) had no statistically significant difference between pre-removal and postoperatively.

Conclusion: Patients with asymptomatic healed fractures benefit in terms of physical function by having their implant removed by one year postoperatively.

References: 1. Jamil W, Allami M, Choudhury MZ, et al. Do orthopaedic surgeons need a policy on the removal of metalwork? A descriptive national survey of practicing surgeons in the United Kingdom. Injury 2008;39:362-7. 2. Richards RH, palmer JD, Clarke NM. Observations on removal of metal implants. Injury 1992;23:258. 3. Busam ML, Esther RJ, Obremsky WT. Hardware removal: indications and expectations. J Am Acad Orthop Surg 2006;14:11320. 4. Hanson B, van der Werken C, Stengel D. Surgeons' beliefs and perceptions about removal of orthopaedic implants. BMC Musculoskelet Disord 2008;9:73. 5. Sanderson PL, Ryan W, Turner PG. Complications of metalwork removal. Injury 1992;23:29-30. 6. Bostman OM. Refracture after removal of condylar plate from the distal third of the femur. J Bone Joint Surg Am 1990;72:1013-8. 7. Deluca PA, Lindsey RW, Ruwe PA. Refracture of bones of the forearm after removal of compression plates. J Bone Joint Surg Am 1988;70:1372-6. 8. Hidaka S, Gustilo RB. Refracture of the forearm bones after plate removal. J Bone Joint Surg Am 1984;66:1241-3. 9. Langkamer VG, Ackroyd CE. Removal of forearm plates. A review of the complications. J Bone Joint Surg Br 1990;72:601-4. 10. Mih $\mathrm{AD}$, Cooney WP, Idler RS, Lewallen DG. Long term follow up of forearm plate diaphyseal plating. Clin Orthop 1994;299:256-8. 11. Rosson JW, Shearer JR. Refracture after removal of plates from the forearm. An avoidable complication. J Bone Joint Surg $\mathrm{Br}$ 1991;73:415-7. 12. Böstman OM, Pihlajamaki H. Routine implant removal after fracture surgery: a potentially reduce consumer of hospital resources in trauma units. J Trauma 1996;41:846-9. 13. Raman R, Roberts CS, Pape HC, et al. Implant retention and removal after internal fixation of the symphysis pubis. Injury 2005;36:827-31. 14. Müller ME, Allgöwer M, Schneider R, et al. Manual of Internal fixation. Techniques recommended by the AO group. New York, NY: Springer;1979. 15. MInkowitz RB, Bhadsavle S, Walsh M, et al. Removal of painful orthopaedic implants after fracture union. J Bone Joint Surg Am 2007;89:1906-12. 16. Beaupre GS, Csongradi JJ. Refracture risk after plate removal in the forearm. J Orthop Trauma 1996;10:87-92. 17. Davison BL. Refracture following plate removal in supracondylar-intercondylar femur fractures. Orthopedics 2003;26:157-9. 18. Gösling T, Hüfner T, Hankemeiser S, et al. Femoral nail removal should be restricted in asymptomatic patients. Clin Orthop Relat Res 2004;423:222-6. 19. Brown OL, Dirschl DR, Obermskey WT. Incidence of hardware-related pain and its effect on functional outcomes after open reduction and internal fixation of ankle fractures. J Orthop Trauma 2001;15:271-4. 20. Townend M, Parker P. Metalwork removal in potential army recruits. Evidencebased changes to entry criteria. J R Army Med Corps 2005;151:2-4. 21. Evers B, Habelt R, Gerngross H. Indication, timing and complications of plate removal after forearm fractures: results of metaanlysis including 635 cases. J Bone Joint Surg Br 2004;86:289. 22. Chla J, Soh CR, Wong HP, et al. Complications following metal removal: a 
follow-up of surgically treated forearm fractures. Singapore Med J 1996;37:268-9. 23. Jago RD, Hindley CJ. The removal of metalwork in children. Injury 1998;29: 439-41. 24. Rumball K, Finnegan M. Refractures after forearm plate removal. J Orthop Trauma 1990;4: 124-9. 25. Vos D, Hanson B, Verhofstad M. Implant removal of osteosynthesis: the Dutch practice. Results of a survey. J Trauma Manag Outcomes 2012;6:6-12. 26. Kahle WK. The case against routine metal removal. J Pediatr Orthop 1994;14:229-37. 27. Brown RM, Wheelwright EF, Chalmers J. Removal of metal implants after fracture surgery - indication and complications. J R Coll Surg Edinb 1993;38:96-100. 28. Kirchhoff C, Braunstein V, Kirchhoff S, et al. Outcome analysis following removal of locking plate fixation of the proximal humerus. BMC Musculoskelet Disord 2008;9:138.

Disclosure: No significant relationships.

\section{PR337}

\section{DO FIBULA NONUNIONS PREDICT LATER TIBIA NONUNIONS?}

\section{S. Ko, S. Chae, W. Choi}

Orthopaedic Surgery, Daegu Catholic University Medical Center, Daegu/KOREA, REPUBLIC OF

Introduction: In patients with a lower leg injury, a tibia shaft fracture is frequently combined with a fibula shaft fracture. Usually, only the tibia is fixed, leaving the fibula to heal on its own (except juxtaarticular fracture). Although many studies have focused on nonunion of the tibia, the fate of the fibula has been given limited attention. Rockwood et all showed that fibula shaft fractures undergo faster and better union than tibia shaft fractures, as the surrounding area has good muscle coverage and blood supply. This study was conducted to determine the incidence of fibula nonunion, to compare the union time of fibula shaft fracture and tibia shaft fracture and to exam whether the finding of a fibular nonunion could predict tibia nonunion.

Material and methods: The relevant ethics committee reviewed and approved the study protocol. A total of 1329 patients with tibiofibula shaft fractures were treated at our hospital from March 1998 to October 2008. Two hundred twenty-four patients (17\%) met inclusion criteria (Table 1). All patients had unilateral fractures. Mean age was 49 years. There were 166 males $(74.1 \%)$ and 58 females (25.9\%). One hundred fifty-three patients were treated with a intramedullary nail, 34 patients were treated with an external fixator, 32 patients were treated with a plate and screws, and 5 remaining patients were treated nonsurgically with a cast or brace (Table 2). There were 37 open fractures and 187 closed fractures. After injury, followup was performed at 1, 2, 3, 6, 9, 12, and 18 months and annually thereafter. Tibia and fibula union determinations were based on the proposal of Treitz et al2: disappearance of pain and tenderness in the tibia and fibula shaft fracture area, full-weight bearing without pseudomotion clinically, and mature callus radiologically with bridging trabeculation across the fracture line. Tibia or fibula nonunion determinations were based on failure to detect union after 3 consecutive monthly radiographs, continuing clinical findings of pain and pseudomotion, as well as sclerosis at the fracture radiologically. Two-sample $t$ tests and $\mathrm{x} 2$ tests were performed using SPSS 14.0, and P values, 0.05 were deemed significant.

Results: One hundred forty-eight patients $(66 \%)$ healed both their tibia and fibula fractures (Table 3). There were 172 patients $(77 \%)$ with a union of the tibia leaving 52 patients $(23 \%)$ with a tibial nonunion. One hundred sixty-nine patients $(75 \%)$ presented with a fibular union leaving 55 with a nonunion (25\%). Among the 148 patients with both tibia and fibula union, union time of the tibia was $33.22 \pm 8.64$ weeks (range: $16-48 \mathrm{wk}$ ) while that of the fibula was $11.79 \pm 2.45$ weeks (range: $8-16 \mathrm{wk}$ ). The time to union for the fibula was earlier than that of the tibia and was statistically significant $(\mathrm{P}=$ 0.002). Among the 169 fibula union patients, 148 patients (88\%) were in the tibia union group and 21 patients $(12 \%)$ were in the tibia nonunion group. Of the 55 fibula nonunion patients, 24 patients $(44 \%)$ were in the tibia union group and 31 patients $(56 \%)$ were in the tibia nonunion group. Fibula nonunion was significantly associated with tibia nonunion $(\mathrm{P}=0.002)$. Regarding the fixation method for tibia shaft fracture, the data is shown in Table 4. In the group that received an intramedullary nail, there are 37 cases of nonunion and 116 unions (a total of 153 cases). Those patients with an external fixation apparatus included 22 unions and 12 nonunion cases (a total of 34 cases). Plate and screws fixation patients included 28 union cases and 4 nonunions (a total of 32 cases). The union group included 3 cases and the nonunion group included 2 cases (a total of 5 cases) from the patients treated with a cast or brace. For these variables, the number of patients in each group was too small to assess statistical significance.

Conclusion: Union time of the fibular fracture was significantly faster than that of the tibia in our series $(\mathrm{P}=0.002)$. Therefore, in fractures of the tibia and fibula shafts, when a nonunion of the fibula is seen, consideration must be given to the fact that a tibial nonunion may develop.

References: 1. Rockwood GA Jr, Green DP, Bucholz RW, et al. Fracture in Adults. 4th ed. Vol 2. Philadelphia, PA: Lippincott-Raven Co.; 1996:2193.

2. Teitz CC, Carter DR, Frankel VH. Problems associated with tibial fractures with intact fibulae. J Bone Joint Surg Am. 1980;62:770-776.

3. Hsiao CW, Wu CC, Su CY, et al. Exchange nailing for aseptic tibial shaft nonunion: emphasis on the influence of a concomitant fibulotomy. Chang Gung Med J. 2006;29:283-290.

4. Sarmiento A, Gersten LM, Sobol PA, et al. Tibial shaft fractures treated with functional braces-experience with 780 fractures. J Bone Joint Surg Br. 1989;71:602-609.

5. Rosenthal RE, MacPhail JA, Oritz JE. Non-union in open tibial fractures. J Bone Joint Surg Am. 1977;59:244-248.

6. Nicoll EA. Fractures of the tibial shaft. A survey of 705 cases. J Bone Joint Surg Br. 1964;46:373-387.

7. Hoaglund FT, States JD. Factors influencing the rate of healing in tibial shaft fractures. Surg Gynecol Obstet. 1967;124:71-76.

8. Jackson RW, Macnab I. Fractures of the shaft of the tibia; a clinical and experimental study. Am J Surg. 1959;97:543-557.

9. Wade PA, Campbell RD Jr. Open versus closed methods in treating fractures of the leg. Am J Surg. 1958;95:599-616.

10. Weissman SL, Herold HZ, Engelberg M. Fractures of the middle twothirds of the tibial shaft. J Bone Joint Surg Am. 1966;48:257-267.

11. DeLee JC, Heckman JD, Lewis AG. Partial fibulectomy for ununited fractures of the tibia. J Bone Joint Surg Am. 1981;63:1390-1395.

12. Thomas KA, Bearden CM, Gallagher DJ, et al. Biomechanical analysis of nonreamed tibial intramedullary nailing after simulated transverse fracture and fibulectomy. Orthopedics. 1997;29:51-57.

Disclosure: No significant relationships. 
PR338

\section{FACTORS INFLUENCING FUNCTIONAL OUTCOMES OF SUBTALAR FUSION FOR POSTTRAUMATIC ARTHRITIS AFTER CALCANEAL FRACTURE}

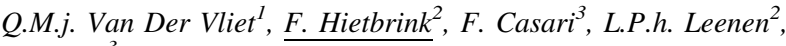 \\ M. Heng ${ }^{3}$
}

${ }^{1}$ Orthopaedic Trauma, Massachusetts General Hospital, Boston/MA/ NETHERLANDS, ${ }^{2}$ Surgery, University Medical Center Utrecht, Utrecht/NETHERLANDS, ${ }^{3}$ Orthopaedic Trauma, Massachusetts General Hospital, Boston/UNITED STATES OF AMERICA

Introduction: Subtalar arthrodesis is a common salvage operation for posttraumatic subtalar arthritis. This study aims to identify factors associated with functional outcomes and quality of life after subtalar fusion for posttraumatic subtalar arthritis after calcaneal fracture.

Material and methods: This is a retrospective study with prospective follow-up by questionnaire in two level 1 trauma centers. 159 patients who underwent subtalar arthrodesis for posttraumatic arthritis after a calcaneal fracture between 2001 and 2016 were identified and contacted for completion of a survey consisting of the Foot and Ankle Ability measure (FAAM), Maryland Foot Score (MFS), PROMIS Physical Function (PROMIS PF, Short Form 10a) questionnaire, EuroQol five-dimensional (EQ-5D) questionnaire, and Visual Analog Scale (EQ-VAS). Exclusion criteria were initial subtalar arthrodesis at an outside facility, primary arthrodesis for fracture, initial arthrodesis earlier than 2001, amputation of the fused foot or leg, $<18$ years at time of fusion, and inability to communicate in English.

Results: 84 patients completed the questionnaires. Median FAAM score was 79 (IQR 48 - 90); median MFS 74 (56 - 86); median PROMIS PF 45 (38 - 51). Quality of life (median EQ-5D 0.78, IQR $0.71-0.84)$ was significantly lower when compared to a reference population $(p<0.001)$. Smoking was independently associated with worse outcomes for all questionnaires. Complications after fusion, high-energy, and ipsilateral injury were predictors for poorer outcomes too.

Conclusion: Acceptable functional outcomes and quality of life were observed after subtalar fusion. Smoking, complications after subtalar fusion, high-energy trauma, and presence of ipsilateral injuries were independently associated with worse functionality and quality of life. References:

Disclosure: No significant relationships.

\section{PR339}

WEIGHT BEARING OR NON-WEIGHT BEARING AFTER SURGICAL TREATMENT OF ANKLE FRACTURES: THE WOW! STUDY. A MULTICENTER RANDOMIZED CONTROLLED TRIAL

D.P.J. Smeeing ${ }^{1}$, R.M. Houwert ${ }^{2}$, J.P. Briet ${ }^{3}$, R.H.h. Groenwold ${ }^{4}$, K. Lansink, L.P.h. Leenen ${ }^{2}$, P. Van Der Zwaal ${ }^{6}$, J.M. Hoogendoorn ${ }^{7}$, E. Verleisdonk ${ }^{8}$, M. Segers ${ }^{9}$, F. Hietbrink ${ }^{2}$

${ }^{1}$ Surgery, St Antonius Hospital, Nieuwegein/NETHERLANDS, ${ }^{2}$ Surgery, UMC Utrecht, Utrecht/NETHERLANDS, ${ }^{3}$ Surgery, Diakonessenhuis Utrecht, Utrecht/NETHERLANDS, ${ }^{4}$ Epidemiology, Julius Center for Health Sciences and Primary Care, Utrecht/ NETHERLANDS, ${ }^{5}$ Surgery, Elisabeth Tweesteden Ziekenhuis, Tilburg/NETHERLANDS, ${ }^{6}$ Orthopaedics, Medisch Centrum Haaglanden, Den Haag/NETHERLANDS, ${ }^{7}$ Surgery, Medisch
Centrum Haaglanden, Den Haag/NETHERLANDS, ${ }^{8}$ Surgery, Diakonessenhuis, Utrecht/NETHERLANDS, ${ }^{9}$ Trauma Surgery, St. Antonius Hospital, Nieuwegein/NETHERLANDS

Introduction: Postoperative care regimens of ankle fracture vary widely and it remains unclear which postoperative treatment is to be preferred. The aim of this multicenter, randomized controlled trial was to provide evidence for the optimal post-operative care regimen after surgical treatment. The null hypothesis was that ankle-specific disability is worse for unprotected weight-bearing and mobilization when compared to protected weight-bearing and unprotected nonweight-bearing.

Material and methods: A multicenter, randomized controlled trial was among patients with a supination exorotation type 2,3 or 4 ankle fracture classified by an expert panel. The primary endpoint of the study was the Olerud Molander Ankle Score (OMAS) which was assessed at twelve weeks after randomization. The secondary endpoints were the health related quality of life using the SF-36v2, time to the return to work and to sports, and the number of complications. Results: The trial was terminated on advice of the DSMB at interim analysis. A total of 115 patients, ranging from 18 to 65 years of age were included. Patients were randomized to unprotected non-weightbearing $(n=37)$, protected weight-bearing $(n=36)$, or unprotected weight-bearing $(n=42)$ treatment. The OMAS was significantly different in the unprotected weight-bearing group after 6 weeks $(p=$ 0.011) compared to the protected weight-bearing and unprotected weight-bearing groups. Unprotected weight-bearing showed a significant earlier return to work $(p=0.028)$ and earlier return to sports in weeks $(p=0.005)$, and no differences were shown in the quality of life scores or number of complications.

Conclusion: Unprotected weight-bearing and mobilization as tolerated shows a better functional outcome on the short-term and earlier return to work and sports.

References: -

Disclosure: No significant relationships.

\section{PR340}

\section{LONG-TERM FUNCTIONAL OUTCOME AND COMPLICATIONS IN 182 SURGICALLY MANAGED PROXIMAL AND MIDDLE PHALANX FRACTURES: A RETROSPECTIVE COHORT STUDY}

$\underline{\text { T. Kootstra }}^{l}$, M. Van Heijl ${ }^{2}$, J. Keizer ${ }^{1}$, R.M. Houwert ${ }^{3}$, P. Wittich ${ }^{1}$, E. Verleisdonk ${ }^{2}$, D. Van Der Velde ${ }^{1}$

${ }^{1}$ Trauma Surgery, St. Antonius Hospital, Nieuwegein/ NETHERLANDS, ${ }^{2}$ Surgery, Diakonessenhuis, Utrecht/ NETHERLANDS, ${ }^{3}$ Utrecht Traumacenter, University Medical Center Utrecht, Utrecht/NETHERLANDS

Introduction: We evaluated functional outcome and complications after operative treatment of proximal and middle phalanx fractures, thumb excluded. Additionally, we registered patient satisfaction.

Material and methods: Consecutive patients treated operatively in two level 2 trauma centers from January 2010 to December 2015 were retrospectively analyzed. One-hundred-and-fifty-nine proximal and 41 middle phalanx fractures could be identified using medical records. Operative fixation was indicated for fracture angulation of $>15$ degrees, shortening of the finger of $>2$ millimeters or clinical rotation. Fractures were reduced and fixed with either K-wires (Kirschner-wires), lag screws or plate fixation. Functional outcome was assessed via phone using the Disabilities of the Arm, Shoulder 
and Hand (DASH) Outcome Measure and the Patient Rated Wrist/ Hand Evaluation (PRWHE), complications were obtained from medical records. Patient satisfaction was also assessed via phone.

Results: Mean follow-up was 3.4 years (Standard Deviation (SD) 1.5; range 1-6) and was achieved for 143 proximal fractures (90\%) and 39 middle fractures $(95 \%)$. In total, $15 \%$ of patients suffered a complication. Mean DASH and PRWHE scores were 4.6 (SD 7.6; range $0-51$ ) and 7.7 (SD 13; range 0-82), respectively. No differences in functional outcomes were found between proximal and middle phalanx fractures and methods of fixation. One-hundred-and-thirty-eight patients $(76 \%)$ reported to be satisfied with their surgery.

Conclusion: In spite of excellent functional outcomes, almost a quarter of the patients (24\%) reported no satisfactory outcome. It is possible the DASH and PRWHE are not sensitive enough to measure fine motor disability. Further research should focus on developing an outcome measure specific to fine motor skill.

References:

Disclosure: No significant relationships.

\section{PR341}

\section{PREOPERATIVE NEGATIVE PRESSURE WOUND THERAPY TO TREAT BLISTERING RELATED TO ANKLE FRACTURES}

A. Barberia, C. Ojeda, M.C. Rodriguez, L. Lopez, M.C. Ortega, J. Vilá Y Rico, L.R. Ramos

Orthopaedic Surgery And Traumatology, Hospital Universitario 12 de Octubre, Madrid/SPAIN

Introduction: When associated to fractures, blisters can delay surgery and favor surgical wound complications, increasing hospital stay and treatment cost. Optimum treatment for fracture related blisters remains unclear. The aim of this study was to assess the effect of preoperative negative pressure wound therapy (NPWT) in this setting. Material and methods: Pilot phase for a prospective clinical trial, including 8 patients admitted between January and September 2017 with ankle fractures complicated by blisters assigned alternately to NPWT or conventional treatment. Information was collected on demographic variables, fracture type and surgical data, as well as blíster type, location and extension during each dressing change. Pain during dressing changes was assessed, and blisters were measured by digital analysis. Early surgical wound complications were registered Results: Both groups were comparable for the collected baseline variables. Blisters were predominantly serous and found on medial side. Although initially the median blister area was larger in the NPTW group (1.657 vs $370 \mathrm{~mm} 2$ ), the area decreased $20 \%$ in the NPTW group while it grew $66 \%$ in controls when performing the first dressing change, Patients treated with NPWT tended to have a shorter surgical delay (11 vs 13 days) and less pain during dressing changes (VAS score 1,4 vs 5,3). Minor wound complications were reported in 2 cases treated with NPWT, although they did not occur on skin affected by blisters.

Conclusion: This pilot phase study showed promising initial results. A larger sample size and cost-effectiveness studies are needed to establish recommendations

References:

Disclosure: No significant relationships.
PR342

\section{CLINICAL RESULTS OF CEDELL'S FRACTURES: SKELETAL TRAUMA 2}

\author{
K. Demiya, T. Noda, S. Yokoo, M. Kiyono, T. Ozaki
}

Orthopaedic Surgery, Department of Orthopaedic Surgery Okayama University, Okayama City/JAPAN

Introduction: Fracture of the posteromedial tubercle of the talus, also known as Cedell's fracture, was first described by Cedell (1) in 1974. It is rare and is an atypical injury that is commonly missed on plain radiographs. To avoid nonunion and chronic hindfoot pain, it is important that this injury be diagnosed early and be treated properly in its clinical course.

Material and methods: Five patients (two males and three females), ranging from 19 to 79 years (mean age of 57 years) were included. The mechanism of injury included 3 cases of falls and 2 cases of traffic accident. In our study, the initial diagnosis was made using plain radiography and $\mathrm{CT}$. We performed open reduction and internal fixation with PLLA or headless screw using a posteromedial approach.

Results: Mean follow-up time was 19.4 months (range from7 to 53 months). After 6 months of treatment, bone union was obtained in all cases and all these patients showed good functional results. These patients could walk and returned to preinjury physical activity.

Conclusion: We should consider the possibility of this fracture when we treat the high energy ankle injuries. Modified radiographs such as an oblique view, in which the foot is $45^{\circ}$ externally rotated, can be effective for the diagnosis of the fracture if a fracture is suspected and the use of CT can also be helpful in ascertaining the diagnosis, determining the size of the fracture fragment and degree of displacement. The posteromedial approach is a good option to make optimal anatomic reduction and rigid fixation.

References: (1) Rupture of the posterior talotibial ligament with the avulsin of a bone fragment from the talus. Cedell CA Acta Orthop Scand 1974

Disclosure: No significant relationships.

\section{PR343}

\section{TOTAL HIP ARTHROPLASTY AND BIPOLAR HEMIARTHROPLASTY FOR FEMORAL NECK FRACTURE: FUNCTIONAL OUTCOMES AND ASSOCIATED COMPLICATIONS}

\section{R. Sapage, M. Maio, P. Mota, C. Branco, R. Sousa, M. Coxo}

Serviço De Ortopedia E Traumatologia, Centro Hospitalar de Trás-osMontes e Alto Douro, Vila Real/PORTUGAL

Introduction: Femoral neck fractures are common injuries in elderly. There is controversy about the best surgical treatment concerning total hip arthroplasty (THA) versus bipolar hemiarthroplasty (BHA).We aimed to evaluate functional outcomes and associated complications of THA and BHA for patients with femoral neck fractures.

Material and methods: A retrospective study was conducted for patients admitted for femoral neck fracture, between January2015April2016.Patients > 70years, with previous autonomous walking ability and submitted to surgery in the first 48 hours were included. 
Mean follow-up time was 12 months.A total of 80 patients were included. Statistical analysis was performed in SPSSv.23, p $<0.05$ was considered of statistical significance.

Results: Thirty-two patients were submitted to THA and 48 to BHA. Mean age, median ASA classification and previous walking ability without external support were slightly different (77vs.86years, p < $0.001 ; 2.4 v s .2 .7, \mathrm{p}=0.029 ; 78.1 \%$ vs.50.0\%, $\mathrm{p}=0.018$ in THAvs.BHA).Median time to surgery was 15.4 hours in both groups $(\mathrm{p}=0.647)$. Operative time was significantly superior in THA (79.7vs.49.9minutes, $\mathrm{p}<0.001$ ). Mean hemoglobin difference was higher in THA $(2.8 \mathrm{vs} .2 .1 \mathrm{~g} / \mathrm{dL}, \mathrm{p}=0.019)$. There was no statistical difference in length of stay (11.6vs.9.2days, $\mathrm{p}=0.771)$ in THA and BHA. Postoperative pain $(2.2 \mathrm{vs} .3 .5, \mathrm{p}=0.030)$ and functional outcome (31.3vs.25.7, $\mathrm{p}=0.019)$ was significantly better in THA. There was no statistical difference concerning surgical reintervention (9.7\%vs.15.9\%, p = 0.434), dislocation (12.9\%vs.13.6\%, $\mathrm{p}=0.927)$, infection (3.2\%vs.6.8\%, $\mathrm{p}=0.495)$, periprosthetic fracture $(0.0 \%$ vs. $4.5 \%, \mathrm{p}=0.229)$ and mortality $(18.8 \%$ vs. $35.4 \%, \mathrm{p}=0.107)$ in THA and BHA.

Conclusion: THA may lead to better functional outcomes and less postoperative pain compared to BHA for femoral neck fractures. We must, however, consider the bias in this study related to patients' previous health state. Longer surgical time and aggression is not negligible either. Although it is suggested in published evidence, we found no difference regarding reoperation and dislocation rates as well as mortality.

References:

Disclosure: No significant relationships.

\section{PR344}

\section{EVALUATION OF IMPLANTS WITH DYNAMISATION OPTION AND TIMING OF DYNAMISATION ACTIVATION FOR FEMORAL SHAFT FRACTURES TREATMENT}

M.B. Mitkovic $^{1}$, S. Milenkovic ${ }^{1}$, I. Micic ${ }^{1}$, M.M. Mitkovic ${ }^{1}$, I. Kostic ${ }^{2}$

${ }^{1}$ Medical Faculty, University of Nis, Nis/SERBIA, ${ }^{2}$ Clinic For Orthopaedics And Traumatology, Clinical Center Nis, Nis/SERBIA

Introduction: Axial dynamization is recognized as method which encourages bone healing. We have no too many internal fixation devices with dynamization option. It is known that dynamization is happened in about $20-25 \%$, according to the literature.

Material and methods: In this work we analysed results of clinical application of two devices: Intramedulary (IM) nail (n15) and Selfdynamisable internal fixator (SIF) (n15). We analyzed complication rate and timing for dynamization activation.

Results: The average operative time was similar in both groops $44-50$ minutes, average fluoroscopy time for IM nail was $1 \mathrm{~min} 44 \mathrm{sec}$ and for SIF 18 SEC. while average blood loss was similar 60-90 milliliters in each group. None of the patients developed complications during the intraoperative period. Healing time also similar 3.9-4.5 months. Healing was achieved in each group. The locking screw breaking occurred 6-18 weeks in one case in each group. Spontaneous axial dynamization was observed in 2 cases in SIF group 6-10 weeks after the operation ( 2 to 4 millimeters).

Conclusion: IM nail and SIF provide possibilities of axial dynamization after internal fixation of femoral shaft fractures. If used IM nail locking screw should be removed at about 2 months postoperatively. If use SIF, no need for additional small surgery for screw removal if antirotation screw placed on proper place as dynamization is activated spontaneously if healing delayed.
References: Mitkovic MB, Milenkovic S, Micic I, Mladenovic D, Mitkovic MM. Results of the femur fractures treated with the new selfdynamisable internal fixator (SIF). Eur J Trauma Emerg Surg. 2012 Apr;38 (2):191-200. Micic I. D., Mitkovic M. B., Park I. H., Mladenovic D. B., Stojiljkovic P. M., Golubovic Z. B., Jeon I. H. Treatment of Subtrochanteric Femoral Fractures Using Selfdynamisable Internal Fixator. Clinics in Orthopedic Surgery 2010 Dec;2 (4)227-31.

Disclosure: At this moment author has licence agreement with producer of selfdynamisable internal fixator

\section{PR345}

\section{THE ASSESSMENT OF PREVALENCE AND CONTRIBUTING FACTORS OF DIFFICULT PERIPHERAL VENOUS ACCESS PERFORMED BY PREHOSPITAL STUFF IN SINA AND SHARIATI HOSPITALS IN 2015-2016}

\section{S. Bijani, P. Payandemehr, E. Karimi, H. Kermanpour}

\section{Emergency Room, Sina hospital, Tehran/IRAN}

Introduction: Peripheral venous access is frequently required in the hospital and prehospital environments. This can sometimes be difficult to obtain, a condition which is terms difficult vascular access (DVA). DVA may lead to untoward consequences, which are occasionally the result of using more invasive alternative methods. So, many studies have been trying to define predictive variables of DVA, but the available data are still inconclusive. In the present study, we aimed to define the rate of DVA in prehospital condition and examine its possible contributors.

Material and methods: In this cross-sectional study a total of 600 prehospital peripheral venous accesses performed in Sina and Shariati Hospitals in 2015-2016 were examined to determine the rate and contributing factors of DVA. DVA was defined as at least two peripheral venous access failures. Probable contributing factors included patient related, operator-related and environment-related variables.

Results: DVA was documented in 125 cases out of the 600 examined patients $(20.83 \%)$. Among the patient-related variables age, heart rate, respiratory rate, systolic and diastolic blood pressure, $\mathrm{O} 2$ saturation, sex, patients' cloth, level of cooperation, severity of the condition, previous venous access at the target point and previous cardiovascular/respiratory disease and trauma were significantly associated with DVA risk. Among operator-dependent variables educational level and last meal time, and among environment-related variables light, time, location and the surrounding insanitation were significant contributors of DVA. The independent variables, however, were higher respiratory rate, low diastolic blood pressure, previous intravenous access in the target limb, last operator meal time and environmental insanity.

Conclusion: Prehospital DVA is a rather common condition in our healthcaresystem. Presence of certain DVA contributors may necessitate higher care and more experienced operators. Keywords: difficult vascular access, risk factor, prehospital

References: 1-Horattas MC, Trupiano J, Hopkins S, Pasini D, Martino C, Murty A. Changing concepts in long-term central venous access: catheter selection and cost savings. American journal of infection control. 2001;29 (1):32-40. 2-Ialongo C, Bernardini S. Phlebotomy, a bridge between laboratory and patient. Biochemia Medica. 2016:17-33. 3-Lamperti M, Pittiruti M. II. Difficult peripheral veins: turn on the lights. British journal of anaesthesia. 2013;110 (6):888-91. 4-Cheung E, Baerlocher MO, Asch M, Myers A. Venous 
access: a practical review for 2009. Canadian family physician Medecin de famille canadien. 2009;55 (5):494-6. 5-Smith B. Peripheral intravenous catheter dwell times: a comparison of 3 securement methods for implementation of a 96-hour scheduled change protocol. Journal of infusion nursing : the official publication of the Infusion Nurses Society. 2006;29 (1):14-7. 6-Anderson NR. When to use a midline catheter. Nursing. 2005;35 (4):28. 7-Gilbert TB, Seneff MG, Becker RB. Facilitation of internal jugular venous cannulation using an audio-guided Doppler ultrasound vascular access device: results from a prospective, dual-center, randomized, crossover clinical study. Critical care medicine. 1995;23 (1):60-5. 8-Maki DG. Reactions associated with midline catheters for intravenous access. Annals of internal medicine. 1995;123 (11):884-6. 9-Taylor RW, Palagiri AV. Central venous catheterization. Critical care medicine. 2007;35 (5):1390-6. 10-Dougherty L. Central venous access devices. Nursing standard. 2000;14 (43): 45-50. 11-Moureau N, Poole S, Murdock MA, Gray SM, Semba CP. Central venous catheters in home infusion care: outcomes analysis in 50,470 patients. Journal of vascular and interventional radiology : JVIR. 2002;13 (10):1009-16. 12-Ng PK, Ault MJ, Ellrodt AG, Maldonado L. Peripherally inserted central catheters in general medicine. Mayo Clinic proceedings. 1997;72 (3):225-33. 13-Tariq M, Huang DT. PICCing the best access for your patient. Critical care. 2006;10 (5):315. 14-Trerotola SO, Kuhn-Fulton J, Johnson MS, Shah H, Ambrosius WT, Kneebone PH. Tunneled infusion catheters: increased incidence of symptomatic venous thrombosis after subclavian versus internal jugular venous access. Radiology. 2000;217 (1):89-93. 15-Debets JM, Wils JA, Schlangen JT. A rare complication of implanted central-venous access devices: catheter fracture and embolization. Supportive care in cancer : official journal of the Multinational Association of Supportive Care in Cancer. 1995;3 (6):432-4. 16-Miller DL, O'Grady NP. Guidelines for the prevention of intravascular catheter-related infections: recommendations relevant to interventional radiology. Journal of vascular and interventional radiology : JVIR. 2003;14 (2 Pt 1):133-6. 17-Robinson MK, Mogensen KM, Grudinskas GF, Kohler S, Jacobs DO. Improved care and reduced costs for patients requiring peripherally inserted central catheters: the role of bedside ultrasound and a dedicated team. JPEN Journal of parenteral and enteral nutrition. 2005;29 (5):374-9. 18-Milling TJ, Jr., Rose J, Briggs WM, Birkhahn R, Gaeta TJ, Bove $\mathrm{JJ}$, et al. Randomized, controlled clinical trial of point-of-care limited ultrasonography assistance of central venous cannulation: the Third Sonography Outcomes Assessment Program (SOAP-3) Trial. Critical care medicine. 2005;33 (8):1764-9. 19-Lok CE, Thomas A, Vercaigne L, Canadian Hemodialysis Catheter Working G. A patient-focused approach to thrombolytic use in the management of catheter malfunction. Seminars in dialysis. 2006;19 (5):381-90. 20-Galloway S, Bodenham A. Long-term central venous access. British journal of anaesthesia. 2004;92 (5):722-34. 21-Denys BG, Uretsky BF, Reddy PS. Ultrasound-assisted cannulation of the internal jugular vein. A prospective comparison to the external landmark-guided technique. Circulation. 1993;87 (5):1557-62. 22-Parkinson R, Gandhi M, Harper $\mathrm{J}$, Archibald C. Establishing an ultrasound guided peripherally inserted central catheter (PICC) insertion service. Clinical radiology. 1998;53 (1):33-6. 23-Chinnock B, Thornton S, Hendey GW. Predictors of Success in Nurse-Performed Ultrasound-Guided Cannulation. The Journal of Emergency Medicine. 2007;33 (4):401-5. 24-Costantino TG, Parikh AK, Satz WA, Fojtik JP. Ultrasonography-Guided Peripheral Intravenous Access Versus Traditional Approaches in Patients With Difficult Intravenous Access. Annals of Emergency Medicine. 2005;46 (5):456-61. 25-Sebbane M, Claret P-G, Lefebvre S, Mercier G, Rubenovitch J, Jreige R, et al. Predicting Peripheral Venous Access Difficulty in the Emergency Department Using Body Mass Index and a Clinical Evaluation of Venous Accessibility. The Journal of Emergency Medicine. 2013;44 (2):299-305. 26-Au AK, Rotte MJ, Grzybowski RJ, Ku BS, Fields JM. Decrease in central venous catheter placement due to use of ultrasound guidance for peripheral intravenous catheters. The American Journal of Emergency Medicine. 2012;30 (9):1950-4. 27-Panebianco NL, Fredette JM, Szyld D, Sagalyn EB, Pines JM, Dean AJ. What You See (Sonographically) Is What You Get: Vein and Patient Characteristics Associated With Successful Ultrasound-guided Peripheral Intravenous Placement in Patients With Difficult Access. Academic Emergency Medicine. 2009;16 (12):1298-303. 28-Stein J, George B, River G, Hebig A, McDermott D. Ultrasonographically Guided Peripheral Intravenous Cannulation in Emergency Department Patients With Difficult Intravenous Access: A Randomized Trial. Annals of Emergency Medicine. 2009;54 (1):33-40. 29-Fields JM, Piela NE, Au AK, Ku BS. Risk factors associated with difficult venous access in adult ED patients. The American Journal of Emergency Medicine. 2014;32 (10):1179-82. 30-Bensghir M, Chkoura K, Mounir K, Drissi M, Elwali A, Ahtil R, et al. Accès veineux périphériques au bloc opératoire : caractéristiques et facteurs prédictifs de difficulté. Annales Françaises d'Anesthésie et de Réanimation. 2012; 31 (7-8):600-4.31-Witting MD. IV Access Difficulty: Incidence and Delays in an Urban Emergency Department. The Journal of Emergency Medicine. 2012;42 (4):483-7. 32-Jacobson AF, Winslow $\mathrm{EH}$. Variables influencing intravenous catheter insertion difficulty and failure: An analysis of 339 intravenous catheter insertions. Heart \& Lung: The Journal of Acute and Critical Care. 2005;34 (5):345-59. 33-Lapostolle F, Catineau J, Garrigue B, Monmarteau V, Houssaye T, Vecci I, et al. Prospective evaluation of peripheral venous access difficulty in emergency care. Intensive Care Medicine. 2007;33 (8):1452-7. 34-Piredda M, Biagioli V, Barrella B, Carpisassi I, Ghinelli R, Giannarelli D, et al. Factors Affecting Difficult Peripheral Intravenous Cannulation in Adults: A Prospective Observational Study. Journal of Clinical Nursing. 2016. 35-Dougherty L. Intravenous therapy in older patients. Nursing Standard. 2013;28 (6):50-8 36-Costantino TG, Kirtz JF, Satz WA. Ultrasound-Guided Peripheral Venous Access vs. the External Jugular Vein as the Initial Approach to the Patient with Difficult Vascular Access. The Journal of Emergency Medicine. 2010;39 (4):462-7. 37-Schoenfeld E, Boniface K, Shokoohi H. ED technicians can successfully place ultrasound guided intravenous catheters in patients with poor vascular access. The American Journal of Emergency Medicine. 2011;29 (5):496-501. 38-de la Torre-Montero J-C, Montealegre-Sanz M, Faraldo-Cabana A, Oliva-Pellicer B, García-Real I, Fenwick M, et al. Venous International Assessment, VIA scale, validated classification procedure for the peripheral venous system. The Journal of Vascular Access. 2014;15 (1):45-50. 39-Fields JM, Piela NE, Ku BS. Association between multiple IV attempts and perceived pain levels in the emergency department. The Journal of Vascular Access. 2014;15 (6):514-8. 40-Brannam L. Emergency Nurses' Utilization of Ultrasound Guidance for Placement of Peripheral Intravenous Lines in Difficult-access Patients. Academic Emergency Medicine. 2004;11 (12):1361-3. 41-Fields JM, Dean AJ, Todman RW, Au AK, Anderson $\mathrm{KL}, \mathrm{Ku} \mathrm{BS}$, et al. The effect of vessel depth, diameter, and location on ultrasound-guided peripheral intravenous catheter longevity. The American Journal of Emergency Medicine. 2012;30 (7):1134- 40. 42-Aulagnier J, Hoc C, Mathieu E, Dreyfus JF, Fischler M, Le Guen $\mathrm{M}$, et al. Efficacy of AccuVein to Facilitate Peripheral Intravenous Placement in Adults Presenting to an Emergency Department: A Randomized Clinical Trial. Academic Emergency Medicine. 2014;21 (8):858-63. 43-Elia F, Ferrari G, Molino P, Converso M, De Filippi G, Milan A, et al. Standard-length catheters vs long catheters in ultrasound-guided peripheral vein cannulation. The American Journal of Emergency Medicine. 2012;30 (5):712-6.

Disclosure: No significant relationships. 


\section{PR346}

\section{THE RELATIONSHIP BETWEEN THE DEGREE OF FRAILTY AND THE POST-OPERATIVE EVOLUTION OF THE ELDERLY PATIENTS WITH FRAILTY FRACTURES}

\author{
A.I. Pislaru ${ }^{1}$, A.C. Ilie $^{1}$, R. Stefaniu ${ }^{1}$, I.D. Alexa ${ }^{1}$, O. Alexa $^{2}$
}

${ }^{1}$ Geriatrics, University of Medicine and Pharmacy Iasi, iasi/ ROMANIA, ${ }^{2}$ Orthopedics And Trauma, University of Medicine and Pharmacy Iasi, iasi/ROMANIA

Introduction: Frailty fractures are a major health care problem in senior population, due to different degrees of incapacity, concomitant diseases and poly-pharmacy, which would severely increase general mortality.

Material and methods: We present a retrospective study of 10 elderly patients over the age of 77 , diagnosed with frailty fractures and transferred from the Orthopedic Department to Geriatric Department for therapeutical and rehabilitation program. We applied Fried criteria to determine frailty, the Charlson Comorbidity Index for concomitant diseases and comprehensive geriatric assessment and we compared the progress of rehabilitation with the degree of frailty to each patient.

Results: All the patients were over 77 years old, the oldest had 94 years old. They had multiple concomitant diseases and poly-pharmacy. Most of the patients had hip fracture, and some had pelvic ring or radius fractures. According to Fried criteria, all patients were fragile (score $\geq 3$ ). The geriatric evaluation showed that most of the patients had malnutrition, and were mildly depressed. We found a strong negative correlation between the degree of frailty and the speed of recovery after fracture in all cases. The degree of sarcopenia and malnutrition were the strongest markers for rehabilitation period.

Conclusion: Frailty is an important factor to consider when starting rehabilitation in very old patients with fragility fractures. Sarcopenia, malnutrition and depression are the most important determinants of a good rehabilitation period, of good evolution of concomitant diseases and an acceptable quality of life.

References: Tom, S. E., Adachi, J. D., Anderson, F. A., Boonen, S., Chapurlat, R. D., Compston, J. E., ... \& Nieves, J. W. (2013). Frailty and fracture, disability, and falls: a multiple country study from the global longitudinal study of osteoporosis in women. Journal of the American Geriatrics Society, 61 (3), 327-334. Van Den Bergh, J. P., Van Geel, T. A., \& Geusens, P. P. (2012). Osteoporosis, frailty and fracture: implications for case finding and therapy. Nature Reviews Rheumatology, 8 (3), 163-172.

Disclosure: No significant relationships.

\section{PR347}

\section{THE FUNCTIONAL OUTCOME OF PUBIC RAMI FRACTURES WITH OR WITHOUT CONCOMITANT INVOLVEMENT OF THE POSTERIOR RING IN ELDERLY PATIENTS}

\section{S. Loggers ${ }^{1}$, P. Joosse ${ }^{2}$, K. Ponsen ${ }^{2}$}

${ }^{1}$ Surgery, NWZ Alkmaar, Alkmaar/NETHERLANDS, ${ }^{2}$ Surgery, Northwest Clinics Alkmaar, Alkmaar/NETHERLANDS

Introduction: Pubic rami fractures are common fractures in a growing osteoporotic geriatric population. [1-2] Concomitant involvement of the posterior pelvic ring is often found when properly looked for. [3-7]. Immobiliztion because of pain makes this patient group vulnerable for complications. [7-9]Mortality rates are proven to be high but long term morbidity outcomes are lacking. [3-5, 10-15] This study aims to review the longer term patient related outcome measurements in order to further establish the consequences of these fractures.

Material and methods: Retrospective analysis of patients aged over 65 years sustaining a pubic rami fracture in the North-West Hospital Group Alkmaar combined with a survey to establish risk factors for morbidity and mortality after 6 months' post trauma. Multiple logistic regression analysis was used to identify risk factors.

Results: 117 patients matched inclusion criteria. 23 cPRF's were identified. Significant deterioration in ambulation and independency was found. $49 \%$ lost their independent mobility status, $34 \%$ of patients were institutionalized at discharge. $40 \%$ of the patients did not experience a full recovery at follow up. 1-year mortality was $23 \%$. cPRF Patients had higher complication rates. Mortality and morbidity did not statistically differ. The strongest predictor for 1-year mortality was a dependent ambulatory status (OR 4,1)

Conclusion: Pubic ramu fractures in elderly patients aged over 65 have a significant impact on mobility and independency. Mortality is similar to hip fracture patients.[16] Immobility and complications during admission are the highest predictor for one-year mortality. Careful evaluation of every patient's mobility status is necessary in order to identify patients at risk for complications and determine their future health care needs.

References: [1] Kannus P, Palvanen M, Niemi S, Parkkari J, Järvinen M. Epidemiology of osteoporotic pelvic fractures in elderly people in Finland: sharp increase in 1970-1997 and alarming projections for the new millennium. Osteoporos Int. 2000;11:443-8 [2] Burge R, Dawson-Hughes B, Solomon DH, Wong JB, King A, Tosteson A. Incidence and economic burden of osteoporosis related fractures in the United States, 2005-2025. J Bone Miner Res. 2007;22:465-75 [3] Rommens PM, Hofmann A. Comprehensive classification of fragility fractures of the pelvic ring: recommendations for surgical treatment. Injury. 2013;44:1733-44 [4] Alnaib M, Waters S, Shanshal Y, Caplan N, Jones S, St Clair Gibson A, Kader D. Combined pubic rami and sacral osteoporotic fractures: a prospective study. J Orthop Traumatol. 2012 Jun;13 (2):97-103 [5] Lau TW, Leung F. Occult posterior pelvic ring fractures in elderly patients with osteoporotic pubic rami fractures. J Orthop Surg (Hong Kong). 2010 Aug;18 (2):153-7 [6] Studer P, Suhm N, Zappe B, Bless N, Jakob M. Pubic rami fractures in the elderly - a neglected injury? Swiss Med Wkly. 2013 Sep 19;143:w13859 [7] Wagner D, Ossendorf C, Gruszka D, Hofmann A, Rommens PM, Fragility fractures of the sacrum: how to identify and when to treat surgically? Eur J Trauma Emerg Surg. 2015 Aug;41 (4):349-62 [8] Babayev M, Lachmann E, Nagler W. The controversy surrounding sacral insufficiency fractures: to ambulate or not to ambulate? Am J Phys Med Rehabil. 2000;79:404-9 [9] Maier GS, Kolbow K, Lazovic D, Horas K, Roth KE, Seeger JB, Maus U. Risk factors for pelvic insufficiency fractures and outcome after conservative therapy. Arch Gerontol Geriatr. 2016 Nov-Dec;67:80-5 [10] Hill RM1, Robinson CM, Keating JF. Fractures of the pubic rami. Epidemiology and five-year survival. J Bone Joint Surg Br. 2001 Nov;83 (8):1141-4 [11] Leung WY, Ban CM, Lam JJ, Ip FK, Ko PS, Prognosis of acute pelvic fractures in elderly patients: retrospective study. Hong Kong Med J. 2001 Jun;7 (2):139-45 [12] Breuil V, Roux $\mathrm{CH}$, Testa J, Albert C, Chassang M, Brocq O, Euller-Ziegler L, Outcome of osteoporotic pelvic fractures: an underestimated severity. Survey of 60 cases. Joint Bone Spine. 2008 Oct;75 (5):585-8 [13] Scheyerer MJ, Osterhoff G, Wehrle S, Wanner GA, Simmen H-P, Werner CML. Detection of posterior pelvic injuries in fractures of the pubic rami. Injury. 2012;43:1326-9 [14] Nüchtern JV, Hartel MJ, Henes FO, Groth M, Jauch SY, Haegele J, Briem D, Hoffmann M, Lehmann W, Rueger JM, Großterlinden LG, Significance of clinical 
examination, CT and MRI scan in the diagnosis of posterior pelvic ring fractures. Injury. 2015 Feb;46 (2):315-9 [15] Morris RO, Sonibare A, Green DJ, Masud T, Closed pelvic fractures: characteristics and outcomes in older patients admitted to medical and geriatric wards, Postgrad Med J 2000;76:646-650 [16] Hu F. Preoperative predictors for mortality following hip fracture surgery: a systematic review and meta-analysis. Injury 2012 Jun43 (6);676-85

Disclosure: No significant relationships.

\section{PR348}

\section{OUTCOMES FOLLOWING TREATMENT OF GERIATRIC DISTAL FEMUR FRACTURES}

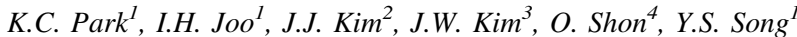

${ }^{1}$ Orthopedic Surgery, Hanyang University Guri Hospital, Guri/ KOREA, REPUBLIC OF, ${ }^{2}$ Orthopaedic Surgery, Asan Medical Center, Ulsan university, Seoul/KOREA, REPUBLIC OF, ${ }^{3}$ Orthopedic Surgery, Haeundae Paik Hospital, Inje University, Busan/KOREA, REPUBLIC OF, ${ }^{4}$ Orthopedic Surgery, Yeungnam University Hospital, Daegu/KOREA, REPUBLIC OF

Introduction: In elderly patients, distal femoral fractures are usually caused by low energy trauma. Surgical treatment is difficult because osteoporosis is often associated. Geriatric distal femur fractures have a high morbidity and mortality. In this study, we analyzed the clinical and radiological results of treatment in geriatric distal femur fractures. Material and methods: We retrospectively reviewed 46 patients over age 60 who sustained a low-energy femur fractures (AO/OTA 33A) treated with ORIF using locked plating from January 2008 through January 2016. Primary outcomes included nonunion, reoperation to promote union and death. Charlson Comorbidity Index (CCI) was calculated based on comorbidities documented in the medical records. Length of hospital stay and CCI were analyzed to find the relation with mortality. In addition, periprosthetic fracture group and nonperiprosthetic fracture group were also compared for this study.

Results: Nonunion was observed in 8 patients (17.4\%) and all achieved bone union after re-operation. The overall mortality rate was $6.5 \%$ and complication rate was $21.7 \%$. There were twenty five periprosthetic fractures (P group) and twenty one non-periprosthetic fractures ( $\mathrm{N}$ group). Comparison between these two groups showed no significant differences in union time; periprosthetic fracture (18.1 weeks) and non-periprosthetic fractures (18.79 weeks). Nonunion and complications between the two groups were not statistically different. However, the mortality rate was different between the two groups ( $\mathrm{P}$ group; $0, \mathrm{~N}$ group; $3, \mathrm{p}=0.026$ ).

Conclusion: Geriatric distal femur fractures have a high incidence of complications including nonunion, delayed union and death. Further research on risk factors for complications and mortality is needed.

References: 1. Kammerlander C, Riedmüller P, Gosch M, et al. Functional outcome and mortality in geriatric distal femoral fractures. Injury 2012;43:1096-101. 2. Streubel PN, Ricci WM, Wong A, Gardner MJ. Mortality after distal femur fractures in elderly patients. Clinical Orthopaedics and Related Research ${ }^{\circledR}$ 2011;469:1188-96. 3. Jordan R, Chahal G, Davies M, Srinivas K. A Comparison of Mortality following Distal Femoral Fractures and Hip Fractures in an Elderly Population. Advances in Orthopedic Surgery 2014;2014.

Disclosure: No significant relationships.
PR349

\section{COULD WE PREDICT COMPLICATIONS AFTER INTRAMEDULLARY NAILING OF DIAPHYSEAL TIBIA FRACTURES?}

\author{
C. Detrembleur, S. Van De Veyver, K. Tribak, D. Putineanu, \\ O. Cornu, J. Manon
}

Orthopaedic And Trauma Department, Cliniques universitaires SaintLuc, UCL, Brussels/BELGIUM

Introduction: Despite a significant evolution in the care of tibial fractures, many complications still remain after intramedullary nailing (IMN). This leads to greater costs for patients and society. The question is : "which risk factors could predict these complications and which ones could be highlighted to take a preventive approach ?

Material and methods: This single centre (Cliniques Universitaires Saint-Luc) and retrospective study enroled 168 patients with 171 fractures, between 2005 and 2015. The independent variables included patients dependent factors (age, sex, BMI, comorbidities, ...) and fracture/surgery dependent factors (pattern of fracture, (non-)reamed IMN, timing, nail diameter, number of screws, ...). An univariate analysis was primarily performed followed by multiple logistic regression model to determine predictors of each complication.

Results: Infection increase significantly $(p<0.05)$ according to the open fracture, the AO classification of the fracture, the antibioprophylaxis $(\mathrm{AB})$ delay and the time to nailing. Another surgical treatment associated with IMN and the ratio nail diameter/reamer diameter are significantly associated to most material damage. Factors increasing the occurence of non-union are tabacco and trauma energy. Conclusion: In addition to current knowledge, this study shows that the $\mathrm{AB}$ delay is a true predictor of infection. Using a nail with a bigger diameter or a nail diameter/reamer diameter ratio in the good range are prognostic datas to predict material damage. This outcome confirms the only study that revealed this in 2016. Keeping these observation in mind could be a way of improving outcomes.

References: 1. Xia, L., et al., A meta-analysis of reamed versus unreamed intramedullary nailing for the treatment of closed tibial fractures. Orthopedics, 2014. 37 (4): p. e332-8. 2. Papakostidis, C., et al., Prevalence of complications of open tibial shaft fractures stratified as per the Gustilo-Anderson classification. Injury, 2011. 42 (12): p. 1408-15. 3. Investigators, S., et al., Study to prospectively evaluate reamed intramedually nails in patients with tibial fractures (S.P.R.I.N.T.): study rationale and design. BMC Musculoskelet Disord, 2008. 9: p. 91. 4. Metsemakers, W.J., et al., Individual risk factors for deep infection and compromised fracture healing after intramedullary nailing of tibial shaft fractures: a single centre experience of 480 patients. Injury, 2015. 46 (4): p. 740-5. 5. Lam, S.W., et al., Systematic review shows lowered risk of nonunion after reamed nailing in patients with closed tibial shaft fractures. Injury, 2010. 41 (7): p. 671-5. 6. Gustilo, R.B., R.M. Mendoza, and D.N. Williams, Problems in the management of type III (severe) open fractures: a new classification of type III open fractures. J Trauma, 1984. 24 (8): p. 742-6. 7. Yokoyama, $\mathrm{K}$., et al., Immediate versus delayed intramedullary nailing for open fractures of the tibial shaft: a multivariate analysis of factors affecting deep infection and fracture healing. Indian J Orthop, 2008. 42 (4): p. 410-9. 8. Thakore, R.V., et al., The Gustilo-Anderson classification system as predictor of nonunion and infection in open tibia fractures. Eur J Trauma Emerg Surg, 2016. 9. Bachoura, A., et al., Infirmity and injury complexity are risk factors for surgical-site infection after operative fracture care. Clin Orthop Relat Res, 2011.469 (9): p. 262130. 10. Antonova, E., et al., Tibia shaft fractures: costly burden of nonunions. BMC Musculoskelet Disord, 2013. 14: p. 42. 11. Paryavi, E., et al., Predictive model for surgical site infection risk after surgery 
for high-energy lower-extremity fractures: development of the risk of infection in orthopedic trauma surgery score. J Trauma Acute Care Surg, 2013.74 (6): p. 1521-7. 12. Matos, M.A., L.G. Lima, and L.A. de Oliveira, Predisposing factors for early infection in patients with open fractures and proposal for a risk score. J Orthop Traumatol, 2015. 16 (3): p. 195-201. 13. Horan, T.C., et al., CDC definitions of nosocomial surgical site infections, 1992: a modification of CDC definitions of surgical wound infections. Am J Infect Control, 1992. 20 (5): p. 271-4. 14. Yokoyama, K., et al., Risk factors for deep infection in secondary intramedullary nailing after external fixation for open tibial fractures. Injury, 2006. 37 (6): p. 554-60. 15. Gaebler, C., et al., Rates and odds ratios for complications in closed and open tibial fractures treated with unreamed, small diameter tibial nails: a multicenter analysis of 467 cases. J Orthop Trauma, 2001. 15 (6): p. 415-23. 16. Court-Brown, C.M., J.F. Keating, and M.M. McQueen, Infection after intramedullary nailing of the tibia. Incidence and protocol for management. J Bone Joint Surg Br, 1992. 74 (5): p. 770-4. 17. Craig, J., et al., Systematic review and meta-analysis of the additional benefit of local prophylactic antibiotic therapy for infection rates in open tibia fractures treated with intramedullary nailing. Int Orthop, 2014. 38 (5): p. 1025-30. 18. Khatod, M., et al., Outcomes in open tibia fractures: relationship between delay in treatment and infection. J Trauma, 2003. 55 (5): p. 949-54. 19. Harley, B.J., et al., The effect of time to definitive treatment on the rate of nonunion and infection in open fractures. J Orthop Trauma, 2002. 16 (7): p. 484-90. 20. Willy, C., et al., [Acute therapeutic measures for limb salvage Part $2:$ Debridement, lavage techniques and anti-infectious strategies]. Unfallchirurg, 2016. 119 (5): p. 388-99. 21. Patka, P., Damage control and intramedullary nailing for long bone fractures in polytrauma patients. Injury, 2017. 48 Suppl 1: p. S7-S9. 22. Siebenrock, K.A., B. Schillig, and R.P. Jakob, Treatment of complex tibial shaft fractures. Arguments for early secondary intramedullary nailing. Clin Orthop Relat Res, 1993 (290): p. 269-74. 23. Henley, M.B., et al., Treatment of type II, IIIA, and IIIB open fractures of the tibial shaft: a prospective comparison of unreamed interlocking intramedullary nails and half-pin external fixators. J Orthop Trauma, 1998. 12 (1): p. 1-7. 24. Bhandari, M., et al., Intramedullary nailing following external fixation in femoral and tibial shaft fractures. $\mathrm{J}$ Orthop Trauma, 2005.19(2): p. 140-4.25. Young, S., et al., Riskfactors for infection after 46,113 intramedullary nail operations in low- and middle-income countries. World J Surg, 2013. 37 (2): p. 349-55. 26. Bandalovic, A., et al., A retrospective study of antibiotic prophylaxis value in surgical treatment of lower limb fracture. Injury, 2015. 46 Suppl 6: p. S67-72. 27. Patzakis, M.J. and J. Wilkins, Factors influencing infection rate in open fracture wounds. Clin Orthop Relat Res, 1989 (243): p. 36-40. 28. Gosselin, R.A., I. Roberts, and W.J. Gillespie, Antibiotics for preventing infection in open limb fractures. Cochrane Database Syst Rev, 2004 (1): p. CD003764. 29. Courtney, P.M., J. Bernstein, and J. Ahn, In brief: closed tibial shaft fractures. Clin Orthop Relat Res, 2011. 469 (12): p. 3518-21. 30. Castillo, R.C., et al., Impact of smoking on fracture healing and risk of complications in limbthreatening open tibia fractures. J Orthop Trauma, 2005. 19 (3): p. 1517. 31. Kortram, K., et al., Risk factors for infectious complications after open fractures; a systematic review and meta-analysis. Int Orthop, 2017. 32. Metsemakers, W.J., et al., Definition of infection after fracture fixation: A systematic review of randomized controlled trials to evaluate current practice. Injury, 2017. 33. Burrus, M.T., B.C. Werner, and S.R. Yarboro, Obesity is associated with increased postoperative complications after operative management of tibial shaft fractures. Injury, 2016. 47 (2): p. 465-70. 34. Moghaddam-Alvandi, A., et al., Cigarette smoking influences the clinical and occupational outcome of patients with tibial shaft fractures. Injury, 2013. 44 (11): p. 1670-1. 35. Adams, C.I., J.F. Keating, and C.M. Court-Brown, Cigarette smoking and open tibial fractures. Injury, 2001. 32 (1): p. 61-5.

Disclosure: No significant relationships.
PR350

\section{RESULT OF OPERATIVE TREATMENT AC DISLOCATION}

\author{
M. Forman, M. Carda, D. Kusý
}

Traumatology, NPK a.s Pardubická nemocnice, Pardubice/CZECH REPUBLIC

Introduction: Acromioclavicular (AC) dislocation is a common injury due to the prominence of the $\mathrm{AC}$ joint on the superolateral aspect of the shoulder. The bony anatomy of the shoulder girdle makes the AC joint vulnerable to direct force, such as a fall onto the shoulder. There are plenty of surgical methods of treatment with different results. We tried to find some method with low rate of complications and good results. We would like to present you our experience with ORIF (hook plate)

Material and methods: We present group of patients (70) with AC dislocation. In this group avarege age was 40 (18-57). To classify injury by AP and Zanca view (10st cephalic tilt) X ray and Rockwood classification of AC dislocation. Relative Constant Shoulder score is used followed by post surgery examination in 1, 3, and 6 months and after hardware removal.

Results: For patients with AC dislocation Rockwood III - IV the surgical treatment has indicated and hook plate yielded good results early after surgery and after hardware removal. However, this method has disadvantage attributable to hardware removal with associated osteolysis of acromion around the hook of the plate. Averege hardware removal time in the group was 6 month. We had only one redislocation after hardware removal. There were three complications asspciated with hook plate method such as wound infections (two times) and migration of hardware.

Conclusion: Therefore, using this method yields quicker return to the normal activity with lower risk of complications linked to the surgical treatment.

References: Fractures in Adults -Rockwood and Green's Surgical Treatment of Orthopaedic Trauma - James P. Stannard, Andrew H. Schmidt, Philip J.Kregor Shoulder and elbow surgery - Donald H. Lee, Robert J. Neviaser

Disclosure: No significant relationships.

\section{PR351}

\section{EPIDEMIOLOGY OF DISTAL RADIUS FRACTURES IN POLYTRAUMA PATIENTS AND THE INFLUENCE OF HIGH TRAUMATIC ENERGY TRANSFER} S. Ferree
R.M. Houwert $^{3}$, L.P. L.h. Leenen
, F. Fietbrink

${ }^{1}$ Surgery, University Medical Center Utrecht, Utrecht/ NETHERLANDS, ${ }^{2}$ Harvard Combined Orthopaedics Residency Program, Surgery, Boston/MA/UNITED STATES OF AMERICA, ${ }^{3}$ Utrecht Traumacenter, Uiversity Medical Center Utrecht, Utrecht/ NETHERLANDS, ${ }^{4}$ Traumatology, University Medical Center Utrecht, Utrecht/NETHERLANDS

Introduction: For several extremity fractures differences in morphology, incidence rate and functional outcome were found when polytrauma patients were compared to patients with an isolated injury. This is not proven for distal radius fractures (DRF). Therefore, this 
study aimed to analyze fracture morphology in relation to energy transfer in both poly- and mono-trauma patients with a DRF.

Material and methods: This was a retrospective cohort study. All patients aged 16 years and older with a DRF were included. Patients with an Injury Severity Score of 16 or higher were classified as polytrauma patients. Injuries were defined as high or low energy. All DRFs were classified using the AO/OTA fracture classification system.

Results: A total of 830 patients with a DRF were included, $12 \%$ were polytrauma. The incidence rate of DRF in polytrauma patients was $3.5 \%$. Ipsilateral upper extremity injury was found in $>30 \%$ of polytrauma and high-energy monotrauma patients, compared to $5 \%$ in low-energy monotrauma patients. More type C DRF were found in polytrauma and high-energy monotrauma patients versus low-energy monotrauma patients. Operative intervention rates for all types of DRF were similar for polytrauma and high-energy monotrauma patients. Non-union rates were higher in polytrauma patients.

Conclusion: Higher energy mechanisms of injury, in polytrauma and high-energy monotrauma patients, were associated with more severe complex articular distal radius fractures and more ipsilateral upper extremity injuries. Polytrauma and high-energy monotrauma patient have a similar fracture morphology. However, polytrauma patients have in addition to more injured body regions also more non-union related interventions than high-energy monotrauma patients.

References: Rockwood CA, Green DP, Bucholz RW. Rockwood and Green's fractures in adults. 7th ed. Philadelphia, PA: Wolters Kluwer Health/Lippincott Williams \& Wilkins; 2010. Scaling CoI. The Abbreviated Injury Scale, 1998 revision (AIS-98) Des Plaines (IL): Association for the Advancement of Automotive Medicine (AAAM). 1998. Diamantopoulos AP, Rohde G, Johnsrud I, Skoie IM, Hochberg M, Haugeberg G. The epidemiology of low- and high-energy distal radius fracture in middle-aged and elderly men and women in Southern Norway. PLoS One. 2012;7:e43367. Banerjee M, Bouillon $\mathrm{B}$, Shafizadeh S, et al. Epidemiology of extremity injuries in multiple trauma patients. Injury. 2013;44:1015-21.

Disclosure: No significant relationships.

\section{PR352}

\section{FUNCTIONAL AND RADIOLOGICAL OUTCOME IN 4-PART PROXIMAL HUMERAL FRACTURES TREATED WITH AFFINIS FRACTURE HEMI-ARTHROPLASTY USING THE CAP-TECHNIQUE}

\section{S. Uyttebroek, G. Putzeys}

\section{Orthopedie, AZ Groeninge Kortrijk, Kortrijk/BELGIUM}

Introduction: The outcome of primary arthroplasty for proximal humeral fractures is dependent of the position and survival of the tuberosities. We analysed the functionality of the shoulder and tuberosity healing in displaced 4-part proximal humeral fractures treated with a hemi-arthroplasty. An adapted technique was used to fix the tuberosities. This cap-technique is to provide better survival of the tuberosities and subsequently better clinical outcomes.

Material and methods: Eleven patients were included in this study. All patients were treated by the same surgeon with the same surgical method. The Affinis Fracture prosthesis was used. After cemented stem placement the tuberosities were reduced and fixed to the metaphyseal part of the prosthesis with non-absorbable heavy sutures. The one-piece continuity of rotator cuff and tuberosities was essential, so it could be reduced as a cap onto the proximal prosthesis. The reduction of the tubercula happened by sequential tensioning of the two circular non-absorbable heavy sutures. Post-operative, the active and passive range of motion and tuberosity healing was evaluated.

Results: The population were 3 male and 8 female patients with a mean age of 77,6 year (55-87). Follow up ranges from 2 months to 8 months follow up. All patients had good clinical outcomes: they had no limitation in performing activities of daily life. 10/11 patient had good radiological results.

Conclusion: Good radiological and clinical results are possible with a primary hemi-arthroplasty using an adapted technique of wire-suture fixation of the tubercula. Higher rates of tuberosity healing will be achieved, as illustrated by our radiological results.

References: 1) Falk Reuther, Bernd Mühlhaüsler, Diethard Wahl, Stefaan Nijs; Functional outcome of shoulder hemiarthroplasty for fractures:

A multicentre analysis. Injury, Int. J. Care Injured 41 (2010) 606-612 2) Xing $\mathrm{Wu}$, Shao-Hua Li, Zheng-Dong Cai and Lie-Ming Lou; Modified hemiarthroplasty for four-part fractures of the

proximal humerus. ANZ J Surg 83 (2013) 165-170 3) Tuyen K. Kiet, Brian T. Feeley, Micah Naimark, Tatiana Gajiu, Sarah L. Hall, Teddy T. Chung, Benjamin Ma. Outcomes after shoulder replacement: comparison between reverse and anatomic total shoulder arthroplasty. 1058-2746 2015 Journal of Shoulder and Elbow Surgery Board of Trustees 4) Aristotelis Kaisidis, Panayiotis G. Pantos, Horst Heger, Dimitrios Bochios, Sotirios Selimas, Vasileios Oikonomoias. Reverse shoulder arthroplasty for the treatment of three and four part fractures of the proximal humerus in patients older than 75 years old. Acta Orthopædica Belgica, Vol. 80 - 1 - 2014

Disclosure: No significant relationships.

\section{PR353}

\section{FRAGILITY HIP FRACTURES IN YOUNG PATIENTS: ARE WE MISSING THE POINT?}

A. Abarquero Diezhandino $^{l}$, C. Ojeda ${ }^{l}, J$. Gallardo $^{2}$, C. García-

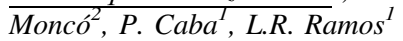

${ }^{1}$ Orthopaedic Surgery And Traumatology, Hospital Universitario 12 de Octubre, Madrid/SPAIN, ${ }^{2}$ Cirugia Ortopedica Y Traumatología, Hospital Universitario 12 Octubre, Madrid/SPAIN

Introduction: Hip fractures are uncommon among patients younger than 65 , and can be caused by high-energy trauma as well as fragility among patients with comorbidities causing secondary osteoporosis. Hip fractures in young patients remains largely unexplored. The goal of this study is to analyse the predisposing factors and prognosis of fragility hip fractures in patients under 65 .

Material and methods: We performed a retrospective study of fragility hip fractures in adults aged 18 to 65 , excluding pathologic and high-energy injuries. We identified 241 patients who suffered fracture between June 2004 and June 2014. Risk factors for osteoporosis were assessed, as well as postoperative complications, new fractures, and mortality for at least one year following the fracture.

Results: The medium age was 56 years old and there were $53,5 \%$ men. $24,9 \%$ of the patients had a history of drug or alcohol abuse and $17,8 \%$ suffered a neuromuscular disease. In total we had $126(52,3 \%)$ intracapsular fractures, more than a half of them, were treated with cannulated screws $(58,7 \%) .115(47,7 \%)$ patients had extracapsular fractures, most of them were treated with cephalomedullary nail. $22,5 \%$ of patients died during follow-up, $8 \%$ of them died in the first year after fracture. $27,7 \%$ of patients suffered postoperative complications and $17 \%$ suffered fragility fractures during follow-up: $5,9 \%$ of the contralateral hip. 
Conclusion: Low-energy fragility fractures are an important entity among younger patients with risk factors of secondary osteoporosis. The complication and mortality rates were high, so, the severity of a low-energy hip fracture should never be underestimated, even in younger patients.

References:

Disclosure: No significant relationships.

\section{PR354}

COMPLICATIONS OF MODULAR MONOPOLAR RADIAL HEAD PROSTHESIS: OUR EXPERIENCE IN THE PAST SEVEN YEARS

\author{
D. Bustamante, V. Jiménez, M.Á. Porras, L. García, D. Cecilia
}

Orthopaedic Surgery And Traumatology, Hospital Universitario 12 de Octubre, Madrid/SPAIN

Introduction: Radial head prostheses are implanted for non-synthesizable radial head fractures, and sequelae derived therefrom, such as lack of consolidation. This prosthesis is being implanted for approximately 20 years, and knowledge about its complications is relatively scarce. Their percentages vary from 15 to $35 \%$, usually related to loosening and oversizing of the components. Osteoarthritis development is also common.

Our objective is to assess the type, frequency and severity of complications after the implantation of this particular prosthesis

Material and methods: Twenty-five patients with 26 radial head prostheses implanted between 2010 and 2016 were reviewed retrospectively. Patients were evaluated clinically and radiographically for a mean follow-up of 30 months.

Results: The same type of prosthesis was implanted (Ascension Modular Radial Head). Three patients $(11,53 \%)$ suffered from continuous pain. Eight cases (30.76\%) showed radiological oversizing, though only three were symptomatic. Ten patients (38.4\%) developed osteoarthritis. Heterotopic ossification was assesed in sixteen cases $(61.53 \%)$, most of them asymptomatic. Stiffness was developed in six patients $(25 \%)$. Fifteen patients $(57.69 \%)$ showed periprosthetic radiolucencies, from which four were symptomatic. Six patients (23\%) developed surgery-related complications: one superficial infection, two symptomatic loosening cases, and three oversizing cases with associated stiffness, from which one patient developed a radial nerve palsy. Three patients $(11,53 \%)$ required revision surgery. Conclusion: Our study obtains similar results to those found in the literature, with $23 \%$ of complications. These results stress the need to improve the surgical technique and design of the implants. Further research is also required to assess long-term results and the potential progression of the radiographic findings.

References: Gauci MO, Winter M, Dumontier C, Bronsard N, Allieu Y.Clinical and radiologic outcomes of pyrocarbon radial head prosthesis: midterm results. J Shoulder Elbow Surg. 2016 Jan;25 (1):98104. Katthagen JC, Jensen G, Lill H, Voigt C.Monobloc radial head prostheses in complex elbow injuries: results after primary and secondary implantation. Int Orthop. 2013 Apr;37 (4):631-9. Chien HY, Chen AC, Huang JW, Cheng CY, Hsu KY. Short- to medium-term outcomes of radial head replacement arthroplasty in posttraumatic unstable elbows: 20 to 70 months follow-up. Chang Gung Med J. 2010 Nov-Dec;33 (6):668-78 Martin Fuentes AM, Cecilia Lopez D, Resines Erasun C. Resultados a medio plazo de la artroplastia monopolar modular de cabeza radial. Rev Esp Cir Ortop Traumatol. 2013;57 (3):217-223

Disclosure: No significant relationships.

\section{PR355}

\section{CLINICAL RESULTS TREATED WITH LCP-DF FOR DISTAL FEMUR FRACTURES -FOCUSING ON SCREW PLACEMENT AND MEDIAL GAP OF FRACTURES}

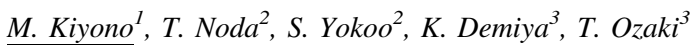

${ }^{1}$ Orthopaedic Surgery, Okayama University, OKAYAMA/JAPAN, ${ }^{2}$ Orthopaedic Surgery, Department of Orthopaedic Surgery Okayama University, Okayama City/JAPAN, ${ }^{3} 2-5-1$, Okayama university hospital, Okayama/JAPAN

Introduction: Plate fixation is one of the standard treatments for distal femoral fracture. There are several reports on the position of the screw and the length of the plate considering the strength of the plate, however, there are few reports focusing on the number of screws not inserted around the fracture site.

Material and methods: This study included 71 distal femoral fractures treated using LCP-DF. In this study, we conducted an evaluation and analysis of (1) bone union rate, (2) plate length, (3) working length of fracture site (4) plate span ratio, (5) number of screws not inserted around the fracture ("empty holes"), and (6) medial bone fragment distance.

Results: Among the simple fracture cases, there were 2 non-union cases. There was 1 case with 1 empty hole, and 1 case with 4 empty holes. Among the comminuted fracture cases, there were 5 non-union cases. There were 2 cases with 1 empty hole, and 3 cases with 2 empty holes. Of 26 simple fracture cases, there were 7 cases with an inner gap of $2 \mathrm{~mm}$ or wider, and the non-union rate was $2 / 7(28.5 \%)$. In addition, there were 13 comminuted fracture cases with medial bone fragment distance of $2 \mathrm{~mm}$ or wider, and the non-union rate was $4 / 13(30.7 \%)$.

Conclusion: Our results also suggested that increasing the number of empty holes around the fracture site may increase the rate of nonunion and indicated that screw placement during plate fixation influences bone union rate. When the gap of the fracture part was 2 $\mathrm{mm}$ or more, the non-union rate worsened.

References: Kolmert L, Wulff K. Epidemiology and treatment of distal femoral fractures in adults. Acta Orthopaedica Scandinavica: 1982;53 (6):957-62. 2. Court-Brown CM, Caesar B. Epidemiology of adult fractures: A review. Injury: 2006;37 (8):691-697. 3. Ricci WM, Streubel PN, Morshed S. Risk Factors for Failure of Locked Plate Fixation of Distal Femur Fractures: An Analysis of 335 Cases. J Orthop Trauma: 2014;28 (2):83-89. 4. Gwathmey, WF, Jones QS, Kahler D. Distal Femoral Fractures: Current Concepts. Journal of the American Academy of Orthopaedic Surgeons: 2010;18 (10) :597-607. Ehlinger M, Ducrot G, Adam P, et al. Distal femur fractures. Surgical techniques and a review of the literature. Orthopaedics \& Traumatology Surgery \& Research: 2013;99:353-360. Smith WR, Ziran BH, Anglen JO et al. Locking Plates: Tips and Tricks. J Bone Joint Surg Am: Perren S, Cordey J, Baumgart F. Technical and Biomechanical Aspects of Screws used for Bone Surgery. J Orthop Trauma: 1992;2:31-48. Cordey J, Rahn B, Perren S. Human torque control in the use of bone screws. Uhthoff $\mathrm{H}$ (ed), Current concepts of internal fixation of fractures $1^{\text {st }}$ ed: 1980:235-243. Weight M, et al.Early Results of the Less Invasive Stabilization System for Mechanically Unstable Fractures of the Distal Femur (AO/OTA Types A2, A3, C2 and C3). J Orthop Trauma: 2004;18 (8):503-8 Lee CH, Shiha KS, Hsu $\mathrm{CC}$ et al. Simulation-based particle swarm optimization and mechanical validation of screw position and number for the fixation stability of a femoral locking compression plate. Medical Engineering \& Physics: 2014;36:57-64. Hunt SB, Buckley RE. Locking Plates: A Current Concepts Review of Technique and Indications for Use. ACTA CHIRURGIAE ORTHOPAEDICAE ET 
TRAUMATOLOGIAE ČECHOSL: 2013;80: 185-191. Hoffmeier KL, Hofmann GO, Muckley T. Choosing a proper working length can improve the lifespan of locked plates. A biomechanical study. Clin Biomech (Bristol, Avon) : 2011;26 (4):405-9. Fracture and dislocation compendium. Orthopaedic Trauma Association Committee for coding and classification. J Orthop Trauma: 199610 (Suppl.1) v-ix, 1-154. Marsh JL, Slongo TF, Agel J, Broderick JS, Creevey W, DeCoster TA, et al. Fracture and dislocation classification compendium_2007: Orthopaedic Trauma Association classification, database and outcomes committee. J Orthop Trauma: 2007;21 (10Suppl):S1-S133. Gautier E, Sommer C. Guidelines for the clinical application of the LCP. Injury: 2003;34:SB63-SB76. Stoffel K, Dieter $\mathrm{U}$, Stachowiak G. Biomechanical testing of the LCP-how can stability in locked internal fixators be controlled?. Injury: 2003;34:SB11SB19. Kanchanomai C, Muanjan P, Phiphobmongkol V. Stiffness and endurance of a locking compression plate fixed on fractured femur. J Appl Biomech: 2010;26 (1):10-6. Chen G, Schmutz B, Wullschleger M, Pearcy MJ, Schuetz MA. Computational investigations of mechanical failures of internal plate fixation. Proc Inst Mech Eng H: 2010;224 (1):119-26. 19. Ries Z, Hansen K, Bottlang M et al. Healing results of periprosthetic distal femur fractures treated with far cortical locking technology: a preliminary retrospective study. Iowa Orthop J: 2013;33:7-11. 20. Panagiotis M. Classification of non-union. Injury: 2005;36S, S30-S37. Elkins J, Marsh JL, Lujan TJ. Motion Predicts Clinical Callus Formation Construct-Specific Finite Element Analysis of Supracondylar Femoral Fractures. J Bone Joint Surg Am: 2016;98:276-84d. Lujan TJ, Henderson CE, Madey SM. Locked Plating of Distal Femur Fractures Leads to Inconsistent and Asymmetric Callus Formation. J Orthop Trauma: 2010;24 (3):156-162. Bottlang M, Doornink J, Lujan TJ. Effects of Construct Stiffness on Healing of Fractures Stabilized with Locking Plates. J Bone Joint Surg Am: 2010;92 Suppl 2:12-22. Henderson CE, Kuhl LL, Fitzpatrick DC. Locking Plates for Distal Femur Fractures: Is There a Problem With Fracture Healing? J Orthop Trauma: 2011;25:S8-S14. Boulton C, Weaver MJ. Predictive factors of distal femoral fracture nonunion after lateral locked plating: A retrospective multicenter case-control study of 283 fractures. Injury: 2014;45 (3):554-559. 26. Michael Z, Mohit B, Daniel MJ. Operative Treatment of Acute Distal Femur Fractures: Systematic Review of 2 Comparative Studies and 45 Case Series (1989 to 2005). J Orthop Trauma: 2006;20 (5):366-371. 27. Editorial. Femoral and tibial blood supply: A trigger for nonunion? Injury: 2014;45:1665-1673. Schandelmaier P, Partenheimer A, Koenemann B, et al. Distal femoral fractures and LISS stabilization. Injury: 2001;32 (Suppl3):55-63. Kregor PJ, Stannard JA, Zlowodzki M, et al. Treatment of distal femur fractures using the less invasive stabilization system: surgical experience and early clinical results in 103 fractures. J Orthop Trauma: 2004;18:509-520. Fankhouser F, Gruber G, Schippinger G, et al. Minimal0invasive treatment of distal femoral fractures with the LISS (Less Invasive Stabilization System): a prospective study of 30 fractures with a follow up of 20 months. Acta Orthop Scand: 2004;75:56-60. Schutz M, Muller M, Regazzoni $\mathrm{P}$, et al. Use of the Less Invasive Stabilization System (LISS in patients with distal femoral (AO33) fractures: a prospective multicenter study. Arch Orthop Trauma Surg: 2005;125:102-108. Wong MK, Leung F, Chow SP. Treatment of distal femoral fractures in the elderly using a less invasive plating technique. Int Orthop: 2005:29:117-120. Vallier HA, Hennessey TA, Sontich JK, et al. Failure of LCP condylar plate fixation in the distal part of the femur. A report of 6 cases. J Bone Joint Surg Am: 2006;88:846-853. Kayali C, Agus H, Turgut A. Successful results of minimally invasive surgery for comminuted supracondylar femoral fractures with LISS: comparative study of multiply injured and isolated femoral fractures. J Orthop SCI: 2007;12:458-465. Gaines RJ, Sanders R, Sagi JC, et al. Titanium versus stainless steel locked plates for distal femur fractures: is there any difference? Paper presented at: OTA 2008 abstract. Femur. Denver, CO: 2008: 10-13. Harvin WH, L Oladeji LO, Della
Rocca DJ, et al. Working length and proximal screw constructs in plate osteosynthesis of distal femur fractures. Injury: 2017. in press. Disclosure: No significant relationships.

\section{PR356}

\section{WHAT IS THE VALUE OF RADIOLOGICAL STUDIES IN THE PROGNOSIS OF INTRA-ARTICULAR CALCANEUS FRACTURES?}

\section{J. Ferrero Recasens $^{\text {I }}$, E. Vacas $^{2}$, G. Luengo Alonso ${ }^{3}$, P. Caba $\overline{D o u s s o u x}^{2}$, J. Vilá Y Rico ${ }^{2}$}

${ }^{1}$ CirugÍa Ortopedica Y TraumatologÍa, HOSPITAL UNIVERSITARIO 12 DE OCTUBRE, MADRID/SPAIN, ${ }^{2}$ Orthopaedic Surgery And Traumatology, Hospital Universitario 12 de Octubre, Madrid/SPAIN, ${ }^{3}$ Cirugia Ortopedica Y Traumatologia, Hospital 12 de Octubre, Madrid/SPAIN

Introduction: The objective of this study is to present a description of the epidemiology of calcaneal fractures in our center and to study the correlation between the radiological-parameters and the clinicalresults of these fractures.

Material and methods: This is a retrospective study of 44 intraarticular calcaneus fractures in 37 patients, operated between January2012-January2016. 79.6\% were men (35) with a middle age of 36.1years (13-58), with a mean follow-up of 20 months (2-50) The mean preoperative Böhler Angle (BA) was $22.8^{\circ}\left(0^{\circ}-54^{\circ}\right)$, with $35 \%$ presenting a value within normality. The mean preoperative Gissane Angle (GA) was $116.6^{\circ}\left(94^{\circ}-137^{\circ}\right)$, presenting a $36 \%$ a value within normality. According to Sanders, 25\% were type2, 47.7\% type 3 and $27.3 \%$ type 4 , being measured by 2 independent evaluators (junior/ senior).

Results: The mean postoperative BA was $31^{\circ}\left(17^{\circ}-50^{\circ}\right)$, the anatomical reduction was achieved in $68 \%$ (30), with an infracorrection in $21 \%$ and an overcorrection in $11 \%$. The average postoperative GA was $128^{\circ}\left(115^{\circ}-142^{\circ}\right)$, obtaining a good reduction in $95 \%$. The mean postoperative AOFAS score was 67points (20-100). There were worse AOFAS results with statistically significant differences in relation to Sanders: type2-73.4 (95\%CI: 72.7-73.9), type 3-71.3 (95\%CI: 70.9-71.7) and type4- 54.7 (95\%CI: 54.2-55.3) The interobserver agreement for Sanders was moderate (k0.47).Regarding the correction of Böhler, significant differences were found in AOFAS. The mean score $(+95 \% \mathrm{CI})$ in patients with corrected angle was 66.8 (95\%CI: 66.4-67.2), infracorrection58.8\% (58\%CI: 57.8-59.1) and overcorrected 86,4 (95\%CI: 85.2-87.3).

Conclusion: Based on the results obtained in our sample, correction of the BA and the Sanders-classification may have a prognostic value independent of results on functional scales (AOFAS) and reincorporation to previous employment. On the other hand, the low values in the AOFA scale are related to lower rates of reincorporation to the work activity.

References: Bruce J, Sutherland A. Surgical versus conservative interventions for displaced intra-articular calcaneal fractures. Cochrane Database Syst Rev 2013;1:CD008628. http://dx.doi.org/ 10.1002/14651858.CD008628.pub2. Review. PubMed PMID:23440830 Jan 31. Brauer CA, Manns BJ, Ko M, Donaldson C, Buckley R (2005) An economic evaluation of operative compared with nonoperative management of displaced intra-articular calcaneal fractures.. J Bone Joint Surg 87:2741-2749 Buckley RE, Tough S, McCormack R, et al. Operative compared with nonoperative treatment of displaced intra-articular calcaneal fractures: a prospective, randomized, controlled multicenter trial. J Bone Joint Surg Am. 
2002;84-A:1733-1744. Bhattacharya R, Vassan UT, Finn P, et al. Sanders classification of fractures of the os calcis. An analysis of inter- and intra-observer variability. $J$ Bone Joint Surg $\mathrm{Br}$. 2005;87:205-208. Humphrey CA, Dirschl DR, Ellis TJ. Interobserver reliability of a CT-based fracture classification system. J Orthop Trauma. 2005; 19:616-622. Loucks C, Buckley R (1999) Bohler's angle: correlation with outcome in displaced intra-articular calcaneal fractures. J Orthop Trauma 13:554-558 Shuler FD, Conti SF, Gruen GS, Abidi NA (2001) Wound healing risk factors after open reduction and internal fixation of calcaneal fractures: does correction of Bohler's angle alter outcomes? Orthop Clin North Am 32:187-192 SooHoo NF, Shuler M, Fleming LL. Evaluation of the validity of the AOFAS clinical rating systems by correlation to the SF-36. Foot Ankle Int. 2003;24:50-55. Epstein N, Chandran S, Chou L. Current concepts review: intra-articular fractures of the calcaneus. Foot Ankle Int. 2012;33:79-86. DeWall M, Henderson CE, McKinley TO, Phelps T, Dolan L, Marsh JL. Percutaneous reduction and fixation of displaced intra-articular calcaneus fractures. J Orthop Trauma 2010;24 (8):466-72. Eastwood DM, Langkamer VG, Atkins RM (1993) Intraarticular fractures of the calcaneum. Part II: open reduction and internal fixation by the extended lateral transcalcaneal approach. J Bone Joint Surg Br 75:189-195 Makki D, Alnajjar HM, Walkay S, et al. Osteosynthesis of displaced intra-articular fractures of the calcaneum: a long-term review of 47 cases. J Bone Joint Surg Br. 2010;92:693-700

Disclosure: No significant relationships.

\section{PR357}

\section{WHEN DO YOU TREAT OPEN FRACTURES WITHOUT EMERGENCY DEBRIDEMENT?}

\author{
A. Kawakubo, T. Kawamura
}

Orthopeadics, Kitasato University, Sagamihara/JAPAN

Introduction: We believe that treatment of open fractures requires debridement within 6 hours after injury (the 'golden hour'). Otherwise, the rate of infection after surgery is significantly increased. But some literature reports that open fractures do not always require this emergency procedure.

Material and methods: Therefore, we compared the cases that underwent emergency debridement (Group E) with cases that did not (Group W), and evaluated the rates of infection after surgery. A total of 96 limbs were evaluated. Mean age was 45.34 years. We evaluated the rate of infection after surgery.

Results: There were 83 limbs in Group E and 13 limbs in group W. The mean waiting period until surgery was 3.6 days (range: 1-14 days). The rate of infection after surgery was 2 limbs (2.4\%) in group $\mathrm{E}$, and 0 limbs $(0 \%)$ in group $\mathrm{W}$. The infection cases were classified as Gustilo IIIa in 1 patient, and IIIc in 1 patient. Both infections resolved.

Conclusion: Group W contained many cases classified as Gustilo I or II, so we have to omit the cases of severe contusion or dirty wounds. Overall, 12 of the fractured limbs $(92 \%)$ in group $\mathrm{W}$ were upper limbs. Many cases of lower limb fracture require emergency surgery in order to stabilize the soft tissue, or due to the severity of the conditions unrelated to open fracture. We suggest that in cases of Gustilo I or II upper limb open fractures, emergency surgery is not always needed under the bad condition.

References:

Disclosure: No significant relationships.

\section{PR358}

\section{PATIENT-REPORTED OUTCOMES AFTER A DISTAL RADIUS FRACTURE IN ADULTS. A PROLONGED FOLLOW UP}

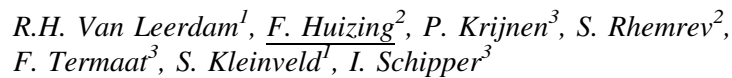

${ }^{1}$ Surgery, Haga Hospital, Den Haag/NETHERLANDS, ${ }^{2}$ Surgery, HMC, Den Haag/NETHERLANDS, ${ }^{3}$ Surgery, Leiden University Medical Center, Leiden/NETHERLANDS

Introduction: The purpose of this study was to investigate the longterm patient-reported functional outcome and health-related quality of life after a distal radius fracture in adults.

Material and methods: We reviewed 823 patients with a distal radius fracture treated either conservatively or operatively in 2012 . The PRWE and EuroQol-5D were sent by mail. Multiple linear regression analysis was conducted with the overall PRWE score as the dependent variable and age, gender, AO fracture type (A, B or C), dominance of the fractured hand and treatment as the independent variables.

Results: 285 patients (35\%) responded after a mean follow-up of 3.8 years. Mean PRWE score was 10.8. Mean EQ-5D index value was 0.88 and the mean EQ VAS score for self-rated health status was 79.8. In the multivariable analysis, PRWE scores were not associated with gender, age, dominance of fracture side or AO fracture type $(\mathrm{p}>$ 0.05). Conservatively treated patients had significant lower PRWE scores compared to operatively treated patients (mean difference -8.9 points, 95\% CI -13.4 - -4.3). There were no significant differences between groups regarding the EQ-5D index value. The EQ VAS score for patients aged 65 and older was significant lower than that of younger patients $(\mathrm{p}=0.04)$.

Conclusion: Long-term outcome after distal radius fractures is generally good. Patients with conservatively treated distal radius fractures have significantly less pain and better wrist function after long-term follow-up than patients with operatively treated fractures. These results need to be confirmed in a large prospective study.

References: 1. Chung KC, Spilson SV. The frequency and epidemiology of hand and forearm fractures in the United States. The Journal of hand surgery. 2001;26:908-915. 2. Chung KC, Kotsis SV, Kim HM. Predictors of functional outcomes after surgical treatment of distal radius fractures. J Hand Surg Am. 2007;32:76-83. 3. Ranjeet N, Estrella EP. Distal radius fractures: does a radiologically acceptable reduction really change the result? Journal of clinical and diagnostic research : JCDR. 2012;6:1388-1392. 4. Rozental TD, Beredjiklian PK, Steinberg DR, et al. Open fractures of the distal radius. The Journal of hand surgery. 2002;27:77-85. 5. Plant CE, Parsons NR, Costa ML. Do radiological and functional outcomes correlate for fractures of the distal radius? Bone Joint J. 2017;99B:376-382. 6. Rothrock NE, Kaiser KA, Cella D. Developing a valid patient-reported outcome measure. Clin Pharmacol Ther. 2011;90:737-742. 7. Kleinlugtenbelt YV, Nienhuis RW, Bhandari M, et al. Are validated outcome measures used in distal radial fractures truly valid? A critical assessment using the Consensus-based Standards for the selection of health Measurement INstruments (COSMIN) checklist. Bone Joint Res. 2016;5:153-161. 8. MacDermid JC, Tottenham V. Responsiveness of the disability of the arm, shoulder and hand (DASH) and patient-rated wrist/hand evaluation (PRWHE) in evaluating change after hand therapy. Journal of hand therapy : official journal of the American Society of Hand Therapists. 2004;17:18-23. 9. Hudak ML, Farrell EE, Rosenberg AA, et al. A multicenter randomized, masked comparison trial of natural versus synthetic surfactant for the treatment of respiratory distress syndrome. 
The Journal of pediatrics. 1996;128:396-406. 10. Brooks R. EuroQol: the current state of play. Health Policy. 1996;37:53-72. 11. Ware JE. The MOS 36-item Short Form Health Survey (SF36). Baltimore: Williams \& Wilkens; 1996. 12. MacDermid JC, Turgeon T, Richards RS, et al. Patient rating of wrist pain and disability: a reliable and valid measurement tool. J Orthop Trauma. 1998;12:577-586. 13. MacDermid J. The patient-rated wrist evaluation (PRWE) user manual. June 2011. 14. Lamers LM, Stalmeier PF, McDonnell J, et al. [Measuring the quality of life in economic evaluations: the Dutch EQ5D tariff]. Nederlands tijdschrift voor geneeskunde. 2005;149:15741578. 15. Marsh JL, Slongo TF, Agel J, et al. Fracture and dislocation classification compendium - 2007: Orthopaedic Trauma Association classification, database and outcomes committee. J Orthop Trauma. 2007;21:S1-133. 16. Müller ME, Müller ME. The Comprehensive classification of fractures of long bones. Berlin; New York: SpringerVerlag; 1990. 17. Walenkamp MM, de Muinck Keizer RJ, Goslings JC, et al. The Minimum Clinically Important Difference of the Patient-rated Wrist Evaluation Score for Patients With Distal Radius Fractures. Clinical orthopaedics and related research. 2015;473:3235-3241. 18. Arora R, Lutz M, Deml C, et al. A prospective randomized trial comparing nonoperative treatment with volar locking plate fixation for displaced and unstable distal radial fractures in patients sixty-five years of age and older. The Journal of bone and joint surgery American volume. 2011;93:2146-2153. 19. Egol KA, Walsh M, Romo-Cardoso S, et al. Distal radial fractures in the elderly: operative compared with nonoperative treatment. The Journal of bone and joint surgery American volume. 2010;92:18511857. 20. Bartl C, Stengel D, Bruckner T, et al. The treatment of displaced intra-articular distal radius fractures in elderly patients. Dtsch Arztebl Int. 2014;111:779-787. 21. Sharma H, Khare GN, Singh $\mathrm{S}$, et al. Outcomes and complications of fractures of distal radius (AO type B and C): volar plating versus nonoperative treatment. $J$ Orthop Sci. 2014;19:537-544. 22. Soong M, van Leerdam R, Guitton TG, et al. Fracture of the distal radius: risk factors for complications after locked volar plate fixation. The Journal of hand surgery. 2011;36:3-9.

Disclosure: No significant relationships.

\section{PR359}

\section{COMBINED INJURIES OF THE SHOULDER GIRDLE: EPIDEMIOLOGICAL DATA AND CONCOMITANT INJURIES OF STERNOCLAVICULAR JOINT, ACROMIOCLAVICULAR JOINT AND CLAVICLE IN GERMANY}

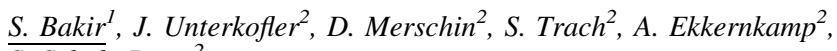
S. Schulz-Drost ${ }^{2}$

${ }^{1}$ Unfallchirurgie Und Orthopädie, Unfallkrankenhaus Berlin gGmbH, Berlin/GERMANY, ${ }^{2}$ Trauma- And Orthopedic Surgery, Unfallkrankenhaus Berlin, Berlin/GERMANY

Introduction: Regarding combined injuries of the shoulder girdle, there are marginal published data. This study reviews combined injuries between clavicle and corresponding joints.

Material and methods: The data are based on ICD-10-codes of all German hospitals, provided by the German Federeal Statistical Office. The ICD-10-codes S42.01, S42.02, S42.03 (clavicle fracture medial, midshaft, lateral) as well as S43.1 (acromioclavicular joint dislocation) and S43.2 (sternoclavicular joint dislocation) are evaluated for a period of three years.

Results: A total number of 100609 cases with one of the named diagnosis is analyzed whereof $1.79 \%$ present combined shoulder girdle injuries. The most common combinations are between lateral clavicle fracture and acromioclavicular joint dislocation $(\mathrm{n}=570)$ as well as between medial and midshaft clavicle fracture $(n=543)$. In addition to soft tissue injuries there are particularly concomitant thoracic and cranial injuries. They are likely attributed to the severity of the trauma mechanism in case of these shoulder girdle injuries. There is interestingly a different frequency of severe concomitant injuries depending on localisaton of the main trauma: craniocerebral injuries are especially associated with traumata of the lateral shoulder girdle whereas serial rib fractures are more common in medial injuries.

Conclusion: The combined injuries which are rarely registrated and seem to occur more prevalent in reality than expected so far. There is the risk of missing further injuries while focussing on a diagnosed mono injury. Therefore, a special attention should be turned to combined injuries at extensive trauma mechanism to avoid negative longterm consequences because of a delayed diagnosis.

References:

Disclosure: No significant relationships.

\section{PR360}

\section{POPULATION-BASED EPIDEMIOLOGY OF 344 CALCANEUS FRACTURES}

M.I. Horodyskyy $^{\text {I }}$, R. Elsoe ${ }^{2}$, I. Kjaer ${ }^{2}$, P. Larsen $^{2}$

${ }^{1}$ Ortopaedics Surgeon, Aalborg Universitets Hospital, Aalborg/ DENMARK, ${ }^{2}$ Ortopaedic, Aalborg Universitets Hospital, Aalborg/ DENMARK

Introduction: The literature lacks recent epidemiology studies of the incidence, trauma mechanism and fracture classification of calcaneus fractures. The purpose of the present study was to provide up-to-date information concerning the incidence of calcaneus fractures in a large and complete population.

Material and methods: Population-based epidemiological study of all patients treated for a calcaneus fracture in a 6-year period from 2005 to 2010 . The study population included an average population of 576,364 . The average population was divided into ten-year agegroups. Retrospective reviews of clinical and radiological records was performed. All fractures were classified according to the AO and Sanders classifications.

Results: A total of 328 patients were treated for 343 calcaneus fractures between 2005 and 2010. The mean age at time of fracture was 47.6 (19.9 SD) years. The mean age for males was 43.0 (18.1SD) years, and for females 57.5 (20.0SD) years. The gender distribution was $68.4 \%$ males and $31.8 \%$ females. The overall incidence of calcaneus fractures between 2005 and 2014 was $9.9 / 100,000 /$ year. For males, the incidence was 13.6/100,000/year, and for females, 6.3/ $100,000 / y e a r$. The incidence divided into age groups and gender shows an increase in incidence among young and middle-aged men. Conclusion: This study shows an incidence of 9.9/100,000/year during a six-year period between 2005 and 2010. The most common fracture type in all age groups was AO type 83-A (extraarticular), representing $51.3 \%$ of all fractures. The predominant mode of injury was fall from above $1 \mathrm{~m}(65.6 \%)$ followed by fall from standing height (8.8\%).

References: There are not references Disclosure: No significant relationships. 
PR361

COMPLICATIONS AFTER PROXIMAL FEMORAL NAIL IMPLANTATION - IS AN INCREASED BODY MASS INDEX A RISK FACTOR?

$\underline{\text { K. Jenny }}^{I}$, D. Sauter ${ }^{2}$

${ }^{1}$ Orthopädie Und Traumatologie, Kantonsspital Münsterlingen, Münsterlingen/SWITZERLAND, ${ }^{2}$ Orthopaedics \& Traumatology, Cantonal Hospital Münsterlingen, Münsterlingen/SWITZERLAND

Introduction: An increasing prevalence of obesity is described worldwide. In countless traumatological or orthopedic procedures, e.g. hip replacement, a high BMI is considered as an independent risk factor. Is a high BMI a risk factor for complications following the implantation of intramedullary nails in the case of pertrochanteric femur fractures?

Material and methods: In the context of a retrospective study we analyzed all patients $(n=524)$ who were treated with a intramedullary nail at our clinic from 2009 to 2016 due to the presence of a pertrochanteric fracture. In 485 cases $(93 \%)$ it was possible to calculate the BMI from the medical record. The AO classification showed 165 (34\%) A1, 206 (42\%) A2 and 114 (24\%) A3 fractures.

Results: The average BMI of all patients was $24.2 \mathrm{~kg} / \mathrm{m} 2$. The patient group with complications showed a significant $(\mathrm{p}=0.003)$ higher BMI $(25.9 \mathrm{~kg} / \mathrm{m} 2)$ than the patient group without complications $(24.0$ $\mathrm{kg} / \mathrm{m} 2$ ). A significant difference was also found in the subgroup analysis, separated by gender. A total of 51 complications occurred in 485 cases $(11 \%)$. Among the patients with complications there were 11 A1 (BMI 25.2kg/m2), 22 A2 (BMI 25.6kg/m2) and 18 A3 (BMI $26.8 \mathrm{~kg} / \mathrm{m} 2)$ fractures. There was no evidence of a significant difference within these subgroups, with regard to the fracture severity.

Conclusion: Implant-related complications in $11 \%$ of the cases treated with proximal femoral nailing are in line with the current literature. With our study we were able to describe a direct relationship between an increased BMI and complications following nail osteosynthesis in pertochenteric femur fractures in detail.

References: Effect of Body Mass Index on Complications and Reoperations After Total Hip Arthroplasty Wagner ER, Kamath AF, Fruth KM, Harmsen WS, Berry DJ. J Bone Joint Surg Am. 2016 Feb 3; 98 (3) Complications After Hip Nailing for Fractures Mavrogenis AF, Panagopoulos GN, Megaloikonomos PD, Igoumenou VG, Galanopoulos I, Vottis CT, Karabinas P, Koulouvaris P, Kontogeorgakos VA, Vlamis J, Papagelopoulos PJ. Orthopedics. 2016 Jan-Feb; 39 (1)

Disclosure: No significant relationships.

\section{PR362}

\section{VARIATIONS AND FIXATION METHODS OF QUADRILATERAL PLATE FRACTURES OF THE ACETABULUM}

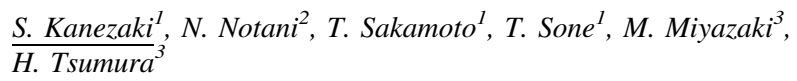

${ }^{1}$ Orthopedic Surgery/advanced Trauma, Emergency And Critical Care Center, Oita University Hospital, Yufu/JAPAN, ${ }^{2}$ Orthopedic Surgery/ Advanced Trauma, Emergency And Critical Care Center, Oita University Hospital, Yufu/JAPAN, ${ }^{3}$ Orthopedic Surgery, Oita University Hospital, Yufu/JAPAN
Introduction: It is challenging to reduce and stabilize displaced quadrilateral plate (QLP) fractures. Although QLP fractures can be fixed with various methods, there are no instruction or classifications for stabilization of QLP based on morphology of QLP fragments. Objectives of this study are to classify QLP fractures based on its morphologic patterns and to review the cases of acetabular fractures with QLP fragments.

Material and methods: From 2013 to 2016, operatively treated 20 cases of acetabular fractures were enrolled. Mean age was 55 yearold. Eleven cases had displacement of QLP fragments (QLP group). We compared quality of reduction between QLP and control group. QLP fractures were classified as "High fracture": a fracture above the line between the caudal edge of upper pubic ramus and the greater sciatic notch, and as "Low fracture" : a fracture below the line. Fracture patterns involving QLP fragments and fixation methods of QLP fragments were investigated.

Results: Eight of ten cases were anatomic in control group. In QLP group, only 3 of 11 were anatomic. There were seven "High fractures". Four cases were classified in "Low fractures". Among high fractures, two fractures were fixed with infrapectineal plates, two with screws, three with buttress plates (BP) and screws, and one without fixation. Among low fractures, one was fixed with posterior plates, two with BPs and screws. Free QLP fragment was fixed only with a BP.

Conclusion: Low fractures were difficult to fix rigidly only with BPs and needed paticular attension. Classifying variations of QLP fragments would help surgeon develop preoperative plans for reliable fixations.

References: 1) White G, Kanakaris NK, Faour O, et al. Quadrilateral plate fractures of the acetabulum: An update. Injury; 44:159-167 (2013) 2) Qureshi AA, Archdeacon MT, Jenkins MA, et al. Infrapectineal Plating for Acetabular Fractures: A Technical Adjunct to Internal Fixation. J Orthop Trauma;18:175-178 (2004) 3) Kistler BJ, Smithson IR, Cooper SA, et al. Are Quadrilateral Surface Buttress Plates Comparable to Traditional Forms of Transverse Acetabular Fracture Fixation? Clin Orthop Relat Res; 472:3353-3361 (2014) Disclosure: No significant relationships.

\section{PR363}

\section{CLINICAL OUTCOMES OF INTRAMEDULLARY NAILING WITH CONTRALATERAL SIDE NAIL IN BOWED ATYPICAL FEMUR FRACTURES}

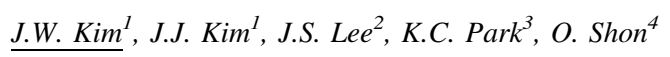

${ }^{1}$ Orthopaedic, Asan Medical Center, Seoul/KOREA, REPUBLIC OF, ${ }^{2}$ Orthopedic Surgery, Haeundae Paik Hospital, Inje University, Busan/KOREA, REPUBLIC OF, ${ }^{3}$ Orthopedic Surgery, Hanyang University Guri Hospital, Guri/KOREA, REPUBLIC OF,

${ }^{4}$ Orthopedic Surgery, Yeungnam University Hospital, Daegu/ KOREA, REPUBLIC OF

Introduction: Diaphyseal AFFs have more varus deformity in the femur compared to subtrochanteric AFFs. The purpose was to determine clinical outcomes of intramedullary nailing (IMN) with contralateral side nail in bowed atypical femur fractures (AFFs). However, currently available intramedullary nail system (IMN) showed a poor fit in severely bowed (varus) femur.

Material and methods: We retrospectively reviewed bowed femur fractures treated with contralateral side of IMN. In total, 17 patients with 18 cases were included. Surgical outcomes involved complication, union time, and femoral bowing, leg length discrepancy (LLD) 
were evaluated. We evaluate of surgical safety with intraoperative and postoperative complications, and compared preoperative and postoperative LLD.

Results: The average age was 76.3 years. There were 12 cases of complete AFFs and 6 cases of incomplete AFFs. Eight patients $(72.7 \%)$ had history of bisphosphonate use with average 71.1 monthuse. All cases achieved bone union without secondary intervention. There were no intraoperative fractures or postoperative complications. The average union time was 24.6 weeks and 12.0. weeks in complete and incomplete AFFs, respectively. The average preoperative femoral bowing in coronal and sagittal plate was $9.8^{\circ}$ and $16.6^{\circ}$, respectively, while postoperative bowing was $6.8^{\circ}, 11.3^{\circ}$, respectively. The average change of angle was $3.0^{\circ}$ in coronal plane and $5.3^{\circ}$ in sagittal plane. The mean change of LLD was $6.5 \mathrm{~mm}$.

Conclusion: The use of contralateral side of nail in bowed femur showed good clinical outcomes without complications. In severely bowed femur, this technique can be a useful.

References: 1. Donnelly E, Meredith DS, Nguyen JT, Gladnick BP, Rebolledo BJ, Shaffer AD, et al. Reduced cortical bone compositional heterogeneity with bisphosphonate treatment in postmenopausal women with intertrochanteric and subtrochanteric fractures. Journal of bone and mineral research : the official journal of the American Society for Bone and Mineral Research. 2012;27:672-678. 2. Koh JS, Goh SK, Png MA, Ng AC, Howe TS. Distribution of atypical fractures and cortical stress lesions in the femur: implications on pathophysiology. Singapore medical journal. 2011;52:77-80. 3. Park JH, Lee Y, Shon OJ, Shon HC, Kim JW. Surgical tips of intramedullary nailing in severely bowed femurs in atypical femur fractures: Simulation with 3D printed model. Injury. 2016;47:1318-1324. 4. Kim JW, Kim H, Oh CW, Kim JW, Shon OJ, Byun YS, et al. Surgical outcomes of intramedullary nailing for diaphyseal atypical femur fractures: is it safe to modify a nail entry in bowed femur? Arch Orthop Trauma Surg. 2017.

Disclosure: No significant relationships.

\section{PR364}

\section{THE NAIL-SHAFT-AXIS OF THE OF PROXIMAL FEMORAL NAIL ANTIROTATION (PFNA) IS AN IMPORTANT PROGNOSTIC FACTOR IN INTERTROCHANTERIC FRACTURES}

\author{
K. Boernert ${ }^{l}$, F.J.P. Beeres ${ }^{1}$, C. Jiamton ${ }^{2}$, R. Babst ${ }^{1}$, B. Link $^{l}$
}

${ }^{1}$ Department Of Orthopaedic And Trauma Surgery, Luzerner Kantonsspital, Luzern/SWITZERLAND, ${ }^{2}$ Institute Of Orthopaedics, Lerdsin Hospital, Bangkok/THAILAND

Introduction: Intertrochanteric fractures are frequent ${ }^{1}$. Internal fixation by intramedullary nailing is commonly considered safe and reliable to treat these fractures ${ }^{2}$. However, postoperative varus displacement and consecutive cut-out are reported in $6.5-21.2 \%^{3,4}$. The primary objective of this study was to analyse the number of complications after osteosynthesis with PFN-A and secondly to identify risk factors for secondary varus displacement.

Material and methods: 101 intertrochanteric fractures treated with PFN-A in a level 1 trauma centre were analysed retrospectively. Follow-up was at least 3 months. We analysed standardised pre-and postoperative radiographs concerning the following parameters: fracture classification, caput-collum-diaphyseal (CCD) angle, adequacy of reduction, blade position, tip-apex distance (TAD) and nailshaft axis.
Results: 24 patients experienced complications. These can be divided into non-implant related complications (33\%) such as surgical site infections, delayed or non-union and implant-related complications $(66.67 \%)$ such as cut-out, nail breakage and tractus irritation symptoms. Furthermore, we could identify risk factors for complications after osteosynthesis with PFN-A. Initial postoperative varus alignment, an improper location of the blade and a TAD $>25 \mathrm{~mm}$ were significant risk factors for postoperative secondary varus displacement and cut-out. Additionally, we identified a lateral nai-shaft axis as significant risk factor.

Conclusion: In addition to already known risk factors for secondary varus displacement and cut-out, this study describes a lateral nailshaft axis as another risk factor. Knowing about this mal-positioning and avoiding it by consequently using a more medial insertion point to prevent medialisation of the proximal fragment by the nail and consecutive varus collapse may help to further decrease postoperative morbidity and mortality.

References: 1. Dhanwal D, Dennison E, Harvey N, Cooper C. Epidemiology of hip fracture: Worldwide geographic variation. Indian $J$ Orthop. 2011;45 (1):15. doi:10.4103/0019-5413.73656. 2. Anglen JO, Weinstein JN, American Board of Orthopaedic Surgery Research Committee JN. Nail or plate fixation of intertrochanteric hip fractures: changing pattern of practice. A review of the American Board of Orthopaedic Surgery Database. J Bone Joint Surg Am. 2008;90 (4):700-707. doi:10.2106/JBJS.G.00517 3. Gadegone WM, Salphale YS. Proximal femoral nail - an analysis of 100 cases of proximal femoral fractures with an average follow up of 1 year. Int Orthop. 2007;31 (3):403-408. doi:10.1007/s00264-006-0170-3 [doi] 4. Fogagnolo F, Kfuri M, Paccola CAJ. Intramedullary fixation of pertrochanteric hip fractures with the short AO-ASIF proximal femoral nail. Arch Orthop Trauma Surg. 2004;124 (1):31-37. doi:10.1007/ s00402-003-0586-9

Disclosure: No significant relationships.

\section{PR365}

\section{OUR EXPERIENCE TREATING ACETATULAR FRACTURES}

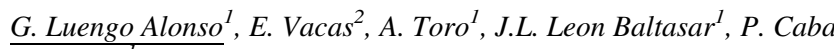 \\ Doussoux
}

${ }^{1}$ Cirugia Ortopedica Y Traumatologia, Hospital 12 de Octubre, Madrid/SPAIN, ${ }^{2}$ Orthopaedic Surgery And Traumatology, Hospital Universitario 12 de Octubre, Madrid/SPAIN

Introduction: Acetabular fractures increase posttraumatic arthritis risk and thus total hip arthroplasty (THA) incidence. These fractures related to high energy mechanism are usually complex, and long surgeries are required, associating complications.

Material and methods: We retrospectively study a cohort of 207 consecutive patients who suffered from acetabular fractures were treated at our center from 2002 to 2014 . 109 were treated surgically, with a minimum follow-up of 3 years. Acetabular fracture pattern was classified according to Judet-Letorunel and AO Classification. We studied variables according to Matta's nomogram and others

Results: Mean aged was 41.01 yo (SD12.44) (range 16-83). Fifteen developed osteoarthritis requiring THA, most of them one or two years after acetabular fractures.

We found significate statistically relationship related to THA after acetabular fractures in: femoral dislocation $(\mathrm{P}<0.01)$, seagull sign $(\mathrm{P}$ $<0.01)$, femoral head cartilage lesion $(\mathrm{P}<0.01)$, marginal acetabular impaction $(\mathrm{P}<0.05)$, and fracture reduction $(\mathrm{P}=0.023)$. No relationship was found in, posterior wall involvement $(\mathrm{P}=0.062)$, 
acetabular roof $(\mathrm{P}=0.142)$, surgical approach $(\mathrm{P}=0.577)$ age $(\mathrm{P}=$ $0.98)$, sex $(P=0.759)$, time to surgery $(P=0.68)$ or type of fracture $(P$ $=0.103)$. Surgical time was not statistically related to infection, avascular necrosis or neurovascular damage.

Conclusion: Femoral head dislocation, seagull sign, femoral head cartilage lesion, marginal acetabular impaction, and fracture reduction are stadistically related with requiring THA. Our experience is similar to others, so predictors and most frequent complications reported could help us to distinguishing which patients should keep a closer surveillance. Functional and clinical outcomes are encouraging to keep fixing these fractures.

References:

Disclosure: No significant relationships.

\section{PR366}

\section{DOES RETROPERITONEAL HEMORRHAGE REMAIN LIFE-THREATENING FOR PATIENTS WITH PELVIC FRACTURE? OVERCOMING A MAJOR PROBLEM IN THE ERA OF INTERVENTIONAL RADIOLOGY}

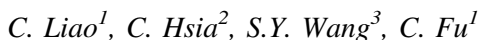

\section{${ }^{1}$ Trauma And Emergency Surgery, Chang Gung Memorial hospital, Taoyuan/TAIWAN, ${ }^{2}$ Surgery, Chang Gung Memorial hospital, Taoyuan/TAIWAN, ${ }^{3}$ Trauma And Emergency Surgery, Chang Gung Memorial Hospital, Taoyuan/TAIWAN}

Introduction: Conventionally, pelvic fracture-related acute retroperitoneal hemorrhage $(\mathrm{ARH})$ is life-threatening and difficult to control. However, the use of angioembolization to treat fracture-associated ARH improves the survival rate of patients with pelvic fractures. We attempted to elucidate the causes of death among patients with pelvic fractures in the era of interventional radiology. The role of angioembolization in the management of patients with pelvic fracture-related ARH was also examined.

Material and methods: We retrospectively reviewed a large case series of patients with pelvic fractures between January 2010 and December 2014. Comparisons were made between patients with and without ARH. In addition, the characteristics of mortality were delineated, whereas the causes of death in patients with pelvic fracture were discussed and analyzed.

Results: A total of 1070 patients' records were reviewed during the 60-month study period, and the overall mortality rate of pelvic fracture was $7.7 \%(82 / 1070)$. However, there were only seven patients who died due to uncontrolled ARH $(0.7 \%)$. The patients with ARH had more injuries to other organs than did the patients without ARH (head: $79.7 \%$ vs. $31.7 \%, p<0.001$; chest: $50.3 \%$ vs. $10.9 \%, p<$ 0.001 ; abdomen: $72.0 \%$ vs. $22.7 \%, p<0.001$; spine: $12.6 \%$ vs. $4.4 \%$, $p<0.001$; extremities: $69.2 \%$ vs. $44.3 \%, p<0.001$ ).

Conclusion: The treatment for pelvic fracture patients declared dead upon arrival is still limited. However, pelvic fracture-related ARH could be controlled effectively with angioembolization. In addition to $\mathrm{ARH}$, injuries to other organs may play a key role in the mortality of patients with pelvic fractures.

References: 1. The Diminishing Role of Pelvic Stability Evaluation in the Era of Computed Tomographic Scanning. (Medicine (Baltimore) 2016 Apr;95 (16):e3421. 2. The selection of diagnostic modalities in the management of pelvic fracture patients requiring transfers. World J Emerg Surg. 2015 Jul 30;10:33

Disclosure: No significant relationships.

\section{PR367}

\section{THE INTRA- AND INTEROBSERVER RELIABILITY OF THE TILE/AO, THE YOUNG\&BURGESS AND FFP CLASSIFICATION IN PELVIC TRAUMA}

\section{D.M. Thiesen, J. Berger-Groch, M.J. Hartel}

Department Of Trauma-, Hand- And Reconstructive Surgery, University Hospital Hamburg Eppendorf, Hamburg/GERMANY

Introduction: The purpose of this study was to measure the inter- and intraobserver reliability of three different classification systems (Tile/ AO, Young\&Burgess, FFP) in pelvic ring fractures. Different experience levels of the observers were included in the evaluation. The reliability of the Rommens classification system (FFP) originally designed for geriatric cases is analysed for the first time.

Material and methods: A total of 156 CT scans of patients with sacrum fractures were analysed. Four reviewers separately reviewed and classified CT-scans of pelvic fracture cases. The four observers had different experience levels ( $2 \mathrm{x}$ senior pelvic trauma surgeon, resident, medical student). The Tile/AO, the Young \& Burgess and FFP-Classification were compared. For intra-observer assessment another blinded reevaluation was carried out after two months. The main objective was to evaluate the reliability of the different classifications with special regard to the experience level of the observers. Results: The overall interobserver agreement for all classification systems is fair (ICC: OTA 0.55 , Y\&B 0.42, FFP 0.58). When collapsing the classifications to their main categories (e.g. type B or C fractures), substantial agreement between the experienced surgeons was shown (kappa: OTA 0.64, Y\&B 0.62, FFP 0.67). The inexperienced observers showed fair agreement in all systems (kappa: OTA 0.23 , Y\&B 0.23, FFP 0.44)

Conclusion: At least for the less experienced pelvic fracture classification systems seem to be unreliable. With increasing experience in pelvic fracture surgery all three classification systems show an increased reliability. The FFP system showed the best reliability for the less experienced participants.

References: 1. Tile M. Acute Pelvic Fractures: I. Causation and Classification. J. Am. Acad. Orthop. Surg. 1996 May;4 (3):143-151. 2. Young JW, Burgess AR, Brumback RJ, et al. Pelvic fractures: value of plain radiography in early assessment and management. Radiology. 1986 Aug;160 (2):445-451. 3. Rommens PM, Hofmann A. Comprehensive classification of fragility fractures of the pelvic ring: Recommendations for surgical treatment. Injury. 2013 Dec;44 (12):1733-1744. 4. Furey AJ, O'toole R V., Nascone JW, et al. Classification of Pelvic Fractures: Analysis of Inter- and Intraobserver Variability Using the Young-Burgess and Tile Classification Systems. Orthopedics. 2009 Jun 1;32 (6):401-406. 5. Koo H, Leveridge M, Thompson $\mathrm{C}$, et al. Interobserver reliability of the young-burgess and tile classification systems for fractures of the pelvic ring. J. Orthop. Trauma. 2008 Jul;22 (6):379-84. 6. Landis JR, Koch GG. The measurement of observer agreement for categorical data. Biometrics. 1977 Mar;33 (1):159-174. 7. Shrout PE, Fleiss JL. Intraclass correlations: uses in assessing rater reliability. Psychol. Bull. 1979 Mar;86 (2):420-428.

Disclosure: No significant relationships. 
PR368

\section{THE EFFECT OF MEDIAL CORTEX POSITIVE SUPPORTIVE REDUCTION FIXING PERTROCHANTERIC FRACTURE OF POSTEROMEDIAL LOSS WITH DYNAMIC HIP SCREW}

W. Choi ${ }^{1}$, M.R. $C h o^{2}$

${ }^{1}$ Orthopaedic Surgery, Daegu Catholic University Medical Center, Daegu/KOREA, REPUBLIC OF, ${ }^{2}$ Orthopadics, Daegu catholic university, daegu/KOREA, REPUBLIC OF

Introduction: We evaluated the radiological and clinical results of medial cortical supportive reduction using dynamic hip screw in patients with OTA $31 \mathrm{~A} 2$ fractures.

Material and methods: We enrolled 100 patients among OTA type 31A2 showing displaced lesser fragments (length of the cortical area longer than $2 \mathrm{~mm}$ ) on pelvis AP view. Positive medial cortical supports were assigned to Group $1(\mathrm{~N}=28)$, neutral medial cortical supports were Group $2(\mathrm{~N}=42)$, and negative medial cortical support were Group $3(\mathrm{~N}=30)$. Radiological results were evaluated by measuring changes in the femoral neck shaft angle and sliding distance of lag screws. Clinical outcomes of each groups were compared by means of the Walking ability score, which was proposed by Ceder. Results: Patients in group 1 showed significantly lower changes in the femoral neck shaft angle and the sliding distance, compared to patients in group 2 and 3. Also, patients with neutral medial cortical support showed significantly lower changes in the femoral neck shaft angle and the sliding distance, compared to patients with negative medial cortical support. Patients with positive medial cortical support showed significantly higher Walking ability scores than patients with negative medial cortical support $(\mathrm{P}=0.00)$. The use of trochanter stabilizing plate (TSP) or fixation using wires for posteromedial wall loss resulted in no significant changes in terms of the femoral neck shaft angle or the sliding distance Conclusion: The positive medial cortical supportive reduction enables reinforcement of the posteromedial stability, and thus prevents excessive force to be applied to the dynamic hip screw.

References:

1. Marmor M, Liddle K, Pekmezci M, Buckley J, Matityahu A. The effect of fracture pattern stability on implant loading in OTA type 31-A2 proximal femur fractures. J Orthop Trauma. 2013;27 (12):6839 .

2. Knobe M, Gradl G, Ladenburger A, Tarkin IS, Pape HC. Unstable intertrochanteric femur fractures: is there a consensus on definition and treatment in Germany? Clin Orthop Relat Res. 2013;471 (9):2831-40.

3. Parker MJ, Pryor GA. Gamma versus DHS nailing for extracapsular femoral fractures. Meta-analysis of ten randomised trials. Int Orthop. 1996;20 (3):163-8.

4. Anglen JO, Weinstein JN, American Board of Orthopaedic Surgery Research C. Nail or plate fixation of intertrochanteric hip fractures: changing pattern of practice. A review of the American Board of Orthopaedic Surgery Database. J Bone Joint Surg Am. 2008;90 (4):700-7.

5. Saudan M, Lubbeke A, Sadowski C, Riand N, Stern R, Hoffmeyer P. Pertrochanteric fractures: is there an advantage to an intramedullary nail?: a randomized, prospective study of 206 patients comparing the dynamic hip screw and proximal femoral nail. J Orthop Trauma. 2002;16 (6):386-93.

6. Madsen JE, Naess L, Aune AK, Alho A, Ekeland A, Stromsoe K. Dynamic hip screw with trochanteric stabilizing plate in the treatment of unstable proximal femoral fractures: a comparative study with the Gamma nail and compression hip screw. J Orthop Trauma. 1998;12 (4):241-8.

7. Jones JB. Screw fixation of the lesser trochanteric fragment. Clin Orthop Relat Res. 1977 (123):107.

8. Cho SH, Lee SH, Cho HL, Ku JH, Choi JH, Lee AJ. Additional fixations for sliding hip screws in treating unstable pertrochanteric femoral fractures (AO Type 31-A2): short-term clinical results. Clin Orthop Surg. 2011;3 (2):107-13.

9. Chang SM, Zhang YQ, Ma Z, Li Q, Dargel J, Eysel P. Fracture reduction with positive medial cortical support: a key element in stability reconstruction for the unstable pertrochanteric hip fractures. Arch Orthop Trauma Surg. 2015;135 (6):811-8.

10. Carr JB. The anterior and medial reduction of intertrochanteric fractures: a simple method to obtain a stable reduction. J Orthop Trauma. 2007;21 (7):485-9.

11. Doppelt SH. The sliding compression screw-today's best answer for stabilization of intertrochanteric hip fractures. Orthop Clin North Am. 1980;11 (3):507-23.

12. Kyle RF, Gustilo RB, Premer RF. Analysis of six hundred and twenty-two intertrochanteric hip fractures. J Bone Joint Surg Am. 1979;61 (2):216-21.

13. Ceder L, Lindberg L, Odberg E. Differentiated care of hip fracture in the elderly. Mean hospital days and results of rehabilitation. Acta Orthop Scand. 1980;51 (1):157-62.

14. Schumpelick W, Jantzen PM. A new principle in the operative treatment of trochanteric fractures of the femur. J Bone Joint Surg Am. 1955;37-A (4):693-8.

15. Clawson DK. Trochanteric Fractures Treated by the Sliding Screw Plate Fixation Method. J Trauma. 1964;4:737-52.

16. Ecker ML, Joyce JJ, 3rd, Kohl EJ. The treatment of trochanteric hip fractures using a compression screw. J Bone Joint Surg Am. 1975;57 (1):23-7.

17. Jacobs RR, Armstrong HJ, Whitaker JH, Pazell J. Treatment of 18 intertrochanteric hip fractures with a compression hip screw and a nail plate. J Trauma. 1976;16 (08):599-603.

18. Larsson S, Friberg S, Hansson LI. Trochanteric fractures. Influence of reduction and implant position on impaction and complications. Clin Orthop Relat Res. 1990 (259):130-9.

19. Steinberg GG, Desai SS, Kornwitz NA, Sullivan TJ. The intertrochanteric hip fracture. A retrospective analysis. Orthopedics. 1988;11 (2):265-73.

20. Kim WY, Han CH, Park JI, Kim JY. Failure of intertrochanteric fracture fixation with a dynamic hip screw in relation to pre-operative fracture stability and osteoporosis. Int Orthop. 2001;25 (6):360-2.

21. Hsu CE, Chiu YC, Tsai SH, Lin TC, Lee MH, Huang KC. Trochanter stabilising plate improves treatment outcomes in AO/OTA 31-A2 intertrochanteric fractures with critical thin femoral lateral walls. Injury. 2015;46 (6):1047-53.

Disclosure: No significant relationships.

\section{PR369}

\section{THE VOLUME-OUTCOME RELATIONSHIP FOR HIP FRACTURES: A SYSTEMATIC REVIEW AND META- ANALYSIS}

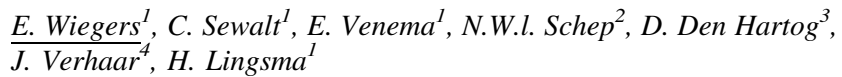

${ }^{1}$ Public Health, Erasmus MC, Rotterdam/NETHERLANDS, ${ }^{2}$ Trauma Unit, Department Of Surgery, Maasstad Ziekenhuis, Rotterdam/ NETHERLANDS, ${ }^{3}$ Surgery, Erasmus MC, Rotterdam/ NETHERLANDS, ${ }^{4}$ Orthopaedic Surgery, Erasmus MC, Rotterdam/ 


\section{NETHERLANDS}

Introduction: In the worldwide increasing elderly population, hip fractures contribute to high mortality and morbidity rates. Several studies that examined the relationship between hospital or surgeon volume and different health outcomes in hip fracture patients reported contradictory results. The aim of this systematic review and metaanalysis is to evaluate the volume-outcome relationship in hip fracture patients.

Material and methods: A search of various databases was performed up to February 2017 and articles that were included evaluated the relationship between hospital or surgical volume and health outcomes in hip fracture patients. Selection of relevant studies, data extraction and critical appraisal of the methodological quality were performed by two independent reviewers. A meta-analysis was performed to pool the adjusted estimates of the effect between volume and the outcomes in-hospital mortality and postoperative complications.

Results: In this systematic review, twenty articles with a total number of 830,767 patients were included. The qualitative analysis of these articles showed inconsistent results. Nine studies were further evaluated in the meta-analyses. There was a trend between hospital volume and in-hospital mortality (OR $0.76,95 \%$ CI $0.56-1.04, \mathrm{I}^{2}=$ $83 \%, \mathrm{p}=0.09$ ) and a non-significant effect in avoiding postoperative complications (OR 0.82, 95\% CI 0.65-1.03, $\mathrm{I}^{2}=71 \%, \mathrm{p}=0.09$ ). For surgeon volume, there was no association between surgeon volume and in-hospital mortality (OR $0.92,95 \%$ CI $0.76-1.12, \mathrm{I}^{2}=61 \%, \mathrm{p}=$ $0.41)$.

Conclusion: This systematic review and meta-analysis did find a potential relationship between hospital volume and in-hospital mortality and postoperative complications. Further research is needed to examine which factors drive this volume-outcome relationship.

References: 1. Kannus P, Parkkari J, Sievanen H, Heinonen A, Vuori I, Jarvinen M. Epidemiology of hip fractures. Bone. 1996;18 (1 Suppl):57S-63S. 2. Simunovic N, Devereaux PJ, Sprague S, Guyatt GH, Schemitsch E, Debeer J, et al. Effect of early surgery after hip fracture on mortality and complications: systematic review and metaanalysis. CMAJ. 2010;182 (15):1609-16. 3. Shervin N, Rubash HE, Katz JN. Orthopaedic procedure volume and patient outcomes: a systematic literature review. Clin Orthop Relat Res. 2007;457:35-41. 4. Vitale MA, Arons RR, Hyman JE, Skaggs DL, Roye DP, Vitale MG. The contribution of hospital volume, payer status, and other factors on the surgical outcomes of scoliosis patients: a review of 3,606 cases in the State of California. J Pediatr Orthop. 2005;25 (3):393-9. 5. Moher D, Liberati A, Tetzlaff J, Altman DG, Group P. Preferred reporting items for systematic reviews and meta-analyses: the PRISMA statement. BMJ. 2009;339:b2535. 6. Sanderson S, Tatt ID, Higgins JP. Tools for assessing quality and susceptibility to bias in observational studies in epidemiology: a systematic review and annotated bibliography. Int J Epidemiol. 2007;36 (3):666-76. 7. Browne JA, Pietrobon R, Olson SA. Hip fracture outcomes: does surgeon or hospital volume really matter? J Trauma. 2009;66 (3):80914. 8. Flood AB, Scott WR, Ewy W. Does Practice Make Perfect? Part I: The Relation between Hospital Volume and Outcomes for Selected Diagnostic Categories. Medical Care. 1984;22 (2):98-114. 9. Forte ML, Virnig BA, Swiontkowski MF, Bhandari M, Feldman R, Eberly LE, et al. Ninety-day mortality after intertrochanteric hip fracture: does provider volume matter? J Bone Joint Surg Am. 2010;92 (4):799-806. 10. Franzo A, Francescutti C, Simon G. Risk factors correlated with post-operative mortality for hip fracture surgery in the elderly: A population-based approach. Eur J Epidemiol. 2005;20 (12):985-91. 11. Genuario J, Koval KJ, Cantu RV, Spratt KF. Does hospital surgical volume affect in-hospital outcomes in surgically treated pelvic and acetabular fractures? Bull NYU Hosp Jt Dis. 2008;66 (4):282-9. 12. Hamilton BH, Hamilton VH. Estimating surgical volume-outcome relationships applying survival models: accounting for frailty and hospital fixed effects. Health Econ. 1997;6 (4):383-95. 13. Hamilton BH, Ho V. Does practice make perfect? Examining the relationship between hospital surgical volume and outcomes for hip fracture patients in Quebec. Med Care. 1998;36 (6):892-903. 14. Hentschker C, Mennicken R. The volume-outcome relationship and minimum volume standards-empirical evidence for Germany. Health Econ. 2015;24 (6):644-58. 15. Hughes RG, Garnick DW, Luft HS, McPhee SJ, Hunt SS. Hospital volume and patient outcomes. The case of hip fracture patients. Med Care. 1988;26 (11):1057-67. 16. Kristensen PK, Thillemann TM, Johnsen SP. Is bigger always better? A nationwide study of hip fracture unit volume, 30-day mortality, quality of in-hospital care, and length of hospital stay. Med Care. 2014;52 (12):1023-9. 17. Lavernia CJ. Hemiarthroplasty in hip fracture care: effects of surgical volume on short-term outcome. J Arthroplasty. 1998;13 (7):774-8. 18. Maceroli M, Nikkel LE, Mahmood B, Ketz JP, Qiu X, Ciminelli J, et al. Total Hip Arthroplasty for Femoral Neck Fractures: Improved Outcomes with Higher Hospital Volumes. J Orthop Trauma. 2016;30 (11):597-604. 19. Metcalfe D, Salim A, Olufajo O, Gabbe B, Zogg C, Harris MB, et al. Hospital case volume and outcomes for proximal femoral fractures in the USA: an observational study. BMJ Open. 2016;6 (4):e010743. 20. Riley G, Lubitz J. Outcomes of surgery among the Medicare aged: surgical volume and mortality. Health Care Financ Rev. 1985;7 (1):37-47. 21. Shah SN, Wainess RM, Karunakar MA. Hemiarthroplasty for femoral neck fracture in the elderly surgeon and hospital volume-related outcomes. J Arthroplasty. 2005;20 (4):503-8. 22. Sund R. Modeling the volume-effectiveness relationship in the case of hip fracture treatment in Finland. BMC Health Serv Res. 2010;10:238. 23. Treskes K, Voeten SC, Tol MC, Zuidema WP, Vermeulen J, Goslings JC, et al. Trauma surgery by general surgeons: Still an option for proximal femoral fractures? Injury. 2017;48 (2):339-44. 24. van Laarhoven JJ, van Lammeren GW, Houwert RM, van Laarhoven CJ, Hietbrink F, Leenen LP, et al. Isolated hip fracture care in an inclusive trauma system: A trauma system wide evaluation. Injury. 2015;46 (6):1042-6. 25. Castronuovo E, Pezzotti P, Franzo A, Di Lallo D, Guasticchi G. Early and late mortality in elderly patients after hip fracture: a cohort study using administrative health databases in the Lazio region, Italy. BMC Geriatr. 2011;11:37. 26. Guida P, Iacoviello M, Passantino A, Scrutinio D. Intra-hospital correlations among 30-day mortality rates in 18 different clinical and surgical settings. Int J Qual Health Care. 2016;28 (6):793-801. 27. Hentschker $\mathrm{C}$, Mennicken R. The volume-outcome relationship and minimum volume standards - Empirical evidence for Germany. Health Econ. 2015;24 (6):644-58. 28. Forte ML, Virnig BA, Swiontkowski MF, Bhandari M, Feldman R, Eberly LE, et al. Ninety-day mortality after intertrochanteric hip fracture: Does provider volume matter? J Bone Jt Surg Ser A. 2010;92 (4):799-806.

Disclosure: No significant relationships.

\section{PR370}

\section{SHORT DELAY TO ANGIOEMBOLIZATION IN BLEEDING} PELVIC FRACTURES: A SINGLE CENTER EXPERIENCE

P. Ciriano Hernandez, A. Moreno Hidalgo, M.I. Orue -Echebarria
Gonzalez, I. Grao Torrente, M.D. Pérez Díaz, F. Turégano Fuentes

Surgery, HOSPITAL GREGORIO MARAÑON, MADRID/SPAIN

Introduction: Reported times to angioembolization (AE) in severe bleeding from pelvic fractures varies between groups, and may contribute to the final outcome. We aimed to assess whether there had been differences in the percentage and delay to $\mathrm{AE}$ between patients 
admitted in weekdays (8h-15h) versus off-hours (weekends and admission after $15 \mathrm{~h}$ ) in our center.

Material and methods: Retrospective review of two cohorts of patients bleeding from pelvic fractures. Patients are divided depending on the time of arrival. We analyzed demographics, mechanism of injury, associated injuries, contrast extravasation (CE) on CT scan, need of embolization, management, ICU stay, and mortality. Correlation between $\mathrm{CE}$ on CT scan and angiography was also assessed.

Results: Overall, 104 patients underwent angiography, and 63 $(60,6 \%)$ had embolization. No differences were found in terms of demographics, mechanism of injury, associated injuries, and requirement of $\mathrm{AE}$ or abdominal surgery. $\mathrm{AE}$ was required in 21/30 patients $(70 \%)$ in the weekday group, and $42 / 74$ (56,8\%) off-hours, with the same median time delay (127 min. vs $128 \mathrm{~min}$, respectively). CE on CT scan was seen in 53 out of 104 patients, and was confirmed by angiography in $45(85 \%)$ of them. 15 out of 104 had AE despite an absence of CE on CT scan. No significant differences were found in mortality $(10,6 \%$ in weekdays vs. $15,4 \%$ off-hours).

Conclusion: There was a short delay from admission to AE even offhours, and a good correlation of CE on CT scan and angiography.

References:

Disclosure: No significant relationships.

\section{PR371}

\section{ASSOCIATED INJURIES WITH TRAUMATIC PELVIC FRACTURES}

I. Perez-Nuñez $^{1}$, L. Alonso Viana ${ }^{1}$, M. Fernandez Alvarez ${ }^{1}$, P. Colsa Gutierrez $^{2}$, V.J. Ovejero Gomez ${ }^{2}$, J.A. Blasco Velarde ${ }^{2}$, D. MoralesGarcia $^{2}$

${ }^{1}$ Traumatology And Orthopedic Surgery, UNIVERSITY HOSPITAL "MARQUES DE VALDECILLA", SANTANDER/SPAIN, ${ }^{2}$ Department Of Surgery, UNIVERSITY HOSPITAL "MARQUES DE VALDECILLA”, SANTANDER/SPAIN

Introduction: Pelvic fractures, by high energy trauma, present a high risk of associated injuries that compromise the functional and vital prognosis of the patients. The aim of the study was to correlate the different types of pelvic fractures, according to the Tile classification, with skeletal, visceral, vascular and nerve injuries; and analyze the impact on the hemodynamic status of these patients.

Material and methods: A preliminary study from June 2013 until January 2016 was carried out, which included patients who suffered high energy traumas. Low-energy traumas and deaths were excluded. For the study of the qualitative data, statistical analysis (Fisher test and analysis of logistic regression) were done. For the comparison of the quantitative and qualitative data Kruskal-Wallis analysis was done. It was evaluated the possible association between the type of pelvic fracture (according to Tile classification), the mechanism, the treatment performed, the associated injuries, the hemodynamic condition at the moment of the hospital admission and the discharge; and the need for transfusion during the stay.

Results: From the total of 42 patients, $69 \%(n=29)$ of them were males, mean age was 48.52 years. $45 \%(n=19)$ suffered traffic accidents and $26.2 \%(\mathrm{n}=11)$ precipitation. There was a different proportion in pelvic injuries, Tile A, B and C of $35.71 \%(n=15)$, $47.61 \%(n=20), 16.6 \%(n=7)$ of cases respectively. $54.8 \%(n=23)$ were surgically operated and $21.4 \%(n=9)$ needed temporary or definitive external fixator. Significant differences were found between fractures Tile A type and scapula fractures $(\mathrm{p}=0,032)$; Tile B and transforaminal fractures of the sacrum $(\mathrm{p}=0.033)$. Statistical trend between the Tile $\mathrm{C}$ and the wall rib injury was found, as in the Tile $\mathrm{B}$ and vertebral fractures. Visceral injuries were statistically associated with Tile B $(\mathrm{p}=0,049)$, being $42 \%(\mathrm{n}=8)$ of them, lung contusions, $15.8 \%(n=3)$ hepatic bruises and $10.5 \%(n=2)$ genito-urinary tract injuries. In vascular injuries, there was one case of Tile B type where existed a section of the left external iliac vein. In addition, $61.9 \%(\mathrm{n}=$ 26) needed blood transfusion along income, presenting $9.5 \%(n=4)$ of patients hypovolemic shock.

Conclusion: Pelvic fractures involve an alteration of hemodynamic status caused by pelvic injury itself, by the associated injuries or by the surgical procedures carried out. Tile A type pelvic fractures presents a statistically significant relationship with scapula fractures; the Tile B type was associated to transforaminal sacral fractures and visceral injuries (pulmonary $42 \%$ and hepatic contusions $15,8 \%$ and genitourinary injuries 10,5\%). The small number of cases of Tile $\mathrm{C}$ type fracture don't allow to perform any statistical association to any pathology, however, this type is the one that presents more cases of hemodynamic and thoracic injuries. There is no statistically significant relationship with $\mathrm{Hb}$ levels at the admission, before any surgical intervention or at the moment of the discharge.

References:

Disclosure: No significant relationships

PR372

\section{LONG-TERM QUALITY OF LIFE AND PHYSICAL FUNCTIONING AFTER PELVIC RING INJURIES} $\frac{\text { H. Banierink, K. Ten Duis, K.W. Wendt, E. Heineman, I.H.f. Reininga, }}{\text { F.F.a. Ijpma }}$

Trauma Surgery, University Medical Center Groningen, Groningen/ NETHERLANDS

Introduction: Aim of this study was to assess the long-term quality of life and physical functioning by using validated patient-reported outcome measures (PROMs) in a large cohort of patients who suffered from pelvic ring injuries. Additionally, results were compared to norm data of the general Dutch population.

Material and methods: All patients treated for pelvic ring injuries between 2007-2016 were included. Demographics, fracture type, injury mechanism, treatment and complications were evaluated. Adult patients received questionnaires concerning physical functioning (SMFA) and quality of life (EQ-5D). Descriptive statistics, Chisquare and independent samples t-test were performed for analyzing the results.

Results: A total of 444 patients (mean age 53 years) were included of which 292 patients were eligible for follow-up by means of PROMs. The response rate was $68 \%$ at a mean follow up of 4.4 (SD 2.6) years. Patients with type A, B or C injuries reported respectively a $13 \%$, $13 \%$ and $10 \%$ decrease in quality of life (EQ-5D) compared to norm data of the Dutch population. They reported a decrease in physical functioning (SMFA function score) of 52\% for type A, $43 \%$ for type $\mathrm{B}$ and $27 \%$ for type $\mathrm{C}$ injuries. For all types of injuries, a $45-48 \%$ decrease on the SMFA bother score was reported.

Conclusion: Pelvic ring injuries affect quality of life and physical functioning, even after years. Insights in the personal impact of these injuries can be gained by means of validated PROMs, and they allow comparison to norm data of the general population.

References:

Disclosure: No significant relationships. 
PR373

\section{OPTIMAL INTRAMEDULLARY NAILING FOR TROCHANTERIC FRACTURES: THE IMPORTANCE OF DISTAL LOCKING SCREW AND REDUCTION POSITION}

\section{T. Waki, Y. Nakanishi, K. Ito, R. Kato, T. Yano, S. Matsushima}

Orthopaedic Surgery, Akashi Medical Center, Akashi/JAPAN

Introduction: When addressing the issue of distal locking in gamma nails, there remains controversy whether to use it. Therefore, the purpose of this study is to investigate the complications using Gamma nail without distal locking screw. Furthermore, the relationship between complication rates and their reduction positions after operation were evaluated.

Material and methods: 146 patients with pertrochanteric fractures, which had been surgically managed with Gamma3 IM Nailing System (Stryker) at our clinic from 2012 to 2016, were investigated. All of them were operated without the distal locking screw. Complications such as delayed healing took longer than 3 months to heal and postoperative peri-implant fractures and cut-out of the lag screw were analyzed. Further, in the lateral view of radiographs, the position of the proximal fragment compared with distal fragment was evaluated after operation. The reduction positions were divided into 3 groups; anterior (Reduction-A), neutral (Reduction-N), and posterior (Reduction-P). In Reduction-A, the anterior cortices of the proximal fragment exist ventrally compared with the distal fragment. In Reduction-N, it achieved anatomically reduction And, in Reduction-P it existed dorsally.

Results: The mean age of the sample was 83.1 years. 94 delayed healing $(64.4 \%), 3$ peri-implant fractures $(2.1 \%)$, and 4 cut-out of the lag screw (2.7\%) were found. After operation, 25 patients $(25.3 \%)$ had reduction P. There was a statistical difference between Complication group and Non-Complication group as for reduction $\mathrm{P}$.

Conclusion: The current study showed a higher number of complications and the position of the proximal fragment could be associated with the complications. We concluded that nailing without distal locking screw could be dangerous and Reduction- P should be avoided.

References:

Disclosure: No significant relationships.

\section{PR374}

\section{THE USEFULNESS OF OTA/AO CLASSIFICATION TO PREDICT PELVIC ARTERIAL BLEEDING IN PATIENTS WITH BLUNT PELVIC TRAUMA}

\author{
M.J. Kim, S.H. Lee
}

Department Of Surgery, Yonsei University College of Medicine, SEOUL/KOREA, REPUBLIC OF

Introduction: Pelvic fractures can lead to life-threatening hemorrhage, which are a common cause of morbidity and mortality in trauma. Therefore, it is important to predict the massive bleeding in patients with pelvic bone fractures. This study aimed to investigate the association between patterns of pelvic fracture according to the OTA/AO classification and pelvic arterial bleeding in patients with blunt pelvic trauma
Material and methods: Medical records of 1417 trauma patients were reviewed retrospectively between January 2013 and August 2017. We enrolled 148 patients aged over 15 years with pelvic fracture due to blunt trauma. Pelvic fracture pattern was classified according to the OTA/AO classification. A linear by linear association model was used to examine linear association between patterns of pelvic fracture and pelvic arterial bleeding

Results: Most common pelvic fracture pattern was type A $(58.8 \%)$, followed by type B (34.5\%), and then by type C $(6.8 \%)$. Of 148 patients, $28(18.9 \%)$ showed pelvic arterial bleeding on angiographic or operative findings. The type $\mathrm{C}$ showed $70 \%$ of pelvic arterial bleeding, $27.5 \%$ in the type B, and $8.0 \%$ in the type A (linear-bylinear association, $p<0.001$ ). In addition, a massive transfusion protocol was used in the type C (30.0\%), type B (9.8\%) and type A (1.1\%) (linear-by-linear association, $p=0.001$ ). A significant linear association was revealed between fracture pattern and in-hospital mortality (type $\mathrm{A}=6.9 \%$, type $\mathrm{B}=15.7 \%$, type $\mathrm{C}=40.0 \%, p=$ 0.004).

Conclusion: A significant linear association exists between patterns of pelvic fracture according to the OTA/AO classification and pelvic arterial bleeding

References: Burgess AR, Eastridge BJ, Young JW, Ellison TS, Ellison PS, Jr., Poka A, et al. Pelvic ring disruptions: effective classification system and treatment protocols. J Trauma. 1990;30 (7):84856 Young JW, Burgess AR, Brumback RJ, Poka A. Pelvic fractures: value of plain radiography in early assessment and management. Radiology. 1986;160 (2):445-51. Heetveld MJ, Harris I, Schlaphoff G, Balogh Z, D'Amours SK, Sugrue M. Hemodynamically unstable pelvic fractures: recent care and new guidelines. World J Surg. 2004;28 (9):904-9 Stambaugh LE, 3rd, Blackmore CC. Pelvic ring disruptions in emergency radiology. Eur J Radiol. 2003;48 (1):71-87 Routt ML, Jr., Nork SE, Mills WJ. High-energy pelvic ring disruptions. Orthop Clin North Am. 2002;33 (1):59-72, viii Blackmore CC, Cummings P, Jurkovich GJ, Linnau KF, Hoffer EK, Rivara FP. Predicting major hemorrhage in patients with pelvic fracture. J Trauma. 2006;61 (2):346-52. Tile M. Pelvic ring fractures: should they be fixed? J Bone Joint Surg Br. 1988;70 (1):1-12. Dalal SA, Burgess AR, Siegel JH, Young JW, Brumback RJ, Poka A, et al. Pelvic fracture in multiple trauma: classification by mechanism is key to pattern of organ injury, resuscitative requirements, and outcome. J Trauma. 1989;29 (7):981-1000; discussion -2 Dalal SA, Burgess AR, Siegel JH, Young JW, Brumback RJ, Poka A, et al. Pelvic fracture in multiple trauma: classification by mechanism is key to pattern of organ injury, resuscitative requirements, and outcome. J Trauma. 1989;29 (7):981-1000; discussion -2.. Costantini TW, Coimbra R, Holcomb JB, Podbielski JM, Catalano RD, Blackburn A, et al. Pelvic fracture pattern predicts the need for hemorrhage control intervention-Results of an AAST multi-institutional study. Journal of Trauma and Acute Care Surgery. 2017;82 (6):1030-8.

Disclosure: No significant relationships.

\section{PR375}

\section{RESULTS OF SURGICAL TREATMENT OF ACETABULAR FRACTURES}

\section{O. Alexa $^{1}$, T.S. Gheorghevici ${ }^{2}$, B. Puha ${ }^{3}$, B. Veliceasa $^{4}$}

${ }^{1}$ Orthopedics And Trauma, University of Medicine and Pharmacy Iasi, iasi/ROMANIA, ${ }^{2}$ Orthopedics And Trauma, Saint Spiridon Emergency Clinical Hospital, Iasi/ROMANIA, ${ }^{3}$ Orthopedics, Gr. T Popa University of Medicine and Pharmacy, Iasi/ROMANIA, ${ }^{4}$ Orthopaedic And Traumatology, University of Medicine and 
Pharmacy Gr.T.Popa - Iasi, Iasi/ROMANIA

Introduction: The aim of the study was to evaluate the results of surgical treatment in acetabular fractures.

Material and methods: One hundred and twenty two patients with acetabular fractures from 2 partener hospitals were treated by open reduction and internal fixation (ORIF) on a 6 years period. The mean age of the patients was 44.3 years, 106 were male and 16 female. According to Letournel classification we recorded: 25 posterior wall fractures, 16 posterior column fractures, 18 transverse fractures, 22 posterior column and posterior wall fractures, 16 transverse and posterior wall fractures, 7 "T"-shaped fractures and 18 both column fractures.

Results: According to Matta classification anatomical reduction was achieved in 61 cases $(50.0 \%)$, imperfect reduction in 36 cases $(29.5 \%)$ and poor in 25 cases $(20.5 \%)$. One year follow-up was available for 92 patients Results showed that clinical results were excellent in 45 cases $(48.9 \%)$, good in 29 cases $(31.5 \%)$, fair results in 10 cases $(10.8 \%)$ and poor results in 8 cases $(8.7 \%)$. Complications were represented by: peroneal nerve palsies (7 patients), rapid massive osteolysis of the femoral head (one case), femoral head osteonecrosis (10 cases), simptomatic heterotopic ossifications (3 cases). Acute deep infection was noted in one case and superficial infection in one case. The overall rate of complications was $25 \%$, close related to the complexity of the fractures, time to reduction of hip dislocation and quality of reduction.

Conclusion: ORIF in acetabular fractures is a good therapeutical option but results will depend by fracture pattern, quality of reduction and patient status.

References: Matta JM. Fractures of the acetabulum: accuracy of reduction and clinical results in patients managed operatively within three weeks after injury. J Bone Joint Surg Am. 1996;78 (11):163245. Moed BR, WillsonCarr SE, Watson JT. Results of operative treatment of fractures of the posterior wall of the acetabulum. J Bone Joint Surg Am. 2002;84-A (5):752-8.

Disclosure: No significant relationships.

\section{PR376}

\section{IMPACT OF VERTEBRAL FRACTURES ON THE FINAL OUTCOME IN POLYTRAUMATIC PATIENTS}

\section{P. González-Tartière ${ }^{I}$, M. Riveiro $^{2}$, M. Vicente Goma-Camps ${ }^{1}$, M. Ramírez-Valencia ${ }^{I}$, A. Matamalas $^{I}$, F. Pellisé ${ }^{1}$}

\section{${ }^{1}$ Spine Unit, Hospital Universitari Vall d'Hebron, Barcelona/SPAIN, ${ }^{2}$ Intensive Care Unit, Hospital Universitari Vall d'Hebron, Barcelona/ SPAIN}

Introduction: Few studies have assessed the impact of vertebral fractures (VF) without associated spinal cord injury (SCI) on polytraumatic patients. The aim of this study was to evaluate its impact on $\mathrm{mid} /$ long-term quality of life (QoL), pain and mental health.

Material and methods: Retrospective analysis of polytraumatic patients admitted in our center between 2010 and 2015 and transversal study of mid/long term QoL and psychological state was performed. Inclusion criteria: $>18$ years, ISS $\geq 16$. Exclusion criteria: GOSE $\leq 3$ at the moment of inclusion, SCI.

Results: $85 / 177$ patients agreed to participate, 32 answered the questionnaires (mean age 50.3; $78.1 \%$ men). 21 had VF (70\% AO A1/ A2). Mean hospital stay 57.6 days (28.09 ICU). $46.9 \%$ of patients presented major complications. VF patients had more injuries on intake (7.6 vs. 4.7). Severe TBI 39.5\%. There were no differences in trauma severity scores, except AIS-head (VF 1.8; no VF 3.1). Mean time from injury to completion of questionnaires was 44.6 months (14-80). A higher rate of patients with VF presented some $(72.2 \%)$ or extreme problems $(16.7 \%)$ in EQ5D pain/discomfort dimension, moreover they had worst global mid/long term QoL (EQ5D index FV $=9.5$; no FV $=0.9$ ) and worst scores in the Posttraumatic Stress Disorder Checklist (PCL FV $=40.5$; no FV $=27$ ).

Conclusion: The odds of suffering pain/discomfort are 12 times higher for polytraumatic patients with VF even years after injury compared to patients with the same trauma severity without VF. This seems to affect their global QoL. Moreover, they suffer more posttraumatic stress symptoms.

References:

Disclosure: No significant relationships.

\section{PR377}

\section{FRACTURE IS PROTECTIVE AGAINST WHIPLASH INJURY FOLLOWING ROAD TRAFFIC ACCIDENT}

\section{K.N. Linton, D. Bennett}

\section{Trauma \& Orthopaedic Surgery, Galway Clinic, Galway City/} IRELAND

Introduction: Much has been written regarding pain and disability following whiplash injury[1]. Several authors have reported on the relationship between insurance claim and whiplash-associated disorders[2]. Our own experience of over 10-years of insurance claim cases suggests that limb fracture may actually be protective of whiplash injury following road traffic accident.

Material and methods: We exported all patient cases from our electronic medical reports (EMR) system and filtered it to include only cases recorded as "medical legal" or "injuries board". From this database of 2,428 cases we extracted 375 cases due to road traffic accident (RTA). When patients present they are required to complete a questionnaire which documents the type of RTA (head-on, side-on, rear-ended), patient demographics (including weight and height), fracture, and the top-3 locations of pain with pain scored on an analogue scale from 0 (none) to 10 (unbearable).

Results: 53\% (199) are male with median age 45 years and $47 \%$ (176) female with median age of 37 years. $26 \%$ are reported as headon, $34 \%$ side-impact with $40 \%$ as rear-ended. $55 \%$ of patients are classed as unemployed with $45 \%$ working at time of claim. In terms of fracture, only $11 \%$ (41) of patients reporting a fracture occurring as a result of the accident. The $89 \%$ (334) of cases with no fracture are twice as likely to report whiplash as their primary source of pain. Conclusion: $16 \%$ of fracture following RTA are due to vertebral fracture. The presence of a peripheral (non-neck) fracture is significantly protective $(\mathrm{p}<0.05)$ for whiplash injury following RTA.

References: [1] Malik AA, Robinson S, Khan WS, Dillon B and Lovell ME. Assessment of Range of Movement, Pain and Disability Following a Whiplash Injury. Open Orthop J. 2017 Jul 19;11:541545.

[2] Boyle E, Cassidy JD, Côté P, Carroll LJ. The relationship between insurance claim closure and recovery after traffic injuries for individuals with whiplash associated disorders. Disabil Rehabil. 2017 May;39 (9):889-896.

Disclosure: No significant relationships. 
PR378

\section{NOT ALL INFRA-ACETABULAR CORRIDORS ARE AMENABLE TO SCREW INSERTION: CT DATA ANALYSIS}

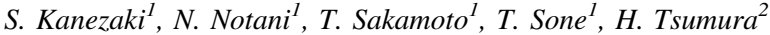

${ }^{1}$ Orthopedic Surgery/advanced Trauma, Emergency And Critical Care Center, Oita University Hospital, Yufu/JAPAN, ${ }^{2}$ Orthopedic Surgery, Oita University Hospital, Yufu/JAPAN

Introduction: The infra-acetabular screw (IAS) is reported to increase the fixation strength of acetabular fractures. However, specifics of Infra-acetabular corridor (IAC) have remained unknown. The purpose of our retrospective study was to reveal details of IAC using CT data analysis.

Material and methods: Traumatized in-patients who had pelvic CT scans from 2014 to 2016 were enrolled. Pediatric patients and adult patients with pelvic fractures and hip prosthesis were excluded. The male/female ratio and distribution of patients' age were equalized (40 male and 40 female patients). IAC was measured on the plane of the inlet view (25 degree caudal) in multi-planar reconstructed CT images. The following morphological measures were obtained: mediallateral width of IAC (MLW), anterior-posterior length of IAC (APL), length from the starting point of IAC to the medial edge of the pelvis (LME), and length from the starting point of IAC to the top of the pubic symphysis (LPS).Bilateral differences and differences between the sexes were also investigated.

Results: Mean MLW/APL/LME/LPS was $3.8 \pm 1.8 / 89.5 \pm 7.1 / 8.7 \pm$ $3.6 / 57.8 \pm 4.8$, respectively. Bilateral difference of MLW was $0.6 \pm$ 0.6. Thirty percent of corridors (48 of 160) were not amenable to IAS placement, because of inadequate width (less than $3.0 \mathrm{~mm}$ ) and/or tortuous shapes. There was no difference in MLW between male and female patients. APL, LME, and LPS were significantly longer in male patients.

Conclusion: CT data analysis revealed the details of IAC. Surgeons should pay attention to the fact that 30 percent of IACs are not amenable to IAS insertion even with perfect reduction in treating acetabular fractures.

References: 1) Culemann U, Marintschev I, Gras F, et al. Infraacetabular corridor-Technical tip for an additional screw placement to increase the fixation strength of acetabular fractures. J Trauma. 2012 Apr;43 (4):470-4 2) Marintschev I, Gras F, Schwarz CE, et al. Biomechanical comparison of different acetabular plate systems and constructs - The role of an infra-acetabular screw placement and use of locking plates. Injury. 2012;43:470-474 3) Ricci WM, Mamczak C, Tynan M, et al. Pelvicinlet and outlet radiographs redefined. J Bone Joint Surg Am. 2010;92A:1947-53

Disclosure: No significant relationships.

\section{PR379}

\section{POLYTRAUMATIZED PATIENT WITH PELVIC FRACTURE: 8 YEARS EXPERIENCE}

\section{J. Martínez Cabañero ${ }^{1}$, F. Martinez Lopez ${ }^{2}$, N. Alegret Monroig ${ }^{2}$}

${ }^{1}$ Anesthesiology, Hospital Parc Tauli, Sabadell/SPAIN,

${ }^{2}$ Anesthesiology, Coorporació Sanitaria Parc Tauli, sabadell/SPAIN

Introduction: Pelvic trauma is usually secondary to high energy blunt trauma. Patients with pelvic fracture $(\mathrm{PF})$ have a mortality rate of $19-33 \%$. Integrated management and a multidisciplinary approach are the key to coordinate effective resuscitation and bleeding control, especially in the first hours after trauma. The aim of this study is to evaluate outcomes at our institution.

Material and methods: Observational study with a prospective data collection of polytraumatized patients (PTP) with associated PF between January 2009 and January 2017. Demographic variables, clinical and analytical data at the emergency room were collected, pelvic fracture type, therapeutic procedures, associated lesions and mortality at 30 days.

Results: 2061 PTP were attended, of which 935 met severity criteria, $12.6 \%$ had associated PF. The $23.7 \%$ admitted with systolic blood pressure $\leq 90 \mathrm{mmHg}$ and $50.8 \%$ heart rate $\geq 100 \mathrm{x}$, presenting $\mathrm{BE}<-$ $626.3 \%$ and lactates $\geq 20 \mathrm{mg} / \mathrm{dL} 67.6 \%$. The mean Injury Severity Score was 20, 19 points. Arteriography was performed in $30.5 \%$ of patients requiring $80 \%$ of angioembolization. Preperitoneal packing was performed in $3.4 \%$ of patients. The main associated lesions were: costal fractures (35.6\%), hemo-pneumothorax (31.3\%), spine lesions $(35.6 \%)$ and TBI (30\%). Mortality at 30 days was $12,7 \%$, with hemorrhagic shock being the main cause $(73.3 \%)$.

Conclusion: Hemodynamic instability and associated injuries are the leading causes of mortality in PTP with PF. A multidisciplinary approach is crucial.

References: Coccolini F, Stahel PF, Montori G, et al. Pelvic trauma: WSES classification and guidelines. World J Emerg Surg. 2017;12 (1):5. doi:10.1186/s13017-017-0117-6. Tran TLN, Brasel KJ, KarmyJones R, et al. Western Trauma Association Critical Decisions in Trauma: Management of pelvic fracture with hemodynamic instability-2016 updates. J Trauma Acute Care Surg. 2016;81 (6):11711174. doi:10.1097/TA.0000000000001230. Cullinane DC, Schiller HJ, Zielinski MD, et al. Eastern Association for the Surgery of Trauma Practice Management Guidelines for Hemorrhage in Pelvic Fracture-Update and Systematic Review. J Trauma Inj Infect Crit Care. 2011;71 (6):1850-1868. doi:10.1097/TA.0b013e31823dca9a. Disclosure: No significant relationships.

\section{PR380}

\section{CLINICAL RESULTS OF OSTEOSYNTHESIS FOR FRACTURES OF THE ACETABULUM IN ELDERLY PATIENTS}

T. Noda ${ }^{1}$, T. Ozaki ${ }^{2}$, Y. Shimamura ${ }^{2}$, Y. Yamakawa ${ }^{2}$, M. Yoshimura ${ }^{2}$, M. Kiyono ${ }^{2}$, S. Yokoo ${ }^{2}$, Y. Mochizuki ${ }^{2}$, K. Demiya ${ }^{2}$

${ }^{1}$ Department Of Musculoskeletal Traumatology, Okayama University Graduate School of Medicine, Dentistry, and Pharmaceutical Sciences, Okayama city/JAPAN, ${ }^{2}$ Orthopaedic Surgery, Department of Orthopaedic Surgery Okayama University, Okayama City/JAPAN

Introduction: Clinico-radiological outcomes among patients over 65 years old treated with open reduction and internal fixation (ORIF) for fractures of the acetabulum were assessed.

Material and methods: 68 patients were included in this study. 48 cases who had more than one year F/U were also investigated by Clinical Assessment. Mean age of the patients was 71 years old. Our strategy is use of single approach as possible, less invasive surgery, buttress plating for the quadrilateral plate and direct reduction and fixation of marginal impaction and roof impaction fragment with osteochondral support graft and/or screw. We investigated fracture type, incidence of roof impaction and marginal impaction, approach, op. time, blood loss, clinico-radiological assessment by Matta criteria and peri-/postoperative complication. 
Results: Incidence of the roof impaction and the marginal impaction in potential fracture types were $48 \%$ and $67 \%$ respectively. Average blood loss was $1255 \mathrm{ml}$, and average operative time was 230 minutes. Postoperative deep infection occurred in four patients. 54\% of the reductions were graded as anatomical $(0-1 \mathrm{~mm}), 37 \%$ as imperfect (2$3 \mathrm{~mm}$ ), and $9 \%$ as poor ( $>3 \mathrm{~mm}$ ). In clinical evaluation (Matta) of 45 patients who had more than 1 year follow-up (average 35 months), $18 \%$ were graded as excellent, $53 \%$ as good, $16 \%$ as fair, and $13 \%$ as poor. 8 patients had moderate to severe posttraumatic arthritis. The only 3 patients of them had a THA conversion (6.7\%).

Conclusion: Our reduction and fixation method was a good option to reduce and maintain the congruency of the acetabulum. We think good outcomes is still possible in the treatment of this difficult fractures.

References:

Disclosure: No significant relationships.

\section{PR381}

\section{EFFECTIVENESS AND WOUND COMPLICATION OF PREPERITONEAL PELVIC PACKING IN PATIENTS WITH HEMODYNAMIC INSTABILITY CAUSED BY PELVIC FRACTURE}

\author{
J.Y. Jang, H. Shim, K.S. Bae
}

Department Of Surgery, Yonsei University Wonju College of Medicine, wonju/KOREA, REPUBLIC OF

Introduction: Despite using a multidisciplinary treatment approach, the mortality with hemodynamic instability due to severe pelvic fracture is still $40-60 \%$. Recently, several studies have showed that preperitoneal pelvic packing (PPP) was useful hemostatic technique for these patients in the acute phase. However, there are few studies about complications after the procedure.

OBJECTIVES: The purpose of present study was to evaluate the efficacy and wound complication of PPP in these patients.

Material and methods: We retrospectively reviewed the medical charts of 62 patients with hemorrhagic shock due to pelvic fracture between March 2011 and May 2017. The patients were divided into PPP group $(n=30)$ and non-PPP group $(n=28)$ according to PPP application.

Results: Thirty patients in the PPP group had significantly lower hemorrhage-induced mortality rate than 28 patients in the non-PPP group $(16.7 \%$ vs $50 \%, \mathrm{p}=0.019)$, although both groups had similar patient characteristics (age, Injury severity score, and initial serum lactate). The logistic regression analysis showed that independent factors associated with hemorrhage-induced mortality were PPP and requirement of packed $\mathrm{RBC}$ for $4 \mathrm{~h}$. Among 25 patients except 5 patients (20\%) who died of bleeding in the PPP group, surgical site infection occurred in 5 patients. Patients with wound complication underwent more frequently pelvic EF with PPP, compared with patients without complication ( $80 \%$ vs $20 \%, \mathrm{p}=0.023$ ).

Conclusion: Although PPP is an effective hemostatic method, possibility of wound complication after the procedure should be considered.

References: Jang JY et al. Preperitoneal pelvic packing in patients with hemodynamic instability due to severe pelvic fracture: early experience in a Korean trauma center. Scand J Trauma Resus. 2016;24. Burlew CC et al. Preperitoneal Pelvic Packing/External Fixation with Secondary Angioembolization: Optimal Care for LifeThreatening Hemorrhage from Unstable Pelvic Fractures. J Am Coll Surgeons. 2011;212 (4):628-35 Burlew CC et al. Preperitoneal pelvic packing reduces mortality in patients with life-threatening hemorrhage due to unstable pelvic fractures. Journal of Trauma and Acute Care Surgery. 2016.

Disclosure: No significant relationships.

\section{PR382}

\section{EXPERIENCES OF SUPRA ACETABULAR COMPRESSION SCREW (SACS) FOR ACETABULAR FRACTURES}

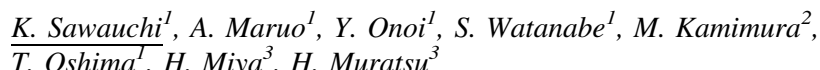

${ }^{1}$ Department Of Orthopaedic Surgery, Steel Memorial Hirohata Hospital, Himeji/JAPAN, ${ }^{2}$ Orthopaedic, Steel Memorial Hirohata Hospital, Himeji/JAPAN, ${ }^{3}$ Orthopaedics, Steel Memorial Hirohata Hospital, Himeji/JAPAN

Introduction: In the treatment of acetabular fractures, the precise reduction of articular surface with rigid fixation is important. Especially, for marginal impaction with anterior column fractures, it's critical to support impacted articular fragment and to compress quadrilateral surface and lateral wall. We have treated these fractures placing supra acetabular compression screw (SACS) for this purpose. Here we report on brief experience of a novel surgical technique with SACS.

Material and methods: From 2015 to 2016, four patients (four men, 59.5 years old), with displaced acetabular fracture, were treated with open reduction and internal fixation using SACS. According to Letournel's classification, ${ }^{1}$ two patients were anterior column, one was both column, and one was anterior with posterior hemitransverse. We reduced medially shifted fragment and impacted articular roof by modified stoppa approach and inserted dynamic condylar screw as SACS above the articular roof from lateral small incision. Additional anterior plates were placed through the lateral window. The quality of reduction was evaluated by X-ray and clinical outcome was evaluated using Matta's criteria. ${ }^{2}$

Results: The mean preoperative and postoperative intra-articular gap were $14.7 \mathrm{~mm}$ and $6.8 \mathrm{~mm}$. The mean preoperative and postoperative intra-articular step-off were $3.6 \mathrm{~mm}$ and $0.8 \mathrm{~mm}$. There was no case of reduction loss. All patients were excellent or good by Matta's criteria. $^{2}$

Conclusion: We consider SACS has two advantages: to compress medial and lateral wall, to support articular fragment and prevent loss of reduction. SACS could be an effective surgical option for displaced anterior column acetabular fractures.

References: 1. Letournel E. Acetabulum fractures: classification and management. Clin Orthop 1980;151:81-106 2. Matta JM. Operative treatment of acetabular fractures through the ilioinguinal approach: a 10-year perspective. Clin Orthop Relat Res 1994;305:10-19

Disclosure: No significant relationships.

\section{PR383}

\section{ACETABULAR FRACTURES- CRITICAL POINTS OF SURGICAL TREATMENT}

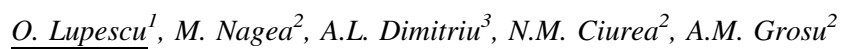

${ }^{1}$ Orthopaedics And Trauma, Clinical Emergency Hospital, University of Medicine and Pharmacy "Carol Davila" Bucharest, Bucharest/ 
ROMANIA, ${ }^{2}$ Orthopaedics And Trauma, CLINICAL EMERGENCY HOSPITAL, BUCHAREST/ROMANIA, ${ }^{3}$ Clinical Emergency Hospital, Orthopaedics And Trauma, University of Medicine and Pharmacy Carol Davila, BUCHAREST/ROMANIA

Introduction: Acetabular fractures represent a real challenge due to their impact upon the long term function of the hip joint, and due to the risk of intra- and peri-operative complications especially that they affect young, active people, therefore treating this fractures require certain skills which must be exercised, regarding the pre-operative planning as well as the execution of surgery

Material and methods: The authors retrospectively analyse 46 cases with acetabular fractures operated in a level 1 Trauma Centre between 01.01.2013- 01.01.2016 withg a follow up of 24 months, in order to establish the main causes of potenbtial errors and to underline the methods for preventing them. This paper analyses the pitfalls revealed by these cases, as well as the measures necessary to enhance the practical skills of the surgeons who perform acetabular surgery.

Results: Pre-op planning matched the intra and post-operative outcome in $88 \%$ of the analysed points; the main plannig issues were wrong approach ( 3 cases) and urecongnising the fracture pattern (2 cases), while post-op complications were : nervous - 3 cases, and intra-articular position of the screws ( 2 cases-demonstrated by postoperative CT scans), requirig secondary screw removal. The most important problems were the reduction of the fractures and the positioning of the screws so as not to interfere with the the articular space

Conclusion: In acetabular fractures, pre-op planning is important for maximum treatment efficacy with minimum of risk; an optimal training of the surgeons insisting on the main points of potential mistakes ensure the success of the procedure and a favourable outcome of the patient

References: 1.E. Letournel and R. Judet, Fractures of the Acetabulum, Springer, New York, NY, USA, 2nd edition, 1993. 2. J. M. Matta, "Fractures of the acetabulum: accuracy of reduction and clinical results in patients managed operatively within three weeks after the injury, "Journal of Bone and Joint Surgery-Series A, vol. 78, no. 11, pp. 1632-1645, 1996. 3.E. Letournel, "Acetabulum fractures: classification and management., " Clinical Orthopaedics and Related Research, no. 151, pp. 81-106, 1980.

Disclosure: No significant relationships.

\section{PR384}

\section{FAILURE OF PLATE FIXATION FOR PUBIC SYMPHYSIS DIASTASIS ASSOCIATED WITH PELVIC RING FRACTURE}

\author{
Y. Yamakawa ${ }^{1}$, T. Noda ${ }^{2}$, K. Demiya $^{3}$, Y. Mochizuki $^{3}$, M. Yoshimura $^{3}$, \\ S. Yokoo ${ }^{3}$, M. Kiyono ${ }^{3}$, T. Ozaki ${ }^{3}$
}

${ }^{1}$ Department Of Emergency Healthcare And Disaster Medicine, Okayama University Graduate School of Medicine, Dentistry, and Pharmaceutical Sciences, Okayama city/JAPAN, ${ }^{2}$ Department Of Musculoskeletal Traumatology, Okayama University Graduate School of Medicine, Dentistry, and Pharmaceutical Sciences, Okayama city/JAPAN, ${ }^{3}$ Orthopaedic Surgery, Department of Orthopaedic Surgery Okayama University, Okayama City/JAPAN

Introduction: We retrospectively reviewed the failure rate and characteristics of anterior plating for pubic symphysis diastasis associated with pelvic fracture.

Material and methods: Nineteen patients are included in this study and there are three open fractures and eight are combined injuries with acetabular fractures. There are ten $\mathrm{B}$ type fractures and nine $\mathrm{C}$ type fractures according to AO/OTA classification. Mean follow-up period is twenty-nine months. Thirteen cases were temporary fixed with external fixator at initial treatment. We investigate the definitive fixation method, the distance of pubic symphysis (at injury, after external fixator, just after internal fixation and at final follow up), failure rate of anterior plating including screw loosening, and Majeed score as clinical result.

Results: $3.5 \mathrm{~mm}$ plate was used in all cases for anterior fixation and three IS screws and eleven plate were used for posterior fixation. The distance of pubic symphysis was $27 \mathrm{~mm}$ at injury, $22 \mathrm{~mm}$ after external fixation, $7 \mathrm{~mm}$ at just after internal fixation and $8 \mathrm{~mm}$ at final follow up. We identified 8 cases of anterior fixation failure $(41 \%)$, one implant removal due to infection. Average Majeed score was 81 points (51-100) and no relevant to anterior fixation failure.

Conclusion: To obtain stable pelvic ring structure, anterior fixation with plate for pubic symphysis diastasis is standard treatment. In this study, failure rate of implant for anterior fixation is high as other report, but no clinical symptom was observed even in those patients. Disclosure: No significant relationships.

\section{PR385}

\section{EPIDEMIOLOGY, PATTERNS, AND OUTCOMES OF PELVIC FRACTURE IN A LEVEL 1 TRAUMA CENTER IN THE MIDDLE EAST}

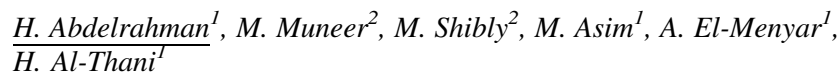

${ }^{1}$ Trauma Surgery, Hamad General Hospital, Doha/QATAR, ${ }^{2}$ Surgery, Hamad General Hospital, Doha/QATAR

Introduction: We aimed to study our experience with pelvic fracture Material and methods: We conducted a retrospective review of trauma registry data to identify all patients who presented with open pelvic fracture between 2011 and 2013. Data were analyzed to look for in-hospital mortality and morbidity.

Results: We identified 297 patients with pelvic fracture. An incidence of nearly 100 cases per year. The mean age was $31.6 \pm 13.2$ and $88.6 \%$ were males. The associated injuries in order were the abdomen $(41.5 \%)$, chest $(31.6 \%)$, spine, upper limbs and lower limbs in a quarter while head injuries were only in $15.8 \%$. The average ISS was $14.9 \pm 9.5$. One third of patients received blood transfusion. The mean length of hospital stay was 18 days while the mean length of both ICU stay and the ventilator days was 4 . The overall mortality was $5.1 \%$. One third of the cohort needed admission to critical care. One patient died at the ED. There were 93 patients with type A, 126 type B, 34 types $\mathrm{C}$ pelvic fracture, 35 patients had acetabular fracture and 9 patients had a hip fracture dislocation. Type $\mathrm{C}$ fractures were significantly associated with more need for blood transfusion (76.5\%), surgical intervention and prolonged hospital stay. The patients groups were not significantly different with regard to age, ISS, complications, ICU LOS, and mortality. The majority of cases were approached conservatively; only 42 patients had surgical fixation.

Conclusion: Pelvic fractures are common in young males and the majority are type A. Conservative non-operative management prevails with low mortality .

References: J Trauma. 2011;71:1850-68.

Disclosure: No significant relationships. 
PR386

\section{FAILURE OF FIXATION OF TROCHANTERIC FRACTURES; SLIDING HIP SCREW OR INTRAMEDULLARY NAIL?}

\author{
M. Timmermans, R.c.l.a. Maayen, M. Leijnen
}

Surgery, Alrijne Ziekenhuis, Leiderdorp/NETHERLANDS

Introduction: Secondary surgery of a trochanteric fracture is a severe event. The aim of this study is to evaluate the patients who needed additional treatment after an intramedullary nail and investigate the risk factors and complications of the additional treatment. To identify the preferred treatment a literature review is performed.

Material and methods: In a retrospective study we included patients with a AO classification 31-A1, 31-A2 and 31-A3 fracture that had been treated at a non-academic teaching hospital in the Netherlands between January 2014 and October 2016. The included patients needed replacement of an intramedullary implant with a Sliding Hip Screw (SHS) to correct non-union or osteosynthesis failure. Data of risk factors and complications were documented from the medical records.

Results: A total of ten fractures in ten patients were included, four AO classification 31-A3 and six 31-A2 fractures. Two patients had an implant failure and eight patients non-union. Reduction results and implant positioning were insufficient in most cases. Eight patients reached complete union after secondary intervention.

Conclusion: Debate remains and no consensus is reached upon the best initial treatment of intertrochanteric fractures. The preferred initial fixation device is still controversial. Our results suggest that reduction result and implant positioning is the most important risk factor to predict non-union and implant failure. A large prospective study is necessary to determine the initial preferred treatment of trochanteric fractures, especially in the AO 31-A2 and AO 31-A3 fractures.

References: Avakian, Z., et al. (2012). "Dynamic hip screws versus proximal femoral nails for intertrochanteric fractures." ANZ journal of surgery 82 (1-2): 56-59. Guerra, M. T., et al. (2014). "Functional recovery of elderly patients with surgically-treated intertrochanteric fractures: preliminary results of a randomised trial comparing the dynamic hip screw and proximal femoral nail techniques." Injury 45: S26-S31. Hsu, C.-E., et al. (2015). "Trochanter stabilising plate improves treatment outcomes in AO/OTA 31-A2 intertrochanteric fractures with critical thin femoral lateral walls." Injury 46 (6): 1047-1053. Hsu, C.-E., et al. (2016). "Integrated risk scoring model for predicting dynamic hip screw treatment outcome of intertrochanteric fracture." Injury 47 (11): 2501-2506. Huang, X., et al. (2013). "Proximal femoral nail versus dynamic hip screw fixation for trochanteric fractures: a meta-analysis of randomized controlled trials." The Scientific World Journal 2013. Nyholm, A. M., et al. (2016). "Lacking evidence for performance of implants used for proximal femoral fractures-A systematic review." Injury 47 (3): 586-594. Disclosure: No significant relationships.

\section{PR387}

\section{THE INVESTIGATION OF TREATMENT AND COMPLICATION OF PERIPROSTHETIC FEMORAL FRACTURE}

M. Yoshida

Myokencho, 2-9, Nagoya/JAPAN

Introduction: The number of periprosthetic femoral fracture is increasing. The purpose of this study is to investigate of how to treatment, postoperative progress, and complications of periprosthetic femoral fracture

Material and methods: Injuries included in the study were Vancouver type A 2cases, type B1 8cases, type B2 5cases, type C 2cases The mean age was 83.6 year, the average time from primary surgery to fracture was 2.1year.

Results: In the type B1, 7cases underwent ORIF with Locking compression plate system (LCP)+cable system. In the type B2, only 1case underwent ORIF, other 4 cases underwent revision surgery for long stem In the type C, 1case underwent ORIF with LCP, another 1 case underwent retroglade nail+additional plate. Regard to complications, 1case was made a wrong diagnosis type B2 as typeB1. 1case of typeC had plate breakage.

Conclusion: In the type $\mathrm{B} 1$ or type $\mathrm{C}$ with transverse or short oblique fracture underwent plate fixation, It may be necessary to pay attention to stress concentration.

References: 1)Andrew N .Fleischman, Antonia F.Chen.Periprosthetic fractures around the femoral stem:overcoming challenges and avoiding pitfalls2015;3 (16):234.1-13 2)Corten K, Vanrykel F, Bellemans $\mathrm{J}$, et al. An algorithm for the surgical treatment of periprosthetic fractures of the femur around awell-fixed femoral component. J Bone Joint Surg Br 2009;91:1424-30 3)Buttaro MA, Farfalli G, Paredes Nunez M, Comba F, Piccaluga F.Locking compression plate fixation of Vancouver type-B1 periprosthetic femoral fractures. J Bone Joint Surg Am.2007;89 (9):1964-9. 4)Gele B.Moloney, Edward R.Westrick, Peter A.Siska, et al. Treatment of periprosthetic femur fractures around a well-fixed hip arthoroplasty implant:span the whole bone. Arch Orthop Trauma Surg (2014)134:914 5)Shunji Nakano, Shinji Yoshioka, Fumitake Tezuka, et al. New Surgical Treatment Using a Docking Nail for postoperative Periprosthetic Femoral Fracture After Total Hip Arthoroplasty. The Journal of Arthoroplasty Vol.28 No. 22013 6)Robinson Jde D et al, Periprosthetic atypical femoral fractures in patients on long-term bisphosphonates; a multicenter retrospective review. J Orthop Trauma 30:170-176, 2016

Disclosure: No significant relationships.

\section{PR388}

\section{A NEW CLASSIFICATION FOR GUSTILO 3B SEVERE OPEN FRACTURE OF THE TIBIA}

\author{
Y. Tsuchida, K. Futamura, N. Yoshida
}

Orthopedic Trauma, Shonan Kamakura General Hospital, Kamakura/ JAPAN

Introduction: In 2016, we classified Gustilo 3B open fracture of the tibia into 4 groups according to bone and soft tissue defects. Flap surgery was necessary in all groups. The degree of bone loss and 
defect increases from Group 1, which has the least bone loss and defects, through Groups 2, 3, and 4, with increasing bone loss and defects, requiring bone reconstruction procedures.

Material and methods: From 2013 to 2017, we treated 30 cases (25 men and three women; mean age of 45.2 yeas) with Gustilo 3B open fractures of the tibia. According to our classification, there were 14 cases in Group 1, three in Group 2, two in Group 3, and 11 in Group

Results: Soft tissue defects in four patients (3, group 1; 1 , group 2) were reconstructed using a pedicle flap and the remaining 26 were reconstructed using a free flap. Bone grafting was almost unnecessary in Group 1, but reconstruction using the Masquelet technique was needed for two patients in Group 2, two patients in Group 3, and 10 patients in Group 4. Bone transport was performed in one case (Group 4). Post-operative infection occurred in four cases in Group 1, four cases in Group 4, and two required amputation. The ability to walk independently was acquired by almost all patients.

Conclusion: According to our classification, treatment of patients in Group 1 is practically associated with no difficulty if soft-tissue reconstruction is completed. Those in Group 4 are more difficult to treat and there is a greater danger of amputation.

References: Tsuchida Y: Bone and Soft Tissue Reconstruction for Gustiolo IIIB/C Severe Open Fracture of the Tibia J. Jpan. S.R.M. 29:1-7, 2016

Disclosure: No significant relationships.

\section{PR389}

\section{SOLUBLE FIBRIN MONOMER COMPLEX CAN BE A USEFUL PREDICTOR OF VENOUS THROMBOEMBOLISM AFTER FRACTURE SURGERY}

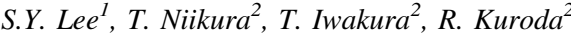

${ }^{1}$ Department Of Orthopaedic Surgery, Showa University School of Medicine, Tokyo/JAPAN, ${ }^{2}$ Department Of Orthopaedic Surgery, Kobe University Graduate School of Medicine, Kobe/JAPAN

Introduction: Venous thromboembolism (VTE), including deep vein thrombosis (DVT) and pulmonary thromboembolism (PTE), is a critical postoperative complication after pelvic and lower extremity fractures. The purpose of this study was to determine whether the soluble fibrin monomer complex (SFMC) could be used for postoperative screening of VTE in patients with lower limb or pelvic fractures.

Material and methods: 105 patients with fractures of the pelvis and/ or lower extremities who were surgically treated were analyzed prospectively. The SFMC and D-dimer levels were measured on postoperative day 7. All patients underwent routine ultrasonography preoperatively and 7 days after surgery. Receiver operating characteristic (ROC) curve analysis was done to evaluate their prognostic accuracy for the development of postoperative VTE. The optimal cutoff levels were set on the basis of having the highest Youden index, and sensitivity and specificity was calculated.

Results: Postoperative VTE was diagnosed in 34 patients $(32.4 \%)$ including 5 patients $(4.8 \%)$. All patients were asymptomatic. The ROC curve analysis revealed diagnostic accuracy for the SFMC test was higher than the D-dimer test. At the optimal SFMC cut-off level $(5.6 \mathrm{ng} / \mathrm{ml})$, sensitivity was $82.4 \%$ and specificity was $78.9 \%$. At the optimal D-dimer cut-off level $(11.0 \mu \mathrm{g} / \mathrm{ml})$, sensitivity was $70.6 \%$ and specificity was $64.8 \%$.

Conclusion: Our results showed the SFMC test measured 7 days after surgery had superior diagnostic power for predicting postoperative
VTE. Sensitivity and specificity of the SFMC test was greater than those of the D-dimer test. SFMC could be a useful marker for screening postoperative VTE in patients with fractures of the pelvis and/or lower extremities.

References:

Disclosure: No significant relationships.

\section{PR390}

\section{THE ACCURACY OF SCREW PLACEMENT FOR ACETABULUM FRACTURES USING WITH 3D-CT NAVIGATION}

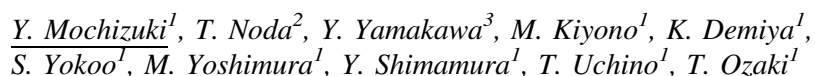

${ }^{1}$ Orthopaedic Surgery, Department of Orthopaedic Surgery Okayama University, Okayama City/JAPAN, ${ }^{2}$ Orthopaedic Surgery,

Department of Musculoskeletal Traumatology, Okayama University Graduate School of Medicine, Okayama City/JAPAN, ${ }^{3}$ Orthopaedic Surgery, Department of Emergency Healthcare and Disaster Medicine, Okayama University Graduate School of Medicine, Okayama City/JAPAN

Introduction: Various screw placement for acetabulum fractures is often difficult because of complex anatomical morphology, however, it becomes very beneficial to set enough fixation stability if we can insert the long screws. 3D-CT navigation system for the screw placement is beneficial for precise screw insertion. We investigated the clinical results of the patients who were inserted screws with $3 \mathrm{D}$ CT navigation.

Material and methods: Our retrospective case series were assessed by the accuracy of screws with 3D-CT navigation for acetabulum fractures. Thirteen patients who sustained acetabulum fractures were included in this study and $3.5 \mathrm{~mm}$ cortical screws or $6.5 \mathrm{~mm}$ cannulated screws were inserted with 3D-CT navigation. We investigated fracture classification, number of screws, clinical complication related to surgical procedure and screw positions which is measured by postoperative CT scan and classified by Smith criteria.

Results: We inserted 1 antegrade pubic screw, 5 anterior column screws, 27 posterior column screws and 6 infra-acetabular screws. 35 of 39 screws $(89.7 \%$ ) were placed in correct position (grade0 or 1$) .4$ screws were in incorrect position and they were all cortical screws. And there was no complication related to screw insertion.

Conclusion: Our study highlights that 3D-CT navigation system reduced the malposition rate of screw insertion for acetabulum fractures. However, we sometimes had difficulty in inserting cortical screws, even though drilling was in correct position. We assumed that this was caused by flexibility of drill or inserting screws in wrong position manually. We should pay much more attention in inserting cortical screws even using 3D-CT navigation.

\section{References:}

Disclosure: No significant relationships 


\section{PR391}

\section{NEW TECHNIQUE FOR DETECTING OSTEOMYELITIS USING PREOPERATIVE 18F-FDG PET/CT}

\author{
M. Takaki, N. Takenaka, K. Mori, S. Harada, T. Asahara, N. Katoh, \\ T. Teramoto, T. Matsushita
}

\section{Traumatology, Fukushima Medical University, Fukushima City/} JAPAN

Introduction: Post-traumatic osteomyelitis is one of the most serious complications of fractures. Especially, infection in Gustilo typeIII open fracture is observed $10 \%$ to $50 \%$ of the time. There are currently no methods for accurately localizing the infection focus of osteomyelitis. We investigated the ${ }^{18} \mathrm{~F}$-fluorodeoxyglucose (FDG) positron emission tomography (PET)/computed tomography (CT) to visualize the infection focus of osteomyelitis accurately. In this study, we compared pathological findings of osteomyelitis with ${ }^{18} \mathrm{~F}-\mathrm{FDG}$ $\mathrm{PET} / \mathrm{CT}$ of osteomyelitis for diagnosing accurate location of osteomyelitis lesion.

Material and methods: We described 13 cases, involving 9 lower limbs and 1 upper limb where osteomyelitis was bacteriologically detected after surgery on limbs. On the other hand, we described 3 cases of nonunion of the lower limb where osteomyelitis was not bacteriologically detected after surgery on limb. We compared regarding surgical pathology and preoperative ${ }^{18} \mathrm{~F}$-FDG PET/CT.

Results: Upon comparing the pathological findings and the ${ }^{18} \mathrm{~F}-\mathrm{FDG}$ PET/CT setting an SUV with a lower level of 2.00 and an upper level of 8.00 , the infection was accurately localized. In the cases of noninfectious nonunion, infection was not detected on both pathological findings and ${ }^{18} \mathrm{~F}-\mathrm{FDG}$ PET/CT findings.

Conclusion: The infection focus of osteomyelitis which was detected by histopathological examination was accurately visualized by ${ }^{18} \mathrm{~F}$ FDG PET/CT setting the SUV cut-off lower level to 2.00 and upper level to 8.00 . We believe this ${ }^{18} \mathrm{~F}-\mathrm{FDG}$ PET/CT technique is helpful for image guided surgery of osteomyelitis.

References: 1) Gustilo RB, Mendoza RM, Williams DN. Problems in management of type III (severe) open fractures: a new classification of typeIII open fractures. J Trauma. 1984;24:742-746. 2) Basu S, Chryssikos T, Moghadam-Kia S, Zhuang H, Torigian DA, Alavi A: Positron emission tomography as a diagnostic tool in infection: present role and future possibilities. Semin Nucl Med. 2009 Jan;39 (1):36-51.

Disclosure: No significant relationships.

\section{PR392}

\section{D PRINTS OF DISPLACED INTRA-ARTICULAR CALCANEAL FRACTURE IMPROVES THE INTER- OBSERVER RELIABILITY OF THE SANDERS CLASSIFICATION}

\section{Misselyn}

\section{Traumatology, UZ Leuven, Leuven/BELGIUM}

Introduction: The Sanders classification is the most used classification of calcaneal fractures, but it has a moderate interobserver agreement. To improve this reliability, several authors tested the added value of 3D imaging but they were not really successful.
Material and methods: After segmentation (virtual disarticulation), 11 intra-articular calcaneal fractures corresponding to different types of the Sanders classification were 3D-printed with a standard 3Dprinter. The 3D-prints and their 2D-CT counterparts of the same fractures were presented separately to 24 observers (trainees, radiologists, foot surgeons). Interobserver agreement for the Sanders classification was assessed by using the kappa coefficient values (Fleiss kappa, Brennan and Prediger weighted kappa). Three versions of the classification were considered: Sanders classification with subclasses, without subclasses and combining Sanders III and IV subclasses. The gold standard for classification was the peroperative findings by a single surgeon.

Results: The 3D print always yielded higher values for agreement and chance-corrected agreement. The (Brennan and Prediger) weighted kappa equaled 0.35 (for 2D) and 0.63 (for 3D) for Sanders with subclasses $(p=0.004), 0.55(2 \mathrm{D})$ and $0.76(3 \mathrm{D})$ for Sanders without subclasses $(\mathrm{p}=0.003)$, and $0.58(2 \mathrm{D})$ and $0.78(3 \mathrm{D})$ for the fusion of Sanders III and IV ( $\mathrm{p}=0.027)$. There was also greater agreement with the peroperative evaluation, $88 \%$ vs $65 \%$ (3D vs $2 \mathrm{D}$, $\mathrm{p}<0.0001$ ), and a higher percentage of Sanders III-IV with 2D compared to $3 \mathrm{D}, 56 \%$ vs $32 \%$ ( $\mathrm{p}<0.0001$ ).

Conclusion: The use of 3D-prints improves the interobserver reliability of the Sanders classification of calcaneal fractures.

References: 1. Schepers T. Calcaneal Fracture Classification: A Comparative Study. J Foot Ankle Surg 48 (2):156-162, 2009. 2. Furey A. Os Calcis Fractures: Analysis of Interobserver Variability in Using Sanders Classification. J Foot Ankle Surg 42 (1): 21-23, 2003. 3. Howells NR, Hughes AW, Jackson M, Atkins RM, Livingstone JA. Interobserver and intraobserver reliability assessment of calcaneal fracture classification systems. J Foot Ankle Surg. 53 (1): 47-51, 2014. 4. Sanders R, Fortin P, Dipasquale T, Walling A. Operative treatment in 120 displaced intraarticular calcaneal fractures. Results using a prognostic computed tomography scan classification. Clin Orthop 290: 87-95, 1993. 5. Brunner A, Heeren N, Albrecht F, Hahn M, Ulmar B, Babst R. Effect of Three-Dimensional Computed Tomography Reconstructions on Reliability of Classification of Calcaneal Fractures. Foot Ankle International doi: 10.3113/ FAI.2012.0727 6. Roll Ch, Schirmbeck J, Schreyer A, Müller F, Neumann C, Nerlich M, Kinner B. How reliable are CT scans for the evaluation of calcaneal fractures? Arch Orthop Trauma Surg 131 (10):1397-1403, 2011. Doi: 10.1007/s00402-011-1312-7. Epub 2011 May 5 7. Howells NR, Hughes AW, Jackson M, Atkins RM, Livingstone JA. Interobserver and intraobserver reliability assessment of calcaneal fracture classification systems. J Foot Ankle Surg 53 (1):4751, 2014. 8. Humphrey CA, Dirschl DR, Ellis TJ. Interobserver reliability of a CT-based fracture classification system. J Orthop Trauma 19 (9):616-622, 2005. 9. Sayed-Noor AS, Agren PH, Wretenberg P. Interobserver reliability and intraobserver reproducibility of three radiological classification systems for intraarticular calcaneal fractures. Foot Ankle Int 32 (9):861-866, 2011. 10. Veltman E, van den Bekerom M, Doornberg J, Verbeek D, Rammelt S, Steller E, Schepers T. Three-dimensional computed tomography is not indicated for the classification and characterization of calcaneal fractures. Injury Int J Care Injured 45: 1117-1120, 2014. 11. Bhattacharya R, Vassan UT, Finn P, Port A, Sanders classification of fractures of the os calcis, an analysis of inter- and intra-observer variability, J Bone Joint Surg (Br) 87-B:205-8, 2007. Doi:10.1302/ 0301-620X.87B2.15260\$2.00 12. Lauder AJ, Inda DJ, Bott AM, Clare MP, Fitzgibbons TC, Mormino MA. Interobserver and intraobserver reliability of two classification systems for intra-articular calcaneal fractures. Foot Ankle Int. 2006 Apr;27 (4):251-5. 13. Durkalski, V. L., Palesch, Y. Y., Lipsitz, S. R., \& Rust, P. F. (2003). Analysis of clustered matched-pair data. Statistics in medicine, 22 (15), 2417-2428. 14. Von Eye A, Von Eye M. On the On the Marginal Dependency of Cohen's $\kappa$. European Psychologist 13 (4):305-315, 2008. 15. Gwet KL. Testing the Difference of Correlated Agreement 
Coefficients for Statistical Significance. Educational and Psychological Measurement 76 (4) 609-637, 2016. 16. R Core Team (2107). R: A language and environment for statistical computing. R Foundation for Statistical Computing, Vienna, Austria. url https://www.R-project.org 17. Roll Ch, Schirmbeck J, Müller F, Neumann C, Kinner B. Value of 3D Reconstructions of CT scans for Calcaneal Fracture Assessment. Foot Ankle Int. 37 (11):1211-1217, 2016. 18. Allon SM, Mears DC. Three dimensional analysis of calcaneal fractures Foot Ankle. 11 (5):254-63, 1991.

Disclosure: No significant relationships.

\section{PR393}

\section{COMPLICATION RATES ASSOCIATED WITH THE SYNTHES 4.5 MM VARIABLE ANGLE LOCKING COMPRESSION DISTAL FEMUR PLATE (VA-LCP)}

\section{B.A. Zelle, K.H. Dang, C.A. Armstrong}

Orthopaedics, UT Health San Antonio, San Antonio/TX/UNITED STATES OF AMERICA

Introduction: Open reduction and internal fixation using locked plating technology remains a standard treatment for distal femur fractures. Recent investigations have questioned the safety of the Synthes VA-LCP and mechanical failure rates as high as $22.2 \%$ have been reported [1]. The objective of this study was to evaluate the safety and efficacy of the VA-LCP. We hypothesize that the VA-LCP is an effective implant with a low mechanical failure rate.

Material and methods: This retrospective clinical study was performed at an urban level 1 trauma center. A total of 102 patients undergoing surgical fixation of their distal femur fracture with the VA-LCP were included. Patients with pathologic fracture and patients younger than 18 years of age were excluded. The primary outcome measure was mechanical hardware failure within 6 months following surgery.

Results: A total of 83 patients were available for their 6-months follow-up. A mechanical failure rate of 7.2\% (6 of 83) was recorded. A total of $93.9 \%(78 / 83)$ returned to their pre-operative ambulatory status. The rate of deep infections requiring hardware removal was $3.6 \%(3 / 83)$.

Conclusion: The VA-LCP is a safe and efficient plating system for distal femur fractures. The clinical and radiographic outcomes associated with the use of this implant compare favorably with the results of other distal femur plating systems that have been reported in the literature.

References: 1. Tank JC, Schneider PS, Davis E, et al. Mechanical Failures of the Synthes Variable Angle Locking Distal Femur Plate. J Orthop Trauma. 2016;30:e7-e11.

Disclosure: The study was partially funded through a research grant from DePuySynthes

\section{PR394}

\section{CHIPPING TECHNIQUE FOR THE TREATMENT OF FEMORAL NONUNIONS ASSOCIATED WITH MAL- ALIGNMENT AND/OR SHORTENING}

G. Sasaki ${ }^{1}, Y$. Watanabe $^{2}$, M. Takaki $^{3}$, Y. Yasui $^{2}$, W. Miyamoto $^{2}$, H. Kawano ${ }^{2}$, T. Matsushita
${ }^{1}$ Orthopaedics, Teikyo University School of Medicine, Tokyo/ JAPAN, ${ }^{2}$ Orthopaedic Surgery, Teikyo University School of Medicine, Tokyo/JAPAN, ${ }^{3}$ Traumatology, Fukushima Medical University, Koriyama, Fukushima/JAPAN

Introduction: To treat long bone nonunion and mal-alignment at the same time is very difficult problem. We have previously reported chipping technique to treat long bone nonunions without bone graft. The purpose of this study is to clarify the effectiveness of the chipping technique for the treatment of femoral nonunions associated with malalignment and/or shortening.

Material and methods: Clinical and radiological outcomes in the patients who recieved operative treatment for femoral nonunion were retrospectively reviewed using the Refractory Fracture Data Registry at the authors' institution. The chipping technique was indicated for patients who had the femoral nonunion with mal-alignment (angular and/or rotational deformity) with or without limb length discrepancy. The deformities were simultaneously corrected by a combination of chipping and temporal external fixator. Then patients with limb length discrepancy had received simultaneously or gradually lengthening after chipping procedure.

Results: A total of 26 femoral nonunions were managed with this procedure. 7 of 12 cases with limb length discrepancy had received gradually lengthening after chipping procedure. The median age of patients were 41 years (range, 19-73 years). The median time from injury or the final surgery to the initial chipping procedure was 24.5 months (range: 5-240 months). All femoral non-unions radiographically healed with one ( 24 cases) or two ( 2 cases) chipping procedures without bone grafting. We could correct preoperative deformities to almost normal alignment.

Conclusion: Chipping and lengthening technique is a possible treatment option for femoral femoral nonunions associated with malalignment and/or shortening.

References: Matsushita T, Watanabe Y. Chipping and lengthening technique for delayed unions and nonunions with shortening or bone loss. J Orthop Trauma 2007;21:404-6. Giannoudis PV, Gudipati S, Harwood P, Kanakaris NK. Long bone non-unions treated with the diamond concept: a case series of 64 patients. Injury 2015;46 (Suppl 8): S48-54. Watanabe Y, Takaki M, Nishizawa Y, Kato N, Takenaka N, Matsushita T. Chipping corrective osteotomy (CCO). Orthop Surg Traumatol 2014;57:833-41 [in Japanese]. Watanabe Y, Matsushita T. Femoral non-union with malalignment: reconstruction and biological stimulation with the chipping technique. Injury 2016;47 (Suppl 6): S47-S52.

Disclosure: No significant relationships.

\section{PR395}

\section{LONG TERM FOLLOW UP AFTER MINIMAL INVASIVE PLATE OSTEOSYNTHESIS FOR PROXIMAL HUMERUS FRACTURES}

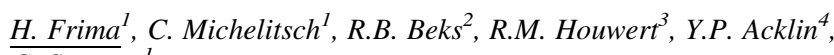 C. Sommer ${ }^{1}$}

${ }^{1}$ Surgery, Kantonsspital Graubünden, Chur/SWITZERLAND,

${ }^{2}$ Utrecht Traumacenter, University Medical Center Utrecht, Utrecht/ NETHERLANDS, ${ }^{3}$ Utrecht Traumacenter, Uiversity Medical Center Utrecht, Utrecht/NETHERLANDS, ${ }^{4}$ Orthopaedic Surgery, Kantonsspital Baselland, Bruderholz, Bruderholz/SWITZERLAND 
Introduction: Minimal invasive plate osteosynthesis (MIPO) has been described as a suitable technique for the treatment of proximal humerus fractures but long term functional results have never been reported. The aim of this study was to describe the long term functional outcome and implant related irritation after MIPO for proximal humerus fractures.

Material and methods: A long term prospective cohort analysis was performed on all patients treated for a proximal humerus fracture using MIPO with a Philos plate (Synthes, Switzerland) between December 2007 and October 2010. The primary outcome was the QuickDASH score. Secondary outcome measures were the Subjective Shoulder Value (SSV), implant related irritation and implant removal. Results: Seventy-eight patients with mean age of 58 years were available for follow up (80\%) with a mean follow up of 8.3 years (SD 0.8 ). The median QuickDASH score was 0 (interquartile range [IQR] $0-4.5$, range $0-86$ ). The median SSV was 97 (IQR $90-100$, range $60-100)$. Forty patients $(52 \%)$ had implant removal of whom 27 (35\%) patients due to implant related irritation. On average the implant was removed after 1.2 years (SD 0.5). Bivariate analysis showed no relevant association of age, trauma mechanism or $\mathrm{AO}$ classification with the QuickDASH score and the SSV.

Conclusion: In our collective, we present excellent functional outcome in the long term follow-up after MIPO for proximal humerus fracture. Never the less, we noticed a relatively high rate of secondary operations due to implant related irritation.

References:

Disclosure: No significant relationships.

\section{PR396}

\section{TREATMENT OF POST TRAUMA SSI FOR IMPLANT RETAINING AND TISSUE PRESERVING STRATEGY BY IMAP (INTRA-MEDULLARY ANTIBIOTICS PERFUSION)}

\author{
A. Maruo $^{1}$, S. Watanabe ${ }^{2}$, K. Sawauchi $^{3}$, Y. Onoi $^{1}$, M. Kamimura $^{4}$,

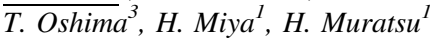

${ }^{1}$ Orthopaedics, Steel Memorial Hirohata Hospital, Himeji/JAPAN, ${ }^{2}$ Department Of Orthopaedic Surgery, Steel Memorial Hirohata Hospital, Himeji-shi, Hyogo/JAPAN, ${ }^{3}$ Department Of Orthopaedic Surgery, Steel Memorial Hirohata Hospital, Himeji/JAPAN,

${ }^{4}$ Orthopaedic, Steel Memorial Hirohata Hospital, Himeji/JAPAN

Introduction: Post traumatic deep surgical site infection (SSI) are difficult to treat, antibiotics does not distribute low vascularized tissue. Aggressive debridement and implant removal was usually required to control infection. We considered high dose antibiotics distribution may eradicate infection with retaining tissue and implant. We have been developed iMAP (intra-medullary antibiotics perfusion) as an alternative local drug delivery system. The purpose of this study was to assess the efficacy of iMAP for post trauma SSI.

Material and methods: From 2000 to 2015, 24 patients (mean age 55, 17 males 7 female) of post traumatic deep SSI was reviewed. Mean follow up period was 4.3 years. Once infection was occurred, $3 \mathrm{~mm}$ needles were applied besides the infection site and aminoglycoside antibiotics (Gentamicin120 300mg, Arbekacin 100 200mg) was continuously instillated to medullary canal with minimal debridement and implant retaining strategy. Intravenous antibiotics were administrated for minimal 4 weeks as an osteomyelitis. Healing rate and clinical outcome were reviewed.

Results: Fractures were fixed by 17 plate, 6 nail and 1 TBW. iMAP was applied 19 tibias, 2 femurs and others. Microorganisms were 11 MSSA, 3 MRSA, 1 MRCNS and others. Mean duration of the iMAP was 17 days. 21 patients retained implant and 20 patients healed by iMAP and 4 patient required to repeated iMAP for recurrence. All patients achieved bone union and free from infection without additional soft tissue or bony reconstruction. No patient underwent amputation nor arthrodesis.

Conclusion: iMAP was an alternative drug delivery system, which achieved high dose antibiotics distribution for infection site and successfully eradicated post traumatic SSI with minimal invasive strategy.

\section{References:}

Disclosure: No significant relationships.

\section{PR397}

\section{A STUDY ABOUT THE CONTOURING ACCURACY OF ANATOMICALLY PRECONTOURED CLAVICLE PLATES WITH ANGULAR STABILITY}

M. Doppelbauer, D. Sauter, M. Schüler

Orthopaedics \& Traumatology, Cantonal Hospital Münsterlingen, Münsterlingen/SWITZERLAND

Introduction: Fractures of the clavicle, which primarily occur in young males, constitute $2.6-4 \%$ of all fractures in adults. The majority $(69-82 \%)$ of these fractures are located in middle third (Kihlström et/al.). Over the last years, there is an increasing trend towards operative treatment of clavicular midshaft fractures. Not at least, because the use of anatomically precontoured clavicle plates.

Material and methods: In the context of a retrospective study, we analyzed 76 patients, who were treated with an LCP-clavicle-plate (Synthes) between 2013-2016. Both the fracture localization as well as the fitting accuracy (in terms of surface area with respect to the clavicle contour) were measured in the postoperative X-ray. We validated our radiologically measuring methods beforehand using original plates on Synbone.

Results: 44 (8-holes) and 32 (7-holes) LCP-clavicle-plates were implanted. The average position of the fractures was $8 \%$ lateralized (in relation to the middle of the clavicle). The plates themselves were placed, without exception, in the center in relation to the fracture, with a consecutive lateralization of the plate. By linear regression (correlation coefficient $r=-0.2451)$ and ANOVA-test $(p=0.0328)$ we have shown statistical significance that the more laterally the plate was placed, a decreasing bone coverage by the plate was the result. Conclusion: Anatomically precontoured clavicle plates show good material properties, but in terms of fitting and modifiability disadvantages have to be accepted. The plate we analyzed only fits well when placed in the middle of the clavicle-however, according to our research almost all fractures occurred lateral of the centerline. These data correspond with our clinical observations of disturbing implants. References:

Disclosure: No significant relationships. 
PR398

\section{MINIMALLY INVASIVE FIXATION OF CALCANEAL FRACTURES USING A REAL-SIZE 3D-PRINTED MODEL}

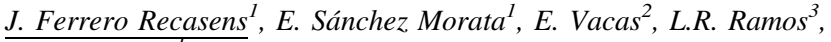 \\ J. Vilá Y Rico
}

${ }^{1}$ CirugÍa Ortopedica Y TraumatologÍa, HOSPITAL UNIVERSITARIO 12 DE OCTUBRE, MADRID/SPAIN, ${ }^{2}$ Cirugia Ortopedica Y Traumatologia, Hospital 12 de Octubre, Madrid/ SPAIN, ${ }^{3}$ Orthopaedic Surgery And Traumatology, Hospital Universitario 12 de Octubre, Madrid/SPAIN

Introduction: The extensile-lateral-approach has been the most common approach for the operative treatment of intrarticular-calcaneal-fractures. Although it allows excellent exposure of the fracture, soft tissue-complications, including flap-necrosis and wound infection, have been a concern. In an attempt to eliminate these complications, minimally-invasive-approaches, such as a sinus-tarsiapproach, have been developed. The objetive are describe the use of a real-size 3-dimensional (3D)-printed calcaneus-model as a preoperative and intraoperative tool for the minimally-invasive plate fixation of calcaneal-fractures.

Material and methods: We present the case of a 35-year-old male with a unilateral calcaneus fracture. A CT scan of calcaneus were taken with a slice thickness of $1 \mathrm{~mm}$. The data acquired from the CT scan are saved in the DICOM (Digital-Imaging-and-Communications-in-Medicine)format. The data are sent to a 3D-printing to produce a realsize calcaneus-model.

Results: A sinus-tarsi approach was performed. The fracture reduction and the overall alignment are checked under C-arm fluoroscopy by comparing it with the real-size model. After evaluating the reduction, it is fixed with a locking plate. The proper positioning of the plate is confirmed using $\mathrm{C}$-arm fluoroscopy comparing the intraoperative image and the images taken before the operation with the plate attached to the real-size model3D.

Conclusion: The use of 3 Dprinting in medicine is growing. In the field of orthopedic surgery, the accurate reproduction of actual osseous anatomy using 3D-models can be invaluable for understanding the characteristics of fractures and deformities, for preoperative contouring of plates. We believe this technique can be useful for the operative treatment of displaced intra-articular-calcaneal-fractures especially for Sanders-type-2 fractures, which will benefit from platefixation.

References: Kline AJ, Anderson RB, Davis WH, Jones CP, Cohen BE. Minimally invasive technique versus an extensile lateral approach for intra-articular calcaneal fractures. Foot Ankle Int. 2013;34 (6):773-780. Rengier F, Mehndiratta A, von Tengg-Kobligk $\mathrm{H}$, et al. 3D printing based on imaging data: review of medical applications. Int J Comput Assist Radiol Surg. 2010;5 (4):335-341. Abdelazeem A, Khedr A, Abousayed M, Seifeldin A, Khaled S. Management of displaced intra-articular calcaneal fractures using the limited open sinus tarsi approach and fixation by screws only technique. Int Orthop. 2014;38 (3):601-606. Zhang T, Su Y, Chen W, Zhang Q, Wu Z, Zhang Y. Displaced intra-articular calcaneal fractures treated in a minimally invasive fashion: longitudinal approach versus sinus tarsi approach. J Bone Joint Surg Am. 2014;96 (4):302309.

Disclosure: No significant relationships.
PR399

\section{SURGICAL TREATMENT OF UNSTABLE LATERAL CLAVICULAR FRACTURES: COMPARISON OF HOOK PLATE VERSUS SUPERIOR PLATE WITH LATERAL EXTENSION. A RETROSPECTIVE COHORT STUDY}

Y. Ochen ${ }^{1}$, H. Frima ${ }^{2}$, R.M. Houwert ${ }^{3}$, M. Van Heijl ${ }^{4}$, E. Verleisdonk ${ }^{4}$, D. Van Der Velde ${ }^{5}$, B.Emmink $^{6}$, J. Keizer $^{5}$

${ }^{1}$ Trauma Surgery, Sint Antonius Ziekenhuis, Nieuwegein/ NETHERLANDS, ${ }^{2}$ Surgery, Kantonsspital Graubünden, Chur/ SWITZERLAND, ${ }^{3}$ Utrecht Traumacenter, Uiversity Medical Center Utrecht, Utrecht/NETHERLANDS, ${ }^{4}$ Surgery, Diakonessenhuis, Utrecht/NETHERLANDS, ${ }^{5}$ Trauma Surgery, St. Antonius Hospital, Nieuwegein/NETHERLANDS, ${ }^{6}$ Trauma Surgery, St Antonius Hospital, Nieuwegein/NETHERLANDS

Introduction: Different fixation methods are used for treatment of unstable lateral clavicle fractures (LCF). The aim of this study was to compare patient-reported functional outcome after open reduction and internal fixation (ORIF) with the clavicle hook plate (CHP) and the superior clavicle plate with lateral extension (SCPLE).

Material and methods: A dual-center retrospective cohort study was performed. All patients operatively treated for unstable LCF between 2011 and 2016, with the CHP $(n=23)$ or the SCPLE $(n=53)$ were eligible for inclusion. The primary outcome was the QuickDASH score. Secondary outcomes were the Numerical Rating Scale (NRS) pain score, complications and implant removal

Results: In total 67 patients (88\%) were available for follow-up and analyses. There was no significant difference in functional outcome scores between the CHP and SCPLE groups (median QuickDASH score, CHP 0.00 (IQR;0.0-0.0), SCPLE 0.00 (IQR;0.0-4.5), $\mathrm{p}=$ 0.073; NRS pain score at rest, $\mathrm{p}=0.373$; NRS pain score during activity, $\mathrm{p}=0.559$ ). The CHP was associated with a higher complication rate of $47 \%$, compared to $8 \%$ in the SCPLE group (relative risk, 5.68; 95\% CI, 1.99-16.26; $\mathrm{p}=<0.001)$. The implant removal rate was $100 \%$ in the CHP group, compared to $42 \%$ in the SCPLE group (relative risk, 2.40; 95\% CI, 1.72-3.35; $\mathrm{p}=<0.001$ ).

Conclusion: Both the CHP and SCPLE result in good patient-reported functional outcome. However, the CHP had a higher complication- and implant removal rate. Therefore, we recommend the use of the SCPLE for the surgical fixation of unstable LCF.

References: 1. Nordqvist A, Petersson C. The incidence of fractures of the clavicle. Clin Orthop Relat Res. United States; 1994 Mar; (300):127-32. 2. Robinson CM. Fractures of the clavicle in the adult. Epidemiology and classification. J Bone Joint Surg Br. England; 1998 May;80 (3):476-84. 3. Postacchini F, Gumina S, De Santis P, Albo F. Epidemiology of clavicle fractures. J shoulder Elb Surg. United States; 2002;11 (5):452-6. 4. Sambandam B, Gupta R, Kumar S, Maini L. Fracture of distal end clavicle: A review. J Clin Orthop trauma. India; 2014 Jun;5 (2):65-73. 5. Oh JH, Kim SH, Lee JH, Shin SH, Gong HS. Treatment of distal clavicle fracture: a systematic review of treatment modalities in 425 fractures. Arch Orthop Trauma Surg. Germany; 2011 Apr;131 (4):525-33. 6. Neer CS 2nd. Fractures of the distal third of the clavicle. Clin Orthop Relat Res. United States; 1968;58:43-50. 7. NEER CS 2nd. Fracture of the distal clavicle with detachment of the coracoclavicular ligaments in adults. J Trauma. United States; 1963 Mar;3:99-110. 8. Ropars M, Thomazeau H, Huten D. Clavicle fractures. Orthop Traumatol Surg Res. France; 2017 Feb;103 (1S):S53-9. 9. Rokito AS, Zuckerman JD, Shaari JM, Eisenberg DP, Cuomo F, Gallagher MA. A comparison of nonoperative and operative treatment of type II distal clavicle fractures. Bull Hosp Jt Dis. United States; 61 (1-2):32-9. 10. Muramatsu K, Shigetomi M, Matsunaga T, Murata Y, Taguchi T. Use of the AO hook-plate for treatment of unstable fractures of the distal 
clavicle. Arch Orthop Trauma Surg. Germany; 2007 Apr;127 (3):191-4. 11. Renger RJ, Roukema GR, Reurings JC, Raams PM, Font J, Verleisdonk EJMM. The clavicle hook plate for Neer type II lateral clavicle fractures. J Orthop Trauma. United States; 2009 Sep;23 (8):570-4. 12. Tambe AD, Motkur P, Qamar A, Drew S, Turner SM. Fractures of the distal third of the clavicle treated by hook plating. Int Orthop. Germany; 2006 Feb;30 (1):7-10. 13. Lee SK, Lee JW, Song DG, Choy WS. Precontoured locking plate fixation for displaced lateral clavicle fractures. Orthopedics. United States; 2013 Jun;36 (6):801-7. 14. Andersen JR, Willis MP, Nelson R, Mighell MA. Precontoured superior locked plating of distal clavicle fractures: a new strategy. Clin Orthop Relat Res. United States; 2011 Dec;469 (12):3344-50. 15. Tiren D, Vroemen JPAM. Superior clavicle plate with lateral extension for displaced lateral clavicle fractures: a prospective study. J Orthop Traumatol. Italy; 2013 Jun;14 (2):115-20. 16. Beirer M, Siebenlist S, Cronlein M, Postl L, HuberWagner S, Biberthaler P, et al. Clinical and radiological outcome following treatment of displaced lateral clavicle fractures using a locking compression plate with lateral extension: a prospective study. BMC Musculoskelet Disord. England; 2014 Nov;15:380. 17. Fleming MA, Dachs R, Maqungo S, du Plessis J-P, Vrettos BC, Roche SJL. Angular stable fixation of displaced distal-third clavicle fractures with superior precontoured locking plates. J shoulder Elb Surg. United States; 2015 May;24 (5):700-4. 18. Vaishya R, Vijay V, Khanna V. Outcome of distal end clavicle fractures treated with locking plates. Chinese $\mathrm{J}$ Traumatol $=$ Zhonghua chuang shang za zhi. China; 2017 Feb;20 (1):45-8. 19. London DA, Stepan JG, Boyer MI, Calfee RP. Performance Characteristics of the Verbal QuickDASH. Vol. 39, The Journal of hand surgery. 2014. 20. Ferreira-Valente MA, Pais-Ribeiro JL, Jensen MP. Validity of four pain intensity rating scales. Pain. United States; 2011 Oct;152 (10):2399-404. 21. Tiren D, van Bemmel AJM, Swank DJ, van der Linden FM. Hook plate fixation of acute displaced lateral clavicle fractures: mid-term results and a brief literature overview. J Orthop Surg Res. England; 2012 Jan;7:2. 22. Lee Y-S, Lau M-J, Tseng Y-C, Chen W-C, Kao H-Y, Wei J-D. Comparison of the efficacy of hook plate versus tension band wire in the treatment of unstable fractures of the distal clavicle. Int Orthop. Germany; 2009 Oct;33 (5):1401-5. 23. Lin H-Y, Wong P-K, Ho W-P, Chuang T-Y, Liao Y-S, Wong C-C. Clavicular hook plate may induce subacromial shoulder impingement and rotator cuff lesion-dynamic sonographic evaluation. J Orthop Surg Res. England; 2014 Feb;9:6. 24. Sun S, Gan M, Sun H, Wu G, Yang H, Zhou F. Does Subacromial Osteolysis Affect Shoulder Function after Clavicle Hook Plating? Biomed Res Int. United States; 2016;2016:4085305. 25. Zhang C, Huang J, Luo Y, Sun H. Comparison of the efficacy of a distal clavicular locking plate versus a clavicular hook plate in the treatment of unstable distal clavicle fractures and a systematic literature review. Int Orthop. Germany; 2014 Jul;38 (7):1461-8. 26. Erdle B, Izadpanah K, Jaeger M, Jensen P, Konstantinidis L, Zwingmann J, et al. Comparative analysis of locking plate versus hook plate osteosynthesis of Neer type IIB lateral clavicle fractures. Arch Orthop Trauma Surg. Germany; 2017 May;137 (5):651-62.

Disclosure: No significant relationships.

\section{PR400}

\section{PERCUTANEOUS PEDICLE SCREW FIXATION (PPSF) IN THE TREATMENT OF THORACIC AND LUMBAR SPINE FRACTURES}

\author{
P. Zogakis ${ }^{I}$, M. Tzurbakis ${ }^{2}$, K. Papagiannakos ${ }^{I}$, G. Fragkeskakis ${ }^{I}$, \\ $\overline{\text { K. Bossinakis }}^{I}$, D. Kourtzis ${ }^{1}$, G. Mpozonelos ${ }^{1}$
}

${ }^{1}$ Orthopaedics, Evaggelismos Hospital, Athens/GREECE,

${ }^{2}$ Orthopaedics, EVAGGELISMOS, ATHENS/GREECE
Introduction: Surgical treatment of Thoracic and Lumbar spine fractures depends on various factors. Pathological features of the vertebral lesion, neurological deficit, general condition of health and presence of polytrauma affects the treatment. (1) Conservative treatment resulting in T-L or Lumbar kyphosis can worsen the quality of life of the patient whereas traditional open surgery may be an overtreatment in some cases, considering possible complications. In this setting, a good option can be a percutaneous minimally invasive surgery. (1) The advantages of percutaneous pedicle screw fixation are: preservation of posterior musculature, less blood loss, shorter operative time, lower infection risk, less postoperative pain, shorter hospital stay and easier implant removal after bone union. Limitations can be dealed by combination with open techniques (2). The objective of this study was to report the results of PPSF technique on these fractures.

Material and methods: Eighteen patients were treated with PPSF from 2015 till now. 15 patients were males, 3 females; average age was 45 years (range from 18 to 82). In all cases, system Pathfinder NXT was used. Follow-up was 19,1 months (7 to 30 months).

Results: Mobilisation of the patients started on the second post-operative day. Post-operative pain was managed with mild painkillers. Limitations in mobilisation in 6 patients were due to co-existent lower limb injuries or non-reversible neurological deficit. No major complications were noticed.

Conclusion: PPSF is a reliable and safe procedure which does not replace the open technique but adds to treatment options (3) by restoring a good sagittal alignment similar to those reported for open surgery (4)

References: 1.Minimally invasive percutaneous fixation in the treatment of thoracic and lumbar spine fractures. 2009 Jun; 18 (Suppl 1): 71-74. 2.Percutaneous fixation of thoracolumbar fractures: current concepts. 2012 Dec;98 (8):900-9 3.Posterior percutaneous reduction and fixation of thoraco-lumbar burst fractures. 2015 May;101 (3):3759 4.Degenerative facet joint changes in lumbar percutaneous pedicle screw fixation without fusion. 2015 May;101 (3):375-9.

Disclosure: No significant relationships.

\section{PR401}

\section{CONTROVERSIES IN TRAUMATIC SPINAL CORD INJURIES MANAGEMENT}

\author{
C. Vale, D. Peixoto, S. Angelo, F.S. Gomes, T. Roseiro, \\ A.M. Carvalho, V. Oliveira, J. Antunes
}

Serviço De Ortopedia, Hospital Distrital da Figueira da Foz, Figueira da Foz/PORTUGAL

Introduction: Traumatic Spinal Cord Injuries (TSCI) have a great morbi-mortality rate in individuals and any polytraumatized patient should be treated as having a potencial injury. Cervical trauma represents $55 \%$ of the lesions in the emergency room, and it requires search for injuries throughout the entire spinal cord.

Material and methods: Review of management of TSCI with discussion of the actual controversies.

Results: Care of a traumatic spinally injured patient is aimed towards the preservation of function and prevention of disability. The actual guidelines in TSCI include imediate immobilization, avoidance of hypotension, early classification with International Standards for Neurologic Classification and rapid referral to specialized centres. MRI role in the early stages stays controversial, except for predicting neurologic outcomes, but CT stays the standard imaging for diagnosis. The role of methylprednisolone as a therapeutic option is still a 
matter of debate and most guidelines do not recomend it, although a recent study considers it as an option in the first 8 hours of injury. For cervical-level injuries they think that early surgical estabilization may improve funtional outcome, but in thoracic and lombar injuries, the time of surgical descompression is still controversial. Neuroprotective and Neuroregenerative therapies are being extensively investigated, with promising initial results.

Conclusion: TSCI management has changed over the last years, and some of the key points in treatment are being recognized. It is important to emphasize that early management could avoid some later complications and could change the course of outcome in these patients.

References: - Wing, P. et al (2008) Early Acute Management in Adults with Spinal Cord Injury, The Journal of Spinal Cord Medicine, volume 31, n 4 , Paralyzed Veterans of America - Bustock, J. et al (2016) Spinal Injury: assessment and initial management, NICE Guideline, NG41, National institute for Health and Care Excellence Fehlings, M. et al (2016) Guidelines for Management of Patients with Spinal Cord Injury: The Use of Methylprednisolone Sodium Succinate, NASS Annual Meeting Proceedings, The Spine Journal - Ahuja, C. et al (2017) Spinal Cord Injury-What are the controversies?, $J$ Orthop Trauma - Purvis, T. et al (2017) The definite risks and questionable benefits of liberal pre-hospital spinal immobilisation, American Journal of Emergency Medicine - Rouanet, C. et al (2017) Traumatic spinal cord injury: current concepts and treatment update, Arq Neuropsiquiatr

Disclosure: No significant relationships.

\section{PR402}

\section{D PRINTED PSI TARGETING - HOW WE DO IT IN RECONSTRUCTION SURGERY?}

E. Csonka ${ }^{1}$, L. Bánki ${ }^{1}$, C. Gombár ${ }^{2}$, T. Tasi ${ }^{1}$, K. Csete ${ }^{1}$, Z. Sádt ${ }^{1}$,

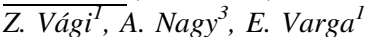

${ }^{1}$ Department Of Traumatology, University of Szeged, Szeged/ HUNGARY, ${ }^{2}$ Department Of Orthopedics, University of Szeged, Szeged/HUNGARY, ${ }^{3}$ Department Of Radiology, University of Szeged, Szeged/HUNGARY

Introduction: Equipments and technologies are continuously evolving in various fields of surgery. The development and use 3 dimensional reconstruction in orthopedic surgery has lead to individualize patient care adjusted to anatomical variances by patient specific instrumets (PSI). Several studies have proved the efficiency and efficacy of PSI use in knee and shoulder arthroplasties or osteotomies. Our PSI workteam in co-operation with software engineers are currently developing a novel software of optimizing the reconstruction technique in shoulder, knee arthroplasties, and osteotomies.

Material and methods: Based on 3 dimensional CT imaging, the anatomical position is evaluated and the precise construction of the implant is designed, then 3 dimensionally printed providing individual prosthesis. Out software, in hallux osteotomies for optimal plane and axis reconstructions, are also eligible for procedural planning in evaluating the ideal angle of osteotomy as well as the angle of correction. Application of post-operative 3D CT imaging are used for controls. Based upon hallux valgus osteotomies, the reconstruction software may provide excellent template for further reconstruction of osteotomies in various anatomical regions.
Results: By the closing time of submission, our pleriminary results are under collection and analysis. The presentation of our results, however, is guaranted by the time of congress date.

Conclusion: We expect that our results will be comparable to prior studies, increasing the accuracy of the procedures without the elongation of procedural time.

References: Warwick Chun-Wang Chan at al.: Patient-specific instrumentation versus conventional instrumentation in total knee arthroplasty Gomes N. Patient-specific instrumentation for total shoulder arthroplasty : Accuracy of patient-specific instrumentation in anatomic and reverse total shoulder arthroplasty

Disclosure: No significant relationships.

\section{PR403}

\section{ALL HIP FRACTURES ARE NOT CREATED EQUAL: IMPACT OF TYPE OF FRACTURE AND LENGTH OF STAY ON TOTAL COST OF TREATMENT}

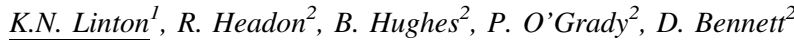 \\ ${ }^{1}$ Trauma \& Orthopaedic Surgery, Galway Clinic, Galway City/ \\ IRELAND, ${ }^{2}$ Trauma \& Orthopaedic Surgery, Mayo University \\ Hospital, Castlebar/IRELAND
}

Introduction: Anecdotal evidence from our trauma \& orthopaedic service suggested that all hip fractures are not created equal. Several authors have reported on time to surgery (TTS), length of stay (LOS) and total cost $[1,2]$. We analyse three years of hip fractures and show how patient demographics and surgical features directly impact discharge destination and cost of treatment.

Material and methods: From a total of 6,584 orthopaedic operations performed between 2014 and 2016 we isolated the 400 neck of femur (NOF) fixations. We captured 12 fields from the logbooks including medical record number, procedure performed and length of operation. Type of fracture was determined from plain radiograph with TTS and LOS derived from the patient administration system. This complete data set was analysed and charted in $\mathbf{R}$.

Results: Hip fracture patients had a wide age range from 10 to 100 years. $63 \%$ were female, median age 79 [20 to 100] years and $37 \%$ male, median age 78 [10 to 99$]$ years. 55\% underwent hemiarthroplasty, 32\% DHS and 13\% IM nail. Length of operation varied significantly depending on procedure; from 45 minutes to over 5 hours. $89 \%$ underwent surgery within one day of admission, though LOS varied from 1 to 116 days (median 15 days). This corresponds to a median cost of $€ 12,000$ [ $€ 800$ to $€ 92,800]$ before we attribute anaesthetics, theatre, radiology and recovery.

Conclusion: Type of fracture and length of stay have a significant effect on cost of treatment. If "money is to follow the patient" this has important implications for the management of our trauma service. References: [1] Soong C, Cram P, Chezar K, Tajammal F, Exconde K, Matelski J, Sinha SK, Abrams HB, Fan-Lun C, Fabbruzzo-Cota C, Backstein D and Bell CM. Impact of an Integrated Hip Fracture Inpatient Program on Length of Stay and Costs. J Orthop Trauma. 2016 Dec;30 (12):647-652. [2] Barinaga G, Wright E, Cagle PJ Jr, Anoushiravani AA, Sayeed Z, Chambers MC, El-Othmani MM and Saleh KJ. Effect of Time of Operation on Hip Fracture Outcomes: A Retrospective Analysis. J Am Acad Orthop Surg. 2017 Jan;25 (1):5560.

Disclosure: No significant relationships. 
PR404

\section{A NOVEL TENSION BAND WIRING TECHNIQUE FOR OLECRANON FRACTURES: TECHNICAL NOTES AND RADIOGRAPHIC OUTCOMES}

\section{N. Yoshida, Y. Tsuchida, K. Futamura}

Department Of Orthopedic Trauma Surgery, Shonankamakura General Hospital, kanagawa/JAPAN

Introduction: Tension band wiring is a common treatment for fixation of olecranon fractures. However, a complication of Kirchner wire migration may be responsible for local pain, secondary displacement, and wound healing problems. Thus, we present a new surgical technique that can avoid Kirchner wire migration. Following reduction of the olecranon fracture and insertion of two Kirchner wires, the sharp proximal end of the Kirchner wire is bent into a question mark shape and the soft wire is passed through the curved part. With this technique, the Kirchner wire is caught and migration is prevented. We determined the effectiveness of this new surgical technique.

Material and methods: We reviewed 48 patients ( 28 females and 20 males) with olecranon fracture undergoing tension band wiring. Mean patient age at surgery was 59.9 years (range, 20-89 years). Of the patients, 18 and 30 underwent surgery with the new and conventional techniques, respectively. All patients were assessed radiologically with elbow x-rays. We compared the distance of Kirchner wire migration in the two groups.

Results: There was a significant difference in the distance of Kirchner wire migration between the two groups. Mean distance was $0.6 \mathrm{~mm}$ [range, $0-6 \mathrm{~mm}$ ] in the new technique, compared to $3.0 \mathrm{~mm}$ [range, $0-18 \mathrm{~mm}]$ in the conventional group $(\mathrm{P}=0.049)$.

Conclusion: The new technique is useful to prevent Kirchner wire migration after tension band wiring for olecranon fracture.

References: Chalidis BE, Sachinis NC, Samoladas EP, et al. Is tension band wiring technique the "gold standard" for the treatment of olecranon fractures? A longterm functional outcome study. J Orthop Surg 2008;22:3-9. Ren YM, Qiao HY, Wei ZJ, et al. Efficacy and safety of tension band wiring versus plate fixation in olecranon fractures: a systematic review and meta-analysis. J Orthop Surg Res 2016;14:137. Wiegand L, Bernstein J, Ahn J. Fractures in brief: Olecranon fractures. Clin Orthop Relat Res 2012;470 (12):3637-3641. Snoddy MC, Lang MF, An TJ, et al. Olecranon fractures: factors influencing re-operation. Int Orthop 2014;38 (8):1711-1716. ChiChuan Wu, Ching-Lung Tai, Chun-Hsiung Shin. Biomechanical Comparison for Different Configurations of Tension Band Wiring Techniques in Treating an Olecranon Fracture. J Trauma 2000;48 (6):1063-1067. den Hamer A, Heusinkveld M, Traa W, et al. Current techniques for management of transverse displaced olecranon fractures. Muscles Ligaments Tendons J 2015;5 (2):129-140. Chan KW, Donnelly KJ. Does K-wire position in tension band wiring of olecranon fractures affect its complications and removal of metal rate? J Orthop 2014;12 (2):111-117. Marco M, Schneider, Tobias E, et al. Tension band wiring in olecranon fractures: the myth of technical simplicity and osteosynthetical perfection. Int Orthop 2014;38 (4):847-855.

Disclosure: No significant relationships.

\section{PR405}

\section{CAN OSTEOCONDUCTIVE CERAMICS BE A SUBSTITUTE FOR ALLOGRAFT IN THE INDUCED MEMBRANE TECHNIQUE?}

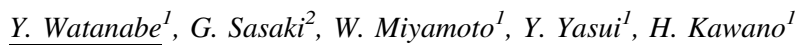

${ }^{1}$ Department Of Orthopaedic Surgery, Teikyo University School of Medicine, Tokyo/JAPAN, ${ }^{2}$ Orthopaedics, Teikyo University School of Medicine, Tokyo/JAPAN

Introduction: In the induced membrane technique (IMT, so-called Masquelet's technique), allograft can be used in combination up to $25 \%$ when the volume of autologous cancellous bone is insufficient. The possibility of osteoconductive ceramics as a substitute of allograft is unknown. The purpose of this study is to know the possibility of using beta-TCP instead of allograft in IMT.

Material and methods: We retrospectively reviewed the clinical and radiological results in consecutive ten patients treated by IMT for the infected non-unions (two femora and eight tibias) between 2014 and 2015 at our institute. In the 1st stage, an antibiotic-loaded PMMA spacer was inserted into the bone defect after an aggressive debridement. The 2 nd stage was performed at 6-8 weeks later, the spacer was removed and the bone defect was filled with a combination of autologous cancellous bone and beta-TCP with a porosity of $75 \%$. The volumetric ratio of cancellous bone and beta-TCP was approximately 1: 1 . Patients were followed up with clinical findings, radiographs and blood test almost every month.

Results: Definitive fixation was the dual plate for four cases, and the intramedullary nail for six. The median size of bone defect was 58 $\mathrm{mm}$ (22-95). All cases were radiographically healed at a median of 6 months (4-12) without recurrence of infection. Healing periods was not affected by age of patient and defect size.

Conclusion: Bone defects of up to $95 \mathrm{~mm}$ can be reconstructed with a combination of autologous cancellous bone and beta-TCP, even if half of the grafted material is beta-TCP not having the osteoinductivity.

References: Masquelet AC, Fitoussi F, Begue T, et al. Reconstruction of the long bones by the induced membrane and spongy autograft. Ann Chir Plast Esthet, 2000 Masquelet AC, Begue T. The concept of induced membrane for reconstruction of long bone defects. Orthop Clin North Am, 2010 Mauffrey C, Giannoudis PV, Conway JD, et al. Masquelet technique for the treatment of segmental bone loss have we made any progress? Injury, 2016 Sasaki G, Watanabe Y, Miyamoto $\mathrm{W}$, et al. Induced membrane technique using beta-tricalcium phosphate for reconstruction of femoral and tibial segmental bone loss due to infection: technical tips and preliminary clinical results, Int Orthop, 2017.

Disclosure: The author received consulting fees for educational lectures from Stryker, Zimmer-Biomet, MDM, and Aimedic.

\section{PR406}

\section{THREE-DIMENSIONAL COMPUTED TOMOGRAPHY MEASUREMENTS OF ACETABULAR FRACTURES}

A.M.l. Meesters $^{1}$, J. Kraeima ${ }^{2}$, H. Banierink ${ }^{3}$, C.H. Slump ${ }^{4}$, K. Ten

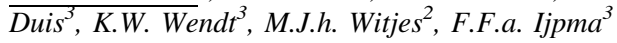

${ }^{1}$ Technical Medicine, University of Twente, Enschede/ NETHERLANDS, ${ }^{2}$ Oral And Maxillofacial Surgery, University Medical Center Groningen, Groningen/NETHERLANDS, ${ }^{3}$ Trauma 
Surgery, University Medical Center Groningen, Groningen/ NETHERLANDS, ${ }^{4}$ Robotics And Mechatronics, University of Twente, Enschede/NETHERLANDS

Introduction: In the current practice, the fracture gap and step-off are still measured in plain radiographs or 2D CT slices. The results can vary widely among physicians, because not everyone measures in a standardized manner. The aim of this study was to develop a standardized 3D CT measurement method for measuring the fracture extent and the quality of the reduction of acetabular fractures.

Material and methods: Ten patients who were operatively treated for an acetabular fracture were included. A 3D computer model of the fracture was created and the fracture was reduced by using the uninjured side of the pelvis as a template. To determine the extent of the fracture, multiple 3D measurements, namely the 3D step-off, 3D gap, 3D displacement, gap deformity and the articular surface area were introduced.

Results: The overall maximum step-off was $14.5 \mathrm{~mm}(0-34.3 \mathrm{~mm})$ and the maximum gap was $28.0 \mathrm{~mm}(0.4-83.0 \mathrm{~mm})$ in the preoperative CT-scans. Post-operatively, the maximum step-off was 4.5 $\mathrm{mm}(0-18.3 \mathrm{~mm})$ and the maximum gap was $7.6 \mathrm{~mm}(0.4-20.4$ $\mathrm{mm})$. It was possible to determine the $3 \mathrm{D}$ displacement for each fracture fragment for each patient pre- and post-operatively. The mean gap deformity was $666 \mathrm{~mm}^{2}$ pre-operatively and $152 \mathrm{~mm}^{2}$ postoperatively, which corresponds with an overall mean fracture reduction rate of $82 \%$.

Conclusion: 3D CT measurements of acetabular fractures are feasible and might be used in addition to the AO/OTA classification system, in order to provide additional information about the fracture extent and quality of the post-operative reduction.

References:

Disclosure: No significant relationships.

\section{PR407}

\section{MEDIAL CLAVICLE FRACTURES; A NEW OPERATIVE TECHNIQUE AND A RETROSPECTIVE COHORT STUDY}

\author{
H. Frima $^{l}$, R.M. Houwert ${ }^{2}$, C. Sommer ${ }^{1}$
}

\section{${ }^{1}$ Surgery, Kantonsspital Graubünden, Chur/SWITZERLAND, ${ }^{2}$ Utrecht Traumacenter, Uiversity Medical Center Utrecht, Utrecht/ NETHERLANDS}

Introduction: Medial clavicle fractures are rare injuries and historically treated non-operatively. Displaced medial clavicle fractures however, have a higher incidence of delayed- or nonunion compared to non- displaced medial clavicle fractures and might benefit from operative treatment. We describe a new technique for treating intraarticular fractures or extra-articular fractures with a small medial fragment using different special locking plates and present the results of our operatively treated patients.

Material and methods: First we describe our technique of treating these fractures with the radial (VA)-LCP ${ }^{\mathrm{TM}}$ Distal Humerus Plate (DePuy Synthes, Switzerland). Second, a retrospective cohort study was performed. All patients operated for a displaced medial clavicle fracture between 2010 and 2017 were included. Primary outcome was the QuickDASH score and the Subjective Shoulder Value (SSV). Secondary outcomes were operative complications including mal- or non-union and implant removal.

Results: All 15 patients were available for follow up. Fourteen patients were included in our analysis. One patient was excluded due to severe concomitant injuries. The mean follow up was 39 months (range 6-79). The mean QuickDASH score was 0,65 (range 0-4.50, SD +/- 1.38) and the mean SSV 96 (range 80-100, SD +/- 6.33). One patient had an early revision and developed an infection after 1,5 years. No mal- or non-unions occurred. Seven patients had their implants removed.

Conclusion: Operative treatment of displaced medial clavicle fractures with well-fitting 'small fragment' locking plates provides an excellent long-term functional outcome. Intra-articular fractures or extra-articular fractures with a small medial fragment can be treated with the radial (VA)-LCP ${ }^{\mathrm{TM}}$ Distal Humerus Plate.

References:

Disclosure: Herman Frima has received financial benefits from Synthes

\section{PR408}

\section{SURGICAL TREATMENT OF UNSTABLE FRACTURES OF THE DISTAL CLAVICLE USING SCORPION® NEO}

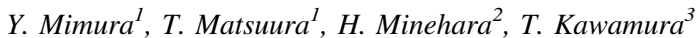

${ }^{1}$ Orthopaedic Surgery, Kitasato university, school of medicine, Sagamihara/JAPAN, ${ }^{2}$ Orthopaedic Surgery/emergency And Critical Care, Kitasato University School of Medicine, Sagamihara/JAPAN, ${ }^{3}$ Orthopeadics, Kitasato University, Sagamihara/JAPAN

Introduction: Distal clavicle fractures of Craig types IIa, IIb and V are unstable and difficult to keep in the reduced position, and thus osteosynthesis is usually indicated. We have been using SCORPION ${ }^{\circledR}$ for surgical treatment of unstable distal clavicle fractures. This device enables reliable fixation without bridging over the acromioclavicular joint if a single screw is inserted into the distal bone piece and held with two arms. Surgery with this device has yielded favorable outcome. Since 2014, we have been using a modified model of this device SCORPION® ${ }^{\circledR}$ NEO. SCORPION ${ }^{\circledR}$ NEO is an improvement from the old type primarily in terms of adoption of the double-arm design, distal movement of the arm, change in arm shape and adoption of an anatomical design. Our experience with treating unstable distal clavicle fracture using SCORPION ${ }^{\circledR}$ NEO is reported.

Material and methods: This study involved 5 patients (4 males and 1 female, mean age 44.2 years) who underwent surgical treatment of unstable distal clavicle fractures with at our hospital. In each case, bone union, complications and three JOA score items (full score $=80$ points) were evaluated.

Results: Bone union was achieved in all cases, with no complications. The JOA score at the time of the last evaluation was 78.8 on average for all cases (75-80).

Conclusion: SCOPION ${ }^{\circledR}$ NEO is a plate which is expected to enable early initiation of ROM training through fixation of distal bone pieces without interfering with the acromioclavicular joint and enabling acquisition of favorable shoulder joint function.

\section{References:}

Disclosure: No significant relationships. 
PR409

\section{INCIDENCE OF POSTTRAUMATIC ARTHRITIS FOLLOWING DISTAL RADIUS FRACTURES IN NON- OSTEOPOROTIC PATIENTS AND THE ASSOCIATION WITH FUNCTION AND PATIENT REPORTED OUTCOMES}

\author{
C.M. Lameijer ${ }^{\text {, }}$,H.J. Ten Duis ${ }^{1}$, M. El Moumni ${ }^{1}$, C.K. Van Der Sluis ${ }^{2}$
}

${ }^{1}$ Traumasurgery, University Medical Center Groningen, Groningen/ NETHERLANDS, ${ }^{2}$ Rehabilitation Medicine, University Medical Center Groningen, Groningen/NETHERLANDS

Introduction: Outcomes of non-osteoporotic patients who sustained a distal radius fracture (DRF) have not gained much attention in recent literature. Aims of this study were to determine incidence of posttraumatic arthritis (PA), to analyze associations of function and Patient Reported Outcomes (PROs) with PA after DRF in non-osteoporotic patients.

Material and methods: Non-osteoporotic patients following a DRF were selected. Radiographs were obtained at follow-up and the degree of PA was determined. Active range of motion and grip strength measurements were performed and 4 questionnaires were answered to assess pain, upper extremity functioning and health status.

Results: Seventy-three patients ( 32 women, 41 men) with a mean age of 33.5 (SD 9.2) years were included. Incidence of PA was $32 \%$ at a median follow-up of 62.0 months. Patients with PA had significantly diminished flexion/extension $\left(12.0^{\circ}, \mathrm{p}=.008\right)$ and ulnar/radial deviation $\left(6.3^{\circ}, \mathrm{p}=.018\right)$. All grip strength measurements were not significantly different between patients with and without PA. Significantly poorer PROs in patients with PA were the MHQ subscales general functioning ( 65 vs. $75, \mathrm{p}=.018$ ), esthetics (94 vs. $100, \mathrm{p}=$ .037 ), satisfaction ( 75 vs. $92, p=.042$ ) and total score of the MHQ ( 83 vs. $91, \mathrm{p}=.044)$, as well as the SF-36 subscale physical functioning (95 vs. $100, \mathrm{p}=.028$ ).

Conclusion: The incidence of PA in non-osteoporotic patients after DRF was considerable, despite relatively short follow-up time. PA was associated with diminished range of motion, but not with grip strength measurements. The PRO MHQ might be a valuable evaluation tool in this patient group.

References:

Disclosure: No significant relationships.

\section{PR410}

\section{DOES ADULT MAXIMAL RADIAL BOWING AND IT'S LOCATION CHANGE IN TURKISH SOCIETY? A DESCRIPTIVE RADIOLOGICAL STUDY}

\section{G. Altun ${ }^{1}$, G. Saka ${ }^{2}$}

${ }^{1}$ Orthopedics And Traumatology, Umraniye Research and Education Hospital, Istanbul/TURKEY, ${ }^{2}$ Orthopedics And Traumatology, Fulya Orthopedics and Spine Center, Istanbul/TURKEY

Introduction: The radius bone consists of a sagittal and coronal bow. The shape of the radius is affected by both the magnitude and the location of the maximum radial bowing. However, it is not known that whether these values show difference in different geographic locations or according to different races or not. The aim of this study is to investigate the effect of results of measurements of maximal radial bowing (MRB)and location of the maximal radial bowing
(LMRB) performed by using standard method in Turkish population.Our study is the first one in the literature evaluating maximum radial bowing and its localization specifically in Turkish population. Material and methods: This study was performed with a total of 200 radius bones (left and right) of 50 healthy female and 50 male volunteers with an atraumatic history. Mean age was 32.4 (range: 20to40) years.

Results: The median amount and location of MRB in our group were $14.76 \pm 1.99 \mathrm{~mm}$ and $60.76 \pm 2.60 \%$; respectively. When our series were compared with the series of Schemitsch and Richards regarding MRB measurements; this rate was found significantly higher in favor of the literature $(\mathrm{p}<0.05)^{1}$.Nevertheless, when our series were compared with these series' LMRB values; the results of our series were found to be significantly higher $(\mathrm{p}<0.05)$

Conclusion: MRB and its location may show difference between geographic locations or different races.It is not known how this bowing shows change in Asian race with lower height compared to especially European race with higher height. This condition may be guiding for design and production of new generation forearm intramedullary nails in this era where the private anatomical implant technology is discussed.

References: 1- Schemitsch EH, Richards RR. The effect of malunion on functional outcome following plate fixation of fractures of both ones of the forearm in the adult. J Bone Joint Surg. (Am) 1992;74:1068-1078.

Disclosure: No significant relationships.

\section{PR411}

\section{UKCLJ FRAME - A NEW CONFIGURATION FOR DISTAL CRURIS EXTERNAL FIXATION}

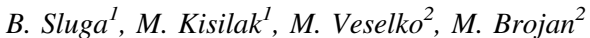

${ }^{1}$ Ko Za Travmatologijo, UKC Ljubljana, Ljubljana/SLOVENIA,

${ }^{2}$ Fakulteta Za Strojništvo, Univerza v Ljubljani, Ljubljana/ SLOVENIA

Introduction: External fixation is most common in distal cruris injuries. Indicated by damage control, soft tissue complications, complex and open fractures this operation must be done quickly, reliably and with thought of definitive care procedures. As delta frame sometimes causes difficulties with build, reposition and dressing/VAC exchanges, our center has developed an alternative configuration alleviating these issues - the UKCLJ frame.

Material and methods: With two Schanz screws placed in proximal tibia, two Steinmann pins placed in calcaneus and four standard carbon fiber bars the frame is assembled. Two long bars cross between the Schanz screws and two short bars lie parallel to the foot, each connected with tube-to-tube links. This configuration was compared with two alternatives (delta and Hoffman). Analysis was done both experimentally in laboratory with synthetic bone as well as numerically by finite elements method in Abaqus/CAE software. The results were qualitatively compared amongst selected configurations. Results: Numeric analysis for a default fracture type showed comparable results between UKCLJ and delta frame, whilst the Hoffman configuration stands out with larger deformation. Bending load produced interfragementary shift values within $1 \%$ for UKCLJ and delta frame, in axial load the UKCLJ frame showed a minimal advantage. Points of maximum frame tension were calculated, the UKCLJ frame produced the lowest maximum values.

Conclusion: The UKCLJ frame is biomechanically comparable to delta frame and can be used as a primary external fixation 
configuration for distal cruris, especially in cases with severe soft tissue damage. Some surgeons may find building and handling of UKCLJ easier than other alternatives.

References: - S. Rammelt, T. Endres, R. Grass, H. Zwipp (2004) The role of external fixation in acute ankle trauma; Foot Ankle Clin. 2004 Sep;9 (3) page 455-74 - D. Seligson, C. Mauffrey, C. S. Roberts: External Fixation in Orthopedic Traumatology. C. Mauffrey et. al.: Practical Biomechanical Considerations about External Fixator. Springer-Verlag London Limited, 2012, page 48-49. - P. S. Cooper et al.: External Fixators of the Foot and Ankle. B. P. Abicht, T. S. Roukis: History and Evolution of External Fixation. Lippincott Williams \& Wilkins, Philadelphia, 2013, page 1-5.

Disclosure: No significant relationships.

\section{PR412}

\section{PROCEDURAL SEDATION AND ANALGESIA VERSUS USUAL CARE FOR THE REDUCTION OF DISTAL RADIUS FRACTURES IN THE EMERGENCY DEPARTMENT}

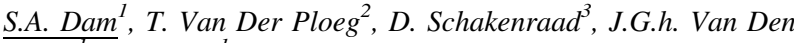 Brand $^{I}$, P. Joosse ${ }^{I}$}

${ }^{1}$ Surgery, Northwest Clincs Alkmaar, Alkmaar/NETHERLANDS, ${ }^{2}$ Statistics, Northwest Clinics Alkmaar, Alkmaar/NETHERLANDS, ${ }^{3}$ Emergency Department, Northwest Clinics Alkmaar, Alkmaar/ NETHERLANDS

Introduction: Distal radius fractures are common injuries and about two-thirds require reduction. Successful reduction can be followed by conservative treatment, inadequate reduction could lead to secondary displacement, repeat reduction or surgical treatment. Procedural sedation and analgesia (PSA), is a safe method to provide sedation and analgesia during a short and painful procedure. We aimed to compare the effect of procedural sedation and analgesia (PSA) to usual care in patients undergoing closed reduction of a distal radius fracture in the emergency department. The effect is measured by quality of reduction (radiologic alignment, number of re-reductions and operative treatment), safety of the procedure, procedure-related factors and patient satisfaction.

Material and methods: We conducted a single-center retrospective matched cohort study in a level 1 trauma centre. We included 44 patients who received PSA for closed reduction of a distal radius fracture in the emergency department, and 111 matched patients based on four age categories. Patient files were accessed to obtain outcome measures.

Results: No significant differences were found between both groups in acceptable alignment $(\mathrm{P}=0.349)$, number of re-reductions $(\mathrm{P}=$ $1.000)$, operative treatment $(\mathrm{P}=0.816)$ or time spent in the emergency department (bootstrapped mean for the PSA group: 260.52 minutes $(95 \% \mathrm{CI}[190.84,333.9])$ and the usual care group: 197.25 minutes $(95 \% \mathrm{CI}[170.09,229.78]))$. No treatment-related serious adverse events were observed.

Conclusion: Our results suggest that procedural sedation and analgesia does not influence radiologic alignment after reduction, number of re-reductions or the indication for operative treatment in patients that need reduction for a displaced distal radius fracture. PSA appears to be a safe method of analgesia.

References: 1. de Putter, C.E. et al. Epidemiology and health-care utilisation of wrist fractures in older adults in The Netherlands, 1997-2009 Injury, Volume 44, Issue 4, 421 - 426 2. Polinder, S., Iordens, G. I., Panneman, M. J., Eygendaal, D., Patka, P., Den Hartog, D., \& Van Lieshout, E. M. (2013). Trends in incidence and costs of injuries to the shoulder, arm and wrist in The Netherlands between 1986 and 2008. BMC Public Health, 13, 531 3. Diaz-Garcia, R. J., \& Chung, K. C. (2012). Common Myths and Evidence in the Management of Distal Radius Fractures. Hand Clinics, 28 (2), 127-133. 4. Brogren E, Petranek M, Atroshi I: Incidence and characteristics of distal radius fractures in a southern Swedish region. BMC Musculoskelet Disord 2007, 8:48. 5. Lutz K., Yeoh K. M., Macdermid J. C., Symonette C., Grewal R. Complications associated with operative versus nonsurgical treatment of distal radius fractures in patients aged 65 years and older. Journal of Hand Surgery. 2014;39 (7):1280-1286. doi: 10.1016/j.jhsa.2014.04.018. 6. Gehrmann SV, Windolf J, Kaufmann RA. Distal radius fracture management in elderly patients: a literature review. J Hand Surg Am. 2008;33 (3):421-429. doi: 10.1016/j.jhsa.2007.12.016. 7. Haase SC, Chung KC. Management of malunions of the distal radius. Hand Clin. 2012;28:207-16. 8. Capo JT, Hashem J, Orillaza NS, Tan V, Warburton M, Bonilla L. Treatment of extra-articular distal radial malunions with an intramedullary implant. J Hand Surg (Br.) 2010;35:892-899. doi: 10.1016/ j.jhsa.2010.02.032 9. Chung KC, Shauver MJ, Birkmeyer JD. Trends in the United States in the treatment of distal radius fractures in the elderly. J Bone Joint Surg. 2009;91:1868-73 10. Sharma H, Khare GN, Singh S, Ramaswamy AG, Kumaraswamy V, Singh AK. Outcomes and complications of fractures of distal radius (AO type B and C): volar plating versus nonoperative treatment. J Orthop Sci. 2014;19:537-544. 11. Forward DP, Davis TR, Sithole JS. Do young patients with malunited fractures of the distal radius inevitably develop symptomatic post-traumatic osteoarthritis? J Bone Joint Surg (Br). 2008;90 (5):629-37 12. Zamzam MM, Khoshhal KI. Displaced fracture of the distal radius in children: Factors responsible for redisplacement after closed reduction. J Bone Joint Surg Br. 2005;87:841-3 13. Jung H-W, Hong H, Jung HJ, et al. Redisplacement of Distal Radius Fracture after Initial Closed Reduction: Analysis of Prognostic Factors. Clinics in Orthopedic Surgery. 2015;7 (3):377-382. doi:10.4055/cios.2015.7.3.377. 14. Chuang P-Y, Yang T-Y, Shen S-H, Tsai Y-H, Huang K-C. The Effects of Dorsal Cortical Comminution on Radiographic Results following Percutaneous Pinning for Extra-Articular Colles' Fracture. BioMed Research International. 2015;2015:714351. doi:10.1155/2015/714351. 15 . Alistair R. Phillips, Anmar Al-Shawi Restoration of the volar cortex: Predicting instability after manipulation of distal radial fractures Injury, 2014-12-01, Volume 45, Issue 12, Pages 1896-1899 16. Swanson ER, Seaberg DC, Mathias S. The use of propofol for sedation in the emergency department. Acad Emerg Med. 1996; 3:234-238. 17. Centers for Medicare \& Medicaid Services (CMS). Revised appendix A, interpretive guidelines for hospitals- state operations manual, anesthesia services. Effective December 2, 2011. Available at: http://www.cms.gov/Regulations-and-Guidance/Guidance/Transmittals/downloads/R74SOMA.pdf. Accessed: November 9, 2012. 18. Godwin SA, Burton JH, Gerardo CJ, et al. Clinical policy: procedural sedation and analgesia in the emergency department. Ann Emerg Med 2014;63:247-58. 19. Cudny ME, Wang NE, Bardas SL, Nguyen CN. Adverse Events Associated With Procedural Sedation in Pediatric Patients in the Emergency Department. Hospital Pharmacy. 2013;48 (2):134-142. doi:10.1310/hpj4802-134. 20. Müller ME, Nazarian S, Koch P (1987) Classification AO des fractures. Tome I. Les os longs. 1st ed. Berlin: Springer- Verlag. 21. Kwaliteitsinstituut voor de Gezondheidszorg CBO. Richtlijn sedatie en/of analgesie (PSA) op locaties buiten de operatiekamer. Deel I: bij volwassen. Deel II: bij volwassenen op de intensive care. http:// www.nvsha.nl/images/secties/psa/volwassenen/

cbo_richtlijn_psa_volwassenen.pdf. Utrecht: Kwaliteitsinstituut voor de Gezondheidszorg CBO, 2012. 22. Kwaliteitsinstituut voor de Gezondheidszorg CBO. Richtlijn sedatie en/of analgesie (PSA) op locaties buiten de operatiekamer. Deel III: bij kinderen. http:// www.nvsha.nl/images/secties/psa/kinderen/cborichtlijn_ psa_kinderen.pdf. Utrecht: Kwaliteitsinstituut voor de Gezondheidszorg CBO, 
2012. 23. Hunt, A. H. (1948). Regional Analgesia. Postgraduate Medical Journal, 24 (276), 539-545. (Böhler block methode) 24. Bear DM, Friel NA, Lupo CL, Pitetti R, Ward WT. Hematoma block versus sedation for the reduction of distal radius fractures in children. J Hand Surg Am. 2015 Jan;40 (1):57-61. doi: 10.1016/ j.jhsa.2014.08.039. Epub 2014 Oct 11. 25. Gaël JP Smits, Maybritt I Kuypers, Lisette AA Mignot, Eef PJ Reijners, Erick Oskam, Karen Van Doorn, Wendy AMH Thijssen, Erik HM Korsten. Procedural sedation in the emergency department by Dutch emergency physicians: a prospective multicentre observational study of 1711 adults. Emerg Med J emermed-2016-205767Published Online First: 21 October 2016 doi:10.1136/emermed-2016-205767 26. Abson, Simon MBBS, Williams, Nicole FRACS, Inglis, Mark FRACS, Antoniou, Georgia BSc (Hons), Cundy, Peter FRACS. Resident Versus Attending Surgeons in Achieving and Maintaining Fracture Reduction in Pediatric Distal Radius Fractures. Journal of Pediatric Orthopaedics. 36 (5):478-482, July/August 2016.

Disclosure: No significant relationships.

\section{PR413}

\section{MEGAPROSTHESES FOR TREATMENT OF COMPLEX KNEE FRACTURES IN NON-ONCOLOGICAL PATIENTS}

\author{
G. Allevi, A. Cordella, L. Memè, P. De Simoni
}

Orthopedic And Trauma Department, Azienda Ospedaliera Ospedali Riuniti Marche Nord, Pesaro/ITALY

Introduction: The increase in the average age is leading to a growing number of patients with poor bone stock having complex articular fractures on a previous arthritis. In these cases treatment options could be: ORIF, retrograde intramedullary neiling, bone graft, revision artrhroplasty. We have evaluated outcome of treatment with megaprostheses in complex articular fractures in elderly non oncological patients with bone loss and low functional requirements

Material and methods: A total of 7 patients between Genuary 2016 and Genuary 2017 have been evaluated after megaprostheses implant following a complex knee fractures. All patient included in this study was age $>80$.years, bone loss, low functional requirements and need for quick functional recovery. Five periprosthetic knee fractures classified according Taylor and Rorabeck classification and two articular multifragment fractures according to $\mathrm{AO}$ classification. Follow up at 1-3-6-12 and 18 months.

Results: All patients had a quick mobilization in the post-operative period. We didn't observe deep infection neither mobilization of the implant. Average knee society score was 86 where follow-up was available and low pain score at 3 months.

Conclusion: The use of knee megaprostheses in case of multifragment fracture or periprosthetic fractures associated with bone loss in elderly patients with low functional requirements and need for quick functional recovery shows favorable results with low complication rates and rapid mobilization.

References: Megaprostheses in the management of trauma of the knee. 2016 May;40 (5):935-43. doi: 10.1007/s00264-015-2991-4. Epub 2015 Sep 25. Megaprostheses in the treatment of periprosthetic fractures of the knee joint: indication, technique, results and review of literature. 2016 May;40 (5):935-43. doi: 10.1007/s00264-015-2991-4. Epub 2015 Sep 25.

Disclosure: No significant relationships.

\section{PR414}

\section{BONE SUBSTITUTES IN FRACTURE TREATMENT- HOW TO APPLY THE 'DIAMOND CONCEPT'}

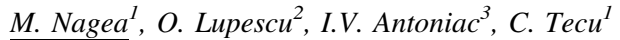

${ }^{1}$ Orthopaedics And Trauma, CLINICAL EMERGENCY HOSPITAL, BUCHAREST/ROMANIA, ${ }^{2}$ Orthopaedics And Trauma, Clinical Emergency Hospital, University of Medicine and Pharmacy " Carol Davila” Bucharest, Bucharest/ROMANIA, ${ }^{3}$ Material Science Engineering, University Politehnica Bucharest, BUCHAREST/ ROMANIA

Introduction: Post-traumatic bone loss became increasingly frequent due to severe injuries, therefore, restoration of functional anatomy regards bone filling with proper bone integration, otherwise surgical reconstruction is useless; that is why not only thorough knowledge about bioceramics, but also standardised algorithms for hadling bone substitutes are necessary. This paper presents the research project 2015-1-RO01-KA202-015230, ERASMUS+ VET “Collaborative learning for enhancing practical skills for patient-focused interventions in gait rehabilitation after orthopedic surgery" which, using e learning as a basic tool, among other subjects, aim to establish the steps for proper indicating and introducing bone fillers.

Material and methods: Surgical experience in a Level 1 Trauma Centre was used as a base for this algorithm; 36 patients (19-62 yrs) who required bone grafting were analysed and an algorithm was developed, including several characteristics of the patient and of the bone defect such as coexistence of associated disorders (especially diabetes and cortisone treatment), the dimensions, the septicity and the vascular supply of the bone defect and the load bearing characteristics.

Results: The surgical treatment consisted of reduction ad osteosynthesis, followed by grafting regarding the above mentioned criteria. The post-operative outcome and complications were described in order to validate the algorithm. Bone healing was obtained in all the cases, and complications were not related to grafting

Conclusion: Orthopaedic practice require bone reconstruction, including methods of covering bone defects, with filling and integration of the bone substitute. Proper surgical training requires guidelines, such as the present algorithm, which enhances the probability of proper graft integration.

References: 1. Giannoudis, PV. et al.Bone substitutes: An update. Injury, Volume 36, Issue 3, S20 - S27

Disclosure: No significant relationships.

\section{PR415}

\section{ANALYSIS OF CORRELATION BETWEEN MANGLED EXTREMITY SEVERITY SCORE (MESS) AND DISABILITIES OF THE ARM SHOULDER AND HAND (DASH) SCORE}

\author{
G. Hohenberger ${ }^{1}$, P. Konstantiniuk ${ }^{2}$, J. Cambiaso-Daniel ${ }^{3}$,

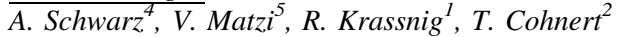

${ }^{1}$ Orthopedics And Trauma Surgery, Medical University of Graz, Graz/AUSTRIA, ${ }^{2}$ Surgery, Medical University of Graz, Graz/ AUSTRIA, ${ }^{3}$ Plastic And Reconstructive Surgery, Medical University of Graz, Graz/AUSTRIA, ${ }^{4}$ Trauma Surgery, AUVA Trauma Hospital, Graz/AUSTRIA, ${ }^{5}$ Surgery, State Hospital Hochsteiermark/Leoben, 
Leoben/AUSTRIA

Introduction: The Mangled Extremity Severity Score (MESS) was developed as decision making tool for limb amputation after trauma. The Disabilities of the Arm Shoulder and Hand (DASH) questionnaire was created to quantify posttraumatic deficiencies of the upper extremity. The main goal of our study was to determine correlations between these two assessments. Secondary endpoint was to evaluate if the defined MESS threshold for amputation ( $\geq 7$ points) was applicable for our collective.

Material and methods: In the retrospective study all patients with upper extremity (UE) traumas were included who had been treated surgically with vascular reconstruction at our level-I trauma centre and a cooperating level-III trauma centre in the interval 2005-2014. The respective MESS was calculated for each participant. All patients were recalled for follow-up examination and functional assessment using the DASH questionnaire.

Results: Fourteen patients could be included in the study. The mean total MESS was 5.9, the mean total DASH score was 30 points. There was no statistically significant correlation between these assessments. In a subgroup of cases with prolonged time of ischemia (seven patients) the mean MESS was 6.9 and the DASH 43.7 points. These values correlated significantly $(\mathrm{p}=.013)$. Four cases had a MESS of at least seven points and did not undergo amputation. Two of them achieved satisfactory outcomes and the other two had a sensorimotor malfunction of the UE due to plexus lesions.

Conclusion: The DASH correlated significantly and positively with the MESS in patients with prolonged ischemia time. Mandatory upper limb amputation is not recommended in patients with MESS $\geq 7$ as $2 / 4$ patients showed satisfactory functional outcomes despite higher MESS values.

References: Prasarn ML, Helfet DL, Kloen P. Management of the mangled extremity. Strategies Trauma Limb Reconstr. 2012 Aug;7 (2):57-66. Bernstein ML, Chung KC. Early management of the mangled upper extremity. Injury. 2007 Dec;38 Suppl 5:S3-7. Korompilias AV, Beris AE, Lykissas MG, Vekris MD, Kontogeorgakos VA, Soucacos PN. The mangled extremity and attempt for limb salvage. J Orthop Surg Res. 2009 Feb 13;4:4. Johansen K, Daines M, Howey T, Helfet D, Hansen ST Jr. Objective criteria accurately predict amputation following lower extremity trauma. J Trauma. 1990 May;30 (5):568-72; discussion 572-3. Mommsen P, Zeckey C, Hildebrand F, Frink M, Khaladj N, Lange N, Krettek C, Probst C. Traumatic extremity arterial injury in children: epidemiology, diagnostics, treatment and prognostic value of Mangled Extremity Severity Score. J Orthop Surg Res. 2010 Apr 15;5:25. Prichayudh S, Verananvattna A, Sriussadaporn S, Sriussadaporn S, Kritayakirana K, Pak-art R, Capin A, Pereira B, Tsunoyama T, Pena D. Management of upper extremity vascular injury: outcome related to the Mangled Extremity

Disclosure: No significant relationships.

\section{PR416}

\section{SELECTIVE FASCIOTOMY FOR THE ACUTE TRAUMATIC LOWER LEG COMPARTMENT SYNDROME; IS IT FEASIBLE?}

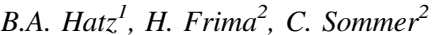

${ }^{1}$ Clinic Of Traumatology, University hospital Zurich, Zürich/ SWITZERLAND, ${ }^{2}$ Surgery, Trauma Unit, Kantonsspital Graubünden, Chur/SWITZERLAND

Introduction: The standard treatment for an acute compartment syndrome (ACS) of the lower leg is a four-compartment fasciotomy.
It can be performed through either one lateral or a lateral and a medial incision, with its known morbidity. Selective fasciotomy might lower this morbidity. The aim of this study was to describe a procedure of selective fasciotomy after pressure measurement and to present the results of a retrospective cohort study.

Material and methods: All patients with an ACS of the lower leg due to a tibia fracture (AO 41 or 42 ), who received either a fourcompartment fasciotomy or a selective fasciotomy after pressure measurement between 2006 and 2016, were included. Every compartment with an intracompartmental pressure of more than $30 \mathrm{mmHg}$ was opened. The primary outcome was any missed compartment syndrome after selective fasciotomy. Secondary outcomes were reoperations for completing four compartment fasciotomy and persistent sensomotor deficits.

Results: 51 patients with a mean age of 43 years $(6-76)$ were included. Of these, $41(80 \%)$ had a selective fasciotomy. There was no missed compartment syndrome. One patient had a reoperation 8 hours after primary selective fasciotomy due to ACS of the superficial and deep flexor compartment. The anterior compartment had to be released in all patients. In $67 \%$ the release of 2 compartments was sufficient. Six patients had postoperative sensomotor deficits at discharge with full recovery during follow up.

Conclusion: Selective fasciotomy is feasible and seems to be safe. Future comparative studies will have to focus on possible benefits of this less invasive treatment.

References:

Disclosure: No significant relationships.

\section{PR417}

\section{BETTER OUTCOME WITH NEGATIVE PRESSURE WOUND THERAPY WITH INSTILLATION TECHNIQUE FOR TREATMENT OF INTRAMEDULLARY POSTTRAUMATIC OSTEOMYELITIS OF LONG BONES}

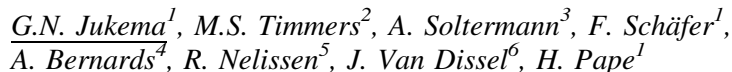

${ }^{1}$ Division Of Traumatology, University Hospital Zürich, Zürich/ SWITZERLAND, ${ }^{2}$ Department Of Surgery, MC Hospital Zuiderzee, Lelystad/NETHERLANDS, ${ }^{3}$ Institute Of Pathology And Molecular Pathology, University Hospital Zürich, Zürich/SWITZERLAND, ${ }^{4}$ Department Of Microbiology, Leiden University Medical Centre, Leiden/NETHERLANDS, ${ }^{5}$ Department Of Orthopedic Surgery, Leiden University Medical Centre, Leiden/NETHERLANDS, ${ }^{6}$ Department Of Infectious Diseases, Leiden University Medical Centre, Leiden/NETHERLANDS

Introduction: Introducing a new technique of negative pressure wound therapy with polyurethane or polyvinylalcohol foam in the medullary canal of bones with instillation technique, a retrospective, case control cohort study for patients with posttraumatic osteomyelitis was performed. Aim was to improve healing of posttraumatic osteomyelitis and to reduce risks of recurrent infection.

Material and methods: With instillation technique of negative pressure wound therapy (NPWT-Instill) the foam is daily instilled with an antiseptic polyhexanid $0.04 \%$ solution. In the period of 1.1999-2.2003 30 patients with posttraumatic osteomyelitis were included and treated. The average time of treatment was 22.4 days (660). In $93.9 \%$ infected wounds became sterile after a mean of 11.4 days (range 3-38). Time for follow up was up to 84 months. For comparison, a historical control group with standard treatment 
(debridement, lavage, gentamycin beads) of 94 patients was evaluated for a period of 20 years (1982-2002).

Results: Although both groups were statistical comparable, in the NPWTi group the rate of recurrence of infection was $10 \%$ vs $58.5 \%$ in the controls. The duration of hospital stay was shorter and the number of surgical procedures was statistical significant reduced (all $\mathrm{p}<$ 0.0001). Histopathological examinations of bone marrow during intramedullary instillation therapy show improved vascularisation (HE/CD 31 Staining's) with ingrowth of new granulation tissue and new bone formation. Long Term follow up with SPECT-CT scan of the recurrence free individuals shows no signs of infection.

Conclusion: Intramedullary Negative Wound Pressure Wound Therapy with instillation technique seems beneficial the treatment of posttraumatic intramedullary osteomyelitis reducing the risk for recurrence.

References: Lehner B, Fleischmann W, Becker R, Jukema GN: First experiences with negative pressure wound therapy and instillation in the treatment of infected orthopaedic implants: a clinical observational study. Int Orthop 2011 Sep;35 (9):1415-20.PMID: 215846432011

Disclosure: No significant relationships.

\section{PR418}

\section{SAFE DORSAL MINIMALLY INVASIVE PLATE OSTEOSYNTHESIS OF THE HUMERUS}

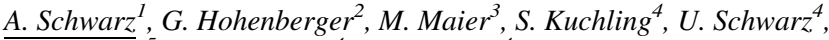

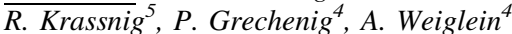

${ }^{1}$ Trauma Surgery, AUVA Trauma Hospital, Graz/AUSTRIA, ${ }^{2}$ Department Of Orthopedics And Trauma Surgery, Medical University Graz, Graz/AUSTRIA, ${ }^{3}$ Institute For Statistics And Mathematics, Vienna University of Economics and Business, Wien/ AUSTRIA, ${ }^{4}$ Institute Of Anatomy, Medical University of Graz, Graz/ AUSTRIA, ${ }^{5}$ Department Of Orthopedics And Trauma Surgery, Medical University of Graz, Graz/AUSTRIA

Introduction: The current literature focusses on minimally invasive plate osteosynthesis (MIPO) of the humeral shaft by various ventral approaches $1,2,3$. The aim of our study was to find a convenient technique for dorsal humerus MIPO as well as to define the relationship of the radial nerve $(\mathrm{RN})$ and the axillary nerve $(\mathrm{AN})$ to the plate

Material and methods: Three minimal incisions from $5 \mathrm{~cm}$ were performed on 20 cadaver upper extremities at the dorsal humerus over the lateral epicondyle (LE), the RN and AN region followed by blunt triceps muscle undermining and $\mathrm{RN}$ visualization with the longest suitable $3.5 \mathrm{~mm}$ LCP plate. Distances and relationships to the nerves and the humeral length (HL) were measured and statistically analyzed.

Results: The RN crossed the plate at $155.16 \mathrm{~mm}$ central (SD 18.09; range: 109-191.5). The AN was on average $4.92 \mathrm{~mm}$ distal to the proximal border of the plate located (SD: 9.63; range: -18.1-14.4) and laid directly on the plate in 13 specimens $(65 \%)$. The mean distance between the AN and RN was $93.49 \mathrm{~mm}$ (SD 20.0; range: 62.08-127.1) with no statistical significant correlation between the HL and gender $(\mathrm{r}=.181, \mathrm{p}=.446 ; \mathrm{W}=60, \mathrm{p}=.456)$.

Conclusion: The MIPO from the dorsal approach is via identification of both nerves using the longest suitable LCP a safe and simple technique. The mean distance of $93.49 \mathrm{~mm}$ between the RN and AN as well as the AN course directly on the plate in $65 \%$ of all cases has to be kept in mind.

References: 1 . Oh HK, Cho DY, Choo SK, Park JW, Park KC, Lee JI. Lessons learned from treating patients with unstable multifragmentary fractures of the proximal humerus by minimal invasive plate osteosynthesis. Arch Orthop Trauma Surg 2015;135:235-42. 2. Rancan M, Dietrich M, Lamdark T, Can U, Platz A. Minimal invasive long PHILOS ${ }^{\circledR}$-plate osteosynthesis in metadiaphyseal fractures of the proximal humerus. Injury 2010;41:1277-83. 3. Yang KH. Helical plate fixation for treatment of comminuted fractures of the proximal and middle one-third of the humerus. Injury 2005;36:75-80.

Disclosure: No significant relationships.

\section{PR419}

\section{PSEUDOANEURYSM OF POPLITEAL ARTERY FOLLOWING TOTAL KNEE ARTHROPLASTY - MANAGEMENT WITH THROMBIN}

\author{
A.K. Raghupathi ${ }^{1}$, A. Joshi ${ }^{2}$
}

${ }^{1}$ Trauma \& Orthopaedics, Southend University Hospital, SouthendOn-Sea/UNITED KINGDOM, ${ }^{2}$ Trauma \& Orthopaedics, Jersey General Hospital, jersey/UNITED KINGDOM

Introduction: Vascular complications after a total knee arthroplasty (TKA) occur in less than $0.5 \%$ of cases. However, when such a complication does occur, it is potentially limb and life threatening. Popliteal artery pseudoaneurysm is one of the sequelae of popliteal artery trauma and also can cause arterial thrombosis, arteriovenous fistulae, or arterial severance. We report a case of a false aneurysm of the popliteal artery after a TKA with unusual presentation, difficult diagnosis and successful management.

Material and methods: A 64 year old male had an unusual presentation of post operative recurrent haemarthrosis of the knee one month after Total knee arthroplasty. Arthroscopic wash outs failed to control this problem. On clinical suspicion, a Duplex ultrasound scan was done which revealed a $5 \mathrm{~cm}$ diameter popliteal artery aneurysm. Femoral angiogram located it to be in the middle of the popliteal artery with a narrow neck. Attempts at balloon occlusion of the aneurysm failed

Results: Thrombin was then injected at multiple positions under ultrasound guidance. This achieved successful occlusion of the aneurysm with subsequent recanalisation of the artery later on. The distal vascular status was never compromised.

Conclusion: Thrombin injection is a safe and reliable modality if used under image guidance in management of pseudo-aneurysm of arteries. The morbidity associated with vascular repair is avoided. Detailed examination is essential in follow up of TKA to avoid missing these rare complications. The clinical presentation of this condition can be confused with that of a postoperative DVT or hematoma formation in early postoperative period.

References: 1. Posttraumatic pseudoaneurysm of popliteal artery following total knee arthroplasty

Disclosure: No significant relationships. 
PR420

BALLOON-TAMP OSTEOPLASTY FOR TIBIAL PLATEAU FRACTURES: QUALITY AND STABILITY OF REDUCTION

\author{
J.W. Schmitt, S. Tiziani, H. Pape, G. Osterhoff
}

Department Of Trauma, University Hospital Zurich, Zurich/ SWITZERLAND

Introduction: Reduction of the articular surface in tibial plateau fractures by the use of a balloon-tamp has been described to be safe in several small case series. However, information on the quality and stability of radiographic reduction has been missing in the literature. Material and methods: Consecutive patients $(\mathrm{N}=14,10$ female, mean age 49 years, SD 18 years) with tibial plateau fractures that were treated by ballon-tamp assisted osteoplasty in combination with screw or plate fixation from $3 / 2011$ to $6 / 2017$ were identified and analyzed. The primary outcome was quality and stability of reduction (parameters: gap, coronal alignment, sagittal alignment, condylar width). Secondary outcome were: perioperative complications, readmissions and the need for revision surgery.

Results: In one patient, the balloon was unable to reduce the impression, which led to a conversion to a conventional approach. Perioperative complications included one deep vein thrombosis and one lung embolus. Revision surgery was necessary in $2 / 13$ patients if implant removal was excluded, one for secondary loss of reduction requiring conversion to total knee arthroplasty and one for persistent meniscal pain that required treatment. At least $3 / 4$ radiographic criteria used to assess the adequacy of reduction on postoperative radiographs were achieved in 13/13 cases, at follow up, at least $3 / 4$ radiographic criteria were achieved in 12/13 cases.

Conclusion: Balloon-tamp assisted reduction of tibial plateau fractures appears to be safe and allows for achieving a high quality of reduction by means of a minimally invasive intervention. Future studies with a larger sample size and longer follow-up should further validate the funtional outcome

References:

Disclosure: No significant relationships.

\section{PR421}

INJURIES TO THE MEDIAL CLAVICLE: THE FIXATION OF THE COSTO-CLAVICULAR LIGAMENT MAKES THE DIFFERENCE!

S. Bakir ${ }^{1}$, D. Merschin ${ }^{2}$, A. Langenbach ${ }^{3}$, S. Trach ${ }^{2}$, J. Unterkofler ${ }^{2}$, $\overline{D . ~ G u ̈ m b e l}^{4}$, A. Ekkernkamp ${ }^{1}$, S. Schulz-Drost ${ }^{1}$

${ }^{1}$ Trauma- And Orthopedic Surgery, Unfallkrankenhaus Berlin, Berlin/
GERMANY, ${ }^{2}$ Trauma- And Orthopedic Surgery, BG Hospital
Unfallkrankenhaus Berlin gGmbH, Berlin/GERMANY, ${ }^{3}$ Trauma
Surgery, University Hospital Erlangen, Erlangen/GERMANY,
${ }^{4}$ Department Of Trauma, reconstructive Surgery And Rehabilitation
Medicine, University Medicine Greifswald, Greifswald/GERMANY

Introduction: The sternoclavicular joint (SCJ) is most stabilized by the costoclavicular ligaments (CLL) followed by the most cited sternoclavicular ligaments (SCL). A combined disruption of SCL and CCL causes severe instability of the medial clavicle and poor outcome if not treated adequately. Alternative to conservative therapy the fixation of CCL by ligament bracing could be considered. Could
TightRope ${ }^{\circledR}$ stabilization of CCL provide sufficient reconstruction of the SCJ?

Material and methods: 7 patients ( 2 female) showed severe and painful instability of the SCJ with disruption of SCL and CCL following a fall on the ipsilateral shoulder 4 of these patients had been treated operatively with TightRope ${ }^{\circledR}$ fixation of the CCL to the first rib and anatomical suture of the SCL. The other 3 patients had been treated conservatively since they preferred this therapy. The outcome of these the two groups had been compared in a retrospective cohort study. Follow up had been carried out after 2, 6, 12, 26 and 52 weeks following the institutional standard with clinical examination and $\mathrm{X}$-ray imaging.

Results: Operative fixation showed better reduction of the SCJ, better function and less pain. The operated patients returned to work in less than 6 months after the procedure whereas 2 of the conservative treated patients did not due to persistent pain and posttraumatic arthrosis.

Conclusion: Innovative stabilization of the CCL with TightRope ${ }^{\circledR}$ additional to a suture of the SCL may enable anatomic reconstruction of the SCJ considering cosmetic and functional results with reduction of posttraumatic arthrosis and pain.

References:

Disclosure: No significant relationships.

\section{PR422}

\section{PRIMARY SHORTENING AND SECONDARY LENGTHENING OF COMPLEX THIRD DEGREE OPEN LOWER LEG FRACTURES FOR LIMB SALVAGE}

\section{$\underline{\text { M. Tryzna }}$}

Surgery, Kantonsspital Chur, Chur/SWITZERLAND

Introduction: Severe $3^{\circ}$ open factures of the lower limb with combined soft tissue and bone loss remain a major treatment challenge. An alternative approach to free flap coverage and bone reconstruction is primary shortening and secondary lengthening of the lower leg (PSSL). The aim of this study was to describe this strategy and present our results of this treatment.

Material and methods: The technique, performing primary shortening at the fracture site with internal plate fixation and soft tissue coverage followed by secondary lengthening and subsequent minimal-invasive plate fixation of the distraction site, is described. Performing a retrospective cohort study, all patients treated with PSSL of the lower leg between 2006 and 2017 were included. The primary endpoint was fracture healing. Secondary endpoints were the time in external fixation (TEF) and the external fixation index (EFI). Results: Five patients, with a mean age of 36 (range 29-55) were included. Healing and union was achieved in all patients. The mean TEF was 72 days (range 52-106) and the mean EFI was $0.79 \mathrm{mo} / \mathrm{cm}$ (0.3-1.1). Limb lengthening started at a mean interval of 47 days (2556) after the initial trauma. The mean time to plate fixation of the distracted site was 7.5 month (3-12). The mean shortening length was $7.5 \mathrm{~cm}(3.5-11)$.

Conclusion: PSSL is a good and safe solution for severe open fractures of the tibia with combined soft tissue and bony defects.

References:

Disclosure: No significant relationships. 
PR423

\section{TREATMENT OF PROXIMAL HUMERUS FRACTURES IN ELDERLY PATIENTS WITH MIROS: OUR EXPERIENCE AND PRELIMINARY RESULTS}

\section{G. Allevi, P. De Simoni}

Orthopedic And Trauma Department, Azienda Ospedaliera Ospedali Riuniti Marche Nord, Pesaro/ITALY

Introduction: Proximal humerus fractures in elderly patients in poor clinical conditions are commonly encountered in clinical practice nevertheless the treatment presents difficulty in management. Regarding this, MIROS system is a valuable device with advantages of quick and easy surgical technique, allowing correction of angular displacement and a stable fixation, and preserving fracture outbreak in order to allow quick mobilization and fast functional recovery.

Material and methods: A total of 54 patients were treated in our department between 1 genuary 2016 and 31 december 2016 using miros system. Inclusion criteria were age $>65$, ASA $>3,2,3,4$ fragments fracture according to Neer classification, low functional requirements. Average follow-up was 19,8 months by x-ray and clinical evaluation performed according Constant Shoulder Score. We evaluated also re-intervention rate, average time between osteosynthesis and shoulder arthroplasty and pin site infection rate.

Results: Follow-up could be performed in 50 of 54 patients treated with Miros. We observed high CS even if the X-ray anatomical result wasn't performed. No deep infection. 4 pin site infection healed after antibiotic therapy. Low re-operation rate.

Conclusion: In our experience and with the data obtained, miros system has proved to be a useful tool for treatment of proximal humerus fractures. Few data in literature and short follow-up do not allow a unique indication of treatment.

References: Percutaneous pinning of three- or four-part fractures of the proximal humerus in elderly patients in poor general condition: MIROS ${ }^{\circledR}$ versus traditional pinning. Int Orthop. 2012 Jun; 36 (6): $1267-1273$

Disclosure: No significant relationships.

\section{PR424}

\section{FIXATION OF PROXIMAL ULNA FRACTURES USING AN INTRAMEDULLARY CANNULATED SCREW}

\author{
W. Bosman ${ }^{1}$, B. Emmink ${ }^{1}$, J. Keizer ${ }^{2}$
}

${ }^{1}$ Trauma Surgery, St Antonius Hospital, Nieuwegein/ NETHERLANDS, ${ }^{2}$ Trauma Surgery, St. Antonius Hospital, Nieuwegein/NETHERLANDS

Introduction: Proximal ulnar fractures are a very common fracture. Traction of the triceps on the proximal fragment often leads to dislocation, making an operative reduction and fixation necessary. In most cases fixation with tension-band-wiring or plate-fixation is used for fixation of the fracture. ${ }^{1}$ As the skin is thin at the proximal ulna these fixation methods lead to hardware irritation and subsequent removal up to $68 \%$ of the cases. ${ }^{1,2}$ In case of a Mayo type I or IIA olecranon fracture, fixation with an intramedullary screw can be chosen. We believe this may lead to excellent function and less reoperations for hardware removal.
Material and methods: A retrospective cohort study (2012-2017) in a single level 2 trauma centre was performed. Patients were identified with Mayo type I or IIA olecranon fracture and treated with an intramedullary $7.3 \mathrm{~mm}$ cannulated screw, between the ages of 16-70. The cases were reviewed for bony healing, operating time, postoperative function and hardware removal. DASH scores were obtained. Results: 13 patients were identified, all had a complete union of their fracture. Mean operating time was $41+/-9$ minutes. Average flexion was 145.7 degrees and the average extension lag was 7.7 degrees. In 5 cases $(38 \%)$ the hardware was removed due to soft tissue irritation. Obtained DASH scores showed an excellent function.

Conclusion: Fixation of simple olecranon fractures with an intramedullary screw is a safe and easy fixation method in young persons, leading to excellent results. Compared to available data, less hardware removal is necessary then with TBW or plate fixation.

References: 1. H. Niéto, A. Billaud, S. Rochet et al. Proximal ulnar fractures in adults: a review of 163 cases. Injury, Int. J. Care Injured 46 S1 (2015) S18-S23 2 S.G. Edwards, M.S. Cohen, L.L. Lattanza et al. Surgeon perceptions and patient outcomes regarding proximal ulna fixation: a multicenter experience. J Shoulder Elbow Surg (2012) 21, 1637-1643

Disclosure: No significant relationships

PR425

COMBINATION OF AN OPEN POSTERIOR FRACTUREDISLOCATION OF THE KNEE WITH AN OPEN LEG FRACTURE: DAMAGE CONTROL APPROACH ON A CATASTROPHIC LIMBLESION

\author{
N.D.F.L. Simões, G. Pina, C. Martins, M.R. Vaz, J.A. Gomes, \\ M. Leirinha
}

Orthopaedics, Centro Hospitalar Tondela-Viseu, Viseu/PORTUGAL

Introduction: Althought being one of the rarest injuries, posterior dislocations of the knee are between the most dangerous because of its potential to cause neurovascular injuries. Open fracture-dislocations of the knee are even rarer presenting with more possible complications.

Material and methods: Case of a 48-year-old man who had a motorbike accident on 13/08/2016. He entered in the ER with a catastrophic lower limb trauma and discussion about damage control approach and guidance to achieve success in this type of orthopaedic trauma.

Results: A Gustilo-Anderson (GA) IIIA posterior knee fracture-dislocation associated with a GA IIIB leg fracture were stated and also a patella and posterolateral condyle fractures. Eco-doppler was performed with no vascular lesion. He was submitted to antibiotherapy, debridement, external fixation (for 10 weeks), transosseous suture of the patella, screw fixation of the femur condyle and wound closure with solear muscle flap . A partial skin flap was later on applied on the leg by the Plastic Surgery Department. 22/11/2016- Femural condyle and patella fractures were healed but there was a nonunion on the leg fracture as shown by the x-ray. External fixator was removed and was submitted to tibia nailing with peroneal osteotomy for fracture site dinamization. Postop knee instability wasn't present possibly due to tissue fibrosis. All fractures are currently consolidated and a MRI to exclude ligament rupture was planned

Conclusion: In knee dislocations, neurovascular assessment should immediately become the focus of the evaluation. Damage control approach is essential to preserve soft tissues and to achieve wound healing. 
References: Bibliography Sanders, Roy. Trauma: Core Knowledge in Orthopaedics. 2nd ed. Philadelphia: Mosby/Elsevier, 2008. 234-52; Campbell, Willis C., S. T. Canale, and James H. Beaty. Campbell's Operative Orthopaedics. 12th ed. Philadelphia, PA: Mosby/Elsevier, 2008. Chapter 41, 1107-1161 and Chapter 52, 2777-2878; Routt, Milton Lee; Gary, Joshua. Skeletal Tauma: Basic Science, Management, and Reconstruction. 5th Ed. Chapter 41, 1107-1161; McKee L, Ibrahim MS, Lawrence T, Pengas IP, Khan WS. Current Concepts in Acute Knee Dislocation: The Missed Diagnosis? The Open Orthopaedics Journal. 2014;8:162-167. Henrichs A. A Review of Knee Dislocations. Journal of Athletic Training. 2004;39 (4):365-369. Schenck RC, Richter DL, Wascher DC. Knee Dislocations: Lessons Learned From 20-Year Follow-up. Orthopaedic Journal of Sports Medicine. 2014;2 (5):73-82.

Disclosure: No significant relationships.

\section{PR426}

DO ALL TIBIAL PLATEAU FRACTURES NEED EXTERNAL FIXATION AS STAGED OPERATIONS?

\section{T. Kawamura}

Orthopeadics, Kitasato University, Sagamihara/JAPAN

Introduction: Tibial plateau fractures have many cases of severe soft tissue injuries. Undergoing the external fixation in early phase for decreasing the swelling of the soft tissue is good strategy to prevent the complications. But it is controversial that low energy injury like geriatric tibial plateau fractures or partial intraarticular fracture like AO type B are needed external fixation. This study aimed to investigate the clinical features and operative results of these fractures.

Material and methods: Twenty displaced tibial plateau fractures (mean age: 54.0 years, range: $28-81$ years) were treated operatively. We investigated the incidences of soft tissue injury, fracture types according to the AO classification, Hohl \& Luck functional and radiological evaluation, preoperative and postoperative complications. The mean follow-up period was 2.1 years (range: 1-6years). eighteen patients $(90 \%)$ were injured by high-energy trauma such as traffic accident or simple fall. Five patients $(25 \%)$ are open fractures. AO $41-\mathrm{B} 1$ in 1 patient, B2 in 1 patient, B3 in 8 patients, $\mathrm{C} 1$ in 1 patient, $\mathrm{C} 2$ in 2 patients, $\mathrm{C} 3$ in 7 patients.

Results: Almost all patients are excellent or good results in Hohl \& Luck functional and radiological evaluation. Complications seen were, infections in one patient $(5 \%)$ which resolved finally and compartment syndrome in one patient $(5 \%)$ which had popliteal injury by traffic accident.

Conclusion: Our results are almost good. It is not always needed external fixation for partial intraarticular fracture like AO type B, although high-energy trauma. But, undergoing external fixation to prevent soft tissue swelling by secondary damage is better for unstable fracture especially AO type C.

References: Management of soft tissue injuries associated with tibial plateau fractures. Borrelli J Jr. et. al J Knee Surg. 2014 Feb;27 (1):59. Staged management of tibial plateau fractures. Dirschl et. al . Am J Orthop. 2007 Apr;36 (4 Suppl):12-7. Staged management of highenergy proximal tibia fractures (OTA types 41): the results of a prospective, standardized protocol. Egol et. al. J Orthop. Trauma 2005 Aug;19 (7):448-55; discussion 456.

Disclosure: No significant relationships.

\section{PR426A}

\section{EMERGENCY STABILIZATION OF PELVIC INJURIES IN GERMANY. IS THERE A STANDARD?}

\author{
S. Zeidler ${ }^{1}$, A. Höch ${ }^{1}$, J. Böhme ${ }^{2}$, C. Josten ${ }^{3}$, F. Stuby ${ }^{4}$
}

${ }^{1}$ Department Of Orthopaedics, Trauma And Plastic Surgery, University of Leipzig, Leipzig/GERMANY, ${ }^{2}$ Department Of Trauma And Septic Surgery, St. Georg Hospital, Leipzig/ GERMANY, ${ }^{3}$ Department Of Orthopeadics, Trauma Surgery, And Plastic Surgery, University of Leipzig, Leipzig/ GERMANY, ${ }^{4}$ Department Of Traumatology And Reconstructive Surgery, BG Hospital Tübingen, Tübingen/GERMANY

Introduction: Instable pelvic ring injuries of type B and $\mathrm{C}$ are reported with a mortality rate of $8.4-18 \%[1]$, in case of hemodynamical instability up to $40 \%[2] .1 / 3$ of the patients die due to uncontrolled bleeding[3]. Given these facts, it is important to establish an effective standard in emergency care for patients with pelvic ring injuries. Besides external mechanical stabilization embolization is a treatment option. The present study was carried out to evaluate frequency and efficiency of used technics.

Material and methods: Data of the German pelvic trauma registry was evaluated between January 2010 to December 2014. Hospitals with missing data sets were excluded. In total, the data of 4485 patients from 10 hospitals were considered. Pelvic ring injuries of 444 patients underwent emergency stabilization and were included. Beside baseline data, ISS, AIS, fracture classification, efficiency of emergency stabilization and mortality were evaluated.

Results: Most Patients had C1 fracture ( $n=144 ; 33,1 \%)$. Average ISS was $29,1 \pm 13,7$. Highest ISS Score had patients with primary stabilization through pelvic c-clamp $(38.3 \pm 13.2)$. Primary stabilization was most of the time made by Fixateur externe ( $n=197 ; 44,3 \%)$, followed by pelvic sling $(n=98 ; 22,1 \%)$ and pelvic binder $(n=78 ; 17,6 \%)$. In 245 patients $(55.20 \%)$ a complementary procedure was performed. Average mortality was $10,40 \%(n=46)$. Patients with primary stabilization by c-clamp had the highest mortality $(29.3 \%)$.

Conclusion: In summary, it can be reported that due to the increased preclinical use of the pelvic binder, the pelvic clamp has found less use in the shock room in recent years. But overall emergency stabilization seems to be inconsistent and there is no standard for external mechanical stabilization of unstable pelvic ring fractures.

References: [1] 1.) Bircher M, Giannoudis PV. Pelvic trauma management within the UK: a reflection of a failing trauma service. Injury. 2004;35:2-6. 2.) Gansslen A, Pohlemann T, Paul C, Lobenhoffer P, Tscherne H. Epidemiology of pelvic ring injuries. Injury. 1996;27(Suppl 1): S-A13-20. 3.) Hauschild O, et. al; Mortality in patients with pelvic fractures: results from the German pelvic injury register. J Trauma. 2008;64:449-455. 4.) Sathy, Ashoke K. MD et al. The Effect of Pelvic Fracture on Mortality After Trauma: An Analysis of 63,000 Trauma Patients; Journal of Bone \& Joint Surgery - 01 December 2009 - Volume 91 - Issue 12 - p 2803-2810 [2] 1.) Heetveld MJ, et al. Hemodynamically unstable pelvic fractures: recentcare and new guidelines. World J Surg. 2004;28:904-9. 2.) Mucha Jr P, Welch TJ. Hemorrhage in major pelvic fractures. Surg Clin North Am. 1988;68:757-73. 3.) Poole GV, Ward EF. Causes of mortality in patients with pelvicfractures. Orthopedics. 1994;17:691-6. 4.) Smith $\mathrm{W}$, et al. Early predictors of mortality in hemodynamically unstablepelvis fractures. J Orthop Trauma. 2007;21:31-6. 5.) Starr AJ, et al. Pelvic ring disruptions: prediction ofassociated injuries, transfusion 
requirement, pelvic arteri-ography, complications, and mortality. J Orthop Trauma. 2002;16:553-61. [3] 1.) Perkins ZB, Maytham GD, Koers L, Bates P, Brohi K, Tai NR. Impact on outcome of a targeted performance improvement programme in haemodynamically unstable patients with a pelvic fracture. Bone Joint J. 2014 Aug;96B(8):1090-7. 2.) Velhamos GC, Toutouzas KG, Vassiliu P, Sarkisyan $\mathrm{GC}$, Linda $\mathrm{SH}$, Sue $\mathrm{H}$, et al. A prospective study on the safety and efficacyof angiographic embolization for pelvic and visceral injuries. $\mathrm{J}$ Trauma. 2002;52:303-508

Disclosure: No significant relationships.

\section{PR427}

\section{TREATMENT OF THE NEW RETROGRADE INTRAMEDULLARY NAILING FOR PROXIMAL HUMERAL FRACTURES}

\section{Uchino}

Orthopaedic Surgery, Kitasato University Medical Center, Kitamoto/ JAPAN

Introduction: To evaluate clinical data after undergoing the new retrograde intramedullary nailing for proximal humeral fractures.

Material and methods: The clinical data of eight patients was performed to evaluate for union period, re-fracture, iatrogenic nerve injury, implant failure, infection, nonunion and functional outcome of the shoulder using Japanese Orthopaedic Association score.

Results: Seven patients achieved union and the average of union period was 3.6 months without any complications. The average of functional score was 96 points. One patient was occurred re-fracture postoperatively according to falling out of the bed.

Conclusion: What the most specific feature of this new retrograde intramedullary nailing is that the rotator cuff is not damaged due to the nail. This new retrograde nailing for the proximal humeral fractures provides sufficient results.

References: 1. SO, Hartmann F, et al. Retrograde nailing versus locking plate osteosynthesis of proximal humeral fractures: a biomechanical study. J Shoulder Elbow Surg 2012; 21 (5): 618-624. 2. Huff LR, Taylor PA, et al. Proximal humeral fracture fixation: a biomechanical comparison of two constructs. J Shoulder Elbow Surg 2013; 22 (1): 129-136. 3. Yamamoto N, Hongo M, et al. Biomechanical analysis of a novel locking plate with smooth pegs versus a conventional locking plate with threaded screws for proximal humerus fractures. J Shoulder Elbow Surg 2013; 22 (4): 445-450. 4. Bae JH, Oh JK, et al. The biomechanical performance of locking olate fixation with intramedullary fibular struct graft augmentation in the treatment of unstable fractures of the proximal humerus. J Bone Joint Surg Br 2011; 93 (7): 937-941.

Disclosure: No significant relationships.

\section{PR428}

\section{INDUCED MEMBRANE TECHNIQUE AS THE FIRST-LINE PROCEDURE FOR TREATING SEVER TIBIAL DEFECTS: A 4-CASE SERIES}

\author{
S. Tomonori ${ }^{1}$, K. Shouzou ${ }^{2}$, N. Notani ${ }^{l}$, T. Sone ${ }^{3}$
}

${ }^{1}$ Orthopedic Surgery/Advanced Trauma, Emergency And Critical Care Center, Oita University Hospital, Yufu/JAPAN, ${ }^{2}$ Orthopedics, Oita university hospital, Yufu/JAPAN, ${ }^{3}$ Orthopedic Surgery/ advanced Trauma, Emergency And Critical Care Center, Oita
University Hospital, Yufu/JAPAN

Introduction: There are numerous reconstruction options for severe bone defects, with no consensus on the optimal method. We use induced membrane (IM) technique as the first-line procedure for treating large tibial bone defect. The purpose of this study is to report a case series treated on the basis of our strategy.

Material and methods: We report 4 cases of tibial bone defect treated by IM technique in our department from April 2013 to October 2017.

Results: Mean age was 66.3 years. Mean follow-up was 23.5 months. The mean tibial defect measured $9.2 \mathrm{~cm}$ in length. The cases included two tibial open fractures, one tibial osteomyelitis and one septic nonunion of the tibia. Two underwent fixations with plates, one with an intramedullary nail and one with a ring-type external fixator. The mean interval between stages was 5.9 weeks. All patients received soft tissue reconstruction with flap at the first stage. Bony fusion was achieved in two cases without any complications. In 1 case, the bone grafts had been infected and vascularized fibula graft was chosen as a salvage operation. The other case underwent tumor endprostheses after debridement of the infected bone grafts. At the final follow up all patients were able to walk with or without canes.

Conclusion: Failure of IM technique could be salvaged in any other methods. It was the reasonable strategy to use IM technique for treating sever tibial bone defects as a first-line procedure and the other methods as a second-line.

References:

Disclosure: No significant relationships.

\section{PR429}

\section{LOSS OF CORRECTION AFTER MINIMAL INVASIVE PERCUTANEUS OPERATIVE TREATMENT OF ANTERIOR AND POSTERIOR COLUMN AFTER A3 AND A4 FRACTURES OF THORACIC AND LUMBAL SPINE}

\author{
N. Eibinger, M. Hackl, M. Benedikt, G. Hohenberger, E. Tackner, \\ P. Puchwein
}

Department Of Orthopedics And Trauma Surgery, Medical University Graz, Graz/AUSTRIA

Introduction: Surgical interventions for thoracolumbar burst fractures without neurological deficit present a major challenge for trauma surgery and do not have a consistent treatment algorithm. The most recent operative procedure for traumatic fracture treatment is the minimally invasive combination of kyphoplasty and percutaneous pedicle screw fixation and allows the reduction of both columns via the dorsal approach. The aim of the study was to evaluate the clinical and radiological results of this method.

Material and methods: A total of 34 patients with traumatic monosegmental A3 and A4 fractures of the thoracic and lumbar spine were included in the study. Patients were treated with kyphoplasty and monoaxial dorsal instrumentation. For the radiological assessment, the vertebral body height and the bisegmental kyphosis angle, were measured preoperatively, postoperatively, before and at a minimum of six months after removal of the internal fixator. In the final examination, the functional outcome was evaluated by the Oswestry Disability Index (ODI) and the segmental pain diagnosis.

Results: The average loss of correction after hardware removal was $5.61 \%$ at the front edge, $0.2 \%$ at the dorsal edge, and $4.54 \%$ at the center of the vertebral body. No significant further loss of height after removal was observed. The bisegmental kyphosis angle decreased on 
average by 5.88 degrees after removal. The average ODI score was $17.7 \%$ and $55.89 \%$ of the patients had no local complaints in the former operative area.

Conclusion: Percutaneous pedicle screw fixation plus kyphoplasty leads to good results with few complications and is a serious alternative to open procedures.

References:

Disclosure: No significant relationships.

\section{PR430}

\section{INTRODUCTION OF A NEW SURGICAL TECHNIQUE FOR THE REPLACEMENT OF THE POSTERIOR CRUCIATE LIGAMENT}

\section{Janko, I. Marzi, J. Frank, D. Schramm, L. Seufert}

Trauma-, hand- And Reconstructive Surgery, University hospital Frankfurt, Frankfut am Main/GERMANY

Introduction: The knee joint has only a minor osseous guidance. The ligaments are the most important stabilizer. The anterior ligament ruptures considerably more easily, faster and thus more frequently than the posterior one.In the last few years, as with the anterior cruciate ligament, an augmentation with a tendon allograft was developed, hoping to achieve the same good results as the replacement of the anterior cruciate ligament.The purpose of this study is to compare two different operation techniques after a posterior cruciate ligament rupture.

Material and methods: The two different operation techniques were compared. The first technique is a two stage operation. First, the posterior cruciate ligament is prepared in supine position of the patient and afterwards in the same operation we turn the patient to prone position with an additional posterior access in the popliteal fossa to attach the allograft and the posterior cruciate ligament.To avoid the repositioning of the patient, we investigated the new method as a one stage operation with a special scoop technique.

Results: The evaluation of the results were done in two ways with a questionnaire and an examination. We found similar results in all examinations for both operation techniques. The only differences where less scarring, due to less invasive access in the operated knee joint and a reduction of the intervention time.

Conclusion: We certainly need a long-term follow up and additional informations, e.g. return to sports to get a more definite evaluation of the superiority of one method over the other. Furthermore we want to contribute to a more individual approach concerning the type and form of the treatment of this injury in the future.

References:

Disclosure: No significant relationships.

\section{PR431}

TEMPORARY EXTERNAL FIXATOR FOR THE INITIAL TREATMENT OF SEVERE LOWER EXTREMITY FRACTURES CAN BE SAFELY USED IN THE OPERATIVE FIELD FOR DEFINITIVE INTERNAL PLATE FIXATION IN TERMS OF POSTOPERATIVE INFECTION

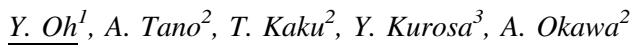

${ }^{1}$ Department Of Orthopaedic And Trauma Research, Tokyo Medical and Dental University, Tokyo/JAPAN, ${ }^{2}$ Department Of Orthopaedic And Spinal Surgery, Tokyo Medical and Dental University, Tokyo/
JAPAN, ${ }^{3}$ Department Of Orthopaedic Surgery, Saku Central Hospital, Nagano/JAPAN

Introduction: A staged approach for severe lower extremity fractures is becoming more common. Some reports described internal fixation (IF) using a temporary external fixator for primary care as an intraoperative reduction tool, to keep reduced alignment and protect soft tissue. However, the infection risk associated with this procedure has not been examined sufficiently. We hypothesized that infection risk of this technique is low and tested the safety.

Material and methods: Between 2012 and 2017, staged internal plate fixation that uses a temporary external fixator for the initial treatment as an intraoperative retention tool was performed for 19 lower extremity fractures in 17 consecutive patients (mean age, $52.5 \pm 16.1$ years) treated with the treatment protocol laid down by 2 hospitals. Three were femur fractures and 16 were lower leg fractures. Mean follow-up period was $36.5 \pm 20.6$ months. Injury severity score (ISS), presence of open fracture, soft tissue management, overlap between the external fixation (EF) pin site and plate area, waiting period (from initial EF to definitive IF), and postoperative infection were assessed. Results: Mean ISS was $15.7 \pm 11.3$. Fourteen (74\%) were open fractures (Gustilo I:2, II:1, IIIa:8, IIIb:3), and tissue grafts were needed in 5 fractures (26\%). The EF pin site overlapped with the plate area in 5 fractures $(26 \%)$. Mean waiting period was $9.1 \pm 3.5$ days. In all 19 fractures, postoperative infection did not occur.

Conclusion: The infection risk associated with staged plate fixation of lower extremity fractures using a temporary external fixator for the initial treatment might be little to no when the waiting period is within about 2 weeks, even when severe open fractures in polytraumatized patients.

References: Shah CM, Babb PE, McAndrew CM, Brimmo O, Badarudeen S, Tornetta P 3rd et al (2014) Definitive plates overlapping provisional external fixator pin sites: is the infection risk increased? J Orthop Trauma 28:518-522. Haidukewych GJ (2002) Temporary external fixation for the management of complex intra- and periarticular fractures of the lower extremity. J Orthop Trauma 16:678-685. Tejwani NC, Achan P (2004) Staged management of high-energy proximal tibia fractures. Bull Hosp Jt Dis 62:62-66. Harwood PJ, Giannoudis PV, Probst C, Krettek C, Pape HC (2006) The risk of local infective complications after damage control procedures for femoral shaft fracture. J Orthop Trauma 20:181-189. Bhandari M, Zlowodzki M, Tornetta P 3rd, Schmidt A, Templeman DC (2005) Intramedullary nailing following external fixation in femoral and tibial shaft fractures. J Orthop Trauma 19:140-144. Hak DJ, Wiater PJ, Williams RM, Pierson CL (2005) The effectiveness of standard povidone iodine surgical preparation in decontaminating external fixator components. Injury 36:1449-1452. Stinner DJ, Beltran MJ, Masini BD, Wenke JC, Hsu JR (2012) Bacteria on external fixators: which prep is best?. J Trauma Acute Care Surg 72:760-764.

Disclosure: The department to which the first author belongs received funding for operating costs from Saku Central Hospital, Medtronic Sofamor Danek Co., Ltd., Stryker Japan Co. Ltd., HOYA Technosurgical Co., Ltd., and A-Z Co. Ltd.

\section{PR432}

\section{INFECTION PROPHYLAXIS FOR OPEN FRACTURES BY HIGH-DOSE LOCAL ANTIBIOTICS ADMINISTRATION FROM INTRA-MEDULLARY ANTIBIOTICS PERFUSION (IMAP)}

S. Watanabe, A. Maruo, Y. Onoi, K. Sawauchi, M. Kamimura, T. Oshima, H. Miya, H. Muratsu

Department Of Orthopaedic Surgery, Steel Memorial Hirohata Hospital, Himeji/JAPAN 
Introduction: The infection control in open fractures is still controversial. We performed a high-dose antibiotics administration by using intra-medullary antibiotics perfusion (iMAP) for perioperative infection control in open fractures, with minimal debridement and one-stage fracture fixation or early conversion to definitive fixation. The purpose of this study was to assess the efficacy of iMAP on infection control in open fractures.

Material and methods: iMAP is a method of antibiotics delivery with the continuous administration of high-dose Aminoglycosides (Gentamicin 120mg/day). We retrospectively reviewed 190 open fractures in lower limbs from 2001 to 2017. iMAP was performed with either external fixation or definitive fracture fixation in open fractures which have been considered as higher-risk of infection (Group P: $\mathrm{n}=36$ ). Rest of cases received intravenous antibiotics administration alone (Group C: $\mathrm{n}=154$ ). Gustilo classification, infection rates, and clinical outcomes were compared between two groups.

Results: In Group P, 19.4\% of patients were classified as Gustilo stage II and $80.6 \%$ were stage III. In Group C, $40.9 \%$ and $28.6 \%$ respectively. Infection rates were $0 \%$ in Group P and $11.7 \%$ in Group C. Despite the stage of Gustilo classification were higher in Group P $(\mathrm{p}<0.05)$, lower infection rate was observed in Group $\mathrm{P}(\mathrm{p}<0.05)$. Union rates were not significantly different between two groups.

Conclusion: For severe open fractures treatment, our novel method; "intra-Medullary Antibiotics Perfusion (iMAP); enabled to deliver antibiotics to low-vascularized tissues and around internal fixation implants, and resulted in the better infection control comparing with conventional method. iMAP would be the alternative effective option for infection control in open fractures.

References:

Disclosure: No significant relationships.

\section{PR433}

\section{MINIMALLY INVASIVE APPROACH FOR INTRA- ARTICULAR CALCANEAL FRACTURES CAUSED BY FALL - SINUS TARSI APPROACH VERSUS EXTENDED L-SHAPED APPROACH}

\section{Y. Sato, M. Yoshida}

Orthopedics, Nagoya Daini Red Cross Hospital, AICHI, NAGOYA/ JAPAN

Introduction: High rate of wound complications are common problem in treating intra-articular calcaneal fractures especially caused by fall. The aim of this study was to compare the outcomes of intraarticular calcaneal fractures treated with open reduction and internal fixation via sinus tarsi approach versus those with extended L-shaped approach.

Material and methods: We performed a retrospective review of intra-articular calcaneal fractures caused by fall treated operatively between 2010 and 2016. A total of 18 patients, 24 fractures were included. 9 fractures between 2010-2013 were treated with extended L-shaped approach (group L), and 15 fractures between 2014-2016 were treated with sinus tarsi approach (group S). We assessed rate of wound complications (skin necrosis, infection, delay of wound healing), radiological outcome (Xp : Bohler's angle, CT : dislocation in posterior facet), Maxfield score as clinical evaluation

Results: The overall wound complication rate was 2 fractures (13\%) in group $\mathrm{S}$ versus 6 fractures $(66 \%)$ in group $\mathrm{L}(\mathrm{P}<0.05)$. No differences were noted in the final postoperative Bohler's angle $\left(22^{\circ}\right.$ in group $\mathrm{S}$ versus $18^{\circ}$ in group L, N.S.) and dislocation of posterior facet (2.0mm in group S versus $2.2 \mathrm{~mm}$ in group L, N.S.). Overall, $80 \%$ of patients in the group $\mathrm{S}$ were satisfied with their result versus $66 \%$ in the group L (N.S.).

Conclusion: Clinical results were similar between calcaneal fractures treated with sinus tarsi approach and those treated with a extended L-shaped approach. However, the sinus tarsi approach had a significantly lower incidence of wound complications .

References: Kline AJ, Anderson RB, Davis WH, et al . Minimally invasive technique versus an extensile lateral approach for intra-articular calcaneal fractures . Foot Ankle Int . 2013 Jun; 34 (6):773-80 Schepers $\mathrm{T}$. The sinus tarsi approach in displaced intra-articular calcaneal fractures : a systematic review . Int Orthop . 2011 May; 35 (5) 697-703 Backes M, Schepers T, Suzan M, et al . Wound infections following open reduction and internal fixation of calcaneal fractures with an extended lateral approach . Int Orhop . 2014 Apr; 38 (4) 767-773

Disclosure: No significant relationships.

\section{PR434}

\section{PICO@: NEGATIVE PRESSURE WOUND THERAPY IN THE TREATMENT OF DIABETIC FOOT IN MULTIDISCIPLINARY TEAM IN THE MANAGEMENT OF DIABETIC FOOT WOUNDS}

\author{
V. Pino Diaz ${ }^{1}$, M.J. Tamayo López ${ }^{2}$, L. Tallón-Aguilar ${ }^{1}$, V. Duran \\ Muñoz-Cruzado ${ }^{2}$, J.T. Gonzalez ${ }^{2}$
}

${ }^{1}$ Emergency Surgery Unit, Virgen del Rocío Universitary Hospital, Seville/SPAIN, ${ }^{2}$ Cirugĺa General Y Digestiva, HOSPITAL UNIVERSITARIO VIRGEN DEL ROCIO, SEVILLA/SPAIN

Introduction: The aim of the present study is to standardize the use of negative pressure therapy using a portable device $\left(\mathrm{PICO}{ }^{\circledR}\right)$ in the healing after transmetatarsal amputation at the Virgen del Rocío University Hospital in Seville.

Material and methods: Prospective study to evaluate the efficacy of our PICO protocol because of a negative wound therapy is well established for treating diabetic foot wounds with reduction in healing time, the number of re-amputations and a higher percentage of satisfactory scarred wounds with a decrease in hospital stays. Indications of usage include transmetatarsal amputation post-debridement wounds for necrotizing fasciitis, foot abscesses, infective heel ulcers and exposed bone, capsule and tendon. Collection of data includes wound surface area, duration of treatment, hospital stays, bacteriology, safety, and patient satisfaction.

Results: A successful outcome in this subgroup of diabetic patients requires a multidisciplinary approach with careful patient selection, appropriate surgical debridement, amputation, targeted antibiotic therapy, and optimization of healing markers in our multidisciplinary team with surgeons, endocrine and nursing specialists in the management of diabetic foot wounds. Portable and more compact systems as well as home-care protocols are also expanding Negative Pressure Wound Therapy's usage beyond the hospital setting. The long-term cost effectiveness of these protocols is still unclear but in the short term, they do facilitate early hospital discharge and reduce hospital stay.

Conclusion: Negative pressure wound therapy seems to be safe and effective in the treatment of some transmetatarsal amputations in the management of diabetic foot wounds.

References:

Disclosure: No significant relationships. 


\section{PR435}

\section{RETROSPECTIVE ANALYSIS OF CECS-SUSPECTED PATIENTS}

R. Maksymiak $^{1}$, E. Ritchie $^{2}$, W.O. Zimmermann ${ }^{3}$, N. Maliko $^{2}$, M. Van Der Werve ${ }^{I}$, R. Hoencamp ${ }^{2}$

${ }^{1}$ Sports Medicine, Alrijne Hospital, Leiderdorp/NETHERLANDS, ${ }^{2}$ Surgery, Alrijne Hospital, Leiderdorp/NETHERLANDS,

${ }^{3}$ Department Of Sports Medicine, Royal Dutch Army and adjunct asscociate professor USUHS, Amersfoort/NETHERLANDS

Introduction: There is still debate on the effectiveness of surgical treatment for Chronic Exertional Compartment Syndrome (CECS). Fasciotomy success-rates as high as $78 \%-98 \%$ have been reported in civilian populations, whereas in military populations up to $44.7 \%$ of patients may have symptom recurrence and up to $27.7 \%$ of patients may not be able to return to full duty. Objective - To analyze characteristics and outcomes of all CECS cases and to identify parameters predicting treatment outcome in favor of conservative or surgical procedure.

Material and methods: CECS-patients from 2013-2017 ( $\mathrm{n}=83$ ) were retrospectively analyzed for characteristics, treatment and outcome.

Results: Preliminary data: There was a delay of 36 months between onset of symptoms and diagnosis. Fascial herniation was present in $11.7 \%$ of patients during physical examination as a comorbidity. The anterior compartment was affected most often $(72.3 \%)$ and patients frequently presented symptoms bilaterally $(85.5 \%)$. After fasciotomy $(n=56)$ (limited) hemorrhage was recorded in $26 \%$ of cases. No other complications such as infection or loss of motor/sensory functionality were recorded. Outcome after fasciotomy was reported as improvement in $92 \%$, no improvement in $6 \%$, with deterioration in only $2 \%$ of cases. $26 \%$ of patients revisited our out-patient clinic with recurring symptoms, and ultimately $14 \%$ had revision surgery. Results of conservative treatment $(n=27)$ are still being gathered.

Conclusion: Fasciotomy for CECS in our clinic has a high success rate of $92.2 \%$ after revision surgery. Deep posterior compartment fasciotomy shows a success rate of $88.9 \%$ in this cohort, which is high compared to reports in the literature (50-65\%) No major complications were reported.

References: Tucker AK. Chronic exertional compartment syndrome of the leg. Current reviews in musculoskeletal medicine 3 (1-4): 32-37, 2010. Bong MR et al. Chronic Exertional Compartment Syndrome: Diagnosis and Management. Bulletin (Hospital for Joint Diseases (New York, N.Y.)) 62 (3-4): 77-84, 2005. Qvarfordt P et al. Intramuscular Pressure, Muscle Blood Flow, and Skeletal Muscle Metabolism in Chronic Anterior Tibial Compartment Syndrome. Clinical Orthopaedics and Related Research 179: 284-290, 1983. Trease L et al. A prospective blinded evaluation of exercise thallium-201 SPET in patients with suspected chronic exertional compartment syndrome of the leg. European Journal of Nuclear Medicine 28 (6): 688-695, 2001. Amendola A et al. The use of magnetic resonance imaging in exertional compartment syndromes. The American Journal of Sports Medicine 18 (1): 29-34, 2010. Pasic $\mathrm{N}$ et al. Assessing Outcomes in Individuals Undergoing Fasciotomy for Chronic Exertional Compartment Syndrome of the Leg. Arthroscopy 31 (4): 707-713, 2015. Godefrooij DA, Zimmermann WO. Ontwikkelingen in de behandeling van chronisch inspanningsgebonden logesyndroom (CECS). Nederlands Militair Geneeskundig Tijdschrift 65: 160-162, 2012. Zimmermann WO, Helmhout PH, Noest R. De enkel-armindex voor en na inspanning bij gezonde militairen en militairen met onderbeenklachten. Nederlands Militair Geneeskundig Tijdschrift 68: 44-49, 2015 Van der Wal WA et al. The natural course of chronic exertional compartment syndrome of the lower leg. Knee Surgery, Sports Traumatology, Arthroscopy 23: 2136-2141, 2013. Davis DE et al. Characteristics of Patients With Chronic Exertional Compartment Syndrome. Foot \& Ankle International 34 (10): 1349-1354, 2013. Ligthert E et al. Het onderbeenspreekuur in het Centraal Militair Hospitaal. Nederlands Militair Geneeskundig Tijdschrift 70: 90-98, 2017. Waterman BR et al. Risk Factors for Chronic Exertional Compartment Syndrome in a Physically Active Military Population. The American Journal of Sports Medicine 41 (11): 2545-2549, 2013. Packer JD et al. Functional Outcomes and Patient Satisfaction After Fasciotomy for Chronic Exertional Compartment Syndrome. The American Journal of Sports Medicine 41 (2): 430-436, 2013. Pedowitz RA et al. Modified criteria for the objective diagnosis of chronic compartment syndrome of the leg. The American Journal of Sports Medicine 18 (1): 35-40, 1990 Van den Brand JGH, Nelson T, Verleisdonk EJMM, van der Werken C. The Diagnostic Value of Intracompartmental Pressure Measurement, Magnetic Resonance Imaging, and Near-Infrared Spectroscopy in Chronic Exertional Compartment Syndrome: A Prospective Study in 50 Patients. The American Journal of Sports Medicine 33 (5): 699-704, 2005. Aweid et al. Systematic Review and Recommendations for Intracompartmental Pressure Monitoring in Diagnosing Chronic Exertional Compartment Syndrome of the Leg. Clinical Journal of Sports Medicine 22 (4): 356-370, 2012. Roberts A, Franklyn-Miller A. The validity of diagnostic criteria used in chronic exertional compartment syndrome: A systematic review. Scandinavian Journal of Medicine \& Science in Sports 22 (5): 585-595, 2012. Van den Brand JGH, Verleisdonk EJMM, van der Werken C. Near Infrared Spectroscopy in the Diagnosis of Chronic Exertional Compartment Syndrome. The American Journal of Sports Medicine 32 (2): 452-456, 2004. Zimmermann WO, Helmhout PH, Beutler A. Prevention and treatment of exercise related leg pain in young soldiers; a review of the literature and current practice in the Dutch Armed Forces. Journal of the Royal Army Medical Corps 163 (2): 94-103, 2017. Compano D et al. Surgical Management for Chronic Exertional Compartment Syndrome of the Leg: A Systematic Review of the Literature. Arthroscopy 32 (7): 1478-1486, 2016. Mafulli N et al. Single minimal incision fasciotomy for chronic exertional compartment syndrome of the lower leg. Journal of Orthopaedic Surgery and Research 11 (1):61, 2016. Drexler M et al. Single minimal incision fasciotomy for the treatment of chronic exertional compartment syndrome: outcomes and complications. Archives of Orthopedic and Trauma Surgery 137 (1): 73-79, 2017. Van Zoest WJF et al. Chronic Deep Posterior Compartment Syndrome of the Leg in Athletes: Postoperative Results of Fasciotomy. International Journal of Sports Medicine 29 (5): 419-423, 2007. Waterman BR et al. Surgical Treatment of Chronic Exertional Compartment Syndrome of the Leg. The Journal of bone and joint surgery. American Volume. 95 (7): 592-596, 2013. Rajasekaran S, Hall MM. Nonoperative Management of Chronic Exertional Compartment Syndrome: A Systematic Review. Current Sports Medicine Reports 15 (3): 191-198, 2016 Isner-Horobeti ME et al. Intramuscular Pressure Before and After Botulinum Toxin in Chronic Exertional Compartment Syndrome of the Leg: A Preliminary Study. The American Journal of Sports Medicine 41 (11): 2558-2566, 2013. Finoff JT, Rajesekaran S. Ultra-Guided, Percutaneous Needle Fascial Fenestration for the Treatment of Chronic Exertional Compartment Syndrome: A Case Report. $P M \& R$ : The Journal of Injury, Function, and Rehabilitation 8 (3): 286-290, 2016. Diebal AR et al. Effects of Forefoot Running on Chronic Exertional Compartment Syndrome: A Case Series. The International Journal of Sports Physical Therapy 6 (4): 312-321, 2011. Roberts A et al. Biomechanical differences between cases with chronic exertional compartment syndrome and asymptomatic controls during marching gait. Gait \& Posture 58: 66-71, 2017. Roberts A et al. Biomechanical differences between cases with suspected chronic exertional compartment syndrome and asymptomatic controls during running. Gait \& Posture 58: 374-379, 2017. Helmhout PH et al. The Effectiveness of a 6-Week Intervention Program Aimed at Modifying Running Style in Patients With Chronic 
Exertional Compartment Syndrome: Results From a Series of Case Studies. The Orthopaedic Journal of Sports Medicine 3 (3): 1-10, 2015. Helmhout PH et al. Modifying Marching Technique in Military Service Members with Chronic Exertional Compartment Syndrome: A Case Series. The International Journal of Sports Physical Therapy 11 (7): 1106-1116, 2016. Qvarfordt P et al. Intramuscular pressure, muscle blood flow, and skeletal muscle metabolism in chronic anterior tibial compartment syndrome. Clinical orthopaedics and related research 179: 284-290, 1983. Amendola A et al. The use of magnetic resonance imaging in exertional compartment syndromes. The American Journal of Sports Medicine 18 (1): 29-34, 1990. Turnipseed WD, Hurschler C, Vanderby R Jr. The effects of elevated compartment pressure on tibial arteriovenous flow and relationship of mechanical and biochemical characteristics of fascia to genesis of chronic anterior compartment syndrome. Journal of vascular surgery 21 (5): 810-816, 1995. Mohler $\mathrm{LR}$ et al. Intramuscular deoxygenation during exercise in patients who have chronic anterior compartment syndrome of the leg. The Journal of Bone and Joint Surgery. American Volume 79 (6): 844-849, 1997. Blackman PG, Simmons LR, Crossley KM. Treatment of chronic exertional anterior compartment syndrome with massage: a pilot study. Clinical Journal of Sport Medicine 8 (1): 14-17, 1998. Trease L et al. A prospective blinded evaluation of exercise thallium-201 SPET in patients with suspected chronic exertional compartment syndrome of the leg. European Journal of Nuclear Medicine 28 (6): 688-695, 2001. Dharm-Datta $S$ et al. Dynamic pressure testing for chronic exertional compartment syndrome in the UK military population. Journal of the Royal Army Medical Corps 159 (2): 114-118, 2013. Dunn JC, Waterman BR. Chronic Exertional Compartment Syndrome of the Leg in the Military. Clinics in Sports Medicine 33 (4): 693-705, 2014. Roberts AJ et al. Outcomes of Surgery for Chronic Exertional Compartment Syndrome in a Military Population. Journal of the Royal Army Medical Corps 161 (1): 42-45, 2015. Finnoff JT, Rajasekaran S. UltrasoundGuided, Percutaneous Needle Fascial Fenestration for the Treatment of Chronic Exertional Compartment Syndrome: A Case Report. $P M \& R$ : The Journal of Injury, Function, and Rehabilitation 8 (3): 286-290, 2016. Meulekamp MZ et al. Short-Term Results of a Rehabilitation Program for Service Members with Lower Leg Pain and the Evaluation of Patient Characteristics. Military Medicine 181 (9): 1081-1087, 2016. Disclosure: No significant relationships.

\section{PR436}

\section{RETROGRADE INTRAMEDULLARY NAILING FOR HUMERAL SHAFT FRACTURES}

\author{
L. Waaijer, B. Emmink, W. Bosman, P. Wittich, J. Keizer
}

Trauma Surgery, St Antonius Hospital, Nieuwegein/ NETHERLANDS

\section{Introduction: \\ Material and methods: \\ Results: \\ Conclusion:}

References: 1. Fan Y et al. Management of humeral shaft fractures with intramedullary interlocking nail versus locking compression plate. Orthopedics. 2015;38:825-9. doi: 10.3928/0147744720150902-62. 2. Kurup H. et al. Dynamic compression plating versus locked intramedullary nailing for humeral shaft fractures in adults. Cochrane Database Syst Rev. 2011 Jun 15;CD005959. doi: 10.1002/ 14651858.CD005959.pub2.

Disclosure: No significant relationships.
PR437

\section{RADIOCARPAL FRACTURE - DISLOCATIONS}

\section{Ira, M. Krticka, A. Bilik, M. Masek}

Department Of Trauma Surgery, University Hospital Brno, Brno/ CZECH REPUBLIC

Introduction: Radiocarpal fracture-dislocations are serious, uncommon injuries involving significant soft-tissue and osseous trauma associated with frequent acute and late complications. The goal of this study is to analyse and compare the outcome and complications after isolated volar and combined approach.

Material and methods: From 2010 to 2015 we observed 9 cases of radiocarpal fracture-dislocations. All patients suffered from highly unstable osteoligamentous injury, two patients presented with open fractures, two with median nerve injury and in 3 cases associated ligamentous injuries were diagnosed. Four patients were treated with combined approach (dorsally - radius plate osteosynthesis, vollary anchor capsule reattachment) a four patient only with isolated volar approach (anchor capsule reattachment in combination with Kirschner wire fixation). In one case closed reduction, external fixation and Kirschner wires stabilization were performed.

Results: Four patients are painless, three patients reported occasional mild wrist, one regular moderate pain after physical activity. Radiographs showed maintained alignment of the radiocarpal joint without significant dorsal or ulnar translation in 8 out 9 cases, signs of radiocarpal arthrosis occured in 4 cases. All patients had returned fully to their previous work duties, no salvage procedures have been so far performed. (Detailed on-going follow-up evaluation will be finished this month)

Conclusion: On the basis of our experience and literature data, we believe that unstable radiocarpal dislocation should be treated with acute reduction of dislocation followed by reattachment of the volar ligaments and fracture osteosynthesis. Unfortunately, even with successful open treatment, patient outcomes can be compromised by stiffness, persistent radiocarpal instability or early arthrosis.

References: Dumontier C, Meyer zu Reckendorf G, Sautet A, Lenoble E, Saffar P, Allieu Y. Radiocarpal dislocations: classification and proposal for treatment. A review of twenty-seven cases. J Bone Joint Surg Am. 2001;83-A (2):212-218 Ilyas A M, Mudgal C S. Radiocarpal fracture-dislocations. J Am Acad Orthop Surg. 2008;16 (11):647-655. [ Mudgal C S, Psenica J, Jupiter J B. Radiocarpal fracture-dislocation. J Hand Surg [Br] 1999;24 (1):92-98.

Disclosure: No significant relationships.

\section{PR438}

\section{SURGICAL MANAGEMENT OF TIBIAL PLATEAU FRACTURES}

G. Croitor, A. Betisor, V. Madan

Orthopedics And Traumatology, Orthopedics and Traumatology Clinic "V. Betisor", State University of Medicine and Pharmacy "N. Testemitanu", Chisinău, Republic of Moldova, Chisinau/MOLDOVA

Introduction: The aim: Analyzing the methods of surgical management of patients with tibial plateau fractures treated in Orthopedics and Traumatology Clinic "V. Betisor" durind 2014-2015 years. 
Material and methods: We analyzed 70 clinical cases: men -26 and women -44 , mean age 52,4 years. Trauma circumstances: habitual trauma -50 cases, traffic accident -10 , precipitation -6 , sport -3 , aggression - 1. For cohort analize Schatzker classification was used: especially type I was meet in 7 cases, II - 20, III - 10, IV - 3, V - 20, VI - 10; 57 close, 3 open. For paraclinic examination were used X-ray and CT. Surgical management consisted of: close reduction, internal fixation - 9 cases (7- percutaneus canulated screws arthroscopic assisted, 2- external fixator), open reduction, internal fixation - 61 cases. Bone graft was applied in 12 cases.

Results: Postoperative follow up was performed at 6, 12, 18, 24 weeks. Patients were evaluated according to the Lysholm Knee Scoring Scale, obtaining an average score of 90 points. Bone healing was achieved in a period of between 10 to 20 weeks. Postoperative complication developed in 8 cases. Results were depending on the stability of osteosynthesis, precocity, rightness of functional reeducation and patient compliance.

Conclusion: Favorable functional results and less complication were meet in cases of individual approach for every patient, the right choice of implants and minimally invasive surgical techniques.

References:

Disclosure: No significant relationships.

\section{PR439}

\section{ACUTE ACHILLES TENDON RUPTURE - COMPARISON BETWEEN OPEN TECHNIQUE AND MINIMALLY INVASIVE TECHNIQUE}

\author{
T. Roseiro' ${ }^{1}$, D. Peixoto ${ }^{2}$, S. Angelo ${ }^{2}$, F.S. Gomes ${ }^{2}$, C. Vale $^{3}$, \\ A.M. Carvalho ${ }^{4}$, J. Corte Real ${ }^{1}$
} ${ }^{1}$ Ortopedia, Hospital Distrital Figueira da Foz, Figueira/PORTUGAL,
${ }^{2}$ Serviço De Ortopedia, Hospital Distrital da Figueira da Foz, Figueira
da Foz/PORTUGAL, ${ }^{3}$ Serviço De Ortopedia, Hospital Distrital
Figueira da Foz, Figueira da Foz/PORTUGAL, ${ }^{4}$ Ortopedia, Hospital
Distrital da Figueira da Foz, Figueira da Foz/PORTUGAL

Introduction: Achilles tendon ruptures occur most frequently between 30-40 years in males. Surgical treatment can be performed through open, minimally invasive and percutaneous techniques.

Material and methods: A retrospective assessment of patients with Achilles tendon rupture treated from 2011 to 2017: intervention, length of hospital stay, complications and functional recovery. The functional evaluation was performed using the AOFAS score. Statistical analysis was performed with SPSS.

Results: 33 patients were included, 24 were treated by the classical technique and 8 were treated by the minimally invasive technique. The mean length of hospital stay was 3,10 days on open technique and 3, 15 days on minimally invasive technique. The complications were 1 deep venous thrombosis and 2 cutaneous necrosis on open technique and 1 deep venous thrombosis and 1 superficial infection on the other.

The difference in length of hospital stay and complication rate was not statistically significant $(\mathrm{p}>0.05)$. The functional results in both were very good without statistically significant differences $(\mathrm{p}>0.05)$. The open technique is associated with higher rates of cutaneous complications while minimally invasive techniques are associated with higher rates of sural nerve injury.

The complication rate was higher in the group treated with the minimally invasive technique, however the result was not statistically significant. The functional outcome was similar between the techniques.

Conclusion: The surgical treatment of Achilles tendon ruptures is associated with good functional results and with minor limitations regardless of the technique used.

Due to the small number of patients under this study, it will be necessary to expand the database.

References: Canale, S. T., Beaty, J. H., \& Campbell, W. C. (2013). Campbell's operative orthopaedics. Philadelphia, PA: Elsevier/ Mosby

Disclosure: No significant relationships.

\section{PR440}

\section{CLOSED REDUCTION OF ELDERLY DISTAL RADIUS FRACTURES ULTRASOUND-GUIDED VERSUS FLUOROSCOPY}

\section{T. Muraoka}

Orthopaedics, Miyazaki prefecture Miyazaki Hospital, MIyazaki city/ JAPAN

Introduction: The purpose of this paper is to assess the accuracy and ability of ultrasound for monitoring closed reduction for elderly distal radius fractures.

Material and methods: Consecutive patients underwent ultrasoundguided closed reduction of acute distal radius fractures between October 2015 and April 2017. The control group was extracted from patients who underwent closed reduction for similar fractures with fluoroscopy between April 2014 and September 2015. At the ultrasound-guided closed reduction, the probe was oriented perpendicular to the longitudinal axis between the index and middle finger in the palmar site. After reduction, we could see volar cortex in fracture site had no gap by using ultrasound. The ultrasound-guided group consisted of 6 patients (mean age 79.2), and the control group consisted of 6 patients (mean age 74.5) .After reduction $X$ ray parameter, latest $\mathrm{X}$ ray parameter and Quick DASH score were compared between the ultrasound-guided group and the control group. We used Radial angle (RA), Palmer tilt (PT), Radial shortening (RS) as X ray parameter.

Results: After reduction $\mathrm{X}$ ray parameter (RA/PT/RS) was $22.5 \%$ $5.7 \% .17 \mathrm{~mm}$ at ultrasound-guided group, $20.6^{\circ} / 0.67^{\circ} / 0.5 \mathrm{~mm}$ at control group. Latest X ray parameter (RA/PT/RS) was $21.5^{\circ} / 3.5^{\circ} / 0.5 \mathrm{~mm}$ at ultrasound-guided group, $18.3 \%-2.0 \% 0.83 \mathrm{~mm}$ at control group. Quick DASH score was 8.3 at ultrasound-guided group and 20.5 at control group. All the score at ultrasound-guided group were superior to that of control group. At the latest PT, we found statistically significant difference $(\mathrm{P}=0.029)$.

Conclusion: Our date suggests that ultrasound assistance can aid reduction of elderly distal radius fractures as well as fluoroscopy. Moreover, ultrasound is easy to use and no radiation exposure.

References: Kodama N, Takemura Y, Ueba H et.al. Ultrasound-assisted closed reduction of distal radius fractures. J Hand Surg Am.2014;39:1287-1294

Disclosure: No significant relationships. 
PR441

\section{OPE FRACTURES-TIME FROM TRAUMA TO SURGICAL DEBRIDEMEMNT IS THE KEY FACTOR FOR INFECTION}

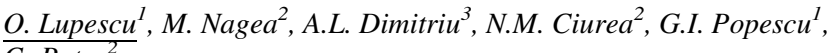

$$
\begin{aligned}
& \text { C. Patru }{ }^{2}
\end{aligned}
$$

${ }^{1}$ Orthopaedics And Trauma, Clinical Emergency Hospital, University of Medicine and Pharmacy " Carol Davila" Bucharest, Bucharest/ ROMANIA, ${ }^{2}$ Orthopaedics And Trauma, CLINICAL EMERGENCY HOSPITAL, BUCHAREST/ROMANIA, ${ }^{3}$ Clinical Emergency Hospital, Orthopaedics And Trauma, University of Medicine and Pharmacy Carol Davila, BUCHAREST/ROMANIA

Introduction: Septic risk after Open Fractures (OF) is unanimously recognized but the factors influencing it have not been yet clearly quantified. The authors evaluate the correlation between time from trauma to initial complete surgical debridement and the rate of infection.

Material and methods: A retrospective study was performed in a Level 1 Trauma Center upon 237 OF (30\% type I, $32 \%$ type II, 38\% type III) operated between 01.06.2011-01.01.2015; the outcome was the incidence of infection within 18 months after trauma correlated with time from trauma to debridement, and time to antibiotics first administration Results: Mean time from trauma to first antibiotic dose was $2.9 \mathrm{hrs}$ (0.8-6 hours) and to initial debridement was $5 \mathrm{hrs}(65 \mathrm{~min}-48 \mathrm{hrs})$. The incidence of infection was $8.3 \%$; time between trauma and antibiotic significantly increased the incidence of sepsis from $4.8 \%$ (antibiotics before $3 \mathrm{hrs}$ ) to $11.8 \%$ (after $3 \mathrm{hrs}$ ), while time from trauma to debridement increased the risk from 3.8\% (debridement before $6 \mathrm{hrs}$ ) to $13.6 \%$-after 6 hrs. It must be underlined that the quality of surgical debridement must be discussed, since there is no practical instrument to measure its' accuracy; it largely depends on the experience of the surgeon and his ability to properly assess the post-traumatic injuries. Conclusion: The current study suggests that, more than time from trauma to antibiotics, time from trauma to surgical debridement is essential for the incidence of infection. Despite its limitations, it suggests that surgical treatment is unfairly underestimated, being at least as important as the antibiotics in open fractures.

References: Singh J, Rambani R, Hashim Z, Raman R, Sharma HK. The relationship between time to surgical debridement and incidence of infection in grade III open fractures. Strategies in Trauma and Limb Reconstruction. 2012;7 (1):33-37.

Disclosure: No significant relationships.

\section{PR442}

\section{DOES TEMPORARY EXTERNAL FIXATION AND STAGED PROTOCOL FOR CLOSED FRACTURES LEAD TO BACTERIAL CONTAMINATION OF THE SURGICAL SITE AND ASSOCIATED COMPLICATIONS? - A PROSPECTIVE TRIAL}

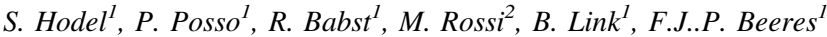 \\ ${ }^{1}$ Orthopaedics And Traumatology, Luzerner Kantonsspital, Luzern/ SWITZERLAND, ${ }^{2}$ Infectiology, Luzerner Kantonsspital, Luzern/ SWITZERLAND}

Introduction: Temporary external fixation is a viable option for numerous conditions and fixations in orthopaedic and trauma surgery.
If the external fixator is left in place it is necessary to disinfect it prior to surgery, yet the subsequent risk for bacterial contamination of the surgical site originating from the external fixator remains unknown. Material and methods: In a prospective study samples were taken at the time of definitive osteosynthesis to assess bacterial contamination of the surgical site and the external fixator in twenty consecutive patients treated with temporary external fixation for closed fractures from October 2016 until March 2017.

Results: Results Twenty patients with twenty external fixators were available for analysis with complete sampling and a mean follow-up of 92 days (range: 81-124). Ten out of 120 cultures of the surgical site $(8.3 \%)$ were positive for bacterial growth in a total of seven patients (35\%). Pathogen's detected were Propionibacterium acnes $(60 \%)$ and Staphylococcus epidermidis $(30 \%)$. No contamination of the external fixator was detected.

Conclusion: We conclude that the presented perioperative management to decontaminate external fixators allows for a safe definitive osteosynthesis in a staged protocol without increasing bacterial contamination of the surgical site. It is safe to leave the external fixator in place for definitive osteosynthesis.

References:

Disclosure: No significant relationships

PR443

\section{NEGATIVE PRESSURE WOUND THERAPY IN INFECTED FRACTURES- INDICATIONS, HANDLING, RESULTS}

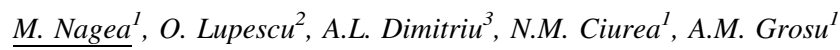

${ }^{1}$ Orthopaedics And Trauma, CLINICAL EMERGENCY HOSPITAL, BUCHAREST/ROMANIA, ${ }^{2}$ Orthopaedics And Trauma, Clinical Emergency Hospital, University of Medicine and Pharmacy " Carol Davila" Bucharest, Bucharest/ROMANIA, ${ }^{3}$ Clinical Emergency Hospital, Orthopaedics And Trauma, University of Medicine and Pharmacy Carol Davila, BUCHAREST/ROMANIA

Introduction: Negative Pressure Wound Therapy (NPWT) therapy has been indicated for severe trauma with extensive soft-tissue injuries, with impaired healing due to the severity of the injury and considerable septic risk, thus needing prolonged treatment

Material and methods: Authors present 15 patients with complex injuries of the limbs, treated ina Level 1 Trauma Centre- 6 crushing trauma with MESS less than 6 and 6 infected fractures with debridement and fracture fixation resulting in considerable soft tissue loss. The patients needed serial debridements and vacuum therapy was applied starting $48 \mathrm{hrs}$ after trauma for an average period of 12 days ( 7 - 27 days). Results: Within the study group, secondary debridements were peformed for all the patients and vaccum system was re-installed after these debridements. No amputations were needed. Literature is reviewed in order to compare the outcome of the patients: the hospital stay, anti-microbial therapy, time to healing of the bone and soft tissues (there is no standard group to be compared with) and the result shows considerable improvements in the outcome of the

Conclusion: Vacuum therapy improves healing of the soft tissues, decreases the risk of infection and so, that of secondary amputation. Hospital stay is also positively influenced, so is the moment of weight bearing, and the number of secondaryy necessary procedures. Therefore it is considered a valuable support in treating trauma of the limbs with severe soft tissue injuries.

References: Peinemann Fr. et al. Negative-Pressure Wound Therapy; Systematic Review of Randomized Controlled Trials., PMC312 Disclosure: No significant relationships. 


\section{PR444}

\section{POSTERIOR ANKLE ARTHROSCOPY}

\section{Lalić}

Department For Orthopedic Surgery And Traumatology, Clinical center of Vojvodina, Novi Sad/SERBIA

Introduction: Arthroscopy in ankle and foot surgery has benefit regarding better visualisation, less tissue trauma and faster recovery. Complications are significantly lower comparing open surgery.

Material and methods: From October 2013.g till 21.March. 2017.g we perform ankle arthroscopy in 61 patients; 22 of that number were posterior ankle arthroscopy. In distribution by sex there were 13 males, and 9 females. Regarding pathology, posterior bony impingement (os trigonum) were found in 4 patients, soft tissue impingement were found in 16 patients, loose bodies in 2 cases. Athletes and recreatives were predominant (18 cases). Average surgery time were 25 minutes. Average hospitalisation was one day. Sutures were removed two weeks after surgery. Partial weight-bearing was allowed at fifth post-operative day, full weight-bearing was achieved at three weeks (from 15 to 30 days). We didn't have infections or wound complications. In one case we have transitory superficial peroneal nerve palsy.

Results: were evaluated by questioners. Average AOFAS score were 52 preoperatively, and 91 postoperatively. According FAOS score, there were improvements in range of motion, decreasing pain, increasing walking distance and improvement in activities of daily living.

Conclusion: Posterior ankle arthroscopy, in our sample, leeds to significant improvement in functional scores postoperatively.

References:

Disclosure: No significant relationships.

\section{PR445}

\section{FRACTURES TREATED WITH PERCUTANEOUS APPROACH}

G. Luengo Alonso ${ }^{1}$, M.A. Porras Moreno ${ }^{2}$, J. Ferrero Recasens ${ }^{1}$, V. Jimenez Diaz ${ }^{1}$, L. Garcia Lamas ${ }^{1}$ D. Cecilia Lopez ${ }^{1}$

${ }^{1}$ Cirugia Ortopedica Y Traumatologia, Hospital 12 de Octubre, Madrid/SPAIN, ${ }^{2}$ Cirugia Ortopedica, Hospital 12 de Octubre, Madrid/SPAIN

Introduction: Scaphoid fractures B1 or B2 according to Herbert's classification, could be treated whether with surgery or orthopedic treatment. The aim of the present study is to analyze results and complications of scaphoid waist fractures treated with percutaneous fixation using a volar approach.

Material and methods: 92 patients with scaphoid waist fractures, treated from 2006 to 2016 using a volar percutaneous fixation. The average follow-up was 16 months (range 12-48). Injuries were classified using Hebert's classification, including types B1 and B2; the rest of fracture types following this classification system were excluded. Politrauma patients, dorsal approach, fractures associated with distal radius injuries, patients treated using another surgical technique and patients transferred to other centers for treatment or follow-up were also excluded. Clinical and radiological assessments were performed at the outpatient clinics. Functional results were evaluated using the DASH questionnaire.

Results: The average time to fracture healing was 6.6 weeks (range 5-11). After 12 months of follow-up, the average wrist range of motion was $70^{\circ}$ of extension (range $58-80$ ) and $75^{\circ}$ of flexion (range 72-86). Regarding functional evaluation, the average score of The DASH questionnaire was 75 in patients that had associated injuries, decreasing to 42 in patients without it. Complications were present in $1.08 \%$ patients during the surgical intervention; $9.78 \%$ patients referred any kind of complication during follow-up. The average timing of surgical intervention was 20-25 minutes

Conclusion: Volar percutaneous fixation is a simple and quick technique for a specialized surgeon characterized by low morbidity and complications rates compared to ORIF, which accelerates patient's functional recovery.

References:

Disclosure: No significant relationships.

\section{PR446}

\section{APPLICATION OF BONE MARROW ASPIRATE CONCENTRATE - A PRELIMINARY DATA}

\section{Lalić}

Depertment For Orthopedic Surgery And Traumatology, Clinical center of Vojvodina, Novi Sad/SERBIA

Introduction: In the treatment of various orthopedic conditions, regenerative therapies recently have been advancing, including PRP and autologous stem cell therapy. Osteoarthritis of the knee is a debilitating disease which can lead to a painfull joint disfunction and fully exclusion of the patient from work and social life .

Material and methods: The purpose of this study was to evaluate preliminary clinical data of treatment of knee osteoarthritis (KL grade 2-4) with stem cells injection. For knee osteoarthritis treatment, 59 patients were included in this study. An average VAS pain pre-intervention decreased statistically significantly three days after intervention (from 7.27 to $2.12, \mathrm{p} \leq 0.05$ ) and persisting over time until one months follow up $=1.2$.

Results: Same results were achieved in average WOMAC score (preintervention $=51.5$; after 1 month $=72$ and after 3 months $=76$ and after 6 months $=79,8$ ).

Conclusion: This procedure was proved as safe providing pain relief and function improvement of treated knee joints.

References:

Disclosure: No significant relationships.

\section{PR447}

\section{EXTERNAL FIXATOR ASSISTED INTRAMEDULLARY NAILING OF THE TIBIA -MINIMALLY INVASIVE REDUCTION TECHNIQUE}

\section{S. Kitada, E. Shoda}

Trauma Center, Hyogo Prefectural Nishinomiya Hospital, Nishimomiya, Hyogo/JAPAN

Introduction: Tibial shaft fracture is often associated with severe soft tissue damage represented by the open fractures. In such cases, 
temporal external fixation is conducted to stabilize the soft tissue around the fracture area. We usually insert the half pins which are used in the intramedullary nailing afterward to maintain the reduction. The purpose of this study is to investigate the clinical results of the external fixator-assisted intramedullary nailing.

Material and methods: In initial surgery of external fixation, half pins are inserted in the posterior area of both proximal and distal fragment. The points of insertion are not on the way of the nailing. Malposition should be reduced as long as possible in initial surgery. Around two weeks later, fixation is converted to the intramedullary nail. The rods and connectors are removed before disinfecting the skin. The half pins remain inserted until the nailing is conducted. We experienced sixteen cases of tibial shaft open fracture which was treated using this technique. The outcomes are the installation period of the external fixation, the number of the bone healing, infection, soft tissue complication and reoperation.

Results: The mean duration of installation of the external fixator was 7.5 days (0-15 days). All cases were healed without infection. There were no revision surgeries due to the complications.

Conclusion: Intramedullary nailing using the external fixator to maintain the reposition is the safe and effective method to avoid the intraoperative damage to the soft tissue.

References:

Disclosure: No significant relationships.

\section{PR448}

\section{A SIMPLE YET EFFECTIVE WAY TO MANAGE COLD- WELD IN TITANIUM LOCKING COMPRESSION PLATES: A CASE REPORT}

\author{
T. Kootstra ${ }^{1}$, W. Bosman ${ }^{2}$, B. Emmink ${ }^{2}$
}

${ }^{1}$ Trauma Surgery, St. Antonius Hospital, Nieuwegein/ NETHERLANDS, ${ }^{2}$ Trauma Surgery, St Antonius Hospital, Nieuwegein/NETHERLANDS

Introduction: Locking compression plates (LCP) are a popular way to fixate long-bone fractures. However, it has been noted that locking screws sometimes become cold-welded to the plate, ${ }^{1}$ secondary to poor screwing technique (without adequate placement of the targeting device) or use of excessive force. ${ }^{2}$ Subsequent hardware removal can be time-consuming, and no single technique is uniformly successful. ${ }^{3}$ In case of a cold-weld, the titanium screws can be obliterated with a carbide drill. This leads to residual metal debris in the wound, subsequently leading to wound healing problems. The current case shows a combined technique to diminish contamination of the wound.

Material and methods: Removal of a titanium LCP distal femur plate was indicated in a 15 year old male, 3 month after fixation of a distal femur fracture. Removal of the proximal screws was unproblematic, but 3 distal screws were found to be cold-welded. A carbide drill was used to obliterate the screwheads. Before drilling, surgical lubricant was applied around the distal screws. Then, a $10 \mathrm{cc}$ plastic syringe was cut in half and the distal end of the syringe was placed over the drill-bit as a collection basket to minimize contamination of the wound by metallic debris.

Results: After all screwheads were obliterated, the wound area was checked under fluoroscopy for any remaining metallic debris. No fragments remained.

Conclusion: Difficulty with removal due to cold welding is common in LCP screws. We describe a simple yet effective way to intercept and remove all metal debris generated by obliterating titanium screws.
References: 1. Bae JH, Oh JK, Oh CW, Hur CR. Technical difficulties of removal of locking screw after locking compression plating. Arch Orthop Trauma Surg 2009;129 (1):91-5 2. Ehlinger M, Adam P, Simon P, Bonnomet F. Technical difficulties in hardware removal in titanium compression plates with locking screws. Orthop Traumatol Surg Res 2009;95 (5):373-6 3. Hak DJ, McElvany M. Removal of broken hardware. J Am Acad Orthop Surg 2008;16 (2):113-20

Disclosure: No significant relationships.

\section{PR449}

\section{ACUTE TREATMENT OF ACETABULAR FRACTURES}

\section{Kishimoto}

Orthopaedics, Nakakawachi Medical Center, Higashiosaka, Osaka/ JAPAN

Introduction: Objective: Among pelvic injuries, acetabular fractures are less commonly associated with vascular injuries and hemorrhagic shock, but in some cases hemodynamic resuscitation is required. The acute treatment of such cases of acetabular fracture is reported here. Material and methods: Since 2004, 56 cases of acetabular fractures were treated at our center; in 9 cases patients exhibited shock and were treated with TAE (T group), whereas the other 47 cases did not (non-T group). We analyzed the age, mechanism of injury and fracture type, using the Judet and Letournel classification, of each case. Results: The average age of the $\mathrm{T}$ group was 58.3 years of age (4 male, 5 female), and for the non- $\mathrm{T}$ group it was 46.8 years old (44 male, 3 female). The age difference between groups was found to be statistically insignificant. In both groups, traffic accidents were the most common mechanism of injury. The fracture types of the T group were 7 both-column, 1 anterior and 1 posterior column + posterior wall; in the non-T group 16 were both-column, 16 posterior wall, 4 posterior column + posterior wall, 3 transverse, 3 anterior column and the rest (3) were miscellaneous. The probability of TAE treatment was high in both-column fractures.

Conclusion: Even in acetabular fractures, the possibility of shock should be considered, especially in cases presenting with both-column fractures.

References:

Disclosure: No significant relationships.

\section{PR450}

\section{DOES THE MINI-PROXIMAL MEDIAL PARATENDINOUS APPROACH IN ITRAMEDULLARY TIBIAL NAILING LEADS TO LESS ANTERIOR KNEE PAIN?}

\author{
A. Dobbelaere ${ }^{1}$, G. Putzeys ${ }^{2}$
}

${ }^{1}$ Orthopaedics And Traumatology, Ghent University Hospital, ghent/ BELGIUM, ${ }^{2}$ Traumatology, AZ Groeninge Kortrijk, Kortrijk/ BELGIUM

Introduction: Anterior knee pain is a common complaint after intramedullary tibial nailing. Patients often experience a cumbersome pain while sitting on their knees. Different approaches exist for intramedullary tibial nailing: paratendinous, transtendinous, suprapatellar. Until now, no evidence exists that one of these approaches is superior to another regarding anterior knee pain. At our institution we 
use a mini-proximal medial paratendinous approach. The incision is located medial from the proximal third of the patellar tendon. The difference with a standard medial paratendinous approach is the incision that doesn't extend more distally than the proximal third of the patellar tendon. The part medial from the distal two thirds of the patellar tendon, is untouched. We believe that this part, is in contact with the ground, while sitting on the knees. The purpose of our study was to examine if there is a difference in anterior knee pain, particularly while sitting on the knees, between patients with a miniproximal medial paratendinous approach and patients with a standard medial paratendinous approach.

Material and methods: Two groups of 20 patients with a tibial diaphyseal fracture, treated with an intramedullary nail between 2012 and 2016, were selected. 20 patients with a mini-proximal medial paratendinous approach and 20 patients with a standard paratendinous approach. Knee function was assessed by the Tegner and Lysholm score. Anterior knee pain was assessed by a visual analogue scale (VAS) in general and particularly while sitting on the knees.

Results: Not available yet, collection of data in progress.

Conclusion: The etiology of anterior knee pain is undoubtedly multifactorial.

References: 1 . Court-Brown CM, Gustilo T, Shaw AD. Knee pain after intramedullary tibial nailing: its incidence, etiology, and outcome. J Orthop Trauma. 1997;11 (2):103-5. 2. Katsoulis E, CourtBrown C, Giannoudis PV. Incidence and aetiology of anterior knee pain after intramedullary nailing of the femur and tibia. J Bone Joint Surg Br. 2006;88 (5):576-80. 3. Obremskey W, Agel J, Archer K, To P, Tornetta P, 3rd. Character, Incidence, and Predictors of Knee Pain and Activity After Infrapatellar Intramedullary Nailing of an Isolated Tibia Fracture. J Orthop Trauma. 2016;30 (3):135-41. 4. Ahmad S, Ahmed A, Khan L, Javed S, Ahmed N, Aziz A. Comparative Analysis Of Anterior Knee Pain In Transpatellar And Medial Parapatellar
Tendon Approaches In Tibial Interlocking Nailing. J Ayub Med Coll Abbottabad. 2016;28 (4):694-7. 5. Brink O. Suprapatellar nailing of tibial fractures: surgical hints. Curr Orthop Pract. 2016;27 (1):107-12. 6. Jones M, Parry M, Whitehouse M, Mitchell S. Radiologic outcome and patient-reported function after intramedullary nailing: a comparison of the retropatellar and infrapatellar approach. J Orthop Trauma. 2014;28 (5):256-62. 7. Song SY, Chang HG, Byun JC, Kim TY. Anterior knee pain after tibial intramedullary nailing using a medial paratendinous approach. J Orthop Trauma. 2012;26 (3):172-7. 8. Toivanen JA, Vaisto O, Kannus P, Latvala K, Honkonen SE, Jarvinen MJ. Anterior knee pain after intramedullary nailing of fractures of the tibial shaft. A prospective, randomized study comparing two different nail-insertion techniques. J Bone Joint Surg Am. 2002;84-A (4):580-5. 9. Vaisto O, Toivanen J, Kannus P, Jarvinen M. Anterior knee pain after intramedullary nailing of fractures of the tibial shaft: an eight-year follow-up of a prospective, randomized study comparing two different nail-insertion techniques. J Trauma. 2008;64 (6):1511-6. 10. Weil YA, Gardner MJ, Boraiah S, Helfet DL, Lorich DG. Anterior knee pain following the lateral parapatellar approach for tibial nailing. Arch Orthop Trauma Surg. 2009;129 (6):773-7. 11. Soraganvi PC, Anand-Kumar BS, Rajagopalakrishnan R, Praveen-Kumar BA. Anterior Knee Pain after Tibial Intramedullary Nailing: Is it Predictable? Malays Orthop J. 2016;10 (2):16-20. 12. Tahririan MA, Ziaei E, Osanloo R. Significance of the position of the proximal tip of the tibial nail: An important factor related to anterior knee pain. Adv Biomed Res. 2014;3:119. 13. Zhang S, Wu X, Liu L, Wang C. Removal of interlocking intramedullary nail for relieve of knee pain after tibial fracture repair. J Orthop Surg (Hong Kong). 2017;25 (1):2309499016684748.

Disclosure: No significant relationships. 


\section{Case Study | Poster}

\section{VISCERAL TRAUMA CASES}

\section{PC001 \\ MULTIDISCIPLINARY APPROACH TO PELVIC TRAUMA IN A TERTIARY CARE CENTRE: A CASE REPORT}

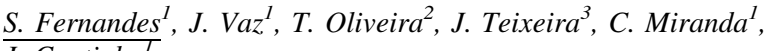 \\ J. Coutinho ${ }^{I}$
}

${ }^{1}$ General Surgery, Centro Hospitalar Lisboa Norte, Lisboa/ PORTUGAL, ${ }^{2}$ Urology, Centro Hospitalar Lisboa Norte, Lisboa/ PORTUGAL, ${ }^{3}$ Orthopedic Surgery, Centro Hospitalar Lisboa Norte, Lisboa/PORTUGAL

Case History: High-energy pelvic trauma often leads to devastating and potentially life-threatening multiorganic lesions. Adequate management may require the collaboration of several highlydifferentiated healthcare professionals. This case concerns a 56-yearold male with multisystemic injury resulting from a suicide attempted on the subway line.

Clinical Findings: Primary evaluation at the emergency room revealed airway patency and adequate ventilation, albeit the presence of right pneumothorax and multiple rib fractures. The patient was hemodynamically stable, despite the presence of an unstable pelvic ring fracture, with extensive deep perineal, scrotal and pelvic floor laceration, with anal sphincter and anterior mesorectum exposure. No neurologic compromise was identified.

Investigation/Results: Evaluation with computed tomography confirmed the right pneumothorax, multiple rib fractures, multiple vertebral fractures without spinal injury, complex and unstable pelvic fracture, with disruption of the pubic symphysis, left sacral wing fracture, bilateral acetabulum and ilio-ischiopubic branches fracture, pelvic floor disruption and complete urethral transection.

Diagnosis: Thoracic and complex pelvic trauma.

Therapy and Progression: The patient underwent multidisciplinary surgical approach: pelvic external fixation; left orchiectomy, reconstruction of proximal left cavernosum injury, direct vision urethral realignment, urethral reconstruction with end-to-end anastomosis, bladder drainage with suprapubic catheter; surgical debridement, pelvic floor reconstruction and left colostomy. Postoperative recovery was on the intensive care unit and general infirmary, with the close collaboration of a multidisciplinary team, including psychological support, physical rehabilitation and nutritional supply. The patient was discharged after two months and is currently autonomous, without urinary drainage and programmed for intestinal reconstruction and sexual rehabilitation.

Comments: A multidisciplinary team approach is of paramount importance to adequately manage multitrauma patients.

References:

Disclosure: No significant relationships.
PC002

\section{LIVER HERNIATION: A COMPLICATION OF THE RIGHT POST-TRAUMATIC DIAPHRAGMATIC HERNIA: CASE REPORT}

\author{
M. Velasquez ${ }^{I}$, A.M. Del Valle ${ }^{1}$, H.E. Munevar ${ }^{1}$, I.F. Cujiño ${ }^{l}$,

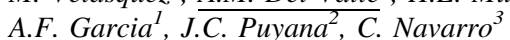

${ }^{1}$ Valle Del Cauca, Fundación Valle del Lili, Cali/COLOMBIA, ${ }^{2}$ Department Of Surgery, University of Pittsburgh Medical Center, Pittsburgh/PA/UNITED STATES OF AMERICA, ${ }^{3}$ Talca, Servicio de salud del Maule, Talca/CHILE

Case History: A 37-year-old male patient with fever, cough and hemoptysis, without dyspnea for two weeks. The only antecedent was a stab wound in the right thoracoabdominal region nine years ago.

Clinical Findings: Physical examination was normal.

Investigation/Results: The patient was evaluated by the thoracic surgeon, who requested complementary lab tests (Leukocytes: 5000, Hemoglobin: 16 g/L, Platelets: 273000, PT 9.8, PTT 25). A chest $\mathrm{x}$-ray showed a radiopaque image in the right lung base in contact with the thoracic wall and the diaphragm. Thorax CT- scan was requested, showed similar findings, being highly suggestive of a solitary fibrous tumor of the pleura.

Diagnosis: Video-Assisted Thoracoscopic Surgery performed through a single port, showed a $6 \mathrm{~cm}$ defect of the right hemidiaphragm $(6 \mathrm{~cm})$, without tumor lesion, with hepatic protrusion, partially strangled.

Therapy and Progression: The hernia defect was dissected with reduction of the liver to abdominal cavity. The hole was repaired with non-absorbable suture; a pleural drainage tube was placed. The postoperative course was uncomplicated. Drainage was removed at 24 hours after surgery. The patient was discharged on the third postoperative day. Ambulatory follow-up was performed on days 15 and 30 without recurrence.

Comments: Delayed presentation of right post-traumatic diaphragmatic hernia is rare and occurs after blunt trauma. Right diaphragmatic perforations after stab-wounds are diagnosed acutely by the signs of lesion of abdominal organs. Frequently, wounds of the right diaphragm are left untreated. The history of a stab-wound in the lower chest must awake the suspicious of a diaphragmatic hernia in patients with intestinal obstruction or thoracic mass.

References: 1. Eren S, Çiriş F. Diaphragmatic hernia: diagnostic approaches with review of the literature. Eur J Radiol. 2005;54 (3):448-459. doi:10.1016/j.ejrad.2004.09.008. 2-Lewis JD, Starnes SL, Pandalakai PK, et al. Traumatic diaphragmatic injury: experience from a level I trauma center. Surgery 2009;146:578 - 583; discussion 583-584. 3- Okan I, Baş G, Ziyade S, et al. Delayed presentation of posttraumatic diaphragmatic hernia. Ulus Travma Acil Cerrahi Derg. 2011;17 (5):435-439. http://www.ncbi.nlm.nih.gov/pubmed/ 22090330. Accessed July 10, 2017. 4- Peer SM, Devaraddeppa PM, Buggi S. Traumatic diaphragmatic hernia-our experience. Int J Surg. 2009;7 (6):547-549. doi:10.1016/j.ijsu.2009.09.003. 5- Kaur R, Prabhakar A, Kochhar S, Dalal U. Blunt traumatic diaphragmatic hernia: Pictorial review of CT signs. Indian $\mathrm{J}$ Radiol Imaging. 2015;25 (3):226-232. doi:10.4103/0971-3026.161433. 6-Starling, S. V., Rodrigues, B. D. L., Martins, M. P. R., Silva, M. S. A. D., \& Drumond, D. A. F. (2012). Non operative management of gunshot wounds on the right thoracoabdomen. Revista do Colegio Brasileiro de Cirurgioes, 39 (4), 286-294. 7-Peker, Y., Tatar, F., Kahya, M. C., Cin, N., Derici, H., \& Reyhan, E. (2007). Dislocation of three segments of the liver due to hernia of the right diaphragm. Hernia, 11 (1), 63-65.

Disclosure: No significant relationships. 
PC003

\section{RHINOCEROS-RELATED TRAUMATIC DIAPHRAGMATIC HERNIA}

M. Sebastian ${ }^{1}$, A. Abdulla ${ }^{1}$, M.I. Abusharia ${ }^{1}, \underline{\text { F.M. Abu-Zidan }}{ }^{2}$

${ }^{1}$ Department Of Surgery, Al-Ain Hospital, Al-Ain/UNITED ARAB EMIRATES, ${ }^{2}$ Surgery, College of Medicine, UAEU University, AlAin/UNITED ARAB EMIRATES

Case History: A 47-year-old lady presented with abdominal pain, bilious vomiting and obstipation of one day duration. She had recurrent attacks of colicky abdominal pain for a week before that. The patient gave history of being admitted to the ICU 3 years before after being gored by a rhinoceros into her chest while working as a veterinary assistant in the zoo.

Clinical Findings: On examination, the abdomen was distended but soft and lax. Bowel sounds were exaggerated.

Investigation/Results: Abdominal $\mathrm{x}$-rays showed multiple air fluid levels. A gastrographin follow through study hold up in the small bowel and did not reach the colon after 7 hours. Abdominal and chest CT scan showed the splenic colonic flexure to be located in the left chest.

Diagnosis: Traumatic left diaphragmatic hernia

Therapy and Progression: Urgent laparotomy showed s healthy splenic flexure of the colon that herniated through a $4 \mathrm{~cm}$ posterolateral defect in the left diaphragm. The colon was reduced and the defect was repaired with non absorbable sutures. Postoperative recovery was smooth. The patient was discharged home 10 days after surgery.

Comments: Animal-related injuries should be analyzed based on the behavior and anatomy of the attacking animal. Rhinoceros-related injuries in humans are extremely rare. Life threatening traumatic diaphragmatic herniation may be delayed for few years. High index of suspicion is needed for its diagnosis.

Disclosure: No significant relationships.

\section{PC004}

\section{LATE PRESENTATION OF TRAUMATIC RUPTURE OF AORTIC VALVE FOLLOWING A MOTORCYCLE ACCIDENT}

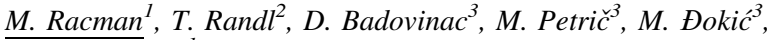 \\ T. Klokočovnik
}

${ }^{1}$ Department Of Cardiovascular Surgery, University Medical Centre of Ljubljana, Ljubljana/SLOVENIA, ${ }^{2}$ Department Of Traumatology, University Medical Centre of Ljubljana, Ljubljana/SLOVENIA, ${ }^{3}$ Department Of Abdominal Surgery, University Medical Centre Ljubljana, Ljubljana/SLOVENIA

Case History: A 45 year old male patient suffered a motorcycle accident. He sustained severe injuries to the thorax, abdomen, spine and facial cranium. Urgent splenectomy was performed. The course of treatment was complicated by a pulmonary embolism and pneumonia. Embolus originated from a lower extremity. Vena cava filter was inserted. Longitudinal osteofixation of the spine was performed later.

Clinical Findings: Two weeks after the accident he patient developed aortic systolic and diastolic murmur.
Investigation/Results: TTE revealed moderate to severe aortic regurgitation.

TEE confirmed perforation of the left coronary cusp of aortic valve with severe aortic insufficiency.

CTA showed no signs of aortic dissection.

Diagnosis: Isolated perforation of left coronary cusp with regards to the rest of the heart and aorta was confirmed.

Therapy and Progression: Operative treatment was required. A mini L- shaped sternotomy was performed. Complete detachment of the left coronary cusp from the aortic annulus was found. Due to the extent of injuries valvuloplasty was not possible. Aortic valve replacement was performed with a mechanical valve size $27 \mathrm{~mm}$. Postoperative period regarding the cardiovascular system was uneventful.

Comments: Traumatic coronary cusp perforation is seldom seen but nevertheless an important cause of aortic valve insufficiency. It is assumed that if blunt force chest trauma occurs during ventricular diastole, the rise of intraaortic pressure against a closed aortic valve may cause rupture or detachment of one or more cusps. The non coronary cusp is most frequently transformed. The traumatic tear in the cusp progresses over time which explains the late presentation in our case.

References: 1. Mehta, S., G. Wilson, K. Suarez and C. D. Chiles (2017). "Aortic valve perforation secondary to blunt force trauma." Proc (Bayl Univ Med Cent) 30 (3): 305-306. 2. Maini, R., R. T. Dadu, D. Addison, L. Cunningham, I. Hamzeh, M. Wall, Jr., N. Lakkis and R. Tabbaa (2015). "Isolated perforation of left coronary cusp after blunt chest trauma." Case Rep Cardiol 2015: 235629. 3. Driessen, R., I. Doodeman, K. Bogaard and S. Reichert (2014). "Contusio cordis, not an innocent diagnosis.” BMJ Case Rep 2014. 4. Li, W., Y. Ni, X. Chen and L. Ma (2011). "Aortic valve tear with severe aortic regurgitation following blunt chest trauma." J Cardiothorac Surg 6: 84.

Disclosure: No significant relationships.

\section{PC005}

\section{FALSE NEGATIVE PERICARDIAL FAST IN A HOLLOW- POINT CARDIAC GUNSHOT WOUND}

G. Medrano, K. Mahendraraj, T. Katsichtis, E. Irizarry, M.E. Lueders

General Surgery, Lincoln Medical and Mental Health Center, Bronx/ NY/UNITED STATES OF AMERICA

Case History: 19 year old man brought to the emergency room after sustaining a thoracoabdominal hollow point bullet gunshot wound.

Clinical Findings: The patient was hemodynamically unstable, in repiratory distress with diminished right breath sounds.

Investigation/Results: Chest $\mathrm{x}$-ray revealed right hemothorax. FAST showed fluid in Morrison's pouch without hemopericardium.

Diagnosis: A right tube thoracostomy yielded $600 \mathrm{cc}$ of blood. Laparotomy revealed a lacerated inferior mesenteric vein, a left hepatic lobe laceration, and a non-expanding left perinephric hematoma. Despite achievement of intraabdoinal hemostasis the patient continued to be hemodynamically unstable raising suspicion of an occult cardiac injury. A median sternotomy was performed and through and through right atrial injuries were discovered and promptly repaired. Blood from the cardiac injury was draining into the right hemithorax. Bulging left hemidiaphragm prompted left lateral thoracotomy. Hemiazygos vein was ligated and diaphragmatic recess was packed to control hemorrhage. 
Therapy and Progression: The patient underwent angioembolization of left first lumbar artery. An Echocardiogram ruled out valvular injuries. Esophagogastroduodenoscopy demonstrated hemorrhagic contusion of the distal esophagus which was managed non-operatively. Tracheostomy and transpyloric Moss feeding tube were placed. Patient had a complete recovery and was discharged home.

Comments: Major vascular and organ injuries from a single hollowpoint-bullet trajectory can be unpredictable, complicating management. Persistent hemodynamic instability in patients with penetrating chest trauma and hemothorax with negative pericardial FAST should raise suspicion for occult cardiac injury. In this case the absence of signs and symptoms of tamponade occured because the right atrium emptied directly into the right chest. Angioembolization can be useful in managing inaccessible vascular injuries.

References: 1. Fackler ML, Sunnchak JS, Malinowski JA, Bowen RE. Bullet fragmentation: a major cause of tissue disruption. J Trauma 1984;24:35-39 2. Fackler ML. Wound ballistics: a review of common misconceptions. JAMA 1988;259:2730-2736 3. Wang ZG, Feng $\mathrm{J}_{1}, \mathrm{Uu}$ YQ. Pathomorphological observations of gunshot wounds. Acta Chir Scand Suppi 1982;508: 185-195 4.- Bowen TE, Bellamy RF. Emergency war surgery: second United States revision of the emergency war surgery NATO handbook, 2nd ed. Washington, DC: United States Department of Defense, United States Government Printing Office, 1988:13-34, 230-238 5.- Harvey EN, KorrIM, Oster G, McMillen JH. Secondary damage in wounding due to pressure changes accompanying the passage of high velocity missiles. Surgery 1947;21 :218-239 6.- Baker, Laura, Ammar Almadani, and Chad G. Ball. \&quot;False negative pericardial Focused Assessment with Sonography for Trauma examination following cardiac rupture from blunt thoracic trauma: a case report.\&quot; Journal of medical case reports 9.1 (2015): 155. 7.- Ball, Chad G., et al. \&quot;A caveat to the performance of pericardial ultrasound in patients with penetrating cardiac wounds. \&quot; Journal of Trauma and Acute Care Surgery 67.5 (2009): 1123-1124. 8.- Udobi, Kahdi F., et al. \&quot;Role of ultrasonography in penetrating abdominal trauma: a prospective clinical study.\&quot; Journal of Trauma and Acute Care Surgery 50.3 (2001): 475-479. 9.- Rozycki, Grace S., et al. \&quot; The role of surgeon-performed ultrasound in patients with possible cardiac wounds.\&quot; Annals of surgery 223.6 (1996): 737.

Disclosure: No significant relationships.

\section{PC006}

\section{HAEMOTHORAX IN SEVERE CHEST INJURIES - WHEN TO DO THORACOSCOPIC LAVAGE ? REPORT OF A CASE}

\section{I.C. Pentara ${ }^{1}$, G. Konstantoudakis ${ }^{\text {I }}$, G. Skazas ${ }^{2}$, I. Massalis ${ }^{1}$, G. Eythymiou ${ }^{1}$, P. Gkanas ${ }^{I}$}

\section{${ }^{1}$ Surgery Department, Nafplio General Hospital, Nafplio/GREECE, ${ }^{2}$ Aneasthesiologist Department, Nafplio General Hospital, Nafplio/ GREECE}

Case History: We report a case of a 45 year-old female patient with severe chest injury (Organ Injury Scaling Grade II) and haemothorax, due to fall from height, we managed with thoracoscopic lavage.
Clinical Findings: The patient suffered severe thoracic pain and shortness of breath. She had diminished air entry to the left and the saturation was $93 \%$. She had multiple bruises to the left chest wall. Investigation/Results: Initial imaging included chest $\mathrm{x}$-ray and E-FAST. Chest CT was done later, on grounds of clinical deterioration. The patient had nine fractured ribs, without a flail segment. Initially the was minimal fluid accumulation in pleural space.

Diagnosis: Multiple rib fractures (5th-11th) with haemothorax observed the next day .

Therapy and Progression: Two ICD were inserted, because of inadequate drainage and eventually thoracoscopic washout was done the 5th day because of retained haemothorax. Intercostal nerve block was done twice during hospitalization and was repeated a week after discharge. No intubation was needed despite the worsening of respiratory function due to pneumonia, which was treated with respiratory exercise and early mobilization. There were no concomitant abdominal injuries. The patient was discharged the 18th day after admission.

Comments: Thoracoscopic lavage is needed in retained haemothorax, to avoid extensive atelectasis, possible empyema and fibrotic changes in pleural cavity. It must be performed in the first 5 to 7 days post injury, because later it becomes organized and therefore difficult to drain. Intercostal nerve block is helpful for early mobilization and subsequently for prevention of complications and favorable outcome. References: 1) Feliciano, David V.; Mattox, Kenneth L.; Moore, Ernest J (2012). Trauma, Seventh Edition (Trauma (Moore)). McGraw-Hill Professional. 2) Yi-Pin Chou, Hsing-Lin Lin and TzuChin Wu. Video-assisted thoracoscopic surgery for retainedhemothorax in blunt chest trauma. Curr Opin Pulm Med 2015 Jul; 21 (4):393398 3) R.T. Villavicencio, J.A. Aucar, M.J. Wall, Jr. Analysis of Thoracoscopy in trauma. Surg Endosc 1999;13:3-9 4) Joseph DuBose, MD, Kenji Inaba, MD, DemetriosDemetriades, MD, PhD, Thomas M. Scalea, MD, James O'Connor, MD, Jay Menaker, MD, Carlos Morales, MD, Agathoklis Konstantinidis, MD, Anthony Shiflett, MD, Ben Copwood, MD, and the AAST Retained Hemothorax Study Group Management of post-traumatic retained hemothorax: A prospective, observational, multicenter AAST study

Disclosure: No significant relationships.

\section{PC007}

\section{LATENT POST-TRAUMATIC DIAPHRAGMATIC HERNIA, A CHALLENGE FOR THE GENERAL SURGEON}

$\frac{\text { E. Migliori }^{1}, \text { E. Mammano }^{2}, \text { E. Tessari }^{2}, \text { L. Pomba }^{2}, \text { G. Mattara }^{2}}{}$,

${ }^{1}$ General Surgery Unit, San Antonio General Hospital, Padova/ ITALY, ${ }^{2}$ Surgery Unit, San Antonio Hospital, Padova/ITALY

Case History: Post-traumatic diaphragmatic hernia is a pathological condition resulting from a closed or penetrating trauma rarely diagnosed, however, often cause of life threatening. It may present as an acute form or stay latent. Latent cases remain misdiagnosed at the time of primary assessment especially because they are too small to be diagnosed with specific radiological investigations. Patient management requires significant multidisciplinary clinical support for nomalize vital parameters. Surgical management is never simple due 
to the function and fragility of the diaphragm tissue. Recurrence risk is high in fact today the use of prosthetic materials with reconstructive intent has allowed to achieve good long-term results

Clinical Findings: We present the clinical case of a 65 year old man assessed in ER for the onset of abdominal pain, diaphoresis and sweating with history of road trauma 5 years before.

Investigation/Results: Diagnostic examination showed extensive right thoracic herniation of small bowel from previous diaphragm rupture.

Diagnosis: Diaphragmatic hernation

Therapy and Progression: Initially threated for medical support the patient underwent operative management with intraoperative finding of a $6 \mathrm{~cm}$ diaphragmatic breach with intestinal laceration of small bowel so we pwerformed an intestinal resection and a direct repair of the diaphragmatic breach. No post-operative complications. Patient was resigned in the $\mathrm{V}$ post-op day nowaday in regulare follow-up Comments: traumatic rupture of the diaphragm still represents a challenge for the general surgeon, especially in the latent form, because it can cause serious complications. The surgical correction must aim at repairing the defect and avoiding recurrence by simultaneously restoring the proper functioning of the diaphragmatic muscle.

References: - 'Recurrence in a laparoscopically repaired traumatic diaphragmatic Hernia: case report and literature review' Nikita R. Bhattet al. Trauma Montlhy2016 Feb; 21 (1): e20421

-'Diaphragmatic rupture: is management with biological mesh feasible?' O.Al-Nouriet al. IJSCR (2012) 349-353

-'Dual mesh repair for a large diaphragmatic hernia defect: an unusual case report' M.Ercanet al IJSCR 28 (2016) 266-269

- The role of computed tomography in the diagnostics of diaphragmatic injury after blunt thoraco-abdominal trauma' A.Gmachowskaet al, PolJ Radiol, 2016; 81: 522-528

Disclosure: No significant relationships.

\section{PC008}

\section{THORACIC TRAUMA WITH RIB FRACTURE DISPLACEMENT: CLINICAL CASE}

R. Lourenco, J. Costa, M. Martins, T.S. Neto, A.C. Carvalho, D. Brito, R. Pinto, J. Oliveira, P. Correia

General Surgery, Hospital Senhora da Oliveira, Guimarães/ PORTUGAL

Case History: Blunt thoracic trauma is an entity very prevalent, counting for $10 \%$ or more trauma cases. Rib fractures are between the most frequent lesions associated to blunt thoracic trauma and can be complicated with pneumothorax, hemothorax or pulmonary contusion. The authors relate a case of a 83-year-old man presented with left thoracic trauma after falling from bed, denying other traumas.

Clinical Findings: Physical examination revealed normal blood pressure, heart rate and saturation level. Thoracic palpation evidenced extensive subcutaneous emphysema in left thoracic wall and auscultation revealed decreased ventricular murmur essentially at left hemithorax.

Investigation/Results: Thorax-CT showed fracture of the fifth to eighth ribs, with multifracture of the sixth and seventh coastal arcs. Fragments of the sixth rib were displaced into pulmonary parenchymal space. Furthermore, it demonstrated a left hemopneumothorax and extensive subcutaneous emphysema.

Diagnosis: We were in the presence of a thoracic trauma with surgical indication. The patient was transferred to cardiothoracic service.
Therapy and Progression: The patient was submitted to left anterolateral thoracotomy, removal of rib fragments and thoracic wall reconstruction. The postoperative period complicated with nosocomial pneumonia requiring non-invasive ventilation. In the remaining hospital stay, the patient presented a good clinical evolution, which led to his discharge after four weeks.

Comments: Patients with closed thoracic trauma associated with rib fractures present considerable mortality and morbidity. The age of the patients and the number of fractures have a direct impact on the prognosis. Surgical treatment is required in less than $10 \%$ of cases. References: Bemelman M, Mark van Baal, Rib Fractures: To Fix or Not to Fix? An Evidence-Based Algorithm. Korean J Thorac Cardiovasc Surg 2017;50:229-234 Talbot B.S., Gange C., Chaturvedi A., Klionsky N., Hobbs S. K., Chaturvedi A., Traumatic Rib Injury: Patterns, Imaging Pitfalls, Complications, and Treatment. RadioGraphics 2017; 37:0000-0000

Disclosure: No significant relationships.

\section{PC009}

\section{DIAPHRAGM RUPTURE AFTER BLUNT CHEST TRAUMA - A CASE REPORT}

X.P. De Sousa, P.S. Ferreira, L. Branco, V. Rigueira, L. Cortez

General Surgery, Hospital São Bernardo, Centro Hospitalar de Setúbal, EPE, Setúbal/PORTUGAL

Case History: We report a case of a 57-year-old male patient who suffered a blunt trauma to the chest by a falling tree, with no evidence of direct trauma to the head or abdomen.

Clinical Findings: At admission GCS 15 and hemodynamically stable with normal peripheral saturation. He complained of chest pain and had dyspnea. A non-penetrating wound on the left hemithorax was present, with pain and crepitation on palpation to chest, dorsal spine and scapula. Chest auscultation showed no alterations.

Investigation/Results: Blood tests were normal and chest $\mathrm{x}$-ray showed no image suggesting hemo/pneumothorax or rib fractures. Chest CT showed small hemo/pneumothorax on the left hemithorax with discrete subcutaneous emphysema, fracture of the 6th and 7th left ribs, areas of pulmonary contusion and herniation of intra-abdominal fat associated to a left hemidiaphragmatic rupture.

Diagnosis: Diaphragm rupture after blunt chest trauma.

Therapy and Progression: Prompt surgery was proposed. An abdominal approach was chosen, after thorough exploration no internal organ injury was detected besides the $3 \mathrm{~cm}$ rupture to the left hemidiaphragm. The diaphragmatic laceration was sutured and chest tube was inserted. The patient was discharged after 10 days.

Comments: Traumatic diaphragmatic injury is usually occult and can easily be missed. Accurate diagnosis is challenging and should be kept in mind in all patients with chest injuries. Treatment should not be delayed because severe complications may occur, such as respiratory dysfunction or incarceration of herniated abdominal content. Transabdominal approach is the most appropriate because it makes simultaneous abdominal injuries easier to treat. Early stage treatment will reduce mortality and improve prognosis.

References: 1 - Sankalp Dwivedi, et al., Treating traumatic injuries of the diaphragm. J Emerg Trauma Shock. 2010 Apr-Jun; 3 (2): 173-176. 2 - Lee WC, et al., Rupture of the diaphragm after blunt trauma. Eur J Surg. 1994 Sep;160 (9):479-83. 3 - Matsevych OY, Blunt diaphragmatic rupture: four year's experience. Hernia. 2008 Feb;12 (1):73-8.

Disclosure: No significant relationships. 


\section{PC010}

\section{OPEN PELVIC RING INJURY WITH RECTUM AVULSION}

\author{
M. Jakopec ${ }^{1}$, A. Kristan ${ }^{2}$
}

${ }^{1}$ Department Of Abdominal Surgery, University Medical centre Ljubljana, Ljubljana/SLOVENIA, ${ }^{2}$ Department Of Traumatology, University Medical centre Ljubljana, Ljubljana/SLOVENIA

Case History: A 41 year male was admitted following a high-speed motorcycle accident.

Clinical Findings: There was a laceration reaching from the left inguinal crease, through the perineum, just past the anus.

Investigation/Results: Imaging revealed a complex pelvic fracture with a dislocation of rami pubis and a complete symphysiolysis, diastasis of the left SI joint and a comminutive fracture of the sacrum. Diagnosis: We placed a supraacetabular external fixator, an osteosynthesis of the symphysis and an SI screw. He was examined by the abdominal surgeon who didn't find any damage to the bowel and decided against stoma formation. An urethral rupture and an avulsion of a corpus cavernosum was found.

Therapy and Progression: The patient was transferred to the ICU, with the perineal wound healing per primam. He was revised on day 9 , because of phlegmon of the perineum. Intraoperatively, we found an intact rectum that has been pulled proximally by the pelvic floor muscles and was contaminating the wound. We laparoscopically formed a stoma, but because of the contamination, VAC had to placed and changed several times. After suturing the wound on day 19, we noticed a purulent discharge and the patient became septic. Several subsequent surgical debridements and daily dressing changes were performed. He needed long term IV antibiotics.

Comments: A stoma was only formed on day 9, after the wound was already severely contaminated by faecal matter. Had the stoma beed placed during the primary operation, the patient would have been spared from several surgical revisions, significant pain and long term antibiotics.

References:

Disclosure: No significant relationships.

\section{PC011}

\section{OPEN THORACIC TRAUMA - A CASE REPORT}

E. Dimitrakopoulou ${ }^{I}$, C. Farazi-Chongouki ${ }^{1}$, C. Iordanou ${ }^{2}$, A. Ninos ${ }^{3}$, M. Vidali ${ }^{2}$, G. Papaioannou ${ }^{2}$, G. Doulgerakis ${ }^{2}$, T. Vathia ${ }^{2}$, K. Mihopoulou ${ }^{2}$, C. Pagaki ${ }^{2}$, A. Stylianou ${ }^{2}$, M. Karamanidi ${ }^{3}$, S. Pierrakakis ${ }^{3}$

${ }^{1}$ Surgery, Thriasio General Hospital of Eleusina, Eleusina/GREECE, ${ }^{2}$ Surgery, GENERAL HOSPITAL OF ELEFSINA THRIASIO, MAGOULA/GREECE, ${ }^{3}$ Surgery, Thriassio Hospital, Athens/ GREECE

Case History: Thoracic injuries account for $20-25 \%$ of deaths due to trauma. Although most chest wall injuries can be managed conservatively or by simple techniques such as tube thoracostomy, the management of open thoracic traumas could be more demanding.

Clinical Findings: A 24 year-old male was brought to our emergency department due to self-injury by a shotgun. There was a $12 \mathrm{~cm}$ open left thoracic trauma.
Investigation/Results: An urgent operative exploration was decided, intraoperative findings included multiple penetrating lung injuries and a total destruction of 6th and 7th intercostal space. The lower bronchopulmonary part of the left upper lobe was resected due to severe air leakage.

Diagnosis: Further exploration revealed small left diaphragmatic perforations and a laparotomy was performed. Intrabdominal findings included multiple bullets, serosal injuries of transverse colon and a small non-expanding mesocolon hematoma.

Therapy and Progression: The patient made a smooth recovery and was discharged in a good condition.

Comments: Traumatic diaphragmatic injuries can often be overlooked due to other severe injuries associated with polytrauma. Although only $3-5 \%$ of patients with traumatic rupture of the diaphragm require laparotomy, a gunshot injury involving the diaphragm requires further exploration.

References: 1. Kuhajda I, Zarogoulidis K, Kougioumtzi I, et al. J Thorac Dis. 2014 Oct;6 (Suppl 4):S461-5. doi: 10.3978/j.issn.20721439.2014.08.51. Review 2. Christofer R. Morse, James S. Alan. The Trauma Handbook of Massachussets General Hospital 2004; Chest Trauma 1, 296-313. 3. Treating traumatic injuries of the diaphragm Sankalp Dwivedi, Pankaj Banode, Pankaj Gharde, Manisha Bhatt, and Sudhakar Ratanlal Johrapurkar1, J Emerg Trauma Shock. 2010 AprJun; 3 (2): 173-176. 4. emedicine.medscape.com

Disclosure: No significant relationships.

\section{PC012}

\section{MASSIVE HEMOTHORAX AND INTRAPERITONEAL HEMORRHAGE DUE TO INFERIOR PHRENIC ARTERY INJURY AFTER BLUNT TRAUMA}

\author{
T. Nakano, T. Ito
}

Department Of Emergency, Shinyurigaoka General Hospital, Kawasaki/JAPAN

Case History: A 91 years-old male fell down indoors thought to be minor trauma was transferred to the emergency department, computed tomography scanning revealed active extravasations of the contrast medium within the intrathoracic and intraperitoneal space. The patient was getting in a shock state, and thoracic drainage was inserted due to suspicion of tension hemothorax from CT findings. However, a sufficient increase in blood pressure was not obtained, and the amount of bleeding from the drain exceeded $1000 \mathrm{~mL}$.

Clinical Findings: Based on the findings of enhanced CT, transcatheter arterial embolization (TAE) was performed considering simultaneous hemorrhage in the thoracic space and intraperitoneal space accompanying damage of the inferior phrenic artery alone.

Investigation/Results: The injured right inferior phrenic artery was successfully embolized with a finely ground gelatin sponge, resulting in stabilization of the patient's clinical condition.

Diagnosis: Simultaneous bleeding from inferior phrenic artery into the thoracic and intraperitoneal space.

Therapy and Progression: He was discharged without complications after rehabilitation on the 39th days.

Comments: Injury to the inferior phrenic artery after blunt trauma is an extremely rare event. This case describes an injury to the right inferior phrenic artery caused by blunt trauma, which was complicated by massive hemothorax and intraperitoneal hemorrhage concurrently, and treated with TAE. TAE represents a safe and effective treatment for this complication. 
References: Yamanashi, Keiji, et al. "A case of delayed hemothorax with an inferior phrenic artery injury detected and treated endovascularly." Clinical case reports 3.7 (2015): 660-663. Aoki, Makoto, et al. "Massive hemothorax due to inferior phrenic artery injury after blunt trauma." World Journal of Emergency Surgery10.1 (2015): 58. Disclosure: No significant relationships.

\section{PC013}

\section{TRAUMATIC RUPTURE OF THE DIAPHRAGM: A CASE REPORT}

N. Guimarães, I. Mónica, M.M. Ferreira, H. Ribeiro, J. Neves, J. Pinho, H. Morais, F. Melo, V. Vieira, N. Azenha, I. Borges, R. Dias, A. Fonseca, J. Cecílio, A. Matos, L. Conceição

General Surgery, Hospital Distrital da Figueira da Foz, Figueira da Foz/PORTUGAL

Case History: Traumatic rupture of the diaphragm (TRD) occurs due to blunt or perforating thoraco-abdominal trauma, with an incidence of $0.8-8 \%$. TRD occur more frequently on the left side, in approximately $75 \%$ of the cases. This report outlines a clinical case of TRD requiring urgent surgical management. We review the pertinent literature, focusing mainly on diagnosis and surgical treatment. We present the case of a 63-year-old male, who was attacked by a cow, resulting in head and thoracoabdominal trauma.

Clinical Findings: On the primary survey the patient was hemodynamically unstable, revealing abdominal tenderness and a transverse abrasion of the abdominal wall.

Investigation/Results: The adjuncts of primary survey revealed hemoperitoneum and multiple rib fractures bilaterally.

Diagnosis: Traumatic rupture of the diaphragm

Therapy and Progression: An exploratory laparotomy was performed: an hemoperitoneum and left diaphragmatic rupture were found. A diaphragmatic suture repair and left thoracic drainage were done. The postoperative period was uneventful.

Comments: Often, the TRD diagnosis goes unnoticed, since the clinic is nonspecific and acute complications are rare, being identified later, due to the development of diaphragmatic hernias. Although rare, acute complications of TRD can jeopardise patient's life imposing urgent intervention.

References:

Disclosure: No significant relationships.

\section{PC014}

DELAYED PRESENTATION AND LAPAROSCOPIC REPAIR OF A TRIPLE POST-TRAUMATIC DIAPHRAGMATIC INJURY $\frac{\text { S. Bozzo, G. Carrara, M. Andretta, R. Pirovano, F. Chiappetta, }}{\text { M. Zago }}$

Chirurgia Generale, POLICLINICO SAN PIETRO, Ponte san pietro/ ITALY
Case History: A 45 years old man was admitted to the ED in shock and severe respiratory distress. One month earlier, he had a motorcycle accident and suffered multiple left ribs fractures, mild hemothorax and an amyelic fracture of the body of D10.

Clinical Findings: At re-admission, the chest X-ray showed the dislocation of the gastric fundus in the left pleural space, hemopneumothorax and dislocation of the mediastinum. A chest tube obtained partial recovery of hemodynamics and respiratory distress. Investigation/Results: A thoracic CT confirmed a large diaphragmatic tear with more than $2 / 3$ of the stomach in the left hemi-thorax. Diagnosis: The diagnosis of delayed post-traumatic diaphragmatic hernia was made.

Therapy and Progression: A 5 ports laparoscopic approach allowed to confirm diagnosis, obtain reduction of the herniated stomach, and the repair of three unexpected radial diaphragmatic tears. Reduction of the strangulated stomach required a pre-cut near the upper border of the larger laceration. Repair of muscular tears was performed with running sutures of nonabsorbable monofilament and of absorbable barbed thread. A video of the procedure highlights technical details. Comments: Laparoscopy is a recognized option for the detection and repair of diaphragmatic post-traumatic injuries. Its use is mandatory for ruling out diaphragmatic post-traumatic injuries in case of thoracoabdominal penetrating trauma. Laparoscopic approach for diaphragmatic injuries is challenging but could be affordable even facing with strangulated hernias or multiple lacerations.

References: J Emerg Trauma Shock. 2010 Apr-Jun; 3 (2): 173-176. Treating traumatic injuries of the diaphragm. Sankalp Dwivedi et al. Disclosure: No significant relationships.

\section{PC015}

\section{SPONTANEOUS RUPTURE OF LIVER HEMATOMA RELATED WITH INCOMPLETE HELLP SYNDROME AT TERM PREGNANCY}

S. Montón, A. Rico, J.L. Moya, I. Poveda, B. Arín, M. Cires

Facultad De Medicina, Hospital García Orcoyen, Estella (Navarra)/ SPAIN

Case History: This is a 32 years old term pregnant who arrived to our Hospital because of a rupture of membranes at term.

Clinical Findings: While we were doing the basic scanning the patient referred a strong epigastric pain, nausea and vomiting. At that moment she had a low blood pressure (140-90) and normal both blood and urine analysis.

Investigation/Results: During an ultrasound examination we found a hematoma beneath Glisson's capsule. In addition the Fetal Monitoring showed us a Cathegory II Tracing.

Diagnosis: Hemoperritoneoum cause for the hepatic cápsula rupture in a term pregnant pacient related with hellp syndrome.

Therapy and Progression: We made an emergency caesarean where we found an important hemoperitoneum because of the rupture of liver hematoma. The treatment was a liver packing by the general surgeon. While we were doing the caesarean the new blood analysis showed a modestly aminotransferases elevation (GPT 255 and GOT 194), low platelet count (84.000) and hemolysis. The result of the 
caesarean was a newborn with Apgar 4-7-10 and arterial cordon blood of 6.87 an venous of 6.99 (nowadays is a healthy child). The pregnant woman was diagnosed as a HELLP Syndrome. She required Intensive Care at the Hospital during 48 hours. During the hospital stay she was given intravenous magnesium sulphate and was support with several blood transfusion, but no antihypertensive drugs were needed. The patient had a good outcome and she was discharged from the hospital, 6 days after the surgery.

Comments: The spontaneous rupture of a liver hematoma is an unusual complication of a HELLP Syndrome. This fact is associated with a high maternal and fetus morbility and even mortality in several reports. So if it takes place, after the diagnosis is confirmed, the multidisciplinary early management is required for a successful outcome.

References:

Disclosure: No significant relationships.

\section{PC016}

\section{TRAUMATIC ABDOMINAL WALL HERNIA AFTER AN EQUESTRIAN ACCIDENT}

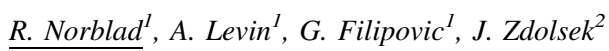

${ }^{1}$ Dept Of Surgery And Dept Of Clinical And Experimental Medicine, Linköping University, Unit for Emergency Surgery and Trauma, Linköping/SWEDEN, ${ }^{2}$ Department Of Hand Surgery, Plastic Surgery And Burns, University Hospital, Plastic Surgery, Linköping/ SWEDEN

Case History: A 42 year old female equestrian was thrown off her horse riding. The patient came to the emergency room due to pain in her right thorax and abdomen, including a sensation of shortness of breath. She had a blurred memory of the accident.

Clinical Findings: The patient had slightly diminished breath sound on both sides, pain at palpation over thorax and at the iliac crest and hip on the right side. No obvious lump or hematoma was detected during primary survey.

Investigation/Results: A Trauma CT from head to pelvis revealed multiple costal fractures on the right side including a minor pneumothorax and lungcontusions, no intraabdominal injuries could be detected. A traumatic abdominal wall hernia (TAWH) at the right iliac crest was present on the abdominal CT scan.

Diagnosis: At exploration, a TAWH grade 5 (complete abdominal wall rupture with herniation) was found at the right iliac crest, with herniation of abdominal content through a defect measuring $75 \mathrm{~mm}$.

Therapy and Progression: Surgery was planned for the second day after admission. A primary repair was done, with interrupted sutures through drill holes in the iliac crest anchoring the oblique muscle layers, thus restoring the continuousness of the abdominal wall. A follow up CT scan after 9 weeks showed god adaptation and no hernia present. Clinical control after 2 months revealed no signs of herniation or asymmetric flanks. The patient was in full health and had taken up horse riding again.

Comments: There is no clear consensus on either diagnosis or treatment algorithm for Traumatic Abdominal Wall Hernia (TAWH). References: Dennis RW, Marshal A, Deshmukh H et al (2009) Abdominal wall injuries occurring after blunt trauma: incidence and grading system. Am J Surg 197:413-417 Pardhan A, Mazahir S, Rao S, Weber D. Blunt traumatic abdominal wall hernias: a surgeon's dilemma. World J Surg 2016;40:231-5 Netto FACS, Hamilton P, Rizoli SB et al (2006) Traumatic abdominal wall hernia: epidemiology and clinical implications. J Trauma 61 (5):1058-1061 Selby RD (1906) Direct abdominal hernia of traumatic origin. JAMA 47:1485-1486

Disclosure: No significant relationships.

\section{PC017}

\section{COCAINE COWBOY-POST-TRAUMATIC COCAINE- INDUCED BOWEL INFARCTION}
K. Mahendraraj ${ }^{1}$, G. Medrano $^{2}$, T. Katsichtis ${ }^{3}$, A. Goparaju $^{4}$, E. Irizarry ${ }^{1}$, V. Katz ${ }^{4}$

${ }^{1}$ General Surgery, Cornell University, Lincoln Medical and Mental Health Center, Bronx/NY/UNITED STATES OF AMERICA, ${ }^{2}$ General Surgery, Cornell University, Lincoln Medical and Mental Health Center, New york/UNITED STATES OF AMERICA, ${ }^{3}$ Department Of Surgery, Cornell University, Lincoln Medical Center Campus, Bronx/UNITED STATES OF AMERICA, ${ }^{4}$ Department Of Surgery, Cornell University, Lincoln Medical Center Campus, Bronx/ NY/UNITED STATES OF AMERICA

Case History: After snorting cocaine to fortify himself, a 58 year old man attacked a gang of teenagers who were harassing him, sustaining a stab wound with a machete in the process.

Clinical Findings: Thoraco-abdominal stab wound at right costal margin.

Investigation/Results: FAST exam showed free intra-abdominal fluid. Urine toxicology positive for cocaine.

Diagnosis: At laparotomy, stab wound noted to have penetrated through right 10th rib, diaphragm, liver, and right kidney. Nonanatomical liver resection of segment 8 was performed with primary repairs of renal and diaphragmatic lacerations. Entire small and large bowel found to be uninjured and viable. After packing, an improvised abdominal dressing was placed. Estimated blood loss was 2 liters.

Therapy and Progression: At second-look, $80 \mathrm{~cm}$ of grossly necrotic distal small bowel was found. Ischemic segment was resected and small bowel left in discontinuity. At third-look, more necrotic small intestine $(70 \mathrm{~cm})$ was found at the proximal ileal staple line. Resection and primary anastomosis was performed. At fourth-look, small bowel anastomosis was viable but new sigmoid colon necrosis was noted, necessitating rectosigmoid resection with descending end-colostomy. At fifth laparotomy, fascia was closed. Six months later, successful colostomy reversal was performed after resecting a strictured rectal stump. He absconded soon after.

Comments: Cocaine-induced mesenteric vasospasm exacerbated an existing low flow state to the bowels from liver hemorrhage, leading to bowel necrosis which evolved over the following days. It is important to maintain a high degree of suspicion for delayed bowel ischemia in patients with cocaine abuse, particularly if there has been a primary systemic insult resulting in hypovolemic shock.

References: 1. Osorio, Javier, et al. "Cocaine-induced mesenteric ischaemia." Digestive surgery 17.6 (2000): 648-651. 2. Nalbandian, $\mathrm{H}$., et al. "Intestinal ischemia caused by cocaine ingestion: report of two cases." Surgery 97.3 (1985): 374-376. 3. Brown, David N., Mitchell J. Rosenholtz, and John B. Marshall. "Ischemic colitis related to cocaine abuse." American Journal of Gastroenterology 89.9 (1994). 4. Freudenberger, Ronald S., Mitchell S. Cappell, and Douglas A. Hutt. "Intestinal infarction after intravenous cocaine administration." Annals of internal medicine 113.9 (1990): 715-716. 5. MIZRAHI, SOLLY, DANI LAOR, and BENJAMIN STAMLER. 
"Intestinal ischemia induced by cocaine abuse." Archives of Surgery 123.3 (1988): 394-394.

Disclosure: No significant relationships.

\section{PC018}

\section{PENETRATING THORACIC INJURY - WHAT WE MUST LEARN TO DO}

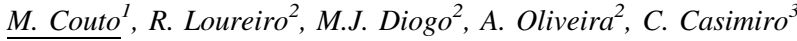 \\ ${ }^{1}$ Cirurgia Geral, Centro Hospitalar Tondela-Viseu, E.P.E., Viseu/ \\ PORTUGAL, ${ }^{2}$ General Surgery, Centro Hospitalar Tondela-Viseu, \\ Viseu/PORTUGAL, ${ }^{3}$ Cirurgia Geral, Centro Hospitalar Tondela- \\ Viseu, Viseu/PORTUGAL
}

Case History: Penetrating abdomino-thoracic wounds can result in significant injury to a patient, with involvement of both thoracic and abdominal organs. Surgical intervention is most of the times needed, both to control the bleeding and to repair the affected organs. The surgical approach may include a laparotomy, a thoracotomy or both, depending on the severity of the lesions and clinical status of the patient. The authors present a case of a man, 91 years old, who presented in the ER with a self-inflicted stab wound.

Clinical Findings: The patient presented in the ER with a knife on the transition of the thorax and epigastric region.

Investigation/Results: He was submitted to an emergency laparotomy, with removal of the knife and exploration of the wound.

Diagnosis: It was observed a grade II laceration of the segments 2 and 3 of the liver and a pericardial laceration.

Therapy and Progression: Since the patient presented bleeding from the pericardial, it was decided to perform a left antero-lateral thoracotomy, with opening of the pericardium. It was identified a small laceration of the right auricula, which was repaired and it was placed a chest drain on the left at the end of the surgery. The patient had a good evolution, with removal of chest drain on the third day after surgery.

Comments: Penetrating injuries of the chest are life-threatening injuries and must be managed in trauma centers who have surgeons with skills to perform an emergency thoracotomy.

References: Griffiths E, Mohamed A, Ball C, BMJ Case Reports, 2010 Berg R, Karamanos E, Inaba K et al. J Trauma Acute Car Surg, 2013, 76 (2)

Disclosure: No significant relationships.

\section{PC019}

\section{PENETRATING CARDIAC STAB WOUND: SOMETIMES YOU CAN TRANSFER SAFELY}

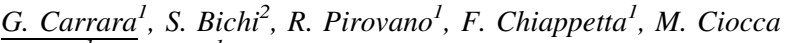 \\ Vasino $^{I}$, M. Zago ${ }^{I}$
}

${ }^{1}$ Chirurgia Generale, POLICLINICO SAN PIETRO, Ponte san pietro/ ITALY, ${ }^{2}$ Cardiochirurgia, ASST PAPA GIOVANNI XXIII BERGAMO, Bergamo/ITALY

Case History: A man arrived at ED of a Level 3 trauma center with a stab wound in the anterior left chest at III-IV rib space. The blade was already extracted.
Clinical Findings: The patient was unconscious, pale, sweaty and in respiratory arrest. BP 66/32, HR 105. BLS manoeuvres regained consciousness and spontaneous breath. At reassessment BP 55/35, HR $115, \mathrm{O}_{2}$ sats $90 \%(\mathrm{FiO} 260 \%)$.

Investigation/Results: EKG was negative for ischemia. Arterial blood gas showed severe acidosis, CX-Ray and TTE revealed a massive pleural effusion and an ab-extrinseco cardiac tamponade of right chambers.

Diagnosis: was hemothorax tamponating heart and hemopericardium. A chest tube was promptly inserted, draining $1600 \mathrm{cc}$, with immediate heart re-expansion. Vital signs normalized and remained stable. While organizing transfer to the referral Center, the patient underwent CT (pneumomediastinum, residual hemothorax, hemopericardium).

Therapy and Progression: He immediately went to OR. A sternotomy was performed with evidence of right ventricle laceration in the anterior wall and posterior right ventricle hematoma. A sutureless repair was done with TachoSil ${ }^{\circledR}$ and BioGlue. Postoperative course was uneventful, the patient discharged after a week.

Comments: Laceration connecting pericardium and pleural spaces allowed drainage of hemopericardium in the thorax and tamponade relief. Prompt chest drainage stabilized the patient. Good communication between centers allowed safe transfer. TachoSil patch permited safe and rapid heart repair.

References: 1-Trauma to the heart: a review of presentation, diagnosis, and treatment. Goldstein et al. J Trauma Acute Care Surg 2017 911-916 2-TachoSil ${ }^{\circledR}$ for postinfarction ventricular free wall rupture Pocar et al. J Thoracic Cardiovasc Surg 2012 866-867

Disclosure: No significant relationships.

\section{PC020}

\section{DELAYED MULTIPLE AORTIC DISSECTIONS IN A PEDESTRIAN WHO WAS HIT BY A CAR WITH MILD IMPACT}

\section{S. Mori, T. Ai, Y. Otomo}

Trauma And Acute Critical Care, Tokyo Medical and Dental University, Tokyo/JAPAN

Case History: An 84-year old female without remarkable medical histories was walking on a street and mildly hit by a car. She fell on the road. She was conscious and quite stable at the first EMS contact except complaining of pain in her bilateral lower leg lacerations. When she was brought to a local hospital, she was completely conscious and her vital sign was stable except mild hypertension.

Clinical Findings: Physical examinations did not reveal any major injuries except small lacerations in her lower legs. Her vital signs were stable and consciousness was consistently stable.

Investigation/Results: X-rays, ultrasound, and EKG showed no findings that indicate major trauma. The patient had irrigation and suture on the leg lacerations in the emergency department. Soon afterward, she started complaining of mild chest discomfort. The echocardiogram revealed mild pericardial effusion. A computed tomography with contrast showed dissection of the ascending aorta. She was transferred to a tertiary trauma center by a helicopter.

Diagnosis: The second CT showed two-part aortic dissections in the ascending aorta and in the descending aorta.

Therapy and Progression: She was planned for elective stent grafting, but she became in respiratory distress and shock. On the day 5 , she deceased because of cardiac tamponade by worsening of the dissection, which was confirmed by a postmortem CT. 
Comments: This patient's injury was initially not considered serious. Physicians should always be reminded of the possibility of vascular injuries regardless of the mechanism or the size of the accident and be cautious of the subtle change of vital signs or complaints of the patient.

References: 1. Late traumatic aortic dissection. Am J Emerg Med. 2012;30:1328.e5-8. 2. Aortic ruptures in seat belt wearers. J Thorac Cardiovasc Surg. 1989;98:355-61. 3. Acute traumatic thoracic aortic rupture in double localization. Ann Cardiol Angeiol. 2014;63:51-4.

Disclosure: No significant relationships.

\section{PC021}

\section{BLUNT DIAPHRAGMATIC RUPTURE: A CASE REPORT}

\section{M.S. Ferreira, S. Carlos, R. Cardoso, J. Simoes, P. Costa}

General Surgery, Hospital Garcia de Orta, Almada/PORTUGAL

Case History: Introduction Blunt diaphragmatic rupture (BDR) is a rare injury, present in about $5 \%$ of trauma patients. Patients often present with high Injury Severity Scores and the most common associated injuries are thoracic and abdominal injuries.

Clinical Findings: Case Report A 49 year old male patient was admitted following a motorcycle accident.

Investigation/Results: Following primary assessment, a chest $\mathrm{x}$-ray showed fluid in the left hemithorax; Focused Assessment with Sonography for Trauma (FAST) scan showed no free fluid in the abdomen, although the spleen could not be identified in the left upper quadrant.

Diagnosis: A CT scan showed 4 rib fractures, scapula fracture, left hemothorax, gastric herniation through a left sided tear in the diaphragm and a grade V splenic lesion.

Therapy and Progression: A thoracostomy tube was placed and the patient underwent a laparotomy. Surgical exploration revealed a grade IV diaphragm rupture, gastric herniation through this rupture and a grade V spleen injury. A splenectomy and diaphragmatic repair were performed. Secondary and tertiary assessment identified a zygomatic arch fracture, and fractures of the 5th finger and toe, yielding an ISS of 38 . The patient had an uneventful post operative recovery.

Comments: Discussion Injuries to the left diaphragm are often associated with spleen injury and lesions to the right diaphragm are often associated with liver injuries. Diagnosis is difficult (particularly on the right side) which may account for the higher incidence of lesions reported on the left side. Diagnosis of diaphragmatic ruptures may be easier in case of displacement of abdominal contents but it may represent a relevant FAST scan pitfall.

References: Scharff, R.F., et al. Traumatic diaphragmatic injuries. Thoracic Surgical Clinics. 2007 Feb;17 (1):81-5. Chughtai, T., et al. Update on managing diaphragmatic rupture in blunt trauma: a review of 208 consecutive cases. Canadian Journal of Surgery. 2009 Jun; 52 (3): 177-181. Reiff, D.A., et al. Identifying injuries and motor vehicle collision characteristics that together are suggestive of diaphragmatic rupture. Journal od Trauma. 2002 Dec;53 (6):1139-45.

Disclosure: No significant relationships.

\section{PC022}

\section{RARE COMPLICATION OF THORACIC AIR GUN SHOT INJURY}

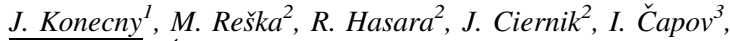
L. Veverkovát

${ }^{1} 1$ st Department Of Surgery, St. Anne‘s University Hospital Brno, Brno/CZECH REPUBLIC, ${ }^{2} 1$ st Departmen Of Surgery, St. Anne's University Hospital, Brno/CZECH REPUBLIC, ${ }^{3} 1$ st Surgery Department, St. Anne's Hospital, Brno/CZECH REPUBLIC, ${ }^{4} 1$ st Surgery Departmen, St. Anne's Hospital, Brno/CZECH REPUBLIC

Case History: 33 years old healthy man without chronic medication had been shot in the left side of the chest by the air gun.

Clinical Findings: The patient was conscious with spontaneous breathing and normal blood pressure. Breathing was symmetric. GCS was 15 . Due to lower oxygen saturation measured by pulse oximetry the patient was intubated. He was hemodynamically stable with symmetric breath sounds and symmetric pupils. The wound was $5 \mathrm{~mm}$ long, placed in the left medioclavicular line in the third intercostal space level.

Investigation/Results: The wound was in the area with risk of cardiac or great vessel injury. To perform the reliable emergent echocardiography was impossible. The urgent surgical exploration of the wound was indicated. The chest $\mathrm{x}$-ray was performed.

Diagnosis: Wound penetrating through the left third intercostal space into the pleural cavity, penetration through the left lung, penetration through the pericardium to the left ventricle. The air gun pellet was not found.

Therapy and Progression: Suture of left ventricle, pulmonary tractotomy, drainage of left hemithorax and closing of left anterolateral thoracothomy was performed. Due to the mydriasis on the left side in the end of the operation the helical CT was indicated. The pellet was found in the right internal carotid artery which was completely occluded. The radiologist attempted to remove the pellet using endovascular approach unsuccessfully. The pellet was pull out using Fogarty catheter through the right carotid arteriotomy.

Comments: This case shows that the air gun shot injury of the chest should be very dangerous and the problem could be also outside of the thorax.

References:

Disclosure: No significant relationships.

\section{PC023}

\section{SEVERE TRAUMATIC BRONSIAL INJURY}

K.B. Ozer, R. Demirhan, A. Ozdemir, H. Eryigit Unaldi, E. Cesur, F.T. Ozlu

Thoracic Surgery, Dr. Lutfi Kirdar Training and Research Hospital, Istanbul/TURKEY

Case History: Tracheobronsial injuries are classified iatrogenic and traumatic. Traumatic rupture of the bronchus is rare but catastrophic. Mostly membranous part of the stem is injured and most common symptom is pneumothorax.

Clinical Findings: 31 y.o. female carried to the emergency room after fallen from $12 \mathrm{mt}$ height. Glascow coma scale (GCS) was eight, and had severe subcutaneus emphysema.

Investigation/Results: The emphysema indicated pneumothorax, so after entubation bilateral chest tube fastly placed and both had air leak. Thorax computed tomography (CT) showed, had multilevel fractures of vertebrae, subcutaneus emphysema, mediastinal emphysema and right persistent pneumothorax. 
Diagnosis: The radiological findings and the persistent air leak on the right gave the impression of tracheobronsial injury. Fiberoptic bronchoscopy was performed. Left endobronsial system was intact, but we detected about $1,5 \mathrm{~cm}$ intermediary bronchus rupture.

Therapy and Progression: With a right thoracotomy approach the bronchial fistula repaired with 4/0After the surgery the follow-up was satisfying, there were no air leak and chest $\mathrm{x}$ - ray showed bilateral lung expansion. On the post operative second day the chest tube terminated. After one week follow-up Chest $\mathrm{x}$ - ray was normal. prolene suture.

Comments: After blunt trauma the chest injuries can result with mortality, initial diagnose and rapid treatment is very important in emergency room.

References: Kiser AC, O'Brien SM, Detterbeck FC. Blunt tracheobronchial injuries: treatment and outcomes. Ann Thorac Surg 2001;71:2059-65. Shankar Hanamantrao Hippargi. Traumatic bronchial rupture: an unusual cause of tension pneumothorax. Int J Emerg Med (2010) 3:193-195

Disclosure: No significant relationships.

\section{PC024}

\section{CHEST WALL STABILIZATION IN VENTILATOR- DEPENDENT TRAUMATIC FLAIL CHEST PATIENT}

\section{P. Carmona Soto, S. Sevilla López, F. Cózar Bernal, A. Bravo Cerro}

Thoracic Surgery, COMPLEJO HOSPITALARIO CIUDAD DE JAEN, JAEN/SPAIN

Case History: 31 Years old male who suffered a fall from a gret height in his work. Due to it was diagnosed and treated of polytrauma. After a month he was in ICU with mechanical ventilation for flail chest.

Clinical Findings: Flail chest with broken ribs from $1^{\mathrm{a}}$ to $10^{\circ}$ of left side. Impossibility of weaning twice with high demands of opioid for analgesia.

Investigation/Results: Due to above information we thought that chest wall stabilization in this patient with vetilator-dependency have to be made.

Diagnosis: Polytrauma with flail chest.

Therapy and Progression: Under general anaesthesia with left side double-lumen tube and right decubitus, lateral rib fractures from $6^{\mathrm{a}}$ to $9^{\text {a }}$ left ribs were fixed. Just one incision was performed and $250 \mathrm{ml}$ of haemothorax was evacuated too. Chest drain was placement and it was taken out 6 days after surgery. Weaning was achieved $72 \mathrm{~h}$ after surgery. Patient was discharged 17 days after surgery. He went to another physical therapy clinic for continue with rehabilitation.

Comments: Operative rib fixation has the potential to reduce ventilator and UCI days in selected patients. Even, this treatment could avoid chest wall deformities and the formation of painful pseudoarthrosis.

References:

Disclosure: No significant relationships.

\section{PC024A}

\section{OCCLUSION OF THE SUBCLAVIAN ARTERY AFTER A CLAVICLE FRACTURE}

\author{
D. Demir, B.L.s. Borger Van Der Burg, R. Hoencamp, \\ G. Stollenwerck
}

Surgery, Alrijne Hospital, Leiderdorp/NETHERLANDS

Case History: A 44-year-old male patient presented nine days after a skiing accident in Austria at our hospital. In Austria an X-ray showed a left clavicle fracture for which conservative treatment was initiated. The patient had an increase of pain at his left shoulder with continuous tickling of his left hand and a colder upper left limb.

Clinical Findings: Physical examination found no motor function or sensory loss of the left arm. The radial artery had a diminished pulse and an absent pulse at the ulnar artery. The left hand was not significantly colder.

Investigation/Results: Radiographic examination revealed a displaced midshaft clavicle fracture, AO-classification $15.2 \mathrm{~B} 3$ and Robinson-classification 2B1. Computed tomography angiography (CTA) showed an occlusion of the left subclavian artery in a length of 3 centimeter, distal of the occlusion the subclavian artery filled through various collaterals.

Diagnosis: Displaced midshaft clavicle fracture with an occlusion of the subclavian artery.

Therapy and Progression: The arterial occlusion and clavicle fracture were treated in one operation session. The amount of callus formation made a venous interposition less suitable; therefore a subclavian-brachial bypass was constructed. The clavicle was fixated with 8-hole locking compression plate (LCP). The follow up at six months showed a full range of motion of the left shoulder and an open bypass.

Comments: Subclavian artery injuries after blunt trauma are uncommon. The combination of a dislocated clavicle fracture and absent distal pulse, advocates further assessment. Open repair can be challenging because to extensive callus formation and close relation of plexus and artery.

References:

Disclosure: No significant relationships.

\section{PC024B}

THORACOSCOPIC DRAINAGE OF HAEMOPERICARDIUM AND PERICARDIAL WINDOW WITHOUT REPARATION OF MIOCARDIAL INJURY AFTER PENETRATING THORACIC TRAUMA

C. Gamo, A. Salcedo, J.J. Serna, C. Miranda, A. Gonzalez, S. De

Lebruisant, R. Rodriguez.

Cali, Hospital Universitario del Valle, Cali/COLOMBIA

Case History: We present a 28 years old man with precordial stab wound

Clinical Findings: Presents tachycardia and dispnea, without other injuries 
Investigation/Results: Presents hemopneumothorax and pericardial effusion seen in the ultrasonography.

Diagnosis: hemopneumothorax and pericardial effusion

Therapy and Progression: Toracostomy is performed with drainage of $800 \mathrm{cc}$ of blood at the insertion and additional $500 \mathrm{cc}$ in the following 24 hours, with stabilization of drainage afterwards. In the presence of pleural compromise the pericardial window can be performed by thoracoscopy. We decide to perform thoracoscopic exploration, wich is delayed 48 hours because of the lack of collaboration by the patient. In the intervention it is evidenced a miocardial injury in the right ventriculum, healed and without active bleeding. We perform thoracoscopic pericardial window and lavage with $1000 \mathrm{cc}$ of isotonic saline obtaining clear liquid, without additional therapeutic intervention. The patient evolves satisfactorily, with toracostomy extraction 48 hours after surgery and hospital discharge. Comments: Pericardial lavage and window in patients with no active bleeding may be enough without necessity of additional therapeutic intervention, avoiding morbidity of a sternotomy.

References: -Haemopericardium in stable patients after penetrating injury: is subxiphoid pericardial window and drainage enough? A prospective study. Navsaria et al, Journal Injury. June 2005 -Sternotomy or Drainage for a Hemopericardium After Penetrating Trauma: A Safe Procedure? Nicol et al, Annals of Surgery feb 2016 Disclosure: No significant relationships.

\section{PC024C}

\section{TRAUMATIC PSEUDOANEURYSM OF THE THORACIC AORTA}

\section{C.L. Palmero, S.G. Modet, L. Ojea, D.S. Relinque}

Cirugia General Y Del Aparato Digestivo, HOSPITAL PUNTA DE EUROPA, ALGECIRAS/SPAIN

Case History: 34 years old pacient who suffered multi-trauma in motorbike traffic accident

Clinical Findings: Important left hydropneumothorax with mediastinal displacement to the right, most likely to strain. Subcutaneous emphysema. Pulmonary involvement affecting practically the whole of the left lung. Immediately caudal aortic rupture at the left subclavian outlet with traumatic pseudoaneurysm of about $3 \mathrm{~cm}$. in the aortic concavity associated with hemomediastinum. Discreet hydropneumothorax right. Right posterior rib fracture from 2nd to 10th.Fracture of left dorsal transverse processes from 1st to 9th.Fracture of 1 D11 body and of D12. Fracture of the distal extremity of the left clavicle

Investigation/Results: In $80 \%$ of cases, the aortic rupture is incomplete and the lesion is limited to a tear of the intima and/or the middle layer, localizing in $90 \%$ of cases at the isthmus level. The ascending aorta at the root level is the second most frequent location (8\%), frequently associated with aortic valve tear, with an untreated mortality of $85 \%$

Diagnosis: Radiologically, in the thoracic CT we can find intramural hematoma without intimal tear, intimal tear, abrupt change in the contour of the aorta, decrease of aortic caliber (pseudocoartation), pseudoaneurysm and, if rupture is complete, extravasation of contrast to the mediastinum

Therapy and Progression: Endovascular balloon occlusion (REBOA) was performed and subsequent surgical intervention Comments: A high percentage of fatalities due to traffic accidents are due to thoracic aortic injury. Those who survive the accident and manage to reach the emergency department have partial transects or traumatic pseudoaneurysms. Endovascular occlusion has been shown to be effective in stabilizing the patient previous surgery

References: T.C. Fabian, J.D. Richardson, M.A. Croce, J.S. Smith Jr., G. Rodman Jr., P.A. Kearney Prospective study of blunt aortic injury: multicenter trial of the American Association for the Surgery of Trauma J Trauma, 42 (1997), pp. 374-380 M.H. Murad, A.Z. Rizvi, R. Malgor, J. Carey, A.A. Alkatib, P.J. Erwin Comparative effectiveness of the treatments for thoracic aortic transaction J Vasc Surg, 53 (2011), pp. 193-199 O. Nzewi, R.D. Slight, V. Zamvar Management of blunt thoracic aortic injury Eur J Vasc Endovasc Surg, 31 (2006), pp. 18-27

Disclosure: No significant relationships.

\section{PC024D}

\section{LAPAROSCOPIC STAB WOUND MANAGEMENT}

\author{
L.O. Ruiz-Yherla, C.L. Palmero, S.G. Modet, D.S. Relinque
}

CirugÍa General Y Del Aparato Digestivo, HOSPITAL PUNTA DE EUROPA, ALGECIRAS/SPAIN

Case History: 75-year-old male with a knife stabbed at the level of the left hypochondrium. Hemodynamically sable, with no signs of generalized peritoneal irritation.

Clinical Findings: Simple chest $x$-ray without pathological findings. Thoracoabdominal CT scan that did not detect pneumoperitoneum, free fluid or lesion of solid organs.

Investigation/Results: It is preferred to perform a non-therapeutic exploratory laparotomy in order to allow the evolution of an initially unnoticed serious lesion. Therefore, Ivatury and Salvino showed the usefulness of laparoscopy in the patient with the penetrating trauma. Both Lin's 2010 study and O'Malley's review published last year conclude that laparoscopy in these cases is safe and decreases the number of non-therapeutic laparotomies. Laparoscopy has a series of advantages such as checking if the wound is penetrating, thoroughly evaluating the abdominal cavity, diagnosing hemoperitoneum and treating it cases of superficial lesions of solid organs, avoiding nontherapeutic exploratory laparotomies and the morbidity that they entail; decreasing hospital stay.

Diagnosis: Laparoscopic intraabdominal images.

Therapy and Progression: Discarded possible diaphragmatic lesion, after creating pneumoperitoneum with Veress needle and introducing umbilical trocar, abdominal cavity was checked in search for possible organic lesion or bleeding. No lesion was observed; therefore the knife was removed under direct laparoscopic vision without evidence of bleeding from the wall.

Comments: In those cases in which patients with penetrating abdominal injuries caused by a knife, provided they are stable and the findings of the imaging tests allow us to do so; it is preferable to 
perform a laparoscopic intraabdominal exploration instead of a midline laparotomy, decreasing the probability of morbidity, recovery time and hospital stay.

References: 1)Ministerio de Salud de Chile. Guía Clínica Politraumatizado., Minsal, Santiago (2007) 2)L. Medina, R. Kaempffer Consider aciones epidemiológicas sobre los traumatismos en Chile Rev. Chil. Cir, 59 (3) (2007), pp. 175-184 3)R. Sanchez, T. Lama, E. Carrillo Trauma abdominal. En Trauma. Sociedad Panamericana de Trauma (2a), Distribuna Editorial, Bogotá (2009), pp. 307-315 4)A. González, A. García Trauma abdominal penetrante. En Trauma $\left(2^{\mathrm{a}}\right)$, Sociedad Panamericana de Trauma. Bogotá. Distribuna Editorial (2009), pp. 317-328 5)A. Pacheco Laparoscopia en trauma penetrante abdominalRev. Chil.Cir, 49 (1) (1997), pp. 88-94 6)G. Schurink, P. Bode, P. van Luijt, A. van Vugt The value of physical examination in the diagnosis of patients with blunt abdominal trauma: a retrospective study Injury, 28 (4) (1997), pp. 261-265 7)Colegio Americano de CirujanosComité de Trauma. ATLS. Programa Avanzado de Apoyo Vital en Trauma Para Médicos. Manual del curso. $\left(7^{\mathrm{a}}\right)$, American College of Surgeons, Chicago (2004)

Disclosure: No significant relationships.

\section{PC024E}

\section{HEPATIC TRAUMA BY FIREARM}

\section{L.O. Ruiz-Yherla, C.L. Palmero, S.G. Modet, D.S. Relinque}

CirugÍa General Y Del Aparato Digestivo, HOSPITAL PUNTA DE EUROPA, ALGECIRAS/SPAIN

Case History: 35 year old male, brought to the emergency room after being shot in the abdomen. After examining the patient, who is cardio-respiratory stable.

Clinical Findings: Right pneumothorax of small size. Right pleural effusion. Metallic foreign body located in the 10th and 11th intercostal space in the posterior region of the right thorax. Hepatic laceration. Pneumoperitoneum and hemoperitoneum.

Investigation/Results: The trauma represents the leading cause of death in individuals between 15 and 40 years of age. The liver is frequently involved in this type of events, with an incidence that varies between 3 and $10 \%$ of traumas. It is the largest solid organ of the abdomen, which makes it vulnerable to both compressive and penetrating trauma, the former being the most frequent. The liver is, after the spleen, the organ that is most frequently injured by abdominal or closed thoracic traumatisms. Mortality from hepatic trauma has been reduced from $60 \%$ of cases in 1940 , to about $15 \%$ at the present times in severe injuries. In less than $4 \%$ of cases, death is directly related to liver injury.

Diagnosis: Scan images.

Therapy and Progression: Surgical treatment: midline laparotomy, liver pathway hemostasis, exploration and lavage of the abdominal cavity.

Comments: In the management of abdominal injuries caused by firearms, speed and patient management are crucial. Surgical exploration will always be done as quickly as possible, without the need for a previous image test if the patient is unstable or we think he or she may become unstable in a few minutes. Complete review of abdominal cavity, identifying possible organic lesions and bleeding foci.

References: 1. Simoni C., 1955; t 2. 4. Delgado RM. Trauma abdominal complicado. Rev Chil Med Intensiv 2006; 15 (2):128. 5. Lobos M, Donoso A, Inostrosa F. Trauma cerrado de abdomen. Bol
Hosp Viña del Mar 2006; 56 (1/2):24-8. 6. Mathonnet M, Peyrou P, Gainant A, Bouvier S. Role of laparoscopy inblunt perforations of the small bowel. Surg Endosc 2004; 18 (4):539-45. 7. Tererta M. Evaluation of peritoneal lavage and local exploration in lower chest and abdominal stab wounds. J Trauma 2005; 59 (6):2-5. 8. Rosemary. Mandatory operation for penetrating wounds of the abdomen. Am J Surg 2008; 195:209-16. 9. Hardonay RM. Vietnam Wound Analysis. J Trauma 1978; 18:635-48. 10. Olson SM, Shermar H. Abdominal Stab Wound protocol prospective study documents applicability as widespread use. Am J Surg 2008; 195:112-6. 11. Benein E, Pellegrino A. Trauma abdominal, indicaciones para la laparotomía. Arch Hosp Vargas 2001; 3 (13):37-40. 12. Kinkel K, Vermeulen B. Blunt abdominal trauma with organ injuries. J Trauma 2006; 60 (2):15-22. 13. Farese S, Scardigno AC, Gaglielmi A. Liver rupture following bullhead cause abdominal trauma. Ann Ital Chir 2005; 76 (1):359-5 Disclosure: No significant relationships.

\section{PC025}

\section{POSTTRAUMATIC PSEUDOANEURYSM ASSOCIATED WITH HANDGUN WOUND}

\author{
A. Fischinger ${ }^{1}$, U. Tominc ${ }^{1}$, M. Cimerman ${ }^{1}$, A. Rajster Koren ${ }^{2}$ \\ ${ }^{1}$ Traumatology, UMC, Ljubljana/SLOVENIA, ${ }^{2}$ Traumatology, \\ University Medical Centre Ljubljana, Ljubljana/SLOVENIA
}

Case History: We report a case of a 41-year-old man who shot himself with a $9 \mathrm{~mm}$ round from a handgun during a shooting competition. The patient treatment was uneventful until massive bleeding from the wound one week after the initial injury.

Clinical Findings: Patient was stable, entry wound was $2 \mathrm{~cm}$ under fibula head, NC status intact, pulse a. dorsalis pedis was confirmed by Doppler ultrasound.

Investigation/Results: Pelvic and lower limb CTA were performed: the vessels are well-transient, without extravasate; multifragmentary fracture of fibula head with some metal foreign bodies. After diagnostic procedures wound debridement and drainage was performed. Antibiotic, antithrombotic and analgesic therapy was prescribed. Because of muscular pain patient received peroneus brace.

Diagnosis: Pseudoaneurysma a. tibialis anterior (ATA) Vulnus sclopetarium cruris dex. Fractura fibulae prox. dex.

Therapy and Progression: One week after the injury swelling and pain suddenly appeared. Emergency revision and drainage of wound was performed. One day after revision massive bleeding from wound occurred, CTA reviled a rupture of pseudoaneurysm ATA. Emergency vascular surgery was done. After surgery patient was stable and there was no additional complications.

Comments: Handgun shooting injuries are common and usually require local wound care, tetanus prophylaxis and short course of oral antibiotics. They are usually easily treated and patients have no special problems. Pseudoaneurysms are very rare, but rupture can be fatal. High level of suspicion about vessel injury is necessary even the CTA is negative.

References: Volgas DA, Stannard JP, Alonso JE; Current orthopaedic treatment of ballistic injuries. Injury. 2005 Mar 36 (3):380-6. Handy Eone Daniel, Ankouane Firmin, Pondy O. Angele, et al; Giant Pseudoaneurysm Associated with Arteriovenous Fistula of the Brachial and Femoral Arteries following Gunshot Wounds: Report of Two Cases, Case Rep Vasc Med. 2015; 2015: 454713. Song JK, Srinivasan J, Gordon DS, Newell DW, Baxter AB.; Internal carotid pseudoaneurysm and cerebral infarction from shotgun pellet penetration and embolization., AJR Am J Roentgenol. 1999 Oct;173 
(4):1116. Bartlett CS, Helfet DL, Hausman MR, et al; Ballistics and gunshot wounds: effects on musculoskeletal tissues. J Am Acad Orthop Surg. 2000 Jan-Feb 8 (1):21-36. Maiden N; Ballistics reviews: mechanisms of bullet wound trauma., Forensic Sci Med Pathol. 2009 5 (3):204-9. Epub 2009 Jul 31 Simpson BM, Wilson RH, Grant RE; Antibiotic therapy in gunshot wound injuries. Clin Orthop Relat Res. 2003 Mar (408):82-5. Loder RT, Farren N.; Injuries from firearms in hunting activities., Injury. 2014 Aug;45 (8):1207-14. Kabak B, Karanfilci M, Ersöz T, Kabak M.; Analysis of sports injuries related with shooting., J Sports Med Phys Fitness. 2016 Jun;56 (6):737-43. Epub 2015 Feb 10

Disclosure: No significant relationships.

\section{PC026}

\section{PULMONARY PACKING AS DAMAGE CONTROL TECHNIQUE IN THORACIC BLUNT TRAUMA}

\author{
J.C. Meneses Pardo ${ }^{1}$, J.A. García Salcedo ${ }^{1}$, G. Supelano Eslait ${ }^{2}$, \\ M. Gutierrez Andreu ${ }^{1}$, P. Gámez ${ }^{1}$, P. Yuste García ${ }^{1}$
}

${ }^{1}$ General And Digestive Surgery, 12 DE OCTUBRE UNIVERSITY HOSPITAL, MADRID/SPAIN, ${ }^{2}$ Digestive And General Surgery, Hospital 12 de Octubre, Madrid/SPAIN

Case History: A 21 year old male was brought to the emergency room after a 12 meters accidental fall with severe cranial, thoracic, and pelvic trauma.

Clinical Findings: On arrival, 60 minutes after the accident: BP: 80/60mmHg HR: 95bpm Glasgow score: 9. Right thorax hypoventilation and weak pulses. Intubation, and vigorous intravenous fluid therapy were instaured. A circunferential compression sheet was applied, and the massive transfusion protocol activated.

Investigation/Results: Thorax Xray: Right hemo-pneumo thorax Pelvic Xray: Multiple fractures Fast: Negative Thoracic drainage: $1000 \mathrm{cc}$ blood debit. With initial transfusion, the patient BP increased (110/70) and a CT scan performed showing pelvic and pulmonar active bleeding.

Diagnosis: Severe cranial, thoracic, pelvic blunt trauma

Therapy and Progression: Pelvic arterial embolization was performed. Thoracic drainage debit increased to $1600 \mathrm{ml}$ so surgical thoracic inspection was mandatory. Simultaneous urgent pelvic fixation, and right anterolateral thoracotomy were performed showing massive pulmonary bleeding. After ineffective hemostatic maneuvers, we decided to perform a thoracic packing. The patient improved, and after 48 hours in the Intensive Care Unit, was again brought to second look surgery. The bleeding had stopped, and the pulmonary lacerations were repaired. Evident pneumomediastinum forced a fibrobroncoscopy, that showed middle lobar bronchial avulsion, that was immediately repaired trough bronchoplasty. Following neurosurgical intervention that required partial frontal lobectomy, he finally recovered from all traumatic injuries.

Comments: Thoracic damage control surgery and specially thoracic packing techniques had recently developed in other countries. Thoracic surgeons must learn and train standard strategies for dealing with the critically ill trauma patient. Pulmonary packing is an effective measure for preventing dead.

References: Rotondo MF, Schwab CW, McGonigal MD, et al. 'Damage control': an approach for improved survival in exsanguinating penetrating abdominal injury. J Trauma 1993;35:375-82. Mashiko K, Matsumoto H, Mochizuki T, Takuhiro K, Hara Y, Katada S. Damage control for thoracic injuries. Nihon Geka Gakkai Zasshi. 2002 Jul;103 (7):511-6. Asensio JA, Petrone P, Roldan G, et al. Has evolution in awareness of guidelines for institution of damage control improved outcome in the management of the posttraumatic open abdomen? Arch Surg 2004;139:209-14. Bouboulis N, Rivas LF, Kuo J, et al. Packing the chest: a useful technique for intractable bleeding after open heart operation. Ann Thorac Surg 1994;57:856-60. Urschel JD, Bertsch DJ, Takita H. Thoracic packing for postoperative hemorrhage. J Cardiovasc Surg (Torino). 1997 Dec;38 (6):673-5. Urschel JD . Conservative management (packing) of hemorrhage complicating mediastinoscopy. Ann Thorac Cardiovasc Surg. 2000 Feb;6 (1):912. Wall MJ Jr1, Soltero E. Damage control for thoracic injuries. Surg Clin North Am. 1997 Aug;77 (4):863-78. Hirshberg A, Wall MJ Jr, Mattox KL. Planned reoperation for trauma: a two year experience with 124 consecutive patients. J Trauma. 1994 Sep;37 (3):365-9. T.H. Yilmaz, E. Degiannis, D. Doll. Temporary treatment of uncontrolled intrathoracic hemorrhaging with abdominal towels in combination with a rescue procedure. Damage control procedure of the chest. Unfallchirurg. 2012 Jan;115 (1):71-4. doi: 10.1007/s00113-010-19136. O'Connor JV, DuBose JJ, Scalea TM. Damage-control thoracic surgery: Management and outcomes. J Trauma Acute Care Surg. 2014 Nov;77 (5):660-665. Mackowski M., Barnett R., Harbrecht B., Miller K., Franklin G., Smith J., Richardson J.D., Benns M. Damage Control for Thoracic Trauma. The American Surgeon September 2014;80: 910-913

Disclosure: No significant relationships.

\section{PC027}

\section{"FALLING DOWN": A RETROPERITONEAL CATASTROPHE}

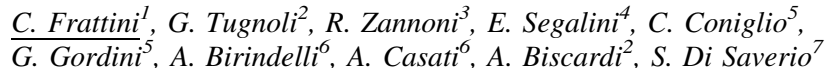

${ }^{1}$ Chirurgia Generale, Università di Pavia - Fondazione IRCCS Policlinico S. Matteo, Pavia/ITALY, ${ }^{2}$ Chirurgia Del Trauma, Ospedale Maggiore Carlo Alberto Pizzardi, Bologna/ITALY, ${ }^{3}$ Radiology, Università degli studi di Parma, Parma/ITALY, ${ }^{4}$ General Surgery, Università di Pavia, Pavia/ITALY, ${ }^{5} \mathrm{Icu}$, Ospedale Maggiore Carlo Alberto Pizzardi, Bologna/ITALY, ${ }^{6}$ Chirurgia Generale, Università di Bologna, Bologna/ITALY, ${ }^{7}$ Surgery, University of Cambridge - Addenbrookes Hospital, Cambridge/UNITED KINGDOM

Case History: A 56 year-old lady, who had chest trauma with multiple left lower rib fractures few weeks earlier, was reported to the ER after fainting.

Clinical Findings: She had signs of hemodynamic shock, metabolic acidosis and severe anemia.

Investigation/Results: FAST-US showed large amount of free fluid, and the patient did not respond to aggressive fluid resuscitation. Despite hemodynamics, a CT scan was performed.

Diagnosis: The exam showed spillage from giant venous retroperitoneal and pelvic varices.

Therapy and Progression: Temporary abdominal packing was placed, then left retroperitoneum was opened with Mattox maneuver, revealing bleeding ruptured varices, repaired with a 5/0 prolene suture. Suturing of pelvic bleeding varices and salpingectomy were also performed and the patient was left with abdominal packing and open abdomen. Postoperative angiography and Angio-CT did not show any blush, nor A-V fistula. No other clear causes for portal hypertension were discovered. In the following hours, persistence of coagulopathy, hemodynamic instability plus development of abdominal-compartment-syndrome required re-laparotomy and re- 
packing. After 10 days, patients' conditions worsened with septic shock and severe hepatic failure. OLT option was excluded given the history of being a good social drinker and the severe sepsis. The patient passed away for MOF on $22^{\text {nd }}$ POD.

Comments: Patients with spontaneous hemoperitoneum and hemorrhagic shock may be challenging for both diagnosis of bleeding source and surgical management. These life-threatening conditions require a prompt multidisciplinary decision-making and a timely surgical management, where Damage Control plays a key-role.

References: Igor Rafael Sincos, Grace Mulatti, Sheila Mulatti, Ilana Cristina Sincos, Sergio Q. Belczak, and Valdir Zamboni. Hemoperitoneum in a Cirrhotic Patient Due to Rupture of Retroperitoneal Varix. HPB Surgery Volume 2009, Article ID 240780, doi:10.1155/ 2009/240780. M. MOLINA-PEREZ, F. RODRIGUEZ-MORENO, E. GONZALEZ-REIMERS, J. PEREZ-PALMA, F. SANTOLARIAFERNANDEZ, H. ESSARDAS-DARYANANI, A. MARTINEZRIERA. Haemoperitoneurn Secondary to Rupture of Retroperitoneal Variceal. HPB Surgery, 1997, Vol. 10, pp. 329-331 Joshua M. Kosowsky, MD and W. Brian Gibler, MD. MASSIVE HEMOPERITONEUM DUE TO RUPTURE OF A RETROPERITONEAL VARIX. The Journal of Emergency Medicine, Vol. 19, No. 4, pp. 347-349, 2000.

Disclosure: No significant relationships.

\section{PC028}

\section{EXTRA-PERITONEAL PELVIC PACKING IN THE "IN EXTREMIS" POLYTRAUMA PATIENT}

P. Yuste García, M. Gutierrez Andreu, G. Supelano Eslait, C. Alegre Torrado, M.C. Nevado, O. García Villar, E. Ferrero

\section{General And Digestive Surgery, 12 DE OCTUBRE UNIVERSITY} HOSPITAL, MADRID/SPAIN

Case History: 29 year old schizofrenic male, was brought to the emergency room after a suicidal attempt jumping from a fifth floor. Clinical Findings: -Pre-Hospitalary Attention: BP: 80/40 HR: 140LPM RR: 30 BPM. Glasgow Score: 8. Unstable pelvis. Cervical Collar, Pelvic Binder, intubation and fluid resucitation were initiated. 25 minutes transport time to the hospital. -Hospitalary Attention: BP: 60/40 HR: 130LPM RR:30 BPM O2Sa:98\%. Normal bilateral ventilation.

Investigation/Results: Thorax Xray: Normal Pelvic Xray: Unstable pelvis with a Tile C3 type fracture FAST: Negative. $\mathrm{pH}$ : 7,31, Hb: $9.8 \mathrm{mg} / \mathrm{dl}$, Lactic Acid: $4.4 \mathrm{mmol} / \mathrm{L}$, Prothrombin Activity $48 \%$. Massive transfusion protocol was activated.

Diagnosis: After primary evaluation, severe hypotension: BP: 40/10: Diagnosis: "IN EXTREMIS" POLYTRAUMA PATIENT

Therapy and Progression: With no available arteriography in the next 45 minutes, the patient was brought to the operating room: Pelvic fixation: 10 minutes Extra-Peritoneal Packing: 10 minutes After transfusion of 5 blood, 3 platelet, and 1 plasma units the patient stabilized. BP: 90/40 HR: 120BPM. After surgery, arteriography was performed, embolizing the obturator, and superior gluteal arteries. In the intensive care unit the patient stabilized and a CT scan was performed: Subdural Haematoma, C7 Stable fracture, sternal and multiple costal fractures. Retroperitoneal haematoma without active bleeding, and complex orthopedic trauma: L1-L2 Fractures. T12-L1 Sub-Luxation: (Chance fracture) an complex pelvic ring unstable fracture.

Comments: Extra-peritoneal packing has been advocated for the unstable "in extremis" polytrauma patient when there's no urgent arteriography available. We recomend the multidisciplinary approach, the damage control strategy, and the early surgical advise in this type of patients.

References:

Disclosure: No significant relationships.

\section{PC029}

\section{THORACOABDOMINAL INJURY AFTER ABDOMINAL PENETRATING TRAUMA}

M. Gutierrez Andreu ${ }^{1}$, O. García Villar ${ }^{2}$, G. Supelano Eslait ${ }^{1}$,

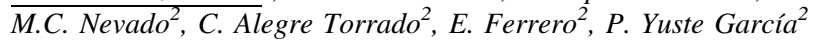

${ }^{1}$ Digestive And General Surgery, Hospital 12 de Octubre, Madrid/ SPAIN, ${ }^{2}$ General And Digestive Surgery, 12 DE OCTUBRE UNIVERSITY HOSPITAL, MADRID/SPAIN

Case History: 31 years old male, usual drug and alcohol user, was brough to the emergency room after being stabbed in the abdomen with a jam knife.

Clinical Findings: -Pre Hospitalary attention: BP: 120/80 HR: 90BPM RR:20BPM Glasgow score:15. 5cm epigastric knife injury. Helicopter Transport time: 60minutes. -Hospitalary attention: BP: 100/70 HR:115, RR:28RPM. Diminished respiratory sounds in the right lower thoracic field. Abdominal exploration: epigastric Knife injury with minimal bleeding. NO peritoneal signs.

Investigation/Results: - $\mathrm{Hb}: \quad 8.5 \mathrm{mg} / \mathrm{dl}$. Lactic Acid: $7.3 \mathrm{mmol} / 1$. Prothtrombin activity: $70 \%$. pH:7.15 -Thorax X ray: Right pleural effusion -Fast: Positive: Minimal subhepatic fluid, no pericardial effusion. -Nasogastric tube: clear. -Urinary catheter: clear. Right thoracic drain was placed, immediatly obteining $1000 \mathrm{cc}$ blood. After primary evaluation: BP:70/40 HR:120 Low conscious level.

Diagnosis: Thoracoabdominal injury after abdominal penetrating trauma

Therapy and Progression: In the operating room: -Massive hemoperitoneum 1.5lts, with segment IV and V hepatic bleeding: controlled with electrocoagulation and hemostatic material. -In the postero-inferior diafragm near the inferior cava vein there was a second bleeding injury: controlled with a direct hemostatic suture. After abdominal bleeding stopped, 6 blood, 4 plasma, and 4 platelet units, the thoracic drainage kept growing and the patient unstable. Right anterolateral thoracotomy was performed. Right diafragmatic arterial bleeding was confirmed and sutured: the patient finally stabilized. Surgical time: 95 minutes.

Comments: After penetrating trauma, the unstable patient represents a big challenge because of the limited diagnostic arsenal. Bleeding can come from different sources. Knowing the mechanism of the injury, may guide the first compartment to tackle. Unstability might force other compartments to be explored.

References:

Disclosure: No significant relationships.

\section{PC030}

\section{ABDOMINAL BLUNT TRAUMA WITH MULTIPLE SOLID ORGANS INJURY: IS NON SURGICAL TREATMENT AN OPTION?}

P. Yuste García, M. Gutierrez Andreu, G. Supelano Eslait, C. Alegre Torrado, M.C. Nevado, O. García Villar, E. Ferrero 
General And Digestive Surgery, 12 DE OCTUBRE UNIVERSITY HOSPITAL, MADRID/SPAIN

Case History: 38 years old male. Brought to the ER after being Knocked down by a car.

Clinical Findings: Pre-Hospitalary Attention: BP: 90/60. HR:90. RR: 20. Glasgow Score 14. On arrival, hypotension: 80/40 responsive to fluids and transfusions. BP after resucitation: 95/60-90BPM.

Investigation/Results: Fast: Positive: Moderate free fluid. CT scan: Hepatic injury: 1) Stage III-IV hepatic injury with no active bleeding. 2) Blunt injury in the VI hepatic segment with active bleeding. Esplenic injury: Mulltiple blunt esplenic injuries with no active bleeding. -Renal injury: 1) Right blunt renal trauma with no excretory lession. 2) Perirenal haematoma. 3) Blunt adrenal injury

Diagnosis: Multiple organ abdominal blunt trauma.

Therapy and Progression: -Urgent arteriography showed arteriovenous hepatic fistula that was inmediatly embolized. -The patient remained stable. "No surgical treatment" was our choice in the urgent setting. Except for a catheter related infection the patient recovered from his injuries with no other procedures. 2 CT scans: 12 days, and 4 months after the accident showed progressive decrease in the size of the injuries until total disappearance. The patient fully recovered and is currently asymptomatic.

Comments: Currently, the "No surgical treatment" option in the abdominal blunt trauma patient is growing, based on the hemodinamic stability, better than the severity of the injuries. The development of intensive care units, the multidisciplinary approach, the urgent endovascular techniques, and the use of standard protocols permit this conservative approach. Free fluid, or severe abdominal injuries must not be taken as absolute guiding principles in the stable patient. The "no surgical treatment" can be done in the right hospitalary setting.

References:

Disclosure: No significant relationships.

\section{PC031}

\section{PARADOXIC AIR EMBOLISM AFTER MASSIVE PENETRATING LIVER INJURY}

M.E. Lueders $^{I}$, G. Tadros $^{2}$, T. Katsichtis $^{2}$

${ }^{1}$ Department Of Surgery, Cornell University, Lincoln Medical Center Campus, Bronx/NY/UNITED STATES OF AMERICA, ${ }^{2}$ Department Of Surgery, Cornell University, Lincoln Medical Center Campus, Bronx/UNITED STATES OF AMERICA

Case History: 27 year old man brought to the ER after being shot with a large caliber bullet to right hypochondrium.

Clinical Findings: The patient was in hemorrhagic shock. Intubation ensued in the ER. There was al large through and through GSW to the right 10th intercostal space in mid-clavicular line.

Investigation/Results: Chest $\mathrm{x}$-ray revealed right sided hemothorax. FAST was positive. Right tube thoracostomy was inserted draining 400cc of blood. During initiation of massive transfusion protocol the patient went for emergent exploratory laparotomy.

Diagnosis: Intraoperatively a large grade 4 penetrating liver injury was found involving Couinaud segments 3,4 and 5 with active bleeding from the liver bed. The bullet had caused a near transection of above segments. Non-viable liver segments were resected. A right diaphragmatic injury was repaired primarily. The raw surface of the bleeding liver bed was thoroughly packed in typical sandwich technique. Intraabdominal hemostasis was subsequently achieved. No other concomitant injuries were found.

Therapy and Progression: Postoperatively patient stabilized. Liver packings were removed on postop day 2 and abdomen was closed. However extubation was delayed because patient did not wake up as expected. A head CT showed multiple small foci of ischemic infarcts in both hemispheres consistent with paradoxic air embolism. Clinically the patient showed bilateral motor weakness. An echocardiogram revealed an undiagnosed ASD with normal LV ejection fraction.

Comments: Paradoxic air embolization in elective hepatic oncological resections has been described. In this case the patient developed multiple small ischemic brain foci caused by air embolism from a large raw surface liver injury with air traversing through an undiagnosed ASD.

References: 1- Poznanska, G., et al., Venous air embolism during major liver surgery: Far more common, than we think. Eur $\mathrm{J}$ Anaesthesiol, 2014. 31 (2): p. 120-1. 2- Lee, S.Y., et al., Paradoxical air embolism during hepatic resection. Br J Anaesth, 2002.88 (1): p. 136-8. 3- Tokunaga, S., et al., Paradoxical air embolism detected by transesophageal echocardiography during hepatic resection. $\mathrm{J}$ Anesth, 1996. 10 (2): p. 147-150. 4- Southern, D.A., J. Arramovic, and K. Fitzsimmons, Air embolism following abdominal stab-wound. Anaesth Intensive Care, 1996. 24 (5): p. 623. 5- Hatano, Y., et al., Venous air embolism during hepatic resection. Anesthesiology, 1990. 73 (6): p. 1282-5. 6- Lai, C.C., et al., Pulmonary artery air embolism after blunt trauma. Resuscitation, 2011. 82 (4): p. 369-70. 7- Milla, F. and M. Cahan, Lethal systemic air embolism in a multi-trauma patient. J Am Coll Surg, 2008. 206 (3): p. 591. 8- Min, S.K., J.H. $\mathrm{Kim}$, and S.Y. Lee, Carbon dioxide and argon gas embolism during laparoscopic hepatic resection. Acta Anaesthesiol Scand, 2007. 51 (7): p. 949-53. 9- Moulton, C.A., et al., Does patient position during liver surgery influence the risk of venous air embolism? Am J Surg, 2001. 181 (4): p. 366-7. 10- Mohammedi, I., et al., Cardiac air embolism after endoscopic retrograde cholangiopancreatography in a patient with blunt hepatic trauma. J Trauma, 2002. 53 (6): p. 11702. 11- Hagen, P.T., D.G. Scholz, and W.D. Edwards, Incidence and size of patent foramen ovale during the first 10 decades of life: an autopsy study of 965 normal hearts. Mayo Clin Proc, 1984. 59 (1): p. 17-20.

Disclosure: No significant relationships.

\section{PC032}

\section{DAMAGE CONTROL SURGERY IN CASE OF THORACOABDOMINAL STAB WOUND}

\author{
A. Szuba, R. Lupiani Moreno, F.J. Jimenez Olmo
}

General Surgery, Hospital del Poniente, El Ejido/SPAIN

Case History: A 45-year-old male brought to the ER with a stab wound in the right hemithorax.

Clinical Findings: Physical examination revealed a penetrating wound to the eighth intercostal space and abdominal tenderness. The thoracic drain is placed confirming the haemothorax and the wound is sutured.

Investigation/Results: Given the initial hemodynamic stability, thoracoabdominal CT is performed which reveals right haemopnuemothorax and perihepatic pneumoperitoneum.

Diagnosis: Thoracoabdominal stab wound.

Therapy and Progression: The patient arrives to the OR unstable, with mixed acidosis, coaulopathy, requires 8 units of RBC and 4 of 
FFP transfusion. Urgent laparotomy was performed, finding the entry wound at the subscapular level, the passage through the pleural cavity (without lung injury), diaphragm, liver (entry in segment VII and exit in IVb), the gallbladder and $1 \mathrm{~cm}$ perforation in the pylorus. Damage control surgery is done: the primary suture of the gastric and diaphragm perforation and the cholecystectomy. At the level of the liver, suture of the portal laceration is needed, bleeding control and biliostasis with local haemostatic patch and urinary Foley catheter with the balloon inflated in the trajectory of the wound. The packing and laparostomy are decided. 48 hours later, the second look and abdominal closure is performed in the absence of new patological findings. The patient progresses favorably and is discharged 40 days after the admission.

Comments: In the penetrating chest wounds below the 7-8 intercostal space it is necessary to rule out intra-abdominal organs lesions. Three phases damage control surgery allows for initial stabilization of the patient and control of hemorrhage, ICU resuscitation and definitive reconstruction.

Disclosure: No significant relationships.

\section{PC033}

\section{SEQUENTIAL PROTOCOL IN THE POLYTRAUMA PATIENT WITH MULTIPLE COMPARTMENT INJURIES}

\author{
M. Gutierrez Andreu, G. Supelano Eslait, M.C. Nevado, C. Alegre
} Torrado, O. García Villar, E. Ferrero, P. Yuste García

General And Digestive Surgery, 12 DE OCTUBRE UNIVERSITY HOSPITAL, MADRID/SPAIN

Case History: 22 years old male. Brought to the ER after a suicidal attempt with multiple stabb wounds.

Clinical Findings: Pre-Hospitalary Attention: BP: 80/40. HR: 110. RR: 8. Glasgow score: 13. Oral intubation, and fluid resucitation where initiated. Hospitalary attention: T: $36{ }^{\circ} \mathrm{C}$ BP: 60/40 HR: 110BPM. Glasgow score 3. SaO2: 92\%. Bilateral hypoventilation, with auscultatory silence in the right hemithorax. No external bleeding. 34 stabb wounds: -In the neck with exposed trachea. -In both hemi-thorax. -In abdomen with evisceration.

Investigation/Results: -Chest X-ray: Right Hemothorax, Pneumomediastinum, subcutaneous enphisema. -pH: 7,25. Hb: 4, 9mg/dl Hto: 14.2\%. Platelet Count: 201000. INR: 2.57. -Nasogastric, and urine tube: Clear

Diagnosis: Multi-compartment open wound polytrauma patient.

Therapy and Progression: Thoracic drain was placed: 2000 cc blood and air obtained. Massive transfusion protocol was initiated, the patient inmediatly received 6 units of red cells, and 2 plasma units. Despite this measures, the patient was still unstable with Noradrenalin $(0,6 \mathrm{mcg} / \mathrm{kg} / \mathrm{min}) \mathrm{BP}: 60 / 40$, HR: $110 \mathrm{lpm}$. In the operating Room: *Right antherolateral thoracotomy: -Hemothorax, internal mamary artery active bleeding: Hemostatic suture -High posterior mediastinal tear with tracheal air leak. After toracotomy the patient stabailized. *Cervicotomy: - $1 \mathrm{~cm}$ Tracheal tear: primary sutured. *Laparotomy: Hemoperitoneum: Gastroepiploic artery bleeding, controlled with hemostatic suture. -3 yeyunal perforations: Intestinal resection and anastomosis. Total Transfusion: 20 red cells, 13 plasma and 2 platelet units.

Comments: Surgical intervention is pritoritary in the unstable patient with multi-compartment injuries. Deciding where to begin, is the most important decision. In this case "thoracotomy first" approach achieved successful outcome. We recomend multidisciplinary approach, damage control strategies, and early surgical advise in this type of patients.

References:

Disclosure: No significant relationships.

\section{PC034}

\section{PANCREATIC TRAUMA: A PURPOSE OF A CASE}

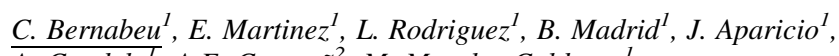
A. Candela', A.F. Compañ ${ }^{2}$, M. Morales Calderon ${ }^{1}$

${ }^{1}$ General Surgery, HOSPITAL DE SAN JUAN DE ALICANTE, SAN JUAN ALICANTE/SPAIN, ${ }^{2}$ Oficina Service, HOSPITAL DE SAN JUAN DE ALICANTE, SAN JUAN ALICANTE/SPAIN

Case History: Pancreatic injuries caused by blunt trauma are rare. Clinical and laboratory findings are nonspecific. Early diagnosis is critical in reducing morbidity and mortality. Late morbidity and mortality are due to complications of pancreatic parenchymal and ductal injury. This pathology occurs because of the compression of pancreas between the vertebral column and anterior abdominal wall; in adults by motor vehicle accidents, in adolescents by bicycle handlebar injuries and in infants because of child abuse. The management of specific pancreatic injury depends on the status of the main pancreatic duct, the degree of parenchymal damage, and the anatomic location of the injury. The complete visualization of the gland and accurate determination of the duct status are key intraoperative maneuvers. Our case was a 19 years male that came to the hospital after a bicycle accident, with severe epigastric abdominal pain and nausea

Clinical Findings: Blood test shows lipase of 37000U/L. The abdominal CT shows a pancreatic laceration and moderate amount of retroperitoneal fluid.

Investigation/Results: No investigation need

Diagnosis: Pancreatic laceration.

Therapy and Progression: We decided urgent surgery. In the OR, we did not visualize evident lesion of the pancreatic duct, deciding conservative management of the gland. During the postoperative period, a low-flow fistula with high lipase biochemistry appears which was solved with conservative management through diet and antibiotic therapy. The patient presented a slow but clear improvement, remaining stable during all the stay, disappearing the fistula. The pancreatic lesion was controlled byimage getting better during the hospitalization until discharge.

Comments: In spite of the inadvertent injury of the pancreatic duct, the conservative therapy was satisfactory

References: 1. Cirillo RL, Koniaris LG. Detecting blunt pancreatic injuries. J Gastrointest Surg. 2002;6:587-598 2. Kao LS, Bulger EM, Parks DL, Byrd GF, Jurkovich GJ. Predictors of morbidity after traumatic pancreatic injury. J Trauma. 2003;55:898-905. 3. Schurink GW, Bode PJ, van Luijt PA, van Vugt AB. The value of physical examination in the diagnosis of patients with blunt abdominal trauma: a retrospective study. Injury. 1997;28:261-265. 4. . 2013 Dec 21; 19 (47): 9003-9011.

Disclosure: No significant relationships.

\section{PC035}

THE TEAM APPROACH TO MANAGEMENT OF THE ABDOMINAL AORTIC ANEURISM IN A PACIENT WITH TRIVIAL TRAUMA . A CASE REPORT 


\section{J. Marante Fuertes ${ }^{1}$, A. Holub ${ }^{2}$}

${ }^{1}$ The Department Of Orthopaedics And Traumatology, In Jerez De La Frontera General Hospital (cádiz, Spain), General Hospital in Jerez de la Frontera, Jerez de la Frontera/SPAIN, ${ }^{2}$ Emergency Room And General Practitioner Resident, General Hospital in Jerez de la Frontera, Cádiz/SPAIN

Case History: The Emergency Ambulance is called to attend a 60year-old male who refers feeling sick and hurts his ankle while he is on his way back from the bathroom in his house minutes after waking up in the morning. On arrival of the Emergency Team the patient is laying on the sofa of his house, feeling nauseous, sweating and vomiting. His son points at the swollen ankle result of the injury but is worried because of finding his father in a bad condition. The patient refers a sudden, severe, sharp and constant low back, flank, abdominal and groin pain.

Clinical Findings: Examination: abdomen: upper abdominal tenderness and a pulsatile abdominal mass of several centimeters od diameter; livid spots from upper abdomen till knees. Blodd pressure : 80/55 mmHg, HR 100, Sp02 98\%. Emergency team starts immediate rapid saline solution intravenous perfusion and transports the patient to the Hospital.

Investigation/Results: In the Hospital the patient is diagnosed of abdominal aortic aneurism by computered tomography (CT) and referred to Vascular Surgery.

Diagnosis: Expanding abdominal aortic aneurism.

Therapy and Progression: Vascular Emergency Surgery: endovascular aneurism repair (EVAR) inserting a stent - graf into the lumen of the aneurismal portion of the aorta.

Comments: Abdominal aortic aneurysms are relatively common and are potentially life-threatening. Patients at greatest risk for AAA are men who are older than 65 years and have peripheral atherosclerotic vascular disease. AAAs are usually asymptomatic until they expand or rupture. The clinical suspicion with rapid life support and transport together with immediate radiological diagnosis with emergency surgery save life.

References: 1. Moll FL, Powell JT, Fraedrich G, et al. Management of abdominal aortic aneurysms: clinical practice guidelines of the European Society for Vascular Surgery. Eur J Vasc Endovasc Surg. 2011;41 (Suppl 1):S1-58. 2. Wanhainen A. How to define an abdominal aortic aneurysm-influence on epidemiology and clinical practice. Scand J Surg. 2008;97 (2):105-9. 3. Wanhainen A, Svensjo S, Mani K. Screening for abdominal aortic aneurysm-areas where information is still inadequate. Scand J Surg. 2008;97 (2):131- 1364. Bush RL, Lin PH, Lumsden AB. Endovascular management of abdominal aortic aneurysms. J Cardiovasc Surg. 2003;44 (4):527-366 5. Chaikof EL, Brewster DC, Dalman RL, et al. The care of patients with an abdominal aortic aneurysm: the Society for Vascular Surgery practice guidelines. J Vasc Surg. 2009;50 (4 Suppl):S2-49

Disclosure: No significant relationships.

\section{PC036}

\section{BULLFIGHT TRAUMA - HOW TO TREAT THE WOUNDS?}

C.N. Marques, R.M. Martelo, M.D.L.A. Rabago, J. Morais, F. Rodrigues

Cirurgia Geral, Hospital Vila Franca de Xira, Vila Franca de Xira/ PORTUGAL
Case History: Male, 68 y Unknown medical history Admitted via emergency room after inferior limb trauma with direct impact from a $550 \mathrm{~kg}$ bull following traditional bull running festival.

Clinical Findings: At observation, patient was drunk, hemodynamically stable and presented a deep puncture wound on the right thigh, with no ongoing haemorrhage. X-Ray presented no fracture. From our experience from bullfight wounds, it was decided to explore the trajectory of the wound in the OR.

Investigation/Results: Patient was submitted to emergency surgery. During inspection, verified transection of medial thigh muscles, preserving main popliteal and femoral vessel and cauterizing the transacted smaller vessels. Repeated pressured wash of the trajectory, with exposure of all traumatically opened aponeurosis and subsequent tenorrhaphy was made. External newly made puncture drainage with passive silicon drain was made for each skin laceration before closing with separated stiches.

Diagnosis: Transection of main medial muscles of right thigh

Therapy and Progression: Post op empirical piperacilin tazobactam was initiated. Good post operatory progress, with physical rehabilitation and evaluation of risk of limb thrombosis. Patient discharged at day 4 , with maintenance of care at the proximity primary care institution. After 1 month complete cicatrisation of the wounds, total mobilization of the limb, with no restriction while walking, standing or resting. No compromise of vascularization or assimetry to contralateral member or alteration of sensitivity.

Comments: Bullfight wounds are highly contaminated gram + and laceration with appearance that frequently minimizes it real extension. It is mandatory its exploration in the OR and external wound drainage with adequate antibiotic therapeutic.

References:

Disclosure: No significant relationships.

\section{PC037}

\section{EMERGENCY VASCULAR REPAIR OF RADIAL ARTERY AFTER GUIDEWIRE RUPTURE DURING CATHETERIZATION FOR CARDIAC INTERVENTION.CASE REPORT}

N. Degermetzoglou $^{1}$, N. Kampouroglou ${ }^{2}$

${ }^{1}$ Vascular Surgery Clinic, 251 HELLENIC AIR FORCE GENERAL HOSPITAL, ATHENS/GREECE, ${ }^{2}$ Cardiology Clinic, PAPAGEORGIOU GENERAL HOSPITAL, THESSALONIKI/ GREECE

Case History: 63 years old male, was admitted and hospitalized in our hospital for coronary atherosclerotic disease. He was transferred in the Cardiac Intervention Department for PTCA. During insertion of the guidewire in the right radial artery, the patient complained for acute pain in the forearm. Fluoroscopy revealed rupture of it and struggle of the radial artery with thrombosis of it. The patient was immediately transferred in the operation theatre for radial artery repair.

Clinical Findings: The patient complained for acute pain in the forearm. Clinical examination revealed loss of pulses in the radial artery and reverse Doppler signal during ultrasound scanning. Swelling, irritation and redness of the forearm skin was significant, while 
pain was reproduced during palpation. The patient also complained for paresthesia of the fingers.

Investigation/Results: Ultrasound scanning revealed thrombosis of the radial artery. The patient was emergently transferred in the operation theatre for repair.

Diagnosis: Right radial artery thrombosis due to guidewire rupture during cardiac catheterization.

Therapy and Progression: The patient underwent, under local anesthesia, exploration of the radial artery in the insertion point. The ruptured guidewire indeed struggled the artery and had caused thrombosis of the peripheral segment of it. It was removed and an thombectomy of the radial artery also took place, which removed a 5 $\mathrm{cm}$ red, fresh thrombus. Inflow and backflow of the artery after thrombectomy were satisfactory. The patient tolerated the operation well and was discharged in the 6th post operative day, after a PTCA intervention that took place during the $2 \mathrm{nd}$ day, through the right common femoral artery.

Comments: Cardiac catheterization is a routine practice in Role 3 Hospitals. Vascular complications in experienced centres are rare but one must be on the alert for the early, acute diagnosis of them and collaboration between intervention cardiologists and vascular surgeons is necessary in order to minimize the complication consequences, morbidity and mortality that are caused from them. Complications due to material failure are extremely rare, because of the quality controls and certificates that needed in order these materials can be used. Thus, this kind of complication is unpredictable but when it happens it should immediately treated and reported in order to avoid it happen again.

References: 1. Ohki T, Veith FJ, Kraas C, et al. Endovascular therapy for upper extremity injury. Semin Vasc Surg 1998;11 (2):106-115 2. Pillai L, Luchette FA, Romano KS, et al. Upper-extremity arterial injury. Am Surg 1997; 63 (3):224-227 3. Shaw AD, Milne AA, Christie J, et al. Vascular trauma of the upper limb and associated nerve injuries. Injury 1995; 26 (8):515-518 4. Stein JS, Strauss E. Gunshot wounds to the upper extremity. Evaluation and management of vascular injuries. Orthop Clin North Am 1995; 26 (1):29-35 Disclosure: No significant relationships.

\section{PC038}

\section{COMPLEX ABDOMINAL WALL INJURIES - THE CLOSING DRAMA}

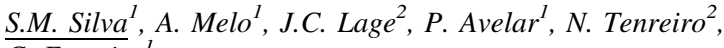
C. Ferreira

${ }^{1}$ Surgery, Centro Hospitalar Trás-os- Montes e Alto Douro, Vila Real/ PORTUGAL, ${ }^{2}$ Surgery, CHTMAD, Vila Real/PORTUGAL

Case History: A 78-year old man admitted to our emergency department with a self-inflicted penetrating abdominal trauma with a shotgun.

Clinical Findings: Initial evaluation in the emergency room revealed a conscious and alert patient, with a penetrating abdominal injury by gunshot, with a single orifice in the anterior abdominal wall. A complete evaluation according to ATLS was performed.Airway, breathing and circulation were

secured and the patient was then evaluated with imaging studies.

Investigation/Results: CT scanning revealed multiple small metallic projectiles located in the subcutaneous layer and some intra-abdominal, an abdominal and thoracic haematoma and some image findings that made us suspect of intestinal perforation.Clinically the haematoma was expanding, so we propose urgent exploratory laparotomy.
Diagnosis: Penetrating abdominal trauma by a shotgun.

Therapy and Progression: Exploratory laparotomy: destruction of the cellular subcutaneous tissue, vascular compromise of the skin of the superior abdominal wall, destruction of the anterior sheath of the rectus muscle with traumatic evisceration. Multiple metallic foreign bodies were removed. Intra-abdominally there was a small haemoperitoneum, small lacerations of the mesentery, with no evidence of major vascular or intra-abdominal organs injury. Postoperative period was longstanding, with the abdominal cavity left open to heal by granulation, with vacuum therapy. Multiple hospitalacquired infections occurred increasing the hospital stay.

Comments: Gunshot wounds are the most common cause of penetrating abdominal trauma, followed by stab and shotgun wounds. Homicide and accidental injury are the most common causes of abdominal injury, while the suicide is uncommon. Intra-abdominal injuries are frequent in gunshot wounds and most of the times an exploratory laparotomy is mandatory for damagecontrol.

References: Walter L. Biffl and Ernest E. Moore. Management guidelines for penetrating abdominal trauma. Curr Opin Crit Care (2010) 16: 609-617.

Disclosure: No significant relationships.

\section{PC039}

\section{SPONTANEOUS RUPTURE OF AN INTERCOSTAL ARTERY}

\author{
C. Afonso $^{1}$, J. Pereira ${ }^{2}$, C. Casimiro $^{3}$
}

${ }^{1}$ Cirurgia Geral, Centro Hospitalar Tondela-Viseu, Vila do Conde/ PORTUGAL, ${ }^{2}$ Cirurgia Geral, Centro Hospitalar Tondela-Viseu, Viseu/PORTUGAL, ${ }^{3}$ General Surgery, Centro Hospitalar TondelaViseu, Viseu/PORTUGAL

Case History: Spontaneous rupture of an intercostal artery is rare without a precipitating event, such as trauma or non-traumatic weakening of the arterial wall. Complicated by hemothorax, hematoma and/or retroperitoneal haemorrhage. It occurs especially in the elderly with cardiovascular disease or under oral anticoagulation.

Clinical Findings: The Authors report a case of a 76-year-old patient who is referred to the Emergency Department for pain associated with the appearance of a mass with a progressive growth in 2 days in the right subscapular region. With no previous history of trauma or fall, ecchymosis or obvious skin changes. Usually medicated with acetylsalicylic acid and a history of heart failure, hemodialysis due to stage 5 chronic renal disease. After administration of 2 units of red blood cells, he was transferred to the Intervention Radiology of the University of Coimbra Hospital, where a embolization with microspheres and microcoils was performed. No complications or bleeding again was observed.

Investigation/Results: Ultrasound revealed a heterogeneous hematoma-compatible collection of about $12 \mathrm{~cm}$. CT showed active hemorrhage of the intercostal artery from the 5th to 6th right ribs. He presented $7 \mathrm{~g} / \mathrm{dl}$ of Hemoglobin with normal vital parameters.

Diagnosis: Spontaneous rupture of an intercostal artery

Therapy and Progression: After administration of 2 units of red blood cells, he was transferred to the Intervention Radiology of the University of Coimbra Hospital, where embolization with microspheres and microcoils was performed. No complications or rebleeding was observed.

Comments: Spontaneous rupture of an intercostal artery is rare, but it can present as a surgical emergency requiring immediate diagnosis and intervention. 
References: 1. Anahita Dua MD, MS, MBA; Arshish Dua; Sarah Jechow; Sapan S. Desai, MD, PhD, MBA; SreyRam Kuy, MD, MHS, Idiopathic Spontaneous Rupture of an Intercostal Artery, WMJ, volume 113 • no. 3 june 2014 2.Cebicci H, et al., CASE REPORT Spontaneous subscapular haematoma in a patient using warfarin therapy BMJ Case Rep 2013. doi:10.1136/bcr-2013-201042 3.J M Moon, S C Lee, B J Chun, Spontaneous intercostal artery bleeding, Emerg Med J January 2008 Vol 25 No 1 4. Raffaella Salmi, at al., MDSUDDEN ACUTE THORACIC PAIN ANd HYPOVOLEMIC SHOCK AS A PRESENTATION OF SPONTANEOUS RUPTURE OF INTERCOSTAL ARTERY: A CASE REPORT, JAGS, FEBRUARY 2012-VOL. 60, NO. 2

Disclosure: No significant relationships.

\section{PC040}

\section{LAPAROSCOPIC SPLENECTOMY AND SPLENIC ARTERY CLIPPING AFTER FAILED ENDOVASCULAR EMBOLIZATION FOR A SPLENIC TRAUMA}

\author{
C. Frattini ${ }^{1}$, G. Tugnoli ${ }^{2}$, R. Zannoni ${ }^{3}$, E. Segalini ${ }^{4}$, A. Casati ${ }^{5}$, \\ A. Birindelli ${ }^{5}$, A. Biscardi ${ }^{2}$, C. Coniglio ${ }^{6}$, M. Podda ${ }^{7}$, A. Rizzuto $^{8}$ \\ S. Di Saverio ${ }^{9}$
}

${ }^{1}$ Chirurgia Generale, Università di Pavia - Fondazione IRCCS Policlinico S. Matteo, Pavia/ITALY, ${ }^{2}$ Chirurgia Del Trauma, Ospedale Maggiore Carlo Alberto Pizzardi, Bologna/ITALY, ${ }^{3}$ Radiology, Università degli studi di Parma, Parma/ITALY, ${ }^{4}$ General Surgery, Università di Pavia, Pavia/ITALY, ${ }^{5}$ Chirurgia Generale, Università di Bologna, Bologna/ITALY, ${ }^{6}$ Icu, Ospedale Maggiore Carlo Alberto Pizzardi, Bologna/ITALY, ${ }^{7}$ Chirurgia Generale, Ospedale San Francesco, Nuoro/ITALY, ${ }^{8}$ Chirurgia Generale, Università degli Studi "Magna Grecia" di Catanzaro, Catanzaro/ ITALY, ${ }^{9}$ Surgery, Uniersity of Cambridge - Addenbrookes Hospital, Cambridge/UNITED KINGDOM

Case History: A 47-year-old man involved in a motorcycle-car impact was referred to our hospital.

Clinical Findings: The vital parameters were stable.

Investigation/Results: A whole body CT scan was performed showing an active bleeding from the spleen, with contrast blushes at the hilum and at the inferior pole (III grade AAST).

Diagnosis: In addition, an aneurysm of the distal part of the splenic artery was described as an incidental finding.

Therapy and Progression: The patient was then transferred in the angiography suite, to attempt a distal embolization of the splenic artery. However, a loop in the proximal tract of the vessel precluded a definitive endovascular treatment. Coils were placed proximally to the aneurysm to reduce the pressure and the bleeding, as a preparation for surgery. The patient underwent a laparoscopic splenectomy. Afterwards, the surgeon isolated the splenic artery from the pancreatic body, and excluded the aneurysm by clipping the artery proximally to the lesion. A follow-up CT scan demonstrated the absence of flow in the aneurysm.

Comments: Endovascular approach is widely accepted as a treatment for both splenic artery aneurysms and active bleeding, however anatomical aberrations may make the procedure impossible to be performed. In such cases, laparoscopic splenectomy is an effective treatment for the bleeding. As for the auneurysm, its exclusion is sufficient to avoid future ruptures.

References: Ryan O. Lakin, MD, James F. Bena, MS, Timur P. Sarac, MD, Samir Shah, MD, Leonard P. Krajewski, MD, Sunita D. Srivastava, MD, Daniel G. Clair, MD, and Vikram S. Kashyap MD.
The contemporary management of splenic artery aneurysms. J Vasc Surg 2011;53:958-65 doi:10.1016/j.jvs.2010.10.055 Wouter Hogendoorn, MD, Anthi Lavida, MBBS, M. G. Myriam Hunink, MD, PhD, Frans L. Moll, MD, PhD, George Geroulakos, MD, PhD, Bart E. Muhs, MD, PhD, and Bauer E. Sumpio, MD, PhD. Open repair, endovascular repair, and conservative management of true splenic artery aneurysms. J Vasc Surg 2014;60:1667-76. Piotr Małczak, Michał Wysocki, Piotr Major, Michał Pędziwiatr, Anna Lasek, Tomasz Stefura, Dorota Radkowiak, Anna Zub-Pokrowiecka, and Andrzej Budzyn' ski, Laparoscopic approach to splenic aneurysms, $\begin{array}{llllll}\text { Vascular 2017, Vol. } 25 & \text { (4) 346-350, DOI: } 10.1177 /\end{array}$ 1708538116682164

Disclosure: No significant relationships.

\section{PC041}

\section{RESUSCITATIVE ENDOVASCULAR BALLOON OCCLUSION OF THE AORTA AS AN ADJUNCT FOR HEMORRHAGIC SHOCK DUE TO UTERINE RUPTURE}

\author{
A. Okada ${ }^{1}$, R. Iiduka ${ }^{2}$
}

${ }^{1}$ Emergency And Critical Care, Kyoto Daini Red Cross Society, Kyoto/JAPAN, ${ }^{2}$ Emergency And Critical Care Medicine, Japanese Red Cross Society Kyoto Daini Hospital, Kyoto/JAPAN

Case History: A 40-year-old Japanese woman was admitted to our Obstetrics and Gynecology Department for the management of placenta accreta at 30 weeks of gestation.

Clinical Findings: She suddenly complained of abdominal pain, and was in shock. Her abdomen was distended.

Investigation/Results: Laboratory data revealed decreased hemoglobin $(7.1 \mathrm{~g} / \mathrm{dL})$ compared to $1 \mathrm{~h}$ previously $(9.8 \mathrm{~g} / \mathrm{dL})$. Sonography revealed massive intra-abdominal bleeding.

Diagnosis: We suspected intra-abdominal bleeding due to uterine rupture.

Therapy and Progression: We decided to perform emergency cesarean section with REBOA, because we predicted life-threatening hemorrhage because of placenta accreta. REBOA improved the systemic blood pressure from $50 \mathrm{mmHg}$ to $120 \mathrm{mmHg}$. Surgery was immediately started. Massive intra-abdominal bleeding caused by uterine rupture. The infant was delivered $4 \mathrm{~min}$ after balloon inflation. A hysterectomy was performed after delivery of the infant. REBOA was performed intermittently during the surgery, and blood pressure was maintained. On admission to the intensive care unit, the patient was hemodynamically stable. She was transferred to the obstetrics ward and subsequently discharged on day 14 without complications. The baby was also discharged uneventfully on day 23 .

Comments: We describe the use of REBOA for life-threatening hemorrhagic shock due to uterine rupture. REBOA may be useful for maternal hemodynamic support, to prevent cardiac arrest. However, REBOA carries potential risks, and therefore, its indication should be considered. References: Clin case rep. 2017 Oct; 5 (10): 1565-1568

Disclosure: No significant relationships.

\section{PC042}

\section{MANAGEMENT OF INTRACARDIAC BULLET WITH CONTRADICTORY MIGRATION IN VENA CAVA: CASE REPORT}

J.P. Carbonell ${ }^{1}$, A.M. Del Valle ${ }^{l}$, H.E. Munevar ${ }^{l}$, A.F. Garcia ${ }^{l}$, R. Ferrada ${ }^{2}$, J.C. Puyana ${ }^{3}$, C. Navarro ${ }^{4}$, J.P. Trochez ${ }^{5}$ 
${ }^{1}$ Valle Del Cauca, Fundación Valle del Lili, Cali/COLOMBIA, ${ }^{2}$ Valle Del Cauca, Clinica Imbanaco, Cali/COLOMBIA, ${ }^{3}$ Department Of Surgery, University of Pittsburgh Medical Center, Pittsburgh/PA/ UNITED STATES OF AMERICA, ${ }^{4}$ Talca, Servicio de salud del Maule, Talca/CHILE, ${ }^{5}$ Valle Del Cauca, Hospital Universitario del Valle, Cali/COLOMBIA

Case History: A 10-year-old girl received a left parasternal gunshot wound without outlet exit.

Clinical Findings: She arrived at the emergency room hemodynamically normal.

Investigation/Results: The E-Fast was positive for pericardial effusion. The patient was transferred to the operating room. A subxiphoid window was positive for blood; the incision was prolonged to a sternotomy. A $7 \mathrm{~mm}$ perforation in the right ventricle was found and sutured. No others cardiac wound and bullets were found.

Diagnosis: In the postoperative evaluation, an echocardiogram and complementary labs were normal. Abdominal x-rays showed bullet in the right lower quadrant, interpreted as a migration projectile to the iliac vein. An exploration of the iliac vessels was performed, but the bullet was not found. Intraoperative fluoroscopy showed displacement to retrohepatic cava vein. The surgery was suspended.

Therapy and Progression: Endovascular management was performed at fifth day post-injury, entering through the right femoral vein. The bullet was found in retrohepatic vena cava and was captured with a steel handle. Through descent, the projectile stopped in the right iliac vein. It was fixed with a second steel handle. The iliacs vessels were accessed by a retroperitoneal incision, and the bullet was removed through right iliac venotomy. The postoperative course was uneventful, and the patient was discharged on the third day.

Comments: Intravascular migration of projectiles are rare, with only 200 reported cases, their diagnosis and treatment are a challenge. Multiple complications can occur when not removed. Surgical removal should be planned, selective, and supported by endovascular methods for technically complex areas.

References: 1. Mattox, K. L., Beall, A. C., Ennix, C. L., \& DeBakey, M. E. (1979). Intravascular migratory bullets. The American Journal of Surgery, 137 (2), 192-195. 2. K Suri, Rajendar \& Jha, Neerod \& Sarwal, Virendar \& Behera, Arunanshu \& Attri, Ashok \& Singh, Shrawan \& Nath Katariya, Rabinder. (1996). Inferior Vena Caval Bullet Embolism. Asian Cardiovascular and Thoracic Annals. DOI: 10.1177/021849239600400313. 3. Ghanaat, M., Goldenberg, C., Walsh, J., \& Sclafani, S. J. (2015). Endovascular management of an intracardiac bullet. Injury, 46 (1), 166-168. 4. Evans, James T. "Bullet migration within the inferior vena cava. (Case Report)." The Free Library 01 January 2003. 20 October $2017<5$. Schroeder, M. E., Pryor, H. I., Chun, A. K., Rahbar, R., Arora, S., \& Vaziri, K. (2011). Retrograde migration and endovascular retrieval of a venous bullet embolus. Journal of Vascular Surgery, 53 (4), 1113-1115

Disclosure: No significant relationships.

\section{PC043}

\section{LAPAROSCOPIC SURGERY AND TRAUMA: APPROACH IN TWO CASES OF PENETRATING AND BLUNT TRAUMA}

\section{Jiménez Fuertes ${ }^{1}$, C. Ferrigni ${ }^{1}$, M. Durán Poveda ${ }^{1}$, D. Garcia $\mathrm{Olmo}^{2}$}

${ }^{1} \mathrm{Hpb}$ Surgery. Emergency Surgery, Rey Juan Carlos University Hospital, Móstoles/SPAIN, ${ }^{2}$ General And Digestive Surgery, Fundacion Jimenez-Diaz University Hospital, Madrid/SPAIN
Case History: The use of laparoscopy has been integrated in surgical practice. However, its inclusion in the management of trauma patients has been slow. CASES Case 1:48 years old man brought to the emergency room after a gunshot wound in the chest. Case 2:21-yearold female patient who came to the hospital after aggression.

Clinical Findings: Case 1: in the physical exam, it was a hole of $1 \mathrm{~cm}$ of the bullet near the left nipple without blowing or bubbling in the wound. Case2:the patient was hemodynamically normal

Investigation/Results: Case1: A portable chest X-ray was performed, which confirmed the absence of pneumothorax or hemothorax, but it was not possible the identification of the projectile in the radiography.An EcoFAST confirmed the absence of fluid. In the rest of the examination, the outlet opening of the projectile was not evidenced. The Thoraco-abdominal-pelvic TC showed the projectile into the subcutaneous tissue of the back. Case2: CT was performed, which showed abdominal free fluid, without pneumoperitoneum without active bleeding associated with bladder lesion.

Diagnosis: Case 1:diaphragm injury Case2:bladder lesion

Therapy and Progression: Case1: After placing a left chest tube, laparoscopy was performed, which showed diaphragm injury. It was repaired. Case2:A laparoscopy exploration was performed, and an injury of intraperitoneal bladder was evident with urine in the peritoneal cavity, which was sutured using this approach. After that, a complete revision of the abdominal cavity was performed without evidence other injuries.

Comments: In selected cases, the laparoscopy approach has some advantages over the open one, and it should be considered as a trauma surgeon tool to diagnose and treat some patients.

Disclosure: No significant relationships.

\section{PC044}

\section{SUCCESSFUL UPPER EXTREMITY ENDOVASCULAR REVASCULARIZATION IN AN AUSTERE ENVIRONMENT}

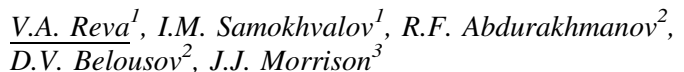

${ }^{1}$ War Surgery Department, Kirov Military Medical Academy, SaintPetersburg/RUSSIAN FEDERATION, ${ }^{2}$ General Surgery, District Military Hospital, Ekaterinburg/RUSSIAN FEDERATION, ${ }^{3} \mathrm{R}$ Adams Cowley Shock Trauma Center, University of Maryland Medical System, Baltimore/MD/UNITED STATES OF AMERICA

Case History: A 27 year-old male sustained a blast injury to his upper extremities. Due to the patients remote location, he was admitted to a Role 2 medical facility 7 hours post-injury.

Clinical Findings: On presentation, he was hemodynamically stable, with multiple closed fractures of both hands, a partial amputation of the right forearm and absent right upper extremity pulses.

Investigation/Results: Plain radiographs confirmed multiple hand fractures and two metallic fragments were observed overlying the right humerus head.

Diagnosis: The patient underwent a completion below-elbow amputation and the right brachial artery exploration, revealing a diminished pulse. Following the insertion of an arterial sheath, a multipurpose 5-Fr catheter was used to obtain a single-shot angiogram, which demonstrated a traumatic sub-total occlusion of the axillary artery.

Therapy and Progression: Using a combination of gentle catheterwire manipulation and serial radiographs, the lesion was traversed and access to normal subclavian artery obtained. A Fluency ${ }^{\circledR}$ Stent Graft 
$(6 \times 100 \mathrm{~mm})$ was then deployed, followed by a completion angiogram, which demonstrated restoration of extremity perfusion. The upper extremity fractures were immobilized with external fixators and the patient was evacuated to the next echelon of care on day 5 with good perfusion of the extremity. CT-angiography on day 30 demonstrated thrombotic occlusion of the stent-graft; however, the extremity was viable and further revascularization was not clinically indicated. He was discharged on day 78 following conversion to internal osteosynthesis.

Comments: Endovascular revascularization of extremity trauma is possible in the austere environment, although techniques need to be refined in order to support a reduced logistical footprint.

References:

Disclosure: No significant relationships.

\section{PC045}

LAPAROSCOPIC DIVERTING SIGMOID COLOSTOMY IN EXTRAPERITONEAL PENETRATING RECTAL INJURIES: A CASE SERIES

\author{
Y. Park, J. Kim, W. Kang, Y. Jo
}

Division Of Trauma Surgery, Department Of Surgery, Chonnam National University Hospital, Chonnam National University Medical School, Gwangju/KOREA, REPUBLIC OF

Case History: Laparoscopy has been a general and effective modality in various situations for management of acute surgical patients. In addition, although some controversies, it's a very attractive modality for the care of trauma patients. We report a case series of extraperitoneal penetrating injuries who were treated by diagnostic laparoscopy and diverting loop sigmoid colostomy.

Clinical Findings: Three patients who had the penetrating injury into the rectum came to the emergency department. Their all vital signs were stable. There were no abdominal tenderness and another abnormal sign on their abdominal physical exam.

Investigation/Results: Some patients had the elevated WBC and CRP. On the digital rectal examination, some bloody spot was noted, however, the perforation site was not palpated. Abdomen CT scan of all patients showed no intraperitoneal perforation. And extraperitoneal air density was revealed in perirectal space.

Diagnosis: We performed the diagnostic laparoscopy and confirmed the extraperitoneal rectal perforation.

Therapy and Progression: Because of the injury location which was too high for transanal approach and too low for intraperitoneal approach, all cases were not appropriate for the primary repair. Therefore, we performed the diverting loop sigmoid colostomy. All of the patients were recovered without any complication, and they had colostomy repair in months.

Comments: Laparoscopic techniques need some training for the liberal use. However, this laparoscopic sigmoid loop colostomy is a very simple technique for performing even though the clinician is not an expert. Therefore, we recommend this procedure for extraperitoneal rectal perforation case.

References: Navsaria PH, Shaw JM, Zellweger R, Nicol AJ, Kahn D. Diagnostic laparoscopy and diverting sigmoid loop colostomy in the management of civilian extraperitoneal rectal gunshot injuries. Br J Surg. 2004;91 (4):460-4.

Disclosure: No significant relationships.

\section{PC046}

\section{SURGICAL MANAGEMENT OF ZONE 3 NECK INJURIES. CASE REPORT}

R. Gràcia ${ }^{l}$, A. Campos Serra ${ }^{2}$, S. Montmany Vioque ${ }^{2}$, P. Rebasa

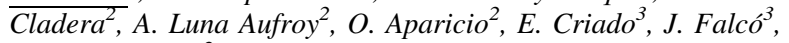
S. Navarro Soto ${ }^{2}$

${ }^{1}$ General And Digestive Surgery, Hospital Universitari Parc Tauli, Sabadell/SPAIN, ${ }^{2}$ General And Digestive Surgery, Hospital Universitari Parc Taulí, Sabadell/SPAIN, ${ }^{3}$ Inteventionist Radiology, Hospital Universitari Parc Taulí, Sabadell/SPAIN

Case History: A 28-year-old male was admitted to our emergency department as a polytrauma code because of a neck knife wound. At his arrival, he was hemodynamically unstable and presented arterial bleeding. The patient was immediately transferred to the operating room.

Clinical Findings: In the OR the surgeons detected incoercible arterial bleeding from the cervical nerve and vessel bundle coming from the skull base. It was impossible to control the bleeding with sutures so they performed a packing with Surgicel ${ }^{\circledR}$ and made compression with the balloon of a urinary catheter $(5 \mathrm{cc})$.

Investigation/Results: Afterward, a CT scan and a cervical arteriography were performed showing no cervical arterial lesion. On the 5th postoperative day, the catheter was removed. The patient was discharged on the 20th postoperative day.

Diagnosis: As a consequence of the aggression, the patient got lesions on the facial, vagus and hypoglossal left nerves.

Therapy and Progression: All these lesions made for the patient impossible to swallow. A tracheostomy and a percutaneous gastrostomy were performed in order to separate the airway from the gastrointestinal tube.

Comments: Zone-3 lesions have an extremely difficult surgical approach. Endovascular techniques may be a useful complement to the surgical approach in order to secure a safe vascular control. Using a urinary catheter as a plugging is a good provisional way to stop the bleeding before performing a definitive endovascular treatment. Our patient had a lesion in the internal jugular vein at the entrance of the jugular foramen. he was hemodynamically unstable, that's why the balloon of the urinary catheter was his best definitive treatment.

Disclosure: No significant relationships.

\section{PC047}

\section{OPEN REPAIR OF RUPTURED ABDOMINAL AORTIC ANEURYSM WITH IDARUCIZUMAB FOR REVERSAL OF DABIGATRAN ANTICOAGULATION}

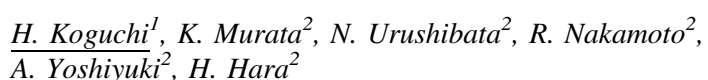

${ }^{1}$ The Shock Trauma And Emergency Medical Center, Matsudo City General Hospital, Matsudo/JAPAN, ${ }^{2}$ Shock Trauma And Emergency Medical Center, Matsudo City General Hospital, Matsudo/JAPAN

Case History: A 67 years old male patient was transported to emergency department for complaining lower abdominal discomfort, dyspnea, and hemodynamically unstable. His medical history was 
hypertension and atrial fibrillation treated with dabigatran $150 \mathrm{mg}$ twice a day. His last dabigatoran dose was taken $12 \mathrm{~h}$ ago.

Clinical Findings: In emergency room, the patient was alert and conscious, pale skin, cold sweat, blood pressure $56 / 44 \mathrm{mmHg}$, heart rates $102 \mathrm{bpm}$. He was responded to a fluid bolus.

Investigation/Results: Ultrasound showed extended abdominal aorta and intimal flap. CT scans showed $7.0 \mathrm{~cm}$ abdominal aortic aneurysm in maximum diameter and retroperitoneal hematoma.

Diagnosis: Ruptured abdominal aortic aneurysm.

Therapy and Progression: After administered $5 \mathrm{~g}$ of idarucizumab intravenously for reversal of dabigatran anticoagulation, an emergency open repair of abdominal aortic aneurysm was performed with normal intraoperative hemostasis, although electrocardiogram temporarily showed PEA (pulseless electrical activity) and chest compression was done. Day 5, heparin treatment was started. Day 9, the patient was extubated. He was successfully alert and oriented. His general condition was gradually improved.

Comments: Idarucizumab, a monoclonal antibody fragment for reversal of dabigatran anticoagulation, was the first specific reversal agent for non-vitamin $\mathrm{K}$ antagonist oral anticoagulants and recently we have been able to use it in life-threatening bleeding and urgent surgery. This was a rare case which a patient received idarucizumab successfully survived an open repair of ruptured abdominal aortic aneurysm.

References: Pollack CV Jr, et al. Idarucizumab for Dabigatran Reversal - Full Cohort Analysis. N Engl J Med. 2017;377 (5):431-41. Disclosure: No significant relationships.

\section{PC048}

\section{USE OF RESUSCITATIVE ENDOVASCULAR BALLON OCLUSIóN OF THE AORTA IN A PATIENT WITH SEVERE HEMORRHAGIC SHOCK AND PRECORDIAL STABWOUND}

\author{
S. De Lebrusant Fernandez ${ }^{1}$, J.J. Serna ${ }^{2}$, O. Bejarano ${ }^{2}$, \\ R. Rodriguez ${ }^{2}$, C. Gamo ${ }^{3}$ A. Gonzalez ${ }^{2}$
}

${ }^{1}$ Cirugia General Y Aparato Digestivo, Hospital Universitario Virgen Macarena, Sevilla/SPAIN, ${ }^{2} \mathrm{Cali}$, Hospital Universitario del Valle, Cali/COLOMBIA, ${ }^{3}$ Madrid, Hospital Universitario de Getafe, Getafe/ SPAIN

Case History: Thirty-three-year-old male patient present with precordial right stabwound located in third intercostal space in the parasternal line. At the arrival with sings of hemorrhagic shock.

Clinical Findings: Trauma Team 1 performed the exposure, dissection of the common femoral artery by open technique and punction of the artery, with introduction $5 \mathrm{Fr}$ catheter clamp to the aortic Zone I. Investigation/Results: It took 5 minutes until complete catheter insertion in the Zone I Aorta with an evident improvement of $35 \mathrm{~mm}$ $\mathrm{Hg}$ of systolic blood pressure, the time of aortic occlusion was 15 minutes at the same time Trauma Team 2 performed sternotomy to control the bleeding by suturing right internal mammary artery.

Diagnosis: Postoperative diagnosis injury right internal mammary artery with a complete section.

Therapy and Progression: The patient was managed in the Intensive Care Unit, at postoperative day five the patient had signs of mediastinitis and purulent pericarditis. Video-assisted thoracic surgery was performed for drainage and decortication. There were no complications associated with the use of REBOA.

Comments: The anatomic structure more commonly injured by this type of trauma is right ventricle. Nevertheless thoracic internal mammary injury always have to be suspected in parasternal wounds.
REBOA implies the temporary insertion con endovascular catheter with balloon insufflation in the aorta to achieve proximal control of hemorraghe Endovascular Occlusion of the aorta is an alternative technique to resuscitative thoracotomy and aortic clamp in a patient with severe hemorrhagic shock, studies have shown positive effects with REBOA use, and it seems to improve overall global survival compared to resuscitative thoracotomy.

References: Biffl WL, Fox CJ, Moore EE. The role of REBOA in the control of exsanguinating torso hemorrhage. J Trauma Acute Care Surg. 2015; 78 (5):1054-8 Moore LJ, Brenner M, Kozar RA et al. Implementation of resuscitative endovascular balloon occlusion of the aorta as an alternative to resuscitative thoracotomy for noncompressible truncal hemorrhage. J Trauma Acute Care Surg. 2015; 79 (4):523-30 Manzano Nuñez R et al. A meta- analysis of resuscitative endovascular balloon occlusion of the aorta (REBOA) or open aortic cross clamping by resuscitative thoracotomy in non compressible torso hemorrhage patients. World J Emerg Surg. 2017;14:12:30 Branco BC, DuBose JJ. Endovascular solutions for the management of penetrating trauma: an update on REBOA and axillo- subclavian injuries. Eur J Trauma Emerg Surg. 2016. 42 (6): 687-694 Quintero B L, Herrera Mario Alain. Trauma de tórax. En: Quintero B Laureano. Trauma. Abordaje inicial en los servicios de urgencias. $4^{\mathrm{a}}$ edición. Santiago de Cali, Colombia. Publicaciones Salamandra; 2008. 186-191 Asencio J et al: One hundred five penetrating cardiac injuries: a 2 year prospective evaluation. J traum. 1998. 22:1073

Disclosure: No significant relationships.

\section{PC049}

\section{APPLICATION OF A HYBRID OR IN 2 POLYTRAUMA PATIENTS WITH HYPOVOLEMIC SHOCK}

\author{
H.C. Wang ${ }^{1}$, H.F. Lin $^{2}$
}

${ }^{1}$ Department Of Thoracic Surgery, Far Eastern Memorial Hospital, New Taipei City/TAIWAN, ${ }^{2}$ Department Of Trauma, Far Eastern Memorial Hospital, New Taipei City/TAIWAN

Case History: A 48 year-old male patient suffering from multiple gun shot wounds in the face, chest, and extremities presented with out-of-hospital cardiac arrest with recovery of spontaneous circulation.

Clinical Findings: After initial resuscitation, because of $300 \mathrm{~mL}$ fresh blood output from left chest tube thoracostomy, he was immediately sent to the hybrid OR due to persistent hypovolemic shock.

Investigation/Results: He received a left anterolateral thoracotomy, and after repair of the lung lacerations, because of ARDS due to aspiration and massive transfusion, ECMO was set up, and angiography was performed to check for active bleeding in the face. After aggressive resuscitation in the intensive unit, he returned to the OR the next day for definitive wound closure.

Diagnosis: Multiple gun shot wounds in the face, chest, and left lower leg resulting in bilateral maxillary fractures with active bleeding, left lung multiple lacerations, and left thigh muscle rupture; hypovolemic shock; Comminuted fractures of C5-6 vertebrae; left tibiofibular fracture.

Therapy and Progression: The patient's consciousness was recovered. However, because of reliance on mechanical ventilation due to cervical myelopathy, tracheostomy was performed. $\mathrm{He}$ is still receiving rehabilitation.

Comments: Identifying ongoing hemorrhage and achieving hemostasis as quickly as possible are central to improving outcomes in severely injured patients. Interventional radiology and surgery are 
complementary interventions for severe trauma, with interventional radiology playing key role in hemostasis when definite repair is not possible. The utilization of a hybrid operating room with angiography capabilities allow for rapid intervention for patients with hemodynamic instability and also reduce transportation between units and should be considered for severe trauma.

References: 1 . Kataoka Y. et al. Hybrid treatment combining emergency surgery and intraoperative interventional radiology for severe trauma. Injury. Int. J. Care Injured 47 (2016)59-63. 2. Kirkpatrick A. et al. The evolution of a purpose designed hybrid trauma operating room from the trauma service perspective: The RAPTOR (resuscitation with angiography percutaneous treatments and operative resuscitations). Injury Int. J. Care Injured 45 (2014) 1413-1421. Disclosure: No significant relationships.

\section{PC050}

\section{ACUTE OCCLUSION OF THE SUBCLAVIAN ARTERY FOLLOWING SURGERY FOR CLAVICLE FRACTURE IN A 36 - YEAR-OLD MALE THAT REQUIRES EMERGENCY REINTERVENTION}

\section{J. Marante Fuertes ${ }^{1}$, A. Holub ${ }^{2}$}

${ }^{1}$ The Department Of Orthopaedics And Traumatology, In Jerez De La Frontera General Hospital (cádiz, Spain), General Hospital in Jerez de la Frontera, Jerez de la Frontera/SPAIN, ${ }^{2}$ Emergency Room, Hospital de Jerez de la Frontera, Jerez de la Frontera/SPAIN

Case History: We present an unusual case of a young man with arterial thrombosis and subclavian pseudoaneurysm secundary to surgery. A 36 -year - old man presents to the Emergency Room with a solid lump in the left supraclavicular zone that progressively increased in the last few days accompanied by signs of left-hand hypoperfusion. No relevant medical history except left clavicle fracture that required surgical intervention two years ago.

Clinical Findings: Clinical examination revealed a cyanotic discoloration of the left distal upper extremitie that was cold on touch suggesting inadequate or obstructed circulation. Clavicular inspection showed an immobile firm and left supraclavicular mass of $2 \mathrm{~cm}$ in diameter that was painful and attached to underlying structures. No left radial pulse could be detected. Oxigen saturation $(\mathrm{StO} 2)$ in the right hand was of $98 \%$ and in the left of $85 \%$; temperature $36,7^{\circ} \mathrm{C}\left(97,8^{\mathrm{a}} \mathrm{F}\right), \mathrm{CPA}$ normal, blood pressure $131 / 92 \mathrm{mmHg}$. . Chest $\mathrm{X}$-rays revealed only the contoured reconstruction plate in the left clavicle from the surgery.

Investigation/Results: Arterial Doppler and CT of the neck and thorax were suggesting of subclavian artery injury by a screw that surpasses the posterior cortical of the clavicle, leaving the distal radial region with no flow, with soft tissue enlargement corresponding to throbosed pseudoaneurysm.

Diagnosis: Arterial thrombosis in the upper limb and thrombosed pseudoaneurysm of the left subclavian artery secundary to osteosyntesis

Therapy and Progression: Removing of the reconstructive plate and the screws.

Comments: The rapid differential diagnosis and clinical suspicion together with the correct emergency surgery are crucial and evoids further complications.

References: 1. Overview of acute arterial occlusion of the extremities (acute limb ischemia). Up2 date. May 2017. 2. Arterial embolism and Thrombosis in Upper Extremity Ischemia. Skeik N, Soo-Hoo SS., Porten BR, et al.Vasc.Endovascular Surg.;2015;49; 100. 3.
Endovascular Therapy for acute limb ischemia. Hynes.BG; Margey RJ.; Ann; Vasc.Surg. 2012.; 26; 110.

Disclosure: No significant relationships.

\section{PC051}

\section{DAMAGE CONTROL SURGERY IN THE ONCOLOGIC PATIENT WITH SPLENIC RUPTURE}

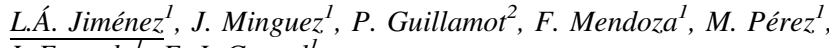 \\ I. Furtado ${ }^{I}$ F..J. Granel ${ }^{I}$
}

${ }^{1}$ Cirugía General, Hospital Príncipe de Asturias, Alcalá de Henares/ SPAIN, ${ }^{2}$ Cirugía General, Hospital Universitario de Móstoles, Móstoles/SPAIN

Case History: We present the case of a 48 -year-old woman with a non-small cell lung carcinoma who need right frontal craniotomy because of cerebral metastasis. After holocranial radiotherapy and systemic chemotherapy, a $11 \mathrm{~cm}$ left suprarenal mass was detected. Before scheduled surgery, she needs emergency care due to acute abdomen secundary to hemoperitoneum.

Clinical Findings: Hemodynamic instability signs, down hemoglobin levels

Investigation/Results: CT scan showin extensive splenic injury, active intraperitoneal bleeding and the adrenal mass infiltrating the spleen (AAST scale grade IV).

Diagnosis: Splenic rupture because of an infiltrant suprarrenal mass Therapy and Progression: Bilateral subcostal incision was performed and the 4 quadrants of the abdomen packed with laparotomy pads, which were removed as the search for the bleeding and intestinal or mesenteric injuries. Medial rotation makes exposure of the hilum of the spleen easier and allows for possible identification of the splenic artery bifurcation. A difficult splenectomy was performed and the left suprarenal removed despite the retroperitoneal infiltration. The time of operation was $126 \mathrm{~min}$, the bleeding during surgery 395 $\mathrm{mL}$ and postoperative hospital stay 8 days. Complications as bleeding or infection by encapsulated organisms did not happened. The AP was splenic metastasis of poorly differentiated carcinoma with pulmonary origin.

Comments: The controversy over when to operate a spontaneous splenic rupture is increased in the oncologic patient, but if you decided, damage control surgery will be done. The debate will spur the continued development of diagnostic and evaluative tools, further minimizing morbidity and mortality caused by splenic injury.

References: 1. C. Castaño Yubera, R. Pérez Martín, Y. Mancebo Rojo, T. Guerra Garijo. Rotura espontánea de bazo. A propósito de un caso Spontaneous splenic rupture. A case report. Semergen 2007;33:485-7 - DOI: 10.1016/S1138-3593 (07)73948-5 2. Roca Martínez FJ. Ecografía clínica del abdomen. Patología del bazo. Ed. JIMS, S.A. Barcelona 1988; 25 3. Lam KY, Tang V. Metastatic tumors to the spleen: a 25 year clinicopathologic study. Arch Pathol Lab Med 2000; 124: 526-530.

Disclosure: No significant relationships.

\section{PC052}

PRIMARY LUNG ABSCESS RUPTURED INTO THE ESOPHAGUS OR LUNG ABSCESS SECONDARY TO ESOPHAGEAL CANCER? 

$\frac{\text { C.A.G.C. Mesquita }}{\text { C.M.S. Antunes }^{2}}$, M.F.L.G. Rosete ${ }^{1}$, A.S.R.S.F. Oliveira ${ }^{1}$,

${ }^{1}$ Centro De Trauma - Serviços De Cirurgia Geral E De Urgência, Centro Hospitalar e Universitário de Coimbra, Coimbra/ PORTUGAL, ${ }^{2}$ Centro De Trauma - Serviço De Imagiologia, Centro Hospitalar e Universitário de Coimbra, Coimbra/PORTUGAL

Case History: Male, 71 years old, transferred from another hospital. Septic shock, well delimited left pulmonary abscess, $11 \mathrm{~cm}$ diameter, of unknown etiology, draining spontaneously to the lower $1 / 3$ of the esophagus.

Clinical Findings: Cough and dyspnea, Sat O2 $<90 \%$, tracheal intubation and ventilation.

Investigation/Results: Esophagoscopy: "At $35 \mathrm{~cm}$ of the incisors, ulcerated fistulous orifice of about $20 \mathrm{~mm}$ in diameter, which communicates to a large necrotic cavity with purulent liquefied content; no apparent aerial communication". Material was collected for study. Diagnosis: Without a definitive diagnosis in a septic patient, the option has been for a damage control strategy, with median laparotomy, gastrotomy and drainage of the pulmonary abscess, through the stomach, esophagus and fistulous tract, with a Foley catheter, subsequently left in continuous washing to the outside, through the abdominal wall.

Therapy and Progression: Without significant clinical improvement, it would be demonstrated 3 weeks later, by a new CT, an occult malignant neoplasm of the esophagus, invading the left lung and left atrium, with associated thrombosis of the pulmonary veins.

Comments: Unfortunately, esophageal cancer can easily spread to adjacent structures, because there is no true anatomic barrier to prevent extension into the mediastinum. This patient died within a few days and the diagnosis was confirmed by autopsy.

References: Kim TJ, et al. Multimodality Assessment of Esophageal Cancer: Preoperative Staging and Monitoring of Response to Therapy. RadioGraphics. 2009; 29:403-421 Rehders A, et al. Esophageal cancer complicated by esophagopulmonary fistula and lung abscess formation: A surgical approach. Thoracic Cancer. 2014;5 (5):468-471 Disclosure: No significant relationships.

\section{PC053}

\section{LAPAROSCOPIC RESECTION FOR TRAUMATIC COLON INJURIES: REPORT OF TWO CASES}

\author{
C. Frattini ${ }^{1}$, G. Tugnoli ${ }^{2}$, R. Zannoni ${ }^{3}$, E. Segalini ${ }^{4}$, A. Birindelli ${ }^{5}$, \\ ${\text { A. } \text { Casati }^{3} \text {, A. Biscardi }}^{2}$, C. Coniglio ${ }^{6}$, A. Rizzuto $^{7}$, M. Podda $^{8}$, S. Di \\ Saverio $^{9}$
}

${ }^{1}$ Chirurgia Generale, Università di Pavia - Fondazione IRCCS Policlinico S. Matteo, Pavia/ITALY, ${ }^{2}$ Chirurgia Del Trauma, Ospedale Maggiore Carlo Alberto Pizzardi, Bologna/ITALY, ${ }^{3}$ Radiology, Università degli studi di Parma, Parma/ITALY, ${ }^{4}$ General Surgery, Università di Pavia, Pavia/ITALY, ${ }^{5}$ Chirurgia Generale, Università di Bologna, Bologna/ITALY, ${ }^{6}$ Icu, Ospedale Maggiore Carlo Alberto Pizzardi, Bologna/ITALY, ${ }^{7}$ Chirurgia Generale, Università degli Studi "Magna Grecia" di Catanzaro, Catanzaro/ ITALY, ${ }^{8}$ Chirurgia Generale, Ospedale San Francesco, Nuoro/ ITALY, ${ }^{9}$ Surgery, Uniersity of Cambridge - Addenbrookes Hospital, Cambridge/UNITED KINGDOM
Case History: Laparoscopy in trauma still has little consideration, even if it could be considered in selected hemodinamically stable patients. Although in literature laparoscopy is widely described to be used in the treatment of perforated diverticulitis, less is written on its use in traumatic perforation. We present two cases of traumatic colonic perforation: a 77 year-old man, involved in a car crash, and a 72 year-old man, who came to the ER for abdominal pain after the insertion of a rectal foreign body.

Clinical Findings: In both cases, patients were suffering from abdominal pain, but their vital signs were normal.

Investigation/Results: They underwent a CT scan, which showed free abdominal air.

Diagnosis: A laparoscopic exploration was performed. In the first case it revealed a laceration of the left colon at the descending-sigmoid junction, while in the second a perforation at the rectosigmoid junction, caused by the rectal foreign body.

Therapy and Progression: A laparoscopic Hartmann's procedure was performed, with resection of the perforated bowel tract using linear staplers, and creation of a colostomy.

Comments: As for perforated diverticulitis, even in stable traumatic patients with colonic perforation laparoscopy is a valid option for treatment, with all the advantages of non open surgery.

References: Vennix S, Boersema GS, Buskens CJ, Menon AG, Tanis PJ, Lange JF, Bemelman WA. Emergency Laparoscopic Sigmoidectomy for Perforated Diverticulitis with Generalised Peritonitis: A Systematic Review. Dig Surg. 2016;33 (1):1-7. doi: 10.1159/ 000441150. Epub 2015 Nov 10. Brad Champagne, Jonah J. Stulberg, Zhen Fan, Conor P. Delaney. The feasibility of laparoscopic colectomy in urgent and emergent

settings Surg Endosc (2009) 23:1791-1796 DOI 10.1007/s00464-0080227-z Ferdinando Agresta, Luca Ansaloni, Gian Luca Baiocchi, Carlo Bergamini, Fabio Cesare Campanile, Michele Carlucci, Giafranco Cocorullo, Alessio Corradi, Boris Franzato, Massimo Lupo, Vincenzo Mandala, Antonino Mirabella, Graziano Pernazza, Micaela Piccoli, Carlo Staudacher, Nereo Vettoretto, Mauro Zago, Emanuele Lettieri, Anna Levati, Domenico Pietrini, Mariano Scaglione, Salvatore De Masi, Giuseppe De Placido, Marsilio Francucci, Monica Rasi, Abe Fingerhut, Selman Uranus, Silvio Garattini. Laparoscopic approach to acute abdomen from the Consensus Development Conference of the Societa' Italiana di Chirurgia Endoscopica e nuove tecnologie (SICE), Associazione Chirurghi

Ospedalieri Italiani (ACOI), Societa' Italiana di Chirurgia (SIC), Societa' Italiana di Chirurgia d'Urgenza e del Trauma (SICUT), Societa' Italiana di Chirurgia nell'Ospedalita' Privata (SICOP), and the European Association for Endoscopic Surgery (EAES). Surg Endosc (2012) 26:2134-2164

DOI 10.1007/s00464-012-2331-3.

Disclosure: No significant relationships.

\section{PC054}

\section{TRAUMATIC LUMBAR HERNIA}

R. Farré, M. Güell, C. Soto

General Surgery, Althaia Xarxa Assistencial de Manresa, Manresa/ SPAIN

Case History: A critically injured subject is rushed into the ER involved in a car accident. Body CT scan revealed: trauma in thorax, 
abdomen, face and traumatic brain injury. These injuries required no immediate surgical procedures.

Clinical Findings: A lumbar hernia is detected, which is initially given conservative treatment. Once the subject's condition is stable, the laparoscopic mesh repair of the lumbar traumatic hernia is performed.

Investigation/Results: Lumbar hernias are described as hernias of the bowel contents through a defect in the lumbar triangle at the lumbar region arising from a non-penetrating abdominal trauma. Clinically, the features of herniation can be missed. However, since CT scan is used on hemodynamically stable trauma patients, hernias can be discovered. Surgery is the treatment of choice for patients with traumatic lumbar herniation however, the timing of surgery has not been established yet. When emergency laparotomy is required for abdominal trauma, a layered closure of the disrupted musculature or a mesh may be performed by laparotomy. If missed initially, repair must be performed as soon as possible.

Diagnosis: Traumatic lumbar hernia

Therapy and Progression: Repair can be open or laparoscopic. The laparoscopic approach is a safer procedure in comparison to the open repair.

Comments: A deferred surgery is an accepted option, once the need of emergency abdominal surgery is ruled out. The laparoscopic approach yields better aesthetic results, low mesh infection and less pain.

References: 1. Mayank Jayant and Robin Kaushik. World J Emerg Med 2012; 3 (1): 74-76. 2. Burick AJ, Parascandola SA. J Laparoendosc Surg 1996 Aug; 6 (4): 259-62. 3. A. Moreno-Egea, JA Torralba-Martínez, G Morales, T Fernandez, E Girela, JL AguayoAbasini. Open vs laparoscopic repair of secondary lumbar hernias: a prospective nonrandomized study. Surg Endosc 2005 Feb; 19 (2): 184-7. 4. Sakarya A, Aydede H, Erhan MY, Kara E, Ilkgul O, Yavuz C. Laparoscopic repair of acquired lumbar hernia. Surg Endosc 2003; 17 (9): 1494.

Disclosure: No significant relationships.

\section{PC055}

\section{PERCUTANEOUS DRAINAGE OF HUGE HEPATIC SUBCAPSULAR HEMATOMA IN CHRONIC RENAL FAILURE: CASE REPORT}

\author{
J. Kim, Y. Park, W. Kang, Y. Jo
}

Division Of Trauma Surgery, Department Of Surgery, Chonnam National University Hospital, Chonnam National University Medical School, Gwangju/KOREA, REPUBLIC OF

Case History: Patients with chronic kidney disease (CKD) display a wide range of derangements in hemostasis. Laparotomy in trauma is a general strategy for the hemostasis, however, this may not be effective in CKD. Therefore, we report a case of huge subcapsular hematoma of the liver which treated a percutaneous drainage and angioembolization.

Clinical Findings: A 37-year old male was transferred to the emergency department after a motor vehicle accident. On arrival, his vital sign was stable, and he presented with abdominal pain.

Investigation/Results: The hemoglobin level was $9.9 \mathrm{~g} / \mathrm{dL}$. CT revealed multiple fractures of left ribs, and liver laceration and large volume subcapsular hematoma of liver involving right and left lobe were detected, and contrast arterial blush was noted in the subcapsular hematoma. There were no absolute indications for laparotomy like pneumoperitoneum.
Diagnosis: Angiography revealed the huge hepatic hematoma and confirmed arterial bleeding in the periphery of the liver.

Therapy and Progression: Gelatin embolization was performed, and no active bleeder further. Even if no further bleeding, the patient presented with abdominal discomfort and dyspnea because of the large size of hematoma. We performed the percutaneous drainage of hematoma at 10 days after the hospital admission. Because of the too large volume of hematoma, even though his drainage duration was somewhat long, he was discharged without any complications.

Comments: Because of minimal regression of hematoma on his follow up CT, we considered of the laparotomy and laparoscopic approach. However, after the careful consideration of hemostatic derangement in the CKD patient, we decided to continue the percutaneous drainage.

References: Ahmed N, Vernick JJ. Management of liver trauma in adults. J Emerg Trauma Shock. 2011;4 (1):114-9.

Disclosure: No significant relationships.

\section{PC056}

\section{ENDOVASCULAR MANAGEMENT OF PERIPHERAL VASCULAR TRAUMA - A WISE CHOICE}

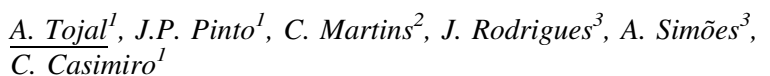

${ }^{1}$ General Surgery, Centro Hospitalar Tondela-Viseu, Viseu/ PORTUGAL, ${ }^{2}$ Orthopaedics, Centro Hospitalar Tondela-Viseu, Viseu/PORTUGAL, ${ }^{3}$ Vascular Surgery, Centro Hospitalar TondelaViseu, Viseu/PORTUGAL

Case History: Extremity arterial injuries are a result of penetrating or blunt traumas and iatrogenic injuries. Endovascular treatment is a minimally invasive option and an alternative to open surgery, which decreases operative time, estimated blood loss and iatrogenic complications by limiting surgical dissection in traumatized operative fields. In situations where definitive repair is not possible with endovascular interventions, it allows control of hemorrhage or damage and facilitate open surgical repair. A traumatic lesion of the main artery in extremities can be treated with stent graft placement, whereas distal branch bleeding can be embolized. A 47-year-old man was transferred from other hospital with a high output active bleeding after hip replacement surgery.

Clinical Findings: The patient's hemodynamics were stabilized after packing the bleeding point and fluid and blood replacement.

Investigation/Results: The patient went to the operating room for selective embolization. Contralateral femoral arterial access was achieved with a 5-F sheath.

Diagnosis: Right iliac and femoral arteriography showed contrast extravasation from the inferior gluteal artery and perforating branches of the deep femoral artery.

Therapy and Progression: Superselective embolization of the bleeding vessels was performed with coils. Final control arteriography confirmed complete exclusion of the bleeding vessels. The wound was closed with drainage system and the patient was discharged without complications to his residence hospital at the third postoperative day to complete the orthopaedic surgery.

Comments: The endovascular approach can be a feasible, safe, and effective option to treat the vascular injuries in the extremities, allowing for timely and minimally invasive management of peripheral active extravasation.

References: Johnson, C. A. (2010). Endovascular Management of Peripheral Vascular Trauma. Seminars in Interventional Radiology, 27 (1), 38-43. http://doi.org/10.1055/s-0030-1247887 
Disclosure: No significant relationships.

\section{PC057}

\section{TRAUMATIC RENAL ARTERY THROMBOSIS}

M. Marone, M. Ferraris, A.M. Socrate

Vascular Surgery, Ospedale Civile di Legnano, Legnano/ITALY

Case History: 61-year-old man, injured by a high energy motorbike accident with impact on the left flank. The patient suffered from, MGRE and had a previous TURP

Clinical Findings: The patient reached the ER with PA $140 / 78$, HR 88, SatO2 98\%, RR 12/min, Hb 13.9 g/dL, Lat 3.7 mmol/L, GCS 15, Creat $1.16 \mathrm{mg} / \mathrm{dL}$. T-pode in situ.

Investigation/Results: Total -body CT scan showed the presence of basin fracture tile B2 with extra abdominal haematoma $(10 \mathrm{x} 4 \mathrm{~cm})$, partial ipodensity of the right kidney and focal defect on the right renal artery. Haematuria at catheterization.

Diagnosis: Given the presence of a focal defect on the right renal artery we have performed a diagnostic angiography that showed thrombosis of the right renal artery.

Therapy and Progression: We have performed a total endovascular angioaspiration of the trombous using 8Fr long introducer and JR4 4fr chateter. Good angiographic result at the end of the procedure. Contrast $60 \mathrm{cc}$.Time frome patient arrival and complete revascularizzation: $5 \mathrm{~h}$ The postoperative period required $72 \mathrm{~h}$ in Intensive Care Unit followed by repair of the basin fracture.

Post-operative TC monitoring demonstrated the e patency of the right renal artery with no perfusion defect on the right kidney. The patient was discharged on day 13. At discharge Creat. $0.97 \mathrm{mg} / \mathrm{dL}$

Comments: Post traumatic blunt renal artery injuries are rare and complex lesions to handle; they require a skilled surgeon both in open and endovascular technique. There are still doubts about the time of warm ischemia that can be tolerated by kidney.

References:

Disclosure: No significant relationships.

\section{PC058}

\section{CONSERVATIVE MANAGEMENT IN PANCREATICODUODENAL TRAUMA}

\section{A. Rico, J.L. Moya, B. Arín, I. Poveda, M. Cires, S. Montón}

General Surgery, Hospital García Orcoyen, Estella-Navarra/SPAIN

Case History: Traumatic pancreaticoduodenal injury still remains challenging with high morbidity and mortality, with an incidence of 0,25-0,4 per 100000 inhabitant per year. Causes of pancreaticoduodenal injuries are blunt trauma in $25 \%$ of cases and penetrating abdominal injuries in $75 \%$ of cases. We present a 35 year old male patient admitted to our service with the diagnosis of a pancreaticoduodenum trauma case after a handlebar blow to the abdomen and its conservative and minimally invasive management.

Clinical Findings: The patient was admitted with abdominal pain, bloating and fever. On admission he remained haemodynamically stable, eupneic at all times. On physical examination, a handlebar mark was observed in the epigastric area. Laboratory results revealed leukocytosis with a deviation to the left

Investigation/Results: Abdominal computed tomography (CT) demonstrated free intraperitoneal fluid and hematoma in the mesenteric root, duodenal wall and around the neck of the pancreas. The patient developed an intraabdominal abscess a few days later

Diagnosis: Pancreatic injury scale according to the American Association for the Surgery of Trauma (grade IV). Drainage: pancreatic fistula, confirmed by the serum amylase elevated

Therapy and Progression: The patient nedeed total parenteral nutrition, antibiotics therapy and drainage for 15 days. The subsequent evolution was positive, the duodenal contusion and pancreatic fistula were resolved with this minimally invasive technique (percutaneous drainage) and a conservative approach

Comments: Optimal management by performing percutaneous drainage, antibiotic therapy and total parenteral nutrition, including a Wirsung duct stent if necessary could resolve some high grade of pancreaticoduodenal injuries without duodenal perforation or hemodynamic instability

References:

Disclosure: No significant relationships.

\section{PC059}

\section{ANTERIOR ABDOMINAL STAB WOUNDS: UTILITY OF DIAGNOSTIC LAPAROSCOPY TO ASSESS INTRA- ABDOMINAL DAMAGES} M. Mogollón, J. Triguero, S. González, C. Plata-Illescas, A. Mansilla-
Rosello, T. Torres

General And Digestive Surgery, Hospital Universitario Virgen de las Nieves, GRANADA/SPAIN

Case History: A 63-year-old male presented to the emergency unit with a $3 \mathrm{~cm}$ infraumbilical wound after self-stabbing.

Clinical Findings: On arrival his blood pressure was controlled and no neurological deficits or external bleeding were detected.

Investigation/Results: The abdomen was soft and not tender.

Diagnosis: Abdominal computed tomography (CT) ruled out presence of pneumoperitoneum and peritoneal effusion with doubt about the penetration of the anterior abdominal fascia.

Therapy and Progression: The patient underwent diagnostic laparoscopy using one $11 \mathrm{~mm}$ supraumbilical port. A $2 \mathrm{~cm}$ parietal peritoneum laceration and omental bleeding were found. All quadrants were explored with no evidence of further damages. The weapon was removed under direct visualization. Postoperative recovery was uneventful. The patient was discharged home on the first postoperative day in a good condition.

Comments: Penetrating abdominal trauma may cause considerable troubles at emergency service. Conventionally, many surgeons still adhere to the traditional mandatory open approach to prevent missed associated injuries, which leads to a high number of unnecessary laparotomies. Laparoscopy can be safely used for diagnostic and therapeutic purposes, minimizing patient morbidity and mortality, hospital stay and reducing medical costs. To optimize results, this procedure should be incorporated in diagnostic and treatment algorithms for trauma patients.

References: 1. Shah M, Galante JM, Scherer LA, et al. The utility of laparoscopic evaluation of the parietal peritoneum in the management of anterior abdominal stab wounds. Injury. 2014;45 (1):128-133. 2. Lin HF, Wu JM, Tu CC, et al. Value of diagnostic and therapeutic 
laparoscopy for abdominal stab wounds:reply. World J Surg. 2013;37 (11):2721-2722

Disclosure: No significant relationships.

\section{PC060}

\section{MULTIDISCIPLINARY APPROACH TO CONSERVATIVE TREATMENT OF SEVERE LIVER LACERATIONS}

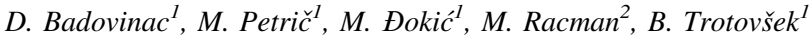

${ }^{1}$ Department Of Abdominal Surgery, University Medical Centre Ljubljana, Ljubljana/SLOVENIA, ${ }^{2}$ Department Of Cardiovascular Surgery, University Medical Centre of Ljubljana, Ljubljana/ SLOVENIA

Case History: A 21 years old male was injured in a motorcycle accident. Full body CT revealed polytrauma and fractures were surgically managed promptly. Severe lacerations of the liver were seen in the abdomen, with surrounding haematomas, but without active bleeding. Patient's clinical condition was stable, he was admitted to ICU. We decided to treat liver lacerations conservatively. During weeks to follow, numerous abdominal ultrasounds and CT scans were performed. Intraparanchymal haematomas were partially in regression.

Clinical Findings: The patient remained stable for one month, when we noticed a drop in haemoglobin levels and hematemesis.

Investigation/Results: Gastroscopy proved blood clots at duodenal papilla. A CT scan demonstrated an $11 \mathrm{~mm}$ wide pseudoaneurysm of the right hepatic artery, actively bleeding, and an enlarged, $8 \mathrm{~cm}$ big haematoma in the right liver. The aneurysm was also compressing and occluding the posterior right portal vein.

Diagnosis: Actively bleeding pseudoaneurysm of the right hepatic artery.

Therapy and Progression: Right hepatectomy was considered, but we decided to exploit conservative measures first. Interventional radiologist performed embolisation of the artery with endovascular spirals. Regular subsequent imaging demonstrated the portal vein becoming patent again and liver function slowly improving. The patient remained stable and three months after the accident, he was discharged.

Comments: Our case demonstrates an example of multidisciplinary cooperation, which managed to overcome the need for abdominal surgery in a severely injured patient with AAST liver injury grade II to III. It is an example of good clinical practice and should be thought of when conservative management of similar patients is considered. References:

Disclosure: No significant relationships.

\section{PC061}

ENDOLUMINAL BULLET. CASE REPORT. REAL CASE OR SCI-FI? R. Gràcia
Cladera $^{2}$, A. Aampos Serra

${ }^{1}$ General And Digestive Surgery, Hospital Universitari Parc Tauli, Sabadell/SPAIN, ${ }^{2}$ General And Digestive Surgery, Hospital Universitari Parc Taulí, Sabadell/SPAIN, ${ }^{3}$ Inteventionist Radiology,
Hospital Universitari Parc Taulí, Sabadell/SPAIN

Case History: A 34-year old male was admitted to our emergency department due to activation of polytrauma code because of a gunshot. At his arrival, the patient was hemodynamically stable and he presented 6 penetrating injuries (two in the thorax, 3 in the abdomen and 1 in the superior extremity).

Clinical Findings: A CT scan was requested showing 2 bullets in the subcutaneous tissue in the thorax, one in the gluteus, another one in the lumbar zone, one in the left superior extremity and the last one, which was intraabdominal, was next to the inferior cava vein, aside from the VII hepatic segment. The CT scan also showed a fracture in the iliac crest and pneumoperitoneum. Given the suspicious of perforation, the surgeons on call decided to perform a laparotomy, detecting a perforation of the small bowel requiring a resection and anastomosis without being able to find the bullet next to the inferior cava vein.

Investigation/Results: Another CT scan was performed 24 hours later

Diagnosis: showing the migration of the bullet to the right renal vein. Therapy and Progression: After much unsuccessful endovascular attempts to remove the bullet, the radiologist decided to place a vena cava filter. The filter was removed on the 20th postoperative day.

Comments: Intravascular migration of bullets has been sporadically described. Intraarterial bullets should be removed by endovascular approach but there is no consensus about intravenous bullets. It has been described endovascular removing and conservative treatment with observation. Given the low incidence, it is recommended an individualized treatment, being important having a good interventionist radiology team for a better management.

Disclosure: No significant relationships.

\section{PC062}

\section{SMALL BOWEL INJURY FROM PENETRATING ABDOMINAL TRAUMA: A LAPAROSCOPIC APPROACH}

\section{A. Marques, R. Loureiro, N. Carrilho, C. Casimiro}

General Surgery, Centro Hospitalar Tondela-Viseu, Viseu/ PORTUGAL

Case History: Laparoscopic surgery has been widely adopted in all areas of General Surgery. In the setting of abdominal trauma, it's mainly used as a diagnostic tool in penetrating injuries, avoiding nontherapeutic laparotomies. One of the main apprehensions regarding laparoscopy in trauma is the potential of missing injuries, which may result in significant morbidity or mortality. The first studies published in the 1990's showed high rates of missed injuries (41-70\%) however, subsequent studies describe rates ranging from 0 to $4 \%$. Laparoscopy as a diagnostic tool in trauma is widely accepted and the discussion is now focused on its therapeutic potential, avoiding the higher morbidity associated with open surgery. In penetrating trauma, laparoscopy can be used for diagnosis and treatment, with the main emphasis on diaphragmatic, hollow viscus and mesentery. The patient must be stable and the surgeon must have experience in laparoscopy. We present the case of a 27 -year-old policeman, with a stab wound to the left flanc.

Clinical Findings: The patient was stable, and the wound penetrated the peritoneal cavity but there was doubt regarding intra-abdominal injuries. 
Investigation/Results: An emergent diagnostic laparoscopy was performed revealing a perforation to the small bowel. A thorough inspection revealed no other injuries or free fluid in peritoneal cavity. Diagnosis: Bowel perforation

Therapy and Progression: The perforation was sutured without the need for laparotomy. The patient was discharged on the $3^{\text {rd }}$ postoperative day without relevant complications.

Comments: Laparoscopy is a viable option in selected trauma cases, allowing the diagnosis and treatment of injuries and avoiding the potential morbidity and mortality associated with non-therapeutic laparotomies.

References: 1. Zafar SN, Onwugbufor MT, Hughes K, et al. Laparoscopic surgery for trauma: the realm of therapeutic management. Am J Surg. 2015;209 (4):627-32 2. Lim KH, Chung BS, Kim JY, et al. Laparoscopic surgery in abdominal trauma: a single center review of a 7-year experience. World J Emerg Surg. 2015;10:16 3. Uranues, S., Popa, D.E., Diaconescu, B. et al. Laparoscopy in Penetrating Abdominal Trauma, World J Surg (2015) 39: 1381. 4. Matsevych, Oleh Yevhenovych et al. Laparoscopic-assisted approach for penetrating abdominal trauma: A solution for multiple bowel injuries, International Journal of Surgery, Volume 44, 94 - 98

Disclosure: No significant relationships.

\section{PC063}

\section{NONOPERATIVE MANAGEMENT OF GRADE III SPLENIC INJURY - CASE REPORT}

\author{
M. Laureano, J. Nobre, I. Gonçalves, E. Ferreira, N. Ventura, \\ S. Hilário, J. Pais, M.C. Santos
}

Cirurgia 2, Centro Hospitalar de Leiria, Leiria/PORTUGAL

Case History: 66-years old male fell down from a tree $(\approx 2 \mathrm{~m})$, resulting in toracoabdominal trauma. Previous history of prostatic benign hypertrophy, hypertension, dyslipidemia and kidney stones.

Clinical Findings: At admission on the emergency room, patient presented with hemodynamic stability $(139 / 84 \mathrm{mmHg}$, HR 66bpm), eupneic, complaining of left thoracic pain. CT showed 4 fractured ribs on the left side with ipsilateral incipient pneumothorax and small pleural effusion. On abdomen, CT showed a grade III splenic injury with a large laceration $(>5 \mathrm{~cm})$, intraparenchimatous hematomas, subcapsular hematoma, subphrenic collection and small amount of free fluid on the left parietocolic groove and Douglas pouch. Preservation of splenic hilar vessels was confirmed.

Investigation/Results: Next day control CT was performed with overlapping results. The posterior imagiologic reevaluation showed favorable evolution. Analytically, there was a drop on hemoglobin level $(2 \mathrm{~g})$ during the first $48 \mathrm{~h}$ with posterior stabilization.

Diagnosis: Grade III splenic injury, 4 broken ribs and pneumothorax. Therapy and Progression: Due to the clinical stability it was decided for conservative treatment with vital signs monitorization and clinical, analytical and imagiological close control. Discharge on the 13th day with no complaints. Follow-up CT 4 months after the trauma showed no evidence of splenic lesion.

Comments: The conservative treatment of splenic injuries has been established mainly for low degree injuries (grade I-II) and is being increasingly accepted for higher grades. Thus we report a successfully nonoperative management grade III splenic injury, performing a close and constant hemodynamic and imaging patient control in order to improve outcoming results.

References: - Cristian Bălălău, Nicolae Bacalbasa, Ion Motofei, Petrisor Banu, Răzvan Scăunasu, Bogdan Popescu, Oana-Denisa
Bălălău, Vlad-Denis Constantin. Noperative Management for Splenic Lesions in Politraumatized Patients - CASE REPORT AND LITERATURE REVIEW. Archives of the Balkan Medical Union, vol. 52, no. 1, pp. 99-105, March 2017 - Branco BC, Tang AL, Rhee P, Fraga GP, Nascimento B, Rizoli S, O'Keeffe T. Tratamento não operatório do trauma de baço grave. Rev Col Bras. Cir. [periódico na Internet] 2013;40 (3). Disponível em URL: - Gerson A Pereira Júnior, Júlia Batista de Carvalho, Geraldo S Prado Neto, Juliana R Guedes. Tratamento não operatório do trauma de vísceras abdominais parenquimatosas. Medicina, Ribeirão Preto 2007; 40 (4): 538-50, out-dez. Disclosure: No significant relationships.

\section{PC064}

\section{LAPAROSCOPIC APPROACH IN THE MANAGEMENT OF STAB WOUNDS IN ANTERIOR ABDOMEN}

\author{
A. Landaluce-Olavarria, B. Ugarte-Sierra, S. Postigo, S. Telletxea, \\ F.J. Ibañez Aguirre
}

General Surgery, hospital Galdacano, Galdacano/SPAIN

Case History: Patient 1 went to the emergency room after an attempted suicide with benzodiazepin intake and a self-stab in the epigastrium $2 \mathrm{cms}$ below sternum xiphoid. Patient 2 went to the emergency after a fortuite stab to the left iliac fossa. .

Clinical Findings: In patient 1, a penetrating stab wound near sternum xifoides was found. Patient 2 had a penetrating stab wound in left iliac fosse

Investigation/Results: Eco and CT was performed

Diagnosis: Patient 1.Ecofast was negative and CT was normal. Patient 2.CT showed an evisceration through the stab wound.

Therapy and Progression: Patient 1. Laparoscopic approach was performed without perforated hollow viscus findings.He recovered 24 hours after laparoscopic exploration and he was transferred to the psychiatry department to treat the mental disorder. Patient 2. Laparoscopic approach was performed to explore abdomen. Only a minimun hemoperitoneum due to abdominal wall bleeding was found. A wall reconstruction by mesh was carried out later. His progress was suitable and he was discharged at 5th day.

Comments: Bibliography reveals that in patients without evident indications for immediate need for laparotomy physical examination is both sensitive and specific for detecting significant intra-abdominal injury. CT is good exploration to rule out an intraabdominal injury but even with a negative initial CT scan, patients with a high likelihood of diaphragmatic or bowel injury should have further observation and serial examinations. CONCLUSIONS 1. Laparoscopic approach is a good option when serial physical examinations is not possible. 2.Laparoscopic approach can avoid a laparotomy approach with the same results than laparotomy and with minimal injury of the tissues References: 1.Selective nonoperative management of penetrating abdominal solid organ injuries. Demetriades D, Hadjizacharia P, Constantinou C, Brown C, Inaba K, Rhee P, Salim A SO Ann Surg. 2006;244 (4):620-8. 2. Practice management guidelines for selective nonoperative management of penetarting abdominal trauma. Como JJ, et al. J .trauma 2010 Mar68 (3):721-33

Disclosure: No significant relationships. 


\section{PC064A}

\section{NON OPERATIVE MANAGEMENT OF PENETRATING EXTRAPERITONEAL RECTAL INJURY}

\author{
C. Gamo, C. Miranda, A. Salcedo, J.J. Serna, S. De Lebruisant, \\ A. Gonzalez, R. Rodriguez
}

\section{Cali, Hospital Universitario del Valle, Cali/COLOMBIA}

Case History: 14 years old woman, with transpelvic gunshot injury 9 hours prior to the arrival to our hospital. Presents transpelvic injury with the projectile entering right gluteal region and allocated in the left side of the pelvis.

Clinical Findings: The patient remains asimptomatic, with laboratory exams without alterations.

Investigation/Results: In the $\mathrm{CT}$ is evidenced a presacral hematoma with minimum extraperitoneal bubbles. We perform a rectosigmoidoscopic study unsuccessful for the presence of fecal material and start antibiotic and analgesic therapy. After 48 hours we repeat rectosigmoidoscopy with oral colonic preparation, evidenciating a healed injury with the appearance of rectal ulcera ulcera without evident perforation.

Diagnosis: Diagnosed as extraperitoneal rectal injury due to gunshot Therapy and Progression: We decide to manage nonoperatively. The patient is discharged after 4 days in-hospital assimptomatic with normal analitic exams. Revised 8 days after the discharge with good evolution and pending a control rectosigmoidoscopy 6 weeks after the injury.

Comments: Rectal extraperitoneal injuries presents high mortallity and morbidity. The treatment is controversial in terms of presacral drain and rectal lavage. The fecal diversion, though, remains established as standard treatment. Nonoperative management has been proposed for nondesctructive, less than $25 \%$ of rectal circumference lessions, with good results published. Nonoperative management may be feasible in selected patiens.

References: -Is fecal diversion necessary for nondestructive penetrating extraperitoneal injuries? Richard P. Gonzalez et al, The journal of trauma oct 2006. -Management of penetrating extraperitoneal rectal injuries: An Eastern Association for the Surgery of Trauma practice management guideline. Journal of trauma and acute care surgery, march 2016

Disclosure: No significant relationships.

\section{PC065}

\section{CASE REPORT- BLUNT THYMIC TRAUMA IN A 6-YEAR- OLD BOY}

\section{Y. Wu}

Trauma And Emergency Surgery Department, Chang Gung Memorial Hospital, Taoyuan County/TAIWAN

Case History: A 5-year-5-month-old boy was transferred to our emergency department from local hospital with a history of blunt chest and abdominal trauma. It was a two-car collision and the patient sat just behind the driver without a child safety chair, where is also the collision point by the approaching car. He was born to a healthy term baby. There was no abnormal finding on antenatal examination and newborn screening, and achieved normal developmental milestones appropriate to his age.
Clinical Findings: On arrival, he had a Glasgow coma score of 15, respiratory rate of 23 per minute, heart rate of 97 beats per minute, and blood pressure of $113 / 51 \mathrm{mmHg}$. Physical examination showed tenderness over upper abdomen but no ecchymosis or open wound over chest or abdominal wall. Other findings included contusions at forehead, left forearm and elbow.

Investigation/Results: The chest radiograph revealed a significant widened mediastinum. Lab data revealed an elevation of liver enzymes and normal hemoglobin. Subsequent computed tomography (CT) showed intracapsular contrast extravasation of thymus, liver laceration without contrast extravasation, and bilateral lung contusion.

Diagnosis: The diagnosis included thysmic bleeding, AAST (American Association for the Surgery of Trauma) grade III liver laceration, and bilateral lung contusion.

Therapy and Progression: During admission, his vital signs remained stable. Dynamic CT was followed 6 days later and no more active bleeding was detected. He was discharged after admission for 1 week. He was followed in our out-patient-clinic uneventfully for 4 months.

Comments: Thymic bleeding is a very rare chest trauma in child. Conservative treatment may be appropriate but close monitoring is required.

References: 1. Takahashi Y, Toyoda Y, Okada Y. Thymic Intracapsular hematoma caused by blunt chest trauma. Ann Thorac Surg. 2003;76:2107. 2. Gschwentner M, Gruber G, Oberladstätter J, Kralinger F, Rieger M. Mediastinal widening after blunt chest trauma in a child: a very rare case of thymic bleeding in a child and possible differential diagnosis. J Trauma. 2007;63:E51-E54.

Disclosure: No significant relationships.

\section{PC066}

\section{NONOPERATIVE MANAGEMENT FOR YOUTH PATIENT IN IV GRADE BLUNT HEPATIC TRAUMA}

\author{
B. Muñoz Jimenez ${ }^{1}$, G. Krasniqi ${ }^{1}$, J. Hernández Gutiérrez ${ }^{1}$, \\ A. Trinidad Borrás $^{1}$, M.J. Lucena González ${ }^{2}$, J.S. Malo Corral, \\ D. Martínez Cecilia ${ }^{I}$, P. Toral Guinea ${ }^{I}$, I. Fraile Alonso ${ }^{I}$ \\ ${ }^{1}$ General Surgery, C.H. Toledo, Toledo/SPAIN, ${ }^{2}$ Radiology, C.H \\ Toledo, Toledo/SPAIN
}

Case History: The liver is the most injured organ in abdominal trauma. The severity of liver injuries is universally classified according to the AAST grading scale. We report a case of severe hepatic trauma.

Clinical Findings: 14 year old male was brought to emergency room with history of road cycle accident. On examination he was abdominal pain. He was conscious, oriented, nauseous, had pallor and sweaty, but hemodynamically stable. Abdominal examination revealed tenderness in the right upper quadrant with guarding.

Investigation/Results: Laborotory investigations revealed a white cell count of $16.9 \times 10^{\wedge} 9 / \mathrm{L}$, Neu $77 \%$, hemoglobin of $9.9 \mathrm{gr} / \mathrm{dL}$., coagulopathy with elevation of serum liver enzymes.

Diagnosis: CT scan was performed which revealed grade IV hepatic trauma with lacerations in segment 6 of the liver associated with a large hemoperitoneum with active bleeding from the inferior liver surfaces in arterial phases.

Therapy and Progression: Despite adequate resuscitation with whole blood. Patient was shifted to ICU and managed conservatively. An effective angioembolization was performed. Patient had complications due to residual hemoperitoneum. He needed to evacuate it by 
percutaneous drainage on two occasions. An abscess in the hepatic bed where the laceration was observed in CT. The boy needed broadspectrum antibiotic. The patient was discharged on the 10th day. During follow-up, abdominal ecography didn't demonstrate progression of the injury.

Comments: Currently, the conservative management of liver trauma is considered the "Gold Standard" and the criteria is often based on hemodynamic stability. Its successful management should be based on early CT recognition of arterial bleeding and prompt use of angiographic embolization to control it.

References: - "Traumatic hepatic artery laceration managed by transarterial embolization in a pediatric patient" Fallon SC, Coker MT, Hernandez JA et al. J Pediatr Surg. 2013 May;48 (5):E9-12. "The rate of success of the conservative management of liver trauma in a developing country" Buci M, Torba A, I. Kajo et al. World Journal of Emergency Surgery (2017) -"WSES classification and guidelines for liver trauma" Coccolini F, Catena F, Moore EE et al. World Journal of Emergency Surgery (2016) 11:50 - "Treatment strategy for hepatic trauma" Wu-Yong Yu a, b, Qu-Jin Li a, Jian-Ping Gong a, * Chinese Journal of Traumatology 19 (2016) 168e171 Disclosure: No significant relationships.

\section{PC067 \\ BLEEDING IN SEVERE PEDIATRIC TRAUMA: IMPORTANCE OF FACTOR XIII}

\author{
R. Roquette, E. Vieira, A.P. Martins, M. Gonçalves
}

Pediatric Surgery, Centro Hospitalar Lisboa Norte, EPE, Lisbon/ PORTUGAL

Case History: We present two cases of trauma-related coagulopathy: 1) 17 years-old male victim of car accident with brain injury (GCS < 8), distal femur fracture without vascular compromise, blunt thoracic trauma with aortic pseudoaneurism and blunt abdominal trauma with grade III hepatic lesion and moderate hemoperitoneum, which was managed conservatively; 2) 12 years-female, with vWB family history, victim of 8 meters fall with blunt abdominal trauma with grade III hepatic lesion and moderate hemoperitoneum, also managed conservatively.

Clinical Findings: 1) At admission, was given packed red blood cells (PRBCs) and fresh frozen plasma (FFP), maintaining hemodynamic stability. In post-operative period for fracture reduction, presented with surgical wound hemorrhage, refractory to compression, with a minimum $\mathrm{Hb}$ of $6 \mathrm{~g} / \mathrm{dL}$ requiring PRBCs; 2) In the first $24 \mathrm{~h}$ after trauma, for worsening hemoperitoneum and lowering hemoglobin, FFP and PRBCs were administered, with repeated administration because of persistent low hemoglobin levels despite stabilized hemoperitoneum extension.

Investigation/Results: For non-expected persistent hemorrhage both patients had complete coagulation investigation, with detection of low XIII factor levels (19-25\%, for normal levels $>75 \%$ ), without other coagulation deficit or platelet dysfunction.

Diagnosis: Hemorrhage induced factor XIII deficiency was assumed as the most probable cause.

Therapy and Progression: Twice daily FFP was administered until factor XIII levels consistently reached above $40 \%$, with controlled hemorrhages.

Comments: Coagulopathy in trauma is likely multifactorial, and if present at admission is associated with poorer outcome. In younger children, variability of maturation of coagulation factors and platelet function must be considered. Recently, XIII factor was described as the first to deplete in severe acute hemorrhage, contributing to its persistence.

References: 1 . Christiaans S, et al. Coagulopathy after severe pediatric trauma: A review. Shock (2014) 41 (6): 476-490 2. Dehmer J, et al. Massive transfusion and blood product use in the pediatric trauma patient. Seminars in Pediatric Surgery (2010) 19, 286-291 3. Shaz B, et al. Transfusion Management of Trauma Patients. Anesth Analg (2009) 108 (6): 1760-1768

Disclosure: No significant relationships.

\section{PC068}

\section{SECONDS FROM DISASTER - COMMON OPERATION, BAD POSTOPERATIVE FOLLOW-UP, MAJOR DAMAGES}

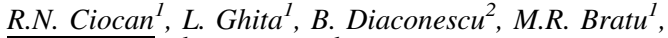 \\ D.L. Bajenaru ${ }^{l}$, R. Marian
}

${ }^{1}$ Surgery, Emergency Hospital Bucharest, Bucharest/ROMANIA,
${ }^{2}$ Surgery Department, Emergency Clinical Hospital, Bucharest/
ROMANIA

Case History: We present an impressive case of a 22-year-old appendectomy patient, poorly traced postoperatively, hospitalized in septic shock with an old, neglected peritonitis, requiring more major surgery, sustained intensive care, important sequelae in turn solved. The case demonstrates that regular operations can lead to disasters if they are not done and tracked properly.

Clinical Findings: The patient was hospitalized 2 weeks after an appendectomy in another hospital, for intense abdominal pain, abdominal distention, abdominal guarding, fever, nausea, altered general condition. Upon palpation the abdomen has a typical appearance of plastic peritonitis in the lower $2 / 3$, tachycardia, hypothension.

Investigation/Results: At admission WBC 28000/mmc, T 810000/mmc, INR 1.2, lipase 317U/l. CT: major multiple fluid collections in pelvis, between bowels, dynamic obstruction.

Diagnosis: The initial diagnostic was mecanoinflammatory obstruction by neglected old general peritonitis probably by unrecognised cecal fistula.

Therapy and Progression: First operation: large abscesses in pelvis, between small bowels, parietocolic, necrotics areas on ascending colon: Right ileo-hemicolectomy, terminal ileostomy. After 8 days with good evolution, with decrease of vasopresors, and septic schock, the patient developped obstruction signs, CT reveal intraabdominal collections and ileon ischemya. Relaparotomy: intraabdomial abscesses, extensive adessions, small bowel ischemia but not infarction. Intensive washing, multiple drainages, Abdominal closure only with skin and heparinotherapy postoperative. Good evolution under major antibiotherapy, discharge at 33 days, anticoagulants for 4 months. Reoperation after 8 months: reverse ileostomy with excellent results.

Comments: This case proves the catastrophic consequences of a poor postoperative follow-up after an operation that is usually benign. Complications developed can be major, at vital risk if not recognized on time.

References:

Disclosure: No significant relationships. 


\section{$\operatorname{PC069}$}

\section{NEGATIVE PRESSURE WOUND THERAPY IN COMPLEX PELVIC INJURY}

\author{
C. Felicio, C. Ramos, C. Lavado, P. Fernandes, J. Coutinho
}

Cirurgia, CHLN - Hospital de Santa Maria, Lisboa/PORTUGAL

Case History: We report the case of a 28 year old female polytrauma patient that was admitted in our Emergency Department with severe injuries after falling off the 4th floor balcony with impalement in the sacral area.

Clinical Findings: After complete assessment the following injuries were identified: minor head trauma, vertebral injuries, extremity lesion, blunt thoracoabdominal trauma, type $\mathrm{C}$ pelvic fracture, comminuted sacral fracture, coccyx avulsion and a large wound with loss of fat and muscle tissue and rectal exposure.

Investigation/Results: The presence of hemorrhagic shock determined the initial approach and injury management: external pelvic fixation, terminal colostomy for adequate fecal diversion and surgical sacral wound cleaning and packing with differed application of negative pressure wound therapy (NPWT).

Diagnosis: The patient was admitted in the intensive care unit in the post operative time.

Therapy and Progression: The management of sacral wound with NPWT was of utmost importance in controlling infection until the definitive lumbopelvic fixation by the Orthopedists and wound closing with musculocutaneous flap of the gluteus maximus by the Plastic Surgeons.

Comments: The multidisciplinar approach was crucial to provide the better treatment in this case and although we are still waiting for a systematic review on the cost-effectiveness of NPWT in traumatic wounds, our results in this case, support its use in this setting.

References: Newton K, et al. Negative pressure wound therapy for traumatic wounds. Cochrane Database of Systematic Reviews 2017, Issue1.Art.No.:CD012522. Petrone P, et al. Treatment of Complex Perineal Trauma. A Review of the Literature. CirEsp.2016;94:313-322.

Disclosure: No significant relationships.

\section{PC070}

\section{IS NON-OPERATIVE MANAGEMENT OF HIGH-GRADE LIVER INJURY A POTENTIAL TREATMENT MODALITY? CASE REPORT}

\section{Torba, R. Madani, S. Ceka, A. Mici, K. Subashi}

Surgery, Trauma University Hospital, Tirana/ALBANIA

Case History: A 38-year-old man was referred to our centre from a regional hospital after a motor vehicle crash. We present a case of grade four blunt liver injury, which was managed successfully nonoperatively.

Clinical Findings: Upon admission, the patient was hemodynamically unstable with a blood pressure of $80 / 50 \mathrm{mmHg}$, heart rate of 120 $\mathrm{bpm}$, and respiratory rate of 28 breaths. He complaining of diffuse abdominal discomfort, distention and shortness of breath, without peritoneal signs.

Investigation/Results: FAST revealed large amounts of free fluid in abdominal cavity and a right pneumohemothorax. Laboratory test showed a red blood cell count of 3.1 million/mm3, hemoglobin 9.2 $\mathrm{g} / \mathrm{dl}$, hematocrit $30.1 \%$, and leukocytosis $\left(26,000 / \mathrm{mm}^{3}\right)$. ALT 1200 U/L and AST $200 \mathrm{U} / \mathrm{L}$. Total body CT scan performed after an initial positive response to resuscitation showed second rib fracture and a right pneumohemothorax, subcapsular liver hematoma, hepatic fracture and free fluid in all peritoneal spaces.

Diagnosis: Blunt liver injury (grade four), subcapsular liver hematoma and hepatic fracture

Therapy and Progression: The patient was selected for non operative management.Serial physical examination, hemodynamic monitoring, serial complete blood test and liver function tests were performed. Intravenous fluids, broad-spectrum antibiotic, analgesic, PPI, anticoagulant therapy, whole blood and fresh frozen plasma were given. On the tenth day free intraabdominal fluid was completely absorbed and liver function tests returned to normal levels. The patient was discharged four weeks later without any events. Ultrasound and CT scan follow-up three, six months and one year after trauma showed gradual reduction of hepatic cyst. The patient remained asymptomatic during one and a half year follow-up.

Comments: High grade liver injuries in hemodynamically stable patients can be managed nonoperatively successfully. Closely hemodynamic monitoring, physical examination of patients, serial complete blood count, liver function test, and surgical team able to perform hepatic surgery on time are preconditions to this management modality.

References: Ward J, Alarcon L, Peitzman AB. Management of blunt liver injury: what is new? Eur J Trauma Emerg Surg. 2015; 41: 229-37. Coimbra R, Hoyt DB, Engelhart S, et al. Nonoperative management reduces the overall mortality of grades 3 and 4 blunt liver injuries. Int Surg. 2006; 91: 251-7.

Disclosure: No significant relationships.

\section{PC071}

\section{TRAUMATIC RUPTURE OF A SPLENIC CYST HYDATID}

\section{N. Ozlem}

Faculty Of Medicine; General Surgery, Ahi Evran University, Kırsehir/TURKEY

Case History: 37 year old female sustained traffic accident presented our emergency department.

Clinical Findings: In her physical examination there was hemodynamic instability, peritoneal irritation sign.

Investigation/Results: An ultrasonographic examination of her abdomen showed intraperitoneal free fluid, a splenic rupture.

Diagnosis: The patient urgently operated; a $11 \times 9 \times 5 \mathrm{~cm}$ ruptured $\mathrm{HC}$ in the spleen was treated by splenectomy.

Therapy and Progression: During surgery the intraabdominal spaces were washed with povidoniodine and saline. Postoperative course of the patient was uneventful. The spleen was an organ involved with hydatidosis in our patient. Pathologic examination of the specimen revealed a splenic hydatid cyst. The patient is symptom free for 18 months.

Comments: Several traumatic ruptured splenic HC case are encountered in the literature. The cases in the literature almost always are not case report but those are one or more than one case in a case series. This case, an extremely infrequent encountered is reporting.Early discovery is important since it is possible to cure viable HCs without dissemination to other organs by conservative surgery. If our patient was examined by ultrasound in a routine check up then her hydatid cyst of spleen would be discovered, the treatment of it would 
be made so no peritoneal dissemination with cystic fluid will be occur.

References: 1. Col C, Col M, Lafci H. Unusual localisations of hydatid disease. Acta Med Austriaca 2003;30:61-4. 2. Derici H, Tansug T, Reyhan E, Bozdag AD, Nazli O. Acute intraperitoneal rupture of hydatid. Cysts World J Surg 2006;30:1879-83. 3. Gunay K, Taviloglu K, Berber E, Ertekin C. Traumatic rupture of hydatid cysts: a 12-year experience from an endemic region. J Trauma 1999;46 (1):164-7. 4. Dar MA, Shah OJ, Wani NA, Khan FA, Shah P. Surgical management of splenic hydatidosis. Surg Today 2002;32 (3):224-9 . 5. Bitton M, Kleiner-Baumgarten A, Peiser J, Barki Y, Sukenik S. Anaphylactic shock after traumatic rupture of a splenic echinococcal cyst. Harefuah 1992;122 (4):226-8, 16. 6. Placer C, Martin R, Sanches E, Soleto E. Rupture of abdominal hydatid cysts. Br J Surg 1988;75:157-61. 7. Kok AN, Yurtman T, Aydin NE. Sudden death due to ruptured hydatid cyst of the liver. J Forensic Sci 1993;38:978-80. 8. Saenz de San Pedro B, Cazana JL, Cobo J, Serrano CL, Quiralte J, Contreras J, et al. Anaphylactic shock by rupture of hydatid hepatic cyst. Follow-up by specific IgE serum antibodies. Allergy 1992;47 (5):568-70. 9. Ozdogan M, Baykal A, Keskek M, Yorgancy K, Hamaloglu E, Sayek I. Hydatid cyst of the spleen: treatment options. Int Surg 2001;86 (2):122-6. 10. Aronson J.K. polyvidone in Meyler's s.ide effects of antimicrobial drugs. disinfectants and antiseptic, pp 2899-2905, 15.Ed., Elsevier Science. Oxford, UK 2006. 11. van Westreenen M, van den Tol PM, Pronk A, Marquet RL, Jeekel J, Leguit P. Perioperative lavage promotes intraperitoneal adhesion in the rat. Eur Surg Res 1999;31 (2):196-201

Disclosure: No significant relationships.

\section{PC072}

\section{A DIAGNOSTIC NEGATIVE ULTRASOUND FINDING IN BLUNT ABDOMINAL TRAUMA}

F.M. Abu-Zidan $^{1}$, H.S. Shalak ${ }^{2}$, M.A. Alhaddad ${ }^{2}$

${ }^{1}$ Surgery, College of Medicine, UAEU University, Al-Ain/UNITED ARAB EMIRATES, ${ }^{2}$ Surgery, Al-Ain Hospital, Al-Ain/UNITED ARAB EMIRATES

Case History: A 50-year-old obese man presented complaining of severe generalized abdominal pain and dysuria of three days duration after he slipped on his abdomen.

Clinical Findings: The patient was haemodynamically stable. The abdomen was distended and tender all over. There was no blood on the urethral meatus

Investigation/Results: FAST scan showed significant intra-peritoneal fluid. The urinary bladder could not be seen. A Foley catheter was inserted which drained 3 liters of clear urine. The abdominal distention became less. Repeated FAST scan was completely negative. Diagnosis: A clinical diagnosis of major intra-peritoneal rupture of the urinary bladder was made. Trauma CT scan confirmed the diagnosis.

Therapy and Progression: Laparotomy closure of a $10 \mathrm{~cm}$ intraperitoneal rupture of the dome of the urinary bladder in 2 layers using Vicryl 3/0.

Comments: The change of a positive FAST to a negative FAST was diagnostic of a major intra-peritoneal urinary bladder rupture even before performing a trauma CT scan. When performing FAST in blunt abdominal trauma, it is more useful to perform FAST before inserting a urinary catheter and it is advised to repeat it.
References:

Disclosure: No significant relationships.

\section{PC073}

\section{ABDOMINAL SEAT-BELT INJURY, THE IMPORTANCE OF EARLY TREATMENT AND PROPER FOLLOW-UP IN A PEDIATRIC PATIENT - A CASE REPORT}

\author{
X.P. De Sousa, P.S. Ferreira, L. Branco, S.P. Carmo, V. Rigueira, \\ L. Cortez
}

General Surgery, Hospital São Bernardo, Centro Hospitalar de Setúbal, EPE, Setúbal/PORTUGAL

Case History: We report a case of a 13-year-old female, victim of a car accident with frontal collision while sitting in the front seat, next to the driver with the seat-belt on. The accident caused a mortal victim.

Clinical Findings: At admission GCS 15, hemodynamically stable, no important wound, limitations or pain to cervical or limb movement were present. She complained of abdominal pain, had an abdominal ecchymosis and pain to palpation of the lower abdominal quadrants. Investigation/Results: Analytically $\mathrm{Hb} 8,9 \mathrm{~g} / \mathrm{dL}$. FAST demonstrated free intra-abdominal fluid with no apparent lesion to massive organs. CT scan showed hemo/pneumoperitoneum, retroperitoneal and anterior abdominal wall hematoma.

Diagnosis: Acute traumatic abdomen with hemo/pneumoperitoneum. Therapy and Progression: Prompt laparotomy showed a voluminous hemoperitoneum, lacerations of the small bowel, mesenteric lacerations with bleeding vessels, a non-expanding retroperitoneal hematoma near the right kidney and destruction of the anterior abdominal wall with dissection of the muscles and fascia. During hospitalization psychological support was needed, due to anxiety and fear of loneliness. She was discharged after 12 days, with follow-up in Pediatric Surgery, Physical Rehabilitation and Psychology consultations, showing progressive improvement.

Comments: This case illustrates the typical seat-belt abdominal injury. In the presence of intra-abdominal injuries prompt laparotomy is indicated. When dealing with pediatric patients, not only physical injuries need to be taken care of. The confrontation with death and near-death experience, may assume big proportions leading to the development of acute and post-traumatic stress disorder. This needs to be kept in mind and appropriate screening and Psychology follow-up is warranted for early treatment to minimize long term sequelae.

References: 1 - M. Torba, et al. Seat belt syndrome, a new pattern of injury in developing countries. Case report and review of literature, $\mathrm{G}$ Chir. 2014 Jul-Aug; 35 (7-8): 177-180. 2 - Velmahos GC, et al. The "seat belt mark" sign: a call for increased vigilance among physicians treating victims of motor vehicle accidents. Am Surg. 1999 Feb;65 (2):181-5. 3 - Jeffrey Odenbach, et al. Screening for post-traumatic stress disorder after injury in the pediatric emergency department - a systematic review protocol. Syst Rev. 2014; 3: 19.

Disclosure: No significant relationships.

\section{PC074}

STAB WOUNDS ON THE ANTERIOR ABDOMINAL WALL: A RARE CAUSE OF LARGE HEMOPERITONEUM. CASE REPORT 
M. Torba, R. Madani, K. Subashi

Surgery, Trauma University Hospital, Tirana/ALBANIA

Case History: A 20-year-old man referred to our hospital from a local hospital with penetrating abdominal trauma. The aim of this paper was to present a rare cause of hemoperitoneum and its management.

Clinical Findings: The patient was hemodynamically stable, had seven stab wounds on the anterior abdominal wall, no peritoneal signs, and no past medical history.

Investigation/Results: Focused Assessment Sonography for Trauma (FAST) detected a large amount of free intraperitoneal fluid. Enhanced contrast abdominal CT scan confirmed the findings of ultrasonography, but not the bleeding source. A complete blood count (CBC) showed a red blood cell count of $3.91 \times 105 / \mu \mathrm{L}$, hemoglobin level of $11.5 \mathrm{~g} / \mathrm{dL}$, hematocrit of $33.1 \%$ and a normal white blood cell count. Coagulation and biochemical parameters were within the normal reference.

Diagnosis: Stab wounds on the anterior abdominal wall with large hemoperitoneum.

Therapy and Progression: About ten hours later, the patient began complaining of diffuse abdominal discomfort and distention, shortness of breath and tachypnea. On physical examination without peritoneal signs, CBC showed a severe drop in $\mathrm{RBC}(2.6 \times 105 / \mu \mathrm{L})$, $\mathrm{Hgb}(6.5 \mathrm{~g} / \mathrm{Dl})$, Hct $(23.2 \%)$ and the normal value of WBC. The patient underwent emergency laparotomy which detected active bleeding from the visceral site of the anterior abdominal wall stab wounds. Suturing of the bleeding vessel with the surrounding tissue was performed. Seven days later the patient was discharged from the hospital in good condition.

Comments: CT scan visualization of the abdominal wall bleeding vessels is often difficult. Carefully physical examination follow-up and serial complete blood count monitoring are important to prevent massive blood loss which leads to an exceedingly high mortality rate. References: Leppäniemi AK, Haapiainen RK. Selective nonoperative management of abdominal stab wounds: prospective, randomized study. World J Surg. 1996; 20:1101-5. Como JJ, Bokhari F, Chiu WC, et al. Practice management guidelines for selective nonoperative management of penetrating abdominal trauma. J Trauma. 2010; 68: 721-33.

Disclosure: No significant relationships.

\section{PC075}

\section{PENETRATING INJURY TO THE LARYNX AND THE HYPOPHARYNX}

\section{A. Hallal ${ }^{1}$, H.M. Farhat ${ }^{2}$}

${ }^{1}$ Department Of Surgery, American University of Beirut Medical Center, Beirut/LEBANON, ${ }^{2}$ General Surgery, american University of Beirut Medical Center, beirut/LEBANON

Case History: A 26-year-old lady sustained a Low Velocity Gunshot to the Neck from Close Range.

Clinical Findings: The bullet entry is $2 \mathrm{~cm}$ lateral to the Thyroid cartilage on the left side (Zone 2) while the exit is a 5-cm wound over the right trapezius muscle. On arrival, her Spo2 was $50 \%$ with hard signs for airway injury (sucking wound, oropharyngeal bleed, subcutaneous emphysema). Airway was secured with an endotracheal tube through the neck.
Investigation/Results: Investigation: CTA Neck: Defect in the subglottic larynx with fracture of the thyroid cartilage. Defect in the posterior aspect of the glottis and supra glottis. Narrowing of the right Vertebral, left common and internal carotid arteries with no evidence of bleeding. Operative Findings: Fractured Thyroid cartilage with injuries to the aretynoid muscles. Surgical tracheostomy created then ETT removed. Rigid esophagoscopy showed a clear perforation at the junction of the hypopharynx/esophagus. the right upper lobe of the thyroid was mobilized identifying an injury through the inferior constrictor muscle into the hypopharynx. The injury was $3 \mathrm{~cm}$ long involving $50 \%$ of the circumference of the hypopharynx esophagus junction. The defect was primarily repaired. A Stent was placed in the thyroid cartilage and covered by muscle flaps.

Diagnosis: Tracheal Transection, Hypopharynx /esophageal injury, Brachial Plexus Injury (Clinical and MRI), vertebral artery thrombosis.

Therapy and Progression: Barium swallow: No leak, good swallow, no aspiration. Right Upper Extremity Paralysis.

Comments: Securing the Airway is always the priority, Definitive surgery comes next. (will add photos at presentation and intraoperative. In addition to investigations done)

References:

Disclosure: No significant relationships.

\section{PC076}

\section{PEDIATRIC ABDOMINAL BLUNT TRAUMA: CASE REPORT}

I.M.F.D. Lobo Gonçalves, F. Mendoza Moreno, M. Perez Gonzales, J. Minguez Garcia, C. Medina Reinoso, H. Aguado Lopez,

F. Hernandez Merlo, F. Noguerales Fraguas, L. Jimenez, Alvarez.

Cirugía General Y Del Aparato Digestivo, Hospital Universitario Príncipe de Asturias, Alcalá de Henares/SPAIN

Case History: Introduction: Duodenal injuries occur in 2-10\% of children with blunt abdominal trauma. The mechanism of action is a direct hit in epigastrium (traffic accidents, sports, bicycle handlebars ...). Case history: We present a case of a 17 year old child, who suffered a blunt epigastric trauma with the handlebars of his bicycle. Clinical Findings: Clinical findings: He arrived at the hospital 60 minutes after the accident complaining of right hypochondrial pain with good vital constants and no other symptoms. After 90 minutes of observation the pain had increased and he presented bad general condition and haematemesis. The CT showed perforation of the posterior wall of the duodenum by the $3^{\text {rd }}$ portion, with leak of oral contrast seen on TC.

Investigation/Results: The current literature was properly reviewed. Diagnosis: Diagnosis: Duodenal perforation due to blunt abdominal trauma.

Therapy and Progression: Therapy: The patient underwent surgery through a transverse laparotomy, where a 2 centimetre perforation was found, as well as a non-expansive retroperitoneal haematoma. A segmental duodenectomy with pancreas preservation and a Roux-enY duodenojejunostomy were performed. Results: As postoperative complications the patient presented abdominal wall haematoma and moderate pancreatic enzyme elevation, which were treated conservatively, and he was discharged 16 days after surgery.

Comments: Comments: The mechanism of action is a direct hit in epigastrium (traffic accidents, sports, bicycle handlebars...). Duodenal injuries after trauma are usually identified late and are associated 
with increasing morbidity and mortality. Its treatment is essentially surgical and its evolution depends on the promptness of diagnosis.

References: 1. Dhua AK, Joshi M. An isolated duodenal perforation in pediatric blunt abdominal trauma: a rare but distinctpossibility. Burns Trauma. 2015 Jun 25;3:4. 2. Bradley M, Bonds B, Dreizin D, Colton K, Shanmuganathan K, Scalea TM, Stein DM. Indirect signs of blunt duodenal injury on computed tomography: Is non-operative managementsafe? Injury. 2016 Jan;47 (1):53-8 3. Ahmad R, Shafique MS, Ul Haq N, Akram Z, Qureshi U, Khan JS. Isolated Duodenal Injuries After Blunt Abdominal Trauma. J Ayub Med Coll Abbottabad. 2016 Apr-Jun;28 (2):400-403. 4. Builes Ramírez S, García Novoa MA, Fernández Soria N, Gómez Gutiérrez M. Duodenal trauma. Consequences of delay in diagnosis. Cir Esp. 2016 Mar;94 (3):204-5

5. Celik A, Altini E, Koksal N, Onur E, Sumer A, Uzun MA, Kayaham M. Management of isolated duodenal rupture due to blunt trauma: case series and literature review. Our J Trauma Emerg Surg. 2010 Dec; 36 (6): 573-8

Disclosure: No significant relationships.

\section{PC077}

GIANT TRAUMATIC HERNIA OF THE ABDOMINAL WALL

A. Suárez Cabrera ${ }^{1}$, A. Curado Soriano ${ }^{1}$, Z. Valera Sánchez ${ }^{1}$, J.R. Naranjo Fernández ${ }^{l}$, A. Domínguez Amodeo ${ }^{l}$, R. Jurado Marchena $^{I}$, E. Navarrete De Carcer ${ }^{1}, F$. Oliva Mompean ${ }^{2}$

${ }^{1}$ General Surgery, University Hospital Virgen Macarena, Seville/ SPAIN, ${ }^{2}$ Cirugía General, Hospital Virgen Macarena, Sevilla/SPAIN

Case History: We present a case of traumatic hernia by car accident. We performed a parietal reconstruction with a posterior separation of components according to the release of transverse muscle associated with a Gore ${ }^{\circledR}$ Bio-A mesh.

Clinical Findings: A 19-year-old male presented with polytrauma due to high-energy traffic accident. He arrived at the emergency room with a generalized abdominal pain and a large tumor and hematoma in the left lower quadrant, vitals were stable. A body axial tomography was performed.

Investigation/Results: Voluminous traumatic hernia, with handles of small and large intestine and pneumoperitoneum. Discrete amount of free liquid in pelvis, not alteration of vascular pedicles.

Diagnosis: Traumatic giant hernia with complete section of the oblique and transverse left musculature, rectum and part of the right rectum.

Therapy and Progression: We performed an exploratory laparotomy, an intestinal resection of sectioned distal ileum and of devitalized sigma was required. Then we repaired the abdominal wall and placed a mesh of Bio A Gore ${ }^{\circledR}$ to give subjection to the wall. At 10 months of follow-up the patient has recovered his academic and sports activity.

Comments: The traumatic hernia is a rupture of the abdominal wall with preservation of the skin associated with a trauma. It's present in $1 \%$ of polytrauma. Its treatment is secondary, the objective is the survival of the patient. The connotations of these patients, as well as the absence of existing clinical evidence, shows a range therapeutic approach that extends from damage control to laparoscopic deferral repair.
References: - Rikki Singal, Usha Dalal, Ashwanir Kumar Dalal, Ashok Kumar Attri. Traumatic anterior abdominal wall hernia: A report of three cases. J. Emerg Trauma Shock. 2011. 4: 142-145. Alfredo Moreno-Egea, Enrique Gibela, Elena Partorio, Jose Luis Aguayo-Albasini. Controversias en el manejo actual de las hernias traumáticas de la pared abdominal. Cir.Esp. 2007. 5: 260-267. Stephanie D. Talutis, Oliver J. Muensterer, Samir Pandya, Whitney McBride and Gustavo Stringel. Laparoscopic-assist management of traumatic abdominal wall hernias in children. Journal of Pediatric Surgery. 2015. 50: 456-461.

Disclosure: No significant relationships.

\section{PC078}

\section{EXPERIENCE OF EXTRACORPOREAL MEMBRANE OXYGENATION (ECMO) IN SINGLE TRAUMA CENTER}

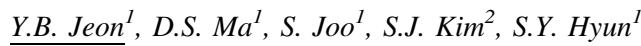

${ }^{1}$ Trauma Surgery, Gachon university Gil hospital, Incheon/KOREA, REPUBLIC OF, ${ }^{2}$ Trauma Surgery, Gachon University Gil Medical Center, Incheon/KOREA, REPUBLIC OF

Case History: We reviewed patients treated with extracorporeal membrane oxygenation (ECMO) from September, 2013 to September, 2017 in a single trauma center. 14 male and 2 female patients were included and average age was 54+/- 18 years old (19-76).

Clinical Findings: There are 11 multiple organ injuries and 5 single organ injuries. Chest, abdomen and pelvis were involved in 3 patients and, 5 patients suffered from chest and abdominal trauma. Remaining patients had 2 chest and pelvis injuries and one abdominal and pelvis injury. Five single organs involved were bronchus, right ventricle, pelvis (2) and spinal cord.

Investigation/Results: Average ISS score was 31.4 and their mechanisms of injuries were motor vehicle accident in 10 , fall down in 3 , crushing injury in 2 and bicycle accident in one patient.

Diagnosis: ECMO was applied at 7+/- 6 days after accident and indications were acute respiratory distress syndrome (6), sudden cardiac arrest (3), hypovolemic shock (3), septic shock (2), acute cor pulmonale (1) and cardiomyopathy (1) sequentially.

Therapy and Progression: Venoarterial (VA) mode of ECMO was used commonly (10 patients). 5 patients $(31.3 \%)$ were weaned from ECMO and 4 patients (25\%) left hospital live. Mortality was compared among modes of ECMO and it showed that 7/10 VA and 2/4 VV (venovenous) failed to survive.

Comments: The mortality ratio of ECMO in trauma patient was lower than expected. Early application to the appropriate patient at the correct time is important to improve results.

References:

Disclosure: No significant relationships.

\section{PC079}

\section{ERAS POST-OP ANALGESIA FOR EMERGENCY CLAMSHELL THORACOTOMY}

\section{T. Egan, E. Coley}

Anaesthesia, The Royal London Hospital, London/UNITED KINGDOM 
Case History: We wish to present the guideline that we have developed at The Royal London Hospital. The busiest MTC in the UK To provide guidelines for a structured, uniform, multidisciplinary approach to patients after emergency thoracotomy with placement of a wound catheters to deliver a continuous infusion of local anaesthetic as part of an opiate sparing multimodal analgesia facilitating rapid extubation. There are both short term and long term benefits for the patients pain control if standard guidelines are followed.

Clinical Findings: The use of local Anaesthetic Infusion (LAI) is an established method of post operative analgesia. This includes the infusion of local anaesthetic directly into the operative site via a specially designed catheter via the use of LAI catheters attached to an infusion device.

Investigation/Results: Emergency clamshell thoracotomy can take place in a range of locations including the pre-hospital environment or early in the patient's trauma management in the emergency department or operating theatre. Patients will have been anaesthetised in this setting to facilitate the procedure. In this scenario, a decision should be taken by the anaesthetist and surgeon about analgesia options that are in the patient's best interests. Epidural and paravertebral analgesia are almost unanimously contraindicated in these patients in view of the potential for trauma-induced coagulopathy and the difficulties in positioning the patient.

Diagnosis: Emergency Clamshell Thoracotomy

Therapy and Progression: Rapid Extubation And Ambulation

Comments: We wish to present the guideline that we have developed at The Royal London Hospital. The busiest MTC in the UK

References: Continuous Local Anesthetic Wound Infusion to Improve Postoperative Outcome. Anesthesiology 2007; 107:369 -71 Liu SS, Richman JM, Thirlby RC, Wu CL. Efficacy of Continuous Wound Catheters Delivering Local Anesthetic for Postoperative Analgesia: A Quantitative and Qualitative Systematic Review of Randomized Controlled Trials. Journal of American College of Surgeons, 2006, 203 (6), 914-932

Disclosure: I teach on a peripheral nerve catheter course run by Halyard who make elastometric pumps and wound catheters and receive renumeration for this

\section{PC080}

\section{DELAYED FORMED COLOCUTANEOUS FISTULA AFTER BLUNT TRAUMA: ACCOMPANIED WITH A VENTRAL HERNIA}

\section{Y. Park, J. Kim, W. Kang, Y. Jo}

Division Of Trauma Surgery, Department Of Surgery, Chonnam National University Hospital, Chonnam National University Medical School, Gwangju/KOREA, REPUBLIC OF

Case History: A post-traumatic colocutaneous fistula is a very rare occurrence secondary to abdominal blunt trauma. Here, we present a case of the colocutaneous fistula with a ventral hernia after blunt abdominal trauma.

Clinical Findings: A 86 year-old man was bumped into a cultivator handle while moving the vehicle. He was brought to the emergency department with abdomen distension and pain.

Investigation/Results: Blood pressure was $90 / 60 \mathrm{mmHg}$. On laboratory findings, hemoglobin was $12.5 \mathrm{~g} / \mathrm{dL}$. An abdominal computed tomography (CT) showed ventral herniation of transverse colonic loop without definite strangulation. Follow up blood pressure was
140/100 and $\mathrm{Hgb}$ was $11.4 \mathrm{~g} / \mathrm{dL}$. He was admitted ward and the clinical sign was improved.

Diagnosis: 10 days later, abdominal wall erythema and swelling appeared with abdominal pain. An abdominal computed tomography (CT) showed out $5.4 \times 2.5 \mathrm{~cm}$ abscess formation in the left abdominal wall with fistula tract to herniated transverse colonic loop.

Therapy and Progression: An emergency operation was performed. In the ventral hernia site, the transverse colon was abutting with abdominal skin and the colocutaneous fistula with abscess was formed. Transverse colon segmental resection and end transverse colostomy were performed. After the operation postoperative wound infection occurred and delayed wound repair was done. 15 days later from the operation, the patient was discharged without any problems. Comments: Blunt abdominal trauma can cause delayed complication such as enterocutaneous fistula. Therefore, serial inspection of the injury site and follow up exam could be a rapid diagnosis and treatment.

References: Wightman JA. Delayed traumatic rupture of colon with colocutaneous fistula. Br Med J. 1967;2 (5544):93-4.

Disclosure: No significant relationships.

\section{PC081}

\section{ISOLATED RUPTURE OF THE GALLBLADDER IN BLUNT ABDOMINAL TRAUMA}

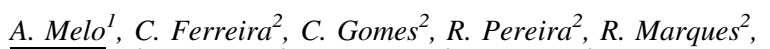

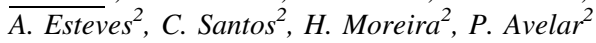

${ }^{1}$ Surgery, Centro Hospitalar Trás-os- Montes e Alto Douro, Vila Real/ PORTUGAL, ${ }^{2}$ Cirurgia Geral, CHTMAD, Vila Real/PORTUGAL

Case History: Gallbladder is a well protected organ due to its relatively small size and anatomic location. Gallbladder injury resulting from blunt abdominal trauma is rare, being found in only $2 \%$ of patients who undergo surgery due to trauma, and isolated gallbladder rupture is even more uncommon. The clinical presentation of gallbladder injury is variable, resulting in a delay in diagnosis and treatment. We report the case of a patient who suffered an isolated gallbladder rupture due to blunt abdominal trauma.

Clinical Findings: A 77-year-old woman presented in the emergency department after falling from her own height in the bathroom. Trauma approach was performed. The patient was hemodynamically stable and had abdominal pain in the right upper quadrant.

Investigation/Results: Computed tomography (CT) showed perihepatic and periesplenic fluid, without other lesions.

Diagnosis: Blunt Abdominal Trauma

Therapy and Progression: The patient presented clinical worsening after 24 hours and an exploratory laparotomy was decided, which identified biliary peritonitis due to isolated rupture of the gallbladder. Other organs showed no sign of injury. Cholecystectomy was performed, with cavity drainage.

Comments: Injuries in the gallbladder resulting from blunt abdominal trauma occur rarely and are usually associated with damage to other abdominal organs. The clinical signs and symptoms that arise from isolated traumatic gallbladder injuries may be vague and insidious, and range from acute abdomen to slowly progressive abdominal complaints. Diagnosis can be made by CT, however it is most often performed by exploratory laparotomy. Cholecystectomy is the recommended treatment.

References: Benjamin Yin Ming Kwan, MD; Paul Plantinga, BSc $(\mathrm{H})$, MD Cand.; and Ian Ross, BSc; Isolated traumatic rupture of the gallbladder, Radiology Case Reports, Volume 10, Issue 1, 2015 Liess 
BD, Awad ZT, Eubanks WS. Laparoscopic cholecystectomy for isolated traumatic rupture of the gallbladder following blunt abdominal injury. J Laparoendosc Adv Surg Tech A. 2006;16 (6):623-5 KwoK-wan Yeung1 Po-Ping Liu2, Isolated Gallbladder Perforation Following Blunt Abdominal Trauma: a case report; J Radiol Sci 2014; 39: 51-55 Ndour O, Moustapha H, Ndoye NA, et al. Isolated gallbladder perforation after blunt abdominal trauma in children. Afr $\mathrm{J}$ Paediatr Surg 2013; 10: 41-42

Disclosure: No significant relationships.

\section{PC082}

\section{RENAL TRAUMA MANAGEMENT IN A SMALL} COUNTRYSIDE HOSPITAL

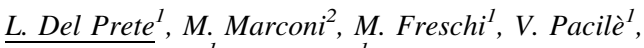
R.A. Pappalardo ${ }^{1}$, M. Oldani ${ }^{1}$

${ }^{1}$ General Surgery, Hospital Santa Maria Delle Stelle, Melzo/ITALY, ${ }^{2}$ General And Emergency Surgery, Hospital Santa Maria Delle Stelle, Melzo/ITALY

Case History: A 54-year-old man felt from the stairs when he was painting and hurt his left flank on a chair nearby the staircase.

Clinical Findings: No loss of consciousness was noted, but hematuria, left flank pain and mass developed. An hour later the patient was admitted in the emergency department of a small countryside hospital and an E-FAST exam was carried out bedside: no abdominal free fluid was noted but the left kidney's parenchima appeared to be irregular.

Investigation/Results: Leukocytosis and $13.3 \mathrm{~g} / \mathrm{dL}$ hemoglobin value were found and physical examination revealed neither abdominal distension nor pain; thirty minutes later laboratory examinations showed loss of three hemoglobin points.

Diagnosis: CT of the abdomen was done which revealed grade four left renal injury, with a huge hematoma extended in the retroperitoneum.

Therapy and Progression: Because the patient's vital signs were unstable, emergency laparotomy was carried out and deep lacerations of the inferior pole and pelvis of the left kidney and injury of the left renal vein were found. A left renal nefrectomy was done and the patient was discharged from the hospital eight days later.

Comments: In renal trauma abdominal evaluation could be misleading even in the presence of a huge retroperitoneal hematoma. Radiologic evaluation must be performed in all blunt trauma with gross hematuria or with hypotension and CT should be preferred for diagnosis. If the patient is unstable, angioembolisation is not available in your hospital or it would take too much time to repair the injury in a time-critical situation, emergency laparotomy and nephrectomy should be considered.

References:

Disclosure: No significant relationships.

\section{PC083}

MVT AND PVT WITH INTESTINAL ISCHEMIA IN A YOUNG PATIENT WITH HISTORY OF THROMBOSIS - A CASE REPORT

\section{A. Hanu, A. Ivanoschi, D.O. Costea}

Surgery, Emergency County Hospital Constanta, Constanta/ ROMANIA

Case History: 41 years old male patient with a history of DVT and pulmonary thromboembolism, with anticoagulant therapy interrupted without medical advice, comes to ER with 48 hours of acute abdominal pain.

Clinical Findings: Physical exam was significant for diffuse abdominal tenderness without peritoneal signs.

Investigation/Results: Laboratory tests showed elevated WBC 22280, CT scans revealed a 50\% PV thrombosis, complete superior MVT, small amount of fluid in the peritoneal cavity. The thrombophilia tests taken after the admission in the ICU were normal.

Diagnosis: Portal vein thrombosis, complete superior mesenteric vein thrombosis, ascitis.

Therapy and Progression: After ICU admission, heparin therapy started (aPTT within normal therapeutical limits). Clinical condition improved at first, but in day 6 he developed fever, associated with abdominal guarding and elevation of WBC. CT revealed pneumatosis intestinalis, besides the thrombosis described initially. He underwent an emergency surgery, intraoperative findings: stercoral peritonitis $(4500 \mathrm{ml})$, intestinal necrosis from Treitz angle until $40 \mathrm{~cm}$ above the ileocecal valve, so an extended enterectomy was performed, with intestinal anastomosis, tissues being to fragile to be taken out in a stomy. The next days, evolution was precarious, developing digestive fistula, which was treated by disbandment of the initial anastomosis, followed by an alimentation ileostomy and a jejunostomy for evacuation. Postoperative, patient remained intubated, mechanical ventilated, on vasopressor support, and despite of ICU efforts, with exitus in day 26.

Comments: Studies show that diagnosis of MVT is commonly associated with a mortality rate $20-40 \%$, with early diagnosis, before the development of intestinal ischemia, after that the prognosis being worse. No characteristic laboratory abnormalities are associated with MVT.

References: 1. Mock JN, Orsay EM. Primary mesenteric venous thrombosis: an unusual cause of abdominal pain in a young, healthy woman. Ann Emerg Med. 1994;23:352-5 2. Hotoleanu C, Andercou $\mathrm{O}$, Andercou A. Mesenteric venous thrombosis with bowel infarction and hyperhomocysteinemia due to homozygous methylenetetrahydrofolate reductase C677T genotype. Vasc Endovascular Surg. 2008;42:477-81 3. Rhee RY, Gloviczki P. Mesenteric venous thrombosis. Surg Clin N Am. 1997;77:327-37 4. Alvi AR, Khan S, Niazi SK, et al. Acute mesenteric venous thrombosis: improved outcome with early diagnosis and prompt anticoagulation therapy. Int J Surg. 2009;7:210-3

Disclosure: No significant relationships.

\section{PC084}

\section{MANAGEMENT OF ISOLATED ADRENAL TRAUMA IN EMERGENCY: REPORT OF TWO CASES}

F. Moreno-Suero ${ }^{1}$, V. Durán-Muñoz-Cruzado ${ }^{1}$, I. Ramallo Solís ${ }^{2}$, L. Tallón-Aguilar ${ }^{1}$, J. Tinoco-González ${ }^{1}$, I. Alarcón-Del Agua ${ }^{l}$,

A. Sánchez-Arteaga ${ }^{1}$, M.J. Tamayo-Lopez ${ }^{1}$, F. Pareja-Ciuró ${ }^{3}$,

J. Padillo-Ruíz ${ }^{3}$, C. Gonzalez De Pedro ${ }^{1}$ 
${ }^{1}$ Emergency Surgery Unit, Virgen del Rocío Universitary Hospital, Seville/SPAIN, ${ }^{2}$ Coloproctology Surgery Unit, Virgen del Rocío Universitary Hospital, Seville/SPAIN, ${ }^{3}$ Digestive And General Surgery Unit, Virgen del Rocío Universitary Hospital, Seville/SPAIN

Case History: We show two clinical cases of isolated adrenal trauma: a man who fell off from a bicycle banging on his right elbow and a woman who fell from her own height hitting against a cube.

Clinical Findings: Both patients are attended in the trauma box according to ATLS principles. They present pain in the site of traumatism; they are conscious and hemodynamically stable, without fracture or peritonism signs.

Investigation/Results: Patients do not show clinical or radiological signs of costal fracture, pneumoperitoneum or other associate damages, so we decide to do an abdominal CT - Scan. In both cases it is detected an isolated adrenal bleeding. Hormonal analysis verifies that adrenal function is normal.

Diagnosis: Adrenal injury without other associated damages.

Therapy and Progression: In both cases, considering the stability hemodynamic of patients and absence of concomitant injuries, the surgical team decides a conservative treatment through close monitoring of patient the first 24 hours. In the control CT, it is verifies that the hematoma has completely disappeared and the biochemical analysis is normal.

Comments: Actually, if the adrenal trauma does not cause hemodynamic repercussions, the initial treatment is monitoring and controls by medical team. Situations that produce hemodynamic repercussion, arterial embolization is considered an effective alternative to laparotomy. In this type of isolated lesions secondary to trauma, we think it is important to confirm their origin and to discard that it could be an incidental finding already existing previously. For this reason, we consider very important to make a complete study with a control CT and analysis of catecholamines.

References: Experience of Emergency Surgery Unit from Virgen del Rocío Universitary Hospital (clinical cases) Liao $\mathrm{CH}$, Ouyang $\mathrm{CH}, \mathrm{Fu}$ CY, Wang SY, Lin KJ, Kuo IM, Hsu CP, Yang SJ, Yuan KC, Hsu YP. The current status and management of blunt adrenal gland trauma. Sugery. 2015 Feb;157 (2):338-43 Lee YS, Jeong JJ, Nam KH, Chung WY, Chang HS, Park CS. Adrenal injury following blunt abdominal trauma. Worl J Surg. 2010 Aug;34 (8):1971-4. Stawicki SP, Hoey BA., Grossman MD et al. Adrenal gland trauma is associated with high injury severity and mortality. Curr Surg. 2003;60:431-6 Sinelnikov AO, Abujudeh HH, Chan D, Novelline, R.A. CT manifestations of adrenal trauma: experience with 73 cases. Emerg Radiol. 2007;13:313-8. Chen KT, Lin TY, Foo NP et al. Traumatic adrenal haematoma: A condition rarely recognised in the emergency department. Injury. 2007;38:584-7. Lee S, Jeong JJ, Nam KH et al. Adrenal injury following blunt abdominal trauma. World $\mathrm{J}$ Surg. 2010;34:1971-4

Disclosure: No significant relationships.

\section{PC085}

\section{PERFORATED SMALL BOWEL AFTER BLUNT TRAUMA OF INGUINAL HERNIA}

A. Raftopoulos, V. Berdelis, M. Karamanidi, E. Dimitrakopoulou,

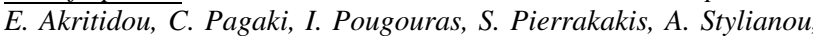
T. Vathia
Surgery, GENERAL HOSPITAL OF ELEFSINA THRIASIO, MAGOULA/GREECE

Case History: A 71 year old male came to emergency department due to acute abdominal pain after blunt abdominal trauma, falling from his height. From his medical history is declared right inguinal hernia.

Clinical Findings: Acute abdomen, with nausea and vomiting.

Investigation/Results: Imaging revealed peritonitis, an inflammatory small bowel loop and right incarcerated hernia.

Diagnosis: Small bowel perforation after violent retreat, peritonitis and acute abdomen.

Therapy and Progression: Emergency exploratory laparotomy revealed small bowel loop perforation in adhesion with the internal inguinal ring. An excision of the affected loop was performed as well as a hernia plastic repair.

Comments: Inguinal hernias are common in men over the age of 45 . Acute traumatic abdominal hernias are considered to be very rare. Bowel perforation in patients with hernias occur from trauma directly to inguinal hernia or from blunt abdominal trauma. There have been only a few case reports that described blunt injury to the inguinal area causing traumatic perforation of the bowel inside the hernia sac. Overall, intestinal and mesenteric injury represent $5 \%$ of patients with blunt abdominal trauma.

References: 1.Large owel Perforation in an Acute Inguinal Hernia Resulting from Blunt Abdominal Trauma Amit Kumar C Jain and S. Viswanath Department of Surgery, St John's Medical College, Sarjapur road, Karnataka, India 2. Case Rep Med. 2015; 2015: 105183. Published online 2015 May 13. doi: 10.1155/2015/105183 An Incarcerated Colon Inguinal Hernia That Perforated into the Scrotum and Exhibited an Air-Fluid Level 3. Chirurg. 1995 Jun;66 (6):637-9. [Perforation of the small intestine in patients with hernia]. Gianom D1, Fenner A. 4. Volume 2014 (2014), Article ID 653847, Case Report Diagnosis of an Inguinal Hernia after a Blunt Inguinal Trauma with an Intestinal Perforation Farès Moustafa, Julien Avouac, MarieAude Vaz, and Jeannot Schmidt

Disclosure: No significant relationships.

\section{PC086}

\section{SELF-INFLICTED PENETRATING CERVICAL TRAUMA: WHEN THE PATIENT KNOWS ANATOMY}

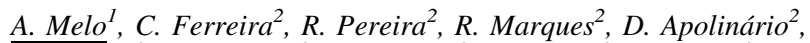

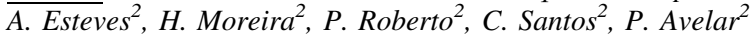

${ }^{1}$ Cirurgia Geral, Centro Hospitalar de Trás-os-Montes e Alto Douro, Vila Real/PORTUGAL, ${ }^{2}$ Cirurgia Geral, CHTMAD, Vila Real/ PORTUGAL

Case History: Diagnosis of the penetrating cervical trauma is a challenge because there are many important anatomical structures involved in this small region, whose damage can be pottencially fatal. We report an uncommon clinical case of a self-inflicted penetrating cervical trauma.

Clinical Findings: A 87-year-old woman was admitted to the emergency room for self-injury by a knife in the anterior region to the neck. The systematic approach showed a permeable airway and no signs of respiratory difficulty. She was hemodynamically stable and 
no visible active bleeding. The knife handle was outside the medial portion of the anterior neck.

Investigation/Results: CT scan revealed that the knife was located to the right of the trachea and esophagus, immediately cranial to the brachiocephalic trunk and in close relation with the right carotid axis, without extravasation of oral or intravenous contrast.

Diagnosis: Penetrating cervical trauma

Therapy and Progression: Cervicotomy was proposed; bronchofibroscopy and upper gastrointestinal endoscopy were performed in the operating room. No significant lesions were identified and the knife was removed without complications. Immediate postoperative period was in the intensive care unit with good clinical evolution.

Comments: Cervical penetrating injuries account for $5-10 \%$ of all cases observed in Emergency. After initial stabilization of the patient, the lesions must be evaluated. In hemodynamically stable patients, the need for surgical intervention is usually determined by integrating both clinical signs and radiological findings. In this case, by the presence of the knife in the injury site, its removal in a controlled environment, with assured airway and thorough surgical exploration, was the treatment of choice.

References: Sebastian Gamba a, Mario Lachat b, Hatem Alkadhi c, Hans-Peter Simmena, Kai Oliver Jensen a; Radiographically occult perforation and dissection of the common carotid artery following stab injury to the neck; Trauma Case Reports; 9 (2017) 17-21 David V. Feliciano, Penetrating Cervical Trauma; World journal of Surgery, June 2015, Volume 39, , pp 1363-1372

Disclosure: No significant relationships.

\section{PC087}

\section{ANORECTAL INJURIES IN TRAUMA PATIENTS}

H. Calis, N. Ozlem

Faculty Of Medicine; General Surgery, Ahi Evran University, Kirsehir/TURKEY

Case History: Traumatic lesions of the anorectum are rare but difficult to treat, requiring experience. These traumas may be fatal. We present three cases managed differently treatment.

Clinical Findings: Case report of three patients admitted to Ahi Evran University Education and Research Hospital in 2016. Patients were treated at the General Surgery Clinic. Patient records have been examined.

Investigation/Results: We present three cases managed differently treatment. First case a male patient, 28 years old, firearm injury, admitted to our hospital in emergency setting for left gluteal open wound with extension to anorectal region and pararectal space, complex pelvic trauma and multiple pelvic fractures. The patient had hypotension and tachycardia. The patient was transported directly to the operating room. There was a full thickness loss of tissue in the anus and lower rectum. Loop colostomy was performed after pelvic hemostasis. Then, the rectum was sutured to the perineum after dissection of the lower rectum. But on the second postoperative day, the sutures opened and the rectum ran up. A restorative surgery was planned for the patient whose condition improved. Second and third cases were also male patients 38 and 43 years old and after bicycle accident transferred to our hospital with the laceration in the anüs. Anal reconstructive surgery was performed after hemostasis.

Comments: The evaluation and management of anorectal injuries is very important for morbidity and mortality. If the patient is stabilized, firstly restorative treatments should be tried according to the condition of the patient.

References: 1.Daniel O. Herzig. Care of the Patient with Anorectal Trauma. Clin Colon Rectal Surg. 2012; 25: 210-213.

Disclosure: No significant relationships.

\section{PC088}

\section{THE IMPACT OF POST-TRAUMATIC STRESS DISORDER IN THE REFUGEE POPULATION}

A. Ntikoudi ${ }^{1}$, A. Papachristou ${ }^{2}$, V. Saridakis ${ }^{3}$

${ }^{1}$ Nursing, KAPODISTRIAN UNIVERSITY OF ATHENS, ATHENS/ GREECE, ${ }^{2}$ Medical Department, KAPODISTRIAN UNIVERSITY OF ATHENS, ATHENS/GREECE, ${ }^{3}$ Surgical Department, GN 2ND YPE, NIKAIA/GREECE

Case History: TWar is the primary reason why refugees suffer from many psychiatric disorders such as PTSD. Moreover, refugees are frequently exposed to a variety of stressors such as socioeconomic disadvantages, poverty, changes in family structure and functioning, losing social support, living in very crowded places, experiencing racism and isolation.

Clinical Findings: This systematic review included research studies published between 2007-2017 from the search databases Medline, Scopus, Cinahl, and PubMed, with keywords "war survivors", "war trauma", "post-traumatic stress disorder", "refugees", "psychiatric disorder".

Investigation/Results: Overall, 16 studies were reviewed and evaluated. The majority of them demonstrated that the most common psychiatric disorders observed among war refugees are post-traumatic stress disorder (PTSD), depression, anxiety, pain and multiple somatic complaints. Moreover, a significant relationship was shown between the development and maintenance of PTSD and sociodemographic features such as gender, age and previous family history of any psychological disorder.

Diagnosis: War violence is highly traumatic, causing multiple, longterm negative outcomes such as PTSD. The findings highlight the need for care of the survivors and their families.

Therapy and Progression: Further research is necessary in order to clarify the role of predictive factors in the development and maintenance of post-traumatic stress in war trauma survivors.

Comments: In conclusion, it is necessary to have large multicenter studies in the future in order to be able to draw reliable conclusions 
about the effects of war in the development and maintenance of PTSD in the refugee population.

References: Alpak, G., Unal, A., Bulbul, F., Sagaltici, E., Bez, Y., Altindag, A., Dalkilic, A., Savas, H.A., 2015. Post-traumatic stress disorder among Syrian refugees in Turkey: A cross-sectional study. International Journal of Psychiatry in Clinical Practice 19, 45-50.

Disclosure: No significant relationships.

\section{PC089}

\section{SUBCUTANEOUS AND SCLERA EMPHYSEMA}

N.F. Oliveira, M. Morgado, P. Carvalho, H. Queimado, L. Ramos, F. Rodrigues

Serviço Cirurgia Geral, Hospital Vila Franca de Xira, Vila Franca de Xira/PORTUGAL

Case History: A 55-year-old man turned to the emergency department after falling $1.5 \mathrm{~m}$ tall. He reported head trauma without loss of consciousness and dorsal trauma with pain in the left costal grid. He denied dyspnoea.

Clinical Findings: Observation: eupneic in ambient air, without alterations on pulmonary auscultation; revealed pain in the palpation of the left costal grid; without evidence of subcutaneous emphysema. Re-observation (after $\mathrm{x}$-rays and skull CT): subcutaneous emphysema in the region of the pectoral muscles, neck, face and temporal region. Investigation/Results: Chest and left chest grid $x$-ray: without hemothorax, pneumothorax or costal arch fractures; signs suggestive of subcutaneous emphysema and an image compatible with the large pectoralis muscle. Computed tomography of the skull: without intracraneal changes but with subcutaneous emphysema in the temporal region, bilaterally. Computed tomography of the thorax: small left pneumothorax, pneumomediastinum, subcutaneous emphysema from the large pectoral muscles to the cervical region and absence of aspects compatible with fracture of costal arches.

Diagnosis: Pneumothorax, pneumomediastinum and subcutaneous emphysema

Therapy and Progression: Thoracic drainage was performed in the 5th left intercostal space. Favorable evolution was observed but initially there was progression of emphysema that was manifested in the sclerotic as well.

Comments: Chest trauma is common and data suggest that approximately $30-50 \%$ of all blunt trauma patients have sustained chest trauma. Thoracic injuries account for $20-25 \%$ of all trauma-related deaths, and complications of it contribute to another $25 \%$ of all deaths. This data and the clinical case presented shows that thoracic trauma is a important entity and it must have the atention of the surgeon at all time during observation/evaluation.

References: John L. Cameron, Andrew M. Cameron. (2017). Current Surgical Therapy, Ed. 12. Elsevier

Disclosure: No significant relationships.

\section{PC090}

\section{DIAPHRAGMATIC HERNIA RESULTING IN LARGE BOWEL OBSTRUCTION 10 YEARS AFTER PENETRATING} TRAUMA

$\underline{\text { D.S. Brito }}^{1}$, A.C. Longras $^{1}$, A. Magalhães ${ }^{2}$, R. Lourenco $^{2}$,

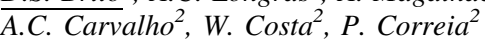

${ }^{1}$ General Surgery, Hospital Senhora da Oliveira, Guimarães/ PORTUGAL, ${ }^{2}$ General Surgery, Hospital da Senhora da Oliveira Guimaraes, Guimaraes/PORTUGAL

Case History: We present the clinical case of a 39 year-old male with history of penetrating left thoracic trauma 10 years before coming to the emergency service.

Clinical Findings: He complained of colicky abdominal pain and constipation, both for stool and gas for 6 days, with a worsening pattern.

Investigation/Results: The abdominal $\mathrm{x}$-ray showed a marked distention of the splenic colonic angle, and the CT reported a large distention of the right and transverse colon, caused by a left diaphragmatic hernia.

Diagnosis: We suspected a left diaphragmatic hernia, with incarceration of left colon.

Therapy and Progression: The patient was performed an urgent laparotomy, wich confirmed the incarceration of the left colon in a diaphragmatic $5 \mathrm{~cm}$ tear. The incarcerated colon was viable, so we reduced the hernia and closed the diaphragmatic tear and placed a left thoracostomy tube for pneumothorax drainage.

Comments: Traumatic diaphragmatic hernias can result from thoracic or abdominal blunt or penetrating trauma. Most of them are diagnosed and treated at the time of trauma, but in $10 \%$ of cases the diaphragmatic trauma is undiagnosed. The diagnosis of a late traumatic diaphragmatic hernia is only made when the patient becomes symptomatic, typically months to years after the traumatic event. So it is important to remember that the patient history is of the most importance, and diaphragmatic hernia must be suspected in cases of significant trauma to the thorax and abdomen.

References: Ganie F.A. et al. Delayed Presentation of Traumatic Diaphragmatic Hernia: a Diagnosis os Suspicion with Increased Morbidity and Mortality. Trauma Monthly. 2013, 18 (1) . Demuro J.B. A delayed traumatic diaphragmatic hérnia presenting with a bowel obstruction 20 years postinjury. Journal of Clinical and Diagnostic Research. 2013, 7 (4): 736-738. Hajong R., Baruah A. Posttraumatic diafragmatic hérnia. Indian J Sur. 2012, 74 (4): 334-335. Disclosure: No significant relationships.

\section{PC091}

\section{PERFURATING ABDOMINAL TRAUMA}

$\frac{\text { N.F. Oliveira, M. Morgado, P. Carvalho, H. Queimado, L. Ramos, }}{\text { F. Rodrigues }}$

Serviço Cirurgia Geral, Hospital Vila Franca de Xira, Vila Franca de Xira/PORTUGAL

Case History: Male, 19 years old, previously healthy person, taken to the emergency department due to perforating abdominal trauma, by metallic object. He reported only lower back pain. On observation: he presented a metallic wire in the left periumbilical region, which perforated the abdominal wall. Abdominal computed tomography described perforation of the abdominal wall at a depth of $9-10 \mathrm{~cm}$, with the extremity of the object in contact with the left psoas muscle, about $15 \mathrm{~mm}$ from the abdominal aorta. He underwent exploratory laparotomy. After laparotomy was identified a perforation of the transverse colon, mesentery and small laceration of the retroperitoneum in the abdominal aorta region. No retroperitoneum hematoma was identified. Segmentar resection of the transverse colon was 
performed. There were no intraoperative or postoperative complications. He was discharged on the 5th postoperative day.

Clinical Findings: Metallic wire in the left periumbilical region, which perforated the abdominal wall.

Investigation/Results: Abdominal computed tomography described perforation of the abdominal wall at a depth of $9-10 \mathrm{~cm}$, with the extremity of the object in contact with the left psoas muscle, about $15 \mathrm{~mm}$ from the abdominal aorta.

Diagnosis: Perfurating abdominal trauma with perfuration of transverse colon and mesentery.

Therapy and Progression: Urgent laparotomy with segmentar resection of the transverse colon

Comments: Perforating abdominal trauma is a potentially lifethreatening condition and most often requires emergent surgical treatment. It requires an initial systematized approach to assess cases requiring immediate surgery and those who need only to be under surveillance. The surgeon, in cases of abdominal trauma, should be prepared for rapid exposure of the intra-abdominal compartment and to control damage.

References: John L. Cameron, Andrew M. Cameron. (2017). John L. Cameron, Andrew M. Cameron. (2017). John L. Cameron, Andrew M. Cameron. (2017). Current Surgical Therapy, Ed. 12. Elsevier

Disclosure: No significant relationships.

\section{PC091A}

\section{SPONTANEOUS SPLENIC RUPTURE AND INSIDIOUS HAEMOPERITONEUM AS FIRST MANIFESTATION OF SEVERE PRIMARY AMYLOIDOSIS. CASE REPORT}

C.M. Ruiz Marin ${ }^{1}$, R. Cobos Cuesta ${ }^{1}$, I. Martínez Casas ${ }^{2}$, F.M. Jiménez Armenteros ${ }^{1}$, R. Ortega Higueruelo ${ }^{1}$, J. Rubio López ${ }^{1}$, J.M. Capitan Vallvey ${ }^{I}$

${ }^{1}$ General And Digestive Surgery, Complejo Hospitalario Jaén, Jaén/ SPAIN, ${ }^{2}$ Ugc Cirugía General, Complejo Hospitalario de jaén, Jaén/ SPAIN

Case History: Undiagnosed amyloid splenic infiltration is a rare cause of spontaneous splenic rupture. We present a case of insidious haemoperitoneum without haemodinamic unstability caused by severe amyloid disease debut.

Clinical Findings: A 50yo female with lower quadrants abdominal pain and two days dyarrea presented in our ED. She reported progressive weight loss $(20 \mathrm{Kg})$ in the past 6 months with anemia and menstrual disorders.

Investigation/Results: Vitals were normal and blood analysis reported anaemia, thrombopenia and leukocytosis, Cretinine $5.8 \mathrm{mg} /$ dl. A CT without contrast reported hepatosplenomegaly, dense free fluyd and pelvic mass infiltrating sacral bone.

Diagnosis: Splenic rupture and primary amyloidosis.

Therapy and Progression: Patient was admitted in Internal Medicine for study. Acute renal insufficiency worsened and leukoerithroblastic disorder was fund in peripheral blood driving to a consultation for haematologyst for iliac crest biopsy and bone marrow aspiration. The patient required blood transfusion without haemoglobin level improvement. The seventh day after admission the patient developed acute abdomen and a second CT was done. Splenic haematoma and increase of free fluyd was reported and laparotomy was indicated. 3 $\mathrm{kg}$ ruptured spleen was found and splenectomy was performed. Pathology results confirmed primary amyloidosis. Treatment was initiated with chemotherapy. Postoperative course was complicated with progressive cardiac, renal failure and metabolic derangement leading to death on 28 postoperative day. No complication was related to surgery but the primary disease.

Comments: Spontaneous splenic rupture secondary to amyloidosis is a rare entity and its diagnosis is difficult if the course is insidious. A more agressive diagnostic approach is necessary in this cases to start early with proper treatment.

References:

Disclosure: No significant relationships.

\section{EMERGENCY SURGERY CASES}

\section{PC092 \\ PRIMARY AORTODUODENAL FISTULA, A RARE LIFE THREATENING DISEASE PRESENTED AS HEMATEMESIS IN THE EMERGENCY ROOM}

G. Supelano Eslait, C. Alegre Torrado, M. Gutierrez Andreu, M.C. Nevado, O. García Villar, E. Ferrero, P. Yuste García

General And Digestive Surgery, 12 DE OCTUBRE UNIVERSITY HOSPITAL, MADRID/SPAIN

Case History: A 65 year old male with no previous history except arterial hypertension was admitted to the emergency room with hematemesis. Two previous episodes of hematemesis and melenas on the last 6 months, one of them asociated with pre-syncope.

Clinical Findings: He showed symptoms of anaemia with generalizad pallor, fatigue and hemoglobin level of $5.4 \mathrm{mg} / \mathrm{dl}$.

Investigation/Results: A gastroscopy was urgently performed showing a full of clots stomach with no evident bleeding or ulcer. In the next two days the upper endoscopy was two times repeated with the same result . In the third in-hospital day he was admited to the intensive care unit after a new episode of hematemesis and syncope with hemodynamic inestability. An angio-CT was performed, with no evident bleeding but an abdominal aortic aneurism adjacent to the duodenum was diagnosed. After 8 upper digestive endoscopies the patient was finally diagnosed with an aortoduodenal fistula.

Diagnosis: Bleeding aortoduodenal fistula

Therapy and Progression: An aortic endovascular repair excluding the aorto-duodenal fistula was urgently performed while the patient was hemodynamically unstable. In the next days he improved until the 8th postoperative day, when a CT scan showed colonic perforation and ischemia. A subtotal colectomy with an aorto-duodenal fistulectomy and mesenteric patch was finally performed. The next day aortic endovascular stent thrombosis developed, and the patient finally died.

Comments: Aortoduodenal fistula is a rare cause of hematemesis. A high index of suspicion is needed, even more when there's no history of aortic aneurism or stent graft placed. Endovascular treatment is the choice in the unstable patient even though it has complications.

References: - Rodrigues dos Santos C, Casaca R, Mendes de Almeida JC, Mendes-Pedro L. Enteric repair in aortoduodenal fistulas: a forgotten but often lethal player. Ann Vasc Surg. 2014 Apr;28 (3):75662. doi: 10.1016/j.avsg.2013.09.004. Epub 2013 Oct 1. Review. PubMed PMID: 24456836. - Lonn L, Dias N, Veith Schroeder T, Resch T. Is EVAR the treatment of choice for aortoenteric fistula? J Cardiovasc Surg (Torino). 2010 Jun;51 (3):319-27. Review. PubMed PMID: 20523281. - Welborn MB 3rd, Seeger JM. Prevention 
and management of sigmoid and pelvic ischemia associated with aortic surgery. Semin Vasc Surg. 2001 Dec;14 (4):255-65. Review. PubMed PMID: 11740833. - Kotsis T, Christoforou P, Asaloumidis N, Papaconstantinou I. Delayed Sigmoid Ischemic Rupture Following Open Repair Abdominal Aortic Aneurysm. Vasc Endovascular Surg. 2017 Aug;51 (6):413-416. doi: 10.1177/1538574417718445. Epub 2017 Jul 5. PubMed PMID: 28677408. - Danneels MI, Verhagen HJ, Teijink JA, Cuypers P, Nevelsteen A, Vermassen FE. Endovascular repair for aorto-enteric fistula: a bridge too far or a bridge to surgery? Eur J Vasc Endovasc Surg. 2006 Jul;32 (1):27-33. Epub 2006 Jan 19. PubMed PMID: 16427330. - Burks JA Jr, Faries PL, Gravereaux EC, Hollier LH, Marin ML. Endovascular repair of bleeding aortoenteric fistulas: a 5-year experience. J Vasc Surg. 2001 Dec;34 (6):1055-9. PubMed PMID: 11743560. -Ho S, Liu B, Loya R, Koury I. Primary Aortoenteric Fistula: A Rare Case of a Massive Gastrointestinal Bleed. Cureus. 2016 Sep 1;8 (9):e766. PubMed PMID: 27725922; PubMed Central PMCID: PMC5045327. -Saers SJ, Scheltinga MR. Primary aortoenteric fistula. Br J Surg. 2005 Feb;92 (2):143-52. Review. PubMed PMID: 15685700.

Disclosure: No significant relationships.

\section{PC093}

\section{RUPTURED ABDOMINAL AORTIC ANEURYSM WITHOUT VASCULAR SURGERY SUPPORT: SHOULD WE GO FOR IT?}

\section{S. Catarino, A.C. Afonso, J. Constantino, J. Pereira, C. Casimiro}

Cirurgia Geral, Centro Hospitalar Tondela-Viseu, Viseu/PORTUGAL

Case History: A 79 years-old female admitted in the ER with syncope and left abdominal pain.

Clinical Findings: The patient was pale, shocked, prostrated and with absent distal pulses.

Investigation/Results: Laboratory revealed $10 \mathrm{~g} / \mathrm{dL}$ of Hemoglobin and 3,2 of lactate. Abdominal CT showed a ruptured infrarenal aortic aneurysm, $8,8 \mathrm{~cm}$ width with retroperitoneal hematoma.

Diagnosis: Ruptured abdominal aortic aneurysm

Therapy and Progression: Patient begun blood transfusion. However, as she remained unstable and with no vascular surgery support in our institution, General Surgery team decided to perform emergent exploratory laparotomy and open repair of AAA. Sigmoidectomy was needed for bowel ischemia. The patient remained with a laparostomy in the ICU for stabilizaton. Re-operation was undertaken at $48 \mathrm{~h}$ : colostomy and abdominal closure was performed. She was discharged home at the 28th postoperative day without further major complications.

Comments: Ruptured AAA, despite advances in operative techniques and peri-operative management, carry a high mortality and complications rate. Open or endovascular aneurysm repair are possible options, with comparable long term mortality. Although endosvascular repair has emerged as the treatment of choice in elective repair of AAA, in the emergent setting open repair still remains the goldstandard, based on prosthesis availability and surgical experience. The authors present a case report of a ruptured AAA in an unstable patient without conditions to transfer for tertiary care, in an institution without emergent vascular surgery support. Experience in vascular procedures is thereby essential to a general surgeon, in order to obtain good results in these cases.

References: McHugh SM, et al.Endovascular versus open repair of ruptured abdominal aortic aneurysm. The Surgeon, 2016, Volume 14, Issue 5, 274-277

Disclosure: No significant relationships.

\section{PC094}

\section{POST-BARIATRIC SURGERY COMPLICATIONS: LESSONS FOR THE EMERGENCY SURGEON}

\author{
J.M. Ryan, G.A. Bass, H.M. Heneghan
}

Dept. Of Bariatric Surgery, St. Vincent's University Hospital, Dublin/ IRELAND

Case History: Medical tourism for bariatric surgery has increased the incidence of complications requiring management by non-bariatric trained emergency surgeons who are unfamiliar with these complications. We present three commonly encountered post-bariatric surgical complications and their management.

Clinical Findings: Case 1 - Slipped gastric band 34 year-old woman presented with a 4 day history of nausea, reflux and abdominal pain. She had a laparoscopic adjustable gastric band inserted in the United Kingdom 3 years previously. Abdominal x-ray revealed a malpositioned ('slipped') gastric band, indicated by the 'polo mint sign'. The band was deflated using the patient's subcutaneous port, giving almost-instant relief. The patient proceeded to laparoscopic band removal during the same admission.

Investigation/Results: Case 2 - Internal hernia 65 year-old woman was admitted with a 1 day history of sudden-onset central abdominal pain, distension, nausea, and vomiting. She had a gastric bypass performed in Belgium 9 years previously. CT revealed internal herniation of most of the small intestine through a $3 \mathrm{~cm}$ Peterson's defect. Emergency laparotomy was performed, the hernia contents were decompressed and reduced, and the defect was closed to prevent recurrence.

Diagnosis: Case 3 - Gastric outlet obstruction 30 year-old woman presented to the ED following laparoscopic gastric plication in Tunisia for a BMI of 30 . She had near-complete gastric outlet obstruction due to oedema of the imbricated stomach and required inpatient observation, corticosteroids, liquid diet and regular antiemetics for 2 weeks.

Therapy and Progression: Not applicable

Comments: Unrecognised complications following bariatric surgery may be catastrophic. Emergency surgeons must arm themselves with familiarity of these conditions.

References: 1. Hossain T, M BP, Kanwar S, Macadam R. Emergency admissions in patients that have undergone bariatric surgery-the experience from a UK district general hospital. Am J Surg 2016;212 (2): 368. 2. Healy P, Clarke C, Reynolds I, Arumugasamy M, McNamara D. Complications of bariatric surgery-What the general surgeon needs to know. The surgeon : journal of the Royal Colleges of Surgeons of Edinburgh and Ireland 2016;14 (2): 91-98.

Disclosure: No significant relationships.

\section{PC095}

THE WALKING DEAD: EARLY SURGERY, ANTIBIOTICS \& O2 TO CONTROL THE FLESH-EATING DISEASE

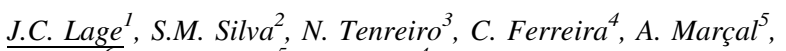 \\ A. Melo ${ }^{6}$, F. Prospero ${ }^{5}$, P. Avelar ${ }^{4}$
}

${ }^{1}$ General Surgery, Centro Hospitalar Tras-os-Montes e Alto Douro, Ourense/SPAIN, ${ }^{2}$ Surgery, Centro Hospitalar Trás-os- Montes e Alto Douro, Vila Real/PORTUGAL, ${ }^{3}$ Surgery, CHTMAD, Vila Real/ PORTUGAL, ${ }^{4}$ Cirurgia Geral, CHTMAD, Vila Real/PORTUGAL, ${ }^{5}$ Vila Real, CHTMAD, Vila Real/PORTUGAL, ${ }^{6}$ Cirurgia Geral, 
Centro Hospitalar de Trás-os-Montes e Alto Douro, Vila Real/ PORTUGAL

Case History: We report the case of a 71-year-old male with a personal history of diabetes and hypertension who arrives to the emergency room in septic shock.

Clinical Findings: The physical exam revealed a perianal abscess as starting point.

Investigation/Results: The patient was admitted to the surgical ward and underwent immediate surgical drainage that revealed a secondary extensive necrotizing fasciitis due to an infraperitoneal rectus perforation by a piece of wood.

Diagnosis: Necrotiziing fasciitis type I (clostridium perfringens, streptococcus milleri and streptococcus anginosus).

Therapy and Progression: A loop colostomy and radical perineal, scrotum and anterior abdominal wall debridement was performed. Broad spectrum antibiotics were started. The patient was admitted in the intensive care unit with cardiovascular, respiratory, hematologic and metabolic dysfunction. Posterior multidisciplinary approach involved vital support care, successive debridement surgeries and hyperbaric oxygen therapy after microbiological agents were identified (clostridium perfringens, streptococcus milleri and streptococcus anginosus). Infection control was achieved and a reconstructive strategy was finally performed (negative pressure wound therapy, miocutaneous flaps and skin grafts) The patient was discharged on the $72^{\circ}$ day.

Comments: Necrotizing fasciitis is a life-threatening rapidly progressive infection of the fascia with secondary necrosis of the subcutaneous tissues. It is a surgical emergency. We highlight the early aggressive surgery as the key point in necrotizing fasciitis treatment. Vital support care, successive surgeries and hyperbaric o2 therapy, when indicated, are also basilar in the final result

References: Necrotizing Fasciitis Treatment \& Management Updated: Apr 14, 2017 Author: Richard F Edlich, MD, PhD, FACS, FACEP, FASPS; Chief Editor: Michael Stuart Bronze

Disclosure: No significant relationships.

\section{PC096}

\section{PRIMARY ABDOMINAL COMPARTMENT SYNDROME DUE TO EXPANSIVE RECTUS MUSCLE SHEATH HEMATOMA}

\section{J.A. Navas-Cuéllar ${ }^{1}$, L. Tallón-Aguilar ${ }^{2}$, J. Tinoco-González ${ }^{2}$, V. Durán-Muñoz-Cruzado ${ }^{2}$, F. Pareja-Ciuró ${ }^{2}$, J. Padillo-Ruíz}

${ }^{1}$ Digestive Surgery Department, Quironsalud Campo de Gibraltar Hospital, Palmones, Los Barrios, Cádiz/SPAIN, ${ }^{2}$ Emergency Surgery Unit, Virgen del Rocío Universitary Hospital, Seville/SPAIN,

${ }^{3}$ Digestive And General Surgery Unit, Virgen del Rocío Universitary Hospital, Seville/SPAIN

Case History: A 67 years old male was brought to emergency department with a 24 hours history of severe abdominal pain. He had atrial fibrillation and an aortic valve, and was on long-term acenocumarol treatment.

Clinical Findings: On examination he was pale, tachycardic at 120 beats/min and his blood pressure was $80 / 55 \mathrm{mmHg}$. He was tender in his right abdomen. The patient was in anuria for the last 12 hours. Investigation/Results: His haemoglobin $(\mathrm{Hb})$ was $6 \mathrm{~g} / \mathrm{dl}$, creatinine was $3.9 \mathrm{mg} / \mathrm{dL}$ and his INR was 4.2 . He was transfused three units of packed cells which improved his systolic blood pressure to 105 . An emergency abdominal scan showed a large right-sided rectus sheath haematoma, without obvious bleeding point. Following transfusion, his $\mathrm{Hb}$ was still $7.5 \mathrm{~g} / \mathrm{dl}$. He continued in anuria and the intraabdominal pressure (IP) was $22 \mathrm{~mm} \mathrm{Hg}$.

Diagnosis: A control abdominal scan showed an increase of the size of the haematoma.

Therapy and Progression: As the patient's condition worsened, a fasciotomy was performed, to evacuate the haematoma, without locating the bleeding point, leaving two dreins in rectus muscle sheath. The IP returned to normal values, and the recovery was sucessfull.

Comments: A primary abdominal compartment syndrome of extrinsic origin, from a rectus muscle sheath haematoma, due to anticoagulation therapy, is a very uncommon condition. Surgical intervention is an alternative option when medical management or radiologist embolization are not effective or available.

References:

Disclosure: No significant relationships.

\section{PC097}

\section{ALTERNATIVE FOR CLOSING THE ABDOMINAL WALL IN DAMAGE CONTROL SURGERY}

A.X. Argote Camacho ${ }^{1}$, F.M. Jiménez Armenteros ${ }^{2}, \underline{\text { A. Szuba }^{3}}$

${ }^{1}$ Ugc Cirugía General, Complejo Hospitalario de jaén, Jaén/SPAIN, ${ }^{2}$ General And Digestive Surgery, Complejo Hospitalario Jaén, Jaén/ SPAIN, ${ }^{3}$ General Surgery, Hospital del Poniente, El Ejido, Almeria, Spain, Aguadulce, Roquetas de Mar/SPAIN

Case History: A 53-year-old male patient, transferred by emergency services to the Intensive Care Unit of the CHU of Jaén, after presenting abdominal GW with an entrance orifice in left hypochondrium and right hypochondrium exit.

Clinical Findings: Intestinal exposition, hemodynamically stable, without vasopressor and with ventilatory support and with GSA pO2 109-pCO2 43.2-pH 7.28-HCO3 19.5.-Hb 10.9-SatO2 97.8\%

Investigation/Results: The patient is immediately taken to the emergency room where exploratory laparotomy is performed without evidence of intra-abdominal organs damage or active bleeding, stable mesocolonous hematoma is identified and abdominal wall injury requires debridement and placement of Omyra-substitution mesh due to the loss of substance-1/3 upper M. rectus abdominis; intervention proceeds without any incident.

Diagnosis: The abdominal surgery requires a tension-free closure to create a suitable tissue reinforcement, suggesting different alternatives such as the use of prosthetic mesh composed of condensed polytetrafluoroethylene (cPTFE), which currently was proven to be resistant to bacterial growth and also has the integration properties to the tissues previously obtained onlyY with polypropylene meshes Therapy and Progression: During the postoperative the patient presents surgical wound infection requires frecuent dressing changes and antibiotic therapy, being discharged after 7 days to continue ambulatory control.

Comments: Gunshot wounds (GW) often cause a great destruction of the tissues, making it difficult to repair them correctly. The use of meshes has become a great help in the struggle with the primary 
closure of the abdominal wall wounds with the loss of substance, as is the case presented.

References:

Disclosure: No significant relationships.

PC098

\section{DAMAGE CONTROL SURGERY AS PART OF ACUTE MESENTERIC ISCHEMIA MANAGEMENT}

A. Garcia-Granero $^{1}$, A. Sala Hernandez ${ }^{1}$, A. Navio Seller ${ }^{1}$, D. Fletcher Sanfeliu, ${ }^{2}$, N. Garcia Gregorio ${ }^{3}$, S. Bonafe ${ }^{1}$, M. Frasson ${ }^{1}$

${ }^{1}$ Colorectal Unit, HOSPITAL UNIVERSITARIO Y POLITECNICO LA FE. VALENCIA, VALENCIA/SPAIN, ${ }^{2}$ Cardiovascular Surgery, HOSPITAL UNIVERSITARIO SON ESPASES, MALLORCA/ SPAIN, ${ }^{3}$ Anaesthesia Service, HOSPITAL UNIVERSITARIO Y POLITECNICO LA FE. VALENCIA, VALENCIA/SPAIN

Case History: The patient was 63-aged male, diagnosed with alcoholic cirrhosis Child b. Came to emergency department for fever and diffuse abdominal pain.

Clinical Findings: Blood test showed creatinine: $4,09 \mathrm{mg} / \mathrm{dL}$, c-reactive proteine: $176,7 \mathrm{mg} / \mathrm{L}$, procalcitonine: $14,83 \mathrm{ng} / \mathrm{mL}$, lactate: $3,50 \mathrm{mmol} / \mathrm{L}$ and leukocytes: $26,00 \times 10^{3} / \mu \mathrm{L}$.

Investigation/Results: Underwent urgent surgery due to computerized tomography scan findings. It showed non occlusive AMI and direct signs of bowel ischemia and neumoperitoneum.

Diagnosis: Non oclusive acute mesenteric ischemia

Therapy and Progression: A double intestinal resection was performed due to clear necrosis signs and intestinal perforation. One meter resection of distal jejunum and proximal ileum and $30 \mathrm{~cm}$ of distal ileum were performed. A simple plastic drape over the bowel, covered with a sterile towel and the use of Ioban over the abdomen was used for the abdominal closure via negative pressure. After 72 hours in critical care unit, ileo-ileal handsewn and ileo-colic handsewn anastomosis were performed in a the second look surgery. Lenght of time in critical care unit was 10 days because of a respiratory distress and pneumonia. He was discharged 16 days after second surgical intervention without any surgical wound complication.

Comments: Damage control is a surgical modality of choice in the critically ill patient with AMI for physiological and technical reasons. Most often, re-exploration should be accomplished within 48-72 h and decisions regarding anastomosis, stoma, or additional resection can be made with plans for sequential abdominal closure. Various abdominal closure techniques have been described, however, the guiding principle is constant traction on the fascia to facilitate closure. References: Acute mesenteric ischemia: guidelines of the World Society of Emergency Surgery. Bala M, Kashuk J, Moore E, Kluger Y, Biffi w, Gomes CA et al. World Journal of Emergency Surgery 2017;12:38. Abdominal damage control surgery and reconstruction: world society of emergency surgery position paper. Godat L, Kobayashi L, Costantini T, Coimbra R. World Journal of Emergency Surgery 2013; 8:53. Indications and procedures for second-look surgery in acute mesenteric ischemia. Meng X, Liu L, Jiang H. Surg Today 2010;40:700-705

Disclosure: No significant relationships.

\section{PC099}

\section{FLESH-EATING BACTERIA: CAN A GOOD OUTCOME BE ACHIEVED?}

\author{
S. Catarino, M. Ferreira, F. Valério, C. Casimiro
}

Cirurgia Geral, Centro Hospitalar Tondela-Viseu, Viseu/PORTUGAL

Case History: A 61 years-old male, previously healthy, was admitted in the surgical wing with the diagnosis of right lower limb cellulitis for medical treatment.

Clinical Findings: In the first $12 \mathrm{~h}$ after admission the patient became hemodynamically unstable with renal and respiratory impairment. Lesions of the right leg become worse.

Investigation/Results: Laboratory revealed leucocitosis, creatinine of 5,6, CPR of 25,96 , procalcitonine of $48,87 \mathrm{mg} / \mathrm{dL}$ and 4,1 of lactate. Diagnosis: Necrotizing fasciitis

Therapy and Progression: Patient was taken to OR for emergent surgical intervention: fasciotomy of the lateral compartment of the leg and extended surgical excisional debridment of the leg and distal tigh. He was admitted in the ICU for stabilization, with need of renal and cardiovascular support. Large spectrum antibiotics were administered and wound care was undertaken for several days. Then Plastic Surgery collaboration was obtained for reconstructive procedures. The patient was discharged home at the 28th postoperative day without further major complications.

Comments: Early surgical treatment of necrotizing fasciitis is the main factor for obtaining favorable outcomes. However its clinical diagnosis is sometimes difficult, which often leads to delays in initiating appropriate treatment. The Laboratory Risk Indicator for Necrotizing Fasciitis (LRINEC) score, based on laboratory data, improves the diagnostic efficiency between necrotizing fasciitis and other soft tissue infections, even in early stages. The present case report shows that, despite the high morbimortality rate associated with necrotizing fasciitis, its timely treatment may lead to more favorable and functional outcomes.

References: Pablo S. Corona, et al. Necrotising fasciitis of the extremities: implementation of new management technologies. Injury, 2016-09-01, Volume 47, Pages S66-S71. Khalid Al Alayed, Charlie Tan and Nick Danema. Red Flags For Necrotizing Fasciitis: A Case Control Study. International Journal of Infectious Diseases, 2015-0701, Volume 36, Pages 15-20. Jinn-Ming Wang and Hwee-Kheng Lim. Necrotizing fasciitis: eight-year experience and literature review. Brazilian Journal of Infectious Diseases, 2014-03-01, Volume 18, Issue 2, Pages 137-143. N. Hodgins, et al. Analysis of the increasing prevalence of necrotising fasciitis referrals to a regional plastic surgery unit: A retrospective case series. Journal of Plastic, Reconstructive \& Aesthetic Surgery, 2015-03-01, Volume 68, Issue 3, Pages 304-311.

Disclosure: No significant relationships.

\section{PC100}

\section{EMERGENCY SURGERY IN A RARE AND NEAR- FULMINANT FIRST PRESENTATION OF CROHN'S DISEASE}

\author{
G. Van Der Wilden, R. Knulst, R.c.l.a. Maayen, A. Ploeg, S. Koch
}

Surgery, Alrijne hospital, Leiderdorp/NETHERLANDS

Case History: A 30-year old female presents to the ED with progressive, diffuse abdominal pain and signs of septic shock. 
Complaints of abdominal pain (RLQ) were apparent for 6 months after a fall from stairs; other complaints included fatigue and weight loss. It acutely changed to nausea, vomiting, no defecation, and progression of the pain in the RLQ. No significant past medical history.

Clinical Findings: Pale, severely dehydrated young woman, 39.8 degrees Celcius, P166/min), RR100/67, RF25/min. On physical exam there was diffuse abdominal pain, active guarding and rebound tenderness, and a large swelling in the RLQ.

Investigation/Results: Hemoglobin 4.1, leukocytes 3.5, CRP 370 Ultrasound and CT abdomen: subhepatic and subcutaneous, possibly communicating, abcesses. Thereisintra-abdominalfluidseen in Douglas, and diffuse in the abdomen, and possibly a mass around the cecum.

Diagnosis: Abdominal perforation of unknown etiology.

Therapy and Progression: We performed an exploratory laparotomy which showed a perforation in the cecum with fecal peritonitis; a right hemicolectomy with ileostomy and drainage of pus followed. Postoperatively ICU admission with severe sepsis. Pathology confirmed Crohn's disease (CD). A relaparotomy was performed after 1week which didn't show abnormalities; milky fluid was drained (enteroccus faecium) and treated (meropenem). Afterwards the patients was discharged to the surgical ward where she started rehabilitation.Additional endoscopy showed no active signs of CD.

Comments: Spontaneous free perforation is uncommon in the natural history of $\mathrm{CD}$ (1.5-9.5\% incidence). ${ }^{1,2}$ It is possible, that $\mathrm{CD}$, as localized but severe IBD predisposed our patient to bowel perforation with minimal trauma (fall from stairs) ${ }^{3}$. An emergency surgical indication, based on an acute intestinal obstruction or perforationperitonitis, could be the first presentation of CD.

References: 1. Greenstein AJ, Sachar DB, Mann D, Lachman P, Heimann T, Aufses AH Jr. Spontaneous free perforation and perforated abscess in 30 patients with Crohn's disease. Ann Surg. 1987 Jan;205 (1):72-6. 2. Ikeuchi H, Yamamura T. Free perforation in Crohn's disease: review of the Japanese literature. J Gastroenterol. 2002;37 (12):1020-7. 3. Johnson GA, Baker J. Colonic perforation following mild trauma in a patient with Crohn's disease. Am J Emerg Med. 1990 Jul;8 (4):340-1.

Disclosure: No significant relationships.

\section{PC101}

\section{MULTIPLE SPONTANEOUS SMALL BOWEL PERFORATIONS}

A.L.A. Rodrigues $^{1}$, R. Camarneiro ${ }^{1}$, M. Magalhães $^{2}$, L. Val Flores ${ }^{2}$, I. Dionísio ${ }^{I}$, P. Calixto ${ }^{I}$, A. Duarte ${ }^{I}$, M. Brito E Melo ${ }^{I}$

${ }^{1}$ General Surgery, Centro Hospitalar do Oeste, Caldas da Rainha/ PORTUGAL, ${ }^{2}$ Internal Medicine, Centro Hospitalar do Oeste, Caldas da Rainha/PORTUGAL

Case History: The authors report the clinical case of a 42-year-old schizophrenic patient, smoker, admitted to the emergency room with diffuse abdominal pain.

Clinical Findings: The patient presented with abdominal rebound tenderness and peritoneal sign.
Investigation/Results: Raised inflammation markers and the abdominal tomography revealed pneumoperitoneum.

Diagnosis: An emergency laparotomy revealed multiple jejunal and ileal perforations.

Therapy and Progression: The patient was subject to small bowel resection complicated with anastomosis dehiscence, respiratory tract infection and intra-abdominal abscess. Histologic specimens showed nonspecific inflammatory findings. The main infectious, inflammatory, congenital and vascular causes of spontaneous perforations were excluded.

Comments: Spontaneous free perforation of the small bowel is uncommon. While diagnosis of the precise cause might be challenging and after exclusion of other causes, it might be attributed to a local transitory ischaemia.

References: Spontaneous free perforation of the small intestine in adults; Freeman H.; World J Gastroenterol 2014 August 7; 20 (29): 9990-9997 Unusual case of multiple spontaneous perforation of small bowel; . 1966 Jul 16; 2 (5506): 155-156

Disclosure: No significant relationships.

\section{PC102}

\section{MECKEL'S ILEITIS - A RARE CAUSE OF GASTROINTESTINAL BLEEDING IN ADULTS}

\section{T.S. Neto, M. Oliveira, A.C. Carvalho, A. Magalhaes, P. Correia}

General Surgery, Hospital Senhora da Oliveira, Guimarães/ PORTUGAL

Case History: Anatomically Meckel's diverticulum (MD) is a true intestinal diverticulum containing all layers of the small intestine. MD is usually asymptomatic, only $2-4 \%$ of the carriers develop a complication over the course of their life. The authors report a case of a 21-year-old man presented with a 4-day history of gastrointestinal bleeding with no abdominal pain. He denied diarrhea or the use of non-steroidal anti-inflammatory drugs.

Clinical Findings: Physical examination revealed paleness of the skin, sinus tachycardia with normal blood pressure. Rectal examination revealed red blood in stools.

Investigation/Results: Laboratory data showed severe anemia with haemoglobin level $3.8 \mathrm{~g} / \mathrm{dL}$. He was trensfused with 5 units of red blood cells. A oesophagogastroduodenoscopy was performed: negative. A colonoscopy was also performed, and showed ulcers in the terminal ileum with red blood in the ileum coming proximally. Although, hemodynamically stable, he kept bleeding and a small bowel capsule were performed and was inconclusive due to the presence of fresh blood. A single-balloon enteroscopy was proposed and it revealed some ulcers in distal ileum. The patient presented a massive recurrent bleeding and he became hypovolemic.

Diagnosis: A laparotomy was performed with simultaneous endoscopy witch identify large Meckel's diverticulum and a peridiverticulum ulcer with visible vessel. An enterectomy with resection of the diverticulum was carried out. 
Therapy and Progression: The postoperatively period was uneventful, without new episodes of bleeding.

Comments: Despite being the most prevalent congenital anomaly of the gastrointestinal tract, MD continues to be a diagnostic challenge. References: Uppal K, Tubbs RS, Matusz P, Shaffer K, Loukas M. Meckel's diverticulum: a review. Clin Anat. 2011;24:416-22 Sagar J, Kummar V, Ahah DK. Meckel's diverticulum: a systemic review. J R Soc Med. 2006;99:501-5

Disclosure: No significant relationships.

\section{PC102A}

\section{ESOFAGIC PERFORATION BY CHOKING}

\author{
C.L. Palmero, S.G. Modet, D.S. Relinque
}

Cirugia General Y Del Aparato Digestivo, HOSPITAL PUNTA DE EUROPA, ALGECIRAS/SPAIN

Case History: Man of 20 years of age, consults to emergency because yesterday to food intake, presents marked dysphagia. Endoscopy:upper esophagus with extensive mucosal laceration. Distal esophagus shows another deeper lesceration .Extracted foreign body. After procedure is decided SCAN:Neumomediastinum.Bilateral pneumothorax .Neumoperitoneum and pneumoretroperitoneum.

Clinical Findings: pneumomediastinum pneumoperitoneum Pneumoretroperitoneum.

Investigation/Results: The most common cause of esophageal perforation today is iatrogenic perforation secondary to endoscopic instrumentation of the esophagus. The other etiologies are: spontaneous rupture, trauma secondary to ingestion of foreign body and others infrequent (tumors, ingestion of caustics, injury by retained drugs, severe esophagitis, difficult endotracheal intubation, thyroidectomy). impaction of food bowls occur frequently, but most resolve spontaneously, including cutting elements, and only about $10-20 \%$ require intervention 22 . When there is foreign body injury, perforator type lesions can occur and the perforation site frequently occurs in areas of physiological narrowing. The injury may occur spontaneously or be induced by the endoscope when trying to remove the foreign body. In addition, it can cause necrosis by pressure and thus weaken the wall, facilitating the injury by the endoscope.

Diagnosis: SCAN IMAGES

Therapy and Progression: SURGICAL TREATMENT: Primary repair of the perforation

Comments: The decision of surgical repair lies in: the location of the lesion, the presence of previous esophageal pathology, the magnitude of the contamination, the viability of the esophagus and the general state of the patient. Localization is important since cervical locations usually with a drainage of the paracervical space is sufficient. The location of perforation in the thoraco-abdominal segment is associated with an increased risk of sepsis, which usually requires surgical resolution.

References: Zwischenberger JB, Savage C, Bidani A. Surgical aspects of esophageal disease: perforation and caustic injury. Am J Respir Crit Care Med 2002; 165: 1037-40. Blom D, Peters J. Esophageal perforation. In: Cameron JL, ed. Current Surgical Therapy. St Louis, MO: Mosby, 2001: 7-12. Sung SW, Park JJ, Kim YT, Kim $\mathrm{JH}$. Surgery in thoracic esophageal perforation: primary repair is feasible. Dis Esophagus 2002; 15: 204-9. Port J, Kent M, Korst R, Bacchetta M, Altorki N. Thoracic Esophageal Perforations: A Decade of Experience. Ann Thorac Surg 2003; 75: 1071-4.

Disclosure: No significant relationships.

\section{PC102B}

\section{¿ IS IT NECESSARY TO PERFORM A SCAN TO REMOVE A FOREIGN BODY FROM THE CHEST?}

\author{
C.L. Palmero, S.G. Modet, L. Ojea
}

Cirugia General Y Del Aparato Digestivo, HOSPITAL PUNTA DE EUROPA, ALGECIRAS/SPAIN

Case History: 35 year old man goes to the emergency because during his workout a nail has been nailed to his chest after bouncing at a table

Clinical Findings: - $\mathrm{x}$ ray: nail is visualized in region thoracic center without evidence pneumotorax, no mediastinal enlargement nor other findings. - images nail over chest

Investigation/Results: Thoracic injuries account for $20 \%$ to $25 \%$ of all trauma deaths, and complications from chest trauma contribute to another $25 \%$ of all deaths. Less than $15 \%$ of these lesions require definitive surgical treatment. More than $80 \%$ of life-threatening chest injuries can be reversed with appropriate measures

Diagnosis: In this case it was sufficient to perform a $\mathrm{x}$ ray to evaluate the damages. If the patient had been unstable or the depth had been greater we would have performed by performing scan,

Therapy and Progression: - images taken on nail in surgery room. Comments: Surgical intervention is rarely necessary in blunt thoracic injuries. . Most can be treated with supportive measures and simple interventions such as this case. In chest wall injuries, fractures, dislocations, and diaphragmatic lesions, indications for immediate surgery include cases with traumatic loss of chest wall integrity and significant diaphragmatic lesions or an unstable patient

References: Manlulu A, Lee T, Thung K, Wong R, Yim A. Current indications and results of VATS in the evaluation and management of hemodynamically stable thoracic injuries. European $\mathbf{J}$ of Cardiothoracic Surgery. 2004;25:1048-53

Disclosure: No significant relationships.

\section{$\mathrm{PC} 103$}

\section{INTESTINAL MALROTATION IN THE ELDERLY: AN UNCOMMON AND DIAGNOSTICALLY CHALLENGING CASE OF SMALL BOWEL OBSTRUCTION}

\author{
L. Viggiani, J. Guerrini, G. Costa, S. Mei, R. Menè, M. Ceolin, \\ H. Kurihara
}

Acute Care And Trauma Surgery Unit, Humanitas Research Hospital, Rozzano, Milano/ITALY

Case History: A 64-years old man was evaluated in our ED for severe abdominal pain, nausea, vomiting, significant weight loss (10kg in one year) and irregular bowel movements. Three weeks before, he underwent laparotomic jejuno-ileal volvulus derotation for the same symptomatology, and CT findings (i.e. whirlpool sign with upstream bowel distension and intestinal loops edema) compatible with Small Bowel Obstruction (SBO) with associated mesenteric volvulus. Recovery was uncomplicated in the first week but characterized by progressive reappearance of preoperative symptomatology. Clinical Findings: Physical examination measured a BMI of 18,8 and vital signs in range of normality. The distended and treatable abdomen presented a scar of a previous median laparotomy and was moderately tender at deep palpation but with normal peristalsis. 
Investigation/Results: Point-of-care ultrasonography and abdominal CT were compatible with SBO relapse. NGT was placed, Gastrografin administered; seven hours later $\mathrm{x}$-ray showed contrast medium in the left colon. Patient was admitted to surgical department for persistent severe postprandial abdominalgia suggestive of angina abdominis. Diagnosis: A more detailed history was taken, revealing the presence of symptoms, even though in a milder form, since childhood. Careful reevaluation of abdominal CT showed signs diagnostic for intestinal malrotation such as duodenal displacement, upper localization of the cecum, inversion in the SMA/SMV relationship.

Therapy and Progression: An explorative laparotomy confirmed our clinical suspect by findings of Ladd band, subsequently Ladd procedure was performed. Complete resolution of pain was achieved with surgery and confirmed after six months follow up. Although nausea and vomiting persist.

Comments: This case report presents an uncommon and diagnostically challenging picture of SBO.

References: Malek MM, Burd RS. The optimal management of malrotation diagnosed after infancy: a decision analysis. Am J Surg 2006; 191:45. Hsu SD, Yu JC, Chou SJ, et al. Midgut volvulus in an adult with congenital malrotation. Am J Surg 2008; 195:705.

Disclosure: No significant relationships.

\section{PC104}

\section{TRANSCATHETER ARTERIAL EMBOLIZATION FOR SPONTANEOUS RUPTURE OF INFERIOR ADRENAL ARTERY ANEURYSM}

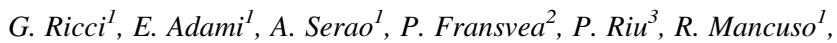
J.R.M. Casella ${ }^{I}$, P. Marini ${ }^{1}$

${ }^{1}$ Emergency Department - Division Of General And Emergency Surgery, San Camillo - Forlanini Hospital, Rome/ITALY, ${ }^{2}$ Chirurgia Generale E D'urgenza, Facoltà di Medicina e Psicologia Università "Sapienza" Roma, Rome/ITALY, ${ }^{3}$ Division Of Interventional Radiology, San Camillo - Forlanini Hospital, Rome/ITALY

Case History: A 76-year-old man was referred to our emergency service for suspected right renal colic due to sudden right flank pain started 12 hours before. In his past medical history poorly controlled hypertension and cholecystectomy for cholelithiasis was reported, patient was using aspirin for primary prevention of cardiovascular disease.

Clinical Findings: Upon admission patient was agitated and pale, on physical examination blood pressure was $133 / 71$ and pulse 110 beats/ min, there was tenderness in the right flank with rebound tenderness and positive Giordano's sign.

Investigation/Results: Routine blood tests were unremarkable except for hemoglobin $(9,3 \mathrm{~g} / \mathrm{dl})$ and white blood cells $\left(18.070 / \mathrm{mm}^{3}\right)$.

Diagnosis: Abdominal ultrasound identified a large right flank mass, then a triphasic CT was done: a large retroperitoneal hematoma due to rupture of a little inferior adrenal artery aneurysm with active bleeding was detected.

Therapy and Progression: Emergency angiography confirmed the diagnosis and transcatheter arterial embolization (TAE) of inferior adrenal artery was performed using a combination of microcoils and gelatin sponge particles. CT performed 24 hours after the procedure confirmed complete cessation of the hemorrhage, the patient did well and was discharged on postoperative day 6. CT obtained 12 months later demonstrated no abnormalities.

Comments: Visceral artery aneurysms are an uncommon form of vascular disease the pathogenesis of which remain incompletely characterized. Aneurysms of adrenal arteries are exceedingly rare. To our knwledge there have been only 9 cases of ruptured adrenal artery aneurysms reported in the literature. Immediate surgical operation versus TAE remains controversial in spontaneous retroperitoneal hemorrhage, our case showed that TAE is safe and effective in emergency setting.

References: 1. Kawashima A, Sandler CM, Ernst RD et al. Imaging of nontraumatic hemorrhage of the adrenal gland. Radiographics 1999;19 (4):949-963. 2. Messina LM, Stanley CJ. Visceral artery aneurysms. Surg Clin North Am 1997;77:425-441. 3. Manners J, Singh R, Page A, Adamson A, McLean D. Radiological treatment of a spontaneously ruptured inferior adrenal artery aneurysm. Nat Rev Urol 2010;7 (12):694-698.

Disclosure: No significant relationships.

\section{PC105}

\section{LAPAROSCOPIC REPAIR OF A GRYNFELT-LESSHAFT HERNIA: CASE PRESENTATION AND REVIEW OF THE LITERATURE}

\section{A. Goparaju $^{1}$, D. Mcphee ${ }^{2}$}

${ }^{1}$ Surgery, Lincoln Medical Center, bronx/NY/UNITED STATES OF AMERICA, ${ }^{2}$ Surgery, Lincoln Medical Center, bronx/UNITED STATES OF AMERICA

Case History: A 42 year old man presented to Lincoln hospital complaining of bilateral chronic back pain. He was initially evaluated for bilateral flank masses in 2012. He had no other associated symptoms. He underwent a left sided laparoscopic repair of a superior lumbar triangle hernia at that time. He now presents 4 years later with right sided pain. He denied fevers, nausea, vomiting, dysuria, change in bowel habits, weight loss.

Clinical Findings: Right sided flank tenderness without rebound or guarding. no abdominal tenderness.

Investigation/Results: CT findings showed lipomatous material herniating below 12th rib, bounded by quadratus lumborum medially and internal oblique laterally on the right side.

Diagnosis: Based on physical exam, history and cross-sectional imaging, a diagnosis of a persistent right sided superior lumbar triangle (grynfeltt-lesshaft hernia) was made.

Therapy and Progression: We elected to perform laparoscopic transabdominal retroperitoneal repair. To perform this the colon was mobilized to expose the gerotas fascia and fat pad, which had found to be herniated through, The hernia was reduced into the retroperitoneum and the defect was cleared of surrounding tissue. an $8 \times 5$ parietex mesh was used to repair the defect and the peritoneum was closed overlying. He has subsequently recovered and has no further complaints.

Comments: This is a rare type of abdominal wall hernia with most surgeons only encountering this once in their careers. While open repair has been historically performed with good outcomes, laparoscopic repair is a more modern technique and found to have decreased pain and decreased length of stay. 
References: 1. Ploneda-Valencia CF, Cordero-Estrada E, CastañedaGonzález LG, et al. Grynfelt-Lesshaft hernia a case report and review of the literature. Annals of Medicine and Surgery. 2016;7:104-106. doi:10.1016/j.amsu.2016.04.002. https://www.ncbi.nlm.nih.gov/pmc/ articles/PMC4840394/2. Sundaramurthy S, Suresh HB, Anirudh AV, Prakash Rozario A. Primary lumbar hernia: A rarely encountered hernia. International Journal of Surgery Case Reports. 2016;20:5356. doi:10.1016/j.ijscr.2015.09.041.

Disclosure: No significant relationships.

\section{PC106}

\section{RECENT ADVANCES IN THORACOSCOPY DO NOT RELEASE TO FASTEN DENTAL PROTHESIS BEFORE A PARTY}

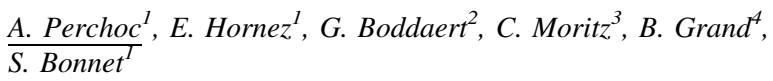

${ }^{1}$ Department Of Digestive Surgery, Percy Military hospital, Clamart/ FRANCE, ${ }^{2}$ Department Of Thoracic Surgery, Percy Military hospital, Clamart/FRANCE, ${ }^{3}$ Digestive Surgery, HIA Percy, Clamart/ FRANCE, ${ }^{4}$ Thoracic Surgry, Percy military hospital, clamart/ FRANCE

Case History: Foreign body ingestion is an uncommon event in adults. In about $80 \%$ of cases, the ingested material passes uneventfully through the gastrointestinal tract; endoscopy is mandatory in about $20 \%$ of cases, and surgery is necessary in less than $1 \%$. This case report illustrates the interest of thoracoscopy to extract esophageal foreign bodies

Clinical Findings: A 67-years-old man presented acute dysphagia after a festive acute alcohol.

Investigation/Results: After a 6 days symptomatic treatment, a CTscan was performed.

Diagnosis: A large intra-esophageal foreign body with parietal remodeling without necrosis or fistula was discovered.

Therapy and Progression: Oesophago-gastro-duodenoscopy was perform and highlighted a large dental prothesis, denture $22 \mathrm{~cm}$ of the dental arches. Endoscopic removing was unsuccessful. This patient was transferred to the thoracic surgery department. At day 10, denture was removed by oesophagotomy done by a right thoracoscopy. The oesophageal repair was done with separate stiches (resorbable wire). Postoperatively, the patient presented a delayed low grade fistula treated by endoscopic closure (clips). After a 3 weeks hospitalization stay, the patient discharged. One month later the clinical assessment and the CT-scan were normal.

Comments: In case of failure of endoscopic procedures, surgery remains the last resort. Gold standard approach is a right thoracotomy. Thoracoscopy is an alternative way allowing to limit the mobidity. Foreign body is extracted through a longitudinal incision in the esophagus, which is closed ideally by a 2 -layer (mucosa and muscularis) primary suture repair, preferably covered by a flap. Concerning this patient with significant predictors of complications (delay $>24$ hours; large dimensions foreign body; age $>50$ years), no significant morbidity was reported.

References: Eisen GM, Baron TH, Dominitz JA, Faigel DO, Goldstein JL, Johanson JF, et al. Guidelines for the management of ingested foreign bodies. Gastrointest Endosc 2002;55:802-6. Hung CW, Hung SC, Lee CJ, Lee WH, Wu KH. Risk factors for complications after a foreign body is retained in the esophagus. J Emerg Med 2012;43:423-7. Sung SH, Jeon SW, Son HS, Kim SK, Jung MK, Cho
$\mathrm{CM}$, et al. Factors predictive of risk for complications in patients with esophageal foreign bodies. Dig Liver Dis 2011;43:632-5.

Disclosure: No significant relationships.

\section{PC107}

\section{HETEROTAXIA: A CHALLENGING DECISION MAKING EVEN IN EMERGENCY CARE SURGERY}

\author{
L. Del Prete ${ }^{I}$, M. Marconi ${ }^{1}$, M. Freschi ${ }^{I}$, V. Pacilè ${ }^{l}$, M. Quarenghi ${ }^{2}$, \\ M. Oldani $^{I}$, R.A. Pappalardo ${ }^{I}$
}

${ }^{1}$ General And Emergency Surgery, Hospital Santa Maria Delle Stelle, Melzo/ITALY, ${ }^{2}$ Radiology Department, ASST Melegnano e Brianza, Vizzolo Predabissi/ITALY

Case History: This case involves a 32-year-old female without any past history, admitted in a small emergency department of a countryside hospital complaining fever and acute severe pain in mesogastrium and left upper quadrant for six hours.

Clinical Findings: Laboratory findings was suitable for infection and an E-FAST exam was carried out bedside with no anatomical correspondence. An abdominal US was consistent for an acute cholecystitis, with the gallbladder positioned in the middle of the abdomen with no spleen on the left side.

Investigation/Results: Despite the patient was eligible for emergency laparoscopic cholecystectomy, she was treated with drugs because of unclear anatomy.

Diagnosis: The next day MRI was performed showing unknown anomalies in abdominal organs including a right-sided stomach, a left-sided midline liver, right-sided spleens, malrotation of the intestine, a short pancreas and anomalies of the inferior vena cava.

Therapy and Progression: For clinical worsening the patient underwent to a laparoscopic cholecystectomy the day after.

Comments: Heterotaxy syndrome is a rare condition involving multiple gastrointestinal, vascular, and cardiac malformations which could make more challenging emergency surgical treatment. It should be suspected if US demonstrates unclear anatomy and a second-level imaging such as CT or MRI is useful to detect anatomical anomalies and to plan surgery based on the patient's anatomy even in acute care surgery.

References:

Disclosure: No significant relationships.

\section{PC108}

\section{INCIDENCE AND MANAGEMENT OF ENDOSCOPIC PERFORATIONS OF THE UPPER GASTROINTESTINAL TRACT}

M.I. Orue-Echebarria González ${ }^{1}$, A. Moreno Hidalgo ${ }^{2}$, J. Morales Bernaldo De Quirós ${ }^{1}$, J. De Tomás Palacios ${ }^{1}$, M. Sanz Sánchez, F. Turégano Fuentes ${ }^{2}$

${ }^{1}$ Surgery, Hospital Gregorio Marañón, Madrid/SPAIN, ${ }^{2}$ Surgery, HOSPITAL GREGORIO MARAÑON, MADRID/SPAIN

Case History: Background: Gastrointestinal (GI) tract perforation during endoscopy is a rare but severe complication. The aim of this study was to describe the management of these perforations in our centre. 
Clinical Findings: Material and Methods: All cases with transmural endoscopic perforations of the upper-GI tract at a tertiary referral center in a 10-year period (2007-2017) were retrospectively analyzed. Demographics, type of endoscopy, site of perforation, management, surgical approach, hospital stay, morbidity and mortality were analyzed.

Investigation/Results: Results: A total of 83.875 upper-GI endoscopies were performed, and $11(0,01 \%)$ patients were diagnosed with GI perforation, all but one during the procedure. Perforations occurred during ERCP, EUS, PEG, anastomotic dilatation, and conventional endoscopy in 4, 4, 1, 1, and 1 patient, respectively. Six occurred at the duodenum, three at the esophagus, and two at the stomach. Suspected perforations were confirmed by CT scan. Therapeutic management involved 6 surgical procedures in the first $24 \mathrm{~h}$ (3 laparoscopic and 3 open), 2 endoscopic procedures, 2 with antibiotics, and 1 case received just supportive care. The median hospital stay was 8 days. Four patients had complications, three of them with a grade $\geq$ III of the Clavien-Dindo classification, and one patient died. Diagnosis: Transmural endoscopic perforations of the upper-GI tract Therapy and Progression: Therapeutic management involved surgical procedures in the first $24 \mathrm{~h}$, endoscopic procedures, or antibiotics.

Comments: Conclusion: The incidence of upper GI endoscopic perforations is low at our center, although it is associated with severe morbidity. Non-operative management and laparoscopy should be considered in selected cases.

References:

Disclosure: No significant relationships.

\section{PC109}

\section{INTERPARIETAL HERNIA - A RARE CAUSE OF INTESTINAL OBSTRUCTION}

\author{
R. Loureiro, A. Marques, J. Constantino, C. Casimiro
}

\section{General Surgery, Centro Hospitalar Tondela-Viseu, Viseu/ PORTUGAL}

Case History: The interparietal hernia is a rare condition in which the hernia sac lies between the layers of the abdominal wall. These hernias occur more frequently in previous incisions or in inguinal area. The diagnosis can be difficult and most patients present with intestinal obstruction.

Clinical Findings: A woman presented to the emergency department complaining with abdominal pain, nausea and vomiting. The physical examination revealed distended abdomen.

Investigation/Results: The abdominal $\mathrm{X}$-rays showed small bowel distension with air fluid levels. An abdominal CT scan was performed revealing small bowel obstruction secondary to an incarcerated hernia in the left upper abdomen. The hernia sac lied posterior to the left rectus abdominis.

Diagnosis: The patient underwent an urgent laparoscopy which showed small bowel herniating through the defect.

Therapy and Progression: The hernia was reduced and mesh repair of the defect was performed laparoscopically. The incarcerated segment of small bowel was edematous with a stenotic area and therefore was resected.

Comments: Interparietal hernias are rare and this is the first case report of an interparietal hernia whose sac lies posterior to the rectus abdominis muscle and isn't related to previous abdominal incisions. A high index of suspicion is needed for its diagnosis and when the hernia is associated to bowel obstruction, urgent surgery is mandatory. Although the laparoscopic approach is not considered the standard treatment for abdominal wall hernias presenting with bowel obstruction, it may be an useful tool in the diagnosis of those cases in which the aetiology is obscure and it can even be used for treatment in some cases.

References: Spaliviero M. et al., Trocar site spigelian-type hernia after robot-assisted laparoscopic prostatectomy. Urology. 2009; 73:1423.e3-1423.e5. Lim SK. et al., A rare case of interparietal incisional hernia from $8 \mathrm{~mm}$ trocar site after robot-assisted laparoscopic prostatectomy. Hernia. 2014. Maurice A. et al., Epigastirc hernia presenting as a giant abdominal interparietal hernia. Int J Surg Case Rep. 2011; 2 (8): 243-245. Townsend, C. et al., Sabiston Textbook of Surgery: The Biological Basis of Modern Surgical Practice, 20th edition, 2016.

Disclosure: No significant relationships.

\section{PC110}

\section{PRIMARY OMENTAL STRANGULATION IN A VIRGIN ABDOMEN, AN ATYPICAL SOURCE OF ABDOMINAL PAIN}

\author{
T. Katsichtis ${ }^{1}$, S. Safadjou ${ }^{1}$, G. Medrano ${ }^{2}$, D. Mcphee ${ }^{3}$
}

${ }^{1}$ Department Of Surgery, Cornell University, Lincoln Medical Center Campus, Bronx/NY/UNITED STATES OF AMERICA, ${ }^{2}$ General

Surgery, Lincoln Medical and Mental Health Center, New york/ UNITED STATES OF AMERICA, ${ }^{3}$ Surgery, Lincoln Medical Center, bronx/NY/UNITED STATES OF AMERICA

Case History: 27 year-old male, with no precious medical or surgical history, presents with right upper quadrant abdominal pain for 5 days. Clinical Findings: Patient presented with tenderness at right upper quadrant upon palpation. No rebound tenderness or other peritoneal signs were identified.

Investigation/Results: Cross sectional imaging demonstrated possible internal hernia containing omentum or mesentery with no herniation of bowel loops. In addition mild mural thickening of a short segment of the ascending colon was demonstrated. There was no evidence of pneumatosis or free air or small bowel obstruction. Due to the concern of internal hernia patient was taken to the operating room for diagnostic laparoscopy.

Diagnosis: On exploration of the peritoneal cavity, there was hemorrhagic fluid at right upper quadrant and the pelvis. Omentum was volvulised and necrotic and adhered to the anterior border of the hepatic flexure of the colon. There was an adhesion band extending from the left upper quadrant to omentum which was transected using LigaSure. The necrotic part of the omentum was resected using the LigaSure. There was no internal hernia and no bowel compromise. Of note patient's colon was found to be redundant.

Therapy and Progression: Patient was started on regular diet postoperative day 1 . He was pain free and was discharged postoperative day 2.

Comments: Omental volvulus and strangulation is an uncommon entity that may lead to abdominal pain. It is commonly secondary to other pathology such us cholecystitis and abdominal wall hernia. In this case the patient presented with primary omental strangulation associated with adhesive band to a redundant colon.

References: Karayiannakis, Anastasios J., et al. "Primary torsion of the greater omentum: report of a case." Surgery today 32.10 (2002): 913-915. Breunung, Nina, and Paul Strauss. "A diagnostic challenge: primary omental torsion and literature review-a case report." World Journal of Emergency Surgery 4.1 (2009): 40. Saraç, A. Murat, et al. "Primary torsion of the omentum mimicking acute appendicitis: 
report of a case." Surgery today 27.3 (1997): 251-253. Al-Husaini, Hiyad, Aideloje Onime, and Soji F. Oluwole. "Primary torsion of the greater omentum." Journal of the National Medical Association 92.6 (2000): 306. Naffaa, Lena N., Nina S. Shabb, and Maurice C. Haddad. "CT findings of omental torsion and infarction: case report and review of the literature." Clinical Imaging 27.2 (2003): 116-118. Disclosure: No significant relationships.

\section{PC111}

\section{MANAGEMENT OF PERFORATED TUMOR OF ILEUM WITH RESPECT TO THE ONCOLOGICAL PRINCIPLES: A CASE REPORT}

\author{
E.J. Barzola Navarro ${ }^{1}$, A. Glagolieva ${ }^{2}$
}

\author{
${ }^{1}$ Digestive Surgery, HM HOSPITALES MADRID, Madrid/SPAIN, \\ ${ }^{2}$ Surgery And Vascular Surgery, PL Shupyk National Medical \\ Academy, kiev/UKRAINE
}

Case History: A 57-year-old male patient was admitted to the emergency department reporting diffuse abdominal pain, which started suddenly in the epigastrium accompanied by nausea.

Clinical Findings: Physical examination revealed signs of peritoneal irritation suspicious for the perforation of the hollow viscus.

Investigation/Results: Chest and abdomen $\mathrm{X}$-rays showed no signs of pneumoperitoneum. In the laboratory data, leukocytosis with neutrophilia were observed. A complete abdominal CT scanning was performed, and a pelvic adhesion was found at the level of pelvis and small extraluminal air bubbles.

Diagnosis: Patient underwent emergency surgery. At the laparotomy, a purulent peritonitis with a moderate amount of liquid was observed and a tumor of approximately $10 \mathrm{~cm}$ at the antimesenteric border of the ileum was found, adhering but not infiltrating to the bottom of the bladder. Oncological resection of the ileum was performed. The pathology report revealed intestinal stromal tumor (GIST) in the ileum of $8 \mathrm{~cm}$, with CD117 +, mitotic index $>$ 10HPF, pT3N0, with free resection margins.

Therapy and Progression: The case was presented to the tumor board and an adjuvant treatment with Imatinib was started. On the follow-up examinations, no evidence of local recurrence was found. Comments: Surgeons usually face several circumstances that may influence the oncological prognosis of patients. Perforated gastrointestinal tumors (GISTs) are rare. Complete surgical resection is the only curative option for the localized GISTs. The prognosis is based on the mitotic count, size, location, and signs of the tumor rupture. As the tumor in the above case was considered high risk, the adjuvant treatment with Imatinib was justified.

References: Efremidou EI, Liratzopoulos N, Papageorgiou MS, Romanidis K, Manolas KJ, Minopoulos GJ. Perforated GIST of the small intestine as a rare cause of acute abdomen: surgical treatment and adjuvant therapy. Case report. Journal of Gastrointestinal and Liver Diseases. 2006 Sep;15 (3):297.

Disclosure: No significant relationships.
PC112

\section{PRACTICAL USE OF IABO (INTRA-AORTIC BALLOON OCCLUSION) IN GENERAL SURGERY}

\section{T. Kubota}

Department Of General Surgery, Tokyo Bay Medical Center, Urayasu/JAPAN

Case History: As general IABO is widely use for traumatic hemorrhagic shock.

Clinical Findings: However we have tried to apply IABO not only trauma but also non-trauma cases, in which IABO really worked.

Investigation/Results: Case 1:47year old male with the status of subacute phase of severe necrotizing pancreatitis had massive gastrointestinal bleeding (hemosuccus pancreaticus) related to ruptured pseudo aneurysm of the GDA (gastro-duodenal artery). Before we started immediateTAE (trans arterial embolization), the patient was in pre-arrest, systolic pressure was under $50 \mathrm{mmHg}$. So we inflated IABO just above celiac axis. After resuscitation, TAE was done successfully.

Diagnosis: Case 2: 69 year old male with advanced pancreatic head cancer undertook pancreatico-duodenectomy. During the surgery, we encountered the massive bleeding from a side hole of the SMA (superior mesenteric artery). At that time, it was difficult to make proximal clump of the SMA. We had two options one was aortic clump using surgical device. The other was IABO. We choose the later, because surgeons hands have been fixed to give a compression to the bleeding point. IABO was inflated just the orifice level of the SMA by another physician. After we got complete hemostasis, the hole of the SMA was easily sutured.

Therapy and Progression: IABO is effective device to control arterial bleeding. It play an important roll in case of critical situation. Comments: We should it is worth to be familiarize ourself to use IABO, or to have a free access to IVR physician.

Disclosure: No significant relationships.

\section{PC112A}

\section{INTESTINAL OBSTRUCCIÓN SECONDARY TO RENAL POLICYSTOSIS. A CASE REPORT}

R. Ortega Higueruelo, J. Rubio López, R. Cobos Cuesta, A. Cozar Ibañez, C.M. Ruiz Marín, F.M. Jiménez Armenteros, J.M. Capitan Vallvey

General And Digestive Surgery, Complejo Hospitalario Jaén, Jaén/ SPAIN

Case History: Intestinal obstruction is a blockage that hinders intestinal transit. The most frequent causes are adhesions, hernias or intestinal tumors. Retroperitoneal masses causing bowel compression are extremely rare.

Clinical Findings: 40yo male came into the emergency room with abdominal pain, vomiting and septic shock and was admitted in ICU. 
Investigation/Results: CT was performed which reported an enlarged right polycystic kidney, normal bowel loops with fluid and hyperemia suggesting an inflammatory process with normal vascular study.

Diagnosis: Septic shock.

Therapy and Progression: The patient is transferred to the ward after improvement but continued with abdominal pain episodes. A new CT was performed on 6thh day in which moderate dilatation of intestinal loops without identifying the cause was reported. Exploratory laparotomy was performed without findings. In the postoperative period, pain and abdominal distension persisted with fecaloid output in the NG tube. An evaluation was requested to Urologists and right cyst punction was performed, after which the patient solved his obstructive sympthoms and was discharged after 3 days.

Comments: Retroperitoneal causes of intestinal obstruction are rare. When suspicion arises the treatment of the cause is mandatory.

References:

Disclosure: No significant relationships.

\section{PC112B}

\section{SUBACUTE DISTAL THROMBOTIC MESENTERIC ISCHAEMIA. AN OLD FELLOW WITH DIFFICULT MANAGEMENT}

I. Martínez Casas ${ }^{1}$, R. Ortega Higueruelo ${ }^{2}$, A.X. Argote Camacho ${ }^{1}$, R. Cobos Cuesta ${ }^{2}$, C.M. Ruiz Marín ${ }^{2}$, F.M. Jiménez Armenteros ${ }^{2}$, J.M. Capitan Vallvey ${ }^{2}$

${ }^{1}$ Ugc Cirugía General, Complejo Hospitalario de jaén, Jaén/SPAIN, ${ }^{2}$ General And Digestive Surgery, Complejo Hospitalario Jaén, Jaén/ SPAIN

Case History: According to guidelines, TAMI has acute but recurrent onset. When untreated will cause mesenteric infarction, intestinal necrosis, an overwhelming inflammatory response and death. Early intervention can everse this process leading to a full recovery. We present a case of a gradual onset TAMI with failure to recognize the disease in early laparotomy.

Clinical Findings: 83yo diabetic male with history of arteriosclerosis, hypertension and bypass surgery, came into our ED for abdominal pain, mainly in RLQ.

Investigation/Results: Analysis showed leukocytosis and a CT informed splenic infarct, distal superior mesenteric arthery trombosis and diverticular disease.

Diagnosis: Subacute Thrombotic Mesenteric Ischaemia.

Therapy and Progression: Vascular consultation for was denied and non-therapeutic exploratory laparotomy was performed. The patient was unable to thrief, with pain after every meal but correct bowel transit. After 16 days another CT showed intestinal pneumatosis and proximal ileon bowel wall enlargement. A second look laparotomy confirmed TAMI and resection plus anastomosis was performed. Patient was discharged on 7th POD

Comments: TAMI is generally described as acute onset recurrent disease. Early diagnosis and prompt treatment are the goals of modern therapy. Endovascular treatment is the first choice treatment whenever possible, but surgery remains the solution specially in distal cases when revascularization is imposible. The timming of surgery is the key and requires good clinical judgement.

Reference: Tilsed, J. V. T., Casamassima, A., Kurihara, H., Mariani, D., Martínez-Casas, I., Pereira, J., et al. (2016, April). ESTES guidelines: acute mesenteric ischaemia. European Journal of Trauma and Emergency Surgery. http://doi.org/10.1007/s00068-016-0634-0 Disclosure: No significant relationships.

\section{PC113}

\section{COMPLETE MECHANICAL SMALL BOWEL OBSTRUCTION SECONDARY TO A CHRONIC VENTRICULOPERITONEAL SHUNT - A RARE COMPLICATION: CASE PRESENTATION AND REVIEW OF THE LITERATURE}

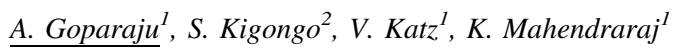

${ }^{1}$ Department Of Surgery, Cornell University, Lincoln Medical Center Campus, Bronx/NY/UNITED STATES OF AMERICA, ${ }^{2}$ Surgery, Lincoln Medical Center, bronx/UNITED STATES OF AMERICA

Case History: A 33 year old man presented with one day history of abdominal pain, nausea, vomiting, abdominal distention. He had a history of VP shunt placed for hydrocephalus as a child that was subsequently revised when he was a teenager.

Clinical Findings: He had worsening generalized abdominal tenderness, and distension.

Investigation/Results: He had no significant leukocytosis or electrolyte abnormalities. He was found on cross-sectional imaging to have a complete bowel obstruction in the mid-distal small intestine associated with the tip of a ventriculoperitoneal shunt.

Diagnosis: $\mathrm{He}$ was diagnosed with a high grade small bowel obstruction associated with VP shunt based on imaging and clinical findings.

Therapy and Progression: A prompt exploration was performed. There was found to be a pseudocapsule formed around the catheter tip with fibrosis adherent to a midjejunal loop. Due to questionable bowel viability, a temporary abdominal closure was performed. Upon reexploration, The bowel loop in question was ischemic and was resected. Abdomen was closed with biologic underlay mesh. The ventriculoperitoneal shunt was externalized and placed to gravity drainage. Subsequently the drain was repositioned into the thoracic cavity. After a prolonged ileus he has recovered and was discharged from the hospital.

Comments: Management of VP shunt in the presence of localized peritonitis secondary to appendicitis has been described and in the absence of generalized peritonitis, conservative management of the catheter has been successful. Intra-abdominal VP shunt complications, while well described in the pediatric population are rare occurrences in the adult population. The clinical situation is challenging and requires a multi-disciplinary approach to minimize morbidity.

References: 1. Starreveld Y, Poenaru D, Ellis P. Ventriculoperitoneal shunt knot: a rare cause of bowel obstruction and ischemia. Canadian Journal of Surgery. 1998;41 (3):239-240. 2. Zhao R, Shi W, Yu J, Gao X, Li H. Complete Intestinal Obstruction and Necrosis as a Complication of a Ventriculoperitoneal Shunt in Children: A Report of 2 Cases and Systematic Literature Review. Elena CR, ed. Medicine. 2015;94 (34):e1375. doi:10.1097/MD.0000000000001375. 3. Tan LA ${ }^{1}$, Kasliwal MK, Moftakhar R, Munoz LF. Ventriculoperitoneal shunt with a rare twist: small-bowel ischemia and necrosis secondary to knotting of peritoneal catheter. J Neurosurg Pediatr. 2014 Sep;14 (3):234-7. doi: 10.3171/2014.6.PEDS1418. Epub 2014 Jul 4. 4. Chung JJ. Intraabdominal complications secondary to ventriculoperitoneal shunts: CT findings and review of the literature. AJR Am J Roentgenol 2009 Nov;193 (5):1311-7

PMID:19843747 5. Yuh S-J, Vassilyadi M. Management of abdominal pseudocyst in shunt-dependent hydrocephalus. Surgical Neurology International. 2012;3:146. doi:10.4103/21527806.103890. 6. Agarwal T, Pandey S, Niranjan A, Jain V, Mishra S, Agarwal V. Unusual complication of ventriculoperitoneal shunt surgery. Journal of Pediatric Neurosciences. 2009;4 (2):122-123. 
doi:10.4103/1817-1745.57340. 7. Apelqvist, J., Willy, C. Fagerdah, A.M. et al. Negative pressure wound therapy - overview, challenges, and perspectives. J Wound Care 2017; 26: 3, Suppl 3 S1-113

Disclosure: No significant relationships.

\section{PC114}

\section{ACUTE MESENTERIC VENOUS THROMBOSIS}

\section{${\text { A. } \text { Logrado }^{I}, \text { J. Constantino }^{2}, \text { J. Pereira }^{I} \text {, C. Casimiro }}^{\text {I }}$}

${ }^{1}$ General Surgery, Centro Hospitalar Tondela-Viseu, Viseu/ PORTUGAL, ${ }^{2}$ Cirurgia Geral, Centro Hospitalar Tondela-Viseu, Viseu/PORTUGAL

Case History: Mesenteric venous thrombosis (MVT) is a mesenteric venous system occlusion, in its main or secondary branches and, in acute form it can cause intestinal infarction. With an incidence of 5\% in cases of intestinal ischemia, MVT is caused by hypercoagulable states, inflammatory disease or trauma.

Clinical Findings: The authors present the case of a 37-year-old woman who resorts to the ER with a sudden onset hypogastric pain, combined with nausea and vomiting. The patient was hemodynamically stable with painful abdomen but no signs of peritoneal irritation. Investigation/Results: Abdominal ultrasound was performed which showed thickened bowel loop and a CT-scan that confirmed the presence of venous thrombosis of the superior mesenteric vein branches with the same extension, adjacent to thickened bowel loop. Diagnosis: Acute mesenteric venous thrombosis

Therapy and Progression: The patient was admitted for observation and started anticoagulation with LMWH. The patient had a good evolution without need for bowel resection. Discharged on day 5 anticoagulated with rivaroxaban. Thrombophilia cause as been excluded and the recent introduction of combined oral contraceptive was determined as the cause of the event. CT-scan six months later showed total venous repermeabilization.

Comments: There are no specific clinical or laboratory abnormalities on MVT. The diagnosis of MVT is made definitively using radiographic studies. Anticoagulation should be established as soon as possible so as to limit the progression and prevent further thrombotic events. The determination of the underlying cause is crucial for the establishment of a definitive therapy.

References:

Disclosure: No significant relationships.

\section{PC115}

DAMAGE CONTROL SURGERY :NEGATIVE PRESSURE WOUND THERAPY (NPWT) MANAGEMENENT OF CELLULITIS IN ABDOMEN WALL DEFICIT FROM FAECAL LOCAL PERITONITIS IN GIANT INCARCERATED HERNIA ON MALIGNANT PANNICULUS:CASE REPORT

\author{
V. Kaldis ${ }^{1}$, T. Zarokostas ${ }^{2}$, I. Skliris ${ }^{2}$, V. Vasilakopoulou ${ }^{2}$, \\ D. Charalampakis ${ }^{2}$, D. Giannakopoulos ${ }^{2}$
}

${ }^{1}$ Department Of General Surgery, PANARKADIKON GENERAL HOSPITAL OF TRIPOLI, TRIPOLI/GREECE, ${ }^{2}$ Department Of General Surgery Argos Medical Center, ARGOLIDAS GENERAL HOSPITAL, ARGOS/GREECE
Case History: Morbid obesity in emergency surgery is associated with increasing comorbidities and mortality. Often develops a bowel herniation that can lead to incarceration and acute exacerbation .This condition must be treated immedialy and presents a surgical challenge .Here we are presenting a case report of 978 yo with intermittent abdominal pain, nausea present in the Emergency Department of our district Hospital .

Clinical Findings: ABGs:normal, WBC 11.000, LACTATE 1.6 $\mathrm{mmol} / \mathrm{L}$, afebrile, no preseptic status, , small abdomen wall cellulitis present, malignant panniculus (group V), ASA 4E, BMI $>40 \mathrm{kr} /$ m2.Admission on Surgery yard.

Investigation/Results: Rx abdomen:Bowel stasis and abdomen CTscan: incarcerated colon with presence of air.

Diagnosis: Incarcerated Transverse colon necrosis in giant wall abdomen hernia sach with local faecal peritonitis with purulent and necrotic tissue on the wall abdomen superficie.

Therapy and Progression: Giant hernia sach resection, necrotic bowel resected, purulent/necrotic wall abdomen (wa) resected, colostomy performed.Partial nylon loop aponeurosis suture perfomed Negative pressure wound therapy on wa deficit applied for 14 days. The patient's length of stay was 48 days . The patient was discharged and recuperated.

Comments: Comorbidities, increased morbility and mortality are associated with malignant obesity surgery in emergency surgery. Preseptic and septic conditions are difficult to be managed intraoperatory .The wound deficit is difficult to manage and these patients must be followed in ICU for rhabdomyoysis and abdominal compartment syndrome. NPWT dressing with repeated procedures in high risk patient is indicated. A chronically infected malignant panniculus with incarcerated hernia must be recognized and treated immedialy. The wall abdomen defect management presents a surgical challenge decision.

References: 1. WSES guidelines for emergency repair of complicated abdominal wall hernias. Sartelli M1, Coccolini F et al. World J Emerg Surg. 2013 Dec 1;8 (1):50. doi: 10.1186/1749-7922-8-50. 2.Choi JJ, Palaniappa NC, Dallas KB, Rudich TB, Colon MJ, Divino CM. Use of mesh during ventral hernia repair in clean-contaminated and contaminated cases: outcomes of 33,832 cases. Ann Surg. 2012;255 (1):176-180. doi: 10.1097/SLA.0b013e31822518e6. [PubMed] [Cross Ref] 3.Review A decade of ventral incisional hernia repairs with biologic acellular dermal matrix: what have we learned? Kissane NA, Itani KM Plast Reconstr Surg. 2012 Nov; 130 (5 Suppl 2):194S202S.

Disclosure: No significant relationships.

\section{PC116}

\section{EMERGENCY LAPAROSCOPIC HEMICOLECTOMY FOR COLO-COLONIC INTUSSUSCEPTION. A CASE REPORT}

\author{
P. Christopoulos, V. Oates, J. O'Dowd, A.M. Mathew, R. Rao
}

Surgical, Airedale General Hospital, Keighley/UNITED KINGDOM

Case History: A 50 year old woman presented to the emergency department with a three-day history of intermittent right sided loin pain. The intermittent abdominal pain was worse on movement and radiated to the groin. There was no associated nausea, vomiting or other systemic symptoms. This patient had no significant past medical or surgical history.

Clinical Findings: On examination there was no renal angle tenderness, the abdomen was soft and yet experienced pain on coughing. Clinical examination of the mildly distended abdomen did not reveal 
any palpable mass. Her abdominal X-ray identified an unremarkable bowel gas pattern. The patient was apyrexial and normotensive. Her routine blood tests were within normal range; with $\mathrm{C}$-reactive protein (CRP) being moderately elevated

Investigation/Results: The abdominal CT identified the colo-colonic intussusception (with no evidence of obstruction) on the right side of the abdomen associated with a $5.3 \mathrm{~cm}$ lipoma acting as the lead point. There was also prominence of the right pelvi-calyceal system which may have been due to extrinsic compression of the right ureter due to the distended bowel.

Diagnosis: A pre-op radiologic diagnosis of bowel intusssusception was made and the patient consented for an emergency operation

Therapy and Progression: The patient went to theatre for a laparoscopic right hemi-colectomy where the intussuscepted segment was resected without reduction. The post-operative period was complicated with post-operative ileus which was managed conservatively. Comments: Adult intussusception is a rare cause of obstructive ileus and differs from childhood intussusception in its presentation, aetiology and treatment. Can present either as an acute bowel obstruction but chronic presentation is not as rare.

References: Maghrebi H. Makini A. Rhaiem R. Atri S. Ayadi M. Jrad M. Jouini M. Kacem M. Bensafta Z. Adult Intussusceptions: Clinical presentation, diagnosis and therapeutic management. International Journal of Surgery Case Reports, 2017; 33: 163-166. Papaziogas B., Koutelidakis I., Christopoulos P., Doulias T., Atmatzidis S., Papadakis G., Atmatzidis K. Adult intussusception caused by an inflammatory polyp. A case report and review of the literature. Surg Chronicles 2011; 16 (11):53-55. Wang Y. Gowing S. Arena G. Adult colo-colonic intussusception caused by congential bands: A case report and literature review. International Journal of Surgery Case Reports 2016; 26: 88-92. Mariscovetere P. Ivatury S.J. White B. Holubar S.D. Intestinal Intussusception: Etiology, Diagnosis and Treatment. Clinics in Colon and Rectal Surgery 2017; 30 (01): 030-039.

Disclosure: No significant relationships.

\section{PC117}

\section{CLINICAL EXPERTISE IN LAPAROSCOPIC APPROACH FOR MANAGEMENT OF ADHESIVE SMALL BOWEL OBSTRUCTION}

\section{A. Sanchez Arteaga ${ }^{l}$, J. Tinoco-González ${ }^{l}$, V. Durán-Muñoz- Cruzado $^{I}$, I. Alarcón-Del Agua ${ }^{I}$, M.J. Tamayo-Lopez ${ }^{1}$, L. Tallón- Aguilar $^{1}$, F. Pareja-Ciuró ${ }^{2}$, J. Padillo-Ruíz ${ }^{2}$}

${ }^{1}$ Emergency Surgery Unit, Virgen del Rocío Universitary Hospital, Seville/SPAIN, ${ }^{2}$ Digestive And General Surgery Unit, Virgen del Rocío Universitary Hospital, Seville/SPAIN

Case History: Adhesions are the main cause of small bowel obstruction (SBO). Laparoscopic approach has become a proper treatment, but not gold standard, after failure in conservative management. We present our experience in laparoscopic approach for management of SBO.

Clinical Findings: Descriptive and retrospective analysis from Trauma and Emergency Surgery Department of University Hospital Virgen del Rocío (October 2016-September 2017). Demographic data, surgical time and outcomes were analyzed.

Investigation/Results: 21 patients underwent surgery because a failure in conservative management of SBO. Median age was 56 years (52-74). A single previous surgery was reported in 14 patients.

Diagnosis: CT-scan findings were: single band occlusion in 14 patients, several changes of bowel loops in 4 cases ( 3 patients underwent surgery without preoperative CT-scan).
Therapy and Progression: Laparoscopic surgery (LS) were performed in 11 patients, open surgery (OS) in 10 patients. Five of the laparoscopic cases were converted to open surgery (need of bowel resection or unfeasibility to set free the adhesions). Median surgical time was: 70 minutes for OS, 45 minutes for LS, and 155 minutes for converted surgeries. Complications related to open surgery group were intraabdominal abscess, evisceration, pneumonia and anastomotic dehiscence. No morbidity was described in the laparoscopic group. Median hospital stay was: 7 days for OS, 5 days for LS, 26 days for converted surgery group.

Comments: Conclusion. Laparoscopic treatment of SBO is feasible and effective in selected patients, with less postoperative morbidity rates. SBO due to single band occlusion may be one of the preferable indication for laparoscopic approach. In unselected cases, conversion rates might be high.

References: - Farinella E, Cirocchi R, La mura F, et al. Feasibility of laparoscopy for small bowel obstruction. World J Emerg Surg. 2009;4:3. - Dindo D, Schafer M, Muller MK, Clavien PA, Hahnloser D. Laparoscopy for small bowel obstruction: the reason for conversion matters. Surg Endosc. 2010;24 (4):792-7. - Otani K, Ishihara S, Nozawa $\mathrm{H}$, et al. A retrospective study of laparoscopic surgery for small bowel obstruction. Ann Med Surg (Lond). 2017;16:34-39.

Disclosure: No significant relationships.

\section{PC118}

\section{BOUVERET SYNDROME TREATED WITH ENDOSCOPIC LITHOTRIPSY}

\author{
J.F. Pérez Martínez, A. Szuba, B. Narbona Calvo
}

General Surgery, Hospital del Poniente, El Ejido/SPAIN

Case History: An 81-year-old woman admitted to the ER for vomiting and abdominal pain. Five months before she was diagnosed with acute cholecystitis, due to the time of evolution and comorbidities was treated with antibiotics.

Clinical Findings: On physical examination, the patient presented a good general condition, no abdominal distention or tenderness.

Investigation/Results: Abdominal CT showed gastric obstruction secondary to a cholecysto-duodenal fistula with total stomach occlusion and a cholelithiasis of approximately $20 \mathrm{~mm}$. In the upper digestive endoscopy, dilated gastric chamber with large amount of bilious fluid and duodenal bulb occlusion by lithiasis was seen without the possibility to extract the calculus.

Diagnosis: BOUVERET SYNDROME.

Therapy and Progression: New upper digestive endoscopy and lithotripsy with Holmium 600 laser are performed, fragmenting the stone into two parts. One of the pieces (the larger) is removed, solving the duodenal occlusion. The other fragment of the lithiasis remains blocked in the cholecystoduodenal fistula. The patient progressed favorably restarting oral tolerance and is discharged on the second day after the procedure. Unfortunately, while she was waiting for the second attempt of endoscopic lithotripsy, she was admitted again with the intestinal obstruction. Given the impossibility of performing the same procedure as urgent and the patient's worse condition we decided the exploratory laparotomy, finding intestinal obstruction secondary to biliary ileous, extracting the calculus from the distal jejunum with gastroenteroanastomosis because of the chronic inflammation of the duodenum seen in the CT scan at the admission. Comments: The procedure will require more studies but it seems that endoscopic lithotripsy may be a therapeutic alternative for Bouveret syndrome. 
Disclosure: No significant relationships.

\section{PC119}

UNUSUAL CASE OF APPENDICITIS, WHAT SHOULD WE DO?

${ }_{\text {J. Hernandez Gutierrez }}{ }^{1}$, A. Aranzana ${ }^{1}$, B. Muñoz Jimenez ${ }^{1}$, A. Trinidad Borrás ${ }^{I}$, I. Fraile Alonso ${ }^{I}$, M.D.P. Sánchez-Camacho González-Carrato $^{2}$, B. Moreno Torres ${ }^{3}$, M.Á. Morlán López ${ }^{I}$

${ }^{1}$ General Surgery, C.H. Toledo, Toledo/SPAIN, ${ }^{2}$ Radiology, C.H. Toledo, Toledo/SPAIN, ${ }^{3}$ Pathology, C.H. Toledo, Toledo/SPAIN

Case History: Enterobius vermicularis is the most common parasitic infection in developed countries. Gastrointestinal infestation with the parasite is common in humans and is usually harmless. Anal pruritus is the most characteristic symptom, but infestation of the appendix can cause symptoms of appendiceal pain.

Clinical Findings: We present a case of a 44 year old male with clinical findings of acute appendicitis, he was admitted to the Emergency room with non-specific abdominal pain that gradually localised to the right iliac fossa and fever.

Investigation/Results: Patient was clinically suspected of having acute appendicitis, investigations including blood tests and abdominal ultrasound confirmed the suspicion. Surgical opinion was sought and the decision was to perform an appendicectomy by minimal invasive approach.

Diagnosis: Enterobius vermicularis was detected in the pathological examinations of specimens.

Therapy and Progression: Laparoscopy showed appendicitis, so appendicectomy was performed following the routine practice. While examining the rest of the abdominal cavity a perforation of the sigmoid colon was discover, so laparotomy was performed. A resection of the sigmoid colon was performed, during this procedure living worms were found. So we deciced to perform a Hartmann procedure and start anthelminthicum treatment.

Comments: Gastro-intestinal infestation with E. vermicularis is very common, especially in young children. The symptoms of appendicitis can be due to Enterobius vermicularis infestation. In Enterobius infections systemic therapy is recommended for patients and their family members.

References: Enterobiasis (pinworm) and trichuriasis (whipworm). Uptodate Bethony. Soil-transmitted helminth infections: ascariasis, trichuriasis, and hookworm. Lancet 2006 Akkapulu. Is Enterobius vermicularis infestation associated with acute appendicitis? Eur J Trauma Emerg Surg 2016

Disclosure: No significant relationships.

\section{PC120}

\section{ROLE OF NEGATIVE PRESSURE THERAPHY IN NECROTIZING SOFT TISSUE INFECTIONS}

\author{
A. Landaluce-Olavarria ${ }^{l}$, B. Ugarte-Sierra ${ }^{l}$, S. Postigo ${ }^{1}$, \\ K. Intxaurraga', B. Estraviz-Mateos ${ }^{2}$, F.J. Ibañez Aguirre ${ }^{l}$
}

${ }^{1}$ General Surgery, hospital Galdacano, Galdacano/SPAIN, ${ }^{2}$ General Surgery, Hospital Urduliz, Urduliz/SPAIN
Case History: CASE 1. 77 years old man. necrotizing soft tissue infection (NSTI) near to the armpit . No risk factors (RF) . CASE 2. 66 years old woman. Eventroplasty 7 days before. RF: obesity CASE 3. 60 years old woman. RF: DM

Clinical Findings: Case 1. NSTI near to the armpit Case 2. NSTI in abdomen Case 3.NSTI in right groin extended to vulva.

Investigation/Results: Laboratory risk indicator for necrotizing fasciitis (LRINEC) $>6$ LRINEC modified (LRINEC mod) $>8$.

Diagnosis: Case 1. LRINEC 9 and LRINEC mod 10. Case 2.LRINEC 7 and LRINEC mod 9. Case 3. LRINEC 9 and LRINEC mod 9

Therapy and Progression: Case 1. Extended debridement + NPT to control the local infection and broad-spectrum antibiotics were supplied. An early re-exploration (within 12 hours) to check the tissues. Length of stay (LoS) 50 days. 11 replacements +2 PICOs Case 2 . Extended debridement + NPT was performed.LoS 12 days. 11 replacements (7 of them ambulatory surgery). Case 3. Extended debridement + NPT was performed. LoS 15 days. 10 replacements (3 of them ambulatory surgery)

Comments: The goal standard treatment of NSTI is the extended debridement + broad-spectrum antibiotics. We have a large suprafascial space and NPT can control this space and besides, it aids to the vascularization and stimulation of inflammatoryresponse. NPT should not be a contraindication in the first stages. Conclusions. 1. NPT improves the inflammatory response in the first stages of NSTI. 2. NPT provides a good control of the extended debridement. 3. The use of NPT decreases further plastic surgery after the control of NSTI. References: 1. Hakkrainen TW, Kopari NM, Phanm TN and Evans HL. Necrotizing soft tissue infections: Review and current concepts in treatment, systems of care, and outcomes. Curr Probl Surg. 2014 AUG 51 (8):344-362. 2. Borschitz T, Schlicht S, Siegel E, Hanke E and Von Stebunt E. Improvement of a clinical Score for necrotiizing fascitis: "Pain Out of prportion" and High CRP Levels Aid the Diagnosis. PLOS ONE/DOI:10.1371/journal.pone. 0132775. Jul 21 $1-13$.

Disclosure: No significant relationships.

\section{PC121}

\section{SPONTANEOUS COMPLETELY REVERSIBLE ACUTE MESENTERIC ISCHEMIA}

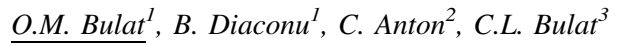

${ }^{1}$ Ivth Surgery, Hospital Sf Spiridon, Iasi/ROMANIA,

${ }^{2}$ Gastroenterology, UMF Grigore T. Popa, Iasi, Iasi/ROMANIA, ${ }^{3}$ Iv Th Surgery, UMF Grigore T. Popa, Iasi, Iasi/ROMANIA

Case History: We report a case of 28 years old male, with personal history of a perforated peptic ulcer operated 5 years ago, and one admission 3 weeks ago for signs of intestinal occlusion treated conservative. He was admitted consecutively for signs of occlusion appeared 9 hours ago.

Clinical Findings: Spontaneous pain and on palpation, distended abdomen. Initial discrepancy between severe abdominal pain and clinical findings.

Investigation/Results: The laboratory revealed elevated white blood cell $(18700 \mathrm{mg} / \mathrm{dl})$ and a lactate level of $22 \mathrm{mmol} / \mathrm{l}$. Abdominal radiography found air-fluid levels in the loops of the small bowel in mesogastrium and abdominal ultrasound revealed dilated loops of intestine until $3 \mathrm{~cm}$, free fluid between the loops and bowel wall thickening of $4 \mathrm{~mm}$.

Diagnosis: We suspected an intestinal occlusion and we decided to operate him. 
Therapy and Progression: We found a mesenteric ischemia on approximately $1,80 \mathrm{~m}$ of small bowel from an entero-enteral adherence in the root of the mesentery. Even that in the beginning we thought that the bowel is compromised, after cutting the adherence, the color reappeared and $20 \mathrm{~cm}$ of the terminal ileum remaining with ischemic aspects. We decided to wait and see, and we leave a laparostomy with negative pressure. After 72 hours the entire intestine was fine.

Comments: Despite a late diagnosis the acute mesenteric ischemia could be completely reversible.

References: 1. Schoots IG, Koffeman GI, Legemate DA, Levy M, Van Gulik TM: Systematic review of survival after acute mesenteric ischemia according to disease aetiology. Br J Surg. 2004, 91: 17-21. 10.1002/bjs.4459. 2. Ottinger LW: The surgical management of acute occlusion of the superior mesenteric artery. Ann Surg. 1978, 188: 721-731. 10.1097/00000658-197812000-00003. 3. Yanar H, Taviloglu K, Ertekin C, Ozcinar B: Planned second-look laparoscopy in the management of acute mesenteric ischemia. World J Gastroenterol. 2007, 13: 3350-3353. 4. Kozuch PL, Brandt LJ: Review article: diagnosis and management of mesenteric ischemia with an emphasis on pharmacotherapy. Aliment Pharmacol Ther. 2005, 23: 201-215. 10.1111/j.1365-2036.2005.02269.x.

Disclosure: No significant relationships.

\section{PC122}

\section{INTRAPERITONEAL FINDING OF A 27X4 CM FOREIGN BODY WITH NO OBVIOUS ENTRY POINT. A CASE REPORT}

\section{Gerogiannis, I. Gkegkes, P. Das, G. Bond-Smith}

General Surgery, Oxford University Hospitals NHS Foundation Trust, Oxford/UNITED KINGDOM

Case History: A 51 yo female was transferred by ambulance to Accident and Emergency Department confused, complaining of mild abdominal pain. She had consumed large amount of alcohol and benzodiazepines, because of family problems.

Clinical Findings: On admission, her Glascow Coma Scale was 11, she was apyrexic and tachycardic. On examination, the abdomen was soft but there was generalised tenderness on deep palpation. The rest of the clinical assessment was unremarkable.

Investigation/Results: $\mathrm{C}$ - reactive protein level in plasma was 219.7 $\mathrm{mg} / \mathrm{L}$ and lactates $2.7 \mathrm{mmol} / \mathrm{L}$ with the rest of blood tests being normal. As she was confused with generalized abdominal pain, a decision was made to proceed with Computed Tomography (CT) Scan of abdomen/pelvis. Report revealed an intraperitoneal tubular foreign body measuring $27 \mathrm{~cm}$ in length and $4 \mathrm{~cm}$ in diameter. Small volume of pelvic fluid and free gas was also detected.

Diagnosis: An urgent laparoscopy identified the foreign body. Vagina and sigmoid colon were found intact. A laparotomy initially didn't help identifying the site of perforation, hence an intraoperative flexible sigmoidoscopy was performed. A 4-cm defect was found in the posterior wall of distal sigmoid colon.

Therapy and Progression: A Hartmann's procedure was performed. Patient recovered well and discharged the 6th post-operative day with safeguarding team involved.

Comments: This was a rare case of a colonic perforation from a large foreign body lying entirely intraperitoneally. Patient was stable and not septic and neither clinical examination nor CT revealed the entry point. Intraoperatively, it was challenging to identify the injury and endoscopy was proven extremely useful.
References: - Lupascu, Cristian et al. "Removal of an Intraperitoneal Foreign Body Using a Single Port Laparoscopic Procedure." JSLS: Journal of the Society of Laparoendoscopic Surgeons 15.2 (2011): 257-260. PMC. Web. 19 Oct. 2017. - Sandhu, A.S. \& Rao, P. Indian J Surg (2008) 70: 86. - Cologne, Kyle G., and Glenn T. Ault. "Rectal Foreign Bodies: What Is the Current Standard?" Clinics in Colon and Rectal Surgery 25.4 (2012): 214-218. PMC. Web. 19 Oct. 2017.

Disclosure: No significant relationships.

\section{PC123}

\section{SEPSIS MANAGEMENT, SOURCE CONTROL IN SUBCUTANEOUS GANGRENE AND NEGATIVE PRESSURE WOUND THERAPY (NPWT): CASE REPORT}

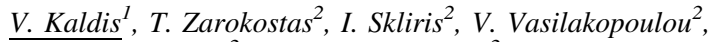
D. Charalampakis ${ }^{2}$, D. Giannakopoulos ${ }^{2}$

${ }^{1}$ Department Of General Surgery, PANARKADIKON GENERAL HOSPITAL OF TRIPOLI, TRIPOLI/GREECE, ${ }^{2}$ Department Of General Surgery Argos Medical Center, ARGOLIDAS GENERAL HOSPITAL, ARGOS/GREECE

Case History: Acute necrotizing soft tissue (ANST) are rare and deleterius. Subcutaneus gangrene begin as papule followed by vesicle evolving in necrotic ulcer. There are two entities hemolytic subcutaneus gangrene (HSSG) and the anaerobic bacteria subcutaneous gangrene (ABSG).We are presenting in our district Hospital 978 yo, transferred from other Medical Center, with diagnosis of left limp cellulitis thru $7 \mathrm{~d}$ antibiotic iv amoxiciline /clindamicine.

Clinical Findings: ABG's:T:38, 5C, Wbc:22.000, RR24, SBP $<80$, tachipnoic, anuric, lethargic, qSOFA:3, in severe sepsis condition, ASA4E, left extremity swollen, in geopardy, from the upper thigh till malleolus.Enormous necrotic ulcer thru the internal superficie of the leg.Arterial/grand safena venus circulation compromized.

Investigation/Results: Sepsis guidelines 2016 applicated 2016 thru iv resuscitation (NACL 0,9\%), antibiotic therapy upgraded empirically in ciprofloxacin /metronidazole.Lower extremity triplex performed.After the patient was haemodinamically stabilized was decided for operation table for source control debridement.

Diagnosis: OR was performed surgical exploration of the swollen erythematous area by excision of infected/necrotic tissues (skin subcutaneous, aponeurosis) were was mandatory the fasciotomy and excision of dead muscles (extensors \& peroneus) of the anterior compartment in proximity of the anterior border of the tibia to medial malleolus.

Therapy and Progression: Relook exploration/excision 3rd day. Acinetobacter Baumani confirmed .Negative pressure wound therapy skin deficit applied for 14 days. Nosocomial length of stay 37 days.The patient discharged. Skin graft plastic surgery denied .

Comments: Sepsis must be diagnosed and treated with the Sepsis guidelines even in the underdiagnosed necrotizing soft tissue necrosis can compromise function and severely affect the prognosis. The tempestive empirical antibiotic therapy is cardinal. The Source control timing in operation table thru surgical exploration and excision is a challenge for the surgeon. NPWT dressing with repeated procedures in high risk patient is indicated.

References: 1.SEPSIS GUIDELINES 2016 2.Stevens DL, Bisno AL, Chambers HF, et al. Practice guidelines for the diagnosis and management of skin and soft tissue infections: 2014 update by the infectious diseases society of America. Clin Infect Dis. 2014 Jul 15. 59 (2):e10-52. [Medline]. [Full Text].

Disclosure: No significant relationships. 
PC124

EMERGENCY SURGERY-NOT FOR THE FAINT HEARTED; IMPROVING OUTCOME FOLLOWING APPENDICECTOMY IN CARDIAC TRANSPLANT PATIENTS. LESSONS LEARNED

${\text { Z. } \text { Rai }^{1}, \text { D. Selvakumar }}^{2}$, M. Nair ${ }^{1}$

${ }^{1}$ General Surgery, North Middlesex University Hospital, London/ UNITED KINGDOM, ${ }^{2}$ General Surgery, North Middlesex University Hospital, london/UNITED KINGDOM

Case History: With the number of heart transplant recipients increasing, the need to provide tailored emergency surgical management also increases. Few cases of acute appendicitis in heart transplant recipients have been reported, we consider the case of a female heart transplant recipient requiring emergency appendicectomy. Appropriate surgical strategy adapted to altered cardiac physiology and implications of post-operative infection in the immunosuppressed are considered.

Clinical Findings: We report the case of a 33 year old female with lower abdominal pain. Past medical history comprised of heart transplant and umbilical hernia repair. Examination demonstrated localised tenderness in the right iliac fossa.

Investigation/Results: Computerised tomography confirmed uncomplicated acute appendicitis and a seroma around hernia repair site.

Diagnosis: Acute uncomplicated appendicitis

Therapy and Progression: Intravenous antimicrobial treatment was commenced and immunosuppressant agents were continued at usual dose. An open appendicectomy was performed under general anaesthesia. The appendix was delivered using an Alexis wound protector. She was discharged three days later.

Comments: The transplanted heart lacks sympathetic and parasympathetic innervation and is unable to utilise neurohormonal pathways to increase output thus becoming dependent on venous return (1). High intra-abdominal pressures during pneumoperitoneum adversely affects venous return and cardiac output (3) thereby necessitating an open approach. Additionally, long term immunosuppressants increase the risk of post-operative wound infection (4); the commonest complication following open appendicectomy. To reduce this, an Alexis wound retractor was used. Conclusion The management of these patients poses a novel challenge and the surgical approach to managing them needs to be tailored specifically, taking into account altered cardiac physiology and the effects of immunosuppression.

References: 1) Kostopanagiotou G., Smyrniotis V., Arkadopoulos N., Theodoraki K., Papadimitriou L., Papadimitriou J. Anesthetic and perioperative management of adult transplant recipients in nontransplant surgery. Anesthesia \& Analgesia. 1999;89 (3):613-622. doi: 10.1097/00000539-199909000-00013 2) Stewart D. The management of acute appendicitis. In: Cameron JL, Cameron AM, editors. Current surgical therapy. Philadelphia: Elsevier Saunders; 2014: 252-255 In: Cameron JL, Cameron AM, editors. 3) O'Malley C, Cunningham AJ. Physiologic changes during laparoscopy. Anesthesiol Clin North America. 2001;19:1-19. 4) Valerio R., Jr., Durra O., Gold M. E. Anesthetic considerations for an adult heart transplant recipient undergoing noncardiac surgery: a case report. AANA Journal. 2014;82 (4):293-299

Disclosure: No significant relationships.
PC125

\section{NON SURGICAL TREATMENT FOR AN ACUTE CHOLECYSTITIS WITH A RARE COMPLICATION OF CHOLECYSTOCUTANEOUS FISTULA}

\author{
A. Szuba $^{l}$, J.F. Pérez Martínez ${ }^{1}$, A.X. Argote Camacho ${ }^{2}$
}

${ }^{1}$ General Surgery, Hospital del Poniente, El Ejido/SPAIN, ${ }^{2}$ General Surgery, Complejo Hospitalario de Jaen, Jaen/SPAIN

Case History: An 81 - year - old woman, admited to the ER for abdominal pain, redness and increased local temperature at the right hypochondrium level which she presented for 4 days. The patient referred she had has the pain at this level for at least 4 months and it was associated with food intolerance.

Clinical Findings: Physical examination reveals redness of the skin and painful tumor in the upper right quadrant.

Investigation/Results: Abdominal ultrasound shows an abscess of the abdominal wall of $11 \times 7 \times 13 \mathrm{~cm}$ with thickening of the parietal peritoneum and wall musculature, destruction of the gallbladder wall with the presence of gas surrounding it and cholelithiasis of $25 \mathrm{~mm}$ in diameter.

Diagnosis: Acute cholecystitis perforated towards the abdominal wall with abscess formation. Cholecystocutaneous fistula.

Therapy and Progression: Given the good general condition of the patient with comorbidity, evolved acute cholecystitis without being able to determine the time elapsed since the onset of symptoms, medical treatment with antibiotic and cutaneous drainage of the abscess was decided. During admission, the patient progresses favorably without the need to perform the cholecystectomy. After discharge, the patient presented recurrence of abdominal wall abscess without associated cholecystitis which was treated with new percutaneous drainage. The patient rejects the elective surgical intervention and five months after the first admission presents the intestinal obstruction secondary to Bouveret's syndrome.

Comments: The cholecystocutaneous fistula with abscess of the abdominal wall is a rare entity that carries a risk of misdiagnosis of a local inflammatory process which can modify the treatment, which in some cases may require cholecystectomy and fistula excision.

References: España Fuente L, Arias Pacheco RD, Bujarrabal Martínez J, Fernández Muñiz PI. Absceso subcutáneo. Presentación atípica de colecistitis aguda. Cir Esp 2016;94:105. Rojas Reyna G, Gómez Cárdenas X, Weisser Jacobs F, Chousleb Kalah A, Cervantes Castro J, Ochoa Gómez JR, Galeazzi Martínez V. Fístula colecistocutánea espontánea. Informe de un paciente. Cir Gen 2002;24:53-56. Guardado Bermúdez F, Aguilar-Jaimes A, Ardisson-Zamora FJ, Guerrero-Silva LA, Villanueva-Rodríguez E, Nubia Alondra Gómezde Leija NA. Fístula colecisto-cutánea espontánea. Cir Cir 2015;83:61-64.

Disclosure: No significant relationships. 


\section{PC126}

\section{IS URGENCY SURGERY A THERAPEUTIC ALTERNATIVE FOR PATIENTS WHIT COLITIS PSEUDOMEMBRANOUS FULMINANT?}

\section{A.X. Argote Camacho' ${ }^{1} \underline{A . S z u b a}^{2}$, C.M. Ruiz Marin ${ }^{1}$, A. Astruc Hoffman ${ }^{1}$}

${ }^{1}$ Ugc Cirugía General, Complejo Hospitalario de jaén, Jaén/SPAIN, ${ }^{2}$ General Surgery, Hospital del Poniente, El Ejido, Almeria, Spain, Aguadulce, Roquetas de Mar/SPAIN

Case History: 65-year-old woman with medical history of Lung Adenocarcinoma EIIB receiving radiotherapy and chemotherapy; admitted to the Oncology Service for abdominal pain, fever and diarrheal stools; analytics with leukocytosis and elevated CRP, abdominal ultrasound without alterations. Antibiotic medical treatment begins: Cefepíme and Ampicillin and serum therapy.

Clinical Findings: The patient presented a torpid evolution, with increased abdominal pain, tachycardia, hypotension and dyspnea. Abdominal CT is performed visualizing dilatation, submucosal thickening and loss of colonic haustration, suggesting colitis with incipient toxic megacolon.

Investigation/Results: It is examined by General Surgery deciding urgent surgical intervention: Exploratory Laparotomy is perfomed evidencing great dilatation of the colon but with healthy colonic wall, with an intraoperative colonoscopy finding mucosa with necrotic ulcer aspect, with whitish vesicles that affects from the cecum to the rectum. Subtotal colectomy is performed with ileostomy without eventualities

Diagnosis: $\mathrm{CP}$ should be taken into account in the differential diagnosis of acute abdomen in immunosuppressed patients with antibiotic treatment. Surgery is a therapeutic option, when fulminating occurs or fails medical treatment.

Therapy and Progression: In the postoperative period antibiotic treatment is completed: Metronidazole + vamcomycin, and is the patient is discharged from hospital. The anatomo-pathological study of the surgical piece reports: CP

Comments: The incidence of Pseudomembranous Colitis (CS) has increased due to the dissemination of hypervirulent strains of Clostridium difficile and the inappropriate use of antibiotics. Although its treatment is medical, we should consider emergency surgery a therapeutic alternative in cases of fulminant colitis as we observe.

References:

Disclosure: No significant relationships.

\section{PC127}

\section{APPENDICULAR COLIC SECONDARY TO OXYURS INFECTION}

\section{Soto Montesinos ${ }^{1}$, J.C. Pastor Mora ${ }^{2}$, M. Sola $^{1}$}

${ }^{1}$ General Surgery, Fundación Althaia Xarxa Assistencial Manresa, Manresa/SPAIN, ${ }^{2}$ General Surgery, Fundación Althaia, Manresa/ SPAIN

Case History: Oxiurasis is intestinal infestation by the parasite Enterobius vermicularis, which rarely migrates to cecal appendix
Clinical Findings: Infestation by E. vermicularis can produce or not, an inflammatory appendicular response, due to the migration of these. It is the first cause of appendicitis for parasites, oscillating frequency between $0.2-4.2 \%$ in developed countries up to $41.8 \%$ in underdeveloped countries.

Investigation/Results: 13 -year-old woman with a history of oxiurasis during childhood and stay in a rural area 1 month prior to admission, who visits the hospital for 4-day abdominal pain in the evolution of the right iliac fossa, It starts abruptly accompanied by vomiting, without diarrhea or fever.

Diagnosis: The patient has selective pain in FID and mild analytical leukocytosis without elevation of the PCR, so an abdominal ultrasound was performed that was inconclusive, and was entered 24 hours in observation for evolutionary control For the persistence of the clinic abdominal CT was performed that shows compatible findings with incipient acute appendicitis, so surgery was indicated.

Therapy and Progression: Exploratory laparoscopy was performed, observing a thickened appendix with little inflammatory signs and normal annexes (supervised by a gynecologist) and so that we decided to perform appendectectomy. During the appendicular section the exit of oxyurs is observed. She presented a postoperative without complications, and was discharged in both days with oral albendazole treatment. The pathological anatomy of the piece showed an appendix without inflammatory signs and with adult forms of $\mathrm{E}$.

Comments: Although infrequent, the appendicular colic for oxyurs is a diagnosis to consider in rural children.

References:

Disclosure: No significant relationships.

\section{PC128}

\section{ENTEROATMOSPHERIC FISTULA; THE CHALLENGE AFTER THE OPEN ABDOMEN}

J. Mateo Retuerta ${ }^{1}$, I. Poveda ${ }^{1}$, J.L. Moya ${ }^{2}$, B. Arín ${ }^{2}$, A. Rico ${ }^{2}$, C. Sanchez ${ }^{1}$, S. Montón ${ }^{2}, M$. Cires ${ }^{2}$

${ }^{1}$ Cirugía General, Complejo Hospitalario de Navarra, Pamplona/ SPAIN, ${ }^{2}$ Cirugía General, Hospital García Orcoyen, Estella/SPAIN

Case History: The enteroatmospheric fistula is defined as the communication between the gastrointestinal tract and the atmosphere, a devastating complication of damage control surgery, associated with significant morbidity and mortality. It has a complex and multifactorial etiology like persistent intraabdominal infection, anastomotic leakage, adhesions and intestinal manipulation

Clinical Findings: We present a case of a 71-year-old patient with a history of left hemicolectomy for recurrent diverticulitis who required new colorectal anastomosis and ileostomy due to suture dehiscence. Investigation/Results: After a poor evolution of the immediate postoperative period of ileostomy closure, the patient required two reinterventions due to signs of peritonitis in the context of an intestinal leak, finding an abdomen that could not be absorbed by a stony adherent block and the impossibility of primary closure, managed like a open abdomen.

Diagnosis: During the immediate postoperative period, an enteroatmospheric fistula was identified due to leakage of bile fluid through the granulation tissue, which required prolonged admission in the intensive care unit, management of sepsis, hydroelectrolytic replacement and total parenteral nutrition.

Therapy and Progression: After daily cures with negative pressure measurements and collection of debit collection systems, it presented a slow and progressive improvement with almost total closure of the 
abdomen and jejunostomy of medium debit in treatment with somatostatin analogues.

Comments: The best treatment of FEA is the prevention of its appearance. The definitive abdominal repair requires a major surgery for the reconstruction of the abdominal wall, which should be delayed between 6 and 12 months after the initial aggression.

References: 1 . The use of negative pressure wound therapy (npwt) in the management of enteroatmospheric fistula - case report and literature review. Rajm und jaguścik, Dominika. Walczak, Jjoanna Porzeżyńska, Piotr W, T Rzeciak. Polski przegląd chirurgiczny. 2015, $87,10,522-527$ 2. Management of enterocutaneous fistulas. William P. Schecter. Surg clin n am 91 (2011) 481-491 3. Controversies in the care of the enterocutaneous fistula. Kurt G. Davis, A, Eric K. Johnson. Surg clin n am 93 (2013) 231-250 4. Metabolic and nutritional support of the enterocutaneous fistula patient: a three-phase approach. Travis M, Polk C, William Schwab. World j surg (2012) 36:524-533 5. Enteroatmospheric fistula: from soup to nuts. Sarah Majercik, Merin Kinikini and Thomas White. Nutrition in clinical practice volume 27 number 4 august 2012 507-512 6. Systematic review and meta-analysis of the open abdomen and temporary abdominal closure techniques in non-trauma Patients. J.J. Atema, S.L. Gans, M.A. Boermeester. World j surg (2015) 39:912-925 7. Practical approaches to definitive reconstruction of complex abdominal wall defects. Rifat Latifi. World J Surg (2016) 40:836-848 8. Enterocutaneous fistula: proven strategies and updates. Irena Gribovskaja-Rupp, Genevieve B. Melton. Clin colon rectal surg 2016;29:130-137 9. "Enteroatmospheric fistulae"-gastrointestinal openings in the open abdomen: a review and recent proposal of a surgical technique. A. Marinis, G. Gkiokas, E. Argyra, G. Fragulidis, G. Polymeneas, D. Voros. Scandinavian journal of surgery 102: 61-68, 2013 10. "Acute postoperative open abdominal wall": nosological concept and treatment implications. Manuel López-Cano, José A Pereira, Manuel Armengol-Carrasco. World j gastrointest surg 2013 december 27; 5 (12): 314-320 11. Management of enteroatmospheric fistulae. Terzi C, Egeli T, Canda AE, Arslan NC. Int wound j 2014; 11 (suppl. 1):17-21 Disclosure: No significant relationships.

\section{PC129}

\section{EMERGENCY SURGERY AND TRAUMA (ESAT) TEAM, A EFFICIENT AND EFFECTIVE APPROACH FOR ACUTE CARE PATIENTS}

\section{P. Lingam}

General Surgery, Khoo Teck Puat Hospital, Singapore/SINGAPORE

Case History: Khoo Teck Puat Hospital (KTPH) is the first hospital in Singapore to commence a dedicated acute surgical unit. It is a .Consultant led service set up in KTPH in November 2014. It consists of 1 Consultant, 2 registrars, 3 Medical officers and 3 House Officers. Clinical Findings: The aim of of this acute surgical unit is to increase the timeliness of clinical assessment and management of emergency general surgical and trauma patients. It also aims to enhance care and satisfaction of general surgical patients. This acute surgical team admits all General Surgery (GS) acute and trauma patients during the daytime. It reviewed referrals from the emergency department in a timely fashion and has a non-refusal policy. The Acute Care surgery team also prioritizes such that patients meet their acuity guidelines appropriate to their surgery

Investigation/Results: In our first 2 years, we see an average of 20 patients per day. Referrals from the emergency department are seen within 90 minutes. A Consultant was present in $100 \%$ of major operative cases. There was also a slight reduction in night time operations by GS residents. It resulted in better coordination and collaboration with Emergency physician, Interventional Radiologist, ICU and anaesthetist for holistic care.

Diagnosis: ESAT improves outcomes.

Therapy and Progression: Benefits that have been noted from the initial years are shorter pre-operative and overall hospital stay for patients, better utilization of resources and increase in elective workload throughout for the respective sub-specialities.

Comments: An ESAT Team enables improved and dedicated experience for surgical trainees in management of acute and trauma cases. References:

Disclosure: No significant relationships.

\section{PC130}

\section{LIFE-THREATENING INFECTION DUE TO PANTON- VALENTINE LEUKOCIDIN POSITIVE COMMUNITY- ACQUIRED METHICILLIN-RESISTANT STAPHYLOCOCCUS AUREUS IN A COLLEGE STUDENT ATHLETE IN JAPAN}

R. Yokomori, J. Tsurukiri, M. Moriya, H. Yamanaka, N. Tsubouchi, Y. Kobabayashi, T. Arai

1163, Tokyo medical university Hachioji Medical Center, Hachioji city, Tokyo/JAPAN

Case History: A 20-years old man was admitted to our emergency center owing to dyspnea. He had no previous medical history.

Clinical Findings: Physical examination revealed the following: Glasgow Come Scale score, E4V4M6; blood pressure, 173/83 mmHg, heart rate,

148 beats/min; respiratory rate, 40 breaths/min; and body temperature $40.4{ }^{\circ} \mathrm{C}$.

Investigation/Results: Chest X-ray revealed bilateral alveolar shadows. Whole body computed tomography revealed ARDS with SPE, SSTIs of the hip and thigh, and infectious iliofemoral DVT that are difficult to operative access.

Diagnosis: CA-MRSA bacteremia, ARDS with SPE, SSTIs of the hip and thigh, and infectious iliofemoral DVT

Therapy and Progression: Thus, the patient underwent aggressive surgical drainage and debridement of SSTIs of the hip and left thigh, and puncture drainage of bilateral knee joint abscess. Intervenous administration of vancomycin combined with clindamycin was started.

Sequential organ failure assessment score was 8 and the patient admitted to our intensive care unit (ICU). No further SSTIs were encountered, and the patient was discharged from our ICU after 12 days in good condition. Pulsed-field gel electrophoresis (PFGE) among MRSA isolates revealed PVL-positive USA300 clone.

Comments: A review of published works was carried out, and only 7 Japanese patients with severe infections, such as SPE or infectious endocarditis, due to PVL-positive CA-MRSA USA300 strain were identified. All of them had not participate in contact sports.

Thus, this is first report of life-threatening infection in due to PVLpositive CA-MRSA in a college student athlete in Japan. Further investigations of CA-MRSA USA300 strains among collegiate athletics to prevent outbreaks in Japan were warranted.

References: 1) Lancet. 2010 May 1; 375 (9725): 1557-1568.

2) N Engl J Med. 2006 Aug 17;355 (7):666-74.

3) Sports Health. 2009 Sep; 1 (5):405-10.

4) Japan. J. Infect. Dis., 66, 416-420, 2013

5) Journal of Chiropractic Medicine (2010) 9, 32-37

6) Sports Health. 2009 Sep; 1 (5):405-10.

Disclosure: No significant relationships. 
PC131

\section{CHOLEDOCHOTOMY WITH PRIMARY CLOSURE OVER BILIARY STENT IN ACUTE SUPPURATIVE CHOLANGITIS}

D. Aveiro, J. Constantino, J. Pereira, C. Casimiro

Cirurgia Geral, Centro Hospitalar Tondela-Viseu, Viseu/PORTUGAL

Case History: The Authors present a case of a 58 year old male, that was referred to the emergency department with abdominal pain, fever and progressive jaundice for a week

Clinical Findings: The patient had right upper quadrant pain and guarding, fever of $40^{\circ}$ celsius and mucocutaneous jaundice

Investigation/Results: Laboratory investigations showed a raised total white blood cell count, C-reactive protein and total $(11 \mathrm{mg} / \mathrm{dL})$ and direct $(9,8 \mathrm{mg} / \mathrm{dL})$ bilirubin. Abdominal ultrasonography and CT scan found signs of cholecystitis with a paracholecystic abscess and choledocholithiasis with moderate biliary tract dilation.

Diagnosis: Acute Cholangitis with associated cholecystitis

Therapy and Progression: The patient underwent urgent laparotomy with abscess drainage, cholecystectomy, choledocotomy with stone retrieval and closure over an anterograde transpapillary biliary stent. During hospitalization the patient recovered of the sepsis and was discharged at the seventh post-operative day with a total bilirubinemia of $1,5 \mathrm{mg} / \mathrm{dL}$.

Comments: Acute suppurative cholangitis is a severe complication of choledocholithiasis, which can be associated with cholecystitis. It demands prompt diagnosis and treatment, mostly consisting in supportive care, antibiotics and drainage of the bile duct, either endoscopically or surgically. For many years, in the surgical approach of choledocholithiasis and its complications the procedure of choice for biliary drainage was external, with choledochostomy with a T-Tube. However, in recent years, the use of biliary stents placed through an anterograde approach with choledochotomy closure seems to have some advantages, such as less morbidity, diminished lenght of hospital stay and an early recovery of the patient. It is a simple procedure, allowing an immediate and effective decompression after bile duct drainage, even in an urgent setting.

References: Takada et al : TG13: Updated Tokyo Guidelines for the management of acute cholangitis and cholecystitis..J Hepatobiliary Pancreat Sci. 2013 Jan;20 (1):1-7. Mangla V et al : A Randomized Trial Comparing the Use of Endobiliary Stent and T-Tube for Biliary Decompression After Laparoscopic Common Bile Duct Exploration. Surg Laparosc Endosc Percutan Tech 2012;22:345-348 Dietrich A et al : Laparoscopic Management of Common Bile Duct Stones: Transpapillary Stenting or External Biliary Drainage? JSLS OctoberDecember 2014 Volume 18 Issue 4 e2014.00277 Lyon M, Menon S, Jain A, Kumar H. Use of biliary stent in laparoscopic common bile duct exploration. 2014. Surg Endosc.

Disclosure: No significant relationships.

\section{PC132}

\section{MISLEADING CLINICAL ASSESSMENT DUE TO TAKO- TSUBO CARDIOMYOPATHY AFTER A LOW-ENERGY RUN OVER} A. Lusilla López ${ }^{1}$, M. Sanz Sánchez ${ }^{1}$, M.D. Pérez Díaz ${ }^{2}$, A. Sánchez-
Arteaga $^{3}$, M. Burneo Esteves ${ }^{4}, F$. Turégano Fuentes

${ }^{1}$ Cirugia General Y Del Ap. Digestivo, Hospital General Universitario Gregorio Marañón, Madrid/SPAIN, ${ }^{2}$ Surgery, HOSPITAL
GREGORIO MARAÑON, MADRID/SPAIN, ${ }^{3}$ Emergency Surgery Unit, Virgen del Rocío Universitary Hospital, Seville/SPAIN, ${ }^{4}$ Cirugía General Y Del Ap. Digestivo, Hospital Quirónsalud Sur, Madrid/SPAIN

Case History: A 56 y.o. woman was admitted to the ED after a run over.

Clinical Findings: Physical examination and vital signs were unremarkable, and a CT scan showed no injuries. A few hours later she developed acute chest pain and hypotension, with elevation of cardiac enzimes and a peak of troponin $\mathrm{T}$ of $609 \mathrm{ng} / \mathrm{L}$ (normal $<14$ ). A transthoracic echocardiogram demonstrated akinesia of medial and distal segments of septum and inferior wall, with a $52 \%$ left ventricle ejection fraction. She was transferred to the cath lab for coronary angiography which showed normal coronary arteries

Investigation/Results: Tako-tsubo cardiomyopathy is a cardiac syndrome that mimics acute coronary syndrome (ACS), and was described in 1990 for the first time in Japan. Its prevalence in patients presenting with ACS ranges from $0.7-2.2 \%$. There is a female predominance especially post-menopause. It usually occurs after a history of emotional or physical stress implicating an abnormal cardiac response to increased catecholamines and causing hypotension which can mislead the clinician assessing a low-energy trauma patient. Supportive treatment usually leads to good long-term results with recovery of left ventricular function.

Diagnosis: Tako-tsubo cardiomyopathy

Therapy and Progression: She was discharged home 3 days afterwards asymptomatic and with a troponin $\mathrm{T}$ of 227.

Comments: Tako-tsubo cardiomyopathy is a rare entity that should be considered in patients admitted after low-energy trauma with no injuries, who suddenly develop acute chest pain, heart failure and elevated cardiac biomarkers. In most cases, heart function easily recovers with supportive treatment.

References: Templin C, Ghadri JR, Diekmann J et al. Clinical Features and Outcomes of Takotsubo (Stress) Cardiomyopathy. N Engl J Med 2015;373 (10):929-38. DOI: 10.1056/NEJMoa1406761. Turley A, Graham R, Hall J. Takotsubo cardiomyopathy in two female patients: two case reports. Cases J 2008;1 (1):325. DOI: 10.1186/ 1757-1626-1-325. Jiang DM, Sunc ZW, Han J. Tako-tsubo cardiomyopathy after a quarrel. Afr Health Sci 2015;15 (4):1349-53. DOI: $10.4314 /$ ahs.v15i4.39.

Disclosure: No significant relationships.

\section{PC133}

\section{PHANTOM RENAL MASS AFTER COLON CANCER RESECTION: A DIAGNOSTIC CHALLENGE}

\author{
$\underline{\text { T. Katsichtis }}^{1}$, M.E. Lueders ${ }^{2}$, E. Irizarry ${ }^{1}$
}

${ }^{1}$ Department Of Surgery, Cornell University, Lincoln Medical Center Campus, Bronx/NY/UNITED STATES OF AMERICA, ${ }^{2}$ Department Of Surgery, Cornell University, Lincoln Medical Center Campus, Bronx/UNITED STATES OF AMERICA

Case History: 63 year-old male with past medical history of stage II colon adenocarcinoma status post proctocolectomy and $\mathrm{J}$ pouch presented to the ED with abdominal pain, fever, melanotic diarrhea and new renal mass.

Clinical Findings: Patient was febrile with diffuse tenderness in the lower abdomen and on rectal exam. He had leukocytosis with WBC of 16 and acute kidney injury. 
Investigation/Results: Non-contrast cross-sectional imaging was performed and demonstrated marked dilatation of small bowel loops proximal to the ileo-rectal anastomosis. A new $5.8 \mathrm{~cm} \mathrm{x} 4.2 \mathrm{~cm}$ solid mass arising from the upper pole of the left kidney, suspicious for metastasis, was found. Due to concern for anastomotic stricture, the patient was taken to the operating room for colonoscopy and possible dilation.

Diagnosis: Colonoscopy demonstrated healthy pouch mucosa with no stricture. Patient's condition improved with antibiotics. Because of high suspicion or renal metastasis, a renal US and a repeat CT scan with intravenous contrast confirmed the left renal lesion with internal vascularity.

Therapy and Progression: Patient's symptoms resolved and was discharged with plan for outpatient biopsy by urology. Upon preoperative imaging in the IR suite, one month after discharge, there was no evidence of the renal mass and biopsy was aborted. Resolution after antibiotic treatment made a diagnosis of focal pyelonephritis more likely.

Comments: Renal masses in the setting of colon cancer should raise suspicion for metastasis. In this case, biopsy was aborted due to lesion resolution. Focal pyelonephritis has common radiologic features with a malignant renal mass, but improves with antibiotic treatment. In any case close follow up is recommended.

References: Hammond, N.A., Nikolaidis, P. and Miller, F.H., 2012. Infectious and inflammatory diseases of the kidney. Radiologic clinics of North America, 50 (2), pp.259-270. Israel, G.M. and Bosniak, M.A., 2008. Pitfalls in renal mass evaluation and how to avoid them. Radiographics, 28 (5), pp.1325-1338. Bhatt, S., MacLennan, G. and Dogra, V., 2007. Renal pseudotumors. American Journal of Roentgenology, 188 (5), pp.1380-1387. Vasen, H.F., et al., Revised guidelines for the clinical management of Lynch syndrome (HNPCC): recommendations by a group of European experts. Gut, 2013. 62 (6): pp. 812-23. Melichar, B., et al., Metastatic colorectal carcinoma and kidney tumors: a report of four cases. Tumori, 2010. 96 (3): pp. 483-6. Julianov, A., H. Stoyanov, and A. Karashmalakov, Late renal metastasis from sigmoid adenocarcinoma. Lancet Oncol, 2004. 5 (12): pp. 726. Kibar, Y., et al., Renal papillae metastasis of sigmoid colon adenocarcinoma. Int J Urol, 2005. 12 (1): pp. 93-5.. Zendel, A., et al., "Vanishing liver metastases"-A real challenge for liver surgeons. Hepatobiliary Surg Nutr, 2014. 3 (5): pp. 295-302

Disclosure: No significant relationships.

\section{PC134}

\section{OPEN ABDOMEN IN A SEPTIC PATIENT WITH DIGESTIVE FISTULAS - MULTI-STAGING APPROACH}

\author{
A. Hanu, A. Ivanoschi, D.O. Costea
}

\section{Surgery, Emergency County Hospital Constanta, Constanta/ ROMANIA}

Case History: 59 years old male patient, transferred from another hospital unit where he was admitted 3 weeks before with diagnosis of perforated pyloric ulcer with generalized peritonitis, and he underwent for excision of the ulcer and suture, developing after 3 days a duodenal fistula. The complication was followed by 2 more surgeries with an attempt of a gastrojejunal bypass and external controlled fistula in the duodenum.

Clinical Findings: In the clinical examination, we noticed: open abdomen with a large amount of bile and gastric fluid in the peritoneal cavity, irritating the organs and also the skin, a total dehiscence of the gastrojejunal anastomosis, and unfunctional duodenostomy.
Investigation/Results: Biological tests showed hypoproteinemia, elevated WBC, elevated amylases and a slight decreased value of Haemoglobine. Chest x-ray on admission was normal.

Diagnosis: Generalized acute peritonitis. Gastrojejunal anastomosis dehiscence. Duodenal fistula.

Therapy and Progression: Due to the irritation of the peritoneal cavity, the weak control of the septic source within one intervention, and to avoid the development of the abdominal compartmental syndrome, the surgical choice we adopted was a multi-staging therapy based on seriated surgeries, leaving the abdomen open, until the final intervention which consisted in reconstruction of the local anatomy of the digestive tract. The clinical and biological conditions of the patient were improved, he was discharged after 30 days, with a programmed incisional hernia.

Comments: Multi-staging surgical therapy gives a better advantage in controlling the septic sources, giving the body enough time to recover between the interventions.

References: 1. Plantefeve G, Hellmann R, Pajot O, Thirion M, Bleichner G, Mentec H. Abdominal compartment syndrome and intraabdominal sepsis: two of the same kind? Acta Clin Belg. 2007;62 (Suppl 1):162-7. doi: 10.1179/acb.2007.62.s1.021 2. Schein M. Surgical management of intra-abdominal infection: is there any evidence? Langenbeck's Arch Surg. 2002;387:1-7. doi: 10.1007/ s00423-002-0276-z 3. Cothren Burlew C. The open abdomen: practical implications for the practicing surgeon. Am J Surg. 2012;204:826-35. doi: 10.1016/j.amjsurg.2012.04.013 4. McCosh AJ., II The treatment of general septic peritonitis. Ann Surg. 1897;25:687-97

Disclosure: No significant relationships.

\section{PC135}

\section{LEAKAGE OF A LARGE BOWEL ANASTOMOSIS AFTER C-SECTION}

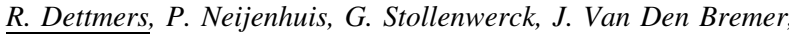 \\ C.Y. Wong
}

Surgery, Alrijne Hospital, Leiderdorp/NETHERLANDS

Case History: Patient underwent a caesarian section 7 days for presentation on our emergency department. She gave bith to a triplet. Furthermore patient was completely healthy.

Clinical Findings: Patient presented at our emergency department wwith abdominal pain and a distended abdomen. She did not defaecate since the caesarian. She suffered from nausea and was not able to eat or drink.

Investigation/Results: Lab findings showed a leukocytotsis of 20.9 x10^9/liter. CT-scan of the abdomen showed a disentended coecum with free air in the abdominal cavity, suspected for coecal perforation, and a colon with a pseudo-obstruction, due to paralytic ileus.

Diagnosis: Large bowel pseudo-obstruction after caesarian.

Therapy and Progression: Patient underwent a explorative emergency laparotomy. We found multiple perforations of the coecum due to distension from the pseudo-obstruction. A right side hemicolectomy with a primarily anastomosis was preformed. Five days after the laparotomy a fascia dehiscence with evisceration was found. Re-laparotomy showed a anastomotic leakage and a gelly fluid though out the entire abdomen, what was assumed to be collagenasis which is seen during labour to weaken the pelvis. We mentioned this could be te reason for the anastomotic leakage and the dehiscence. A ileostomy was made and the abdomen was closed. Three days after the re-do surgery fluid was found on CT-scan. We placed 2 tubes trough a small 
minilaparotomy incision at McBurney and flushed the abdomen with natriumsaline for 7 days. We removed the drains and patient could recover at home 1 month after the first laparotomy.

Comments: - Pseudo-obstruction ather caesarian is rare - When preforming a colectomy after a caesarian a primairy anastomosis is not advisable.

References: 2009 Feb;31 (2):167-71. Cecal perforation due to paralytic ileus following primary caesarean section.

Disclosure: No significant relationships.

\section{PC135A}

\section{STREPTOCOCCUS PYOGENES: AN UNCOMMON CAUSE OF SPONTANEOUS BACTERIAL PERITONITIS IN A YOUNG HEALTHY WOMAN}

\author{
J.M. Canga Presa, V. Olmos Juste, M.L. De La Hoz Riesco, \\ L.M. Alcoba García, C. Gavilanes Calvo, I.M. Marco López, \\ A. Álvarez Martínez, M. Beltrán Martos, A. Cavero Ibiricu, I. Oliva \\ López, C. Santamaría Revuelta, M.V. Diago Santamaría
}

Cirugía General Y Del Aparato Digestivo, Complejo Asistencial Universitario de León, León/SPAIN

Case History: 45 year old female, without previous illnesses, is admitted at ER complaining of lower abdominal pain and fever for 6hours. Initial laboratory tests: normal. Hemoculture was performed. She remained at observation care unit for 24hours. Abdominal pain and fever persisted. New blood tests showed leukocytosis, coagulopathy and elevated CRP.

Clinical Findings: Rebound tenderness in lower abdomen.

Investigation/Results: CT-scan: dilated bowel loops in pelvis. Small amount of free fluid in pouch of Douglas. The patient had a gynecological assessment, with normal ultrasound images.

Diagnosis: Acute abdomen of unknown origin

Therapy and Progression: Exploratory laparoscopy: normal appearance of appendix, ovaries and sigmoid colon. Edematous terminal ileum. Purulent fluid in pouch of Douglas and both subphrenic recesses. Lavage and drainage. After 48 hours in postoperative ICU, she was discharged to surgical department, receiving treatment with ceftriaxone + metronidazole. Streptococcus pyogenes was identified in hemocultures. Therapy was changed to Penicillin G. Intraabdominal and vaginal cultures: negative.

Comments: Primary peritonitis in the absence of risk factors is uncommon (less than $1 \%$ of peritonitis). Most of them are retrospectively diagnosed, once secondary causes are excluded. S. pyogenes is a causative agent of pharyngitis, erysipelas and necrotizing fasciitis. Primary peritonitis is caused by Streptococcus pneumoniae in most cases, meanwhile $\mathrm{S}$. pyogenes is unusual, and it is rarely diagnosed in healthy patients. Peritonitis due to S. pyogenes described in young woman suggests a vaginal origin, despite the lack of gynaecological symptoms. Also could be caused by hematogenous spread from other origins. It is a severe infection, requiring high index of suspicion and an early diagnosis to start antibiotherapy immediately.

References: 1. Park JY, Moon SY, Son JS, Lee MS, Jung MH. Unusual primary peritonitis due to Streptococcus pyogenes in a young healthy woman. J Korean Med Sci. 2012;27 (5):553-5. 2. Capdevila O, Pallares R, Grau I, Tubau F, Liñares J, Ariza J, Gudiol F. Pneumococcal peritonitis in adult patients: report of 64 cases with special reference to emergence of antibiotic resistance. Arch Intern Med 2001; 161: 1742-8. 3. Moskovitz M, Ehrenberg E, Grieco R, Chamovitz B, Burke M, Snyder D, Book M. Primary peritonitis due to group A streptococcus. J Clin Gastro- enterol 2000; 30: 332-5. 4. Brivet FG, Smadja C, Hilbert U, Vons C, Jacobs F, Gordji-Therani H, Musset D.
Usefulness of abdominal CT scan in severe peritoneal sepsis linked to primary peritonitis. Scand J Infect Dis 2005; 37: 76-8. 5. Farooq A, Ammori BJ. Laparoscopic diagnosis and management of primary bacterial peritonitis. Surg Laparosc Endosc Percutan Tech 2005;15: 36-7. 6. Gavala A, Klimopulos S, Exarchos D, Konstantinidis K, Daniil Z, Zakyn- thinos SG, Zakynthinos E. Persistent primary peritonitis due to group A Streptococcus and E. coli. Intensive Care Med 2002; 28: 1829-31. 7. Auskalnis S, Bogusevicius A, Maleckas A, Butrimavicius S, Toker I. Primary peritonitis caused by group A betahemolytic streptococcus. Medicina (Kaunas) 2004; 40: 969-74.

Disclosure: No significant relationships.

\section{PC136}

\section{HEPATIC PORTAL VENOUS GAS DUE TO SMALL BOWEL ISCHAEMIA: A CONSERVATIVE STRATEGY}

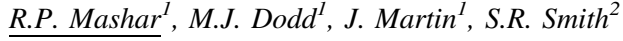

${ }^{1}$ General Surgery, University Hospitals of South Manchester, Manchester/UNITED KINGDOM, ${ }^{2}$ General Surgery, University Hospital South Manchester, Manchester/UNITED KINGDOM

Case History: A 67-year-old gentleman, during an admission for the treatment of new onset atrial fibrillation, developed colicky central and upper abdominal pain associated with nausea, vomiting, abdominal distension and absolute constipation. His past medical history included coronary artery disease, with 2 previous myocardial infarctions and cardiac arrest following ventricular fibrillation.

Clinical Findings: On examination, his abdomen was soft and nontender.

Investigation/Results: Computed tomography (CT) of his abdomen and pelvis showed dilated proximal and mid small bowel with no transition point. There was no free intraperitoneal gas. The small bowel demonstrated contrast enhancement but there was gas in the portal and superior mesenteric veins suggesting a recent small bowel ischaemic event. Associated with this, there was noted hepatic portal venous gas in the left lobe of the liver

Diagnosis: Portal vein and hepatic portal venous gas (HPVG) secondary to transient ischaemia of the small bowel following a paroxysm of atrial fibrillation

Therapy and Progression: He was treated with bowel rest, analgesia, antibiotics, treatment dose enoxaparin and fluid resuscitation, as his abdomen was only mildly tender, with a raised lactate on serial arterial blood gases, which resolved. He was commenced on warfarin and later discharged.

Comments: HPVG results from pneumatosis intestinalis (PI). Mucosal injury results in trans-mural tracking of intraluminal gas into the portomesenteric systems. Bowel ischaemia is the most common aetiology. Generally considered a peri-mortem sign, HPVG usually prompts emergency laparotomy or palliative care. In selected cases, however, there may be a role for non-operative management of HPVG, but further work is required to elucidate an appropriate riskstratification tool.

References: 1. Mohammed, A. H., Mohammed, A. H., Khot, U. P. \& Thomas, D. Portal venous gas-case report and review of the literature. Anaesthesia 62, 400-404 (2007). 2. Abboud, B., El Hachem, J., Yazbeck, T. \& Doumit, C. Hepatic portal venous gas: physiopathology, etiology, prognosis and treatment. World J. Gastroenterol. 15, 3585-3590 (2009). 3. Pineda Bonilla, J. J., Diehl, D. L., Babameto, G. P. \& Smith, R. E. Massive hepatic portal venous gas and gastric pneumatosis secondary to gastric ischemia. Gastrointest. Endosc. 78, 540; discussion 541 (2013). 4. Ohtsubo, K. et al. Pneumatosis 
intestinalis and hepatic portal venous gas caused by mesenteric ischemia in an aged person. J. Gastroenterol. 36, 338-340 (2001). 5. McElvanna, K., Campbell, A. \& Diamond, T. Hepatic portal venous gas - three non-fatal cases and review of the literature. Ulster Med. J. 81, 74-78 (2012). 6. Wayne, E. et al. Management algorithm for pneumatosis intestinalis and portal venous gas: treatment and outcome of 88 consecutive cases. J. Gastrointest. Surg. Off. J. Soc. Surg. Aliment. Tract 14, 437-448 (2010). 7. Morisaki, T., Ohba, K., Yoshida, A., Mizuta, Y. \& Nakao, K. [A case of hepatic portal venous gas caused by transient type ischemic enteritis]. Nihon Shokakibyo Gakkai Zasshi Jpn. J. Gastro-Enterol. 107, 407-415 (2010). 8. Nevins, E. J. et al. A rare case of ischaemic pneumatosis intestinalis and hepatic portal venous gas in an elderly patient with good outcome following conservative management. Int. J. Surg. Case Rep. 25, 167-170 (2016). 9. Greenstein, A. J. et al.Pneumatosis intestinalis in adults: management, surgical indications, and risk factors for mortality. J. Gastrointest. Surg. Off. J. Soc. Surg. Aliment. Tract 11, 1268-1274 (2007).

Disclosure: No significant relationships.

\section{PC137}

\section{NECROTIZING FASCIITIS OF THE UPPER EXTREMITY}

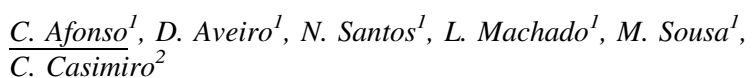

${ }^{1}$ Cirurgia Geral, Centro Hospitalar Tondela-Viseu, Viseu/ PORTUGAL, ${ }^{2}$ General Surgery, Centro Hospitalar Tondela-Viseu, Viseu/PORTUGAL

Case History: Necrotizing fasciitis is a rapidly progressive, potentially lethal bacterial infection involving skin, subcutaneous tissue, and superficial fascia. Encompasses a broad spectrum of presentations, and is associated with a poor outcome. The elderly, diabetic, intravenous drug abusers, immunocompromised represent some of the risk groups that are more susceptible. It can affect any body part; however, commonly the extremities, abdominal wall and perineum are favored.

Clinical Findings: The Authors present a case of a 80 year old male, diabetic, presenting in the Emergency Department with edema, subcutaneous emphysema and necrotic tissue of the upper left limb.

Investigation/Results: The diagnosis was made based on clinical examination findings. The patient was in septic shock. An aggressive surgical strategy was adopted with disarticulation of the upper limb at the shoulder blade associated to IV broad-spectrum antibiotic (meropenem). Diagnosis: Upper limb Necrotizing fasciitis

Therapy and Progression: He was admitted to the ICU and submitted to multiple surgical debridements. The bacteriological results of the specimens collected showed a soft tissue infection caused by a Lancefield's Group A beta hemolitic Streptococcus. The patient was grafted with skin mesh, with full coverage of the debrided areas. He needed intense rehabilitation to recover the pre-illness physical status. Comments: Upper limb Necrotizing fasciitis is a one possible presentation of this life-threatening disease. Early use of IV broadspectrum antibiotic and surgical treatment is a crucial. However, there is still a high morbidity and mortality associated.

References: 1. A. G. Angoules et al, Necrotizing fasciitis of upper and lower limb: a systematic review, Injury, Int. J. Care Injured (2007), 38S, S18-25 2. Schecter et al, Necrotizing fasciitis of the upper extremity, The Journal of Hand Surgery, vol.7, $\mathrm{n}^{\circ}$, January 1982 3. Nazerani $S$ et al, Necrotizing fasciitis of the upper extremity, Trauma Mon. 2012; 17 (2)

Disclosure: No significant relationships.

\section{PC138}

\section{JEJUNOILEAL DIVERTICULA: A BROAD-SPECTRUM OF COMPLICATIONS}

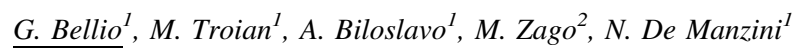

${ }^{1}$ Sc Chirurgia Generale, Azienda Sanitaria Universitaria Integrata di Trieste, Trieste/ITALY, ${ }^{2}$ Chirurgia Generale Ed Oncologica, Policlinico San Pietro, Bergamo/ITALY

Case History: Between 2013 and 2017, 7 patients ( 4 females and 3 males) were treated for complicated jejunoileal diverticula at the Departments of General Surgery of Trieste and Bergamo. Patients with complicated Meckel's diverticulum were not included in this series.

Clinical Findings: The median age was 74 years (46-85). Four patients presented with an acute inflammatory process, whereas two patients complained intestinal obstruction and one presented with severe anemia. Pain was the most constant symptom, being present in six patients, but its localization was different for each case. Only two patients complained rebound abdominal tenderness. Two patients lamented fever and vomiting, whereas one had diarrhea. Six cases out of 7 , small bowel diverticula were associated with colonic diverticula. Investigation/Results: Lab tests and abdominal CT scan were performed in every patient. Leukocytosis was recorded in 4 patients out of 7 and CPR elevation in 5 patients.

Diagnosis: Radiologic findings at abdominal CT scan allowed for a definite diagnosis just in one case. In the other 6 patients, the diagnosis was reached intraoperatively.

Therapy and Progression: One patient presented with uncomplicated small bowel diverticulitis at CT scan and was managed conservatively with antibiotics. In the other patients, a surgical procedure was carried out and small bowel resection was performed in 3 patients.

Comments: A complication from jejunoileal diverticula should be always suspected in patients admitted for unspecific abdominal pain with non-diagnostic CT scan, especially when colonic diverticula are associated.

References: Grana L, Pedraja I, Mendez R, Rodriguez R. Jejuno-ileal diverticulitis with localized perforation: CT and US findings. European Journal of Radiology 2009;71:318-323 Mantas D, Kykalos S, Patsouras D, Kouraklis G. Small intestine diverticula: is there anything new? World J Gastrointest Surg 2011;3:49-53 Spasojevic M, Naesgaard JM, Ignjatovic D. Perforated midgut diverticulitis: revisited. World J Gastroenterol 2012;18:4714-4720

Disclosure: No significant relationships.

\section{PC139}

\section{NEW STRATEGIES IN THE MANAGEMENT OF ACUTE MESENTERIC ISCHEMIA}

$\frac{\text { J. Galvanin, A. Ardito, G. Costa, M. Ceolin, S. Mei, H. Kurihara, }}{\text { J. Guerrini }}$

Acute Care And Trauma Surgery Unit, Humanitas Research Hospital, Rozzano (MI)/ITALY

Case History: 88-year-old woman was admitted to our Emergency Department for acute abdominal pain. The previous week admission for the same complains and first diagnosis of atrial fibrillation. Anticoagulant and rate-control therapy were started. 
Clinical Findings: Abdominal tenderness was observed in the left flank; electrocardiogram confirmed atrial fibrillation.

Investigation/Results: Point-of-care ultrasonography: small bowel segment without peristaltis. No free fluid. Laboratory: WBC count $12200 / \mu \mathrm{L}, \mathrm{C}$-reactive protein was $7.5 \mathrm{mg} / \mathrm{dL}$. Serum lactate level was 1.7. Negative Troponin-T. Abdominal MDCT revealed arterial thrombosis mesenteric ischemia with complete thrombosis of superior mesenteric artery and embolism of one of its collaterals. Reduced enhancement and wall thickening of jejunal segment. No radiologic approach available, due to uncertain embolism dating.

Diagnosis: Small bowel segmental ischemia

Therapy and Progression: Conservative treatment was initially undertaken. Worsening of clinical and laboratoristic status, led to a first laparotomical exploration. $14 \mathrm{~cm}$ of ischemic jejunal segment were resected and anastomosis wasn't performed. A temporary abdominal closure via a NPWT device was used and planned a second look 48h later During relaparotomy, Indocyanine green was infused and fluorescence real-time angiography was performed in order to evaluate intestinal microcirculation No ischemic progression was found, indicating correct margins of the first resection, so lateral to lateral anastomosis was performed. Histopathological study confirmed the clinical and surgical diagnosis of segmental small bowel ischemia.

Comments: Are open abdomen and second-time anastomosis right treatments for intestinal ischemia in elderly patients or is possible to use indocyanine green angiography to measure the viability of bowel at first exploration, reduce the number of unnecessary resections and decrease anastomotic leak rates?

References: Tilsed JVT, Casamassina A et al. ESTES guidelines: acute mesenteric ischemia, Eur J Trauma Emerg Surg, 2016 Bala M, Kashuk J et al. Acute mesenteric ischemia: guidelines of the WSES, World Journal of Emergency Surgery (2017) Toshiyuki I et al. Successful treatment of NOMI with indocyanine green fluorescence and open-abdomen management, Clinic J Gastroenterol, 2017 Xianzhi M et al. Indications and procedures for second-look surgery in acute mesenteric ischemia, Surg Today, 2010 Nowak K et al. Ischemic and injured bowel evaluation by Fluorescence imaging, Colorectal Disease, 2015 Ryer EJ, Kalra M et al. Revascularization for acute mesenteric ischemia, J Vascular Surg, 2012

Disclosure: No significant relationships.

\section{PC140}

\section{PALLIATIVE MANAGEMENT OF PNEUMOPERITONEUM}

A. Suárez Cabrera ${ }^{1}$, J.A. Lopez Ruiz ${ }^{2}$, B.L. López Durán ${ }^{1}$, L. TallónAguilar $^{3}$, B.M. De La Cuadral, M.S. Ramírez, E.P. Margallo ${ }^{1}$, F. Oliva Mompean ${ }^{2}$

${ }^{1}$ Cirugía De Urgencia, Hospital Universitario Virgen Macarena, Sevilla/SPAIN, ${ }^{2}$ General Surgery, University Hospital Virgen Macarena, Seville/SPAIN, ${ }^{3}$ Emergency Surgery Unit, Virgen del Rocío Universitary Hospital, Seville/SPAIN

Case History: We present two cases, a 86 years old men and a88 years old woman, pluripathologic with poor quality of life, came to our service for pain and abdominal distension, being diagnosed of pneumoperitoneum with suspicion of abdominal viscera perforation. Due to the poor clinical situation of the patients and the low expectations of survival we decided palliative treatment.

Clinical Findings: Sudden and severe abdominal pain and distension, in both cases with hemodynamic stability.
Investigation/Results: Abdominal $\mathrm{CT} 1^{\text {st }}$ patient: Large pneumoperitoneum chamber that compresses all structures of the abdominal cavity Abdominal $\mathrm{CT} 2^{\text {nd }}$ patient: Extensive pneumoperitoneum chamber, air adjacent to the duodenal bulb. No free liquid in significant amount.

Diagnosis: Pneumoperitoneum with suspicion of hollow viscera perforation.

Therapy and Progression: Palliative treatment through percutaneous drainage through Pleurecath was decided in both cases. After drainage, both patients reported significant improvement in pain and decreased abdominal distension. The first patient evolved favorably and presented complete resolution of the pneumoperitoneum in the control CT. The patient was discharged on the 8th day, eating normally and with established intestinal transit. The second patient required progressive palliative sedation during the 72 hours after drainage, dying on the 4 th day due to respiratory failure.

Comments: The radiological finding of free air in the abdominal cavity is generally considered a surgical emergency and is associated in more than $90 \%$ of cases with hollow viscera perforation. We propose a therapeutic option for palliative purposes in patients who are not subsidiary to surgical treatment

References: - Tony L. Weaver, Ross F. Goldberg and John A. Stauffer. Needle before the knife: Nonoperative maanagement of pneumoperitoneum with image-guided aspiration after gastrointestinal perforation. Surgical laparoscopid.2014; Volume 4, number2. Costaza Chiapponi, Urban Stocker, Marcus Koner and Roland Ladurner. Emergency percutaneous needle descompression for tension pneumoritoneum.BMC Gastroenterology. 2011. 48:11. - Zaj Khan and JR Nowel. Conservative management of tension pneumoperitoneum. Ann R Coll Surg Engl. 2002.84:164-165.

Disclosure: No significant relationships.

\section{PC141}

\section{OCCURENCE AND MANAGEMENT OF FROSTBITES IN MIGRATING POPULATION}

A. Ntikoudi ${ }^{1}, \underline{\text { A. Papachristou }^{2}}$, E. Ntikoudi $^{1}$, V. Saridakis ${ }^{3}$

${ }^{1}$ Nursing, KAPODISTRIAN UNIVERSITY OF ATHENS, ATHENS/ GREECE, ${ }^{2}$ Medical Department, KAPODISTRIAN UNIVERSITY OF ATHENS, ATHENS/GREECE, ${ }^{3}$ Surgical Department, GN 2ND YPE, NIKAIA/GREECE

Case History: Migration routes are often associated with prolonged cold exposure and further extreme environmental conditions. Frostbite is a freezing injury where localized damage affects the skin and other tissues.

Clinical Findings: It concerns the population in transit, men, and women of various ages. Several environmental (temperature, wind, snow, wetness, cold objects, altitude) and individual (behavior, health, physiology) predisposing factors are being monitored related to vulnerable populations having a chronic disease (cardiovascular, diabetes, and depression), children and the elderly. Frostbites cause different types of discomfort and functional limitations that may persist for years and eventually public health issues are involved. Investigation/Results: The initial actions include the primitive and usually risky management of the condition during the migration route due to minimum patients' awareness and mistreatment. Because of 
the lack of antiseptic, anti-inflammatory and antibiotic agents, the injury may deteriorate to severe infection.

Diagnosis: The holistic approach is set upon classification of frostbites injuries according to the depth of injury and amount of tissue damage, based on acute physical findings and ranging from partial skin freezing, erythema, edema, skin desquamation to hemorrhagic blisters form, skin necrosis and further extension into subcutaneous tissues, muscle, bone, and tendon.

Therapy and Progression: Initial field management includes prevention of further cold injury, hypothermia, dehydration and refreezing, while the core hospital treatment includes rapid rewarming of the injured area in warm water, gentle early conservative treatment (leave most blisters intact, no early surgery, or amputations), meticulous local care, adequate pain relief, and physical therapy.

Comments: Keywords: cold, frostbite, injury, migration, vulnerable, population

References: Keywords: cold, frostbite, injury, migration, vulnerable, population

Disclosure: No significant relationships.

\section{PC142}

\section{URGENT SURGICAL REVASCULARIZATION IN MESENTERIC ISCHEMIA}

C. Domínguez Sánchez ${ }^{1}$, J.A. Lopez Ruiz ${ }^{2}$, L. Tallón-Aguilar ${ }^{3}$, S. De Lebruisant $^{4}$, B. De Soto Cardenal ${ }^{5}$, B.M. De La Cuadra ${ }^{6}$, M. Sanchez Ramirez $^{7}$, J. López Pérez ${ }^{8}$, E. Perez Margallo ${ }^{2}, F$. Oliva Mompean ${ }^{7}$

${ }^{1}$ General Surgery, University Hospital Virgen Macarena, Seville/ SPAIN, ${ }^{2}$ Emergency Surgery Unit, Hospital Universitario Virgen Macarena, Seville/SPAIN, ${ }^{3}$ Emergency Surgery Unit, Virgen del Rocío Universitary Hospital, Seville/SPAIN, ${ }^{4}$ Cali, Hospital Universitario del Valle, Cali/COLOMBIA, ${ }^{5}$ General Surgery, HOSPITAL UNIVERSITARIO VIRGEN MACARENA, sevilla/ SPAIN, ${ }^{6}$ General Surgery, H.U.V.Macarena, Sevilla/SPAIN, ${ }^{7}$ Cirugía De Urgencia, Hospital Universitario Virgen Macarena, Sevilla/ SPAIN, ${ }^{8}$ Emergency Surgery Unit, Hospital Universitario Virgen Macarea, Seville/SPAIN

Case History: Observational, descriptive and retrospective study based on a clinical case. It is a patient of 62 years, smoker and excessive drinker. He goes to the ER for intense abdominal pain, hours of evolution and abrupt onset. He denied fever or traffic alterations

Clinical Findings: A CT scan of the abdomen is performed with contrast, observing a dilatation of small intestine with levels and an anomalous arrangement of bowel loops with wall edema.

Investigation/Results: Given the clinical worsening with significant pain, tachycardia and increased leukocytosis, surgical intervention was decided urgently.

Diagnosis: We found an important mesenteric ischemia with necrosis of two meters of small intestine and ischemia of the rest, except for the first jejunal and terminal ileum sections. The palpation of the artery presented a woody touch. No pulse.

Therapy and Progression: Due to this situation a mesenteric endarterectomy with Fogarty 5Fr was decided. After checking for revascularization, we resected the segment of the small necrotic intestine, with ileostomy and mucosal fistula. Arteriography at 48 hours. No image of thrombosis or bleeding. 5th postoperative day we performed a "second look", finding a segment of $20 \mathrm{~cm}$ necrotic proximal small intestine. Correct mesenteric circulation. New resection and closure of the ileostomy and reconstruction transit are proceeded.

Comments: Despite advancements in clinical algorithms, laboratory investigations, and improved imaging techniques, AMI remains a clinical diagnostic challenge with a non-specific, high-mortality presentation. The possibility of surgical revascularization is infrequent, with good results even less frequently.

References: - Acosta S. Epidemiologyof mesenteric vascular disease: clinical implication. Semin Vasc Surg. 2010 Mar;23 (1):4-8. Yasuhara H, Niwa H, Takenoue T, Naka S. Factors influencing mortality of acude intestinal infarction associated with SIRS. Hepatogastroenterology. 2005 Sep-Oct;52 (65):1474-8. - Cosse C, Sabbagh C, Kamel S, Galmiche A; Regimbeau JM. Procalcitonin and intestinal ischemia: a review of the literature. World J Gastroenterol. 2014 Dec 21;20 (47):17773-8. - Cudnik MT, Darbha S, Jones J, Macedo J, Stockton SW, Hiestand BC. The diagnosis of acute mesenteric ischemia: A systematic review and meta-analysis. Acad Emerg Med. 2013 Nov;20 (11):1087-100.

Disclosure: No significant relationships.

\section{PC143}

\section{ABDOMINAL EMERGENCY IN NON VISCERAL CANCER - A RARE COMPLICATION IN A COMMON TUMOR}

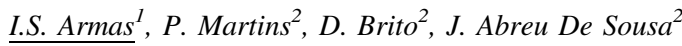

${ }^{1}$ Surgical Oncology Department, Instituto Português de Oncologia do Porto, Porto/PORTUGAL, ${ }^{2}$ Surgical Oncology, Instituto Portugues de Oncologia do Porto, Porto/PORTUGAL

Case History: Male, 79 years old, with an extensive inferior lobe pulmonary Adenocarcinoma, stage III at diagnosis, EGFR negative, ALK negative, doing chemotherapy. Admitted for the beginning of corticotherapy and holocraneal radiotherapy in the context of symptomatic new detected cerebral metastasis with cerebral edema.

Clinical Findings: Ten days after admission started left hypochondria abdominal pain, no other associated symptoms, without systemic repercussion, no fever. He had decompression pain in the left upper quadrant, no other findings in the physical exam.

Investigation/Results: The abdominal X-ray showed a not clear minimal line of pneumoperitoneum, the abdominal CT-scan showed a small pneumoperitoneum, probably traducing small intestine perforation in the left upper quadrant.

Diagnosis: With a pneumoperitoneum from hollow viscera perforation, the patient was proposed to an exploratory laparotomy.

Therapy and Progression: We found a perforated jejunal lesion, probably a metastasis, with an adjacent small amount of biliary content. We did an enterectomy and drained the abdominal cavity. We solved the abdominal complication but the patient died weeks later from his primary disease progression. Histology confirmed the jejunal metastasis.

Comments: It is a case of perforated jejunal metastasis from primary pulmonar Adenocarcinoma. In this bad prognosis stage IV tumors, sometimes is difficult to decide which procedures are plausible. In this case, an abdominal complication without systemic repercussion and with an easy surgical solution, was mandatory to explore. This case shows a rare kind of metastasis from pulmonary carcinoma, with a perforation due to metastasis ulceration and corticotherapy. Also shows the difficult management of this patients in their disease progression.

References:

Disclosure: No significant relationships. 


\section{PC144}

\section{THE POLYTRAUMATIZED PATIENT WITH A FIREGUN WOUND. CLINICAL EMERGENCY MANAGEMENT AN DECISION MAKING}

\section{J.A. Marante Fuertes $^{1}$, A. Holub $^{2}$, J. Marante Fuertes ${ }^{3}$}

${ }^{1}$ Emergency Service, General Hospital in Molina de Segura, Murcia, Spain, Molina de Segura, Murcia, Spain/SPAIN, ${ }^{2}$ Emergency Room And General Practitioner Resident, General Hospital in Jerez de la Frontera, Cádiz/SPAIN, ${ }^{3}$ The Department Of Orthopaedics And Traumatology, In Jerez De La Frontera General Hospital (cádiz, Spain), General Hospital in Jerez de la Frontera, Jerez de la Frontera/ SPAIN

Case History: A 70 - year- old male is brought to to Emergency Service by a Ambulance Emergency Team after being accidentally shut in his lowe extremity with a firegun by his relative.

Clinical Findings: The patient arrives at the emergency room stable and conscious with extra support of inyravenous liquids. Glasgow Coma Score on his arrival was $15 \backslash 15$. Blood pressure 90\55. Heart rate of 78 . Lower extremity with compressive bandage that controlled the massive bleeding.

Investigation/Results: Urgent Computerized tomography and plain radiograghy revealed left tibial bone massive destruction (comminuted fracture) with severe tissue destruction.

Diagnosis: Massive bone and tissue destruction of the lower left extremity. Hypovolemic shock.

Therapy and Progression: With a patient stable but severe injury with massive bone an issue damage with empiric antybiontc perfusion the decision to made $s$ to amputate the left extremity to safe the patients life. The amputation is performed with revascularization, microvascular tecnique.

Comments: The multidisciplinary approach should be make while dealing with the extremity amputations. Usually in programmed surgeries the patient undergoing amputation should be examined by psiquiatrist for cognitive and psichical ability but the emergency surgery in a life threatening situation is impossible and gains more value the posterior psychological help.

References: 1. Janos P. Ertl P. Lower extremity amputations techniques. April 04; 2009 (8); 34- 4623; 56

Disclosure: No significant relationships.

\section{PC145}

\section{EMERGENCY DIAPHRAGMATIC HERNIAS}

\author{
M. Güell Farre, R. Farré, C. Soto
}

General Surgery, Althaia Xarxa Assistencial de Manresa, Manresa/ SPAIN

Case History: From January 2009 to December 2014 we performed 62 interventions of patients with diaphragmatic hernia, of which 12 were urgent. The mean age of the 12 patients who took emergency intervention was 81.16 years (range 62-94). All the patients were previously diagnosed, the reason to go to the emergency room in all cases was retrosternal pain and vomiting, a thoracoabdominal CT scan was performed, reporting an incarcerated hiatus hernia, so emergency surgical intervention was decided.
Clinical Findings: Of the 12 interventions, only one was by laparoscopy, the remainder by laparotomy. In 1 case, the thoracoabdominal CT reported gastric perforation and a distal esophagogastrectomy was performed with esophagoyeyunal anastomosis; in another case, the hiatus hernia repair and splenectomy were performed, since the spleen was intrathoracic and the maneuvers presented bleeding. In 7 cases the hiatus was repaired and some type of plication (4 Nissen and 3 Toupet) were hiatus hernias type 2 or 3 , in 6 cases the diaphragm was repaired with mesh, another 3 cases were made defect closure and reinforcement with mesh. 10 cases required entry into the immediate postoperative period in the ICU. Two patients who died of esophagostractomy and the patient of splenectomy died. The average stay is 9.4 days.

Investigation/Results: The literature states that paraesophageal hernias (type 2-3) should be offered surgery, since 30\% present complications, post-traumatic and congenital hernias consist of a repair of the defect either primary closure if possible or placement of material replacement prosthetic. We present a series of 12 cases in 5 years of emergency surgery of diaphragmatic hernias, representing $19.35 \%$ of the cases that intervened during that period of time, surgery that, as we can see in our series, is not without complications.

Diagnosis: Diaphramatic hernia

Therapy and Progression: Treatment is emergency surgery

Comments: Paraesophageal hernias must be intervened in an elective way, since we present a series in which $20 \%$ of the cases are emergency surgery, all cases were previously known but the surgery had been disregarded in most cases by age, mprtaly was $2 \%$

References: 1. Oddsdottir M: Paraesophageal hernia. Surg Clin North Am 80:1243, 2000. 2. De Meester TR, Bonavina L: Paraesophageal Hiatal hernia. In Nyhus LM, Condon RE (eds): Hernia . Philadelphia, Lippincott 1989, p684.

Disclosure: No significant relationships.

\section{PC146}

\section{NUTCRACKER SYNDROME: DIFFERENTIAL DIAGNOSIS} FOR ACUTE APPENDICITIS- CASE STUDY

\section{R. Chaves-Marcos ${ }^{1}$, L. Tallón-Aguilar ${ }^{1}$, V. Durán-Muñoz-Cruzado ${ }^{2}$, F. Pareja-Ciuról, J. Padillo-Ruíz ${ }^{2}$}

${ }^{1}$ Emergency Surgery Unit, Virgen del Rocío Universitary Hospital, Seville/SPAIN, ${ }^{2}$ Digestive And General Surgery Unit, Virgen del Rocío Universitary Hospital, Seville/SPAIN

Case History: Female, 24 years, denies previous diseases. She suffered from severe abdominal pain in the right lower quadrant (RLQ) which started 2 days ago, with progressive worsening, nausea and vomiting. She denies fever, urinary symptoms or gynecologic complaints. The previous week, in treatment with antibiotics because of a urinary lower tract infection.

Clinical Findings: Pain in deep palpation in the RLQ without signs of peritoneal irritation. Laboratory test were unchanged. Alvarado score was 5.

Investigation/Results: Abdominal ultrasound showed no alterations but it could not identify the appendix. A CT scan was then requested. The angle formed by the aorta and the superior mesenteric artery (SMA) was reduced to $27^{\circ}$ compromising venous return of the left renal vein causing pelvic venous congestion with severe uterine varices.

Diagnosis: She was diagnosed with Nutcracker Syndrome (NCS) ${ }^{1}$

Therapy and Progression: She remained hospitalized for pain control and she is waiting for endovascular surgery. 
Comments: NCS is a rare condition, even though, the prevalence is still unknown. It seems to be more prevalent in young adults and females ${ }^{2}$. The most common way of manifestation is asymptomatic hematuria ${ }^{1}$. In other cases, it causes a severe abdominal pain as seen in acute appendicitis. Appendicitis is the most common abdominal emergency requiring emergency surgery ${ }^{3}$, so it is important to make a good differential diagnosis, having into account that its symptoms are frequently overlap with other diseases. The use of Alvarado score, laboratory test and physical examination are useful to suspect it $^{4}$, however, in cases of doubt, it is necessary an imaging test that can manifested other conditions.

References: 1. Gulleroglu et al. Nutcracker syndrome. World Journal of Nephrology. 2014 Nov; 3 (4): 277-81 2. Siddiqui W.J et al. Left Renal Vein Compression Syndrome: Cracking the nut of clinical dilemas- three cases and review of literature. American Journal of Case Reports. 2017 Jul; 18: 754-9. 3. Shogilev et al. Diagnosing Appendicitis: Evidenced-based review of the Diagnostic approach in 2014. Western Journal of Emergency Medicine. 2014 Nov; 7; 859-71. 4. Gorter R et al. Diagnosis and management of acute appendicitis. EAES consensus development conference 2015. Surg Endosc. 2016; 30: 4668-90.

Disclosure: No significant relationships.

\section{PC147}

\section{A RARE CASE OF INTESTINAL OBSTRUCTION CAUSED BY PSYLLIUM SEEDS}

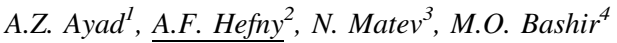

${ }^{1}$ Emergency Department, Al Rahba Hospital, Abu Dhabi/UNITED ARAB EMIRATES, ${ }^{2}$ Department Of Surgery, College of Medicine and Health Sciences, Al Ain/UNITED ARAB EMIRATES,

${ }^{3}$ Radiology Department, Al Rahba Hospital, Abu Dhabi/UNITED

ARAB EMIRATES, ${ }^{4}$ Surgery Department, Al Rahba Hospital, Abu Dhabi/UNITED ARAB EMIRATES

Case History: 21-year-old man presented to the Emergency Department complaining of lower abdominal pain and constipation for 5 days. Blood investigations and $\mathrm{x}$ ray abdomen were normal. Patient was diagnosed to have constipation and was discharged home on laxative therapy. Two days later, the patient presented with the same complaint. He did not pass stool, but he was passing flatus.

Clinical Findings: His vital signs were normal. Abdomen was distended but soft. No scars of previous operations were seen. Intestinal sounds were audible. P/R examination was normal.

Investigation/Results: Repeated abdominal $\mathrm{X}$ ray has shown huge fecal loading filling the whole colon. CT scan of the abdomen has shown marked dilatation of the whole colon with fecal matters, the sigmoid colon was markedly distended and filled with gas and fecal matters. The wall of the colon was thickened and highly enhanced post contrast. No clear evidence of level of colonic obstruction was noted.

Diagnosis: Patient was admitted to the surgical ward for conservative management. The patient received repeated enemas and laxatives. The patient admitted that he ingested psyllium seeds prior to constipation (psyllium seeds are known in Bangladesh to increase the health status of the people consuming it). He said that he was feeling weak during the fasting hours of Ramadan and he ingested the seeds without adequate fluid because of fasting.

Therapy and Progression: The patient received repeated enemas and laxatives. He passed a huge amount of fecal matters during his stay in the hospital and was discharged home two days later without any operative intervention.
Comments: The Food and Drug Administration (FDA) considered the use of psyllium as a laxative in granular dosage form not generally recognized as safe and effective. This rule was due to reports of esophageal obstruction related to its usage. It is important to instruct patients who are receiving psyllium ingredients as a laxative to drink a good amount of fluids to avoid the development of fecal impaction or intestinal obstruction.

References: 1. Fisher RE. Psyllium Seeds: Intestinal Obstruction. Cal West Med 1938; 48:190 2. Pérez-Piqueras J, Silva C, Jaqueti J, Sáez MA, Martinez D, Santa JM, Peralba I. Endoscopic diagnosis and treatment of an esophageal bezoar resulting from bulk laxative ingestion. Endoscopy 1994; 26:710. 3. Chen YA, Cervini P, Kirpalani A, Vlachou PA, Grover SC, Colak E. Small bowel obstruction following computed tomography and magnetic resonance enterography using psyllium seed husk as an oral contrast agent. Can J Gastroenterol Hepatol. 2014 Jul-Aug;28 (7):391-5 4. Herrle F, Peters T, Lang C, von Fluee M, Kern B, Peterli R. Bolus obstruction of pouch outlet by a granular bulk laxative after gastric banding. Obes Surg. 2004 Aug;14 (7):1022-4.

Disclosure: No significant relationships.

\section{PC148}

\section{CRITICAL MANAGEMENT OF INTRAABDOMINAL FOREIGN BODY}

C. Cózar Lozano, L.O. Carrión Retuerto, A. Nonnemacher San Julián, L. Oliver García, B. Méndez Gómez, O. Blasco Delgado

General Surgery, Virgen del Puerto Hospital, Plasencia (Cáceres)/ SPAIN

Case History: 78-year-old male suffers an accident while working with a brush cutter.

Clinical Findings: Hemodynamically stable at the ER, abdominal tenderness without guarding, and an incised-contused wound on the left upper abdominal quadrant.

Investigation/Results: Abdominal x-ray showed a $4 \mathrm{cms}$ foreign body on the upper left hemiabdomen with metallic appearance, without pneumoperitoneum.

Diagnosis: Intraabdominal foreign body.

Therapy and Progression: The patient underwent surgical exploration under local anesthesia, without observing peritoneal continuity solution. The wound was washed, closed and drained. Control abdominal X-ray showed persistence of the foreign body and therefore abdominal CT scan was requested, confirming a metallic foreign body with a distal end on the left anterolateral aortic wall between the celiac trunk and the superior mesenteric artery (AMS). The patient was transfered to Vascular Surgery Department where laparotomy was performed and the needle housed in AMS origin was extracted, requiring repair of the first portion of infrapancreatic AMS. The patient was discharged on the sixth postoperative day without complications.

Comments: In patients with open wounds, once the initial assessment of the patient is performed following the $\mathrm{ABC}$ method, and if hemodynamically stable, CT is the gold standard both to exactly stablish diagnosis and to rule out other abdominal injuries.

References: Colwell C, E Moore E. Initial evaluation and management of abdominal stab wounds in adults. Moreira ME (Ed). UpToDate. Waltham, MA: UpToDate Inc. (Accessed on October 03, 2016)

Disclosure: No significant relationships. 


\section{PC148A}

\section{AMYAND'S HERNIA. A RARE FORM OF CRURAL HERNIA}

R. Cobos Cuesta ${ }^{l}$, A.I. Rueda López ${ }^{l}$, I. Martínez Casas ${ }^{l}$, C.M. Ruiz, Marín $^{\prime}$, R. Ortega Higueruelo ${ }^{l}$, F.M. Jiménez Armenteros ${ }^{I}$, J.M. Capitan Vallvey

${ }^{1}$ General And Digestive Surgery, Complejo Hospitalario Jaén, Jaén/ SPAIN, ${ }^{2}$ Ugc Cirugía General, Complejo Hospitalario de jaén, Jaén/ SPAIN

Case History: 87-year-old man with personal history of hypertension, arrived to the emergency department suffering two days of pain and right inguinal tumour. He also reported vomiting, diarrhoea and fever. Clinical Findings: Temperature $38{ }^{\circ} \mathrm{C}$, hemodynamically normal. On palpating abdomen was soft, pain in right lower quadrant, mass in right lump which was irreducible. No other findings.

Investigation/Results: Blood analysis only showed leucocytosis, RCP 230mg/L. Urine analysis: nitrites + Echo / CT: incarcerated crural hernia, hiatal hernia, bilateral renal cysts, marked prostatic hypertrophy. Intraoperative findings: crural sac with dark haemorrhagic fluid and inflamed and necrotic appendix without macroscopic perforation inside.

Diagnosis: Right crural hernia with strangulation of vermiform appendix. Acute appendicitis.

Therapy and Progression: Appendectomy and hernioplasty with preperitoneal polypropylene plug.

Comments: Amyand's hernia is a rare case of inguinal hernia (incidence to $0.28-1 \%$ ). The main characteristic of this inguinal hernia is the vermiform appendix, which is inflamed inside the hernial sac and promote $0.13 \%$ of appendicitis. The particularity in our case was that the hernia diagnosed was crural and not inguinal. This kind of hernia are mostly diagnosed during surgery, in the suspicion of a complicated inguinal hernia, being its preoperative diagnosis rare. The correct treatment includes appendectomy and hernia repair, not being precluded the use of extraperitoneal mesh. From of knowledge, there are only few cases reported in the literature.

References:

Disclosure: No significant relationships.

\section{PC149}

\section{MASSIVE PNEUMOPERITONEUM AS A RESULT OF THE RESUSCITATION MANEUVER}

\author{
A. Szuba ${ }^{1}$, R. Lupiani Morenol ${ }^{1}$ A.X. Argote Camacho ${ }^{2}$
}

${ }^{1}$ General Surgery, Hospital del Poniente, El Ejido/SPAIN, ${ }^{2}$ General Surgery, Complejo Hospitalario de Jaen, Jaen/SPAIN

Case History: We present a case of a 47-year-old female, an ex drug addict who suffers a loss of consciousness after heroin consumption. Her husband does mouth-to-mouth resuscitation without cardiac massage. 24 hours later the patient presents abdominal pain and distension that increase progressively. No dyspnea or chest pain.

Clinical Findings: 48 hours from the event the physical examination reveals an important increase in the abdominal perimeter, generalized abdominal pain without peritoneal irritation and cervical subcutaneous emphysema.

Investigation/Results: In the simple chest/abdomen X-ray, massive tension pneumoperitoneum and pneumomediastinum were observed.
CT scan confirms these findings ruling out the pneumotorax and the presence of intra-abdominal free fluid, suggesting hollow viscera perforation as the first possibility. The upper digestive endoscopy to verify the esophageal perforation was denied by the Gastroenterologist.

Diagnosis: MASSIVE PNEUMOPERITONEUM DUE TO BAROTRAUMA

Therapy and Progression: An urgent supraumbilical laparotomy was performed evidencing the pneumoperitoneum without other pathological findings in the abdominal cavity. On the second postoperative day, esophagogastric transit and brochoscopy were performed, ruling out the esophageal perforation and finding a small hematoma at the subglotic level (a possible air entance location in the valve mechanism - into the mediastinum and the abdominal cavity. The patient was released two weeks after the surgery.

Comments: An extensive pneumoperitoneum is uncommon in adults and usually is due to the gastric or colonic perforation. It can be also secondary to the necrotising enterocolitis, trauma intestinal perforation or mechanical ventilation however it is unusual to be found after a mouth-to-mouth resuscitation as a result of high pressure of inflated air.

References: Hull A, Buckley P, Wills B. Massive Pneumoperitoneum. West J Emerg Med 2010;11:92. Levine MS, Scheiner JD, Rubesin SE, et al. Diagnosis of pneumoperitoneum on supine abdominal radiographs. AJR Am J Roentgenol 1991;156:731-5 Milanchi S, Wood D. Rigler's sign in a patient with massive pneumoperitoneum. Emerg Med J. 2006;23:884. Rampton JW. The football sign. Radiology. 2004;231:81-2 ElGendy K, Salem A. Massive pneumoperitoneum following colonoscopic sigmoid perforation. BMJ Case Rep 2014 Milanchi S, Wood D. Rigler's sign in a patient with massive pneumoperitoneum. Emerg Med J. 2006;23:884. Disclosure: No significant relationships.

\section{PC150}

\section{HANDLEBAR HERNIA, UNUSUAL PRESENTATION OF ABDOMINAL TRAUMA}

J. López Sánchez, M.D.J. Rodríguez Perdomo, F.C. Parreño

Manchado, O. Abdel-Lah Fernández, L. Muñoz Bellvís

CirugÍa General Y Del Aparato Digestivo, COMPLEJO ASISTENCIAL UNIVERSITARIO DE SALAMANCA, Salamanca/ SPAIN

Case History: A 41-year-old man who came to the emergency department for abdominal trauma to the handlebar of his bicycle. He reported abdominal pain in the right flank. The patient presented good general condition, hemodynamic stability and was afebrile.

Clinical Findings: Physical examination showed an excoriation and weakness on the right flank.

Investigation/Results: Laboratory tests revealed leukocytosis with neutrophilia. The CT reported traumatic abdominal wall hernia.

Diagnosis: Traumatic abdominal wall hernia

Therapy and Progression: Direct suture of the abdominal wall was performed. The patient had a favorable postoperative period.

Comments: Traumatic abdominal wall hernia (TAWH) is defined as disruption of the muscle-aponeurotic layers due to non-penetrating trauma. It presents a lower incidence $(1 \%)$ and within the etiological causes, it appears the traffic accidents (49\%). Less common are the consequences of falls or trauma to handlebars ${ }^{1,2,3}$. About 200 cases have been reported in the literature. More than 50\% are not diagnosed on physical examination and imaging studies should be performed 
(CT is the gold standart). Diagnostic failure may lead to complications and ignore intra-abdominal injuries ${ }^{1,2}$. The treatment is controversial, being able to perform urgent or delayed surgery, open approaches (laparotomy or local exploration) or laparoscopic 2, 3, 4, 5, 6,7 . The techniques of raffia or mesh are accepted ${ }^{1,7}$. In our case, we performed a local approach and muscle aponeurotic raffia. TAWH should be suspected when closed abdominal trauma appears. The diagnosis is made with anamnesis, physical examination and complementary tests. There is controversy about the treatment, having to individualize each case.

References: 1. A. Matalon S, Askari R, D. Gates J, Patel K, D. Sodickson A, Khurana B. Don't forget the abdominal wall: imaging spectrum of abdominal wall injuries after nonpenetrating trauma. RadioGraphics 2017; 37: 1218-1235. 2. Akbaba S, Haldum Gündogdu R, Temel H, Oduncu M. Traumatic abdominal wall hernia: early o delayed repair? Indian J Surg 2015; 77: 963-966. 3. Burt BM, Afifi HY, Wantz GE, Barie PS. Traumatic lumbar hernia: report of cases and comprehensive review of the literature. J Trauma 2004;57 (6):1361-1370. 4. Netto FA, Hamilton P, Rizoli SB, Nascimento B, Brenneman FD, Tien $\mathrm{H}$ et al (2006) Traumatic abdominal wall hernia: epidemiology and clinical implications. J Trauma 61:1058-1061. 5. Kumar A, Hazrah P, Bal S, Seth A, Parshad R (2004) Traumatic abdominal wall hernia: a reappraisal. Hernia 8 (3):277-280. 6. BenderJS, DennisRW, AlbrechtRM (2008)Traumaticflankhernias: acute and chronic management. Am J Surg 195 (3):414-417. 7. S. Al Beteddini O, Abdulla S, Omari O. Traumatic abdominal wall hernia: a case report and literature rewiew, International Journal of Surgery Case Reports 24 (2016); 55-57.

Disclosure: No significant relationships.

\section{PC151}

\section{SPILLED GALLSTONES AS AN UNUSUAL CAUSE OF INTRA-ABDOMINAL ABSCESS - CASE REPORT}

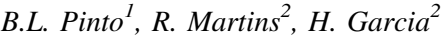

${ }^{1}$ General Surgery, Hospital Prof. Dr. Fernando Fonseca, Parede/ PORTUGAL, ${ }^{2}$ General Surgery, Hospital Prof. Dr Fernando Fonseca, Venteira/PORTUGAL

Case History: The technical advance of laparoscopic cholecystectomy has provided patients with a better treatment for gallstones with faster recovery and better patient satisfaction. However, problems as bile duct injuries and spilled gallstones have become more frequent, and the latter is often ignored as a risk for potential complications. The risk of gallbladder perforation is reported to be $10-40 \%$ in different series. Furthermore, unretrieved stone spillage reported in the range of $6-30 \%$ of laparoscopic cholecystectomies. Complications from these stones occur in $0.08-0.3$ of patients and although they usually present in the first postoperative months, they can appear several years later. Abscess formation accounts for $60 \%$ of the complications. We report the case of an 88 year old women that present to the emergency department with a 1 week history of an abdominal wall abscess located in the right hypocondrium and fever. Her surgical history was significant for an open cholecystectomy for acute cholecystitis approximately 15 years before

Clinical Findings: Abdominal abscess located under the previous cholecystectomy scar.

Investigation/Results: Laboratory evaluation showed leucocytosis, neutrophilia and C-Reactive protein elevation. She underwent a and computed tomography scan which showed an intra-abdominal abscess located in the previous gallbladder location extending to the abdominal wall, furthermore, there was evidence of a retro-hepatic collection without signs of active infection

Diagnosis: Spilled gallstones complicated by abdominal abscess

Therapy and Progression: The patient was submitted to an urgent abscess drainage and several gallbladder stones were retrieved. The retro-hepatic collection was later drained by a CT-guided percutaneous procedure. The patient had an uneventful recovery.

Comments: Spilled gallstones are often ignored as a risk for complications after cholecystectomy. This case is a reminder of the importance of avoiding gallbladder perforation and of the infrequent complications associated with it

References: Helme, S., Samdani, T., \& Sinha, P. (2009). Case report Complications of spilled gallstones following laparoscopic cholecystectomy: a case report and literature overview, 4, 1-4. https://doi.org/10.4076/1752-1947-3-8626 Moga, D., Periæanu, Æ., Popenåiu, A., Sora, D., \& Magdu, H. (2016). Right Retroperitoneal and Subhepatic Abscess; Late Complications Due to Spilled Stones During Laparoscopic Cholecystectomy - Case Report, (1), 67-70. Saklani, A. P., Vinayagam, R., \& Blackett, R. L. (2004). review of the literature, (August 2003), 77-80. https://doi.org/10.1136/ pmj.2003.006023 Rammohan, A., Srinivasan, U. P., Jeswanth, S., \& Ravichandran, P. (2012). CASE REPORT - OPEN ACCESS International Journal of Surgery Case Reports Inflammatory pseudotumour secondary to spilled intra-abdominal gallstones CASE REPORT OPEN ACCESS. International Journal of Surgery Case Reports, 3 (7), 305-307. https://doi.org/10.1016/j.ijscr.2012.03.013 Yethadka, R., Shetty, S., \& Vijayakumar, A. (2014). Attitudes and Practices of Surgeons towards Spilled Gallstones during Laparoscopic Cholecystectomy: An Observational Study, 2014. Singh, K., Wang, M. L., Ofori, E., Widmann, W., Alemi, A., \& Nakaska, M. (2012). CASE REPORT - OPEN ACCESS International Journal of Surgery Case Reports Gallstone abscess as a result of dropped gallstones during laparoscopic cholecystectomy CASE REPORT - OPEN ACCESS. International Journal of Surgery Case Reports, 3 (12), 611-613. https://doi.org/10.1016/j.jiscr.2012.07.017

Disclosure: No significant relationships.

\section{PC152}

\section{AN UNUSUAL CASE OF PERFORATED CECAL DIVERTICULUM}

C. Farazi-Chongouki, A. Ninos, C. Iordanou, I. Pougouras, A. Raftopoulos, M. Karamanidi, V. Mperdelis, E. Akritidou, E. Dimitrakopoulou, S. Pierrakakis, E. Papakonstantinou, A. Natsiopoulou

Surgery, Thriassio Hospital, Athens/GREECE

Case History: A 56-year-old Caucasian woman, with BMI $>30$, presented to our Emergency department with a 48-hour history of RIF pain associated with mild fever and diarrhea. The pain was constant but become worse during the last 8 hours.

Clinical Findings: The abdominal examination revealed rebound tenderness in her RLQ.

Investigation/Results: Blood investigations showed a haemoglobin level of $13.6 \mathrm{~g} / \mathrm{dl}$, total white blood cell count of 17.0 with neutrophilia of 14.1, normal electrolytes and slightly elevated liver function tests. An abdominal CT scan was performed that illustrated a large mass measuring $5,8 \times 4.3 \times 3,9 \mathrm{~cm}$ in the RIF, involving the cecum, ileum, the ileocolic valve and probably the appendix.

Diagnosis: The provisional diagnosis was an inflamatory mass, probably appendicitis. 
Therapy and Progression: The patient underwent a midline laparotomy. Intraoperative findings matched the CT imaging. The mass in the RIF involved a perforated cecal diverticulum in the anterior wall of the cecum, with a $6 \mathrm{~cm}$ external circumference. The appendix was normal and not included in the abscess. There were also enlarged mesenteric lymph nodes. A right hemicolectomy was performed. The patient's post-operative course was uneventful and she was discharged on the 6 th postoperative day.

Comments: The cecal diverticulum is a quite rare occurrence, with an an incidence of 1/50 to 1/300 during an appendicectomy. It has a higher prevalence in Eastern societies. In most cases it is misdiagnosed as an appendicitis. A relative prolonged period of RIF pain and absence of nausea and vomiting may alarm the clinician towards a different diagnosis. Surgical management depends on the specific intra-operating findings.

References: 1. Chiu PW, Lam CY, Chow TL, Kwok SP: Conservative approach is feasible in the management of acute diverticulitis of the right colon. Aust NZ J Surg 2001, 71:634-636. 2. Papapolychroniadis C, Kaimakis D, Fotiadis P, Karamanlis E, Stefopoulou M, Kouskauras K, Dimitriadis A, Harlaftis N:Perforated diverticulum of the caecum: A difficult preoperative diagnosis. Report of two cases and review of the literature. Tech Coloproctol 2004, 8:S116-S118. 3. Kurer MA: Solitary caecal diverticulitis as an unusual cause of right iliac fossa mass: case report. J Medical Case Reports 2007, 1:132. 4. Conolly D, McGookin R, Gidwai A, Brown MG: Inflamed solitary caecal diverticulum- it is not appendicitis, what should I do? Ann R Coll Surg Engl 2006, 88:672-674. 5. L.-R. Shyung, S.-C. Lin, S.-C. Shih, C.-R. Kao, and S.-Y. Chou, "Decision making in right-sided diverticulitis, "World Journal of Gastroenterology, vol. 9, no. 3, pp. 606-608, 2003. 6. Yang HR, Huang HH, Wang YC, Hsieh CH, Chang PK, Jeng LB, Chen RJ: Management of right colon diverticulitis: A 10-year experience. World J Surg 2006, 30:1929-1934.

Disclosure: No significant relationships.

\section{PC153}

\section{STRANGULATED FEMORAL HERNIA: A DIFFERENT PRESENTATION}

D. Aveiro, S. Catarino, R. Simao, C. Casimiro

Cirurgia Geral, Centro Hospitalar Tondela-Viseu, Viseu/PORTUGAL

Case History: A 68 year old male, schizophrenic, was referred to the emergency department for exuberant inflammatory signs and necrosis of skin of the right groin for 3 days. He stated that it all had started 15 days earlier with inguinal swelling, redness, mild inguinal pain and later on constipation, that he had neglected.

Clinical Findings: At examination the patient presented a necrotizing infection of the right inguinal area with drainage of faecal matter and cellulitis, which extended to the right flank and thigh. He had no abdominal pain.

Investigation/Results: Laboratory studies showed leukocytosis (14.600) and C - reactive protein of 15.93. Abdominal CT - Scan indicated suspicion of perfurated hollow viscera within an inguinal hernia sac, without intra-abdominal liquid or free air.

Diagnosis: Strangulated femoral hernia with necrotizing infection and faecal fistulae
Therapy and Progression: The patient underwent exploratory laparotomy that confirmed strangulated femoral hernia, with necrosis of the bowel and fistulization to the skin, without intraperitoneal contamination. A segmental resection of ileal loop was made, with end to end anastomosis and closure of the femoral ring with suture. Surgical debridement of the inguino-femoral region right was also made. This area was subsequently closed with negative pressure therapy for one week in the ward and followed-up as outpatient with full cicatrization of the skin.

Comments: Femoral hernias, although of low incidence, are the ones that have a higher risk of imprisonment. When they are not diagnosed and subjected to rapid treatment, its complications can be lifethreatening. This case illustrates a rare manifestation, resulting from the negligence of the patient.

References: Dahlstrand U, Wollert S, Nordin P, Sandblom G, Gunnarsson U. Emergency Femoral Hernia Repair. A Study Based on a Na;onal Register. Ann Surg 2009;249:672-6

Arkoulis N, Savanis G, Simatos G. Richter type strangulated femoral hernia containing caecum and appendix masquerading as a groin abcess. J Surg Case Rep. Jun 2012;2012 (6):6

Marwah S, Marwah N, Sandhu DS, Karwasra RK. Strangulated Femoral Hernia Presen;ng as Parietal Wall Emphysema. Indian J Gastroenterol. 2005;24:218-219

Disclosure: No significant relationships.

\section{PC154}

\section{A RARE CASE OF GASTRIC PERFORATION}

M.L. Serra, A. Pimentel, T.C. Santos, R.R. Lages, C. Ribeiro, L. Sardo, J. Esteves, R. Moreira, S. Cordeiro, M. Martins, E. Oliveira, A. Vieira

General Surgery, Centro Hospitalar do Baixo Vouga, Aveiro/ PORTUGAL

Case History: Bochdalek hernias are congenital diaphragmatic hernias resulting from the failure of posterolateral diaphragmatic foramina to fuse properly in utero. Symptomatic Bochdalek hernias in adults are rare but may lead to incarcerated bowel, intraabdominal organ dysfunction or severe pulmonary disease.

Clinical Findings: 81 years old, female, presented to the Emergency Room with abdominal pain, vomits and fatigue, starting 3 days before. Physical examination revealed a distended, tympanic and tender abdomen; peritoneal irritation signs were present. The patient was hypotensive, tachycardic and oliguric. After fluid resuscitation, computed tomography was performed and showed signs of left diaphragmatic hernia and pneumoperitoneum.

Investigation/Results: The patient underwent an emergent laparotomy. Observed gastric perforation along the greater curvature, due to strangulated left Bochdalek hernia, containing great omentum, left colon, spleen, left kidney (all viable) and stomach. An atypical gastrectomy was performed, associated to closure of the diaphragmatic defect with interrupted sutures. The patient was left laparostomized and admitted to the Intensive Care Unit. The laparostomy was revisited 24 hours later: Segmental ischemia of the terminal ileum and colon was observed, which led to corresponding excision and end stomas (No anastomosis).

Diagnosis: Bochdalek Hernia 
Therapy and Progression: There was progressive improvement of the patient's clinical status, and after 3 more laparostomy revisions a definitive closure was performed. However, due to maintained needs of norepinephrine, ischemic intestinal complications were observed and the patient died on the 18th post-operative day.

Comments: Adult Bochdalek hernias are rare, but represent a wellrecognized clinical entity. Accurate diagnosis and urgent surgery when symptomatic are essential for a favorable outcome.

References: Yagmur, Yusuf, et al. "Bochdalek hernia: A rare case report of adult age." Annals of Medicine and Surgery 5 (2016): 72-75. Disclosure: No significant relationships.

\section{PC155}

\section{GALLBLADDER VOLVULUS MANAGEMENT: A RARE CASE PRESENTATION}

\section{A. Glagolieva $^{1}$, E.B. Navarro ${ }^{2}$}

${ }^{1}$ Surgery And Vascular Surgery, P.L. Shupyk National Medical Academy of Postgraduate Education, KYIV/UKRAINE, ${ }^{2}$ General Surgery, Hospital Quironsalud-Sur, Madrid/SPAIN

Case History: A female patient aged 85 years with a history of atrial fibrillation and sigmoid volvulus was admitted to the emergency department complaining of pain in the epigastrium with irradiation to the right hypochondrium, accompanied by nausea and vomiting.

Clinical Findings: Physical examination revealed tenderness in the right hypochondrium with signs of peritoneal irritation.

Investigation/Results: Laboratory analyses showed discrete leukocytosis and the abdominal sonography demonstrated severely dilated gallbladder and its edematous wall with no signs of gallstones.

Diagnosis: Considering the symptoms of acute abdomen caused by acute complicated cholecystitis, an urgent exploratory laparoscopy was performed. A gallbladder volvulus with an ischemic-necrotic appearance of the gallbladder was observed.

Therapy and Progression: A decompression and devolvulation by more than $180^{\circ}$ was performed followed by standard cholecystectomy. The postoperative period was unremarkable and the patient was discharged on the postoperative day 3 . The pathology report described acute ischemic acalculous cholecystitis.

Comments: Discussion Volvulus or acute gallbladder torsion is a rare pathology; it develops more frequently in elderly women being usually found during the explorative surgery in cases of acute abdomen with a presumptve diagnosis of acute cholecystitis. It is defined as a rotation of the gallbladder about its mesentery along the axis formed by the cystic duct and the cystic artery. Unlike standard procedure for acute cholecystitis, optimal surgical treatment pathway in the above case should involve decompression, devolvulation and obtainment of critical view of safety during the sunsequent cholecystectomy.

References: 1. Boonstra EA, van Etten B, Prins TR, Sieders E, van Leeuwen BL. Torsion of the Gallbladder. J Gastrointest Surg. 2012;16 (4):882-4. 2. Kimura T, Yonekura T, Yamauchi K, Kosumi T, Sasaki T, Kamiyama M. Laparoscopic treatment of gallbladder volvulus: case report and literature review. J Laparoendosc Adv Surg Tech A 2008; 18:330-4.

Disclosure: No significant relationships.

\section{PC156}

\section{ILEO-ILEAL INTUSSUSCEPTION IN THE ADULT}

\section{A. Logrado, L. Duarte, J. Constantino, C. Casimiro}

General Surgery, Centro Hospitalar Tondela-Viseu, Viseu/ PORTUGAL

Case History: Intussusception is a process in which a segment of intestine invaginates into the adjoining intestinal lumen, causing bowel obstruction. It is a disorder most frequently found in children, and only $5 \%$ of cases occur in adulthood. Around 80 to $90 \%$ of intussusceptions in the adult are associated to organic lesions, mostly benign tumours.

Clinical Findings: The authors present the case of a 70 years-old lady, admitted on the post-op of a laparoscopic distal splenopacreatectomy for cystic tumour of the pancreas. She presented with sudden and intense abdominal pain, accompanied with nausea and vomiting. Investigation/Results: CT scan revealed extensive intussusception of the small bowel related to an $18 \mathrm{~mm}$ lesion, probably a lipoma.

Diagnosis: Ileo-ileal intussusception

Therapy and Progression: She underwent laparotomy that confirmed the ileo-ileal intussusception, without ischemia, and with a parietal lipoma as the transition point. Manual reduction of the intussusception was performed and also lipoma enucleation. Postoperative course without complications. Pathology report confirmed a lipoma.

Comments: In the adult, the diagnosis of intussusception implies generally the need for bowel resection. Besides, when the intussusception occurs on small bowel, when there is no ischemia and the cause can be identified as benign in nature, manual reduction and lesion enucleation remain a valid and safe option for theses patients. References: TRANSIENT AND NON-TRANSIENT INTUSSUSCEPTIONS OF THE LARGE BOWEL IN ADULTS: TWO CASE REPORTS

René Vobořil, Jan Fanta, Petr Bačkovský, Daniel Ehrenberger, Jana Vobořilová ACTA MEDICA (Hradec Králové) 2015; 58 (2): 66-68 Disclosure: No significant relationships.

PC157

\section{ASCENDıNG COLON CANCER AS A COLOCUTANEOUS FISTULA TO THE ABDOMiNAL WALL AT THE SITE OF FLANK AREA}

\section{N. Ozlem}

Faculty Of Medicine, General Surgery, Ahi Evran University, Kırsehir/TURKEY

Case History: 71 YO Male presented with 3 subcutaneous masses in RLQ, flank region, 3 draining fistula openings for 18 months with foulsmelling discharge, intense abdominal pain, distention, fatique, loworalintake, inability, bed ridden.

Clinical Findings: A punch biopsy from the fistulous opening revealed a mucinious carcinoma of the colon (MCC)

Investigation/Results: he operated urgently; a mass, $20 * 15 \mathrm{~cm}$ in diameter, located superiorly liver edge, inferiorly iliac bone pelvic floor, medially smv \& arteria, VCI, infiltrating psoas muscle. MCC\&mesentery, partially tumourmass has been resected, ileotransversostomi has been reconstructed. 
Diagnosis: the reasons of non curative resection were smv\&a, iliac bone, vena cava inferior infiltration doubtly, the anaesthesist could not managed hypothermia and arytmia, a R2 resection has been completed only, another operation has been planned for R0 resection.The patient was died for septic shock in poday3.

Therapy and Progression: However, direct skin invasion is rare with no defined prevalence or other epidemiologic data and even case reports are scarce. Surgical excision remains the mainstay of treatment, although clinical T4b lesions can be considered for neoadjuvant therapy. Multivisceral resection is associated with improved overall survival, being negative margins (R0)the most important prognostic factor. Mortality rate seems to be comparable or even lower than single organ resections.Even so, this approach appears to be underutilized, apparently related to several patient related factors (sex, age, localization of tumor)and even regional variation. Our patient proved to be a challenge not only because of her multiple comorbidities and advanced age but also due to this bizarre presentation. Ultimately, we decided on a more aggressive approach owing to poor quality of life after the development of the colo-cutaneous fistula.

Comments: an ACC with a colocutaneous fistula is a late presentation and rare occurence.

References: 1.Locally advanced colon cancer with cutaneous invasion: case report Tenreiro et al. BMC Res Notes (2017) 10:113 2.B. Bogdani\} et al.: Perforated Colon Cancer to Abdominal Wall, Coll. Antropol. 36 (2012) 1: 335-338

Disclosure: No significant relationships.

\section{PC158}

\section{INTESTINAL OBSTRUCTION BY AGANGLIONIC MEGACOLON IN THE ADULT}

\author{
C. Domínguez Sánchez ${ }^{1}$, J.A. Lopez Ruiz, L. Tallón-Aguilar ${ }^{2}$, \\ B.L. López Durán', A. Suárez Cabrera', B.M. De La Cuadra', \\ M. Sanchez Ramirez ${ }^{2}$, E. Perez Margallo ${ }^{I}, J$. López Pérez ${ }^{I}, F$. Oliva \\ Mompean
}

${ }^{1}$ General Surgery, University Hospital Virgen Macarena, Seville/ SPAIN, ${ }^{2}$ Emergency Surgery Unit, Virgen del Rocío Universitary Hospital, Seville/SPAIN

Case History: Hirschsprung's disease $(\mathrm{HH})$ in the adult is a rare entity, often under-diagnosed or inadvertent. We present the case of a 54-year-old male with a history of intervention by a cerebral meningioma and a bowel resection in infancy whose cause we have no information. He comes to our hospital for intermittent constipation of long evolution, with generalized abdominal pain and bloating Clinical Findings: $\mathrm{X}$-ray of the abdomen where the dilatation of the colon is objectified. Abdominal CT scan shows dilatation of sigmoid colon with signs of generalized intestinal pneumatosis.

Investigation/Results: We decided urgent surgical intervention, confirming a very dilated redundant sigma that occupies practically all of the peritoneal cavity. We decided to perform a total colectomy with ileo-rectal anastomosis.

Diagnosis: The anatomo-pathological study describes an aganglionic megacolon/Hirschsprung's disease, with a decrease in the number of ganglion cells in the myenteric and submucosal plexus.

Therapy and Progression: Favorable postoperative evolution Comments: Adult HD is a rare condition, there are a very limited number of cases described. Most cases are diagnosed during the neonatal period. The case report describes an atypical presentation of the disease. Resection of the aganglionic segment and restoration of intestinal continuity is the treatment of choice. In our case, due to the massive dilatation of the whole colon, we chose total colectomy.

References: - Qiu J-F, Shi Y-J, Hu L, Fang L, Wang H-F, Zhang M-C. Adult Hirschsprung's disease: report of four cases. International Journal of Clinical and Experimental Pathology. 2013;6 (8):16241630. - A.A. Bakari, B.M. Gali, A.G. Ibrahim, H.A. Nggada, N. Ali, D. Dogo, et al.Case report: congenital aganglionic megacolon in Nigerian adults: two case reports and review of the literatura. Niger $\mathbf{J}$ Clin Pract, 14 (2011), pp. 249-252.

Disclosure: No significant relationships.

\section{PC159}

\section{DE GARENGEOT'S HERNIA: ONE CASE REPORT}

J.C. Gamero Huamán ${ }^{1}$, J.A. López Ruíz ${ }^{2}$, B.M. De La Cuadra ${ }^{3}$, B. De Soto Cardenal $^{4}$, M. Sanchez Ramirez ${ }^{5}$, E.P. Margallo ${ }^{6}$, J. López. Pérez ${ }^{7}, F$. Oliva Mompean ${ }^{7}$

${ }^{1}$ Sevilla, Hospital Universitario Virgen Macarena, sevilla/SPAIN, ${ }^{2}$ General Surgery, HOSPITAL UNIVERSITARIO VIRGEN MACARENA, SEVILLA/SPAIN, ${ }^{3}$ General Surgery, H.U.V.Macarena, Sevilla/SPAIN, ${ }^{4}$ General Surgery, HOSPITAL UNIVERSITARIO VIRGEN MACARENA, sevilla/SPAIN, ${ }^{5}$ Cirugía De Urgencia, Hospital Universitario Virgen Macarena, Sevilla/ SPAIN, ${ }^{6}$ Cirugía General Y Del Aparato Digestivo, Hospital Universitario Virgen Macarena, Seville/SPAIN, ${ }^{7}$ Emergency Surgery Unit, Hospital Universitario Virgen Macarea, Seville/SPAIN

Case History: A 88 years old female came to the Emergency department complaining of abdominal pain and an irreducible painful mass located in right femoral area. No history or clinical evidence of bowel obstruction.

Clinical Findings: An irreducible painful mass located in right femoral area, accompanied with erythema. No peritonitis symptoms. Investigation/Results: Laboratory test showed WBC of $7200 \mu \mathrm{L}$ and CRP of $140 \mathrm{mg} / \mathrm{dL}$. Basic metabolic panel within normal values.

Diagnosis: De Garengeot's Hernia incarcerated

Therapy and Progression: The patient underwent emergency open surgery. An incision on the hernia sac was performed and after dissection, it showed a cecal appendix without clinical signs of appendicitis. Prophylactic appendectomy and inguinal repair of the femoral were made. Lichtenstein hernia technique was performed. On the immediate postoperative, the patient presented a seroma without further complication. She was discharge on the second post-operative day.

Comments: De Garengeot hernia is very uncommon pathology and usually found during surgery. There is no established standard treatment. However, the literature reports that appendectomy should be done when there are signs of infection. It not clear if this is mandatory if no signs of infection are found. Moreover the hernia repair could be a simple suture herniorrhaphy if signs of infections are presented or mesh hernioplasty if they do not. De Garengeot hernia is a rare occurrence that it is usually diagnosed during surgery. The surgical management will depend on a currently signs of infection that will condition the mesh placement.

References: Hussain, A; Slesser, A; et al. A De Garengeot hernia masquerading as a strangulated femoral hernia. 2014. Inter. J. Surg. Case Reports. 656-658. Gonzalez Alcolea, N; Martinez Arrieta, F; et al. De Garengeot's hernia: incarcerated femoral hernia containing the vermiform appendix. Report of two cases and literature review. 2017. Cir Esp. 95. 170-178.

Disclosure: No significant relationships. 


\section{SKELETAL TRAUMA CASES}

\section{PC160}

\section{BOWEL ENTRAPMENT WITHIN FRAGMENTS OF A CRESCENT FRACTURE}

A. Konstas ${ }^{1}$, D. Klapsakis ${ }^{2}$,D. Kitridis ${ }^{3}$, M. Savvidis $^{3}$

${ }^{1}$ Orthopaedic, Nikaia General Hospital, Piraeus/GREECE, ${ }^{2}$ General Surgery, Nikaia General Hospital, Piraeus/GREECE, ${ }^{3}$ Orthopaedic, 424 General Military Hospital, Thessaloniki/GREECE

Case History: A 67-year-old male was involved in a car accident and transferred to the Emergency Unit conscious and hemodynamically stable.

Clinical Findings: Shortening and medial rotation of the left leg, and clinical signs of injury of the left L5 nerve root. The rectal examination revealed blood. We applied a pelvic binder without tension. Investigation/Results: The whole-body CT scan with contrast showed fracture-dislocation both to the pubic symphysis and to the left sacroiliac joint. The sigmoid was entrapped within the fragments of the crescent fracture, and a small amount of free air was apparent. Diagnosis: $\mathrm{C} 1.2$ fracture-dislocation of the left hemipelvis, with sigmoid entrapment and rupture.

Therapy and Progression: An exploratory laparotomy and a diverted colostomy were performed. We re-applied the pelvic binder with light pressure. At the fourth day, we performed internal fixation of the ilium with two $4.5 \mathrm{~mm}$ LCP plates and fixation of the pubic symphysis with a $3.5 \mathrm{~mm}$ reconstruction plate, through an ilioinguinal approach. The degloving injuries to the lower lumbar and the gluteus region bilaterally were addressed with debridement, drains and deep suturing. The patient remained to the ICU for a week and was discharged after ten days. He was mobilized in sitting position for six weeks and started partial weight-bearing for six more weeks. The colostomy was repaired after three months, with no complications.

Comments: Bowel entrapment within a pelvic fracture site is a rare complication, which can remain undiagnosed and follow a severe course. Therefore, a high index of suspicion and effective treatment are crucial in patients with such fractures.

References: Kekez T, Augustin G. Small bowel entrapment associated with pelvic fracture: a case report with review of the literature and differentiation of clinical picture. Eur J Emerg Med. 2012 Feb;19 (1):60-1 Stubbart JR, Merkley M. Bowel entrapment within pelvic fractures: a case report and review of the literature. J Orthop Trauma 1999; 13:145-148. Crowther A, McMaster J, Abercrombie J, Hahn D: Sacral fracture associated with small bowel entrapment: A case report. J Orthop Trauma 2006, 20:580-3. Levine J, Crampton R: Major abdominal injuries associated with pelvic fractures. Surg Gynecol Obstet 1963, 116:223-6. Charnley GJ, Dorrell JH. Small bowel entrapment in an iliac wing fracture. Injury 1993; 24:627-628. Disclosure: No significant relationships.

\section{PC161}

A RARE COMBINATION OF ELBOW DISLOCATION WITH IPSILATERAL DIAPHYSEAL HUMERAL AND FOREARM FRACTURE

B. Puha, B. Veliceasa, O. Alexa, A. Filip

Orthopaedic And Traumatology, University of Medicine and Pharmacy Gr.T.Popa - Iasi, Iasi/ROMANIA
Case History: The case describes a 64 year-old patient who is transported to the emergency room after a trauma falling from moving wagon.

Clinical Findings: Pain, local deformity, bruise, located at the left upper limb with increased pain, edema and loss of mobility to the left elbow with normal neurovascular status.

Investigation/Results: Arm, elbow, forearm X-Ray: Humeral shaft fracture, diaphyseal fracture of both forearm bones and elbow dislocation

Diagnosis: Humeral fracture type 12-A3 Forearm shaft fracture type 22-A3 Posterolateral elbow dislocation

Therapy and Progression: We started with the humeral shaft fracture and preformed a close reduction and anterograde nailing. Next we fixed the forearm by ORIF with 2 DCP plates. Finally when both the forearm and humeral fractures were stable, we reduced the elbow dislocation by usual (classical) reduction maneuver and a light splint will was applied at 90 degrees flexion.

Comments: A literature search on this injury pattern showed it to be quite rare. A number of cases describing an associated proximal or distal forearm fracture with an elbow dislocation have been published (1). All of these reported dislocations are either posterolateral or posterior and are managed by closed reduction of the elbow and open reduction and internal fixation of the forearm fractures (2). Elbow dislocation with ipsilateral radial and ulnar shaft fractures is rare and an associated humeral shaft fracture has not been reported previously. Different from all other cases was our approach of the injury by the fact that we first fixed the fractures and finally reduced the elbow witch was stable and maintain stable after surgery.

References: 1. S.F. Viegas, W. Gogan, S. RileyFloating dislocated elbow: case report and review of the literatureJ Trauma, 29 (1989), pp. 886-888 2. Goni V, Behera P, Meena UK, raj Gopinathan N, Akkina N, Arjun RH. Elbow dislocation with ipsilateral diaphyseal forearm bone fracture: A rare injury report with literature review. Chinese Journal of Traumatology. 2015 Apr 30;18 (2):113-5.

Disclosure: No significant relationships.

\section{PC162}

\section{COMBINED ANTERIOR AND POSTERIOR APPROACHES FOR THE TREATMENT OF TIBIAL PLATEAU FRACTURES}

\section{Fujita}

Orthopedic Surgery, Toyohashi Municipal Hospital, Toyohashi, Aichi/JAPAN

Case History: Between January 2011 and January 2017, Eleven patients ( 9 males, 2 females) were surgically treated using combined anterior and posterior approaches to the tibial plateau fracture with fracture of posterior column and/or posterior cruciate ligament attachment. Patient mean age at the time of operation was 46 years. According to Schatzker classification, there were 1 case of type I, 2 cases of type II, 2 cases of type IV, 3 cases of type V and 3 cases of type VI.

Clinical Findings: Severe tibial plateau fracture with posterior instability of proximal tibial fracture requires combined anterior and posterior reduction and fixation. We investigated combined anterior and posterior approaches for the treatment of tibial plateau fractures. Investigation/Results: The mean follow-up period was 25 months (range 7-60). Average range of knee flexion angle was 129 degrees (range 100-145). All fractures healed. The mean Rasmussen functional grading score was 26 (range 21-30): excellent in 5 patients, good in 5, and fair in 1 . The mean Rasmussen anatomical grading 
score was 15 (range 12-18): excellent in 4 patients, good in 5, and fair in 2. One patient sustained suppurative osteomyelitis and had healed by removal of all implants, debridement and Masquelet technique.

Diagnosis: ALL fractures were diagnosed severe tibial plateau fracture with posterior instability of proximal tibial fracture.

Therapy and Progression: Combined anterior and posterior approaches for the treatment of severe tibial plateau fracture with posterior instability is useful and effective.

Comments: Rigid fixation of anterior and posterior aspect of proximal tibial fracture obtains a good result.

References: Schattzker J, et al., The tibial plateau fracture. The Toronto experience 1968-1975. Clin Ortop Relat Res, 138: 94-104, 1979. Rasmussen PS, et al., Tibial Condylar Fractures. Impairment of knee joint stability as an indication for surgical treatment. J Bone Joint Surg., 55A, 1331-1350, 1973.

Disclosure: No significant relationships.

\section{PC163}

\section{PRIMARY ELBOW TEP AFTER COMMINUTION FRACTURE OF DISTAL HUMERUS IN AN ELDERLY PATIENT}

\section{$\underline{J . K o s}^{1}$, R. Beden ${ }^{2}$}

${ }^{1}$ Department For Traumatology, University cinical center Ljubljana, Ljubljana/SLOVENIA, ${ }^{2}$ Department For Traumatology, University clinical center Ljubljana, Ljubljana/SLOVENIA

Case History: 75-year old healthy right-handed woman fell on ice and hurt her left arm.

Clinical Findings: At presentation she had a swollen left elbow with no nevro-vascular deficiency. With X-ray imaging we diagnosed a multyfragmentary distal humerus fracture that was later more precisely visualised with a CT imaging.

Investigation/Results: The decision was made to go for a CoonradMorrey total elbow arthroplasty This kind of operation was done only a couple of times before in our hospital, but the surgeon had a lot of experience with elbow trauma osteosintesis.

Diagnosis: AO-13 C 3.3

Therapy and Progression: After waiting 8 days, for the swelling to subside, the operation was carried out with deperiostation of triceps from olecranon. It went uneventully, ulnar nerve was protected. Aftercare consisted of analgesia, cryotherapy and rehabilitation passive/active motion. Patient was discharged 12 days after operation. At the time of discharge ROM was $10^{\circ}-110^{\circ}$. During the next checkups, she briefly complained of some tingling sensations in the 5 th finger that later vent away. After 1 month we allowed $1 \mathrm{~kg}$ weight bearing, and 3 months after operation $5 \mathrm{~kg}$. In the beginning the patient had problems with muscle strength of fingers, which got better after a while. 6 months after operation the patient had flexion/extension $15^{\circ}-135^{\circ}$ with full pro/supination. She had no problems or pain with daily activities and there was no sign of prosthesis loosening.

Comments: Even if not done routinely, elbow TEP in elderly patients with low demands is a good way to achieve quick and satisfying results after complicated distal humerus fracture with poor bone stock.

References:

Disclosure: No significant relationships.

\section{PC164}

\section{CATASTROPHIC FINGERS: 6-MONTHS FOLLOW UP OF TWO CASES TREATED WITH A VARIATION OF DYNAMIC EXTERNAL FIXATOR}

\author{
F. Teodonno, P. Vadillo Cardona, P. Crespo Lastras, H. Garlito \\ Díaz, R. Serrano Serrano, D. López Dorado, A. Abad Arevalillo, \\ J.C. Marquez Ambite
}

Cirugia Ortopedica Y Traumatologia, Hospital Universitario Infanta Elena, Valdemoro/SPAIN

Case History: Two cases of catastrophic fingers are presented: A 34-year-old man with a semiamputation of the second finger of the non-dominant hand and a 56-year-old man with a crush injury of the second finger of the dominant hand.

Clinical Findings: Case 1: A complex wound is observed, concerning severe tendon injury and lesion of the radial neurovascular bundle. Case 2: A complex wound with tendon and neurovascular injury is observed.

Investigation/Results: Case 1: Hand X-ray: Intraarticular conminuted fracture of F2 associated to a luxation of the PIP joint. Case 2: Hand X-ray: Comminuted fracture of F2.

Diagnosis: Case 1: Intraarticular conminuted fracture-dislocation of the middle phalanx Gustilo type IIIC. Case 2: Comminuted fracture of the middle phalanx Gustilo type IIIC.

Therapy and Progression: Case 1: Four K wires are passed through the cap of a needle and an external fixation is performed. An interfragmentary $\mathrm{K}$ wire is passed through the fracture. At six weeks' review, the external fixator is removed. ROM of $0-70^{\circ}$ of the PIP joint and ROM of $0-60^{\circ}$ of the DIP joint is observed. Case 2: A fixation of the fracture with four $\mathrm{K}$ wires assembled as an external fixator is realized. At six weeks' review, the external fixator is removed. ROM of $0-60^{\circ}$ of the PIP joint and ROM of $5-30^{\circ}$ of the DIP joint is observed.

Comments: The external fixator proposed is efficient, easy to build and cost-effective. It allows the reduction of the fracture while preserving the mobilization of the finger. It is a good treatment for catastrophic fingers.

References: • Crowley et al, A Novel External Fixator Used to Treat Open Comminuted Fractures of the Middle and Distal Phalanges With Concomitant FDP Avulsion Injury, Techniques in Hand \& Upper Extremity Surgery 2014 Volume 18, Number 3. • Liodaki et al, Management of difficult intra-articular fractures or fracture dislocations of the proximal interphalangeal joint. The Journal of Hand Surgery (European Volume) 2015, Vol. 40E (1) 16-23. • Faheem Khadim et al, A simple dynamic external fixator for complex phalangeal fractures, Journal of Plastic Surgery And Hand Surgery 2013, Vol 47, Iss. 2. • Benjamin Kapur et al, An Alternative Technique for External Fixation of Traumatic Intra-articular Fractures of Proximal and Middle Phalanx, Techniques in Hand \& Upper Extremity Surgery 2015, Volume 19, Number 4. • Walter FL, Papandrea RF. A mini external fixator for hand and finger fractures constructed from readily available materials. Tech Hand Up Extrem Surg. 2011;15:215-218. • Dailiana Z, Agorastakis D, Varitimidis S, Bargiotas K, Roidis N, Malizos KN. Use of a mini-external fixator for the treatment of hand fractures. J Hand Surg 2009;34A:630-6. • Hynes MC, Giddins GEB. Dynamic external fixation for pilon fractures of the interphalangeal joints. J Hand Surg Br. 2001, 26: 122-4. • Suzuki Y, Matsunaga T, Sato $\mathrm{S}$, et al. The pins and rubbers traction system for treatment of comminuted intra-articular fractures and fracture-dislocations in the hand. J Hand Surg [Br]. 1994;19:98-107.

Disclosure: No significant relationships. 


\section{PC165}

\section{"SHOWING OFF MAY LEAD TO TEARING OFF... A COMPLETE TRICEPS RUPTURE, A RARE TENDON INJURY"}

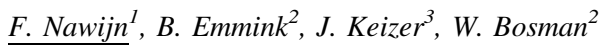

${ }^{1}$ Surgery, University Medical Center Utrecht, Utrecht/ NETHERLANDS, ${ }^{2}$ Trauma Surgery, St Antonius Hospital, Nieuwegein/NETHERLANDS, ${ }^{3}$ Trauma Surgery, St. Antonius Hospital, Nieuwegein/NETHERLANDS

Case History: A 48-year-old fitness athlete with no use of anabolic steroids, presented to the emergency department after fall from unstable chair while performing a one arm push-up.

Clinical Findings: Physical examination revealed pain and swelling of the posterior aspect of the right arm just above the elbow. In addition a palpable painful gap over the distal triceps tendon was observed with an inability to extend the elbow against gravity.

Investigation/Results: A radiograph of the elbow showed no fracture. Diagnosis: Complete triceps tendon rupture.

Therapy and Progression: The patient was surgical treated within six days of trauma. The Krackow suture technique was used to fixate the distal triceps tendon to the olecranon, with the arm in full extension. Drill holes in the proximal ulna were made through which fibrewire could be tunneled. Postoperatively the patient was treated with a brace, increasing the elbow flexion gradually during the first eight weeks. Three months after surgery $150^{\circ}$ flexion was possible with a 5 loss of extension. The Disabilities of the Arm, Shoulder and Hand (DASH) score, at three months follow-up was 10.5.

Comments: Only $1 \%$ of all tendon injuries affect the triceps tendon, making triceps ruptures very rare, with a high incidence in middleaged man and athletes. ${ }^{1,2}$ The mechanism of injury is most often sudden deceleration force upon an contracted triceps muscle. ${ }^{3,4}$ An acute rupture is easily missed due to a low degree of suspicion. ${ }^{3} \mathrm{We}$ demonstrated that acute triceps tendon rupture treated with Krackow suture technique results in an excellent functional outcome at three month follow-up.

References: 1. Morrey BF, Sanchez-Sotelo J, Morrey ME. Morrey's the Elbow and its Disorders. 5th ed. Philadelphia: Elsevier; 20182. Shuttlewood K, Beazley J, Smith CD. Distal triceps injuries (including snapping triceps): A systematic review of the literature. World Journal of Orthopedics 2017 June 18; 8 (6): 507-513 3. Sharma SC, Singh R, et al. Missed diagnosis of triceps tendon rupture: a case report and review of literature. Journal of Orthopaedic Surgery 2005; 13 (3):307309 4. Jaiswal A, Kacchap ND, et al. Rupture of the triceps tendon - a case series. Chinese Journal of Traumatology 2016; 19:235-238 Disclosure: No significant relationships.

\section{PC166}

INTRAOPERATIVE INJURY OF A SINGLE VESSEL ARTERIAL BLOOD SUPPLY IN THE FOOT (PERONEA ARTERIA MAGNA VARIATION) IN A PATIENT WITH TRIMALLEOLAR FRACTURE

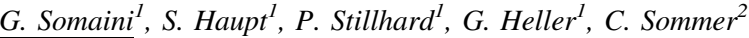

\footnotetext{
${ }^{1}$ Surgery, Kantonsspital Graubünden, Chur/SWITZERLAND,

${ }^{2}$ Surgery, Trauma Unit, Kantonsspital Graubünden, Chur/

SWITZERLAND
}

Case History: The dorsolateral approach has become popular for osteosynthesis of malleolar fractures. because of some advantages like having an excellent view to the fibula and Volkmann triangle at the same time. But there are anatomical variations in arterial blood supply to the leg, which have to be known.

Clinical Findings: A 60-year-old patient came to our emergencyroom with a trimalleolar fracture. We decided to stabilize the fracture immediately with an anti-glide-plate in the Volkmann fragment, lag screw and neutralization plate in the lateral malleolus via dorsolateral approach, and a tension-band-wiring in the medial malleolus.

Investigation/Results: A few hours after surgery, the right foot was pale, pulseless and in extreme pain. CT- angiography showed an injury of the distal peroneal artery combined with constitutional variation of a peroneal arteria magna as the only blood supply of the right foot.

Diagnosis: Intraoperative injury of a peronea arteria magna variation in a patient with trimalleolar fracture

Therapy and Progression: The artery was repaired instantly. Soft tissue conditioning followed during several weeks. 8 months postoperatively implant removal was performed. The patient regained painless function of her foot and ankle joint.

Comments: Anatomic variations have been described in about $6 \%$ of the population. A peroneal arteria magna as the only vessel supplying the foot has been reported in $0.2-8.3 \%$. An injury of an artery of the lower leg has little relevance with normal anatomy. In our case careful clinical and immediate radiologic examination led to an early diagnosis of the above mentioned anomaly.

References: Kim, D : Surgical significance of popliteal arterial variants. A unified angiographic classification. - Annals of surgery 1989;210 (6):776-81

Disclosure: No significant relationships.

\section{PC167}

\section{ADULT TYPE I MONTEGGIA FRACTURE DISLOCATION AND ASSOCIATED OLECRANON FRACTURE - A CASE REPORT}

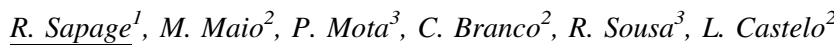

${ }^{1}$ Serviço De Ortopedia E Traumatologia, Centro Hospitalar de Trásos-Montes e Alto Douro, Vila Real/PORTUGAL, ${ }^{2}$ Ortopedia E Traumatologia, Centro Hospitalar Trás os Montes e Alto Douro, Vila Real/PORTUGAL, ${ }^{3}$ Orthopedics And Traumatology, Centro Hospitalar Trás os Montes e Alto Douro, Vila Real/PORTUGAL

Case History: We present a 39-year-old female who suffered a motor vehicle accident with projection.

Clinical Findings: She presented with pain, swelling and evident deformity at the elbow joint. No neurological injury was evident.

Investigation/Results: Radiographs

Diagnosis: She was diagnosed with a closed type I Monteggia fracture dislocation and associated olecranon fracture.

Therapy and Progression: She was promptly taken to surgery where we perform an open reduction and internal fixation of ulna shaft fracture using a $3.5 \mathrm{~mm}$ DCP plate and internal fixation of olecranon with tension band. Radial head dislocation was then reduced in closed manner and elbow stability was achieved. It was maintained an above-the-elbow splinting for 3 weeks, patient then began progressive passive and active motion of range exercises. For prominent and symptomatic hardware referred to olecranon, we did its removal after 8 months. At 1.5 years follow-up, patient is asymptomatic with $10^{\circ}$ extension deficit. 
Comments: Monteggia lesion is a recognized serious injury defined as fracture at any segment of the ulna associated to radial head dislocation. It is rare in adults. It may be part of a complex injury pattern with other associated lesions as olecranon fracture. According to Bado classification, type I includes an anterior radial head dislocation and is the most common in children and yound adults. Optimal treatment of Monteggia fractures involves recognition of the injury pattern and elaboration of a surgical plan to address all its components. Anatomic stable fixation must be achieved in order to optimize outcomes. Complications are common and may be related to the injury spectrum itself and to inadequate fixation.

References: Adult Monteggia and Olecranon Fracture Dislocations of the Elbow. Hand clinics. 2015;31 (4):565-80.

Disclosure: No significant relationships.

\section{PC168}

\section{FOUR PART TIBIAL FRACTURE AFTER MOTOR-VEHICLE} ACCIDENT

\section{J. Cuarental García, G. Luengo Alonso, V. Rodríguez Vega}

Cirugía Ortopédica Y Traumatología, Hospital 12 de Octubre, Madrid/SPAIN

Case History: 49 year-old male, brought to our center after a high energy mechanism. During initial evaluation he was hemodynamically stable, and Glasgow scale was 14 points.

Clinical Findings: $\mathrm{He}$ presented facial trauma and right leg deformity.

Investigation/Results: Initial $\mathrm{x}$-rays showed no chest or pelvic ring injuries. Limb X-ray showed several fractures. Using CT scan, isolated left ischiopubian fracture was diagnosed.

Diagnosis: Four part diaphyseal tibia fracture (42-C1). Right distal femur fracture (32-B3). Non-displaced proximal ulna affecting left upper extremity (22-A1), associated to scaphoid fracture (Herbert B2). And finally, Gustilo I opened patella fracture.

Therapy and Progression: Initially, an intramedullary nail was implanted to his right femur and external fixation was implanted to treat right tibia fracture due to its complexity, apart from washing and suturing left knee wounds. Two days later, the external fixation was removed and another intramedullary nail was implanted in tibia through a suprapatelar approach. Later on, ulna fracture was osteosynthesied (LCP plate and a lag screw) and scaphoid was treated with a headless screw. After 7 months he is asymptomatic with no pain, and good range of motion (knee: $0-110^{\circ}$, ankle $30^{\circ}$ flexion- $10^{\circ}$ extension). By that time, $\mathrm{x}$-rays images showed fracture consolidation of all fractures

Comments: Initial treatment in a four part diaphyseal tibia fracture should be external fixation (early appropriate care strategy). Definitive treatment should be nailing if possible, due to blood supply in this kind of fractures should be carefully manage. Different studies report good long-term results in function although knee pain has been reported as result of the suprapatellar approach.

References:

Disclosure: No significant relationships.

\section{PC169}

\section{BILATERAL QUADRICEPS TENDON RUPTURE IN A HEALTHY MAN, AFTER LOW ENERGETIC TRAUMA. A CASE REPORT}

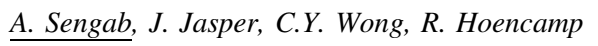

Surgery, Alrijne Ziekenhuis, Leiderdorp/NETHERLANDS

Case History: We present the case of an 82-year-old healthy male with bilateral quadriceps tendon rupture (BQTR) without any predisposing risk factors.

Clinical Findings: The patient presented 2 days in a row at the ED. The first day, after a bike vs MVA, with pain when palpating along the left femur. Plain radiographs of femur and knee showed no fractures and patella in normal position. The patient was discharged with crutches but tripped at a doorstep and returned the next day unable to bear weight on both legs. Examination showed bilateral suprapatellar gaps and inability to raise the extended knees.

Investigation/Results: As diagnosis was clear on physical examination alone, no further investigations were necessary.

Diagnosis: Bilateral quadriceps tendon rupture

Therapy and Progression: The patient was admitted for surgical repair by patellar drill holes and transosseous bunnel sutures. Postoperative treatment consisted of a leg extension splint ( 2 weeks) and hinged knee brace ( 6 weeks) until 90 degrees flexion in both knees was possible. At final follow-up the patient had good function and muscle strength in both knees.

Comments: BQTRs are rare and predominantly occur in patients with predisposing risk factors such as chronic renal failure, corticosteroid use and diabetes mellitus. Only 9 similar cases have been reported in literature. The typical triad of symptoms suggestive of QTRs are inability to actively raise the extended knee, a suprapatellar gap and painful swelling which is present in almost $60 \%$ of patients. Early, surgical repair of complete QTRs lead to the best functional outcome, and must be considered as a possible diagnosis in presence of the triad. References:

Disclosure: No significant relationships.

\section{PC170}

\section{OPEN SUPRA AND INTERCONDYLAR FEMUR FRACTURE} WITH BONE LOSS - LOOSING THE RACE

\section{F.S. Alves, T. Aguiar, J. Castro, J. Cabral, J. Pedro}

Ortopaedics And Traumatology, Centro Hospitalar Lisboa Norte Hospital de Santa Maria, Lisbon/PORTUGAL

Case History: A 46 year old male patient presented with an open fracture type 3A Gustillo-Anderson of the distal femur with bone loss after falling into an elevator shaft.

Clinical Findings: The paitent had no motor, vascular or nervous lesion.

Investigation/Results: Open distal femur fractures with bone loss are rare and complex injuries that usually are produced by high impact trauma. Bone cominution and soft tissue associated lesions frequently lead to complications and poor treatment outcomes. Every internally stabilized fracture represents a race between fractures healing and implant failure. 
Diagnosis: Computer tomography (TC-scan) demonstrated an highly cominuted fracture classified as a $\mathrm{AO} 33-\mathrm{C} 3$

Therapy and Progression: After initial general trauma evaluation, open fracture antibiotic protocol was initiated and surgical debridement and external transarticular fixation was ensued. Following softtissue recovery at two weeks, the patient fracture was fixed with a long distal femur LISS implant. Non radiographic union and implant failure was identified one year post operatively. The patient was reoperated: plate removal, fracture focus debridement and reosteosynthesis with an external LCP plate and an internal LC-DCP plate and bone graft from the contralateral femur.

Comments: There is a paucity of studies defining the best treatment choice for these rare and complex fracture involving the knee. Although lateral or medial plate and screws are frequently refered as the standard of treatment, with this case report we demonstrate that a locked plate implant with minimally invasive insertion does not guarantee successful fracture repair. The multiplicity of injury patterns, bone quality, mechanic and biologic factors interplay in each case determining who wins the race.

References: Henderson CE, Kuhl L, Fitzpatrick D, et al. Locking Plates for Distal Femur Fractures: Is there a Problem with Fracture Healing. J Orthop Trauma 2011;25:S8-S14

Kayali C, Agus H, Turgut A. Successful results of minimally invasive surgery for comminuted supracondylar femoral fractures with LISS: comparative study of multiply injured and isolated femoral fractures. J Orthop Sci. 2007;12:458-465.

Wong MK, Leung F, Chow SP. Treatment of distal femoral fractures in the elderly using a less invasive plating technique. Int Orthop. 2005:29: 117-120.

Disclosure: No significant relationships.

\section{PC171}

\section{SUCCESSFUL REMOVAL OF DISTAL PART OF BROKEN INTRAMEDULLARY FEMORAL NAIL WITH CLOSED TECHNIQUE}

\author{
R. Sapage, M. Maio, P. Mota, C. Branco, R. Sousa, B. Barbosa
}

Serviço De Ortopedia E Traumatologia, Centro Hospitalar de Trás-osMontes e Alto Douro, Vila Real/PORTUGAL

Case History: We present a 79-year-old male who sustained a comminuted subtrochanteric femoral fracture following a fall. He was first treated with routine closed reduction and fixation with a short cephalomedullary nail. We unsuccessful tried to drill one of the distal holes, possibly creating local nail fragility.

Clinical Findings: One month later he was admitted to our care for persistent atraumatic hip pain and lower limb functional impairment. Investigation/Results: He was found to have a broken nail implant distal to the femoral fracture site.

Diagnosis: Broken intramedullary femoral nail

Therapy and Progression: The patient was submitted to nail exchange and was positioned supine on a traction table. Proximal blade, distal screw and proximal nail fragment were removed with ease utilizing the nail extraction set. A long antegrade guide wire serving as a hook was passed through the length of the remaining nail. After several attempts, we successfully extract it. At 3 months followup, the patient is asymptomatic walking with external support.

Comments: Proximal femur fracture is a common fracture among elderly. These are routinely treated with cephalomedullary nailing. With their increasing use for complex fractures, rate of nail breakage increases. Its removal, especially a retained distal fragment, is a challenging procedure. Various techniques have been described with satisfactory outcomes. Some procedures for removal of broken distal femoral nails can cause damage to surrounding bone and soft tissue and even to knee joint. As we report, closed techniques can be technically demanding. Even so, this method of nail extraction should be attempted as a primary procedure associated with less surgical aggression and morbidity.

References:

Disclosure: No significant relationships.

\section{PC172}

\section{ANGRY BULL GIVES YOU WINGS - LUXATIO ERECTA}

I. Jurčević, J. Kolodziej, D. Kikić, V. Gajski

Surgery, General Hospital, Nova Gradiška/CROATIA

Case History: Luxatio erecta, an uncommon form of shoulder dislocation $(0.5-1 \%)$, is an inferior displacement of the humeral head with loss of

articulation with the glenoid fossa caused by severe arm hyperabduction. 24year old man presented in Emergency department after being injured in a bull attack, in which he was thrown against a fence. Clinical Findings: Clical examination has shown both arm "locked" in abduction. Paraesthesia of both upper extremity was reported. There were no circulatory impairments.

Investigation/Results: Radiological examination has shown a bilateral inferior dislocation of the humeral head and greater tuberosity fractures. Electroneurography has shown bilateral brachial plexus injury.

Diagnosis: Bilateral inferior glenohumeral dislocation associated with greater tuberosity fractures and brachial plexus injury

Therapy and Progression: Immediate closed reduction and immobilisation under intravenous anesthesia was performed. In order to facilitate the implementation of physical therapy, open reduction and fixation of a greater tuberosity of right humerus with two titanium screws has been performed. Two weeks after surgery, physical therapy treatment was initiated. Five months after physical rehabilitation the functional result of both shoulder was excellent.

Comments: Special attention should be paid to identifying possible bone, rotator cuff, neurological and vascular lesions which can complicate the patient's recovery.

References: 1. Camarda L, Martorana U, D'Arienzo M. A case of bilateral luxatio erecta. J Orthop Traumatol. 2009 Jun; 10 (2): 97-9. 2. Petty K, Price J, Kharasch M, Novack J. Bilateral luxatio erecta - a case report. J Emerg Med. 2014 Feb; 46 (2): 176

Disclosure: No significant relationships.

\section{PC172A}

\section{TRAUMATIC AMPUTATION BY CRUSHING}

\section{Lara Palmero, L. Ojea, S.G. Modet, D.S. Relinque}

CirugÍa General Y Del Aparato Digestivo, HOSPITAL PUNTA DE EUROPA, ALGECIRAS/SPAIN

Case History: 40-year-old male who was involved in a workplace accident in which he was run over by a crane, crushing his lower 
limbs. The lower left limb presented great loss of substance and almost total amputation of it.

Clinical Findings: Comminuted fracture of the lower left limb tibia and fibula. Leg left dislocated from the thigh and separated by $9 \mathrm{~cm}$. Complete section of left popliteal artery with aneurysmal formation. Fracture of the right ankle external malleolus.

Investigation/Results: Injuries affecting the extremities account for approximately $80 \%$ of all vascular trauma. In our country it seems that $38 \%$ affects the lower limbs and $26 \%$ the upper limbs. Regarding etiology, iatrogenic lesions predominate in our environment (38\%), due to the rise of interventional techniques (radiology and cardiac hemodynamics), followed by traffic accidents (24\%) and workplace accidents (18\%). There are other mechanisms of contusion such as crushing, elongation and deceleration.

Diagnosis: Scan images.

Therapy and Progression: Surgical treatment: supracondylar amputation of the lower left limb.

Comments: In the case of trauma with open fractures, once the patient has been confirmed to be hemodynamically stable, antibiotics and tetanus prophylaxis are administered, and the fracture is stabilized; which will help us to reduce the bleeding and perform a better exploration afterwards. In our case, the patient had almost total removal of the left leg, and according to the CT scan, a comminuted fracture in the tibia and fibula accompanied by popliteal artery section and severe soft tissue injuries, so it was decided to perform a supracondylar amputation on said member.

References: 1. Choudry U., Moran S., Karacor Z.: Soft-tissue coverage and outcome of Gustilo grade IIIB midshaft tibia fractures: A 15-year experience. Plast Reconstr Surg 122. 479-485.2008; 2. Zhang Y., Fang W., Lou C., et al: Unilateral external fixator combined with simple internal fixation for severe open tibia-fibular fracture. Zhonghua Wai Ke Za Zhi 40. 855-857.2002; 3. Muangman P., Engrav L.H., Heimbach D.M., et al: Complex wound management utilizing an artificial dermal matrix. Ann Plast Surg 57. 199-202.2006; 4. Gaspar K., Erdei I., Peter Z., et al: Role of acellular dermal matrix allograft in minimal invasive coverage of deep burn wound with bone exposed-case report and histological evaluation. Int Wound $\mathrm{J} 3$. 51-58.2006; 5. Menzoian J.O., Doyle J.E., Cantelmo N.L., et al: A comprehensive approach to extremity vascular trauma. Arch Surg 120. 801-805.1985; 6. Garfien E, Haddock N, Saadeh P, et al. Soleal perforators: recipient options in lower extremity reconstruction. Presented at Northeastern Society of Plastic Surgeons. Philadelphia, PA; October 2-5, 2008. 7. Haddock N., Garfein E., Saadeh P., et al: The lower extremity Allen Test (LEAT). J Reconstruct Microsurg 25. 2009; 8. Isenberg J.S., Sherman R.: Zone of injury: a valid concept in microvascular reconstruction of the traumatized lower limb? Ann Plast Surg 36. 270-272.1996;

Disclosure: No significant relationships.

\section{PC173}

\section{FRACTURE-RELATED INFECTION OF THE CLAVICLE AFTER OSTEOSYNTHESIS: THREE-DIMENSIONAL PLANNING AS A STANDARD FOR BONE DEFECT RECONSTRUCTION}

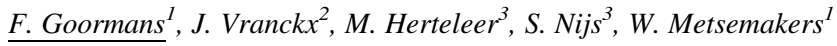

${ }^{1}$ Trauma Surgery, University Hospitals Leuven, Leuven/BELGIUM, ${ }^{2}$ Department Of Plastic And Reconstructive Surgery, University Hospitals Leuven, Leuven/BELGIUM, ${ }^{3}$ Traumatology, UZ Leuven, Leuven/BELGIUM
Case History: We present a case of a 17-year-old female patient who sustained a left clavicle fracture after a fall. After failure of the primary osteosynthesis, a revision plate osteosynthesis was performed. Spontaneous evacuation of seropurulent discharge occurred postoperatively, which was initially treated with antibiotics. After 2 weeks of continued drainage, an operative debridement was performed.

Clinical Findings: Another 2 weeks later, the patient presented to our emergency department with a wound fistula and shoulder pain during mobilization.

Investigation/Results: C-reactive protein (CRP) was $1,5 \mathrm{mg} / \mathrm{L}$ and the white blood cell (WBC) count was $9.98 \times 10^{9} / \mathrm{L}$. Plain radiography and $\mathrm{CT}$ showed a localized translucent area in the midclavicular region, indicating osteomyelitis.

Diagnosis: Irrigation and debridement were performed. This was followed by the removal of osteosynthetic material and necrotic bone. Histopathology confirmed the diagnosis of osteomyelitis. Intra-operative cultures indicated the presence of Pseudomonas.

Therapy and Progression: To reconstruct the bone defect an osteomyocutaneous fibula flap was chosen in combination with an osteosynthesis. For the exact anatomical reconstruction, three-dimensional planning by computed tomography (CT) was used. In the preoperative planning software, the models of the contralateral anatomy were mirrored and used as reconstruction template. Plain radiographs and a CT-scan obtained at 18 months postoperatively confirmed complete bony consolidation. Three-dimensional evaluation showed restoration of the anatomy of the shoulder girdle. Clinically there was no shoulder pain and the patient reported a full return to activities.

Comments: To the best of our knowledge, this is the first case published on three-dimensional planning for free fibula flap transfer for clavicle bone defects in case of fracture-related infection.

References: Hingsammer AM, Lazaros V, Dominik MC, Fürnstahl P. Three-dimensional corrective osteotomies of mal-united clavicles - is the contralateral anatomy a reliable template for reconstruction? Clin. Anat. 2015;28 (7):865-871. Kim DS, Lee DW, Jang YH, Yeom JS, Banks SA. Effects of short malunion of the clavicle on in vivo scapular kinematics. J. Shoulder Elb. Surg. 2017;26 (9):e286-e292. Melean PA, Zuniga A, Marsalli M, Fritis NA, Cook ER, Zilleruelo M, et al. Surgical treatment of displaced middle-third clavicular fractures: A prospective, randomized trial in a working compensation population. J. Shoulder Elb. Surg. 2015;24 (4):587-592. Metsemakers WJ, Kuehl R, Moriarty TF, Richards RG, Verhofstad M, Borens O, et al. Infection after fracture fixation: current surgical and microbiological concepts. Injury [epub ahead of print]. 2016. Vlachopoulos L, Schweizer A, Meyer DC, Gerber C, Fürnstahl P. Three-dimensional corrective osteotomies of complex malunited humeral fractures using patient-specific guides. J. Shoulder Elb. Surg. 2016;25 (12):2040-2047.

Disclosure: No significant relationships.

\section{PC174}

\section{OPEN PELVIC FRACTURE WITH EXTENSIVE LOWER LIMBS DEGLOVING INJURY: ATTITUDE COUNTS}

\author{
P.F. Liao, Y.J. Chen
}

Orthopedics, Far Eastern Memorial Hospital, New Taipei City/ TAIWAN

Case History: Two complex open pelvic fractures were treated in a tertiary medical center in Taiwan, with similar trauma mechanism (run over by a trunk). 
Clinical Findings: Open pelvic fracture and huge contaminated wounds from low abdomen to lower limbs with degloved skin flaps were presented at first sight.

Investigation/Results: Infection rapidly progressed and patient died within one month due to refractory pelvic sepsis in one case. The other has survived for more than 70 days, was predicted to ambulate independently in someday.

Diagnosis: Focused abdominal sonography in trauma (FAST) and whole body computed tomography (CT) were performed for initial assessment.

Therapy and Progression: In case one, colostomy was delayed for 30 hours and the open wound was wet dressed. However, imperfect sterilization led to acute infection. Poor tissue perfusion and exudate accelerated soft tissue necrosis and bone sequestration. Rotational muscle flap, hip disarticulation and hemipelvectomy was performed, but in vain and patient ended up wtih multiple organ failure. The other was treated with early colostomy and wound bed protection by full thickness skin graft harvested from devitalized skin flap. Infection was controlled yet.

Comments: Degloved skin flap made infection control more complicated. Better outcome would be achieved by early colostomy/cystostomy, vigorous debridement, fracture stabilization and aggressive wound protection to prevent wound infection, reducing exudate and tissue necrosis, and offer time for further skin reconstruction. Harvested full thickness skin graft from devitalized skin flap could be a good treatment option. We emphasize again the principle of treating open pelvic fracture with large soft tissue compromise would be early intervention and a competitive attitude.

References: 1 . Does colostomy prevent infection in open blunt pelvic fractures? A systematic review. J Trauma. 2006;60:1145-1148. 2. Current management of severe pelvic and perineal trauma. Journal of Visceral Surgery (2012) 149, e227-e238 3. Treatment Outcomes of Open Pelvic Fractures Associated with Extensive Perineal Injuries. Clinics in Orthopedic Surgery 2013;5:263-268 4. Factors of Pelvic Infection and Death in Patients with Open Pelvic Fractures and Rectal Injuries. Surg Infect (Larchmt). 2017 Aug/Sep;18 (6):711-715. 5. Management and outcome of open pelvic fractures: a retrospective study of 41 cases. Injury. 2011 Oct;42 (10):1003-7.

Disclosure: No significant relationships.

\section{PC175}

\section{TREATMENT OF SYMPTOMATIC SEVERE MAL-UNION OF SCAPULA, RIBS, AND CLAVICLE AFTER TRAUMA. A CASE REPORT}

\section{T. Malovrh, A. Fischinger}

Department Of Traumatology, University Medical Centre Ljubljana, Ljubljana/SLOVENIA

Case History: A 17-year-old right-handed male presented with unclear history. He complained of 4-month lasting right shoulder pain with severely impaired arm function, left chest pain and some difficulties in breathing. He denied any traumatic event.

Clinical Findings: There was marked asymmetry of shoulder girdle, with deformed contours of the right clavicle and scapula. Shoulder was medialised with impaired function; painfull pasive and active anteflexion and abduction of $70^{\circ}$, with almost no scapulo-thoracic movement. He could hardly reach mouth with his right hand.

Investigation/Results: X-ray and CT scan showed fracture of the right scapular body, with severe inward angulation of $75^{\circ}$ and its impingement into the deformed proximal posterolateral chest wall (duplex fractures of the right ribs 2 to 5). There were also angulated fracture of the mid-third clavicle $\left(60^{\circ}\right)$ and displaced avulsion of the coracoid process. Fractures were radiographically healed. On spirometry his forced vital capacity (FVC) was $3300 \mathrm{ml}$ ( $87 \%$ of predicted value).

Diagnosis: Displaced, mal-united fractures of the right scapula, ribs (2-5), clavicle and coracoid process

Therapy and Progression: Patient was operated through classic Judet approach. Scapula was lifted from the chest, ribs were osteotomized and fixed by locking plates. Scapula and clavicle were also osteotomized and fixed. Patient recovered well, with good radiographical and functional outcome. FVC improved to $4400 \mathrm{ml}(100 \%)$. Comments: Most scapula and ribs fractures are managed non-operatively. There is some evidence that support operations on fractured scapular neck or body with marked displacement. (1-3) We successfully operatively treated a symptomatic patient with severely displaced and mal-united combined fractures of scapular body, ribs and clavicle.

References: 1. Cole PA, Gauger EM, Schroder LK. Management of Scapular Fractures. J Am Acad Surg 2012;20:130-41. 2. Bartonicek J, Fric V. Scapular body fractures: results of operative treatment. Int Orthop 2011;35:747-53. 3. Cole PA, Talbot M, Schroder LK, Anavian J. Extra-articular Malunions of the Scapula: A Comparison of Functional Outcome Before and After Reconstruction. J Orthop Trauma 2011;25:649-56

Disclosure: No significant relationships.

\section{PC176}

THE RESULTS OF COVERSION HIP ARTHROPLASTY FOR FAILED INTERNAL FIXATION OF FEMORAL NECK FRACTURES. -THE SHORT-TERM RESULT USING CEMENTLESS COLLARED CORAIL STEM

\author{
M. Tomita, H. Sakanaka, T. Kita, K. Takayama, A. Masuda
}

Orthopaedics, Seikeikai Hospital, Sakai City/JAPAN

Case History: Internal fixation is the treatment of choice for the most of stable type of femoral neck fracture. However, it is reported a certain re-operation rate because of fixation failure, avascular necrosis of the femoral head, especially in the cases of older patients or unstable type of fracture. To rescue failed internal fixation, hip arthroplasty (THA or BHA) is considered to be "golden standard" in turn. Compared to internal fixation, hip arthroplasty has such advantage as more significant improvements in pain, early ambulation, and lower rates of re-operation. However, the complications such as newly femoral sub-trochanteric fracture or stem failure are reported in the past literatures.

Clinical Findings: We hereby report the result of consecutive 4 cases of hip arthroplasty using unique stem after failed internal fixation performed in our institution. All failed implants were retrieved and revised with cementless collared Corail stem.

Investigation/Results: The short-term follow-up (average 1year) revealed the successful clinical and radiographic outcome. No implants was loosened.

Diagnosis: Based on the results of the present report, we argue the indication of adequate type of stems, and report the satisfactory clinical and radiographic results of cementless collared Corail stem. Therapy and Progression: Use of this type of stem has a capacity to avoid complications such as sub-trochanteric or shaft fractures induced by excessive rasping or remnant holes on conversion hip arthroplasty. Comments: These cases with good clinical and radiographic results are now under follow-up, and further long-term outcome will be reported. 
References: 1) Røkkum M. (1999) Total hip replacement with an entirely hydroxyapatite-coated prosthesis. J Arthroplasty 14 (6): 689-700 2) Vidalain JP. (2011) Twenty-year results of the cementless Corail stem. Int Orthop. 35 (2): 189-94

Disclosure: No significant relationships.

\section{PC177}

\section{SCAPULAR FRACTURE WITH INTRATORACIQUE DISPLACEMENT AS A ISOLATED INJURY AFTER LOW ENERGY TRAUMA IN ADULT PATIENT}

\section{Auñon Martin, A. Capel Agundez, G. Muñoz Garcia, P. Caba} Doussoux

Orthopedic Surgery Service, Hospital 12 de Octubre, Madrid/SPAIN

Case History: A 32-year-old male, with no medical history of interest, comes to the emergency department after falling snowboarding. It presents pain and functional limitation in left shoulder.

Clinical Findings: At the exploration, there is pain in the clavicle and shoulder, elevation of the clavicle and shoulder is limited and there are signs compatible with acromioclavicular dislocation. Neurovascular examination was normal. A radiological study was carried out in the emergency room, evidencing a grade III acromioclavicular dislocation and non-displaced longitudinal fracture of the scapula body and a conservative treatment was decided by means of a sling.

Investigation/Results: The patient came to the clinic for follow-up at 7 days presenting blockage of mobility, with elevation of the shoulder, and the accomplishment of CT was indicated.

Diagnosis: The CT scan shows a longitudinal fracture of the scapula, with intrathoracic displacement, without rib fractures or pneumothorax. Therapy and Progression: A thoracotomy is performed dorsolateral to locate the intrathoracic fragment of the scapula extracting the fragment, given the size of the fragment is decided its removal, achieving a complete arch of scapulothoracic mobility.

At 6 weeks the fracture healing is complete and sports activity resumes after three months.

Comments: The presence of intrathoracic displacement is a complication described with a very low frequency, more published in the pediatric age and with associated thoracic lesions. The treatment described ranges from closed reductions to open reduction and synthes. This case is remarkable for the mechanism of low energy and the isolated presence of this lesion, probably the presence of scapulotracal block should lead to maintain a high index of suspicion.

References: Cole PA, Gauger EM, Schroder LK. Management of scapular fractures. J Am Acad Orthop Surg. 2012 Mar; 20 (3): 130-41. Tadros AM, Lunsjo K, Czechowski J, Abu-Zidan FM. Causes of delayed diagnosis of scapular fractures. Injury. 2008 Mar; 39 (3): 314-8. Harris RD, Harris JH Jr. The prevalence and significance of missed scapular fractures in blunt chest trauma. AJR Am J Roentgenol. 1988 Oct; 151 (4): 747-50. Armitage BM, Wijdicks CA, Tarkin IS, Schroder LK, Marek DJ, Zlowodzki M, Cole PA. Mapping of scapular fractures with three-dimensional computed tomography. J Bone Joint Surg Am. 2009 Sep; 91 (9): 2222-8. Jones CB, Cornelius JP, Sietsema DL, Ringler JR, TJ Enders. Modified Judet approach and minifragment fixation of scapular body and glenoid neck fractures. J Orthop Trauma. 2009 Sep; 23 (8): 558-64.

Herrera DA, Anavian J, Tarkin IS, Armitage BA, Schroder LK, Cole PA. Delayed operative management of fractures of the scapula. J Bone Joint Surg Br. 2009 May; 91 (5): 619-26 Khallaf F, Mikami A, Al-Akkad M. The use of surgery in displaced scapular neck fractures. Med Princ Pract. 2006; 15 (6): 443-8. Egol KA, Connor PM,
Karunakar MA, Sims SH, Bosse MJ, Kellam JF. The floating shoulder: clinical and functional results. J Bone Joint Surg Am. 2001 Aug; 83-A (8): 1188-94. Hollinshead R, James KW. Scapulothoracic dislocation (locked scapula). A case report. J Bone Joint Surg Am. 1979 Oct; 61 (7): 1102-3. Ward WG, Weaver JP, Garrett WE Jr. Locked scapula. A case report. J Bone Joint Surg Am. 1989 Dec; 71 (10): 1558-9. Walker JS, Walker BB. Scapular dislocation (locked scapula). Ann Emerg Med 1990 Nov; 19 (11): 1329-31. Nettrour LF, Krufky EL, Mueller RE, Raycroft JF. Locked scapula: intrathoracic dislocation of the inferior angle. A case report. J Bone Joint Surg Am. 1972 Mar; 54 (2): 413-6. Shin SJ, Wang SI, Kim JR. Lung injury caused by greenstick fracture of the scapular body in a 6-year-old boy. Skeletal Radiol. 2016 Apr; 45 (4): 555-8. van Schie-van der Weert EM, van Laanen JH, Kraan GA, de Vries MR. Intrathoracic displacement of a scapular fracture: a case report. J Bone Joint Surg Am. 2012 Feb 1; 94 (3): e16. Blue JM, Anglen JO, Helikson MA. Fracture of the scapula with intrathoracic penetration. A case report. J Bone Joint Surg Am. 1997 Jul; 79 (7): 1076-8. Park HY, Jang HJ, South YJ. Scapular body fracture and concomitant inferior angle apophyseal separation with intrathoracic displacement: a case report. J Pediatr Orthop B. 2016 Nov 11. Schwartzbach CC, Seoudi H, Ross AE, Hendershot K, Robinson L, Malekzadeh A. Fracture of the scapula with intrathoracic penetration in a skeletally mature patient. A case report. J Bone Joint Surg Am. 2006 Dec; 88 (12): 2735-8. Porte AN, Wirtzfeld DA, Mann C. Intrathoracic scapular impaction: an unusual complication of scapular fractures. Can J Surg. 2009 Jun; 52 (3): E623.

Disclosure: No significant relationships.

\section{PC178}

A SURGICAL CHALLENGE: IPSILATERAL FEMORAL NECK FRACTURE FIXATION IN A PATIENT WITH ABOVEKNEE AMPUTATION

\section{J. Jasper $^{1}$, T.T.c.f. Van Dongen ${ }^{2}$, R. Hoencamp ${ }^{3}$}

${ }^{1}$ Surgery, Alrijne Ziekenhuis, Leiderdorp/NETHERLANDS,

${ }^{2}$ Department Of Surgery, Alrijne Hospital, Leiderdorp/

NETHERLANDS, ${ }^{3}$ Surgery, Alrijne Hospital, Leiderdorp/ NETHERLANDS

Case History: Hip fracture fixation surgery in patients with aboveknee amputations poses a problem to surgeons in terms of obtaining traction and rotation for fracture reduction. The lack of the foot in these patients makes stable reposition challenging. This study highlights this problem and provides a unique technique to overcome it. Case history

A 78-year-old man with above-knee amputation presented with a history of falling.

Clinical Findings: The ipsilateral leg was painfull and the patient was unable to stand on it with his prosthesis.

Investigation/Results: Radiographic imagery revealed a Garden I collum fracture. A Dynamic hip screw fixation of the fracture was planned although the position of the patient on the fracture table was a dilemma.

Diagnosis: Ipsilateral femoral neck fracture in a patient with aboveknee amputation

Therapy and Progression: The distal femur was fixated with a transverse Steinman nail. With sterile ropes the nail was secured to the fracture table which allowed traction on the femur. The femur was endorotated for reduction using a sterile rope to the lateral side of the Steinman nail and securing the rope as soon as a good reduction was 
achieved. A good reduction of the fracture was achieved, unfortunately the fracture was unstable and dislocated secondary. There was no other option than to convert to a hemi-arthroplasty of the hip.

Comments: Patients with above knee amputation poses a challenge when these patients are in need of fixation of a femoral neck fracture of the ipsilateral hip. This case report shows and illustrates a unique technique for fixation of ipsilateral hip fractures in these patients

References: Al-Harthy A, Abed R, Campbell AC. Manipulation of hip fracture in the below-knee amputee. Injury. 1997;28 (8):p. 570. Rethnam U, Yesupalan RS, Shoaib A, Ratnam TK. Hip fracture fixation in a patient with below-knee amputation presents a surgical dilemma: a case report. Journal of Medical Case Reports. 2008;2, article 296 Aqil A, Desai A, Dramis A, Hossain S. A simple technique to position patients with bilateral above-knee amputations for operative fixation of intertrochanteric fractures of the femur: a case report. Journal of Medical Case Reports. 2010;4, article 390 Dynamic hip screw fixation versus multiple screw fixation for intracapsular hip fracture. Jettoo P1, James P2. J Orthop Surg (Hong Kong). 2016 Aug;24 (2):146-9. Illustrative photographs will be shown on the poster presentation

Disclosure: No significant relationships.

\section{PC180}

SURGICAL TREATMENT OF COMPLEX
METACARPOPHALANGIC DISLOCATION OF THE INDEX
WITH LATERAL APPROACH: SKELETAL TRAUMA CASES 2

J.M. Pereira, M. Quesado, M. Silva, J.D.D. Carvalho, H. Nogueira, J. Alves

Orthopaedics, Centro Hospitalar Tâmega e Sousa, Penafiel/ PORTUGAL

Case History: The metacarpophalangic dislocation is rare, but the peripheral position of the index make him more susceptible to lesion.The treatment of complex lesions is invariably surgical, and the approaches classically used are dorsal or volar. A 16-year-old male suffered a fall on his right hand.

Clinical Findings: In the Emergency with pain and deformity in hyperextension of the metacarpohalangic joint of the index.In the volar portion was evident the prominence of the head of the 2 nd metacarpal.

Investigation/Results: Complex metacarpophalangic dislocations with interposed osteochondral fragments should be approached surgically. In the surgical treatment, the classically approaches are the dorsal or volar.In this case, the lateral approach, with the preservation of the neurovascular bundle and the collateral ligament allowed the visualization and re-insertion of volar plate, as well the access to the volar and dorsal structures.

Diagnosis: Radiologically with metacarpophalangic dislocation of the second finger. It appeared that there was a small osteochondral fragment associated with dorsal localization.

Therapy and Progression: A lateral approach was taken on the metacarpophalangic joint and interposition of the volar plate and the osteochondral fragment of the head block the reduction. The reduction and fixation of the osteochondral fragment was done with a $1.7 \mathrm{~mm}$ screw oriented retrograde and re-insertion of the volar plate. At week 6 , the patient presented fracture consolidation.

Comments: The authors believe that the location of the operative scar in the lateral approach allows to reduce the risk of adhesions to the tendon structures, as well as cicatricial retractions with implications in the joint mobility arch.

References:

Disclosure: No significant relationships.

\section{PC181}

\section{POSTERIOR HIP DISLOCATION ASSOCIATED WITH LARGE AND INVERTED FEMORAL HEAD FRACTURE}

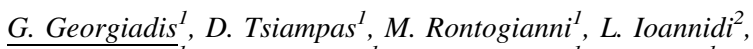 \\ $\overline{\text { A. Tsimpoukis }}^{1}$, M. Bourazani ${ }^{1}$, K. Giannakaki ${ }^{1}$, A. Tzima ${ }^{1}$, \\ A. Borodimos ${ }^{l}$
}

${ }^{1}$ Orthopaedic, Filiates General Hospital, Filiates/GREECE, ${ }^{2}$ Upper Gi And General Surgery, University College London Hospitals NHS Foundation Trust, London/UNITED KINGDOM

Case History: A 32 year-old male was tranferred to our emergency department after being involved in a head-on car accident.

Clinical Findings: On admission, the left lower extremity was painful and held in fixed flexion and internal rotation. No other major injuries were evident following initial assessment of the patient by the trauma team.

Investigation/Results: The posterior hip dislocation was confirmed by emergency x-rays, in which a large concominant head fracture was evident as well. In order to evaluate the position and magnitude of the fragment, emergency CT of the left hip was performed, which demonstrated an intrarticular fragment of approximately $1 / 3$ of the head diameter, originating from the medial - anterior region of the femoral head.

Diagnosis: Traumatic posterior hip dislocation associated with a large Pipkin type 1 fracture

Therapy and Progression: Patient underwent emergent closed reduction under general anesthesia. The reduction was successful, but post-reduction $\mathrm{c}$-arm images demonstrated non-concentric head position due to complete inversion of the intra-articular fragment. The patient was scheduled for open reduction. Due to the position and the magnitude of the fragment, an approach through the anterior capsule was chosen and the fragment was fixed with two cannulated screws. At one year follow-up, the patient was painless and fully active. Two years post-injury, he started experiencing mild pain in the anterior hip pain, which has been attributed to post-traumatic degenerative changes, without evidence of femoral head osteonecrosis in MRI scan.

Comments: Malreduced pipkin fractures of this extent are only occasionally seen. There is controversy about the optimal treatment of these rare injuries.

References: 1.Wang CG, Li YM, Zhang HF, Li H, Li ZJ. Anterior approach versus posterior approach for Pipkin I and II femoral head fractures: A systemic review and meta-analysis. Int J Surg. 2016 Mar;27:176-81 2.Massè A, Aprato A, Alluto C, Favuto M, Ganz R. Surgical hip dislocation is a reliable approach for treatment of femoral head fractures. Clin Orthop Relat Res. 2015 Dec;473 (12):3744-51 3.Park KS, Lee KB, Na BR, Yoon TR. Clinical and radiographic outcomes of femoral head fractures: excision vs. fixation of fragment in Pipkin type I: what is the optimal choice for femoral head fracture? J Orthop Sci. 2015 Jul;20 (4):702-7 4.Lin D, Lian K, Chen Z, Wang L, Hao J, Zhang H. Emergent surgical reduction and fixation for Pipkin type I femoral fractures. Orthopedics. 2013 Jun;36 (6):778-82 5.Chen ZW, Zhai WL, Ding ZQ, Lian KJ, Kang LQ, Guo LX, Liu H, Lin B. Operative versus nonoperative management of Pipkin type-II fractures associated with posterior hip dislocation. Orthopedics. 2011 
May 18;34 (5):350. 6.Uzel AP, Laflamme GY, Rouvillain JL. Irreducible Pipkin II femoral head fractures: Is transgluteal approach the best strategy? Orthop Traumatol Surg Res. 2010 Oct;96 (6):695-701. 7.Zhi-wen Chen, Bin Lin, Wen-liang Zhai, Zhi-min Guo, Zhou Liang, Jia-Peng Zheng, Ke-jian Lian, and Zheng-qi Ding. Conservative versus surgical management of Pipkin type I fractures associated with posterior dislocation of the hip: a randomised controlled trial. Int Orthop. 2011 Jul; 35 (7): 1077-1081. 8.Giannoudis PV, Kontakis G, Christoforakis Z, Akula M, Tosounidis T, Koutras C. Management, complications and clinical results of femoral head fractures. Injury. 2009 Dec;40 (12):1245-51

Disclosure: No significant relationships.

\section{PC182}

\section{THE IMPORTANCE OF CERVICAL SPINE IMMOBILIZATION, EVALUATION AND TREATMENT IN A SPINE INJURY IN THE PATIENT WITH POLITRAUMA}

\section{A. Holub ${ }^{1}$, J. Marante Fuertes ${ }^{2}$}

${ }^{1}$ Emergency Room And General Practice, General Hospital in Jerez de la Frontera, Cádiz/SPAIN, ${ }^{2}$ The Department Of Orthopaedics And Traumatology, In Jerez De La Frontera General Hospital (cádiz, Spain), General Hospital in Jerez de la Frontera, Jerez de la Frontera/ SPAIN

Case History: We present a case af 28 - year old male that suffers a motorbike accident with head trauma and is taken to the hospital by ambulance with cervical spine properly immobilized.

Clinical Findings: On arrival at the Emergency Room the patient's Glasgow coma score is $15 / 15$, normotensive, BP 120/75, HR 87, Sp02 97\%., stable. No thoracic nor abdominal injury. The patient is admitted to the ward for observation due to the cerebral contusion. He denied having neck pain.

Investigation/Results: The inicial cervival spine radiological examination (anteroposterior and lateral) are labeled as normal. Few hours later the patient refers tenderness at the lower cervical region and reduced power and sensation from $\mathrm{C} 6$ below. We repeat the radiological examination adding computered tomography $\mathrm{Ct}$ and magnetic resonance MR to the study that show a burst fracture of C6 vertebral body with Subaxial Spine Injury Classification of 5 requiering ungent surgery.

Diagnosis: C6 vertebral body fracture.

Therapy and Progression: The patient undergoes the anterocervical plating surgery and after 6 months of follow up presents satisfactory rates of recovery.

Comments: In the Emergency Department it is easy to miss the cervical fracture mostly because the plain radiography is not the best method to diagnose them thats why the clinical findings are so importante at that stage. A missed cervical fractures may lead to severe disability or even be life threatening if the spinal cord is involved. That is way it is so importante for all medical personal involved to sospect, identyfy and diagnose a cervical fracture.

References: 1. Duane TM, Dechert T, Wolfe LG, Aboutanos MB, Malhotra AK, Ivatury RR. Clinical Examination and its reliability in identifying cervical spine fractures. J Trauma. 2007;62:1405-10. 2. Sharma OP, Oswanski MF, Yazdi JS, Jindal S, Taylor M. Assessment for additional spinal trauma in patients with cervical spine injury. Am Surg. 2007;73:70-4. 3. Allen BL, Jr, Ferguson RL, Lehmann TR, O'Brien RP. A mechanistic classification of closed indirect fractures and dislocations of the lower cervical spine. Spine. 1982;7:1-27.2. Disclosure: No significant relationships.

\section{PC183}

\section{ISOLATED CEREBRAL FATTY EMBOLISM: REVIEW OF TWO CASES}

$\frac{\text { I. Auñon Martin }}{\text { I }}$, A. Capel Agundez ${ }^{2}$, P. Caba Doussoux ${ }^{3}$, G. Muñoz

${ }^{1}$ Ortopédica Surgery Service, Hospital 12 de Octubre, Madrid/SPAIN, ${ }^{2}$ Orthopedic Surgery Service, Hospital 12 de Octubre, Madrid/ SPAIN, ${ }^{3}$ Orthopaedic Surgery And Traumatology, Hospital Universitario 12 de Octubre, Madrid/SPAIN

Case History: They are two patients, 28 and 32 years old, one of them with no history of interest and the other with a diagnosis of schizophrenia, who suffer high energy injuries .

Clinical Findings: The first patient initially presented a GCS 14/15 thoracic and abdominal trauma associated with orthopedic trauma with both floating knees, the left tibial fracture was open IIIC according to Gustilo. In the initial evolution, external fixation of the orthopedic lesions was performed and amputation of the left leg was required. The second patient had initial GCS 13/15, moderate chest trauma and orptopedic trauma with femur fracture and complex pelvic fracture. The fractures were stabilized on arrival with external fixation. Both patients had a persistent deterioration in their level of consciousness following the withdrawal of sedation. The respiratory evolution was satisfactory precociously.

Investigation/Results: Both patients presented brain CT without alterations and cerebral MR with findings consistent with massive fat embolism

Diagnosis: Patients were diagnosed with Fat Embolism Syndrome in the context of polytrauma. In both patients the cerebral involvement was isolated or very predominant. In both patients, the presence of permeable foramen ovale was discarded with echocardiography.

Therapy and Progression: Both patients received supportive treatment. In the following weeks one of them regained the usual level of consciousness,

at two months there was significant bradypsychia that improved in evolution.

The other patient had a fluctuating level of consciousness, severe bradypsychia with only partial improvement in the following months. Comments: Fat embolism syndrome may present incompletely and cases of cerebral fat embolism may be underdiagnosed if only CT is performed

References: 2015 Apr;77 (Suppl 1):46-8.

Disclosure: No significant relationships.

\section{PC184}

\section{TURNER'S SYNDROME AND ITS INCREASED FRACTURE} RATES. CLINICAL CASE PRESENTATION

\section{A. Holub ${ }^{1}, \underline{\text { J. Marante Fuertes }}{ }^{2}$}

${ }^{1}$ Emergency Room And General Practice, General Hospital in Jerez de la Frontera, Cádiz/SPAIN, ${ }^{2}$ The Department Of Orthopaedics And Traumatology, In Jerez De La Frontera General Hospital (cádiz, Spain), General Hospital in Jerez de la Frontera, Jerez de la Frontera/ SPAIN

Case History: 29- year old female diagnosed with Turner's Syndrome and medical history of lumbar and thoracic spine surgery and 
bilateral femoral shaft fracture 5 years ago treated with plate osteosynthesis suffers a fatal fall in her bathroom and arrives at the Emergency Room with inability to walk and severe pain in her left lower limb.

Clinical Findings: Examination: unilateral thigh pain exacerbated by activity, with motion of her left lower limb limited. Glasgow Coma Score 15 . Normotensive. No head, thoracic or abdominal injury. No neurovascular deficit in the lower limb were found.

Investigation/Results: Radiological examination revealed: left displaced femoral shaft fracture just right above the proximal extremity of the plate that stabilized the previous femoral fracture of the patient. Diagnosis: Displaced femoral shatf peri- implant fracture in the left limb.

Therapy and Progression: We procede to the fracture fixation: as a first step challenge was the extraction of the metal plate uses in previous the surgical treatment requiring the high speed surgical motor system to remove the screws. During the surgery fisiological saline solution was required in high amount for irrigation. As a second step we used antegrade intramedullary nailing.

Comments: Patients with Turner's Syndrome have increased fracture risk and decreased bone density. Bone fragility is recognized as one of the major comorbidities in Turner syndrome so that it is crucial to prevent fractures in those patients. It is frecuent to find periprosthesic and peri - implant fractures in those patient and the goals of treatment include surgical stabilization, implant replacement, or both to restore function.

References: 1. S. Rabin, F . Talavera, S, Agnew, Periprosthesic and preri - implant fractures treatment and management. Aug. 2016 (7); 65; 4795 2. Han TS, Cadge B, Conway GS. Hearing impairment and low bone mineral density increase the risk of bone fractures in women with Turner's syndrome. Clin Endocrinol (2006) 65:643-7.10.1111/ j.1365-2265.2006.02643 3. Bakalov VK, Bondy CA. Fracture risk and bone mineral density in Turner syndrome. Rev Endocr Metab Disord (2008) 9:145-51.10.1007/s11154-008-9076-2 4. Nissen N, Gravholt $\mathrm{CH}$, Abrahamsen B, Hauge EM, Jensen JE, Mosekilde L, et al. Disproportional geometry of the proximal femur in patients with Turner syndrome: a cross-sectional study. Clin Endocrinol (2007) 67:897-903.10.1111/j.1365-2265.2007.02984 5. Hansen S, Brixen K, Gravholt $\mathrm{CH}$. Compromised trabecular microarchitecture and lower finite element estimates of radius and tibia bone strength in adults with turner syndrome: a cross-sectional study using high-resolutionpQCT. J Bone Miner Res (2012) 27:1794-803.10.1002/jbmr.1624 Disclosure: No significant relationships.

\section{PC185}

\section{HIP FRACTURE IN A 35- YEAR -OLD FEMALE IN HER THIRD TRIMESTER OF PREGNANCY REQUIRING URGENT SURGERY. A CLINICAL CASE REPORT}

\section{J.A. Marante Fuertes ${ }^{1}$, A. Holub ${ }^{2}$, J. Marante Fuertes ${ }^{3}$}

${ }^{1}$ Emergency Service, General Hospital in Molina de Segura, Murcia, Spain, Molina de Segura, Murcia, Spain/SPAIN, ${ }^{2}$ Emergency Room, Hospital de Jerez de la Frontera, Jerez de la Frontera/SPAIN, ${ }^{3}$ The Department Of Orthopaedics And Traumatology, In Jerez De La Frontera General Hospital (cádiz, Spain), General Hospital in Jerez de la Frontera, Jerez de la Frontera/SPAIN

Case History: 35 - year- old female in her third trimester of pregnancy, with no significante medical history, presented to to Emergency Room of her Hospital with a 3 week long left hip pain associated with knee pain that became more severe in the last few hours resulting in acute inability to walk.
Clinical Findings: . Examination: left hip pain exacerbated by activity, hip motion limited. Review of systems was negative for other symptoms. Evaluated by physical therapy, she was assigned physical exercises and recommended to use a walker. Given her unrelenting pain, which had progressively worsened over time, an X -rays of the pelvis and hips was performed that revealed subcapital left hip fracture and necrosis of femoral head that needed to undergo a surgical treatment.

Investigation/Results: Radiologycal examinatión: subcapital left hip fracture and necrosis of femoral head

Diagnosis: Subcapital left hip fracture and necrosis of femoral head in a pregnant women (third trimester of pregnancy).

Therapy and Progression: Surgical treatment: intramedullar nail and screws The patients presented a favorable evolution with full recovery of the movement along the first year of follow- up.

Comments: At so early age and related with the pregnacy the hip fractures are mostly caused because of the transient osteoporosis of the pregnancy. The screening and the early diagnosis during the rutinary check - up it's crucial in prevention of the side effects of the hip fracture at the early age.

References: 1. Ergin T, Selam E et al. Transient Osteoporosis of Pregnancy case report. J. Turk Ger Gynecol Assoc 2010. 163 - 1642. Vester H. et al Fractures due to transit osteoporosis of pregnancy. Orthopedics, 2013, Jul. $36912-6$.

Disclosure: No significant relationships.

\section{PC186}

\section{THE POLYTRAUMATIZED PATIENT: TEMPORARY STABILIZATION AND EXTERNAL FIXATION OF MULTIPLE LIMB FRACTURES AND ITS MANAGEMENT. HOW TO PRIORITIZE?}

\section{J. Marante Fuertes $^{1}$, A. Holub ${ }^{2}$}

${ }^{1}$ The Department Of Orthopaedics And Traumatology, In Jerez De La Frontera General Hospital (cádiz, Spain), General Hospital in Jerez de la Frontera, Jerez de la Frontera/SPAIN, ${ }^{2}$ Emergency Room, General Hospital in Jerez de la Frontera, Cádiz/SPAIN

Case History: We present a case of a 18-year-old male with no relevent medical history who while driving his car suffers multiple frontal collisión with another vehicle involved. His injuries are mostly fractures of the lower limb. Presenting this case we want to put an emphasis on the surgical priorities in damage control in polytrauma. Clinical Findings: On his arrival at the emergency room his Glasgow Coma Score is 15, nbormotensive, BP (blood pressure) 110/60, HR (heart rate) 95, Sp02 (oxygen saturation) 98\%. No head, thoracic or abdominal injury.

Investigation/Results: Radiological examination (plain $\mathrm{x}$-ray and computered tomography $\mathrm{CT}$ ) revealed: 1 . Left comminuted tibial and fibular shalf fracture (open fracture grade I of Gustillo and Anderson) 2. Left comminuted supracondylar femur fracture (open fracture grade II of Gustillo and Anderson) 3. Left hip dislocation. 4. Left posterior column acetabular fracture

Diagnosis: Multiple fractures and hip dislocation.

Therapy and Progression: With the patient in stable condition with no neurovascular damage in the left limb found we procede to the treatement: first and immediate hip dislocation reduction with traction pinning was performed followed by external fixation with Triax system of tibial and fibular shalf fracture and finally we have proceded with reduction and and stabilization of the supracondylar fracture with minimally invasive internal fixation (and its further 
external fixation (Hoffman system) . As the last step the acetabular fracture was treated with deferred internal fixation.

Comments: Its crucial to know how to prioritize fractures in polytrauma patients because orthopedic surgery plays a key role in the treatment.

References: 1. Giannoudis PV. Aspects of current management. Surgical priorities in damage control polytrauma. J Bone Joint SurgBr 2003;85:478-483. 2. Vallier HA, Super DM, Moore TA, Wilber JH. Do patients with multiple system injuries benefit from early fixation of unstable axial fractures? The effects of timing of surgery on initial hospital course. J Orthop Trauma 2013;27:405-412. 3. Tschernen H, Regel G, Pape HC, Pohlemann T, Krettek C. Internal fixation of multiple fractures in patients with polytrauma. Clin Orthop Rel Res 2005;347:62-78.

Disclosure: No significant relationships.

\section{PC187}

\section{A POOR OUTCOME OF AN OPEN PROXIMAL FEMUR FRACTURE - A CASE OF INFECTED PSEUDOARTHROSIS OF THE FEMUR AFTER OPEN SUBTROCHANTERIC FRACTURE}

\section{V.R. Spassoff, B. Tasev, H. Hristov}

Trauma Orthopaedic, pirogov hospital, sofia/BULGARIA

Case History: A 40-year old female sustained 3B open subtrochanteric fracture of the left femur with massive soft-tissue defects of both gluteal regions. She was treated initially with an angled blade plate in another institution.

Clinical Findings: Postoperatively she developed signs of osteomyelitis - the plate was removed and thorough debridement was performed, the soft-tissue defect was covered and after 3 months IM nail was introduced. Over a period of 18 months the fracture did not heal, so the nail was removed and the patient was treated functionally for 6 months.

Investigation/Results: At the end of this period there were no clinical and laboratory data for infection, and the bony defect was bridged with an antibiotic-coated IM nail and a $9,5 \mathrm{~cm}$ frozen cortical allograft. After failing to achieve union, both the nail and graft were removed, and antibiotic spacer was placed for 6 months, followed by total hip replacement.

Diagnosis: Infected pseudoarthrosis of the femur

Therapy and Progression: spacer, revision hip replacement, antibiotic

Comments: The open tractures of the proximal femur are challenge, according to a long term treatment and poor results.

References: 1. Bone LB, Johnson KD, Weigelt J, Scheinberg R. Early versus delayed stabilization of femoral fractures: a prospective randomized study. J Bone Joint Surg Am. 1989;71:336-40 2.Giannoudis PV, Smith RM, Bellamy MC, et al. Stimulation of the inflammatory system by reamed and unreamed nailing of femoral fractures. An analysis of the second hit. J Bone Joint Surg Br. 1999;81:356-61. 3. Gustilo RB, Anderson J.t. prevention of infection in the treatment of one thousand and twenty - five open fractures of long bones: retrospective and prospective analyses. J. Bone Joint Surg. am.1976 Jun; 58 (4):453-458. 4. Pape HC, Tornetta 3rd P, Tarkin I, Tzioupis C, Sabeson V, Olson SA. Timing of fracture fixation in multitrauma patients: the role of early total care and damage control surgery. J Am Acad Orthop Surg. 2009; 17 (9):541-9.

Disclosure: No significant relationships.

\section{PC188}

\section{HOFFA FRACTURE ACCOMPANIED BY DISSECTION OF THE POPLITEAL ARTERY}

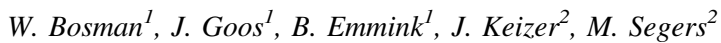

${ }^{1}$ Trauma Surgery, St Antonius Hospital, Nieuwegein/ NETHERLANDS, ${ }^{2}$ Trauma Surgery, St. Antonius Hospital, Nieuwegein/NETHERLANDS

Case History: A 37-year-old male motor rider drove approximately $40 \mathrm{~km} / \mathrm{h}$ when he was hit by an accelerating car.

Clinical Findings: At arrival in the nearest hospital the patient was alert but complained of a painful left knee. Primary survey revealed a hemodynamically stable patient and secondary survey pain and swelling of both knees with a small laceration approximately $10 \mathrm{~cm}$ caudally of the left tuberositas tibiae.

Investigation/Results: X-rays and CT-scan showed a communitive tibial plateau fracture of the left knee, and a Hoffa fracture of the lateral femoral condyle of the right knee with a posterior dislocation of $8 \mathrm{~mm}$. A few hours after admittance the patient developed a pale, cold and pulseless right foot. CTA-scan showed a dissection of the popliteal artery with a length of approximately $5 \mathrm{~cm}$ starting $6.5 \mathrm{~cm}$ cranially of the knee. Crural arteries were intact.

Diagnosis: Hoffa fracture and dissection of the popliteal artery Therapy and Progression: Arterial reconstruction was performed using a bovine patch. Postoperatively the patient was heparanized with $12.500 \mathrm{IE} / 24 \mathrm{~h}$. Four days later, the Hoffa fracture was fixed using two HCS screws and the tibial plateau fracture was fixed using double plating.

Comments: Intra-articular unicondylar fractures of the medial or lateral femoral condyle were firstly reported by Albert Hoffa. ${ }^{1}$ These so-called Hoffa fractures are rare and to date literature is still limited. To our knowledge, we report the first patient with a Hoffa fracture accompanied by a popliteal dissection. Emergency physicians and orthopedic traumasurgeons should be aware that shear forces needed for a Hoffa fracture may lead to an arterial dissection.

References: 1. Hoffa A. Lehrbuch der Frakturen und Luxationen, 4th ed. Stuttgart: Ferdinand Enke- Verlag, 1904, p. 453.

Disclosure: No significant relationships.

\section{PC189}

\section{IMPLANT LOOSENING AND MIGRATION WITH BLADDER PERFORATION AFTER SYMPHYSEAL PLATING - A CASE REPORT}

\section{R.A. Mazzucchelli ${ }^{1}$, P. Potocnik ${ }^{1}, J$. Erhardt $^{2}$, P. Zurmühle ${ }^{I}$}

${ }^{1}$ Orthopaedics And Traumatology, Kantonsspital St. Gallen, St. Gallen/SWITZERLAND, ${ }^{2}$ Departement Orthopädie, Spitalregion RWS, Grabs/SWITZERLAND

Case History: A 40-year old male sustained a Type C pelvic ring injury with bilateral disruption of the iliosacral joints, comminuted fracture of the right pubic rami and symphyseal diastasis. The pelvic ring was fixed anteriorly with a 3.5 low profile plate over a Stoppa approach and posteriorly with two 3.5 reconstruction plates over bilateral anterolateral approaches. Postoperative recovery was uneventful with good clinical outcome. The plate showed signs of breakage on follow-up X-ray 1 year postoperatively. 3 years later the 
patient suddenly complained about increasing low abdominal pain and haematuria without any history of recent trauma.

Clinical Findings: The abdomen was soft with tenderness above the symphysis. Blood samples were normal.

Investigation/Results: $\mathrm{X}$-ray examination of the abdomen revealed partial loosening of one of the right-sided symphyseal screws with protrusion into the small pelvis. On CT-scan $2 \mathrm{~cm}$ of the screw were found to be located inside the bladder.

Diagnosis: Screw loosening with bladder perforation after open reduction and internal fixation of the pubic symphysis.

Therapy and Progression: The patient underwent cystotomy and bladder repair over the previous Stoppa approach. The screw was extracted through the bladder and complete implant removal of the anterior pelvic ring was performed. The postoperative course was uneventful.

Comments: Implant loosening or breakage after ORIF of the anterior pelvic ring is a well-known complication. Screw migration into hollow visceral organs is extremely rare. A review of the literature shows only 3 similar cases. In conclusion, regular radiological follow-up examinations after symphyseal plating are indicated particularly if hardware failure is noticed. The role of implant removal remains controversial.

References: 1 . Heetveld MJ et al. Spontaneous expulsion of a screw during urination: an unusual complication 9 years after internal fixation of pubic symphysis diastasis. Urology. 2003 Mar;61 (3):645. 2. Fridman M et al. Spontaneous urinary voiding of a metallic implant after operative fixation of the pubic symphysis: a case report. JBJS Am. 2003 Jun;85-A (6):1129-32. 3. Yadav S et al. Spontaneous urinary voiding of metallic screws in a patient with symphyseal plating for type II pelvic ring disruption. Chinese Journal of Traumatology. 2013;16 (4):230-232. 4. Sagi HC et al. Comparative radiographic and clinical outcome of two-hole and multi-hole symphyseal plating. J Orthop Trauma. 2008 Jul;22 (6):373-8

Disclosure: No significant relationships.

\section{PC190}

\section{A PEDIATRIC FOREARM FRACTURE WITH A TWIST: A PROXIMAL RADIOULNAR TRANSLOCATION COMBINED WITH RADIAL HEAD FRACTURE}

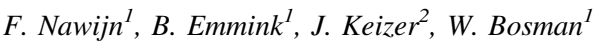 \\ ${ }^{1}$ Trauma Surgery, St Antonius Hospital, Nieuwegein/ \\ NETHERLANDS, ${ }^{2}$ Trauma Surgery, St. Antonius Hospital, \\ Nieuwegein/NETHERLANDS
}

Case History: A 10-year-old girl presented to the emergency department after fall onto an outstretched hand.

Clinical Findings: Physical examination revealed pain and slight swelling of the elbow. The vascular and neurological integrity of the arm was intact.

Investigation/Results: The anteroposterior and lateral radiographs showed a posterior elbow dislocation and a radial head fracture.

Diagnosis: Proximal radioulnar translocation combined with radial head fracture.

Therapy and Progression: An attempt at closed reduction was unsuccessful. Within 24 hours, open reduction was used to reduce the elbow joint after which the radial head was fixated by Kirschner wires. After surgery the elbow joint was stable. The elbow was immobilized for four weeks in a long arm cast. The Kirschner wires were removed four weeks after initial surgery. Three months after surgery, union of the radial head was achieved and full range of motion was regained with a general Disabilities of the Arm, Shoulder and Hand (DASH) score of 11.3.

Comments: Dislocation of the elbow in a pediatric patient is very rare; only $3 \%$ of all elbow injuries in children are dislocations (1). Within this category the proximal radioulnar translocation is an extremely uncommon injury. Proximal radioulnar translocation is often missed on initial radiographs. Persistent restriction of forearm rotation with seemingly normal elbow configuration must trigger to take a closer look at the relationship between the ulna, radius and distal humerus. The clinical importance of this case is that early diagnosis and surgical treatment, of a proximal radioulnar translocation associated with a radial head fracture results in an excellent functional outcome.

References: (1) Flynn J, Skaggs DL, Waters PM. Rockwood and Wilkins' Fractures in Children. 8th ed. Philadelphia: Lippincott Williams \& Wilkins; 2015

Disclosure: No significant relationships.

\section{PC191}

\section{SEPTIC ARTHRITIS OF THE PUBIC SYMHPYSIS REQUIRING SURGICAL IMAGE - GUIDED DRAINAGE. A CASE REPORT}

\section{J. Marante Fuertes ${ }^{I}$, A. Holub $^{2}$, A. Pérez Alcántara ${ }^{I}$}

${ }^{1}$ The Department Of Orthopaedics And Traumatology, In Jerez De La Frontera General Hospital (cádiz, Spain), General Hospital in Jerez de la Frontera, Jerez de la Frontera/SPAIN, ${ }^{2}$ Emergency Room And General Practitioner Resident, General Hospital in Jerez de la Frontera, Cádiz/SPAIN

Case History: We present a case of 21 - year - old male with no relevant medical history. Semiprofesional football player who presents at the Emergency Room with fever and pelvic and groin pain that has been increasing for the past 2 weeks.

Clinical Findings: Examination: groin and pubic pain which irradiates to the genitals and increases when hip is mobilized producing gait claudication Review of systems was negative for chills, fatigue, headache, chest pain, palpitation, parestesias or any other symptoms. Investigation/Results: Plain radiography was normal, as well as urine and bood tests that revealed only some leucocytosis. As the clinical suspicion was pelvic region as a source of sepsis an urgent bone gammagraphy was made with Tc 99 that reveals pathological captation at the pubis. The confirm the diagnosis the Magnetic Resonance (MR) is made and shows retropubic abscess.

Diagnosis: Septic arthritis of the pubic symphysis with retropubic abscess.

Therapy and Progression: The treatment was surgical abscess image - guided drainage and a prolonged course of intravenous and oral antibiotics (intravenous flucloxacillin $1 \mathrm{~g}$ six times a day for six weeks with further four months of oral flucloxacillin at $1 \mathrm{~g}$ three times a day).

Comments: Septic arthritis of the pubic symphysis also called osteomyelitis is the infection which involves pubic symphysis and its joint. It is a rare condition, representing less than one percent of all cases of osteomyelitis. It affects most frequently young athletes and women undergoing gynecologic or urologic surgery. Awareness and early recognition can prevent disease progression.

References: 1. Ross JJ, Hu LT. Septic arthritis of the pubic symphysis: review of 100 cases. Medicine (Baltimore) 2003;82 (5):340-5 2. 
Yoshida S, Nakagomi K, Goto S. Abscess formation in the prevesical space and bilateral thigh muscles secondary to osteomyelitis of the pubis-basis of the anatomy between the prevesical space and femoral sheath. Scand J Urol Nephrol. 2004;38 (5):440-1. 3. Pauli S, Willemsen P, Declerck K, et al. Osteomyelitis pubis versus osteitis pubis: a case presentation and review of the literature. Br J Sports Med. 2002;36 (1):71-3. 4. Choi H, McCartney M, Best TM. Treatment of osteitis pubis and osteomyelitis of the pubic symphysis in athletes: a systematic review. Br J Sports Med. 2011;45 (1):57-64 Disclosure: No significant relationships.

\section{PC192}

\section{RARE ASSOCIATION OF COMPLEX BILATERAL OPEN LOWER LEG INJURY IN A POLYTRAUMA CAR ACCIDENT PATIENT: A CASE PRESENTATION}

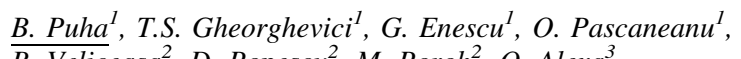

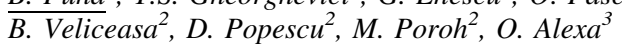

${ }^{1}$ Orthopedics, Gr. T Popa University of Medicine and Pharmacy, Iasi/ ROMANIA, ${ }^{2}$ Orthopaedic And Traumatology, University of Medicine and Pharmacy Gr.T.Popa - Iasi, Iasi/ROMANIA, ${ }^{3}$ Orthopedics And Trauma, University of Medicine and Pharmacy Iasi, iasi/ROMANIA

Case History: We are presenting our delayed management of multiple open fractures, high velocity trauma car accident victim.

Clinical Findings: Materials and methods A 50 year-old female patient with polytrauma, is admitted to the emergency room with multiple fractures of both lower leg, thoracic and cervical spine fractures.

Investigation/Results: Full body CT scan was performed.

Diagnosis: We identified a type 2 fracture of the odontoid process, burst L1 fracture type ASIA E, right pneumothorax, right tibial pilon fracture $43 \mathrm{C} 2 \mathrm{AO}$, right talar neck fracture Hawkins IV, left tibial pilon fracture 43C1AO, left non-displaced talar fracture, left Gustilo type III B opened Sanders 4 calcaneal fracture.

Therapy and Progression: Surgical debridement and splinting of both legs was performed. The patient was transferred to the neurosurgery department and underwent emergency surgical stabilisation of the spine fracture. After 14 days in the ICU, she was transferred to the orthopaedics department presenting acute osteitis of left calcaneus. Open reduction and internal fixation was performed of both distal tibia and talus fractures. The late definitive fixation of the open intraarticular fracture of the calcaneus with acute osteitis did not allow anatomical reduction but only the debridement and fixation with $\mathrm{k}$ wires.

Comments: Despite delayed surgical treatment, clinical and radiological results 6 weeks were good, favored by atraumatic surgical techniques thus increasing the probability of a fair result.

References: Tomás-Hernández J. High-energy pilon fractures management: State of the art. EFORT Open Rev. 2017 Mar 13;1 (10):354361. doi: 10.1302/2058-5241.1.000016.

Disclosure: No significant relationships.
PC193

\section{SURGICAL TREATMENT OF COMMINUTED FRACTURE OF THE TALUS NECK THROUGH A COMBINED APPROACH}

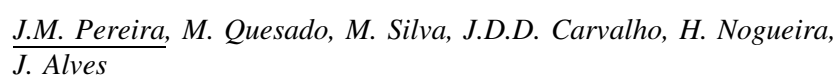

Orthopaedics, Centro Hospitalar Tâmega e Sousa, Penafiel/ PORTUGAL

Case History: A 22-year-old male patient was transferred from another Hospital with the diagnosis of fracture-dislocation of talus, Hawkins type III, resulting from a motorcycle accident.

Clinical Findings: Talus neck fractures are rare and occur as a result of high energy trauma. The principles for surgical treatment are defined, but approach and osteosynthesis methods are still a topic of debate. Anatomic reduction is essential to avoid post-traumatic arthrosis and preserve the vascularity.

Investigation/Results: The combined approach allowed not only the reduce of the fracture but also allow the use of a simplified method of fixation.

Diagnosis: X-Ray and CT with comminuted fracture of the head and neck of the talus.

Therapy and Progression: A double approach (antero-lateral and medial)was performed, with care to preserve vascularization. Cleansing of free articular bone fragments, fracture focus grafting, anatomical reduction and fixation with two $5.0 \mathrm{~mm}$ cannulated screws with retrograde orientation were performed. In the surgical planning of talus lesions is essential an imaging method that allows analysis of the fracture. Conventional CT is recommended for all patients with this type of injury.

Comments: The incidence of avascular necrosis of the talus is associated with the Hawkins classification.The patient had a Hawkins type III lesion, so the probability of developing avascular necrosis is high (up to 70\%). Several surgical approaches have been described for the treatment of talus neck fractures, however, the combined approach allows the preservation of the tissues and allows a precise reduction of the bone fragments.

References:

Disclosure: No significant relationships.

\section{PC194}

\section{POSTTRAUMATIC CLAVICLE EPIPHYSIOLYSIS}

G. Luengo Alonso, J. Ferrero Recasens, E. Vacas, R. Viña, R. Marti

Cirugia Ortopedica Y Traumatologia, Hospital 12 de Octubre, Madrid/SPAIN

Case History: We present a case of an 11-year-old boy who suffered a direct blow to his left shoulder. He was brought to the emergency room complaining about shoulder pain. An initial x-ray was performed without any fractures signs. He came to our clinics one week later. Clinical Findings: Physical exam showed antalgic posture with pain localized in his right clavicle. After reviewing x-rays, we decided to perform a CT scan of his shoulder, confirming the presence of the clinically suspected posterior sternoclavicular pathology. 
Investigation/Results: After CT scan we could not confirm weather it was a case of pure dislocation or an epiphysiolysis type I or II. Apart from that, the clavicle was marking proximal vena cava.

Diagnosis: The patient underwent successful open reduction, where we could finally diagnosed a type I Salter and Harris fracture, and internal fixation of the medial clavicle physeal fracture, after an initial gentle attempt at closed reduction was unsuccessful.

Therapy and Progression: After surgery the chil was wearing an 8 bandage and we confirmed ten days after surgery, the good result we had, by performing a CT control scan. Nowadays he is doing well with no sequels.

Comments: In the skeletally immature population, these injuries have been described as either dislocations of the SC joint or fractures of the medial clavicular physis. These physeal injuries are important to recognize as the displaced epiphysis can block reduction of the sternoclavicular joint.

Disclosure: No significant relationships.

\section{PC195}

\section{NON OPERATIVE MANAGEMENT FOR GRADE V LIVER BLUNT TRAUMA}

\author{
J.C. Garcia Hernandez ${ }^{1}$, D. Muñiz Tolivia ${ }^{2}$, I. Fernández-Burgos ${ }^{1}$,

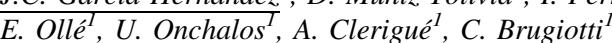

${ }^{1}$ Surgery, HOSPITAL COMARCAL DE INCA, INCA/SPAIN, ${ }^{2}$ Icu, HOSPITAL COMARCAL DE INCA, Inca/SPAIN

Case History: 48 y.o. female, bicycle crash. Admitted to ED, right abdominal and chest pain. ATLS protocols were onset.

Clinical Findings: Right chest and upper right upper quadrant abdominal pain. No tenderness, No chest flail. Reduced right breath sounds. BP 115/63, HR 64, RR 22.

Investigation/Results: Haemoglobin 12.3. CXR: right neumothorax. FAST: + free fluid in abdomen. CT: grade V liver laceration, free fluid, no iv contrast leakage. Right adrenal enlargement.

Diagnosis: Grade V liver laceration. Right neumothorax.

Therapy and Progression: ICD was placed. Based on haemodynamic stability, the patient was admitted for ICU conservative management. During her stay, chest tube was retired at the third day, $\mathrm{CT}$ was performed with no additional findings or injury progression. Discharged from ICU after 48 hours. No signs of biliary peritonitis were present. Laparoscopic abdominal washout was considered unnecessary.

Comments: Nonoperative management of blunt hepatic injuries currently is the modality of choice in hemodynamically stable patients, irrespective of the grade of injury or patient age. Biliary leakage is an increasing complication for non operative blunt liver injuries. Delayed laparoscopic washout prevents bile peritonitis and decreases inflammatory response.

References:

Disclosure: No significant relationships.

\section{PC196}

\section{PSEUDARTHROSIS OF THE CAPITATE DUE TO DELAYED DIAGNOSIS OF AN ISOLATED FRACTURE: SHORT-TERM FUNCTIONAL OUTCOME AFTER SURGICAL FIXATION}

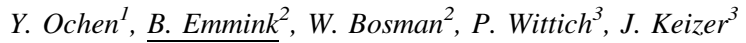 \\ ${ }^{1}$ Trauma Surgery, Sint Antonius Ziekenhuis, Nieuwegein/ \\ NETHERLANDS, ${ }^{2}$ Trauma Surgery, St Antonius Hospital, \\ Nieuwegein/NETHERLANDS, ${ }^{3}$ Trauma Surgery, St. Antonius \\ Hospital, Nieuwegein/NETHERLANDS
}

Case History: A 14-year old girl presented to the emergency department after a fall onto her left hand. Shortly after the fall she developed pain, swelling and was unable to fully flex the hand.

Clinical Findings: There were no apparent signs of altered alignment, displacement or rotational deformities. A carpal bone fracture was suspected, yet the radiographs did not reveal any fracture. She was treated for a contusion with a splint. After one week she had improved range of motion and there were no further signs of tenderness. She returned to the outpatient clinic four months later due to persisting pain.

Investigation/Results: A MRI scan revealed a transverse fracture and non-union of the capitate. In addition, the CT-scan showed multiple sclerotic subcortical cysts.

Diagnosis: Pseudarthrosis of the capitate.

Therapy and Progression: The fracture was treated by open reduction and internal fixation, after pseudarthrosis debridement and cancellous proximal tibial bone grafting. Six months after surgery, union of the capitate and an excellent functional outcome (assessed with DASH score) was attained.

Comments: Isolated fractures of the capitate are rare injuries, easily missed on plain radiographs and delay of diagnosis can lead to complications. Persisting tenderness over the capitate should be an indication to perform an additional CT or MRI scan. Displaced fractures and cases of non-union can be treated by - open reduction and internal fixation, with good functional outcome. This case provides a detailed overview of the surgical fixation method applied for the treatment of pseudarthrosis of an isolated capitate fracture.

References:

Disclosure: No significant relationships.

\section{PC197}

TIBIAL PLAFOUND BILATERAL FRACTURE IN A YOUNG MALE AFTER HIGH - ENERGY IMPACT AND ITS DIFERENT MANAGEMENT

\section{A. Holub ${ }^{1}$, J. Marante Fuertes ${ }^{2}$}

${ }^{1}$ Emergency Room And General Practice, General Hospital in Jerez de la Frontera, Cádiz/SPAIN, ${ }^{2}$ The Department Of Orthopaedics And Traumatology, In Jerez De La Frontera General Hospital (cádiz, Spain), General Hospital in Jerez de la Frontera, Jerez de la Frontera/ SPAIN

Case History: We present a case of a 30 - year old patient who arrives at the Emergency Department brought by an ambulance after falling from 4 meters of height complaining of severe bilateral burning foot pain developing local bruising and swelling soon aftyer the injury. The patient arrives with double splints that stabilized the injuries. 
Clinical Findings: 30 - year old male with no relevant medical history. On his arrival at the emergency room the patient's Glasgos Coma Score is 15 , nornotensive, blood pressure 110/65, heart rate 78, sp02 97\%. No head, thoracic nor abdominal injery. No lose of consciousness. No dates for hypovolemic shock were found. LOWER LIMBS EXAMINATION: bilateral haematomas with swelling and pain rated with 4 on a scale of 1-10 after analgesic in continuous infusion administrated by a rescue team. Due to a suspicion for lower limb bilateral fracture radiological examination is made.

Investigation/Results: Radiologic evaluation reveals: tibial plafound fracture in his left ankle and tibial plafound fracture with medium and posterior malleolar fracture in his right ankle.

Diagnosis: Bilateral tibial plafound fracture (AO /OTA Classification of 43 - B3).

Therapy and Progression: Right ankle: external fixation Left ankle: minimally invasive technique in the absence of skin lesions: using several small incisions (anteromedial approach that enabled the joint surface reconstruction followed by proximal incisión of anterolateral tibia to the final introducction of premodelled plate). Radiological control was satisfactory.

Comments: Tibial plafoud fractures account for $<10 \%$ of lower extremity injuries. In this case we can compare a different surgical approach in two tibial plafound fractures.

References: 1. Leonard M, Magill P, Khayyat G. Minimally - invasive treatment of high velocity intra - articular fractures of distal tibia. Int Ortop (SICOT) 2009; 33:1149 - 53. 2. He X, Hu Y, Ye P, Huang L, Zhang F, Ruan Y. The operative treatment of complex pilon fractures: A strategy of soft tissue control. Indian J. Orthop. 2013 Sep; 47 (5): 487 - 92. 3. Villaseñor Villaseñor LE, OLEA Leyva MA et all. Clinical result of the tibial pilon bilateral fracture with minimally invasive technique. Orthopedic Acta 2009; 23 (3):163 - 166. Disclosure: No significant relationships.

\section{PC198}

\section{A BOY WITH A TWISTED KNEE}

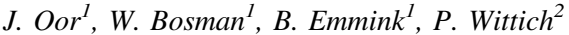

${ }^{1}$ Trauma Surgery, St Antonius Hospital, Nieuwegein/ NETHERLANDS, ${ }^{2}$ Trauma Surgery, St. Antonius Hospital, Nieuwegein/NETHERLANDS

Case History: A 14-year-old boy with no past medical history was presented to the emergency department with a painful left knee, which had acutely developed during jumping around on a trampoline. Clinical Findings: Physical examination revealed a painful left knee, which was kept in Bonnet position (slight flexion), and the boy was unable to actively extend the lower leg or bear weight on the leg. Investigation/Results: Conventional radiology showed a suprapatellar fat pad with minimal lateralization of the patella, and no fractures were seen. Patient was discharged with a posterior splint and at the outpatient clinics, an ultrasound of the left knee was performed, demonstrating hemarthrosis with an intact quadriceps- and patellar tendon, but signs of a ruptured medial retinaculum. A magnetic resonance imaging (MRI) scan of the knee demonstrated suprapatellar hemarthrosis and an osteochondral lesion of the lateral femoral condyle, with a corpus liberum of $2 \times 1 \mathrm{~cm}$ located in the lateral articular space.

Diagnosis: Osteochondral lesion following patellar luxation.

Therapy and Progression: Due to the size and location of the lesion (weight-baring part of lateral condyle), patient was scheduled for operative correction. Following a lateral parapatellar approach, the osteochondral chip was reimplanted and fixated using two absorbable compression screws. Five months following surgery, the function of the left knee was completely restored.

Comments: Osteochondral lesions are probably an underreported complication following patellar luxation. Studies using MRI-scanning and/or arthroscopy describe incidences up to $67 \%$, especially in children $^{1,2}$. In case an osteochondral defect is suspected in patients with clinical signs of hemarthrosis, an MRI scan should be performed. Operative correction is indicated for lesions of the weight-bearing part of the condyle or lesions $>1 \mathrm{~cm}^{1}$.

References: 1. Rockwood, Charles A., David P. Green, James D. Heckman, and Robert W. Bucholz. 2001. Rockwood and Green's fractures in adults. Philadelphia: Lippincott Williams \& Wilkins. 2. Kirsch MD, Fitzgerald SW, Friedman H, Rogers LF. Transient lateral patellar dislocation: diagnosis with MR imaging. AJR Am J Roentgenol. 1993 Jul;161 (1):109-13.

Disclosure: No significant relationships.

\section{PC199}

\section{AN UNUSUAL CASE OF BILATERAL TIBIAL TUBERCLE AVULSIóN FRACTURE IN A 13-YEAR-OLD ADOLESCENT WHILE SPORT PRACTICING}

\author{
J.A. Marante Fuertes ${ }^{1}$, A. Holub ${ }^{2}$, J. Marante Fuertes ${ }^{3}$
}

${ }^{1}$ Emergency Service, General Hospital in Molina de Segura, Murcia, Spain, Molina de Segura, Murcia, Spain/SPAIN, ${ }^{2}$ Emergency Room, General Hospital in Jerez de la Frontera, Cádiz/SPAIN, ${ }^{3}$ The Department Of Orthopaedics And Traumatology, In Jerez De La Frontera General Hospital (cádiz, Spain), General Hospital in Jerez de la Frontera, Jerez de la Frontera/SPAIN

Case History: We present a case of a 13 - year old adolescent patient who suffers a soprt injury while we was playing football suffering a $\mathrm{du}_{i}$ irect blow to anteromedial tibia with his knees in extension and varus forced.

Clinical Findings: 13 - year old obessed adolescent patient with no other relevent medical history arrives to to Emergency Room brought by an ambulance with severe knees pain. LOWER LIMBS exam with careful inspection of both knees showed acute swelling and pain at palpation. To confirm a suspicion of fracture radiological examination is realized.

Investigation/Results: Radiological examination reveals: bilateral tibial avulsion fracture.

Diagnosis: Bilateral tibial tubercle avulsion fracture (according to the Salter and Harris clasification : type III)

Therapy and Progression: The patient undergoes an urgent ORIF surgery (Open Reduction and Internal Fixation).

Comments: A tibial tubercle avulsion fracture is usually an injury to the knee occurring in adolescence, during the transitional phase of physeal closure just prior to completion of growth. This fracture most often is an isolated injury related to pushoff or landing while jumping as the quadriceps eccentrically contracts to support the individual's weight. The correct surgery with early physiotherapy plays the key role in the full recovery.

References: 1. Arredondo-Gómez E, López Hernández JD, Chávez Martínez F. [Fracture due to bilateral avulsion of the tuberosity of the shin bone (tibia). A case report]. Acta Ortop Mex. 2007;21 (3):154-8. 2. Tulic G, Sopta J, Bumbasirevic M, Todorovic A, Vucetic C. Simultaneous bilateral avulsion fracture of the tibial tubercle in adolescent: a case report. J Pediatr Orthop B. 2010;19 (1):118-21. 3. Hamilton SW, Gibson PH. Simultaneous bilateral avulsion fractures 
of the tibial tuberosity in adolescence: A case report and review of over 50 years of literature. Knee. 2006;13 (5):404-7. 4. Ergün M, Taşkiran E, Ozgürbüz C. Simultaneous bilateral tibial tubercle avulsion fracture in a basketball player. Knee Surg Sports Traumatol Arthrosc. 2003;11 (3):163-6.

Disclosure: No significant relationships.

\section{PC200}

\section{CARE MUST BE TAKEN FOR THE TREATMENT OF IPSILATERAL FRACTURES OF THE DISTAL FEMUR AND FEMORAL SHAFT USING ANTEGRADE INTRAMEDULLARY NAIL COMBINED WITH DISTAL LOCKING PLATE}

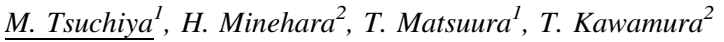

${ }^{1}$ Orthopaedic Surgery, Kitasato University School of Medicine, Sagamihara/JAPAN, ${ }^{2}$ Orthopaedic Surgery/emergency And Critical Care, Kitasato University School of Medicine, Sagamihara/JAPAN

Case History: Case1: $53 \mathrm{~F}$ car accident Case2: $55 \mathrm{M}$ motor cycle accident

Clinical Findings: Case1: Distal fracture site was healed at 6 months, however, the fracture line was visible at shaft with large callus. Radiograph showed slow but steady biological activity at every follow up in the clinic. It healed at 1.5 years. Back to work without problems. Case2:: Radiograph revealed slowly ongoing biological activity at shaft area in the clinic. Fractures healed at 2 years. Patient still has limited ROM on his right knee with a flexion of 110 degrees. Investigation/Results: The treatment of such fractures by the antegrade nail with a plate, due to the prevention of the interference of interlocking screws, working length of the nail can be rather short and sometimes fixation may not be rigid enough although the distal fractures heal well with LCP. The retrograde nail is not suitable for the small distal fragment. Care must be taken for the delayed healing especially on the shaft area in such cases

Diagnosis: Case 1 Left femur shaft fracture (AO 32-A3), distal femur fracture (AO 33 A1) Case 2 Right femur shaft fracture (AO 32-B3), distal femur fracture (AO 33 A2)

Therapy and Progression: Case $1 \mathrm{LCP}$ DF + antegrade nail (day 0) Case 2 External fixation (day0) LCP DF + antegrade nail (day3)

Comments: The ipsilateral fractures of the distal femur and femoral shaft are usually occurred by high energy trauma and difficult to treat. 2 delayed union cases of such fractures fixed by antegrade intramedullary nail combined with distal locking plate were reported.

References: 1) Wood EG, Savioie FH, vander Griend RA. Treatment of ipsilateral fractures of the distal femur and femoral shaft. J Orthop Trauma. 1991; 5 (2): 177-83. 2) Butler MS, Brumback RJ, Ellison TS et al. Interlocking intramedullary nailing for ipsilateral fractures of the femoral shaft and distal part of the femur. J Bone Joint Surg Am. 1991 Dec; 73 (10): 1492-502.

Disclosure: No significant relationships.

PC201

\section{PELVIC FRACTURE WITH PERSISTENT RHABDOMYOLYSIS - AN AUTOPSY CASE REPORT}

T. Miyake, N. Kanda, H. Okada, S. Yoshida, F. Yamaji, H. Okamoto, S. Ogura

Emergency And Disaster Medicine, Gifu University, Gifu-shi/JAPAN

Case History: A 83-year-old male cyclist struck by a car was transported to our hospital.

Clinical Findings: The patient had a hemorrhagic shock.

Investigation/Results: He was examined by $\mathrm{X}$ rays and whole-body $\mathrm{CT}$, and found extravasation in right internal iliac artery.

Diagnosis: The patient was diagnosed as multiple trauma including pelvic fracture with right acetabulum, hepatic injury, multiple rib fractures, lumbar vertebral fractures of transverse process.

Therapy and Progression: Since he had a hemorrhagic shock, massive transfusion and transcatheter arterial embolization (TAE) were required. Twelve hours after injury, right above-knee amputation was performed, because serum creatine kinase was elevated by right lower leg ischemia. However, serum creatine kinase, potassium and myoglobin were still elevated after the amputation. Therefore, hemodiafiltration to treat the acute renal injury due to elevated creatine kinase and myoglobin was performed on hospital day 2. In addition, sustained low efficiency hemodialysis and plasma exchange therapies were performed on hospital day 3. Despite these treatments, the patient's hemodynamics was not improved and he died on hospital day 8 . The autopsy revealed necrotic iliopsoas and digestive tract.

Comments: Pelvic fracture with high energy trauma represents high mortality rate especially in elderly patient. We herein report a case that persistent rhabdomyolysis occurred after pelvic ring fracture despite of continuous renal replacement therapy. The cause of the patient's death was thought persistent rhabdomyolysis and severe hypotension due to iliopsoas necrosis and peritonitis caused by necrotizing digestive tract. These might be caused by inferior mesenteric and internal iliac artery injury via TAE.

References: Pollanen MS, Fatal rhabdomyolysis after torture by reverse hanging. Forensic Sci Med Pathl. 2016 Jun; 12 (2): 170-3. doi: 10. 1007/s12024-016-9752-6. Epub 2016 Feb 18

Disclosure: No significant relationships. 


\section{Author Index}

Abad Arevalillo A. PC164

Abarquero Diezhandino A. PR353

Abbasi H. O003

Abdel-Lah FernándezO. PC150

Abdelrahman H. PR283, PR385

Abdulla A. PC003

Abdurakhmanov R.F. PC044

Abe R. PR231

Abeid A. PR191

Abreu De Sousa J. PC143

Abu-Zidan F.M. PC003, PC072, PR257

Abusharia M.I. PC003

Achatz G. PR264A

Acklin Y.P. PR395

Acuña-Castroviejo D. PR080, PR081

Adamenko V.N. PR305

Adami E. PC104, PR014, PR310

Afonso A.C. PC093

Afonso C. PC039, PC137

Agnes A. PR319

Agresta F. PR017

Agrogianni X. PR031

Aguado Lopez H. PC076

Aguiar T. PC170

Aguilar L.T. PR026, PR043, PR065

Aguiló Lucía J. PR075

Aguir S. PR227

Ahadov T. PR103

Ahl R. O066

Ahmed K. O098

Ai T. PC020

Aiboshi J. PR032

Aiolfi A. O024

Aitken R.J. PR013, PR239

Ajiko M.M. PR128

Akahoshi K. PR032

AkaraborwornO. PR188

Akers K. PR253

Akhadov T. O035, O037, PR102, PR105, PR108, PR111, PR112,

PR116, PR126, PR281

Akhlebinina M. PR108, PR126, PR281

Akritidou E. PC085, PC152

Al Thani H. PR123

Al-Thani H. O039, O098, PR005, PR178, PR180, PR191, PR192, PR278, PR279, PR283, PR385

Alarcón-Del Agua I. O011, PC084, PC117, PR025, PR034, PR054, PR065, PR074, PR082, PR083, PR084, PR158

Alcoba García L.M. PC135A

Aldea A. PR076

Alegre Torrado C. PC028, PC029, PC030, PC033, PC092, PR035, PR046, PR184

Alegret Monroig N. PR190, PR232, PR379

Alegret N. PR140, PR150

Alexa I.D. PR346

AlexaO. PC161, PC192, PR346, PR375

Alfici R. PR069

Älgå A. PR152

Alhaddad M.A. PC072

Alhassani A. O098

Allen B. PR137, PR182

Allen G. PR251

Allen K. PR123

Allevi G. PR413, PR423
Almasri S. PR050

Almre I. PR042

Almusawi J. O014

Alonso Viana L. PR208, PR371

Altun G. O004, O084, PR410

Álvarez Martínez A. PC135A

Alvarez Sarrado E. PR085

Alvarez Sánchez M.D.C. PR075

Alves F.S. PC170

Alves J. PC180, PC193

Alves P.H.F. PR316

Alvite Canosa M. PR075

Ambagtsheer I. O042

Ambrona Zafra D. PR317

Amcheslavskii V. PR109

Ammar A. PR192

Anastasiu M. PR265

Andela A. PR172

Andersson P. PR226

Andretta M. PC014

Andruszkow H. PR162

Aneste E. PR308

Angelo S. PR280, PR401, PR439

Ansaloni L. PR138

Anton C. PC121

Antoniac I.V. PR414

Antunes C.M.S. PC052

Antunes J. PR311, PR401

Aparicio J. PC034

AparicioO. PC046

Apolinário D. PC086

Apostolides M. PR289

Arai T. PC130

Arand C. O085

Aranzana A. PC119

Ardito A. O017, PC139, PR243

Argote Camacho A.X. PC097, PC112B, PC125, PC126, PC149

Arieta-Araunabeña S.T. PR078

Armas I.S. PC143

Armstrong C.A. PR393

ArnautO. PR001

Arzuaga-Esquino M. PR073

Arín B. PC015, PC058, PC128

Asahara T. PR391

Ashkenazi I. PR061, PR069

Asim M. O039, O098, PR180, PR279, PR385

Askarynezhadbehzady R. O003

Assunção A. PR311

Astruc Hoffman A. PC126

Asturias S. PR168

Attili F. O092

Auñon Martin I. PC177, PC183, PR010, PR173

Avaro J.P. PR227

Aveiro D. PC131, PC137, PC153

Avelar P. PC038, PC081, PC086, PC095, PR047

Averianov S. PR109

Avram M. PR287

Avramenko A. PR227

Axelrad A. O034

Ayad A.Z. PC147

Azenha N. PC013

Babst R. PR008, PR235, PR364, PR442

Badovinac D. PC004, PC060

Badrin A. O096

Bae K.S. PR381

Bagnenko S.S. PR210 
Bahouth H.B. PR290

Bailey K. O096

Bajenaru D.L. PC068

Bakir S. PR241, PR359, PR421

Bakota B. O028

Balakrishnan N. O068A

Balcells E. PR160

Balducci G. O071

Ballesteros Sanz M..A. O078

Baltaga R. PR001

Baltazar Branco C. PR179

Bandholm T.Q. O036

Banerjee A. O030, O052, O070, PR006

Banierink H. PR372, PR406

Bar Haim R. PR070

Barberia A. PR341

Barbieri C. PR094

Barbosa B. PC171

Barea Mendoza J. O078, PR175

Baron R. PR099

BarzelO. PR061

Barzola Navarro E.J. PC111

Bascuas Rodrigo B. PR043

Bashir M.O. PC147

Bass G.A. O050A, PC094, PR052, PR229

Bassi N. PR307, PR324

Bastian J.D. O009

Bayona J.C. O013

Beck S. O081

Becker A. PR248

Becker J. O010

Beden R. PC163

Beerekamp S. O080

Beeres F.J..P. PR008, PR235, PR364, PR442

BejaranoO. PC048

Bejenaru I. PR287

Beks R.B. O025, PR395

Bellio G. PC138

Belousov D.V. PC044

Beltrán M. PR075

Beltrán Martos M. PC135A

Benedikt M. PR429

Beneker J. PR261

Bengulescu I. PR076

Benini B. PR014, PR310

Benjamin E. O024, O032

Bennett D. PR377, PR403

Berdel H.O. O074

Berdelis V. PC085

Berezhnaya M. PR109

Berezhnoy Y. PR109

Berger-Groch J. O007, PR367

Bergmeister H. O081

Berlin Y. PR248

Bernabeu C. PC034

Bernal Tirapo J. PR035, PR184

Bernard A.C. O074

Bernard C. PR251

Bernards A. PR417

Bernini C.O. PR316

Bersztel A. PR138

Bessant G. O067

Betisor A. PR438

Beuran M. PR022, PR101, PR287

Bhashyam A.R. PR351

Bianchini S. PR233
Bichi S. PC019

Biert J. O006

Bijani S. PR345

Bilik A. PR437

Biloslavo A. O017, PC138

Bini R. PR086, PR090

Bird N. PR309

Birindelli A. PC027, PC040, PC053

Biscardi A. PC027, PC040, PC053

Blansfield J. O068A

Blasco DelgadoO. PC148

BlascoO. PR207

Blasco Velarde J.A. PR208, PR371

Bloemers F.W. PR240

Blokhuis T. PR129

Boddaert G. PC106, PR227, PR260

Bodzay T. PR245

Boernert K. PR364

Bolandparvaz S. O003

Bonafe S. PC098

Bonariol L. PR307, PR324

Bond-Smith G. PC122

Bonde A. O048

Bonilla-Escobar F. PR181

Bonilla-Escobar F.J. PR132, PR187

Bonin-Font F. PR060

Bonnet S. O054, PC106, PR260

Böhme J. PR335A, PR426A

Booms P. PR058

Bordu S.I. PR079

Borger Van Der Burg B.L.S. O046, PC024A, PR213, PR219

Borges I. PC013

Borodimos A. PC181

Borrello A. PR319

Bosman W.-M. PC165, PC188, PC190, PC196, PC198, PR424, PR436, PR448

Bossers S.M. PR240

Bossinakis K. PR400

Bossola M. O092

Bountouris I. PR024, PR045, PR214

Bourazani M. PC181

Bowyer M. PR213, PR231

Bozzo S. O093, PC014, PR041

Bragaglia L. O071

Bramnik Z. PR070

Branco C. PC167, PC171, PR343

Branco L. PC009, PC073

Brand A.G. PR335

Brandariz Gil L. PR035, PR046

Brathwaite C.E.M. O034

Bratu M.R. PC068, PR101

Bravo Cerro A. PC024, PR285

Briet J.P. PR339

Brinck T. PR147, PR177

Brito D. PC008, PC143

Brito D.S. PC090

Brito E Melo M. PC101

Brito M. PR270

Brojan M. PR411

Bronkhorst M. O031

Brooks A. PR154

Broughton K. PR013, PR239

Brouwers L. O006

Brown C. PR253

Brugiotti C. PC195

Bruna M. PR075 
Brzozowski R. PR258

Brügger L. PR020

Bubuianu A.L. PR048

Bueno-Larano P. PR080, PR081

Bulat C.L. PC121

BulatO.M. PC121

Burke P. O068A

Burneo Esteves M. O064, PC132, PR155, PR163

Bustamante D. PR131, PR354

Bustos Jimenez M. PR064

Butscher A. O009

Bánki L. PR402

Bækgaard J.S. O033, PR141

Böhme J. O087, PR335A, PR426A

Bühren V. O010

Caba Doussoux P. PC177, PC183, PR173, PR356, PR365

Caba P. PR353

Caballero M. PR075

Cabral J. PC170

Calis H. PC087, PR033, PR139, PR274

Calixto P. PC101

Camarneiro R. PC101

Cambiaso-Daniel J. PR415

Campbell M. O019A, PR088

Campos Serra A. O049, PC046, PC061, PR140

Candela A. PC034

Candinas D. PR020, PR030

Canga Presa J.M. PC135A

Capel Agundez A. PC177, PC183

Capitan Vallvey J.M. PC091A, PC112A, PC112B, PC148A

Čapov I. PC022, PR295

Caragounis E.-C. PR138

Caratozzolo E. PR307, PR324

Carbonell J.P. O013, PC042

Carda M. PR350

Cardoso R. PC021

Carlos S. PC021

Carmo S.P. PC073

Carmona Soto P. PC024, PR285

Carrara G. O093, PC014, PC019, PR041

Carrilho N. PC062

Carrión L. PR207

Carrión Retuerto L.O. PC148

Cartu D. PR079

Carvalho A.C. PC008, PC090, PC102

Carvalho A.M. PR280, PR401, PR439

Carvalho J.D.D. PC180, PC193

Carvalho M. PR148

Carvalho P. PC089, PC091

Casari F. PR338

Casati A. PC027, PC040, PC053

Casella J.R.M. PC104, PR014

Casimiro C. PC018, PC039, PC056, PC062, PC093, PC099,

PC109, PC114, PC131, PC137, PC153, PC156, PR238

Castell Gómez J.T. PR075

Castelo L. PC167

Castle N. PR005

Castro J. PC170

Catarino S. PC093, PC099, PC153, PR238

Cavero Ibiricu A. PC135A

Cazolari P.G. PR315

Ceballos Esparragón J. PR075

Cecilia D. PR354

Cecilia Lopez D. PR445

Cecílio J. PC013

Ceka S. PC070
Cendoya I. PR075

Ceolin M. O017, PC103, PC139, PR036, PR243

Ceresoli M. PR087

Cervellera M. PR233

Cesur E. PC023

Cevallos C.Y. PR292, PR299

Cevik A.A. PR257

Chae S. PR336, PR337

Chainiramol P. PR188

Chan G. O028

Chang S.W. PR138

Chapman J.D. PR159

Chapman M.P. O050, O075, PR216

Charalampakis D. PC115, PC123

Charitonova A. PR039

Chatziantoniou C. PR289

Chaveli Diaz C. PR093

Chaves-Marcos R. PC146

Chen Y. O094

Chen Y.-D. O012

Chen Y.J. PC174

Cheng V. O032

Chiappetta F. PC014, PC019

Chica J. PR292

Chichom-Mefire A. O061, PR148

Chico Fernández M. O078, PR165, PR175

Chinna S. PR154

Chiotoroiu A. PR287

Cho M.R. PR368

Choi W. PR336, PR337, PR368

Choynowski M. O096

Christie J.D. PR156

Christie S..A. O061, PR128, PR148

Christopoulos P. PC116

Christou C. $\mathrm{O} 062$

Ciernik J. PC022

Cimerman M. PC025, PR332

Ciocan R. PR101

Ciocan R.N. PC068

Ciocca Vasino M. PC019, PR041

Cipressi C. PR233

Cires M. PC015, PC058, PC128

Ciriano Hernandez P. PR268, PR269, PR370

Cirocchi R. O059

Cisneros J.M. PR082

Ciurea N.M. O043, PR205, PR383, PR441, PR443

Clerigué A. PC195

Cobos Cuesta R. PC091A, PC112A, PC112B, PC148A

Cobuccio L. PR017

Coccolini F. PR138

Codognotto E. PR090

Cohen M.J. O070

Cohnert T. PR415

Coleman J.R. O030, O052, PR006

Coley E. PC079

Colleoni R. PR315

Colsa Gutierrez P. PR208, PR371

Colsa P. O073, PR015, PR016, PR018, PR019, PR266

Comino-Pardo A. PR080, PR081

Compañ A.F. PC034

Conceição L. PC013

Coniglio C. PC027, PC040, PC053

Constantino J. PC093, PC109, PC114, PC131, PC156

Consunji R. PR123, PR191, PR192

Coppola S. O093

Cordeiro S. PC154 
Cordella A. PR413

CornuO. PR349

Correia P. PC008, PC090, PC102

Corte Real J. PR439

Cortez L. PC009, PC073

Costa G. O017, O071, PC103, PC139, PR017, PR036, PR243

Costa J. PC008

Costa P. PC021

Costa W. PC090

Costamagna G. O092

Costea D.O. PC083, PC134

Cotirlet A. PR055

Cotton B.A. O070

Coutinho J. PC001, PC069, PR270

Couto M. PC018

Coxo M. PR179, PR343

Cozar Ibañez A. PC112A

Cozza V. O092, PR063, PR319

Craus Miguel A. PR091, PR317

Crespo Lastras P. PC164

Criado E. PC046, PC061

Croitor G. PR438

Crouch R. PR196

Cruz Vigo F. PR035, PR184

Csete K. PR256, PR402

Csonka E. PR193A, PR245, PR402

Cuarental García J. PC168, PR173

Cujiño I.F. PC002

Curado Soriano A. PC077

Curbelo-Peña Y. PR250

Cózar Bernal F. PC024, PR285

Cózar Lozano C. PC148, PR207

Dam S.A. PR412

Damaskos D. PR246

Damous S.H.B. PR316

Dang K.H. PR393

Das P. PC122

Dauer E.D. PR303

Davidson A. PR217

Davies E. O042

De Carbonnieres A. O054

De Graaf M.W. PR151

De Groot M. O038

De Jong L. PR326

De Jong M. O025, PR230

De Jongh M.A.C. O060, O065

De La Cuadra B.M. PC140, PC142, PC158, PC159, PR026, PR043, PR044, PR051, PR092

De La Hoz M.L. PR075

De La Hoz Riesco M.L. PC135A

De Lebruisant S. PC024B, PC064A, PC142

De Lebrusant Fernandez S. PC048

De Leeuw M.A. PR240

De Lesquen H. PR227

De Loos E.R. PR275, PR282

De Manzini N. PC138

De Moya M. PR141

De Muinck Keizer R.J.O. O080

De Munter L. O065

De Pedro C.G. PR064

De Simoni P. PR413, PR423

De Soto Cardenal B. PC142, PC159, PR026, PR027, PR028

De Sousa X.P. PC009, PC073

De Tomás Palacios J. PC108, PR021

De Vries J. O060

De-Soto-Cardenal B. PR025
Deans C. PR246

Dedu R. PR265

Degermetzoglou N. PC037, PR024, PR045, PR214

Degrate L. PR087

Dekker L. PR144

Del Prete L. PC082, PC107

Del Valle A.M. O013, PC002, PC042

Delgado Plasencia L. PR075

Demetriades D. O024, O032

Demir D. PC024A

Demirhan R. PC023

Demiya K. PR169, PR342, PR355, PR380, PR384, PR390

Den Hartog D. PR183, PR228, PR369

Denisov A.V. PR210, PR305

Depesa C. PR237

Desoucy E. PR217

Detrembleur C. PR349

Dettmers R. PC135

Di Cosimo C. PR014, PR310

Di Flumeri G. O092

Di Giorgio A. O092, PR063, PR319

Di Grezia M. O092, PR063, PR319

Di Saverio S. PC027, PC040, PC053

Diaconescu B. PC068, PR101

Diaconu B. PC121

Diago Santamaría M.V. PC135A

Dias R. PC013

Dicker R.A. O061, PR128, PR148

Dijkgraaf M. O038

Dimitrakopoulou E. PC011, PC085, PC152

Dimitriu A.L. O043, PR205, PR383, PR441, PR443

Diogo M.J. PC018

Dionísio I. PC101

Dissak-Delon F. O061, PR148

Divković D. PR110, PR124

Dobbelaere A. PR450

Dodd M.J. PC136

Dogan E.M. O041, PR138, PR209

Dogjani A. PR318, PR321

Đokić M. PC004, PC060

Dolly K. PR003

Dominguez L.B. PR255

Domínguez Amodeo A. PC077

Domínguez Sánchez C. PC142, PC158, PR051, PR322

Dongen T.V. PR219

Doppelbauer M. PR397

Doulgerakis G. PC011

Duarte A. PC101

Duarte L. PC156

Dubose J.J. O046, PR213

Duffy C.C. O050A

Duman S. O084

Dumanic M. O081

Dumas R.P. PR156

Dumbrava B.-D. PR052

Duran Muñoz-Cruzado V. PR064, PR434

Durso J. O032

Durán Muñoz-Cruzado V.M. O011, PR082

Durán Poveda M. PC043, PR075, PR077

Durán-Muñoz-Cruzado V. PC084, PC096, PC117, PC146, PR025,

PR034, PR054, PR065, PR074, PR083, PR084, PR158, PR322

Dvortsevoj S. PR252

Eakins J. O021

Eckhart F. PR008

Edwards M. O006, O025

Eefting D. PR213 
Egan T. PC079

Egloff M.P. O009

Eguaras I. O076, PR012, PR023, PR093

Eibinger N. PR429

Eid A.I. O089, PR237

Eilenberg M. O081

Eisenhauer B. PR234

Ekkernkamp A. O055, O056, O058, PR224, PR241, PR254, PR259, PR261, PR263, PR264, PR359, PR421

El Hechi M. O018

El Moumni M. PR151, PR172, PR409

El Rifai A.Y. O018

El-Menyar A. O039, O098, PR005, PR149, PR178, PR180, PR191, PR192, PR278, PR279, PR283, PR385

Ellabib M. PR279

Elsoe R. PR360

Emmink B. PC165, PC188, PC190, PC196, PC198, PR399, PR424, PR436, PR448

Endo A. O047

Ene D. PR101

Enescu G. PC192

Erginy C. PR039

Erhardt J. PC189

Eryigit Unaldi H. PC023

Escames-Rosa G. PR080, PR081

Eskesen T.G. O033, PR141

Espert Ibáñez J.J. PR075

Esteves A. PC081, PC086

Esteves J. PC154

Estraviz-Mateos B. PC120

Etoundi Mballa G.A. O061, PR148

Etxart Lopetegi A. PR075

Euthymiou G. PR029, PR300

Eythymiou G. PC006

Ezra S. PR061

Fadden E. PR088

Fadden S.J. PR309

Fagenholz P.J. O089, PR141

Fakler J.K.M. PR007, PR277, PR335

Falcó J. PC046, PC061

Falkenberg M. PR138

Farazi-Chongouki C. PC011, PC152

Farhat H.M. PC075

Farré R. PC054, PC145

Fattori L. PR037, PR087, PR094, PR097

Faulconer E.R. PR217

Fazendin J. O021

Felicio C. PC069, PR270

Ferguson J. PR301

Feria González A. PR155

Fernandes P. PC069

Fernandes S. PC001

Fernandez Alvarez M. PR208, PR371

Fernandez M. PR269

Fernandez Martinez M. PR268

Fernandez Vazquez M.L. PR268

Fernandez-Moreno M.D.C. PR072, PR323

Fernandez-Velilla B. O076, PR012, PR023

Fernández Balaguer P. PR075

Fernández García F. PR075

Fernández Vega L. PR091

Fernández-Burgos I. PC195

Ferrada R. O013, PC042

Ferraris M. PC057

Ferree S. PR351

Ferreira C. PC038, PC081, PC086, PC095, PR047
Ferreira E. PC063

Ferreira M. PC099

Ferreira M.M. PC013

Ferreira M.S. PC021

Ferreira P.S. PC009, PC073

Ferrero E. PC028, PC029, PC030, PC033, PC092, PR046, PR184

Ferrero Recasens J. PC194, PR356, PR398, PR445

Ferrigni C. PC043

Filip A. PC161

Filipov A. PR136

Filipovic G. PC016

Findlay C.A. PR159

Finkbeiner R. PR293, PR296

Fischer H. PR235

Fischinger A. PC025, PC175, PR247

Fleming C.D. O050, PR216

Fletcher Sanfeliu D. PR085, PC098

Flores Cortes M. PR064

Foco M. O092, PR063

Follet L. PR186

Fonseca A. PC013

Forman M. PR350

Forsman D. PR209

Foss N.B. O036

Fragkeskakis G. PR400

Fragoso M. PR006

Fraguero M.-J.P. PR095

Fraile Alonso I. PC066, PC119

Fraile J.J. PR078

Frank B. PR303

Frank J. O069, PR430

Fransvea P. O071, PC104, PR014, PR017, PR310

Frasson M. PC098, PR085

Frattini C. PC027, PC040, PC053

Freiman T. PR009

Freschi M. PC082, PC107

Frezza B. O071

Friemert B. PR264A

Frima H. PR334, PR395, PR399, PR407, PR416

Frolke J.P. O025

Fu C.-Y. PR366

Fujita M. PC162

Fukui T. PR331

Funabiki T. PR220

Furrer M. PR334

Furtado I. PC051

Furuya S. PR130

Futamura K. O008, PR388, PR404

Gagauz I. PR068

Gajski V. PC172

Gakidis I. PR289

Galbete A. PR012, PR023

Gallardo J. PR353

Gallego-Vila Á. PR095, PR098

Galo T. PR110, PR124

Galvagno S.M. PR314

Galvanin J. PC139

Galvez A. O021

Gamero Huamán J.C. PC159, PR027, PR028, PR051

Gamo C. PC024B, PC048, PC064A

Gamundi Cuesta M.B. PR091

Ganescu R. PR287

Garcia A.F. O013, PC002, PC042

Garcia Gregorio N. PC098

Garcia H. PC151

Garcia Hernandez J.C. PC195 
Garcia Lamas L. PR445

GarciaOlmo D. PC043

Garcia Perez J.M. PR171

Garcia Sevilla A. O064, PR155

Garcia-Granero A. PC098, PR085

Garcia-Leon N. PR250

Garciafilia Cabrera L. O063, O077

Garcià Juarraz C. PR165

Garcés-Albir M. PR072, PR323

García A.F. PR292, PR299, PR306

García Fuentes C. PR165, PR175

García L. PR354

GarcíaOlmo D. PR075, PR077

García Salcedo J.A. PC026

García Sáez I. O078

García Ureña M.A. PR075

García VillarO. PC028, PC029, PC030, PC033, PC092, PR035, PR046

García-Fernández N. PR065, PR074

García-Moncó C. PR353

García-Moreno Nisa F. PR075

García-Rivera C. PR158

Garlito Díaz H. PC164

Garrigue D.H. PR211

Gaspar B. PR287

Gattuso M.I. PR094

Gaudric J. O054

Gavilanes Calvo C. PC135A

Gavrankapetanovic I. PR200

Gavril L. PR055

Geeraedts L. O042, PR144, PR164

Gefen A. PR061

Gehweiler D. O085

Georgescu E.F. PR079

Georgiadis G. PC181

Georgis P.A. PR289

Gerogiannis I. PC122, PR057

Ghahramani Z. PR174

Ghanaati S. PR058

Gheju I. PR287

Gheorghevici T.S. PC192, PR375

Ghita L. PC068

Gianchandani Moorjani R. PR163

Giannakaki K. PC181

Giannakopoulos D. PC115, PC123, PR320

Giannakopoulos G. O042, PR164

Giannakopoulos G.F. PR240

Gianotti L.V. PR037, PR087, PR094, PR097

Gichuki S.M. PR189

Giesbrecht G. PR204

Gil Rodriguez J. O077

Girshausen R. PR162

Givon A. PR142

Gkanas P. PC006, PR029, PR300

Gkegkes I. PC122

Gkrilias P. PR134

Glagolieva A. PC111, PC155

Gleiss A. PR329

Goena Iglesias I.M. PR075

Goganau A.M. PR079

Goikoetxea A. O076, PR012, PR023, PR093

Goldenberg-Sandau A. PR303

Golikov D.E. PR039, PR119, PR133, PR298

Gombár C. PR402

Gomes C. PC081

Gomes F.S. PR401, PR439
Gomes J.A. PR425

Gondert M. PR261

Gonzalez A. PC024B, PC048, PC064A

Gonzalez Argente F.X. PR091, PR171, PR317

Gonzalez De Pedro C. PC084

Gonzalez J.T. PR064, PR434

Gonzàles Lòpez A. PR165

González Gómez C. PR075

González S. PC059

González Valverde F.M. PR075

González-Argenté F.J. PR060

González-Tartière P. PR376

Gonçalves I. PC063

Gonçalves M. PC067

Goormans F. PC173

Goos J. PC188

Goparaju A. PC017, PC105, PC113

Gordini G. PC027

Gorelik A.L. PR039, PR103, PR104, PR119, PR122, PR133, PR298

Goslings J.C. O027, O080

Gracia Roman R. O049

Gradica F. PR318, PR321

Grand B. PC106

Grande-Posa L. PR078, PR089, PR095, PR098

Granel F..J. PC051

Grao Torrente I. PR370

Grechenig P. PR418

Groenwold R. O025

Groenwold R.H.H. PR339

Grosso E. PR262, PR333

Grosu A.M. O043, PR383, PR443

Grunert R. O087

Gràcia R. PC046, PC061

Guerrini J. O017, PC103, PC139, PR036, PR243

Gui D. O092, PR063, PR319

Guillamot P. PC051

Guimarães N. PC013

Guixà-Gener M. PR250

Gulle H. PR301

Gultekin S.S. PR056

Gumaa D. PR062

Gupta S. PR003, PR314

Gurghis R. PR068, PR308

Gurney J.M. PR253

Gutierrez Andreu M. PC026, PC028, PC029, PC030, PC033, PC092, PR035, PR046, PR173, PR184

Guzmán-Ahumada J. PR095

Gámez P. PC026

Gárgyán I. PR245, PR256

Gómez De La Portilla A. PR075

Gómez Pérez R. PR075

Gómez-Bravo M.Á. PR158

Görig T. O056, PR224, PR259, PR263

Güell Farre M. PC145

Güell M. PC054

Gümbel D. O055, O056, O058, PR224, PR241, PR254, PR259,

PR263, PR264, PR421

Güsgen C. O095, PR312

Ha S.C. O094

Haak T. O031

Haase D.J. PR314

Hackl M. PR429

Hagan N. O074

Hagiwara T. PR221

Hajdu S. PR329 
Hallal A. O018, PC075, PR050

Haltmeier T. O032, PR020, PR030

Hamming J.F. PR213

Han K. O089

Handolin L. PR138, PR147, PR177

Hanu A.-L. PC083, PC134

Hara H. PC047, PR206, PR304

Harada S. PR391

Harcuba R. O082

Hartel M.J. O007, PR367

Hartwell J. PR303

Harvin J. PR303

Hasadia R. PR142

Hasara R. PC022

Hatz B.A. PR416

Haupt S. PC166

Haverkamp F. PR204, PR242

Haverkort J.J.M. PR313

Haverkort M. PR230

Headon R. PR403

Hebron D. PR138

Hedeman Joosten P.P.A. O046

Heeres M. O038

Hefny A.F. PC147, PR325

Heimel P. O081

Heineman E. PR151, PR372

Hekman E.E.G. O088

Helfrich F. O050, O075

Hellebrekers P. O038, O040

Heller G. PC166

Heller H. PR144

Heneghan H.M. PC094, PR229

Heng M. PR338

Hennig F.F. O023, PR198, PR293, PR296

Henning E. O056, PR224, PR259, PR263, PR264

Henrich D. O069, PR058

Henriques C. PR238

Herijgers P. O083

Hermans E. O006

Hernandez Gutierrez J. PC119

Hernandez Merlo F. PC076

Hernando Sanz A. O076

Hernández Gutiérrez J. PC066

Herrera J. O076, PR012, PR023

Herrera-Vizcaíno C. PR058

Herrmann V. O082

Hershko D. PR248

Herteleer M. O083, PC173, PR161

Hesselink L. O038, O040

Hettiarachchi N. PR088

Heyde C.-E. O026, PR335

Hietbrink F. O025, O038, O040, PR223, PR313, PR338, PR339,

PR351

Hildebrand F. PR115, PR162

Hilário S. PC063

Hirani N. PR191

Hirose T. PR153, PR220

Hirschmann M. PR198

Hishikawa S. O045

Hjortnaes J. PR222

Hoareau G. PR217

Hodel S. PR442

Hoefer I. O038

Hoekstra H. PR161

Hoencamp R. O046, O051, PC024A, PC169, PC178, PR213, PR219, PR435
Hohenberger G. PR415, PR418, PR429

Hohmann T. O087, PR335A

Holena D.N. PR156

Holmgren K. PR226

Holub A. PC035, PC050, PC144, PC182, PC184, PC185, PC186, PC191, PC197, PC199

Holzgang M. PR030

Honan D.M. O050A

Hondo K. PR032, PR231, PR271, PR273

Hoogendoorn J.M. PR339

Horchinova E. PR096

Hornez E. O054, PC106, PR227, PR260

Horodyskyy M.I. PR360

Horst K. PR115, PR162

Houwert R.M. O025, PR223, PR339, PR340, PR351, PR395, PR399, PR407

Hristov H. PC187

Hsia C.-C. PR366

Hu P. PR314

Huang Y. PR156

Hughes B. PR403

Huisman A. O038

Huizing F. PR358

Huizinga E.P. O051

Hulsewé K.W.E. PR275, PR282

Humenberger M. O081, PR329

Hung Y.L. O090

Hustinx P.A. PR275

Hyder A. PR123, PR191

Hyun S.Y. PC078, PR284

Höch A. O087, PR335A, PR426A

Hörer T.M. O041, O046, PR138, PR209, PR213

Ibañez Aguirre F.J. PC064, PC120

Ibáñez-Aguirre F.J. PR073

Idenburg F.J. O051

Idoguchi K. PR138, PR220

Iiduka R. PC041, PR215

Ijpma F.F.A. PR372, PR406

Ilie A.C. PR346

Inaba K. O024, O032, O045

Ingelsrud L.H. O036

Ingeman A. O068

Íñigo J.J. PR093

Inokuchi K. PR330

Inoue J. O044

Intxaurraga K. PC120

Inui T. O002, PR330

Ioannidi L.-E. PC181

Iordanou C. PC011, PC152

Iorga C. PR076

Iorga C.R. PR076

Ira D. PR437

Irizarry E. PC005, PC017, PC133

Irungu E. PR189

Isabel Grifo Albalat I. PR075

Ishida K. PR220

Ishida T. PR138, PR220

Ishii W. PR215

Isla A. O014

Ito K. PR120, PR373

Ito T. PC012

Ivakhov G. PR038

Ivanoschi A. PC083, PC134

Ivantsova A. PR111

Ivchenko D. O029, PR004

Ivković K. PR110, PR124 
Iwakura T. PR327, PR331, PR389

Iwami T. PR130

Izawa J. PR153

Izawa Y. O045, PR194

Jacobs L. O042

Jagdish J. PR325

Jakopec M. PC010

Jang J.Y. PR381

Janko M. PR430

Jansson K. PR209

Jarvers J.-S. O026, PR335

Jasper J. PC169, PC178

Jenny K. PR361

Jensen K.O. PR157, PR267

Jeon Y.B. PC078, PR284

Jeong I. PR294

Jiamton C. PR364

Jimenez Alvarez L. PC076

Jimenez Diaz V. PR445

Jimenez Morillas P. PR091, PR171

JimenezOlmo F.J. PC032

Jimenez Viñas C. PR171

Jiménez Armenteros F.M. PC091A, PC097, PC112A, PC112B,

PC148A

Jiménez Fuertes M. PC043, PR075, PR077

Jiménez L.Á. PC051

Jiménez Moragas J.M. O078

Jiménez Morillas P. PR075

Jiménez Rodríguez R.M. PR075

Jiménez V. PR354

Jiménez-Ramírez A. PR060

Jo Y. PC045, PC055, PC080

Johnson E.D. PR187, PR203

Johnson M.A. PR217

Jones C. PR309

Jonkers I. O083

Jonuzaj E. PR318, PR321

Joo I.H. PR348

Joo S. PC078, PR284

Joosse P. PR347, PR412

Joshi A. PR249, PR419

Josten C. PR007, PR277, PR335, PR335A, PR426A

Jover Navalón J.M. PR075

Juillard C. O061, PR128, PR148

Jukema G.N. PR417

Jurado Marchena R. PC077

Jurčević I. PC172

Jønsson L.R. O036

Kaafarani H. O089, PR141, PR237

Kabir U. O096

Kaewsaengrueang K. PR188

Kahn T. PR335

Kaiser J. O079

Kaji M. PR032

Kaku T. PR431

Kalayci M.U. O015

Kaldis V. PC115, PC123, PR320

Kalff J.C. O095

Kamer L. O022, O085

Kamimura M. PR382, PR396, PR432

Kampouroglou N. PC037

Kanda N. PC201

Kanezaki S. PR195, PR362, PR378

Kang W. PC045, PC055, PC080

Kapustin S. PR004

Karamanidi M. PC011, PC085, PC152
Karamanos D. PR045, PR214

Karaseva O.V. PR066, PR096, PR039, PR103, PR104, PR119, PR122, PR133, PR281, PR298

Karimi E. PR345

Kasotakis G. O068A

Kastberg A. PR138

Kastner P. PR202

Katayama Y. PR153

Kato R. PR120, PR373

Kato S. PR059, PR185, PR272

Katoh N. PR391

Katsaros K. PR029, PR300

Katsichtis T. PC005, PC017, PC031, PC110, PC133

Kattakayam A. PR099

Katz V. PC017, PC113

Katzengold R. PR061

Kaufman E.J. PR156

Kawakubo A. PR357

Kawamura T. PC200, PR357, PR408, PR426

Kawano H. PR330, PR394, PR405

Kawashima S. PR059, PR272

Kayser Mata S. O063

Kayser S. PR049

Keel M. O009

Kehler M. O030

Keizer J. O031, PC165, PC188, PC190, PC196, PR340, PR399, PR424, PR436

Kellett S. PR196

Kelley K. PR303

Kermanpour H. PR345

Kessel B. PR138, PR142

Kettner S. PR329

Khan M. O032

Kharitonova A. PR122

Khor D. O024

Khuri S. PR290

Kigongo S. PC113

Kiguchi T. PR153

Kikić D. PC172

Kim D. PR294

Kim G. PR294

Kim J. PC045, PC055, PC080

Kim J.J. PR348, PR363

Kim J.W. PR348, PR363

Kim M.J. PR374

Kim S.J. PC078, PR284

Kincses Z. PR127

Kindler C. PR209

King D. O089

Kishimoto M. PR449

Kisilak M. PR411

Kita T. PC176

Kitada S. PR328, PR447

Kitamura T. PR130, PR153

Kitano T. PR185

Kitridis D. PC160

Kiyohara K. PR153

Kiyono M. PR169, PR342, PR355, PR380, PR384, PR390

Kjaer I. PR360

Kjossev K. O059

Klapsakis D. PC160

Kleinveld S. PR358

Klem T. PR326

Klocker J. O057

Klokočovnik T. PC004

Kluger Y. PR290 
Knulst R. PC100

Ko S. PR336, PR337

Kobabayashi Y. PC130

Koch S. PC100

Koenderman L. O038, O040

Koguchi H. PC047

Koh C.-C. O094

Kolodziej J. PC172

Koltovich A. O029, PR004

Kon Y. PR138, PR220

Kon Z.N. PR003

Kondo H. PR220

Konecny J. PC022, PR295

Kongkaewpaisan N. O089, PR237

Kongwibulwut M. O089

Konrad C. PR008

Konstantara F. PR289

Konstantiniuk P. PR415

Konstantoudakis G. PC006, PR029, PR300

Konstas A. PC160

Kontradowitz K. PR058

Kootstra J.J. O088

Kootstra T. PR340, PR448

Kopač C. PR332

Korac Z. O028

Kordež D. PR247

Kos J. PC163

Kostic I. O005, PR344

Kostikova T. PR126, PR135

Kotakos C. PR024

Kourtzis D. PR400

Kovalenko M. PR011, PR122

Košir S. PR247

Kraeima J. PR406

Kramer W.L.M. PR129

Krasniqi G. PC066

Krassnig R. PR415, PR418

Krijnen P. PR358

Krinner S. O023, PR198, PR293, PR296

Kristan A. PC010

Kristensen M.T. O036, O068

Krticka M. O079, PR437

Kubota T. PC112

Kucejko R. O072

Kuchling S. PR418

Kuijper M. PR326

Kujirai D. PR194

Kunitatsu K. PR059, PR272

Kuorikoski J. PR147, PR177

Kurata Y. O002

Kurihara H. O017, PC103, PC139, PR036, PR243

Kuroda R. PR331, PR389

Kurosa Y. PR431

Kusý D. PR350

Kószó B. PR245

Křivohlávek M. O082

La Greca A. O092, PR063, PR319

Lage J.C. PC038, PC095, PR047

Lages R.R. PC154

Lal D.P.C.K.A. PR113

Lalić I. PR444, PR446

Lameijer C.M. PR409

Lan T.-Y. PR118

LandaluceOlavarría A. PR075

Landaluce-Olavarria A. PC064, PC120, PR073

Langat E. PR189
Langenbach A. O023, PR198, PR293, PR296, PR421

Lansink K. O006, O060, PR339

Lara Fernández Y. PR043, PR051, PR092

Lara Palmero C. PC172A

Laroye C. PR211

Larsen P. PR360

Larzon T. PR138

Latifi R. O039, PR149

Laureano M. PC063

Lavado C. PC069, PR270

Lavanchy J.L. PR030

Lawler J. O096

Lazar M.C. PR079

Lazaridis I. PR045, PR214

Le T.D. PR253

Lecky F. PR183, PR228

Lee C.-H. PR118

Lee J. PR284

Lee J.M. O089

Lee J.S. PR363

Lee S.H. PR374

Lee S.Y. PR328, PR331, PR389

Leenen L.P.H. O025, O038, O040, PR129, PR176, PR222, PR223,

PR230, PR313, PR338, PR339, PR351

Leenhouts P.A. O080

Lefering R. PR115, PR198, PR296

Leijnen M. PR386

Leirinha M. PR425

Leli R. PR086, PR090

Lenk M. O087

Leon Baltasar J.L. PR365

Levin A. PC016

Liao C.-A. PR286

Liao C.-H. PR366

Liao P.F. PC174

Lichtveld R.A. PR176, PR222

Liew Y.K. PR143

Lim K.X. PR106

Lin H.-F. O012

Lin H.F. PC049

Linacero Martín S. PR075

Lingam P. PC129

Lingsma H. PR183, PR228, PR369

Link B.-C. PR235, PR364, PR442

Linton K.N. PR377, PR403

Lintzeris I. PR031

Liotta G. PR310

Llaquet Bayo H. O049, PR140

Llompart-Pou J.A. O078

Lobo Gonçalves I.M.F.D. PC076

Lodoli C. O092, PR063, PR319

Loggers S. PR347

Logrado A. PC114, PC156

Lokerman R.D. PR176

Long K. PR132

Longras A.C. PC090

Lopez De Heredia Armentia E. PR075

Lopez L. PR341

Lopez Perez J. PR043

Lopez Ruiz J.A. PC140, PC142, PC158, PR025, PR028, PR043, PR044, PR051, PR092, PR322

Lopez-Mozos F. PR072, PR323

Lorenz P. PR267

Loureiro R. PC018, PC062, PC109

Lourenco R. PC008, PC090

Lucena González M.J. PC066 
Lueders M.E. PC005, PC031, PC133

Luengo Alonso G. PC168, PC194, PR356, PR365, PR445

Luna Aufroy A. PC046, PC061

Lunevicius R. PR137, PR182

Lupescu D. O043

LupescuO. O043, PR205, PR383, PR414, PR441, PR443

Lupiani Moreno R. PC032, PC149

Lusilla López A. O064, PC132, PR155, PR163, PR269

Lustenberger T. PR009, PR167

López Dorado D. PC164

López Durán B.L. PC140, PC158, PR026, PR044

López Pérez J. PC142, PC158, PC159, PR026, PR027, PR028, PR044, PR051, PR092

López Ruíz J.A. PC159, PR026, PR027

López Sánchez J. PC150

Ma D.S. PC078, PR284

Maas M. O080

Maayen R.C.L.A. PC100, PR386

Machado L. PC137

Macleod J. PR189

Madan V. PR438

Madani R. PC070, PC074

Madrazo Z. PR075

Madrid B. PC034

Madurska M.J. PR138

Magalhaes A. PC102

Magalhães A. PC090

Magalhães M. PC101

Magalini S. O092, PR063, PR319

Magdalena López J.C. PR075

Magrach Barcenilla L.A. PR075

Mahendraraj K. PC005, PC017, PC113

Maher Z. PR303

Mahmood I. O098, PR279

Mahmoudi Nezhad G.S. PR302

Maier M. PR418

Maio M. PC167, PC171, PR179, PR343

Makovec G. PR332

Maksymiak R. PR435

Malaquias J. PR270

Malgras B. O054

Malik H. PR099

Malik S. PR123

Maliko N. PR435

Malo Corral J.S. PC066

Malovrh T. PC175

Mammano E. PC007

Manchev V. PR138

Mancuso R. PC104, PR014, PR310

Manea M.L. PR079

Manley N. PR303

Manning J. PR138

Manon J. PR349

Mansilla-Rosello A. PC059, PR080, PR081

Mantelas M. PR045, PR214

Manu N. PR166, PR309

Manzano R. PR292, PR299

Manzhurtsev A. O035, O037, PR102, PR105, PR111, PR112, PR116, PR281

Marante Fuertes J. PC035, PC050, PC144, PC182, PC184, PC185, PC186, PC191, PC197, PC199

Marante Fuertes J.A. PC144, PC185, PC199

Marco López I.M. PC135A

Marconi M. PC082, PC107

Marenco De La Cuadra B. PR025, PR027, PR028, PR322
Margallo E.P. PC140, PC159, PR026, PR027, PR028, PR044, PR092

Marian R. PC068, PR101

Marini C.P. O034

Marini P. PC104, PR014, PR017, PR310

Markovtsova A.V. PR181, PR203

Marone M. PC057

Marosán P. PR127

Marques A. PC062, PC109

Marques C.N. PC036, PR193

Marques R. PC081, PC086

Marquez Ambite J.C. PC164

Marte G. PR307, PR324

Martelo R.M. PC036, PR193

Marti R. PC194, PR131

Martin E. PR075

Martin J. PC136

Martin Martin G.P. PR317

Martin Roman L. O077, PR049, PR268

Martinez E. PC034

Martínez Casas I. PC091A, PC112B, PC148A

Martinez Lopez F. PR232, PR379

Martinez Moreno C.M. O063, PR049

Martinez Soriano F. PR085

Martins A.P. PC067

Martins C. PC056, PR425

Martins M. PC008, PC154

Martins P. PC143

Martins R. PC151

Marttala J. PR138

Martí-Obiol R. PR072

Martínez Cabañero J. PR190, PR379

Martínez Cecilia D. PC066

Martínez Núñez S. PR064

Martínez-Nuñez S. PR034

Martínez-Solà A. PR098

Maruo A. PR382, PR396, PR432

Marzi F. PR319

Marzi I. O069, PR009, PR058, PR167, PR234, PR430

Marçal A. PC095

Masek M. PR437

Mashar R.P. PC136

Mashbari H. PR303

Mason J. PR088

Massa G. O071, PR017

Massalis I. PC006, PR029, PR300

Massani M. PR307, PR324

Masuda A. PC176

Maszkowski M. PR138

Mata Sancho F. PR075

Matamalas A. PR376

Mateo J. O076, PR012

Mateo Retuerta J. PC128, PR093

Matev N. PC147

Mathew A.M. PC116

Mato T. O045

Matos A. PC013, PR238

Matsuda M. PR276

Matsuhima K. PR231

Matsui K. PR330

Matsumoto J. PR138, PR220

Matsumura Y. PR138, PR218, PR220

Matsushima K. O024, O045

Matsushima S. PR120, PR373

Matsushita T. PR391, PR394

Matsuura T. PC200, PR408 
Matsuyama T. PR153

Mattara G. PC007

Matzaroglou C. PR134

Matzi V. PR415

Maure Blesa L. PR165

May C. O009

Mayr W. O081

Mazzucchelli R.A. PC189

Mccracken B. O021, O072

Mcfall M. O067

Mcgreevy D. PR138

Mcphee D. PC105, PC110

Medina C. PR160

Medina E. PR181

Medina Reinoso C. PC076

Medinskiy P. PR121

Medrano G. PC005, PC017, PC110

Meesters A.M.L. PR406

Meesters B. PR275

Mehmood A. PR123, PR191

Mei S. O017, PC103, PC139, PR036, PR243

Meier S.L. PR234

Mel'Nikov A.V. PR104

Melnikov I. O035, PR105, PR108, PR116, PR126

Melo A. PC038, PC081, PC086, PC095, PR047

Melo F. PC013

Membrilla-Fernández E. PR078, PR089, PR095, PR098

Memè L. PR413

Menaker J. PR003

Mendes A. PR280

Mendoza A. O089

Mendoza F. PC051

Mendoza Moreno F. PC076

Menegozzo C.A.M. PR315, PR316

Meneses Pardo J.C. PC026

Menne A.R. PR314

Menshchikov P. O035, O037, PR102, PR105, PR111, PR112,

PR116, PR281

Menè R. PC103, PR036

Merschin D. O055, O058, PR241, PR254, PR359, PR421

Mesquita C.A.G.C. PC052

Metsemakers W.-J. PC173

Metzger J. PR235

Mica L. PR157, PR267

Michelitsch C. PR334, PR395

Michlovska L. O079

Michos P.T. PR289

Michos T.P. PR289

Mici A. PC070

Micic I. O005, PR344

Micu N. PR265

Migliori E. PC007

Mihailescu A. PR048

Mihopoulou K. PC011

Mikhailovskaya E.M. PR218

Milenkovic S. O005, PR344

Miletic M. O028

Mimura Y. PR408

Minehara H. PC200, PR408

Mingler B. O081

Minguez Garcia J. PC076

Minguez J. PC051

Miranda C. PC001, PC024B, PC064A

Misra N. O019A, O067, PR088, PR099, PR137, PR159, PR182,

PR309

Misselyn D. PR392
Mitish V. PR121

Mitkovic M.B. O005, PR344

Mitkovic M.M. O005, PR344

Mitrakos I. PR320

Miya H. PR382, PR396, PR432

Miyake T. PC201

Miyamoto W. PR394, PR405

Miyazaki M. PR362

Mizobata Y. PR221

Moayad W. PR152

Mochizuki Y. PR169, PR380, PR384, PR390

Modet S.G. PC024C, PC024D, PC024E, PC102A, PC102B, PC172A

Mogollón M. PC059

Mohammed M. O068A

Mohseni S. O003, O066

Molina Díaz I. O078

Molina J. PR082

Mollazehi M. PR191

Monchal T. PR260

Monforte X. PR301

Montejo González J.C. PR165, PR175

Montmany Vioque S. O049, PC046, PC061, PR140

Montuori M. PR037, PR094, PR097

Montón S. PC015, PC058, PC128

Moody N. PR154

Moore E.E. O030, O050, O052, O070, O075, PR006, PR216

Moore H.B. O050, O070, O075

Moori P.L. PR309

Morais H. PC013

Morais J. PC036, PR193

Morales Bernaldo De Quirós J. PC108, PR021

Morales Calderon M. PC034

Morales D. O073, PR015, PR016, PR019, PR266

Morales García D. PR018

Morales Soriano R. PR171

Morales-Garcia D. PR208, PR371

Morales-Soriano R. PR060

Moreira H. PC081, PC086

Moreira R. PC154

Moreno Bargueiras A. PR046, PR184

Moreno Hidalgo A. PC108, PR370

Moreno Torres B. PC119

Moreno-Suero F. PC084

Morgado M. PC089, PC091

Mori K. PR391

Mori S. PC020, PR032, PR236

Morishita K. PR032, PR231, PR271, PR273

Moritz C. PC106, PR260

Moriya M. PC130

Morlán López M.Á. PC066, PC119

Morrison J.J. O046, PC044, PR138, PR210, PR218, PR305

Morton B. PR088

Mota P. PC167, PC171, PR179, PR343

Motiei M. PR061

Motwani G. PR148

Mouemel S.M. PR192

Moya J.L. PC015, PC058, PC128

Moynihan A.L. PR052, PR229

Mperdelis V. PC152

Mpozonelos G. PR400

Mucenic S.C. PR079

Mueller T.S. PR202

Muhrbeck M. PR242

Mulders M.A.M. O027

Mun D. PR040 
Muneer M. PR385

Munevar H.E. O013, PC002, PC042

Muntaner N. PR060

Muraoka T. PR440

Murata K. PC047, PR206, PR304

Muratsu H. PR382, PR396, PR432

Murthi S.B. PR314

Mustafa F. O098

Mutafchiyski V. O059

Muthee S.W. PR189

Muñiz Tolivia D. PC195

Muñoz Bellvís L. PC150

Muñoz Garcia G. PC177, PC183

Muñoz Jimenez B. PC066, PC119

Mácsai A. PR256

Méndez Gómez B. PC148

Mónica I. PC013

Nagea M. O043, PR205, PR383, PR414, PR441, PR443

Nagy A. PR402

Nagy S. PR127

Nair M. PC124

Naja K. O018

Nakagawa Y. PR153

Nakama R. PR194, PR197

Nakamoto R. PC047, PR206, PR304

Nakanishi Y. PR120, PR373

Nakano T. PC012

Nalbandyan R. PR121

Nanwani K. O078

Naranjo Fernández J.R. PC077

Naranjo M.P. PR292, PR299

Narbona Calvo B. PC118

Nardai G. PR245

Narsule C. O068A

Nasirov F. O091, O097

Nasu T. PR059, PR272

Natroshvili A. O091, O097

Natsiopoulou A. PC152

Navaratne L. O014

Navarrete De Carcer E. PC077

Navarro C. O013, PC002, PC042

Navarro E.B. PC155

Navarro Morales L. PR064

Navarro Soto S. O049, PC046, PC061, PR140

Navas-Cuéllar A. PR084

Navas-Cuéllar J.A. PC096, PR100

Navio Seller A. PC098

Nawijn F. PC165, PC190, PR351

Neff L. PR217

Negoi I. PR022

Neijenhuis P. PC135

Nekuda V. O079

Nelissen R. PR417

Nerlander M.P. PR152

Nespoli L.C. PR037

Nessen S.C. PR253

Neto T.S. PC008, PC102

Nevado M.C. PC028, PC029, PC030, PC033, PC092, PR035, PR046

Neves J. PC013

Nevins E.J. PR309

Niikura T. PR328, PR331, PR389

Nijs S. O083, PC173, PR161

Nikishov S. PR125, PR135

Nikolaev K. O029, PR004, PR252

Nilsson K.F. O041, PR138, PR209
Ninos A. PC011, PC152

Ninov B. PR136

Nistri C. PR307, PR324

Nitta M. PR130

Nobre J. PC063

Noda T. O086, PR169, PR221, PR342, PR355, PR380, PR384,

PR390

Nogueira H. PC180, PC193

Noguerales Fraguas F. PC076

Nonnemacher San Julián A. PC148, PR207

Norblad R. PC016

Nordestgaard A.T. O089, PR237

Noser H. O022, O085

Notani N. PR195, PR362, PR378, PR428

Notario-Fernandez P. PR080, PR081

Novo F.C.F. PR315

Ntikoudi A. PC088, PC141

Ntikoudi E. PC141

Nunns G.R. O030, O052, O070, PR006

Nwanna-NzewunwaO. PR148

Nzamushe J.R. PR211

Nürnberger S. O081

O’Connor J.V. PR003

O'Dowd J. PC116

O'Grady P. PR403

Oak S. PR168

Oates V. PC116

Obelchak I. PR252

Ochen Y. PC196, PR399

Ochogavia Segui A. PR091

Oda S. PR231

Oe K. PR328, PR331

Oehme F. PR235

Ogawa K. O086

Ogura S. PC201

Ogura T. PR194, PR197

Oh Y. PR431

Ojea L. PC024C, PC102B, PC172A

Ojeda C. PR341, PR353

Oka T. PR032

Okada A. PC041

Okada H. PC201

Okada Y. PR215

Okamoto H. PC201

Okawa A. PR431

Okawara K. PR276

Okishio Y. PR059, PR272

Oldani M. PC082, PC107

Oliva López I. PC135A

Oliva Mompean F. PC077, PC140, PC142, PC158, PC159, PR025, PR026, PR027, PR028, PR043, PR044, PR051, PR092, PR322

Oliveira A. PC018

Oliveira A.S.R.S.F. PC052

Oliveira E. PC154

Oliveira J. PC008

Oliveira M. PC102

Oliveira N.F. PC089, PC091

Oliveira T. PC001

Oliveira V. PR401

Oliver Garcia L. PR207

Oliver García L. PC148

Olivero G. PR086, PR090

Ollé E. PC195

Olmos Juste V. PC135A

Olona Casas C. PR075

OlshaO. PR069 
Omi E. PR303

Onchalos U. PC195

Onoi Y. PR382, PR396, PR432

Oor J. PC198

Oosthuizen G. PR138

Ordoñez C.A. PR292, PR299

Ortega Garcia F.J. PR010

Ortega Higueruelo R. PC091A, PC112A

Ortega J. PR072, PR323

Ortega M.C. PR341

Orti Rodriguez R. O064, PR163

Orue -Echebarria Gonzalez M.I. PR370

Orue-Echebarria González M.I. PC108

Oshima T. PR382, PR396, PR432

Osinloye W. PR009

Osmanov E. O097

Ossendorf C. PR267

Osterhoff G. O001, PR267, PR420

Otegi I. O076, PR012, PR023

Otomo Y. O019, O044, O047, PC020, PR032, PR071, PR231, PR236, PR271, PR273

Ovejero Gomez V.J. PR208, PR371

Ovejero V. PR016, PR019

Owattanapanich N. PR199

Ozaki T. O086, PR169, PR342, PR355, PR380, PR384, PR390

Ozdemir A. PC023

Ozer K.B. PC023

ÖzkurtulO. PR007, PR277

Ozlem N. PC071, PC087, PC157, PR033, PR056, PR139, PR274, PR288

Ozlu F.T. PC023

Öztürk B. O068

Pacilè V. PC082, PC107

Padillo-Ruíz J. O011, PC084, PC096, PC117, PC146, PR025, PR034, PR065, PR074, PR082, PR083, PR084, PR100, PR158, PR322

Pagaki C. PC011, PC085

Pais J. PC063

Pajenda S. O081

Palau-Figueroa T. PR250

Palm H. O068

Palma Caucig N. O063

Palmero C.L. PC024C, PC024D, PC024E, PC102A, PC102B

Panero F. PR090

Panzera R. PR319

Papa L. PR014, PR310

Papachristou A. PC088, PC141

Papadima E. PR029, PR300

Papadopoulos V. O062, PR146

Papagiannakos K. PR400

Papaioannou G. PC011

Papakonstantinou E. PC152

Pape H.-C. O001, PR115, PR267, PR417, PR420

Pape M. PR164

Pappalardo R.A. PC082, PC107

Paraschiakos F. O038

Pareja Ciuró F. PR064

Pareja-Ciuró F. O011, PC084, PC096, PC117, PC146, PR025, PR034, PR054, PR065, PR074, PR082, PR083, PR084, PR100,

PR158, PR322

Park K.C. PR348, PR363

Park Y. PC045, PC055, PC080

Parreño Manchado F.C. PC150

Parva E. PR174

Parvanov Y. PR136

PascaneanuO. PC192
Paspaliaris C. PR320

Pastor Mora J.C. PC127

Patel K. PR303

Patel S. PR166

Paterson H. PR246

Paterson-Brown S. PR246

Patias P. PR029, PR300

Patricia Pérez De Villarreal P. PR075

Patru C. PR205, PR441

Pauletti B. PR307

Paun S. PR022, PR287

Payandemehr P. PR345

Paydar S. O003, PR174, PR302

Pedersen A.B. O068

Pedro J. PC170

Peixoto D. PR280, PR401, PR439

Pek J.H. PR143

Peleg K. PR142

Pellisé F. PR376

Pentara I.C. PC006

Pepe G. O092, PR063, PR319

Peponis T. PR141

Peralta R. O098, PR123, PR191, PR192, PR283

Perchoc A. PC106, PR260

Pereira J. PC039, PC093, PC114, PC131, PR238

Pereira J.M. PC180, PC193

Pereira R. PC081, PC086

Perez Gonzales M. PC076

Perez J.A. PR207

Perez Margallo E. PC142, PC158, PR043, PR051

Perez-Nuñez I. PR208, PR371

Perl M. O010

Petrič M. PC004, PC060

Petrone P. O034

Petrov H. O059

Petukhov V. PR038

Peña S. PR203

Peštál A. PR295

Pfeifer R. O001

Phadnis J. O028

Pieroh P. O087, PR335A

Pierrakakis S. PC011, PC085, PC152

Pijnenburg A.M. PR275

Pilati P. PC007

Pimentel A. PC154

Pina G. PR425

Pineño Flores C. PR091, PR171, PR317

Pinho J. PC013

Pino Diaz V. PR434

Pino Sánchez F.I. O078

Pinto B.L. PC151

Pinto J.P. PC056

Pinto R. PC008

Pirouzram A. PR138

Pirovano R. O093, PC014, PC019, PR041

Pislaru A.I. PR346

Placer A. PR160

Plata-Illescas C. PC059, PR080, PR081

Plate J. PR223

Ploeg A. PC100

Plénier I. PR211

Poblete B. PR008

Pochtarnik A.A. PR218

Podda M. PC040, PC053

Polinder S. O065

Polyakova U. PR112 
Pomba L. PC007

Ponchietti L. PR075

Pons P. O042

Pons-Fragero M.J. PR089, PR098

Ponsen K.-J. PR347

Popa E. PR055

Popal Z. PR240

Popescu D. PC192

Popescu G.I. PR441

Popivanov G. O059

Popovtzer R. PR061

Poroh M. PC192

Porras M.Á. PR354

Porras Moreno M.A. PR445

Posso P. PR442

Post J.R. PR203

Postigo S. PC064, PC120

Postigo-Morales S. PR073

Pothmann C.E.M. PR157

Potocnik P. PC189

Pougouras I. PC085, PC152

Poulos I. PR320

Pourbaix E.F. PR211

Poveda I. PC015, PC058, PC128

Prat-Fabregat S. PR160

Prieto Calvo M. PR075

Prionas A. O062, PR146

Prospero F. PC095

Puchwein P. PR429

Puha B. PC161, PC192, PR375

Putineanu D. PR349

Putzeys G. PR186, PR352, PR450

Puyana J.C. O013, PC002, PC042, PR132, PR168, PR181, PR187, PR203, PR292, PR299

Pylarinou P. PR024

Pérez Alcántara A. PC191

Pérez Díaz M.D. O064, PC132, PR049, PR075, PR155, PR269, PR370

Pérez M. PC051

Pérez Martínez J.F. PC118, PC125

Pérez Sánchez E. PR155, PR163

Pérez-Santiago L. PR072, PR323

Quarenghi M. PC107

Queimado H. PC089, PC091

Quesado M. PC180, PC193

Qurashi K. O014

Rabago M.D.L.A. PC036, PR193

Racman M. PC004, PC060

Radu P.A. PR076

Radulescu M. PR079

Raftopoulos A. PC085, PC152

Raghupathi A.K. PR249, PR419

Rai Z. PC124

Rajster Koren A. PC025

Ramallo Solís I. PC084, PR025

Ramires A. PR270

Ramos C. PC069

Ramos L. PC089, PC091

Ramos L.R. PR341, PR353, PR398

Ramírez M.S. PC140, PR026, PR044, PR092

Ramírez-Valencia M. PR376

Randl T. PC004

Ranke H. O055, O058, PR241, PR254

Rao R. PC116

Rasmussen L.S. O033

Ravaii H. O003
Rawand H. PR152

Rebasa Cladera P. O049, PC046, PC061

Redeen S. PR226

Redl H. PR301

Reetz D. O025

Reilly P.M. PR 156

Reininga I.H.F. PR151, PR372

Reinoso J. PR203

Reinsoo A. PR042

Reis H.Ç. O084

Relinque D.S. PC024C, PC024D, PC024E, PC102A, PC172A

Relja B. PR167

Restrepo-Lopera C. PR181

Reva V.A. PC044, PR138, PR210, PR218, PR305

Rey Simó I. PR075

Rey Varcalcel C. O063, O064, O077, PR075

Reška M. PC022, PR295

Rhemrev S. PR358

Ribarov R. O059

Ribeiro C. PC154

Ribeiro H. PC013

Ricci G. PC104, PR014, PR017, PR310

Richards G.R. O085

Richardsen I. PR312

Rico A. PC015, PC058, PC128

Rigueira V. PC009, PC073

Ringrose T. PR196

Ritchie E. PR435

Riu P. PC104

Riveiro M. PR376

Rivera J. PR075

Rizzuto A. PC040, PC053

Roberto P. PC086

Rocha M.C. PR316

Rock N.D. O068

Rodrigo B.B. PR092

Rodrigues A.L.A. PC101

Rodrigues F. PC036, PC089, PC091, PR193

Rodrigues J. PC056

Rodriguez C. PR132, PR181, PR187

Rodriguez F. PR306

Rodriguez J. PR015, PR016, PR019, PR266

Rodriguez J.A.P. PR078

Rodriguez L. PC034

Rodriguez M.C. PR341

Rodriguez R. PC024B, PC048, PC064A

Rodríguez Padilla A. PR075

Rodríguez Perdomo M.D.J. PC150

Rodríguez Vega V. PC168

Rohner M. PR008

Roig Bataller A. PR075

Roine R. PR147

Rojnoveanu G. PR001, PR053, PR068, PR308

Romano A. PR233

Rommens P.M. O022, O085

Ronan R. PR229

Ronchetta C. PR086

Rontogianni M. PC181

Roozendaal N.C. PR275, PR282

Roquette R. PC067

Roseiro T. PR280, PR401, PR439

Rosenthal M.G. O089

Rosete M.F.L.G. PC052

Roshal L. PR121

Rossi M. PR442

Roukema G. PR326 
Rubio López J. PC091A, PC112A, PC112B, PC148A

Rubio-Manzanares-Dorado M. PR034, PR084

Rueda López A.I. PC091A, PC148A

Ruehle A. PR235

Ruffolo C. PR307, PR324

Ruiz C. PR049

Ruiz Marín C.M. PC091A, PC112A, PC112B, PC126, PC148A

Ruiz Marín M. PR075

Ruiz-Tovar J. PR075

Ruiz-Yherla L.O. PC024D, PC024E

Ruíz-Artola V. PR089

Ryan J.M. PC094

Saar S. PR042

Sabinina T. PR109

Sadeghi M. PR138, PR209

Sader R. PR058

Safadjou S. PC110

Saillant N. O089, PR141

Sainz B. PR093

Sainz Cabrejas J. PR165, PR175

Saito J. O002

Saka G. O004, O084, PR410

Sakamoto T. PR195, PR362, PR378

Sakanaka H. PC176

Sakurai A. PR327, PR328, PR331

Sala Hernandez A. PC098

Saladich-Cubero M. PR250

Salamea J.C. PR203

Salas R. PR015, PR016, PR019

Salcedo A. PC024B, PC064A

Salim E. PR325

Saluvere P. PR042

Samokhvalov I.M. PC044, PR218, PR305

Samson D. PR149

Samuels J. O052

Samuels J.M. O030, PR006

Samà L. O017

Sanchez A. PR292, PR299

Sanchez Arteaga A. O064, PC117, PR083

Sanchez C. PC128

Sanchez P. O076, PR012, PR023

Sanchez Ramirez M. PC142, PC158, PC159, PR025, PR027, PR028, PR043, PR051

Sanchez S. O068A

Sancho Muriel J. PR085

Sancho-Insenser J. PR078, PR089, PR095, PR098

Sander A.L. O069

Sangji N.F. O089

Sangthong B. PR188

Santamaría Revuelta C. PC135A

Santos C. PC081, PC086

Santos M.C. PC063

Santos N. PC137

Santos T.C. PC154

Santurro L. PR037, PR094, PR097

Sanz Sanchez M. PR268

Sanz Sánchez M. O077, PC108, PC132, PR155, PR269

Sapage R. PC167, PC171, PR179, PR343

Sara M.J. PR093

Sarani B. O066

Saratzis N. PR045, PR214

Sardo L. PC154

Sarici İ.S. O015

Saridakis V. PC088, PC141

Saris T.T.F. PR313

Sasaki G. PR394, PR405
Sasaki T. PR328

Sasazawa S. PR197

Sato K. PR114

Sato Y. PR433

Satorras A. PR075

Sattout A. PR159

Sauaia A. O050, O070, O075, PR216

Sauter D. PR117, PR361, PR397

Savvidis M. PC160

Sawaguchi T. O022

Sawamura S. PR327

Sawano M. PR276

Sawauchi K. PR120, PR382, PR396, PR432

Sazhin A. PR038

Sağlam N. O084

Scalea T.M. PR003, PR314

Scanting D. O021, O072

Schaaf S. O095

Schafroth B. PR008

Schakenraad D. PR412

Schecter W. PR142

Schep N.W.L. O027, O080, PR369

Schipper I. PR358

Schmelzer M.K. PR157

Schmitt J.W. PR420

Schnüriger B. O032, PR020, PR030

Schober P. PR240

Scholler D. O042

Schramm D. PR430

Schreuder M. PR170

Schuit S. PR228

Schulz-Drost S. O023, O055, O056, O058, PR198, PR224, PR241, PR254, PR259, PR261, PR263, PR264, PR291, PR293, PR296,

PR359, PR421

Schulze R. O068A

Schwab R. O095, PR312

Schwarz A. PR415, PR418

Schwarz U. PR418

Schwitter L. O081

Schäfer F.-P. PR417

Schüler M. PR117, PR397

Scott S. PR159

Seamon M. PR303

Sebastian M. PC003

Segalini E. PC027, PC040, PC053

Segers M. PC188, PR339

Segol G. PR248

Segura Sampedro J.J. PR171

Segura-Sampedro J.J. PR060, PR091, PR317

Seifert V. PR009

Seinen S. PR277

Seisdedos L. PR021

Sekayan T. PR123

Sekiya K. PR071

Selles C.A. O080

Selvakumar D. PC124

Semenov E.A. PR210, PR305

Semenova J.B. PR104

Semenova N. O035, O037, PR102, PR105, PR111, PR116, PR281

Senent-Boza A. PR084

Sengab A. PC169

Serao A. PC104, PR014, PR310

Sergeeva V. O037

Serna J.J. PC024B, PC048, PC064A

Serova N. PR125, PR135

Serra M.L. PC154 
Serrano Serrano R. PC164

Sethu C. O053

Seufert L. PR430

Sevilla López S. PC024, PR285

Sewalt C. PR183, PR228, PR369

Shahzad K. PR137, PR182

Shalak H.S. PC072

Shandru S. PR001

Sharpe J. PR303

Shepetko-Dombrovskyi G. PR067

Shepetko-DombrovskyiO. PR067

Shevelev P.Y. PR210

Shibly M. PR385

Shim H. PR381

Shimamura Y. PR169, PR380, PR390

Shimazu T. PR130, PR153

Shinyama N. PR221

Shiraishi A. O019, O044

Shoda E. PR328, PR447

ShonO.-J. PR348, PR363

Shouzou K. PR428

Shulutko A. O091, O097

Siboni S. O024, O032

Sidorov S. PR135

Siebenrock K. O009

Sillesen M. O033, O048

Silliman C.C. O030, O052, O070, PR006, PR216

Silva F. PR280

Silva M. PC180, PC193

Silva S.M. PC038, PC095, PR047

Simao R. PC153

Simmen H.P. PR157, PR267

Simoes J. PC021

Simon M.A. PR217

Simões A. PC056

Simões N.D.F.L. PR425

Sintonen H. PR147

Sjodahl R. PR226

Sjolin G. O066

Skazas G. PC006

Skliris I. PC115, PC123, PR320

Skoog P. PR138

Slawik S. PR274A

Slezak P. PR301

Slovenc S. PR247

Sluga B. PR411

Slump C.H. PR406

Smeeing D.P.J. PR313, PR339

Smith S.R. PC136

Smith-Williams J. PR309

Socrate A.M. PC057

Sojka K. O079

Sola M. PC127

Soldevila Verdeguer C. PR317

Sologub E. PR096

Soltermann A. PR417

Solà A.M. PR095

Somaini G. PC166

Sommer C. PC166, PR334, PR395, PR407, PR416

Sommer K. O069

Sone T. PR195, PR362, PR378, PR428

Song Y.S. PR348

Sosa Quesada Y. PR075

Sosef N. PR170

Soto C. PC054, PC145

Soto Montesinos C. PC127
Sousa M. PC137

Sousa R. PC167, PC171, PR179, PR343

Spanjol Pandelo I. PR226

Spassoff V.R. PC187

Spazierer D. PR301

Spiegl U. O026, PR335

Spijkerman R. O040, PR129

Spiteri N. O067

Sprengel K. O001, PR157, PR267

Sram J. O082

Staresinic M. O028

Stavrides K. PR045, PR214

Stefaniu R. PR346

Stein D.M. PR003

Steinmetz J. O033

Stettler G.R. O030, O052, O070

Stevenson R. PR154

Stickel M. PR235

Stillhard P. PC166

Stockinger M. PR329

Stoica B. PR022

Stojiljkovic P. O005

Stollenwerck G. PC024A, PC135

Stradymov E. PR038

Strambu V. PR076

Streltov L. PR053

Stuby F. PR335A, PR426A

Stumpp P. PR335

Stylianou A. PC011, PC085

Störmann P. PR009

Subashi K. PC070, PC074

Subirana L. PR232

Sugimoto M. PR206, PR304

Sugrue M. O096, PR244

Sumislawski J.J. O070

Supelano Eslait G. PC026, PC028, PC029, PC030, PC033, PC092,

PR035, PR046, PR184

Surlin V. PR079

Sutalo S. PR301

Suzuki M. PR071

Suzuki T. O019, O044, PR231

Suárez Cabrera A. PC077, PC140, PC158, PR044

Suárez-Artacho G. PR054

Svenningsen P. O048

Szabo S. PR287

Szuba A. PC032, PC097, PC118, PC125, PC126, PC149

Sádt Z. PR402

Sánchez Casado M. O078

Sánchez Manuel F.J. PR075

Sánchez Morata E. PR398

Sánchez-Arteaga A. O011, PC084, PC132, PR025, PR034, PR054, PR065, PR074, PR082, PR084, PR158, PR163

Sánchez-Camacho González-Carrato M.D.P. PC119

Sánchez-Guillén L. PR085

Söderlund T. PR147, PR177

Søreide J.A. O016

Søreide K. O016

Süveges G. PR245

Tabakov M. O059

Tackner E. PR429

Tadros G. PC031

TagadiucO. PR308

Takaki M. PR391, PR394

Takayama K. PC176

Takayama W. O047

Takenaka N. PR391 
Takiguchi N. PR185

Tallón-Aguilar L. O011, PC084, PC096, PC117, PC140, PC142, PC146, PC158, PR025, PR027, PR028, PR034, PR044, PR051, PR054, PR064, PR065, PR074, PR082, PR083, PR084, PR092, PR100, PR158, PR322, PR434

Talving P. PR042

Tamayo López M.J. PR064, PR434

Tamayo-Lopez M.J. O011, PC084, PC117, PR025, PR034, PR054, PR074, PR082, PR083, PR084, PR158

Tamayo-López M.J. PR065

Tamini N. PR037, PR094

Tamverkeras P. PR274A

Tan E. PR204, PR242

Tanase I. PR022

Tangl S. O081

Tano A. PR431

Tasev B. PC187

Tasi T. PR402

Taub E. PR303

Taximi S. PR029, PR300

Taylor J.V. O019A, O067, PR099, PR137, PR166, PR182, PR274A, PR309

Tecu C. PR414

Tefft A. O074

Teichman A. O021

Teixeira J. PC001

Telickiy S.Y. PR210, PR305

Telletxea S. PC064

Ten Berg M. O038

Ten Duis H.J. PR409

Ten Duis K. PR372, PR406

Tengberg L.T. O036

Tenreiro N. PC038, PC095, PR047

Teodonno F. PC164

Ter Bogt N.C.W. O065

Terada C. O086

Teramoto T. PR391

Termaat F. PR358

Terra M. PR240

Tesoriero R.B. PR003

Tessari E. PC007

Teuben M.P.J. O020, PR129, PR201

Thannheimer A. O010

The Traumaregister Dgu A. PR167

Theofanidou S. PR024, PR045, PR214

Thiesen D.M. O007, PR367

This A. O009

Thomassen I. PR170

Thongkhao K. PR188

Thorsen K. O016

Thorwart K. PR058

Tibbits E. PR217

Tigkiropoulos K. PR045, PR214

Tilley E. PR149

Timmermans M. PR386

Timmers M.S. PR417

Timofeeva A.V. PR039, PR103, PR104, PR119, PR133, PR298

Tinoco-González J. O011, PC084, PC096, PC117, PR025, PR034, PR054, PR065, PR074, PR082, PR083, PR084, PR100, PR158, PR322

Tintari S. PR308

Tiziani S. PR420

Toivola A. PR138

Tojal A. PC056

Tollens T. O083

Tomazevic M. PR332
Tominc U. PC025

Tomita K. PR220

Tomita M. PC176

Tomonori S. PR428

Tonini V. PR233

Toor A. O042, PR164

Toor E.J. PR144

Tooulias A. O062, PR146

Topaz G. PR061

Topaz M. PR061

Toral Guinea P. PC066

Torba M. PC070, PC074

Toro A. PR365

Torrent-Jansà L. PR095, PR098

Torres T. PC059

Trach S. O055, PR241, PR254, PR359, PR421

Traumcat Working Group T. PR160

Tremps C. PR 150

Tribak K. PR349

Triguero J. PC059

Trillo Parejo P. PR075

Trinidad Borrás A. PC066, PC119

Trochez J.P. O013, PC042

Troian M. PC138

Tropeano G. PR319

Trotovšek B. PC060

Tryzna M. PR422

Tsekoura M. PR134

Tsepis E. PR134

Tsiampas D. PC181

Tsimpoukis A. PC181

Tsolakidis A. O062

Tsoulfas G. O062, PR045, PR146

Tsubouchi N. PC130

Tsuchida Y. PR388, PR404

Tsuchiya M. PC200

Tsuji H. O002

Tsumura H. PR195, PR362, PR378

Tsurukiri J. PC130

Tuboly E.S. PR193A

Tugnoli G. PC027, PC040, PC053

Tuncer A. PR033

Tur J. O034

Turégano Fuentes F. O063, O064, O077, PC108, PC132, PR021, PR049, PR075, PR155, PR163, PR269, PR370

Tyler S.L. PR251

Tzima A. PC181

Tzurbakis M. PR400

Türkmen İ. O084

Ublinskiy M. O035, O037, PR102, PR105, PR108, PR112, PR116, PR126, PR281

Ublinsky M. PR111

Uchino M. PR427

Uchino T. PR169, PR390

Ueda K. PR059, PR185, PR272

Ueda Y. O002, PR114

Ugarte B. PR075

Ugarte-Sierra B. PC064, PC120, PR073

Uggowitzer P. O081

Uityama E.M. PR315

Um J.Y. PR154

Umakoshi K. PR220

Umemoto M. PR185

Unterkofler J. O055, O058, PR241, PR254, PR359, PR421

Urushibata N. PC047, PR206, PR304

Ushakov M. PR125 
Ussia A. PR233

Utiyama E.M. PR316

Utkina K. PR039, PR119, PR133

Uyttebroek S. PR352

Vacas E. PC194, PR131, PR173, PR356, PR365, PR398

Vaccari S. PR233

Vadillo Cardona P. PC164

Vaisnora L. PR020

Val Flores L. PC101

Vale C. PR280, PR311, PR401, PR439

Valera Sánchez Z. PC077

Valle Beltran A. PR150, PR232

Vallverdú-Cartie H. PR250

Valverde Navarro A. PR085

Valério F. PC099

Van De Veyver S. PR349

Van Delft E.A.K. PR107, PR170

Van Delft-Schreurs K. O060

Van Den Brand J.G.H. PR412

Van Den Bremer J. PC135

Van Der Bij G.J. PR107

Van Der Linde R.A. O088

Van Der Meij J.E. PR144, PR212

Van Der Ploeg T. PR412

Van Der Sluijs R. PR222

Van Der Sluis C.K. PR409

Van Der Velde D. PR340, PR399

Van Der Vliet Q.M.J. PR338, PR351

Van Der Werve M. PR435

Van Der Wilden G. PC100

Van Der Zwaal P. PR339

Van Dissel J. PR417

Van Dongen T.T.C.F. O046, O051, PC178, PR213

Van Doremalen R.F.M. O088

Van Gent T. O006

Van Gool M.H. PR275, PR282

Van Heijl M. PR176, PR222, PR340, PR399

Van Helden S.H. O088

Van Leerdam R.H. PR358

Van Loon B. O042

Van Rein E.A.J. PR176, PR222

Van Roozendaal L.M. PR275, PR282

Van Solinge W. O038

Van Vugt R. PR275

Van Wessem K. O038

Van Wessem K.J.P. PR313

Van Wijck S. O089

Vanakesa T. PR042

Vancleef S. O083

Vander Sloten J. O083

Vanni E. PR243

Varga E. PR193A, PR245, PR256, PR402

Vargas Muñoz A.M. PR190

Vasilakopoulou V. PC115, PC123, PR320

VasiukovaO. O037

Vathia T. PC011, PC085

Vaz J. PC001

Vaz M.R. PR425

Veen H. PR242

Velasquez M. PC002

Veliceasa B. PC161, PC192, PR375

Velmahos G. O089

Velmahos G.C. PR141

Venema E. PR183, PR228, PR369

Ventura N. PC063

Verboket R.D. PR058
Verhaar J. PR369

Verhofstad M. O060

Verleisdonk E.-J. PR339, PR340, PR399

Vermeulen J. PR107

Ververeli C. PR024

Veselko M. PR411

Veverková L. PC022, PR295

Vicente Goma-Camps M. PR376

Vico-Arias A. PR080, PR081

Vicol D. PR265

Vidal M. PR299

Vidali M. PC011

Vidovic D. O028

Vieira A. PC154

Vieira E. PC067

Vieira V. PC013

Viejo Martinez E. PR268

Viggiani L. PC103, PR036

Vilaça L. PR311

Vilá Y Rico J. PR341, PR356, PR398

Vissers Y.L.J. PR275, PR282

Viña R. PC194, PR131

Vojtova L. O079

Von Rüden C. O010

Von Schreeb J. PR152

Von Websky M. O095

Vozian M. PR068

Vranckx J. PC173

Vági Z. PR402

Waaijer L. PR436

Wadsworth P. PR274A

Wagner D. O022, O085, 0087

Wagner N. PR167

Wahlen B.M. PR005, PR279, PR283

Waki T. PR120, PR373

Waksman I. PR070

Walenkamp M.M.J. O027

Wang H.-L.C. PC049

Wang S.Y. O090, PR366

Wannatoop T. PR138

Ward R.G. PR137, PR182

Waseem S. O053

Watanabe S. PR382, PR396, PR432

Watanabe Y. PR330, PR394, PR405

Weber C.D. PR115

Weber T. PR277

Weigeldt M. O055, O058, PR254, PR261

Weiglein A. PR418

Wendorf R. PR224, PR259, PR263

Wendt K.W. PR151, PR172, PR372, PR406

Wenzel L. O010

Wiebe D.J. PR156

Wiegers E. PR183, PR228, PR369

Wijaya R. PR040

Williams T.K. PR217

Williamson J. O072

Willms A.G. O095, PR312

Wing H. O068A

Witjes M.J.H. PR406

Wittich P. PC196, PC198, PR340, PR436

Wladis A. PR242

Woltmann A. O010

Wong C.Y. PC135, PC169

Wong K.Y. O053

$\mathrm{Wu}$ Y. PC065

Wutzler S. PR167 
Yablokov I.P. PR210

Yamada H. PR185

Yamaguchi S. PR303

Yamaji F. PC201

Yamakawa Y. PR169, PR380, PR384, PR390

Yamanaka H. PC130

Yanaga K. PR231

Yano T. PR120, PR373

Yasui Y. PR394, PR405

Yeh D. PR141

Yokomori R. PC130

Yokoo S. PR169, PR342, PR355, PR380, PR384, PR390

Yoshida M. PR387, PR433

Yoshida N. PR388, PR404

Yoshida S. PC201

Yoshimura M. PR169, PR380, PR384, PR390

Yoshioka Y. PR271, PR273, PR297

Yoshiyuki A. PC047, PR206, PR304

Younis B. O098

Yuste García P. PC026, PC028, PC029, PC030, PC033, PC092,

PR035, PR046, PR173, PR184

Zabkowski T. PR258

Zacharopoulos N. PR045, PR214

Zago M. O093, PC014, PC019, PC138, PR017, PR041

Zaitseva N. O037

Zannoni R. PC027, PC040, PC053
ZarainObrador L. PR163

Zarokostas T. PC115, PC123, PR320

Zboncak M. O079

Zdolsek J. PC016

Zeidler S. PR426A

Zeina A.-R. PR069

Zelle B.A. PR393

Zhao H. PR303

Zheleznyak I.S. PR210, PR218

Zheng D.J. PR128

Zhestkov K. O029

Zhirnova N.A. PR210

Ziev_Ner I. PR061

Zilkens E. O001

Zilony N. PR061

Zimmermann W.O. PR435

Zlatev B. O059

Zlobec I. PR020

Zogakis P. PR400

Zubarev A. PR252

Zubritskij V. PR004

Zugna D. O017

Zulueta J. O063

Zurmühle P. PC189

Zurzu M. PR076 